\title{
The Life-Cycle Assessment of a Single- Storey Retail Building in Canada
}

\author{
by \\ Kevin Van Ooteghem \\ A thesis \\ presented to the University of Waterloo \\ in fulfillment of the \\ thesis requirement for the degree of \\ Master of Applied Science \\ in \\ Civil Engineering
}

Waterloo, Ontario, Canada, 2010

CKevin Van Ooteghem 2010 


\section{AUTHOR'S DECLARATION}

I hereby declare that I am the sole author of this thesis. This is a true copy of the thesis, including any required final revisions, as accepted by my examiners.

I understand that my thesis may be made electronically available to the public. 


\begin{abstract}
In North America, the operation of buildings accounts for approximately one third of the total energy use and greenhouse gas emissions annually. Office buildings are responsible for roughly $35 \%$ of the total commercial/institutional secondary energy use in Canada, followed by retail buildings at $17 \%$ (NRCan, OEE, 2010).

In recent years, a number of researchers from around the world have conducted life-cycle assessment (LCA) studies to investigate the impacts of buildings on the environment. Most studies have focused on three types of buildings: office buildings, single residential dwellings, and multi-unit residential apartments. There have been almost no comprehensive LCA studies of retail buildings, specifically single-storey retail buildings. This is a problem, since compared to office buildings, single residential dwellings, and multi-unit residential apartments, retail buildings consume approximately 1.2, 2.0, and 2.3 times more energy per floor area respectively (NRCan, OEE, 2010). In addition, retail buildings usually undergo major resource intensive renovations far sooner than other building types. Therefore, the primary goal of this study was to conduct a comprehensive LCA for the components of a singlestorey retail building located in Toronto, Canada, to determine which building components contribute the most towards the total life-cycle energy use and global warming potential (GWP) after 50 years.

Using the latest LCA techniques, the total life-cycle energy use and GWP was calculated for 220 different building components including: exterior infill walls, roofs, structural systems, floors, windows, doors, foundations, and interior partition walls. Also, a comprehensive LCA study was conducted for five single-storey retail buildings (including a pre-engineered steel building system which is lacking in the literature), in order to determine which components of a single-storey retail building are responsible for the most environmental damage.

For a typical single-storey retail building located in Toronto, Canada, the operating energy (and GWP) accounts for about $91 \%$ (88\%) and the total embodied energy (and GWP) accounts for about $9 \%(12 \%)$ of the total energy (and GWP) after 50 years. The roof alone is responsible for nearly half of the total embodied energy and GWP of the entire building. The LCA study also found that after 50 years, the total energy (and GWP) of the five case study buildings only differed at most by $6 \%$ (7\%), regardless of the choice of structural system, or whether the building was made predominately of steel or wood building components. This thesis concludes with a prioritized list of recommendations for reducing the total life-cycle energy use and GWP of a single-storey retail building in Canada.
\end{abstract}




\section{Acknowledgements}

First and foremost I would like to thank my supervisor Dr. Lei Xu for his guidance and enthusiastic support of this project from the beginning. His insight and expertise in cold-formed steel design was sincerely appreciated and his support during the completion of this thesis was invaluable. Throughout this process he has always encouraged me to pursue my interests on this study.

I would also like to express my sincere gratitude to Dr. John Straube. Dr. Straube has continued to be a source of encouragement and his enthusiasm for this work has been contagious. Our many conversations over the last two years have proven to be a great source of inspiration for this work. His expertise in building science has been invaluable.

Dr. Bryan Tolson also deserves credit for his work reviewing this thesis. I would like to thank him for his efforts in this regard.

Also, I would also like to thank the Canadian Sheet Steel Building Institute (CSSBI) and its members. Without their support, this project would not have been completed. From the very beginning, they have been the largest supporter of this research. In particular, I would like to especially thank Dr. Steven Fox for his help and expertise along the way. Dr. Fox has provided me ample opportunity to grow on a professional level and for that I sincerely thank him.

Over the last two years I have had the opportunity to collaborate with Associated Professor Terri Meyer Boake and her graduate student Chris Black, from the School of Architecture at the University of Waterloo. Terri has been a tremendous help and her knowledge and expertise in the area of sustainable building was much appreciated. Chris was responsible for creating the renderings of the five case study retail buildings, as well as the architectural plans referenced in this thesis. I would like to thank him for this and acknowledge his assistance.

Finally, most of all I would like to thank my family and friends for their unwavering support and encouragement throughout my many years of school. Without their support, none of this would be possible. 


\section{Table of Contents}

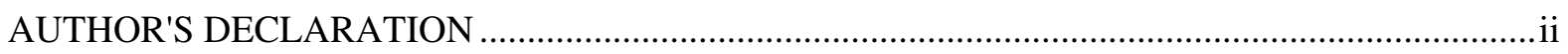

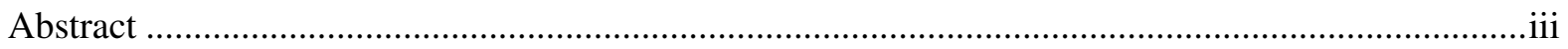

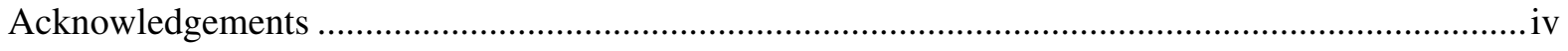

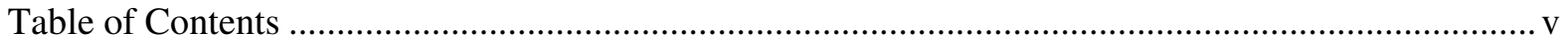

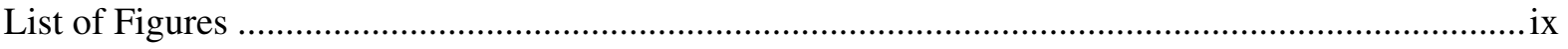

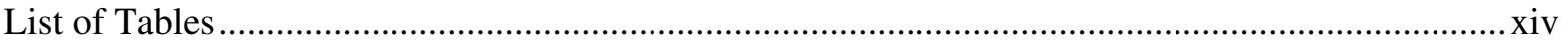

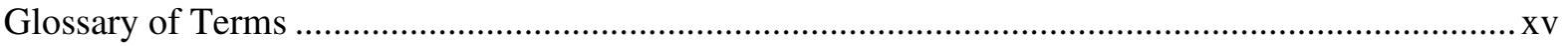

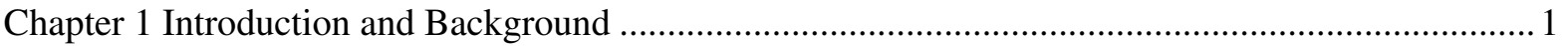

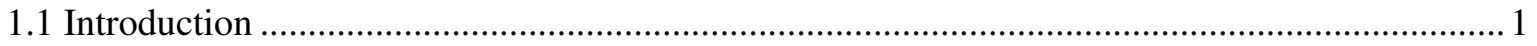

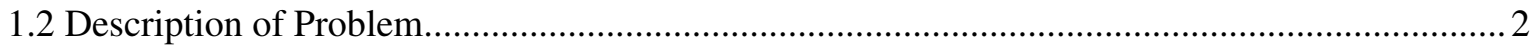

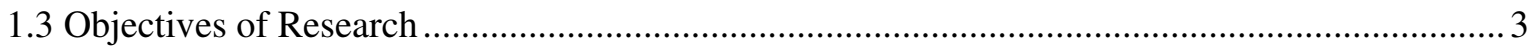

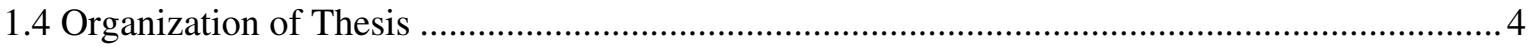

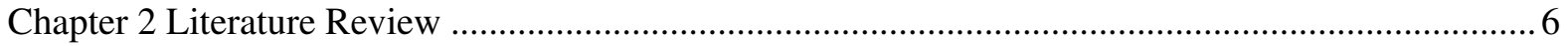

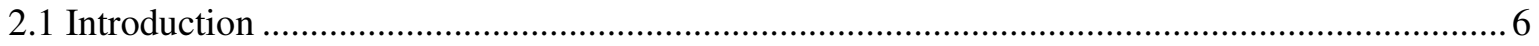

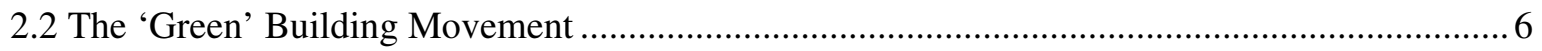

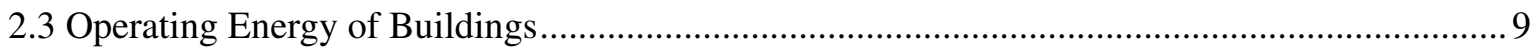

2.3.1 Background on Operating Energy Statistics for Buildings.................................................. 9

2.3.2 Operating Energy Statistics for Canadian Commercial Buildings ..................................... 10

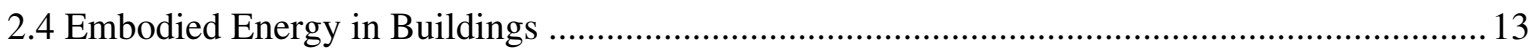

2.4.1 Background on Embodied Energy in Buildings ............................................................. 13

2.4.2 Embodied Energy of Common Building Materials .......................................................... 15

2.4.3 Problems with Measures of Embodied Energy …............................................................. 17

2.5 Operating Global Warming Potential (GWP) of Buildings................................................... 17

2.5.1 Background on Operating GWP Statistics for Buildings ............................................... 18

2.5.2 Operating GWP Statistics for Canadian Commercial Buildings ....................................... 18

2.6 Embodied Global Warming Potential (GWP) in Buildings ................................................... 21

2.6.1 Background on Embodied GWP in Buildings.............................................................. 21

2.6.2 Embodied GWP of Common Building Materials............................................................. 22

2.6.3 Problems with Measures of Embodied GWP ................................................................. 24

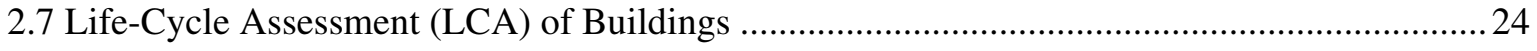

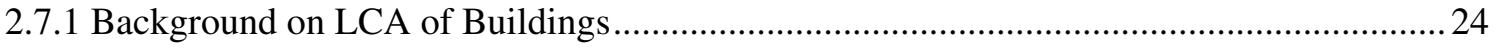




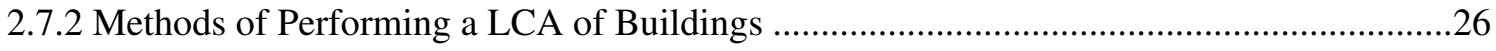

2.7.3 Previous LCA Studies of Commercial Buildings .............................................................28

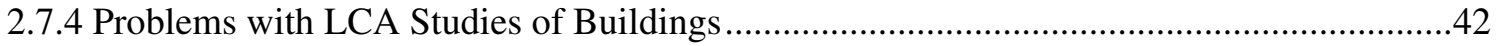

Chapter 3 Methodology: Life-Cycle Assessment of Building Components ......................................45

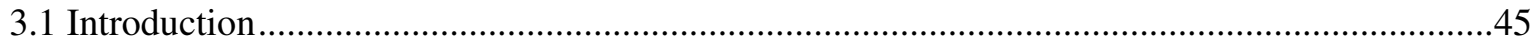

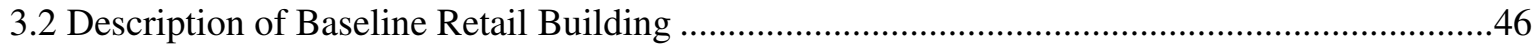

3.3 Identifying Building Components for the LCA ….....................................................................

3.3.1 Description of Exterior Infill Wall Enclosures ..............................................................50

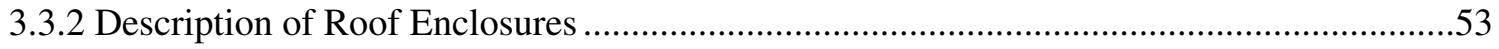

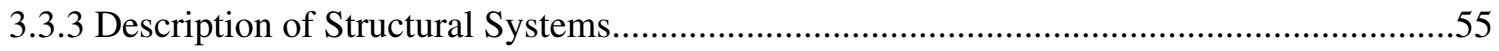

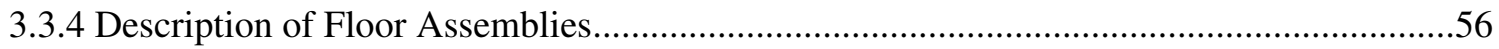

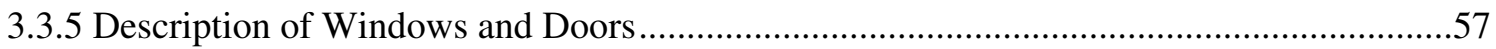

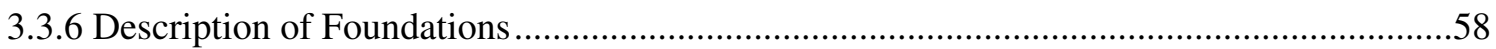

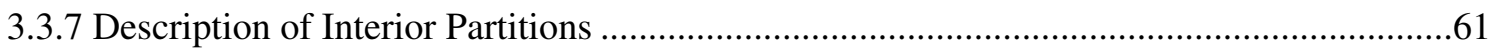

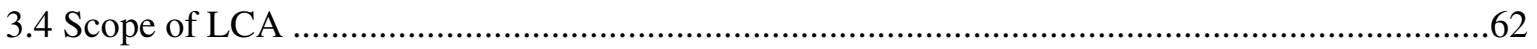

3.5 Evaluating Embodied Energy and Embodied GWP ...............................................................64

3.6 Evaluating Operating Energy and Operating GWP ............................................................67

3.7 Calculating the Thermal Resistance of Building Enclosures ..................................................69

3.7.1 Background on Heat Transfer in Buildings ....................................................................69

3.7.2 Calculating One-Dimensional Heat Flow Using the Tabular Method ................................70

3.7.3 Calculating Two-Dimensional Heat Flow Using THERM ..............................................72

3.7.4 Calculating Two-Dimensional Heat Flow Using ASHRAE Standard 90.1-2007 ..............74

3.7.5 Explanation of Differences in Thermal Resistance between THERM and ASHRAE

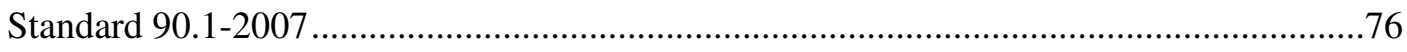

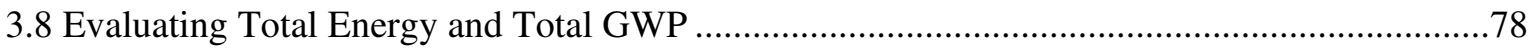

Chapter 4 Methodology: Life-Cycle Assessment of Whole Buildings..............................................80

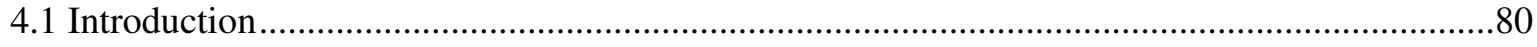

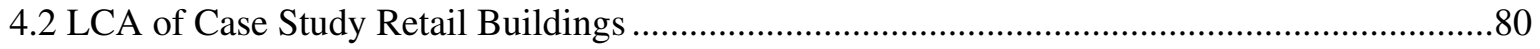

4.2.1 Description of Typical Hot-Rolled Steel Structure Retail Building (Case Study \#1)..........81

4.2.2 Description of Typical Heavy Timber Structure Retail Building (Case Study \#2) .............83

4.2.3 Description of Typical Pre-Engineered Steel Retail Building (Case Study \#3) ..................85

4.2.4 Description of Predominately Steel Retail Building (Case Study \#4) ................................87 
4.2.5 Description of Predominately Timber Retail Building (Case Study \#5) ............................. 88

Chapter 5 Results: Life-Cycle Assessment of Whole Buildings ....................................................... 91

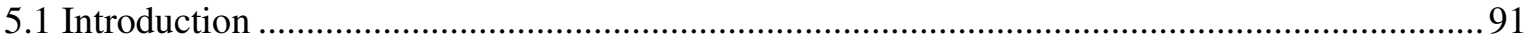

5.2 LCA Results for the Baseline Retail Building (Case Study \#1) ...............................................91

5.2.1 Operating Energy and GWP of the Baseline Retail Building (Case Study \#1) ..................92

5.2.2 Embodied Energy and GWP of the Baseline Retail Building (Case Study \#1) .................95

5.2.3 Total Energy and GWP of the Baseline Retail Building (Case Study \#1) ..........................98

5.2.4 Comparison of the Baseline Retail Building (Case Study \#1) to Average Canadian Retail

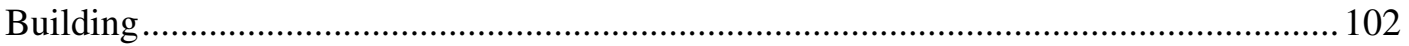

5.3 LCA Results for Case Study Retail Buildings.................................................................... 103

5.3.1 Operating Energy and GWP of Case Study Retail Buildings........................................... 104

5.3.2 Embodied Energy and GWP of Case Study Retail Buildings ........................................ 105

5.3.3 Total Energy and GWP of Case Study Retail Buildings .................................................. 109

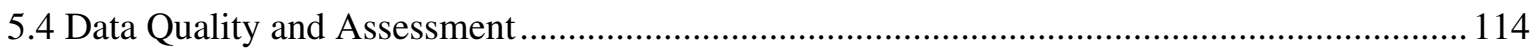

Chapter 6 Results: Life-Cycle Assessment of Building Components ............................................. 115

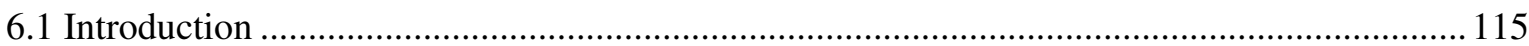

6.2 Interpreting the LCA Graphs of Building Components .................................................. 115

6.3 LCA Results for Exterior Infill Wall Enclosures ................................................................ 116

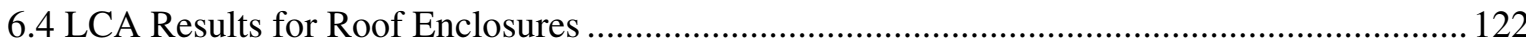

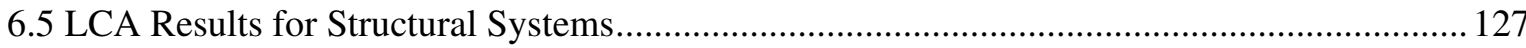

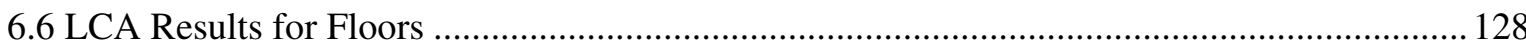

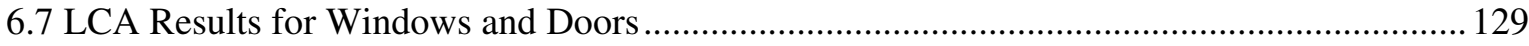

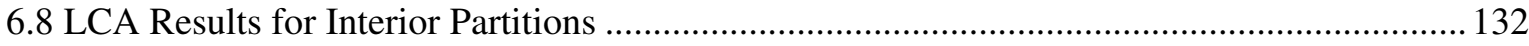

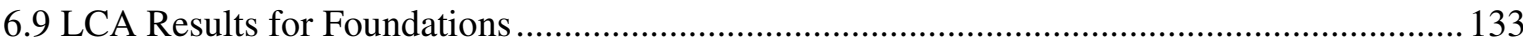

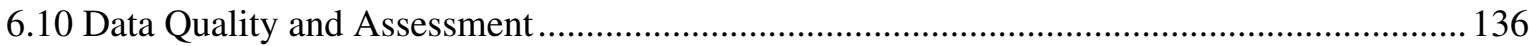

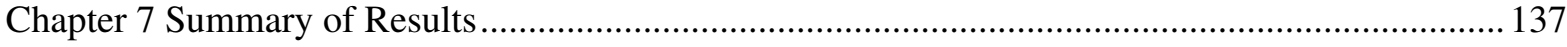

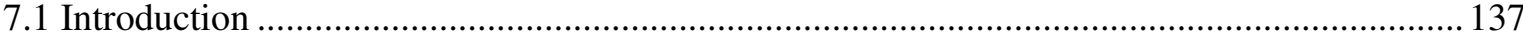

7.2 Sensitivity Analysis of Total Embodied Effects verses Operating Effects .............................. 137

7.3 Magnitudes of Order in the LCA of Buildings ................................................................... 143

7.4 A Summary of Design Strategies to Reduce the Total Life-Cycle Energy Use and GWP of

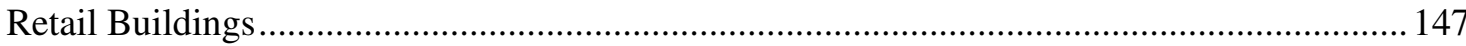

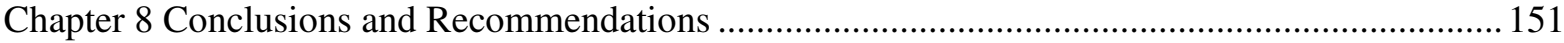

vii 


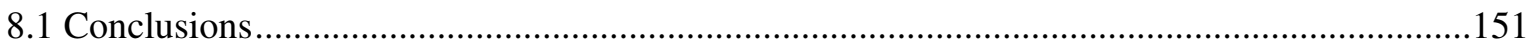

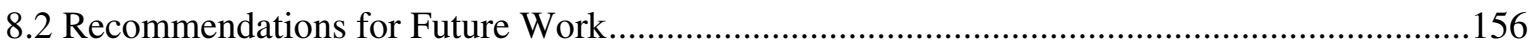

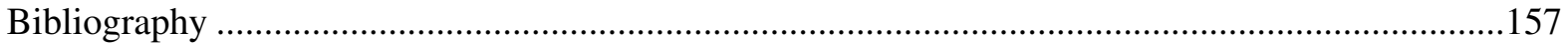

Appendix A Description of Baseline Retail Building (Case Study \#1) ...........................................161

Appendix B Life-Cycle Assessment Data for Building Components..............................................174

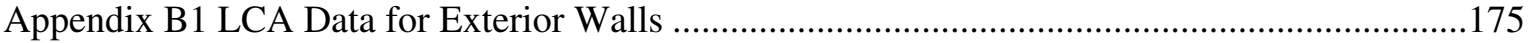

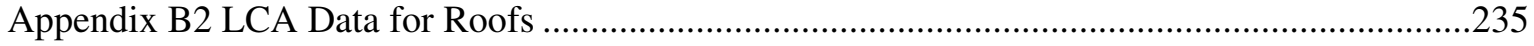

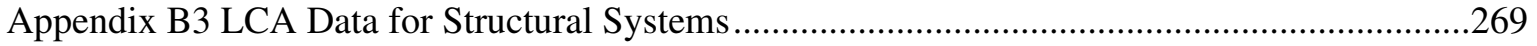

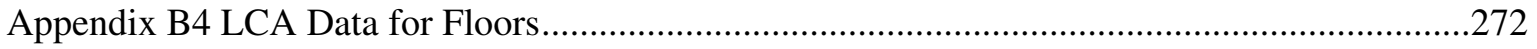

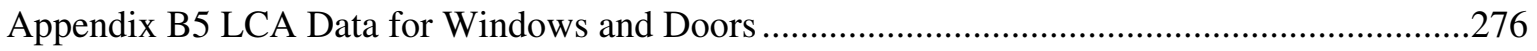

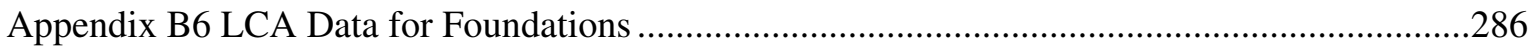

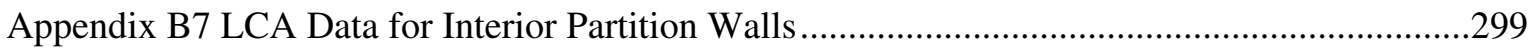

Appendix C Life-Cycle Assessment Results for Case Study Buildings ............................................305 


\section{List of Figures}

Figure 2-1: Canada's Greenhouse Gas Emissions from 1990-2007 ................................................... 7

Figure 2-2: The Transformation from Primary to Secondary to Tertiary Energy ............................... 10

Figure 2-3: Breakdown of Total Secondary Energy Use by Sector in Canada in 2007 ...................... 11

Figure 2-4: Breakdown of Total Commercial/Institutional Secondary Energy Use by Activity

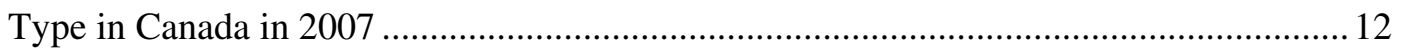

Figure 2-5: Breakdown of Retail Secondary Energy Use by End Use in Canada in 2007.................. 12

Figure 2-6: Commercial/Institutional Sector Average Annual Operating Energy Intensity by

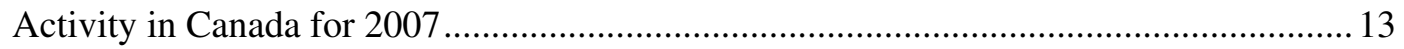

Figure 2-7: Breakdown of Total Secondary GWP by Sector in Canada in 2007 ............................... 19

Figure 2-8: Breakdown of Commercial/Institutional Total Secondary GWP by Activity Type in

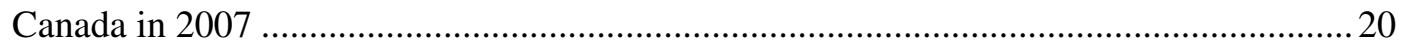

Figure 2-9: Breakdown of Retail Secondary GWP by End Use in Canada in 2007.......................... 20

Figure 2-10: Commercial/Institutional Sector Average Annual Operating GWP Intensity by

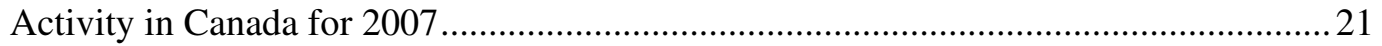

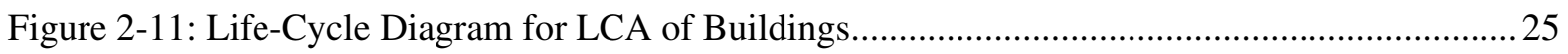

Figure 2-12: Typical Life-Cycle Energy Use of a Building ...........................................................28

Figure 2-13: Total Energy for a Range of Residential and Non-Residential Buildings from a Literature Review of LCA Studies. 30

Figure 2-14: Breakdown of the Initial Embodied Energy for Steel Structural Office Building Located in Toronto

Figure 2-15: Breakdown of Initial Embodied Energy for Wood, Steel, and Concrete Structure Office Buildings Located in Toronto. 32

Figure 2-16: Initial Embodied Energy for Wood, Steel, and Concrete Structural Systems for Office Building Located in Toronto. .33

Figure 2-17: Breakdown of Initial Embodied Energy verses Recurring Embodied Energy for Wood Structure Office Building after 50 Year Lifespan in Toronto 34

Figure 2-18: Breakdown of Total Embodied Energy for Wood Structure Office Building after 50 Year Lifespan in Toronto .34

Figure 2-19: Life-Cycle Energy Use for Wood, Steel, and Concrete Structure Office Buildings after 50 Year Lifespan in Toronto. .35 
Figure 2-20: Life-Cycle Energy Use (Averaged for Wood, Steel, and Concrete Structure Office

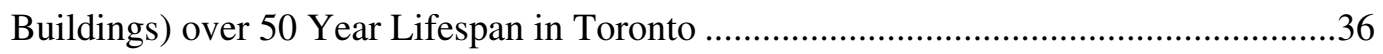

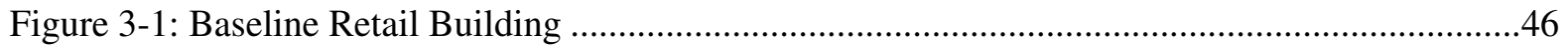

Figure 3-2: Typical Assembly Layers for Exterior Infill Wall Enclosures..........................................51

Figure 3-3: Typical Assembly Layers for Roof Enclosures................................................................53

Figure 3-4: Typical Assembly Layers for Floor Systems ............................................................57

Figure 3-5: Typical Components of Windows and Doors .................................................................57

Figure 3-6: Typical Components of Isolated Footings with Piers …................................................59

Figure 3-7: Typical Components of Perimeter Footings and Foundation Walls .................................59

Figure 3-8: Typical Components of Slab-On-Grade ............................................................................6

Figure 3-9: Typical Assembly Layers for Interior Partition Walls ...................................................61

Figure 3-10: Life-Cycle Assessment System Boundaries and Outputs ..............................................63

Figure 3-11: Assembly Layers for Concrete Masonry Unit Wall \#1 (CMU-W1) ................................71

Figure 3-12: Assembly Layers for Cold-Formed Steel Stud Wall \#1 (SS-W1) ....................................73

Figure 3-13: Example of Two-Dimensional Heat Flow through SS-W1 Using THERM ...................73

Figure 4-1: Case Study \#1 - Typical Hot-Rolled Steel Structure Single-Storey Retail Building.........82

Figure 4-2: Case Study \#2 - Typical Heavy Timber Structure Single-Storey Retail Building ............84

Figure 4-3: Case Study \#3 - Typical Pre-Engineered Steel Single-Storey Retail Building .................86

Figure 4-4: Case Study \#4 - Predominately Steel Single-Storey Retail Building ..............................87

Figure 4-5: Case Study \#5 - Predominately Timber Single-Storey Retail Building ...........................89

Figure 5-1: Rendering and eQUEST Model of Baseline Retail Building (Case Study \#1) .................92

Figure 5-2: Breakdown of Annual Energy Consumption for the Baseline Retail Building

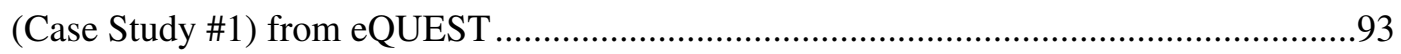

Figure 5-3: Monthly Energy Consumption for the Baseline Retail Building from eQUEST ...............95

Figure 5-4: Total Life-Cycle Embodied Energy of the Baseline Retail Building (Case Study \#1)

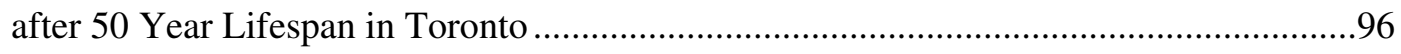

Figure 5-5: Total Life-Cycle Embodied GWP of the Baseline Retail Building (Case Study \#1) after 50 Year Lifespan in Toronto . .98

Figure 5-6: Total Life-Cycle Energy Breakdown of the Baseline Retail Building (Case Study \#1) after 50 Year Lifespan in Toronto . .99

Figure 5-7: Total Life-Cycle GWP Breakdown of the Baseline Retail Building (Case Study \#1) after 50 Year Lifespan in Toronto 100 
Figure 5-8: A Scaled Diagram of Total Life-Cycle Energy Use and GWP for a Typical Retail

Building after a 50 Year Lifespan in Toronto 102

Figure 5-9: Total Life-Cycle Embodied Energy of the Case Study Buildings after 50 Year Lifespan in Toronto 107

Figure 5-10: Total Life-Cycle Embodied GWP of the Case Study Buildings after 50 Year Lifespan in Toronto 108

Figure 5-11: Total Life-Cycle Energy Consumption and GWP of the Case Study Buildings after 50 Year Lifespan in Toronto.

Figure 5-12: Total Life-Cycle Energy Breakdown of the Typical Heavy Timber Structure Retail Building (Case Study \#2) after 50 Year Lifespan in Toronto

Figure 5-13: Total Life-Cycle Energy Breakdown of the Typical Pre-Engineered Steel Retail Building (Case Study \#3) after 50 Year Lifespan in Toronto

Figure 5-14: Total Life-Cycle GWP Breakdown of the Typical Heavy Timber Structure Retail Building (Case Study \#2) after 50 Year Lifespan in Toronto

Figure 5-15: Total Life-Cycle GWP Breakdown of the Typical Pre-Engineered Steel Retail Building (Case Study \#3) after 50 Year Lifespan in Toronto

Figure 6-1: Total Life-Cycle Energy and GWP of Concrete Masonry Unit Exterior Infill Walls (CMU-W) after 50 Year Lifespan in Toronto.

Figure 6-2: Total Life-Cycle Energy and GWP of Concrete Tilt-Up Exterior Infill Walls (CTU-W) after 50 Year Lifespan in Toronto......

Figure 6-3: Total Life-Cycle Energy and GWP of Structural Insulated Panel Exterior Infill Walls (WSIP-W \& MSIP-W) after 50 Year Lifespan in Toronto 118

Figure 6-4: Total Life-Cycle Energy and GWP of Cold-Formed Steel Stud Exterior Infill Walls (SS-W) after 50 Year Lifespan in Toronto 118

Figure 6-5: Total Life-Cycle Energy and GWP of Wood Stud Exterior Infill Walls (WS-W) after 50 Year Lifespan in Toronto.

Figure 6-6: Total Life-Cycle Energy and GWP of Pre-Engineered Steel Building (PENG-W) and Opaque Spandrel Panel Exterior Infill Walls (CWALL-W) after 50 Year Lifespan in Toronto... 119

Figure 6-7: Total Life-Cycle Energy and GWP of Concrete Hollow Core Roofs (CHC-R) after 50 Year Lifespan in Toronto. 123 
Figure 6-8: Total Life-Cycle Energy and GWP of Open Web Steel Joist Roofs (OWSJ-R) after 50

Year Lifespan in Toronto

Figure 6-9: Total Life-Cycle Energy and GWP of Cold-Formed Steel Roofs (CFS-R) after 50

Year Lifespan in Toronto

Figure 6-10: Total Life-Cycle Energy and GWP of Glulam Joist Roofs (GLU-R) after 50 Year

Lifespan in Toronto

Figure 6-11: Total Life-Cycle Energy and GWP of Structural Insulated Panel Roofs (WSIP-R \&

MSIP-R) after 50 Year Lifespan in Toronto

Figure 6-12: Total Life-Cycle Energy and GWP of Pre-Engineered Steel Building Roofs (PENG-R) after 50 Year Lifespan in Toronto.

Figure 6-13: Total Life-Cycle Energy and GWP of Structural Systems after 50 Year Lifespan in Toronto .

Figure 6-14: Total Life-Cycle Energy and GWP of Floors (FL) after 50 Year Lifespan in Toronto .

Figure 6-15: Total Life-Cycle Energy and GWP of Windows (W) after 50 Year Lifespan in Toronto .

Figure 6-16: Total Life-Cycle Energy and GWP of Doors (D) after 50 Year Lifespan in Toronto ...131

Figure 6-17: Total Life-Cycle Energy and GWP of Interior Partition Walls (WS-P, SS-P, \&

CMU-P) after 50 Year Lifespan in Toronto

Figure 6-18: Total Life-Cycle Energy and GWP of Isolated Footing and Concrete Pier

Foundations (IF-FDN) after 50 Year Lifespan in Toronto

Figure 6-19: Total Life-Cycle Energy and GWP of Strip Footing and Concrete Wall Foundations

(SF-FDN) after 50 Year Lifespan in Toronto.

Figure 6-20: Total Life-Cycle Energy and GWP of Concrete Slab-On-Grades (SOG-FDN)

after 50 Year Lifespan in Toronto.

Figure 7-1: A Sensitivity Analysis of the Total Embodied Energy Use for the Components of a

Typical Retail Building after a 50 Year Lifespan in Toronto

Figure 7-2: A Sensitivity Analysis of the Total Embodied GWP for the Components of a Typical

Retail Building after a 50 Year Lifespan in Toronto

Figure 7-3: A Sensitivity Analysis of the $\Delta$ Total Life-Cycle Energy Use for the Components of a Typical Retail Building after a 50 Year Lifespan in Toronto. 141 
Figure 7-4: A Sensitivity Analysis of the $\Delta$ Total Life-Cycle GWP for the Components of a Typical Retail Building after a 50 Year Lifespan in Toronto

Figure 7-5: Orders of Magnitude for Energy Consumption of a Typical Retail Building after 50 Year Lifespan in Toronto... 144

Figure 7-6: Orders of Magnitude for Global Warming Potential of a Typical Retail Building after 50 Year Lifespan in Toronto... 146 


\section{List of Tables}

Table 2-1: Initial Embodied Energy of Common Building Materials ................................................16

Table 2-2: Initial Embodied $\mathrm{CO}_{2}$ of Common Building Materials ...................................................23

Table 2-3: A Summary of the Prominent LCA Tools and What They Do ..........................................27

Table 2-4: A Summary of Relevant LCA Studies of Commercial Buildings .....................................41

Table 3-1: Description of Baseline Retail Building Components......................................................48

Table 3-2: Range of Exterior Infill Wall Design Strategies............................................................51

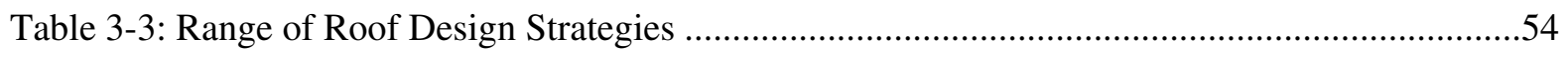

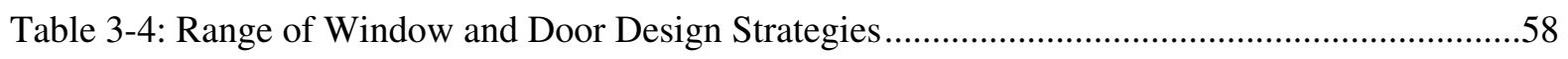

Table 3-5: Range of Foundation Design Strategies ........................................................................60

Table 3-6: Range of Interior Partition Wall Design Strategies .......................................................62

Table 3-7: Calculating Thermal Resistance of CMU-W1 Using the Tabular Method .........................72

Table 4-1: Building Component Quantities for Case Study \#1 .......................................................83

Table 4-2: Building Component Quantities for Case Study \#2 ........................................................84

Table 4-3: Building Component Quantities for Case Study \#3 ..........................................................86

Table 4-4: Building Component Quantities for Case Study \#4 .........................................................8

Table 4-5: Building Component Quantities for Case Study \#5 ........................................................90

Table 5-1: Annual Operating Energy and GWP Results for Case Study Buildings ..........................105

Table 6-1: A Summary of the $\Delta$ Total Energy and $\Delta$ Total GWP from the Baseline Case for the Alternative Building Components in this Study after 50 Years ......................................120

Table 6-2: Comparison of Embodied LCA Data in this Study to ATHENA® EcoCalculator............136

Table 7-1: Alternative Design Strategies to Reduce the Total Life-Cycle Energy Use of the Baseline Retail Building after a 50 Year Lifespan in Toronto .........................................148

Table 7-2: Alternative Design Strategies to Reduce the Total Life-Cycle GWP of the Baseline Retail Building after a 50 Year Lifespan in Toronto 150 


\section{Glossary of Terms}

\begin{tabular}{|c|c|}
\hline $\begin{array}{l}\circledR \text { ental } \\
\text { stimator } \\
\text { ngs }\end{array}$ & $\begin{array}{l}\text { The only life-cycle assessment software in North America that is capable of } \\
\text { evaluating whole buildings and individual building components based on } \\
\text { internationally accepted LCA methodology. Refer to website: } \\
\text { (http://www.athenasmi.org/tools/impactEstimator/) }\end{array}$ \\
\hline $\begin{array}{l}\text { Carb } \\
\text { Equi } \\
\left(\mathrm{CO}_{2}\right.\end{array}$ & $\begin{array}{l}\text { Carbon dioxide equivalency }\left(\mathrm{CO}_{2} \text { eq. }\right) \text { is a measure of the equivalent amount } \\
\text { of } \mathrm{CO}_{2} \text { that would have the same global warming potential (GWP) as a } \\
\text { mixture of } \mathrm{CO}_{2} \text { and other greenhouse gases in the Earth's atmosphere. }\end{array}$ \\
\hline Em & $\begin{array}{l}\text { The total energy (usually primary energy) associated with the acquisition, } \\
\text { processing, manufacturing, transportation, construction, repair, replacement, } \\
\text { and end-of-life effects of whole buildings or building materials. The total } \\
\text { embodied energy is the sum of the initial embodied energy and the recurring } \\
\text { embodied energy and is usually measured in MJ (or GJ) of energy. }\end{array}$ \\
\hline & $\begin{array}{l}\text { The energy (usually primary energy) associated with the demolition and } \\
\text { recycling/disposal of whole buildings or building materials. }\end{array}$ \\
\hline & $\begin{array}{l}\text { The 'Quick Energy Simulation Tool' (eQUEST) is an energy modelling } \\
\text { software program for buildings that is available free of charge. eQUEST is } \\
\text { based on the latest DOE-2 building simulation engine. Refer to website: } \\
\text { (http://www.doe2.com/equest/) }\end{array}$ \\
\hline & $\begin{array}{l}\text { A term that was developed to compare one greenhouse gas to another in terms } \\
\text { of their ability to trap heat in the Earth's atmosphere. GWP is measured in } \\
\text { mass of } \mathrm{CO}_{2} \text { equivalent. For buildings, the total GWP is the sum of the initial } \\
\text { embodied GWP and the recurring embodied GWP and is typically expressed } \\
\text { in either kg of } \mathrm{CO}_{2} \text { eq. or tonnes of } \mathrm{CO}_{2} \text { eq. }\end{array}$ \\
\hline $\begin{array}{l}\text { G } \\
(\mathbf{C}\end{array}$ & $\begin{array}{l}\text { Gases in the Earth's atmosphere that absorb and emit radiation and are the } \\
\text { fundamental cause behind the greenhouse effect. Some common GHG's } \\
\text { include carbon dioxide, methane, nitrous oxide, ozone, and water vapour. }\end{array}$ \\
\hline
\end{tabular}




\begin{tabular}{|c|c|}
\hline $\begin{array}{l}\text { Initial Embodied } \\
\text { Energy (or GWP) }\end{array}$ & $\begin{array}{l}\text { The energy (or GWP) used to acquire raw materials and manufacture, } \\
\text { transport, and install building products in the initial construction of a building. }\end{array}$ \\
\hline LEED & $\begin{array}{l}\text { The 'Leadership in Energy and Environmental Design' green building rating } \\
\text { system encourages global adoption of sustainable building practices through a } \\
\text { performance based point system. LEED is operated by the U.S. Green } \\
\text { Building Council (the Canadian Green Building Council in Canada). Refer to } \\
\text { web site: (http://www.cagbc.org/leed/what/index.php) }\end{array}$ \\
\hline $\begin{array}{l}\text { Life-Cycle } \\
\text { Assessment (LCA) }\end{array}$ & $\begin{array}{l}\text { "A method used to quantify environmental burdens based on inventory of } \\
\text { environmental factors for a product, process, or activity from the abstraction of } \\
\text { raw materials to their final disposal" (Lee, O'Callaghan, \& Allen, 1995). }\end{array}$ \\
\hline Operating Energy & $\begin{array}{l}\text { The use of either renewable or non-renewable energy in buildings to meet their } \\
\text { demands for heating, cooling, lighting, ventilation, etc. during the occupancy } \\
\text { phase of its life. Typically operating energy in buildings is measured in either } \\
\text { kWh or GJ of energy. }\end{array}$ \\
\hline Primary Energy & $\begin{array}{l}\text { "Encompasses the total requirements for all uses of energy. This includes } \\
\text { secondary energy use. Additionally, primary energy use refers to the energy } \\
\text { required to transform one form of energy to another (e.g. coal to electricity). It } \\
\text { also includes the energy used to bring energy supplies to the consumer (e.g. } \\
\text { pipeline). Further, it entails the energy used to feed industrial production } \\
\text { processes (e.g. the natural gas used by the chemical industries)" (NRCan, } \\
2009 \text { ). }\end{array}$ \\
\hline $\begin{array}{l}\text { Recurring } \\
\text { Embodied Energy } \\
\text { (or GWP) }\end{array}$ & $\begin{array}{l}\text { The energy (or GWP) associated with maintaining, repairing, and replacing } \\
\text { materials and components over the lifetime of the building. }\end{array}$ \\
\hline
\end{tabular}




\begin{tabular}{|c|c|}
\hline $\begin{array}{l}\text { Secondary Energy } \\
\text { (or Site Energy) }\end{array}$ & $\begin{array}{l}\text { "Energy used by the final consumer in various sectors of the economy. This } \\
\text { includes, for example, the energy used by vehicles in the transportation sector. } \\
\text { Secondary energy also encompasses energy required to heat and cool homes or } \\
\text { businesses in the residential and commercial/institutional sectors. It also } \\
\text { comprises energy required to run machinery in the industrial and agricultural } \\
\text { sectors" (NRCan, 2009). }\end{array}$ \\
\hline THERM & $\begin{array}{l}\text { A two-dimensional (finite element based) heat flow analysis software program } \\
\text { for building enclosures that is available free of charge. It was developed by the } \\
\text { Lawrence Berkeley National Laboratory. Refer to web site: } \\
\text { (http://windows.lbl.gov/software/therm/therm.html) }\end{array}$ \\
\hline $\begin{array}{l}\text { Total Life-Cycle } \\
\text { Energy (or GWP) }\end{array}$ & $\begin{array}{l}\text { The sum of the total embodied energy (or GWP) and the total operating energy } \\
\text { (or GWP) of a building over a specified lifespan. The total life-cycle energy } \\
\text { (or GWP) is usually expressed in } \mathrm{MJ} \text { (or kg of } \mathrm{CO}_{2} \text { eq.). }\end{array}$ \\
\hline
\end{tabular}




\section{Chapter 1 \\ Introduction and Background}

\subsection{Introduction}

The building industry in North America is changing. Over the past few decades, there has been an industry movement towards the design and construction of more energy efficient buildings. In North America, LEED ${ }^{\circledR}$ has become the foremost green building protocol and continues to encourage an aggressive reduction of energy use and greenhouse gas (GHG) emissions within the building industry. Over the past decade, the number of LEED ${ }^{\circledR}$ certified buildings in North America has increased at an exponential rate. There is a clear and growing market demand for energy efficient buildings. In the coming years, architects and engineers will need to adopt a more holistic design approach that fully considers the total life-cycle environmental impacts of the buildings that they design.

The building industry has an overwhelming impact on the environment. In the United States, buildings are responsible for around 39\% of primary energy use, $38 \%$ of all carbon dioxide emissions, and nearly $40 \%$ of all raw material use annually (USGBC, 2010). The trends in Canada are much the same. In Canada, the building sector as a whole accounts for approximately $29 \%$ of the total secondary energy use (NRCan, OEE, 2010). This is effectively equal to the secondary energy consumption of the entire transportation sector in Canada. Of this, retail buildings are responsible for about $17 \%$ of the total commercial/institutional secondary energy use, second only to office buildings which consume roughly 35\% (NRCan, OEE, 2010). However, compared to office buildings in Canada, retail buildings on average have a higher energy intensity, consuming approximately $23 \%$ more energy per square meter of floor space (NRCan, OEE, 2010). Despite the need for energy efficient retail buildings, issues of sustainability have rarely been addressed for retail buildings in Canada. Of the 137 total LEED® Canada certified commercial buildings (excluding single family homes less than three storeys), only 10 (7\%) are retail buildings compared to $43(31 \%)$ office buildings (CaGBC, 2009).

Operational energy use in buildings is only one part of the problem. Over the life of a building, the total energy use is a combination of both the operational energy use and the embodied energy of the building materials. In a typical building today, about $85 \%$ of the total life-cycle energy use after 50 years is a result of the building operations, while only about $15 \%$ is due to the energy that is embodied in the materials (Cole \& Kernan, 1996). Given this, there is a misconception among some building professionals who place a disproportionally large emphasis on material selection as a means 
of achieving significant reductions in total life-cycle energy use. That being said, as the operating energy use of buildings continues to decrease through a combination of conservation and the use of renewable energy sources, an intelligent allocation of building materials will gain increasing importance.

\subsection{Description of Problem}

Over the past two decades, a number of researchers around the world have conducted life-cycle assessment (LCA) studies to investigate the impacts of buildings on the environment. However, the vast majority of these studies have focused on three types of buildings: office buildings, single residential dwellings, and multi-unit residential apartments. In Canada, these buildings account for $15 \%, 45 \%$, and $10 \%$ of the secondary energy use in the building sector (NRCan, OEE, 2010). Despite retail buildings accounting for $7 \%$ of the secondary energy use in the building sector in Canada, they have not historically been the focus of many LCA studies (NRCan, OEE, 2010). The lack of focus on retail buildings is a problem, since compared to office buildings, single residential dwellings, and multi-unit residential apartments, retail buildings consume approximately 1.2, 2.0, and 2.3 times more energy per floor area respectively (NRCan, OEE, 2010). In addition, retail buildings usually undergo major renovations or demolition far sooner than office buildings or residential dwellings. Also, a large majority of retail buildings are single-storey buildings. There is currently a lack of understanding in the literature of the life-cycle environmental impacts of single-storey commercial buildings. Single-storey buildings have a very different roof-to-wall area ratio than multi-storey buildings. For this reason, among others, it is important to investigate single-storey retail buildings within the framework of a comprehensive LCA to identify ways of reducing their life-cycle energy consumption and global warming potential (GWP).

In addition to the fact that few LCA studies have been conducted for retail buildings, there is still as widespread lack of knowledge on the part of many building industry professionals when it comes to material/assembly selection in low-energy buildings. Specifically, some building professionals have an inherent bias against the use of certain materials or assemblies in low-energy buildings, based solely on the initial embodied energy of an individual material. However, buildings and their component parts are far from homogonous systems in terms of material use. Comparing the life-cycle environmental impacts of different building components (or entire buildings) based solely on a comparison of the initial embodied energy of an individual building material is a gross simplification that is flawed. 
Historically, LCA studies for buildings have primarily focused on a comparison of either different wall assemblies or structural systems. In most cases, these studies focus on a comparison of wood, steel, and concrete alternatives. This approach inevitably distils to a comparison of the embodied energy of wood, steel, and concrete. However, this approach places a disproportionately large emphasis on one material as an effect gauge of determining the life-cycle environmental burdens of an overall building assembly (or an entire building).

Buildings are more than just a structural system or a wall assembly. They are complex systems with numerous components and sub-assemblies that act together as part of a greater system. Few studies have investigated a wider range of building components with the goal of determining the relative impacts of all of the components of a building, not just the wall assembly and the structural system. The few comprehensive studies that have been done all vary drastically in their approach, the building components that are studied, and their degree of complexity. There needs to be a sensitivity analysis of a large range of building components within one comprehensive study using the latest LCA techniques, to develop a more complete understanding of the relative environmental impacts of all the components of a building, specifically in a single-storey retail building.

\subsection{Objectives of Research}

The purpose of this study is to conduct a comprehensive LCA of the energy use and GWP of a singlestorey retail building in Canada. A sensitivity analysis of numerous building components will be performed to determine which components of a single-storey retail building have the greatest impact on the environment. Using these findings, a range of different retail building types in Canada will be analyzed and compared based on their life-cycle environmental burdens. A key objective will be to rank the components of a single-storey retail building in order of the damage that they cause to the environment. The ultimate goal of this research is to develop a list of recommendations for reducing the life-cycle energy use and GWP of single-storey retail buildings in Canada.

The specific objectives of this research study are:

1. To review previous LCA studies of embodied energy, embodied GWP, operating energy, and operating GWP in commercial buildings.

2. To identify a comprehensive list of alternative design strategies for a single-storey retail building across the following seven areas:

a. The exterior infill wall enclosures 
b. The roof enclosures

c. The floor assemblies

d. The windows and doors

e. The structural systems (beams and columns)

f. The foundations

g. The interior partitions

3. To calculate the total life-cycle energy and total GWP of each alternative building component identified in objective 2, over a 50 year lifespan in Toronto, Canada.

4. Using the findings from Objective 3, perform a sensitivity analysis of the total life-cycle energy use and total GWP for a typical Canadian retail building, in order to determine which building components have the greatest negative impact on the environment after 50 years.

5. Using the results from Objective 3 and 4, conduct a LCA of energy use and GWP for a range of common Canadian retail building types, in order to identify if there is a significant difference in the total life-cycle energy use and total GWP of these buildings after 50 years.

6. To use the results of this study to develop a list of recommendations for reducing the total life-cycle energy use and GWP of single-storey retail buildings in Canada.

\subsection{Organization of Thesis}

The organization of this thesis closely parallels the list of objectives that were presented in the previous section.

Chapter 2 contains a literature review of previous LCA studies of buildings. As virtually no significant LCA studies have been published for retail buildings specifically, most of the literature review deals with commercial buildings in general. There is also a discussion of energy and GWP trends in the Canadian building sector, along with a presentation of some background terminology.

The method employed for calculating the total life-cycle energy use and total GWP of the extensive list of building components in this study is discussed in Chapter 3. A detailed description of the building components considered is also presented, along with the method that was followed for calculating both the embodied effects and the operating effects for each building component. The scope of the LCA is also discussed. 
Chapter 4 presents a detailed description of the five single-storey retail buildings looked at in this study.

The results of the comprehensive LCA of energy use and GWP for the five case study retail buildings are presented in Chapter 5, along with a discussion of the results. In Chapter 6, the LCA results for the extensive list of buildings components are presented.

Chapter 7 contains a summary of the data presented in the previous two chapters and distils it into a list of key findings. A list of recommendations for reducing the total life-cycle energy use and total GWP of a retail building in Canada is also presented.

Provided in Chapter 8 are concluding remarks for this study, as well as a list of recommendations for future work.

There is also a detailed Appendix with supplementary information and results at the end of this thesis. 


\section{Chapter 2 \\ Literature Review}

\subsection{Introduction}

The life-cycle assessment of whole buildings and their components has been a growing area of research over the last 15 years. In the past, the focus of the vast majority of these studies has been on residential (single family dwellings and multi-unit apartments) and multi-storey office buildings. Significant strides have been made in the understanding of material effects and operating effects in these types of buildings. However, limited research has been conducted on the life-cycle environmental impact of single-storey retail buildings.

A large proportion of commercial buildings in North America are single-storey buildings. As well, retail buildings tend to be some of the least energy efficient buildings constructed today. Not only do they use more energy per square meter than office buildings, but they tend to undergo energy intensive renovations on a more frequent basis.

Unfortunately, the number of LCA studies dealing with retail buildings specifically are very scarce. Therefore, in this section a literature review of the important LCA studies of commercial buildings over the past 15 years will be conducted. The goal is to develop an understanding of the literature as it pertains to commercial buildings in general. Having an understanding of the relevant LCA studies of commercial buildings is an important first step towards a more complete understanding of the lifecycle environmental impacts of single-storey retail buildings. As well, some important terms and concepts relating to energy use in buildings will be discussed.

\subsection{The 'Green' Building Movement}

Since the 1800's, scientists have predicted that a rise in carbon dioxide $\left(\mathrm{CO}_{2}\right)$ concentrations in the atmosphere could result in unprecedented global climate change, due to an increase in global temperatures. As Dr. Gilbert Plass (a physicist at John Hopkins university and a pioneering researcher on the relationship between $\mathrm{CO}_{2}$ and climate change) describes "humanity is conducting a large-scale experiment on the atmosphere, the results of which will not be available for several generations" (Fleming, 1998).

In terms of total GHG emissions, Canada emitted the seventh highest amount of GHG in 2005 of any country in the world and was second only to Australia in terms of GHG emissions per capita 
(NRTEE, 2009). In 1997, the Kyoto Protocol agreement was signed under the United Nations Framework Convention on Climate Change (UNFCCC) by 37 industrialized nations including Canada (as well as a host of other nations) with the goal of reducing global climate change. The Protocol is aimed at tackling the problem of global climate change by providing member countries with binding targets for reducing their GHG emissions. Under the Kyoto Protocol, Canada has agreed to reduce its GHG emissions to $6 \%$ below what they were in 1990 by the year 2012. Figure 2-1 illustrates Canada's GHG emissions from 1990 to 2007. Given the most recent data (for 2007) Canada currently stands about $34 \%$ above its Kyoto target. Given that the Kyoto commitment period is from 2008 to 2012, it seems highly unlikely that Canada will be able to meet its Kyoto commitment.

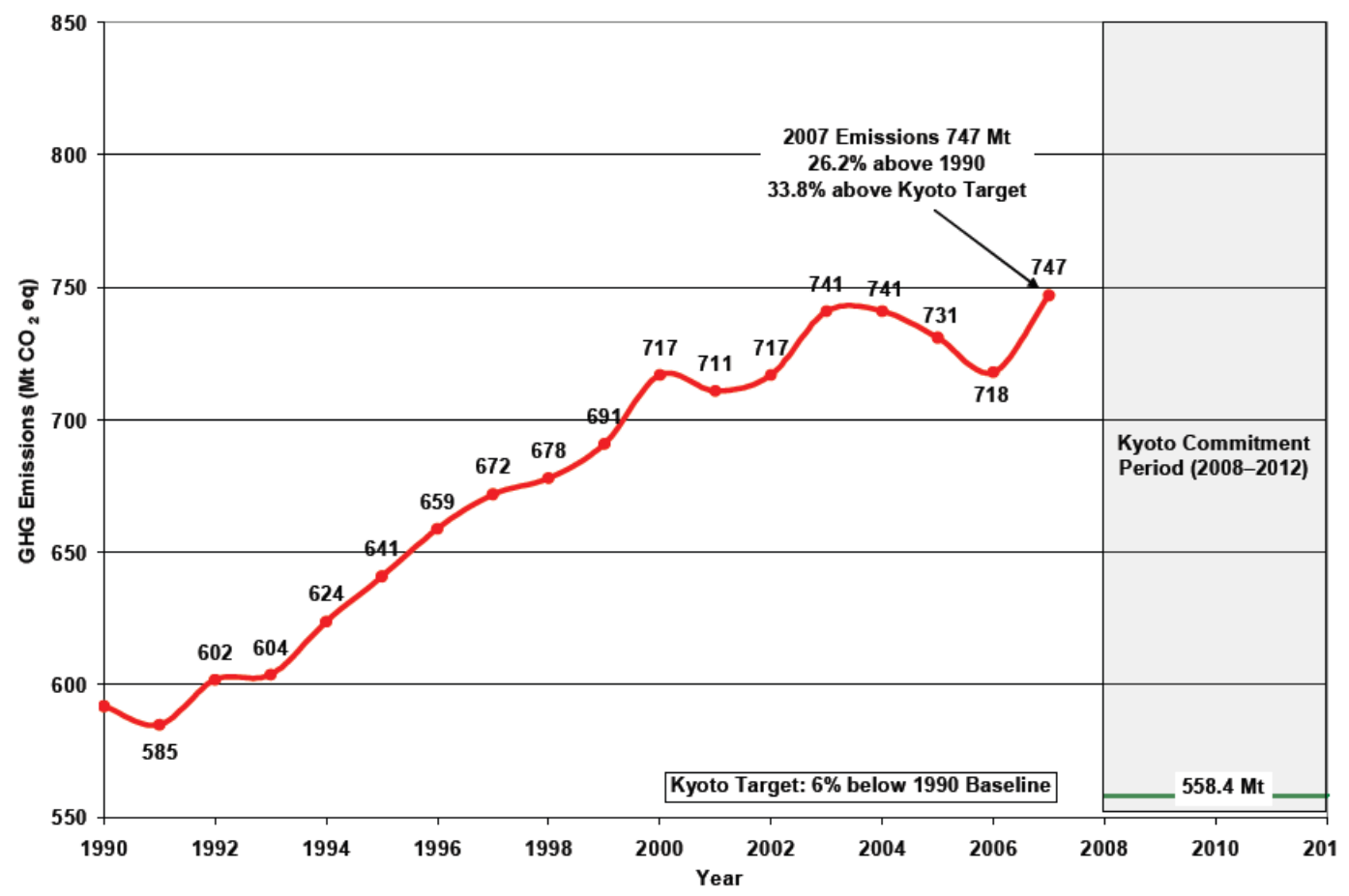

Figure 2-1: Canada's Greenhouse Gas Emissions from 1990-2007 (Environment Canada, 2009)

That being said, one of the single biggest contributors to $\mathrm{CO}_{2}$ emissions worldwide is the building industry. In fact, the construction and operation of buildings accounts for over a third of the world's energy consumption and $40 \%$ of all the mined recourses (Straube J. F., 2006). In addition, the vast majority of buildings constructed in the developed world in the last 30-50 years have a shorter service 
life than older buildings (Straube J. F., 2006). This means that 'modern' building practices have resulted in inferior buildings from the standpoint of performance and durability. This often requires modern buildings to go through many resource intensive renovations over their lifespan.

The term 'sustainability' is used a lot in the building industry, often without a complete understating of what it means. The 'Brundtland Report' to the United Nations in 1987 defined the concept of sustainability as "development that meets the needs of the present without compromising the ability of future generations to meet their own needs" (The World Commission on Environment and Development, 1987). "A subset of sustainable development, sustainable construction, addresses the role of the built environment in contributing to the overarching vision of sustainability" (Kibert, 2005). In terms of the building industry today, Straube (2006) provides a working definition of a 'green' building: "a building that uses energy and material more effectively both in production and operation while polluting and damaging natural systems as little as possible" (Straube J. F., 2006).

The push for more sustainable buildings in recent years has led to the development of numerous 'green' building protocols around the world. In North America, the most prominent 'green' building protocol is LEED. LEED stands for "Leadership in Energy and Environmental Design" and aims to encourage the adoption of sustainable building practices through the creation of performance criteria. LEED was first developed by the United States Green Building Council (USGBC) in 1998 and was later adopted by the Canadian Green Building Council (CaGBC). LEED operates on a points based system, whereby a score is awarded for performance in five key areas: sustainable site development, water efficiency, energy efficiency, materials selection, and indoor environmental quality (CaGBC, 2009). As of May 2010, 249 buildings in Canada (excluding residential projects less than $600 \mathrm{~m}^{2}$ ) had achieved LEED certification and the number is increasingly rapidly (CaGBC, 2009). "Despite the success of LEED and the green building movement in general, challenges abound when implementing sustainability principles within the well-entrenched, traditional construction industry" (Kibert, 2005). At the end of the day, the reluctance of the building industry to change will likely present the biggest obstacle in the struggle to reach a new level of sustainability in the built environment. 


\subsection{Operating Energy of Buildings}

Perhaps one of the biggest objectives of many of the green building protocols today is the pursuit of an aggressive reduction in the operating energy use of buildings. In general, there are two forms of energy available: non-renewable energy (e.g. coal, oil, natural gas, etc.) and renewable energy (e.g. solar, wind, water, etc.). Today, the vast majority of buildings depend on non-renewable energy to meet their demands for heating, cooling, lighting, ventilation, etc.

To begin a discussion of energy use in buildings, it is important to have an understanding of the relevant terms and definitions. In this section, some background terms relating to energy will be presented, along with a discussion of the energy use trends in the Canadian building sector.

\subsubsection{Background on Operating Energy Statistics for Buildings}

Energy use is typically expressed in terms of Joules $(\mathrm{J})$. However, since the quantity of energy use in buildings is relatively large, it is often more convenient to express the energy use in terms of Megajoules (MJ), Gigajoules (GJ), or Petajoules (PJ). To help put these terms in context, one PJ is equivalent to "the energy required by almost 9,000 households (excluding transportation requirements) over one year" (NRCan, 2009).

According to Harvey (2006) there are three forms of energy: primary, secondary, and tertiary (or final end-use) energy. Primary energy is "energy as it occurs in nature" (Harvey, 2006). Examples of primary energy are oil, natural gas, coal, and uranium as they exist in the ground. Harvey (2006) explains that "to be useful to humans, these forms of energy need to be extracted and transformed into secondary energy" (Harvey, 2006). Examples of secondary energy are things like electricity and refined petroleum. Finally, tertiary energy (or end-use energy) are "things like warmth, motion, mechanical power, or light" (Harvey, 2006). Whenever energy is transformed, transported, or utilized there are losses. Harvey (2006) illustrates the relationships between primary, secondary, and tertiary energy in Figure 2-2.

Natural Resources Canada (NRCan) provides a similar distinction between primary and secondary energy:

"Secondary energy use is the energy used by the final consumer in various sectors of the economy. This includes, for example, the energy used by vehicles in the transportation sector. Secondary energy use also encompasses energy required to heat and cool homes 
or businesses in the residential and commercial/institutional sectors. In addition, it comprises energy required to run machinery in the industrial and agricultural sectors.

Primary energy use encompasses the total requirements for all uses of energy. This includes secondary energy use. Additionally, primary energy use refers to the energy required to transform one form of energy to another (e.g. coal to electricity). It also includes the energy used to bring energy supplies to the consumer (e.g. pipeline). Further, it entails the energy used to feed industrial production processes (e.g. the natural gas used by the chemical industries)" (NRCan, 2009).

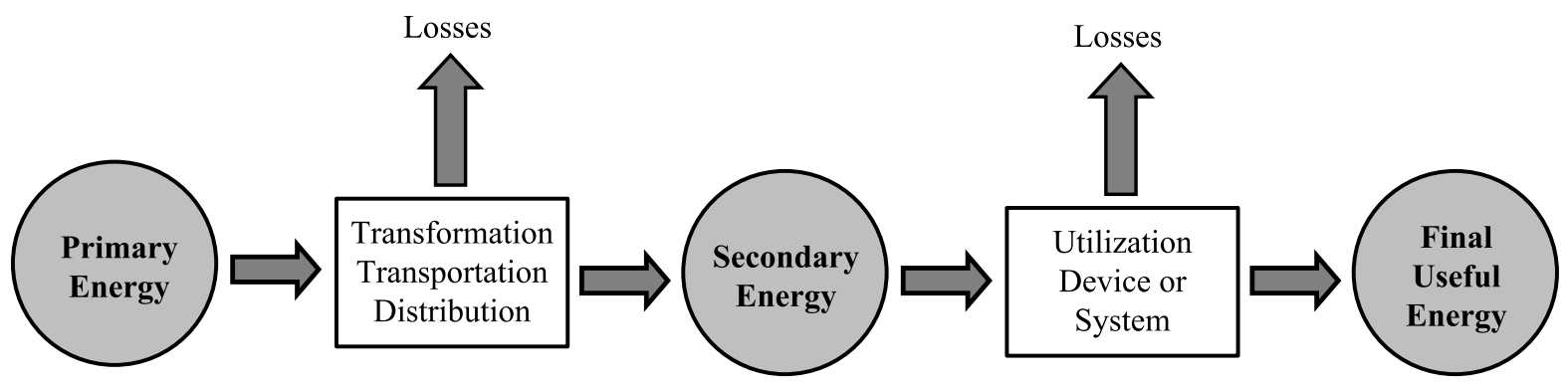

Figure 2-2: The Transformation from Primary to Secondary to Tertiary Energy (Harvey, 2006)

In the context of buildings, the operating energy can be defined as the amount of energy (renewable or non-renewable) that is required to maintain the functions of the building (e.g. heating, cooling, lighting, ventilation, equipment, etc.) and the activities of the occupants.

\subsubsection{Operating Energy Statistics for Canadian Commercial Buildings}

Each year the Office of Energy Efficiency (OEE) at National Resources Canada (NRCan) publishes the Energy Use Data Handbook (NRCan, OEE, 2010). The OEE completes an annual audit of the energy use by sector in Canada. The most recent data available is for 2007. According to the OEE, in 2007 the total primary energy consumed in Canada was about 12,786 PJ and the total secondary energy use was about $8,870.5 \mathrm{PJ}$ (or about $69 \%$ of the total primary energy use) (NRCan, 2009). The OEE also provides a detailed breakdown of the energy consumption trends in each sector of the Canadian economy. Figure 2-3 illustrates the breakdown of the total secondary energy use by sector in Canada in 2007. 


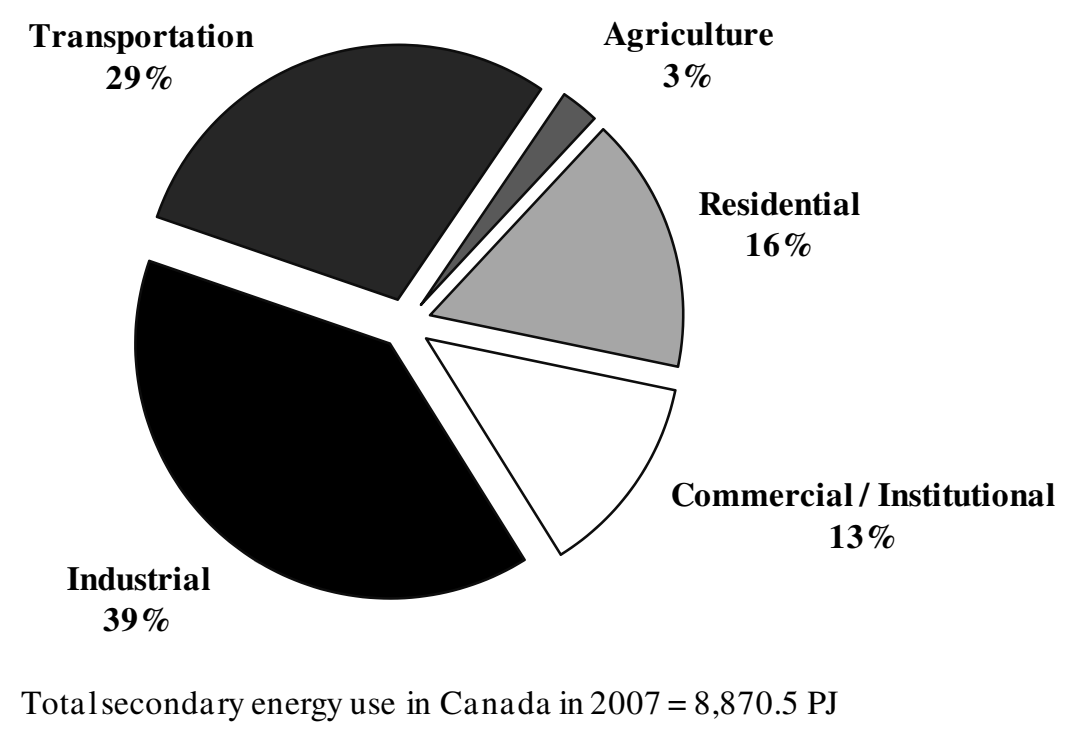

Figure 2-3: Breakdown of Total Secondary Energy Use by Sector in Canada in 2007 (NRCan, OEE, 2010)

By adding the residential secondary energy use (16\%) with the commercial/institutional secondary energy use (13\%), then the total secondary energy use of the building sector (not including material related effects or transportation) accounts for around $29 \%$ of the total secondary energy use in Canada. This is essentially equal to the secondary energy use of the entire transportation sector in Canada. However, the primary energy use changes the relative proportions and buildings are more significant.

Figure 2-4 illustrates the breakdown of the total commercial/institutional secondary energy use by activity type in Canada in 2007. Office buildings are responsible for about $35 \%$ of the total secondary energy use in the commercial/institutional sector in Canada. This is interesting as office buildings have historically been the major focus of studies dealing with energy use in buildings. Interestingly enough, retail buildings are responsible for the next highest amount of secondary energy consumption at $17 \%$. Despite the fact that retail buildings are responsible for the highest percentage of secondary energy use in Canada next only to office buildings, they have not historically been the focus of studies dealing with energy use in buildings.

Looking specifically at the case of retail buildings now, Figure 2-5 illustrates the breakdown of secondary energy use by end use in Canada in 2007 for retail buildings. Not surprisingly, space 
heating accounts for half of the energy use in retail buildings in Canada. Canada is a cold climate and buildings located here require a significant amount of energy for space heating.

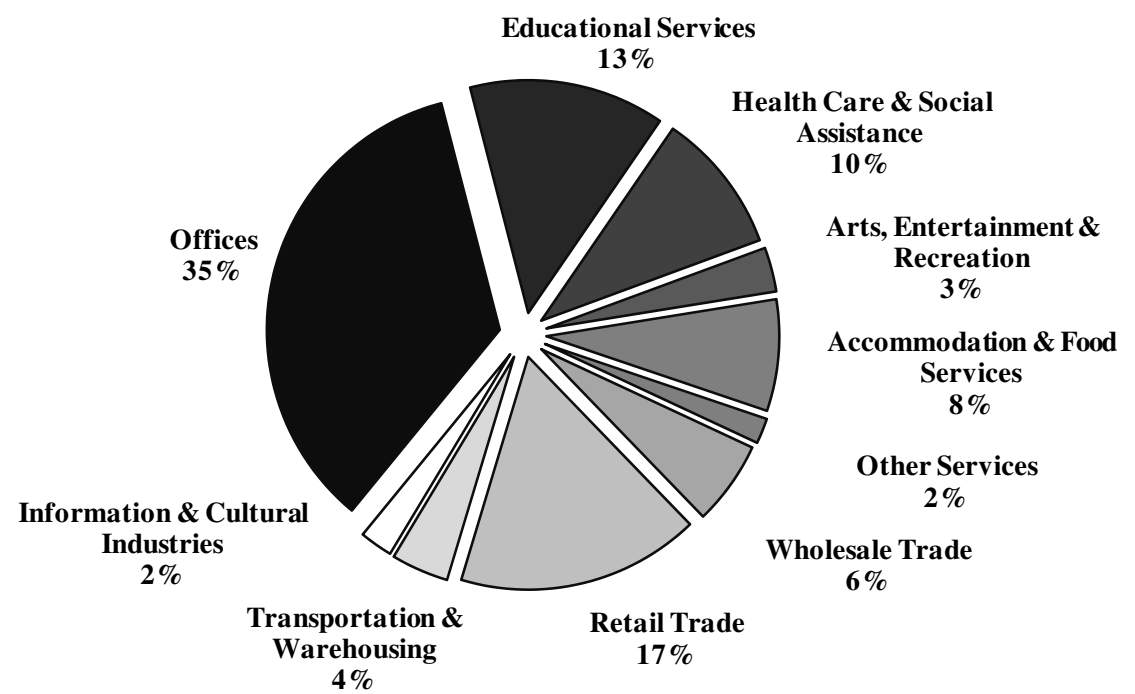

Totalcommercial/institutional secondary energy use in Canada in 2007 $=1,141.6 \mathrm{PJ}$

Figure 2-4: Breakdown of Total Commercial/Institutional Secondary Energy Use by Activity Type in Canada in 2007 (NRCan, OEE, 2010)

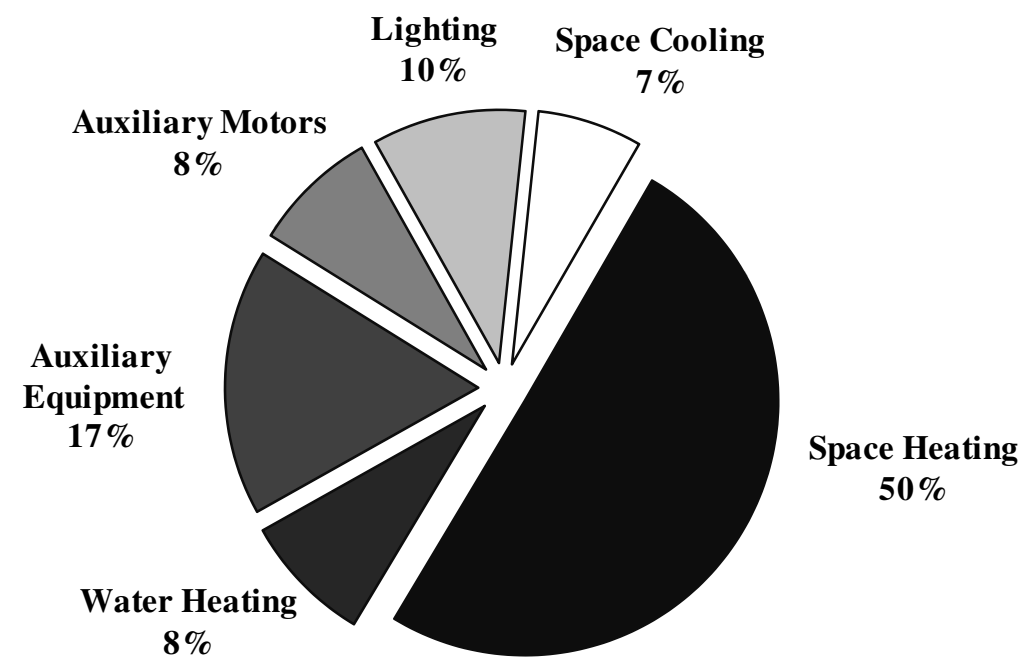

Total retail secondary energy use in Canada in $2007=191.1 \mathrm{PJ}$

Figure 2-5: Breakdown of Retail Secondary Energy Use by End Use in Canada in 2007 (NRCan, OEE, 2010) 
An interesting and useful way to compare the energy consumption of the various commercial/institutional type buildings in Canada is to consider their energy intensity. Energy intensity is essentially a measure of the amount of energy that is consumed per square meter of floor area. Figure 2-6 illustrates the average annual operating energy intensity by activity type for the commercial/institutional sector in Canada in 2007. Retail buildings actually consume about 1.2 times more operating energy per square meter than office buildings.

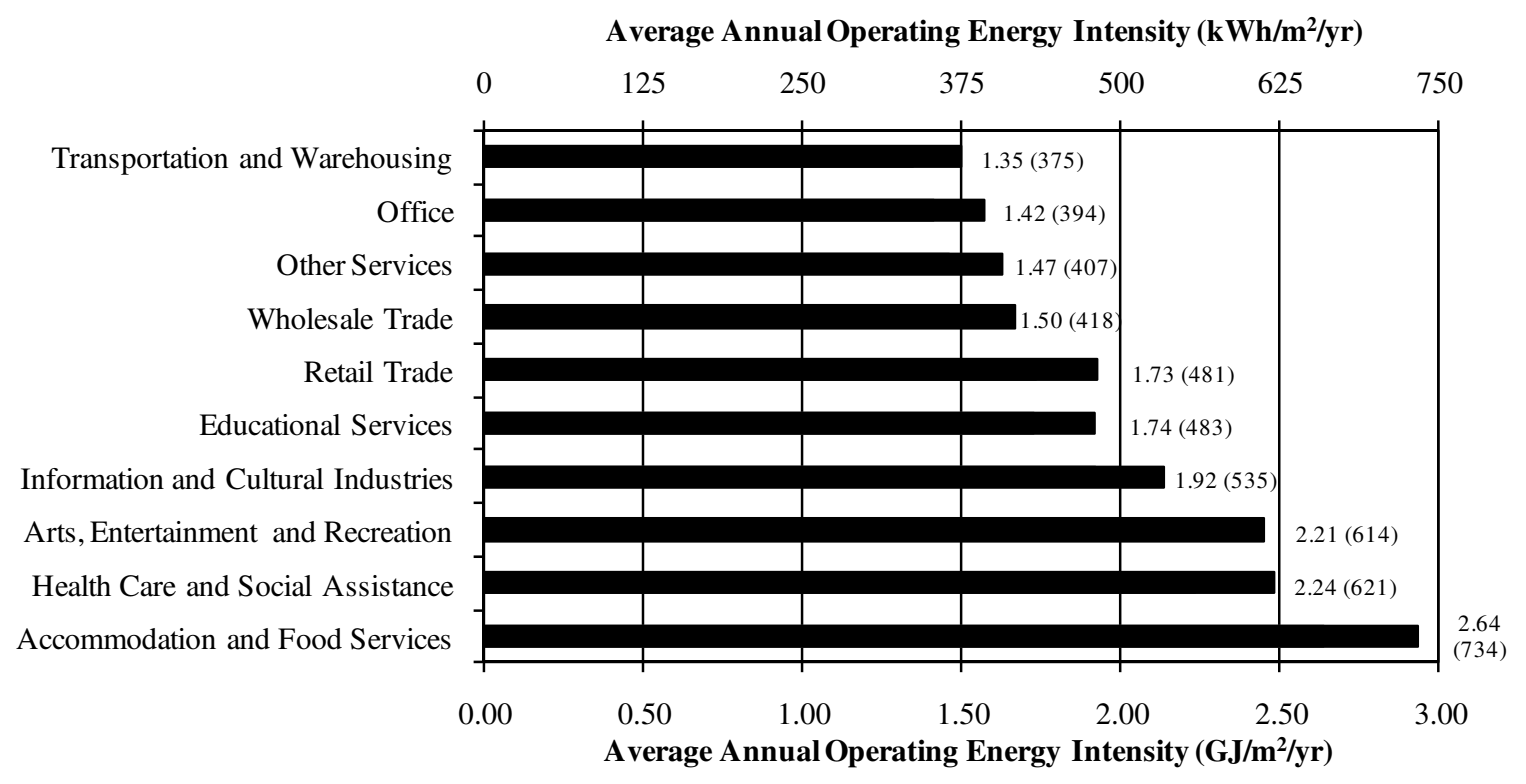

Figure 2-6: Commercial/Institutional Sector Average Annual Operating Energy Intensity by Activity in Canada for 2007 (NRCan, OEE, 2010)

\subsection{Embodied Energy in Buildings}

The operating energy consumption of buildings has been studied for years. Statistics are kept and reported by Natural Resources Canada each year for every sector of the Canadian economy. However, the energy associated with the acquisition, processing, manufacturing, transportation, construction, and repair/replacement of building materials is less familiar. The amount of energy that is associated with these activities is called embodied energy.

\subsubsection{Background on Embodied Energy in Buildings}

Embodied energy refers to "the total energy consumed in the acquisition and processing of raw materials, including manufacturing, transportation, and final installation" (Kibert, 2005). The 
embodied energy can be calculated for a building material, a component of a building, or even an entire building project. The embodied energy of a particular building material is usually reported in terms of MJ of primary energy per $\mathrm{kg}$ ( or $^{3}$ ) of material. There are two kinds of embodied energy: initial embodied energy and recurring embodied energy. The initial embodied energy of a building refers to "the energy used to acquire raw materials and manufacture, transport and install building products in the initial construction of a building" (Cole \& Kernan, 1996). On the other hand, recurring embodied energy is "the energy associated with maintaining, repairing and replacing materials and components over the lifetime of the building" (Cole \& Kernan, 1996). The total life-cycle embodied energy is simply the sum of the initial embodied energy plus the recurring embodied energy.

Embodied energy can be divided into direct embodied energy and indirect embodied energy. In terms of a building project, the direct embodied energy is the energy needed to transport building materials to the site and then construct the building. The indirect embodied energy is the energy required to extract and process the raw materials, the energy required to manufacture the building materials, and any related transportation energy. Determining the indirect embodied energy requires inputs from a large array of other industries. According to Harvey (2006), "there are a number of indirect energy inputs of first order, second order, third order, and so on. Fully accounting for the embodied energy in building materials requires accounting for a very large succession of linkages" (Harvey, 2006).

As mentioned, the total embodied energy (initial embodied energy plus recurring embodied energy) can be calculated for a building material, building component, or even an entire building. "Products with greater embodied energy usually have higher environmental impact due to the emissions and greenhouse gases associated with energy consumption" (Kibert, 2005). "The embodied energy depends on the energy intensity of the industries involved in producing building materials, while transportation energy depends on the energy intensity of transportation and the distances transported" (Harvey, 2006). "As the energy intensities (in the industrial and transportation sectors) improve, the embodied energy in new buildings will decrease" (Harvey, 2006).

In theory, calculating the embodied energy of a building is relatively straightforward if the embodied energies of the individual building materials are known. In reality, calculating the embodied energy of a building is anything but straightforward. The problem today is that the embodied energies of the various building materials are not well known. There is a lack of reliable information on the embodied energy of building materials. In fact, there is often a wide range of variability in these numbers from one source to the next. No industry standard for the embodied energy of building 
materials exits. Furthermore, since the embodied energy of a material depends on the local industries that manufacture them, the energy generation profile of the region where the materials are being produced, the availability of raw materials, etc., the embodied energy numbers vary from country to country and even from one region to the next. However, in theory calculating the embodied energy of a building "requires determining the embodied energy per unit mass or per unit volume of all the materials that go into a building, multiplying by the amounts of each material used, accounting for energy used during construction and adding all these terms" (Harvey, 2006).

\subsubsection{Embodied Energy of Common Building Materials}

To date, there are no industry standard embodied energy numbers for the various building materials. In fact, the embodied energy of a building material can vary (sometimes significantly) depending on the location that it is produced, the energy intensity of the manufacturing industries, the availability of raw materials, and numerous other factors. In addition, the embodied energies of the various building materials continually change as industries reduce their energy consumption and get more efficient at producing their products. That being said, it is still valuable to have an understanding of the range of values that are currently cited in the literature for the embodied energy of some common building materials. Table 2-1 lists a range of values for the embodied energy of some common building materials.

It can be seen that some materials like virgin aluminum have a relatively high embodied energy (201.0 to $217.0 \mathrm{MJ} / \mathrm{kg}$ ). Insulation materials also tend to have a high embodied energy although little mass is used. For example, polystyrene insulation ranges from about 88.6 to $117.0 \mathrm{MJ} / \mathrm{kg}$ in Table $2-1$. Depending on the recycled content, general virgin steel can vary from about 15.4 to $35.3 \mathrm{MJ} / \mathrm{kg}$. Other naturally occurring materials such as stone have a relatively low embodied energy (0.8 to 6.8 $\mathrm{MJ} / \mathrm{kg}$ ). One important material to make mention of is concrete. In Table 2-1 the embodied energy of cement (4.6 to $15.0 \mathrm{MJ} / \mathrm{kg}$ ) is listed as well as the embodied energy of concrete (1.1 to $4.5 \mathrm{MJ} / \mathrm{kg}$ ). Concrete is actually about $75 \%$ aggregates, $10 \%$ water, and only $15 \%$ cement. Therefore, even though the embodied energy of cement can be relatively high, when combined with water and aggregates (which have very low embodied energy) the concrete mixture has a relatively low embodied energy. However, in building projects concrete is usually used in far greater mass than any other building material. Therefore, when the embodied energy of concrete is multiplied by the quantity of concrete, the total embodied energy of concrete used in a project can be significant. Once again, the embodied energy numbers presented here are by no means standard values for the industry. They have been 
collected from a sample of the literature and represent a range of values that one would find if they consulted the literature. It was difficult to find embodied energy numbers for building materials in Canada or the United States specifically, so most of the data in Table 2-1 was taken from comprehensive studies done in New Zealand and the United Kingdom.

Table 2-1: Initial Embodied Energy of Common Building Materials

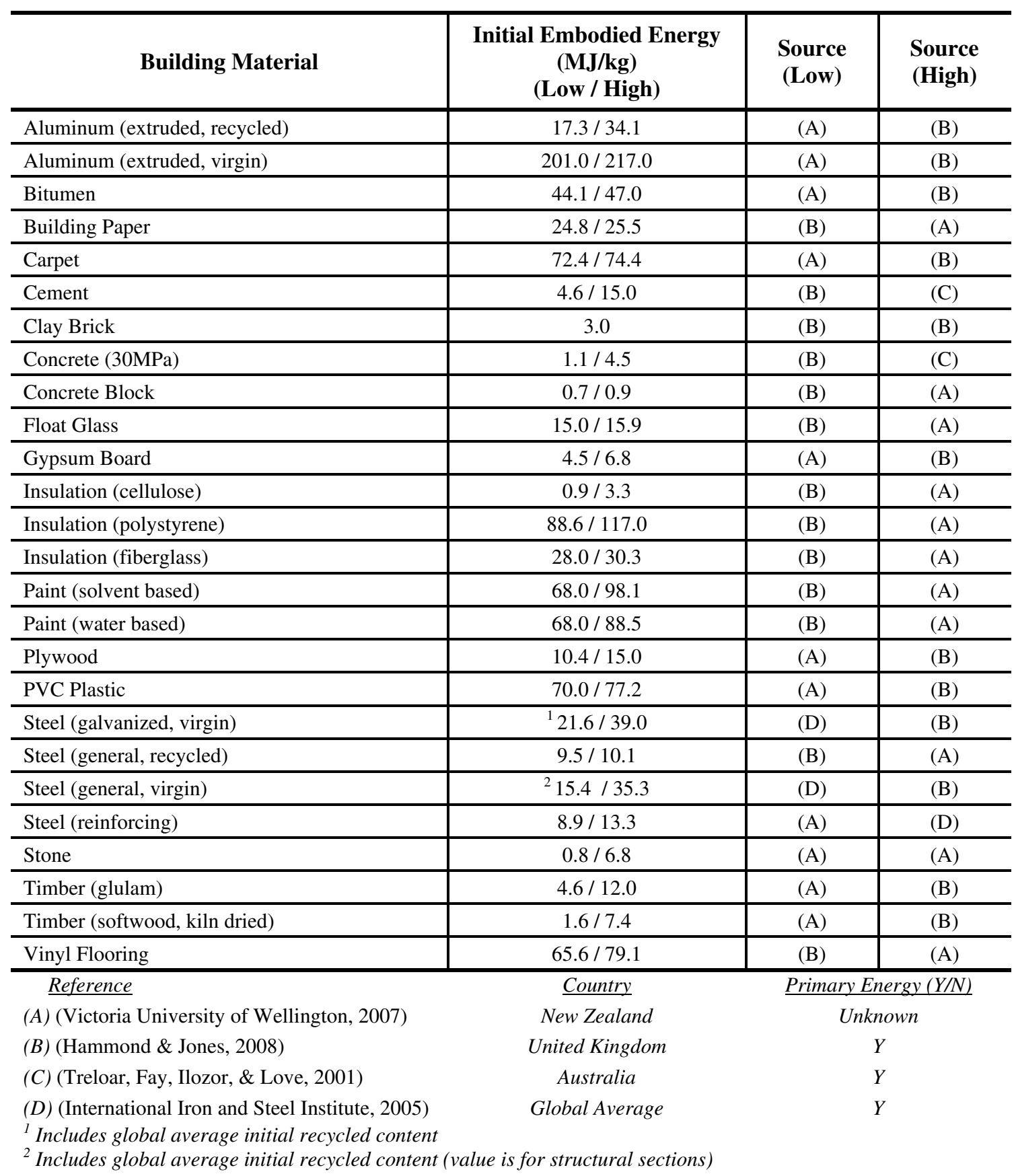




\subsubsection{Problems with Measures of Embodied Energy}

It can not be stressed enough the variability of embodied energy numbers for building materials. The process of calculating the embodied energy of a building material depends on many factors. For example, to calculate the embodied energy of a material, one must rely on the manufacturers to accurately and comprehensively account for the energy use associated with every aspect of their manufacturing process. In reality, this is rarely the case. More often than not, a detailed accounting of the total energy associate with the raw material extraction, transportation, processing, and manufacturing of a building material is not done. Therefore, this makes it difficult to determine the true embodied energy of a building material with any certainty of accuracy.

Also, the various industries that produce building materials are continually updating their processes and becoming more efficient. Therefore, the true embodied energy of a building material is not static. It can vary from one year to the next and depends heavily on the specific location in which it is produce, as the energy generation, transportation, and manufacturing process in one location can vary drastically from the next.

When deciding on a material to use in a building project, it is a gross simplification to simply compare the embodied energy of two alternative materials and pass judgement on which is better for the environment. Recall that the embodied energy numbers are presented in terms of MJ of primary energy per $\mathrm{kg}\left(\right.$ or $\mathrm{m}^{3}$ ) of material. Therefore, one must also accurately determine the material quantities involved in order to determine the total embodied energy of a material that is used in a project. For example, even though the embodied energy per kg of concrete is relatively low, concrete tends to be one of the most significantly used materials in construction projects. As well, it weighs a great deal more than other building materials like insulation, which has a significantly higher embodied energy per $\mathrm{kg}$, but weighs far less than concrete. Therefore, without a full accounting of materials in a project, it is difficult to make a comparison based solely on embodied energy.

\subsection{Operating Global Warming Potential (GWP) of Buildings}

In addition to understanding the consumption of energy in buildings, it is also useful to look at the related GHG emissions. In recent years, as the problem of global climate change has been thrust to the forefront of the public agenda, there has been an increasing effort to quantify and ultimately minimize the release of GHG emissions from buildings. Similar to the operating energy use, buildings also produce GHG emissions from their operation. In this section, some background terms relating to 
GHG emissions will be presented, along with a discussion of the GHG emission trends in the Canadian building sector.

\subsubsection{Background on Operating GWP Statistics for Buildings}

The main reason why there has been an increase in the global temperature in recent decades is because of the presence of greenhouse gases in the atmosphere. Some of the more abundant greenhouse gases include: carbon dioxide, methane, nitrous oxide, ozone, and water vapour. These gases are unique in that they are 'spectrally selective' materials. Essentially, these gases in the Earth's atmosphere allow shortwave solar radiation to pass through them uninhibited, while at the same time not allowing longwave terrestrial radiation to pass back out. The net effect is a rise in global temperatures due to some of the terrestrial radiation being 'trapped' in the Earth's atmosphere by these spectrally selective gases.

As mentioned previously, greenhouse gases actually refer to more than just carbon dioxide. However, carbon dioxide is the most abundant GHG in the Earth's atmosphere. Therefore, scientists have developed the term call Global Warming Potential (GWP). The term, GWP was developed to compare one GHG to another in terms of their ability to trap heat in the Earth's atmosphere. GWP is measured in mass of $\mathrm{CO}_{2}$ equivalent. Carbon dioxide equivalency $\left(\mathrm{CO}_{2}\right.$ eq. $)$ is a measure of the equivalent amount of $\mathrm{CO}_{2}$ that would have the same GWP as a mixture of $\mathrm{CO}_{2}$ and other GHGs in the Earth's atmosphere. Often for the case of buildings the GWP is expressed in either $\mathrm{kg}$ of $\mathrm{CO}_{2}$ eq. or tonnes of $\mathrm{CO}_{2}$ eq.

\subsubsection{Operating GWP Statistics for Canadian Commercial Buildings}

Similar to operating energy, each year the OEE at NRCan publishes the GWP data for each sector of the Canadian economy. The data is published annually in the Energy Use Data Handbook (NRCan, OEE, 2010). The most recent data available is for 2007. According to the OEE, in 2007 the total GWP released in Canada was estimated to be about $746.7 \mathrm{Mt}$ of $\mathrm{CO}_{2}$ eq. and the total secondary GWP was about $501.6 \mathrm{Mt}$ of $\mathrm{CO}_{2}$ eq. (or about $67 \%$ of the total GWP). Figure 2-7 illustrates the breakdown of the total secondary GWP by sector in Canada in 2007. 


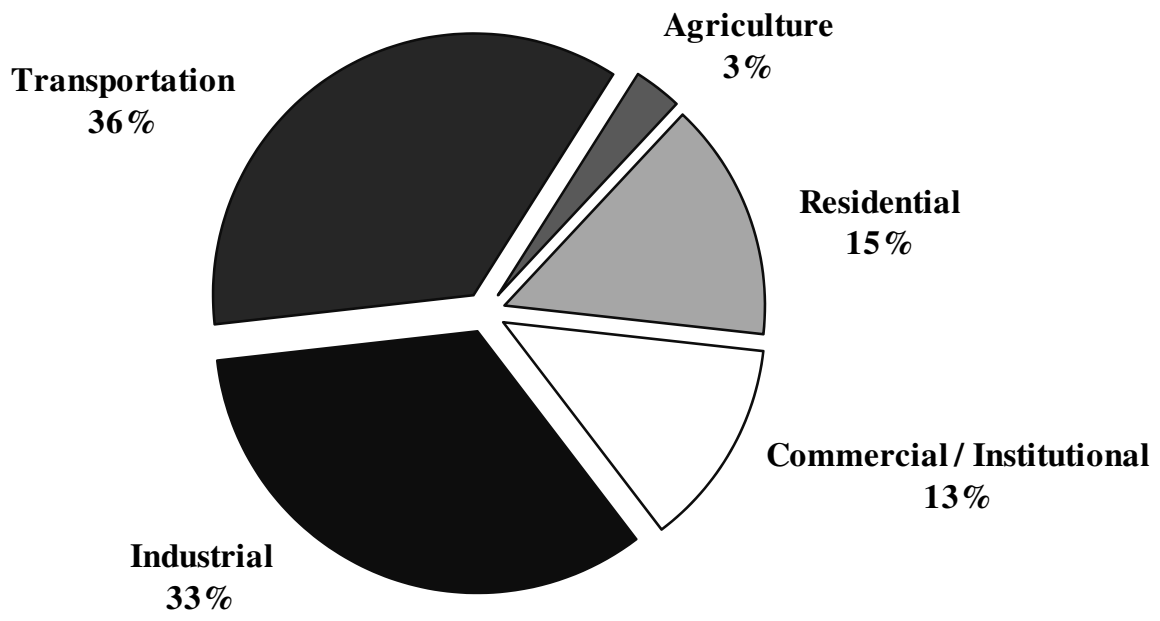

Total global warming potential(GWP) in Canada in $2007=501.6 \mathrm{Mt}$ of $\mathrm{CO}_{2}$ eq.

Figure 2-7: Breakdown of Total Secondary GWP by Sector in Canada in 2007 (NRCan, OEE, 2010)

By adding the residential and commercial/institutional sectors together, the building sector is responsible for about $28 \%$ of the total secondary GWP in Canada. This represents a significant percentage of the total GWP each year.

Next, Figure 2-8 illustrates the breakdown of commercial/institutional total secondary GWP by activity type in Canada in 2007. Office buildings account for about 35\% of the total secondary GWP followed by retail buildings at $17 \%$.

Focusing now on retail buildings specifically, Figure 2-9 illustrates the breakdown of the retail secondary GWP by end use in Canada in 2007. Not surprisingly space heating accounts for nearly half of the total secondary GWP of retail buildings. Notice how the trends in secondary GWP are very similar to the trends presented earlier for the secondary energy. There is a close relationship between energy use and GWP, as the release of greenhouse gases often accompanies the consumption of energy.

Next, Figure 2-10 illustrates the average annual GWP intensity by activity type for the commercial/institutional sector in Canada in 2007. Similarly to energy use, retail buildings produce about 1.2 times the GWP per square meter of floor area than office buildings. 


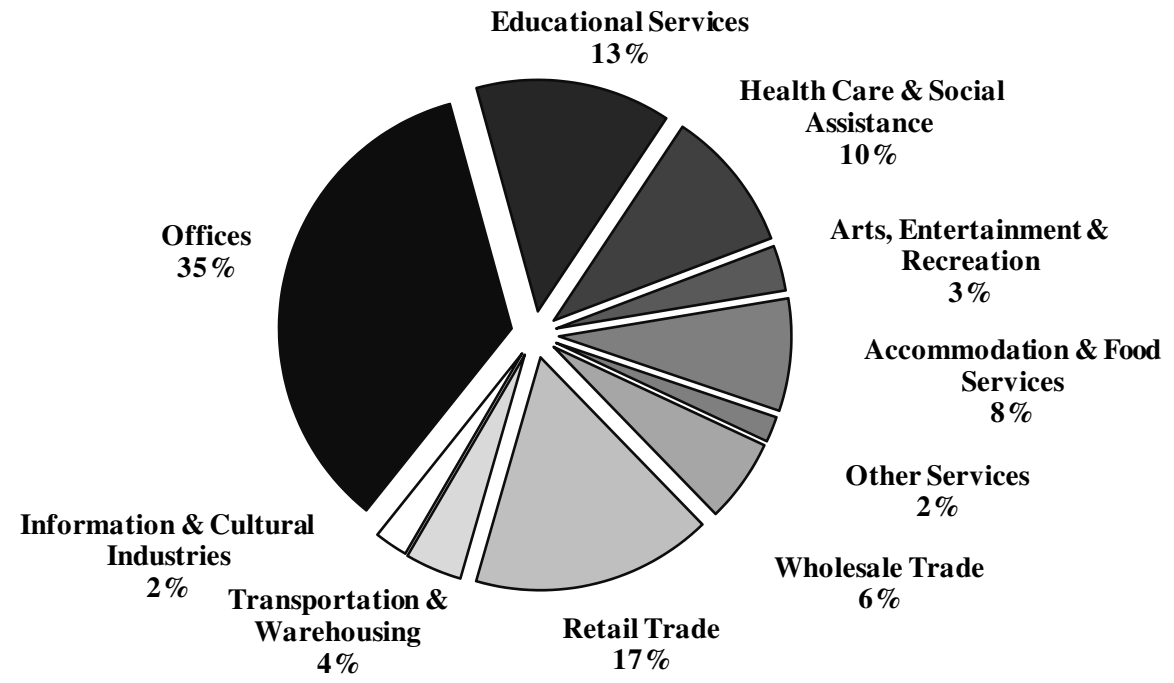

Totalcommercial/institutional global warming potential(GWP) in Canada in $2007=64.5 \mathrm{Mt}$ of $\mathrm{CO}_{2}$ eq.

Figure 2-8: Breakdown of Commercial/Institutional Total Secondary GWP by Activity Type in Canada in 2007 (NRCan, OEE, 2010)

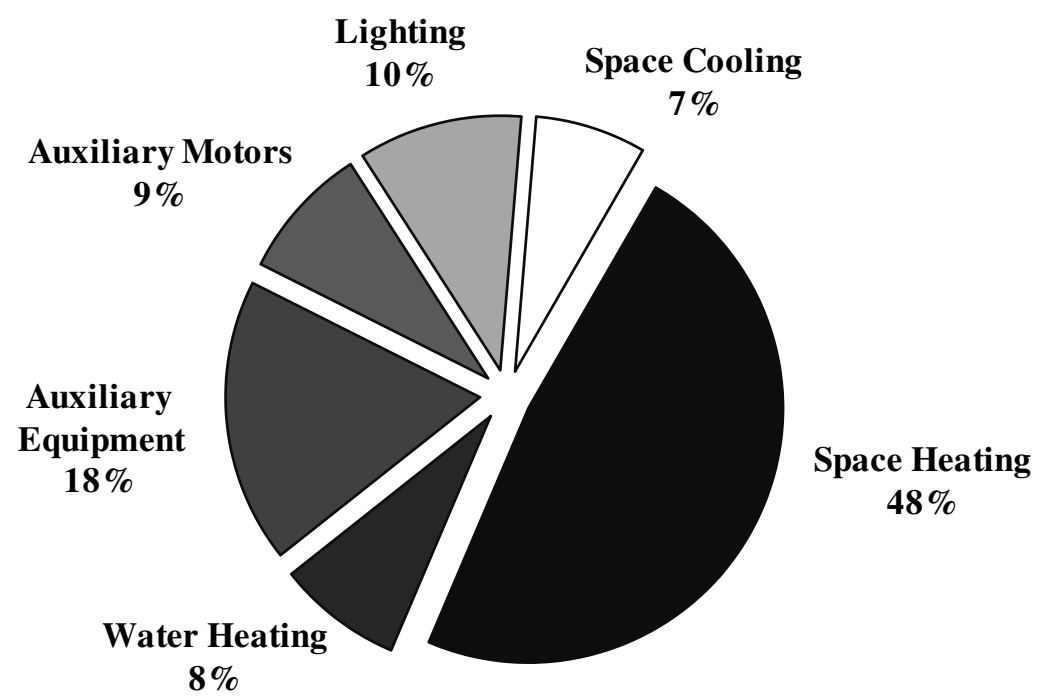

Total retail global warming potential(GWP) in Canada in $2007=10.7 \mathrm{Mt}$ of $\mathrm{CO}_{2}$ eq.

Figure 2-9: Breakdown of Retail Secondary GWP by End Use in Canada in 2007 (NRCan, OEE, 2010) 


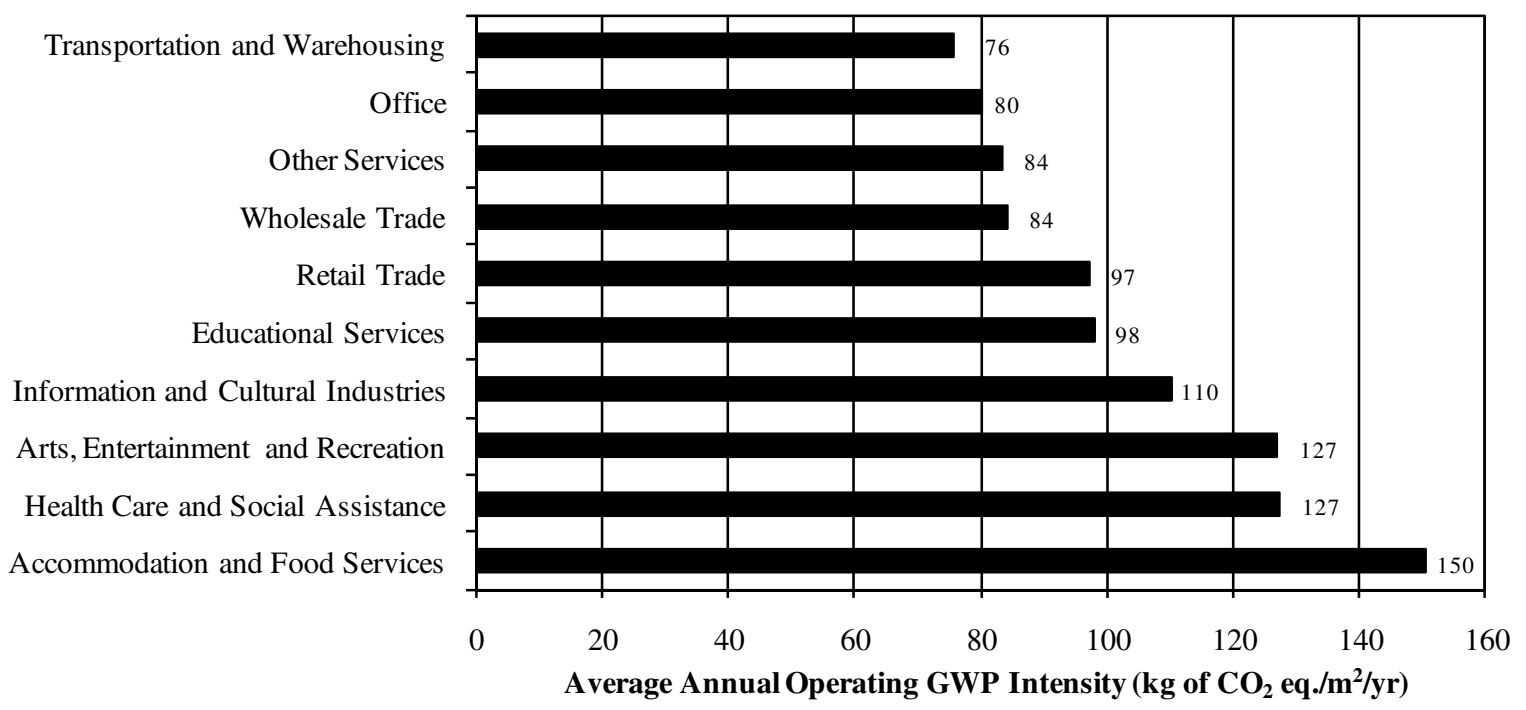

Figure 2-10: Commercial/Institutional Sector Average Annual Operating GWP Intensity by Activity in Canada for 2007 (NRCan, OEE, 2010)

\subsection{Embodied Global Warming Potential (GWP) in Buildings}

Recall that the energy use in buildings can be divided into operating energy and embodied energy. A similar division can be done for the GWP of buildings. Not only is there a release of GHG during the operating phase of a building, but there are also GHG emissions associated with the acquisition, processing, manufacturing, transportation, construction, and repair/replacement of building materials. The amount of GWP that is associated with these effects is called embodied GWP.

\subsubsection{Background on Embodied GWP in Buildings}

Embodied GWP refers to the total GWP produced in the acquisition and processing of raw materials, including manufacturing, transportation, and final installation. The embodied GWP can be calculated for a building material, a component of a building, or even an entire building project. The embodied GWP of a particular building material is usually reported in terms of $\mathrm{kg} \mathrm{of} \mathrm{CO}_{2}$ eq. per $\mathrm{kg}$ (or $\mathrm{m}^{3}$ ) of material. Similar to energy use, there are two kinds of embodied GWP: initial embodied GWP and recurring embodied GWP.

The embodied GWP of a building material depends on the GHG emissions of the industries that produce them. An accurate measure of embodied GWP is extremely difficult to determine for the various building materials. The same concerns and limitations that have already been discussed for 
the embodied energy numbers in previous sections, also apply to the case of embodied GWP numbers here. A repeat of that discussion will not be provided. However, it is important to note that the GWP numbers for building materials are even more difficult to find and exhibit a greater range of values than is the case for the embodied energy numbers. It is far easier to look at energy bills and determine how much energy was required to operate a manufacturing plant that produced a certain building material. However, since consumers do not pay for GHG emissions, there is no easy way of tracking the exact amount of GHG that is emitted during the production of a building material. These numbers can be estimated from the energy use, but such data is much harder to find and can vary significantly from one source to the next. Once again, there are no industry standard values for the embodied GWP of the various building materials. In the next section some common values for the embodied GWP of some common building materials will be presented.

\subsubsection{Embodied GWP of Common Building Materials}

To date, there are no industry standard embodied GWP numbers for the various building materials. Recall that the embodied GWP of a building material can vary (sometimes significantly) depending on the location that it is produced, the GWP intensity of the manufacturing industries, the availability of raw materials, and numerous other factors. In addition, the embodied GWP of the various building materials continually change as industries reduce their GWP emissions and get more efficient at producing their products. That being said, it is still valuable to have an understanding of the range of values that are currently cited in the literature for the embodied GWP of some common building materials. Table 2-2 lists a range of values for the embodied GWP of some common building materials.

Materials that have a high embodied energy also tend to have high embodied GWP. Those materials include things like virgin aluminum ( 8.4 to $11.2 \mathrm{~kg}$ of $\mathrm{CO}_{2}$ eq. $/ \mathrm{kg}$ ). General virgin steel has less embodied GWP (1.2 to $2.8 \mathrm{~kg}$ of $\mathrm{CO}_{2}$ eq. $/ \mathrm{kg}$ ) and can have less than this depending on the recycled content. Naturally occurring building materials like stone tend to have the least embodied GWP (0.1 $\mathrm{kg}$ of $\mathrm{CO}_{2}$ eq./kg). Similar to embodied energy, embodied GWP values have been listed for both cement and concrete.

Once again, the embodied GWP numbers presented here are by no means standard values for the industry. They have been collected from a sample of the literature and represent a range of values that one would find if they consulted the literature. It was extremely difficult to find embodied GWP 
numbers for building materials in Canada or the United States specifically, so most of the data in Table 2-2 was taken from comprehensive studies done in New Zealand and the United Kingdom.

Table 2-2: Initial Embodied $\mathrm{CO}_{2}$ of Common Building Materials

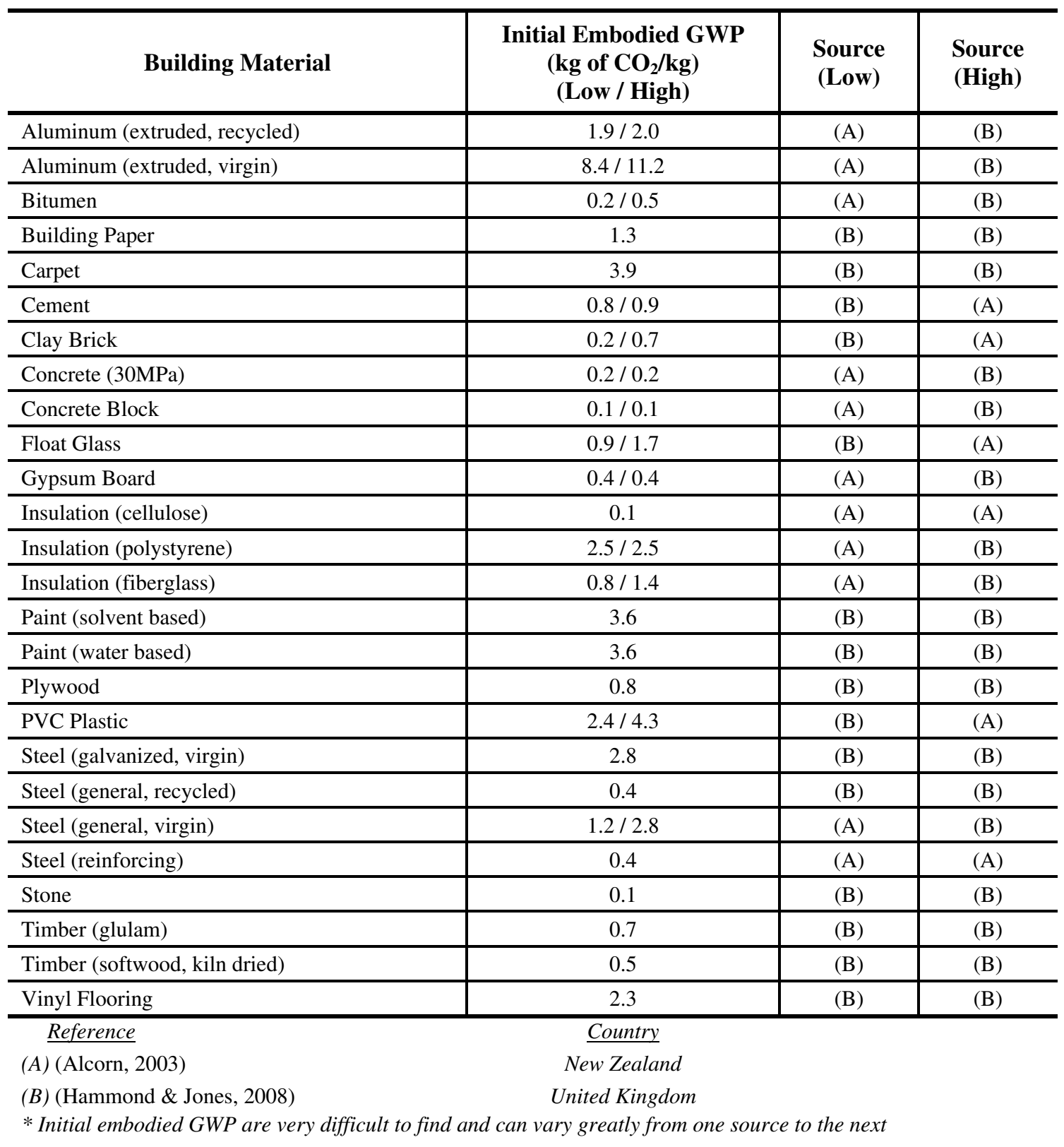




\subsubsection{Problems with Measures of Embodied GWP}

The same problems and concerns that applied to embodied energy numbers also are applicable to embodied GWP numbers. These problems include: reliance on manufactures to accurately report embodied GWP numbers, continual improvements in the manufacturing process, and variation in numbers based on location for example.

Once again, it is important to accurately account for all the material quantities in a building project, in order to get an accurate measure of the total embodied GWP. Passing judgement on the GWP of different materials without considering the greater scope of a building project is a gross simplification that is flawed. In the next section, the proper way of accounting for the true life-cycle impact of both the operating effects and embodied effects in buildings will be presented.

\subsection{Life-Cycle Assessment (LCA) of Buildings}

In recent years, the process of tracking the environmental burdens of various products and processes from cradle-to-grave has been gaining in popularity. Across most industries today, there continues to be a push to reduce energy consumption and to decrease the environmental impacts associated with all aspects of production and operation. However, only recently has the initiative to reduce the cradleto-grave environmental impacts in the building industry gained popularity.

Buildings are complex, multi-facet systems that have a direct and indirect impact on the environment. Their influence spans multiple industries and uncovering the relationships between the built and natural environment is not an easy task. Therefore, "given the complexities of interactions between the built and the natural environment, life-cycle assessment represents a comprehensive approach to examining the environmental impacts of an entire building" (Scheuer, Keoleian, \& Reppe, 2003).

\subsubsection{Background on LCA of Buildings}

The European Committee for Standardization has defined LCA to be "a method used to quantify environmental burdens based on inventory of environmental factors for a product, process, or activity from the abstraction of raw materials to their final disposal" (Lee, O'Callaghan, \& Allen, 1995). The International Organization for Standardization states that "LCA addresses the environmental aspects and potential environmental impacts (e.g. use of resources and the environmental consequences of releases) throughout a product's life-cycle from raw material acquisition through production, use, end-of-life treatment, recycling and final disposal (i.e. cradle-to-grave) (ISO, 2006). Essentially, "LCA is a method for determining the environmental and resource impacts of a material, product, or 
even a whole building over its entire life" (Kibert, 2005). Typically, "the upstream (extraction, production, transportation, and construction), use, and downstream (deconstruction and disposal) flows of a product or service are inventoried. Next, the global and/or regional impacts are calculated based on energy consumption, GWP, and other measures" (Scheuer, Keoleian, \& Reppe, 2003).

Although LCA techniques vary from once source to the next, ISO 14040 has emerged in recent years as the standard of choice. According to the International Organization for Standardization, ISO 14044 outlines the requirements of conducting a LCA and generally involves the following four phases:

1. Defining the goal and scope of the LCA

2. A life-cycle inventory (LCI) of the materials and their associated environmental impacts

3. A life-cycle impact assessment of the product or process using the LCI data

4. Interpretation of the results

The first stage of a LCA (goal and scope definition) defines the purpose, scope, and system boundaries of the LCA (ISO, 2006). In their LCA study of a new university building located on the University of Michigan campus, Scheuer, Keoleian, \& Reppe, (2003) outlined the following system boundary for their LCA.

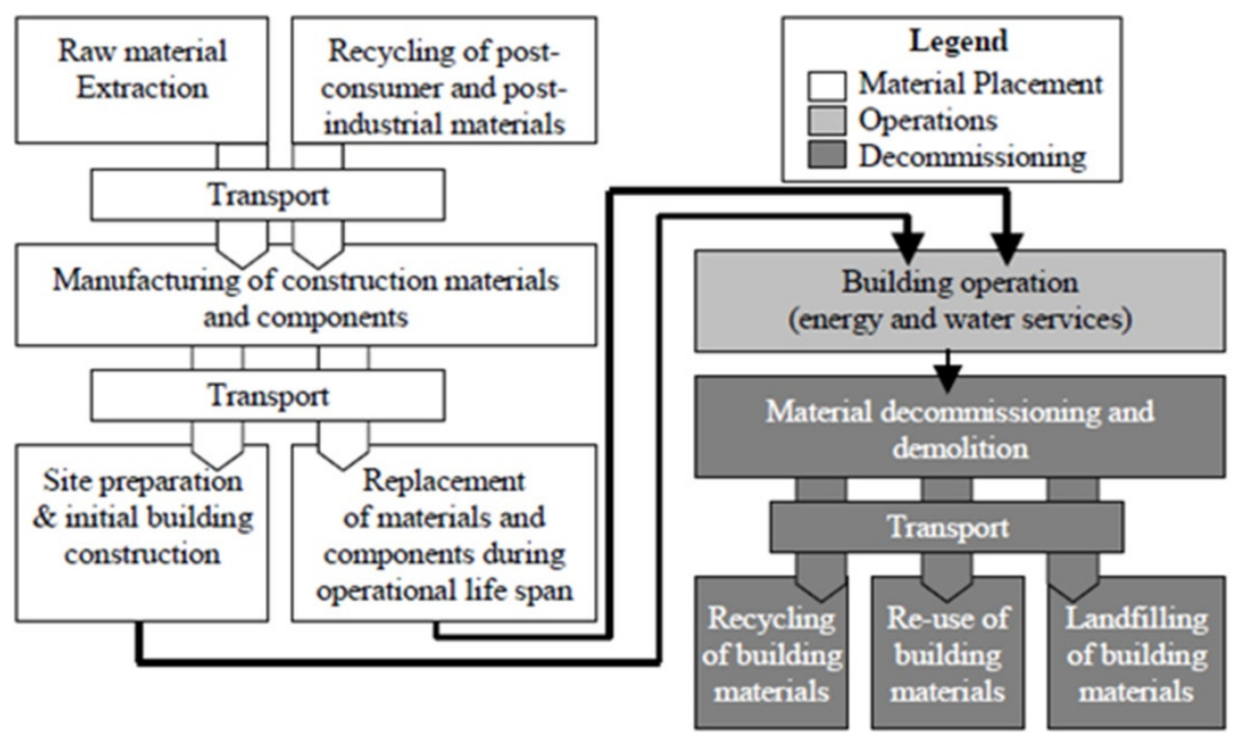

Figure 2-11: Life-Cycle Diagram for LCA of Buildings (Scheuer, Keoleian, \& Reppe, 2003) 
Stage two of a LCA (the LCI of materials) involves the collection of data to quantify the material and energy inputs and outputs of a system (ISO, 2006). Specifically, a LCI is "an objective data-based process of quantifying energy and raw material requirements, air emissions, water borne effluents, solid waste, and other environmental releases incurred throughout the life-cycle of a product, process, or activity" (Lee, O'Callaghan, \& Allen, 1995).

In stage three of a LCA, a life-cycle impact assessment is conducted using the LCI data to calculate the significance of the potential environmental impacts of the product or process. Finally, the last stage of a LCA involves interpreting the results, which is important to do in order to provide a list of findings and recommendations.

For the case of a building specifically, a life-cycle assessment involves evaluating the environmental burdens of a building throughout its lifespan. This involves calculating the environmental burdens associated with all aspects of a building from manufacturing the building materials, to constructing the building, to operating the building, to renovating or disposing of it at the end of its life. Generally speaking, this most often involves calculating the embodied effects and the operating effects of a building (or components of a building) from cradle-to-grave.

Unfortunately there is no industry standard that is followed in the building industry when it comes to conducting a LCA. In fact, from country to country and within the literature, there is considerable variation in the scope of the LCA studies, their system boundaries, and in particular, the LCI data. Collecting a detailed database of material and energy inputs and outputs for all the various building materials, construction techniques, transportation methods, etc. is enormously complex. This data is regional specific, as the methods, techniques, and energy generation (or energy use profile) of one country (or region) to the next can differ dramatically. Therefore, the LCI data in one country (or region) can be entirely different, even for the same building material, in another country (or region).

\subsubsection{Methods of Performing a LCA of Buildings}

In recent years, numerous software programs have been developed (and continue to be developed) to simplify the LCA process. At the heart of these LCA programs is their LCI database of materials. Most developers of these programs have spent significant resources populating a LCI database for the various building materials. Trusty and Horst (2005) conducted a review of the most prominent LCA software tools in North America, as well as other international tools. Table 2-3 was taken from their study and summarizes the key LCA programs that are available today and what they do. 
Table 2-3: A Summary of the Prominent LCA Tools and What They Do (Trusty \& Horst, 2005)

\section{LCA Tools and What They Do}

\begin{tabular}{|c|c|c|}
\hline \multicolumn{3}{|c|}{$\begin{array}{l}\text { Level 1A Tools: Focus is on individual products or simple building assemblies (intended for } \\
\text { use by LCA practitioners) }\end{array}$} \\
\hline SimaPro & Netherlands & \multirow{4}{*}{$\begin{array}{l}\text { While the countries of origin vary, these } \\
\text { tools can be used in different regions by } \\
\text { selecting or incorporating the appropriate } \\
\text { data. But the task is best done by LCA } \\
\text { practitioners for whom the tools are } \\
\text { intended. }\end{array}$} \\
\hline $\mathrm{GaBi}$ & Germany & \\
\hline Umberto & Germany & \\
\hline TEAM & France & \\
\hline \multicolumn{3}{|c|}{$\begin{array}{l}\text { Level 1B Tools: Focus is on individual products or simple building assemblies (intended for } \\
\text { those who want results, but detailed LCA work is done in the background) }\end{array}$} \\
\hline BEES & USA & $\begin{array}{l}\text { Combines LCA and life cycle costing. } \\
\text { Includes both brand-specific and generic } \\
\text { data. }\end{array}$ \\
\hline LCAiT & Sweden & $\begin{array}{l}\text { Streamlined LCA tool for product designers } \\
\text { and manufactures. }\end{array}$ \\
\hline TAKE-LCA & Finland & $\begin{array}{l}\text { LCA tool for comparison of HVAC } \\
\text { products, including energy content of the } \\
\text { product and energy consumption. }\end{array}$ \\
\hline \multicolumn{3}{|c|}{$\begin{array}{l}\text { Level } 2 \text { Tools: Focus on whole building or complete building assemblies or elements (tend to } \\
\text { apply from early conceptual through detailed design stages of project) }\end{array}$} \\
\hline $\begin{array}{l}\text { ATHENA Environmental } \\
\text { Impact Estimator (EIE) }\end{array}$ & Canada/USA & \multirow{7}{*}{$\begin{array}{l}\text { All of these tools use data and incorporate } \\
\text { building systems that are specific to the } \\
\text { country or regions for which they were } \\
\text { designed. }\end{array}$} \\
\hline BRI LCA (energy and $\mathrm{CO}_{2}$ ) & Japan & \\
\hline EcoQuantum & Netherlands & \\
\hline Envest & United Kingdom & \\
\hline $\begin{array}{l}\text { Green Guide to } \\
\text { Specifications }\end{array}$ & United Kingdom & \\
\hline LISA & Australia & \\
\hline LCADesign & Australia & \\
\hline \multicolumn{3}{|c|}{$\begin{array}{l}\text { Level } 3 \text { Tools: Whole building assessment frameworks encompassing a broad range of } \\
\text { environmental, economic, and social concerns relative to sustainability }\end{array}$} \\
\hline BREEAM & United Kingdom & $\begin{array}{l}\text { Uses LCA results from the Level } 2 \text { Green } \\
\text { Guide. }\end{array}$ \\
\hline GBTool & International & $\begin{array}{l}\text { Experimental platform that accepts LCA } \\
\text { results or performs rudimentary LCA } \\
\text { calculations using built-in calculators. }\end{array}$ \\
\hline Green Globes & Canada/USA & $\begin{array}{l}\text { Assigns a high percentage of resource use } \\
\text { credits based on evidence that a design } \\
\text { team has conducted LCA using recognized } \\
\text { Level } 1 \text { or } 2 \text { tools. }\end{array}$ \\
\hline
\end{tabular}

Note: This table was taken from (Trusty \& Horst, 2005)

A similar critical review of building environmental assessment tools was conducted by Haapio and

Viitaniemi (2008). In their study, they found that "the comparison of the tools and their results is 
difficult, if not impossible. For example, the tools are designed for assessing different types of buildings, they emphasise different phases of the life cycle, and they rely on different databases, guidelines, and questionnaires" (Haapio \& Viitaniemi, 2008). This highlights one of the major problems with conducting a LCA of a building: there is no clear industry standard to follow in terms of methodology or scope. The process remains extremely complicated and the results can vary, sometimes significantly, from one study to the next. As Trusty and Horst (2005) describe, "because LCA attempts to track a complex world, it remains a complex methodology" (Trusty \& Horst, 2005).

\subsubsection{Previous LCA Studies of Commercial Buildings}

Over the past 15 years, there have been an increasing number of LCA studies of buildings. Historically, most of these studies have investigated the relationships between embodied energy and operating energy for the case of residential (single dwelling or multi-unit apartment) buildings and multi-storey office buildings. Extremely few LCA studies have looked at single-storey retail buildings, which is one of the reasons that this type of building was chosen for this study.

Most of the LCA studies tend to focus on calculating the relationships between embodied energy (initial and recurring) and operating energy of a building over a set lifespan. Figure 2-12 illustrates the typical life-cycle energy use of a building.

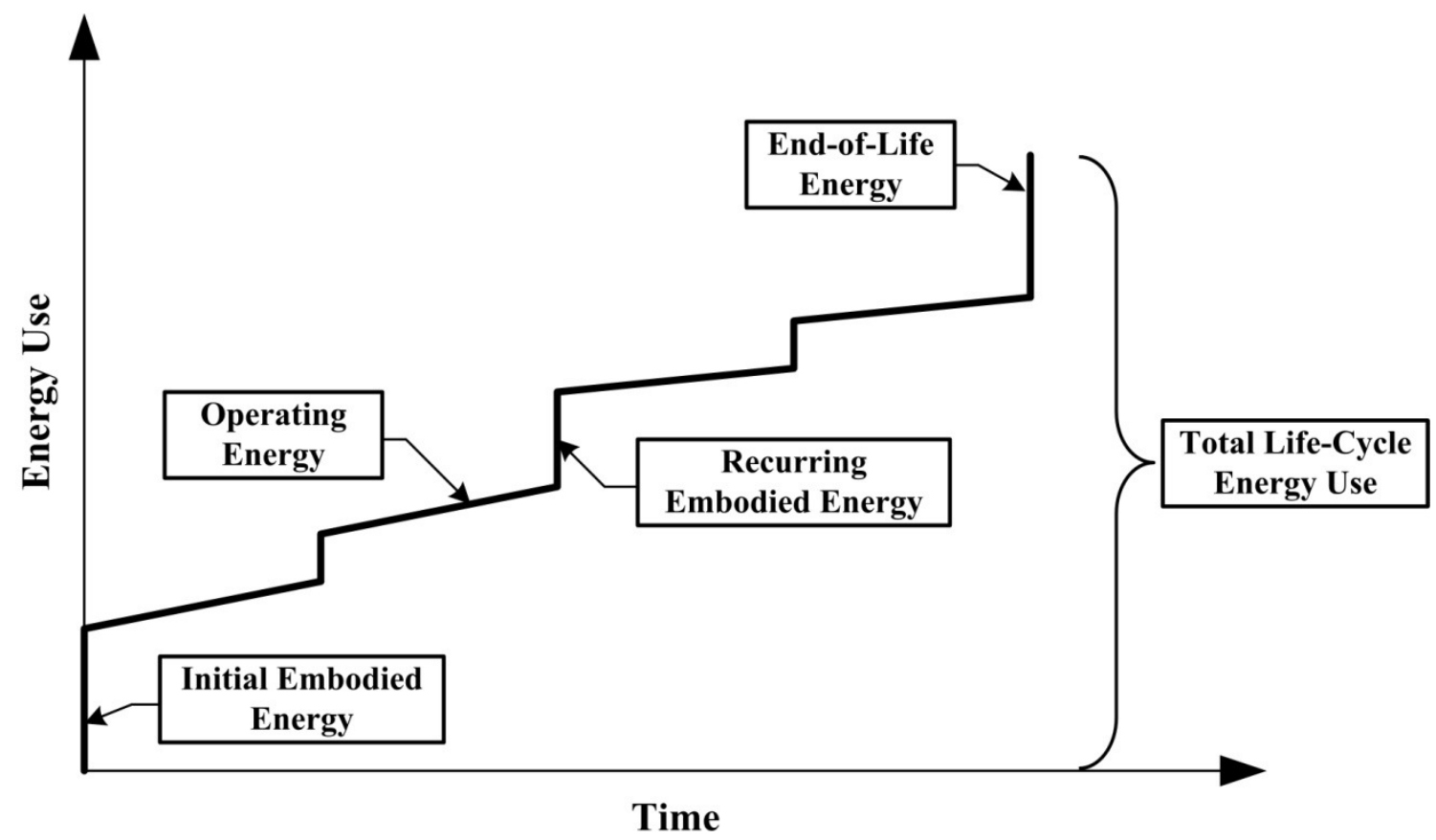

Figure 2-12: Typical Life-Cycle Energy Use of a Building (Itard \& Klunder, 2007) 
It can be seen how there is an initial amount of embodied energy that is incurred at time 0 due to the construction of the building. Then over time there is a linear increase in the total energy use due to the building operations. At certain intervals of the building's life, as materials and systems need repair and replacement, there are discrete intervals of recurring embodied energy (and possibly an end-oflife energy for decommissioning the building, depending on the scope of the LCA). At the end of the building's lifespan, the total energy use is simply the summation of the initial embodied energy, the recurring embodied energy (and the end-of-life energy if applicable), and the total life-cycle operating energy. The way in which each of these phases is calculated varies from one study to the next.

\subsubsection{Sartori and Hestne's Study}

In order to place the findings of this study into the appropriate context, it is important to have an understanding of the results from previous LCA studies of buildings. Sartori and Hestnes (2007) conducted a literature survey of the total life-cycle energy use of 60 different buildings (both residential and non-residential) from nine different countries. They focused on gathering information on the total embodied energy and operating energy of these buildings (no data was collected on GWP). The majority of buildings that were surveyed were either residential or office buildings. From their literature review, they concluded that despite climate and other differences between the case study buildings, a linear relationship between operating energy and total energy was found (Sartori \& Hestnes, 2007). In other words, the operating energy of a building has the single greatest impact on the total life-cycle energy of a building.

Sartori and Hestnes (2007) also discussed one of the systemic problems with the literature dealing with the LCA of buildings. In their literature review, Sartori and Hestnes (2007) found that there was a wide variation in how the data was presented from one study to the next. For instance, the 60 case study buildings that they looked at, all varied in terms of their lifespan, whether energy data was presented in terms of secondary or primary energy, whether only the initial embodied energy or total embodied energy was calculated, and whether end-of-life effects (such as recycling) were considered. These variations in methodology from one study to the next highlight the major problem with the literature dealing with the LCA of buildings. There is no standard methodology that is followed from one LCA study to the next. This creates a huge problem when trying to compare the results from one LCA study to another. Regardless, a review of the literature is still important, as the general trends between embodied energy and operating energy remain consistent from study to study. 
Figure 2-13 illustrates the range of total energy for the buildings examined by Sartori and Hestnes (2007) in their comprehensive literature review. It is important to note that only those studies that reported the energy in terms of primary energy (not secondary energy) were included in this graph. The total energy in each case has been divided into the total embodied energy and the total operating energy, annualized for one year. Their data gathering clearly shows the dominance of operating energy compared to embodied energy that is common throughout the literature. They demonstrated that a linear relationship between operating and total energy exists, despite climate and other contextual differences (Sartori \& Hestnes, 2007). In their study, the also showed that in the design of low energy buildings (i.e. buildings with a lower operating energy than the typical case), that low energy buildings have a net decrease in total life-cycle energy, but generally also have an increase in total embodied energy (Sartori \& Hestnes, 2007). This is because extra materials such as insulation (which is high in embodied energy) are used to help decrease the operating energy of the building.

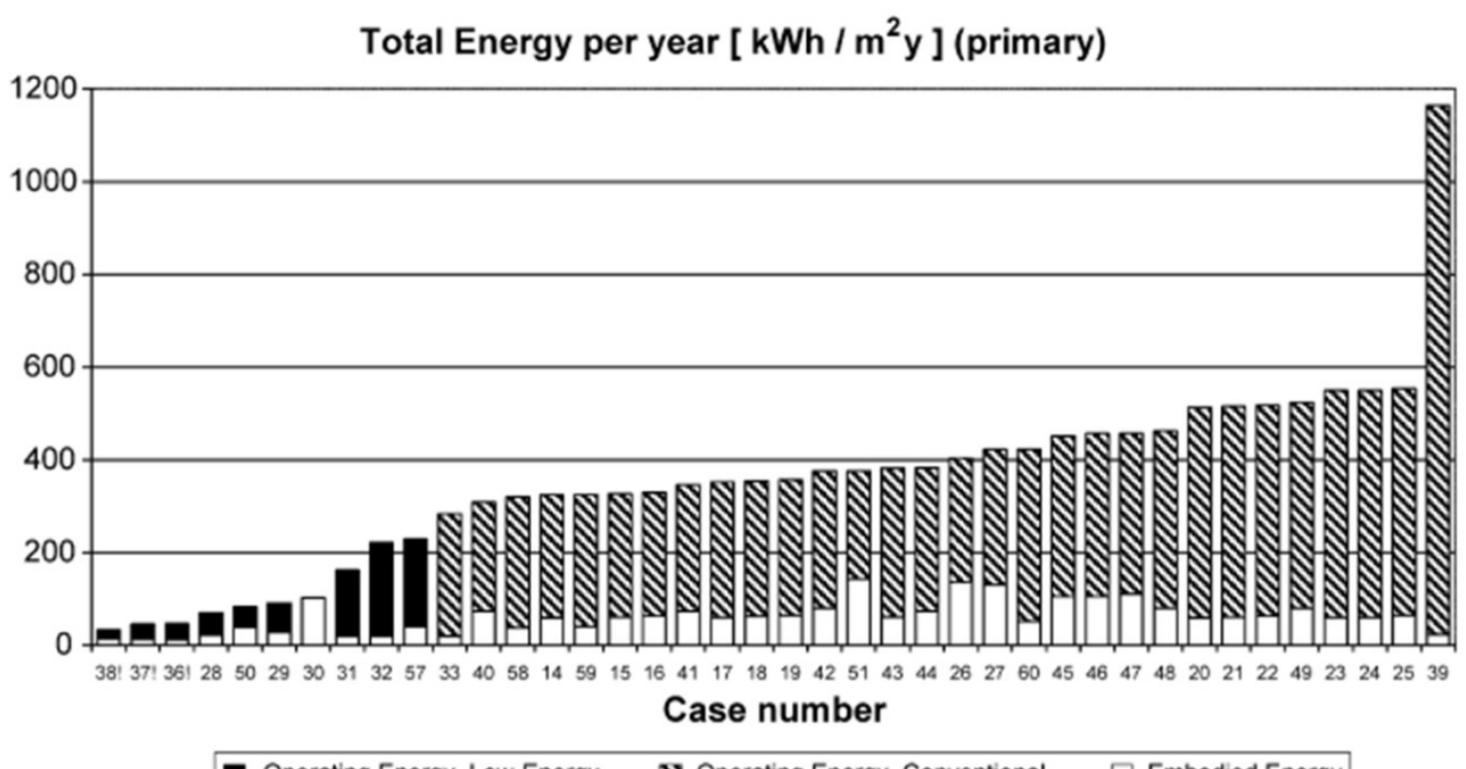

Operating Energy, Low-Energy

N Operating Energy, Conventional

Embodied Energy

Note: $1 \mathrm{kWh}=0.0036 \mathrm{GJ}$

Figure 2-13: Total Energy for a Range of Residential and Non-Residential Buildings from a Literature Review of LCA Studies (presented in terms of primary energy) (Sartori \& Hestnes, 2007) 


\subsubsection{Cole and Kernan's Study}

One of the most influential LCA studies of buildings was conducted by Cole and Kernan (1996). Their investigation of the life-cycle energy use in office buildings was one of the first studies to comprehensively investigate the relationships between embodied energy and operating energy (this study did not look at GWP). Since then, this paper has been referenced in almost every LCA study of buildings in the last 15 years.

In their investigation, Cole and Kernan looked at the relationships between initial embodied energy, recurring embodied energy, and operating energy for the case of a 4,620 $\mathrm{m}^{2}\left(50,000 \mathrm{ft}^{2}\right)$ three-storey generic office building, located in Toronto and Vancouver and constructed from alternative wood, steel, and concrete structural systems (with and without underground parking).

Figure 2-14 illustrates the breakdown of the initial embodied energy for the steel structure office building located in Toronto as determined by Cole and Kernan (1996). They found that the structure, envelope, and services accounted for $25 \%, 27 \%$, and $23 \%$ of the initial embodied energy of the threestorey office building respectfully. The initial embodied energy was less impacted by the finishes $(12 \%)$, construction $(7 \%)$, and the site work $(6 \%)$.

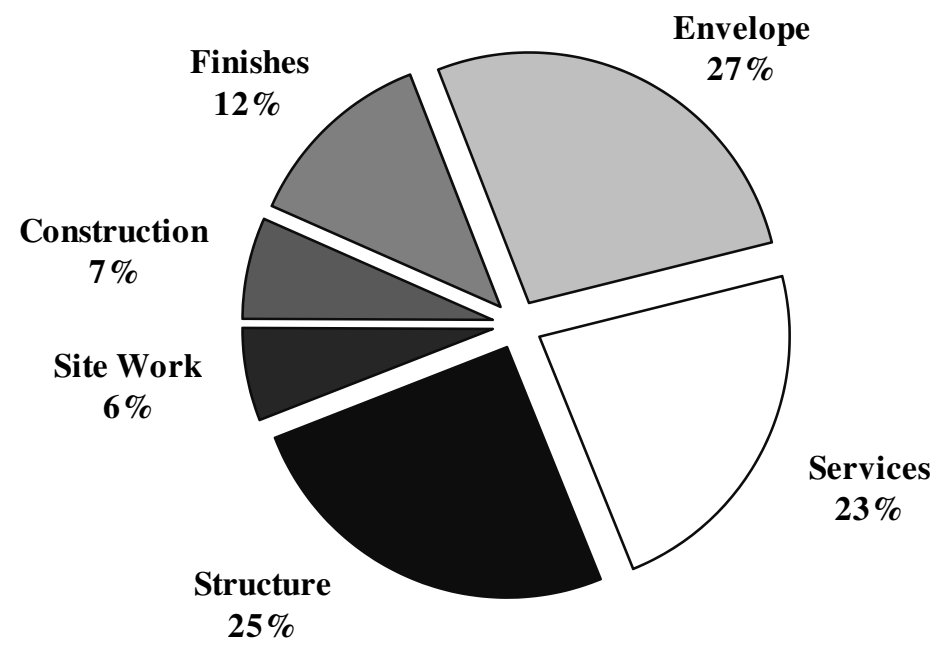

Totalinitial embodied energy $=4.86 \mathrm{GJ} / \mathrm{m}^{2}$

Figure 2-14: Breakdown of the Initial Embodied Energy for Steel Structural Office Building Located in Toronto (Cole \& Kernan, 1996)

A similar breakdown of initial embodied energy was calculated for the wood and concrete structure office buildings as well. Figure 2-15 illustrates the initial embodied energy breakdown for the three 
office buildings (wood, steel, and concrete structure) as determined by Cole and Kernan (1996). It can be seen that there is a slight variation in the initial embodied energy of the structural systems, but that very little variation exists across the three different buildings for the site work, construction, finishes, envelope, and services (HVAC, conveyance, etc.). In essence, the components of the building that are common to all three buildings (i.e. other than the structure) tend to have a moderating effect on the total initial embodied energy of the buildings.

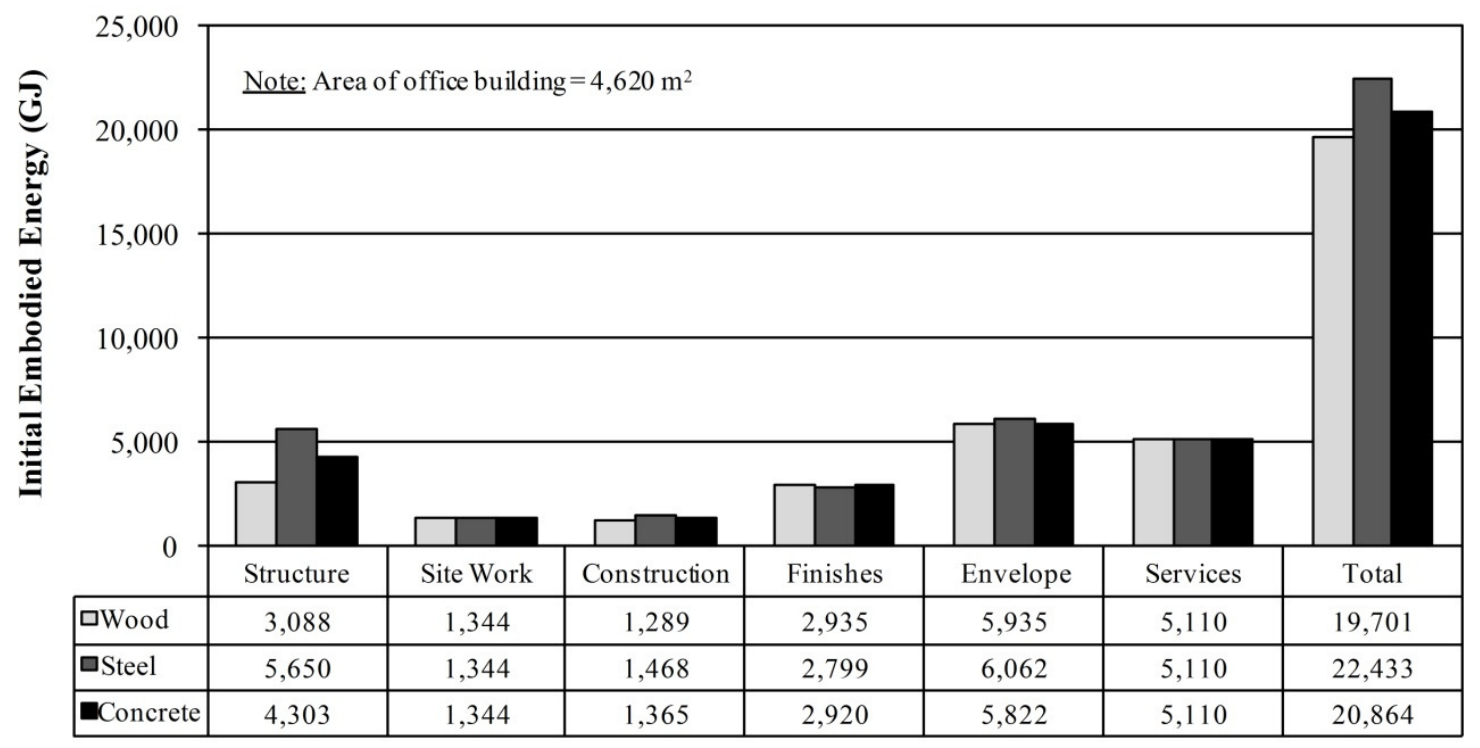

Figure 2-15: Breakdown of Initial Embodied Energy for Wood, Steel, and Concrete Structure Office Buildings Located in Toronto (Cole \& Kernan, 1996)

Looking specifically at the initial embodied energy of the structural systems for the three types of buildings, Figure 2-16 illustrates the initial embodied energy. Cole and Kernan (1996) found that the steel structure had about 1.83 and 1.31 times more initial embodied energy than the wood and concrete structural systems.

Cole and Kernan (1996) also looked at the relationships between the initial embodied energy and the recurring embodied energy for the three types of buildings. Figure 2-17 illustrates the relationships between the initial embodied energy and the recurring embodied energy for the case of the wood structure office building (the steel and concrete buildings are similar). One can see that somewhere between 25 years and 50 years, the recurring embodied energy becomes greater than the initial embodied energy. By the end of a 100 year lifespan, the recurring embodied energy is about 3.4 times greater than the initial embodied energy. Therefore, Cole and Kernan (1996) found that recurring 
embodied energy is a significant percentage of the total embodied energy of an office building after about 25 years.

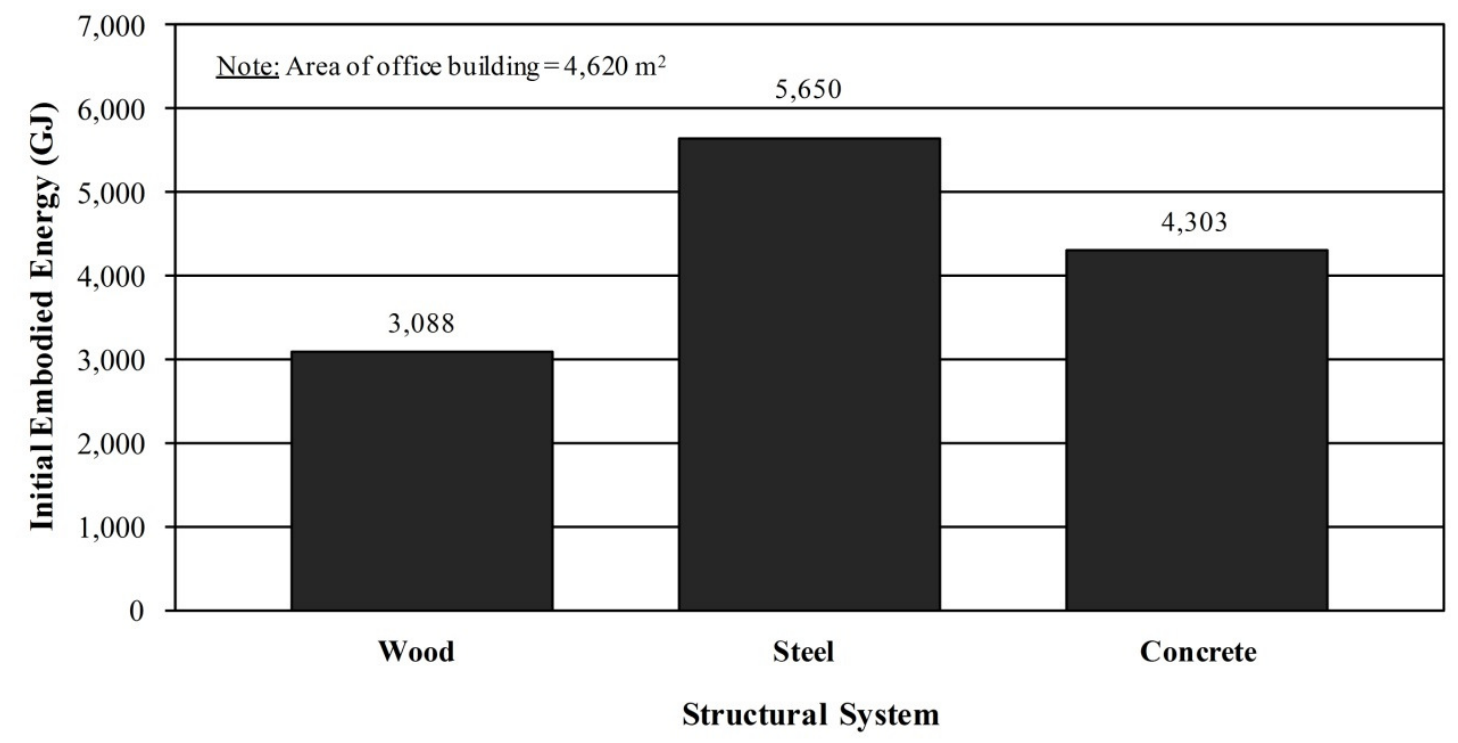

Figure 2-16: Initial Embodied Energy for Wood, Steel, and Concrete Structural Systems for Office Building Located in Toronto (Cole \& Kernan, 1996)

The relationships between the initial embodied energy and the recurring embodied energy were also determined for the steel and concrete structure office buildings. Figure 2-18 illustrates the relationships between the initial and recurring embodied energy as determined by Cole and Kernan (1996). The most important point to note is that after about 50 years, the finishes, envelope, and services completely dominate the total embodied energy of the three buildings, since there is far more recurring embodied energy associated with these building components than there is with the structure, site work, or construction. One can imagine that the structure of the building is erect at time 0 and then little to no repair/maintenance is ever done. On the contrary, the finishes in the building are often repaired or replaced many times over the life of a building. Therefore, there is far more recurring embodied energy associated with the finishes over any significant length of time. These findings by Cole and Kernan (1996) show the insignificance of the embodied energy of the structure compared to the other components of the building like the finishes, envelope, and services. 


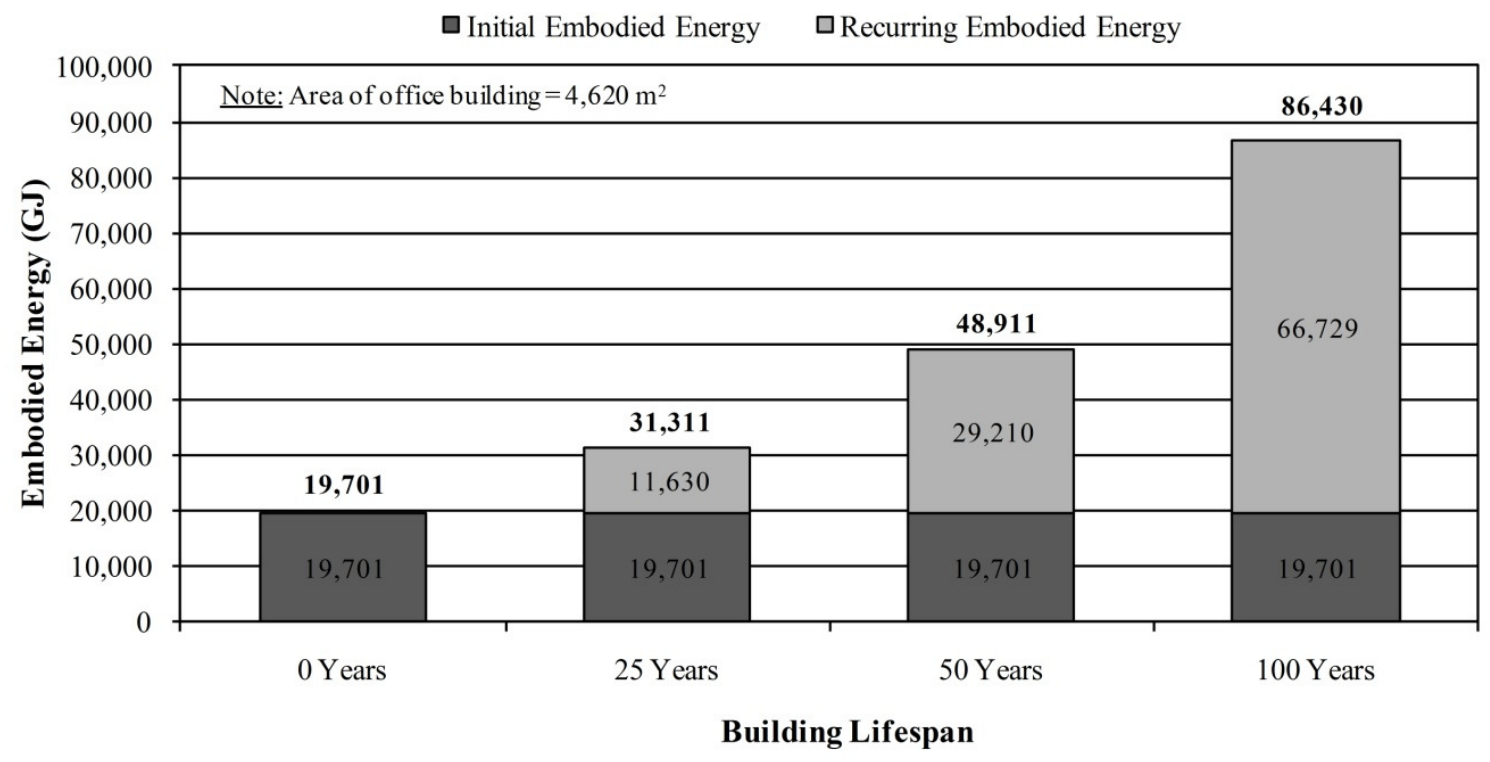

Figure 2-17: Breakdown of Initial Embodied Energy verses Recurring Embodied Energy for Wood Structure Office Building after 50 Year Lifespan in Toronto (Cole \& Kernan, 1996)

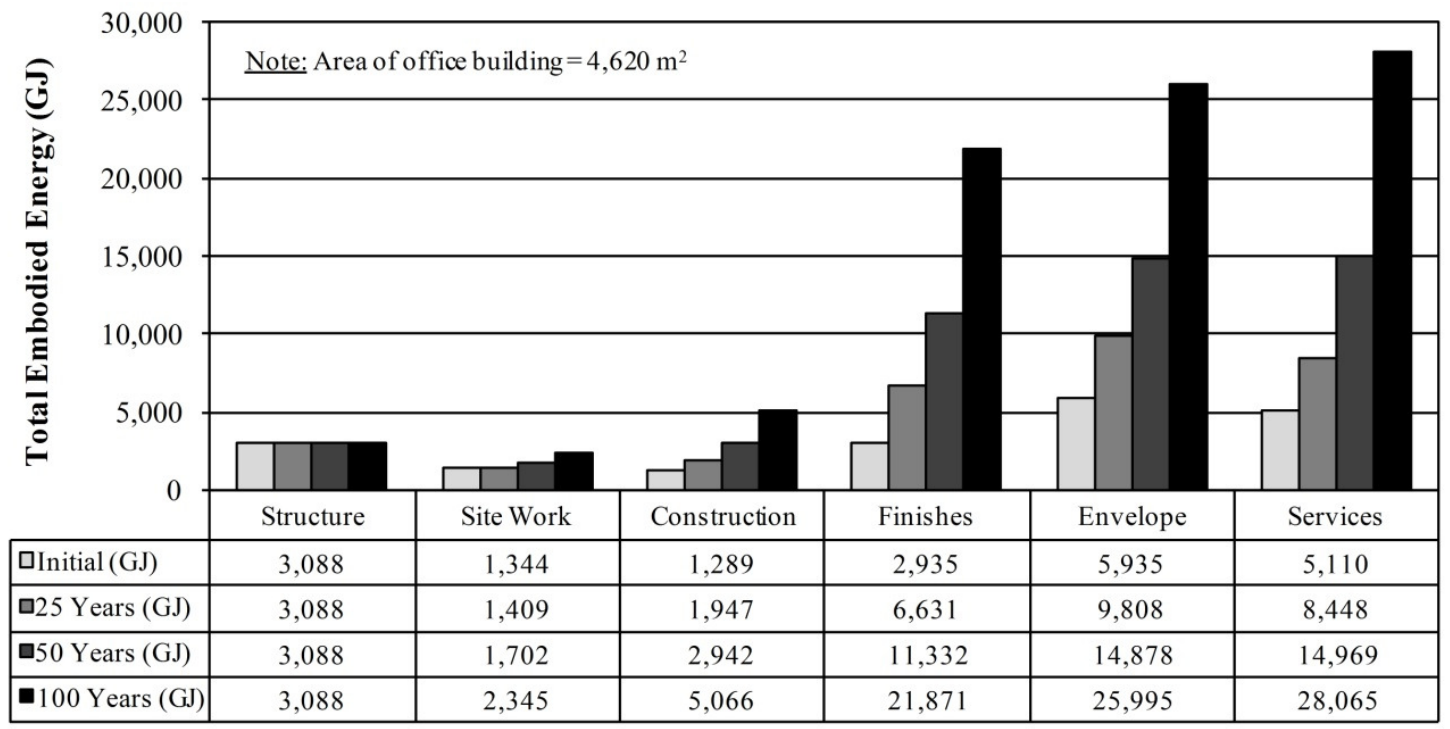

Figure 2-18: Breakdown of Total Embodied Energy for Wood Structure Office Building after 50 Year Lifespan in Toronto (Cole \& Kernan, 1996)

Cole and Kernan (1996) also calculated the operating energy of the three different office buildings. Figure 2-19 illustrates the relationships between the operating energy and the embodied energy for the three buildings. Cole and Kernan (1996) showed that after 50 years, the operating energy 
represents about $85 \%$ of the total energy, compared to only about $15 \%$ for the total embodied energy. They also determined that any differences in the embodied energy of the three different buildings are far outweighed by the similarities in the operating energy. In other words, the operating energy has a moderating effect, as it is very similar for the three buildings despite any differences in the material used for the structural system. In fact, the total energy of the steel building is only 1.01 times greater than either the wood or concrete structure office building. Therefore, Cole and Kernan (1996) showed that after 50 years of operation, the total energy is essentially identical for the three office buildings, despite the type of structural systems that is chosen.

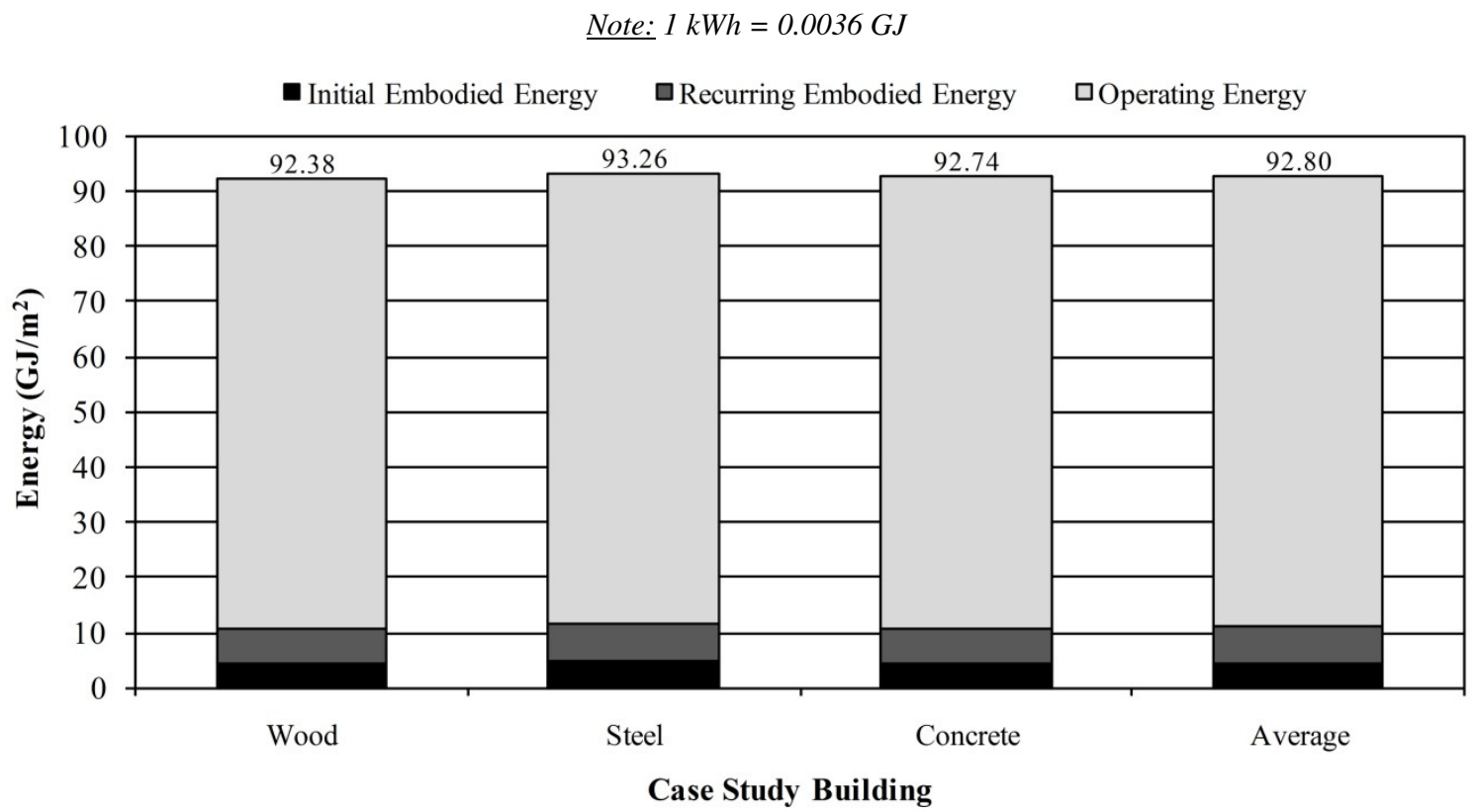

Figure 2-19: Life-Cycle Energy Use for Wood, Steel, and Concrete Structure Office Buildings after 50 Year Lifespan in Toronto (Cole \& Kernan, 1996)

Finally, Figure 2-20 was created from the data that Cole and Kernan (1996) found for the three-storey generic office building located in Toronto. The values for the wood, steel, and concrete structure buildings were averaged and the results were plotted. In this case, a linear relationship between the recurring embodied energy and time was assumed for the purposes of this figure. This figure shows how after about 10 years, that operating energy of the building begins to complete dominate the total energy of the office building. After 50 years, the operating energy represents about $88 \%$ of the total energy, compared to only about $7 \%$ for the recurring embodied energy and $5 \%$ for the initial embodied energy. 
Note: $1 \mathrm{kWh}=0.0036 \mathrm{GJ}$

\section{Life-Cycle Energy Use (Toronto)}

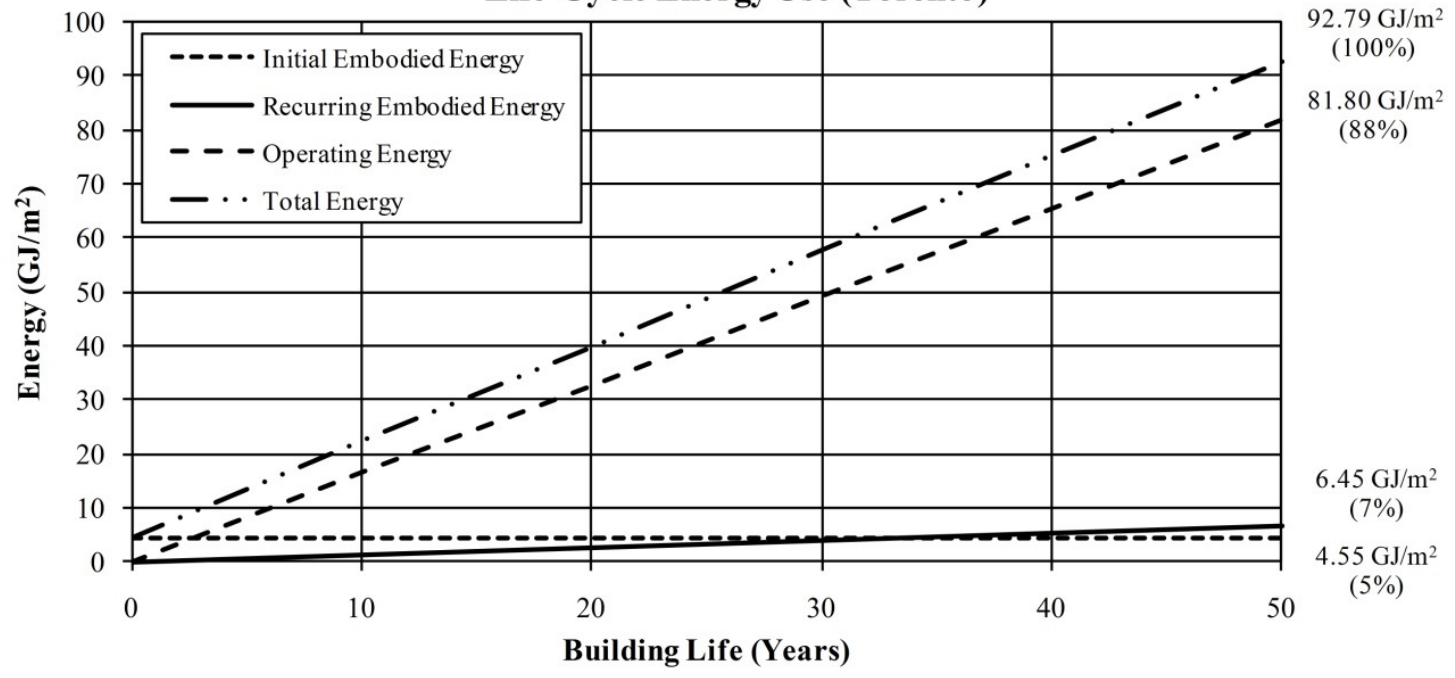

Figure 2-20: Life-Cycle Energy Use (Averaged for Wood, Steel, and Concrete Structure Office Buildings) over 50 Year Lifespan in Toronto (Cole \& Kernan, 1996)

In summary, the main conclusions from this very important and influential LCA study of commercial office buildings by Cole and Kernan (1996) are:

1. After about 50 years, the total embodied energy associated with the finishes, envelope, and services far outweighs that of the structure, site work, and construction due to higher amounts of recurring embodied energy.

2. Structure can represent a significant proportion of the initial embodied energy of a commercial office building, but after 50 years the operating energy of the building represents about $85 \%$ of the total energy compared to only about $15 \%$ for the total embodied energy of the building.

3. Not until the operating energy is reduced by about $50 \%$ from typical values, does the embodied energy of the building become significant.

4. Strategies for reducing the life-cycle energy use of an office building should focus on those design considerations that significantly reduce the building's operating energy. Only when the operating energy has been significantly reduced should the emphasis be directed at reducing the building's embodied energy. 


\subsubsection{Ding's Study}

An important LCA study that is relevant to this project was conducted by Ding (2007). In this study, Ding (2007) calculated the total energy consumption of 20 public secondary schools in New South Wales, Australia. These schools ranged in size from 1,300 $\mathrm{m}^{2}$ to $16,000 \mathrm{~m}^{2}$. In this study, Ding (2007) looked at the initial embodied energy, the recurring embodied energy, and the operating energy (not the end-of-life energy or GWP) of the schools.

In this project, the lifespan of the schools was assumed to be 60 years. The average initial embodied energy per square meter of gross floor area of the 20 schools was found to be about $7.83 \mathrm{GJ} / \mathrm{m}^{2}$. The average recurring embodied energy was determined to be about $8.19 \mathrm{GJ} / \mathrm{m}^{2}$ over the 60 year lifespan. In this study, the author also listed a range of values for the initial embodied energy and the recurring embodied energy from their literature review. For commercial buildings, the initial embodied energy per square meter of gross floor area was found to range from $3.4 \mathrm{GJ} / \mathrm{m}^{2}$ to $19.0 \mathrm{GJ} / \mathrm{m}^{2}$ (Ding, 2007). Ding (2007) also noted that the recurring embodied energy per square meter of gross floor area from the literature review ranged between $6.32 \mathrm{GJ} / \mathrm{m}^{2}$ to $20.40 \mathrm{GJ} / \mathrm{m}^{2}$ for commercial buildings. In this study, Ding (2007) found the average annual operating energy to be about $0.55 \mathrm{GJ} / \mathrm{m}^{2}$ for the 20 schools in Australia. Recall that the average annual operating energy for an educational services building in Canada is about $1.73 \mathrm{GJ} / \mathrm{m}^{2}$ (NRCan, OEE, 2010).

Ding (2007) found that the total embodied energy represented about 38\% of the total energy after 60 years, compared to $62 \%$ for the operating energy. According to Ding (2007), the total embodied energy represents about 37 years of operating energy. Ding (2007) also noted that in the literature, the total embodied energy range from 15 to 37 years of operating energy. So, one can see that the significance of embodied energy relative to operating energy in this study is relatively high compared to the literature. This is likely due to the fact that little heating energy is required for these schools due to them being located in a warm climate in Australia. If these schools were located in a cold climate, like in Canada, then it would be reasonable to expect that the operating energy would be higher.

\subsubsection{Junnila et al.'s Study}

In this study, the authors conducted a LCA of a newly constructed office building in Finland and in the Midwest region of the United States. In both instances, the lifespan of the office building was taken to be 50 years. The office building in Finland was a four-storey, concrete frame building with a 
gross floor area of about 4,400 $\mathrm{m}^{2}$. The office building in the United States was a five-storey, concrete frame building with a gross floor area of about $4,400 \mathrm{~m}^{2}$. In this study, the energy, $\mathrm{CO}_{2}$ emissions, and other measures were reported.

After 50 years of operation, Junnila et al. (2006) calculated the breakdown of total energy use for the Finnish office building to be: materials $(6.4 \%)$, construction $(2.1 \%)$, use-phase $(87.1 \%)$, maintenance (4.1\%), end-of-life $(0.3 \%)$. In terms of $\mathrm{CO}_{2}$ emissions, this breakdown was: materials $(9.8 \%)$, construction (1.5\%), use-phase (83.0\%), maintenance (5.3\%), end-of-life $(0.4 \%)$.

Similarly, after 50 years of operation, Junnila et al. (2006) calculated the breakdown of total energy use for the United States office building to be: materials (8.7\%), construction (1.5\%), use-phase (82.9\%), maintenance $(6.0 \%)$, end-of-life $(0.9 \%)$. In terms of $\mathrm{CO}_{2}$ emissions, this breakdown was: materials $(7.7 \%)$, construction (1.5\%), use-phase (85.0\%), maintenance $(5.0 \%)$, end-of-life $(0.8 \%)$.

After 50 years, the Finnish building consumed about 35\% less total energy and emitted about 49\% less total $\mathrm{CO}_{2}$ than the office building in the United States (Junnila, Horvath, \& Guggemos, 2006). In either case, despite the differences in climate and location, the two offices buildings had a similar breakdown of embodied energy (and $\mathrm{CO}_{2}$ emissions) and operating energy (and $\mathrm{CO}_{2}$ emissions).

\subsubsection{Scheuer et al.'s Study}

In this study, the authors conducted a comprehensive LCA of a $7,300 \mathrm{~m}^{2}$, six-storey university building with a projected lifespan of 75 years. The building is located on the University of Michigan campus.

A complete inventory of all the materials was conducted covering the building structure, envelope, interior structure and finishes, as well as the utility and sanitary systems (Scheuer, Keoleian, \& Reppe, 2003). As well, energy modelling was conducted to determine the primary energy use associated with the heating, cooling, ventilation, lighting, hot water, and sanitary water consumption. Demolition and end-of-life effects were also accounted for.

The total life-cycle primary energy intensity over the building's lifespan was calculated by the authors to be about $316 \mathrm{GJ} / \mathrm{m}^{2}$. The production of the building materials, their transportation to site, and construction of the building was responsible for about $2.2 \%\left(7.0 \mathrm{GJ} / \mathrm{m}^{2}\right)$ of the total life-cycle primary energy of the building. HVAC, electricity, and water services (i.e. building operations) accounted for $97.7 \%\left(309 \mathrm{GJ} / \mathrm{m}^{2}\right)$ of the total life-cycle primary energy. Only about $0.2 \%(0.63$ $\mathrm{GJ} / \mathrm{m}^{2}$ ) of the total life-cycle primary energy intensity was attributed to the building demolition. In 
this case, the operating phase primary energy demand exceeds the total embodied energy after only about 3.1 years.

In this particular study, the authors also calculated the GWP of the building. The total life-cycle GWP over the building's lifespan was calculated to be about 18.5 tonnes of $\mathrm{CO}_{2}$ eq. $/ \mathrm{m}^{2}$. They found that the operating phase alone accounted for about $96.5 \%$ (17.8 tonnes of $\mathrm{CO}_{2}$ eq. $\left./ \mathrm{m}^{2}\right)$ of the total life-cycle GWP. The production of the building materials, their transportation to site, and construction of the building was responsible for about 3.2\% (0.59 tonnes of $\mathrm{CO}_{2}$ eq. $\left./ \mathrm{m}^{2}\right)$ of the total life-cycle GWP. Only about $0.2 \%$ (0.04 tonnes of $\mathrm{CO}_{2}$ eq. $\left./ \mathrm{m}^{2}\right)$ of the total life-cycle GWP was attributed to the building demolition.

The findings in this study are much the same as other LCA studies of buildings. The operating phase was by far the greatest contributor to the total life-cycle energy and GWP. The authors noted that one of the greatest limitations on the applicability of LCA research is that it is difficult to do without the building having already been constructed. In fact, they state that "in order for life-cycle modeling to fulfill its potential in assisting design decisions, there is a need for detailed data on specific building systems and components" (Scheuer, Keoleian, \& Reppe, 2003).

\subsubsection{John et al.'s Study}

This study is one of the most comprehensive LCA studies of multi-storey buildings that have been conducted to date. The study was completed for the Ministry of Agriculture and Forestry in New Zealand.

In this study, the authors modelled the life-cycle performance of four similar office buildings constructed from concrete, steel, timber, and timber-plus (similar to timber building but timber was also used for the exterior cladding, windows, and ceiling). All the buildings were based on an actual six-storey 4,200 $\mathrm{m}^{2}$ office building located in Ne Zealand. Both the primary energy and the GWP were calculated over the life of the building for the various building components. The study assumed a 60 year lifespan for all the buildings.

The authors determined that in every case, the life-cycle operating energy and operating GWP of all four buildings contributed towards the total life-cycle energy and total GWP far more than the total life-cycle embodied energy and embodied GWP. In particular, the total life-cycle operating energy (and total life-cycle operating GWP) of the concrete, steel, timber, and timber-plus buildings were $89 \%$ (72\%), $87 \%(73 \%), 91 \%(86 \%)$, and $94 \%$ (95\%) of the total life-cycle energy (and total life- 
cycle GWP) (John, Nebel, Perez, \& Buchanan, 2008). On the other hand, the total life-cycle embodied energy (and total life-cycle embodied GWP) of the concrete, steel, timber, and timber-plus buildings were $10 \%(25 \%), 12 \%(25 \%), 8 \%(18 \%)$, and $6 \%(13 \%)$ of the total life-cycle energy (and total life-cycle GWP) (John, Nebel, Perez, \& Buchanan, 2008).

Once more, this study showed that for a typical multi-storey office building, the operating effects far outweigh the embodied effects after 60 years, even for buildings that use different materials.

\subsubsection{A Summary of Previous LCA Studies of Buildings}

So far, a brief summary of the LCA studies of buildings that are relevant to this project have been presented. However, it would also be useful to have a summary of a wider scope of LCA studies of commercial buildings, in order to understand the range of values for the total life-cycle energy and GWP of commercial buildings. In this section, the results from a number of LCA studies of buildings have been summarized in Table 2-4.

Only those LCA studies that focus on commercial type buildings have been included in Table 2-4. These include office buildings, schools, and mixed-use buildings (combination of office, retail, and other). A preference was taken towards those LCA studies that looked at buildings located in Canada. Both the operating effects and the embodied effects were recorded for each study. It was decided not to include residential buildings in this review, as residential buildings tend to have a different operating schedule than commercial buildings and are generally constructed using different systems. Therefore, a focus on commercial type buildings was taken.

From Table 2-4, it can be seen that the values for annual total life-cycle operating energy per gross floor area varied from 0.23 to $4.23 \mathrm{GJ} / \mathrm{m}^{2} / \mathrm{yr}$. Similarly, the operating GWP varied from 0.02 to 0.24 tonnes of $\mathrm{CO}_{2}$ eq. $/ \mathrm{m}^{2} / \mathrm{yr}$. The total life-cycle embodied energy per gross floor area varied from 3.42 to $22.45 \mathrm{GJ} / \mathrm{m}^{2}$. Likewise, the total embodied GWP varied from 0.20 to 0.89 tonnes of $\mathrm{CO}_{2} \mathrm{eq} / \mathrm{m}^{2}$. 
Table 2-4: A Summary of Relevant LCA Studies of Commercial Buildings

\begin{tabular}{|c|c|c|c|c|c|c|c|c|c|c|}
\hline \multirow[b]{2}{*}{ Source } & \multirow[b]{2}{*}{ Location } & \multirow{2}{*}{$\begin{array}{c}\text { Type of } \\
\text { Building } \\
\text { (\# of } \\
\text { Storeys) }\end{array}$} & \multirow{2}{*}{$\begin{array}{c}\text { Gross } \\
\text { Floor } \\
\text { Area } \\
\left(\mathrm{m}^{2}\right)\end{array}$} & \multirow[b]{2}{*}{$\begin{array}{l}\text { Lifespan } \\
\text { (years) }\end{array}$} & \multicolumn{2}{|c|}{$\begin{array}{l}\text { Annual Total Life-Cycle } \\
\text { Operating Effects per } \\
\text { Gross Floor Area }\end{array}$} & \multicolumn{4}{|c|}{$\begin{array}{c}{ }^{2} \text { Total Life-Cycle Embodied Effects per Gross Floor } \\
\text { Area (after Lifespan) }\end{array}$} \\
\hline & & & & & $\begin{array}{c}1 \text { Operating } \\
\text { Energy } \\
\left(\mathbf{G J} / \mathrm{m}^{2} / \mathbf{y r}\right)\end{array}$ & $\begin{array}{c}\text { Operating } \\
\text { GWP } \\
\text { (tonnes of } \\
\mathrm{CO}_{2} \\
\text { eq. } / \mathrm{m}^{2} / \mathrm{yr} \text { ) }\end{array}$ & $\begin{array}{l}\text { Initial } \\
\text { Embodied } \\
\text { Energy } \\
\left(\mathbf{G J} / \mathbf{m}^{2}\right)\end{array}$ & $\begin{array}{l}\text { Recurring } \\
\text { Embodied } \\
\text { Energy } \\
\left(\mathbf{G J} / \mathbf{m}^{2}\right)\end{array}$ & $\begin{array}{l}\text { Total } \\
\text { Embodied } \\
\text { Energy } \\
\left(\mathbf{G J} / \mathbf{m}^{2}\right)\end{array}$ & \begin{tabular}{|c} 
Total \\
Embodied \\
GWP \\
(tonnes of \\
$\left.\mathrm{CO}_{2} \mathrm{eq} . / \mathrm{m}^{2}\right)$
\end{tabular} \\
\hline $\begin{array}{c}(\text { Cole \& } \\
\text { Kernan, 1996) }\end{array}$ & $\begin{array}{l}\text { Toronto, } \\
\text { Canada }\end{array}$ & $\begin{array}{c}{ }^{3} \text { OFF, } \\
\text { Timber } \\
\text { Frame (3) }\end{array}$ & 4,620 & 50 & ${ }^{4} 1.64(\mathrm{U})$ & N/A & 4.26 & 6.32 & 10.58 & N/A \\
\hline $\begin{array}{c}(\text { Cole \& } \\
\text { Kernan, 1996) }\end{array}$ & $\begin{array}{l}\text { Toronto, } \\
\text { Canada }\end{array}$ & $\begin{array}{c}{ }^{3} \text { OFF, } \\
\text { Steel } \\
\text { Frame (3) }\end{array}$ & 4,620 & 50 & ${ }^{4} 1.64(\mathrm{U})$ & N/A & 4.86 & 6.60 & 11.46 & N/A \\
\hline $\begin{array}{c}(\text { Cole \& } \\
\text { Kernan, 1996) }\end{array}$ & $\begin{array}{l}\text { Toronto, } \\
\text { Canada }\end{array}$ & $\begin{array}{c}{ }^{3} \mathrm{OFF}, \\
\text { Concrete } \\
\text { Frame (3) }\end{array}$ & 4,620 & 50 & ${ }^{4} 1.64(\mathrm{U})$ & N/A & 4.52 & 6.42 & 10.94 & N/A \\
\hline $\begin{array}{c}5 \text { (Sartori \& } \\
\text { Hestnes, 2007) }\end{array}$ & Varies & Varies & Varies & Varies & $\begin{array}{l}0.23 \text { to } \\
4.23(\mathrm{P})\end{array}$ & N/A & N/A & N/A & $\begin{array}{c}0.04 \\
\mathrm{GJ} / \mathrm{m}^{2} / \mathrm{yr} \text { to } \\
0.5 \mathrm{GJ} / \mathrm{m}^{2} / \mathrm{yr}\end{array}$ & N/A \\
\hline $\begin{array}{l}\text { (Junnila, } \\
\text { Horvath, \& } \\
\text { Guggemos, } \\
\text { 2006) }\end{array}$ & Finland & $\begin{array}{c}\text { OFF, } \\
\text { Concrete } \\
\text { Frame (4) }\end{array}$ & 4,400 & 50 & $0.93(\mathrm{U})$ & ${ }^{6} 0.05$ & 4.50 & 2.16 & ${ }^{7} 6.84$ & ${ }^{6} 0.51$ \\
\hline $\begin{array}{c}\text { (Junnila, } \\
\text { Horvath, \& } \\
\text { Guggemos, } \\
\text { 2006) }\end{array}$ & $\begin{array}{l}\text { Midwest, } \\
\text { U.S.A. }\end{array}$ & $\begin{array}{c}\text { OFF, } \\
\text { Concrete } \\
\text { Frame (5) }\end{array}$ & 4,400 & 50 & $1.35(\mathrm{U})$ & ${ }^{6} 0.10$ & 8.32 & 4.91 & ${ }^{7} 13.98$ & ${ }^{6} 0.89$ \\
\hline $\begin{array}{c}\text { (Morrison } \\
\text { Hershfield Ltd., } \\
2009)\end{array}$ & $\begin{array}{l}\text { Ottawa, } \\
\text { Canada }\end{array}$ & $\begin{array}{c}\text { MIX, } \\
\text { Concrete } \\
\text { Frame (2) }\end{array}$ & 462 & 60 & N/A & N/A & N/A & N/A & ${ }^{7} 14.12$ & ${ }^{7} 0.75$ \\
\hline $\begin{array}{c}\text { (Morrison } \\
\text { Hershfield Ltd., } \\
\text { 2009) }\end{array}$ & $\begin{array}{l}\text { Winnipeg, } \\
\text { Canada }\end{array}$ & $\begin{array}{c}\text { OFF, } \\
\text { Steel and } \\
\text { Wood } \\
\text { Frame (4) }\end{array}$ & 3,030 & 60 & N/A & N/A & N/A & N/A & ${ }^{7} 18.69$ & ${ }^{7} 0.80$ \\
\hline $\begin{array}{c}\text { (Morrison } \\
\text { Hershfield Ltd., } \\
\text { 2009) }\end{array}$ & $\begin{array}{l}\text { Alberta, } \\
\text { Canada }\end{array}$ & $\begin{array}{c}\text { MIX, } \\
\text { Concrete } \\
\text { and Steel } \\
\text { Frame (6) }\end{array}$ & 8,882 & 60 & N/A & N/A & N/A & N/A & ${ }^{7} 11.21$ & ${ }^{7} 0.81$ \\
\hline $\begin{array}{c}\text { (Morrison } \\
\text { Hershfield Ltd., } \\
\text { 2009) }\end{array}$ & $\begin{array}{l}\text { Vancouver, } \\
\text { Canada }\end{array}$ & $\begin{array}{c}\text { MIX, } \\
\text { Concrete } \\
\text { and Steel } \\
\text { Frame (4) }\end{array}$ & 1,360 & 60 & N/A & N/A & N/A & N/A & ${ }^{7} 12.57$ & ${ }^{7} 0.68$ \\
\hline $\begin{array}{l}\text { (Treloar, Fay, } \\
\text { Ilozor, \& Love, } \\
\text { 2001) }\end{array}$ & $\begin{array}{c}\text { Melbourne, } \\
\text { Australia }\end{array}$ & $\begin{array}{c}\text { OFF, } \\
\text { Concrete } \\
\text { Frame (3) }\end{array}$ & 6,480 & N/A & N/A & N/A & 10.70 & N/A & N/A & N/A \\
\hline${ }^{8}$ (Ding, 2007) & $\begin{array}{c}\text { New South } \\
\text { Wales, } \\
\text { Australia }\end{array}$ & $\begin{array}{c}\mathrm{SCH} \\
\text { (varies) }\end{array}$ & Varies & 60 & $\begin{array}{l}0.29 \text { to } \\
1.61(\mathrm{P})\end{array}$ & N/A & $\begin{array}{c}2.95 \text { to } \\
12.96\end{array}$ & $\begin{array}{c}5.87 \text { to } \\
9.49\end{array}$ & $\begin{array}{l}8.83 \text { to } \\
22.45\end{array}$ & N/A \\
\hline $\begin{array}{c}\text { (Scheuer, } \\
\text { Keoleian, \& } \\
\text { Reppe, 2003) }\end{array}$ & $\begin{array}{l}\text { Michigan, } \\
\text { U.S.A. }\end{array}$ & $\begin{array}{c}\mathrm{SCH}, \\
\text { Steel } \\
\text { Frame (6) }\end{array}$ & 7,300 & 75 & $4.12(\mathrm{P})$ & 0.24 & N/A & N/A & 7.63 & 0.63 \\
\hline $\begin{array}{c}\text { (John, Nebel, } \\
\text { Perez, \& } \\
\text { Buchanan, } \\
\text { 2008) }\end{array}$ & $\begin{array}{c}\text { New } \\
\text { Zealand }\end{array}$ & $\begin{array}{c}\text { OFF, } \\
\text { Steel } \\
\text { Frame (6) }\end{array}$ & 4,200 & 60 & $0.55(\mathrm{P})$ & 0.02 & 0.52 & 0.52 & ${ }^{7} 5.09$ & 0.45 \\
\hline
\end{tabular}


Table 2-4 (Cont.): A Summary of Relevant LCA Studies of Commercial Buildings

\begin{tabular}{|c|c|c|c|c|c|c|c|c|c|c|}
\hline \multirow[b]{2}{*}{ Source } & \multirow[b]{2}{*}{ Location } & \multirow{2}{*}{$\begin{array}{l}\text { Type of } \\
\text { Building } \\
\text { (\# of } \\
\text { Storeys) }\end{array}$} & \multirow{2}{*}{$\begin{array}{l}\text { Gross } \\
\text { Floor } \\
\text { Area } \\
\left(\mathbf{m}^{2}\right)\end{array}$} & \multirow[b]{2}{*}{$\begin{array}{c}\text { Lifespan } \\
\text { (years) }\end{array}$} & \multicolumn{2}{|c|}{$\begin{array}{c}\text { Annual Total Life-Cycle } \\
\text { Operating Effects per } \\
\text { Gross Floor Area }\end{array}$} & \multicolumn{4}{|c|}{$\begin{array}{c}{ }^{2} \text { Total Life-Cycle Embodied Effects per Gross Floor } \\
\text { Area (after Lifespan) }\end{array}$} \\
\hline & & & & & $\begin{array}{l}{ }^{1} \text { Operating } \\
\text { Energy } \\
\left(\mathbf{G J} / \mathbf{m}^{2} / \mathbf{y r}\right)\end{array}$ & $\begin{array}{c}\text { Operating } \\
\text { GWP } \\
\text { (tonnes of } \\
\mathrm{CO}_{2} \\
\text { eq. } / \mathrm{m}^{2} / \mathrm{yr} \text { ) }\end{array}$ & $\begin{array}{l}\text { Initial } \\
\text { Embodied } \\
\text { Energy } \\
\left(\mathbf{G J} / \mathbf{m}^{2}\right)\end{array}$ & $\begin{array}{l}\text { Recurring } \\
\text { Embodied } \\
\text { Energy } \\
\left(\mathbf{G J} / \mathbf{m}^{2}\right)\end{array}$ & $\begin{array}{l}\text { Total } \\
\text { Embodied } \\
\text { Energy } \\
\left(\mathbf{G J} / \mathbf{m}^{2}\right)\end{array}$ & \begin{tabular}{|c|} 
Total \\
Embodied \\
GWP \\
$($ tonnes of \\
$\mathrm{CO}_{2}$ eq. $\left./ \mathrm{m}^{2}\right)$ \\
\end{tabular} \\
\hline $\begin{array}{l}\text { (John, Nebel, } \\
\text { Perez, \& } \\
\text { Buchanan, } \\
\text { 2008) }\end{array}$ & $\begin{array}{c}\text { New } \\
\text { Zealand }\end{array}$ & $\begin{array}{c}\text { OFF, } \\
\text { Concrete } \\
\text { Frame (6) }\end{array}$ & 4,200 & 60 & $0.54(\mathrm{P})$ & 0.02 & 3.28 & 0.41 & 4.15 & 0.45 \\
\hline $\begin{array}{l}\text { (John, Nebel, } \\
\text { Perez, \& } \\
\text { Buchanan, } \\
\text { 2008) }\end{array}$ & $\begin{array}{c}\text { New } \\
\text { Zealand }\end{array}$ & $\begin{array}{c}\text { OFF, } \\
\text { Timber } \\
\text { Frame (6) }\end{array}$ & 4,200 & 60 & $0.57(\mathrm{P})$ & 0.02 & 2.76 & 0.45 & 3.42 & 0.20 \\
\hline $\begin{array}{c}\text { (Yohanis \& } \\
\text { Norton, 2002) }\end{array}$ & UK & $\begin{array}{c}\text { OFF, } \\
\text { Steel } \\
\text { Frame (1) }\end{array}$ & 584 & N/A & N/A & N/A & 9.5 & N/A & N/A & N/A \\
\hline \multicolumn{5}{|c|}{ Range } & $\begin{array}{c}0.23 \text { to } \\
4.23(\mathrm{P})\end{array}$ & $\begin{array}{c}0.02 \text { to } \\
0.24\end{array}$ & $\begin{array}{c}0.52 \text { to } \\
12.96\end{array}$ & $\begin{array}{c}0.45 \text { to } \\
9.49\end{array}$ & $\begin{array}{c}3.42 \text { to } \\
22.45\end{array}$ & $\begin{array}{c}0.20 \text { to } \\
0.89\end{array}$ \\
\hline
\end{tabular}

Note: $1 \mathrm{kWh}=0.0036 \mathrm{GJ}$

Note: OFF $=$ Office, $\mathrm{SCH}=$ School, MIX = Mixed-Use (Office, Retail, Other)

${ }^{1}(\mathrm{P})=$ Primary Energy, $(\mathrm{S})=$ Secondary Energy, $(\mathrm{U})=$ Unknown

${ }^{2}$ Assumed that embodied energy was calculated in terms of primary energy (unclear in most studies)

${ }^{3}$ Office buildings with no underground parking

${ }^{4}$ Cole and Kernan (1996) also calculated operating energy for same building located in Vancouver, Canada to be $47.95 \mathrm{GJ} / \mathrm{m}^{2}\left(0.96 \mathrm{GJ} / \mathrm{m}^{2} / \mathrm{yr}\right)$

${ }^{5}$ Sartori and Hestnes (2007) conducted a literature review of 60 buildings (both residential and non-residential) from nine different countries. The range of values in this table were estimated from their graphs

${ }^{6} \mathrm{GWP}$ is presented in terms of $\mathrm{CO}_{2}$ not $\mathrm{CO}_{2}$ equivalent

${ }^{7}$ Also includes energy (and/or GWP) for end-of-life

${ }^{8}$ In this study, 20 public secondary schools were examined

\subsubsection{Problems with LCA Studies of Buildings}

As alluded to thus far, there are several problems with the way that LCA's of buildings have been conducted in the literature that make it difficult (if not impossible) to compare one LCA study to the next. Some of the more important limitations and discrepancies between the LCA studies from the literature are discussed in the paragraphs below.

Perhaps the most systemic problem with the LCA studies of buildings in the literature today is the lack of consistency when it comes to the life-cycle inventories (LCI) of building materials. Only in recent years has there been a concerted effort to quantify the embodied energy and GWP of the numerous building materials. However, to date there is still no industry standard LCI that is recognized. Without an industry standard LCI, it is virtually impossible to compare the results of one 
LCA study to the next. Each study in the literature tends to use different embodied energy and GWP numbers for the various building materials (see Table 2-1and Table 2-2). Historically it has been left up to the LCA researchers to establish a range of possible values for the embodied energy and GWP of the various building materials and to estimate the expected lifespan of the materials. The significant variation in LCI data from one LCA study to the next makes it extremely difficult to compare results.

Another problem with the LCA studies of buildings in the literature is the confusion between primary energy and secondary energy. It is extremely important to distinguish between the two when talking about the embodied energy and the operating energy of a building. Secondary energy is the energy used by the final consumer. In terms of buildings, this is the heating, cooling, lighting, etc. energy use of a building. On the contrary, primary energy is a measure of the total energy including the energy used by the final consumer (i.e. the secondary energy) as well as the energy used in transforming one energy form to another (like coal to electricity), the energy used by providers in providing energy to the market, and more. In other words, the primary energy is a complete measure of the total energy, as it includes the energy requirements upstream of the final end use, such as the energy generation and transportation. In the literature, many of the studies neglect to mention if their results are in terms of primary or secondary energy. This is a problem, as the results cannot be compared with any degree of certainty from one LCA study to the next.

The absence of any data on the GWP of buildings in the literature is also a problem. It is difficult to find comprehensive LCA studies of energy use in buildings, but it is nearly impossible to find detailed LCA studies of the GWP of buildings. Those studies that do attempt to quantify the GWP of buildings either present the results in terms of $\mathrm{CO}_{2}$ emissions or $\mathrm{CO}_{2}$ equivalent emissions. Carbon dioxide equivalency $\left(\mathrm{CO}_{2}\right.$ eq.) is a measure of the equivalent amount of $\mathrm{CO}_{2}$ that would have the same GWP as a mixture of $\mathrm{CO}_{2}$ and other greenhouse gases in the Earth's atmosphere. Therefore, it includes a measure of other gases such as methane, nitrous oxide, ozone, even water vapour, which all have an ability to trap heat in the Earth's atmosphere and therefore contribute towards global warming. Those studies that only report $\mathrm{CO}_{2}$ emissions fail to account for these other gases.

The level of complexity varies significantly between LCA studies in the literature. Some studies are very detailed and comprehensive, while others only attempt a simple approximation of the life-cycle environmental effects. For instance, some studies consider a wide range of building components such as the exterior walls, roofs, windows, doors, structure, interior finish, services, foundations, etc. 
However, other studies only consider a few of these components in detail. The number of building materials that are considered in each case also differs. Some studies provide a detail material takeoff, while others only estimate the materials in the building on a gross scale. Not every LCA study considers the end-of-life effects, construction effects, transportation effects, or recurring effects. In other words, the scope of the various LCA studies can be drastically different, making a direct comparison between studies very challenging. On top of this, some LCA studies make use of advanced LCA software programs to conduct their analysis. Others tend to use simple material takeoffs and hand calculations to come up with an estimate of embodied effects and operating effects for a building.

A big problem that everyone who conducts a LCA of a building faces is a lack of information at the design phase of the project (which is when a detailed LCA would be most helpful). It is difficult to estimate the total life-cycle environmental effects of a building accurately when the building has yet to be designed or built. A detailed and accurate LCA of a building is possible only after it is designed or built. However, at this point one could argue that a LCA is not as useful, as the impact of the building on the environment has already been set. Scheuer, Keoleian, \& Reppe (2003) state that one of the greatest limitations of LCA research for buildings is that it is difficult to do without the building having already been constructed. In fact, "in order for life-cycle modeling to fulfill its potential in assisting design decisions, there is a need for detailed data on specific building systems and components" (Scheuer, Keoleian, \& Reppe, 2003). This is currently lacking in the literature.

Finally, a significant deficiency in the literature is the lack of information on the life-cycle environmental burdens of single-storey retail buildings. In fact, no relevant LCA studies dealing with single-storey retail buildings could be located in the literature. Most LCA studies for commercial buildings have been done for multi-storey buildings (usually office buildings or mixed-use buildings). 


\section{Chapter 3 \\ Methodology: Life-Cycle Assessment of Building Components}

\subsection{Introduction}

The LCA process in this study was carried out in accordance with the four phase approach suggested in ISO 14044 (ISO, 2006). According to ISO 14044, the first phase of any LCA study is to define a goal (ISO, 2006). The primary goal of this study was to conduct a comprehensive LCA for the components of a single-storey retail building located in Toronto, Canada, to determine which building components contribute the most towards the total life-cycle energy use and GWP after 50 years.

To date, the vast majority of LCA studies of buildings tend to focus on residential and multi-storey office buildings. Despite the fact that single-storey commercial buildings represent a large proportion of buildings in North America, there is little to no research on the LCA of these types of buildings. There is a need for a comprehensive LCA study of single-storey commercial buildings in Canada, specifically single-storey retail buildings. In particular, there is a need for a study that looks at a broad scope of building components for a single-storey retail building and that puts the life-cycle impacts of these various components into perspective. That is the goal of this study.

In order to evaluate the components of a single-storey retail building and to put the life-cycle impacts of the various components into perspective, the following two part methodology was followed:

1. First, a comprehensive LCA was conducted for the major components of a single-storey retail building in Canada. It is worth mentioning that many of the building components that were studied are also found in residential buildings. These building components can be divided into seven categories: exterior infill walls, roofs, floors, windows and doors, structural systems (beams and columns), foundations, and interior partitions. A description of these building components, along with an explanation of the method that was followed to calculate their total life-cycle energy use and GWP, is presented here in Chapter 3.

2. Next, the focus was expanded to include entire building systems rather than only individual building components. A detailed LCA was performed for five single-storey retail buildings located in Toronto. The energy use and GWP of the various components of a single-storey retail building (exterior infill walls, roofs, floors, windows and doors, structural systems, foundations, and interior partitions) were compared to the overall energy use and GWP of an entire building. The five case study retail buildings are described in Chapter 4. 


\subsection{Description of Baseline Retail Building}

In order to conduct a comprehensive LCA of energy use and GWP for the components of a singlestorey retail building, it was useful to establish a baseline building. The baseline building was created to represent the features of a typical single-storey retail building that would be constructed in Canada today. Using the baseline retail building as a datum, alternative design strategies were explored for the various building components (exterior infill walls, roofs, floors, windows and doors, structural systems, foundations, and interior partitions).

The baseline retail building was established based on a combination of ASHRAE Standard 90.1-2007 (ASHRAE, 2007) requirements for climate zone 6 (Toronto, Canada) and the RSMeans Assemblies Cost Data (RSMeans, 2003). A rendering of the baseline retail building can be seen in Figure 3-1.

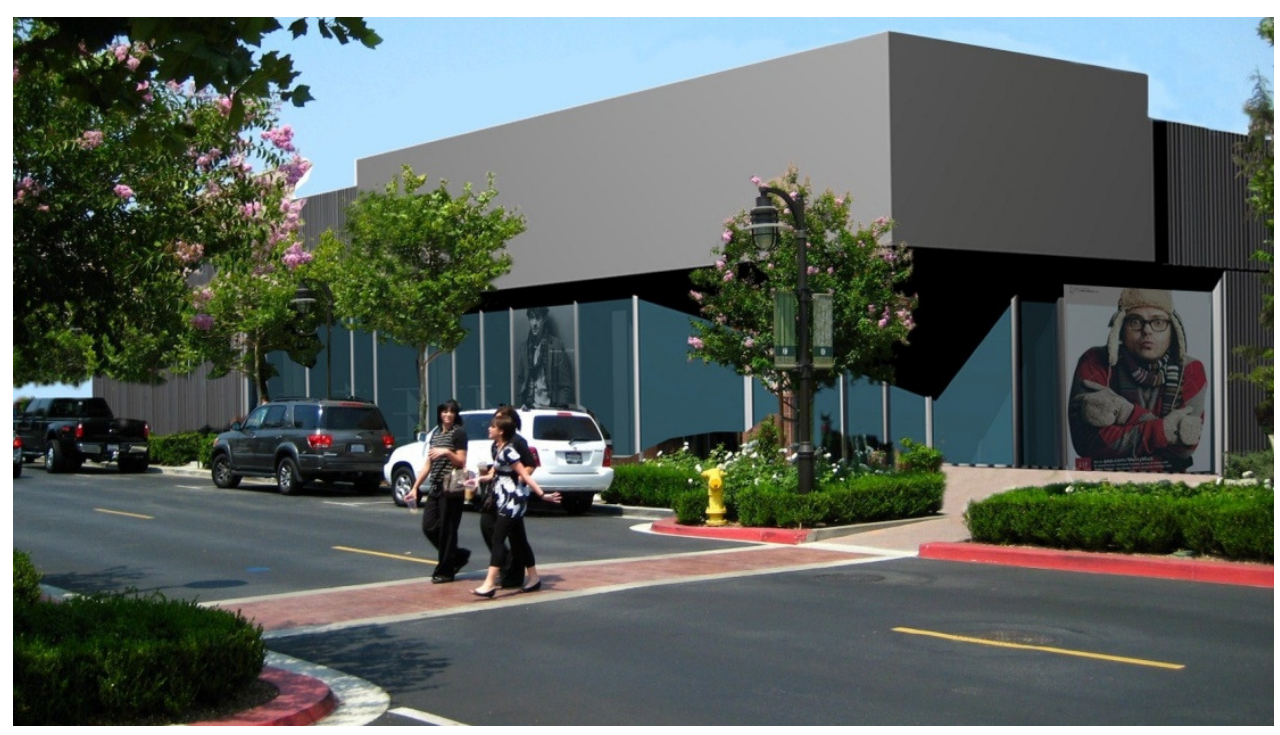

Figure 3-1: Baseline Retail Building

A detailed description of the baseline retail building can be found in Appendix A. This includes architectural floor plans, sections, elevations, structural drawings, as well as a summary of the key building descriptors. However, a brief summary of some important features of the baseline retail building are presented next.

- Located in Toronto, Ontario, Canada

- 50 year lifespan

- Stand alone retail building 
- Single-storey with a small mezzanine for offices

- Gross floor area (not including mezzanine) of $6,300 \mathrm{ft}^{2}\left(586 \mathrm{~m}^{2}\right)$

- Building orientation: rectangular shape (long dimension aligned along E-W axis)

- Approximately $17 \%$ window-to-wall ratio

- Hours of operation: Monday to Saturday, 8am-9pm and Sunday, 9am-6pm

- Cooling equipment: direct expansion (DX) coils (electric)

- Heating equipment: combustion furnace (natural gas)

- System type: packaged single zone DX with furnace (central packaged single zone air conditioner with combustion furnace)

- Thermostat set-points:

- Occupied spaces: cool to $76.0^{\circ} \mathrm{F}\left(24.4^{\circ} \mathrm{C}\right)$ and heat to $70.0^{\circ} \mathrm{F}\left(21.1^{\circ} \mathrm{C}\right)$

- Unoccupied spaces: cool to $82.0^{\circ} \mathrm{F}\left(27.8^{\circ} \mathrm{C}\right)$ and heat to $64.0^{\circ} \mathrm{F}\left(17.8^{\circ} \mathrm{C}\right)$

- Zoning: $100 \%$ perimeter zone

- Designed for NBCC 2005 structural loads

A summary of the building components that make up the baseline retail building including: the exterior infill wall, roof, structure, mezzanine floor, windows, doors, interior partitions, and foundations can be found in Table 3-1. As mentioned earlier, these building components were chosen to represent typical assemblies that would be specified for a single-storey retail building in Canada. Although the building components for the baseline retail building were chosen as representative of common practice in Canada, there are countless alternative strategies that could have been chosen. The goal of this study is to investigate the components of a single-storey retail building within a comprehensive LCA, in order to determine which components have the greatest impact on the environment. Therefore, in the next section a range of alternative design strategies for the components of a typical single-storey retail building in Canada are presented. 
Table 3-1: Description of Baseline Retail Building Components

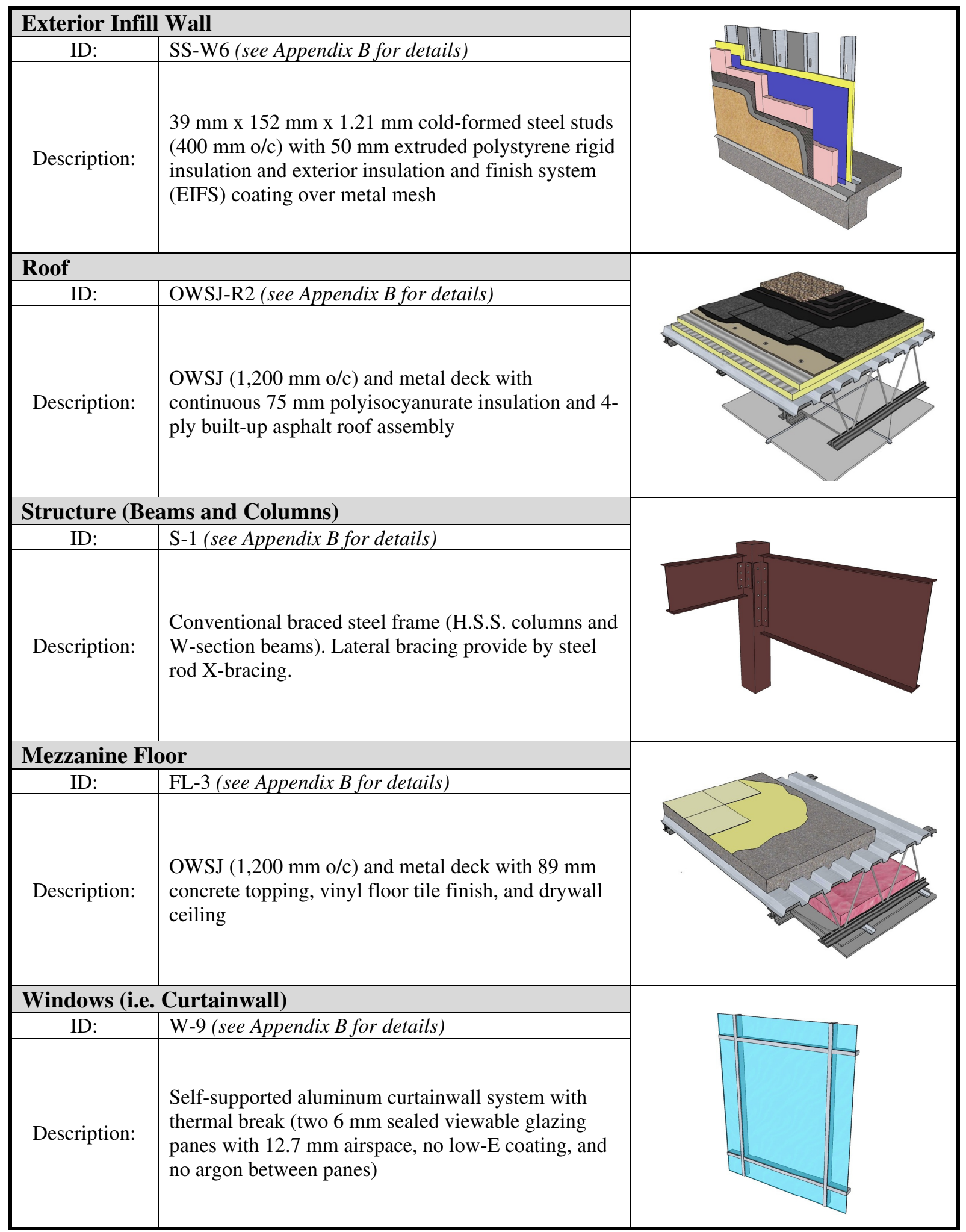


Table 3-1 (Cont.): Description of Baseline Retail Building Components

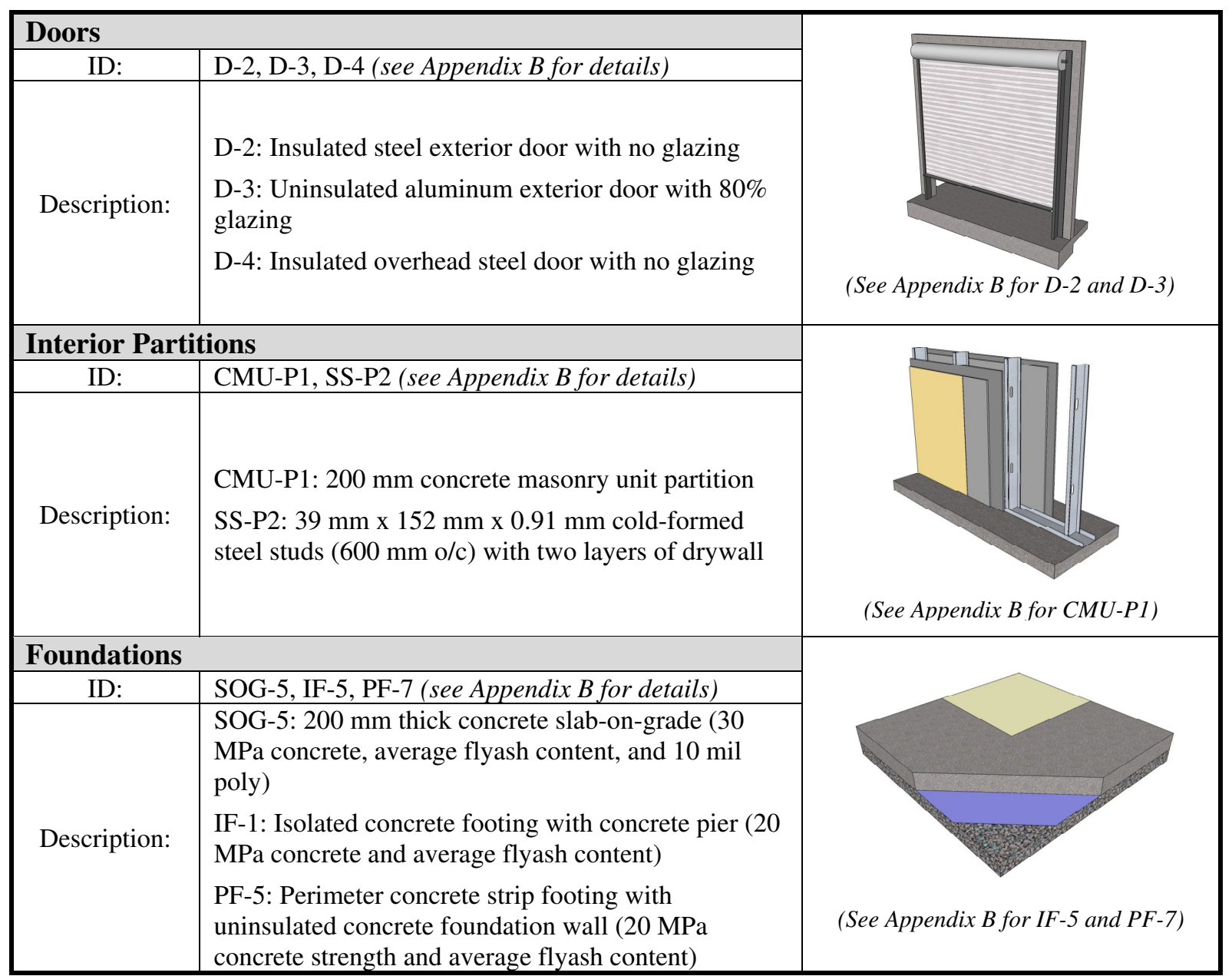

\subsection{Identifying Building Components for the LCA}

As stated in the introduction of this chapter, the primary goal of this LCA study is to understand the breakdown of energy use and GWP as it pertains to the components of a single-storey retail building in Canada. In order to do this, 220 different building components were analyzed within the framework of a comprehensive LCA. The LCA of these building components was performed over a 50 year lifespan for a commercial building located in Toronto, Canada. These building components represent the spectrum of common assemblies that are used in commercial buildings in Canada, but many of the components are also used in residential buildings. A detailed description of each of the 220 different building components that were studied can be found in Appendix B. 
The building components chosen for this study were selected based on two criteria: discussions with building industry professionals and from the RSMeans Assemblies Cost Data (RSMeans, 2003) manual. The RSMeans manual lists a range of typical assemblies for commercial buildings in Canada. The remainder of this section will introduce the range of buildings components that were examined in this study.

\subsubsection{Description of Exterior Infill Wall Enclosures}

A total of 109 commercial exterior infill wall enclosures were examined in this study. The wall assemblies represent a broad sample of exterior infill walls that are typically used in commercial buildings in Canada. Aside from resisting lateral loads, these walls were not designed for load bearing applications. A complete description of the wall enclosures (including all of the assembly layers and a list of the building material quantities) that were chosen for this study can be found in Appendix B-1.

The various wall assemblies have been classified based on their structural framing material and have been placed into the following eight categories:

- Concrete masonry unit walls (CMU-W)

- Concrete tilt-up walls (CTU-W)

- Wood structural insulated panel walls (WSIP-W)

- Metal structural insulated panel walls (MSIP-W)

- Cold-formed steel stud walls (SS-W)

- Wood stud walls (WS-W)

- Pre-engineered steel building walls (PENG-W)

- Aluminum curtainwalls (CWALL-W)

Displayed in Figure 3-2 are the typical assembly layers that were modelled for each of the exterior infill wall enclosures. In general, each wall consisted of: an exterior cladding material, an air space, exterior installed rigid insulation (if applicable), a water barrier membrane, the structural framing (with cavity insulation if applicable), and an interior finish. The air barriers (AB), vapour barriers/retarders (VB/VR), and water barriers (WB) were selected and located within the wall assemblies based on building science principles for a cold-climate in Canada. 


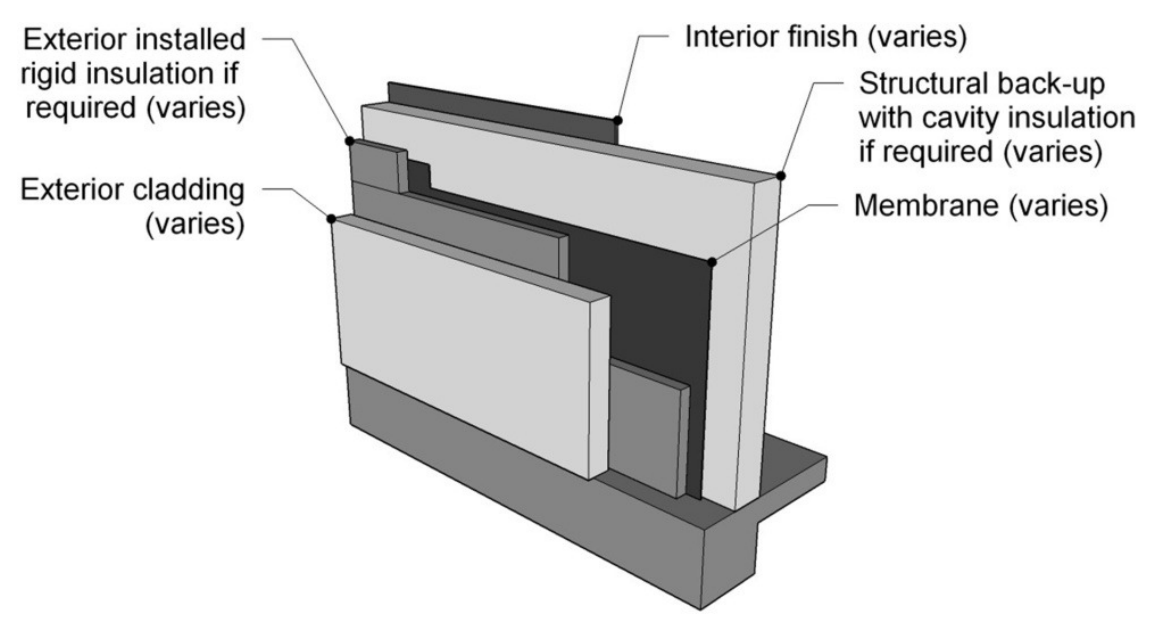

Figure 3-2: Typical Assembly Layers for Exterior Infill Wall Enclosures

The wall types examined in this study are listed in Table 3-2, along with a breakdown of the different options that were explored. It is important to note that most wall assemblies are a collection of multiple sub-assemblies, layers, and materials that all act together as part of a greater system. A change to one of these layers can have a significant impact on the performance of the entire enclosure (both in terms of thermal resistance and total energy use or GWP). One of the goals of this study is to compare the LCA results for various different types of walls. Therefore, it is not enough to simple look at one or two different steel stud walls for example. The interdependency of the layers within a wall assembly means that a change to one layer or material can have an impact on the remaining layers. It was important to look at a broad range of different wall enclosures, including several variations of the same wall. It was important to consider an array of strategies for each wall type that included different cladding materials, insulation strategies, structural materials, and finishes.

Table 3-2: Range of Exterior Infill Wall Design Strategies

\begin{tabular}{|c|c|c|}
\hline Wall Type & Variables & Options \\
\hline \multirow[t]{3}{*}{ CMU-W } & Cladding & $\begin{array}{ll}\text { - } & \text { Standard (Ontario) clay brick cladding } \\
\text { - } & \text { Split-faced concrete brick cladding } \\
\text { - } & 125 \mathrm{~mm} \text { concrete pre-cast cladding } \\
\text { - } & \text { Pine wood bevel siding } \\
\text { - } & 26 \text { ga. }(0.46 \mathrm{~mm}) \text { galvanized corrugated cold-formed steel (CFS) cladding } \\
- & \text { EIFS coating over metal mesh }\end{array}$ \\
\hline & Insulation & $\begin{array}{ll}\text { - } & 50 \mathrm{~mm} \text { exterior installed extruded polystyrene rigid insulation } \\
\text { - } & 100 \mathrm{~mm} \text { exterior installed extruded polystyrene rigid insulation } \\
\text { - } & 140 \mathrm{~mm} \text { fibreglass batt insulation installed between CFS studs }\end{array}$ \\
\hline & Structure & - $\quad 200 \mathrm{~mm}$ standard weight concrete block with grouted 15M rebars @ $400 \mathrm{~mm} \mathrm{o} / \mathrm{c}$ \\
\hline
\end{tabular}


Table 3-2 (Cont.): Range of Exterior Infill Wall Design Strategies

\begin{tabular}{|c|c|c|}
\hline Wall Type & Variables & Options \\
\hline \multirow{4}{*}{ CTU-W } & Cladding & $\begin{array}{ll}\text { - } & \text { None } \\
\text { - } & 50 \mathrm{~mm} \text { concrete front wythe } \\
\text { - } & \text { Standard (Ontario) clay brick cladding } \\
\text { - } & \text { Split-faced concrete brick cladding }\end{array}$ \\
\hline & Insulation & $\begin{array}{ll}- & \text { None } \\
- & \text { Same options as CMU } \\
\end{array}$ \\
\hline & $\begin{array}{l}\text { Interior } \\
\text { Finish }\end{array}$ & $\begin{array}{ll}- & \text { Latex/alkyd based paint } \\
- & \text { Regular gypsum board } \\
- & 26 \text { ga. }(0.46 \mathrm{~mm}) \text { galvanized corrugated CFS cladding } \\
\end{array}$ \\
\hline & Structure & $\begin{array}{l}\text { - } 150 \mathrm{~mm} \text { concrete tilt-up wall (30 MPa, 9\% flyash) with reinforcement and } \\
\text { miscellaneous steel angles }\end{array}$ \\
\hline \multirow{3}{*}{ WSIP-W } & Cladding & - $\quad$ Same options as CMU \\
\hline & Insulation & $\begin{array}{l}\text { - } \quad 50 \mathrm{~mm} \text { exterior installed extruded polystyrene rigid insulation } \\
\text { - } \quad 100 \mathrm{~mm} \text { extruded polystyrene rigid insulation between two layers of OSB } \\
\text { - } \quad 150 \mathrm{~mm} \text { extruded polystyrene rigid insulation between two layers of OSB } \\
\end{array}$ \\
\hline & Structure & $\begin{array}{l}\text { - Wood structural insulated panel comprised of } 12 \mathrm{~mm} \text { OSB, extruded polystyrene } \\
\text { insulation (thickness varies), and } 12 \mathrm{~mm} \text { OSB with } 200 \mathrm{~mm}, 14 \text { ga. }(1.90 \mathrm{~mm}) \\
\text { galvanized CFS Z-girts @ } 1,200 \mathrm{~mm} \mathrm{o} / \mathrm{c}\end{array}$ \\
\hline \multirow[t]{2}{*}{ MSIP-W } & Insulation & $\begin{array}{l}\text { - } 150 \mathrm{~mm} \text { polyurethane foam insulation installed between two corrugated CFS } \\
\text { sheets } \\
\text { - } 100 \mathrm{~mm} \text { polyurethane foam insulation installed between two corrugated CFS } \\
\text { sheets } \\
\text { - } \quad 75 \mathrm{~mm} \text { polyurethane foam insulation installed between two corrugated CFS sheets }\end{array}$ \\
\hline & Structure & $\begin{array}{l}\text { - Metal structural insulated panel comprised of } 26 \text { ga. }(0.46 \mathrm{~mm}) \text { galvanized } \\
\text { corrugated CFS cladding, polyurethane foam insulation (thickness varies), and } 26 \\
\text { ga. }(0.46 \mathrm{~mm}) \text { galvanized corrugated CFS cladding with } 200 \mathrm{~mm}, 14 \mathrm{ga} .(1.90 \\
\mathrm{mm}) \text { galvanized CFS Z-girts @ } 1,200 \mathrm{~mm} \text { o/c }\end{array}$ \\
\hline \multirow{3}{*}{ SS-W } & Cladding & - $\quad$ Same options as CMU \\
\hline & Insulation & - $\quad$ Same options as CMU \\
\hline & Structure & $\begin{array}{l}\text { - } \quad 39 \mathrm{~mm} \text { x } 152 \mathrm{~mm} 18 \text { ga. }(1.21 \mathrm{~mm}) \text { CFS studs @ } 400 \mathrm{~mm} \\
-\quad 39 \mathrm{~mm} \text { x } 152 \mathrm{~mm} 16 \text { ga. }(1.52 \mathrm{~mm}) \text { CFS studs @ } 600 \mathrm{~mm} \\
\end{array}$ \\
\hline \multirow{3}{*}{ WS-W } & Cladding & - $\quad$ Same options as CMU \\
\hline & Insulation & - $\quad$ Same options as CMU (except wood studs not CFS studs) \\
\hline & Structure & $\begin{array}{l}\text { - } \quad 38 \mathrm{~mm} \times 140 \mathrm{~mm} \text { wood studs @ } 400 \mathrm{~mm} \text { o/c } \\
\text { - } \quad 38 \mathrm{~mm} \text { x } 140 \mathrm{~mm} \text { wood studs @ } 600 \mathrm{~mm} \mathrm{o} / \mathrm{c}\end{array}$ \\
\hline \multirow[t]{2}{*}{ PENG-W } & Insulation & $\begin{array}{ll}\text { - } & \text { None } \\
\text { - } & 140 \mathrm{~mm} \text { fibreglass batt insulation (compressed at girt locations) } \\
\text { - } & 150 \mathrm{~mm} \text { extruded polystyrene rigid insulation between two corrugated CFS sheets } \\
\text { - } & 250 \mathrm{~mm} \text { extruded polystyrene rigid insulation between two corrugated CFS sheets }\end{array}$ \\
\hline & Structure & $\begin{array}{l}\text { - } 26 \text { ga. }(0.46 \mathrm{~mm}) \text { galvanized corrugated CFS cladding with } 200 \mathrm{~mm}, 14 \text { ga. }(1.90 \\
\text { mm) galvanized CFS Z-girts @ } 1,200 \mathrm{~mm} \text { o/c }\end{array}$ \\
\hline \multirow{3}{*}{ CWALL-W } & Cladding & $\begin{array}{ll}\text { - } & \text { Painted metal spandrel panel } \\
\text { - } & \text { Opaque glazing spandrel panel } \\
\end{array}$ \\
\hline & Insulation & $\begin{array}{ll}\text { - } & \text { None } \\
\text { - } & 90 \mathrm{~mm} \text { high density fiberglass insulation with metal backpan } \\
\end{array}$ \\
\hline & Structure & $\begin{array}{l}\text { - Self-supporting aluminum curtainwall system with thermal break (100 mm deep } \\
\text { mullions spaced } 2,000 \mathrm{~mm} \text { o/c vertically and } 1,500 \mathrm{~mm} \text { o/c horizontally }\end{array}$ \\
\hline
\end{tabular}




\subsubsection{Description of Roof Enclosures}

A total of 58 different commercial roof enclosures were examined in this study. In the literature, very little research has been carried out on the LCA of roof enclosures, especially for single-storey commercial buildings. The roof enclosures that were included in this study represent a broad sample of typical roof enclosures for a commercial building in Canada. A complete description of the roofs (including all of the assembly layers and the material quantities) can be found in Appendix B-2.

The various roof assemblies have been classified based on their structural framing material and have been placed into the following seven categories:

- Concrete hollow core roof (CHC-R)

- Open web steel joist roofs (OWSJ-R)

- Cold-formed steel roofs (CFS-R)

- Glulam roofs (GLU-R)

- Wood structural insulated panel roofs (WSIP-R)

- Metal structural insulated panel roofs (MSIP-R)

- Pre-engineered steel building roofs (PENG-R)

Displayed in Figure 3-3 is the typical assembly layers that were identified for each of the roofs. In general, each roof enclosure consisted of: a roof covering, a roof coverboard (if required), insulation, a water barrier membrane, the roof deck, the roof structure, and a suspended acoustic tile ceiling.

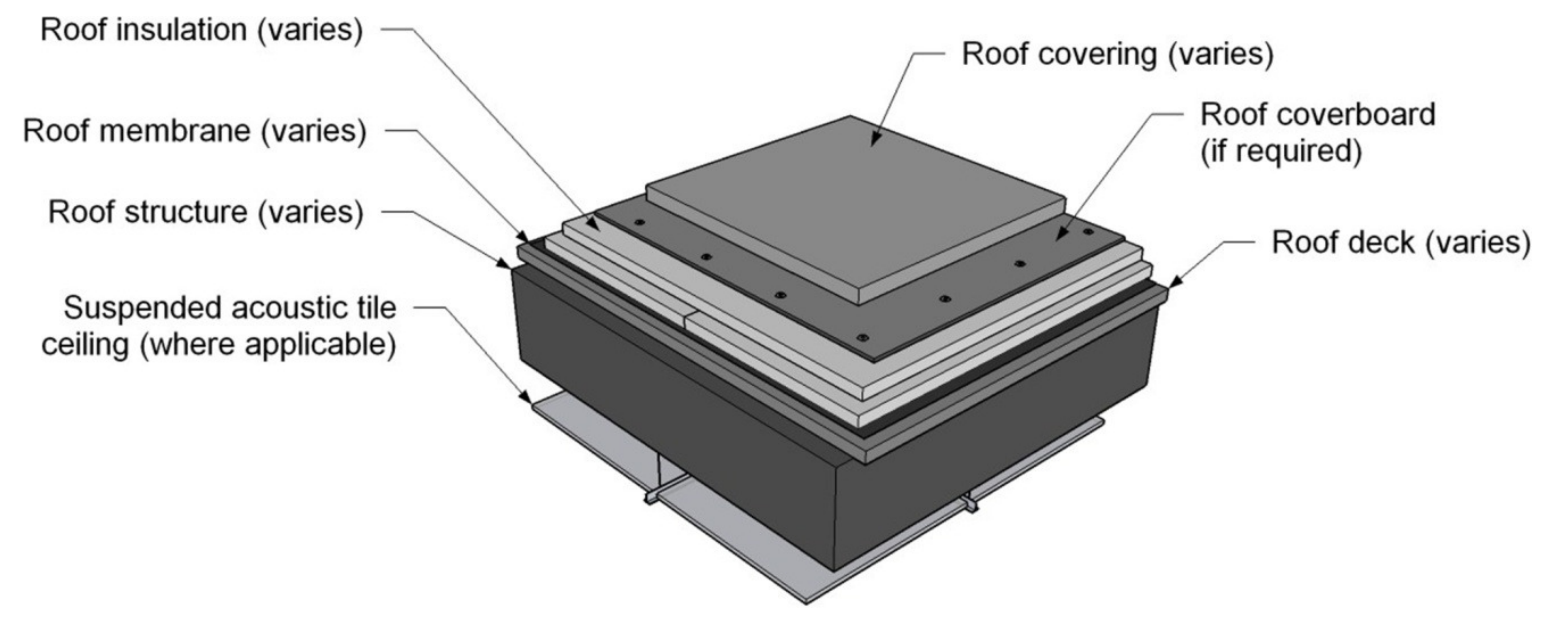

Figure 3-3: Typical Assembly Layers for Roof Enclosures 
The roof types examined in this study are listed in Table 3-3, along with a breakdown of the different options that were explored. Similar to the exterior infill walls, a wide range of different roofs were identified, including many variations of the same roof type. It was acknowledged that even within the same type of roof, there could be significant variation in terms of the thermal resistance, the total energy use, and GWP. It was important to consider an array of strategies for each roof type that included different roof coverings, insulation strategies, and structural materials.

\section{Table 3-3: Range of Roof Design Strategies}

\begin{tabular}{|c|c|c|}
\hline Roof Type & Variables & Options \\
\hline \multirow{3}{*}{ CHC-R } & $\begin{array}{l}\text { Roof } \\
\text { Covering }\end{array}$ & $\begin{array}{ll}- & \text { SBS modified bitumen membrane roof assembly } \\
- & \text { 4-ply built-up asphalt roof assembly with gravel ballast } \\
\text { - } & \text { EPDM roof assembly with gravel ballast } \\
\text { - } & \text { PVC membrane roof assembly with gravel ballast } \\
- & \text { Commercial } 26 \text { ga. }(0.46 \mathrm{~mm}) \text { galvanized standing seam steel roof } \\
- & \text { Green roof assembly }\end{array}$ \\
\hline & Insulation & $\begin{array}{ll}\text { - } & 75 \mathrm{~mm} \text { polyisocyanurate insulation } \\
\text { - } & 100 \mathrm{~mm} \text { extruded polystyrene rigid insulation (green roof assembly only) } \\
\text { - } & 150 \mathrm{~mm} \text { polyisocyanurate insulation } \\
\text { - } & 200 \mathrm{~mm} \text { extruded polystyrene rigid insulation (green roof assembly only) }\end{array}$ \\
\hline & Structure & $\begin{array}{l}\text { - } 200 \mathrm{~mm} \text { concrete hollow core roof slab }(45+\mathrm{MPa}, 9 \% \text { flyash, typical } \\
\text { reinforcement) }\end{array}$ \\
\hline \multirow{3}{*}{ OWSJ-R } & $\begin{array}{c}\text { Roof } \\
\text { Covering }\end{array}$ & - $\quad$ Same options as CHC \\
\hline & Insulation & - $\quad$ Same options as $\mathrm{CHC}$ \\
\hline & Structure & $\begin{array}{l}\text { - } \quad 550 \mathrm{~mm} \text { open web steel joists @ } 1,200 \mathrm{~mm} \text { o/c with } 39 \mathrm{~mm} \text { x } 22 \text { ga. }(0.76 \mathrm{~mm}) \\
\text { galvanized corrugated CFS deck }\end{array}$ \\
\hline \multirow[b]{3}{*}{ CFS-R } & $\begin{array}{c}\text { Roof } \\
\text { Covering }\end{array}$ & - $\quad$ Same options as CHC \\
\hline & Insulation & - $\quad$ Same options as $\mathrm{CHC}$ \\
\hline & Structure & $\begin{array}{l}\text { - } 1 \text { (1) - } 39 \mathrm{~mm} \text { x } 245 \mathrm{~mm}, 16 \text { ga. }(1.52 \mathrm{~mm}) \text { galvanized CFS C-joist @ } 600 \mathrm{~mm} \text { o/c } \\
\text { with } 19 \mathrm{~mm} \text { plywood deck } \\
\text { - }(2)-39 \mathrm{~mm} \times 245 \mathrm{~mm}, 16 \text { ga. }(1.52 \mathrm{~mm}) \text { galvanized CFS C-joists back-to-back @ } \\
600 \mathrm{~mm} \text { o/c with } 19 \mathrm{~mm} \text { plywood deck } \\
\text { - } 600 \mathrm{~mm} \text { deep CFS trusses spaced } @ 600 \mathrm{~mm} \text { with } 19 \mathrm{~mm} \text { plywood deck } \\
-\quad 762 \mathrm{~mm} \text { deep CFS trusses spaced @ } 1,200 \mathrm{~mm} \text { with } 39 \mathrm{~mm} \times 22 \mathrm{ga} .(0.76 \mathrm{~mm}) \\
\text { galvanized corrugated CFS deck }\end{array}$ \\
\hline \multirow{3}{*}{ GLU-R } & $\begin{array}{c}\text { Roof } \\
\text { Covering }\end{array}$ & - $\quad$ Same options as $\mathrm{CHC}$ \\
\hline & Insulation & - $\quad$ Same options as CHC \\
\hline & Structure & $\begin{array}{l}\text { - } 80 \mathrm{~mm} \times 494 \mathrm{~mm} 24 \mathrm{f}-\mathrm{E} \text { D-Fir-L glulam joists @ } 1,800 \mathrm{~mm} \text { o/c with } 38 \mathrm{~mm} \\
\text { tongue and groove solid wood plank decking }\end{array}$ \\
\hline
\end{tabular}


Table 3-3 (Cont.): Range of Roof Design Strategies

\begin{tabular}{|c|c|c|}
\hline Roof Type & Variables & Options \\
\hline \multirow[b]{2}{*}{ WSIP-R } & $\begin{array}{c}\text { Roof } \\
\text { Covering }\end{array}$ & - $\quad$ Same options as CHC \\
\hline & Structure & $\begin{array}{l}\text { Wood structural insulated panel comprised of } 12 \mathrm{~mm} \text { OSB, extruded polystyrene } \\
\text { insulation (thickness varies), and } 12 \mathrm{~mm} \text { OSB with } 229 \mathrm{~mm}, 14 \mathrm{ga} .(1.90 \mathrm{~mm}) \\
\text { galvanized CFS Z-shape purlins @ } 1,200 \mathrm{~mm} \mathrm{o} / \mathrm{c}\end{array}$ \\
\hline \multirow{3}{*}{ MSIP-R } & $\begin{array}{c}\text { Roof } \\
\text { Covering }\end{array}$ & $\begin{array}{ll}- & \text { None } \\
- & \text { Green roof assembly }\end{array}$ \\
\hline & Insulation & $\begin{array}{l}\text { - } 150 \mathrm{~mm} \text { polyurethane foam insulation installed between two corrugated CFS } \\
\text { sheets } \\
\text { - } 100 \mathrm{~mm} \text { polyurethane foam insulation installed between two corrugated CFS } \\
\text { sheets } \\
\text { - } \quad 75 \mathrm{~mm} \text { polyurethane foam insulation installed between two corrugated CFS sheets }\end{array}$ \\
\hline & Structure & $\begin{array}{l}\text { - Metal structural insulated panel comprised of } 26 \text { ga. }(0.46 \mathrm{~mm}) \text { galvanized } \\
\text { corrugated CFS cladding, polyurethane foam insulation (thickness varies), and } 26 \\
\text { ga. }(0.46 \mathrm{~mm}) \text { galvanized corrugated CFS cladding with } 229 \mathrm{~mm}, 14 \text { ga. }(1.90 \\
\mathrm{mm}) \text { galvanized CFS Z-shape purlins @ } 1,200 \mathrm{~mm} \text { o/c }\end{array}$ \\
\hline \multirow[t]{2}{*}{ PENG-R } & Insulation & $\begin{array}{ll}\text { - } & \text { None } \\
\text { - } & 150 \mathrm{~mm} \text { fibreglass batt insulation } \\
\text { - } & 150 \mathrm{~mm} \text { extruded polystyrene rigid insulation between two corrugated CFS sheets } \\
\text { - } & 250 \mathrm{~mm} \text { extruded polystyrene rigid insulation between two corrugated CFS sheets }\end{array}$ \\
\hline & Structure & $\begin{array}{l}\text { - } 26 \text { ga. }(0.46 \mathrm{~mm}) \text { galvanized corrugated CFS cladding with } 200 \mathrm{~mm}, 16 \text { ga. }(1.52 \\
\mathrm{mm}) \text { galvanized CFS Z-shape purlins @ } 1,200 \mathrm{~mm} \text { o/c }\end{array}$ \\
\hline
\end{tabular}

It is important to note that not all roof joists can span the same distance. Some reach their optimum design state when spanning longer distances and some at shorter spans. To account for this variability, each roof joist was designed for a typical span that it would likely be used for, rather than for one standard span for all. This ensured that unfair advantage/disadvantage was not placed on one system over another, by designing it for a span for which it was not intended. Each roof was designed for loads according to Part 4 of the NBCC 2005 (Canadian Commission on Building and Fire Codes, 2006) using the typical design span.

\subsubsection{Description of Structural Systems}

Three different structural systems were examined in this study. The structural systems that were looked at are described in more detail in Appendix B-3.

For the purposes of this study, the structural system is defined as the primary structural components of a building including the beams and columns. In this study, the wall framing and roof purlins are included in the exterior infill wall and roof assemblies respectfully. As this study is specifically dealing with a single-storey retail building, it was important to identify the most common types of 
structural systems that are used in these types of buildings today. Those structural systems include: conventional hot-rolled steel systems, heavy timber systems, and pre-engineered steel building systems. Previous LCA studies have examined many of the conventional types of structural systems including: hot-rolled steel, timber, and concrete structures. However, there is almost no research on the environmental impact of pre-engineered steel building systems, in comparison to other conventional structural systems. Pre-engineered building systems are highly optimized structural systems from a materials and cost perspective. It is thought that this high level of material optimization will translate into a structural system that is very competitive in terms of total life-cycle energy use and GWP. Therefore, one of the major contributions of this study will be the detailed LCA of a pre-engineered steel building system, which is currently lacking in the literature.

It should be noted that structural systems are very unique to the characteristics of an individual project. Therefore, rather than trying to evaluate the total life-cycle energy use and GWP for countless different arrangements of beams and columns here, the results in Appendix B-3 were calculated per $\mathrm{m}^{2}$ of floor area for each type of structural system. A full LCA of the three different structural systems as applied to a typical single-storey retail building in Canada will be discussed in more detail in Chapter 4.

\subsubsection{Description of Floor Assemblies}

A total of five different commercial floors were examined in this study. A complete description of the floor assemblies (including all of the assembly layers and the material quantities) that were examined can be found in Appendix B-4.

In this study, only single-storey retail buildings were examined. Therefore, only the most common floor assemblies were examined. The floor assemblies in this study were restricted to typical floor systems that would be used for a mezzanine level where offices are located. Each floor was designed for structural loads according to Part 4 of the NBCC 2005 (Canadian Commission on Building and Fire Codes, 2006) using a typical design span (similar to the method described for the roof enclosures).

Figure 3-4 illustrates the typical assembly layers that were modelled for each of the floor systems. In general, each floor system consisted of: vinyl floor tile secured with adhesive, a floor deck, a floor structure (with fiberglass batt insulation if required), and two layers of regular gypsum board with steel resilient channels (if required). 


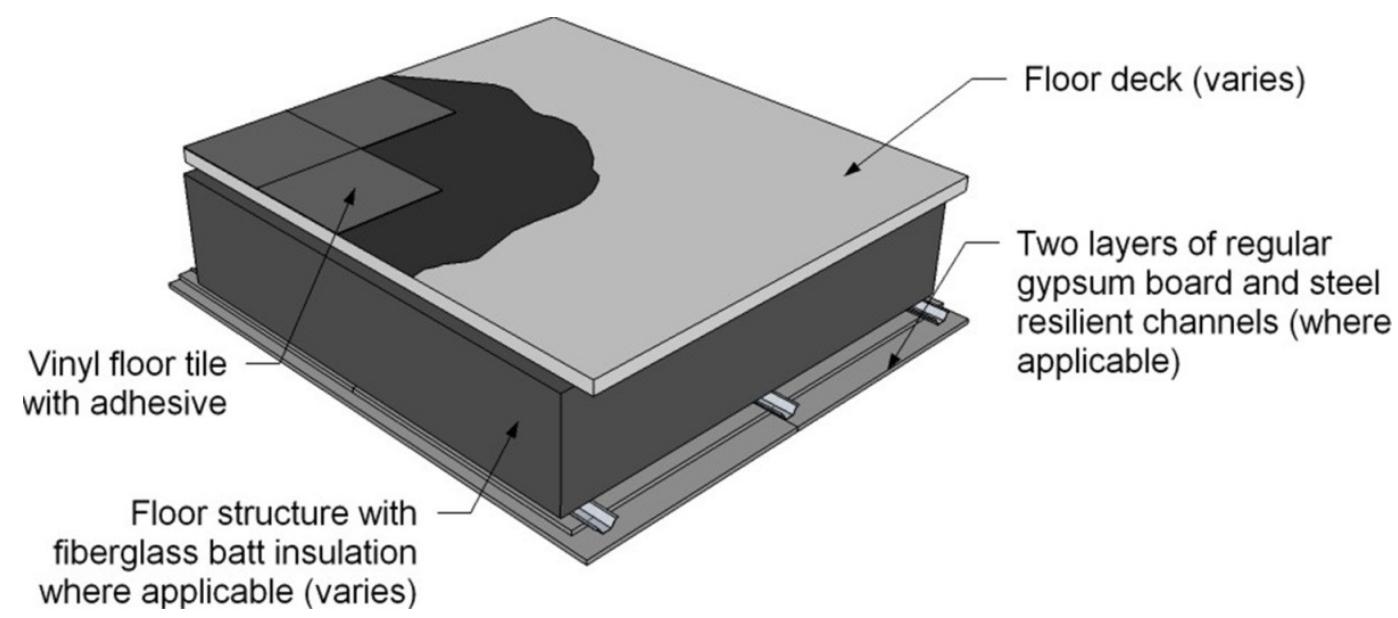

Figure 3-4: Typical Assembly Layers for Floor Systems

\subsubsection{Description of Windows and Doors}

A total of nine different windows and six different doors were examined in this study. The windows and doors were chosen to represent both what is typically used in commercial buildings in Canada today, as well as some more progressive alternatives. A complete description of the windows and doors that were chosen for this study can be found in Appendix B-5. Figure 3-5 displays the typical components that were modelled for the windows and doors in this study.

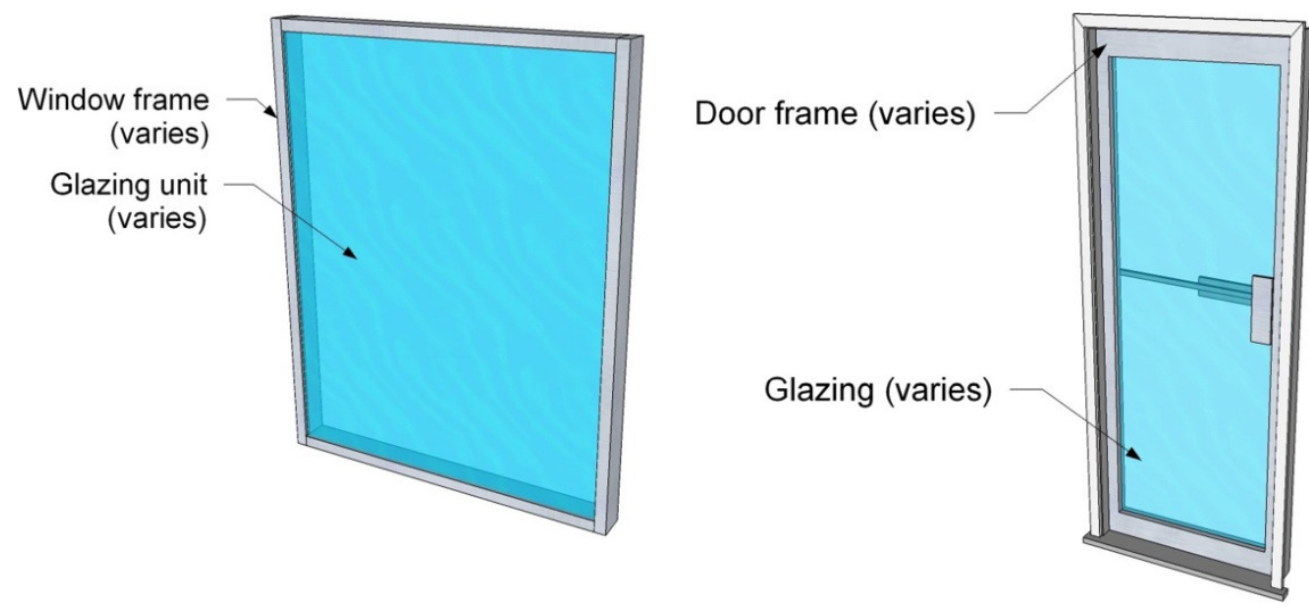

Figure 3-5: Typical Components of Windows and Doors

In general, each window consisted of a window frame and a glazing unit. No operable windows were considered. Each door consisted of a door frame and glazing (if applicable). The list of doors 
examined in this study included: exterior doors, interior doors, and an overhead sectional door. In each case, the frame material and the characteristics of the glazing were varied when populating the list of windows and doors to examine in this study.

The windows and doors examined in this study are listed in Table 3-4, along with a breakdown of the different options that were explored. In general, the frame material was varied in each case as well as the glazing (from typical glazing to a more progressive alternative).

\section{Table 3-4: Range of Window and Door Design Strategies}

\begin{tabular}{|c|c|c|}
\hline Type & Variables & Options \\
\hline \multirow{2}{*}{$\begin{array}{c}\text { WINDOWS } \\
\text { (W) }\end{array}$} & $\begin{array}{l}\text { Frame } \\
\text { material }\end{array}$ & $\begin{array}{ll}\text { - } & \text { Aluminum with thermal break } \\
\text { - } & \text { PVC clad wood with thermal break } \\
\text { - } & \text { PVC with thermal break } \\
\text { - } & \text { Wood with thermal break } \\
\text { - } & \text { Self-supporting aluminum curtainwall grid system with thermal break }\end{array}$ \\
\hline & Glazing & $\begin{array}{l}\text { - Typical sealed double pane glazing unit with } 12.7 \mathrm{~mm} \text { airspace (no argon between } \\
\text { panes) and no low-E coating } \\
\text { - Sealed double pane glazing unit with } 12.7 \mathrm{~mm} \text { argon space (argon gas between } \\
\text { panes) and tin based low-E coating }(\mathrm{e}=0.05 \text { ) } \\
\text { - Two } 6 \mathrm{~mm} \text { sealed viewable glazing panes with } 12.7 \mathrm{~mm} \text { airspace (no argon } \\
\text { between panes and no low-E coating) (aluminum curtainwall only) }\end{array}$ \\
\hline DOORS (D) & Type & $\begin{array}{ll}\text { - } & \text { Solid wood, no glazing } \\
\text { - } & \text { Insulated steel, no glazing } \\
\text { - } & \text { Uninsulated aluminum, } 80 \% \text { glazing }\end{array}$ \\
\hline
\end{tabular}

\subsubsection{Description of Foundations}

Seven different isolated concrete footing and pier combinations, eight different concrete strip footing and foundation wall combinations, and six different concrete slab-on-grades were examined in this study. All of the foundation options considered in this study can be found in Appendix B-6.

Displayed in Figure 3-6 to Figure 3-8 are the typical components that were identified for each of the foundation systems. Figure 3-6 illustrates a typical isolated concrete footing and pier that was identified for this study. Figure 3-7 shows the typical parameters that were identified for the concrete strip footings and foundation walls. Likewise, Figure 3-8 illustrates the typical parameters for a slabon-grade. The goal was to populate a list of common foundation systems that are used in commercial buildings in Canada. Typical sizes were selected based on design experience. 


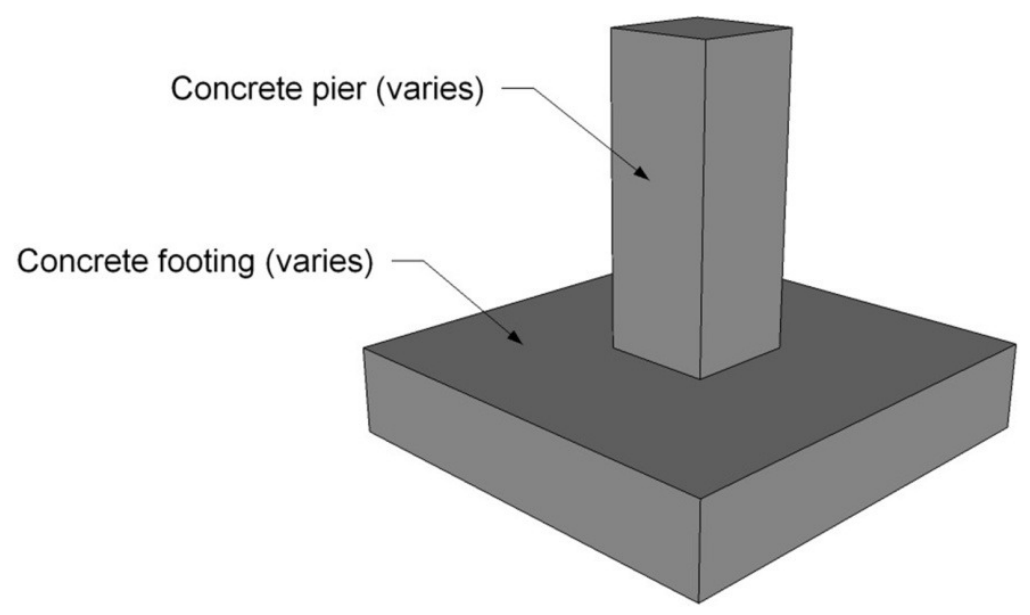

Figure 3-6: Typical Components of Isolated Footings with Piers

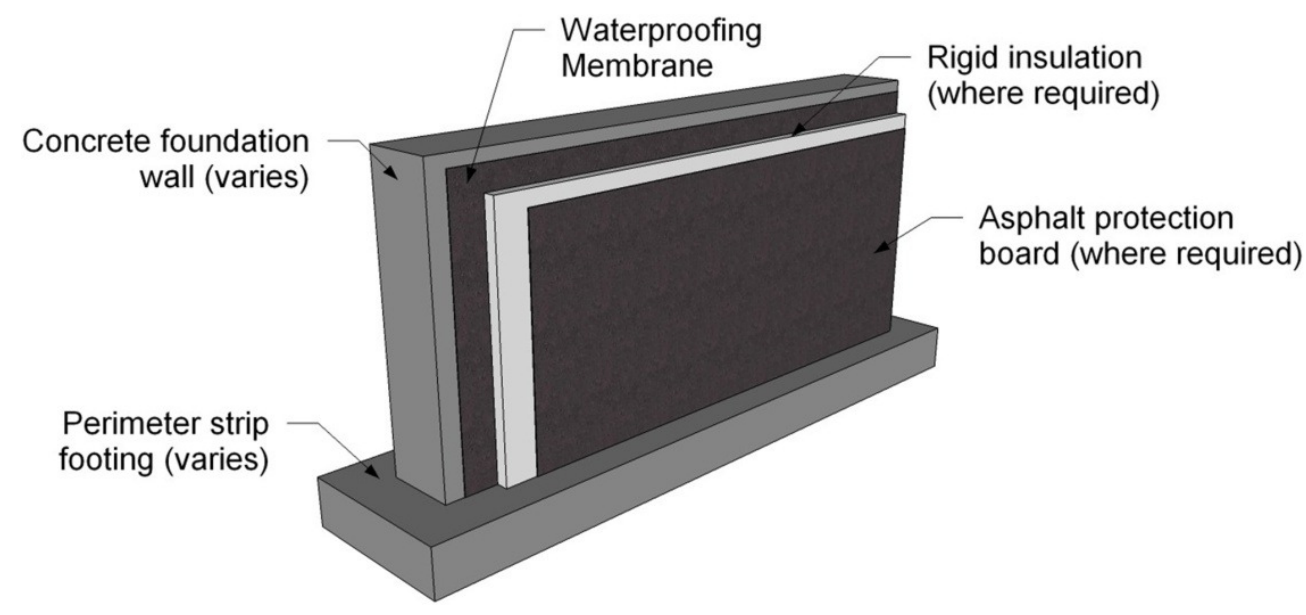

Figure 3-7: Typical Components of Perimeter Footings and Foundation Walls

The foundation components examined in this study are listed in Table 3-5, along with a breakdown of the different options that were explored. In each case, a number of different options were identified. Variables such as concrete strength, flyash content, insulation, and size were among the options that were explored. Obviously footing size and concrete strength vary depending on the structural loads that must be resisted and the nature of the soil conditions. However, it is still useful to identify a range of typical options for a single-storey retail building. 


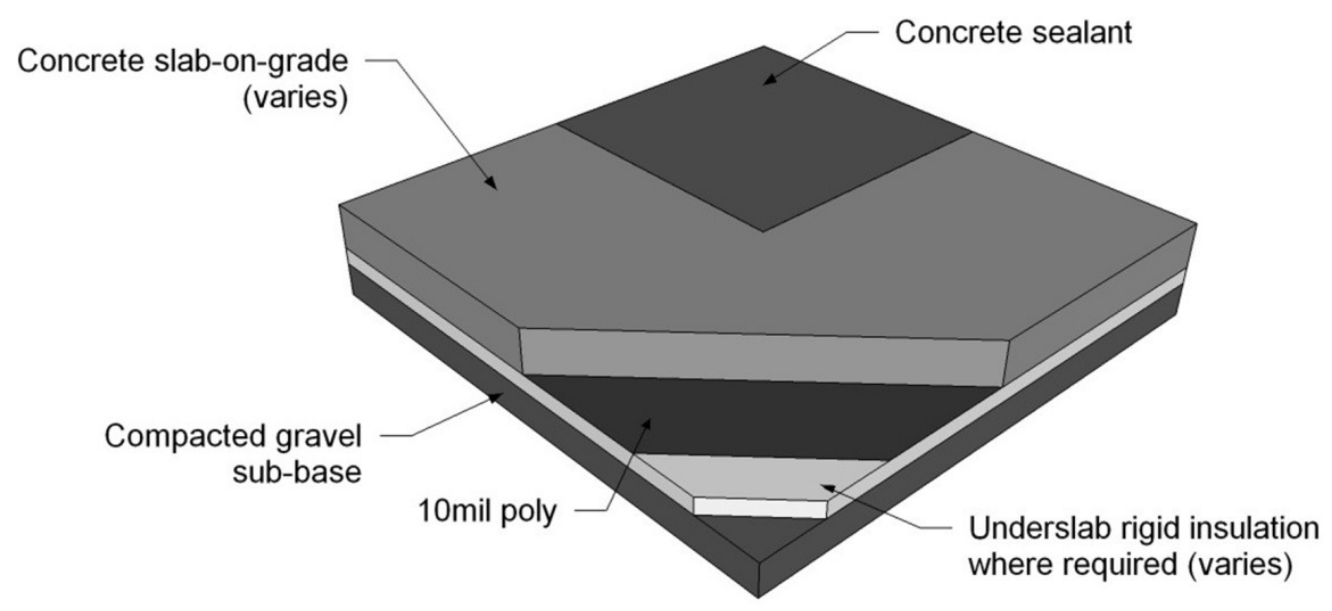

Figure 3-8: Typical Components of Slab-On-Grade

Table 3-5: Range of Foundation Design Strategies

\begin{tabular}{|c|c|c|}
\hline $\begin{array}{l}\text { Foundation } \\
\text { Type }\end{array}$ & Variables & Options \\
\hline \multirow{3}{*}{ IF-FDN } & Strength & $\begin{array}{l}-\quad 20 \mathrm{MPa} \\
-\quad 30 \mathrm{MPa}\end{array}$ \\
\hline & $\begin{array}{l}\text { Flyash } \\
\text { Content }\end{array}$ & $\begin{array}{ll}- & \text { Average flyash content }(9 \%) \\
- & \text { High flyash content }(35 \%)\end{array}$ \\
\hline & Footing Size & $\begin{array}{ll}- & 1,200 \mathrm{~mm} \times 1,200 \mathrm{~mm} \times 350 \mathrm{~mm} \\
- & 1,500 \mathrm{~mm} \times 1,500 \mathrm{~mm} \times 350 \mathrm{~mm} \\
- & 1,800 \mathrm{~mm} \times 1,800 \mathrm{~mm} \times 350 \mathrm{~mm} \\
- & 2,400 \mathrm{~mm} \times 2,400 \mathrm{~mm} \times 400 \mathrm{~mm}\end{array}$ \\
\hline \multirow{3}{*}{ PF-FDN } & Strength & $\begin{array}{l}-\quad 20 \mathrm{MPa} \\
-\quad 30 \mathrm{MPa}\end{array}$ \\
\hline & $\begin{array}{l}\text { Flyash } \\
\text { Content }\end{array}$ & $\begin{array}{ll}- & \text { Average flyash content }(9 \%) \\
- & \text { High flyash content }(35 \%)\end{array}$ \\
\hline & $\begin{array}{l}\text { Exterior } \\
\text { Insulation }\end{array}$ & $\begin{array}{ll}- & \text { None } \\
- & 50 \mathrm{~mm} \text { extruded polystyrene rigid insulation }\end{array}$ \\
\hline \multirow{3}{*}{ SOG-FDN } & $\begin{array}{c}\text { Slab } \\
\text { Thickness }\end{array}$ & $\begin{array}{l}-\quad 100 \mathrm{~mm} \\
-\quad 200 \mathrm{~mm} \\
\end{array}$ \\
\hline & $\begin{array}{c}\text { Flyash } \\
\text { Content }\end{array}$ & $\begin{array}{ll}- & \text { Average flyash content }(9 \%) \\
- & \text { High flyash content }(35 \%)\end{array}$ \\
\hline & $\begin{array}{l}\text { Under Slab } \\
\text { Insulation }\end{array}$ & $\begin{array}{ll}- & \text { None } \\
- & 50 \mathrm{~mm} \text { extruded polystyrene rigid insulation }\end{array}$ \\
\hline
\end{tabular}




\subsubsection{Description of Interior Partitions}

Nine different interior partition walls were examined in this study. A detailed description of each interior partition wall (including all of the assembly layers and the material quantities) can be found in Appendix B-7.

The various interior partition walls have been classified based on their structural framing material and have been placed into the following three categories:

- Concrete masonry unit partition walls (CMU-P)

- Cold-formed steel stud partition walls (SS-P)

- Wood stud partition walls (WS-P)

Figure 3-9 displays the typical assembly layers that were identified for each of the interior partition walls. In general, each interior partition wall consisted of an interior finish on either side of the wall and the structural framing (with cavity installed fiberglass batt insulation if required). The interior partition walls were not designed for load bearing applications.

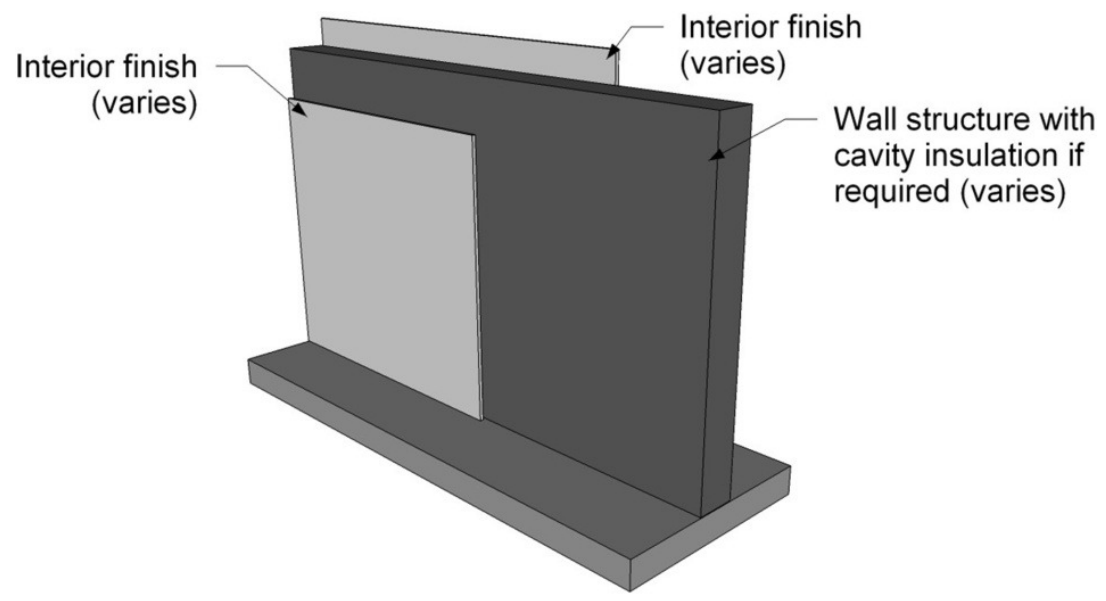

Figure 3-9: Typical Assembly Layers for Interior Partition Walls

The interior partition walls examined in this study are listed in Table 3-6, along with a breakdown of the different options that were explored. The primary variables considered were the framing material, the stud spacing, and the presence of insulation for fire-rating and sound dampening. 
Table 3-6: Range of Interior Partition Wall Design Strategies

\begin{tabular}{|c|c|c|}
\hline Type & Variables & Options \\
\hline CMU-P & None & $\begin{array}{l}\text { - Only one concrete masonry unit interior partition wall was examined. It consisted } \\
\text { of } 200 \mathrm{~mm} \text { standard weight concrete block with grouted 15M rebars @ } 400 \mathrm{~mm} \\
\mathrm{o} / \mathrm{c}\end{array}$ \\
\hline \multirow{2}{*}{ SS-P } & Structure & $\begin{array}{l}\text { - } \quad 39 \mathrm{~mm} \text { x } 152 \mathrm{~mm} 20 \text { ga. }(0.91 \mathrm{~mm}) \text { CFS studs @ } 400 \mathrm{~mm} \\
\text { - } \quad 39 \mathrm{~mm} \text { x } 152 \mathrm{~mm} 20 \text { ga. }(0.91 \mathrm{~mm}) \mathrm{CFS} \text { studs @ } 600 \mathrm{~mm}\end{array}$ \\
\hline & Insulation & $\begin{array}{ll}\text { - } & \text { None } \\
\text { - } & 140 \mathrm{~mm} \text { fibreglass batt insulation installed between CFS studs }\end{array}$ \\
\hline \multirow[t]{2}{*}{ WS-P } & Structure & $\begin{array}{l}\text { - } \quad 38 \mathrm{~mm} \times 140 \mathrm{~mm} \text { wood studs @ } 400 \mathrm{~mm} \\
\text { - } \quad 38 \mathrm{~mm} \text { x } 140 \mathrm{~mm} \text { wood studs @ } 600 \mathrm{~mm} \\
\end{array}$ \\
\hline & Insulation & - $\quad$ Same as SS \\
\hline
\end{tabular}

Up to this point, the baseline retail building has been discussed along with the range of alternative building components to be considered in the comprehensive LCA. The remainder of this chapter will concentrate on outlining the method that was followed in carrying out the LCA study. In particular, the scope of the LCA will be identified, as well as the methods that were followed for calculating the life-cycle embodied energy, embodied GWP, operating energy, and operating GWP of a typical single-storey retail building in Canada.

\subsection{Scope of LCA}

According to ISO 14044, the first step in performing any LCA study is defining the goal and scope (ISO, 2006). The goal of this LCA study has already been discussed: to conduct a comprehensive LCA for the components of a single-storey retail building located in Toronto, Canada, to determine which building components contribute the most towards the total life-cycle energy use and GWP after 50 years. In this section, the scope of the LCA study will be presented.

From one LCA study to the next, there can be substantial variation in the scope of the analysis. Some LCA studies only consider the immediate effects, such as the on-site construction and operating energy of a building (and its components). Other studies get into more detail and look back up the supply chain to account for the environmental effects that are associated with mining the natural resources, manufacturing the building materials, transporting them to the construction site, and so on. Therefore, it is very important to specify the system boundaries as well as the outputs of the LCA analysis. Figure 3-10 illustrates the system boundaries and outputs for the LCA in this study. 


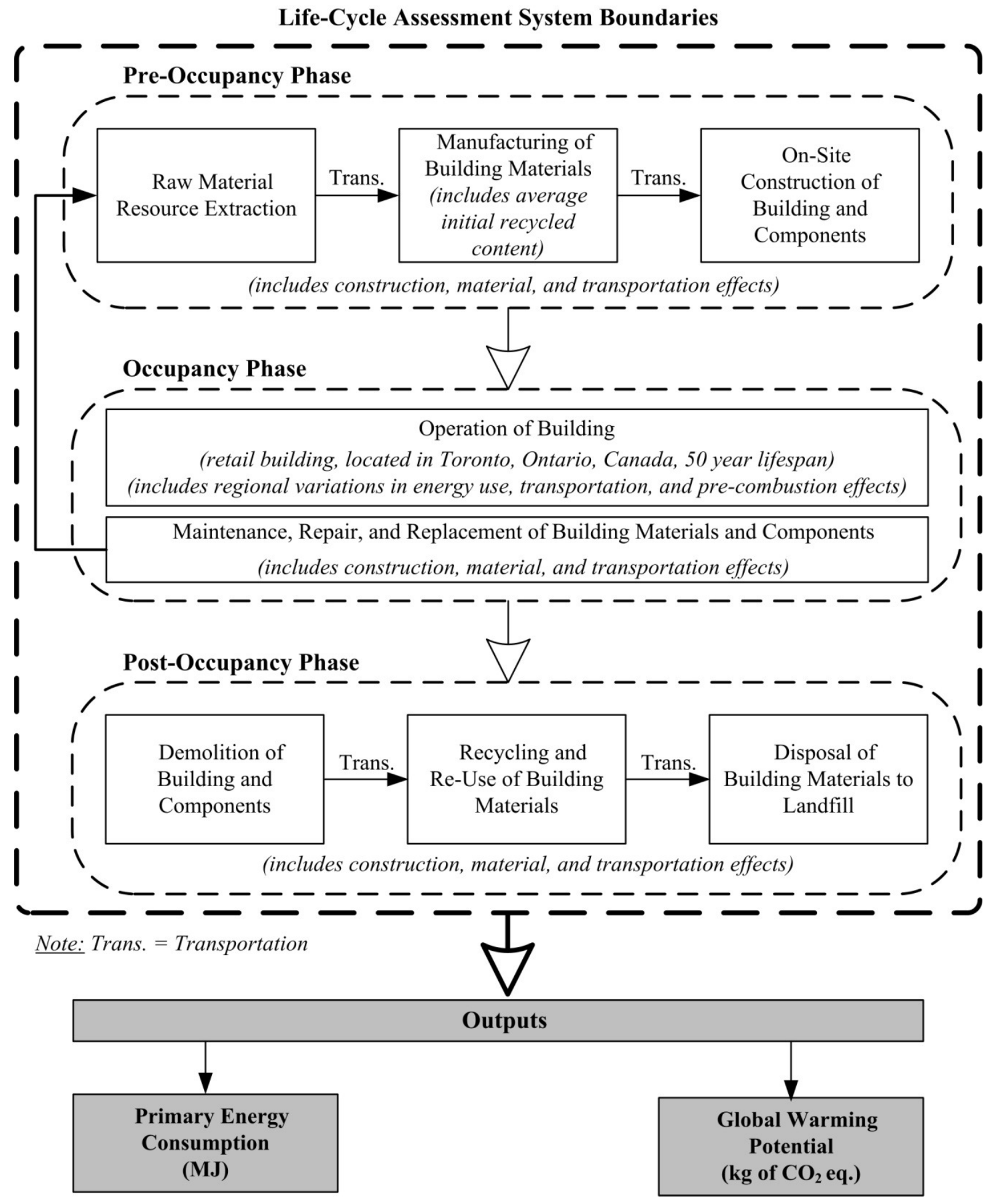

Figure 3-10: Life-Cycle Assessment System Boundaries and Outputs 
In the LCA of the components of a single-storey retail building in this study, the system boundaries can essentially be divided into three categories: the pre-occupancy phase, the occupancy phase, and the post-occupancy phase. The outputs of this LCA study include the total primary energy consumption and the total GWP for all three phases.

The pre-occupancy phase includes the effects of mining the raw materials, manufacturing the building materials, and constructing the building (and its components) on-site. The occupancy phase is concerned with the operation of the building over its lifespan (i.e. the non-renewable resources used for heating, cooling, ventilating, lighting, etc.) as well as the effects of maintenance, repair, and replacement of the various building components over their lifespan. The post-occupancy phase deals with the demolition of the building, the recycling and re-use of the building materials, and the disposal of the remaining waste materials. At each stage, the total transportation effects are included, along with the construction and material effects. It is also important to note that the primary energy consumption and the GWP associated with each of the three phases were calculated based on region specific data. In this case, Toronto was used as the location for the LCA study. Therefore, the energy generation profile (i.e. percentage of energy from hydroelectric, nuclear, coal, oil, natural gas, etc.) of Toronto was used, as well as the transportation and repair/maintenance profiles of a building located in Toronto. In other words, the outputs were generated from location specific data.

The outputs of this LCA study were generated for a building with a 50 year lifespan. A 50 year lifespan is commonly used in LCA studies of buildings and allows for a reasonable cycle of maintenance, repair, and replacement of building components.

As mentioned, the two main outputs from this LCA study are primary energy consumption and GWP. Recall that primary energy includes "the effects of energy used by the final consumer (secondary energy use), non-energy uses, intermediate uses of energy, energy in transforming one energy form to another (e.g. coal to electricity), and energy used by suppliers in providing energy to the market (e.g. pipeline fuel)" (Natural Resources Canada, 2008). Some LCA studies only consider the secondary energy in their outputs. However, by using primary energy as one of the outputs of this study, the result is a more complete analysis of the actual energy use across all three phases.

\subsection{Evaluating Embodied Energy and Embodied GWP}

Now that the goal and scope of the LCA have been identified, a discussion of the methods for conducting the LCA can proceed. From the literature review it is known that energy use and GWP for 
a building can be divided into embodied effects and operating effects. The method for evaluating the embodied effects in this study will be presented first, followed by a discussion of how the operating effects were accounted for in the next section.

Accurately accounting for the environmental damage caused by a building over its lifespan is a very complex task. Buildings and their components are far from homogonous assemblies and often are comprised of an extensive array of materials that are intertwined within numerous sub-assemblies. Accurately identifying, quantifying, and optimizing all of the materials used over the lifespan of a building from an environmental perspective is extremely complex. A major source of difficult arises from the fact that buildings and their component parts are comprised of numerous different materials and their effects are linked to multiple different industries. Accounting for the energy use and GWP at each stage of the raw material acquisition, processing, manufacturing of building materials, transportation, construction, repair, replacement, and end-of-life effects of whole buildings (and their components) is computationally intense. Fortunately, there is a growing list of computer programs available to calculate the cradle-to-grave environmental impacts of both building materials and whole building projects.

In North America, the ATHENA® Environmental Impact Estimator (ATHENA® EIE) for Buildings is the only software tool available that is specific to the North American industry, can evaluate both whole buildings and individual assemblies, and is based on internationally recognized LCA methods. The ATHENA $®$ EIE for Buildings is quickly becoming the standard for LCA calculations in the North American building industry. The ATHENA® EIE for Buildings v4.0.64 (The Athena Institute, 2010) was used in this study to calculate the embodied energy and embodied GWP for each alternative building component of a single-storey retail building over a 50 year lifespan in Toronto, Canada.

The ATHENA® Institute is a non-profit organization that has been around for over a decade. The Institute is dedicated to improving the sustainability of the built environment by providing a tool that can be used at the conceptual design phase of a building to evaluate and compare alternative design options within a comprehensive LCA methodology.

Recall that according to ISO 14044, the second stage of any LCA study is the definition of a lifecycle inventory LCI) of the materials and their associated environmental impacts (ISO, 2006). As one might imagine, calculating the environmental impacts of all the materials in a building project would be incredibly complex and time consuming. In fact, without access to the energy use and GWP data 
from the manufactures of the building materials, this would be impossible. Fortunately, the ATHENA ${ }^{\circledR}$ Institute has spent a great deal of money, time, and resources compiling a comprehensive LCI database for the various building materials and continues to update the database as new information becomes available. A major strength of the LCI database is the fact that it is regionally specific to North America and considers variations in manufacturing technology, energy generation, recycled content, and transportation depending on the location of the building project. The ATHENA ${ }^{\circledR}$ LCI database is widely considered to be the most comprehensive and relevant LCI database for the North American building industry and is the main engine used in the ATHENA® EIE for Buildings.

Using its comprehensive LCI database, the ATHENA® EIE for Buildings v4.0.64 considers the full life-cycle impacts of (The Athena Institute, 2009):

- Building type and lifespan

- Material manufacturing, including resource extraction and initial recycled content

- Related transportation effects

- On-site construction effects

- Regional variation in energy use, energy generation, transportation, and other factors

- Maintenance, repair, and replacement effects over the building's lifespan

- Demolition, disposal, and recycling effects at the end of the building's lifespan

- Operating energy emissions and pre-combustion effects over the building's lifespan

The ATHENA® EIE is able to summarize the complex LCA calculations into a series of useful measures. For the purposes of this study, the primary energy consumption and the GWP were the two important outputs that were calculated.

Each building component identified in this study was individually modeled as accurately as possible using the standard inputs in the ATHENA ${ }^{\circledR}$ EIE for Buildings v4.0.64. A bill of materials was then generated in the ATHENA® EIE for each case. This bill of materials was compared to expected results and any discrepancies were overcome by adjusting the material quantities for the building component via. the user specified additional materials input feature of the software. It should be mentioned that the ATHENA® EIE is currently unable of calculating the embodied effects associated 
with the mechanical, electrical, and plumping services in a building directly. Therefore, the embodied effects associated with these services have not been accounted for in this study.

Ultimately, calculating the life-cycle primary energy consumption and GWP of a building and its component parts is not an exact science. Some degree of uncertainty is inevitable given the complexity of the calculations and the inherent degree of uncertainty in the LCI data. However, the ATHENA® EIE for Buildings v4.0.64 provides the best method for estimating these effects for the North America building industry today. According to the ATHENA® Institute, the ATHENA® EIE for Buildings is able to "model well over 1000 structural and envelope assembly combinations and is generally applicable to more than $90 \%$ of the typical North American building stock" (The Athena Institute, 2008). However, there is one significant limitation of this software. The ATHENA® EIE for Buildings is unable of calculating the operating energy consumption and operating GWP of a building directly. In fact, the total energy use and total GWP of a building is a combination of the embodied energy, embodied GWP, operating energy, and operating GWP. Therefore, since the ATHENA® EIE is only capable of calculating the embodied effects, additional means had to be employed to calculate the operating effects. The method for calculating the operating effects for the components of a building will be discussed next.

\subsection{Evaluating Operating Energy and Operating GWP}

Recall that the total energy use of a building is a combination of the total embodied energy and the total operating energy. The ATHENA® EIE for Buildings is able to calculate the embodied energy of the building materials, but is unable to calculate the operating energy consumption of a building directly. It does have a calculator that converts operating energy (i.e. secondary energy) into primary energy and GWP over a building's lifespan. However, additional software programs are required to determine the appropriate fuel consumption due to building operations to input into the ATHENA® EIE converter. There are a number of different computer programs that are available to do this and they all vary in terms of their difficulty to use and their comprehensiveness. The 'Quick Energy Simulation Tool' (eQUEST) computer software provides an excellent combination of both a user friendly interface and detailed building energy simulation capability.

eQUEST is based on the latest DOE-2 building simulation engine. DOE-2 is the most widely respected building energy simulation program available today. It has been around since the 1970's and has been funded in large part by ASHRAE, NASA, and the United States Department of Energy. 
eQUEST allows a user to perform sophisticated hourly energy simulations of a building to predict its operating energy use. Among other inputs, eQUEST allows for a detailed description of a building's geometry, layout, envelope, operating schedule, space conditioning systems (such as HVAC and lighting), climatic data, and much more. The result is a comprehensive and detailed output of monthly and annual energy use for the building. Recall from the literature review that two kinds of energy were identified: secondary energy (i.e. operating energy) and primary energy. Embodied energy is expressed in terms of primary energy. However, eQUEST (and other building energy modelling programs) calculate secondary energy. Therefore, once the annual energy use of a building has been determined from eQUEST, it can be entered into the ATHENA® EIE converter to calculate the resulting total primary energy consumption and total GWP. By converting the operating energy (i.e. secondary energy) into primary energy, the results can be compared directly with the embodied energy results from the ATHENA® EIE for Buildings.

Calculating the embodied energy for each of the 220 different building components in this study is relatively straightforward using the ATHENA® EIE for Buildings. However, estimating the impact on the operating energy of a building for each of the 220 different building components is less straightforward. In order to get an estimate of the operating energy for each of the 220 different building components in this study, an energy model was created for the baseline retail building using QUEST v3.63 (Hirsch, 2009). Using this model, the 50 year operating energy use was estimated for the baseline retail building. Once the breakdown of natural gas and electricity use was determined from eQUEST, the numbers were input in the ATHENA® EIE converter and the total operating primary energy use and GWP was determined for the baseline retail building. These values became the datum for all subsequent energy models in this study.

Next, using the eQUEST model of the baseline retail building as the datum, a new eQUEST model was created for each of the 220 different building components. In each model, all of the other building variables were held constant, except that one of the 220 different building components was substituted for the corresponding component in the baseline retail building model. In each case, the 50 year operating energy use of the modified baseline retail building was simulated in eQUEST. By doing this for each of the 220 different building components, the 50 year operating energy of the modified baseline retail building could be compared to the 50 year operating energy of the baseline retail building. The difference (either an increase in energy or a decrease in energy) from the baseline could be found and the difference attributed to the corresponding substitution of a particular building 
component. Therefore, one-by-one the impact on the 50 year operating energy of the baseline retail building due to the systematic substitution of the 220 different building components could be determined. In each case, the annual electricity and natural gas use of the modified baseline retail building from eQUEST was input into the ATHENA® EIE converter and the primary operating energy and GWP for each of the 220 building components was determined. In this way, the impact that each of the 220 different building components in this study had on the operating energy use of a single-storey retail building after 50 years could be estimated.

\subsection{Calculating the Thermal Resistance of Building Enclosures}

In the previous section, a method was presented for calculating the impact on the operating energy of a typical single-storey retail building for each of the 220 different building components. In each case, an eQUEST model was created and the 50 year operating energy of the modified baseline retail building was determined. However, in order to create the eQUEST models, the thermal resistance of the different building components had to be determined. In particular, it was important to get an accurate measure of the thermal resistance for each exterior infill wall, roof, window, door, and slabon-grade in order to determine the corresponding operating energy and GWP related to each case.

This section begins with a brief overview of some basic principles of heat transfer through buildings and some of the underlying assumptions that were made for this study. This is followed by a comparison of the primary methods that were used in this study to calculate the thermal resistance of the different building components.

\subsubsection{Background on Heat Transfer in Buildings}

Accurately accounting for heat transfer through the building enclosure is critical to calculating the total life-cycle energy and GWP of a building. In addition, "understanding heat transfer and the temperature distribution through building materials and assemblies is important for assessing energy use, thermal movements, durability, and the potential for moisture problems" (Straube \& Burnett, 2005).

A number of LCA studies have estimated that the operating energy of a typical building (either residential or commercial) is upwards of $85 \%$ or more of the total life-cycle energy after 50 years. With operating energy contributing so heavily towards the overall environmental burdens of a building, it is critical to accurately calculate the resistance to heat flow through each alternative building enclosure, in order to determine which systems use less energy over time. 
Thermal resistance is a measure of the ability of a building material (or assembly) to resist heat flow though it. In the imperial system, thermal resistance is expressed in terms of R-value and has units of $\left(\mathrm{hr} \cdot \mathrm{ft}^{2} \cdot{ }^{0} \mathrm{~F}\right) / \mathrm{Btu}$. In the $\mathrm{SI}$ system, thermal resistance is expressed in terms of $\mathrm{R}_{\mathrm{SI}^{-}}$-value and has units of $\left(\mathrm{m}^{2} \cdot \mathrm{K}\right) / \mathrm{W}$.

Alternatively, the heat flow through an enclosure assembly is sometimes expressed in terms of the overall heat transfer coefficient (U-value or $\mathrm{U}_{\mathrm{SI}^{-}}$-value). The overall heat transfer coefficient is "a system measure of the amount of heat flow that will occur across a unit area of an enclosure system or other assembly for a unit temperature difference" (Burnett \& Straube, 2005). The overall heat transfer coefficient is simply the inverse of the thermal resistance and is often referred to in these types of calculations.

Heat flow through the building enclosure can occur in one, two, or three dimensions. It can also be steady state or transient, where temperature and/or heat flow vary with time. The appropriate method for calculating the thermal resistance of a building enclosure depends on the type of problem at hand. That being said, there are several ways of calculating the thermal resistance of the building enclosure. The three most common methods are discussed next, as well as the method that was chosen for this study.

\subsubsection{Calculating One-Dimensional Heat Flow Using the Tabular Method}

The tabular (or series) method is the simplest of the three methods and as such, has a limited range of usefulness. It is appropriate to use when the enclosure assembly can be considered to be a onedimensional, steady-state heat flow system, where no significant thermal bridges (a short circuit for heat flow) exist such as wood, steel, or concrete penetrations through the enclosure.

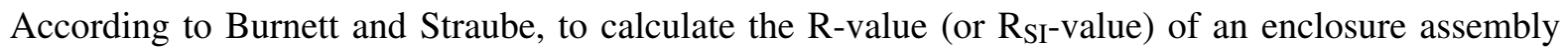
using the tabular method, the following steps should be taken (Burnett \& Straube, 2005):

1. List each material in the enclosure assembly, its conductivity (k), and its thickness $(l)$

2. Calculate the conductance $(\mathrm{C})$ of each layer using $\mathrm{C}=\mathrm{k} / l$

3. Calculate the thermal resistance of each layer $\left(\mathrm{R}_{\mathrm{SI} \text { Layer }}\right) \mathrm{using} \mathrm{R}_{\mathrm{SI} \text { Layer }}=1 / \mathrm{C}$

4. Sum the individual thermal resistances of each layer $\left(\mathrm{R}_{\mathrm{SI}}\right.$ Layer $)$ to get the overall thermal resistance $\left(\mathrm{R}_{\mathrm{SI} \text { Overall }}\right)$ of the entire assembly: $\mathrm{R}_{\mathrm{SI} \text { Overall }}=\sum \mathrm{R}_{\mathrm{SI} \text { Layer }}$ 
5. Take the inverse of the overall thermal resistance to get the overall heat transfer coefficient $\left(\mathrm{U}_{\mathrm{SI}}\right.$ Overall $): \mathrm{U}_{\mathrm{SI} \text { Overall }}=1 / \mathrm{R}_{\mathrm{SI} \text { Overall }}$

Figure 3-11 shows an example of a wall assembly in this study, where the thermal resistance can be reasonably approximated using the tabular method. There are no significant thermal bridges through this assembly, so a one-dimensional analysis can be applied with reasonable accuracy. Table 3-7 illustrates how an application of the tabular method can result in an approximation of the overall resistance to heat flow through the wall system.
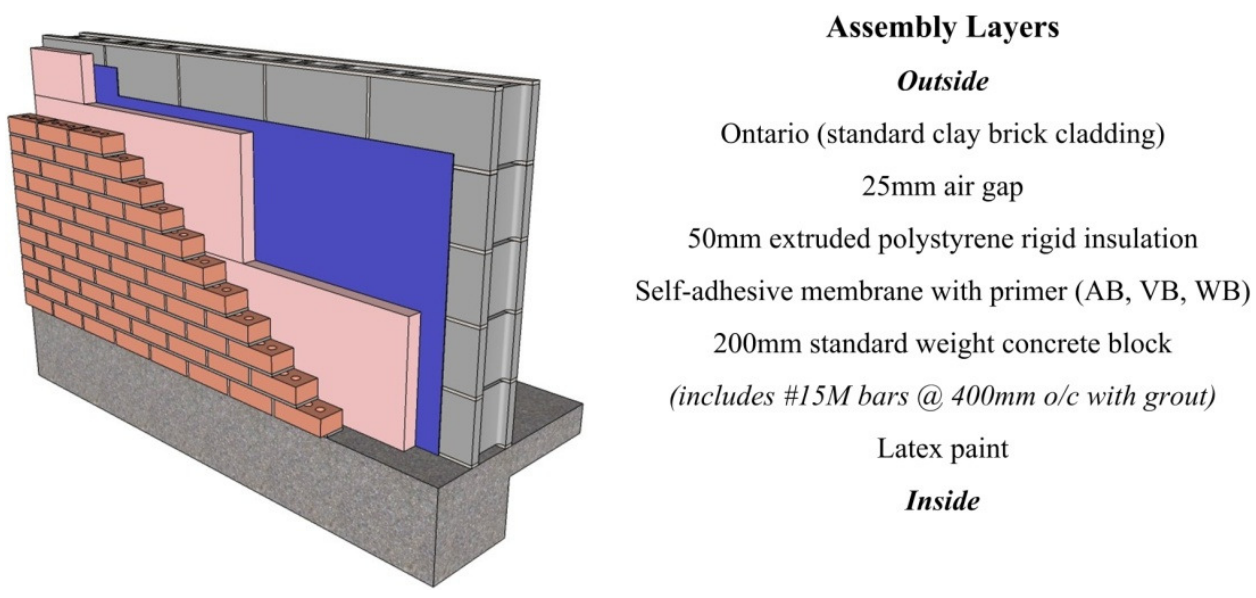

Figure 3-11: Assembly Layers for Concrete Masonry Unit Wall \#1 (CMU-W1)

As mentioned earlier, this method has its limitations. It can only be used to calculate the thermal resistance of very simple building enclosures. In this study, it was not appropriate to assume steady state, one-dimensional heat flow for all of the building enclosures. Many of the building enclosures in this study had thermal bridging, which requires at least a two-dimensional analysis to accurately calculate the overall thermal resistance. In this study, the tabular method was used to verify the results of other methods and not as the primary method for calculating the thermal resistance of the enclosures. The two primary methods that were used to calculate the two-dimensional heat flow through the building enclosures in this study are discussed next. 
Table 3-7: Calculating Thermal Resistance of CMU-W1 Using the Tabular Method

\begin{tabular}{|c|c|c|c|c|}
\hline Layer Material & $\begin{array}{c}\text { Conductivity } \\
(\mathbf{k}) \\
\mathbf{W} /(\mathbf{m} \cdot \mathbf{K})\end{array}$ & $\begin{array}{c}\text { Thickness } \\
(l) \\
\text { m }\end{array}$ & $\begin{array}{c}\text { Conductance } \\
(\mathbf{C}) \\
\mathbf{W} /\left(\mathbf{m}^{2} \cdot \mathbf{K}\right)\end{array}$ & $\begin{array}{c}\text { Resistance } \\
\left(\mathbf{R}_{\text {SI Layer }}\right) \\
\left(\mathbf{m}^{2} \cdot \mathbf{K}\right) / \mathbf{W}\end{array}$ \\
\hline $\begin{array}{l}\text { Exterior air film (moving air, winter } \\
\text { conditions, } \varepsilon=0.90 \text { ) }\end{array}$ & - & - & 33.40 & 0.03 \\
\hline Ontario (standard) clay brick cladding & 1.30 & 0.09 & 14.44 & 0.07 \\
\hline $25 \mathrm{~mm}$ air gap & - & - & 5.19 & 0.19 \\
\hline $\begin{array}{l}\text { 50mm extruded polystyrene rigid } \\
\text { insulation }\end{array}$ & 0.03 & 0.05 & 0.58 & 1.72 \\
\hline Self-adhesive membrane with primer & N/A & N/A & N/A & N/A \\
\hline $\begin{array}{l}\text { 200mm standard weight concrete block } \\
\text { with solid grouted cores @ 400mm o/c }\end{array}$ & - & - & 5.10 & 0.20 \\
\hline Latex paint & N/A & N/A & N/A & N/A \\
\hline Interior air film (still air, $\varepsilon=0.90$ ) & - & - & 8.35 & 0.12 \\
\hline
\end{tabular}

\subsubsection{Calculating Two-Dimensional Heat Flow Using THERM}

One-dimensional, steady-state heat flow calculations using the tabular method no longer accurately predict the heat flow through an enclosure, when one of the following conditions exist (Burnett \& Straube, 2005):

1. Thermal bridging - a short circuit for heat flow through the building enclosure when a structural member such as wood, steel, or concrete penetrates through the enclosure.

2. Thermal mass - storage of heat in thermally massive materials (such as concrete and stone) which is stored and released throughout the day. This process is not steady-state.

3. Air leakage - a loss of air through the building enclosure which results in excess heat loss that would not be accounted for by a one-dimensional analysis.

Given any of these conditions, a two or three-dimensional heat flow analysis is required. In this study, two-dimensional heat flow analysis was required due to all three of the above conditions being true.

There are many readily available computer programs that can perform a two-dimensional heat flow analysis of building enclosures. These programs rely on a finite element analysis to predict the heat 
flow in two dimensions. In this study, THERM v5.2.14 (LBNL, 2009) was used. THERM was developed by the Lawrence Berkeley Laboratory in California and is well respected within the building science industry. In this study, each alternative building enclosure was modelled in THERM to get an estimate of the overall heat transfer coefficient ( $U_{\text {SI Overall). }}$.

Figure 3-12 shows an example of a typical wall assembly from this study that was modelled in THERM, to determine the overall heat transfer coefficient $\left(U_{\text {SI Overall }}\right.$ ). Figure 3-13 shows both the THERM model that was created for this wall assembly, as well as a plot of the resulting temperature distribution (heat flow) through the enclosure.
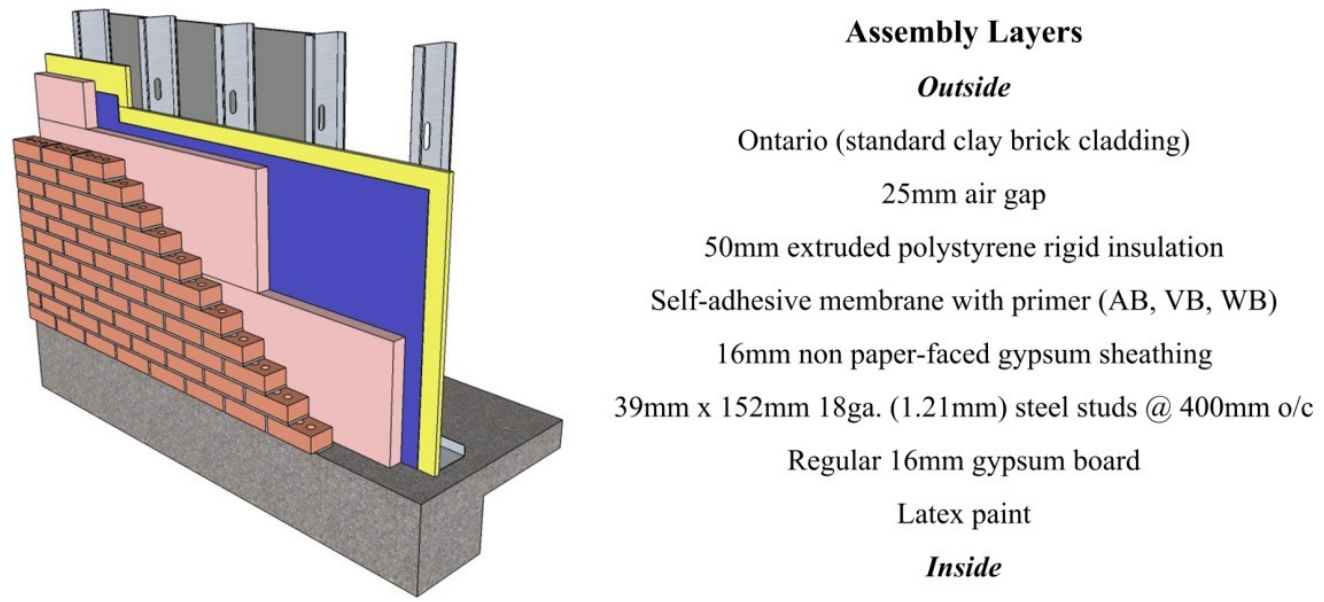

Figure 3-12: Assembly Layers for Cold-Formed Steel Stud Wall \#1 (SS-W1)

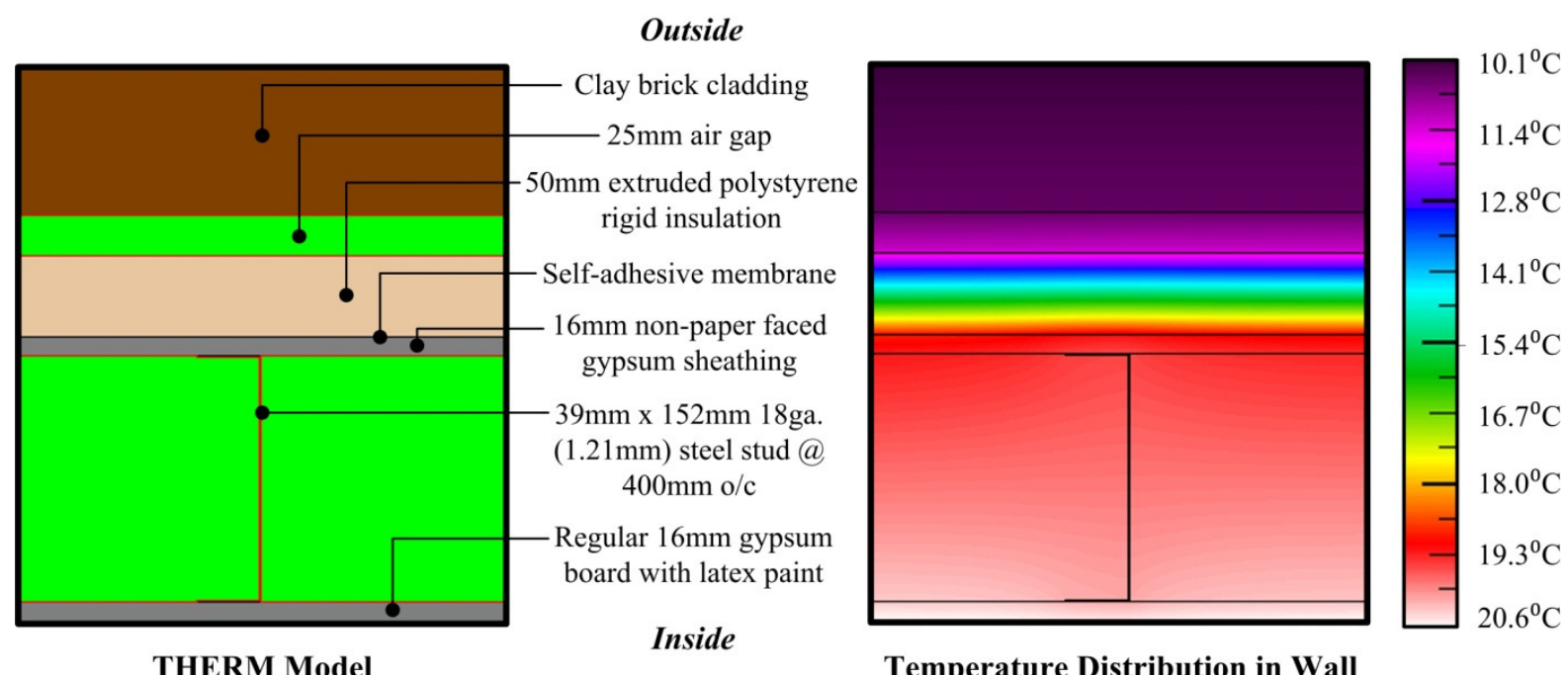

Figure 3-13: Example of Two-Dimensional Heat Flow through SS-W1 Using THERM 
A similar model was constructed for each building enclosure component in this study, for which an approximation of the thermal resistance was required. The thermal resistance of each building component can be found in Appendix B.

For each THERM model in this study, both the geometric properties of the building enclosure, as well as the conductivity $(\mathrm{k})$ of each different building material were specified. For each different building material there is a range of possible values for the conductivity based on numerous parameters (such as the moisture content of a material, the specific chemical or physical composition of the material, etc.). In this study, the conductivity of the individual building materials were primarily taken from the THERM database, ASHRAE Standard 90.1-2007 (ASHRAE, 2007), and "Building Science for Building Enclosures" (Straube \& Burnett, 2005). In each THERM model, appropriate interior and exterior air films were included on the exterior cladding surface and the interior finish surface of the assemblies. In each case, an adiabatic boundary condition was also specified at either end of the enclosure, as only a portion of the entire building enclosure was modelled in THERM.

Although a two-dimensional heat flow analysis is reasonably accurate for the types of building enclosures that were modelled in this study, there are some sources of error. For example, the heat loss through the sill plate and top plate of the wood stud and steel stud walls was not accounted for. Neither was the heat loss due to additional framing around window and door openings. The THERM model only considered a typical section cut through the building enclosure, not all of the unique framing effects. Also, thermal bridging at the corners of buildings (where two walls intersect) was a source of heat loss that was not accounted for in this study. To accurately account for the heat loss through these irregularities (such as sill plates, top plates, framing effects around openings and at corners of walls), a three-dimensional, or even four-dimensional (which includes thermal mass effects) heat flow analysis would be required. This level of accuracy is beyond the scope of this project.

\subsubsection{Calculating Two-Dimensional Heat Flow Using ASHRAE Standard 90.1-2007}

In addition to calculating the thermal resistance of each building enclosure in this study using THERM, ASHRAE Standard 90.1-2007 was also used for comparison/verification of the results.

The original American Society of Heating, Refrigerating and Air-Conditioning Engineers (ASHRAE) Standard was first published in 1975. The purpose of the Standard is "to provide minimum requirements for the energy-efficient design of buildings, except low-rise residential buildings" 
(ASHRAE, 2007). The ASHRAE Standard 90.1-2007 is the most recent publication and it outlines the minimum energy-efficient requirements for the design and construction of new buildings (and their systems) and new portions of buildings (and their systems). This includes provisions for the envelope of buildings and the systems and equipment of buildings. Compliance with the ASHRAE Standard is completely voluntary and it is not enforceable under any building codes in Canada at this time. However, the ASHRAE 90.1 Standard is widely adopted within the building industry, so it was important to evaluate the thermal resistance of the building enclosures in this study using this method as well. Section 5 of ASHRAE Standard 90.1-2007 deals with the building envelope. Generally speaking, this section provides guidelines for estimating the thermal resistance of walls, roofs, fenestrations (windows), doors, and foundations (slab-on-grades).

For the purposes of this study, ASHRAE climate zone 6 (Toronto, Canada) was assumed. Generally speaking, for each building enclosure type (exterior infill walls, roofs, fenestrations, doors, and foundations), a base assembly is specified in the ASHRAE Standard. This base assembly is usually representative of a typical assembly that would be specified in a commercial type building. To determine the thermal resistance of a specific building enclosure using the ASHRAE Standard, the general procedure involves:

1. Identifying the ASHRAE base assembly that most closely approximates the building enclosure in question.

2. Following the various guidelines and tables in the ASHRAE Standard to modify the ASHRAE base assembly until it accurately represents the building enclosure in question.

3. Calculating the thermal resistance of the building enclosure in question.

As mentioned earlier, both THERM and ASHRAE Standard 90.1-2007 were used to determine the thermal resistance of the building enclosures identified in this study. However, there were some exceptions to this, when only one of the two methods was used. The following list identifies which method was used to calculate the thermal resistance of the building enclosures in this study:

- Exterior infill walls: THERM and ASHRAE Standard 90.1-2007

- Roofs: THERM and ASHRAE Standard 90.1-2007

- Fenestration (windows) and doors: ASHRAE Standard 90.1-2007 only

- Foundations (slab-on-grades): THERM only 
When calculating the thermal resistance of the exterior infill walls and roofs, both the THERM and ASHRAE Standard 90.1-2007 values were calculated. Both values have been listed for each applicable building component in Appendix B.

In practice, there are a huge number of different windows and doors that could be specified. These types of enclosures can vary in a number of ways including: frame material, number of glazing layers, argon-filled verses air-filled, low-E coatings, and fixed verses operable. The ASHRAE Standard lists the overall U-value (including the frame effects and glazing effects) for a large variety of fenestration types. Rather than trying to model a large number of these very unique and complex types of enclosures using THERM, typical U-values were simply selected from the supplementary information on fenestrations found in the ASHRAE Standard (ASHRAE Fundamentals SI, 2009). Therefore, when it came to calculating the thermal resistance of the windows and doors in this study, only the ASHRAE Standard 90.1-2007 values were used.

When if came to calculating the thermal resistance of the foundations (slab-on-grades) in this study, only the THERM results were used and not the ASHRAE Standard values. The ASHRAE Standard uses what they call an 'F-factor' (rather than an R-value) for specifying the thermal resistance of slabon-grades. The F-factor is a measure of the perimeter heat loss around the slab-on-grade. In this study, THERM was the sole means of calculating the thermal resistance of the slab-on-grades, not the F-factor method as specified in the ASHRAE Standard.

In cases where the thermal resistance was calculated using both the THERM and the ASHRAE Standard, some slight differences were noticed. The next section will discuss some of the reasons that the results from these two methods are slightly different.

\subsubsection{Explanation of Differences in Thermal Resistance between THERM and ASHRAE Standard 90.1-2007}

As mentioned in the previous section, when it came to calculating the thermal resistance of the exterior infill wall and roof enclosures in this study, two methods were used: THERM and ASHRAE Standard 90.1-2007. The thermal resistance of each exterior infill wall and roof in this study can be found in Appendix B. Both the results from THERM as well as the results that were calculated using the ASHRAE Standard 90.1-2007 have been included for comparison purposes. An explanation of the differences between the two methods will be presented next. As well, an explanation of the method that was ultimately chosen for this study will also be discussed. 
In many cases, the ASHRAE Standard values were found to be somewhat generic and simplified values, due to the fact that they must be applicable to a wide range of alternative enclosures. In many cases, so long as the major components of a wall or roof assembly are indentified in ASHRAE (i.e. the structure and the insulation), then the thermal resistance of that assembly can be calculated with reasonable accuracy. However, the generic approached to calculating the thermal resistance of building enclosures using the ASHRAE method often neglects the unique differences between assemblies. This can have a tendency to underestimate the true thermal resistance of an enclosure.

\section{Exterior Infill Wall Enclosures}

In almost every case, the thermal resistances of the walls calculated using THERM were found to be slightly higher than the values calculated using the ASHRAE Standard. Typically, the thermal resistance that was calculated using THERM was between $1 \%$ and $15 \%$ higher than the thermal resistance that was calculated for the same wall enclosure using the ASHRAE Standard 90.1-2007. The maximum difference between the two methods in any case was found to be about $23 \%$. The reason that the THERM values are higher than the ASHRAE values is because the ASHRAE method does not consider the thermal resistance of the cladding material or any air spaces within the enclosure. On the contrary, the THERM values include the thermal resistance contributions from every single layer of the enclosure assembly, including the different cladding materials and the air spaces. Therefore, the THERM method is slightly more comprehensive than the ASHRAE method, in that every single layer of the assembly was included in the calculation of thermal resistance. Thus, in this study the THERM $\mathrm{R}_{\mathrm{SI}}$-values were used instead of the ASHRAE values, as it was felt that the THERM values were even more accurate that the ASHRAE values. The ASHRAE values were included in Appendix B along with the THERM values for comparison.

\section{Roof Enclosures}

Similar to the wall enclosures, the thermal resistances of the roofs that were calculated using THERM were found to be slightly higher in most cases than the values calculated using the ASHRAE Standard. Typically, the thermal resistance that was calculated using THERM was between $1 \%$ and $10 \%$ higher than the thermal resistance calculated for the same roof enclosure using the ASHRAE Standard 90.1-2007. The maximum difference between the two methods in any case was found to be about $12 \%$. The reason that the THERM values are slightly higher than the ASHRAE values is because the ASHRAE method does not consider the thermal resistance of the roof covering or the 
roof deck (other than for the case of a metal deck). On the contrary, the THERM values include the thermal resistance contributions from every single layer of the roof assembly, including the different roof coverings and roof deck materials. Thus, in this study the THERM $\mathrm{R}_{\mathrm{SI}}$-values were used instead of the ASHRAE values, as it was felt that the THERM values were more accurate. The ASHRAE values were again included in Appendix B along with the THERM values for comparison.

Therefore, in many instances, the thermal resistance values that were calculated using the ASHRAE Standard were found to be slightly conservative, in that they underestimated the true thermal resistance of the enclosures. The ASHRAE Standard is somewhat generic and simplified, due to the fact that it must be applicable to a wide range of similar enclosures that only differ in some small way. Although the omissions by the ASHRAE Standard are small in terms of the overall thermal resistance of the building enclosures (usually less than 10\%), this study is concerned with evaluating the life-cycle environmental burdens of many similar enclosures and requires a higher degree of accuracy. This higher degree of accuracy was provided by the THERM method. Every single assembly layer (including the cladding materials, air spaces, roof coverings, and roof decking) were input and modelled in THERM. Thus, in this study the THERM $\mathrm{R}_{\mathrm{SI}^{-}}$-values were used instead of the ASHRAE values, as it was felt that the THERM values were more accurate that the ASHRAE values. However, the ASHRAE values were still calculated and included in the results for comparison purposes.

\subsection{Evaluating Total Energy and Total GWP}

Thus far the discussion has focused on methods of calculating embodied energy, embodied GWP, operating energy, and operating GWP for buildings and their components. In this section, the two results are combined as a method for calculating the total life-cycle energy and total GWP of a building and its components is discussed.

The total energy or total GWP is a combination of the total embodied energy, total embodied GWP, total operating energy, and total operating GWP. This can be explained using the example of a wall enclosure. Suppose there is a choice of using two different wall enclosures on a building project. These two walls will be made up of different materials. Therefore, there will be a difference in the embodied energy or embodied GWP between the two walls. However, depending on the assembly layers (such as the cladding material, insulation, structural framing, etc.) these two walls will also have a different thermal resistance $\left(\mathrm{R}_{\mathrm{SI}}\right.$-value). Therefore, over the lifespan of a building, both of 
these walls will have a different influence on the operating energy of the building. It is important to note that just because a building assembly has a higher embodied energy or embodied GWP this does not necessarily mean that it will have a higher operating energy or operating GWP. For example, adding insulation to a wall assembly will increase the embodied energy of the wall, but will result in a lower operating energy. This is also true of other building components such as the roof, windows, and foundations for example. The total energy and total GWP accounts for both the materials effects and the operating effects of a building assembly.

The total embodied energy (and total embodied GWP) and the 50 year operating energy (and operating GWP) for the baseline retail building can be calculated. Then, by systematically substituting the 220 different building components in this study for the corresponding building component in the baseline retail building, the difference in the total energy or total GWP from the baseline building can be determined in each case. Therefore, the difference in the total energy from the baseline retail building for each of the 220 different building components in this study was calculated according to Eq. 1 for a 50 year lifespan.

$$
\Delta \text { T.E. }=\Delta \text { T.E.E. }+\Delta \text { T.O.E. }
$$

Where $\Delta \mathrm{T} . \mathrm{E}=$ difference in the total energy from the baseline retail building after 50 years due to changing baseline building component to an alternative building component, $\Delta$ T.E.E. $=$ difference in the total embodied energy from the baseline retail building (from ATHENA® EIE for Buildings) after 50 years, and $\Delta$ T.O.E. $=$ difference in total operating energy from baseline building (from eQUEST) after 50 years.

Likewise, the difference in the total GWP from the baseline retail building for each of the 220 different building components in this study was calculated according to Eq. 2 for a 50 year lifespan.

$$
\Delta \mathrm{T} . \mathrm{GWP}=\Delta \mathrm{T} . \mathrm{E} \cdot \mathrm{GWP}+\Delta \mathrm{T} \cdot \mathrm{O} \cdot \mathrm{GWP}
$$

Where $\Delta \mathrm{T} . \mathrm{GWP}=$ difference in the total GWP from the baseline retail building after 50 years due to changing baseline retail building component to an alternative building component, $\Delta$ T.E.GWP $=$ difference in the total embodied GWP from the baseline retail building (from ATHENA® EIE for Buildings) after 50 years, and $\triangle$ T.O.GWP $=$ difference in total operating GWP from baseline building (from eQUEST) after 50 years. 


\section{Chapter 4 \\ Methodology: Life-Cycle Assessment of Whole Buildings}

\subsection{Introduction}

In Chapter 3, a method was outlined for calculating the total life-cycle energy use and GWP of 220 individual building components that are typically used in single-storey retail buildings. A baseline retail building was established and the process of systematically replacing the baseline retail building components with the 220 different building components in this study was discussed. However, so far the discussion has only focused on individual building components. It would be useful to have an understanding of the relationships between the individual building components as they pertain to an entire building project. In this section, a method is presented for calculating the total life-cycle energy use and GWP of five different single-storey retail buildings, located in Toronto, with a 50 year lifespan. The goal is to determine whether there is a significant difference in the life-cycle energy use and GWP of different types of single-storey retail buildings. Also, a list of recommendations for reducing the environmental burdens of a single-storey retail building will be developed, once a detailed breakdown of energy use and GWP in an entire building project is determined.

\subsection{LCA of Case Study Retail Buildings}

In Chapter 3, a method was discussed for calculating the life-cycle energy use and GWP of 220 individual building components. In this section, the scope of the LCA study is broadened to included entire building systems. In this section, the five case study single-storey retail buildings that were investigated within the framework of a comprehensive LCA are introduced. These five case study buildings have been designed specifically for this study. They are the result of collaboration between the author of this study and a colleague from the School of Architecture at the University of Waterloo.

As mentioned in the introduction, five different single-storey retail buildings were developed for this study. When selecting the case study buildings, the goal was to identify the most common types of single-storey retail buildings that are constructed in Canada today. The five case study buildings presented next are identical to the baseline retail building in every respect (see description of baseline retail building in Chapter 3 and Appendix A), except as outlined here. The five retail buildings include: 
1. A typical hot-rolled steel structure, single-storey retail building

2. A typical heavy timber structure, single-storey retail building

3. A typical pre-engineered steel, single-storey retail building

4. A predominately steel, single-storey retail building

5. A predominately timber, single-storey retail building

The first three case study buildings represent common types of single-storey retail buildings in Canada today. The predominately steel and predominately timber case study buildings were developed to investigate the influence of material selection on the life-cycle energy use and GWP of a single-storey retail building. As mentioned, all of these buildings are identical to the baseline retail building in this study, except for the differences that will be outlined in the next sections.

\subsubsection{Description of Typical Hot-Rolled Steel Structure Retail Building (Case Study \#1)}

A large proportion of single-storey retail buildings in Canada are built with a conventional hot-rolled steel structure. For this reason, it was important to select this type of building to examine within a comprehensive LCA.

Recall the baseline retail building that was introduced in Chapter 3. The baseline retail building in this study was chosen to be one of the five case study buildings. Specifically, Case Study \#1: Typical HotRolled Steel Structure Retail Building is exactly the same as the baseline retail building in every respect.

This building is comprised of $350 \mathrm{~W}$ hollow structural steel columns and W-section beams. The structure is a series of braced frames, which rely on cross bracing and a concrete masonry stair tower for lateral stability. For the foundations, isolated concrete footings with piers were designed at every column location. As well, strip footings and concrete foundation wall were specified where required. A $200 \mathrm{~mm}$ (8 in.) thick slab-on-grade with 10mil poly was also used in this case. The exterior infill walls are comprised of cold-formed steel studs with exterior installed rigid insulation and an exterior insulation and finish system (EIFS). This assembly is very common in single-storey retail buildings, which is why it was chosen here. An open web steel joist roof with metal deck was designed in this case. A 4-ply built-up asphalt roof assembly was specified, along with $75 \mathrm{~mm}$ (3 in.) of insulation. The mezzanine floor is made up of open web steel joists with a metal deck and a concrete topping. A common floor finish in retail applications is vinyl tile, which was used in this building. The interior 
partitions are cold-formed steel studs with two layers of drywall finish. The windows are a selfsupported aluminum curtainwall system with thermal break (two $6 \mathrm{~mm}$ sealed viewable glazing panes with $12.7 \mathrm{~mm}$ airspace, no low-E coating, and no argon between panes). The doors are a combination of opaque steel doors, aluminum doors with glazing, and an insulated overhead steel door in the shipping and receiving area. As Case Study \#1 is exactly the same as the baseline retail building in this study, a detailed description of this building can be found in Appendix A. An illustration of Case Study \#1 (i.e. the baseline retail building) can bee seen in Figure 4-1.

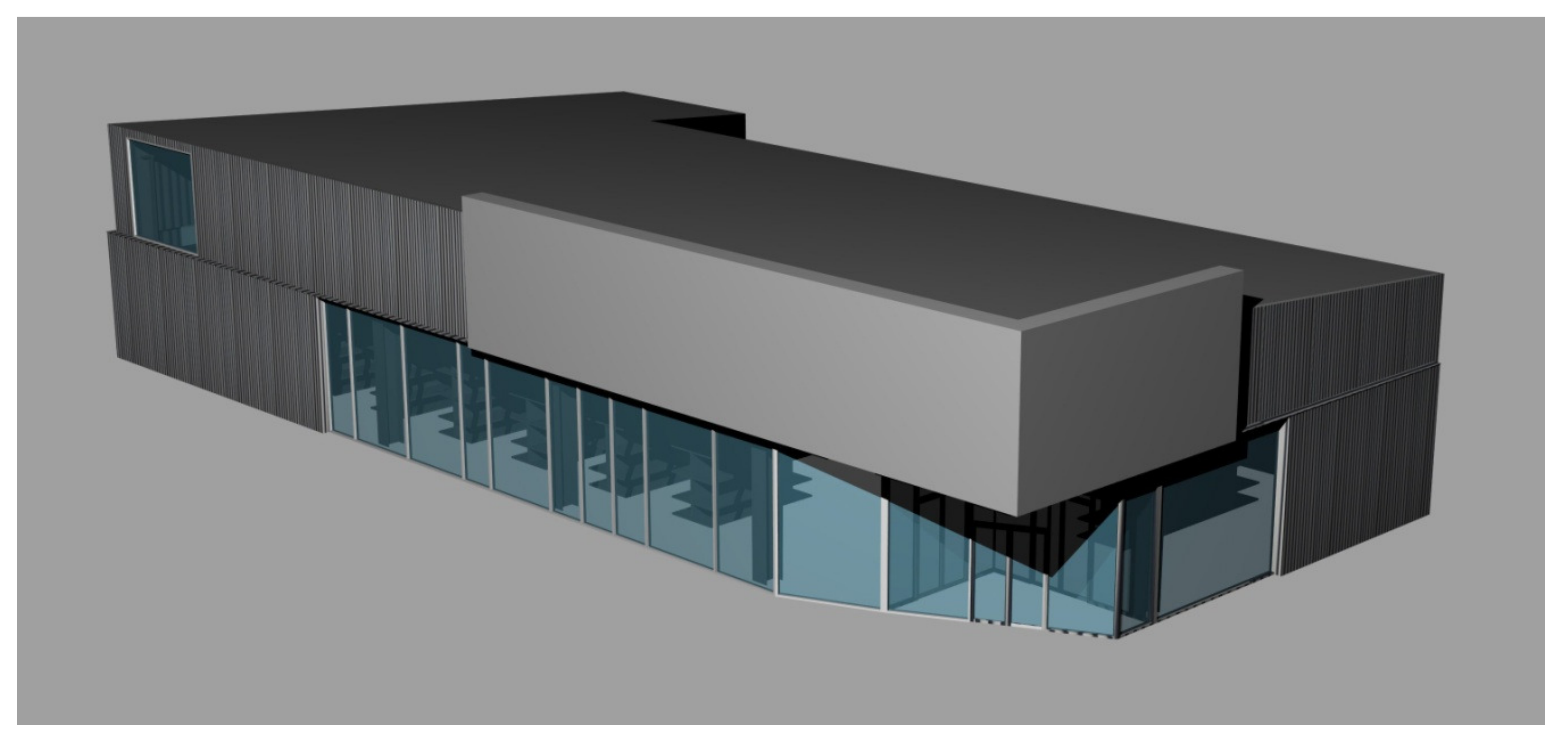

Figure 4-1: Case Study \#1 - Typical Hot-Rolled Steel Structure Single-Storey Retail Building

Table 4-1 contains a breakdown of the various building components that were specified for Case Study \#1, as well as the estimated quantity of each component. The building components have been listed by their ID, so Appendix B can be referenced for a further description of these components. 
Table 4-1: Building Component Quantities for Case Study \#1

\begin{tabular}{|c|c|c|c|}
\hline \multirow[b]{2}{*}{ Building Component } & \multicolumn{3}{|c|}{ Building Component Quantities } \\
\hline & ID & $\begin{array}{c}\text { Estimated } \\
\text { Quantity }\end{array}$ & Unit \\
\hline Exterior Infill Wall Enclosure & BASE-W & 581.0 & sq.m \\
\hline Roof Enclosure (Includes Roof Joists, JOIST-1) & BASE-R & 586.0 & sq.m \\
\hline Structural System - 350W Hot-Rolled Steel & - & - & - \\
\hline Beams (Includes BM-1, BM-2, BM-3, GIRT-1) & S-1 & 11.8 & tonnes \\
\hline Columns (Includes COL-A) & S-1 & 3.3 & tonnes \\
\hline Hot-Rolled Steel Connection Plates & N/A & 0.3 & tonnes \\
\hline Fasteners & N/A & 0.2 & tonnes \\
\hline Miscellaneous Hot-Rolled Steel & N/A & 1.2 & tonnes \\
\hline Mezzanine Floor (Includes Floor Joists, JOIST-1) & FL-3 & 48.0 & sq.m \\
\hline Windows & - & - & - \\
\hline Curtainwall (Façade) & W-9 & 128.0 & sq.m \\
\hline Curtainwall (Interior Vestibule) & W-9 & 34.5 & sq.m \\
\hline Windows & $\mathrm{W}-1$ & 20.3 & sq.m \\
\hline Doors & - & - & - \\
\hline Overhead Doors & D-4 & 1.0 & doors \\
\hline Exterior Doors - Opaque & D-2 & 1.0 & doors \\
\hline Exterior Doors - Glazing & D-3 & 6.0 & doors \\
\hline Interior Doors & D-6 & 9.0 & doors \\
\hline Interior Partitions & - & - & - \\
\hline Fire Rated Stair Tower & CMU-P1 & 84.0 & sq.m \\
\hline Insulated Interior Stud Wall Partition & SS-P3 & 75.0 & sq.m \\
\hline Uninsulated Interior Stud Wall Partition & SS-P1 & 52.0 & sq.m \\
\hline $6 \mathrm{~mm}$ Tempered Glass & N/A & 5.7 & sq.m \\
\hline Foundations & - & - & - \\
\hline Slab-On-Grade & SOG-FDN4 & 586.0 & sq.m \\
\hline Is olated Footings with Concrete Piers & IF-FDN1 & 15.0 & units \\
\hline Strip Footings with Concrete Foundation Wall & SF-FDN5 & 160.0 & $\mathrm{~m}$ \\
\hline
\end{tabular}

\subsubsection{Description of Typical Heavy Timber Structure Retail Building (Case Study \#2)}

The second type of single-storey retail building considered in this study is a heavy timber structure building. This is the same building as Case Study \#1, except that a heavy timber structure is used instead of a hot-rolled steel structure.

In this study, Case Study \#2: Typical Heavy Timber Structure Retail Building is exactly the same as Case Study \#1, with the exception of the structural system. The heavy timber structure is comprised of Douglas-Fir-Larch (D-Fir-L) glulam columns and beams. The structure is a series of braced frames, which rely on cross bracing and a concrete masonry stair tower for lateral stability. In every other respect (the foundations, walls, roofs, floor, interior partitions, windows, and doors), Case Study \#2 is exactly the same as Case Study \#1. The difference between Case Study \#1 and \#2 comes down to the structural system only. An illustration of Case Study \#2 can bee seen in Figure 4-2. Table 4-2 contains a breakdown of the various building components that were specified for Case Study \#2. 


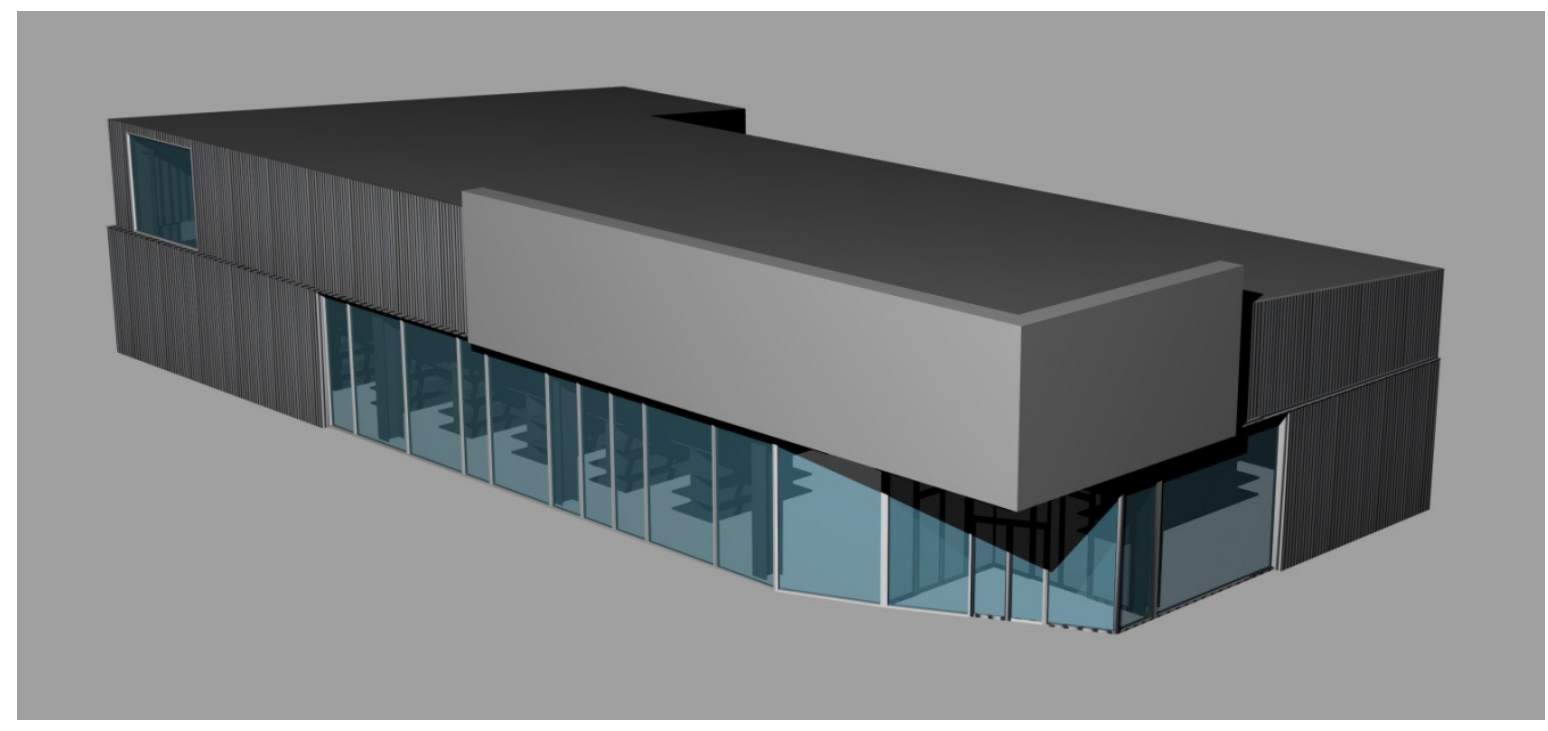

Figure 4-2: Case Study \#2 - Typical Heavy Timber Structure Single-Storey Retail Building

Table 4-2: Building Component Quantities for Case Study \#2

\begin{tabular}{l|c|c|c}
\hline \multirow{2}{*}{ Building Component } & \multicolumn{3}{l}{ Building Component Quantities } \\
\cline { 2 - 4 } & ID & $\begin{array}{c}\text { Estimated } \\
\text { Quantity }\end{array}$ & Unit \\
\hline Exterior Infill Wall Enclosure & BASE-W & 581.0 & sq.m \\
\hline Roof Enclosure (Includes Roof Joists, JOIST-1) & BASE-R & 586.0 & sq.m \\
\hline Structural System - 24f-E Glulam Timber & - & - & - \\
\hline Beams (Includes BM-1, BM-2, BM-3, GIRT-1) & S-2 & 25.4 & cu.m \\
\hline Columns (Includes COL-A) & S-2 & 8.0 & cu.m \\
\hline Hot-Rolled Steel Connection Plates & N/A & 0.3 & tonnes \\
\hline Fasteners & N/A & 0.2 & tonnes \\
\hline Miscellaneous Hot-Rolled Steel & N/A & 1.2 & tonnes \\
\hline Mezzanine Floor (Includes Floor Joists, JOIST-1) & FL-3 & 48.0 & sq.m \\
\hline Windows & - & - & - \\
\hline Curtainwall (Façade) & W-9 & 128.0 & sq.m \\
\hline Curtainwall (Interior Vestibule) & W-9 & 34.5 & sq.m \\
\hline Windows & W-1 & 20.3 & sq.m \\
\hline Doors & - & - & - \\
\hline Overhead Doors & D-4 & 1.0 & doors \\
\hline Exterior Doors - Opaque & D-2 & 1.0 & doors \\
\hline Exterior Doors - Glazing & D-3 & 6.0 & doors \\
\hline Interior Doors & D-6 & 9.0 & doors \\
\hline Interior Partitions & - & - & - \\
\hline Fire Rated Stair Tower & CMU-P1 & 84.0 & sq.m \\
\hline Insulated Interior Stud Wall Partition & SS-P3 & 75.0 & sq.m \\
\hline Uninsulated Interior Stud Wall Partition & SS-P1 & 52.0 & sq.m \\
\hline 6mm Tempered Glass & N/A & 5.7 & sq.m \\
\hline Foundations & SOG-FDN4 & 586.0 & sq.m \\
\hline Slab-On-Grade & IF-FDN1 & 15.0 & units \\
\hline Isolated Footings with Concrete Piers & SF-FDN5 & 160.0 & m \\
\hline Strip Footings with Concrete Foundation Wall & & - & - \\
\hline & & & \\
\hline
\end{tabular}

* See Appendix B for a detailed description of the building components corresponding to the building component ID 


\subsubsection{Description of Typical Pre-Engineered Steel Retail Building (Case Study \#3)}

The third common type of single-storey retail building in Canada is the pre-engineered steel retail building. The components of these buildings are designed and built off-site, then transported to the building location and erected. Typically, these buildings are comprised of a series of rigid steel frames, with corrugate cold-formed steel wall cladding and a standing seam steel roof. Fiberglass batt insulation is usually installed between the wall girts and roof purlins. The main advantages of preengineered buildings are: they are highly optimized structures in terms of cost and material use, they are less expensive than other systems, and they have a shorter construction time. For these reasons they have become popular in the single-storey retail building market. However, almost no research has been conducted at this point on the LCA of pre-engineered steel building systems. It is unknown how these types of buildings compare to conventional systems like the previous two buildings.

In this case, a company that specializes in the design of pre-engineered steel buildings was asked to design a building for the purposes of this study. The company provided a design, including a series of construction drawings and material quantities that met the specifications of this project. Case Study \#3: Typical Pre-Engineered Steel Retail Building was designed to have exactly the same characteristics as the previous two buildings, except for the differences that will be discussed next.

The structure of Case Study \#3 is made up of a series of 350W hot-rolled steel rigid frames. Cross bracing is provided for lateral stability between the frames. Isolated concrete footings with piers were designed at every column location. As well, strip footings and concrete foundation wall were specified where required. A $200 \mathrm{~mm}$ (8 in.) thick slab-on-grade with $10 \mathrm{mil}$ poly was also specified in this case. The exterior infill wall consists of cold-formed steel girts with galvanized cold-formed steel cladding. Fiberglass batt insulation is installed within the wall cavity and is compressed at the girt locations. The roof consists of cold-formed steel purlins and a galvanized standing seam steel roof with fiberglass batt insulation compressed at the purlin locations. The mezzanine floor is comprised of cold-formed steel joists with a metal deck, concrete topping, and vinyl floor tile. The interior partitions are cold-formed steel studs with two layers of drywall finish. The windows are a selfsupported aluminum curtainwall system with thermal break (two $6 \mathrm{~mm}$ sealed viewable glazing panes with $12.7 \mathrm{~mm}$ airspace, no low-E coating, and no argon between panes). The doors are a combination of opaque steel doors, aluminum doors with glazing, and an insulated overhead steel door in the shipping/receiving area. An illustration of Case Study \#3 can bee seen in Figure 4-3. Table 4-3 contains a breakdown of the various building components that were specified for Case Study \#3. 


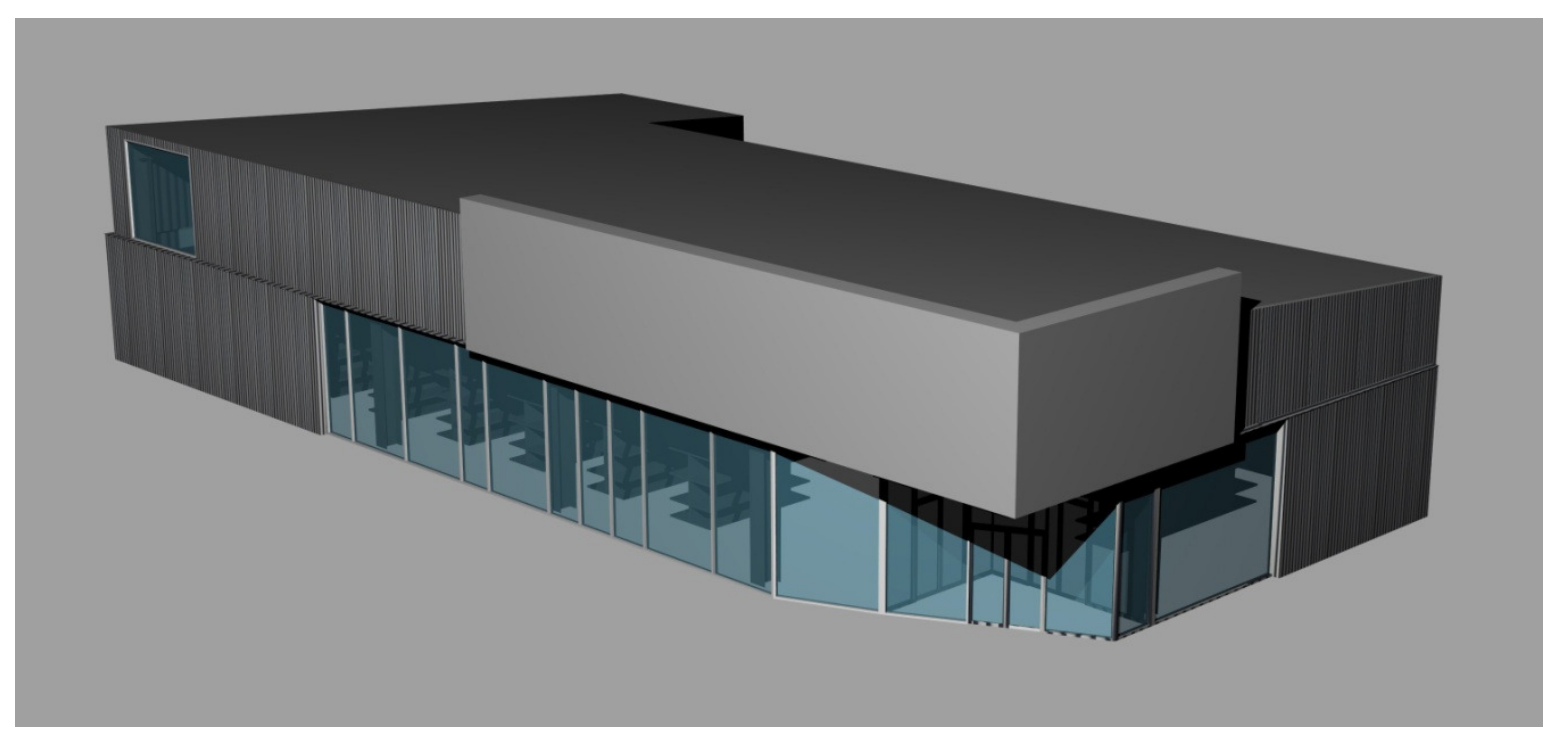

Figure 4-3: Case Study \#3 - Typical Pre-Engineered Steel Single-Storey Retail Building

Table 4-3: Building Component Quantities for Case Study \#3

\begin{tabular}{l|c|c|c}
\hline \multirow{2}{*}{ Building Component } & \multicolumn{3}{|l}{ Building Component Quantities } \\
\cline { 2 - 4 } & ID & $\begin{array}{c}\text { sstimated } \\
\text { Quantity }\end{array}$ & Unit \\
\hline Exterior Infill Wall Enclosure (Includes Girts) & PENG-W2 & 581.0 & sq.m \\
\hline Roof Enclosure (Includes Roof Joists) & PENG-R2 & 586.0 & sq.m \\
\hline Structural System - Pre-Engineered Steel & - & - & - \\
\hline Beams and Columns (Hot-Rolled Steel) & N/A & 13.1 & tonnes \\
\hline Fasteners & N/A & 0.2 & tonnes \\
\hline $\begin{array}{l}\text { Additional Hot-Rolled Steel } \\
\text { (Including Hot-Rolled Steel Connection Plates) }\end{array}$ & N/A & 1.3 & tonnes \\
\cline { 2 - 4 } Additional Cold-Formed Steel & N/A & 1.2 & tonnes \\
\hline Mezzanine Floor (Includes Floor Joists) & N/A & 1.8 & tonnes \\
\hline Windows & N/A & 48.0 & sq.m \\
\hline Curtainwall (Façade) & - & - & - \\
\hline Curtainwall (Interior Vestibule) & W-9 & 128.0 & sq.m \\
\hline Windows & W-9 & 34.5 & sq.m \\
\hline Doors & W-1 & 20.3 & sq.m \\
\hline Overhead Doors & - & - & - \\
\hline Exterior Doors - Opaque & D-4 & 1.0 & doors \\
\hline Exterior Doors - Glazing & D-2 & 1.0 & doors \\
\hline Interior Doors & D-3 & 6.0 & doors \\
\hline Interior Partitions & D-6 & 9.0 & doors \\
\hline Fire Rated Stair Tower & - & - & - \\
\hline Insulated Interior Stud Wall Partition & CMU-P1 & 84.0 & sq.m \\
\hline Uninsulated Interior Stud Wall Partition & SS-P3 & 75.0 & sq.m \\
\hline 6mm Tempered Glass & SS-P1 & 52.0 & sq.m \\
\hline Foundations & N/A & 5.7 & sq.m \\
\hline Slab-On-Grade & - & - & - \\
\hline Isolated Footings with Concrete Piers & S0G-FDN4 & 586.0 & sq.m \\
\hline Strip Footings with Concrete Foundation Wall & IF-FDN1 & 15.0 & units \\
\hline & SF-FDN5 & 160.0 & m \\
\hline
\end{tabular}

* See Appendix B for a detailed description of the building components corresponding to the building component ID 


\subsubsection{Description of Predominately Steel Retail Building (Case Study \#4)}

The fourth single-storey retail building looked at in this study was a predominately steel retail building. The goal was to create a building that used steel building components wherever it was possible to do so. The objective was to investigate the influence of material selection on the life-cycle energy use and GWP of a single-storey retail building constructed primarily of steel, compared to other buildings where a broad mix of materials were used.

For Case Study \#4: Predominately Steel Retail Building, the structural system, foundations, mezzanine floor, interior partitions, windows, and doors are exactly the same as Case Study \#1. However, in Case Study \#4 the exterior infill walls are comprised of cold-formed steel studs with exterior installed rigid insulation and a galvanized cold-formed steel cladding. An open web steel joist roof with metal deck was designed in this case, along with a galvanized standing seam steel roof with $75 \mathrm{~mm}$ (3 in.) of insulation. Otherwise, Case Study \#4 is exactly the same as Case Study \#1. An illustration of Case Study \#4 can bee seen in Figure 4-4.

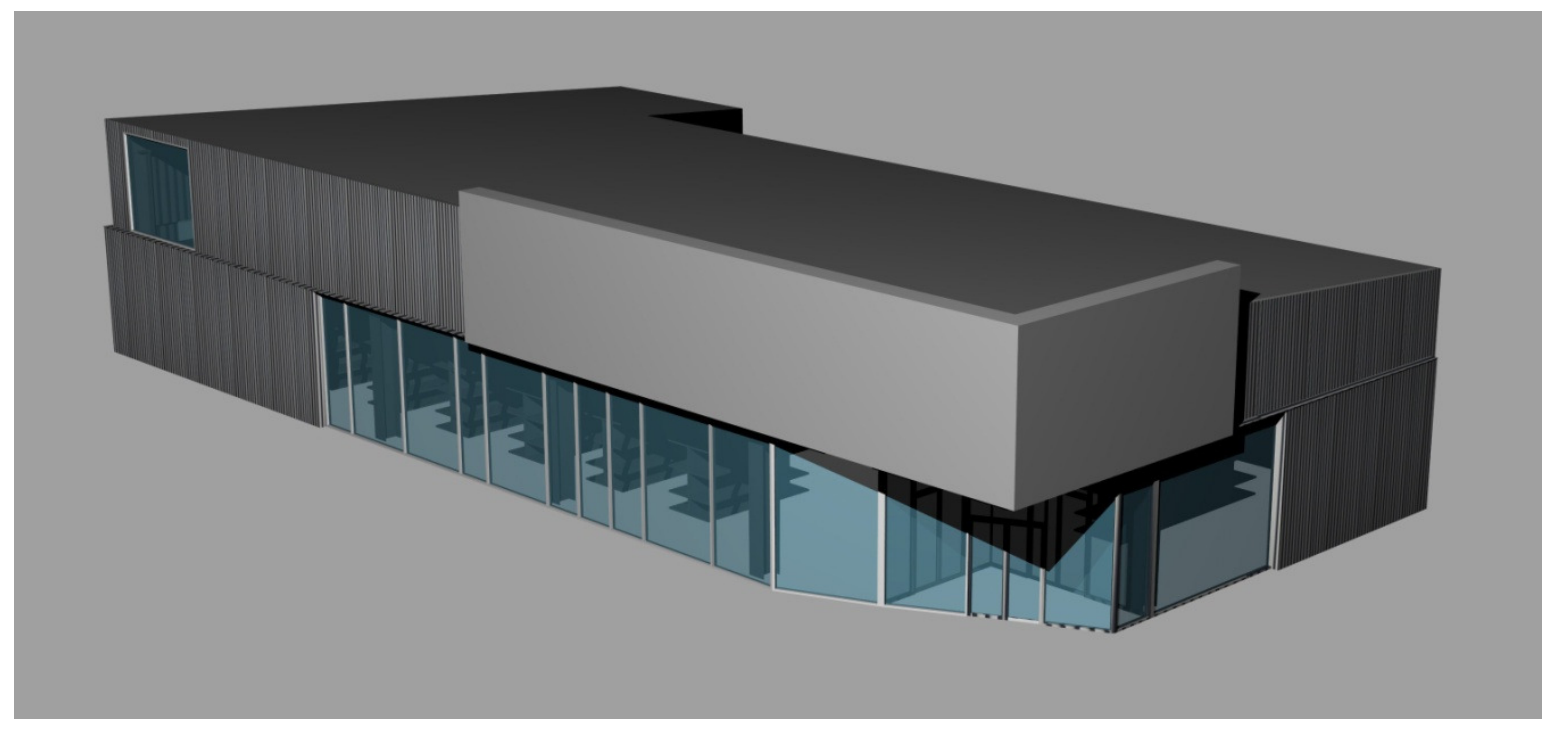

Figure 4-4: Case Study \#4 - Predominately Steel Single-Storey Retail Building

Table 4-4 contains a breakdown of the various building components that were specified for Case Study \#4. The building components have been listed by their ID, so Appendix B can be consulted for a detailed description of each building component. 
Table 4-4: Building Component Quantities for Case Study \#4

\begin{tabular}{|c|c|c|c|}
\hline \multirow[b]{2}{*}{ Building Component } & \multicolumn{3}{|c|}{ Building Component Quantities } \\
\hline & ID & $\begin{array}{c}\text { Estimated } \\
\text { Quantity }\end{array}$ & Unit \\
\hline Exterior Infill Wall Enclosure & SS-W17 & 581.0 & sq.m \\
\hline Roof Enclosure (Includes Roof Joists, JOIST-1) & OWSJ-R5 & 586.0 & sq.m \\
\hline Structural System - 350W Hot-Rolled Steel & - & - & - \\
\hline Beams (Includes BM-1, BM-2, BM-3, GIRT-1) & S-1 & 11.8 & tonnes \\
\hline Columns (Includes COL-A) & S-1 & 3.3 & tonnes \\
\hline Hot-Rolled Steel Connection Plates & N/A & 0.3 & tonnes \\
\hline Fasteners & N/A & 0.2 & tonnes \\
\hline Miscellaneous Hot-Rolled Steel & N/A & 1.2 & tonnes \\
\hline Mezzanine Floor (Includes Floor Joists, JOIST-1) & FL-3 & 48.0 & sq.m \\
\hline Windows & - & - & - \\
\hline Curtainwall (Façade) & W-9 & 128.0 & sq.m \\
\hline Curtainwall (Interior Vestibule) & W-9 & 34.5 & sq.m \\
\hline Windows & $\mathrm{W}-1$ & 20.3 & sq.m \\
\hline Doors & - & - & - \\
\hline Overhead Doors & D-4 & 1.0 & doors \\
\hline Exterior Doors - Opaque & D-2 & 1.0 & doors \\
\hline Exterior Doors - Glazing & D-3 & 6.0 & doors \\
\hline Interior Doors & D-6 & 9.0 & doors \\
\hline Interior Partitions & - & - & - \\
\hline Fire Rated Stair Tower & CMU-P1 & 84.0 & sq.m \\
\hline Insulated Interior Stud Wall Partition & SS-P3 & 75.0 & sq.m \\
\hline Uninsulated Interior Stud Wall Partition & SS-P1 & 52.0 & sq.m \\
\hline 6mm Tempered Glass & N/A & 5.7 & sq.m \\
\hline Foundations & - & - & - \\
\hline Slab-On-Grade & SOG-FDN4 & 586.0 & sq.m \\
\hline Isolated Footings with Concrete Piers & IF-FDN1 & 15.0 & units \\
\hline Strip Footings with Concrete Foundation Wall & SF-FDN5 & 160.0 & $\mathrm{~m}$ \\
\hline
\end{tabular}

* See Appendix B for a detailed description of the building components corresponding to the building component ID

\subsubsection{Description of Predominately Timber Retail Building (Case Study \#5)}

The fifth single-storey retail building considered was a predominately timber retail building. The goal was to create a building using timber building components wherever timber was the best choice. Once again, the objective was to investigate the influence of material selection on the life-cycle energy use and GWP of a single-storey retail building constructed primarily of timber, compared to other buildings where a broad mix of materials were used.

For Case Study \#5: Predominately Timber Retail Building, the structural system and foundations are exactly the same as Case Study \#2. However, in Case Study \#5 the exterior infill walls are comprised of wood studs with exterior installed rigid insulation and wood siding. The roof structure consists of glulam joists with tongue and groove solid wood plank decking. Since no timber roof coverings are commonly used in commercial buildings, a 4-ply built-up asphalt roof assembly was specified along with $75 \mathrm{~mm}$ (3 in.) of insulation. This is a very common roof assembly for single-storey retail 
buildings and was also used for Case Study \#1 and \#2. The mezzanine floor is made up of glulam joists with tongue and groove solid wood plank decking and a vinyl tile floor finish. The interior partitions are wood studs with two layers of drywall finish. The windows are timber frame with a thermal break (two sealed viewable glazing panes with $12.7 \mathrm{~mm}$ airspace, no low-E coating, and no argon between panes). The doors are a combination of opaque wood doors, wood doors with glazing, and an insulated overhead steel door in the shipping and receiving area. An illustration of Case Study \#5 can bee seen in Figure 4-5.

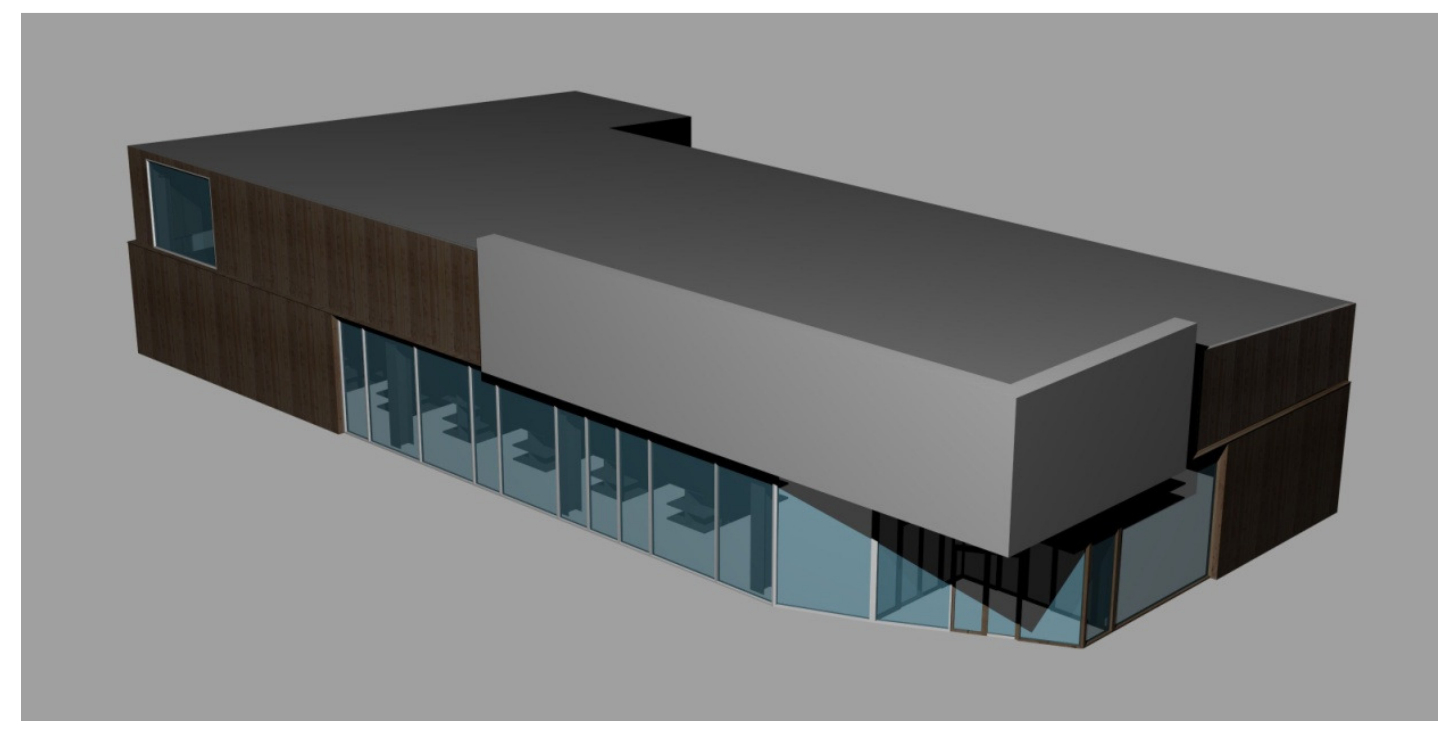

Figure 4-5: Case Study \#5 - Predominately Timber Single-Storey Retail Building

Table 4-5 contains a breakdown of the various building components that were specified for Case Study \#5. The building components have been listed by their ID, so Appendix B can be consulted for a detailed description of each building component.

In this chapter, five single-storey retail buildings were presented. These five buildings were examined within the framework of a comprehensive LCA, in order to determine the relative significance of the various building components in relation to the overall environmental burdens of an entire building. In Chapter 3 a method for calculating the life-cycle energy use and GWP of entire buildings and individual building components was discussed. In addition, 220 different building components that are commonly used in single-storey retail buildings in Canada were identified. The remainder of this study will focus on the results of the life-cycle assessment of the 220 different building components and the five case study buildings, as well as an interpretation of the results. 
Table 4-5: Building Component Quantities for Case Study \#5

\begin{tabular}{|c|c|c|c|}
\hline \multirow[b]{2}{*}{ Building Component } & \multicolumn{3}{|c|}{ Building Component Quantities } \\
\hline & ID & $\begin{array}{c}\text { Estimated } \\
\text { Quantity }\end{array}$ & Unit \\
\hline Exterior Infill Wall Enclosure & WS-W4 & 581.0 & sq.m \\
\hline Roof Enclosure (Includes Roof Joists, JOIST-1) & GLU-R2 & 586.0 & sq.m \\
\hline Structural System - 24f-E Glulam Timber & - & - & - \\
\hline Beams (Includes BM-1, BM-2, BM-3, GIRT-1) & S-2 & 25.4 & cu.m \\
\hline Columns (Includes COL-A) & S-2 & 8.0 & cu.m \\
\hline Hot-Rolled Steel Connection Plates & N/A & 0.3 & tonnes \\
\hline Fasteners & N/A & 0.2 & tonnes \\
\hline Miscellaneous Hot-Rolled Steel & N/A & 1.2 & tonnes \\
\hline Mezzanine Floor (Includes Floor Joists, JOIST-1) & FL-2 & 48.0 & sq.m \\
\hline Windows & - & - & - \\
\hline Curtainwall (Façade) & W-9 & 128.0 & sq.m \\
\hline Curtainwall (Interior Vestibule) & W-9 & 34.5 & sq.m \\
\hline Windows & $\mathrm{W}-4$ & 20.3 & sq.m \\
\hline Doors & - & - & - \\
\hline Overhead Doors & D-4 & 1.0 & doors \\
\hline Exterior Doors - Opaque & D-1 & 1.0 & doors \\
\hline Exterior Doors - Glazing & D-3 & 6.0 & doors \\
\hline Interior Doors & D-5 & 9.0 & doors \\
\hline Interior Partitions & - & - & - \\
\hline Fire Rated Stair Tower & CMU-P1 & 84.0 & sq.m \\
\hline Insulated Interior Stud Wall Partition & WS-P3 & 75.0 & sq.m \\
\hline Uninsulated Interior Stud Wall Partition & WS-P1 & 52.0 & sq.m \\
\hline 6mm Tempered Glass & N/A & 5.7 & sq.m \\
\hline Foundations & - & - & - \\
\hline Slab-On-Grade & SOG-FDN4 & 586.0 & sq.m \\
\hline Isolated Footings with Concrete Piers & IF-FDN1 & 15.0 & units \\
\hline Strip Footings with Concrete Foundation Wall & SF-FDN5 & 160.0 & $\mathrm{~m}$ \\
\hline
\end{tabular}

* See Appendix B for a detailed description of the building components corresponding to the building component ID 


\section{Chapter 5 \\ Results: Life-Cycle Assessment of Whole Buildings}

\subsection{Introduction}

Over the life of a building, the total energy use and total GWP is a combination of the embodied energy and embodied GWP of the building materials and the operating energy and operating GWP of the building. In a typical multi-storey office building, about $85 \%$ of the total life-cycle energy use after 50 years is a result of the building operations, while only about $15 \%$ is due to the energy that is embodied in the materials (Cole \& Kernan, 1996).

A similar relationship between embodied effects and operating effects is thought to exist for the case of a single-storey retail building, but the data to support this is difficult to find. Therefore, this study examined the breakdown of embodied energy, embodied GWP, operating energy, and operating GWP for five different single-storey retail buildings, located in Toronto, Canada, with a 50 year lifespan. The five retail buildings included: a typical hot-rolled steel structure retail building, a typical heavy timber structure retail buildings, a typical pre-engineered steel retail building, a predominately steel retail building, and a predominately timber retail building.

Similar to the studies of multi-storey office buildings (Cole \& Kernan, 1996), the operating effects of the five single-storey retail buildings in this study were found to dominate over the lifespan of the buildings. However, some interesting relationships in terms of the embodied energy and embodied GWP for the components of the retail buildings were found. The aim of this chapter is to present a summary of the LCA results for the five case study retail buildings. It is important to develop an understanding of the energy use and GWP for whole buildings first, before moving onto a detailed analysis of the individual building components. Having an understanding of the energy use and GWP of an entire building will allow the detailed analysis of the 220 different building components in this study to be placed in context. Therefore, the results for the LCA study of the five case study buildings will be presented first, followed by a discussion of the life-cycle energy use and GWP of the 220 alternative building components in Chapter 6.

\subsection{LCA Results for the Baseline Retail Building (Case Study \#1)}

A LCA was performed for the baseline retail building (Case Study \#1) described in Chapter 3 and Appendix A, in order to establish a datum of total embodied energy, embodied GWP, operating 
energy, and operating GWP for a single-storey retail building. Recall that the baseline retail building is a typical hot-rolled steel structure building. This building represents a typical single-storey retail building that would be constructed in Canada today. The remainder of this section will present the results of the comprehensive LCA study for the baseline retail building. A summary of the LCA results can be found in Appendix $\mathrm{C}$.

\subsubsection{Operating Energy and GWP of the Baseline Retail Building (Case Study \#1)}

Based on Cole \& Kernan (1996), the operating energy of a typical Canadian office building represents upwards of $85 \%$ of the total life-cycle energy after 50 years. An analysis of operating energy and operating GWP was conducted for the baseline retail building in this study using eQUEST and the ATHENA® EIE converter. A rendering of the baseline retail building, along with the corresponding eQUEST model are illustrated in Figure 5-1.
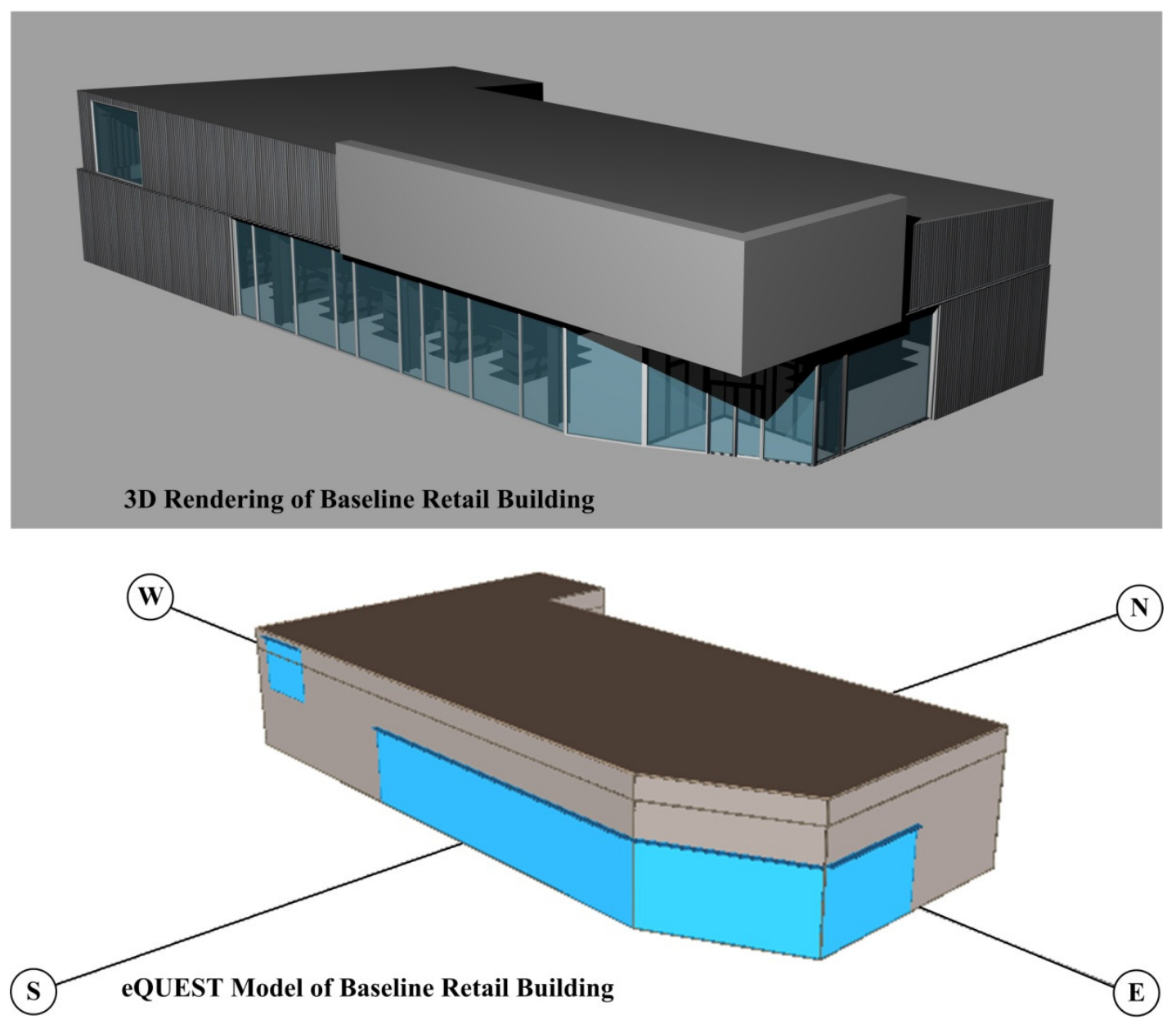

Figure 5-1: Rendering and eQUEST Model of Baseline Retail Building (Case Study \#1) 
The electricity and natural gas use for the baseline retail building were determined from eQUEST. A breakdown of the annual energy consumption for the baseline retail building is presented in Figure $5-2$.

Annual Electricity Consumption $=78,947 \mathrm{kWh}$

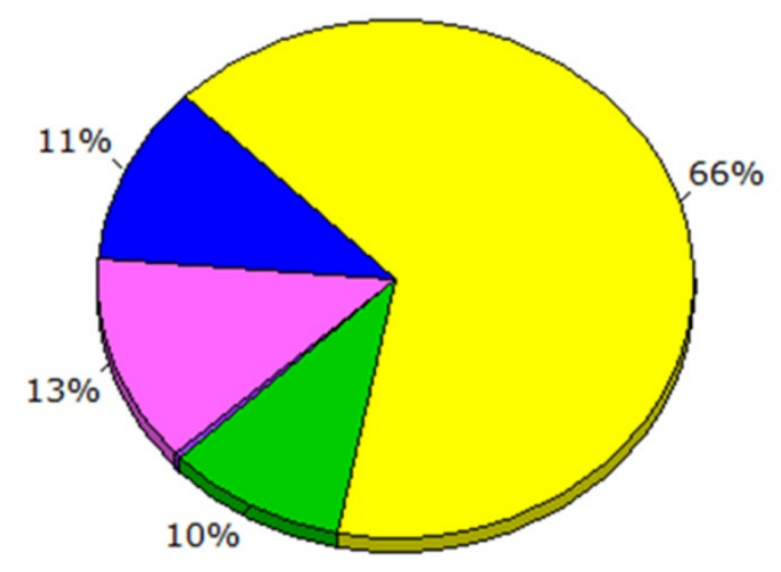

Electricity

Area Lighting

Task Lighting

Misc. Equipment
Exterior Usage

Pumps \& Aux.

Ventilation Fans
Annual Natural Gas Consumption $=376.46 \mathrm{MBtu}$

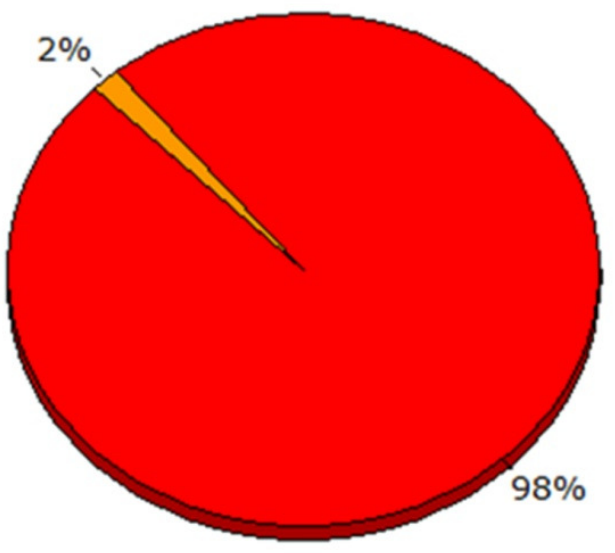

Natural Gas

Water Heating

Ht Pump Supp.

Space Heating

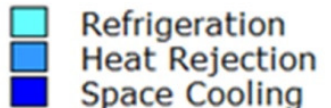

Heat Rejection

\section{Figure 5-2: Breakdown of Annual Energy Consumption for the Baseline Retail Building (Case Study \#1) from eQUEST}

The eQUEST simulations found the annual electricity consumption of the baseline retail building to be about 79,000 kWh/year. Clearly, Figure 5-2 indicates that area lighting is responsible for the largest proportion of electricity use on an annual basis. In fact, area lighting is responsible for approximately $66 \%$ of the annual electricity use for the entire baseline retail building. This result is expected for a retail building. Electricity use in a retail type building is primarily due to lighting, given that a prominent display of retail merchandise is often a primary concern. The eQUEST results for the baseline retail building also indicate that ventilation fans (13\%), space cooling (11\%), and miscellaneous equipment such as office equipment (10\%) are also significant consumers of electricity on an annual basis. Recall that the cooling equipment for the baseline retail building in this study was taken to be direct expansion (DX) coils, which operate on electricity. Therefore, space cooling load appears in the form of electricity use in the eQUEST results. 
Focusing now on the annual natural gas consumption in Figure 5-2, the eQUEST simulations found the annual natural gas consumption of the baseline retail building to be about $376 \mathrm{MBtu} / \mathrm{year}$. About $98 \%$ of this was due to space heating. This is expected as the heating equipment for the baseline retail building was specified as a natural gas combustion furnace, as the building is located in Toronto, Canada (which is a cold climate). Therefore, it is naturally expected to see a significant amount of energy for space heating as demonstrated herein.

Using the ATHENA® EIE converter, the annual electricity use and natural gas use were converted into a total of about 1,014 GJ/year of annual primary energy use. Over a 50 year lifespan, the baseline retail building therefore consumes about 50,700 GJ of primary energy. Similarly, using the ATHENA® EIE converter, this translates into about 46 tonnes of $\mathrm{CO}_{2}$ eq./year (or 2,310 tonnes of $\mathrm{CO}_{2}$ eq. over a 50 year lifespan). The total electricity use and the total natural gas use represent about $56.9 \%(47.3 \%)$ and $43.1 \%(52.7 \%)$ of the annual operating energy use (and operating GWP) respectfully. A breakdown of the annual operating energy use (and operating GWP) for the baseline retail building can be further divided into: space heating (assuming a natural gas furnace) $=42.4 \%$ $(51.8 \%)$, area lighting $=37.3 \%(31.0 \%)$, ventilation fans $=7.4 \%(6.2 \%)$, space cooling $($ assuming DX electric coils $)=6.4 \%(5.4 \%)$, miscellaneous equipment $=5.6 \%(4.6 \%)$, water heating $=0.7 \%$ $(0.9 \%)$, and pumps and auxiliary $=0.2 \%(0.1 \%)$.

The annual energy use for the baseline retail building from eQUEST can also be displayed in terms of the monthly energy consumption in Figure 5-3. The electricity consumption from area lighting is relatively constant each month, as is the ventilation fans and the miscellaneous equipment. However, the electricity consumption due to space cooling goes up in the summer months. This is expected, as space cooling is only required during the summer in a single-storey, $100 \%$ perimeter zone building such as this.

Figure 5-3 also shows the monthly distribution of natural gas use for the baseline retail building. Notice that in the summer months when the space cooling electricity use is the highest, this corresponds to when the natural gas use for space heating is the least. This makes sense, as there is no need to operate the furnace for space heating during the warm summer months. As expected, the natural gas use due to space heating is highest during the winter months. Recall that operating energy and operating GWP are only one part of the equation. The results for the total embodied energy and embodied GWP of the baseline retail building will be presented in the next section. 


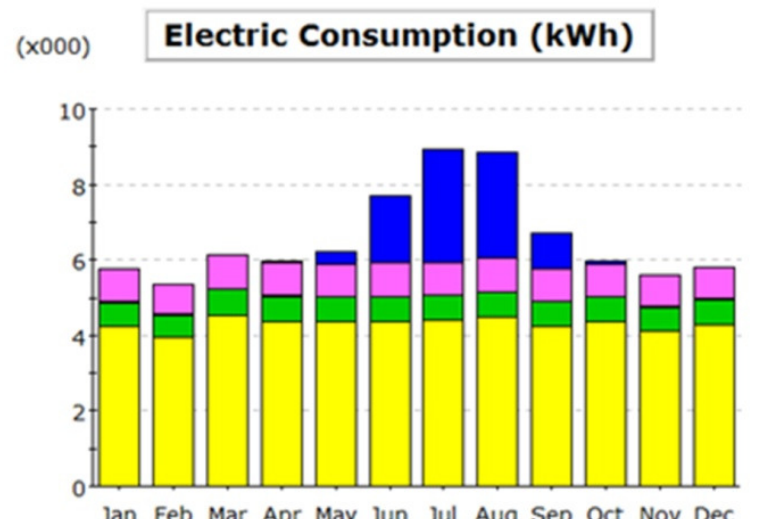

Jan Feb Mar Apr May Jun Jul Aug Sep Oct Nov Dec

Area Lighting Task Lighting

Misc. Equipment
Exterior Usage Pumps \& Aux. Ventilation Fans

\section{$(\times 000,000) \quad$ Gas Consumption (Btu)}

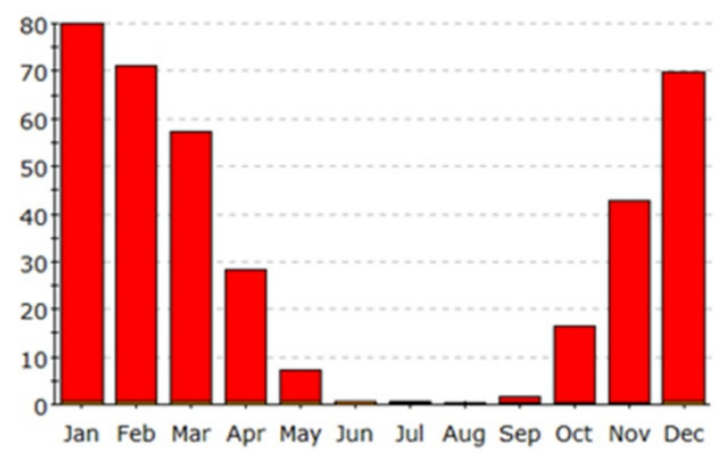

Water Heating Ht Pump Supp. Space Heating
Refrigeration Heat Rejection Space Cooling

Figure 5-3: Monthly Energy Consumption for the Baseline Retail Building from eQUEST

\subsubsection{Embodied Energy and GWP of the Baseline Retail Building (Case Study \#1)}

Using the ATHENA® EIE for Buildings v4.0.64, the total life-cycle embodied energy (initial embodied energy + recurring embodied energy) of the baseline retail building was calculated for a 50 year lifespan in Toronto. The results for the various components of the building are presented in Figure 5-4 in terms of the total primary energy.

The total embodied energy of the baseline retail building was calculated to be about 5,247 GJ. This includes both the initial embodied energy as well as the recurring embodied energy. The key observation to note is the relatively large total embodied energy of the roof compared to the other components of the baseline retail building. In fact, the roof alone represents about $52 \%$ of the total embodied energy of the entire building. This is primarily due to two factors: the roof-to-wall area ratio in a single-storey building and the relatively high total embodied energy of the roof structure compared to the other components of the building.

In a single-storey building like in this study, the roof-to-wall area ratio is typically much larger than in a multi-storey building. This ratio is dependent on the geometric proportions of the building. In a single-storey building, the roof is a larger proportion of the total enclosure area than in a multi-storey building. The roof-to-wall area ratio for the baseline retail building in this study is about 1.0 
(including the window area in the calculation would result in a roof-to-vertical enclosure area ratio of about 0.80 ). In multi-storey buildings, these ratios are typically much less than 1.0. Therefore, in a single-storey retail building like in this study, the roof is a larger proportion of the total enclosure area and therefore plays a significant role in terms of the total embodied energy.

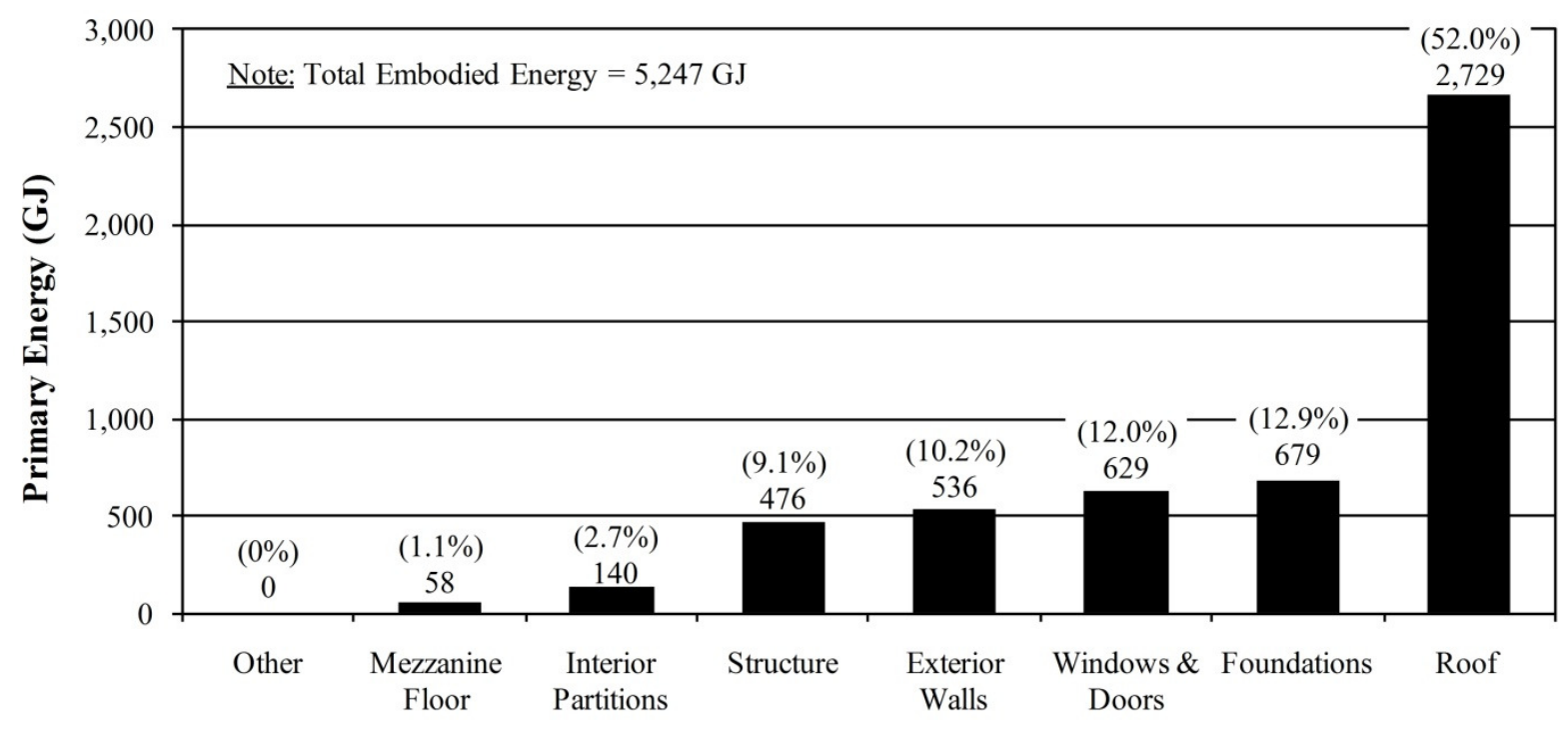

Building Component

\section{Figure 5-4: Total Life-Cycle Embodied Energy of the Baseline Retail Building (Case Study \#1) after 50 Year Lifespan in Toronto}

In addition to the roof being a large proportion of the total enclosure area, the embodied energy per $\mathrm{m}^{2}$ of roof is relatively high compared to the embodied energy per $\mathrm{m}^{2}$ of wall. For the exterior infill wall enclosures identified in Appendix B, the total embodied energy $\mathrm{m}^{2}$ of wall ranged from 0.49 $\mathrm{GJ} / \mathrm{m}^{2}$ to $3.17 \mathrm{GJ} / \mathrm{m}^{2}$. The average embodied energy per $\mathrm{m}^{2}$ of exterior infill wall for the walls identified in Appendix B was about $1.42 \mathrm{GJ} / \mathrm{m}^{2}$. On the contrary, the total embodied energy per $\mathrm{m}^{2}$ of roof ranged from $0.74 \mathrm{GJ} / \mathrm{m}^{2}$ to $5.18 \mathrm{GJ} / \mathrm{m}^{2}$ for the roof enclosures identified in Appendix B. The average embodied energy per $\mathrm{m}^{2}$ of roof for the roofs in Appendix B was about $2.67 \mathrm{GJ} / \mathrm{m}^{2}$. Therefore, on average the total embodied energy per $\mathrm{m}^{2}$ of enclosure for the roofs could be up to about two times greater than that of the exterior infill walls. This is because many of the roof coverings have to be replaced or repaired many times over a 50 year lifespan with the exception of the commercial standing seam steel roof. Also, many roof enclosures tend to use asphalt-based materials which are both high in embodied energy and need to be replaced/repaired often. On the 
contrary, the exterior infill walls tend to have less recurring embodied energy associated with them, as the cladding materials for example are not replaced/repaired as often as the roof covering. Therefore, the roof enclosures tend to have significantly higher recurring embodied energy than the walls, which results on average in a higher total embodied energy per $\mathrm{m}^{2}$ of enclosure.

On its own, the total embodied energy of the roof is responsible for over half of the total embodied energy of the entire single-storey retail building. If the total embodied energy of the roofs, exterior infill walls, and windows/doors are grouped together (referred to as the building enclosure), then the total embodied energy of the enclosure would be responsible for about $74 \%$ of the total embodied energy of the building. Compared to the structural system, which is responsible for only about $9 \%$ of the total embodied energy of the building, the enclosure has a far greater impact on the environment than the structural system. The foundations actually represent about $13 \%$ of the total embodied energy of the building, which is more than that of the entire structural system.

In this study, the floor area refers to the mezzanine floor, not the slab-on-grade. The impacts from the slab-on-grade are included in the foundation results. In this building, the floor was only responsible for about $1 \%$ of the total embodied energy after 50 years. However, this is because the floor area in this building was relatively small (only a $50 \mathrm{~m}^{2}$ mezzanine). The total embodied energy of the floors in Appendix B ranged from $0.56 \mathrm{GJ} / \mathrm{m}^{2}$ to $1.21 \mathrm{GJ} / \mathrm{m}^{2}$ of floor area. Therefore, in buildings with a larger proportion of floor area, the total embodied energy of the floor could potentially represent a much higher percentage of the total embodied energy of the building.

Next, a comparison of the total embodied GWP of the building components is made for the baseline retail building. The LCA results for the embodied GWP are displayed in Figure 5-5.

It can be seen that the relationships between the various building components for the baseline retail building in terms of embodied GWP are much the same as the relationships for embodied energy. Again, the roof enclosure represents the most significant contribution towards the total embodied GWP of the building. The building enclosure (roof, exterior infill walls, windows, and doors) is responsible for about $63 \%$ of the total embodied GWP of the building. The total embodied GWP of the building is slightly less influenced by the enclosure than was the case with the total embodied energy. This is mainly due to the fact that the embodied GWP of the foundations accounts for about $25 \%$ of the total embodied GWP of the building (compared to only $13 \%$ of the total embodied energy of the building). This is primarily because the foundations are comprised almost entirely of concrete 
and steel, which tend to have higher embodied GWP than many other building materials when used in large quantities.

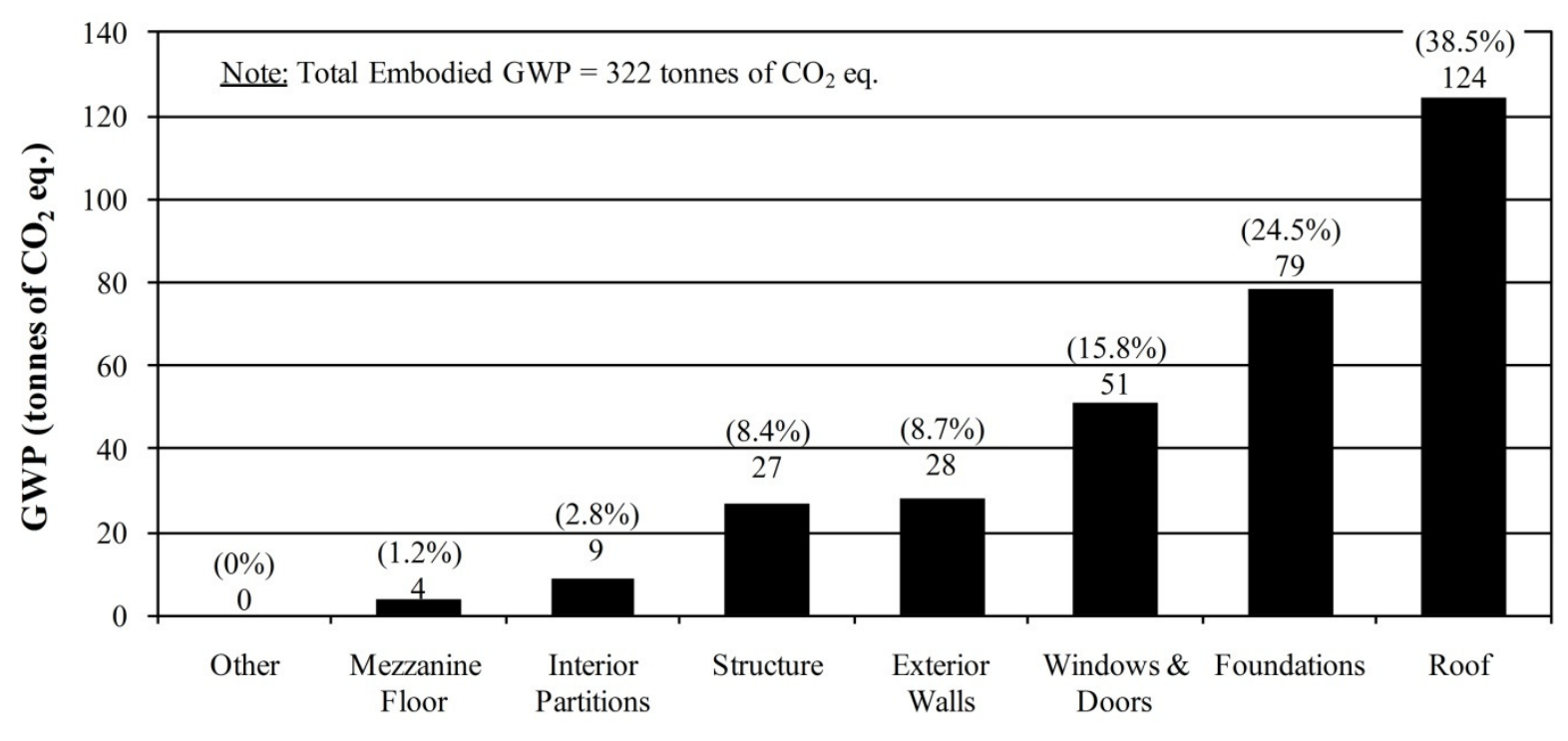

Building Component

\section{Figure 5-5: Total Life-Cycle Embodied GWP of the Baseline Retail Building (Case Study \#1) after 50 Year Lifespan in Toronto}

Therefore, total life-cycle embodied energy and embodied GWP of the baseline retail building was calculated for a 50 year lifespan. Next, the total embodied energy and embodied GWP are compared to the total operating energy and operating GWP for the baseline retail building, in order to determine the relative importance of each to the total energy and total GWP of the building.

\subsubsection{Total Energy and GWP of the Baseline Retail Building (Case Study \#1)}

The operating energy, operating GWP, embodied energy, and embodied GWP of the baseline retail building have already been discussed. In this section, the results will be compared in order to determine the relative proportion of the total life-cycle energy use and total GWP that is attributed to the operations of the building verses the embodied effects.

Figure 5-6 illustrates the breakdown of the total life-cycle energy use for the baseline retail building. Over a 50 year lifespan, the operating energy is equal to about 50,700 GJ of primary energy use and the total embodied primary energy is about 5,247 GJ. This results in a total life-cycle primary energy use of about 55,947 GJ after 50 years for the baseline retail building. 


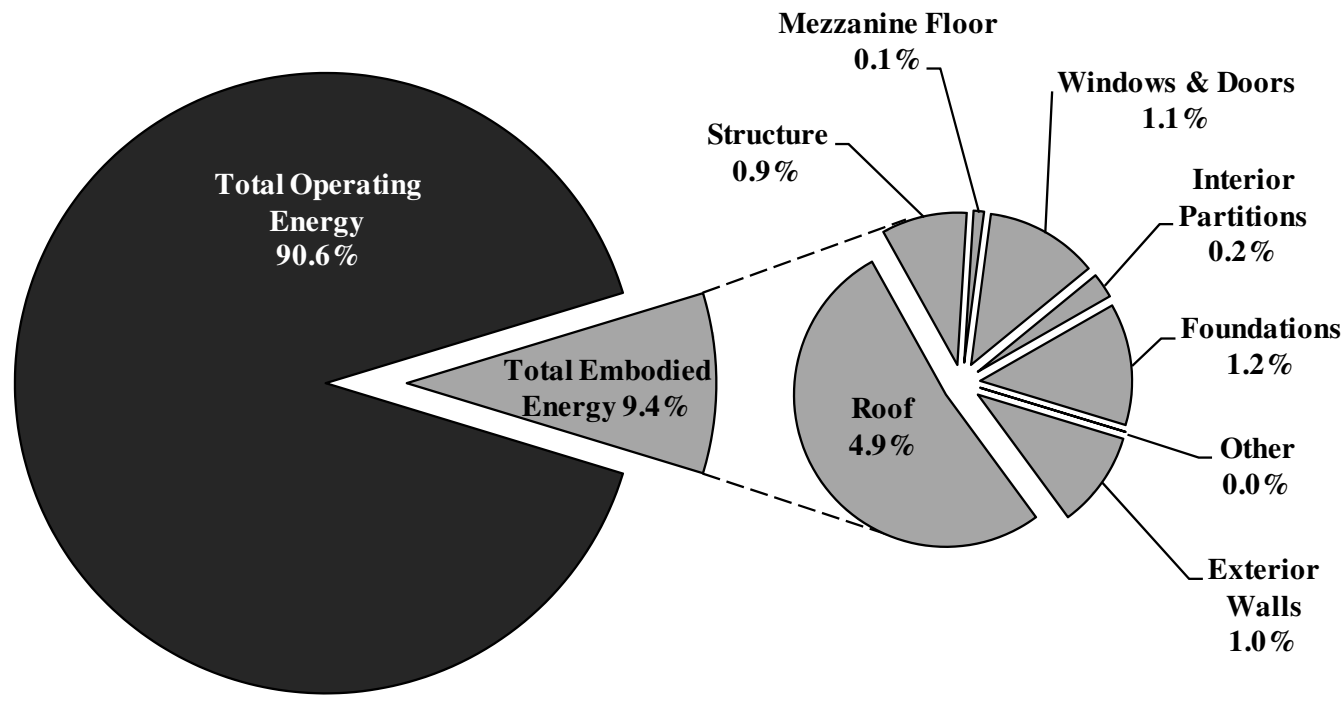

Total 50 Year Embodied Energy = 5,247 GJ

Total 50 Year Operating Energy $=50,700 \mathrm{GJ}$

Total 50 Year Energy = 55,947 GJ

Figure 5-6: Total Life-Cycle Energy Breakdown of the Baseline Retail Building (Case Study \#1) after 50 Year Lifespan in Toronto

Also indicated in Figure 5-6 is the distribution of embodied energy to operating energy for the baseline retail building. Some very interesting results can be derived from the Figure. First, after a 50 year lifespan the operating energy accounts for nearly $91 \%$ of the total life-cycle energy use. The total embodied energy is only about 9\%. Recall that Cole and Kernan (1996) found the operating energy to be $85 \%$ and the total embodied energy to be $15 \%$ for a typical multi-storey office building in Toronto, with a 50 year lifespan. Therefore, after 50 years for the case of a single-storey, $100 \%$ perimeter zone retail building, located in Toronto, the embodied effects account for less than $10 \%$ of the total lifecycle energy use.

In the past, many people have argued that the choice of a structural system has a significant role to play in designing a low-energy building, simply due to the fact that a particular structural system has less embodied energy than another. This argument is most often made as the basis for selecting a wood-based system over a steel or concrete-based system. However, the results in Figure 5-6 clearly show that as less than $1 \%$ of the total life-cycle energy use after 50 years is due to the embodied energy of the structural system, the structural material is essentially negligible. In other words, the maximum savings in terms of life-cycle energy use after 50 years is limited to about $1 \%$ of the total 
energy for a typical retail building in Canada. In fact, reducing the annual operating energy use of the building by only $5 \%$ would save around five times as much energy over 50 years as is currently embodied in the entire structural system. Looking at the big picture, the embodied energy of the structural system is simply not a factor when the operating energy of the building is so much more significant. Only when the operating energy of the building is decreased drastically, would the embodied energy of the structural system gain greater importance.

Looking at the other components of the retail building in Figure 5-6, it can be seen that the total embodied energy of the roof accounts for about $5 \%$ of the total energy use of the baseline retail building after 50 years. Grouping the roof, exterior infill walls, windows, and doors together, the embodied energy of the exterior enclosure is responsible for about $7 \%$ of the total energy.

Next, looking at the breakdown of total GWP for the baseline retail building after 50 years, similar trends can be observed in terms of operating effects verses embodied effects as illustrated in Figure $5-7$.

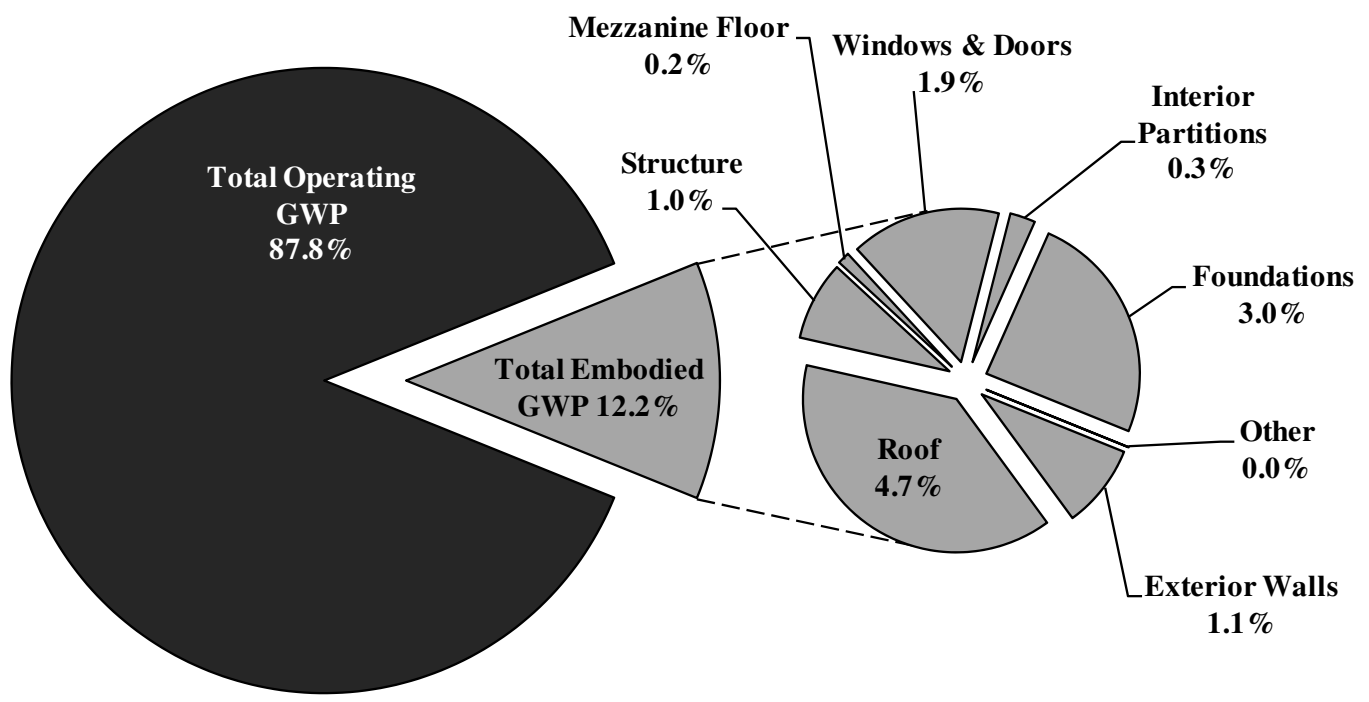

Total 50 Year Embodied GWP $=322$ tonnes of $\mathrm{CO}_{2}$ eq. Total 50 Year Operating $\mathrm{GWP}=2,310$ tonnes of $\mathrm{CO}_{2}$ eq. Total 50 Year $\mathrm{GWP}=2,632$ tonnes of $\mathrm{CO}_{2}$ eq.

Figure 5-7: Total Life-Cycle GWP Breakdown of the Baseline Retail Building (Case Study \#1) after 50 Year Lifespan in Toronto 
The total operating GWP represents about $88 \%$ of the total life-cycle GWP after 50 years, compared to only about $12 \%$ for the embodied GWP. The relative significance of embodied effects verses operating effects has gone up slightly for GWP compared to primary energy, due to the fact that the GWP of the foundations is relatively higher. The foundations are responsible for about $3 \%$ of the total GWP of the retail building after 50 years. The exterior enclosure is responsible for about $8 \%$ of the total GWP of the building, while the structural system accounts for a negligible $1 \%$.

Therefore, for a typical single-storey retail building in Canada, the operating energy and operating GWP are by far the most significant contributors towards the total life-cycle energy use and total GWP after a 50 year lifespan. Not until the operating energy and operating GWP of the building are reduced by around 50\% from typical values today, would the embodied energy and embodied GWP of the building components even begin to become a concern.

The relationship between operating effects and embodied effects for the baseline retail building are very significant. Understanding that the embodied effects play a minor role in terms of the total lifecycle effects in a typical retail building today, can go along way to designing better performing retail buildings from an energy and GWP standpoint. These relationships are not exclusive to retail type buildings. In fact, the conclusions that have been drawn for the baseline retail building thus far could conceivably be applied to any single-storey commercial building in Canada with a 50 year lifespan.

The LCA results of operating effects verses embodied effects for a typical single-storey retail building in Canada can be displayed in another way. Figure 5-8 displays the relative proportions of the embodied effects verses the operating effects for the components of the baseline retail building. Essentially, the percentage of the total life-cycle energy use and total GWP for the components of the baseline retail building have been represented as a scaled proportion of the total area of the building (or emission cloud for the case of GWP) in Figure 5-8. Again, one can see that when plotted to scale, the embodied effects are small compared to the operating effects. This figure simply serves to display these relationships in a different way. 


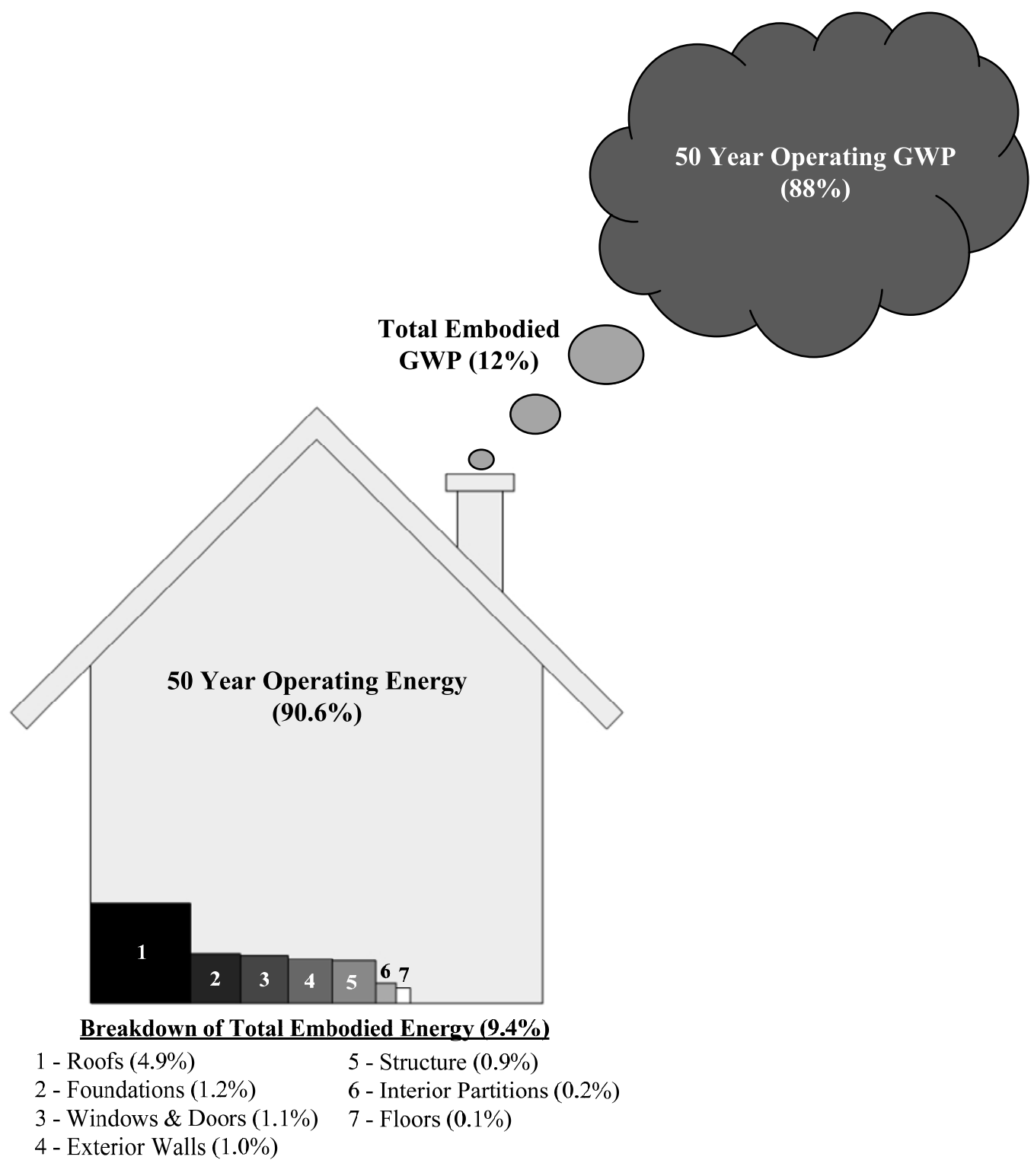

Figure 5-8: A Scaled Diagram of Total Life-Cycle Energy Use and GWP for a Typical Retail Building after a 50 Year Lifespan in Toronto

\subsubsection{Comparison of the Baseline Retail Building (Case Study \#1) to Average Canadian Retail Building}

It is important to compare the LCA results for the baseline retail building to the average retail building in Canada, in order to determine how accurately this baseline retail building represents the typical retail building in Canada. 
Recall that after a 50 year lifespan in Toronto, the total operating energy of the baseline retail building was found to be about 50,700 GJ of primary energy use. Given a total floor area of about 581 $\mathrm{m}^{2}$, this translates into about $1,745 \mathrm{MJ} / \mathrm{m}^{2} / \mathrm{yr}$ of primary energy use. The total operating GWP of the baseline retail building was found to be about 2,310 tonnes of $\mathrm{CO}_{2}$ eq. after 50 years $\left(80 \mathrm{~kg}\right.$ of $\mathrm{CO}_{2}$ eq. $\left./ \mathrm{m}^{2} / \mathrm{yr}\right)$.

The average retail building in Canada uses about $1,740 \mathrm{MJ} / \mathrm{m}^{2} / \mathrm{yr}$ of energy and emits about $97 \mathrm{~kg}$ of $\mathrm{CO}_{2}$ eq. $/ \mathrm{m}^{2} / \mathrm{yr}$ (NRCan, OEE, 2010). The baseline building in this study consumes about the same energy per year and emits approximately $18 \%$ less $\mathrm{CO}_{2}$ eq. per year than the average retail building in Canada. Therefore, the baseline retail building in this study was deemed to adequately represent the life-cycle operating energy and operating GWP of a typical retail building in Canada.

Unfortunately, there is no data available when it comes to the embodied energy and embodied GWP of single-storey retail buildings. Therefore, it is difficult (if not impossible) to provide a comparison of the embodied energy or embodied GWP of the baseline retail building in this study to the average retail building in Canada.

So far the LCA results for the baseline retail building (Case Study \#1) have been presented. In the next section, a comparison of the LCA results for the remaining four retail buildings will be discussed.

\subsection{LCA Results for Case Study Retail Buildings}

Thus far, the LCA results have been discussed for the baseline retail building (Case Study \#1). In this section, the LCA results will be presented for the remaining four case study retail buildings.

The objective here is to investigate what affect changing the structural system from a typical hotrolled steel structure (Case Study \#1), to a typical heavy timber structure (Case Study \#2), to a typical pre-engineered steel structure (Case Study \#3) has on the total life-cycle energy use and GWP of a retail building. Also, the impact on the total life-cycle energy use and GWP will be determined for the case when a predominately steel building (Case Study \#4) and a predominately timber building (Case Study \#5) are designed.

All of the case study buildings are identical to the baseline retail building (Case Study \#1), except for the specific variables that have been changed such as the structural system. Otherwise, all of the buildings have the same dimensions, layout, mechanical systems, operating hours, etc. A detail description of all the case study buildings, along with the LCA results, can be found in Appendix C. 
Based on the results from the previous section for the baseline retail building, it is expected that the operating effects will dominate over the lifespan of the other four case study buildings. The remainder of this section will present the relationships between embodied effects and operating effects for all of the case study buildings.

\subsubsection{Operating Energy and GWP of Case Study Retail Buildings}

In the same way as the baseline retail building, the annual operating energy of each case study building was calculated using eQUEST. Using the ATHENA® EIE converter, the annual electricity use and natural gas use was then converted into an equivalent amount of annual primary energy and GWP. The annual operating energy and GWP results for all five case study buildings are listed in Table 5-1.

The trends in electricity use and natural gas use were found to be the same for all five case study buildings. Area lighting dominated the annual electricity use and space heating was responsible for almost all of the annual natural gas use.

Case Study \# 4 (the predominately steel retail building) was found to have the highest annual operating energy use (and operating GWP) at about 1,040 GJ/yr (48 tonnes of $\mathrm{CO}_{2}$ eq./yr). Case Study \#3 (the typical pre-engineered steel structure retail building) actually had the least annual operating energy use (and operating GWP) at approximately 1,009 GJ/yr (46 tonnes of $\mathrm{CO}_{2}$ eq./yr). These results are essentially identical. It is important to note that the operating effects are highly dependent on the degree of thermal resistance (i.e. R-value or RSI-value) provided by the exterior infill walls and roof. Therefore, the R-values (and RSI-values) are also listed in Table 5-1 alongside the operating energy and GWP results for each case study building. For each case study building, the exterior walls and roofs were designed based on typical practice. Wherever possible, the thermal resistances were taken to be as close as possible to Case Study \#1, in order to ensure an equal comparison. However, in some instances the thermal resistances deviated slightly from Case Study \#1 (either higher or lower). However, for the purposes of this study, these slight deviations were deemed to be acceptable.

In any case, the annual operating energy and operating GWP of all five case study buildings only differed at most by $3 \%$ and $4 \%$ respectfully. Therefore, given the level of accuracy required in this study, it can be said that all of these buildings had almost identical annual operating energy and operating GWP. Therefore, assuming that the thermal resistance of the exterior walls and roof are 
similar, the impact of changing the structural system from a timber system to a steel system has a negligible impact on the annual operating effects. The same is true of the predominately steel and predominately timber retail buildings. So long as the thermal resistance of the exterior walls and roof are similar, the differences in annual operating effects are minimal.

Table 5-1: Annual Operating Energy and GWP Results for Case Study Buildings

\begin{tabular}{|c|c|c|c|c|c|c|c|}
\hline \multirow{2}{*}{$\begin{array}{c}\text { Case } \\
\text { Study \# }\end{array}$} & \multirow{2}{*}{$\begin{array}{c}\text { Building } \\
\text { Description }\end{array}$} & \multicolumn{2}{|c|}{$\begin{array}{l}* \text { R-Value } \\
\text { (RSI-Value) }\end{array}$} & \multicolumn{2}{|c|}{ Data from eQUEST } & \multicolumn{2}{|c|}{$\begin{array}{c}\text { Data from } \\
\text { ATHENA } ® \text { EIE Converter }\end{array}$} \\
\hline & & $\begin{array}{l}\text { Exterior } \\
\text { Walls }\end{array}$ & Roof & $\begin{array}{c}\text { Annual } \\
\text { Electricity Use } \\
(\mathbf{k W h} / \mathbf{y r})\end{array}$ & $\begin{array}{c}\text { Annual } \\
\text { Natural Gas } \\
\text { Use } \\
\text { (MBtu/yr) }\end{array}$ & $\begin{array}{c}\text { Annual } \\
\text { Primary } \\
\text { Energy Use } \\
\text { (GJ/yr) }\end{array}$ & $\begin{array}{c}\text { Annual GWP } \\
\text { (tonnes of } \\
\left.\mathrm{CO}_{2} \mathrm{eq} \cdot / \mathrm{yr}\right)\end{array}$ \\
\hline $\begin{array}{c}1 \\
\text { (Baseline } \\
\text { Retail } \\
\text { Building) }\end{array}$ & $\begin{array}{l}\text { Typical Hot- } \\
\text { Rolled Steel } \\
\text { Structure Retail } \\
\text { Building }\end{array}$ & $\begin{array}{l}15.6 \\
(2.7)\end{array}$ & $\begin{array}{l}20.8 \\
(3.7)\end{array}$ & 78,947 & 376.46 & 1,014 & 46 \\
\hline 2 & $\begin{array}{l}\text { Typical Heavy } \\
\text { Timber } \\
\text { Structure Retail } \\
\text { Building }\end{array}$ & $\begin{array}{l}15.6 \\
(2.7)\end{array}$ & $\begin{array}{l}20.8 \\
(3.7)\end{array}$ & 78,947 & 376.46 & 1,014 & 46 \\
\hline 3 & $\begin{array}{l}\text { Typical Pre- } \\
\text { Engineered } \\
\text { Steel Structure } \\
\text { Retail Building }\end{array}$ & $\begin{array}{l}17.9 \\
(3.2)\end{array}$ & $\begin{array}{l}17.8 \\
(3.1)\end{array}$ & 79,341 & 370.65 & 1,009 & 46 \\
\hline 4 & $\begin{array}{l}\text { Predominately } \\
\text { Steel Retail } \\
\text { Building }\end{array}$ & $\begin{array}{l}13.0 \\
(2.3)\end{array}$ & $\begin{array}{l}17.8 \\
(3.1)\end{array}$ & 79,839 & 393.53 & 1,040 & 48 \\
\hline 5 & $\begin{array}{l}\text { Predominately } \\
\text { Timber Retail } \\
\text { Building }\end{array}$ & $\begin{array}{l}14.9 \\
(2.6)\end{array}$ & $\begin{array}{l}23.1 \\
(4.1)\end{array}$ & 79,420 & 376.21 & 1,016 & 46 \\
\hline
\end{tabular}

* Note: The exterior walls and roofs were designed based on typical practice for each type of building. Wherever possible, the R-values were taken to be as close as possible to the R-values of Case Study \#1. However, in some circumstances the $R$-values deviate slightly from Case Study \#1. The differences in $R$-values do affect the annual operating energy and GWP of the buildings, but this was assumed to be acceptable for the purposes of this study.

\subsubsection{Embodied Energy and GWP of Case Study Retail Buildings}

In this section, the results are presented for the total embodied energy and total embodied GWP of the five case study buildings. Figure 5-9 displays the total life-cycle embodied energy of each of the five case study buildings after a 50 year lifespan in Toronto using the ATHENA® EIE for Buildings. 
The total embodied energy of each case study building has been divided into the relevant building components: exterior walls, roof, structure, interior partitions, floor, windows, doors, foundations, and other. Case Study \#3 (the typical pre-engineered steel retail building) was found to have the least total embodied energy of all the buildings (2,927 GJ). This result is logical as pre-engineered steel buildings are highly engineered and optimized structures from a material standpoint. This is primarily achieved to save material and construction costs. However, this high degree of material optimization has the added benefit of creating a building that uses less material and therefore, has less embodied energy than other types of buildings. Although pre-engineered steel buildings are comprised mostly of steel (a material with a relatively high embodied energy), little recurring embodied energy is associated with this system as most of the building components can last 50 years with minimal repair/replacement. From an embodied energy standpoint, pre-engineered buildings show excellent potential as building systems for low-energy building applications.

Case Study \#1 (the typical hot-rolled steel structure retail building) was found to have the highest total embodied energy of all five buildings (5,247 GJ). One might understandably expect Case Study \#4 (the predominately steel retail building) to have a higher total embodied energy, but this is not the case. The reason for this is that the predominately steel retail building had a standing seam steel roof which had significantly less embodied energy than the 4-ply built-up asphalt roof that was specified for the typical hot-rolled steel structure retail building. This observation leads to a very important conclusion for single-storey commercial buildings.

Of all the building components, the roof has by far the largest total embodied energy. Roof coverings are often made of asphalt-based materials which are very high in embodied energy and must be replaced often. Case Studies \#1, \#2, and \#5 all have a 4-ply built-up asphalt roof system. On the contrary, Case Studies \#3 and \#4 have a commercial standing seam steel roof. The steel roof requires less maintenance, repair, and replacement than the asphalt-based roof. Therefore, the recurring embodied energy of the steel roof system is much smaller after 50 years. Although steel as a material has a relatively high embodied energy, steel roofs perform much better than asphalt-based roof systems due to less recurring embodied energy. It should be noted that if Case Study \#5 (the predominately timber retail building) were to have a commercial standing seam steel roof instead of a 4-ply built-up asphalt roof, it would actually have the least total embodied energy of all five buildings. This just serves to reinforce the importance of the roof in the calculations of total embodied energy for a single-storey building. Generally speaking, if the concern is to reduce the total embodied 
energy of a single-storey building, then the attention should focus on reducing the total embodied energy of the roof.

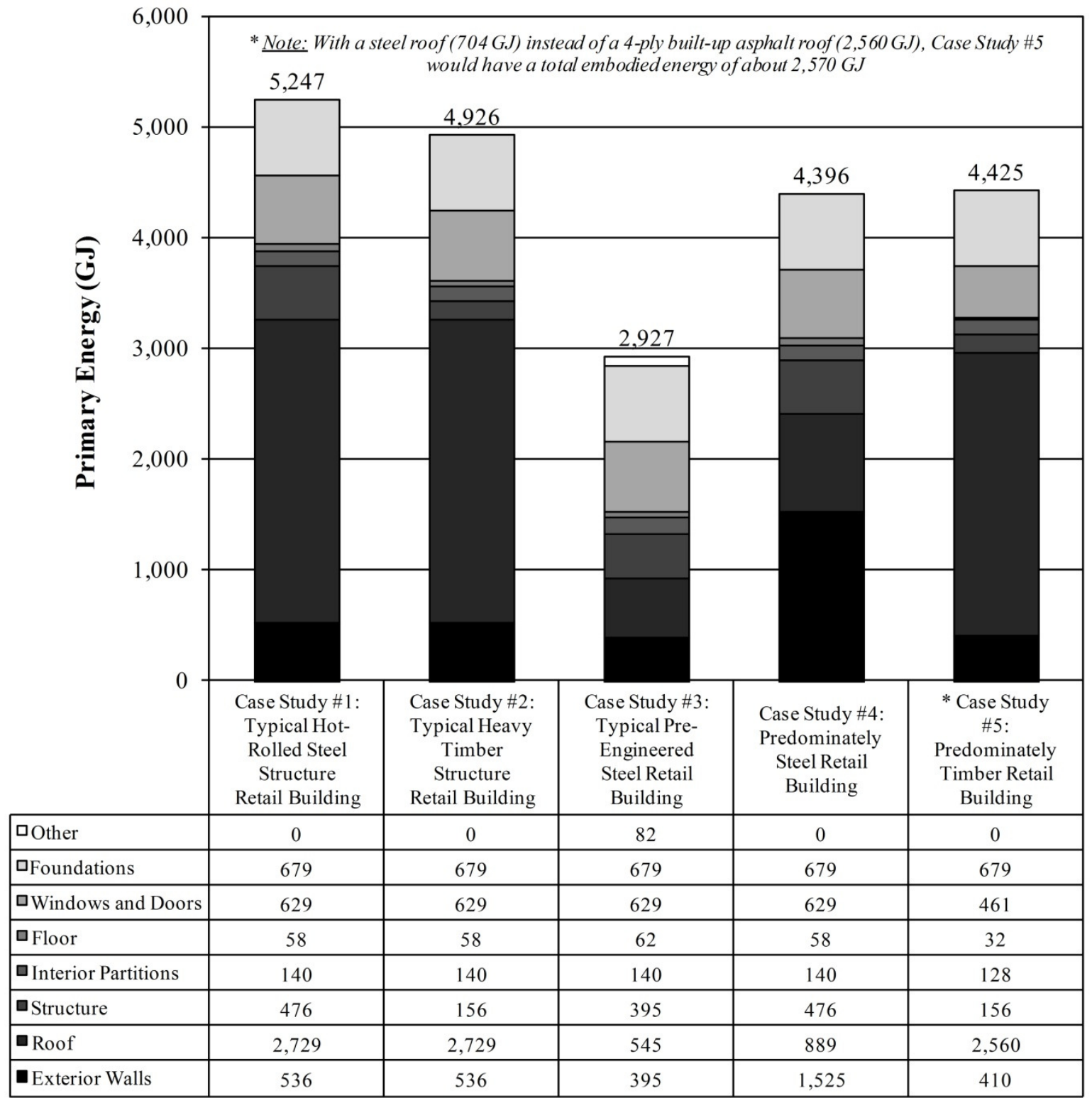

Figure 5-9: Total Life-Cycle Embodied Energy of the Case Study Buildings after 50 Year Lifespan in Toronto

With the discussion of the total embodied energy for the five case study buildings completed, the focus can now be shifted to a comparison of the total embodied GWP. As before, the embodied GWP 
was calculated using the ATHENA® EIE for buildings and the results for the five case study buildings are displayed in Figure 5-10.

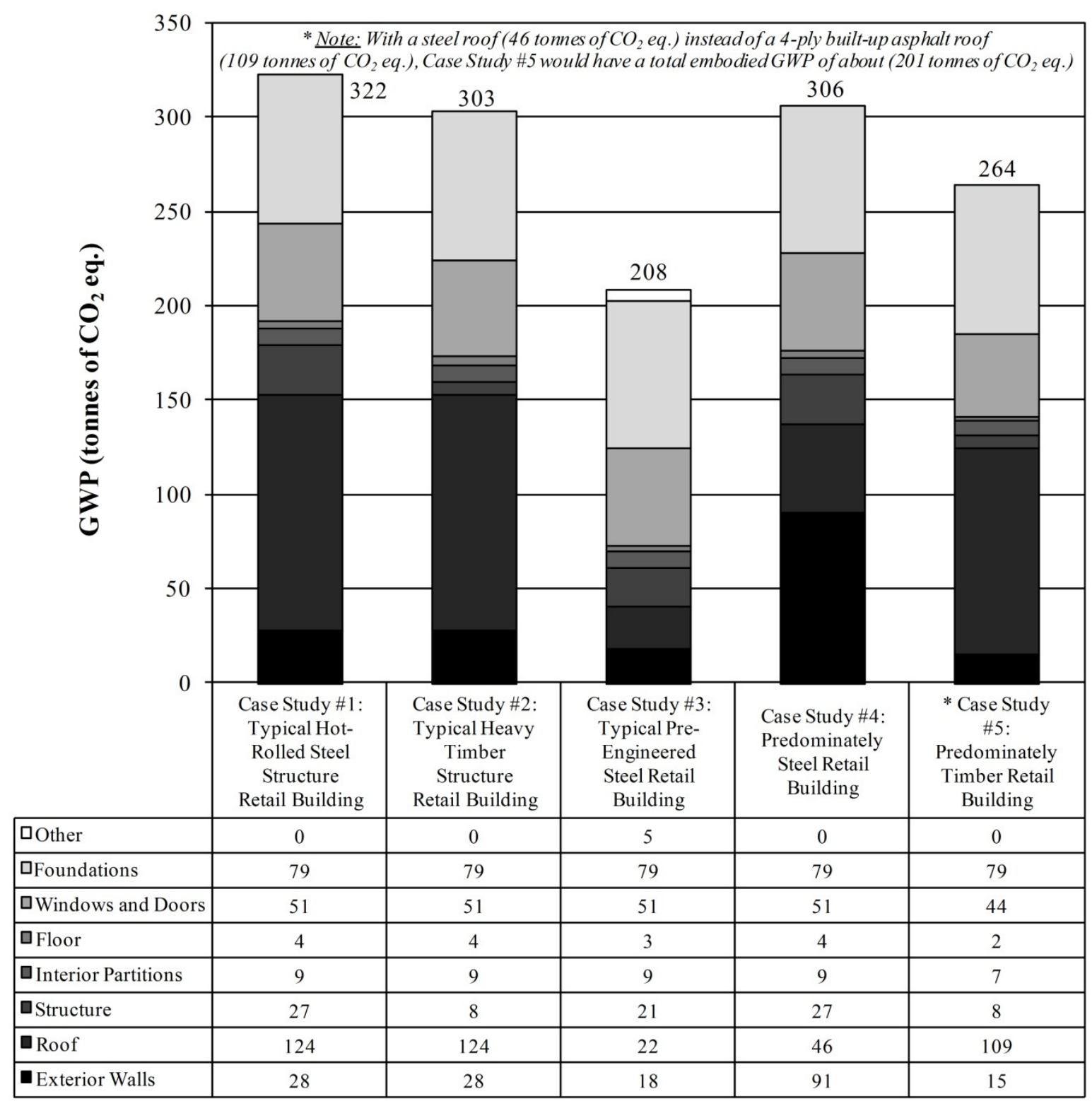

Figure 5-10: Total Life-Cycle Embodied GWP of the Case Study Buildings after 50 Year Lifespan in Toronto

Similar to the total embodied energy, Case Study \#3 (the typical pre-engineered steel retail building) was found to have the least total GWP after 50 years (208 tonnes of $\mathrm{CO}_{2}$ eq.). Again, this can be attributed to the high degree of material optimization that is inherent to these types of buildings. On 
the contrary, Case Study \#1 (the typical hot-rolled steel structure retail building) was found to have the highest total embodied GWP (322 tonnes of $\mathrm{CO}_{2}$ eq.). Again, the roof plays a significant role in this. The standing seam steel roof had much less embodied GWP compared to the 4-ply built-up asphalt roof systems, due to less recurring GWP. It should be pointed out that if Case Study \#5 (the predominately timber retail building) were to switch from a 4-ply built-up asphalt roof to a steel roof, it would actually have the least total embodied GWP of any building after 50 years. Figure 5-10 also indicates that in most cases, the embodied GWP of the foundations was the highest single contributor towards the total embodied GWP next to the roof. This suggests that a concerted effort to optimize the foundation systems for low-energy buildings should be undertaken, if reducing the embodied GWP is a concern.

Now that the total life-cycle operating energy, operating GWP, embodied energy, and embodied GWP for the five case study buildings has been discussed, the results will be combined to determine which case study building has the least total energy and total GWP after 50 years.

\subsubsection{Total Energy and GWP of Case Study Retail Buildings}

So far it can be seen that the annual operating energy and operating GWP do not significantly differ among the five case study buildings. It was also observed that the embodied energy (and embodied GWP) does differ between the five buildings, sometimes by as much as $44 \%$ (35\%). In this section, the total operating energy, operating GWP, embodied energy, and embodied GWP are combined to determine the total life-cycle energy and total GWP of the five case study buildings.

Displayed in Figure 5-11 are the total life-cycle energy and GWP for the five case study buildings after a 50 year lifespan in Toronto. After a 50 year lifespan, the total energy and GWP of the five case study buildings differs at most by only $6 \%$ and $7 \%$ respectfully. This is a very important result as it indicates that regardless of the choice of structural system, or whether the building is primarily made of steel or timber building components, the differences in total energy and GWP after 50 years are minimal. The operating energy and operating GWP completely dominate the total energy and total GWP of the buildings after 50 years.

It was found that Case Study \#3 (the typical pre-engineered steel retail building) had the least total energy and total GWP of any building. However, the energy and GWP savings for Case Study \#3 were minimal compared to the other buildings. Regardless, a pre-engineered steel retail building performs at least as well as other types of buildings (and in this case slightly better). It is difficult to 
say that in every case a pre-engineered steel building would perform better than other types of buildings in terms of life-cycle energy use and GWP. This is highly dependent on the thermal resistance of the enclosure among other variables. Historically, pre-engineered steel buildings have been plagued by massive thermal bridging problems at the location of wall girts and roof purlins. Therefore, a concerted effort has to be taken to limit the thermal bridging problems in pre-engineered steel buildings, in order for them to be competitive with other conventional building types.

It is important to note that since the differences in the total energy and GWP among the five case study buildings are so small, it is totally plausible that under slightly different circumstances (for example a slight change to the thermal resistance of the enclosure) that the rankings in Figure 5-11 could differ. However, this study proved that the type of building does not significantly impact the total life-cycle energy use and GWP. Only once the operating effects of a typical retail building are reduced significantly (by at least $50 \%$ ), would a comparison of the total embodied effects of different building types become important.

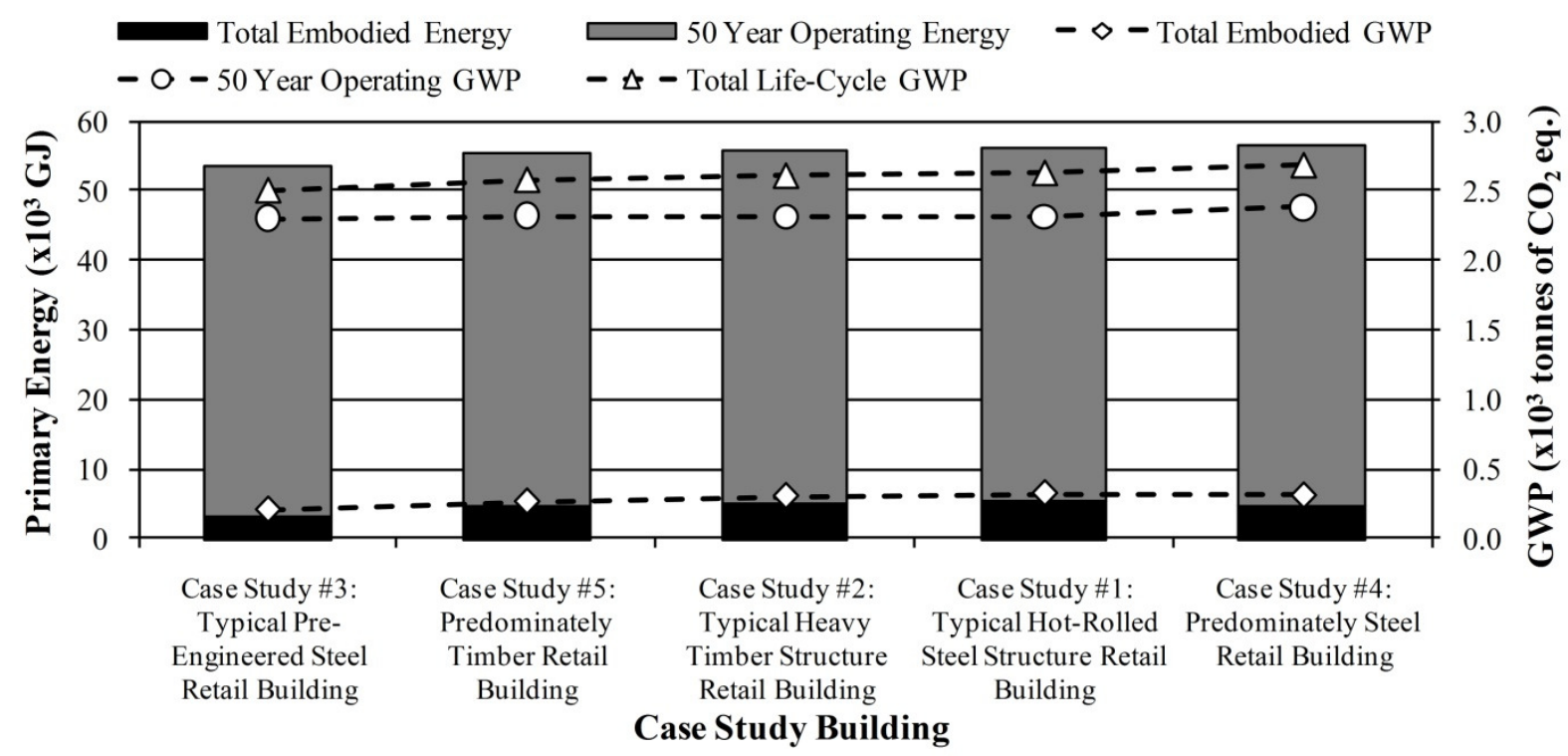

Note: Total Life-Cycle Energy = Total Embodied Energy + 50 Year Operating Energy

\section{Figure 5-11: Total Life-Cycle Energy Consumption and GWP of the Case Study Buildings after 50 Year Lifespan in Toronto}

To reinforce the importance of operating effects for the different building types, a breakdown of operating energy and embodied energy has been plotted for Case Study \#2 and \#3 in Figure 5-12 and 
5-13 respectfully (recall that a similar breakdown for Case Study \#1 has already been plotted previously).

Figure 5-12 illustrates the breakdown of total life-cycle operating energy and embodied energy for Case Study \# 2 (the typical heavy timber structure retail building). The total operating energy in this case represents about $91 \%$ of the total life-cycle energy, while the total embodied energy is only responsible for about $9 \%$.

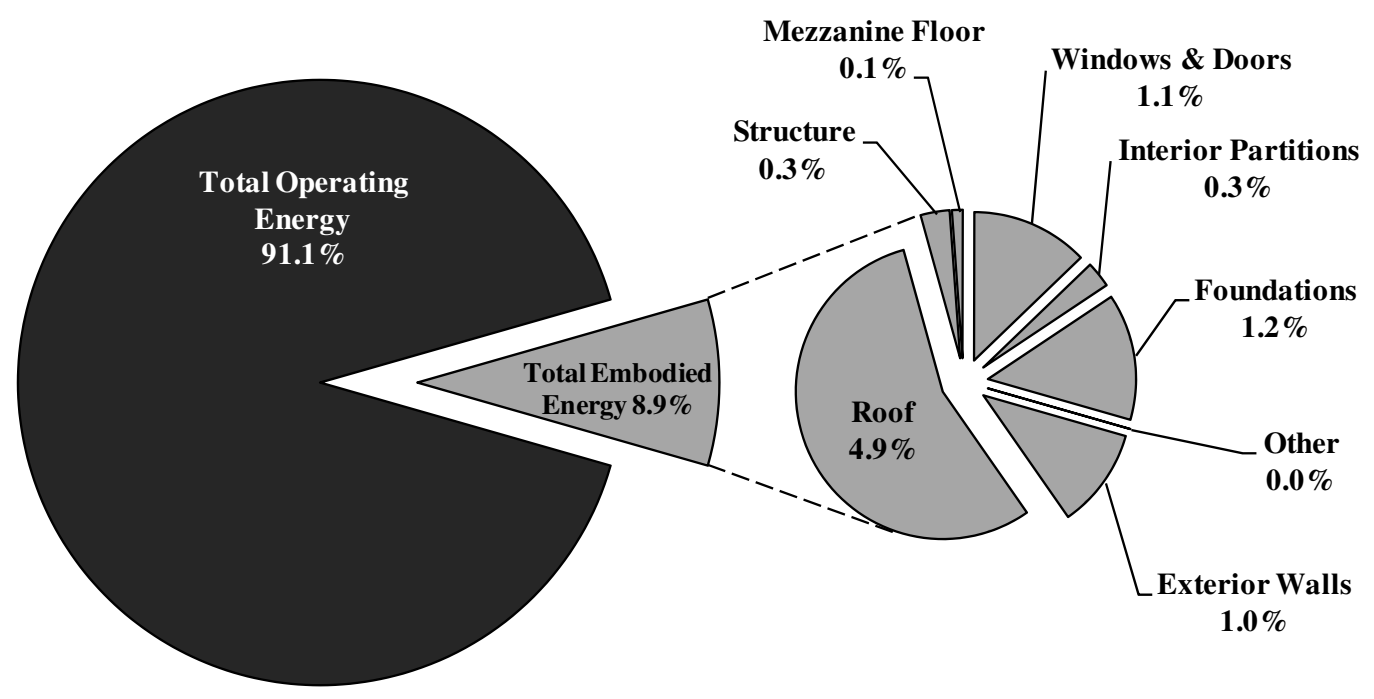

Total 50 Year Embodied Energy =4,926 GJ

Total 50 Year Operating Energy = 50,700 GJ

Total 50 Year Energy = 55,626 GJ

\section{Figure 5-12: Total Life-Cycle Energy Breakdown of the Typical Heavy Timber Structure Retail Building (Case Study \#2) after 50 Year Lifespan in Toronto}

Similarly, Figure 5-13 illustrates the breakdown of total life-cycle operating energy and embodied energy for Case Study \# 3 (the typical pre-engineered steel retail building). The total operating energy in this case represents about $94 \%$ of the total life-cycle energy, while the total embodied energy is only responsible for about $6 \%$.

A similar breakdown of the total life-cycle operating GWP and embodied GWP can be done. Figure 5-14 and Figure 5-15 illustrate the results for Case Study \#2 and \#3 respectfully. Again, the dominance of the operating GWP compared to the embodied GWP is clear. 


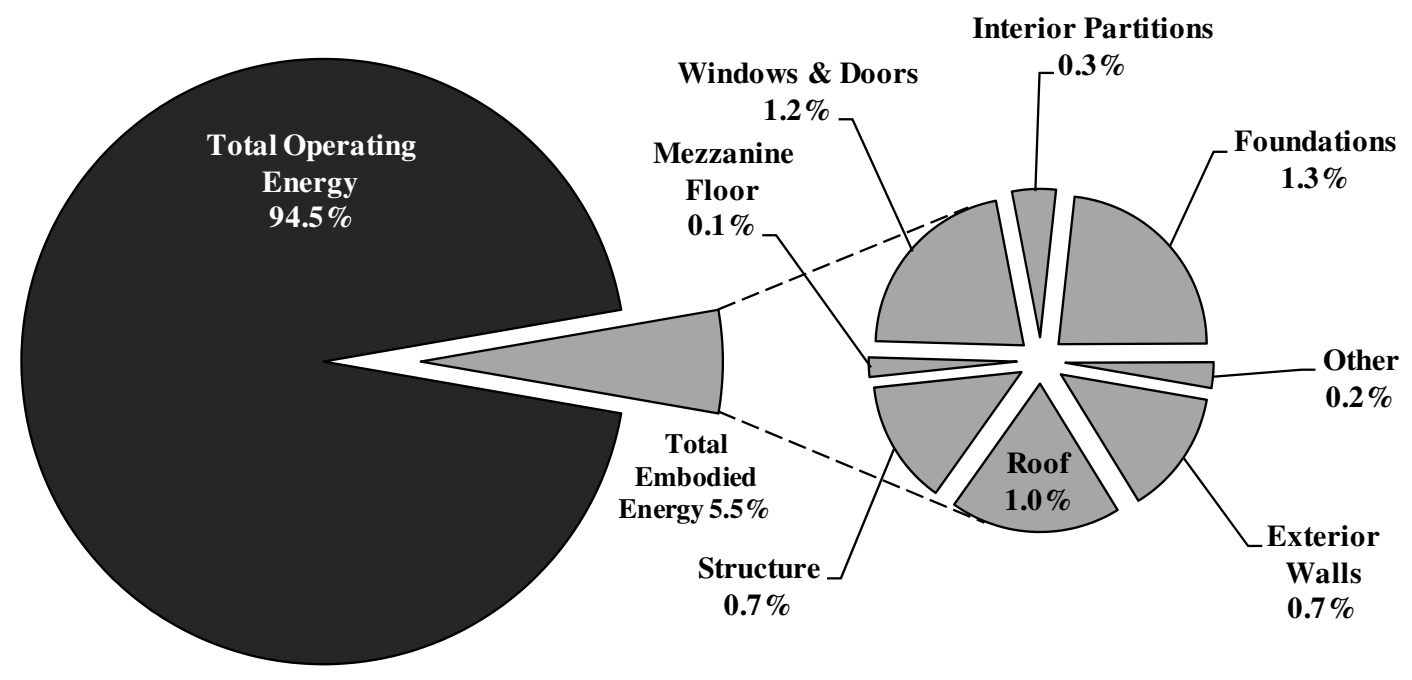

Total 50 Year Embodied Energy = 2,927 GJ

Total 50 Year Operating Energy = 50,470 GJ

Total 50 Year Energy = 53,396 GJ

Figure 5-13: Total Life-Cycle Energy Breakdown of the Typical Pre-Engineered Steel Retail Building (Case Study \#3) after 50 Year Lifespan in Toronto

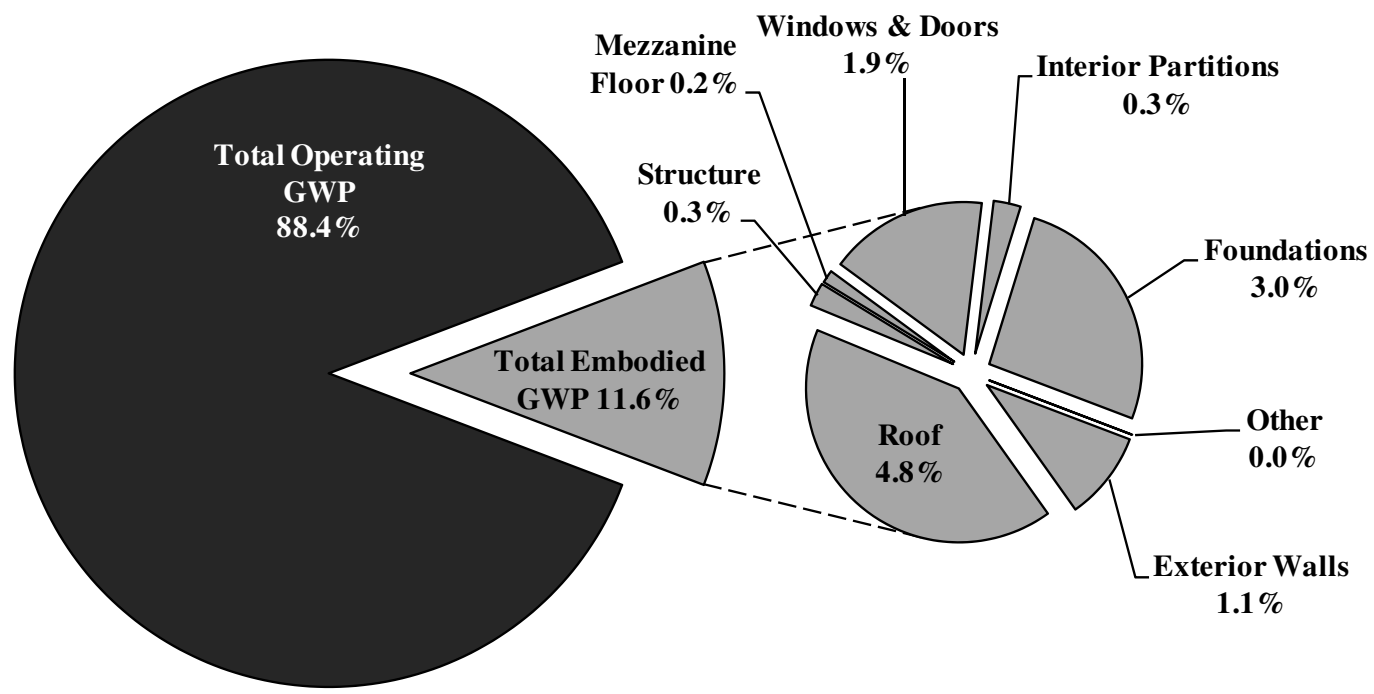

Total 50 Year Embodied GWP $=303$ tonnes of $\mathrm{CO}_{2}$ eq.

Total 50 Year Operating GWP $=2,310$ tonnes of $\mathrm{CO}_{2}$ eq.

Total 50 Year GWP $=2,613$ tonnes of $\mathrm{CO}_{2}$ eq.

Figure 5-14: Total Life-Cycle GWP Breakdown of the Typical Heavy Timber Structure Retail Building (Case Study \#2) after 50 Year Lifespan in Toronto 


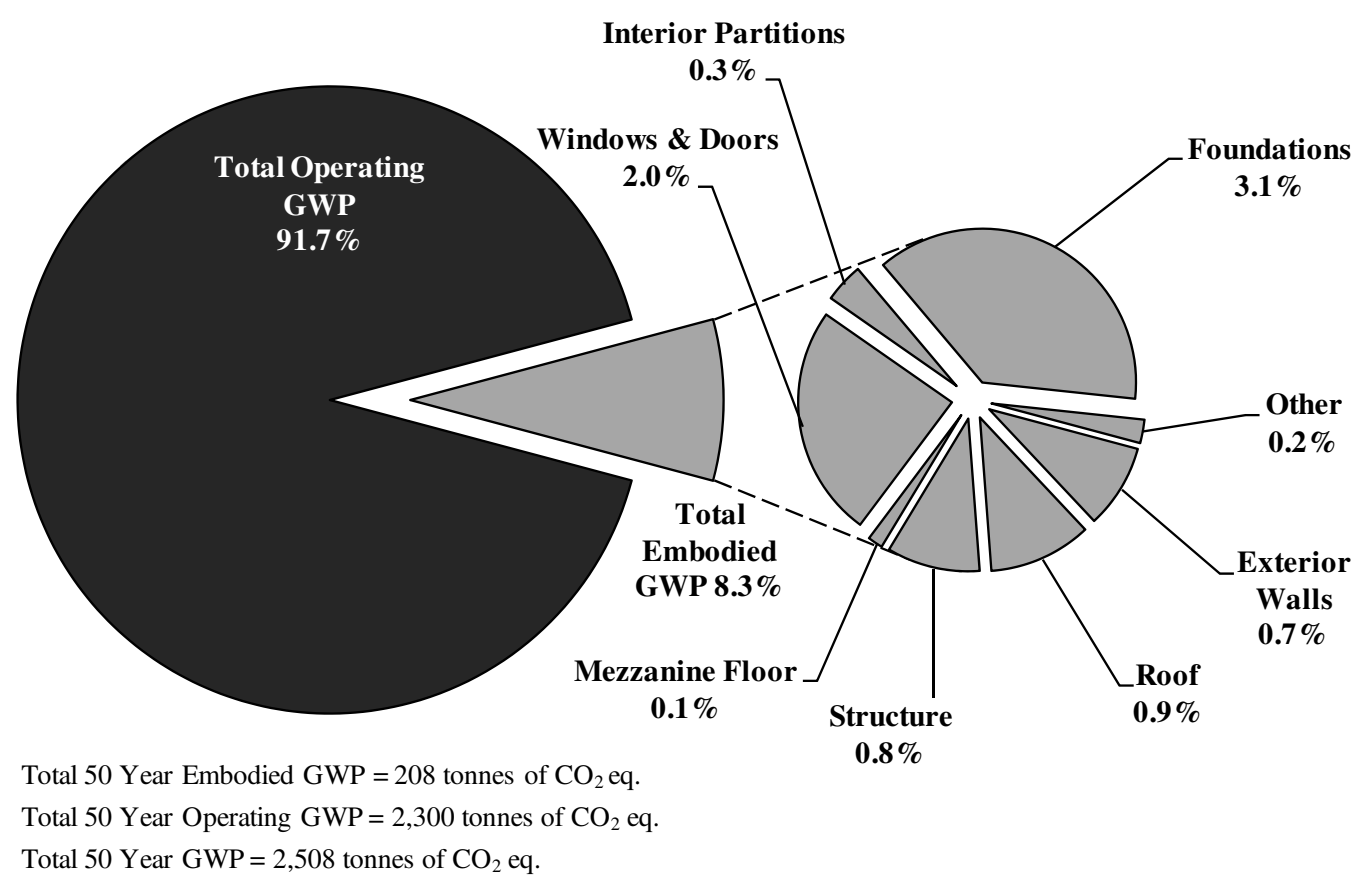

Figure 5-15: Total Life-Cycle GWP Breakdown of the Typical Pre-Engineered Steel Retail Building (Case Study \#3) after 50 Year Lifespan in Toronto

These LCA results for the five case study retail buildings in Canada are very informative. The importance of operating effects verses embodied effects is obvious. Practically speaking, any building designer who is concerned with reducing the life-cycle energy and GWP of a single-storey retail building should focus primarily on reducing the operating energy and operating GWP of the building. Only once the operating effects can be reduced by at least $50 \%$ from typical values today, does a comparison of embodied effects become relevant.

That being said, as aggressive reductions in operating effects are achieved through such means as the LEED ${ }^{\circledR}$ certification process, it will be important to know which building components (exterior walls, roofs, floors, structural systems, foundations, windows, doors, and interior partitions) consume the most amount of energy and have the most GWP. The remainder of this study will examine in greater detail some of the differences between these various building components in terms of operating and embodied effects. However, one should not forget that in a typical retail building today, operating effects account for upwards of $90 \%$ of the total effects, while embodied effects only represent about $10 \%$. 


\subsection{Data Quality and Assessment}

Before moving onto a detailed discussion of the LCA results for the 220 different building components in this study, it would be prudent to briefly compare the LCA results for the five singlestorey retail buildings in this study to the literature.

Recall from Table 2-4 in Chapter 2 that the annual total life-cycle operating energy per gross floor area for the LCA studies of commercial buildings in the literature ranged from about 0.23 to 4.23 $\mathrm{GJ} / \mathrm{m}^{2} / \mathrm{yr}$. The annual total life-cycle operating energy per gross floor area for the five single-storey retail buildings in this study ranged from about 1.74 to $1.75 \mathrm{GJ} / \mathrm{m}^{2} / \mathrm{yr}$, which is within the range from the literature. Likewise, the annual total life-cycle operating GWP per gross floor area for the LCA studies of commercial buildings in the literature ranged from about 0.02 to 0.24 tonnes of $\mathrm{CO}_{2}$ eq. $/ \mathrm{m}^{2} / \mathrm{yr}$. The annual total life-cycle operating GWP per gross floor area for the five single-storey retail buildings in this study was about 0.08 tonnes of $\mathrm{CO}_{2}$ eq. $/ \mathrm{m}^{2} / \mathrm{yr}$, which is also within the range from the literature. Also, in the literature the total life-cycle embodied energy per gross floor area at the end of the building's lifespan ranged from about 3.42 to $22.45 \mathrm{GJ} / \mathrm{m}^{2}$ for a commercial building. In this study, the equivalent values for the five single-storey retail buildings ranged from about 5.04 to $9.03 \mathrm{GJ} / \mathrm{m}^{2}$, which is within the range from the literature. Likewise, the total life-cycle embodied GWP per gross floor area at the end of the building's lifespan from the commercial buildings in the literature ranged from about 0.20 to 0.89 tonnes of $\mathrm{CO}_{2}$ eq. $/ \mathrm{m}^{2} / \mathrm{yr}$, compared to a range of about 0.36 to 0.55 tonnes of $\mathrm{CO}_{2}$ eq. $/ \mathrm{m}^{2} / \mathrm{yr}$ for the five single-storey retail buildings in this study. Therefore, the LCA results in this study fall within the range of LCA results for commercial buildings from the literature. 


\section{Chapter 6 \\ Results: Life-Cycle Assessment of Building Components}

\subsection{Introduction}

To this point, the LCA results have been presented for five different single-storey retail buildings with a 50 year lifespan in Toronto. The dominance of operating energy and operating GWP on the total life-cycle energy and total GWP has been established. Specifically, about $90 \%$ of the total lifecycle energy use and total GWP of a single-storey retail building after 50 years is a result of building operations, while only $10 \%$ or less is attributed to the embodied effects. However, as the building industry continues to strive for reductions in the annual operating energy and operating GWP of buildings, it will become increasingly important to consider the embodied energy and embodied GWP of the building components.

In this chapter, a brief overview of the total energy and total GWP for the 220 different building components that were examined in this study will be conducted. Recall that these building components are grouped into the following categories: exterior infill walls, roofs, structural systems, floors, windows, doors, interior partitions, and foundations. The LCA calculations are performed over a 50 year lifespan for the case of a building located in Toronto, Canada.

The ultimate objective of this chapter is to examine a wide array of strategies within each of the building component categories, in order to rank the alternative strategies in order of increasing total energy use and total GWP. By completing a detailed LCA of a wide array of strategies, this will serve as a reference to building professionals who are interested in a relative comparison of the total lifecycle energy use and total GWP of different walls, roofs, structural systems, floors, windows, doors, interior partitions, and foundations. The intent of this chapter is not to deal specifically with a direct comparison of one building component to another, but rather to present the range of values that are possible for the various categories of building components. Thus, the results in this chapter are a summary of the detailed LCA results that can be found in Appendix B.

\subsection{Interpreting the LCA Graphs of Building Components}

Interpreting the graphs of the total life-cycle energy and GWP presented in this chapter is relatively straightforward. The baseline retail building (Case Study \#1) was used as the datum. Each building component of the baseline building was then systematically replaced with a new building component 
(for example the baseline exterior infill wall was replaced with a new exterior infill wall). The difference in the total embodied energy ( $\Delta$ embodied energy) and the difference in the total operating energy ( $\Delta$ operating energy) from the baseline case after 50 years was plotted. Similarly, the difference in the total embodied GWP ( $\Delta$ embodied GWP) and the difference in the total operating GWP ( $\Delta$ operating GWP) from the baseline case was also plotted.

The key point to note when interpreting these graphs is that the values (both embodied energy and operating energy) have been plotted relative to a baseline component. In each case, the baseline component is also plotted on the graphs and is highlighted in grey. The range of building components were potted in order of increasing total energy (embodied energy + operating energy). Therefore, those building components that are plotted to the left of the baseline component (highlighted in grey) consume less total energy after 50 years than the baseline component. Conversely, those building components that are plotted to the right of the baseline building component, consume more total energy after 50 years than the baseline component. In this way, one can quickly tell from these graphs how the various building components compare to one another, based on their relative comparison to a baseline building component.

\subsection{LCA Results for Exterior Infill Wall Enclosures}

In this section, the LCA results for the collection of exterior infill walls in Appendix B will be presented. The $\Delta$ total energy and $\Delta$ total GWP of the different types of exterior infill walls examined in this study relative to the baseline wall (BASE-W) can be seen in Figure 6-1 to Figure 6-6. Recall that a detailed description of these walls can be found in Appendix B.

From outside to inside, BASE-W is comprised of: an exterior insulation and finish system (EIFS), 64 $\mathrm{mm}$ of extruded polystyrene rigid insulation, a self-adhesive membrane with primer, $16 \mathrm{~mm}$ non paper-faced gypsum sheathing, $39 \mathrm{~mm} \times 152 \mathrm{~mm}$ cold-formed steel studs spaced at $600 \mathrm{~mm}$ on center, regular $16 \mathrm{~mm}$ gypsum board, and a latex paint finish. After 50 years, the total embodied energy of BASE-W is about $0.927 \mathrm{GJ} / \mathrm{m}^{2}$ of primary energy and the total embodied GWP is about 49 $\mathrm{kg}$ of $\mathrm{CO}_{2}$ eq. $/ \mathrm{m}^{2}$ (results are per $\mathrm{m}^{2}$ of exterior wall). 


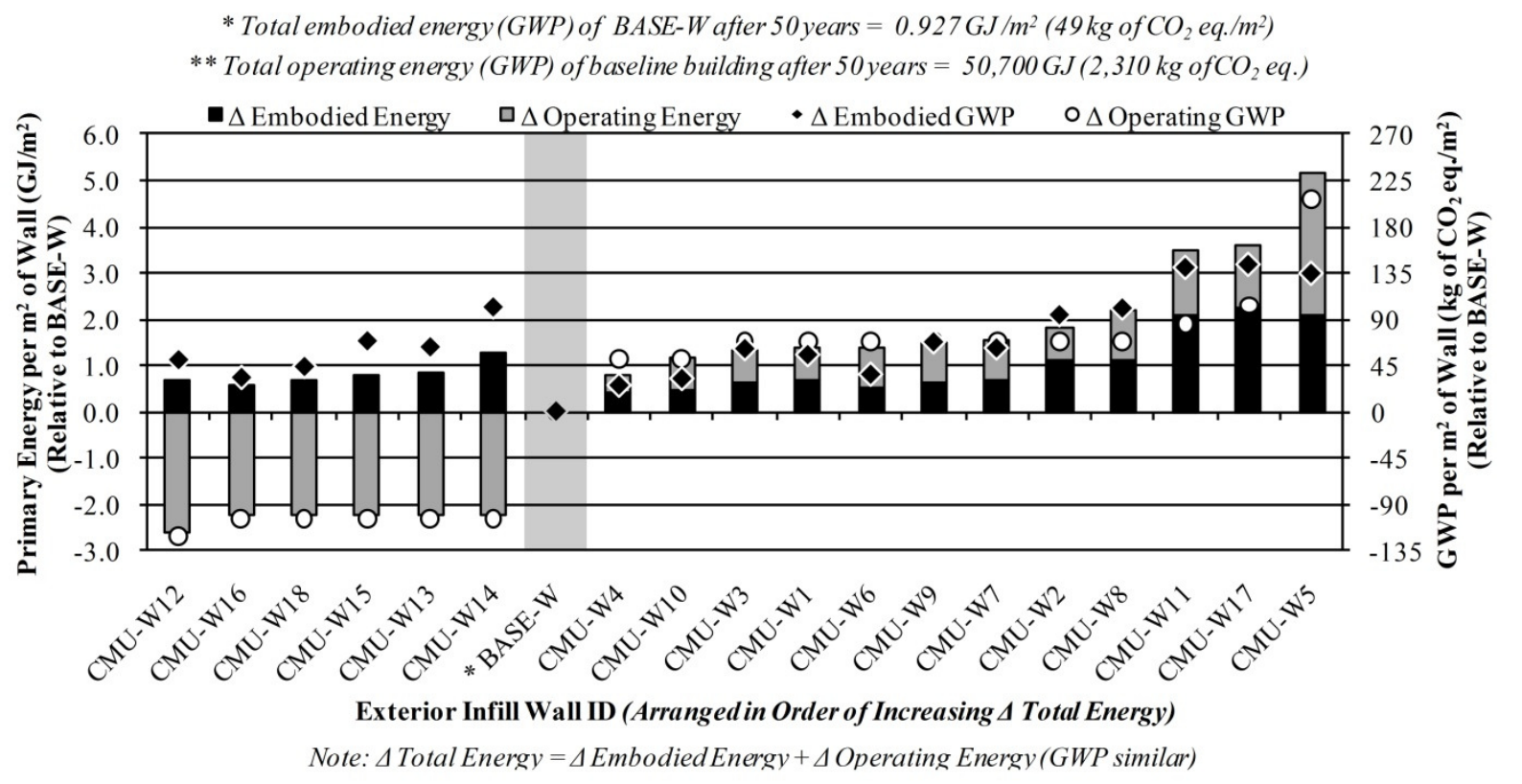

Figure 6-1: Total Life-Cycle Energy and GWP of Concrete Masonry Unit Exterior Infill Walls (CMU-W) after 50 Year Lifespan in Toronto
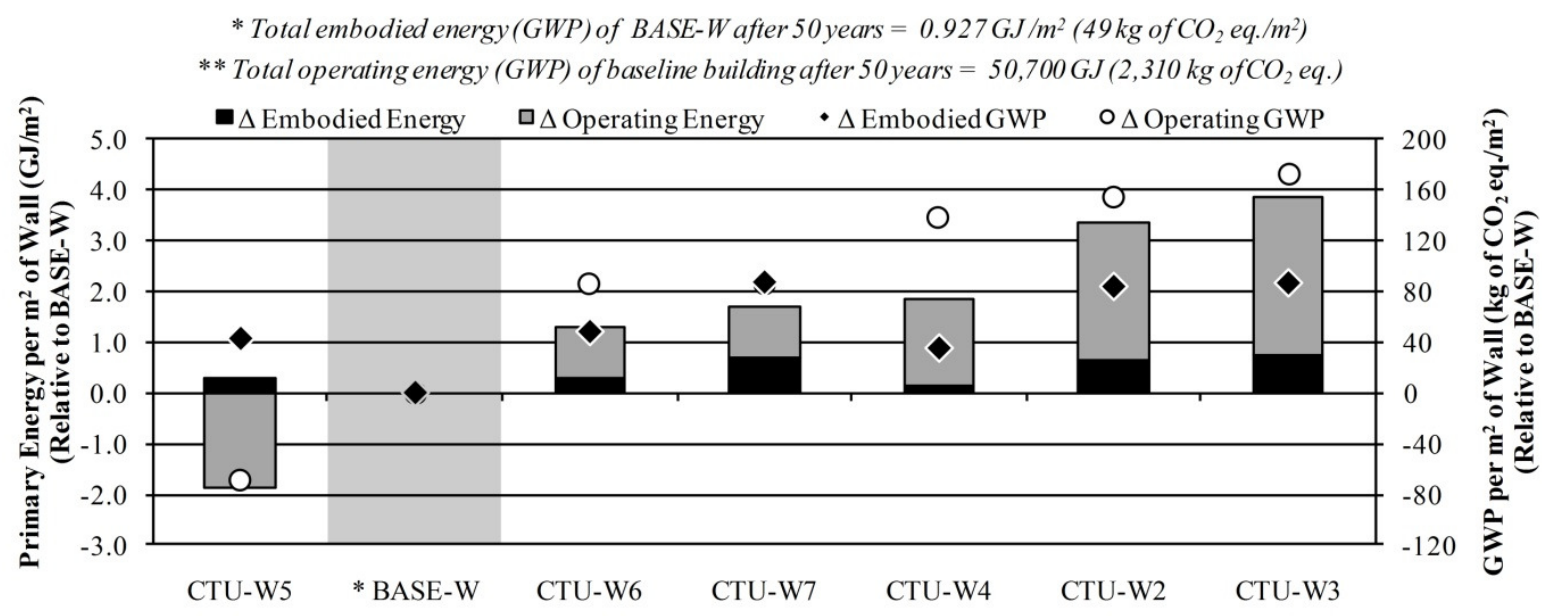

Exterior Infill Wall ID (Arranged in Order of Increasing 4 Total Energy)

Note: $\Delta$ Total Energy $=\Delta$ Embodied Energy $+\Delta$ Operating Energy (GWP similar)

(CTU-W1 was excluded from graph due to very high operating energy \& operating GWP)

Figure 6-2: Total Life-Cycle Energy and GWP of Concrete Tilt-Up Exterior Infill Walls (CTUW) after 50 Year Lifespan in Toronto 


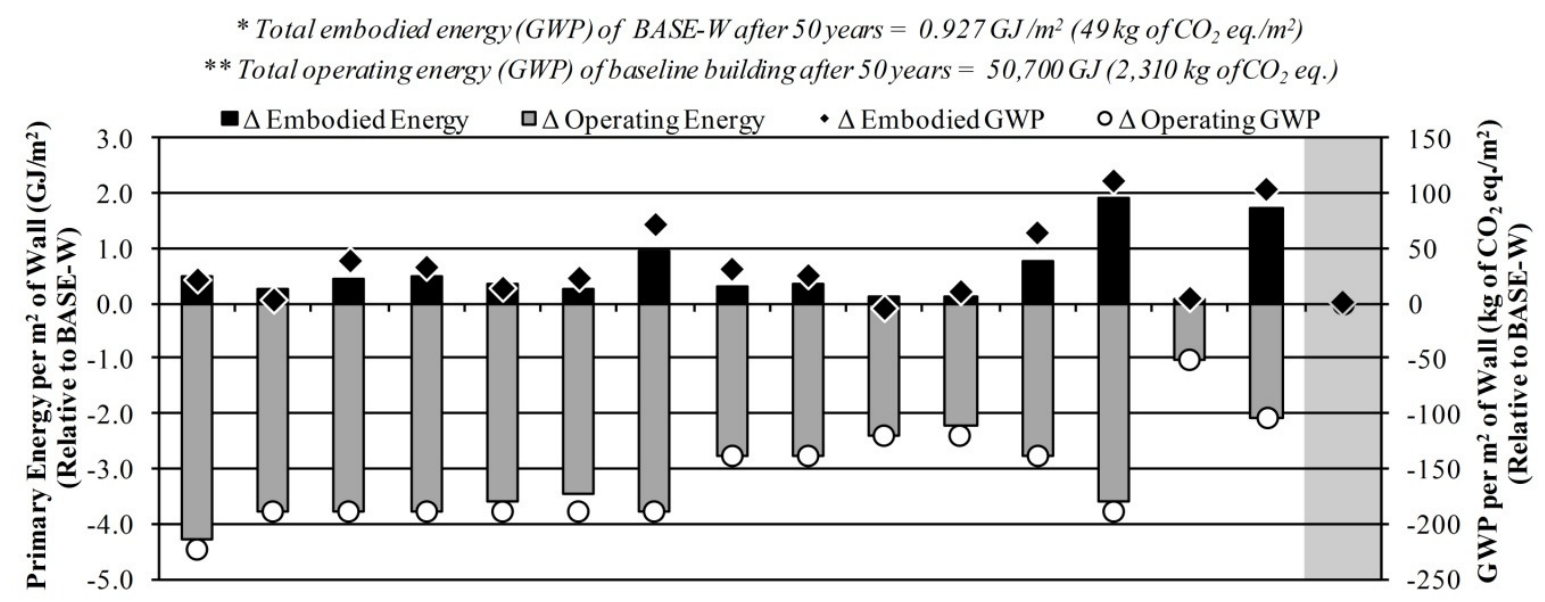
Figure 6-3: Total Life-Cycle Energy and GWP of Structural Insulated Panel Exterior Infill
Walls (WSIP-W \& MSIP-W) after 50 Year Lifespan in Toronto

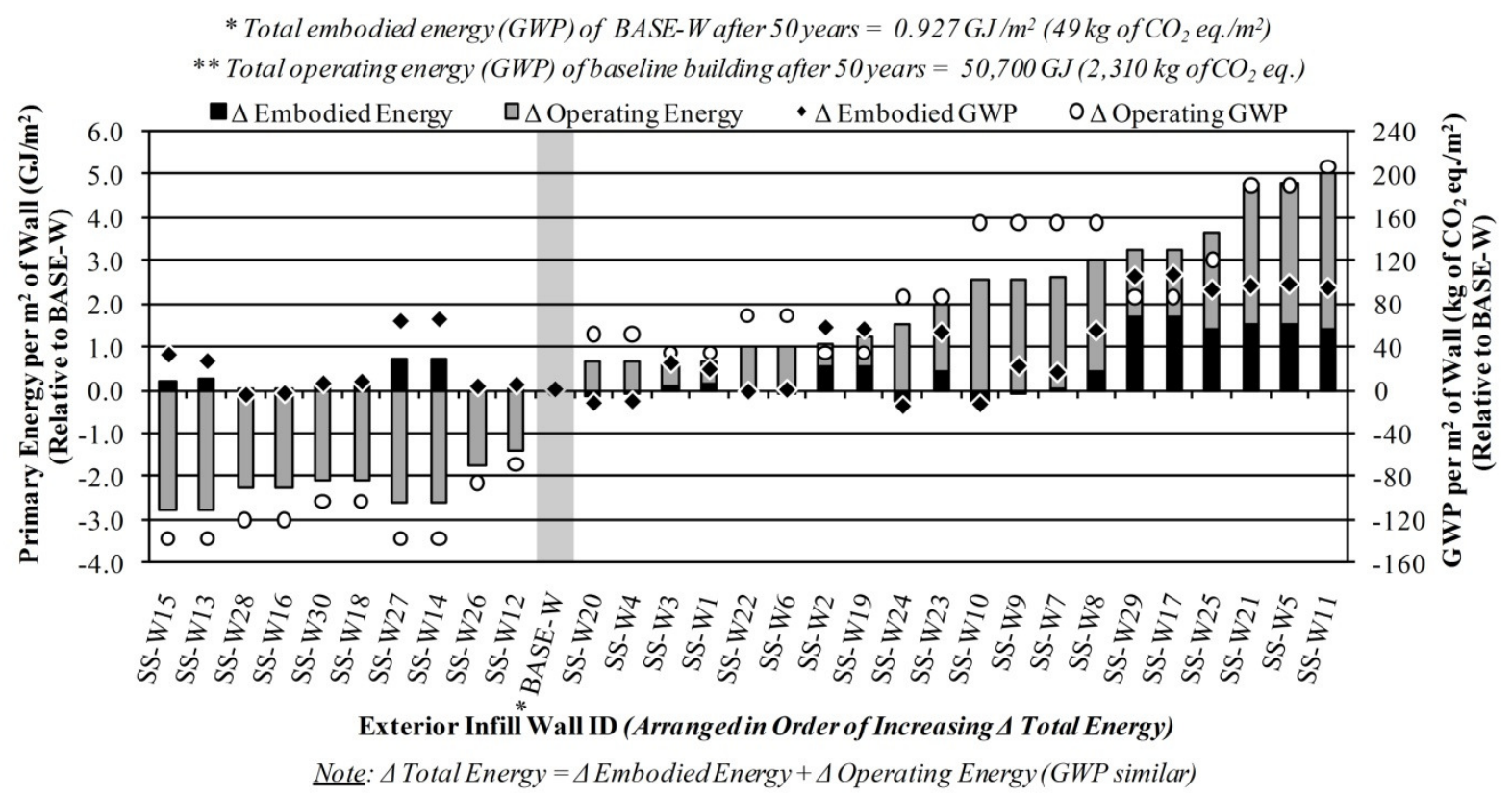

Figure 6-4: Total Life-Cycle Energy and GWP of Cold-Formed Steel Stud Exterior Infill Walls (SS-W) after 50 Year Lifespan in Toronto 

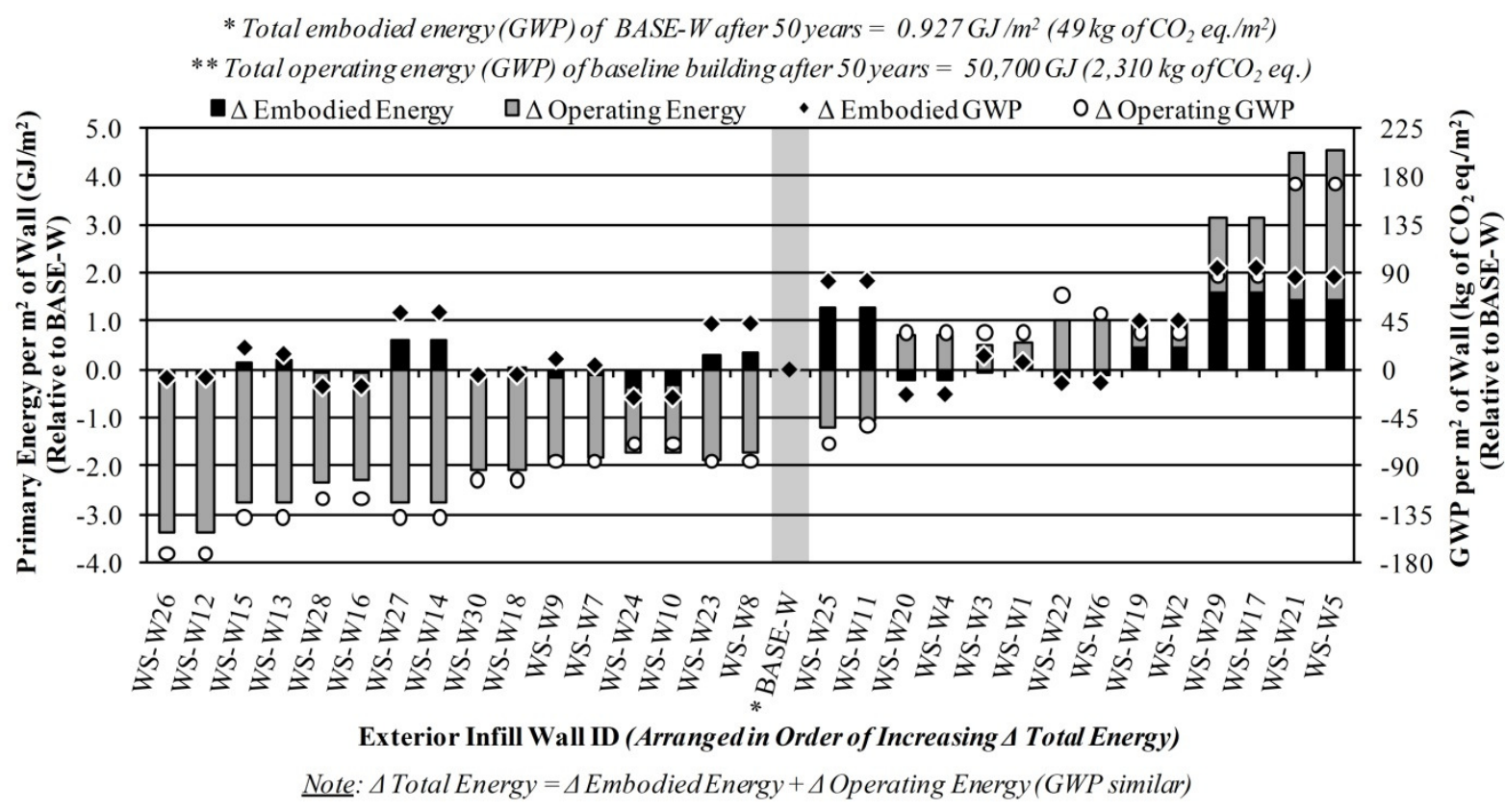

Figure 6-5: Total Life-Cycle Energy and GWP of Wood Stud Exterior Infill Walls (WS-W) after 50 Year Lifespan in Toronto

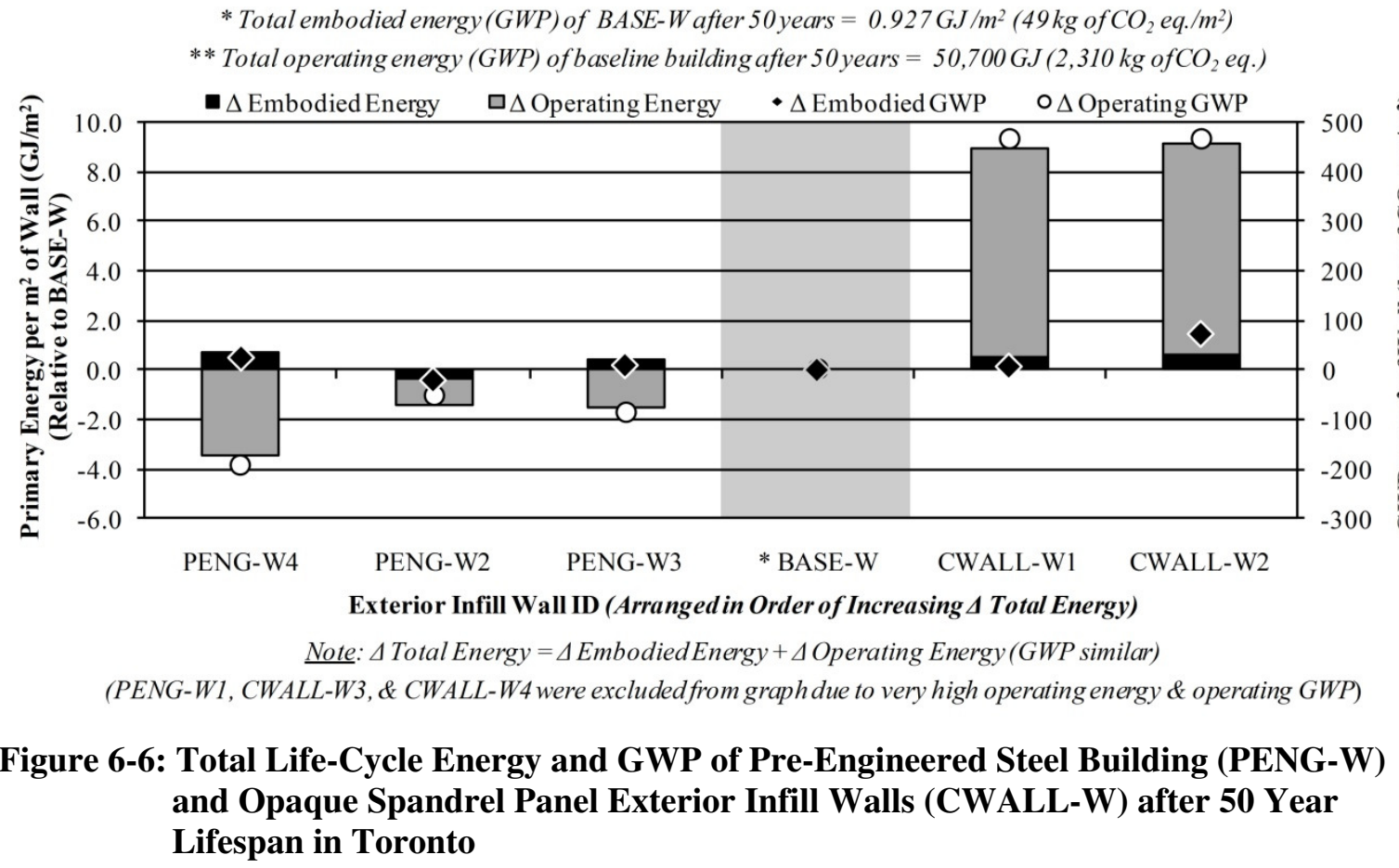


Table 6-1 summarizes the data presented in the preceding figures, along with a summary of the data for the additional building components that will be presented in subsequent sections. The average, minimum, and maximum values for the $\Delta$ total energy and $\Delta$ total GWP from the baseline datum have been calculated for the 220 different building components in this study. Table 6-1 allows for a quick comparison of the range of values that are possible for the different types of exterior infill walls and other building components in this study, with respect to the $\Delta$ total energy and $\Delta$ total GWP. Negative values indicate a savings in energy and GWP and positive values indicate an increase.

Table 6-1: A Summary of the $\Delta$ Total Energy and $\Delta$ Total GWP from the Baseline Case for the Alternative Building Components in this Study after 50 Years

\begin{tabular}{|c|c|c|c|c|c|c|}
\hline \multirow{2}{*}{ Building Component } & \multicolumn{3}{|c|}{$\Delta$ Total Primary Energy $\left(\mathrm{GJ} / \mathrm{m}^{2}\right)$} & \multicolumn{3}{|c|}{$\Delta$ Total GWP $\left(\mathrm{kg}\right.$ of $\left.\mathrm{CO}_{2} \mathrm{eq} . / \mathrm{m}^{2}\right)$} \\
\hline & Average & Min & Max & Average & Min & Max \\
\hline \multicolumn{7}{|l|}{ Exterior Infill Walls } \\
\hline $\begin{array}{l}\text { Concrete Masonry Unit } \\
\text { Walls (CMU-W) }\end{array}$ & 0.911 & $\begin{array}{c}-1.879 \\
(\mathrm{CMU}-\mathrm{W} 12) \\
\end{array}$ & $\begin{array}{c}5.180 \\
\text { (CMU-W5) }\end{array}$ & 92 & $\begin{array}{c}-70 \\
(\mathrm{CMU}-\mathrm{W} 12) \\
\end{array}$ & $\begin{array}{c}342 \\
\text { (CMU-W5) }\end{array}$ \\
\hline $\begin{array}{l}{ }^{1} \text { Concrete Tilt-Up Walls } \\
(\mathrm{CTU}-\mathrm{W})\end{array}$ & 1.751 & $\begin{array}{c}-1.597 \\
(\mathrm{CTU}-W 5)\end{array}$ & $\begin{array}{c}3.848 \\
(\mathrm{CTU}-\mathrm{W} 3)\end{array}$ & 159 & $\begin{array}{c}-26 \\
(\mathrm{CTU}-\mathrm{W} 5)\end{array}$ & $\begin{array}{c}259 \\
(\mathrm{CTU}-\mathrm{W} 3)\end{array}$ \\
\hline $\begin{array}{l}\text { Wood Structural Insulated } \\
\text { Panel Walls (WSIP-W) }\end{array}$ & -2.606 & $\begin{array}{c}-3.810 \\
(\text { WSIP-W12) } \\
\end{array}$ & $\begin{array}{c}-0.322 \\
\text { (WSIP-W5) }\end{array}$ & -125 & $\begin{array}{c}-203 \\
\text { (WSIP-W12) } \\
\end{array}$ & $\begin{array}{c}0 \\
\text { (WSIP-W5) } \\
\end{array}$ \\
\hline $\begin{array}{l}\text { Metal Structural Insulated } \\
\text { Panel Walls (MSIP-W) }\end{array}$ & -2.085 & $\begin{array}{c}-3.189 \\
(\mathrm{MSIP}-W 1) \\
\end{array}$ & $\begin{array}{c}-0.961 \\
(\mathrm{MSIP}-W 3) \\
\end{array}$ & -109 & $\begin{array}{c}-168 \\
(\mathrm{MSIP}-\mathrm{W} 1) \\
\end{array}$ & $\begin{array}{c}-48 \\
(\mathrm{MSIP}-\mathrm{W} 3) \\
\end{array}$ \\
\hline $\begin{array}{l}\text { Cold-Formed Steel Stud } \\
\text { Walls (SS-W) }\end{array}$ & 0.837 & $\begin{array}{c}-2.521 \\
(\mathrm{SS}-\mathrm{W} 15) \\
\end{array}$ & $\begin{array}{c}5.022 \\
(\mathrm{SS}-\mathrm{W} 11) \\
\end{array}$ & 65 & $\begin{array}{c}-126 \\
(\mathrm{SS}-\mathrm{W} 28) \\
\end{array}$ & $\begin{array}{c}301 \\
(\mathrm{SS}-\mathrm{W} 11) \\
\end{array}$ \\
\hline Wood Stud Walls (WS-W) & -0.465 & $\begin{array}{c}-3.393 \\
(\text { WS-W26) }\end{array}$ & $\begin{array}{c}4.520 \\
\text { (WS-W5) }\end{array}$ & -13 & $\begin{array}{c}-180 \\
(\mathrm{WS}-\mathrm{W} 26)\end{array}$ & $\begin{array}{c}259 \\
\text { (WS-W5) }\end{array}$ \\
\hline $\begin{array}{l}{ }^{2} \text { Pre-Engineered Steel } \\
\text { Building Walls (PENG-W) }\end{array}$ & -1.768 & $\begin{array}{c}-2.759 \\
(\mathrm{PENG}-\mathrm{W} 4) \\
\end{array}$ & $\begin{array}{c}-1.165 \\
\text { (PENG-W3) } \\
\end{array}$ & -105 & $\begin{array}{c}-165 \\
\text { (PENG-W4) }\end{array}$ & $\begin{array}{c}-77 \\
\text { (PENG-W3) }\end{array}$ \\
\hline $\begin{array}{l}{ }^{3} \text { Opaque Curtainwalls } \\
(\mathrm{CWALL}-\mathrm{W})\end{array}$ & 9.008 & $\begin{array}{c}8.920 \\
(\mathrm{CWALL}-\mathrm{W} 1) \\
\end{array}$ & $\begin{array}{c}9.096 \\
(\mathrm{CWALL}-\mathrm{W} 2) \\
\end{array}$ & 504 & $\begin{array}{c}472 \\
(\mathrm{CWALL}-\mathrm{W} 1) \\
\end{array}$ & $\begin{array}{c}537 \\
\text { (CWALL-W2) } \\
\end{array}$ \\
\hline \multicolumn{7}{|l|}{ Roofs } \\
\hline $\begin{array}{l}\text { Concrete Hollow Core } \\
\text { Roofs (CHC-R) }\end{array}$ & -2.675 & $\begin{array}{c}-4.850 \\
(\mathrm{CHC}-\mathrm{R} 10)\end{array}$ & $\begin{array}{c}-0.194 \\
(\mathrm{CHC}-\mathrm{R} 2) \\
\end{array}$ & -102 & $\begin{array}{c}-210 \\
(\mathrm{CHC}-\mathrm{R} 10)\end{array}$ & $\begin{array}{c}14 \\
(\mathrm{CHC}-\mathrm{R} 2) \\
\end{array}$ \\
\hline $\begin{array}{l}\text { Open Web Steel Joist Roofs } \\
\text { (OWSJ-R) }\end{array}$ & -2.537 & $\begin{array}{c}-4.602 \\
\text { (OWSJ-R10) } \\
\end{array}$ & $\begin{array}{c}-0.171 \\
\text { (OWSJ-R2) } \\
\end{array}$ & -112 & $\begin{array}{c}-223 \\
\text { (OWSJ-R10) } \\
\end{array}$ & $\begin{array}{c}0 \\
\text { (OWSJ-R2) } \\
\end{array}$ \\
\hline $\begin{array}{l}\text { Cold-Formed Steel Joist } \\
\text { Roofs (CFS-R) }\end{array}$ & -2.341 & $\begin{array}{c}-4.925 \\
(\mathrm{CFS}-\mathrm{R} 10) \\
\end{array}$ & $\begin{array}{c}-0.240 \\
(\mathrm{CFS}-\mathrm{R} 13) \\
\end{array}$ & -102 & $\begin{array}{c}-243 \\
(\mathrm{CFS}-\mathrm{R} 10) \\
\end{array}$ & $\begin{array}{c}-1 \\
(\mathrm{CFS}-\mathrm{R} 13) \\
\end{array}$ \\
\hline $\begin{array}{l}\text { Glulam Joist Roofs } \\
\text { (GLU-R) }\end{array}$ & -3.123 & $\begin{array}{c}-5.161 \\
(\text { GLU-R10) }\end{array}$ & $\begin{array}{c}-0.827 \\
(\mathrm{GLU}-\mathrm{R} 2) \\
\end{array}$ & -150 & $\begin{array}{c}-255 \\
(\mathrm{GLU}-\mathrm{R} 10)\end{array}$ & $\begin{array}{c}-44 \\
(\mathrm{GLU}-\mathrm{R} 2)\end{array}$ \\
\hline $\begin{array}{l}\text { Wood Structural Insulated } \\
\text { Panel Roofs (WSIP-R) }\end{array}$ & -3.186 & $\begin{array}{c}-4.627 \\
(\text { WSIP-R5) } \\
\end{array}$ & $\begin{array}{c}-1.314 \\
(\mathrm{WSIP}-\mathrm{R} 2) \\
\end{array}$ & -168 & $\begin{array}{c}-218 \\
(\text { WSIP-R6) } \\
\end{array}$ & $\begin{array}{c}-83 \\
(\mathrm{WSIP}-\mathrm{R} 2) \\
\end{array}$ \\
\hline $\begin{array}{l}\text { Metal Structural Insulated } \\
\text { Panel Roofs (MSIP-R) }\end{array}$ & -4.313 & $\begin{array}{c}-5.180 \\
(\mathrm{MSIP}-\mathrm{R} 1) \\
\end{array}$ & $\begin{array}{c}-3.313 \\
(\mathrm{MSIP}-\mathrm{R} 3) \\
\end{array}$ & -182 & $\begin{array}{c}-225 \\
(\mathrm{MSIP}-\mathrm{R} 1) \\
\end{array}$ & $\begin{array}{c}-124 \\
(\mathrm{MSIP}-\mathrm{R} 3) \\
\end{array}$ \\
\hline $\begin{array}{l}{ }^{4} \text { Pre-Engineered Steel } \\
\text { Building Roofs (PENG-R) }\end{array}$ & -3.754 & $\begin{array}{c}-4.580 \\
(\text { PENG-R4) }\end{array}$ & $\begin{array}{c}-3.339 \\
(\mathrm{PENG}-\mathrm{R} 2)\end{array}$ & -173 & $\begin{array}{c}-222 \\
(\text { PENG-R4) }\end{array}$ & $\begin{array}{c}-144 \\
(\mathrm{PENG}-\mathrm{R} 2)\end{array}$ \\
\hline
\end{tabular}


Table 6-1 (Cont.): A Summary of the $\Delta$ Total Energy and $\Delta$ Total GWP from the Baseline Case for the Alternative Building Components in this Study after 50 Years

\begin{tabular}{|c|c|c|c|c|c|c|}
\hline \multirow{2}{*}{ Building Component } & \multicolumn{3}{|c|}{$\Delta$ Total Primary Energy $\left(G J / m^{2}\right)$} & \multicolumn{3}{|c|}{$\Delta$ Total GWP $\left(\mathrm{kg}\right.$ of $\left.\mathrm{CO}_{2} \mathrm{eq} . / \mathrm{m}^{2}\right)$} \\
\hline & Average & Min & Max & Average & Min & Max \\
\hline Structural Systems (S) & -0.343 & $\begin{array}{l}-0.547 \\
(\mathrm{~S}-2)\end{array}$ & $\begin{array}{l}-0.139 \\
(\mathrm{~S}-3)\end{array}$ & -22 & $\begin{array}{c}-33 \\
(\mathrm{~S}-2)\end{array}$ & $\begin{array}{c}-11 \\
(\mathrm{~S}-3)\end{array}$ \\
\hline Floors (FL) & -0.527 & $\begin{array}{l}-0.646 \\
(\mathrm{FL}-5)\end{array}$ & $\begin{array}{l}-0.448 \\
(\text { FL-4) }\end{array}$ & -37 & $\begin{array}{c}-55 \\
(\mathrm{FL}-5)\end{array}$ & $\begin{array}{c}-6 \\
(\mathrm{FL}-1)\end{array}$ \\
\hline Windows $(\mathbf{W})$ & -10.399 & $\begin{array}{c}-18.082 \\
(\mathrm{~W}-8)\end{array}$ & $\begin{array}{l}-2.621 \\
(\mathrm{~W}-3)\end{array}$ & -476 & $\begin{array}{l}-935 \\
(W-8)\end{array}$ & $\begin{array}{c}37 \\
(\mathrm{~W}-3)\end{array}$ \\
\hline${ }^{5}$ Doors (D) & -17.404 & $\begin{array}{c}-107.796 \\
(D-1)\end{array}$ & $\begin{array}{c}33.342 \\
(\mathrm{D}-4)\end{array}$ & 122 & $\begin{array}{l}-273 \\
(\mathrm{D}-1)\end{array}$ & $\begin{array}{l}1,158 \\
(D-4)\end{array}$ \\
\hline $\begin{array}{l}\text { Interior Partitions } \\
\text { (WS-P, SS-P, \& CMU-P) }\end{array}$ & -0.056 & $\begin{array}{c}-0.196 \\
(\mathrm{WS}-\mathrm{P} 2)\end{array}$ & $\begin{array}{c}0.394 \\
(\mathrm{CMU}-\mathrm{P} 1) \\
\end{array}$ & -5 & $\begin{array}{c}-16 \\
(\mathrm{WS}-\mathrm{P} 2)\end{array}$ & $\begin{array}{c}29 \\
(\mathrm{CMU}-\mathrm{P} 1) \\
\end{array}$ \\
\hline \multicolumn{7}{|l|}{ Foundations } \\
\hline $\begin{array}{l}{ }^{6} \text { Isolated Footing and } \\
\text { Concrete Pier (IF-FDN) }\end{array}$ & 0.882 & $\begin{array}{c}-0.091 \\
(\mathrm{IF}-\mathrm{FDN} 2) \\
\end{array}$ & $\begin{array}{c}3.224 \\
(\text { IF-FDN7) }\end{array}$ & 110 & $\begin{array}{c}-22 \\
(\mathrm{IF}-\mathrm{FDN} 2)\end{array}$ & $\begin{array}{c}393 \\
(\mathrm{IF}-\mathrm{FDN} 7)\end{array}$ \\
\hline $\begin{array}{l}{ }^{7} \text { Strip Footing and } \\
\text { Concrete Wall (SF-FDN) }\end{array}$ & 0.273 & $\begin{array}{c}-0.044 \\
(\text { SF-FDN6) }\end{array}$ & $\begin{array}{c}0.550 \\
(\mathrm{SF}-\mathrm{FDN} 3)\end{array}$ & 16 & $\begin{array}{c}-10 \\
(\text { SF-FDN6) } \\
\end{array}$ & $\begin{array}{c}44 \\
(\mathrm{SF}-F D N 3) \\
\end{array}$ \\
\hline $\begin{array}{l}\text { Slab-On-Grades } \\
\text { (SOG-FDN) }\end{array}$ & -1.184 & $\begin{array}{c}-2.976 \\
\text { (SOG-FDN3) }\end{array}$ & $\begin{array}{c}-0.056 \\
\text { (SOG-FDN5) }\end{array}$ & -77 & $\begin{array}{c}-194 \\
\text { (SOG-FDN3) }\end{array}$ & $\begin{array}{c}-12 \\
\text { (SOG-FDN5) }\end{array}$ \\
\hline
\end{tabular}

* Note: Baseline building components are not included in the average, min, and max calculations

1 Results for CTU-W1 were not considered in table due to very high operating energy and operating GWP

${ }^{2}$ Results for PENG-W1 were not considered in table due to very high operating energy and operating GWP

${ }^{3}$ Results for CWALL-W3 \& CWALL-W4 were not considered in table due to very high operating energy and operating GWP

${ }^{4}$ Results for PENG-R1 were not considered in table due to very high operating energy and operating GWP

${ }^{5}$ Numbers are expressed for one individual door not per $m^{2}$

${ }^{6}$ Numbers are expressed for one isolated footing and pier combination not per $m^{2}$

${ }^{7}$ Numbers are expressed per $m$ of strip footing and wall not per $m^{2}$

If the average values in Table 6-1 are used as an indicator of overall performance, then the different types of exterior infill walls in this study can be ranked in order of increasing total energy relative to the baseline case. Arranged in order of the highest savings in total energy to the highest increase in total energy after 50 years, the exterior infill walls in Table 6-1 would be ranked: WSIP-W, MSIP-W, PENG-W, WS-W, SS-W, CMU-W, CTU-W, and CWALL-W. In essence, the results indicate that on average the WSIP-W walls were the best performing exterior infill wall enclosure after 50 years and that the CWALL-W walls were the worst performing in terms of energy use. However, it may be misleading to draw any general conclusions about the competitiveness of a given wall type relative to other wall types based entirely on the average values in Table 6-1. The results in Table 6-1 also indicate that for each different type of exterior infill wall, a range of values were possible for the $\Delta$ total energy and $\Delta$ total GWP in each case, depending on the exact composition of the wall (i.e. the 
type of cladding material used, cavity insulation verses exterior installed rigid insulation, etc.). Therefore, the minimum and maximum values for the $\Delta$ total energy and $\Delta$ total GWP in each case are more representative of the competitiveness of a given wall type. Thus, the minimum and maximum values in Table 6-1 indicate the range of possible values for a given wall type and should be referenced when drawing any general conclusions about the relative competitiveness of a given wall type to another.

That being said, some important observations can be made for the exterior infill walls examined in this study based on the results in Table 6-1 and Figure 6-1 to Figure 6-6. In general, there was found to be a strong correlation between the thermal resistance of the exterior infill walls in this study and the $\Delta$ total energy and $\Delta$ total GWP. It was found that an increase in total energy and total GWP corresponded in general to a decrease in the thermal resistance of the exterior infill walls. Therefore, thermal resistance (i.e. R-value or RSI-value) played a more significant role in terms of energy use and GWP over the life of the building than any differences in embodied effects between the building materials in the walls. In other words, in almost every case there were far more significant energy and GWP savings to be had because of savings in operating effects than from savings in embodied effects for the various exterior infill walls examined in this study. In general, the best performing walls after 50 years were the ones that were able to reduce the total operating energy and total operating GWP due to an increased thermal resistance compared to the BASE-W. A similar comparison can also be done for the roof enclosures in this study.

\subsection{LCA Results for Roof Enclosures}

Presented in this section are the LCA results for the various roof enclosures from Appendix B. Figure 6-7 to Figure 6-12 illustrate the $\Delta$ total energy and $\Delta$ total GWP of the different types of roof enclosures examined in this study relative to the baseline roof (BASE-R).

From outside to inside, BASE-R is comprised of: gravel ballast, a 4-ply built-up asphalt roof, $12 \mathrm{~mm}$ roof coverboard, $75 \mathrm{~mm}$ of continuous polyisocyanurate insulation, $39 \mathrm{~mm}$ galvanized corrugated steel deck, open web steel joists spaced at 1,200 $\mathrm{mm}$ on center, and a suspended acoustic ceiling. After 50 years, the total embodied energy of BASE-R is about $4.684 \mathrm{GJ} / \mathrm{m}^{2}$ of primary energy and the total embodied GWP is about $213 \mathrm{~kg}$ of $\mathrm{CO}_{2}$ eq. $/ \mathrm{m}^{2}$ (results are per $\mathrm{m}^{2}$ of roof). 


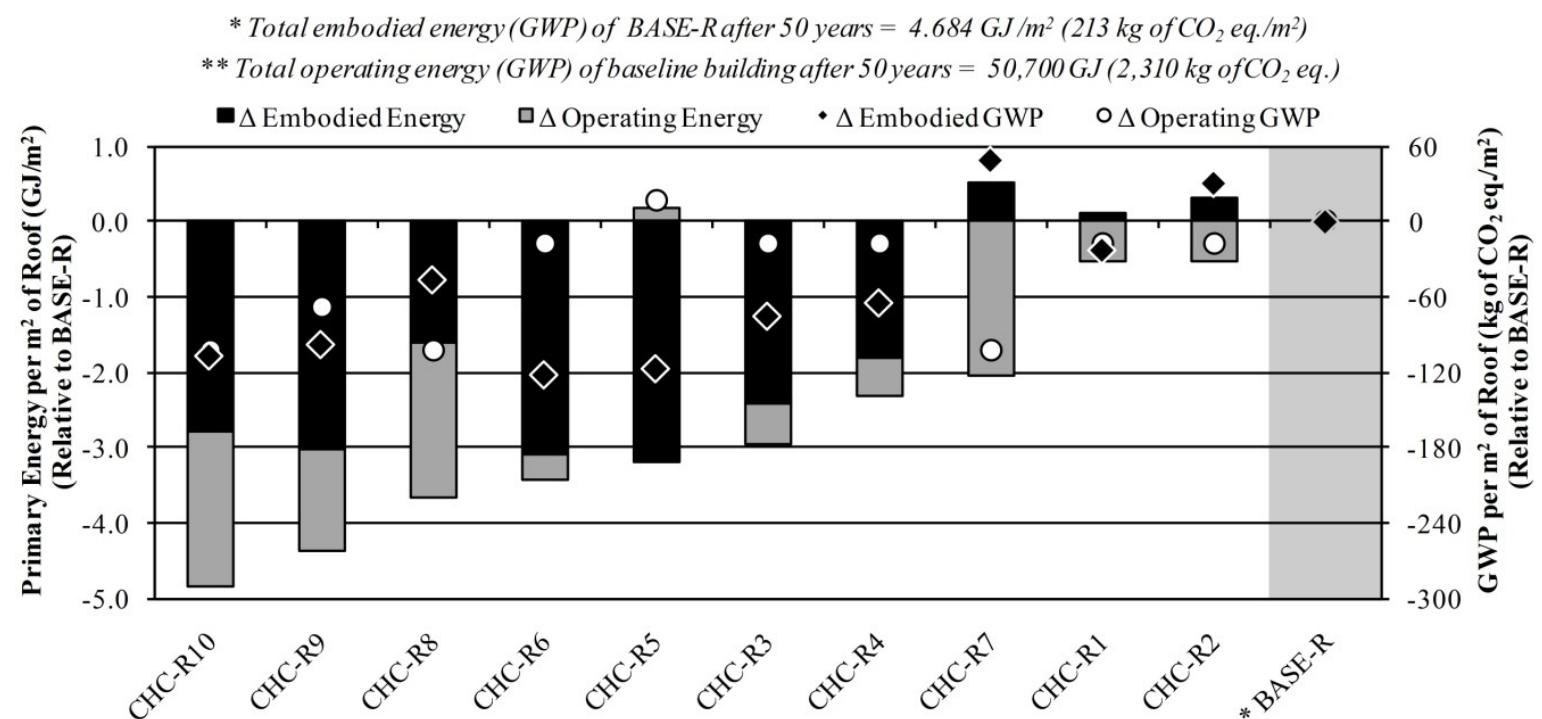

Roof ID (Arranged in Order of Increasing 4 Total Energy)

Note: $\Delta$ Total Energy $=\Delta$ Embodied Energy $+\Delta$ Operating Energy $(G W P$ similar $)$

Figure 6-7: Total Life-Cycle Energy and GWP of Concrete Hollow Core Roofs (CHC-R) after 50 Year Lifespan in Toronto

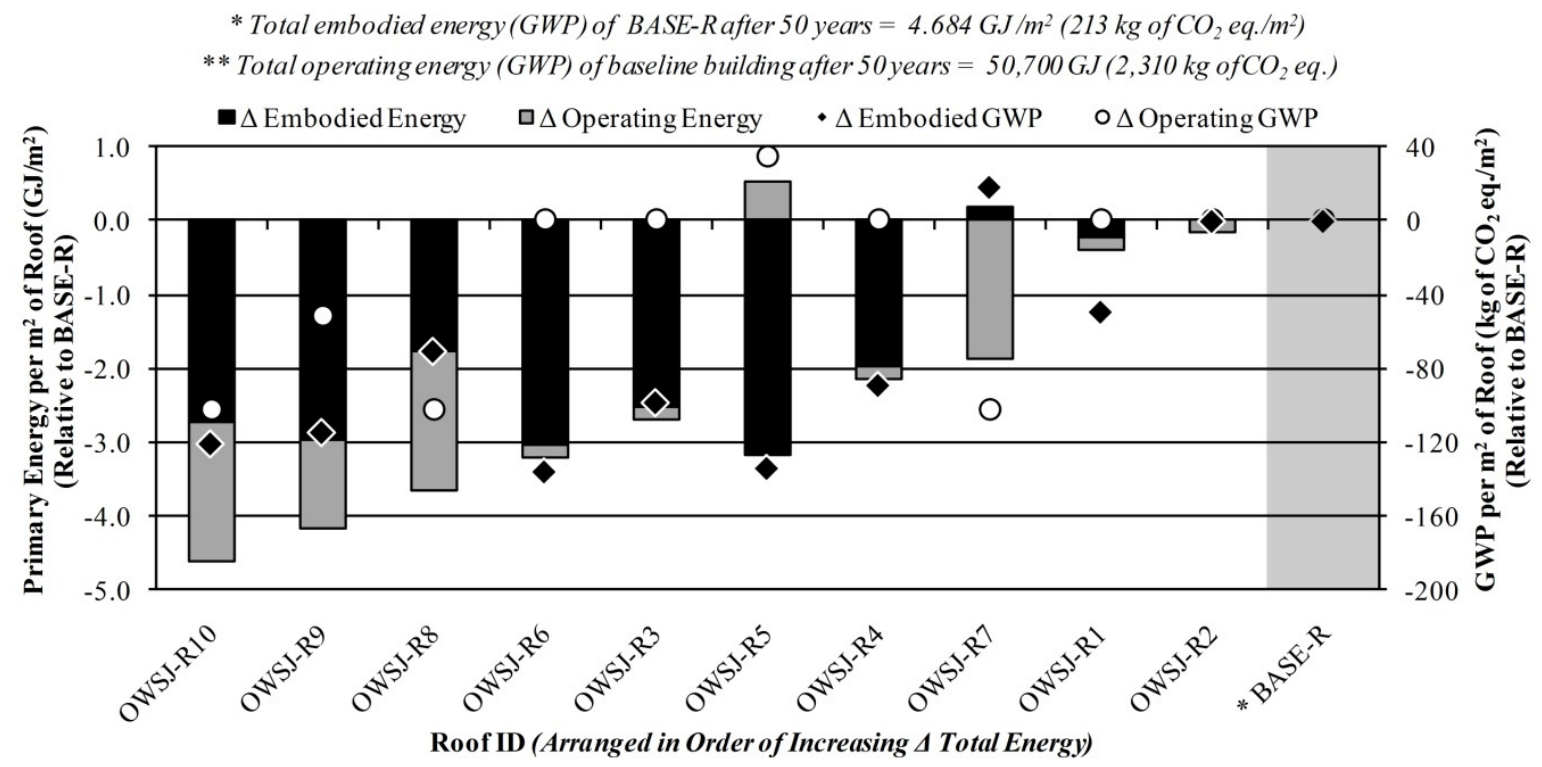

Note: $\triangle$ Total Energy $=\Delta$ Embodied Energy $+\Delta$ Operating Energy (GWP similar)

Figure 6-8: Total Life-Cycle Energy and GWP of Open Web Steel Joist Roofs (OWSJ-R) after 50 Year Lifespan in Toronto 


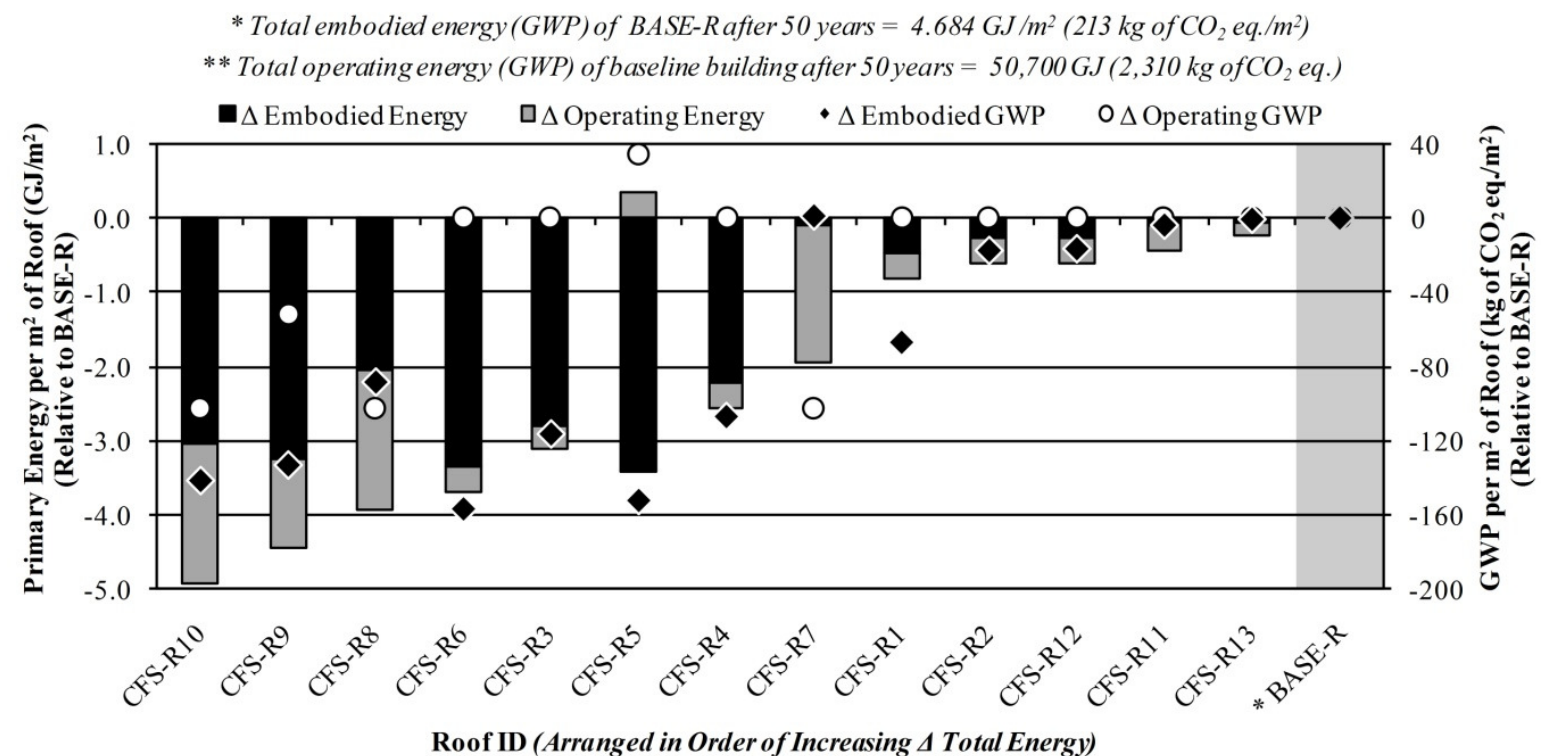

Note: $\Delta$ Total Energy $=\Delta$ EmbodiedEnergy $+\Delta$ Operating Energy (GWP similar)

Figure 6-9: Total Life-Cycle Energy and GWP of Cold-Formed Steel Roofs (CFS-R) after 50 Year Lifespan in Toronto

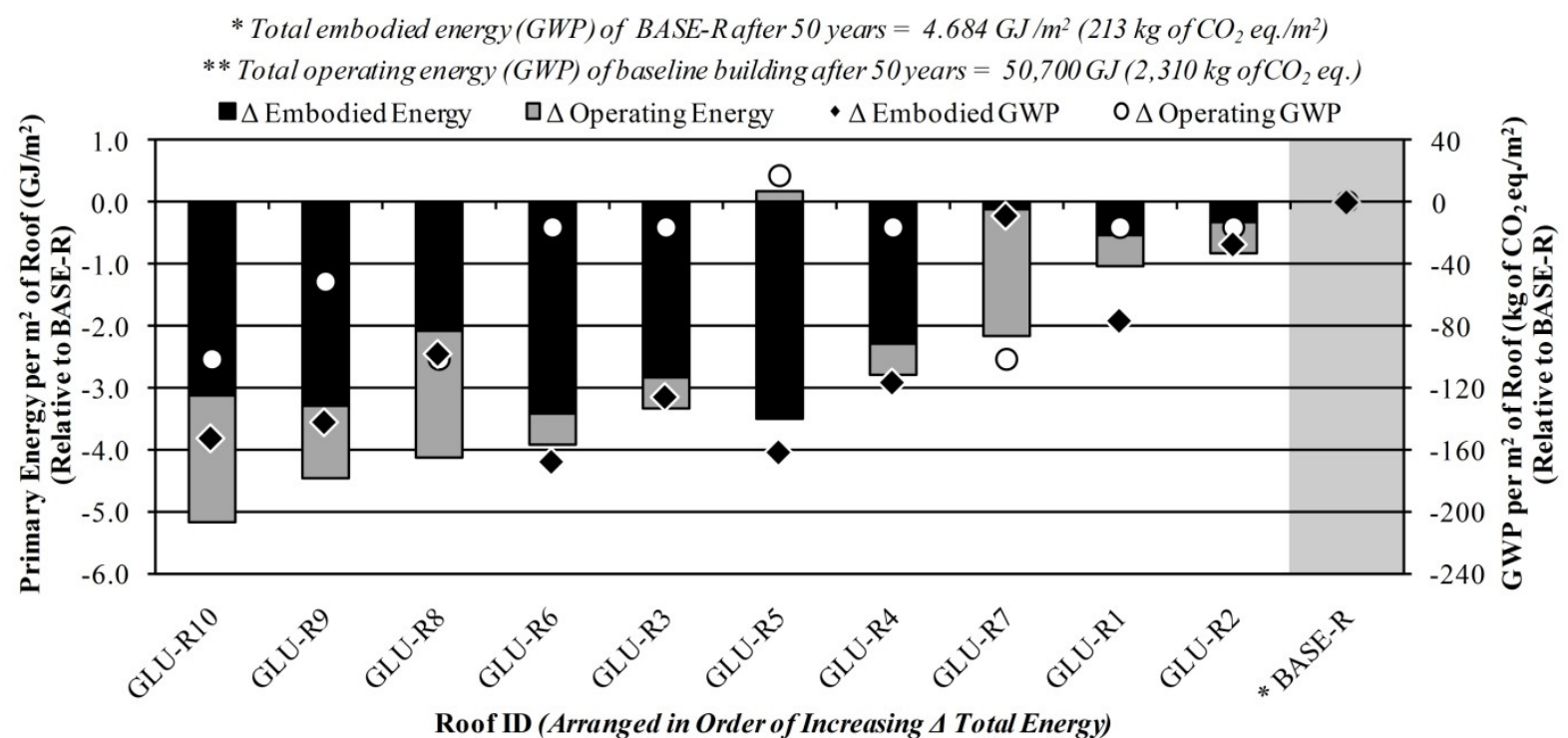

Note: $\Delta$ Total Energy $=\Delta$ Embodied Energy $+\Delta$ Operating Energy (GWP similar)

Figure 6-10: Total Life-Cycle Energy and GWP of Glulam Joist Roofs (GLU-R) after 50 Year Lifespan in Toronto 


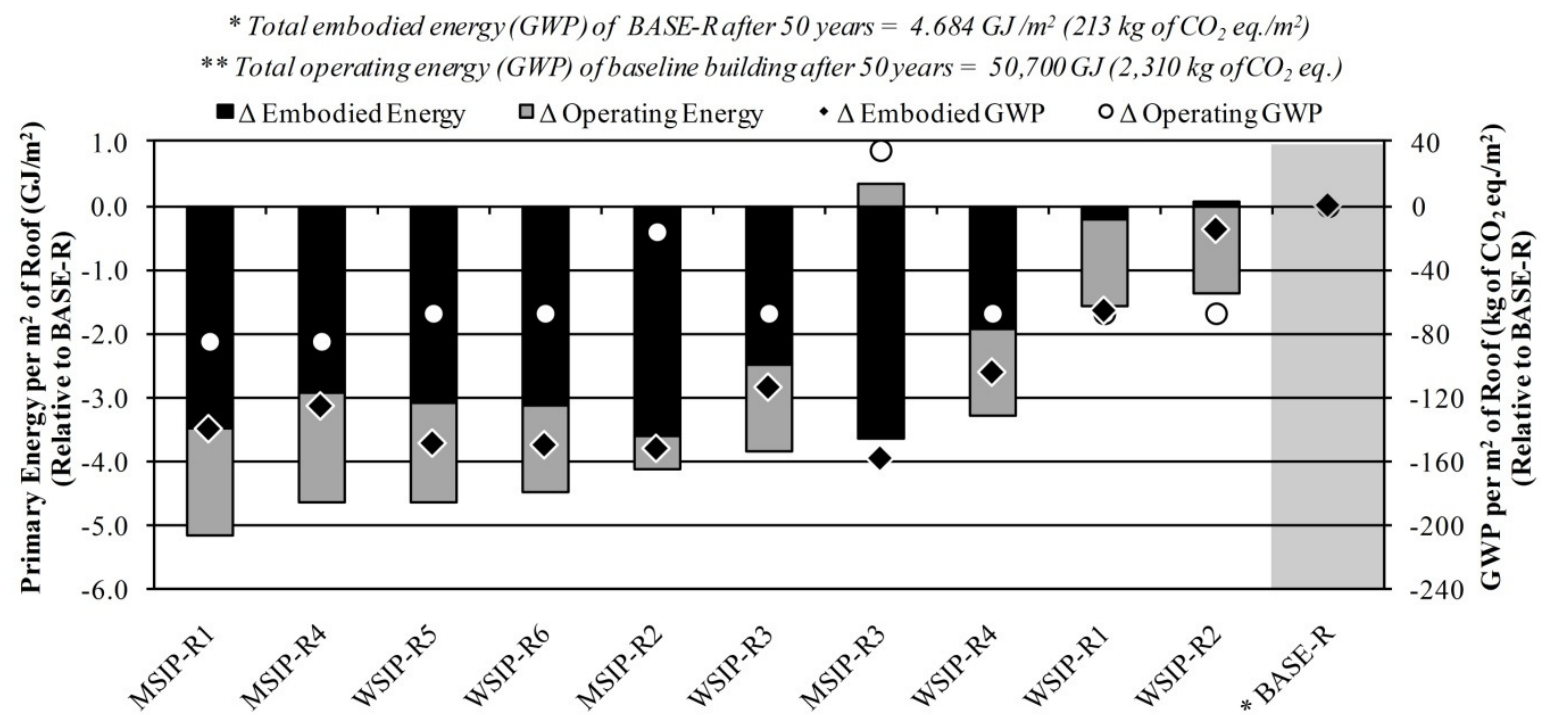

Roof ID (Arranged in Order of Increasing 4 Total Energy)

Note: $\Delta$ Total Energy $=\Delta$ Embodied Energy $+\Delta$ Operating Energy (GWP similar)

Figure 6-11: Total Life-Cycle Energy and GWP of Structural Insulated Panel Roofs (WSIP-R \& MSIP-R) after 50 Year Lifespan in Toronto

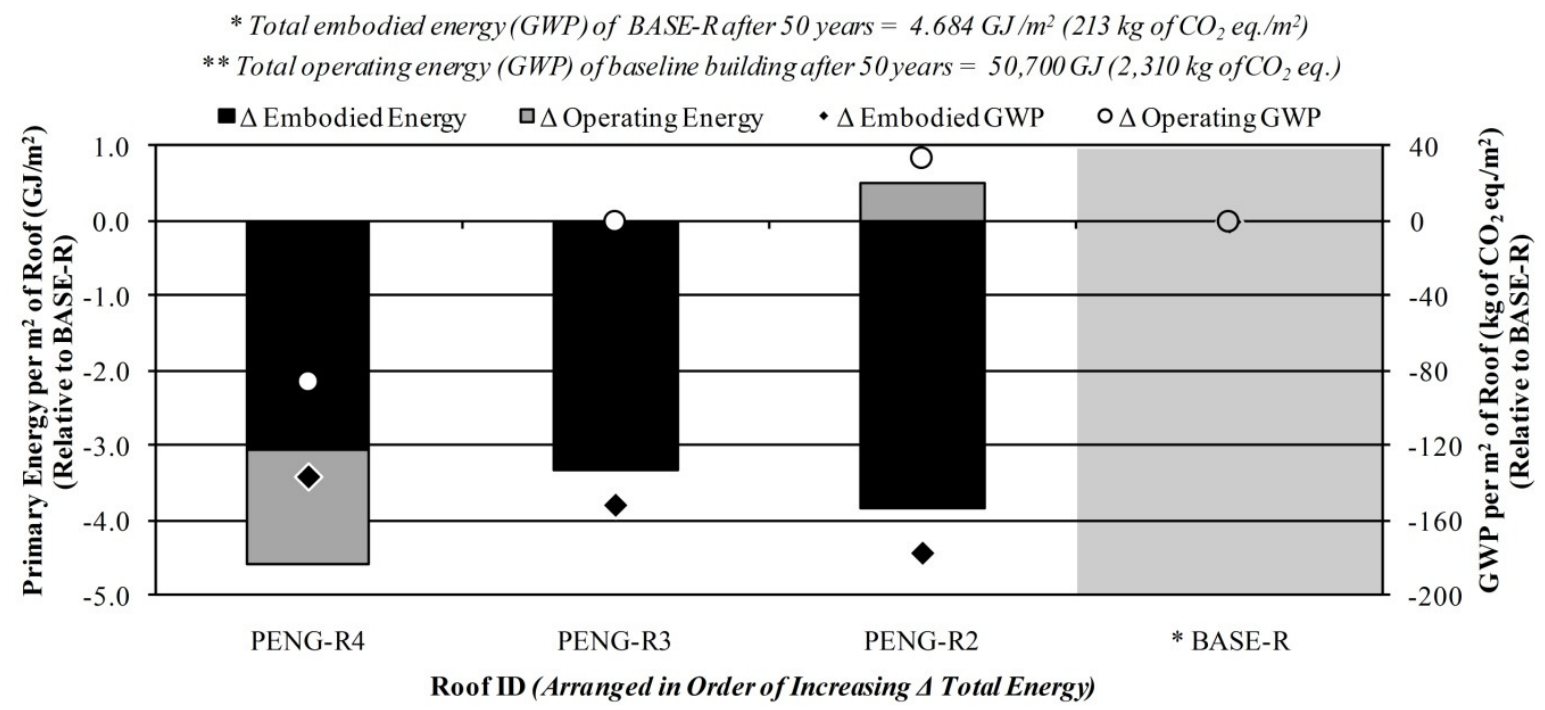

Note: $\Delta$ Total Energy $=\Delta$ Embodied Energy $+\Delta$ Operating Energy (GWP similar)

(PENG-Rl was excluded from graph due to very high operating energy \& operating GWP)

Figure 6-12: Total Life-Cycle Energy and GWP of Pre-Engineered Steel Building Roofs (PENG-R) after 50 Year Lifespan in Toronto 
Table 6-1 also illustrates the range of possible values for the $\Delta$ total energy and $\Delta$ total GWP of the different types of roof enclosures in this study. Similar to the case of the exterior infill walls, the minimum and maximum values for the $\Delta$ total energy and $\Delta$ total GWP of each roof have been included for comparison.

Based on the results of this study, some general conclusions can be made about the various types of roof enclosures that were examined. First, there is a stronger correlation between the embodied energy and embodied GWP of the various roofs and the $\Delta$ total energy and $\Delta$ total GWP than was the case for the exterior infill walls. In fact, in many instances the embodied effects of the various roof enclosures outweighed the operating effects. This result suggests that for the roof enclosures, the building materials have a more significant affect on the $\Delta$ total energy and $\Delta$ total GWP than was observed for the exterior infill walls. That is not to say that the operating energy and operating GWP of the roof enclosures did not have an impact on the $\Delta$ total energy and $\Delta$ total GWP. In fact, an increase in the total energy and total GWP of the roofs corresponded in general to a decrease in the thermal resistance of the roof enclosures. However, this relationship was less marked than for the case of the exterior infill walls.

Another very important observation to note has to do with the roof covering. The results of this study suggest that significant savings can be achieved in terms of the total energy and total GWP if a green roof or a steel roof covering is used as opposed to an asphalt-based roof covering. This presumably has to do with the fact that green roofs have significantly less embodied effects compared to asphaltbased roof coverings and that steel roof systems tend to be very durable systems that need to be repaired or replaced less often.

Two of the most promising roof types that were looked at in this study were the WSIP-R and MSIP-R roofs. Structural insulated panel roofs tend to have a higher thermal resistance than other conventional types of roofs and they also tend to have similar or less embodied effects. Therefore, the WSIP-R and MSIP-R roofs performed very well in terms of total energy and total GWP after a 50 year lifespan in this study.

It should be noted that PENG-R roofs also show significant promise as relatively good systems in terms of energy use and GWP. These roofs tend to be significantly lower in embodied effects compared to other conventional roof types. However, one of the biggest drawbacks to conventional pre-engineered steel roofs is their poor thermal resistance due to massive thermal bridging problems. Therefore, if the thermal resistance of these types of enclosures can be improved, then these types of 
roof systems look promising in terms of life-cycle energy use and GWP compared to conventional roof enclosures.

\subsection{LCA Results for Structural Systems}

A comparison of the $\Delta$ total energy and $\Delta$ total GWP for the different structural systems from Appendix B is presented next. Figure 6-13 illustrates the $\Delta$ total energy and $\Delta$ total GWP of the three different structural systems examined in this study.

In this case, the $\Delta$ total energy and $\Delta$ total GWP of the different structural systems were compared relative to S-1 (typical hot-rolled steel structural system). This was the structural system that was used in the baseline retail building. After 50 years, the total embodied energy of S1 was found to be about $0.813 \mathrm{GJ} / \mathrm{m}^{2}$ and the total embodied GWP was about $46 \mathrm{~kg}$ of $\mathrm{CO}_{2}$ eq. $/ \mathrm{m}^{2}$ (results are per $\mathrm{m}^{2}$ of structural system).

It is important to note that the three structural systems only differed in terms of the total embodied energy and total embodied GWP. Changing the structural system did not impact the operating energy and operating GWP of the baseline building at all. Therefore, although S-2 and S-3 have less total energy and total GWP than S-1 after 50 years, in the overall scheme of things these differences are minimal. Recall that negative values in Figure 6-13 denote that those structural systems have less total energy and total GWP than the baseline structural system after 50 years. Therefore, it can be seen in Figure 6-13 that both the typical heavy timber structural system (S-2) and the pre-engineered steel building structural system (S-3) had less total energy and total GWP than the baseline case.

Table 6-1 also indicates the range of values that were calculated for the $\Delta$ total energy and $\Delta$ total GWP of the different structural systems examined in this study. Average, minimum, and maximum values have been calculated for the range of different structural systems. 


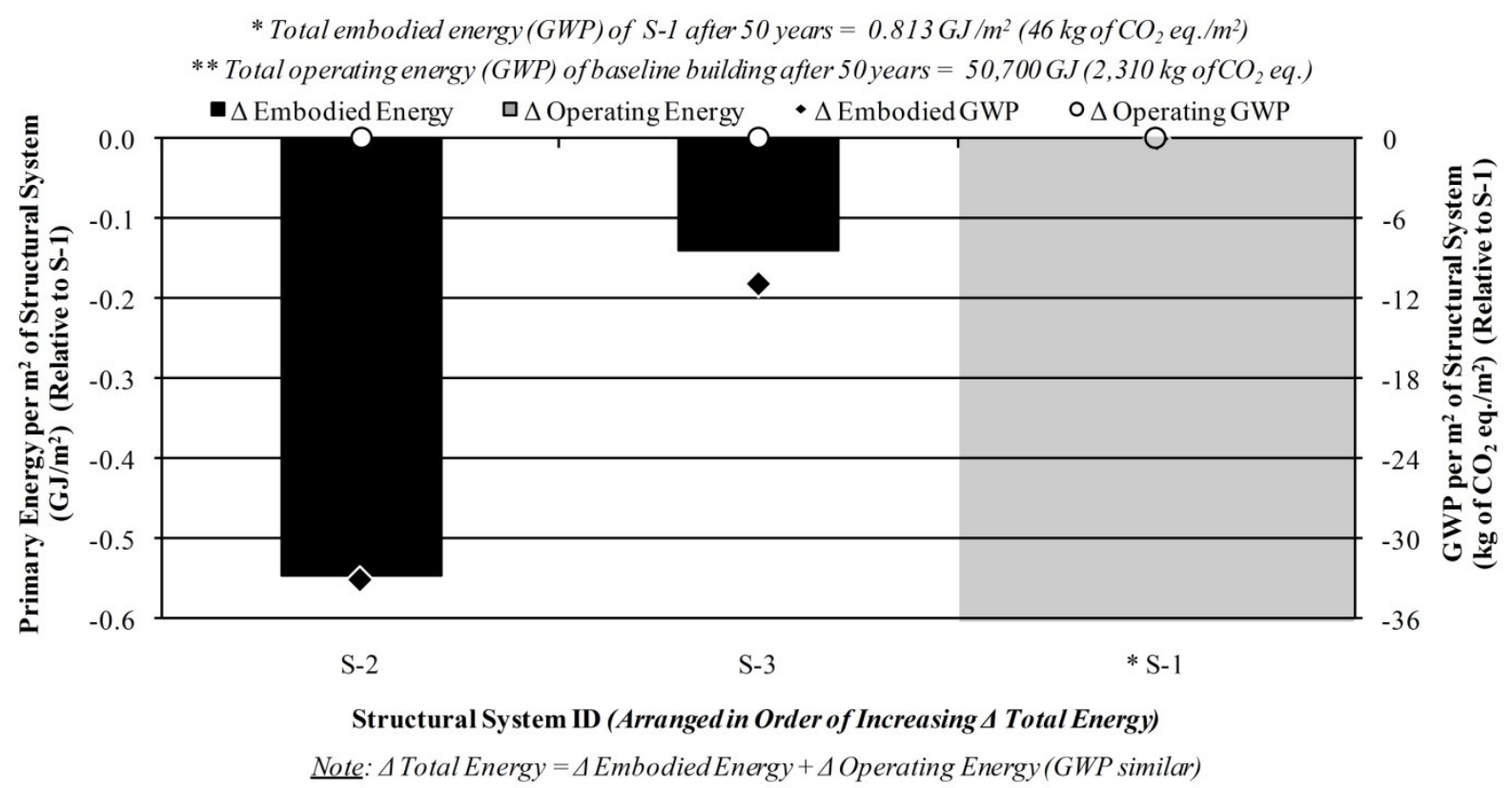

Figure 6-13: Total Life-Cycle Energy and GWP of Structural Systems after 50 Year Lifespan in Toronto

\subsection{LCA Results for Floors}

In this section, the LCA results are compared for the different floor assemblies from Appendix B. Figure 6-14 illustrates the $\Delta$ total energy and $\Delta$ total GWP of the different floor assemblies examined in this study. Table 6-1 also contains the range of values that were calculated for the $\Delta$ total energy and $\Delta$ total GWP of the different floor assemblies.

In this case, the $\Delta$ total energy and $\Delta$ total GWP of the different floor assemblies were compared relative to FL-3. This was the floor assembly that was used in the baseline retail building. FL-3 consists of: vinyl floor tile, $89 \mathrm{~mm}$ reinforced concrete topping, $39 \mathrm{~mm}$ galvanized corrugated steel deck, open web steel joists spaced at 1,200 mm on center, $90 \mathrm{~mm}$ fiberglass batt insulation, galvanized steel resilient channels, two layers of $12 \mathrm{~mm}$ gypsum board, and finished with latex paint. After 50 years, the total embodied energy of FL-3 was found to be about $1.205 \mathrm{GJ} / \mathrm{m}^{2}$ and the total embodied GWP was about $86 \mathrm{~kg}$ of $\mathrm{CO}_{2}$ eq. $/ \mathrm{m}^{2}$ (results are per $\mathrm{m}^{2}$ of floor).

All of the floor assemblies had less total energy and total GWP than FL-3. It is important to note that the floor assemblies only differed in terms of their total embodied energy and total embodied GWP. Changing the floor assembly did not significantly impact the operating energy or operating GWP of 
the baseline building in this case. It is interesting to note that the baseline floor assembly (FL-3) performed the worst of all the floor assemblies that were examined in this study. This is an interesting observation because this floor type is one of the most common types of floor systems used in commercial buildings today.

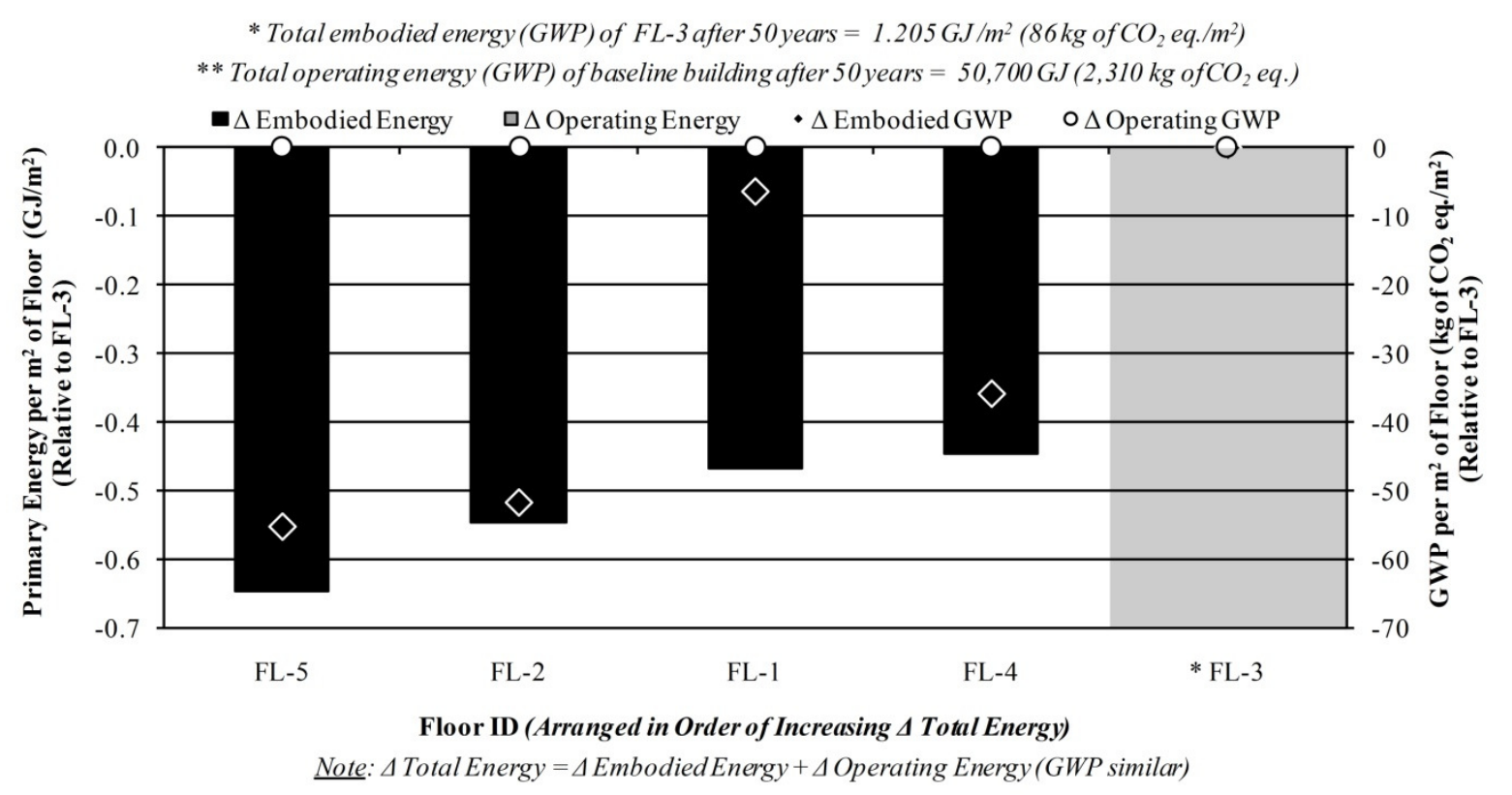

Figure 6-14: Total Life-Cycle Energy and GWP of Floors (FL) after 50 Year Lifespan in Toronto

\subsection{LCA Results for Windows and Doors}

A detailed LCA was conduced for the different windows and doors in Appendix B. The results of this LCA are displayed in Figure 6-15 and Figure 6-16. As well, Table 6-1 contains the range of values that were calculated for the $\Delta$ total energy and $\Delta$ total GWP of the different windows and doors.

In this case, the $\Delta$ total energy and $\Delta$ total GWP of the different windows were compared relative to $\mathrm{W}-1$. W-1 consists of a thermally broken aluminum window frame, with a sealed double pane glazing unit filled with air (no low-E coating and no argon gas). After 50 years, the total embodied energy of $\mathrm{W}-1$ was found to be about $8.657 \mathrm{GJ} / \mathrm{m}^{2}$ and the total embodied GWP was about $537 \mathrm{~kg}$ of $\mathrm{CO}_{2}$ eq. $/ \mathrm{m}^{2}$ (results are per $\mathrm{m}^{2}$ of window).

Figure 6-15 illustrates that the windows with high performance glazing (argon filled, low-E coating) outperformed the corresponding windows with typical glazing (air filled, no low-E coating). This is 
because the high performance glazing resulted in windows with a higher thermal resistance. The results found a strong correlation between the thermal resistance of the windows and the $\Delta$ total energy and $\Delta$ total GWP. In fact, it was found that an increase in total energy and total GWP corresponded to a decrease in the thermal resistance of the windows.

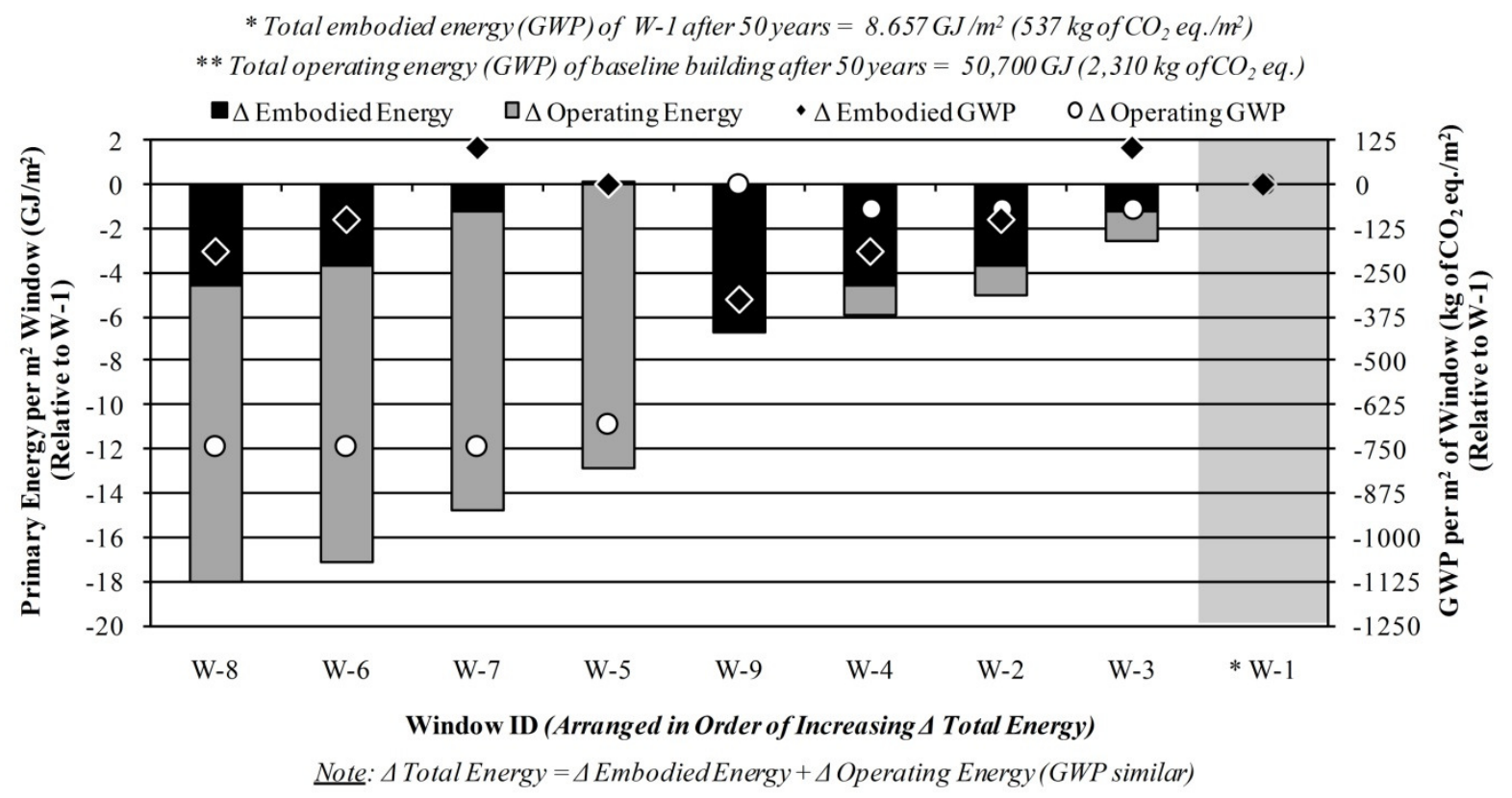

\section{Figure 6-15: Total Life-Cycle Energy and GWP of Windows (W) after 50 Year Lifespan in Toronto}

These results show that per $\mathrm{m}^{2}$ of enclosure, windows actually have a relatively large total energy and total GWP compared to the exterior infill walls and roofs. In fact, both the embodied effects and the operating effects of the windows are relatively high. This suggests that when designing a building, the area of windows should be optimized from both a daylighting perspective, but also from an embodied energy and embodied GWP perspective. Having a very large window-to-wall ratio (say $>50 \%$ ) could not only increase the operating requirements of the building, but also increase the embodied energy and embodied GWP of the building significantly. It is interesting to note that many of the retail buildings and office buildings in North America are made almost entirely of glass/aluminum curtainwall. The results from this study suggest that significant energy and GWP savings could result from a better balance of glass curtainwall with opaque enclosures in commercial buildings. 
Figure 6-16 illustrates the $\Delta$ total energy and $\Delta$ total GWP for the doors in Appendix B. In this case, the values were plotted relative to D-2, which is an $813 \times 2,134 \mathrm{~mm}$ insulated steel exterior door. After 50 years, the total embodied energy of D-2 was found to be about $8.335 \mathrm{GJ} /$ door and the total embodied GWP was about $290 \mathrm{~kg}$ of $\mathrm{CO}_{2}$ eq./door.

D-5 and D-6 are interior doors and were found to have no significant impact on the operating energy or operating GWP of the building. D-4 is an insulated sectional steel overhead door and has a significantly higher embodied energy and embodied GWP than the other doors. It can be seen from Figure 6-16 that D-1 (solid wood exterior door with no glazing) had a significant savings in terms of operating energy and operating GWP. After 50 years, D-1 resulted in a $\Delta$ total energy of about -108 $\mathrm{GJ} /$ door and a $\Delta$ total GWP of about $-273 \mathrm{~kg}$ of $\mathrm{CO}_{2}$ eq./door. The significance of the energy and GWP savings for D-1 was surprising. To date, very little research can be found in the literature on the primary energy and GWP of different types of doors. The results from this study suggest that further research should be conducted in this respect. Only three exterior doors were considered in this study (D-1, D-2, and D-3) and a larger sample of doors should be considered.

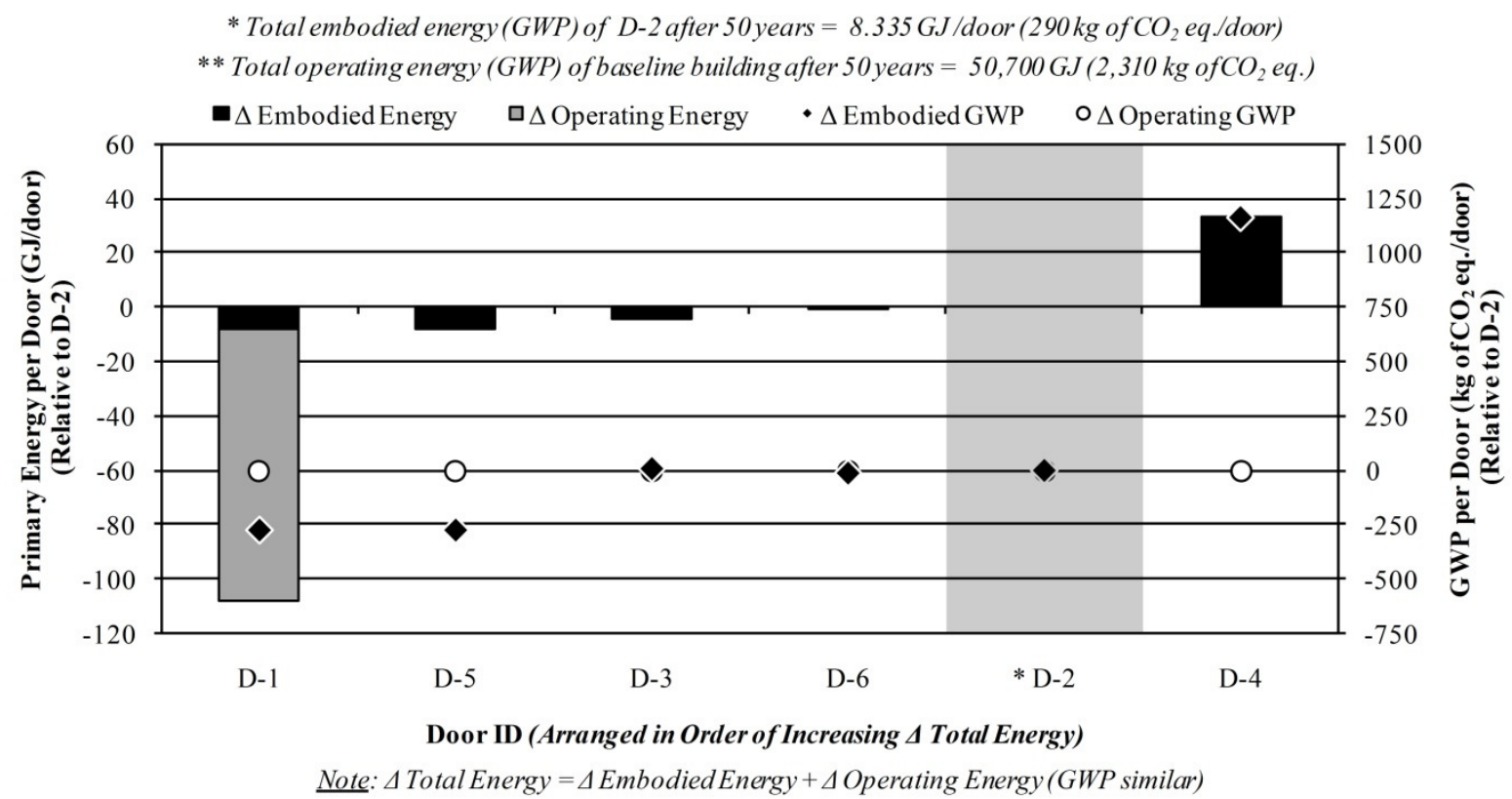

Figure 6-16: Total Life-Cycle Energy and GWP of Doors (D) after 50 Year Lifespan in Toronto 


\subsection{LCA Results for Interior Partitions}

The range of interior partition walls examined in this study is outlined in Appendix B. Table 6-1 displays the range of values that were calculated for the $\Delta$ total energy and $\Delta$ total GWP and Figure 6-17 illustrates the results for the different interior partitions.

The $\Delta$ total energy and $\Delta$ total GWP of the different interior partitions were compared relative to SSP3. From one side to the next, SS-P3 consists of: latex paint, two layers of regular $12 \mathrm{~mm}$ gypsum board, 39 x $152 \mathrm{~mm}$ cold-formed steel studs spaced at $400 \mathrm{~mm}$ on center, $140 \mathrm{~mm}$ fiberglass batt insulation, two layers of regular $12 \mathrm{~mm}$ gypsum board, and latex paint. After 50 years, the total embodied energy of SS-P3 was found to be about $0.523 \mathrm{GJ} / \mathrm{m}^{2}$ and the total embodied GWP was about $30 \mathrm{~kg}$ of $\mathrm{CO}_{2}$ eq. $/ \mathrm{m}^{2}$ (results are per $\mathrm{m}^{2}$ of wall).

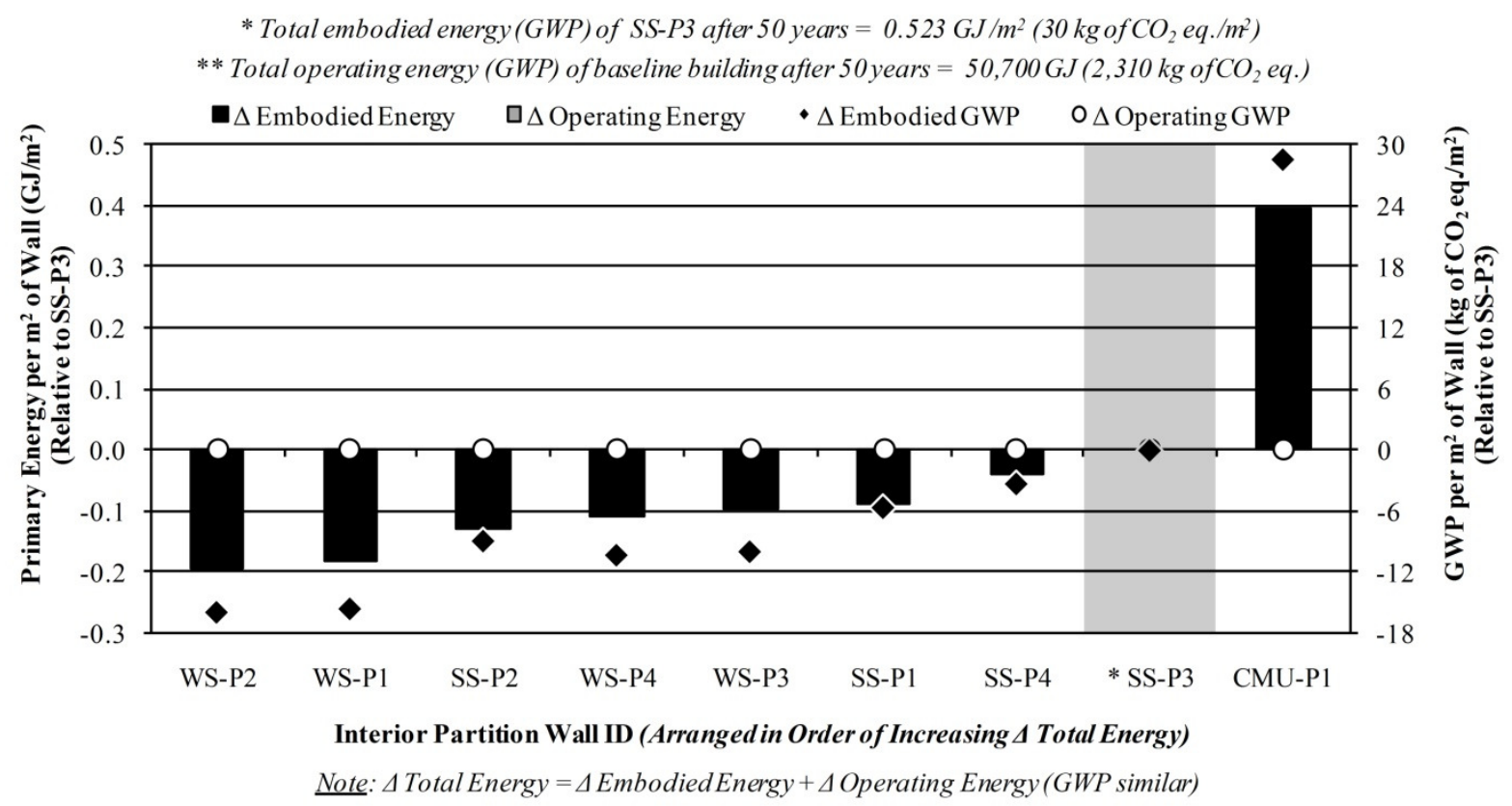

\section{Figure 6-17: Total Life-Cycle Energy and GWP of Interior Partition Walls (WS-P, SS-P, \& CMU-P) after 50 Year Lifespan in Toronto}

In every case, the interior partitions did not have an affect on the operating energy or operating GWP of the building. Therefore, the differences between the various partition walls are solely a result of the differences in embodied effects. Generally speaking, the wood stud partition walls performed slightly better than the cold-formed steel stud equivalent. However, the concrete masonry unit partition wall 
had a significantly higher total energy and total GWP than either the wood stud or cold-formed steel stud partition walls.

\subsection{LCA Results for Foundations}

Lastly, in this section the LCA results are compared for the different foundations from Appendix B. Figure 6-18 illustrates the $\Delta$ total energy and $\Delta$ total GWP of the different isolated footings and concrete pier foundations (IF-FDN) examined in this study. Table 6-1 displays the range of values that were calculated for the $\Delta$ total energy and $\Delta$ total GWP for the different foundation components.

In this instance, the $\Delta$ total energy and $\Delta$ total GWP of the different IF-FDN's were compared relative to IF-FDN1. IF-FDN1 is a $1,200 \times 1,200 \times 350 \mathrm{~mm}$ isolated concrete footing with a $450 \times 450 \times$ $1,200 \mathrm{~mm}$ concrete pier (concrete is $20 \mathrm{MPa}$ with $9 \%$ flyash content). After 50 years, the total embodied energy of IF-FDN1 was found to be about $1.609 \mathrm{GJ} /$ unit and the total embodied GWP was about $175 \mathrm{~kg}$ of $\mathrm{CO}_{2}$ eq./unit (results are per footing and pier combination).

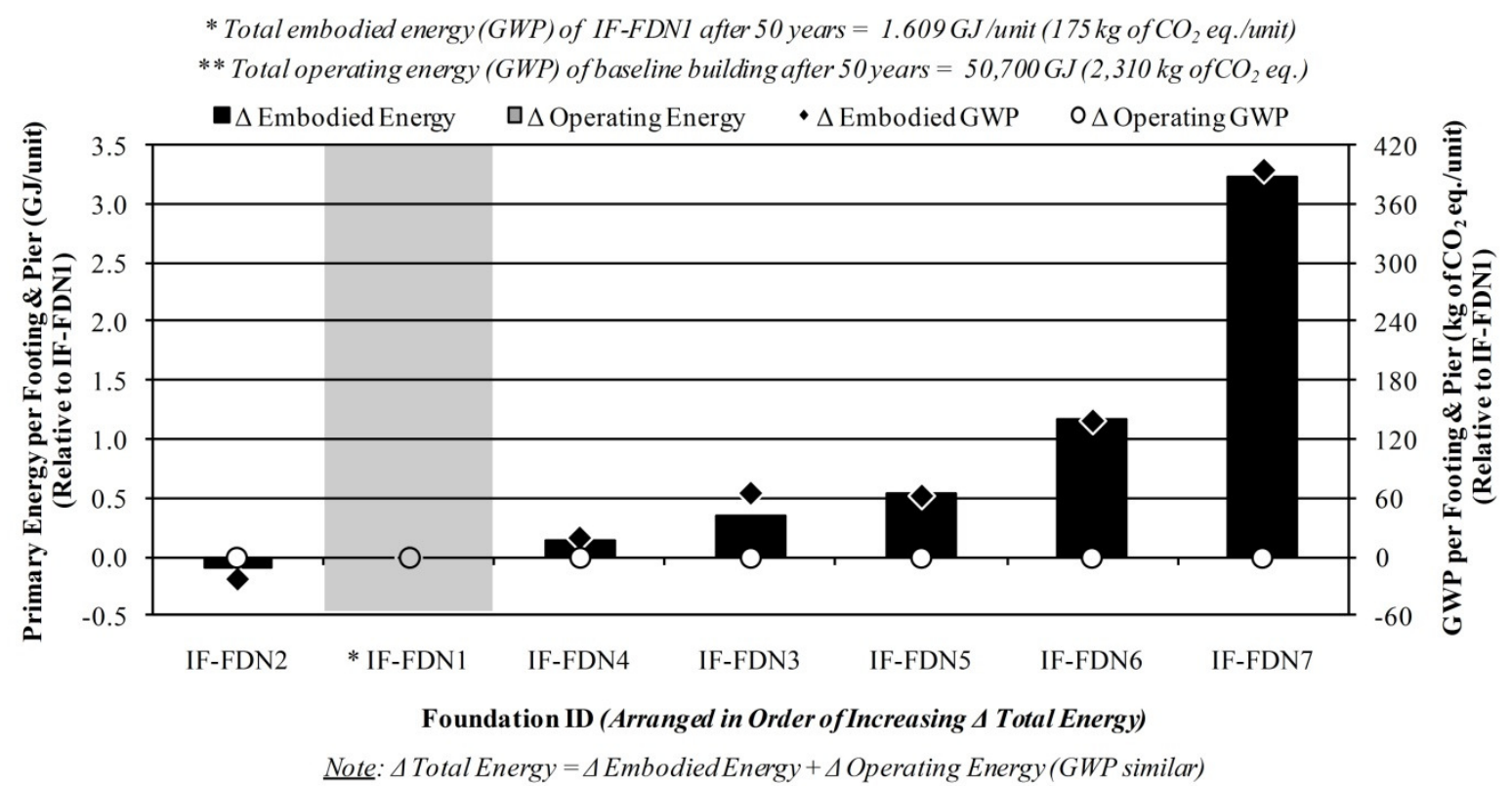

Figure 6-18: Total Life-Cycle Energy and GWP of Isolated Footing and Concrete Pier
Foundations (IF-FDN) after 50 Year Lifespan in Toronto

Since the different IF-FDN's did not have any affect on the operating energy or operating GWP of the building, the differences in Figure 6-18 are a result of embodied effects alone. The variable that had 
the most significant affect on the $\Delta$ total energy and $\Delta$ total GWP of the IF-FDN's in this study was the size of the footing and pier. The larger footings and piers required more concrete and reinforcement, which resulted in a higher total energy and total GWP. Generally speaking, the use of higher percentages of flyash resulted in a lower total energy and total GWP.

Figure 6-19 illustrates the $\Delta$ total energy and $\Delta$ total GWP of the different strip footings and concrete foundations walls (SF-FDN) examined in this study. The values in this case are plotted relative to SFFDN5, which consists of a $600 \times 200 \mathrm{~mm}$ concrete strip footing with a 1,200 x $200 \mathrm{~mm}$ concrete foundation wall with waterproofing (concrete is $20 \mathrm{MPa}$ with $9 \%$ flyash content). After 50 years, the total embodied energy of SF-FDN5 was found to be about $1.097 \mathrm{GJ} / \mathrm{m}$ and the total embodied GWP was about $94 \mathrm{~kg}$ of $\mathrm{CO}_{2}$ eq./m (results are per linear m of footing and wall).

Once again, the different SF-FDN's did not have any affect on the operating energy or operating GWP of the building. Therefore, the differences observed in Figure 6-19 are solely based on differences in the embodied effects. The larger foundation components tended to result in a higher total energy and total GWP due to more concrete and reinforcement being required. The use of higher percentages of flyash was once again found to slightly decrease the total energy and total GWP.

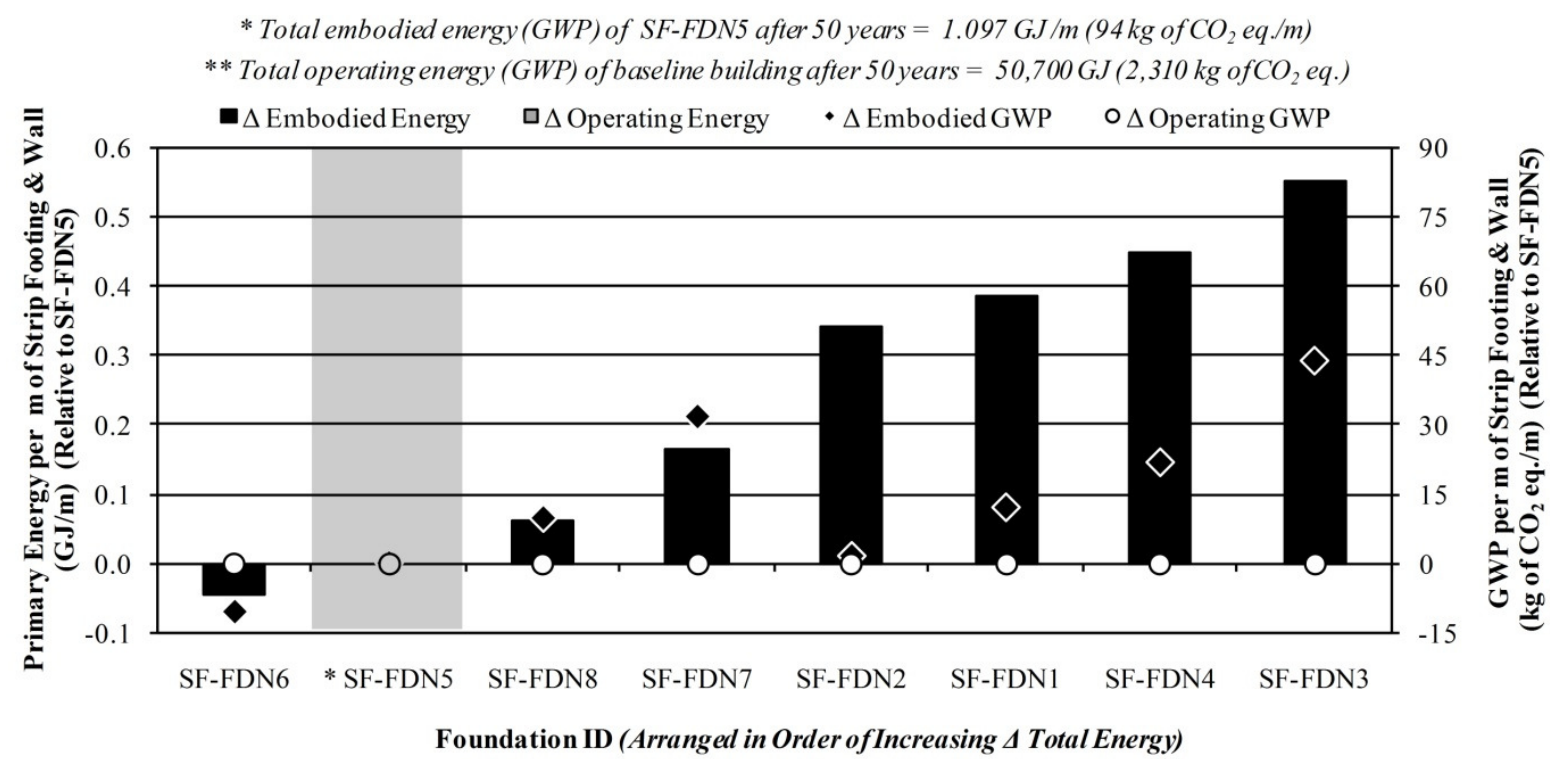

Note: $\Delta$ Total Energy $=\Delta$ Embodied Energy $+\Delta$ Operating Energy (GWP similar)

Figure 6-19: Total Life-Cycle Energy and GWP of Strip Footing and Concrete Wall Foundations (SF-FDN) after 50 Year Lifespan in Toronto 
Lastly, Figure 6-20 illustrates the $\Delta$ total energy and $\Delta$ total GWP of the different concrete slab-ongrades (SOG-FDN) examined in this study. The values in this case are plotted relative to SOG-FDN4. SOG-FDN4 is a $200 \mathrm{~mm}$ thick, reinforced concrete slab, on a poly vapour barrier, and with a concrete sealant finish (concrete is $30 \mathrm{MPa}$ with $9 \%$ flyash content). After 50 years, the total embodied energy of SOG-FDN4 was found to be about $0.515 \mathrm{GJ} / \mathrm{m}^{2}$ and the total embodied GWP was about $65 \mathrm{~kg}$ of $\mathrm{CO}_{2}$ eq. $/ \mathrm{m}^{2}$ (results are per $\mathrm{m}^{2}$ of slab-on-grade).

The best performing slab-on-grades were found to be the ones where under slab insulation was specified. This was because significant savings in operating effects were achieved. Also, in cases where a thinner slab-on-grade was specified, there was a slight savings in embodied effects.

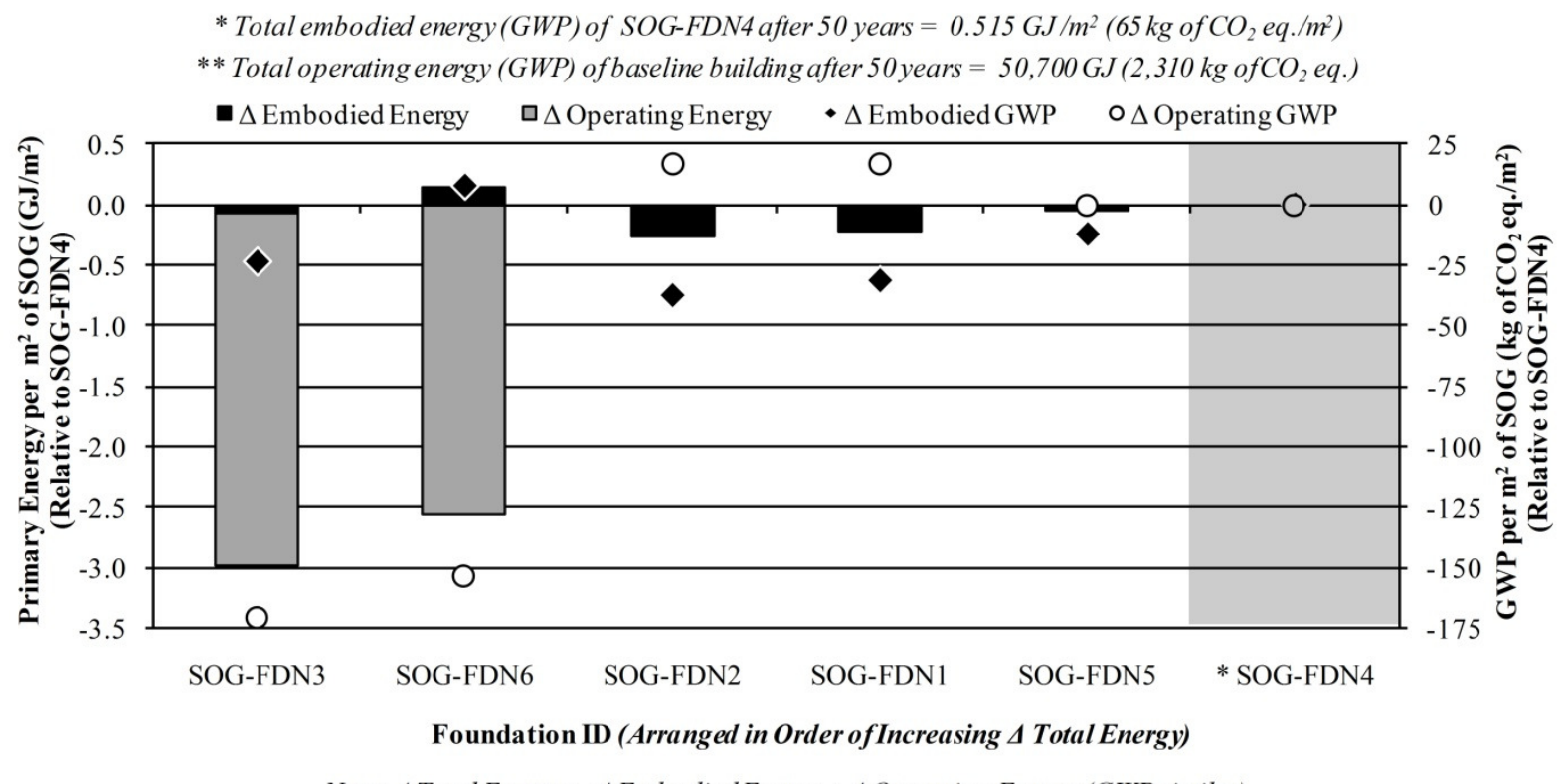

Note: $\Delta$ Total Energy $=\Delta$ Embodied Energy $+\Delta$ Operating Energy (GWP similar)

\section{Figure 6-20: Total Life-Cycle Energy and GWP of Concrete Slab-On-Grades (SOG-FDN) after 50 Year Lifespan in Toronto}

The goal of this chapter was to present the simplified LCA results for the range of different building components that were examined in this study. These buildings components have been ranked in order of increasing total energy, so that a building professional can quickly compare one system to the next from a life-cycle energy and GWP perspective. By summarizing the comprehensive LCA results in this way, a building professional is able to quickly make LCA decisions that otherwise would be extremely exhaustive both in time and effort. 


\subsection{Data Quality and Assessment}

Now that the LCA results have been presented for the 220 different building components in this study, it is useful to compare the data to the values for similar components in the literature. Unfortunately, this task is extremely difficult. There are an endless number of unique building components that can be generated and so, the LCA data tends to differ from one study to the next. Therefore, it is difficult to directly compare the LCA results for the 220 building components in this study to the literature. However, the ATHENA® Institute has published a free program on their website called the ATHENA® EcoCalculator for Assemblies (The ATHENA Institute, 2010). It lists the embodied energy and embodied GWP for 400 common building components. The results do not include operating effects and were generated for a low-rise office building with a 60 year lifespan, but will be used for comparison in this study. Table 6-2 indicates that the embodied LCA data in this study is relatively close to the range of values listed in the ATHENA® EcoCalcualtor for Assemblies.

Table 6-2: Comparison of Embodied LCA Data in this Study to ATHENA® EcoCalculator

\begin{tabular}{|c|c|c|c|c|}
\hline \multirow[b]{2}{*}{ Building Component } & \multicolumn{2}{|c|}{ ATHENA® EcoCalculator } & \multicolumn{2}{|c|}{ This Study } \\
\hline & $\begin{array}{c}\text { Primary Energy } \\
\left(\mathrm{MJ} / \mathrm{m}^{2}\right)\end{array}$ & $\begin{array}{c}\mathrm{GWP} \\
\left(\mathrm{kg} \text { of } \mathrm{CO}_{2} \text { eq. } / \mathrm{m}^{2}\right) \\
\end{array}$ & $\begin{array}{c}\text { Primary Energy } \\
\left(\mathrm{MJ} / \mathrm{m}^{2}\right)\end{array}$ & $\begin{array}{c}\mathrm{GWP} \\
\left(\mathrm{kg} \text { of } \mathrm{CO}_{2} \text { eq. } / \mathrm{m}^{2}\right)\end{array}$ \\
\hline Exterior Infill Walls & $599-2,659$ & $20-212$ & $491-3,172$ & $23-193$ \\
\hline Concrete Masonry Unit Walls & $1,228-2,659$ & $72-212$ & $1,365-3,172$ & $75-193$ \\
\hline Concrete Tilt-Up Walls & $983-2,414$ & $68-208$ & $542-1,677$ & $52-137$ \\
\hline Wood Structural Insulated Panel Walls & $1,224-2,401$ & $34-169$ & $1,037-2,820$ & $44-160$ \\
\hline Metal Structural Insulated Panel Walls & N/A & N/A & $999-1,180$ & $53-71$ \\
\hline Cold-Formed Steel Stud Walls & $599-1,945$ & $28-172$ & $680-2,631$ & $33-156$ \\
\hline Wood Stud Walls & $602-1,851$ & $20-156$ & $570-2,513$ & $23-145$ \\
\hline Pre-Engineered Steel Building Walls & N/A & N/A & $491-1,610$ & $23-74$ \\
\hline Opaque Curtainwalls & $1,156-1,876$ & $46-133$ & $1,300-1,590$ & $49-122$ \\
\hline Roofs & $1,399-9,050$ & $50-306$ & $738-5,184$ & $29-263$ \\
\hline Concrete Hollow Core Roofs & N/A & N/A & $1,477-5,184$ & $90-263$ \\
\hline Open Web Steel Joist Roofs & $1,449-9,050$ & $53-306$ & $1,516-4,866$ & $77-232$ \\
\hline Cold-Formed Steel Joist Roofs & N/A & N/A & $1,252-4,615$ & $57-214$ \\
\hline Glulam Joist Roofs & $1,399-8,850$ & $50-287$ & $1,201-4,551$ & $46-205$ \\
\hline Wood Structural Insulated Panel Roofs & N/A & N/A & $1,564-4,735$ & $63-198$ \\
\hline Metal Structural Insulated Panel Roofs & N/A & N/A & $1,029-1,736$ & $55-88$ \\
\hline Pre-Engineered Steel Building Roofs & N/A & N/A & $738-1,639$ & $29-76$ \\
\hline Structural Systems & $114-1,260$ & $4-68$ & $266-813$ & $13-46$ \\
\hline Floors & $370-1,390$ & $10-106$ & $559-1,205$ & $30-86$ \\
\hline Windows & $2,764-6,521$ & $213-356$ & $1,895-8,661$ & $209-642$ \\
\hline Interior Partitions & $314-1,078$ & $10-60$ & $327-917$ & $14-59$ \\
\hline
\end{tabular}

* Note: Doors and foundations have been omitted from table due to lack of comparison data 


\section{Chapter 7 \\ Summary of Results}

\subsection{Introduction}

In Chapter 5, the LCA results were presented for five single-storey retail buildings with a 50 year lifespan in Toronto. It was shown that after 50 years, operating energy and operating GWP accounts for about $90 \%$ of the total energy and total GWP, while embodied energy and embodied GWP is only responsible for about $10 \%$. It was also determined that among the five buildings, there was very little difference in the total life-cycle energy use and total GWP after 50 years.

Next, focus was shifted from an analysis of whole buildings to a comparison of individual building components. In Chapter 6, 220 different building components including: exterior infill walls, roofs, structural systems, floors, windows and doors, interior partitions, and foundations were analyzed within the framework of a comprehensive LCA of energy use and GWP. Within each building component category, numerous alternative strategies were analyzed. The goal was to determine the range of possible values for the life-cycle energy and GWP of the different building components.

In this chapter, the data from Chapters 5 and 6 will be summarized into a series of useful measures to help reinforce the key findings. In essence, the data summary in this chapter will serve as an overview of the more detailed analysis that has been conducted in previous chapters. The goal here is to enable the reader to quickly grasp the most important concepts of this study through the use of a few key figures and tables. The ultimate objective is to provide a clear and simple summary of an otherwise incredibly complex and time consuming LCA study. In doing so, the final step of the LCA process according to ISO 14044 will be completed: an interpretation of the LCA results (ISO, 2006).

\subsection{Sensitivity Analysis of Total Embodied Effects verses Operating Effects}

In Chapter 6, the results from a detailed LCA of 220 different building components were presented. These results are very useful if one has several specific building components in mind that one wishes to compare. However, to get a better understanding of the greater picture, it is useful to plot the results for all of the 220 different building components on one graph.

Figure 7-1 illustrates the results of a sensitivity analysis of the total embodied energy for the components of the baseline retail building after 50 years. In this figure, the various building components of the baseline retail building were systematically replaced with each alternative building 
component in this study, such that a new estimate of the total embodied energy of the building could be determined. In this way, an approximation of the total embodied energy for each of the 220 different building components was calculated, appropriately scaled for the baseline retail building as a whole. In other words, the total embodied energy of each alternative building component has been multiplied (i.e. weighted) by the appropriate material quantities in order to take into consideration scale effects for the entire building. In doing so, some interesting observations can be made about the range of possible values for the weighted embodied energy of the building components.

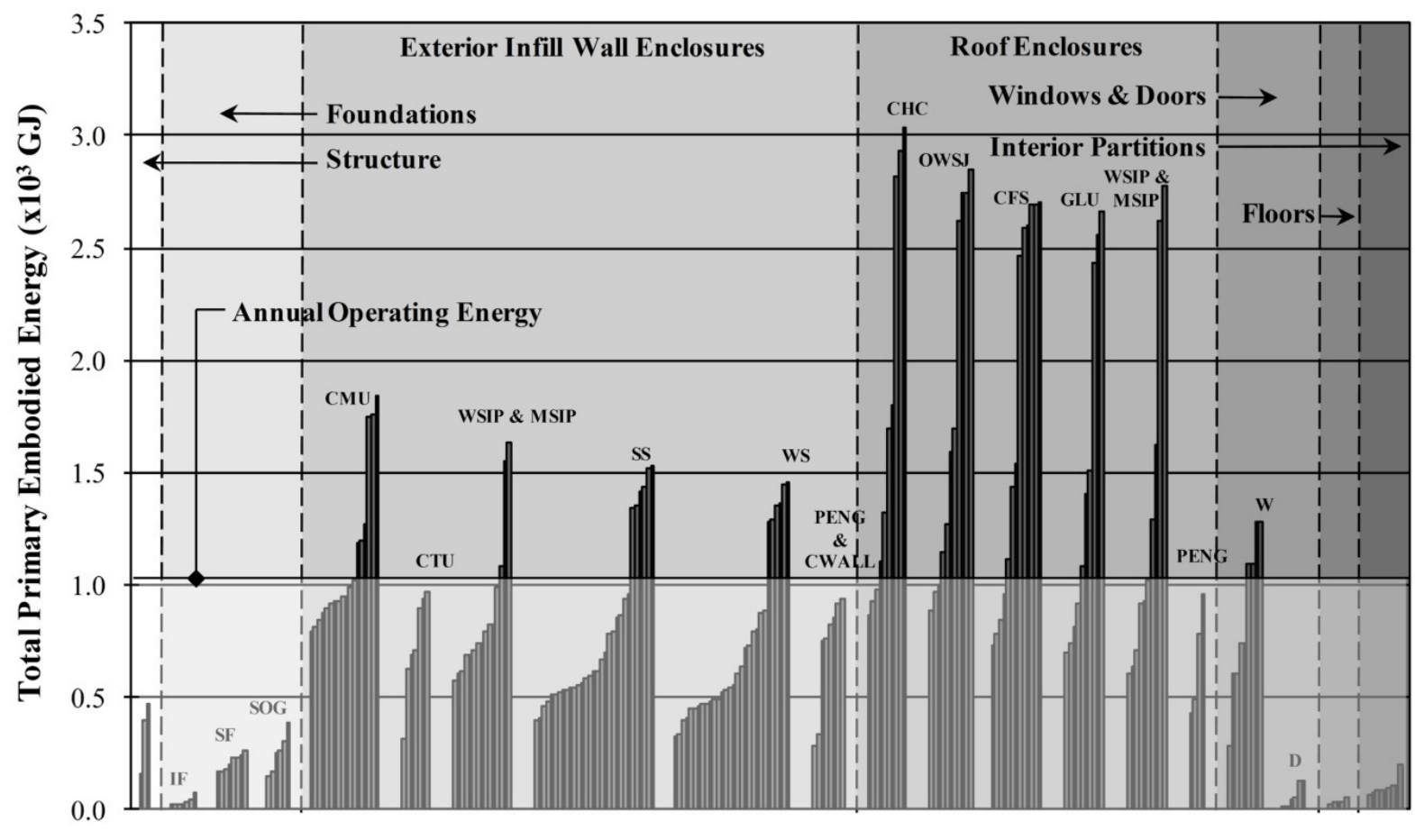

\section{Building Components}

Note: Values were generated using the baseline retail building (located in Toronto with a 50 year lifespan) and the range of building components identified in Appendix $B$

\section{Figure 7-1: A Sensitivity Analysis of the Total Embodied Energy Use for the Components of a Typical Retail Building after a 50 Year Lifespan in Toronto}

From Figure 7-1, it can be seen that within each building component category (structure, foundations, exterior walls, roofs, windows and doors, floors, and interior partitions) the various different alternatives from Appendix B have been grouped together. For example, for the exterior walls the different alternatives are grouped into CMU, CTU, WSIP \& MSIP, SS, WS, PENG, and CWALL type walls. Also, the annual operating energy of the baseline retail building has been plotted for 
comparison. This allows one to quickly approximate the number of equivalent years of operating energy that is associated with the total embodied energy of the different building components.

Quickly one can begin to see how little the total embodied energy of the structural system, foundations, doors, interior partitions, and floors matters compared to the total embodied energy of the exterior walls, roofs, and windows. It also becomes clear how much variation is possible in the total embodied energy of different alternatives within the same category of building component. For example, depending on which OWSJ one chooses, the total embodied energy can vary from as little as 0.9 years of equivalent operating energy to 2.9 years.

The multitude of conclusions and comparisons that could be drawn from this figure are too numerous to explain in detail here. The real usefulness of this figure is its ability to show the relative importance of the different building components and the range of possible values for the total embodied energy within each category.

In a similar way, Figure 7-2 illustrates the results from a sensitivity analysis of the total embodied GWP for the components of the baseline retail building after 50 years. Again, an approximation of the total embodied GWP for each of the 220 different building components was calculated for the case of the baseline retail building, in the same way that the total embodied energy was calculated (i.e. multiplied by the appropriate material quantities in order to take into consideration scale effects for the entire building). Once more, the annual operating GWP was also plotted for comparison.

Similar to the case of total embodied energy, there is a range of possible values for the total embodied GWP within each building component category. It is apparent in this case as well, that the total embodied GWP of the structural system, foundations, doors, interior partitions, and floors is insignificant compared to the total embodied GWP of the exterior walls, roofs, and windows. This figure is able to show the relative importance of the different building components and the range of possible values for the total embodied GWP within each category.

Now, it has been shown throughout this study that operating energy and operating GWP are a much greater concern than embodied energy and embodied GWP in a typical retail building today. Although a comparison of the total embodied energy and total embodied GWP are useful, it does not tell the whole story. For example, it has been shown that some building components come with an increase in total embodied energy and total embodied GWP, but result in an even greater decrease in operating energy and operating GWP after 50 years. To account for this, the $\Delta$ total life-cycle energy 
for each of the 220 different building components was plotted in Figure 7-3. In this case, the baseline retail building was used as the datum. Each of the building components of the baseline retail building was systematically replaced with the building components in Appendix B and the difference in embodied energy ( $\Delta$ embodied energy) and operating energy ( $\Delta$ operating energy) was calculated. Recall that $\Delta$ total energy is equal to $\Delta$ embodied energy plus $\Delta$ operating energy.

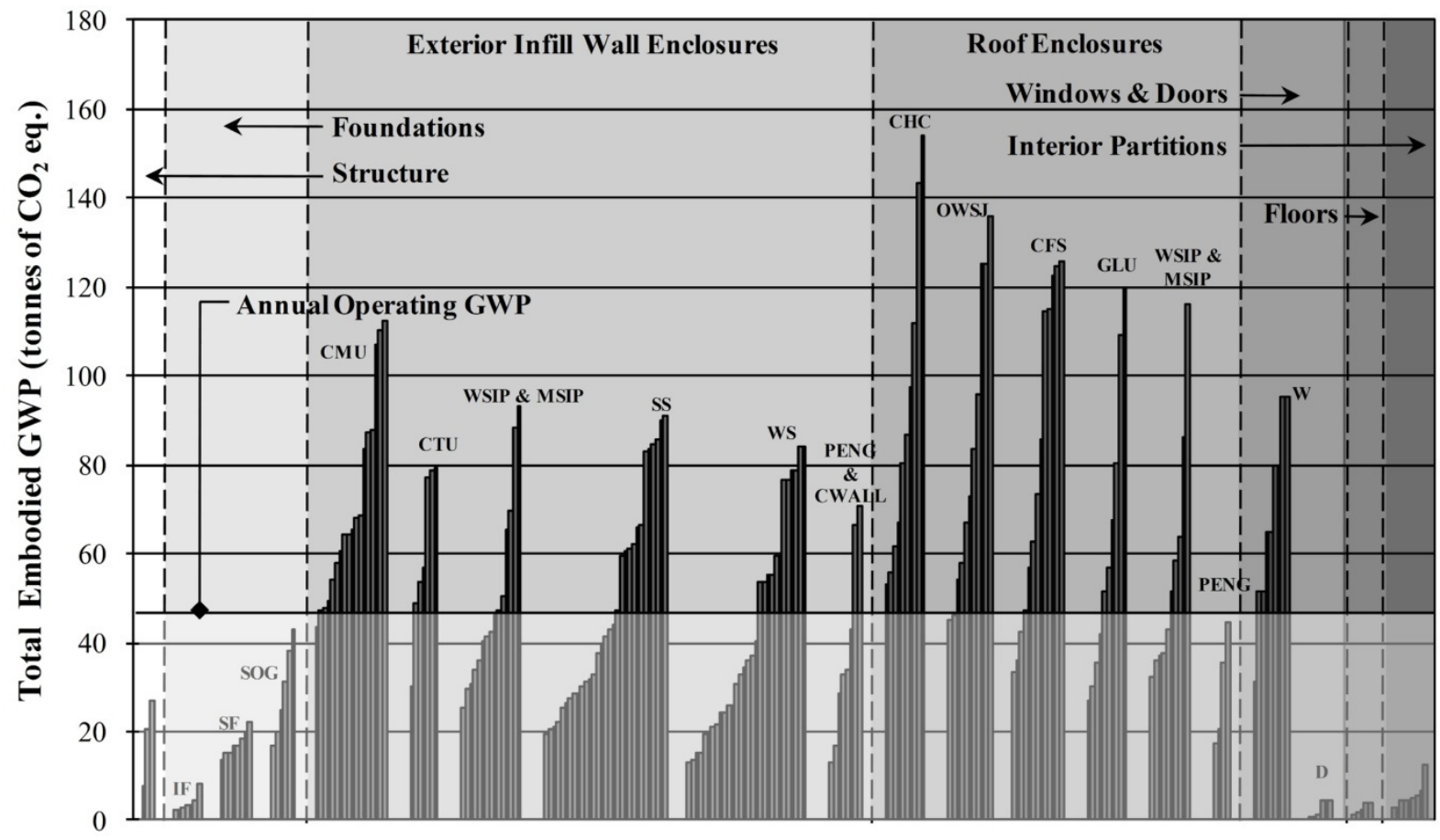

Building Components

Note: Values were generated using the baseline retail building (located in Toronto with a 50 year lifespan) and the range of building components identified in Appendix $B$

\section{Figure 7-2: A Sensitivity Analysis of the Total Embodied GWP for the Components of a Typical Retail Building after a 50 Year Lifespan in Toronto}

Figure 7-3 is divided into the various different building component categories in much the same way as the previous two figures. Also, the annual operating energy for the baseline retail building has also been plotted. One will notice that the vertical axis of the graph has both positive and negative values. In some cases, certain substitutions of building components resulted in an increase in the total energy from the baseline case. In these cases, the values are plotted above the horizontal axis. The horizontal axis represents the baseline retail building (since the $\Delta$ total energy compared to the baseline retail 
building is 0). In other circumstances, certain substitutions of building components resulted in a decrease in the total energy from the baseline case and are plotted below the horizontal axis. Similar to before, the $\Delta$ total energy values have been scaled accordingly for the baseline retail building and represent total values for the building (i.e. they account for the appropriate quantities and numbers of the various building components in the baseline retail building).

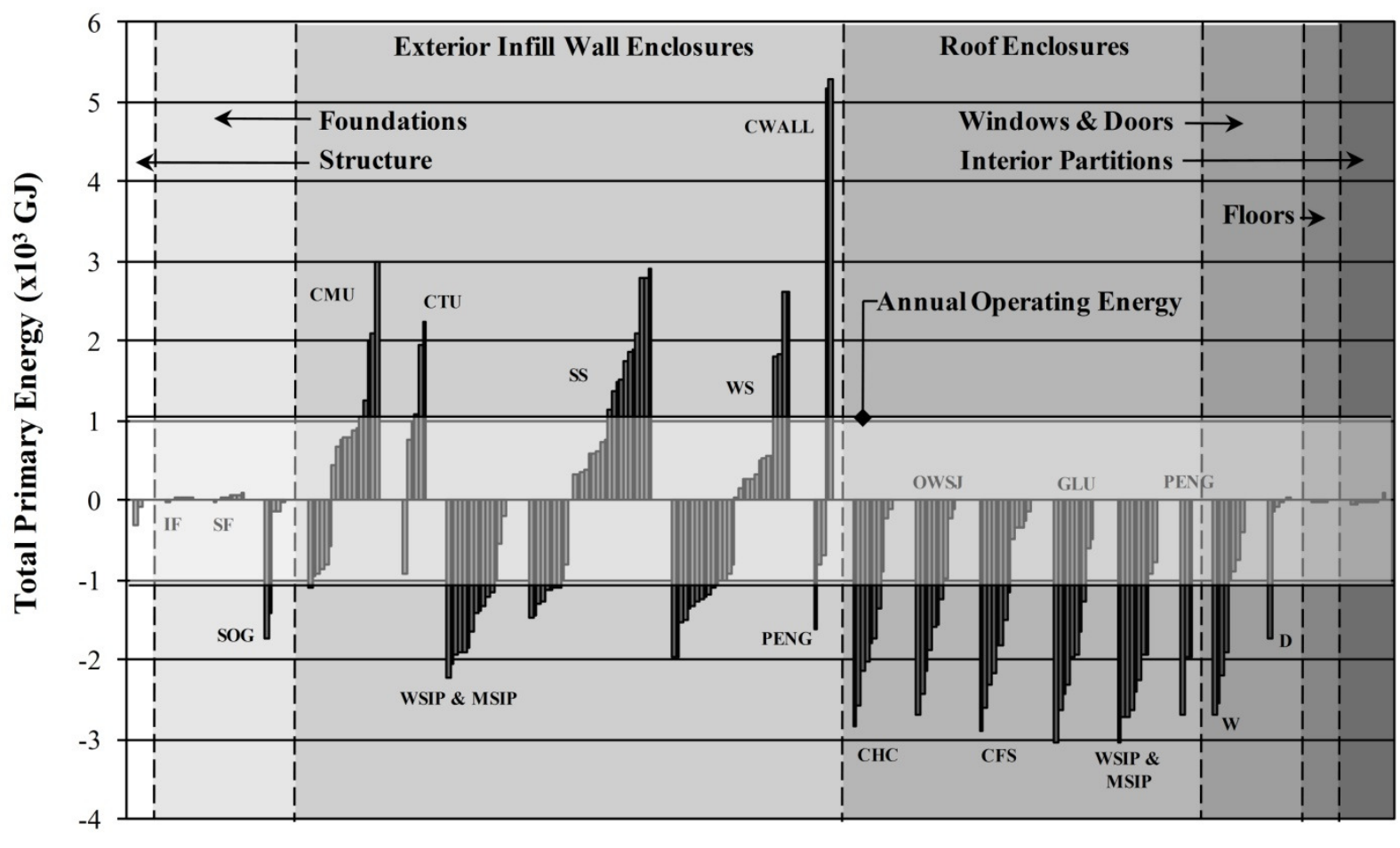

Building Components

Note: Values were generated using the baseline retail building (located in Toronto with a 50 year lifespan) and the range of building components identified in Appendix B

Interpreting the Figure

- Values plotted above the $\mathrm{x}$-axis, represent those building components that resulted in an increase in the total energy use $(\Delta$ embodied energy $+\Delta$ operating energy) of the baseline building after 50 years

- Values plotted below the $\mathrm{x}$-axis, represent those building components that resulted in a decrease in the total energy use ( $\Delta$ embodied energy $+\Delta$ operating energy) of the baseline building after 50 years

\section{Figure 7-3: A Sensitivity Analysis of the $\Delta$ Total Life-Cycle Energy Use for the Components of a Typical Retail Building after a 50 Year Lifespan in Toronto}

From Figure 7-3, one can understand the possible range of $\Delta$ total energy associated with the baseline retail building, depending on the specific choice of building components. It is evident that building components that have no impact on the operating energy of a building (like the structural system), 
tend to pale in comparison to those building components that do have a direct influence on the operating energy of a building (like the exterior walls and roof). It can be seen that the exterior walls, roofs, and windows have the greatest variation in $\Delta$ total energy from the baseline. Depending on the specific exterior wall, roof, or window, it can be observed in Figure 7-3 that this could be equivalent to $+/$ - about three years of operating energy. It is interesting to note that the curtainwalls (CWALL) result in the greatest potential increase in total energy compared to the baseline. In fact, using certain curtainwall enclosures can result in an increase of as much as five years of operating energy compared to the baseline.

A similar figure was created for the $\Delta$ total GWP. Figure 7-4 illustrates the $\Delta$ total life-cycle GWP for each of the 220 different building components from Appendix B. Again, similar to before these values represent either an increase in total GWP (plotted above the horizontal axis) or a decrease in total GWP (plotted below the horizontal axis) compared to the baseline retail building components. The horizontal axis represents the baseline retail building components. The annual operating GWP has also been plotted.

Once again it is evident that those building components that tend to have an influence on the operating GWP of a building (like the exterior walls, roofs, and windows), have the greatest range in $\Delta$ total GWP. Those building components that tend not to affect the operating energy of a building show less range in $\Delta$ total GWP.

It can be seen in Figure 7-4 that there is the potential for a substantial amount of variation within each building component category. Depending on the building component, there could be a relatively insignificant deviation in the total GWP from the baseline, or an increase/decrease of up to four years of operating GWP (an increase of six years of operating GWP for the case of the curtainwall enclosures).

Once again, the purpose of these figures is to summarize what otherwise is a very complex and time consuming task of evaluating the LCA of the various components of a retail building over 50 years. These figures are a useful reference at the beginning stages of a building design process, as the priorities for reducing the total energy use and total GWP of a building can quickly be set. For example, these results would suggest that the efforts of the design team be spent on reducing the total energy and total GWP of the exterior infill walls, roofs, and windows of a building rather than worrying about the structural system, foundations, or interior partitions. Also, using these figures these decisions can quickly be placed in terms of the number of equivalent years of operating energy. 
Therefore, given the expected lifespan of a building in question, one could reference these figures and quickly determine how many years of operating energy could be saved by specifying one building component strategy over the next. One could also quickly see how little a particular decision has on the total life-cycle energy use and GWP compared to one year of operating energy.

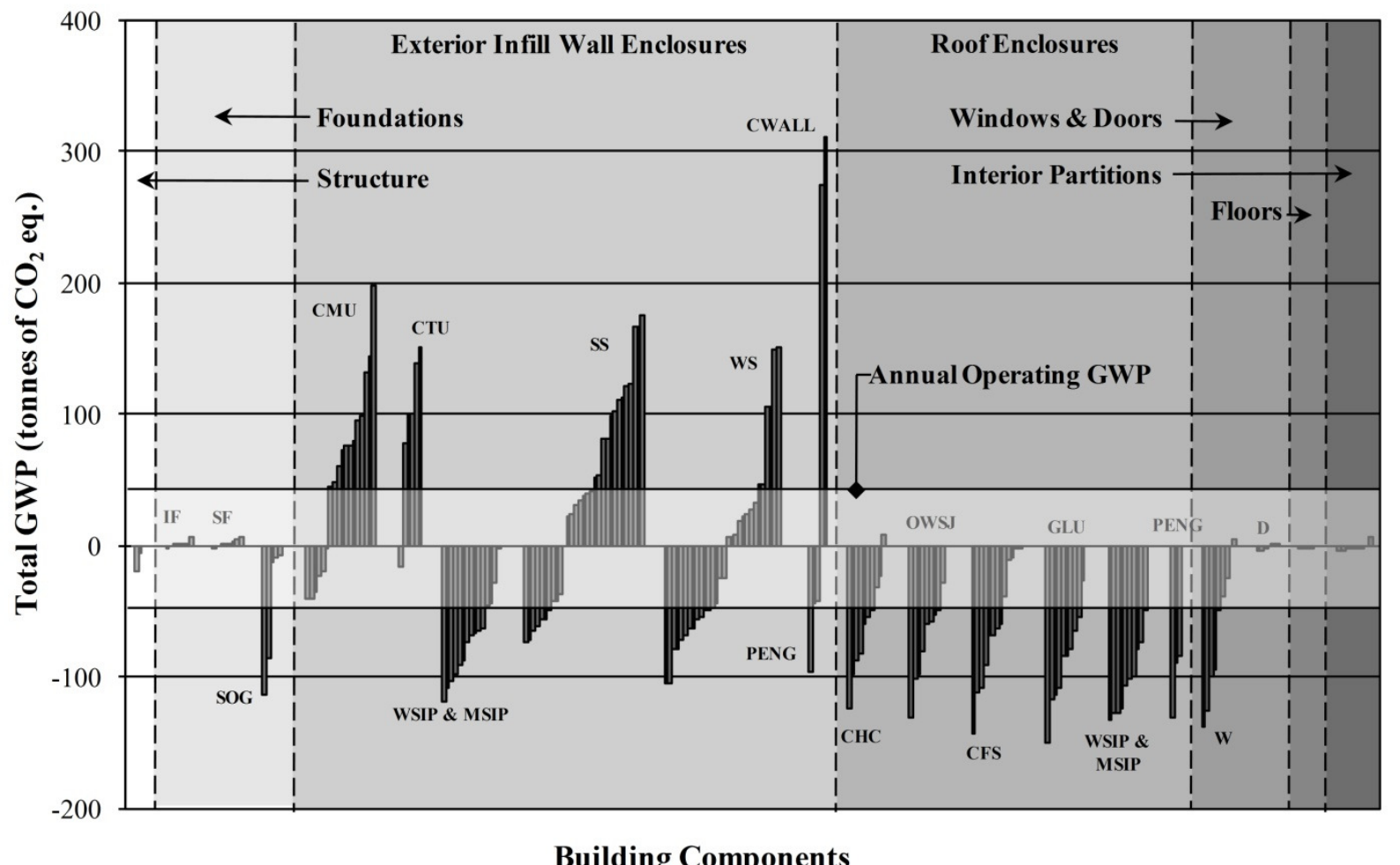

Note: Values were generated using the baseline retail building (located in Toronto with a 50 year lifespan) and the range of building components identified in Appendix B

\section{Interpreting the Figure}

- Values plotted above the $\mathrm{x}$-axis, represent those building components that resulted in an increase in the total GWP ( $\Delta$ embodied GWP $+\Delta$ operating GWP) of the baseline building after 50 years

- Values plotted below the $\mathrm{x}$-axis, represent those building components that resulted in a decrease in the total GWP ( $\Delta$ embodied GWP $+\Delta$ operating GWP) of the baseline building after 50 years

\section{Figure 7-4: A Sensitivity Analysis of the $\Delta$ Total Life-Cycle GWP for the Components of a Typical Retail Building after a 50 Year Lifespan in Toronto}

\subsection{Magnitudes of Order in the LCA of Buildings}

Throughout this study, the relationships between embodied energy, embodied GWP, operating energy, and operating GWP have been discussed. It has been shown that after 50 years, operating energy and operating GWP are a much greater concern than embodied energy and embodied GWP. 
However, as the operating energy and operating GWP of buildings continues to decrease in the coming years, the embodied energy and embodied GWP will become an increasing concern. In this section, the embodied energy and embodied GWP of the components of a building are compared to various reductions in the operating energy and operating GWP of a typical retail building.

Compared to the 50 year operating energy of a typical retail building, the embodied energy of the various building components is relatively insignificant (less than 10\%). However, suppose one was able to reduce the 50 year operating energy of a building by $50 \%$ or more. At what level of operating energy reduction would the effects be on par with the embodied energy of the building components? Figure 7-5 displays the relationships between the embodied energy of the building components and various levels of operating energy for the case of the baseline retail building.

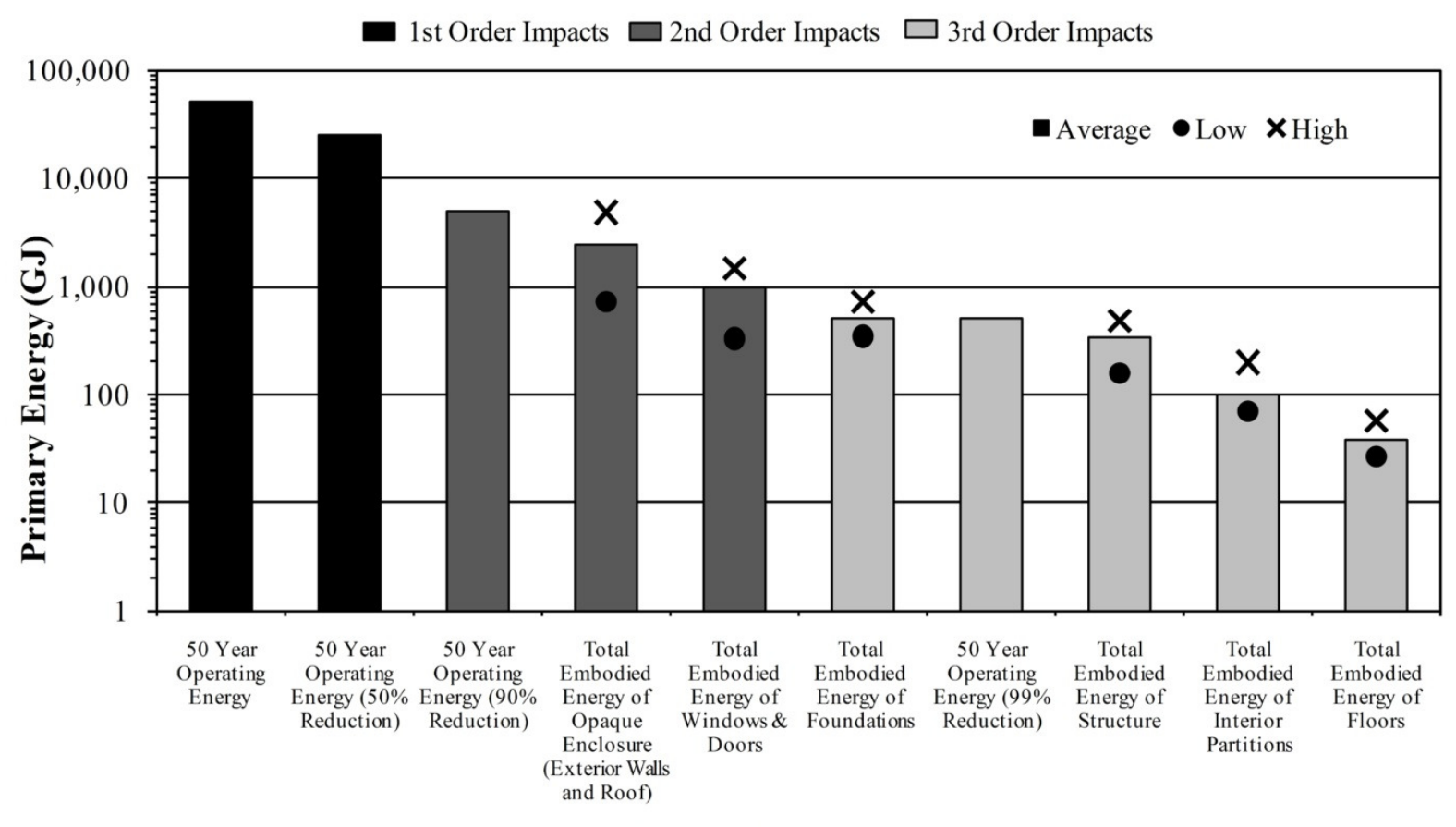

Building Component

Note: Values were generated using the baseline retail building (located in Toronto with a 50 year lifespan) and the range of building components identified in Appendix $B$

\section{Figure 7-5: Orders of Magnitude for Energy Consumption of a Typical Retail Building after 50 Year Lifespan in Toronto}

The results in Figure 7-5 were generated using the baseline retail building with a 50 year lifespan, as well as the range of building components identified in previous chapters. The results in Figure 7-5 
have been plotted on a log-scale. This means that for every increment on the vertical axis, this corresponds to an increase by a factor of ten in primary energy. The building components have been classified based on the quantity of primary energy that is associated with them. In particular, the $1^{\text {st }}$ order impacts have $\geq 10,000 \mathrm{GJ}$, the $2^{\text {nd }}$ order impacts have $<10,000 \mathrm{GJ}$ and $\geq 1,000 \mathrm{GJ}$, and the $3^{\text {rd }}$ order impacts have $<1,000$ GJ of primary energy after 50 years. It can be seen that a low, average, and high value has been plotted for each building component category. These values were generated from the data in the previous section for the weighted embodied energy of the 220 different building components examined in this study. They represent the range of possible values for each building component.

Using Figure 7-5 some important observations can be made about the level of operating energy reduction that would be required to be on par with the embodied energy of the building components. First, notice how the 50 year operating energy of the baseline retail building is a $1^{\text {st }}$ order impact. Essentially, this means that there is around 100 times more energy associated with the 50 year operating energy of the baseline retail building, than for a $3^{\text {rd }}$ order impact such as the total embodied energy of the foundations, structure, interior partitions, and floors. Compared to the $2^{\text {nd }}$ order impacts, the 50 year operating energy is responsible for around 10 times more energy after 50 years than the total embodied energy of opaque enclosure (exterior walls and roof) or the windows and doors.

Suppose that the 50 year operating energy of the baseline retail building was reduced by $50 \%$ through a combination of conservation, better design, and improved construction practices. Notice in Figure 7-5 that the 50 year operating energy reduced by 50\% has been plotted. Interestingly, the 50\% reduction in operating energy is still a $1^{\text {st }}$ order impact. Even if the typical 50 year operating energy was reduced by half, the amount of energy associated with building operations would still be about 50 to 100 times more than a $3^{\text {rd }}$ order impact and about 10 times more than a $2^{\text {nd }}$ order impact.

Now suppose that the 50 year operating energy of the baseline retail building was reduced by an aggressive $90 \%$ (extremely unlikely today). The 50 year operating energy reduced by $90 \%$ has been plotted. Only once the 50 year operating energy of the baseline retail building has been reduced by $90 \%$ does it become on par with a $2^{\text {nd }}$ order impact like the opaque enclosure or the windows and doors. Even at a $90 \%$ reduction in the 50 year operating energy, the operating effects still consume about 10 times more energy than a $3^{\text {rd }}$ order impact.

It is not until the 50 year operating energy of the baseline retail building has been reduced by $99 \%$ (almost impossible to do today), that the operating effects are on par with a $3^{\text {rd }}$ order impact. Clearly, 145 
a significant reduction in the operating energy is the most effective way to reduce the life-cycle energy consumption of a building. Not until the operating energy has been reduced by around $90 \%$ does the embodied energy of the building components begin to become a real concern.

A similar discussion can be done for the case of the life-cycle operating GWP verses embodied GWP for the baseline retail building. Figure 7-6 displays the relationships between the embodied GWP of the building components and various levels of operating GWP for the baseline retail building.

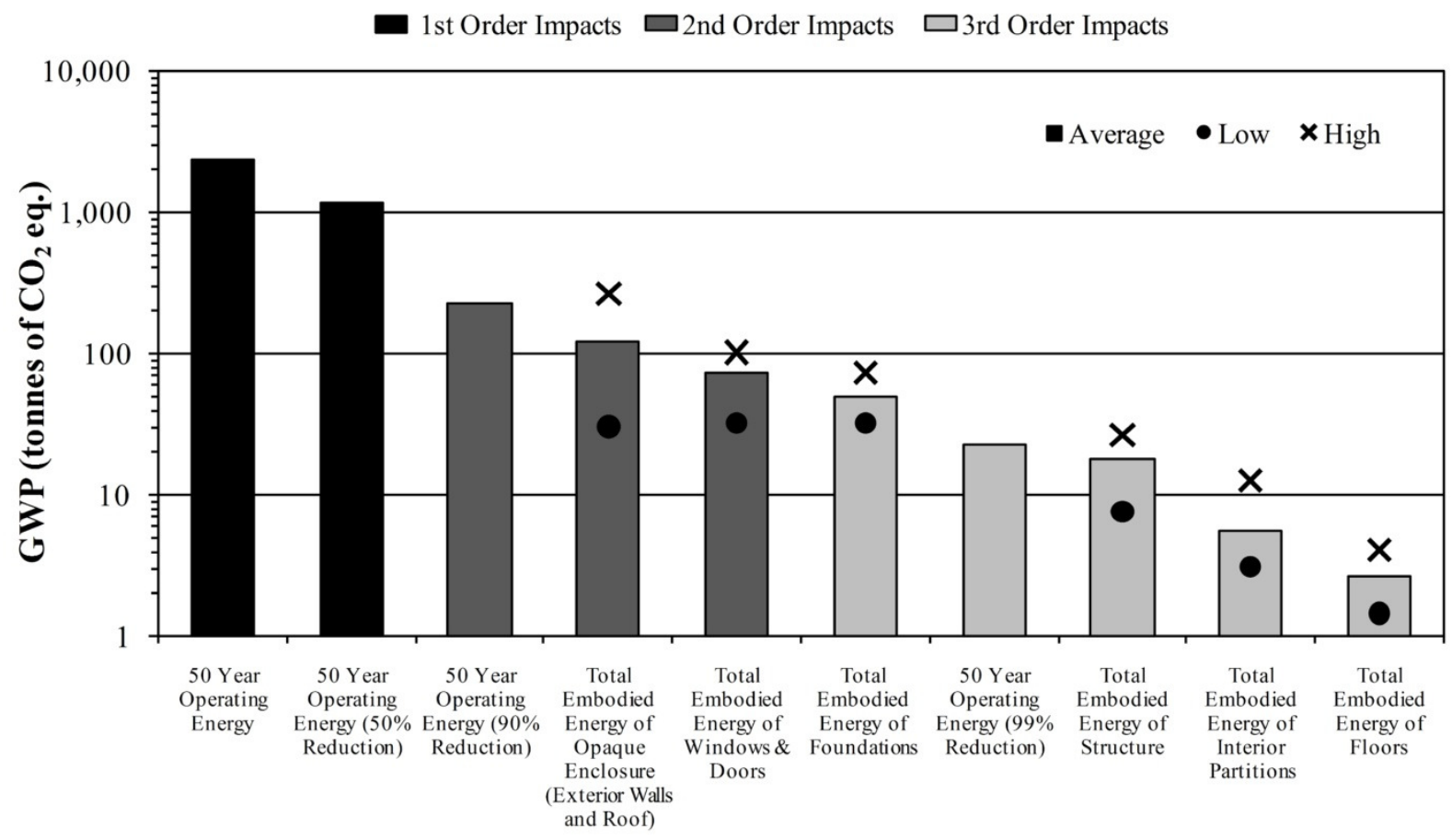

Building Component

Note: Values were generated using the baseline retail building (located in Toronto with a 50 year lifespan) and the range of building components identified in Appendix $B$

\section{Figure 7-6: Orders of Magnitude for Global Warming Potential of a Typical Retail Building after 50 Year Lifespan in Toronto}

Again, the GWP has been plotted on a log-scale. The building components have been classified based on the quantity of GWP that is associated with them. In particular, the $1^{\text {st }}$ order impacts have $\geq 1,000$ tonnes of $\mathrm{CO}_{2}$ eq., the $2^{\text {nd }}$ order impacts have $<1,000$ tonnes of $\mathrm{CO}_{2}$ eq. and $\geq 100$ tonnes of $\mathrm{CO}_{2}$ eq., and the $3^{\text {rd }}$ order impacts have $<100$ tonnes of $\mathrm{CO}_{2}$ eq. after 50 years. Similar to before, a low, average, and high value has been plotted for each building component, based on the sensitivity analysis of the weighted embodied GWP of the 220 building components in this study. 
Similar to the primary energy, the same relationships between the various reductions in operating effects and the embodied effects for the GWP are observed. Not until the typical 50 year operating GWP is reduced by around $90 \%$ does it become on par with a $2^{\text {nd }}$ order impact. Likewise, the typical 50 year operating GWP has to be reduced by around $99 \%$ before it is on par with a $3^{\text {rd }}$ order impact. Once again, the importance of reducing the operating GWP relative to the embodied GWP is apparent.

\subsection{A Summary of Design Strategies to Reduce the Total Life-Cycle Energy Use and GWP of Retail Buildings}

Given the relationships between the operating energy, operating GWP, embodied energy, and embodied GWP for a typical single-storey retail building in Toronto with a 50 year lifespan, some design strategies for reducing the life-cycle effects can be explored.

Table 7-1 lists a number of alternative design strategies to reduce the total life-cycle energy use of the baseline retail building after a 50 year lifespan. Notice how the design strategies are divided based on the building component, as well as into low-impact, mid-impact, and high-impact design strategies. The low-impact design strategies are those changes to the baseline retail building that would result in $\leq 5 \%$ savings in the total life-cycle energy use of the building. Likewise, the mid-impact design strategies correspond to a savings in total life-cycle energy between 5\% and 25\%. High-impact design strategies are those that result in $>25 \%$ savings in total energy.

The first key observation to note is that none of the design strategies, other than reducing the annual operating energy by $>28 \%$, can achieve the highest-level of energy savings. In other words, the only individual design strategy to decrease the total energy of the baseline retail building by more than $25 \%$ would be to reduce the operating energy. Not even a combination of all the best design strategies for the other building components would be able to achieve a $25 \%$ reduction in the total life-cycle energy use of the baseline building.

Looking now at the mid-impact design strategies, it can be seen that switching from the baseline roof enclosure (BASE-R) to the roof enclosure with the lowest total life-cycle energy (MSIP-R1), would result in a savings of just over 3,000 GJ of primary energy after 50 years. No other substitution of a single building component is able to achieve a mid-impact energy savings for the baseline building (other than a reduction in operating energy). 


\section{Table 7-1: Alternative Design Strategies to Reduce the Total Life-Cycle Energy Use of the Baseline Retail Building after a 50 Year Lifespan in Toronto}

\begin{tabular}{|c|c|c|c|}
\hline \multicolumn{4}{|c|}{$\begin{array}{l}\text { Design strategies are divided into low, mid, and high-impact strategies that correspond to a different percentage } \\
\text { reduction in the total life-cycle energy use of the baseline retail building }\end{array}$} \\
\hline $\begin{array}{l}\text { Building } \\
\text { Component }\end{array}$ & $\begin{array}{c}\text { Low-Impact Design Strategies } \\
\leq 5 \%(\leq 2,800 \mathrm{GJ})\end{array}$ & $\begin{array}{l}\text { Mid-Impact Design Strategies } \\
\qquad 5 \% \text { and } \leq 25 \% \\
(>2,800 \mathrm{GJ} \text { and } \leq 14,000 \mathrm{GJ})\end{array}$ & $\begin{array}{l}\text { High-Impact Design Strategies } \\
\quad>25 \%(>14,000 \mathrm{GJ})\end{array}$ \\
\hline Operations & $\begin{array}{l}\text { Reduce annual operating energy by } \leq \\
5.5 \% \text { [Energy Savings } \leq-2,789 \text { GJ] }\end{array}$ & $\begin{array}{l}\text { Reduce annual operating energy by }> \\
5.5 \% \text { and } \leq 27.6 \% \text { [Energy Savings } \\
\text { between }-2,789 \text { GJ \& }-13,993 \text { GJ] }\end{array}$ & $\begin{array}{l}\text { Reduce annual operating energy by }> \\
27.6 \% \text { [Energy Savings }>-13,993 \\
\text { GJ] }\end{array}$ \\
\hline $\begin{array}{l}\text { Exterior Infill } \\
\text { Wall } \\
\text { Enclosures }\end{array}$ & $\begin{array}{l}\text { Switch from baseline wall enclosure } \\
\text { (BASE-W) to the wall enclosure with } \\
\text { the lowest total life-cycle energy } \\
\text { (WSIP-W12) [Energy Savings }= \\
-2,214 \text { GJ] }\end{array}$ & $\begin{array}{l}\text { Unable to achieve this level of energy } \\
\text { reduction using only the exterior } \\
\text { infill wall design strategies in } \\
\text { Appendix B }\end{array}$ & $\begin{array}{l}\text { Unable to achieve this level of energy } \\
\text { reduction using only the exterior } \\
\text { infill wall design strategies in } \\
\text { Appendix B }\end{array}$ \\
\hline $\begin{array}{c}\text { Roof } \\
\text { Enclosures }\end{array}$ & $\begin{array}{l}\text { Switch from baseline roof enclosure } \\
\text { (BASE-R) which has a 4-ply built-up } \\
\text { asphalt roof covering to (OWSJ-R5) } \\
\text { which has a galvanized standing } \\
\text { seam steel roof [Energy Savings }= \\
-1,556 \mathrm{GJ} \text { ] }\end{array}$ & $\begin{array}{l}\text { Switch from baseline roof enclosure } \\
\text { (BASE-R) to the roof enclosure with } \\
\text { the lowest total life-cycle energy } \\
\text { (MSIP-R1) [Energy Savings }=-3,035 \\
\text { GJ] }\end{array}$ & $\begin{array}{l}\text { Unable to achieve this level of energy } \\
\text { reduction using only the roof design } \\
\text { strategies in Appendix B }\end{array}$ \\
\hline Structure & $\begin{array}{l}\text { Switch from conviential hot-rolled } \\
\text { steel structural system }(\mathrm{S}-1) \text { to heavy } \\
\text { timber glulam structural system } \\
\text { (S-2) [Energy Savings }=-320 \mathrm{GJ} \text { ] }\end{array}$ & $\begin{array}{l}\text { Unable to achieve this level of energy } \\
\text { reduction using only the structural } \\
\text { system design strategies in Appendix } \\
\text { B }\end{array}$ & $\begin{array}{l}\text { Unable to achieve this level of energy } \\
\text { reduction using only the structural } \\
\text { system design strategies in Appendix } \\
\text { B }\end{array}$ \\
\hline $\begin{array}{l}\text { Mezannine } \\
\text { Floor }\end{array}$ & $\begin{array}{l}\text { Switch from baseline mezzanine } \\
\text { floor (FL-3) to the mezzanine floor } \\
\text { with the lowest total life-cycle energy } \\
\text { (FL-5) [Energy Savings }=-31 \text { GJ] }\end{array}$ & $\begin{array}{l}\text { Unable to achieve this level of energy } \\
\text { reduction using only the mezzanine } \\
\text { floor design strategies in Appendix B }\end{array}$ & $\begin{array}{l}\text { Unable to achieve this level of energy } \\
\text { reduction using only the mezzanine } \\
\text { floor design strategies in Appendix B }\end{array}$ \\
\hline $\begin{array}{l}\text { Windows \& } \\
\text { Doors }\end{array}$ & $\begin{array}{l}\text { Switch from aluminum curtainwall } \\
\text { with no low-E coating and no argon } \\
\text { (W-9) to (W-8) which has a wood } \\
\text { frame, low-E coating, and argon gas } \\
\text { [Energy Savings }=-1,679 \text { GJ] }\end{array}$ & $\begin{array}{l}\text { Unable to achieve this level of energy } \\
\text { reduction using only the } \\
\text { window/door design strategies in } \\
\text { Appendix B }\end{array}$ & $\begin{array}{l}\text { Unable to achieve this level of energy } \\
\text { reduction using only the } \\
\text { window/door design strategies in } \\
\text { Appendix B }\end{array}$ \\
\hline $\begin{array}{c}\text { Interior } \\
\text { Partitions }\end{array}$ & $\begin{array}{l}\text { Switch all interior partitions in the } \\
\text { baseline retail building (incuding } \\
\text { stair tower) to WS-P2 which uses the } \\
\text { least amount of life-cycle energy } \\
\text { [Energy Savings }=-42 \text { GJ] }\end{array}$ & $\begin{array}{l}\text { Unable to achieve this level of energy } \\
\text { reduction using only the interior } \\
\text { partition design strategies in } \\
\text { Appendix B }\end{array}$ & $\begin{array}{l}\text { Unable to achieve this level of energy } \\
\text { reduction using only the interior } \\
\text { partition design strategies in } \\
\text { Appendix B }\end{array}$ \\
\hline Foundations & $\begin{array}{l}\text { Switch from } 200 \mathrm{~mm} \text { thick concrete } \\
\text { SOG with no under slab insulation } \\
\text { (SOG-FDN4) to } 100 \mathrm{~mm} \text { thick } \\
\text { concrete SOG with } 50 \mathrm{~mm} \text { insulation } \\
\text { (SOG-FDN3) [Energy Savings }= \\
-1,744 \text { GJ] }\end{array}$ & $\begin{array}{l}\text { Unable to achieve this level of energy } \\
\text { reduction using only the foundation } \\
\text { design strategies in Appendix B }\end{array}$ & $\begin{array}{l}\text { Unable to achieve this level of energy } \\
\text { reduction using only the foundation } \\
\text { design strategies in Appendix B }\end{array}$ \\
\hline
\end{tabular}

Total life-cycle energy of baseline retail building = 55,947 GJ $(100 \%)$

Total life-cycle operating energy of baseline retail building $=50,700 \mathrm{GJ}(90.6 \%)$

Total life-cycle embodied energy of baseline retail building $=5,247 \mathrm{GJ}(9.4 \%)$

There are a number of different low-impact design strategies that could be implemented in order to save $\leq 5 \%$ of the total energy for the baseline retail building. For example, switching from a $200 \mathrm{~mm}$ 
thick concrete slab-on-grade with no under slab insulation (SOG-FDN4) to a $100 \mathrm{~mm}$ thick concrete slab-on-grade with $50 \mathrm{~mm}$ of under slab insulation (SOG-FDN3) would result in a savings of over $1,700 \mathrm{GJ}$ of primary energy after 50 years. Similarly, switching from the baseline wall enclosure (BASE-W) to the wall enclosure with the lowest total life-cycle energy (WSIP-W12) would save over 2,200 GJ of primary energy.

Clearly, the best strategy to reduce the total life-cycle energy use of the baseline retail building would be to implement as many of the energy saving design strategies in Table 7-1 as possible. Only focusing on one or two design strategies probably won't save a great deal of energy in the end. The most effective way to reduce the total life-cycle energy of the baseline retail building is to find ways of reducing the annual operating energy (such as shortening the hours of operation, providing a better balance between daylighting and space heating, increasing the efficiency of the mechanical systems, implementing passive heating, cool, and ventilation strategies, etc.).

A similar list of design strategies for reducing the total GWP of the baseline retail building can also be developed. Table 7-2 lists the alternative design strategies for reducing the total life-cycle GWP of the baseline retail building after a 50 year lifespan.

Essentially the same trends apply for the case of total GWP as were just discussed for total energy. The only single strategy for reaching a high-impact of GWP savings is to reduce the annual operating GWP by more than about 29\%. Switching from the baseline roof (BASE-R) to the roof enclosure with the lowest total life-cycle GWP, would save about 150 tonnes of $\mathrm{CO}_{2}$ eq. after 50 years. A number of low-impact GWP saving design strategies are possible. For example, switching from aluminum curtainwall with no low-E coating and no argon gas (W-9) to a wood frame window with a low-E coating and filled with argon gas (W-8) would save about 90 tonnes of $\mathrm{CO}_{2}$ eq. after 50 years. Only if all of the low-impact design strategies (excluding the operating GWP) were implemented, would one be able to achieve a mid-impact GWP savings. Similar to the case of primary energy, the best approach to saving the most about of total GWP would be a combination of as many design strategies as possible, with a focus on reducing the annual operating GWP. 
Table 7-2: Alternative Design Strategies to Reduce the Total Life-Cycle GWP of the Baseline Retail Building after a 50 Year Lifespan in Toronto

\begin{tabular}{|c|c|c|c|}
\hline \multicolumn{4}{|c|}{$\begin{array}{l}\text { Design strategies are divided into low, mid, and high-impact strategies that correspond to a different } \\
\text { percentage reduction in the total life-cycle GWP of the baseline retail building) }\end{array}$} \\
\hline $\begin{array}{l}\text { Building } \\
\text { Component }\end{array}$ & $\begin{array}{l}\text { Low-Impact Design Strategies } \\
\leq 5 \%\left(\leq 130 \text { tonnes of } \mathrm{CO}_{2} \text { eq. }\right)\end{array}$ & $\begin{array}{l}\text { Mid-Impact Design Strategies } \\
>5 \% \text { and } \leq 25 \% \\
\left(>130 \text { and } \leq 660 \text { tonnes of } \mathrm{CO}_{2}\right. \\
\text { eq. })\end{array}$ & $\begin{array}{l}\text { High-Impact Design Strategies } \\
>25 \%\left(>660 \text { tonnes of } \mathrm{CO}_{2} \text { eq. }\right)\end{array}$ \\
\hline Operations & $\begin{array}{l}\text { Reduce annual operating GWP by } \leq \\
5.6 \% \text { [GWP Savings } \leq-130 \text { tonnes } \\
\text { of } \mathrm{CO}_{2} \text { eq. }\end{array}$ & $\begin{array}{l}\text { Reduce annual operating GWP by }> \\
5.6 \% \text { and } \leq 28.5 \% \text { [GWP Savings } \\
\text { between } 130 \& 658 \text { tonnes of } \mathrm{CO}_{2} \\
\text { eq.] }\end{array}$ & $\begin{array}{l}\text { Reduce annual operating GWP by } \geq \\
28.5 \% \text { [GWP Savings }>-658 \text { tonnes } \\
\text { of } \mathrm{CO}_{2} \text { eq.] }\end{array}$ \\
\hline $\begin{array}{l}\text { Exterior Infill } \\
\text { Wall } \\
\text { Enclosures }\end{array}$ & $\begin{array}{l}\text { Switch from baseline wall enclosure } \\
\text { (BASE-W) to the wall enclosure with } \\
\text { the lowest total life-cycle GWP } \\
\text { (WSIP-W12) [GWP Savings }=-118 \\
\text { tonnes of } \mathrm{CO}_{2} \text { eq.] }\end{array}$ & $\begin{array}{l}\text { Unable to achieve this level of GWP } \\
\text { reduction using only the exterior } \\
\text { infill wall design strategies in } \\
\text { Appendix B }\end{array}$ & $\begin{array}{l}\text { Unable to achieve this level of GWP } \\
\text { reduction using only the exterior } \\
\text { infill wall design strategies in } \\
\text { Appendix B }\end{array}$ \\
\hline $\begin{array}{l}\text { Roof } \\
\text { Enclosures }\end{array}$ & $\begin{array}{l}\text { Switch from baseline roof enclosure } \\
\text { (BASE-R) which has a 4-ply built-up } \\
\text { asphalt roof covering to (OWSJ-R5) } \\
\text { which has a galvanized standing } \\
\text { seam steel roof [ GWP Savings }=-59 \\
\text { tonnes of } \mathrm{CO}_{2} \text { eq.] }\end{array}$ & $\begin{array}{l}\text { Switch from baseline roof enclosure } \\
\text { (BASE-R) to the roof enclosure with } \\
\text { the lowest total life-cycle GWP } \\
\text { (GLU-R10) [GWP Savings }=-149 \\
\text { tonnes of } \mathrm{CO}_{2} \text { eq.] }\end{array}$ & $\begin{array}{l}\text { Unable to achieve this level of GWP } \\
\text { reduction using only the roof design } \\
\text { strategies in Appendix B }\end{array}$ \\
\hline Structure & $\begin{array}{l}\text { Switch from conviential hot-rolled } \\
\text { steel structural system }(\mathrm{S}-1) \text { to heavy } \\
\text { timber glulam structural system } \\
(\mathrm{S}-2) \text { [GWP Savings }=-19 \text { tonnes of } \\
\mathrm{CO}_{2} \text { eq.] }\end{array}$ & $\begin{array}{l}\text { Unable to achieve this level of GWP } \\
\text { reduction using only the structural } \\
\text { system design strategies in Appendix } \\
\text { B }\end{array}$ & $\begin{array}{l}\text { Unable to achieve this level of GWP } \\
\text { reduction using only the structural } \\
\text { system design strategies in Appendix } \\
\text { B }\end{array}$ \\
\hline $\begin{array}{l}\text { Mezannine } \\
\text { Floor }\end{array}$ & $\begin{array}{l}\text { Switch from baseline mezzanine } \\
\text { floor (FL-3) to the mezzanine floor } \\
\text { with the lowest total life-cycle energy } \\
\text { (FL-5) [GWP Savings }=-3 \text { tonnes of } \\
\mathrm{CO}_{2} \text { eq.] }\end{array}$ & $\begin{array}{l}\text { Unable to achieve this level of GWP } \\
\text { reduction using only the mezzanine } \\
\text { floor design strategies in Appendix B }\end{array}$ & $\begin{array}{l}\text { Unable to achieve this level of GWP } \\
\text { reduction using only the mezzanine } \\
\text { floor design strategies in Appendix B }\end{array}$ \\
\hline $\begin{array}{l}\text { Windows \& } \\
\text { Doors }\end{array}$ & $\begin{array}{l}\text { Switch from aluminum curtainwall } \\
\text { with no low-E coating and no argon } \\
(\mathrm{W}-9) \text { to }(\mathrm{W}-8) \text { which has a wood } \\
\text { frame, low-E coating, and argon gas } \\
\text { [GWP Savings }=-90 \text { tonnes of } \mathrm{CO}_{2} \\
\text { eq.] }\end{array}$ & $\begin{array}{l}\text { Unable to achieve this level of GWP } \\
\text { reduction using only the } \\
\text { window/door design strategies in } \\
\text { Appendix B }\end{array}$ & $\begin{array}{l}\text { Unable to achieve this level of GWP } \\
\text { reduction using only the } \\
\text { window/door design strategies in } \\
\text { Appendix B }\end{array}$ \\
\hline $\begin{array}{l}\text { Interior } \\
\text { Partitions }\end{array}$ & $\begin{array}{l}\text { Switch all interior partitions in the } \\
\text { baseline retail building (including } \\
\text { stair tower) to WS-P2 which uses the } \\
\text { least amount of life-cycle GWP } \\
\text { [GWP Savings }=-3 \text { tonnes of } \mathrm{CO}_{2} \\
\text { eq.] }\end{array}$ & $\begin{array}{l}\text { Unable to achieve this level of GWP } \\
\text { reduction using only the interior } \\
\text { partition design strategies in } \\
\text { Appendix B }\end{array}$ & $\begin{array}{l}\text { Unable to achieve this level of GWP } \\
\text { reduction using only the interior } \\
\text { partition design strategies in } \\
\text { Appendix B }\end{array}$ \\
\hline Foundations & $\begin{array}{l}\text { Switch from } 200 \mathrm{~mm} \text { thick concrete } \\
\text { SOG with no under slab insulation } \\
\text { (SOG-FDN4) to } 100 \mathrm{~mm} \text { thick } \\
\text { concrete SOG with } 50 \mathrm{~mm} \text { insulation } \\
\text { (SOG-FDN3) [GWP Savings }=-114 \\
\text { tonnes of } \mathrm{CO}_{2} \text { eq.] }\end{array}$ & $\begin{array}{l}\text { Unable to achieve this level of GWP } \\
\text { reduction using only the foundation } \\
\text { design strategies in Appendix B }\end{array}$ & $\begin{array}{l}\text { Unable to achieve this level of GWP } \\
\text { reduction using only the foundation } \\
\text { design strategies in Appendix B }\end{array}$ \\
\hline
\end{tabular}

Total life-cycle GWP of baseline retail building = 2,632 tonnes of $\mathrm{CO}_{2}$ eq. (100\%)

Total life-cycle operating GWP of baseline retail building $=2,310$ tonnes of $\mathrm{CO}_{2}$ eq. $(87.8 \%)$

Total life-cycle embodied GWP of baseline retail building $=322$ tonnes of $\mathrm{CO}_{2}$ eq. $(12.2 \%)$ 


\section{Chapter 8 \\ Conclusions and Recommendations}

\subsection{Conclusions}

A detailed review of the literature determined that there is a need for a comprehensive life-cycle assessment (LCA) study of single-storey commercial buildings in Canada, specifically single-storey retail buildings. Multi-storey office buildings and residential buildings have been the focus of the vast majority of LCA studies in the past, despite the fact that retail buildings consume more energy per floor area than both these types of buildings. As well, second only to office buildings, retail buildings in Canada are responsible for the largest percentage of energy use in the commercial/institutional building sector. This thesis addresses the need for a study that looks at a broad scope of building components for a single-storey retail building and that puts the life-cycle impacts of the various components into perspective.

That being said, the primary goal of this study was to conduct a comprehensive LCA study for the components of a single-storey retail building located in Toronto, Canada, to determine which building components contribute the most towards the total life-cycle energy use and global warming potential (GWP) after 50 years. In order to accomplish this goal, a two part methodology was followed.

First, the total life-cycle energy use and GWP was calculated for 220 different building components grouped into the following categories: exterior infill walls, roofs, structural systems, floors, windows, doors, foundations, and interior partition walls. Next, the scope of the study was broadened to include the LCA of five different single-storey retail buildings including: a typical hot-rolled steel structure retail building (i.e. the baseline retail building), a typical heavy timber structure retail building, a preengineered steel retail building, a predominately steel retail building, and a predominately timber retail building. For both the analysis of the individual building components and the entire buildings, the LCA calculations included the embodied and operating effects related to a pre-occupancy, occupancy, and post-occupancy phase.

Although a detailed analysis of 220 different individual building components was conducted in this study, the major thrust of this research work was focused on establishing the overall relationships between energy use and GWP for single-storey retail buildings as a whole. A detailed comparison of the LCA results for the individual building components was left to future work. 


\section{LCA Results for a Typical Single-Storey Retail Building (i.e. Baseline Retail Building)}

A detailed LCA was conducted for a typical single-storey retail building, with a 50 year lifespan, located in Toronto, Canada. The relationships between operating energy, operating GWP, embodied energy, and embodied GWP were determined. Like previous studies of commercial type buildings (Cole \& Kernan, 1996), operating effects accounted for the vast majority of the total effects as compared to embodied effects.

\section{Total Energy and Total GWP}

After a 50 year lifespan in Toronto, Canada, the typical single-storey retail building in this study was found to have a total life-cycle primary energy use of about 55,947 GJ and a total life-cycle GWP of around 2,632 tonnes of $\mathrm{CO}_{2}$ eq.

As a percentage of the total life-cycle energy use (and total life-cycle GWP) the operating energy (and operating GWP) accounted for about 91\% (88\%) and the embodied energy (and embodied GWP) accounted for about 9\% (12\%) after 50 years. Therefore, it was determined that the best strategy for reducing the total life-cycle energy use and GWP of a typical single-storey retail building in Canada, is to decrease the energy use and GWP associated with the operating phase of the building. Any strategies that either directly or indirectly reduce the operating effects of the building should be a priority, if reducing the total life-cycle environmental impacts is a concern. In fact, the operating effects of a typical single-storey retail building in Canada would have to be reduced by about $90 \%$ from typical values today before a concerted effort to reduce embodied effects would be justified. That being said, as a percentage of the total energy (and total GWP) of the entire building, the following breakdown of the total embodied energy (and total embodied GWP) for the components of a single-storey retail building in Canada was found: roof $=4.9 \%(4.7 \%)$, foundations $=1.2 \%(3.0 \%)$, windows and doors $=1.1 \%(1.9 \%)$, exterior infill walls $=1.0 \%(1.1 \%)$, structure $=0.9 \%(1.0 \%)$, interior partitions $=0.2 \%(0.3 \%)$, and mezzanine floor $=0.1 \%(0.2 \%)$.

\section{Total Operating Energy and Total Operating GWP}

In a typical single-storey retail building in Canada, the operating effects were found to account for around $90 \%$ of the total effects after 50 years. In this study, the total electricity use and the total natural gas use represented about $56.9 \%(47.3 \%)$ and $43.1 \%(52.7 \%)$ of the annual total operating energy (and total operating GWP) respectfully. A breakdown of the annual total operating energy use (and total operating GWP) for the typical single-storey retail building in this study can be further 
divided into: space heating (assuming a natural gas furnace) $=42.4 \%(51.8 \%)$, area lighting $=37.3 \%$ $(31.0 \%)$, ventilation fans $=7.4 \%(6.2 \%)$, space cooling $($ assuming DX electric coils $)=6.4 \%(5.4 \%)$, miscellaneous equipment $=5.6 \%(4.6 \%)$, water heating $=0.7 \%(0.9 \%)$, and pumps and auxiliary $=$ $0.2 \%(0.1 \%)$.

\section{Total Embodied Energy and Total Embodied GWP}

In a typical single-storey retail building in Canada, the embodied effects were found to account for around $10 \%$ of the total effects after 50 years. The results of this study found that the roof accounted for nearly half of the total embodied energy of the entire building and about $40 \%$ of the total embodied GWP after 50 years. This is an important finding as the embodied effects of the roof were found to account for a significantly greater percentage of the total embodied effects compared to any other individual building component and were found to account for a significantly greater percentage of the total embodied effects compared to multi-storey buildings. The following breakdown of total embodied energy (and total embodied GWP) for a typical single-storey retail building was found in this study: roof $=52.0 \%(38.5 \%)$, foundations $=12.9 \%(24.5 \%)$, windows and doors $=12.0 \%$ $(15.8 \%)$, exterior infill walls $=10.2 \%(8.7 \%)$, structure $=9.1 \%(8.4 \%)$, interior partitions $=2.7 \%$ $(2.8 \%)$, and mezzanine floor $=1.1 \%(1.2 \%)$.

\section{The Impact of Material Selection on the LCA of a Typical Single-Storey Retail Building}

A detailed LCA of five single-storey retail buildings with a 50 year lifespan, located in Toronto, Canada was conducted. These five retail buildings included: a typical hot-rolled steel structure retail building (Case Study \#1), a typical heavy-timber structure retail building (Case Study \#2), a typical pre-engineered steel retail building (Case Study \#3), a predominately steel retail building (Case Study \#4), and a predominately timber retail building (Case Study \#5). In each case, the operating effects were found to far outweigh the embodied effects after a 50 year lifespan.

\section{Total Energy and Total GWP}

A comprehensive LCA study was conduced for each of the five single-storey retail buildings in this study. After a 50 year lifespan, the total energy (and total GWP) of the five case study buildings was found to only differ at most by $6 \%$ (7\%). Regardless of the material chosen for the structural system, or whether the building was primarily made of steel or timber building components, the range in the total energy and total GWP of the five case study buildings after 50 years was minimal. In each case, the operating effects far outweighed the embodied effects after 50 years. Therefore, as the annual 
operating energy (and operating GWP) of the five buildings only differed by about $3 \%(4 \%)$, the similarity in operating effects moderated any differences in embodied effects between the buildings due to using different building materials.

That being said, the typical pre-engineered steel building (Case Study \#3) was found to have the least total energy and total GWP of any building after 50 years. However, the energy and GWP savings were minimal compared to the other buildings and was highly dependent on the thermal resistance of the enclosure. Therefore, it is possible that under slightly different conditions that another building would have less total energy and total GWP after 50 years. However, the typical pre-engineered steel retail building was found to have the least total energy and total GWP of any building in this study.

\section{Total Operating Energy and Total Operating GWP}

The annual operating energy (and operating GWP) of the five single-storey retail buildings in this study only differed at most by 3\% (4\%). Therefore, using steel or timber building components did not significantly affect the operating energy or operating GWP of a typical single-storey retail building, so long as the thermal resistance of the enclosure could be kept relatively consistent.

\section{Total Embodied Energy and Total Embodied GWP}

After a 50 year lifespan, the total embodied energy (and total embodied GWP) of the five singlestorey retail buildings in this study differ by as much as $44 \%$ (35\%). The typical pre-engineered steel retail building (Case Study \#3), was found to have the least total embodied energy and total embodied GWP of all the buildings. This was due in large part to the high degree of material optimization that is inherent to these types of buildings.

The standing seam steel roof performed significantly better after 50 years than the asphalt-based, PVC, and EPDM roofs. Standing seam steel roofs have significantly less recurring embodied energy and recurring embodied GWP and show great promise in comparison to other roof coverings with higher recurring embodied effects. If a standing seam steel roof was to replace the 4-ply built-up asphalt roof in the predominately timber retail building (Case Study \#5), then this building would have the least total embodied energy and total embodied GWP of all five buildings. The typical hotrolled steel structure retail building (Case Study \#1) was found to have the highest total embodied energy and total embodied GWP of all five buildings. 


\section{LCA Results for Individual Building Components}

In general, a strong correlation was observed between the thermal resistance of the exterior infill walls in this study and the $\Delta$ total energy and $\Delta$ total GWP compared to the baseline datum. In fact, it was found that an increase in total energy and total GWP corresponded in general to a decrease in the thermal resistance of the exterior infill walls. In almost every case there were far more significant energy and GWP savings to be had because of savings in operating effects than from savings in embodied effects for the various exterior infill walls examined in this study.

For the case of the roof enclosures examined in this study, the magnitude of the embodied effects outweighed the operating effects in many instances. This result suggests that for the roof enclosures, the building materials have a more significant affect on the $\Delta$ total energy and $\Delta$ total GWP than was observed for the exterior infill walls. However, there was still a correlation between a decrease in the thermal resistance of the roof enclosures and an increase in the total energy and total GWP of the roofs. The results also indicate that significant savings could be achieved in terms of the total energy and total GWP if a green roof or a steel roof covering were used as opposed to an asphalt-based roof covering.

The choice of structural system, floor assembly, or interior partition wall type did not have any impact on the operating energy and operating GWP of the baseline building at all. Therefore, the $\Delta$ total energy and $\Delta$ total GWP compared to the baseline datum were minimal in these types of building components. The differences between the various options for these types of building components were solely a result of the differences in embodied effects.

The results of this study found a strong correlation between decreasing thermal resistance of the windows and increasing total energy and total GWP. Therefore, high performance glazing (argon filled, low-E coating) outperformed the corresponding windows with typical glazing (air filled, no low-E coating). The results also found that per $\mathrm{m}^{2}$ of enclosure, windows actually have a relatively large total energy and total GWP compared to the exterior infill walls and roofs. A larger sample of doors must be examined before conclusive remarks can be made about the $\Delta$ total energy and $\Delta$ total GWP of different types of doors.

The best performing foundation components in this study were the ones that used less material. Therefore, smaller concrete footings, piers, foundation walls, and thinner slab-on-grades generally performed better in the LCA calculations. Also, higher percentages of flyash in the concrete mix 
resulted in greater energy and GWP savings. The best performing slab-on-grades were the ones where under slab insulation was specified, as significant savings in operating effects resulted.

\subsection{Recommendations for Future Work}

Based on the findings of this study, the following recommendations for future work have been compiled:

1. Determine how the relationships between embodied energy, embodied GWP, operating energy, and operating GWP change for a different building lifespan $(5,25,100$ years for example).

2. Determine how the results of this study would differ if the retail building were located elsewhere in Canada or in the United States.

3. Further investigate the LCA of pre-engineered steel buildings since they showed great promise in this study as an effective system to use in low-energy building applications due to their high degree of material optimization. For example, the savings in construction energy that would result from using a pre-engineered steel building system compared to other conventional systems might be significant due to shorter construction times and prefabrication benefits.

4. Compare the LCA results for a single-storey retail building to a multi-storey retail building in order to determine which building components have the most impact on the total life-cycle energy and GWP.

5. Calculate the embodied energy and embodied GWP that is associated with the mechanical, electrical, and plumbing components of a building. The operating effects of these components have been included in this study, but their embodied energy and embodied GWP has not been calculated here. 


\section{Bibliography}

Alcorn, A. (2003). Embodied Energy and $\mathrm{CO}_{2}$ Coefficients for NZ Building Materials. Wellington, New Zealand: Centre for Building Performance Research, Victoria University of Wellington.

ASHRAE. (2007). ASHRAE Standard 90.1-2007: Energy Standard for Buildings Except Low-Rise Residential Buildings I-P Edition. Atlanta, GA: American Society of Heating, Refrigerating and Air-Conditioning Engineers, Inc.

ASHRAE Fundamentals SI. (2009). Related Commerical Resources, ASHRAE Handbook CD, Fundamentals SI, Chapter 15-Fenstration. In ASHRAE, ASHRAE Standard 90.1-2007: Energy Standard for Buildings Except Low-Rise Residential Buildings. Atlanta, GA: American Society of Heating, Refrigerating and Air-Conditioning Engineers, Inc.

Burnett, E. F., \& Straube, J. F. (2005). Building Science for Building Enclosures. Westford, MA: Building Science Press.

CaGBC. (2009). LEED Canada. Retrieved June 02, 2010, from Canada Green Building Council Web Site: http://www.cagbc.org/leed/what/index.php

CaGBC. (2009, December 31). LEED Projects. Retrieved March 26, 2010, from Canadian Green Building Council Web Site: http://www.cagbc.org/leed/leed_projects/index.php

Canadian Commission on Building and Fire Codes. (2006). User's Guide-National Building Code 2005: Structural Commentaries (Part 4 of Division B). Ottawa, ON: National Research Council Canada.

Cole, R. J., \& Kernan, P. C. (1996). Life-Cycle Energy Use in Office Buildings. Building and Environment, 31, 307-317.

Ding, G. K. (2007). Life cycle energy assessment of Australian secondary schools. Building Research \& Information, 35 (5), 487-500.

Environment Canada. (2009). Canada's 2007 Greenhouse Gas Inventory - A Summary of Trends. Gatineau, Quebec: Environment Canada.

Fleming, J. R. (1998). Historical Perspectives on Climate Change. New York: Oxford University Press.

Haapio, A., \& Viitaniemi, P. (2008). A critical review of building environmental assessment tools. Environmental Impact Assessment Review , 28, 469-482. 
Hammond, G., \& Jones, C. (2008). Inventory of Carbon \& Energy (ICE). Retrieved May 26, 2010, from Sustainable Energy Research Team (SERT), University of Bath: http://www.bath.ac.uk /mech-eng/sert/embodied/

Harvey, L. D. (2006). A Handbook on Low-Energy Buildings and District-Energy Systems. Sterling, VA: Earthscan.

Hirsch, J. J. (2009). eQUEST. Retrieved March 29, 2010, from DOE2 Website: http://www.doe2.com /eQuest/

International Iron and Steel Institute. (2005, November). World Steel Association Web Site. Retrieved June 23, 2009, from World Steel Life-Cycle Inventory Data: http://www.worldsteel.org /index.php?action=lcaform

ISO. (2006). CAN/CSA-ISO 14040:06 Environmental management - Life cycle assessment Principles and framework. Mississauga: Canadian Standards Association .

Itard, L., \& Klunder, G. (2007). Comparing environmental impacts of renovating housing stock with new construction. Building Research \& Information, 35 (3), 252-267.

John, S., Nebel, B., Perez, N., \& Buchanan, A. (2008). Environmental Impacts of Multi-Storey Buildings Using Different Construction Materials. Christchurch, New Zealand: University of Canterbury.

Junnila, S., Horvath, A., \& Guggemos, A. A. (2006). Life-Cycle Assessment of Office Buildings in Europe and the United States. Journal of Infrastructure Systems , 10-17.

Kibert, C. J. (2005). Sustainable Construction - Green Building Design and Delivery. Hoboken, New Jersey: John Wiley \& Sons Inc.

LBNL. (2009, March 12). THERM. Retrieved April 08, 2010, from Lawrence Berkeley National Laboratory Web Site: http://windows.lbl.gov/software/therm/therm.html

Lee, J. J., O'Callaghan, P., \& Allen, D. (1995). Critical review of life cycle analysis and assessment techniques and their application to commerical activities. Resources, Conservation and Recycling , 13, 37-56.

Morrison Hershfield Ltd. (2009). A Life Cycle Assessment Study of Embodied Effects for Existing Historic Buildings. Merrickville, Ontario: The Athena Sustainable Materials Institute. 
Natural Resources Canada. (2008). Energy Efficiency Trends in Canada, 1990 to 2005. Ottawa: Natural Resources Canada's Office of Energy Efficiency.

NRCan. (2009). Energy Efficiency Trends in Canada 1997 to 2007. Ottawa, Ontario: Office of Energy Efficiency, Natural Resources Canada.

NRCan, OEE. (2010, March 26). Statistics and Analysis, Energy Use Data Handbook Tables. Retrieved May 27, 2010, from Natural Resources Canada, Office of Energy Efficiency Web Site: http://oee.nrcan.gc.ca/corporate/statistics/neud/dpa/home.cfm?attr=0

NRTEE. (2009, April 16). Fact Sheet 1: Canadian GHG Emissions. Retrieved June 01, 2010, from National Round Table on the Environment and the Economy Web Site: http://www.nrteetrnee.com/eng/publications/carbon-pricing/carbon-pricing-eng.php

RSMeans. (2003). Assemblies Cost Data 2004. Kingston, MA: Construction Publishers \& Consultants.

Sartori, I., \& Hestnes, A. (2007). Energy use in the life cycle of conventional and low-energy buildings: a review article. Energy and Buildings , 39, 249-257.

Scheuer, C., Keoleian, G. A., \& Reppe, P. (2003). Life cycle energy and environmental performance of a new university building: modelling challenges and design implications. Energy and Buildings , 35, 1049-1064.

Straube, J. F. (2006). Green Building and Sustainability. Westford, MA: Building Science Press.

Straube, J., \& Burnett, E. (2005). Building Science for Building Enclosures. Wsetford, MA: Building Science Press.

The Athena Institute. (2009, October 28). An Overview of the Athena Impact Estimator for Buildings. Retrieved January 28, 2010, from The Athena Institute website: http://www.athenasmi.org /tools/impactEstimator/index.html

The ATHENA Institute. (2010, June 1). EcoCalculator Overview. Retrieved June 15, 2010, from The ATHENA Institute Web Site: http://www.athenasmi.org/tools/ecoCalculator/

The Athena Institute. (2010, February 18). The Impact Estimator for Buildings. Retrieved March 29, 2010, from Athena Institute Website: http://www.athenasmi.org/tools/impactEstimator /index.html 
The Athena Institute. (2008, December 18). The Inner Workings of the Athena Impact Estimator for Buildings: Transparency Document. Retrieved January 28, 2010, from The Athena Institute website: http://www.athenasmi.org/tools/impactEstimator/innerWorkings.html

The World Commission on Environment and Development. (1987). Our Common Future. Geneva, Switerland: Oxford University Press.

Treloar, G., Fay, R., Ilozor, B., \& Love, P. (2001). Building Materials Selection: Greenhouse Strategies for Built Facilities. Facilities , 19 (3/4), 139-149.

Trusty, W., \& Horst, S. (2005). LCA Tools Around the World. Building Design \& Construction, 1215.

USGBC. (2010). Green Building Research. Retrieved March 18, 2010, from U.S. Green Building Council Website: http://www.usgbc.org/DisplayPage.aspx?CMSPageID=1718

Victoria University of Wellington. (2007, September 20). Resources - Table of Embodied Energy Coefficients. Retrieved May 26, 2010, from Centre for Building Performance Research: http://www.victoria.ac.nz/cbpr/resources/index.aspx

Yohanis, Y. G., \& Norton, B. (2002). Life-cycle operational and embodeid energy for a generic singstorey office building in the UK. Energy, 27, 77-92. 


\section{Appendix A}

\section{Description of Baseline Retail Building (Case Study \#1)}

Appendix A provides a detailed description of the baseline retail building (Case Study \#1 - Typical Hot-Rolled Steel Structure Retail Building) that was used in this study. This includes drawings of the floor plans, sections, building elevations, structural drawings, as well as a detailed list of building descriptors that were used for the energy modeling. Where noted, Appendix B should be consulted for a more complete description of certain building components.
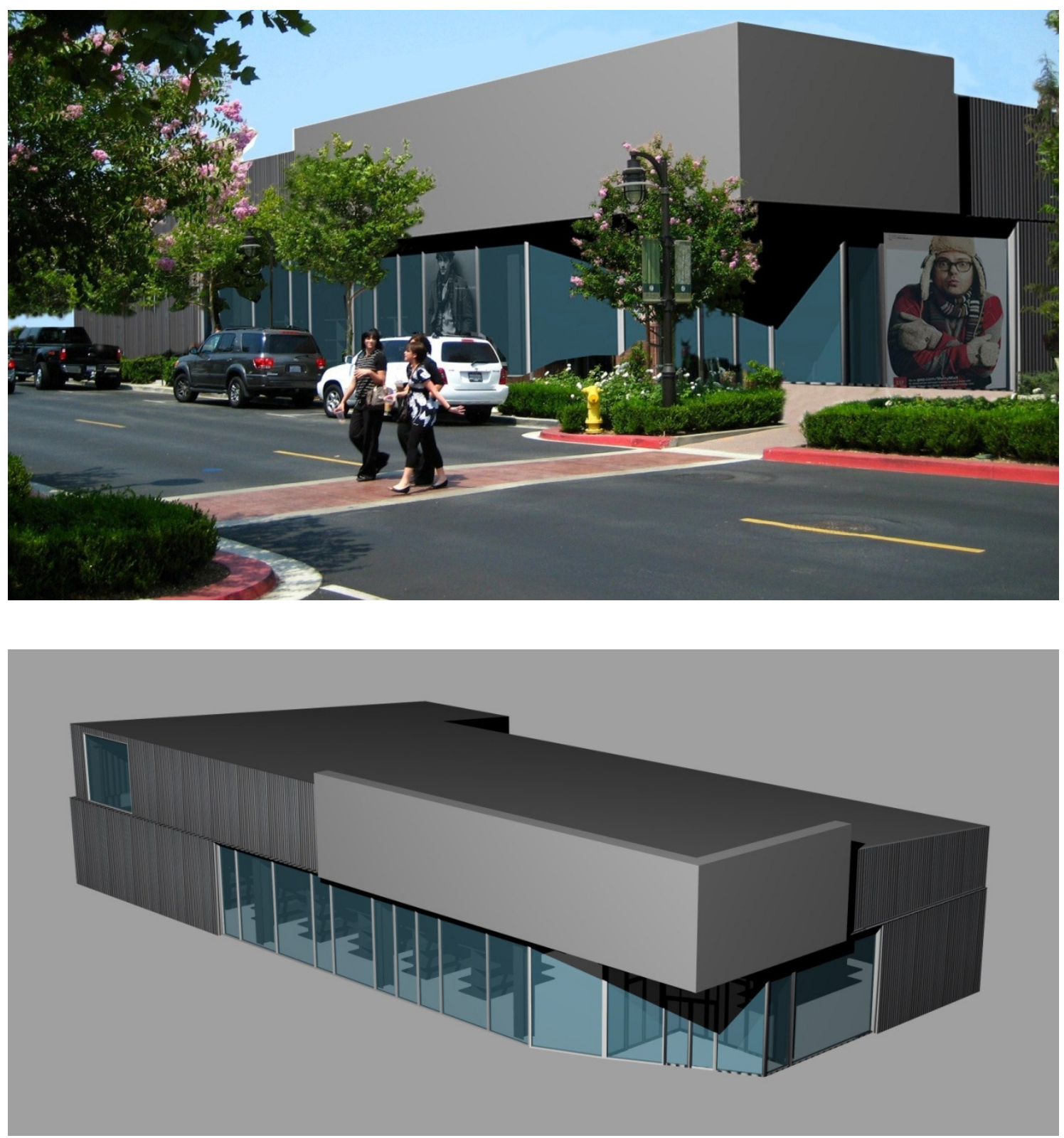


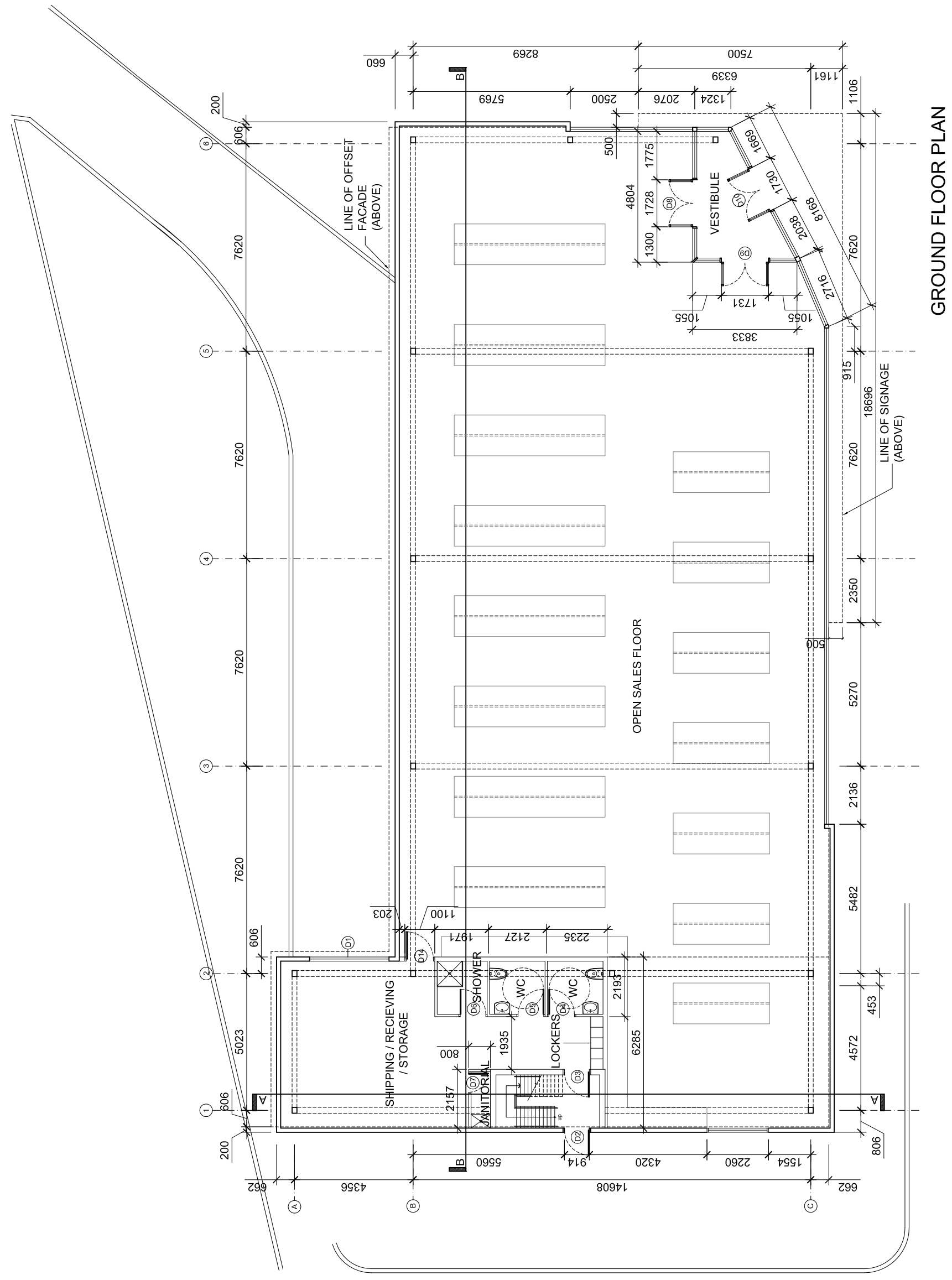




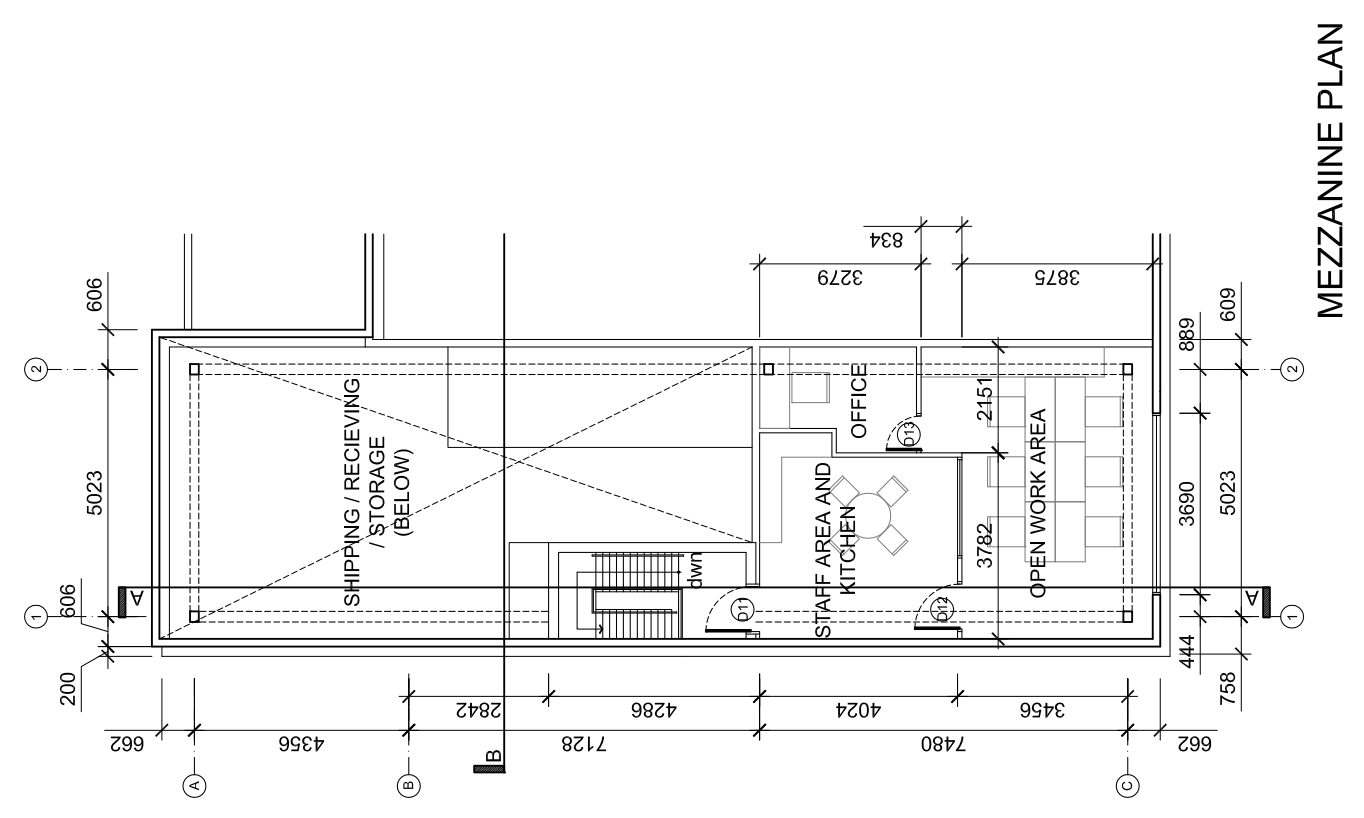




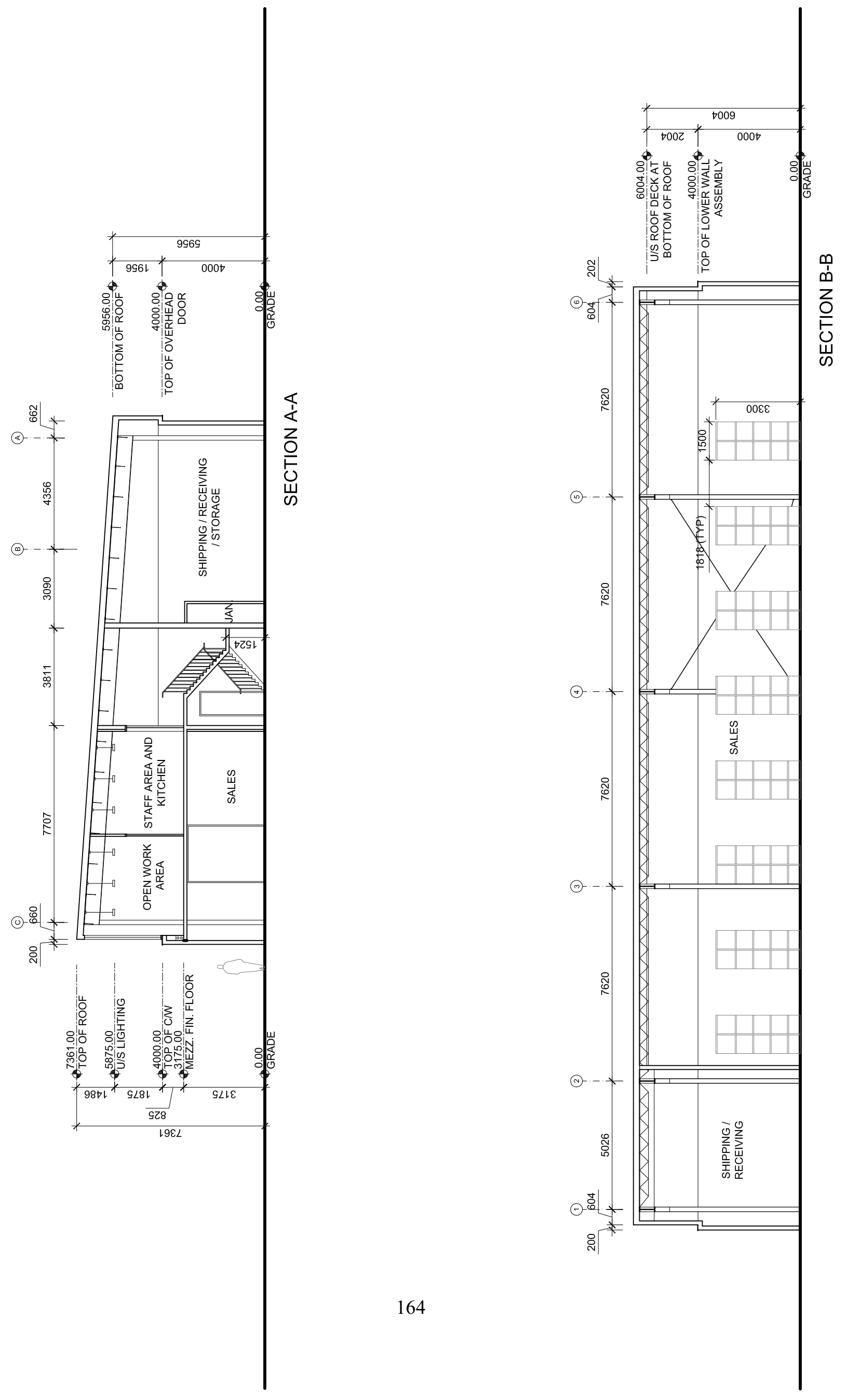



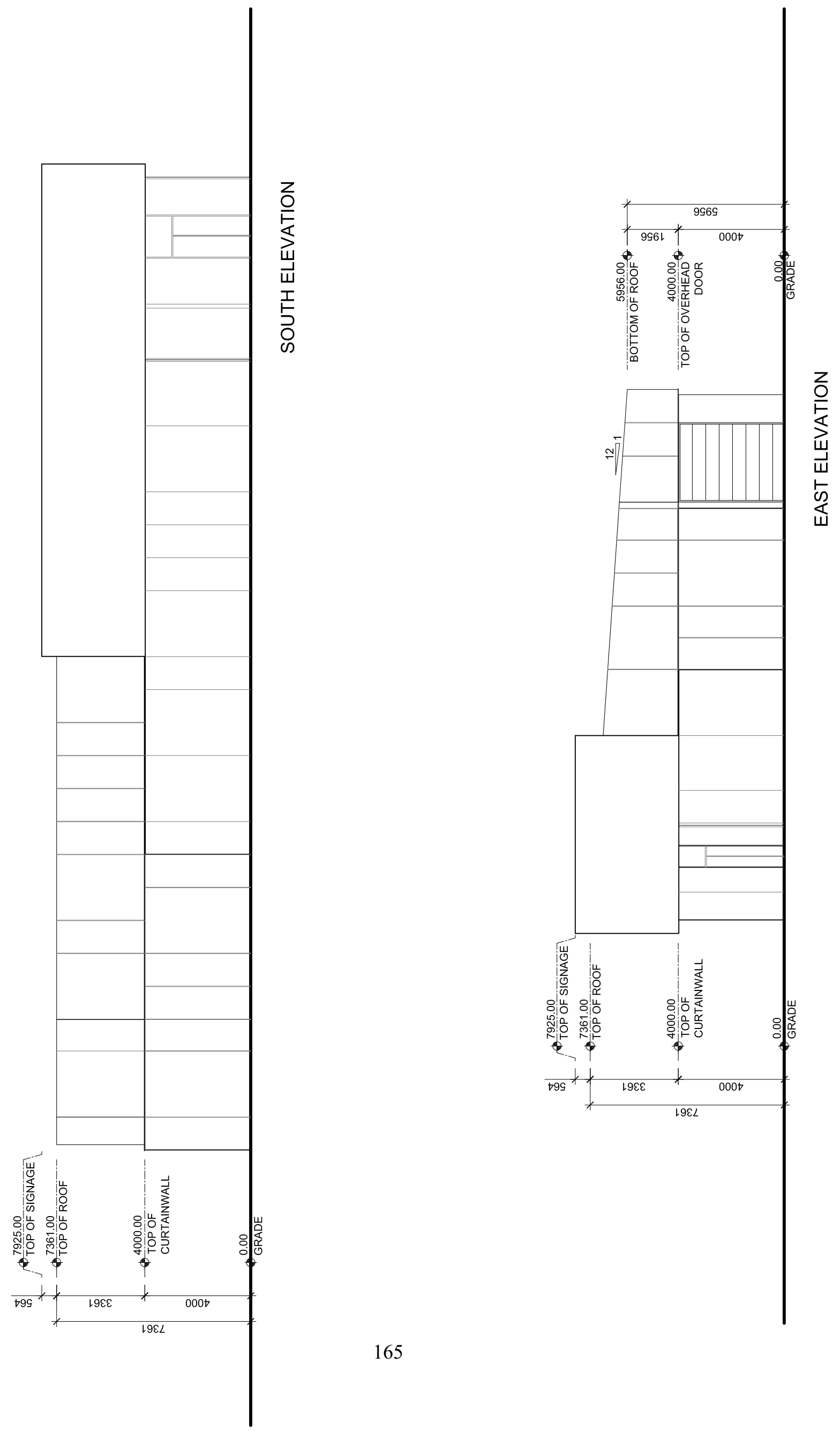

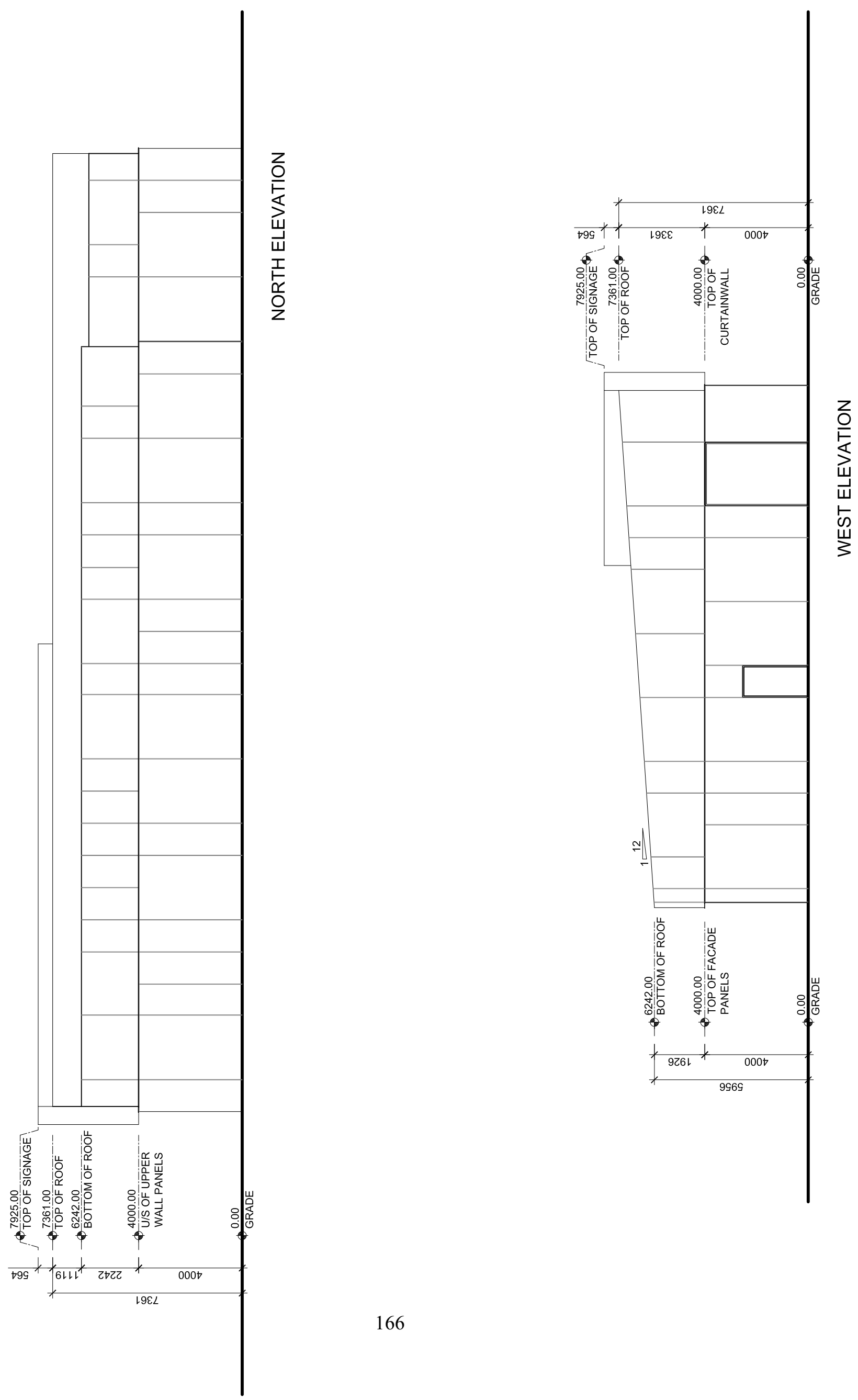

166 


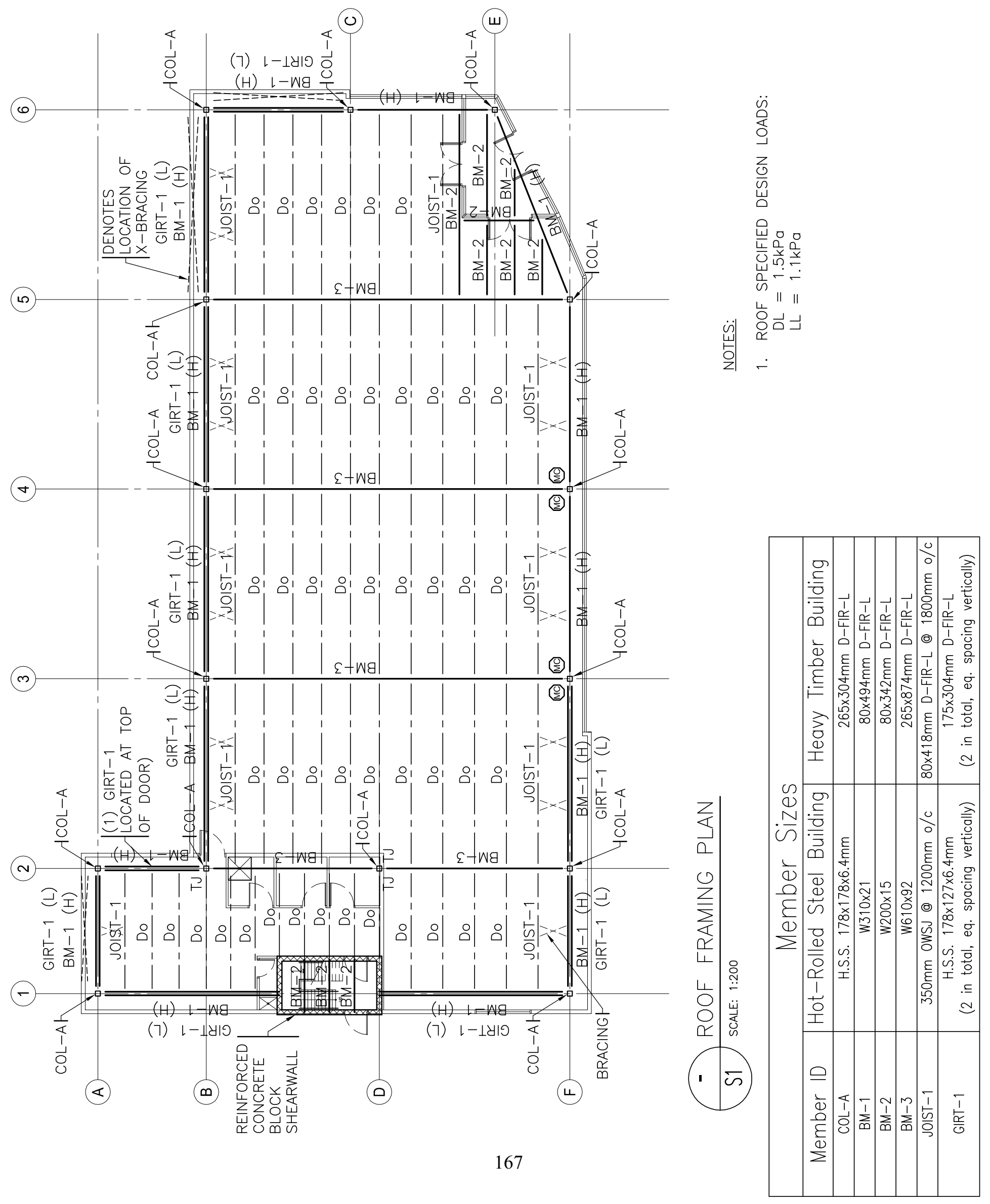




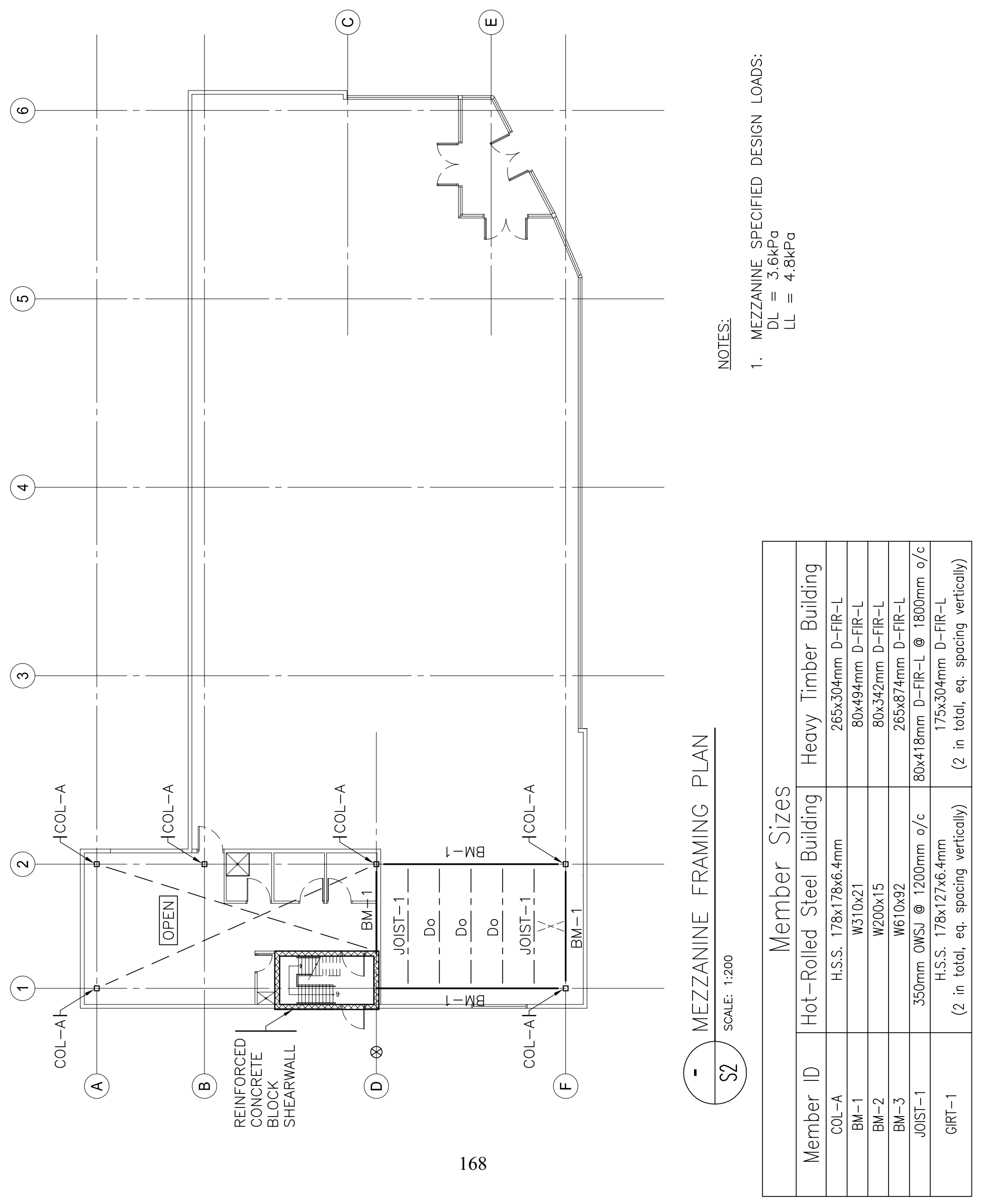



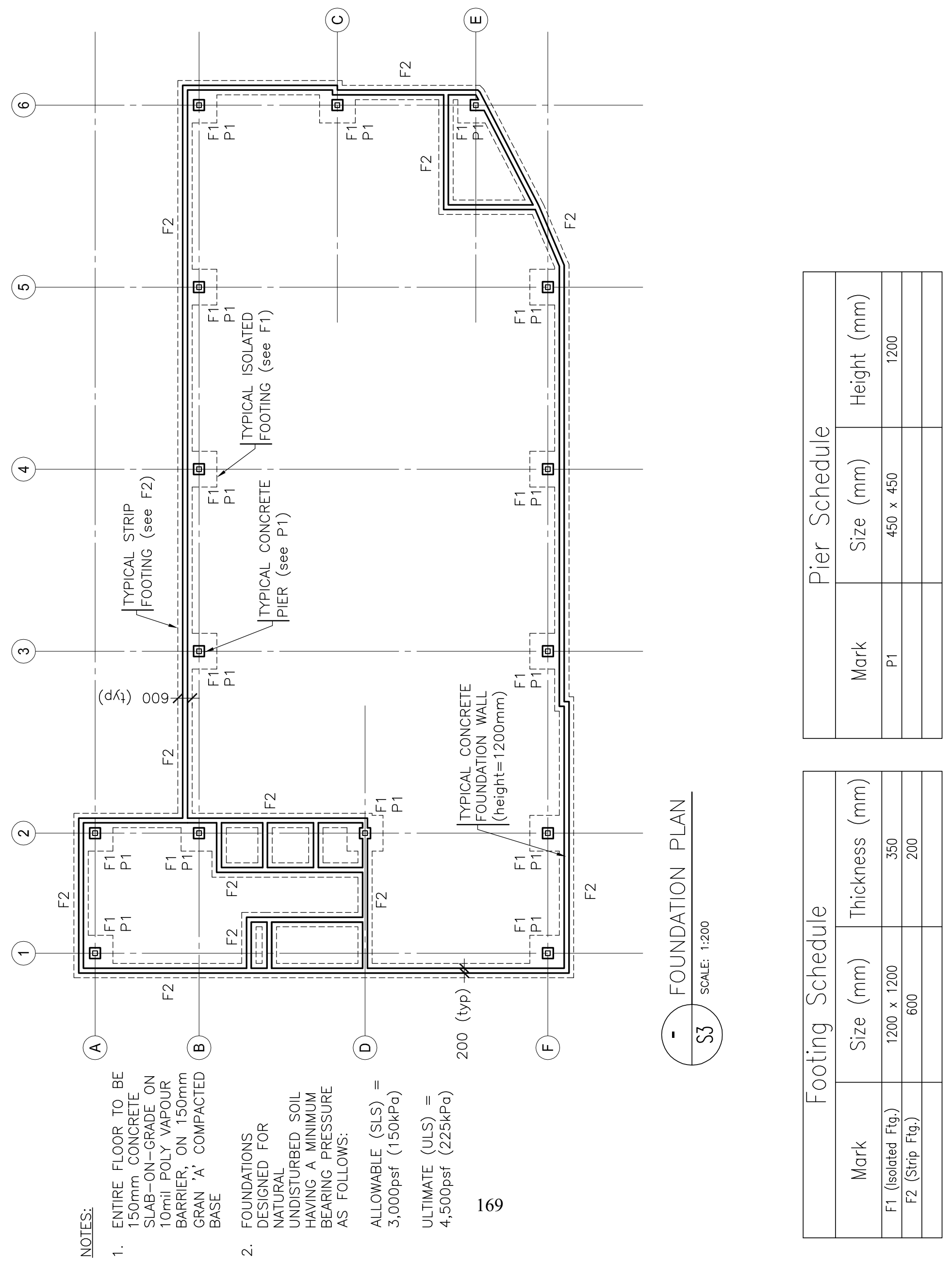


\begin{tabular}{|c|c|}
\hline \multicolumn{2}{|r|}{ General Descriptors } \\
\hline Building Type & - $\quad$ Stand alone retail \\
\hline Location & - Toronto, Ontario, Canada \\
\hline Number of Stories & - 1 storey with mezzanine for offices \\
\hline Gross Floor Area & - $\quad 586 \mathrm{~m}^{2}\left(6,300 \mathrm{ft}^{2}\right)$ \\
\hline Gross Dimensions & $\begin{array}{l}\text { - Length x width (not including shipping and receiving area): } 36.8 \mathrm{~m} \mathrm{x} 15.8 \mathrm{~m} \\
\text { (120.7 ft x } 51.8 \mathrm{ft}) \\
\text { - } \quad \text { Mid-height of roof: } 6.7 \mathrm{~m}(22.0 \mathrm{ft}) \\
\text { - } \quad \text { Floor to ceiling height: } 5.6 \mathrm{~m}(18.4 \mathrm{ft})\end{array}$ \\
\hline Hours of Occupancy & $\begin{array}{l}\text { - } \quad \text { Monday to Saturday 8am to } 9 \mathrm{pm} \\
\text { Sunday 9am to 6pm } \\
\text { - Closed during statutory holidays }\end{array}$ \\
\hline Building Orientation & - $\quad$ Long dimension aligned along E-W axis \\
\hline Roof Slope & - $\quad$ Mono-slope roof with 1:12 pitch \\
\hline $\begin{array}{l}\text { Percentage of Gross } \\
\text { Floor Area by Activity } \\
\text { Type }\end{array}$ & 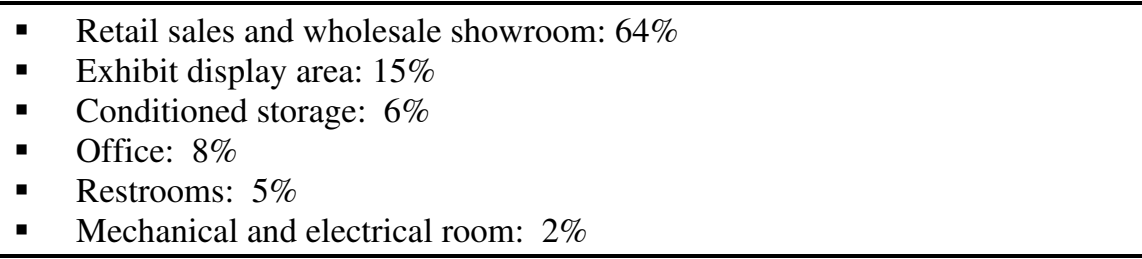 \\
\hline $\begin{array}{l}\text { Design Maximum } \\
\text { Occupancy by Activity } \\
\text { Type }\end{array}$ & $\begin{array}{l}\text { - } \text { Retail sales and wholesale showroom: } 27.9 \mathrm{~m}^{2} / \text { person }\left(300 \mathrm{ft}^{2} / \text { person }\right) \\
\text { - } \text { Exhibit display area: } 4.6 \mathrm{~m}^{2} / \text { person }\left(50 \mathrm{ft}^{2} / \text { person }\right) \\
\text { - } \text { Conditioned storage: } 46.5 \mathrm{~m}^{2} / \text { person }\left(500 \mathrm{ft}^{2} / \text { person }\right) \\
\text { - } \text { Office: } 18.6 \mathrm{~m}^{2} / \text { person }\left(200 \mathrm{ft}^{2} / \text { person }\right) \\
\text { - } \text { Restrooms: } 27.9 \mathrm{~m}^{2} / \text { person }\left(300 \mathrm{ft}^{2} / \text { person }\right) \\
\text { Mechanical and electrical room: } 185.9 \mathrm{~m}^{2} / \text { person }\left(2,000 \mathrm{ft}^{2} / \text { person }\right)\end{array}$ \\
\hline $\begin{array}{l}\text { Infiltration } \\
\text { (Shell Air Tightness) }\end{array}$ & $\begin{array}{l}\text { - } \quad \text { Core zone: } 5.08 \times 10^{-6} \mathrm{~m}^{3} / \mathrm{s} / \mathrm{m}^{2}\left(0.001 \mathrm{cfm} / \mathrm{ft}^{2}\right) \\
\text { - } \\
\end{array}$ \\
\hline Limitations & $\begin{array}{l}\text { - Shelving, furniture, and retail merchandise not accounted for in models } \\
\text { Mechanical equipment not accounted for in embodied energy (and GWP) } \\
\text { calculations }\end{array}$ \\
\hline
\end{tabular}

\begin{tabular}{|c|c|}
\hline \multicolumn{2}{|r|}{ Structural Design Assumptions } \\
\hline Structural Loads & 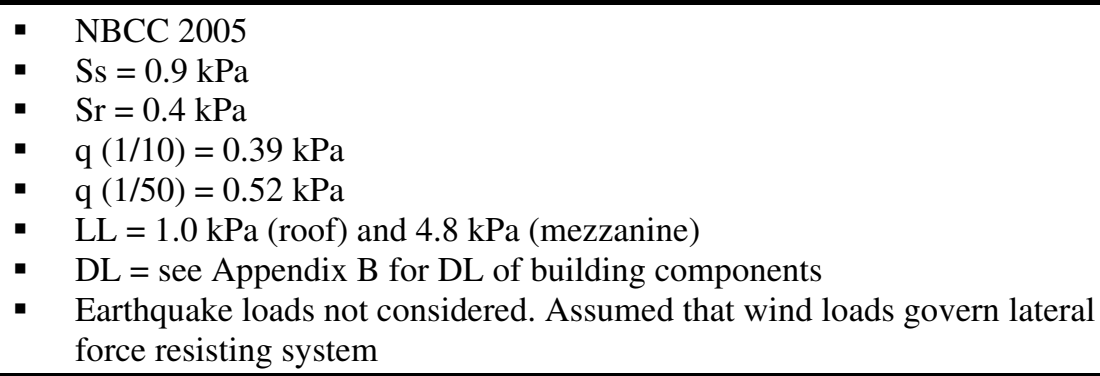 \\
\hline Building Codes & $\begin{array}{ll} & \text { Hot-Rolled Steel: CAN/CSA S16-01 } 9^{\text {th }} \text { ed. } \\
\text { - } & \text { Cold-Formed Steel: CSA S136-07 } \\
\text { - } & \text { Concrete: CSAN/CSA-086-01 } \\
\end{array}$ \\
\hline Deflections & $\begin{array}{l}\text { - Max allowable roof deflection: L/240 } \\
\text { - Max allowable horizontal deflection of building: } 64 \mathrm{~mm} \text { (2.5 in) }\end{array}$ \\
\hline
\end{tabular}




\section{Structural Design Assumptions (Cont.)}

\begin{tabular}{|c|c|}
\hline Columns & $\begin{array}{l}\text { Height }=7.4 \mathrm{~m}(24.3 \mathrm{ft}) \\
\text { - } \\
\text { Pin-pin connections assumed } \\
\text { Bearing not considered } \\
\text { minimum connection widths, typical practice, and least weight per } \mathrm{m}\end{array}$ \\
\hline Beams & $\begin{array}{l}\text { - } \quad \text { Length = varies (see drawings) } \\
\text { - } \quad \text { Bimply supported pin-pin connections assumed } \\
\text { connection widths, typical practice, and least weight per m }\end{array}$ \\
\hline $\begin{array}{l}\text { Lateral Force Resisting } \\
\text { System }\end{array}$ & $\begin{array}{l}\text { - Combination of reinforced concrete masonry unit stair tower and 300W steel } \\
\text { rod x-bracing (i.e. braced frame system) }\end{array}$ \\
\hline
\end{tabular}

\begin{tabular}{|c|c|}
\hline \multicolumn{2}{|r|}{ Description of Building Components } \\
\hline Exterior Infill Walls & - $\quad$ [BASE-W] - CFS studs @ 400 mm o/c with EIFS (see Appendix B) \\
\hline Roofs & $\begin{array}{l}\text { - } \quad \text { [BASE-R]-OWSJ @ 1,200 mm o/c and metal deck with continuous } \\
\text { polyisocyanurate insulation and 4-ply built-up asphalt roof assembly (see } \\
\text { Appendix B) } \\
\text { - No roof overhang assumed }\end{array}$ \\
\hline Structure & $\begin{array}{l}\text { - }[\mathrm{S}-1]-\text { Conventional braced steel frame (H.S.S. columns and W-section } \\
\text { beams) (see Appendix B) }\end{array}$ \\
\hline Mezzanine Floor & $\begin{array}{l}\text { - } \quad \text { Mezzanine includes office space (not modeled in eQUEST) } \\
\text { - } \quad \text { FL-3] - OWSJ @ 1,200 mm o/c and metal deck with concrete topping, vinyl } \\
\text { floor tile finish, and drywall ceiling (see Appendix B) }\end{array}$ \\
\hline Windows & $\begin{array}{l}\text { - }[\mathrm{W}-1] \text { - Aluminum window frame with thermal break, typical double glazing } \\
\text { unit with airspace, no low-E coating, no argon, fixed (see Appendix B) } \\
\text {-W-9] - self-supported aluminum curtainwall system with thermal break, } \\
\text { double glazing unit with airspace, no low-E coating, no argon (see Appendix } \\
\text { B) } \\
\text { - Window-to-wall ratio: } 17 \% \\
\text { - Typical window overhang }=0.3 \mathrm{~m}(1.0 \mathrm{ft} \text { ) } \\
\text { - No window blinds or drapes to provide shade }\end{array}$ \\
\hline Doors & $\begin{array}{l}\text { - } \quad \text { D-2] - Insulated steel exterior door with no glazing (see Appendix B) } \\
\text { - } \quad \text { D-3] - Uninsulated aluminum exterior door with } 80 \% \text { glazing (see } \\
\text { Appendix B) } \\
\text { - } \\
\text { - Ap-4] - Insulated sectional overhead steel door with no glazing (see } \\
\text { - } \quad[\mathrm{D}-6]-\text { Steel interior door with no glazing (see Appendix B) }\end{array}$ \\
\hline Interior Partitions & $\begin{array}{l}\text { - } \quad \text { CMU-P1] for stair tower - Reinforced } 200 \mathrm{~mm} \text { concrete masonry unit wall } \\
\text { (see Appendix B) } \\
\text { - } \\
\text { (SS-P2] elsewhere - CFS studs @ } 600 \mathrm{~mm} \text { o/c with two layers of drywall } \\
\text { (see Appendix B) }\end{array}$ \\
\hline
\end{tabular}




\section{Description of Building Components (Cont.)}

- Assume natural undisturbed soil with minimum bearing pressure of:

- $\quad$ Allowable (SLS) $=150 \mathrm{kPa}(3,000 \mathrm{psf})$

- $\quad$ Ultimate $(\mathrm{ULS})=215 \mathrm{kPa}(4,500 \mathrm{psf})$

- Design load for isolated footings $=150 \mathrm{kN}$ (34 kips)

- All isolated footings assumed to be the same size for simplicity

Foundations

- [SOG-5] - $200 \mathrm{~mm}$ thick concrete slab, $30 \mathrm{MPa}$, average flyash content, 10 mil poly vapor barrier, earth contact (see Appendix B)

- $\quad$ [IF-1]-1,200 mm x 1,200 mm x $350 \mathrm{~mm}$ isolated footing with $450 \mathrm{~mm} \times 450$ mm x 1,200 mm concrete pier, $20 \mathrm{MPa}$, average flyash content (see Appendix B)

- [PF-5]-600 mm $200 \mathrm{~mm}$ strip footing with 1,200 mm $x 200 \mathrm{~mm}$ concrete foundation wall, $20 \mathrm{MPa}$, average flyash content (see Appendix B)

\begin{tabular}{l|l}
\hline Finish Materials & - As per building components in Appendix B \\
\hline
\end{tabular}

\section{Mechanical Systems}

\begin{tabular}{l|ll}
\hline Hours of Operation & - $\begin{array}{l}\text { Mechanical systems operate } 1 \text { hour before and } 1 \text { hour after hours of } \\
\text { occupancy } \\
\text { Off during statutory holidays }\end{array}$ \\
\hline
\end{tabular}

HVAC

\begin{tabular}{|c|c|}
\hline Cooling Equipment & - $\quad$ Direct expansion (DX) coils (electric) \\
\hline Heating Equipment & - Combustion furnace (natural gas) \\
\hline System Type & $\begin{array}{l}\text { - Packaged single zone DX with furnace (central packaged single zone air } \\
\text { conditioner with combustion furnace) } \\
\text { - Ducted return air }\end{array}$ \\
\hline Thermostat Set-Points & $\begin{array}{l}\text { - Occupied spaces: Cool to } 24.4^{\circ} \mathrm{C}\left(76.0^{\circ} \mathrm{F}\right), \text { Heat to } 21.1^{\circ} \mathrm{C}\left(70.0^{\circ} \mathrm{F}\right) \\
\text { - } \quad \text { Unoccupied spaces: Cool to } 27.8^{\circ} \mathrm{C}\left(82.0^{\circ} \mathrm{F}\right) \text {, Heat to } 17.8^{\circ} \mathrm{C}\left(64.0^{\circ} \mathrm{F}\right)\end{array}$ \\
\hline Air Flow & - $\quad$ Minimum design air flow: $2.54 \times 10^{-3} \mathrm{~m}^{3} / \mathrm{s} / \mathrm{m}^{2}\left(0.50 \mathrm{cfm} / \mathrm{ft}^{2}\right)$ \\
\hline Zoning & $\begin{array}{l}\text { - } \quad 0 \% \text { core zone } \\
\text { - } \quad 100 \% \text { perimeter zone }\end{array}$ \\
\hline
\end{tabular}

\section{Hot Water}

- Heater fuel: natural gas

- Heater type: storage

DHW Equipment - Hot water use: $1.9 \mathrm{~L} /$ person/day $(0.50 \mathrm{gal} / \mathrm{person} /$ day $)$

- Supply water temperature: $57.2^{\circ} \mathrm{C}\left(135.0^{\circ} \mathrm{F}\right)$

- Inlet water temperature: equal to ground temperature 


\begin{tabular}{l|c|c|c|c}
\hline \multicolumn{1}{c|}{ Load Profiles by Space } \\
\hline $\begin{array}{l}\text { Space } \\
\text { wholesil sales and }\end{array}$ & $\begin{array}{c}\text { Interior Lighting } \\
\mathrm{W} / \mathrm{m}^{2}\left(\mathrm{~W} / \mathrm{ft}^{2}\right)\end{array}$ & $\begin{array}{c}\text { Office Equipment } \\
\mathrm{W} / \mathrm{m}^{2}\left(\mathrm{~W} / \mathrm{ft}^{2}\right)\end{array}$ & $\begin{array}{c}\text { Misc. Electric } \\
\text { Loads } \\
\mathrm{W} / \mathrm{m}^{2}\left(\mathrm{~W} / \mathrm{ft}^{2}\right)\end{array}$ & $\begin{array}{c}\text { Misc. Natural Gas } \\
\text { Loads W/m } \\
\left(\mathrm{Btuh} / \mathrm{ft}^{2}\right)\end{array}$ \\
\hline Exhibit display area & $11.08(1.03)$ & - & $2.69(0.25)$ & - \\
\hline Conditioned storage & $12.80(1.19)$ & - & $2.69(0.25)$ & - \\
\hline Office & $13.34(1.24)$ & $5.0(0.46)$ & $8.07(0.75)$ & - \\
\hline Restrooms & $8.28(0.77)$ & - & $1.08(0.10)$ & - \\
\hline $\begin{array}{l}\text { Mechanical and } \\
\text { electrical room }\end{array}$ & $8.71(0.81)$ & - & $1.08(0.10)$ & - \\
\hline
\end{tabular}

Additional Loads/Notes

\begin{tabular}{l|cl}
\hline Building Occupants & - & Average occupant heat gain of $132 \mathrm{~W} /$ person \\
\hline Exterior Lighting & - & Not considered in models \\
\hline Daylight Controls & - & No daylighting controls \\
\hline
\end{tabular}




\section{Appendix B}

\section{Life-Cycle Assessment Data for Building Components}

This Appendix contains detailed LCA data for the 220 different building components that were examined in this study. For each building component, there is a detailed description of the assembly, a summary of the important LCA data, as well as a detailed list of the material quantities that were assumed.

Appendix B is divided into the following sub-sections:

- Appendix B-1: Exterior Infill Walls (pg 175)

- Appendix B-2: Roofs (pg 235)

- Appendix B-3: Structural Systems (pg 269)

- Appendix B-4: Floors (pg 272)

- Appendix B-5: Windows and Doors (pg 276)

- Appendix B-6: Foundations (pg 286)

- Appendix B-7: Interior Partition Walls (pg 299)

For each building component group in this Appendix, there is a brief introduction as well as a graph that summaries the embodied energy and embodied GWP of the relevant building components. When reading this Appendix, it may be useful to reference the table below, which lists common thickness and gauges.

Common Thicknesses and Gauges

\begin{tabular}{c|c|c}
\hline Gauge & $\begin{array}{c}\text { Nominal } \\
\text { Thickness } \\
(\mathrm{mm})\end{array}$ & $\begin{array}{c}\text { Nominal } \\
\text { Thickness } \\
\text { (in) }\end{array}$ \\
\hline 8 & 4.176 & 0.164 \\
\hline 10 & 3.416 & 0.135 \\
\hline 12 & 2.657 & 0.105 \\
\hline 14 & 1.897 & 0.075 \\
\hline 16 & 1.591 & 0.060 \\
\hline 18 & 1.214 & 0.048 \\
\hline
\end{tabular}

\begin{tabular}{c|c|c}
\hline Gauge & $\begin{array}{c}\text { Nominal } \\
\text { Thickness } \\
(\mathrm{mm})\end{array}$ & $\begin{array}{c}\text { Nominal } \\
\text { Thickness } \\
\text { (in) }\end{array}$ \\
\hline 20 & 0.912 & 0.036 \\
\hline 22 & 0.759 & 0.030 \\
\hline 24 & 0.607 & 0.024 \\
\hline 25 & 0.531 & 0.021 \\
\hline 26 & 0.455 & 0.018 \\
\hline 28 & 0.378 & 0.015 \\
\hline 29 & 0.343 & 0.014 \\
\hline
\end{tabular}


Appendix B-1

LCA Data for Exterior Infill Walls 


\section{LCA Data for Concrete Masonry Unit Walls}

This section contains a detailed description of each concrete masonry unit (CMU) exterior infill wall that was examined in this study (18 in total). The assembly layers are listed for each wall, along with a detailed description of the material quantities from the ATHENA® Environmental Impact Estimator for Buildings.

A breakdown of the total primary energy consumption and the total global warming potential (GWP) for each wall is also included. In each case, the results were calculated for an area of wall equal to $50.9 \mathrm{~m}^{2}$, which represents a typical bay size for a single-storey retail building. The results are also expressed on a per $\mathrm{m}^{2}$ basis in each case. The data has been calculated for two different lifespans: at the completion of initial construction and after 50 years.

As a summary, the figure below illustrates a comparison of the total embodied energy (and GWP) after 50 years for the various walls in this section. For comparison purpose, the building component used in the baseline retail building has been shaded in grey.

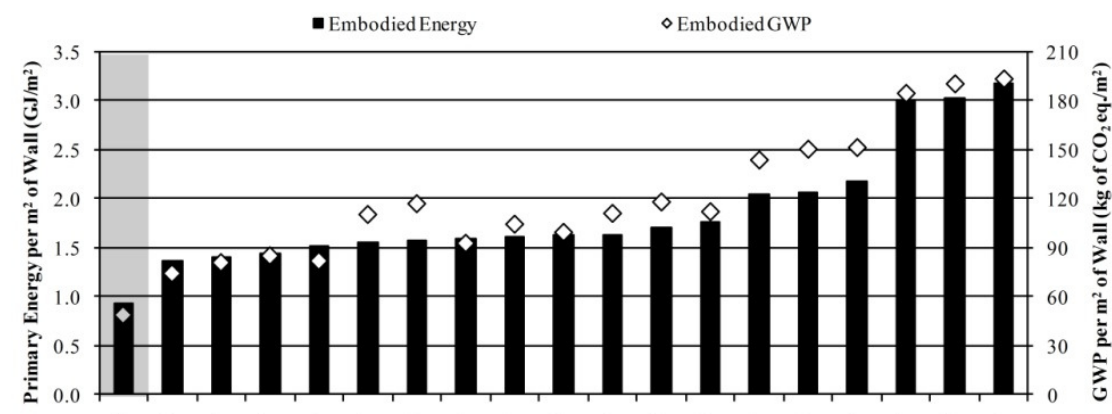

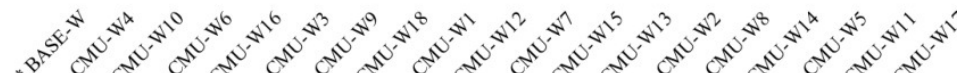
Exterior Infill Wall ID (Arranged in Order of Increasing Embodied Energy)

\section{Concrete Masonry Unit Wall \#1 (CMU-W1)}

Building Component Description:

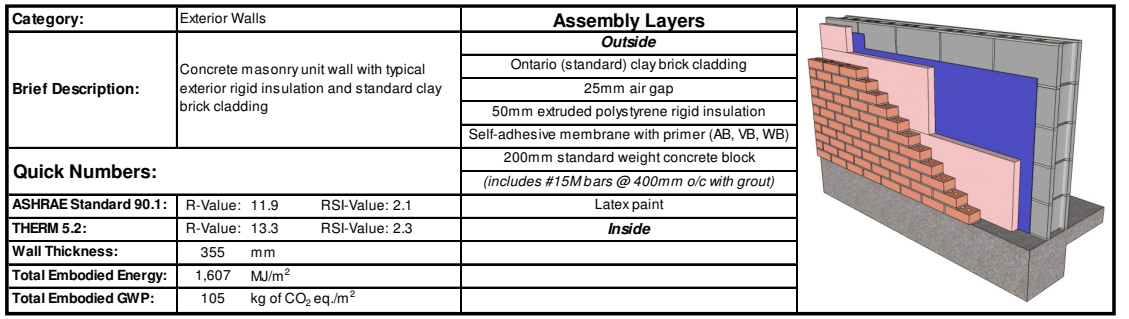

Life-Cycle Assessment Results:

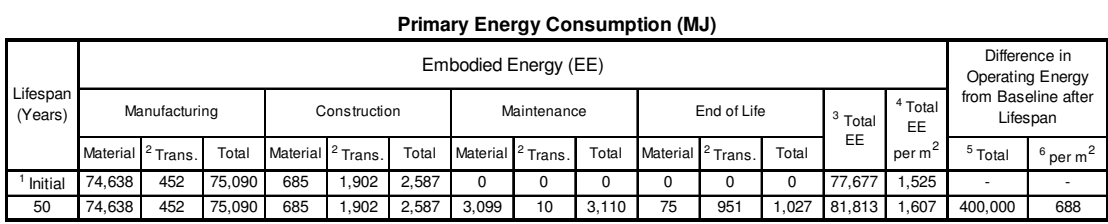

Global Warming Potential ( $\mathrm{kg}$ of $\mathrm{CO}_{2}$ eq.)

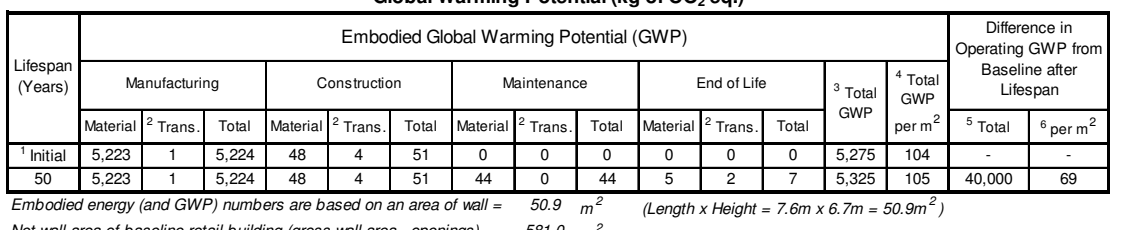

Net wall area of baseline retil building (gross wall area- openings) $=581.0 \mathrm{~m}^{2}$

ATHENA $®$ EIE Material List:

(Includes all materials after 50 years)

\begin{tabular}{|l|c|c|}
\hline \multicolumn{1}{|c|}{ Material List } & Quantities & Unit \\
\hline 3 mil Polyethylene & 54.0 & $\mathrm{~m} 2$ \\
\hline Cold Rolled Sheeet & 10.3 & $\mathrm{~kg}$ \\
\hline Concrete Blocks & 648.0 & $\mathrm{Blocks}$ \\
\hline Extruded Polystyrene & 104.3 & $\mathrm{~m} 2(25 \mathrm{~mm})$ \\
\hline Modififed Bitumen membrane & 68.2 & $\mathrm{~kg}$ \\
\hline Mortar & 3.5 & $\mathrm{~m} 3$ \\
\hline Nails & 3.1 & $\mathrm{~kg}$ \\
\hline Ontario (Standard) Brick & 53.5 & $\mathrm{~m} 2$ \\
\hline Rebar, Rod, Light Sections & $1,092.8$ & $\mathrm{~kg}$ \\
\hline Solvent Based Alkyd Paint & 19.6 & $\mathrm{~L}$ \\
\hline Water Based Latex Paint & 66.3 & $\mathrm{~L}$ \\
\hline
\end{tabular}

Notes:
${ }^{1}$ Initial $=$ Time ' 0 ' (i.e. at the completion of initial construction)

${ }^{2}$ Trans. $=$ Transportation

${ }^{3}$ Total EE (or Total GWP) $=$ Total embodied energy (or total embodied GWP of building component after lifespan (i.e. total mal
construction + total maintenance + total enc-of life effects)

${ }^{4}$ Total EE (or Total GWP) per $\mathrm{m}^{2}=$ Total EE (or Total GWP) of building
component / area of building component that was modelled in ATHENAQ EIE ${ }^{5}$ Total Difference in Operating Energy (or GWP) from Baseline after Lifespan = The interence in the total ifiecycle eperating energy (or GWP component instead of the baseline component

${ }^{6}$ Total Difference in Operating Energy (or GWP) from Baseline after Lifespan per $\mathrm{m}^{2}=$ Total difference in operating energy (or GWP) from

* Total operating primary energy use of baseline retail building after 50 years
$50,700 \mathrm{GJ}\left(1,745 \mathrm{M} / \mathrm{m} \mathrm{m}^{2} \mathrm{yr}\right)$

* Total operating $\mathrm{GWP}$ of baseline retal
of $\mathrm{CO}_{2}$ eq. $\left(80 \mathrm{~kg}\right.$ of $\mathrm{CO}_{2}$ eq. $\left./ \mathrm{m}^{2} \mathrm{yr}\right)$ 


\section{Concrete Masonry Unit Wall \#2 (CMU-W2)}

Building Component Description:

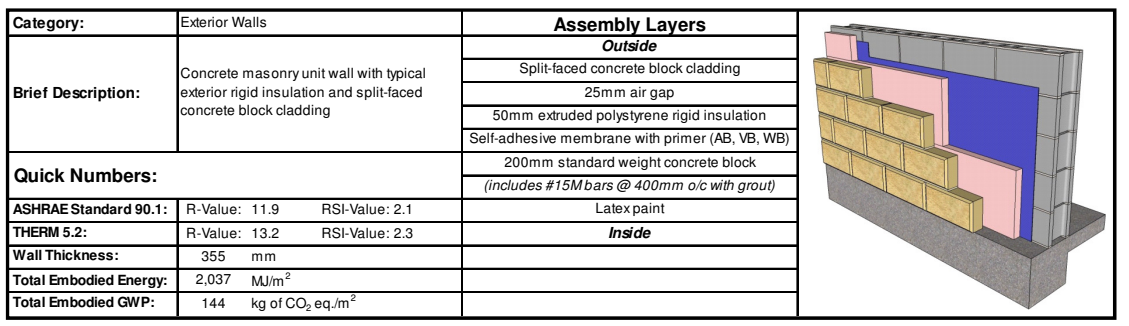

Life-Cycle Assessment Results:

Primary Energy Consumption (MJ)

\begin{tabular}{|c|c|c|c|c|c|c|c|c|c|c|c|c|c|c|c|c|}
\hline \multirow{3}{*}{\begin{tabular}{|l} 
Lifespan \\
(vears)
\end{tabular}} & \multicolumn{14}{|c|}{ Embodied Energy (EE) } & \multirow{2}{*}{\multicolumn{2}{|c|}{$\begin{array}{c}\text { Difference in } \\
\text { Operating Energy } \\
\text { from Baseline after } \\
\text { Lifespan }\end{array}$}} \\
\hline & \multicolumn{3}{|c|}{ Manufacturing } & \multicolumn{3}{|c|}{ Construction } & \multicolumn{3}{|c|}{ Maintenance } & \multicolumn{3}{|c|}{ End of L Life } & \multirow{2}{*}{$\begin{array}{c}{ }^{3} \text { Total } \\
\mathrm{EE}\end{array}$} & \multirow{2}{*}{\begin{tabular}{|c}
${ }^{4}{ }^{4}$ Total \\
$\mathrm{EE}$ \\
per m
\end{tabular}} & & \\
\hline & Material & ${ }^{2}$ Trans. & \begin{tabular}{|l|l|} 
Total \\
\end{tabular} & \begin{tabular}{|l|} 
Material \\
\end{tabular} & ${ }^{2}$ Trans. & Total & \begin{tabular}{|l|} 
Material \\
\end{tabular} & ${ }^{2}$ Trans. & Total & \begin{tabular}{|l|} 
Material \\
\end{tabular} & $\mid{ }^{2}$ Trans. & \begin{tabular}{|l|l|} 
Total \\
\end{tabular} & & & ${ }^{5}$ Total & ${ }^{6}$ per m ${ }^{2}$ \\
\hline Initial & 93,666 & 1,015 & 94,681 & 685 & 2,713 & \begin{tabular}{|l|l|}
3,397 \\
\end{tabular} & 0 & 0 & 0 & 0 & 0 & 0 & 98,078 & \begin{tabular}{|l|}
1,926 \\
\end{tabular} & & \\
\hline 50 & \begin{tabular}{|l|l|}
33,666 \\
\end{tabular} & \begin{tabular}{|l|l|}
1,015 \\
\end{tabular} & \begin{tabular}{|l|l|}
94,681 \\
\end{tabular} & 685 & \begin{tabular}{|l|l|}
2,713 \\
\end{tabular} & \begin{tabular}{|l|l|}
3,397 \\
\end{tabular} & 3,099 & 10 & \begin{tabular}{|l|l|}
3,110 \\
\end{tabular} & 76 & \begin{tabular}{l|l}
2,477 \\
\end{tabular} & 2,553 & \begin{tabular}{|l|l|}
103,7 \\
\end{tabular} & \begin{tabular}{|l|l|} 
& 2,037 \\
\end{tabular} & 400,000 & 688 \\
\hline
\end{tabular}

Global Warming Potential ( $\mathrm{kg}$ of $\mathrm{CO}_{2}$ eq.)

ฟ

\begin{tabular}{|c|c|c|c|c|c|c|c|c|c|c|c|c|c|c|c|c|}
\hline \multirow{3}{*}{$\begin{array}{c}\text { Lifespan } \\
\text { (Years) }\end{array}$} & \multicolumn{14}{|c|}{ Embodied Global Warming Potential (GWP) } & \multirow{2}{*}{\multicolumn{2}{|c|}{$\begin{array}{c}\text { Difference in } \\
\text { Operating GWP from } \\
\text { Baseline after } \\
\text { Lifespan }\end{array}$}} \\
\hline & \multicolumn{3}{|c|}{ Manufacturing } & \multicolumn{3}{|c|}{ Construction } & \multicolumn{3}{|c|}{ Maintenance } & \multicolumn{3}{|c|}{ End of Life } & \multirow{2}{*}{$\begin{array}{l}{ }^{3} \text { Total } \\
\text { GWP }\end{array}$} & \multirow{2}{*}{$\begin{array}{l}{ }^{4} \text { Total } \\
\text { GWP } \\
\text { per m}{ }^{2}\end{array}$} & & \\
\hline & Material & Trans. & Total & Material & Trans. & Total & Material & ${ }^{2}$ Trans. & Total & Material & ${ }^{2}$ Trans. & Total & & & ${ }^{5}$ Total & ${ }^{6} \operatorname{per~m}^{2}$ \\
\hline Initial & 7,209 & 2 & 7,210 & 48 & 5 & 53 & 0 & 0 & 0 & 0 & $\overline{0}$ & 0 & 7,263 & 143 & & \\
\hline 50 & 7,209 & 2 & 7,210 & 48 & 5 & 53 & 44 & 0 & 44 & 5 & 5 & 10 & 7,317 & 144 & 40,000 & 69 \\
\hline
\end{tabular}

ATHENA ® EIE Material List:

\begin{tabular}{|l|c|c|}
\multicolumn{1}{|c|}{ Material List } & Quantities & Unit \\
\hline 3 mil Polyethylene & 54.0 & $\mathrm{~m} 2$ \\
\hline Cold Rolled Sheet & 10.3 & $\mathrm{~kg}$ \\
\hline Concrete Blocks & 648.0 & Blocks \\
\hline Extruded Polystyrene & 104.3 & $\mathrm{~m} 2(25 \mathrm{~mm})$ \\
\hline Modified Bitumen membrane & 401.5 & $\mathrm{~kg}$ \\
\hline Mortar & 6.4 & $\mathrm{~m} 3$ \\
\hline Nails & 3.1 & $\mathrm{~kg}$ \\
\hline Rebar, Rod, Light Sections & $1,092.8$ & $\mathrm{~kg}$ \\
\hline Solvent Based Alkyd Paint & 19.6 & $\mathrm{~L}$ \\
\hline Spplit-faced Concrete Block & $1,238.2$ & Blocks \\
\hline Water Based Latex Paint & 66.3 & $\mathrm{~L}$ \\
\hline
\end{tabular}

Notes:
${ }^{1}$ Initial $=$ Time 'o' (i.e. at the completion of initial construction)

${ }^{2}$ Trans. = Transportation

${ }^{3}$ Total EE (or Total GWP) $=$ Total embodied energy (or total embodied GWP) of building component after lifespan (i.e. total man
construction + total maintenance + total end-of-life effects)

${ }^{4}$ Total EE (or Total GWP) per $\mathrm{m}^{2}=$ Total EE (or Total GWP) of building
component/ area of building component that was modelled in ATHENA@ EIE

${ }^{5}$ Total Difference in Operating Energy (or GWP) from Baseline after Lifespan = The difference in the total lifeccycle operating energy (or GWP)
from the baseline retail building after lifespan, due to using this building
component instead of the baseline component

${ }^{6}$ Total Difference in Operating Energy (or GWP) from Baseline after Lifespan per $\mathrm{m}^{2}=$ Total difference in operating energy (or GWP) from

* Total operating primary energy use of baseline retail building after 50 years =
$50,700 \mathrm{GJ}\left(1,745 \mathrm{MJ} / \mathrm{m}^{2} / \mathrm{yr}\right)$

of $\mathrm{CO}_{2}$ eq. $\left(80 \mathrm{~kg}\right.$ of $\mathrm{CO}_{2}$ eq./m? $\mathrm{m}^{2} \mathrm{yr}$ )

\section{Concrete Masonry Unit Wall \#3 (CMU-W3)}

Building Component Description:

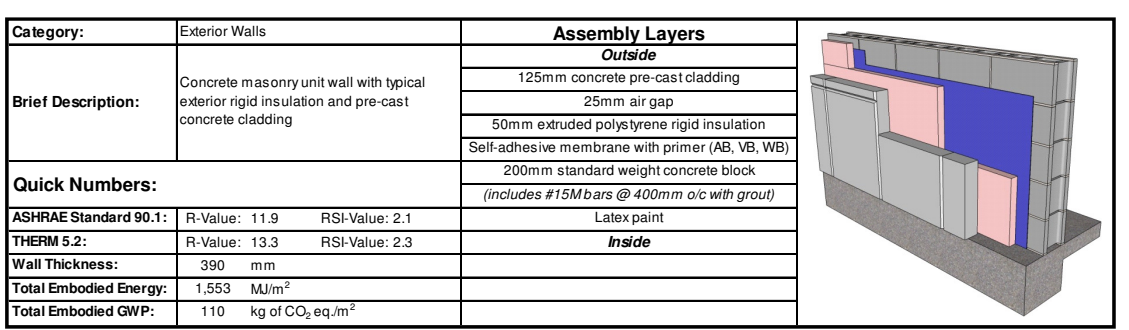

Life-Cycle Assessment Results:

Primary Energy Consumption (MJ)

\begin{tabular}{|c|c|c|c|c|c|c|c|c|c|c|c|c|c|c|c|c|}
\hline \multirow{3}{*}{ (Years } & \multicolumn{14}{|c|}{ Embodied Energy (EE) } & \multirow{2}{*}{\multicolumn{2}{|c|}{$\begin{array}{l}\text { Difference in } \\
\text { Operating Energy } \\
\text { from Baseline after } \\
\text { Lifiespan }\end{array}$}} \\
\hline & \multicolumn{3}{|c|}{ Manufacturing } & \multicolumn{3}{|c|}{ Cons } & \multicolumn{3}{|c|}{ Maintenance } & \multicolumn{3}{|c|}{ End of Lifie } & \multirow{2}{*}{$\begin{array}{c}{ }^{3} \text { Total } \\
\text { EE }\end{array}$} & \multirow{2}{*}{$\begin{array}{c}{ }^{4} \text { Total } \\
\text { EE } \\
\text { per } \mathrm{m}^{2}\end{array}$} & & \\
\hline & Material & \begin{tabular}{|l|l|}
${ }^{2}$ Trans. \\
\end{tabular} & Total & Material & ${ }^{2}$ Trans. & Total & Material & ${ }^{2}$ Trans. & Total & Materia & 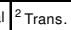 & Total & & & ${ }^{5}$ Tot: & ${ }^{6} \operatorname{perm}^{2}$ \\
\hline & 325 & 1,043 & 72,368 & 685 & 1,842 & 2,526 & 0 & 0 & 0 & 0 & 0 & 0 & 74,894 & 1,471 & & \\
\hline
\end{tabular}

Global Warming Potential (kg of $\mathrm{CO}_{2}$ eq.)

\begin{tabular}{|c|c|c|c|c|c|c|c|c|c|c|c|c|c|c|c|c|}
\hline \multirow{3}{*}{$\begin{array}{l}\text { Lifespana } \\
\text { (Years) }\end{array}$} & \multicolumn{14}{|c|}{ Embodied Global Warming Potential (GWP) } & \multirow{2}{*}{\multicolumn{2}{|c|}{\begin{tabular}{|c} 
Difierence in \\
Operating GWP from \\
Baseline after \\
Lifespan
\end{tabular}}} \\
\hline & \multicolumn{3}{|c|}{ Manutacturing } & \multicolumn{3}{|c|}{ Construction } & \multicolumn{3}{|c|}{ Maintenance } & \multicolumn{3}{|c|}{ End of Life } & \multirow{2}{*}{$\begin{array}{l}{ }^{3} \text { Total } \\
\text { GWP }\end{array}$} & \multirow{2}{*}{$\begin{array}{l}{ }^{4} \text { Total } \\
\text { GWP } \\
\text { perm }\end{array}$} & & \\
\hline & Material & ${ }^{2}$ Trans. & Total & Material & ${ }^{2}$ Trans. & Total & Material & ${ }^{2}$ Trans. & Total & Material & ${ }^{2}$ Trans. & \begin{tabular}{|l|} 
\\
\end{tabular} & & & ${ }^{5}$ Total & ${ }^{6}{ }^{\text {perm }}{ }^{2}$ \\
\hline Initial & 5,523 & 2 & 5,525 & 48 & 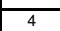 & 51 & 0 & $\Gamma_{1}$ & 0 & 0 & 0 & 0 & 5,576 & 110 & & \\
\hline 50 & \begin{tabular}{|l|l|}
5,523 \\
\end{tabular} & 2 & \begin{tabular}{|l|l|}
5,525 \\
\end{tabular} & 48 & 4 & 51 & 44 & 0 & 44 & 5 & 2 & 7 & 5,626 & 110 & 40,000 & 69 \\
\hline
\end{tabular}

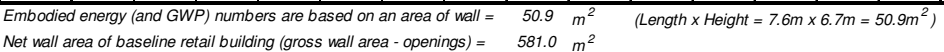

$\begin{gathered}\text { ATHENA } \\
\text { (Includes all materials after 50 years) }\end{gathered}$
\begin{tabular}{|l|c|c|}
\multicolumn{1}{|c|}{ Material List } & Quantities & Unit \\
\hline 3 mil Polyythylene & 54.0 & $\mathrm{~m} 2$ \\
\hline Concrete 30 MPa (flyash avg.) & 6.7 & $\mathrm{~m} 3$ \\
\hline Concrete Blocks & 648.0 & Blocks \\
\hline Extruded Polystyrene & 104.3 & $\mathrm{~m} 2(25 \mathrm{~mm})$ \\
\hline Modified Bitumen membrane & 68.2 & $\mathrm{~kg}$ \\
\hline Mortar & 2.1 & $\mathrm{~m} 3$ \\
\hline Nails & 3.1 & $\mathrm{~kg}$ \\
\hline Rebar, Rod, Light Sections & $1,496.8$ & $\mathrm{~kg}$ \\
\hline Solvent Based Alkyd Paint & 19.6 & $\mathrm{~L}$ \\
\hline Water Based Latex Paint & 66.3 & $\mathrm{~L}$ \\
\hline
\end{tabular}

Notes:
${ }^{1}$ Initial $=$ Time 'o' (i.e. at the completion of initial construction)

${ }^{2}$ Trans. $=$ Transportation

${ }^{3}$ Total EE (or Total GWP) $=$ Total embodied energy (or total embodied GWP) of building component after lifespan (i.e. total man
construction + total maintenance +total end-of-life effects)

${ }^{4}$ Total EE (or Total GWP) per $\mathrm{m}^{2}=$ Total EE (or Total GWP) of building
component / area of building component that was modelled in ATHENAQ EIE ${ }^{5}$ Total Difference in Operating Energy (or GWP) from Baseline after fitespan = The difierence in the total life-cycle operating energy (or GWP
from the baseline retail building after lifespan, due to using this building Total Difference in Operating Energy (or GWP) from Baseline after Lifespan per $m^{2}=$ Total difference in operating energy (or GWP) from

* Total operating primary energy use of baseline retail building after 50 years $=$
$50,700 \mathrm{G} J\left(1,745 \mathrm{MJ}^{2} \mathrm{~m}^{2} \mathrm{yr}\right)$ ${ }^{*}$ Total operating $\mathrm{GWP}$ of baseline re
of $\mathrm{CO}_{2}$ eq. $\left(80 \mathrm{~kg}\right.$ of $\mathrm{CO}_{2}$ eq. $\left./ \mathrm{m}^{2} \mathrm{yr}\right)$ 


\section{Concrete Masonry Unit Wall \#4 (CMU-W4)}

Building Component Description:

\begin{tabular}{|c|c|c|c|}
\hline \multirow{4}{*}{ Brief Description: } & \multirow{4}{*}{$\begin{array}{l}\text { Concrete masonnrunit wall with typical } \\
\text { exterior rigid insulation and pine wood } \\
\text { bevel siding }\end{array}$} & Assembly Layers & \\
\hline & & $\begin{array}{l}\text { Outside } \\
\text { Latex paint }\end{array}$ & \\
\hline & & Pine wood bevel siding & \\
\hline & & 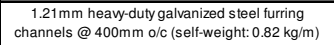 & \\
\hline \multicolumn{2}{|l|}{ Quick Numbers: } & \begin{tabular}{|l|}
$50 \mathrm{~mm}$ extruded polystyrene rigid insulation \\
Selt-adhesive membrane with primer (AB, VB, WB)
\end{tabular} & \\
\hline No Significant Thermal $B$ & Bridge Through Exterior Insulation: & $200 \mathrm{~mm}$ standard weight concrete block & \\
\hline ASHRAEStandard 90.1: & R-Value: 11.9 RSI-Value: 2.1 & (includes \#15Mbars @ 400mm o/c with grout) & \\
\hline THERM 5.2: & R-Value: 13.9 & Latexpaint & \\
\hline Wall Thickness: & $290 \mathrm{~mm}$ & Inside & \\
\hline $\begin{array}{l}\text { Total Embodided Energy: } \\
\text { Totat Embolided GW: }\end{array}$ & $1,365 \quad \mathrm{M} / \mathrm{m}^{2}$ & & \\
\hline
\end{tabular}

\section{Life-Cycle Assessment Results:}

Primary Energy Consumption (MJ)

\begin{tabular}{|c|c|c|c|c|c|c|c|c|c|c|c|c|c|c|c|c|}
\hline \multirow{3}{*}{\begin{tabular}{|c} 
Lifespan \\
(Years)
\end{tabular}} & \multicolumn{14}{|c|}{ Embodied Energy (EE) } & \multirow{2}{*}{\multicolumn{2}{|c|}{$\begin{array}{c}\text { Difference in } \\
\text { Operating Energy } \\
\text { from Baseline after } \\
\text { Lifespan }\end{array}$}} \\
\hline & \multicolumn{3}{|c|}{ Manufactu } & \multicolumn{3}{|c|}{ Constrin } & \multicolumn{3}{|c|}{ Maintenan } & \multicolumn{3}{|c|}{ Endo } & \multirow{2}{*}{${ }^{3}$ Total } & \multirow{2}{*}{\begin{tabular}{|c}
${ }^{4}$ Total \\
$\mathrm{EE}$ \\
per m
\end{tabular}} & & \\
\hline & Materia & ${ }^{2}$ Trans. & Total & Materia & ${ }^{2}$ Trans. & Total & Material & \begin{tabular}{|l|}
${ }^{2}$ Trans. \\
\end{tabular} & Total & Material & \begin{tabular}{|l|}
${ }^{2}$ Trans. \\
\end{tabular} & Total & & & ${ }^{5}$ Total & ${ }^{6}$ per $\mathrm{m}^{2}$ \\
\hline Initial & $63,37 \mathrm{C}$ & 470 & 63,840 & 685 & 1,252 & 1,936 & 0 & 0 & 0 & 0 & 0 & 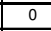 & 65,776 & 1,292 & & \\
\hline 50 & $63,37 \mathrm{C}$ & 470 & 63,840 & 685 & $\begin{array}{l}, 252 \\
\end{array}$ & 1,936 & \begin{tabular}{|l|l|}
3,099 \\
\end{tabular} & 10 & 3,110 & 75 & 541 & 616 & \begin{tabular}{|l|l|}
69,501 \\
\end{tabular} & 1,365 & 200,000 & 344 \\
\hline
\end{tabular}

Global Warming Potential ( $k g$ of $\mathrm{CO}_{2}$ eq.)

$\underset{\infty}{-}$

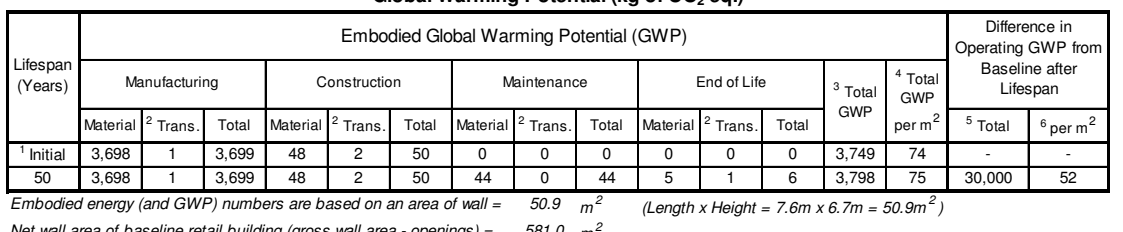

ATHENA ® EIE Material List:

(Includes all materials after 50 years)

\begin{tabular}{|l|c|c|}
\hline \multicolumn{1}{|c|}{ Material List } & Quantities & Unit \\
\hline 3 mil Polyethyllene & 54.0 & $\mathrm{~m} 2$ \\
\hline Concrete Blocks & 648.0 & Blocks \\
\hline Extruded Polystyrene & 104.3 & $\mathrm{~m} 2(25 \mathrm{~mm})$ \\
\hline Galvanized Sheet & 106.1 & $\mathrm{~kg}$ \\
\hline Modified Bitumen membrane & 68.2 & $\mathrm{~kg}$ \\
\hline Mortar & 2.1 & $\mathrm{~m} 3$ \\
\hline Nails & 4.5 & $\mathrm{~kg}$ \\
\hline Pine Wood Bevel Siding & 160.4 & $\mathrm{~m} 2$ \\
\hline Rebar, Rod, Light Sections & $1,092.8$ & $\mathrm{~kg}$ \\
\hline Solvent Based Alkyd Paint & 19.6 & $\mathrm{~L}$ \\
\hline Water Based Latex Paint & 132.5 & $\mathrm{~L}$ \\
\hline
\end{tabular}

Notes:
${ }^{1}$ Initial $=$ Time 'o' (i.e. at the completion of initial construction)

${ }^{2}$ Trans. $=$ Transportation

${ }^{3}$ Total EE (or Total GWP) = Total embodied energy (or total embodied GWP) of building component after lifespan (i.e. total mal
construction + total maintenance + total end-of-life effects)

${ }^{4}$ Total EE (or Total GWP) per $\mathrm{m}^{2}=$ Total EE (or Total GWP) of building

component/ area of building component that was modelled in ATHENAQ EIE Total Difference in Operating Energy (or GWP) from Baseline after
Lifespan = The difference in the total lifecycle operating energy (or GWP) from the baseline retail building after lifespan, due to using this building rom the baseline retail building after lifesp
component instead of the baseline component

Total Difference in Operating Energy (or GWP) from Baseline after
Lifespan per $\mathrm{m}^{2}=$ Total difference in operating energy (or GWP) from Lifespan per $\mathrm{m}^{2}=$ Total difference in operating energy (or
baseline after lifespan $/$ net wall area of baseline retail building "Total operating primary energy use of baseline retail building after 50 years
$50,700 \mathrm{GJ}\left(1,745 \mathrm{MJ} / \mathrm{m}^{2} \mathrm{yr}\right)$

"Total operating GWP of baseline reta

\section{Concrete Masonry Unit Wall \#5 (CMU-W5)}

Building Component Description:

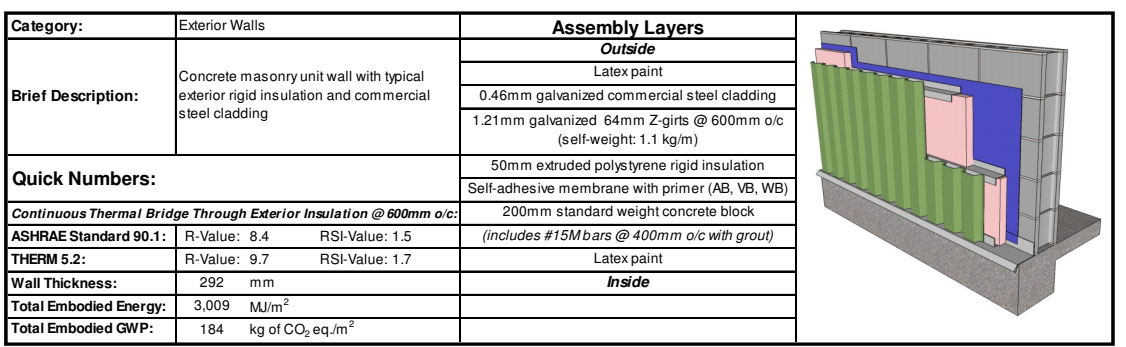

Life-Cycle Assessment Results:

Primary Energy Consumption (MJ)

\begin{tabular}{|c|c|c|c|c|c|c|c|c|c|c|c|c|c|c|c|}
\hline \multirow{3}{*}{$\begin{array}{l}\text { iespan } \\
\text { (Years }\end{array}$} & \multicolumn{13}{|c|}{ Embodied Energy (EE) } & \multirow{2}{*}{\multicolumn{2}{|c|}{$\begin{array}{c}\text { Difference in } \\
\text { Operating Energy } \\
\text { from Baseline after } \\
\text { Lifespan }\end{array}$}} \\
\hline & \multicolumn{2}{|c|}{ Manutacturing } & \multicolumn{3}{|c|}{ Construction } & \multicolumn{3}{|c|}{ Maintenance } & \multicolumn{3}{|c|}{ End of Life } & \multirow{2}{*}{$\begin{array}{c}{ }^{3} \text { Total } \\
\text { EE }\end{array}$} & \multirow{2}{*}{$\begin{array}{l}{ }^{4} \text { Total } \\
\text { EE }\end{array}$} & & \\
\hline & \begin{tabular}{|l|l|} 
Material & 2 \\
\end{tabular} & \begin{tabular}{|l|l|l|l} 
Total & \\
\end{tabular} & Material & ns. & Total & Material & Trans. & Total & Material & 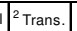 & Total & & & ${ }^{5}$ Total & ${ }^{6}$ perm ${ }^{2}$ \\
\hline & \begin{tabular}{|l|l|}
147,220 & 422 \\
14200
\end{tabular} & 147,642 & \begin{tabular}{|l|l|}
685 \\
695
\end{tabular} & \begin{tabular}{|l|l|l|l|}
1,152 \\
1152
\end{tabular} & $\frac{1,836}{1,926}$ & 0 & 0 & 0 & 0 & 0 & 0 & $\begin{array}{l}149,478 \\
152107\end{array}$ & 2,936 & & \\
\hline
\end{tabular}

Global Warming Potential ( $\mathrm{kg}$ of $\mathrm{CO}_{2}$ eq.

\begin{tabular}{|c|c|c|c|c|c|c|c|c|c|c|c|c|c|c|c|c|}
\hline \multirow{3}{*}{$\begin{array}{l}\text { Litespas) } \\
\text { (Years) }\end{array}$} & \multicolumn{14}{|c|}{ Embodied Global Warming Potential (GWP) } & \multirow{2}{*}{\multicolumn{2}{|c|}{$\begin{array}{c}\text { Difference in } \\
\text { Operating GWPP from } \\
\text { Baseline after } \\
\text { Lifespan }\end{array}$}} \\
\hline & \multicolumn{3}{|c|}{ Manutacturing } & \multicolumn{3}{|c|}{ Construction } & \multicolumn{3}{|c|}{ Maintenance } & \multicolumn{3}{|c|}{ End of Life } & \multirow{2}{*}{$\begin{array}{l}{ }^{3} \text { Total } \\
\text { GWP }\end{array}$} & \multirow{2}{*}{$\begin{array}{l}{ }^{4} \text { Total } \\
\text { GWP } \\
\text { per m}{ }^{2}\end{array}$} & & \\
\hline & Naterial & ${ }^{2}$ Trans. & Total & Material & ${ }^{2}$ Trans. & Total & Material & ${ }^{2}$ Trans. & Total & Material & ${ }^{2}$ Trans. & Total & & & \begin{tabular}{|l|l|}
${ }^{5}$ Total \\
\end{tabular} & ${ }^{6} \operatorname{perm}^{2}$ \\
\hline Initial & 9,284 & 1 & 9,285 & 48 & 2 & 50 & 0 & 0 & 0 & 0 & 0 & 0 & 9,335 & 183 & & \\
\hline 50 & 9,284 & 1 & 9,285 & 48 & 2 & 50 & 44 & 0 & 44 & 5 & 1 & 6 & 9,384 & 184 & 120,000 & 20 \\
\hline
\end{tabular}

Net wall area of baseline retail building (gross wall are - openings) $=581.0 \mathrm{~m}^{2}$

ATHENA $®$ EIE Material List:

(Includes all materials after 50 years)

\begin{tabular}{|l|c|c|}
\multicolumn{1}{|c|}{ Material List } & Quantities & Unit \\
\hline 3 mil Polyythylene & 54.0 & $\mathrm{~m} 2$ \\
\hline Commercial $0.46 \mathrm{~mm}$ Steel Cladding & 168.0 & $\mathrm{~m} 2$ \\
\hline Concretete Blocks & 648.0 & Blocks \\
\hline Extruded Polystyrene & 104.3 & $\mathrm{~m} 2(25 \mathrm{~mm})$ \\
\hline Galvanized Studs & 101.0 & $\mathrm{~kg}$ \\
\hline Modilied Bitumen membrane & 68.2 & $\mathrm{~kg}$ \\
\hline Mortar & 2.1 & $\mathrm{~m} 3$ \\
\hline Nails & 3.1 & $\mathrm{~kg}$ \\
\hline Rebar, Rod, Light Sections & $1,092.8$ & $\mathrm{~kg}$ \\
\hline Screws Nuts \& Bolts & 1.3 & $\mathrm{~kg}$ \\
\hline Solvent Based Alkyd Paint & 19.6 & $\mathrm{~L}$ \\
\hline Water Based Latex Paint & 132.5 & $\mathrm{~L}$ \\
\hline
\end{tabular}

Notes:
${ }^{1}$ Initial = Time 'o' (i.e. at the completion of initial construction)

${ }^{2}$ Trans. $=$ Transportation

Total EE (or Total GWP) $=$ Total embodied energy (or total embodied GWP)
of building component after lifespan (i.e. total manufacturing + tota

of building component atrer lifespan (i.e. total mant
construction + total maintenance + total end-of-lifie effects)

${ }^{4}$ Total EE (or Total GWP) per $\mathrm{m}^{2}=$ Total EE (or Total GWP) of building
component / area of building component that was modelled in ATHENAQ EIE

${ }^{5}$ Total Difference in Operating Energy (or GWP) from Baseline after from the baseline retail building after lifespan, due to using this building component instead of the baseline component

Total Difference in Operating Energy (or GWP) from Baseline after Lifespan per $\mathrm{m}^{2}=$ Total difference in operating energy (or GWP) from

Total operating primary energy use of baseline retail building after 50 years =

Total operating GWP of baseline retail building after 50 years $=2,310$ tonnes
of $\mathrm{CO}_{2}$ eq $\left(80 \mathrm{~kg}\right.$ of $\mathrm{CO}_{2}$ eq. $\left./ \mathrm{m}^{2} \mathrm{xr}\right)$ 


\section{Concrete Masonry Unit Wall \#6 (CMU-W6)}

Building Component Description:

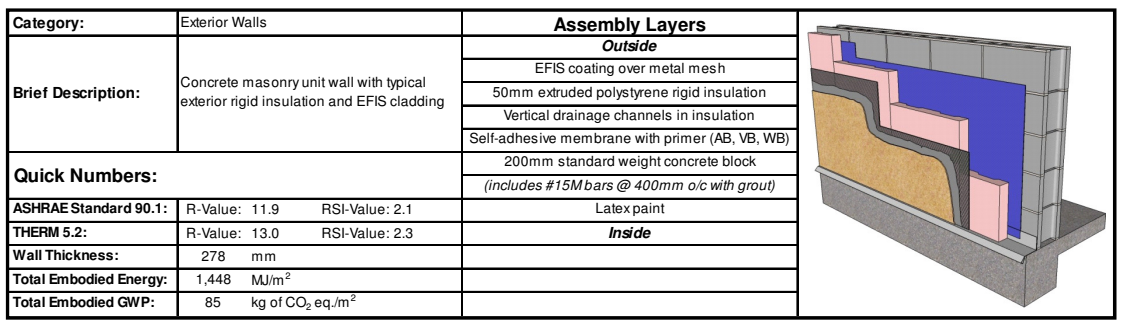

Life-Cycle Assessment Results:

Primary Energy Consumption (MJ)

\begin{tabular}{|c|c|c|c|c|c|c|c|c|c|c|c|c|c|c|c|c|}
\hline \multirow{3}{*}{\begin{tabular}{|l} 
Lifiespan \\
(Years)
\end{tabular}} & \multicolumn{14}{|c|}{ Embodied Energy (EE) } & \multirow{2}{*}{\multicolumn{2}{|c|}{$\begin{array}{c}\text { Difference in } \\
\text { Operating Energy } \\
\text { from Baseline afte } \\
\text { Lifespan }\end{array}$}} \\
\hline & \multicolumn{3}{|c|}{ Manufacturing } & \multicolumn{3}{|c|}{ Construction } & \multicolumn{3}{|c|}{ Mainten } & \multicolumn{3}{|c|}{ End of Life } & \multirow{2}{*}{$\begin{array}{l}3 \text { Total } \\
\mathrm{EE}\end{array}$} & \multirow{2}{*}{$\begin{array}{l}{ }^{4} \text { Total } \\
\text { EE } \\
\text { per m }\end{array}$} & & \\
\hline & Materiz & ${ }^{2}$ Trans. & \begin{tabular}{|l|} 
Total \\
\end{tabular} & \begin{tabular}{|l|} 
Materia \\
\end{tabular} & \begin{tabular}{|l|}
${ }^{2}$ Trans. \\
\end{tabular} & Total & Material & \begin{tabular}{|l|}
${ }^{2}$ Trans. \\
\end{tabular} & Total & Material & 11 ${ }^{2}$ Trans. & \begin{tabular}{|l|l|} 
Total \\
\end{tabular} & & & ${ }^{5}$ Total & ${ }^{6} \operatorname{per} \mathrm{m}^{2}$ \\
\hline Initial & 67,23 & \begin{tabular}{|l|l|}
477 \\
\end{tabular} & 67,714 & 685 & \begin{tabular}{|l|l|}
1,244 \\
\end{tabular} & 1,929 & 0 & 0 & 0 & 0 & 0 & 0 & 699,643 & 1,368 & & \\
\hline 50 & $\begin{array}{ll}67,23 \\
\end{array}$ & \begin{tabular}{|l|l|}
477 \\
\end{tabular} & 67,714 & 685 & \begin{tabular}{|l|l|}
1,244 \\
\end{tabular} & 1,929 & 3,099 & 10 & 3,110 & 75 & 890 & 965 & 718 & 1,448 & 50,000 & 861 \\
\hline
\end{tabular}

Global Warming Potential ( $\mathrm{kg}$ of $\mathrm{CO}_{2}$ eq.)

テั

\begin{tabular}{|c|c|c|c|c|c|c|c|c|c|c|c|c|c|c|c|c|}
\hline \multirow{3}{*}{\begin{tabular}{|c} 
Lifespan \\
(Years)
\end{tabular}} & \multicolumn{14}{|c|}{ Embodied Global Warming Potential (GWP) } & \multirow{2}{*}{\multicolumn{2}{|c|}{\begin{tabular}{|c} 
Difference in \\
Operating GWPP from \\
Baseline after \\
Lifespan
\end{tabular}}} \\
\hline & \multicolumn{3}{|c|}{ Manufacturing } & \multicolumn{3}{|c|}{ Construction } & \multicolumn{3}{|c|}{ Maintenance } & \multicolumn{3}{|c|}{ End of Life } & \multirow{2}{*}{$\begin{array}{l}{ }^{3} \text { Total } \\
\text { GWP }\end{array}$} & \multirow{2}{*}{$\begin{array}{l}{ }^{4} \text { Total } \\
\text { GWP } \\
\text { per m }\end{array}$} & & \\
\hline & Material & ${ }^{2}$ Trans. & 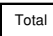 & Material & ${ }^{2}$ Trans. & Total & Material & 2 Trans. & Total & Material & $2^{2}$ Trans. & Total & & & ${ }^{5}$ Total & ${ }^{6}{ }_{\text {perm }}^{2}$ \\
\hline \begin{tabular}{|l|l|} 
'Initial \\
\end{tabular} & 4,243 & 1 & 4,244 & 48 & 2 & 50 & 0 & 0 & 0 & 0 & 0 & 0 & 4,295 & 84 & & \\
\hline 50 & 4,243 & 1 & 4,244 & 48 & 2 & 50 & 44 & 0 & 44 & 5 & 2 & 7 & 4,345 & 85 & 40,000 & 69 \\
\hline
\end{tabular}

(a)

\section{ATHENA $®$ EIE Material List:}

\begin{tabular}{|l|c|c|}
\hline \multicolumn{1}{|c|}{ Material List } & Quantities & Unit \\
\hline \#115 Organic Felt & 219.4 & $\mathrm{~m} 2$ \\
\hline 3 mil Polyethylene & 54.0 & $\mathrm{~m} 2$ \\
\hline Concrete Blocks & 648.0 & Blocks \\
\hline Extruded Polystyrene & 104.3 & $\mathrm{~m} 2(25 \mathrm{~mm})$ \\
\hline Galvanized Sheet & 51.4 & $\mathrm{~kg}$ \\
\hline Modified Bitumen membrane & 68.2 & $\mathrm{~kg}$ \\
\hline Mortar & 2.1 & $\mathrm{~m} 3$ \\
\hline Nails & 4.6 & $\mathrm{~kg}$ \\
\hline Rebar, Rod, Light Sections & $1,092.8$ & $\mathrm{~kg}$ \\
\hline Solvent Based Alkyd Paint & 19.6 & $\mathrm{~L}$ \\
\hline Stucco over metal mesh & 136.0 & $\mathrm{~m} 2$ \\
\hline Water Based Latex Paint & 132.5 & $\mathrm{~L}$ \\
\hline
\end{tabular}

Notes:
${ }^{1}$ Initial = Time 'o' (i.e. at the completion of initial construction)

${ }^{2}$ Trans. $=$ Transportation

Total EE (or Total GWP) = Total embodied energy (or total embodied GWP) of building component after lifespan (i.e. total mat
construction + total maintenance + total enc-offlife effects)

${ }^{4}$ Total EE (or Total GWP) per $\mathrm{m}^{2}=$ Total EE (or Total GWP) of building
component/ area of building component that was modelled in ATHENAQ EIE

Total Difference in Operating Energy (or GWP) from Baseline after
Lifespan = The difference in the total lifecycle operating energy (or GWP) Lifespan = The difiererence in the total lifiecycle operating energy (or GWP)
from the baseline retail building after lifespan, due to using this building from the baseline retail building after lifesp
component instead of the baseline component

Total Difference in Operating Energy (or GWP) from Baseline Lifespan per $\mathrm{m}^{2}=$ Total difference in operating energy (or GWP) fro

* Total operating primary energy use of baseline retail building after 50 years =

"Total operating $\mathrm{GWP}$ of baseline retail building after 50 years $=2,310$ tonnes
of $\mathrm{CO}_{2} \mathrm{eq}$. $\left(80 \mathrm{~kg}\right.$ of $\left.\mathrm{CO}_{2} \mathrm{eq} / \mathrm{m}^{2} \mathrm{yr}\right)$

\section{Concrete Masonry Unit Wall \#7 (CMU-W7)}

Building Component Description:

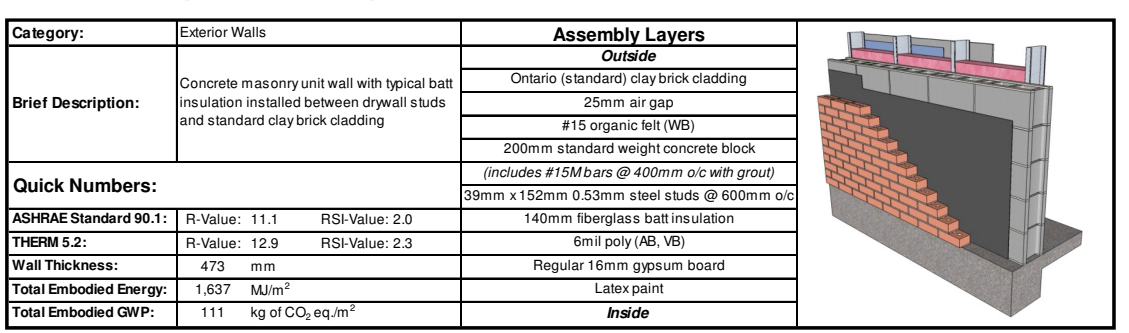

Life-Cycle Assessment Results:

Primary Energy Consumption (MJ)

\begin{tabular}{|c|c|c|c|c|c|c|c|c|c|c|c|c|c|c|c|c|}
\hline \multirow{3}{*}{$\begin{array}{l}\text { Litespar } \\
\text { (Years) }\end{array}$} & \multicolumn{14}{|c|}{ Embodied Energy (EE) } & \multirow{2}{*}{\multicolumn{2}{|c|}{$\begin{array}{l}\text { Difference in } \\
\text { Operating Energy } \\
\text { from Baseline after } \\
\text { Lifespan }\end{array}$}} \\
\hline & \multicolumn{3}{|c|}{ Manufacturing } & \multicolumn{3}{|c|}{ Construction } & \multicolumn{3}{|c|}{ Maintenance } & \multicolumn{3}{|c|}{ End of Life } & \multirow{2}{*}{$\begin{array}{c}{ }^{3} \text { Total } \\
\text { EE }\end{array}$} & \multirow{2}{*}{\begin{tabular}{|c|}
${ }^{4}$ Total \\
EE \\
per m
\end{tabular}} & & \\
\hline & Material & ${ }^{2}$ Trans. & Total & Material & \begin{tabular}{|l|}
${ }^{2}$ Trans. \\
\end{tabular} & \begin{tabular}{|l|} 
Total \\
\end{tabular} & Material & \begin{tabular}{|l|}
${ }^{2}$ Trans. \\
\end{tabular} & Total & \begin{tabular}{|l|l|} 
Material \\
\end{tabular} & \begin{tabular}{l|l}
${ }^{2}$ Trans. \\
.
\end{tabular} & Total & & & ${ }^{5}$ Total & ${ }^{6}$ per $\mathrm{m}^{2}$ \\
\hline & 77,793 & 520 & 78,313 & 844 & 2,084 & 2,928 & 0 & 0 & 0 & 0 & 0 & 0 & 81,241 & 1,595 & & \\
\hline & 7,793 & 520 & 78,313 & 844 & 2,084 & 2,928 & $\begin{array}{l}1,047 \\
\end{array}$ & 10 & 1,057 & 75 & 4,008 & 1.083 & 381 & & 0,000 & 861 \\
\hline
\end{tabular}

Global Warming Potential ( $\mathrm{kg}$ of $\mathrm{CO}_{2}$ eq $)$

\begin{tabular}{|c|c|c|c|c|c|c|c|c|c|c|c|c|c|}
\hline \multirow{3}{*}{$\left|\begin{array}{c}\text { Lifiespan } \\
\text { (Years) }\end{array}\right|$} & \multicolumn{11}{|c|}{ Embodied Global Warming Potential (GWP) } & \multirow{2}{*}{\multicolumn{2}{|c|}{$\begin{array}{c}\text { Difference in } \\
\text { Operating GWP from } \\
\text { Baseline after } \\
\text { Lifespan }\end{array}$}} \\
\hline & Manufactur & & Constructio & & Maintenan & & & End of Lifie & & ${ }^{3}{ }^{3}$ otat & ${ }^{4}$ Tot & & \\
\hline & 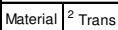 & Total & 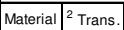 & Total & 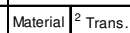 & Total & Material & 1. ${ }^{2}$ Trans. & Total & & per $\mathrm{m}$ & & \begin{tabular}{|l|l|l} 
Total & 6 perm \\
\end{tabular} \\
\hline
\end{tabular}
\begin{tabular}{|c|c|c|c|c|c|c|c|c|c|c|c|c|c|c|c|c|}
\hline 'Initial & 5,572 & 1 & 5,573 & 58 & 4 & 62 & 0 & 0 & 0 & 0 & 0 & 0 & 5,635 & 111 & - & - \\
\hline 50 & 5,572 & 1 & 5,573 & 58 & 4 & 62 & 18 & 0 & 18 & 5 & 2 & 7 & 5,660 & 111 & 40,000 & 69 \\
\hline
\end{tabular} Embodied energy (and GWP) numbers are based on an area of wall $=50.9 \mathrm{~m}^{2} \quad$ (Length $x$ Height $=7.6 \mathrm{~m} \times 6.7 \mathrm{~m}=50.9 \mathrm{~m}^{2}$ )

(Q)

ATHENA ${ }^{\circledR}$ EIE Material List:

(Includes all materials after 50 years)

${ }^{1}$ Initial $=$ Time $0^{\prime}$ (i.e. at the completion

\begin{tabular}{|l|c|c|}
\hline \multicolumn{1}{|c|}{ Material List } & Quantities & Unit \\
\hline \#15 Organic Felt & 51.9 & $\mathrm{~m} 2$ \\
\hline $16 \mathrm{~mm}$ Regular Gypsum Board & 56.0 & $\mathrm{~m} 2$ \\
\hline 6 mil Polyyethllene & 54.0 & $\mathrm{~m} 2$ \\
\hline Batt. Fiberglass & 289.8 & $\mathrm{~m} 2(25 \mathrm{~mm})$ \\
\hline Cold Rolled Sheet & 10.3 & $\mathrm{~kg}$ \\
\hline Concrete Blocks & 648.0 & $\mathrm{Blocks}$ \\
\hline Galvanized Studs & 138.3 & $\mathrm{~kg}$ \\
\hline Joint Compound & 55.9 & $\mathrm{~kg}$ \\
\hline Mortar & 3.5 & $\mathrm{~m} 3$ \\
\hline Nails & 3.7 & $\mathrm{~kg}$ \\
\hline Ontario (Standard) Brick & 53.5 & $\mathrm{~m} 2$ \\
\hline Paper Tape & 0.6 & $\mathrm{~kg}$ \\
\hline Rebar, Rod, Light Sections & $1,092.8$ & $\mathrm{~kg}$ \\
\hline Screws Nuts \& Bolts & 2.6 & $\mathrm{~kg}$ \\
\hline Water Based Latex Paint & 66.3 & $\mathrm{~L}$ \\
\hline
\end{tabular}

${ }^{2}$ Trans. $=$ Transportation

Total EE (or Total GWP) = Total embodied energy (or total embodied GWP) of building component after lifespan (i.e. total mat
construction + total maintenance + total end-ofl-life effects) ${ }^{4}$ Total EE (or Total GWP) per $\mathrm{m}^{2}=$ Total EE (or Total GWP) of building
component/area of building component that was modelled in ATHENA@ EIE Total Difference in Operating Energy (or GWP) from Baseline after Lifespan = The difference in the total lif-cycle operating energy (or GWP)
from the baseline retail building after lifespan, due to using this building com the baseline retall building atter lifese
component instead of the baseline component

Total Difference in Operating Energy (or GWP) from Baseline aftor Lifespan per $\mathrm{m}^{2}=$ Total difference in operating energy (or GWP) from "Total operating primary energy use of baseline retail building after 50 years =

"Total operating $\mathrm{GWP}$ of baseline retail building after 50 years $=2,310$ tonnes
of $\mathrm{CO}_{2} e q .\left(80 \mathrm{~kg}\right.$ of $\mathrm{CO}_{2}$ eq. $\left./ \mathrm{m}^{2} \mathrm{yr}\right)$ 


\section{Concrete Masonry Unit Wall \#8 (CMU-W8)}

Building Component Description:

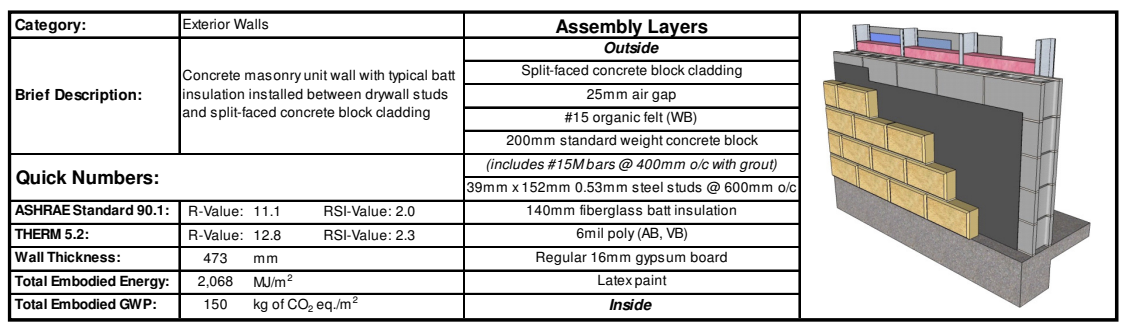

\section{Life-Cycle Assessment Results:}

Primary Energy Consumption (MJ)

\begin{tabular}{|c|c|c|c|c|c|c|c|c|c|c|c|c|c|c|c|c|}
\hline \multirow{3}{*}{$\begin{array}{l}\text { Lifespan } \\
\text { (Years) }\end{array}$} & \multicolumn{14}{|c|}{ Embodied Energy (EE) } & \multirow{2}{*}{\multicolumn{2}{|c|}{$\begin{array}{c}\text { Difference in } \\
\text { Operating Energy } \\
\text { from Baseline after } \\
\text { Lifespan }\end{array}$}} \\
\hline & \multicolumn{3}{|c|}{ Manufacturing } & \multicolumn{3}{|c|}{ Construction } & \multicolumn{3}{|c|}{ Maintenance } & \multicolumn{3}{|c|}{ End of L Life } & \multirow{2}{*}{$\begin{array}{c}{ }^{3} \text { Total } \\
\mathrm{EE}\end{array}$} & \multirow{2}{*}{$\begin{array}{l}{ }^{4} \text { Total } \\
\mathrm{EE} \\
\text { per m} \mathrm{m}^{2}\end{array}$} & & \\
\hline & Material & ${ }^{2}$ Trans. & \begin{tabular}{|l|l|} 
Total \\
\end{tabular} & \begin{tabular}{|l|} 
Material \\
\end{tabular} & ${ }^{2}$ Trans. & Total & \begin{tabular}{|l|} 
Material \\
\end{tabular} & ${ }^{2}$ Trans. & \begin{tabular}{|l|l|} 
Total \\
\end{tabular} & \begin{tabular}{|l|} 
Material \\
\end{tabular} & $\mid{ }^{2}$ Trans. & \begin{tabular}{|l|} 
Total \\
\end{tabular} & & & ${ }^{5}$ Total & ${ }^{6}$ per m ${ }^{2}$ \\
\hline Initial & 96,821 & 1,083 & 97,904 & 844 & \begin{tabular}{|l|l|}
2,894 \\
\end{tabular} & \begin{tabular}{|l|l|}
3,738 \\
\end{tabular} & 0 & 0 & 0 & 0 & 0 & 0 & 101,643 & 1,996 & & \\
\hline 50 & 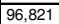 & \begin{tabular}{|l|l|}
1,083 \\
\end{tabular} & 97,904 & 844 & \begin{tabular}{|l|l|}
2,894 \\
\end{tabular} & 3,738 & 1,047 & 10 & \begin{tabular}{|l|l|}
1,057 \\
\end{tabular} & 76 & \begin{tabular}{|l|l}
2,534 \\
\end{tabular} & 2,61 & \begin{tabular}{|l|l|}
105,309 \\
\end{tabular} & 2,068 & 600,000 & 1,033 \\
\hline
\end{tabular}

Global Warming Potential ( $\mathrm{kg}$ of $\mathrm{CO}_{2}$ eq.)

\begin{tabular}{|c|c|c|c|c|c|c|c|c|c|c|c|c|c|c|c|c|}
\hline \multirow{3}{*}{$\begin{array}{l}\text { Lifespan } \\
\text { (Years) }\end{array}$} & \multicolumn{14}{|c|}{ Embodied Global Warming Potential (GWP) } & \multirow{2}{*}{\multicolumn{2}{|c|}{$\begin{array}{c}\text { Difference in } \\
\text { Operating GWP from } \\
\text { Baseline after } \\
\text { Lifespan }\end{array}$}} \\
\hline & \multicolumn{3}{|c|}{ Manufacturing } & \multicolumn{3}{|c|}{ Construction } & \multicolumn{3}{|c|}{ Maintenance } & \multicolumn{3}{|c|}{ End of Lite } & \multirow{2}{*}{$\begin{array}{l}{ }^{3} \text { Total } \\
\text { GWP }\end{array}$} & \multirow{2}{*}{$\begin{array}{l}{ }^{4} \text { Total } \\
\text { GWP } \\
\text { per m}\end{array}$} & & \\
\hline & Material & ${ }^{2}$ Trans. & Total & Material & $\left.\right|^{2}$ Trans. & Total & Materia & 2 Trans. & \begin{tabular}{|l|l} 
Total \\
\end{tabular} & Material & ${ }^{2}$ Trans. & Total & & & ${ }^{5}$ Total & ${ }^{6} \mathrm{perm}^{2}$ \\
\hline 1 Initial & 7,558 & 2 & 7,5600 & 58 & 6 & 64 & 0 & 0 & 0 & 0 & 0 & 0 & 7,624 & 150 & & \\
\hline 50 & 7,558 & 2 & 7,7560 & 58 & 6 & 64 & 18 & 0 & 18 & 5 & 5 & 10 & 7,652 & 150 & 40,000 & 69 \\
\hline
\end{tabular}

\section{ATHENA ® EIE Material List:}

\begin{tabular}{|l|c|c|}
\multicolumn{1}{|c|}{ Material List } & Quantities & Unit \\
\hline \#15 Organic Felt & 51.9 & $\mathrm{~m} 2$ \\
\hline 16mm Regular Gypsum Board & 56.0 & $\mathrm{~m} 2$ \\
\hline 6 mil Polyethylene & 54.0 & $\mathrm{~m} 2$ \\
\hline Batt. Fiberglass & 289.8 & $\mathrm{~m} 2(25 \mathrm{~mm})$ \\
\hline Cold Rolled Sheet & 10.3 & $\mathrm{~kg}$ \\
\hline Concrete Blocks & 648.0 & Blocks \\
\hline Galvanized Studs & 138.3 & $\mathrm{~kg}$ \\
\hline Joirt Compound & 55.9 & $\mathrm{~kg}$ \\
\hline Modified Bitumen membrane & 333.3 & $\mathrm{~kg}$ \\
\hline Mortar & 6.4 & $\mathrm{~m} 3$ \\
\hline Nails & 3.7 & $\mathrm{~kg}$ \\
\hline Paper Tape & 0.6 & $\mathrm{~kg}$ \\
\hline Rebar, Rod, Light Sections & $1,092.8$ & $\mathrm{~kg}$ \\
\hline Screws Nuts \& Bolts & 2.6 & $\mathrm{~kg}$ \\
\hline Split-faced Concrete Block & $1,238.2$ & $\mathrm{Blocks}$ \\
\hline Water Based Latex Paint & 66.3 & $\mathrm{~L}$ \\
\hline
\end{tabular}

\section{Concrete Masonry Unit Wall \#9 (CMU-W9)}

Building Component Description:

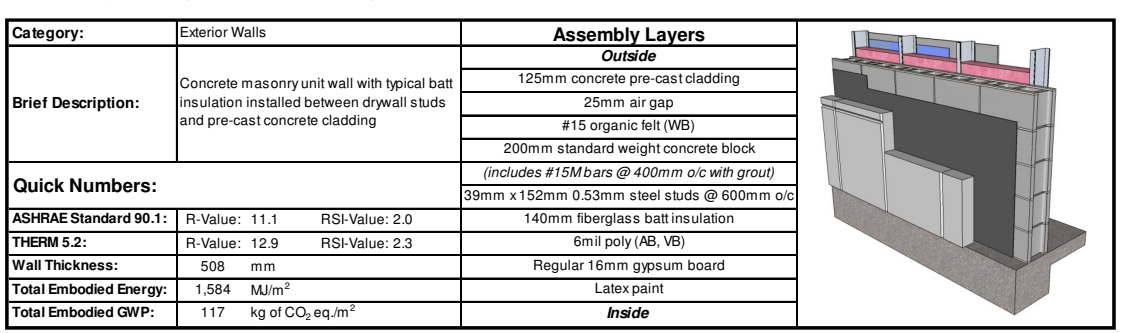

Life-Cycle Assessment Results:

Primary Energy Consumption (MJ)

\begin{tabular}{|c|c|c|c|c|c|c|c|c|c|c|c|c|c|c|c|c|}
\hline \multirow{3}{*}{$\begin{array}{l}\text { Lifespan } \\
\text { (Years) }\end{array}$} & \multicolumn{14}{|c|}{ Embodied Energy (EE) } & \multirow{2}{*}{\multicolumn{2}{|c|}{$\begin{array}{c}\text { Difference in } \\
\text { Operating Energy } \\
\text { from Baseline after } \\
\text { Lifespan }\end{array}$}} \\
\hline & \multicolumn{3}{|c|}{ Manufacturing } & \multicolumn{3}{|c|}{ Construction } & \multicolumn{3}{|c|}{ Maintenance } & \multicolumn{3}{|c|}{ End of Life } & \multirow{2}{*}{$\begin{array}{c}{ }^{3} \text { Total } \\
\text { EE }\end{array}$} & \multirow{2}{*}{\begin{tabular}{|c|}
${ }^{4} \mathrm{~T}$ Total \\
$\mathrm{EE}$ \\
per m²
\end{tabular}} & & \\
\hline & \begin{tabular}{|l|} 
Material \\
\end{tabular} & \begin{tabular}{|l|l|}
${ }^{2}$ Trans. \\
\end{tabular} & Total & Material & ${ }^{2}$ Trans. & Total & Material & 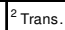 & Total & \begin{tabular}{|l|} 
Material \\
\end{tabular} & \begin{tabular}{|l|l|}
${ }^{2}$ Trans. \\
\end{tabular} & \begin{tabular}{|l|l|} 
Total \\
\end{tabular} & & & ${ }^{5}$ Total & ${ }^{6}$ perm $\mathrm{m}^{2}$ \\
\hline & 84,480 & , 1111 & 75,591 & 844 & \begin{tabular}{ll|}
2,023 \\
\end{tabular} & 2,867 & 0 & 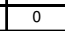 & 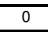 & 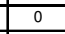 & 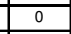 & 0 & 78,458 & 1,541 & & \\
\hline
\end{tabular}

Global Warming Potential (kg of $\mathrm{CO}_{2}$ eq.)

\begin{tabular}{|c|c|c|c|c|c|c|c|c|c|c|c|c|c|c|c|c|}
\hline \multirow{3}{*}{$\begin{array}{l}\text { Lifespan } \\
\text { (Years) }\end{array}$} & \multicolumn{14}{|c|}{ Embodied Global Warming Potential (GWP) } & \multirow{2}{*}{\multicolumn{2}{|c|}{$\begin{array}{l}\text { Difference in } \\
\text { Operating GWP from } \\
\text { Baseline after } \\
\text { Lifespan }\end{array}$}} \\
\hline & \multicolumn{3}{|c|}{ Manutacturing } & \multicolumn{3}{|c|}{ Construction } & \multicolumn{3}{|c|}{ Maintenance } & \multicolumn{3}{|c|}{ End of Life } & \multirow{2}{*}{$\begin{array}{l}{ }^{3} \text { Total } \\
\text { GWP }\end{array}$} & \multirow{2}{*}{$\begin{array}{l}{ }^{4} \text { Total } \\
\text { GWP } \\
\text { per m }\end{array}$} & & \\
\hline & Material & ${ }^{2}$ Trans. & Total & Material & ${ }^{2}$ Trans. & Total & Material & ${ }^{2}$ Trans. & Total & Material & ${ }^{2}$ Trans. & Total & & & ${ }^{5}$ Total & ${ }^{6}$ per m ${ }^{2}$ \\
\hline Initial & 5.872 & 2 & 5.874 & 58 & 4 & 62 & 0 & 0 & 0 & 0 & 0 & 0 & \begin{tabular}{|l}
5,936 \\
\end{tabular} & \begin{tabular}{|l|l|}
117 \\
\end{tabular} & & \\
\hline 50 & \begin{tabular}{|l|l|}
5,872 \\
\end{tabular} & 2 & $\mid$ & 58 & 4 & 62 & $\begin{array}{l}18 \\
\end{array}$ & 0 & 18 & 5 & 2 & 7 & 5,961 & 117 & 40,000 & 69 \\
\hline
\end{tabular}

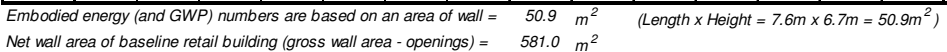

ATHENA $®$ EIE Material List:

Notes:
1 Initial $=$ Time ' 0 ' (i.e. at the completion of initial construction)

${ }^{2}$ Trans. = Transportation

Total EE (or Total GWP) = Total embodied energy (or total embodied GWP) of
construnction + total maintenance e tototal end-of-life effects)

${ }^{4}$ Total EE (or Total GWP) per $\mathrm{m}^{2}=$ Total EE (or Total GWP) of building
component/ area of building component that was modelled in ATHENAQ EIE component/ area of building component that was modelled in ATHENA@ EIE Total Difference in Operating Energy (or GWP) from Baseline after
Lifespan $=$ The difference in the total life-cycle operating energy (or GWP) Lifespan = The eifiterence in the total life-cycle operating energy (or GWP)
from the baseline retail buildining ater lifespan, due to using this building component instead of the baseline component

Total Difference in Operating Energy (or GWP) from Baseline after Lifespan per $\mathrm{m}^{2}=$ = Total difiference en in operating energy (or GWP) from "Total operating primary energy use of baseline retail building after 50 years
$50,700 \mathrm{GJ}\left(1,745 \mathrm{MJ} / \mathrm{m}^{2} \mathrm{yr}\right)$ *Total operating GWP of baseline retail building after 50 years $=2,310$ tonnes
of $\mathrm{CO}_{2}$ eq. $\left(80 \mathrm{~kg}\right.$ of $\mathrm{CO}_{2}$ eq. $\left./ \mathrm{m}^{2} \mathrm{Mr}\right)$

\begin{tabular}{|l|c|c|}
\hline \multicolumn{1}{|c|}{ Material List } & Quantities & Unit \\
\hline \#15 Organic Felt & 51.9 & $\mathrm{~m} 2$ \\
\hline $16 \mathrm{~mm}$ Regular Gypsum Board & 56.0 & $\mathrm{~m} 2$ \\
\hline 6 mil Polyethylene & 54.0 & $\mathrm{~m} 2$ \\
\hline Batt. Fiberglass & 289.8 & $\mathrm{~m} 2(25 \mathrm{~mm})$ \\
\hline Concrete $30 \mathrm{MPa}$ (flyash avg.) & 6.7 & $\mathrm{~m} 3$ \\
\hline Concrete Blocks & 648.0 & $\mathrm{Blocks}$ \\
\hline Galvanized Studs & 138.3 & $\mathrm{~kg}$ \\
\hline Joint Compound & 55.9 & $\mathrm{~kg}$ \\
\hline Mortar & 2.1 & $\mathrm{~m} 3$ \\
\hline Nails & 3.7 & $\mathrm{~kg}$ \\
\hline Paper Tape & 0.6 & $\mathrm{~kg}$ \\
\hline Rebar, Rod, Light Sections & $1,496.8$ & $\mathrm{~kg}$ \\
\hline Screws Nuts \& Boolts & 2.6 & $\mathrm{~kg}$ \\
\hline Water Based Latex Paint & 66.3 & $\mathrm{~L}$ \\
\hline
\end{tabular}

Notes:
1 'Initial = Time 'o' (i.e. at the completion of initial construction)

${ }^{2}$ Trans. $=$ Transportation

${ }^{3}$ Total EE (or Total GWP) $=$ Total embodied energy (or total embodied GWP of building component after lifespan (i.e. total man
construction + total maintenance + total end-of-life effects)

${ }^{4}$ Total EE (or Total GWP) per $\mathrm{m}^{2}=$ Total EE (or Total GWP) of building
component / area of building component that was modelled in ATHENAQ EIE Total Difference in Operating Energy (or GWP) from Baseline after Lifespan = The difference in the total lifi-cycle operating energy (or GWP)
from the baseline retail building after lifespan, due to using this building
component instead of the easeline compont component instead of the baseline component

- Total Difference in Operating Energy (or GWP) from Baseline after * Total operating primary energy use of baseline retail building after 50 years =

"Total operating $\mathrm{GW}$ of baseline reta Lifespan per $\mathrm{m}^{2}=$ Total difference in operating energy (or GWP) from 


\section{Concrete Masonry Unit Wall \#10 (CMU-W10)}

Building Component Description:

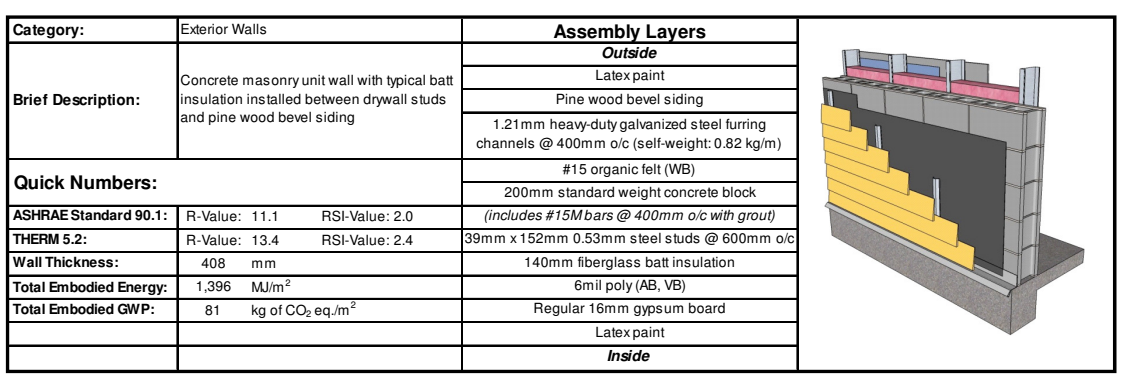

Life-Cycle Assessment Results:

Primary Energy Consumption (MJ)

\begin{tabular}{|c|c|c|c|c|c|c|c|c|c|c|c|c|c|c|c|c|}
\hline \multirow{3}{*}{\begin{tabular}{|l} 
Lifespana \\
(Years)
\end{tabular}} & \multicolumn{14}{|c|}{ Embodied Energy (EE) } & \multirow{2}{*}{\multicolumn{2}{|c|}{$\begin{array}{c}\text { Difference in } \\
\text { Operating Energy } \\
\text { from Baseline after } \\
\text { Lifespan }\end{array}$}} \\
\hline & \multicolumn{3}{|c|}{ Manutacturing } & \multicolumn{3}{|c|}{ Construction } & \multicolumn{3}{|c|}{ Maintenance } & \multicolumn{3}{|c|}{ End of Lifie } & \multirow{2}{*}{$\begin{array}{c}{ }^{3} \text { Total } \\
\mathrm{EE}\end{array}$} & \multirow{2}{*}{\begin{tabular}{|c|}
${ }^{4}$ Total \\
$\mathrm{EE}$ \\
per m
\end{tabular}} & & \\
\hline & Material & ${ }^{2}$ Trans. & Total & Materia & ${ }^{2}$ Trans. & Total & Materia & \begin{tabular}{|l|}
${ }^{2}$ Trans. \\
\end{tabular} & \begin{tabular}{|l|l|l|} 
Total & \\
\end{tabular} & Material & \begin{tabular}{|l|}
${ }^{2}$ Trans. \\
\end{tabular} & Total & & & ${ }^{5}$ Total & ${ }^{6}$ per m ${ }^{2}$ \\
\hline Initial & 66,525 & 538 & 67,063 & 844 & 1,433 & 2,277 & 0 & 0 & 0 & 0 & 0 & 0 & 69,340 & 1,362 & & \\
\hline 50 & 66,525 & \begin{tabular}{|l|}
538 \\
\end{tabular} & \begin{tabular}{|l|l|}
67,063 \\
\end{tabular} & 844 & $\begin{array}{l}, 433 \\
\end{array}$ & 2,277 & 1,047 & 10 & 1,057 & 75 & 598 & 673 & 771,070 & 1,396 & 400,000 & 688 \\
\hline
\end{tabular}

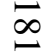

Global Warming Potential ( $\left(\mathrm{kg}\right.$ of $\mathrm{CO}_{2}$ eq.)

\begin{tabular}{|c|c|c|c|c|c|c|c|c|c|c|c|c|c|c|c|c|}
\hline \multirow{3}{*}{$\begin{array}{l}\text { Lifespar } \\
\text { (Years }\end{array}$} & \multicolumn{14}{|c|}{ Embodied Global Warming Potential (GWP) } & \multirow{2}{*}{\multicolumn{2}{|c|}{$\begin{array}{c}\text { Difference in } \\
\text { Operating GWP from } \\
\text { Baseline after } \\
\text { Lifespan }\end{array}$}} \\
\hline & \multicolumn{3}{|c|}{ Manufacturing } & \multicolumn{3}{|c|}{ Construction } & \multicolumn{3}{|c|}{ Maintenance } & \multicolumn{3}{|c|}{ End of Life } & \multirow{2}{*}{$\begin{array}{l}{ }^{3} \text { Total } \\
\text { GWP }\end{array}$} & \multirow{2}{*}{$\begin{array}{l}{ }^{4} \text { Total } \\
\text { GWP } \\
\text { perm }\end{array}$} & & \\
\hline & Materia & ${ }^{2}{ }^{2}$ Trans. & Total & Material & ${ }^{2}$ Trans. & Total & Material & 1. ${ }^{2}$ Trans. & Total & Material & $a^{2}$ Trans. & Total & & & ${ }^{5}$ Total & ${ }^{6} \operatorname{per~m}^{2}$ \\
\hline 1 Initial & 4,047 & 1 & 4,048 & 58 & 3 & 61 & 0 & 0 & 0 & 0 & 0 & 0 & 4,109 & 81 & & \\
\hline 50 & 4,047 & 1 & 4,048 & 58 & 3 & 61 & 18 & 0 & 18 & 5 & 1 & 6 & 4,133 & 81 & 30,000 & 52 \\
\hline
\end{tabular}

\begin{tabular}{|c|c|c|}
\hline \multicolumn{3}{|c|}{$\begin{array}{c}\text { ATHENA } ® \text { EIE Material List: } \\
\text { (Includes all materials after } 50 \text { years) }\end{array}$} \\
\hline Material List & Quantities & Unit \\
\hline$\# 15$ Organic Felt & 51.9 & $\mathrm{~m} 2$ \\
\hline $16 \mathrm{~mm}$ Regular Gypsum Board & 56.0 & $\mathrm{~m} 2$ \\
\hline 6 mil Polyethylene & 54.0 & $\mathrm{~m} 2$ \\
\hline Batt. Fiberglass & 289.8 & $\mathrm{~m} 2(25 \mathrm{~mm})$ \\
\hline Concrete Blocks & 648.0 & Blocks \\
\hline Galvanized Sheet & 106.1 & $\mathrm{~kg}$ \\
\hline Galvanized Studs & 138.3 & $\mathrm{~kg}$ \\
\hline $\begin{array}{l}\text { Joint Compound } \\
\end{array}$ & 55.9 & $\mathrm{~kg}$ \\
\hline Mortar & 2.1 & $\mathrm{~m} 3$ \\
\hline Nails & 5.0 & $\mathrm{~kg}$ \\
\hline Paper Tape & 0.6 & $\mathrm{~kg}$ \\
\hline Pine Wood Bevel Siding & 160.4 & $\mathrm{~m} 2$ \\
\hline Rebar, Rod, Light Sections & 1,092.8 & $\mathrm{kg}$ \\
\hline Screws Nuts \& Bolts & 2.6 & $\mathrm{~kg}$ \\
\hline Water Based Latex Paint & 132.5 & L \\
\hline
\end{tabular}
*Total operating primary energy use of baseline retail building after 50 years
$50,700 \mathrm{GJ}\left(1,745 \mathrm{MJ} \mathrm{m}^{2} \mathrm{yr}\right)$

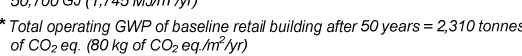

\section{Concrete Masonry Unit Wall \#11 (CMU-W11)}

Building Component Description:

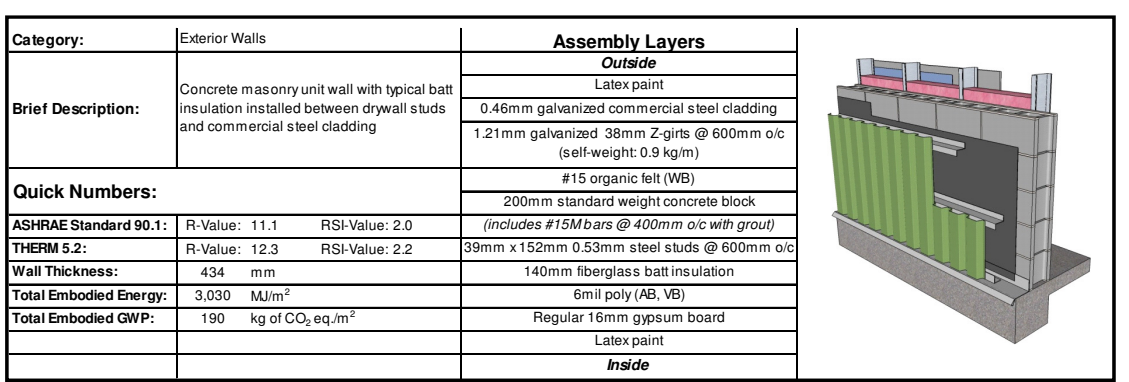

\section{Life-Cycle Assessment Results:}

Primary Energy Consumption (MJ)

\begin{tabular}{|c|c|c|c|c|c|c|c|c|c|c|c|c|c|c|c|}
\hline \multirow{3}{*}{$\begin{array}{c}\text { Lifespan } \\
\text { (Years) }\end{array}$} & \multicolumn{13}{|c|}{ Embodied Energy (EE) } & \multirow{2}{*}{\multicolumn{2}{|c|}{$\begin{array}{l}\text { Ditierence in } \\
\text { Operating Energy } \\
\text { from Basaline atter } \\
\text { Lifespan }\end{array}$}} \\
\hline & \multicolumn{2}{|c|}{ Manufacturing } & \multicolumn{3}{|c|}{ Construction } & \multicolumn{3}{|c|}{ Maintenance } & \multicolumn{3}{|c|}{ End of Life } & \multirow{2}{*}{$\begin{array}{c}{ }^{3} \text { Total } \\
\mathrm{EE}\end{array}$} & \multirow{2}{*}{\begin{tabular}{|l|}
${ }^{4}$ Total \\
EE \\
per m
\end{tabular}} & & \\
\hline & \begin{tabular}{|l|l|} 
Material & ${ }^{2}$ Trans \\
\end{tabular} & \begin{tabular}{|l|l|} 
Total \\
\end{tabular} & Material & 2 Trans. & Total & Material & ${ }^{2}$ Trans. & Total & Material & ${ }^{2}$ Trans. & Total & & & $\begin{array}{l}{ }^{5} \text { Total } \\
\end{array}$ & ${ }^{6}{ }^{6}$ perm ${ }^{2}$ \\
\hline itial & 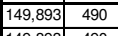 & 150,383 & 844 & $\begin{array}{ll}1,331 \\
\end{array}$ & 2,175 & 0 & 0 & 0 & 0 & 0 & 0 & 152,55 & 2,996 & & \\
\hline
\end{tabular}

\section{Global Warming Potential (kg of $\mathrm{CO}_{2}$ eq.)}

\begin{tabular}{|c|c|c|c|c|c|c|c|c|c|c|c|c|c|c|}
\hline \multirow{3}{*}{$\begin{array}{l}\text { Lifespan } \\
\text { (Years) }\end{array}$} & \multicolumn{12}{|c|}{ Embodied Global Warming Potential (GWP) } & \multirow{2}{*}{\multicolumn{2}{|c|}{$\begin{array}{c}\text { Difference in } \\
\text { Operating GWP from } \\
\text { Baseline after } \\
\text { Lifespan }\end{array}$}} \\
\hline & \multicolumn{2}{|c|}{ Manufacturing } & \multicolumn{2}{|c|}{ Construction } & \multicolumn{3}{|c|}{ Maintenance } & \multicolumn{3}{|c|}{ End of Life } & \multirow{2}{*}{$\begin{array}{l}{ }^{3} \text { Total } \\
\text { GWP }\end{array}$} & & & \\
\hline & 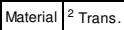 & Total & \begin{tabular}{|l|l|} 
Material & 2 \\
Trans.
\end{tabular} & Total & Material & \begin{tabular}{l|l|}
${ }^{2}$ Trans. \\
\end{tabular} & Total & Material & 1) ${ }^{2}$ Trans. & Total & & & & ${ }^{5}$ Total \\
\hline
\end{tabular}
\begin{tabular}{|l|c|c|c|c|c|c|c|c|c|c|c|c|c|c|}
\hline & & &
\end{tabular}

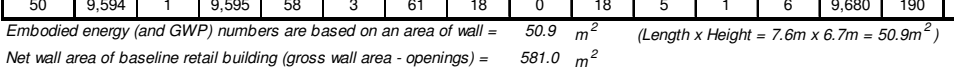

\begin{tabular}{|c|c|c|}
\hline Material List & Quantities & Unit \\
\hline$\# 15$ Organic Felt & 51.9 & $\mathrm{~m} 2$ \\
\hline $16 \mathrm{~mm}$ Regular Gypsum Board & 56.0 & $\mathrm{~m} 2$ \\
\hline 6 mil Polyethylene & 54.0 & $\mathrm{~m} 2$ \\
\hline Batt. Fiberglass & 289.8 & $\mathrm{~m} 2(25 \mathrm{~mm})$ \\
\hline Commercial $0.46 \mathrm{~mm}$ Steel Cladding & 168.0 & $\mathrm{~m} 2$ \\
\hline Concrete Blocks & 648.0 & Blocks \\
\hline Galvanized Studs & 219.1 & $\mathrm{~kg}$ \\
\hline Joint Compound & 55.9 & $\mathrm{~kg}$ \\
\hline Mortar & 2.1 & $\mathrm{~m} 3$ \\
\hline Nails & 3.7 & $\mathrm{~kg}$ \\
\hline Paper Tape & 0.6 & $\mathrm{~kg}$ \\
\hline Rebar, Rod, Light Sections & $1,092.8$ & $\mathrm{~kg}$ \\
\hline Screws Nuts \& Bolts & 3.9 & $\mathrm{~kg}$ \\
\hline Water Based Latex Paint & 132.5 & $\mathrm{~L}$ \\
\hline
\end{tabular}

Notes:
${ }^{1}$ IInitial $=$ Time 'o' (i.e. at the completion of intial construction)

Total EE (or Total GWP) = Total embodied energy (or total embodied GWP) of building component after lifespan (i.e. total man
construction t total maintenance + total end-of-life effects) ${ }^{4}$ Total EE (or Total GWP) per $\mathrm{m}^{2}=$ Total EE (or Total GWP) of building
component/ area of building component that was modelled in ATHENA EIE ${ }^{5}$ Total Difference in Operating Energy (or GWP) from Baseline after Lifespan = The difiference in the totat lififccycle operating energy (or GWP)
from the baseline retail building after lifespan, due to using this building
component instead of the baseline component ${ }^{6}$ component instead of the baseline component Lifespan per $\mathrm{m}^{2}=$ Total difference in operating energy (or GWP) from
baseline after lifespan $/ \mathrm{net}$ wall area of baseline retail building * Total operating primary energy
$50,700 \mathrm{GJ}\left(1,745 \mathrm{MJ} / \mathrm{m}^{2} /(\mathrm{r})\right.$

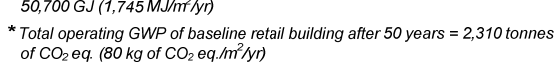
${ }^{2}$ Trans. = Transportation

${ }^{6}$ Total Difference in Operating Energy (or GWP) from Baseline after
Lifespan per $\mathrm{m}^{2}=$ Total difference in (operating energy (or GWP) ftor

Water Based Latex 


\section{Concrete Masonry Unit Wall \#12 (CMU-W12)}

Building Component Description:

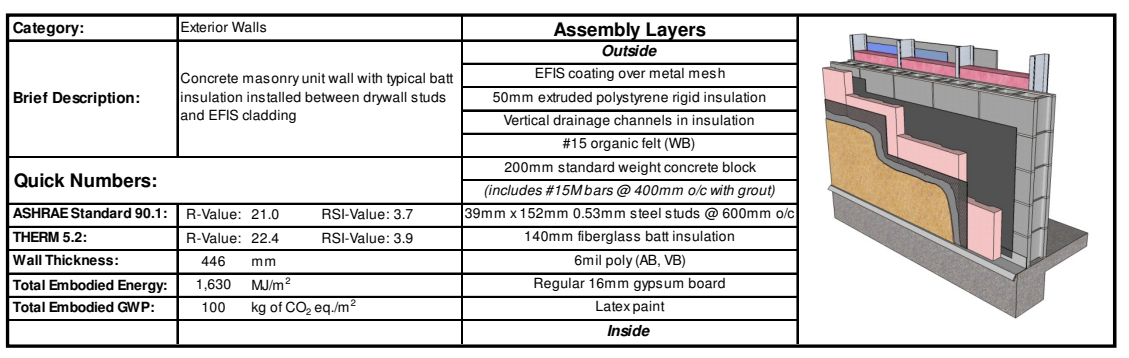

Life-Cycle Assessment Results:

Primary Energy Consumption (MJ)

\begin{tabular}{|c|c|c|c|c|c|c|c|c|c|c|c|c|c|c|c|c|}
\hline \multirow{4}{*}{$\begin{array}{l}\text { Lifespapa) } \\
\text { (Years) }\end{array}$} & \multicolumn{14}{|c|}{ Embodied Energy (EE) } & \multirow{3}{*}{\multicolumn{2}{|c|}{$\begin{array}{c}\text { Difference in } \\
\text { Operating Energy } \\
\text { from Baseline after } \\
\text { Lifespan }\end{array}$}} \\
\hline & \multirow{2}{*}{\multicolumn{3}{|c|}{ Manufactu }} & \multirow{2}{*}{\multicolumn{3}{|c|}{ istruction }} & \multirow{2}{*}{\multicolumn{3}{|c|}{ Maintenance }} & \multirow{2}{*}{\multicolumn{3}{|c|}{ Endo }} & \multirow{3}{*}{$\begin{array}{c}{ }^{3} \text { Total } \\
\mathrm{EE}\end{array}$} & \multirow{3}{*}{$\left|\begin{array}{c}{ }^{4} \text { Total } \\
\mathrm{EE} \\
\text { per } \mathrm{m}^{2}\end{array}\right|$} & & \\
\hline & & & & & & & & & & & & & & & & \\
\hline & Material & ${ }^{2}$ Trans. & Total & Material & ${ }^{2}$ Trans. & Total & Material & ${ }^{2}$ Trans. & Total & Material & ${ }^{2}$ Trans. & Total & & & \begin{tabular}{|l|l|}
${ }^{5}$ Total \\
\end{tabular} & ${ }_{6}^{6} \mathrm{perm}^{2}$ \\
\hline Initial & 78,065 & 546 & 78,612 & \begin{tabular}{|l|l|}
844 \\
\end{tabular} & 8 & 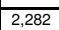 & 0 & 0 & 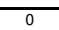 & 0 & 0 & 0 & 80,894 & 1,589 & & \\
\hline 50 & \begin{tabular}{|l}
778,005 \\
\end{tabular} & 546 & 78,612 & 844 & 1,438 & \begin{tabular}{|l|l|}
2,288 \\
\end{tabular} & \begin{tabular}{|l|l}
1,047 \\
\end{tabular} & 10 & 1,057 & 75 & 957 & \begin{tabular}{|l}
1,032 \\
\end{tabular} & 82,983 & 1,630 & $-1,500,0$ & $-2,58$ \\
\hline
\end{tabular}

Global Warming Potential ( $\mathrm{kg}$ of $\mathrm{CO}_{2}$ eq.)

i

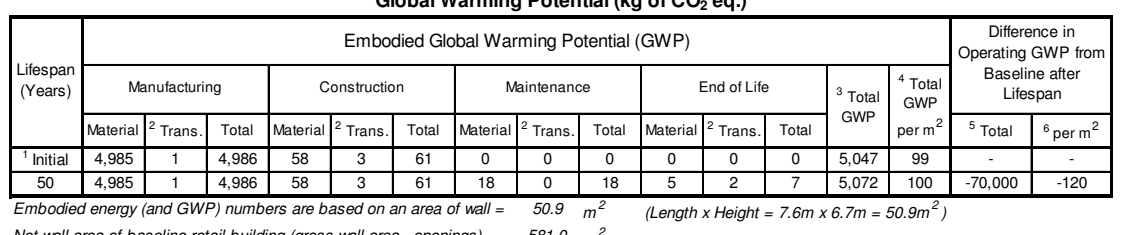

Net well area of beline retail building (oross wall area - openings) $=50.9 \mathrm{~m}^{2}$

ATHENA $®$ EIE Material List:

\begin{tabular}{|c|c|c|}
\hline Material List & Quantities & Unit \\
\hline$\# 15$ Organic Felt & 271.3 & $\mathrm{~m} 2$ \\
\hline $16 \mathrm{~mm}$ Regular Gypsum Board & 56.0 & $\mathrm{~m} 2$ \\
\hline 6 mil Polyethylene & 54.0 & $\mathrm{~m} 2$ \\
\hline Batt. Fiberglass & 289.8 & m2 (25mm) \\
\hline Concrete Blocks & 648.0 & Blocks \\
\hline Extruded Polystyrene & 104.3 & $\mathrm{~m} 2(25 \mathrm{~mm}$ \\
\hline Galvanized Sheet & 51.4 & $\mathrm{~kg}$ \\
\hline Galvanized Studs & 138.3 & $\mathrm{~kg}$ \\
\hline Joint Compound & 55.9 & $\mathrm{~kg}$ \\
\hline Mortar & 2.1 & $\mathrm{~m} 3$ \\
\hline Nails & 8.3 & $\mathrm{~kg}$ \\
\hline Paper Tape & 0.6 & $\mathrm{~kg}$ \\
\hline Rebar, Rod, Light Sections & $1,092.8$ & $\mathrm{~kg}$ \\
\hline Screws Nuts \& Bolts & 2.6 & $\mathrm{~kg}$ \\
\hline Stucco over metal mesh & 136.0 & $\mathrm{~m} 2$ \\
\hline Water Based Latex Paint & 132.5 & 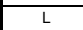 \\
\hline
\end{tabular}

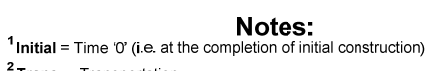

Total EE (or Total GWP) = Total embodied energy (or total embodied GWP)
of building component atter lifespan (i.e. total manufacturing + totat construction t total maintenance t tolat endo-ilife effects

${ }^{4}$ Total EE (or Total GWP) per $\mathrm{m}^{2}=$ Total EE (or Total GWP) of building
component/ area of building component that was modelled in ATHENAQIEIE from the baseline retalib building after lifespan, due to using this building
component instead of the baseline component

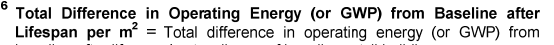

* Total operating primary energy use of baseline retail building after 50 years
$50,700 \mathrm{GJ}\left(1,745 \mathrm{M} \mathrm{Mm}^{2} \mathrm{yr}\right)$

*Total operating $\mathrm{GWP}$ of baseline retail building after 50 years $=2,310$ tonnes
of $\mathrm{CO}_{2}$ eq. $80 \mathrm{~kg}$ of $\mathrm{CO}_{2}$ eq, $\left./ \mathrm{m}^{2} / \mathrm{yr}\right)$ Lifespan $=$ The difference in the total life-cycle operating energy (or GWP)

\section{Concrete Masonry Unit Wall \#13 (CMU-W13)}

Building Component Description:

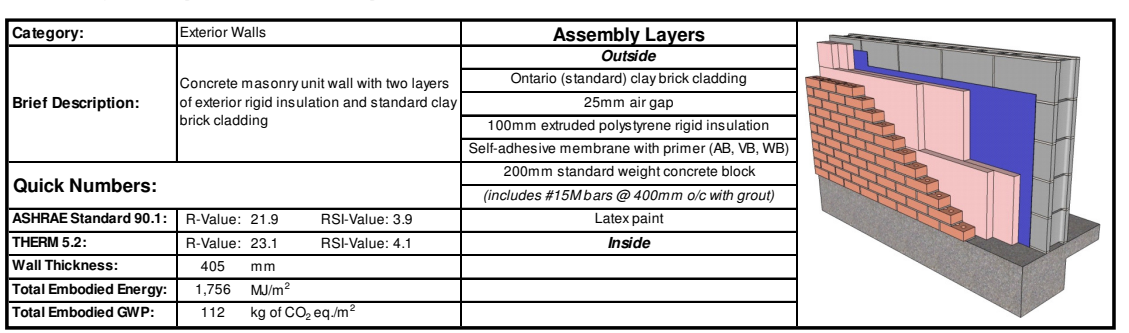

\section{Life-Cycle Assessment Results:}

Primary Energy Consumption (MJ)

\begin{tabular}{|c|c|c|c|c|c|c|c|c|c|c|c|c|c|c|c|c|}
\hline \multirow{3}{*}{$\begin{array}{l}\text { Lifespan } \\
\text { (Years) }\end{array}$} & \multicolumn{14}{|c|}{ Embodied Energy (EE) } & \multirow{2}{*}{\multicolumn{2}{|c|}{$\begin{array}{c}\text { Difference in } \\
\text { Operating Energy } \\
\text { from Baseline after } \\
\text { Lifespan }\end{array}$}} \\
\hline & \multicolumn{3}{|c|}{ Manufacturing } & \multicolumn{3}{|c|}{ Construction } & \multicolumn{3}{|c|}{ Maintenance } & \multicolumn{3}{|c|}{ End of L Life } & \multirow{2}{*}{$\begin{array}{c}{ }^{3} \text { Total } \\
\text { EE }\end{array}$} & \multirow{2}{*}{$\begin{array}{l}{ }^{4} \text { Total } \\
\mathrm{EE} \\
\mathrm{per}^{2}\end{array}$} & & \\
\hline & Material & \begin{tabular}{|l|}
${ }^{2}$ Trans. \\
\end{tabular} & Total & \begin{tabular}{|l|} 
Material \\
\end{tabular} & \begin{tabular}{|l|}
${ }^{2}$ Trans. \\
\end{tabular} & Total & Material & ${ }^{2}$ Trans. & Total & \begin{tabular}{|l|} 
Material \\
\end{tabular} & \begin{tabular}{l|l}
${ }^{2}$ Trans. \\
.
\end{tabular} & Total & & & ${ }^{5}$ Total & ${ }^{6}$ per $\mathrm{m}^{2}$ \\
\hline Initial & 82,221 & 452 & \begin{tabular}{|l|l|}
82,673 \\
\end{tabular} & 685 & \begin{tabular}{|l|}
1,915 \\
\end{tabular} & \begin{tabular}{|l|l|}
2,599 \\
\end{tabular} & 0 & 0 & 0 & 0 & 0 & 0 & 85,272 & $\begin{array}{l}1,675 \\
\end{array}$ & & \\
\hline 50 & 82,221 & 452 & \begin{tabular}{|l|l|}
82,673 \\
\end{tabular} & 685 & \begin{tabular}{|l|l|}
1,915 \\
\end{tabular} & 2,599 & 3,099 & 10 & 3,110 & 75 & 961 & $\frac{1,036}{10}$ & & 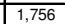 & & \\
\hline
\end{tabular}

Global Warming Potential (kg of $\mathrm{CO}_{2}$ eq.)

\begin{tabular}{|c|c|c|c|c|c|c|c|c|c|c|c|c|c|c|c|c|}
\hline \multirow{3}{*}{$\begin{array}{l}\text { Lifespan } \\
\text { (Years) }\end{array}$} & \multicolumn{14}{|c|}{ Embodied Global Warming Potential (GWP) } & \multirow{2}{*}{\multicolumn{2}{|c|}{$\begin{array}{l}\text { Difference in } \\
\text { Operating GWP from } \\
\text { Baseline after } \\
\text { Lifespan }\end{array}$}} \\
\hline & \multicolumn{3}{|c|}{ Manufacturing } & \multicolumn{3}{|c|}{ Construction } & \multicolumn{3}{|c|}{ Maintenance } & \multicolumn{3}{|c|}{ End of Life } & \multirow{2}{*}{$\begin{array}{l}{ }^{3} \text { Total } \\
\text { GWP }\end{array}$} & \multirow{2}{*}{$\begin{array}{l}{ }^{4}{ }^{4} \text { Total } \\
\text { GWP } \\
\text { perm }\end{array}$} & & \\
\hline & Material & ${ }^{2}$ Trans. & Total & Material & ${ }^{2}$ Trans. & Total & Material & ${ }^{2}$ Trans. & Total & Material & | ${ }^{2}$ Trans. & Total & & & ${ }^{5}$ Total & ${ }^{6} \mathrm{perm}^{2}$ \\
\hline${ }^{1}$ Initial & 5,610 & 1 & 5,611 & 48 & 4 & 51 & 0 & 0 & 0 & 0 & 0 & 0 & 5,663 & 111 & & \\
\hline 50 & 5,610 & 1 & 5,611 & 48 & 4 & 51 & 44 & 0 & 44 & 5 & 2 & 7 & 5,713 & 112 & 60,0 & -103 \\
\hline
\end{tabular}

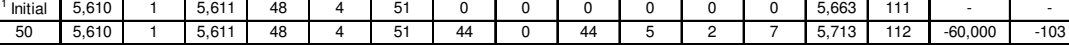
Embodied energy (and GWP) numbers are based on an area of wall $=50.9 \mathrm{~m}^{2} \quad$ (Length $x$ Height $=7.6 \mathrm{~m} \times 6.7 \mathrm{~m}=50.9 \mathrm{~m}^{2}$ )

ATHENA $®$ EIE Material List:

\begin{tabular}{|l|c|c|}
\hline \multicolumn{1}{|c|}{ Material List } & Quantitites & Unit \\
\hline 3 mil Polyythylene & 54.0 & $\mathrm{~m} 2$ \\
\hline Cold Rolled Sheet & 10.3 & $\mathrm{~kg}$ \\
\hline Concrete Blocks & 648.0 & Blocks \\
\hline Extruded Polystyrene & 208.6 & $\mathrm{~m} 2(25 \mathrm{~mm})$ \\
\hline Modified Bitumen membrane & 68.2 & $\mathrm{~kg}$ \\
\hline Mortar & 3.5 & $\mathrm{~m} 3$ \\
\hline Nails & 3.1 & $\mathrm{~kg}$ \\
\hline Ontario (Standard) Brick & 53.5 & $\mathrm{~m} 2$ \\
\hline Rebar, Rod, Light SSections & $1,092.8$ & $\mathrm{~kg}$ \\
\hline Solvent Based Alkyd Paint & 19.6 & $\mathrm{~L}$ \\
\hline Water Based Latex Paint & 66.3 & $\mathrm{~L}$ \\
\hline
\end{tabular}

Notes:
${ }^{1}$ Initial $=$ Time ' $O$ ' (i.e. at the completion of initial construction)

${ }^{3}$ Total EE (or Total GWP) $=$ Total embodied energy (or total embodied GWP) of building component after lifespan (i.e. total manufacturing + tota
construction + total maintenance + total enc-oflifife effects)

${ }^{4}$ Total EE (or Total GWP) per $\mathrm{m}^{2}=$ Total EE (or Total GWP) of building
component/ area of building component that was modelled in ATHENAQ EIE ${ }^{5}$ Total Difference in Operating Energy (or GWP) from Baseline after Lifespan = The difference in the total life-cycle operating energy (or GWP)
from the baseline retail building after lifespan, due to using this building component instead of the baseline component

${ }^{6}$ Total Difference in Operating Energy (or GWP) from Baseline after Lifespan per $\mathrm{m}^{2}=$ Total difference in operating energy (or GWP) from * Total operating primarn energy use of baseline retail building after 50 years
$50,700 \mathrm{G}\left(1,745 \mathrm{MJ} / \mathrm{m}^{2} \mathrm{yr}\right)$

"Total operating GWP of baseline retat
of $\mathrm{CO}_{2}$ eq.
$\left(80 \mathrm{~kg}\right.$ of $\mathrm{CO}_{2}$ eq. $\left./ \mathrm{m}^{2} \mathrm{yr}\right)$ 


\section{Concrete Masonry Unit Wall \#14 (CMU-W14)}

Building Component Description:

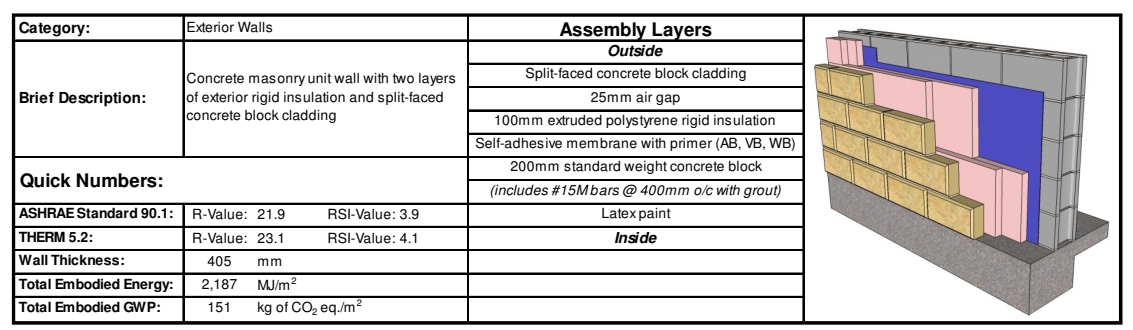

Life-Cycle Assessment Results:

Primary Energy Consumption (MJ)

\begin{tabular}{|c|c|c|c|c|c|c|c|c|c|c|c|c|c|c|c|}
\hline \multirow{3}{*}{\begin{tabular}{|l} 
Lifespan \\
(Years)
\end{tabular}} & \multicolumn{13}{|c|}{ Embodied Energy (EE) } & \multirow{2}{*}{\multicolumn{2}{|c|}{$\begin{array}{c}\text { Difference in } \\
\text { Operating Energy } \\
\text { from Baseline afte } \\
\text { Lifespan }\end{array}$}} \\
\hline & \multicolumn{2}{|c|}{ Manutacturing } & \multicolumn{3}{|c|}{ Construction } & \multicolumn{3}{|c|}{ Mainte } & \multicolumn{3}{|c|}{ End of Life } & \multirow{2}{*}{$\begin{array}{c}{ }^{3} \text { Total } \\
\mathrm{EE}\end{array}$} & \multirow{2}{*}{$\begin{array}{l}{ }^{4} \text { Total } \\
\text { EE } \\
\text { per m }\end{array}$} & & \\
\hline & 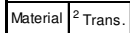 & \begin{tabular}{|l|l} 
Total \\
\end{tabular} & \begin{tabular}{|l|l|} 
Materia| \\
\end{tabular} & ${ }^{2}$ Trans. & \begin{tabular}{|l|l|} 
Total \\
\end{tabular} & Material & \begin{tabular}{|l|}
${ }^{2}$ Trans. \\
\end{tabular} & Total & Material & \begin{tabular}{|l|l|}
${ }^{2}$ Trans. \\
\end{tabular} & \begin{tabular}{|l|l|} 
Total \\
\end{tabular} & & & ${ }^{5}$ Total & ${ }^{6}{ }_{\mathrm{perm}}^{2}{ }^{2}$ \\
\hline Initial & \begin{tabular}{|l|l|}
101,249 & 1,016 \\
\end{tabular} & 102,264 & 685 & 2,7 & 3,409 & 0 & 0 & 0 & 0 & 0 & 0 & 105,674 & 2,075 & & \\
\hline 50 & \begin{tabular}{|l|l|}
101,249 & 1,016 \\
\end{tabular} & 102,264 & 685 & 2,725 & \begin{tabular}{|l|l}
3,409 \\
\end{tabular} & 3,099 & 10 & 3,110 & 76 & 2,487 & 2,563 & 111,346 & $\mid 2,187$ & 300,00 & $-2,238$ \\
\hline
\end{tabular}

Global Warming Potential ( $\mathrm{kg}$ of $\mathrm{CO}_{2}$ eq.)

$\ddot{\omega}$

\begin{tabular}{|c|c|c|c|c|c|c|c|c|c|c|c|c|c|c|c|c|}
\hline \multirow{3}{*}{$\begin{array}{l}\text { Lifespan } \\
\text { (Years) }\end{array}$} & \multicolumn{14}{|c|}{ Embodied Global Warming Potential (GWP) } & \multirow{2}{*}{\multicolumn{2}{|c|}{$\begin{array}{c}\text { Difference in } \\
\text { Operating GWP from } \\
\text { Baseline after } \\
\text { Lifespan }\end{array}$}} \\
\hline & \multicolumn{3}{|c|}{ Manufacturing } & \multicolumn{3}{|c|}{ Construction } & \multicolumn{3}{|c|}{ Maintenance } & \multicolumn{3}{|c|}{ End of Life } & \multirow{2}{*}{$\begin{array}{l}{ }^{3} \text { Total } \\
\text { GWW }\end{array}$} & \multirow{2}{*}{$\begin{array}{l}{ }^{4} \text { Total } \\
\text { GWP } \\
\text { per m}{ }^{2}\end{array}$} & & \\
\hline & Material & ${ }^{2}$ Trans. & 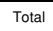 & Material & ${ }^{2}$ Trans. & Total & Material & ${ }^{2}$ Trans. & Total & Material & ||$^{2}$ Trans. & Total & & & ${ }^{5}$ Total & ${ }^{6}$ per m $^{2}$ \\
\hline$\overline{|l| n i t i a l}$ & $\begin{array}{l}7,596 \\
\end{array}$ & 2 & 7,598 & 48 & 5 & 53 & 0 & 0 & 0 & 0 & $\overline{0}$ & 0 & 77,651 & $\begin{array}{l}150 \\
\end{array}$ & & \\
\hline 50 & 7,596 & 2 & 7,598 & 48 & 5 & 53 & 44 & 0 & 44 & 5 & 5 & 10 & 7,704 & 151 & $-60,000$ & -103 \\
\hline
\end{tabular}

ine retail building (gross wall area - openings) = 581.0

\section{ATHENA ® EIE Material List:}

\begin{tabular}{|l|c|c|}
\hline \multicolumn{1}{|c|}{ Matuludes all material Lials after 50 years) } & Quantities & Unit \\
\hline 3 mil Polyethylene & 54.0 & $\mathrm{~m} 2$ \\
\hline Cold Rolled Sheet & 10.3 & $\mathrm{~kg}$ \\
\hline Concrete Blocks & 648.0 & Blocks \\
\hline Extruded Polystyrene & 208.6 & $\mathrm{~m} 2(25 \mathrm{~mm})$ \\
\hline Modified Bitumen membrane & 401.5 & $\mathrm{~kg}$ \\
\hline Mortar & 6.4 & $\mathrm{~m} 3$ \\
\hline Nails & 3.1 & $\mathrm{~kg}$ \\
\hline Rebar, Rod, Light Sections & $1,092.8$ & $\mathrm{~kg}$ \\
\hline Solvent Based Alkyd Paint & 19.6 & $\mathrm{~L}$ \\
\hline Split-faced Concrete Block & $1,238.2$ & Blocks \\
\hline Water Based Latex Paint & 66.3 & $\mathrm{~L}$ \\
\hline
\end{tabular}

Notes:
${ }^{1}$ Initial $=$ Time ' $O$ ' (i.e. at the completion of initial construction)
${ }^{2}$ Trans. $=$ Transportation

${ }^{3}$ Total EE (or Total GWP) $=$ Total embodied energy (or total embodied GWP) of building component after lifespan (i.e. total manufacturing + tota
construction + total maintenance+total end-oflife effects)

${ }^{4}$ Total EE (or Total GWP) per $\mathrm{m}^{2}=$ Total EE (or Total GWP) of building
component/ area of building component that was modelled in ATHENA@ EIE ${ }^{5}$ Total Difference in Operating Energy (or GWP) from Baseline after Lifespan = The difiference in the total lifecycle operating energy (or GWP)
from the baselien retali builing after lifespan, due to using this building
component instead of the baseline component

Total Difference in Operating Energy (or GWP) from Baseline after
Lifespan per $\mathrm{m}^{2}=$ Total difference in operating energy (or GWP) from Lifespan per $m^{2}=$ Total difference in operating energy
baseline after lifespan $/$ net wall area of baseline retail building

* Total operating primary energy use of baseline retail building after 50 years

of $\mathrm{CO}_{2}$ eq. (80 $\mathrm{kg}$ of of $\mathrm{CO}_{2} \mathrm{eq}$. $/ \mathrm{m}^{2} \mathrm{yr}$ )

\section{Concrete Masonry Unit Wall \#15 (CMU-W15)}

Building Component Description:

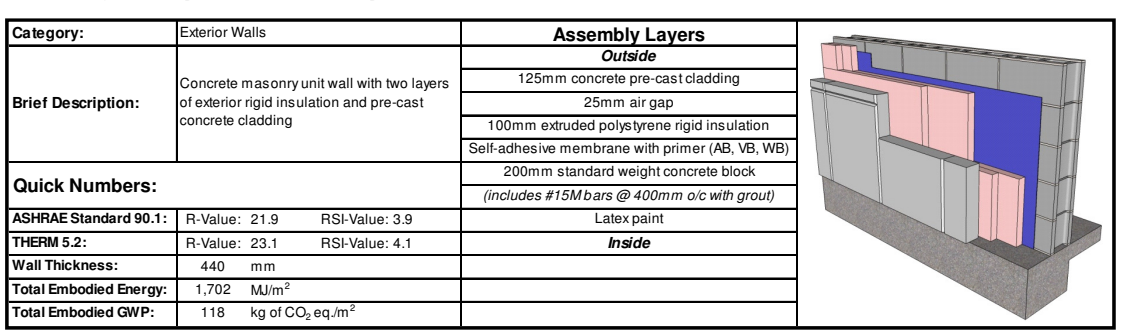

Life-Cycle Assessment Results:

Primary Energy Consumption (MJ)

\begin{tabular}{|c|c|c|c|c|c|c|c|c|c|c|c|c|c|c|c|c|}
\hline \multirow{3}{*}{$\begin{array}{l}\text { Lifespan } \\
\text { (Years) }\end{array}$} & \multicolumn{14}{|c|}{ Embodied Energy (EE) } & \multirow{2}{*}{\multicolumn{2}{|c|}{$\begin{array}{c}\text { Difference in } \\
\text { Operating Energy } \\
\text { from Baseline after } \\
\text { Lifespan }\end{array}$}} \\
\hline & \multicolumn{3}{|c|}{ Manufacturin } & \multicolumn{3}{|c|}{ Const } & \multicolumn{3}{|c|}{ Maintenance } & \multicolumn{3}{|c|}{ End of Life } & \multirow{2}{*}{$\begin{array}{c}{ }^{3} \text { Total } \\
\mathrm{EE}\end{array}$} & \multirow{2}{*}{$\begin{array}{l}{ }^{4} \text { Total } \\
\mathrm{EE} \\
\mathrm{per}^{2}\end{array}$} & & \\
\hline & ial & $\left.\right|^{2}{ }^{2}$ Trans. & Total & Material & 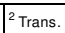 & Total & \begin{tabular}{|l|} 
Material \\
\end{tabular} & $\begin{array}{l}{ }^{2} \text { Trans. } \\
\end{array}$ & Tota & Materia & \begin{tabular}{l|l} 
al & 2 \\
\end{tabular} & \begin{tabular}{|l|} 
Total \\
\end{tabular} & & & ${ }^{5}$ Total & ${ }^{6}$ perm ${ }^{2}$ \\
\hline & 8,908 & 043 & 79,951 & 685 & $\begin{array}{l}1,854 \\
\end{array}$ & 2,538 & 0 & 0 & 0 & 0 & 0 & 0 & 82,489 & 1,620 & & \\
\hline
\end{tabular}

Global Warming Potential (kg of $\mathrm{CO}_{2}$ eq.)

\begin{tabular}{|c|c|c|c|c|c|c|c|c|c|c|c|c|c|c|c|c|}
\hline \multirow{3}{*}{ Lifespan } & \multicolumn{14}{|c|}{ Embodied Global Warming Potential (GWP) } & \multirow{2}{*}{\multicolumn{2}{|c|}{$\begin{array}{l}\text { Difference in } \\
\text { Operating GWP from } \\
\text { Baseline after } \\
\text { Lifespan }\end{array}$}} \\
\hline & \multicolumn{3}{|c|}{ Manutacturing } & \multicolumn{3}{|c|}{ Construction } & \multicolumn{3}{|c|}{ Maintenance } & \multicolumn{3}{|c|}{ End of Lifie } & \multirow{2}{*}{$\begin{array}{l}{ }^{3} \text { Total } \\
\text { GWP }\end{array}$} & \multirow{2}{*}{$\begin{array}{c}{ }^{4} \text { Total } \\
\text { GWP } \\
\text { per m }{ }^{2}\end{array}$} & & \\
\hline & Material & ${ }^{2}$ Trans. & Total & Material & ${ }^{2}$ Trans. & Total & Material & Trans. & Total & Material & 2 Trans. & Total & & & ${ }^{5}$ Total & ${ }^{6}$ per $\mathrm{m}^{2}$ \\
\hline Initial & 5.910 & 2 & 5,991 & 48 & 4 & 51 & 0 & 0 & 0 & 0 & 0 & 0 & \begin{tabular}{|l}
5,964 \\
\end{tabular} & $\begin{array}{l}117 \\
17\end{array}$ & & \\
\hline 50 & 5,910 & 2 & 5,912 & 48 & 4 & 51 & 44 & 0 & 44 & 5 & 2 & 7 & 6,014 & 118 & 60,000 & -103 \\
\hline
\end{tabular}

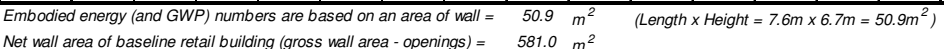

ATHENA ® EIE Material List:

(Includes all materials after 50 years)

Notes:
${ }^{1}$ Initial $=$ Time ' $O$ ' (i.e. at the completion of initial construction)

\begin{tabular}{|l|c|c|}
\hline \multicolumn{1}{|c|}{ Material List } & Quantities & Unit \\
\hline 3 mil Polyyethylene & 54.0 & $\mathrm{~m} 2$ \\
\hline Concrete 30 MPa (flyash avg.) & 6.7 & $\mathrm{~m} 3$ \\
\hline Concrete Blocks & 648.0 & Blocks \\
\hline Extruded Polystyrene & 208.6 & $\mathrm{~m} 2(25 \mathrm{~mm})$ \\
\hline Modified Bitumen membrane & 68.2 & $\mathrm{~kg}$ \\
\hline Mortar & 2.1 & $\mathrm{~m} 3$ \\
\hline Nails & 3.1 & $\mathrm{~kg}$ \\
\hline Rebar, Rod, Light Sections & $1,496.8$ & $\mathrm{~kg}$ \\
\hline Solvent Based Alkyd Paint & 19.6 & $\mathrm{~L}$ \\
\hline Water Based Latex Paint & 66.3 & $\mathrm{~L}$ \\
\hline
\end{tabular}

${ }^{2}$ Trans. = Transportation

${ }^{3}$ Total EE (or Total GWP) = Total embodied energy (or total embodied GWP of building component after lifespan (i.e. total man
construction + total maintenance + total end-ofl-life effects)

${ }^{4}$ Total EE (or Total GWP) per $\mathrm{m}^{2}=$ Total EE (or Total GWP) of building
component/area of building component that was modelled in ATHENAQ EIE ${ }^{5}$ Total Difference in Operating Energy (or GWP) from Baseline after Lifespan = The difference in the total life-cycle operating energy (or GWP)
from the baseline retail building after lifespan, due to using this building component instead of the baseline component

Total Difference in Operating Energy (or GWP) from Baseline after Lifespan per $\mathrm{m}^{2}=$ Total difference in operating energy (or GWP) from * Total operating primary energy use of baseline retail building after 50 years =

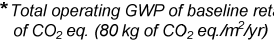




\section{Concrete Masonry Unit Wall \#16 (CMU-W16)}

Building Component Description:

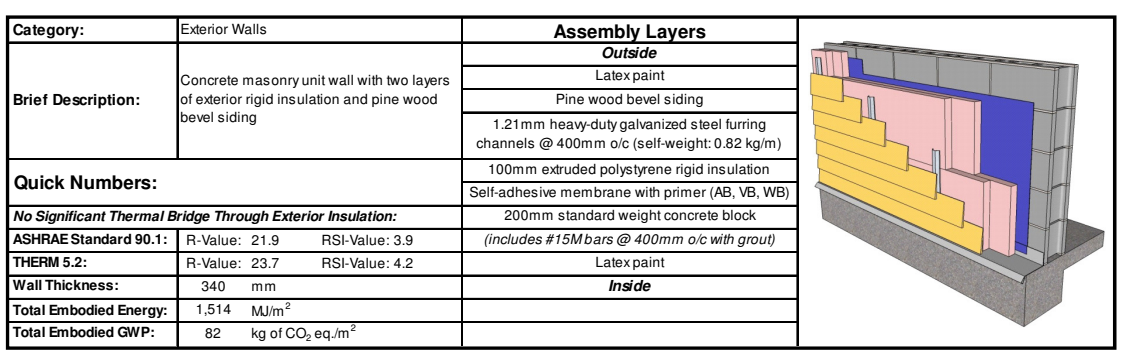

Life-Cycle Assessment Results:

Primary Energy Consumption (MJ)

\begin{tabular}{|c|c|c|c|c|c|c|c|c|c|c|c|c|c|c|c|c|}
\hline \multirow{3}{*}{$\mid$\begin{tabular}{|l} 
Lifiespan \\
(vears)
\end{tabular}} & \multicolumn{14}{|c|}{ Embodied Energy (EE) } & \multirow{2}{*}{\multicolumn{2}{|c|}{$\begin{array}{c}\text { Difference in } \\
\text { Operating Energy } \\
\text { from Baseline afte } \\
\text { Lifespan }\end{array}$}} \\
\hline & \multicolumn{3}{|c|}{ Manufacturing } & \multicolumn{3}{|c|}{ Construct } & \multicolumn{3}{|c|}{ Maintenance } & \multicolumn{3}{|c|}{ End of $L$} & \multirow{2}{*}{$\begin{array}{c}{ }^{3} \text { Total } \\
\mathrm{EE}\end{array}$} & \multirow{2}{*}{$\begin{array}{c}{ }^{4} \text { Total } \\
\mathrm{EE} \\
\mathrm{BE}^{2} \mathrm{r}^{2}\end{array}$} & & \\
\hline & Material & ${ }^{2}$ Trans. & \begin{tabular}{|l|l} 
Total \\
\end{tabular} & Material & ${ }^{2}$ Trans. & Total & Material & ${ }^{2}$ Trans. & Total & Material & \begin{tabular}{|l|}
${ }^{2}$ Trans. \\
\end{tabular} & Total & & & \begin{tabular}{|l|}
${ }^{5}$ Total \\
\end{tabular} & ${ }^{6}$ per m $^{2}$ \\
\hline Initial & 70,953 & 470 & 71,423 & f & 1,264 & 48 & 0 & 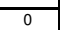 & 0 & 0 & 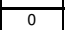 & 0 & 73,371 & 1,441 & & \\
\hline 50 & 70,953 & 470 & 71,423 & 685 & 1,264 & 1,948 & 3,099 & 10 & 3,110 & 75 & 551 & 626 & 77,107 & 1,514 & 300,00 & $-2,238$ \\
\hline
\end{tabular}

Global Warming Potential ( $\mathrm{kg}$ of $\mathrm{CO}_{2}$ eq.)

$\stackrel{\infty}{\oplus}$

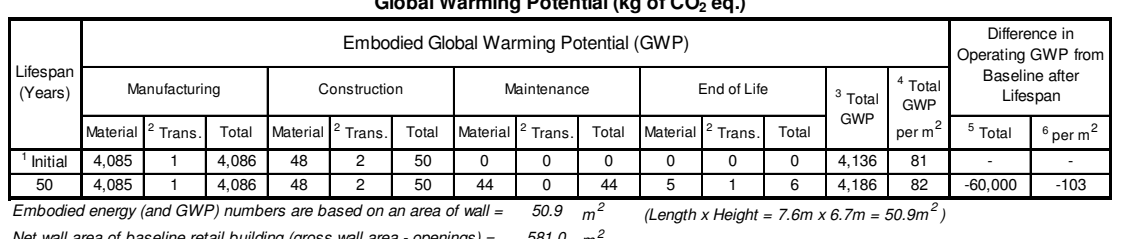

ATHENA ® EIE Material List:

\begin{tabular}{|l|c|c|}
\hline \multicolumn{1}{|c|}{ (Includes all materials after 50 years) } \\
\hline 3 mil Polyethylene & Quantitites & Unit \\
\hline Concrete Blocks & 54.0 & $\mathrm{~m} 2$ \\
\hline Extruded Polystyrene & 648.0 & Blocks \\
\hline Galvanized Sheet & 208.6 & $\mathrm{~m} 2(25 \mathrm{~mm})$ \\
\hline Modified Bitumen membrane & 106.1 & $\mathrm{~kg}$ \\
\hline Mortar & 68.2 & $\mathrm{~kg}$ \\
\hline Nails & 2.1 & $\mathrm{~m} 3$ \\
\hline Pine Wood Bevel Siding & 4.5 & $\mathrm{~kg}$ \\
\hline Rebar, Rod, Light Sections & 160.4 & $\mathrm{~m} 2$ \\
\hline Solvent Based Alkyd Paint & $1,092.8$ & $\mathrm{~kg}$ \\
\hline Water Based Latex Paint & 19.6 & $\mathrm{~L}$ \\
\hline
\end{tabular}

$$
\begin{aligned}
& \text { Notes: } \\
& { }^{1} \text { Initial }=\text { Time ' } O \text { ' (i.e. at the completion of initial construction) }
\end{aligned}
$$

${ }^{2}$ Trans. $=$ Transportation

Total EE (or Total GWP) $=$ Total embodied energy (or total embodied GWP) of building component after lifespan (i.e. total manu
construction + total maintenance + total end-oflifie effects)

${ }^{4}$ Total EE (or Total GWP) per $\mathrm{m}^{2}=$ Total EE (or Total GWP) of building
component/ area of building component that was modelled in ATHENAQ EIE ${ }^{5}$ Total Difference in Operating Energy (or GWP) from Baseline after The difierence in the total litecycle operating energy (or GWP from the baseline retail building after lifespan,
component instead of the baseline component Total Difference in Operating Energy (or GWP) from Baseline after
Lifespan per m $^{2}=$ Total difiterence in operating energy (or GWP) from
baseline after lifespan / net wall area of baseline retail building Total operating primary energy use of baseline retail building after 50 years $=$
$50,700 \mathrm{GJ}\left(1,745 \mathrm{MJ} / \mathrm{m}^{2} \mathrm{yr}\right)$

"Total operating $\mathrm{GWP}^{\circ}$ of baseline retail building after 50 years $=2,310$ tonnes

\section{Concrete Masonry Unit Wall \#17 (CMU-W17)}

Building Component Description:

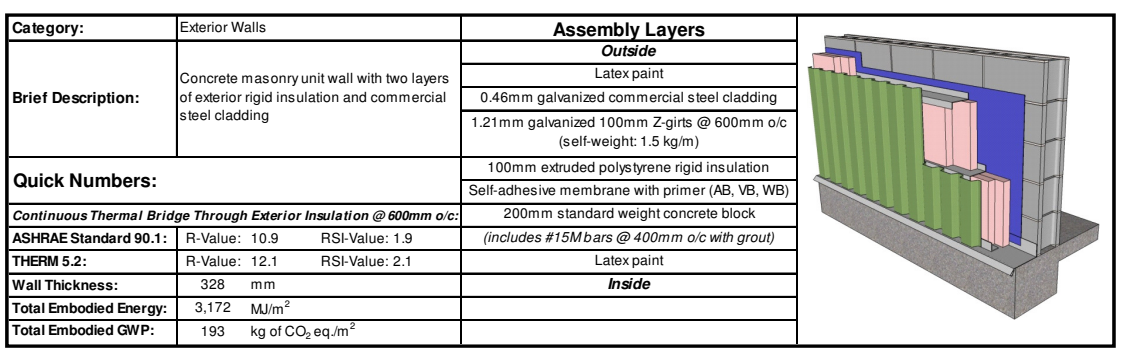

Life-Cycle Assessment Results:

Primary Energy Consumption (MJ)

\begin{tabular}{|c|c|c|c|c|c|c|c|c|c|c|c|c|c|c|c|c|}
\hline \multirow{3}{*}{\begin{tabular}{|} 
Lifespan \\
(Years)
\end{tabular}} & \multicolumn{14}{|c|}{ Embodied Energy (EE) } & \multirow{2}{*}{\multicolumn{2}{|c|}{$\begin{array}{l}\text { Difference in } \\
\text { Operating Energy } \\
\text { from Baseline after } \\
\text { Lifespan }\end{array}$}} \\
\hline & \multicolumn{3}{|c|}{ Manufacturing } & \multicolumn{3}{|c|}{ Construction } & \multicolumn{3}{|c|}{ Maintenance } & \multicolumn{3}{|c|}{ End of Life } & \multirow{2}{*}{$\begin{array}{c}{ }^{3} \text { Total } \\
\mathrm{EE}\end{array}$} & \multirow{2}{*}{$\begin{array}{c}{ }^{4} \text { Total } \\
\text { EE }\end{array}$} & & \\
\hline & Material & ${ }^{2}$ Trans. & Total & Material & ${ }^{2}$ Trans. & Total & Material & ${ }^{2}$ Trans. & Total & Naterial & rans. & Total & & & ${ }^{5}$ Total & per \\
\hline & 526 & \begin{tabular}{|l|l|}
424 \\
\end{tabular} & 50 & 685 & 1,167 & 1,852 & 0 & $\frac{0}{10}$ & 0 & 0 & 0 & 0 & 802 & 3,099 & & \\
\hline
\end{tabular}

Global Warming Potential (kg of $\mathrm{CO}_{2}$ eq.)

\begin{tabular}{|c|c|c|c|c|c|c|c|c|c|c|c|c|c|c|c|c|}
\hline \multirow{3}{*}{$\begin{array}{l}\text { Lifespan } \\
\text { (Years) }\end{array}$} & \multicolumn{14}{|c|}{ Embodied Global Warming Potential (GWP) } & \multirow{2}{*}{\multicolumn{2}{|c|}{\begin{tabular}{|c} 
Difference in \\
Operating GWPP from \\
Baseline after \\
Lifespan
\end{tabular}}} \\
\hline & \multicolumn{3}{|c|}{ Manufacturing } & \multicolumn{3}{|c|}{ Construction } & \multicolumn{3}{|c|}{ Maintenance } & \multicolumn{3}{|c|}{ End of Life } & \multirow{2}{*}{${ }^{3}$ GWtal } & \multirow{2}{*}{\begin{tabular}{|l}
${ }^{4}$ Total \\
GWP \\
per $\mathrm{m}^{2}$
\end{tabular}} & & \\
\hline & Material & ${ }^{2}$ Trans. & Total & \begin{tabular}{|l|} 
Material \\
\end{tabular} & ${ }^{2}$ Trans & Total & \begin{tabular}{|l|} 
Material \\
\end{tabular} & \begin{tabular}{|l|}
${ }^{2}$ Trans. \\
\end{tabular} & Total & \begin{tabular}{|l|} 
Material \\
\end{tabular} & \begin{tabular}{l|l}
${ }^{2}$ Trans. \\
.
\end{tabular} & \begin{tabular}{|l|l|} 
Total \\
\end{tabular} & & & \begin{tabular}{|l|l|}
${ }^{5}$ Total \\
\end{tabular} & ${ }^{6} \operatorname{perm} \mathrm{m}^{2}$ \\
\hline${ }^{1}$ Initial & \begin{tabular}{|l}
9,731 \\
\end{tabular} & 1 & 9,731 & 48 & 2 & 50 & 0 & 0 & 0 & 0 & 0 & 0 & \begin{tabular}{|l|l|}
9,781 \\
\end{tabular} & 192 & & \\
\hline 50 & \begin{tabular}{|l|l}
9,731 \\
\end{tabular} & 1 & 9,731 & 48 & 2 & 50 & 44 & 0 & 44 & 5 & 1 & 6 & 9,831 & 193 & \begin{tabular}{|l|l|}
60,000 \\
\end{tabular} & 103 \\
\hline
\end{tabular}
(a)

ATHENA $®$ EIE Material List:

(ncludes all materials after 50 years)

Material List

$$
\begin{aligned}
& \text { Notes: } \\
& { }^{1} \text { Initial }=\text { Time ' } 0 \text { ' (i.e. at the completion of initial construction) }
\end{aligned}
$$

\begin{tabular}{|l|c|c|}
\hline \multicolumn{1}{|c|}{ Material List } & Quantities & Unit \\
\hline 3 mil Polyethylene & 54.0 & $\mathrm{~m} 2$ \\
\hline Commercial 0.46mm Steel Cladding & 168.0 & $\mathrm{~m} 2$ \\
\hline Concrete Blocks & 648.0 & Blocks \\
\hline Extruded Polystyrene & 208.6 & $\mathrm{~m} 2(25 \mathrm{~mm}$ \\
\hline Galvanized Studs & 131.3 & $\mathrm{~kg}$ \\
\hline Modifed Bitumen membrane & 68.2 & $\mathrm{~kg}$ \\
\hline Mortar & 2.1 & $\mathrm{~m} 3$ \\
\hline Nails & 3.1 & $\mathrm{~kg}$ \\
\hline Rebar, Rod, Light Sections & $1,092.8$ & $\mathrm{~kg}$ \\
\hline Screws Nuts \& Bolts & 1.3 & $\mathrm{~kg}$ \\
\hline Solvent Based Alkyd Paint & 19.6 & $\mathrm{~L}$ \\
\hline Water Based Latex Paint & 132.5 & $\mathrm{~L}$ \\
\hline \multicolumn{3}{|c}{} \\
\hline \multicolumn{2}{|l}{}
\end{tabular}

${ }^{2}$ Trans. $=$ Transportation

Total EE (or Total GWP) = Total embodied energy (or total embodied GWP) of builling component after lifespan (i.e. total manu
construction + total maintenance + total end-of lifie effects)

${ }^{4}$ Total EE (or Total GWP) per $\mathrm{m}^{2}=$ Total EE (or Total GWP) of building component/ area of building component that was modelled in ATHENAQ EIE Total Difference in Operating Energy (or GWP) from Baseline after from the baseline retail building after lifespan, due to using this building component instead of the baseline component

${ }^{6}$ Total Difference in Operating Energy (or GWP) from Baseline after
Lifespan per $\mathrm{m}^{2}=$ Total difference in operating energy (or GWP) from Lifespan per $\mathrm{m}^{2}=$ Total difference in operating energy
baseline after lifespan $/$ net wall area of baseline retail building

* Total operating primary energy use of baseline retail building after 50 years =
$50,700 \mathrm{G}\left(1.74 \mathrm{M} / \mathrm{V} / \mathrm{m}^{2} \mathrm{yr}\right)$ * $T$ Total operating $\mathrm{GWP}$ of baseline retail building after 50 years $=2,310$ tonnes
of $\mathrm{CO}_{2}$ eq. $\left(80 \mathrm{~kg}\right.$ of $\mathrm{CO}_{2}$ eq. $\left./ \mathrm{m}^{2} \mathrm{yr}\right)$ 


\section{Concrete Masonry Unit Wall \#18 (CMU-W18)}

Building Component Description:

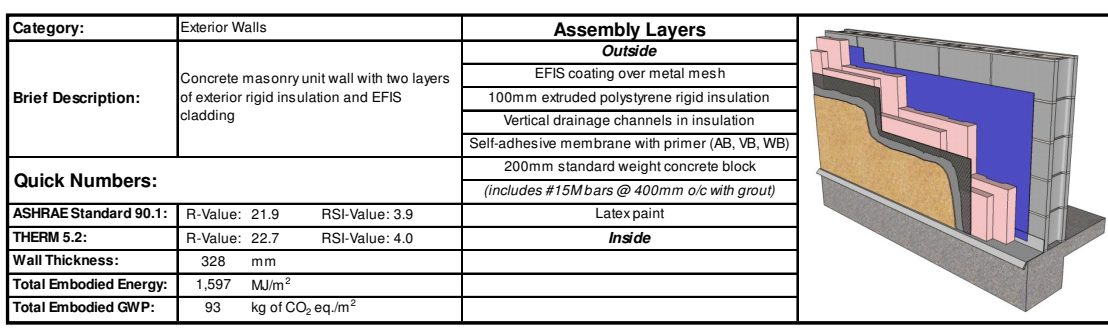

Life-Cycle Assessment Results:

Primary Energy Consumption (MJ)

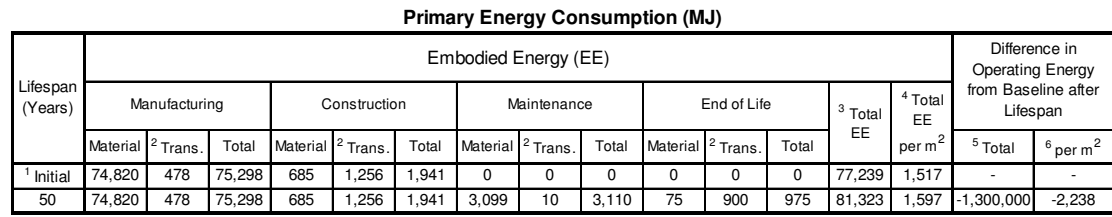

Global Warming Potential ( $\mathrm{kg}$ of $\mathrm{CO}_{2}$ eq.)

$\bar{\infty}$

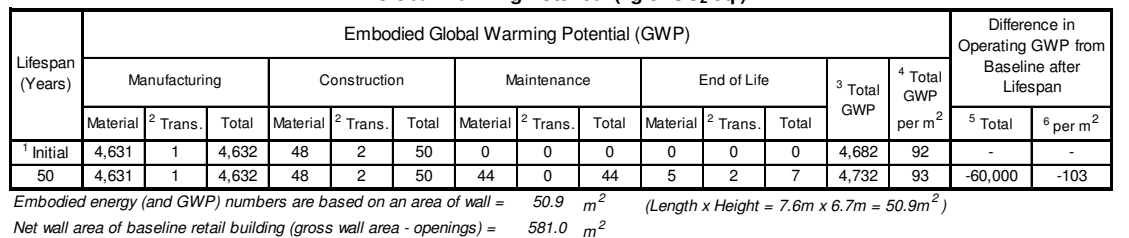

Net wall area of baseline retail building (gross wall area - openings) $=58.0 \mathrm{~m}^{2}$

ATHENA @ EIE Material List:
(nncludes all materials after 50 years)
\begin{tabular}{|l|c|c|}
\hline \multicolumn{1}{|c|}{ Material List } & Quantities & Unit \\
\hline \#15 Organic Felt & 219.4 & $\mathrm{~m} 2$ \\
\hline 3 mil Polyethylene & 54.0 & $\mathrm{~m} 2$ \\
\hline Concrete Blocks & 648.0 & Blocks \\
\hline Extruded Polystyrene & 208.6 & $\mathrm{~m} 2(25 \mathrm{~mm})$ \\
\hline Galvanized Sheet & 51.4 & $\mathrm{~kg}$ \\
\hline Modified Bitumen membrane & 68.2 & $\mathrm{~kg}$ \\
\hline Mortar & 2.1 & $\mathrm{~m} 3$ \\
\hline Nails & 4.6 & $\mathrm{~kg}$ \\
\hline Rebar, Rod, Light Sections & $1,092.8$ & $\mathrm{~kg}$ \\
\hline Solvent Based Alkyd Paint & 19.6 & $\mathrm{~L}$ \\
\hline Stucco over metal mesh & 136.0 & $\mathrm{~m} 2$ \\
\hline Water Based Latex Paint & 132.5 & $\mathrm{~L}$ \\
\hline
\end{tabular}

Notes:
1 Initial $=$ Time ' 0 ' (i.e. at the completion of initial construction)

romention

${ }^{3}$ Total EE (or Total GWP) = Total embodied energy (or total embodied GWP) of building component after lifespan (i.e. total man

Total EE (or Total GWP) per $\mathrm{m}^{2}=$ Total EE (or Total GWP) of building component/area or bulding component hat was modeled in ATHENA@ EI ${ }^{5}$ Total Difference in Operating Energy (or GWP) from Baseline after from the baseline retail building after lifespan, due to using this building component instead of the baseline component

${ }^{6}$ Total Difference in Operating Energy (or GWP) from Baseline after Lifespan per $\mathrm{m}^{2}=$ Total difference in operating energy (or GWP) from

* Total operating primary energy use of baseline retail building after 50 years
$50,700 \mathrm{GJ}\left(1,745 \mathrm{MJ} / \mathrm{m}^{2} \mathrm{yr}\right.$ )

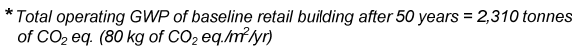

\section{LCA Data for Concrete Tilt-Up Walls}

This section contains a detailed description of each concrete tilt-up (CTU) exterior infill wall that was examined in this study ( 7 in total). The assembly layers are listed for each wall, along with a detailed description of the material quantities from the ATHENA® Environmental Impact Estimator for Buildings.

A breakdown of the total primary energy consumption and the total global warming potential (GWP) for each wall is also included. In each case, the results were calculated for an area of wall equal to $50.9 \mathrm{~m}^{2}$, which represents a typical bay size for a single-storey retail building. The results are also expressed on a per $\mathrm{m}^{2}$ basis in each case. The data has been calculated for two different lifespans: at the completion of initial construction and after 50 years.

As a summary, the figure below illustrates a comparison of the total embodied energy (and GWP) after 50 years for the various walls in this section. For comparison purpose, the building component used in the baseline retail building has been shaded in grey.

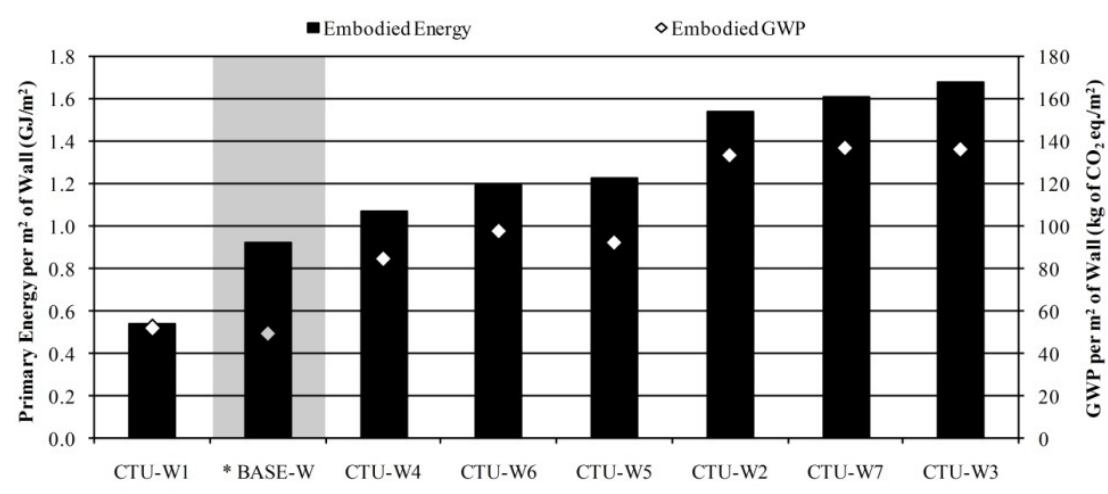

Exterior Infill Wall ID (Arranged in Order of Increasing Embodied Energy) 


\section{Concrete Tilt-Up Wall \#1 (CTU-W1)}

Building Component Description:

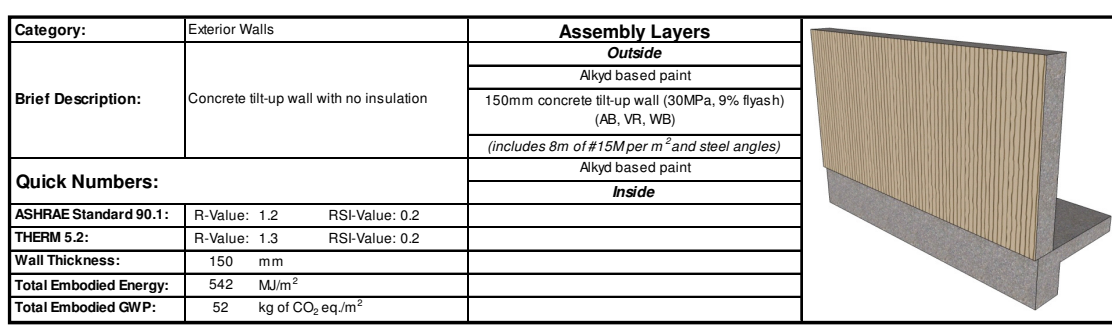

Life-Cycle Assessment Results:

Primary Energy Consumption (MJ)

\begin{tabular}{|c|c|c|c|c|c|c|c|c|c|c|c|c|c|c|c|c|}
\hline \multirow{3}{*}{\begin{tabular}{|l} 
Liffspan \\
(Years)
\end{tabular}} & \multicolumn{14}{|c|}{ Embodied Energy (EE) } & \multirow{2}{*}{\multicolumn{2}{|c|}{$\begin{array}{c}\text { Difference in } \\
\text { Operating Energy } \\
\text { from Baseline afte } \\
\text { Lifespan }\end{array}$}} \\
\hline & \multicolumn{3}{|c|}{ Manutacturing } & \multicolumn{3}{|c|}{ Construction } & \multicolumn{3}{|c|}{ Mainten } & \multicolumn{3}{|c|}{ End of Life } & \multirow{2}{*}{$\begin{array}{c}{ }^{3} \text { Total } \\
\mathrm{EE}\end{array}$} & \multirow{2}{*}{\begin{tabular}{|c|}
${ }^{4}$ Total \\
EE \\
per m
\end{tabular}} & & \\
\hline & Material & ${ }^{2}$ Trans & \begin{tabular}{|l|} 
Total \\
\end{tabular} & Materia & ${ }^{2}$ Trans. & Total & \begin{tabular}{|l|} 
Material \\
\end{tabular} & ${ }^{2}$ Trans. & \begin{tabular}{|l|} 
Total \\
\end{tabular} & Material & ${ }^{2}$ Trans. & Total & & & ${ }^{5}$ Total & ${ }^{6}{ }^{\text {per m }}{ }^{2}$ \\
\hline Initital & 26,093 & 924 & 27,017 & \begin{tabular}{|l|}
0 \\
\end{tabular} & 0 & 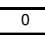 & \begin{tabular}{|l}
0 \\
\end{tabular} & 0 & 0 & 0 & 0 & 0 & 27,017 & 531 & & \\
\hline 50 & 26,0 & 924 & 27,017 & 0 & 0 & 0 & 0 & 0 & 0 & 1 & 582 & 583 & 01 & 42 & $2.58 \mathrm{E}+07$ & 44, \\
\hline
\end{tabular}

Global Warming Potential ( $\mathrm{kg}$ of $\mathrm{CO}_{2}$ eq.)

$\ddot{\infty}$

\begin{tabular}{|c|c|c|c|c|c|c|c|}
\hline \multirow{3}{*}{$\begin{array}{l}\text { Lifespan } \\
\text { (Years) }\end{array}$} & \multicolumn{7}{|c|}{ Embodied Global V } \\
\hline & \multicolumn{3}{|c|}{ Manufacturing } & \multicolumn{3}{|c|}{ Construction } & \\
\hline & Material & ${ }^{2}$ Trans & Total & Material & ${ }^{2}$ Trans. & Total & \\
\hline Initial & 2,636 & 2 & 2,638 & 0 & 0 & 0 & \\
\hline \multirow{2}{*}{\multicolumn{8}{|c|}{$\begin{array}{l}\text { Embodied energy (and GWP) numbers are based on an area of wall = } \\
\text { Net wall area of baseline retail building (gross wall area - openings) = }\end{array}$}} \\
\hline & & & & & & & \\
\hline \multicolumn{8}{|c|}{$\begin{array}{c}\text { ATHENA } ® \text { EIE Material List: } \\
\text { (Includes all materials after 50 years) }\end{array}$} \\
\hline \multicolumn{4}{|c|}{ Material List } & \multicolumn{2}{|c|}{ Quantities } & \\
\hline \multicolumn{4}{|c|}{ Concrete $30 \mathrm{MPa}$ (flyash av) } & \multicolumn{2}{|r|}{8.0} & \multicolumn{2}{|c|}{$\mathrm{m} 3$} \\
\hline \multicolumn{4}{|c|}{ Rebar, Rod, Light Sections } & & & \\
\hline \multicolumn{4}{|c|}{ Solvent Based Alkyd Paint } & & 116.5 & \multicolumn{2}{|c|}{$\mathrm{L}$} \\
\hline
\end{tabular}

${ }^{2}$ Trans. = Transportation

\section{ing Potential (GWP)}

\begin{tabular}{l|l}
\hline Maintenance & End of L
\end{tabular}

\begin{tabular}{|c|c|c|c|c|c|}
\hline & \multirow{2}{*}{\multicolumn{2}{|c|}{$\begin{array}{c}\text { Difference in } \\
\text { Operating GWP fror } \\
\text { Baseline after } \\
\text { Lifespan }\end{array}$}} \\
\hline \multicolumn{2}{|l|}{ of L Life } & \multirow{2}{*}{$\begin{array}{l}{ }^{3} \text { Total } \\
\text { GWP }\end{array}$} & \multirow{2}{*}{$\begin{array}{l}{ }^{4} \text { Total } \\
\text { GWP } \\
\text { per m}\end{array}$} & & \\
\hline ans & Total & & & \begin{tabular}{|l|}
${ }^{5}$ Total \\
\end{tabular} & ${ }^{6}$ per m ${ }^{2}$ \\
\hline & 0 & 2,638 & 52 & & \\
\hline & 1 & 2,639 & 52 & 1, 1,420,000 & 2,444 \\
\hline
\end{tabular}

$50.9 \mathrm{~m}^{2}$
$581.0 \mathrm{~m}^{2}$

$$
\begin{aligned}
& \text { Notes: } \\
& { }^{1} \text { Initial = Time 'O' (i.e. at the completion of initial construction) }
\end{aligned}
$$

${ }^{3}$ Total EE (or Total GWP) $=$ Total embodied energy (or total embodied GWP) of building component after lifespan (i.e. total manufacturing + total
construction + total maintenance + total end-oflife effects)

${ }^{4}$ Total EE (or Total GWP) per $\mathrm{m}^{2}=$ Total EE (or Total GWP) of building
component/area of building component that was modelled in ATHENA@ EIE ${ }^{5}$ Total Difference in Operating Energy (or GWP) from Baseline after Lifespan $=$ The difference in the total lifecycle operating energy (or GWP from the baseline retail building after lifespan,
component instead of the baseline component

${ }^{6}$ Total Difference in Operating Energy (or GWP) from Baseline after Lifespan per $\mathrm{m}^{2}=$ Total difference in operating energy (or GWP) from

* Total operating primary energy use of baseline retail building after 50 years =
$50,700 \mathrm{G}\left(1,745 \mathrm{MJ} / \mathrm{m}^{2} / \mathrm{yr}\right)$

Total operating $\mathrm{GWP}$ of baseline reta
of $\mathrm{CO}_{2}$ eq. $\left(80 \mathrm{~kg}\right.$ of $\mathrm{CO}_{2}$ eq. $\left./ \mathrm{hr}^{2} \mathrm{yrn}\right)$

\section{Concrete Tilt-Up Wall \#2 (CTU-W2)}

Building Component Description:

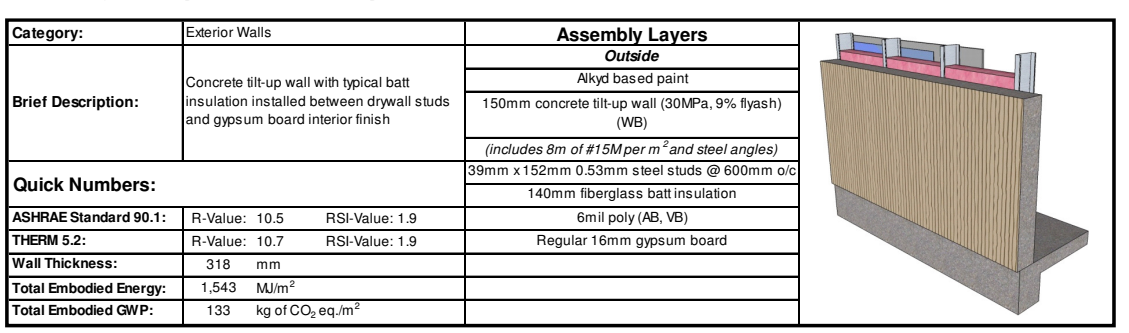

Life-Cycle Assessment Results:

Primary Energy Consumption (MJ)

\begin{tabular}{|c|c|c|c|c|c|c|c|c|c|c|c|c|c|c|c|c|}
\hline \multirow{4}{*}{$\mid \begin{array}{l}\text { Lifespan } \\
\text { (Years) }\end{array}$} & \multirow{2}{*}{\multicolumn{14}{|c|}{ Embodied Energy (EE) }} & \multirow{3}{*}{\multicolumn{2}{|c|}{$\begin{array}{c}\text { Difference in } \\
\text { Operating Energy } \\
\text { from Baseline after } \\
\text { Lifespan }\end{array}$}} \\
\hline & \multirow{2}{*}{\multicolumn{3}{|c|}{ Manufacturing }} & & & & & & & & & & & & & \\
\hline & & & & \multicolumn{3}{|c|}{ Construction } & \multicolumn{3}{|c|}{ Maintenance } & \multicolumn{3}{|c|}{ End of Life } & \multirow{2}{*}{$\mid \begin{array}{c}{ }^{3} \text { Total } \\
{ }^{2}\end{array}$} & \multirow{2}{*}{\begin{tabular}{|c|}
${ }^{4}$ Total \\
EE \\
per m
\end{tabular}} & & \\
\hline & \begin{tabular}{|l|} 
Material \\
\end{tabular} & \begin{tabular}{|l|}
${ }^{2}$ Trans. \\
\end{tabular} & Total & \begin{tabular}{|l|} 
Material \\
\end{tabular} & ${ }^{2}$ Trans. & Total & Material $\left.\right|^{2}$ & \begin{tabular}{|l|}
${ }^{2}$ Trans. \\
\end{tabular} & Total & \begin{tabular}{|l|} 
Material \\
\end{tabular} & \begin{tabular}{|l|}
${ }^{2}$ Trans. \\
\end{tabular} & Total & & & ${ }^{5}$ Total & ${ }^{6}$ per m ${ }^{2}$ \\
\hline Initial & 73,841 & \begin{tabular}{|c|}
1,973 \\
\end{tabular} & 75,814 & \begin{tabular}{|c|}
160 \\
\end{tabular} & \begin{tabular}{|l|}
1,288 \\
\end{tabular} & 1,448 & 0 & 0 & 0 & 0 & 0 & 0 & 77,262 & \begin{tabular}{|l|}
1,517 \\
\end{tabular} & & \\
\hline 50 & 73.841 & 1973 & 75,814 & 160 & 1,288 & 1,448 & 0 & 0 & 0 & 2 & 1301 & 130 & & & 00 & \\
\hline
\end{tabular}

Global Warming Potential (kg of $\mathrm{CO}_{2}$ eq.)

\begin{tabular}{|c|c|c|c|c|c|c|c|c|c|c|c|c|c|c|c|c|}
\hline \multirow{3}{*}{$\begin{array}{l}\text { Lifespan } \\
\text { (Years) }\end{array}$} & \multicolumn{14}{|c|}{ Embodied Global Warming Potential (GWP) } & \multirow{2}{*}{\multicolumn{2}{|c|}{$\begin{array}{l}\text { Difference in } \\
\text { Operating GWP fron } \\
\text { Baseline after } \\
\text { Lifespan }\end{array}$}} \\
\hline & \multicolumn{3}{|c|}{ Manutacturing } & \multicolumn{3}{|c|}{ Construction } & \multicolumn{3}{|c|}{ Maintenance } & \multicolumn{3}{|c|}{ End of Lite } & \multirow{2}{*}{$\begin{array}{l}{ }^{3} \text { Total } \\
\text { GWP }\end{array}$} & \multirow{2}{*}{$\begin{array}{c}{ }^{4} \text { Total } \\
\text { GWP } \\
\text { per m }\end{array}$} & & \\
\hline & Material & ${ }^{2}$ Trans. & Total & Material & 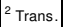 & Total & Material & ${ }^{2}$ Trans. & Total & Material & ${ }^{2}$ Trans. & Total & & & $\begin{array}{ll}{ }^{5} \text { Total } \\
\end{array}$ & ${ }^{6} \operatorname{perm}^{2}$ \\
\hline Initial & 6,758 & 4 & 6,761 & 10 & 2 & 13 & 0 & 0 & 0 & 0 & 0 & 0 & 6,774 & \begin{tabular}{|l|l|l|ll}
133 \\
\end{tabular} & & \\
\hline 50 & 6,758 & 4 & 6,761 & 10 & 2 & 13 & 0 & 0 & 0 & 0 & 3 & 3 & \begin{tabular}{ll|l}
6,777 \\
\end{tabular} & 133 & 90,000 & 155 \\
\hline
\end{tabular}

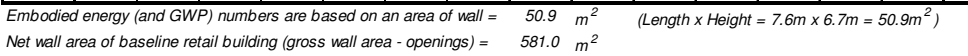

ATHENA ® EIE Material List:

(Includes all materials after 50 years)

Notes:
${ }^{1}$ Initial $=$ Time ' $O$ ' (i.e. at the completion of initial construction)

\begin{tabular}{|l|c|c|}
\hline \multicolumn{1}{|c|}{ Material List } & Quantities & Unit \\
\hline 16mm Regular Gypsum Board & 56.0 & $\mathrm{~m} 2$ \\
\hline 6 mil Polyethylene & 54.0 & $\mathrm{~m} 2$ \\
\hline Batt. Fiberglass & 289.8 & $\mathrm{~m} 2(25 \mathrm{~mm})$ \\
\hline Concrete 30 MPa (flyash av) & 8.0 & $\mathrm{~m} 3$ \\
\hline Galvanized Studs & 138.3 & $\mathrm{~kg}$ \\
\hline Joint Compound & 55.9 & $\mathrm{~kg}$ \\
\hline Nails & 3.7 & $\mathrm{~kg}$ \\
\hline Paper Tape & 0.6 & $\mathrm{~kg}$ \\
\hline Rebar, Rod, Light Sections & 347.6 & $\mathrm{~kg}$ \\
\hline Screws Nuts \& Bolts & 2.6 & $\mathrm{~kg}$ \\
\hline Solvent Based Alkyd Paint & 58.3 & $\mathrm{~L}$ \\
\hline Water Based Latex Paint & 66.3 & $\mathrm{~L}$ \\
\hline
\end{tabular}

${ }^{2}$ Trans. = Transportation

${ }^{3}$ Total EE (or Total GWP) $=$ Total embodied energy (or total embodied GWP) of building component after lifespan (i.e. total manu
construction + total maintenance + total end-of-life effects)

${ }^{4}$ Total EE (or Total GWP) per $\mathrm{m}^{2}=$ Total EE (or Total GWP) of building
component /area of building component that was modelled in ATHENAQ EIE ${ }^{5}$ Total Difference in Operating Energy (or GWP) from Baseline after Lifespan $=$ The difierence in the total life-cycle operating energy (or GWP
from the baseline retaib building atter lifespan, due to using this building component instead of the baseline component

${ }^{6}$ Total Difference in Operating Energy (or GWP) from Baseline after Lifespan per $\mathrm{m}^{2}=$ Total difference in operating energy (or GWP) from
baseline affer lifespan / net wall area of baseline retail buliling * Total operating primary energy use of baseline retail building after 50 years =

* Total operating $\mathrm{GWP}$ of baseline retal
of $\mathrm{CO}_{2}$ eq. $\left(80 \mathrm{~kg}\right.$ of $\mathrm{CO}_{2}$ eq. $\left./ \mathrm{m}^{2} \mathrm{yr}\right)$ 


\section{Concrete Tilt-Up Wall \#3 (CTU-W3)}

Building Component Description:

\begin{tabular}{|c|c|c|c|c|}
\hline Category: & \multicolumn{2}{|l|}{ Exxerior Walls } & Assembly Layers & \multirow[t]{10}{*}{ athent } \\
\hline \multirow{3}{*}{ Brief Description: } & \multirow{2}{*}{\multicolumn{2}{|c|}{ 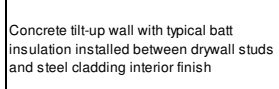 }} & $\begin{array}{l}\text { Outside } \\
\text { Akyd based paint }\end{array}$ & \\
\hline & & & $\begin{array}{l}150 \mathrm{~mm} \text { concretet tilt-up wall (30MPa, 9\% flyash) } \\
\text { (WB) }\end{array}$ & \\
\hline & & & (includes $8 \mathrm{~m}$ of \#15Mperm $\mathbf{m}^{2}$ and steel angles) & \\
\hline \multicolumn{3}{|l|}{ Quick Numbers: } & $39 \mathrm{~mm} \times 152 \mathrm{~mm} 0.53 \mathrm{~mm}$ steel studs @ $600 \mathrm{~mm}$ o/c & \\
\hline ASHRAE Standard 90.1: & R-Value: 10.1 & RSIVValue: 1.8 & 6mil poly (AB, VB) & \\
\hline THERM 5.2: & R-Value: 10.3 & RSI-Value: 1.8 & $0.46 \mathrm{~mm}$ galvanized commercial steel cladding & \\
\hline Wall Thickness: & $340 \mathrm{~mm}$ & & & \\
\hline Total Embodied Energy: & $1,677 \quad \mathrm{~m} / \mathrm{m}^{2} \mathrm{z}$ & & & \\
\hline Total Embodied GWP: & $136 \mathrm{~kg}$ of & eq/m & & \\
\hline
\end{tabular}

Life-Cycle Assessment Results:

Primary Energy Consumption (MJ)

\begin{tabular}{|c|c|c|c|c|c|c|c|c|c|c|c|c|c|c|c|c|}
\hline \multirow{3}{*}{\begin{tabular}{|l} 
Lifespan \\
(Years)
\end{tabular}} & \multicolumn{14}{|c|}{ Embodied Energy (EE) } & \multirow{2}{*}{\multicolumn{2}{|c|}{$\begin{array}{c}\text { Difference in } \\
\text { Operating Energy } \\
\text { from Baseline afte } \\
\text { Lifespan }\end{array}$}} \\
\hline & \multicolumn{3}{|c|}{ Manufacturing } & \multicolumn{3}{|c|}{ Construction } & \multicolumn{3}{|c|}{ Mainte } & \multicolumn{3}{|c|}{ End of Life } & \multirow{2}{*}{$\begin{array}{c}{ }^{3} \text { Total } \\
\mathrm{EE}\end{array}$} & \multirow{2}{*}{\begin{tabular}{|l}
${ }^{4}$ Total \\
EE \\
per $\mathrm{m}^{2}$
\end{tabular}} & & \\
\hline & Material & \begin{tabular}{l|l}
$1{ }^{2}$ Trans. \\
Thans
\end{tabular} & \begin{tabular}{|l|l|} 
Total \\
\end{tabular} & \begin{tabular}{|l|} 
Materia \\
\end{tabular} & \begin{tabular}{|l|}
${ }^{2}$ Trans. \\
\end{tabular} & Total & Nateria & \begin{tabular}{|l|}
${ }^{2}$ Trans. \\
\end{tabular} & \begin{tabular}{|l|l|} 
Total \\
\end{tabular} & Material & 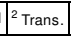 & \begin{tabular}{|l|l|} 
Total \\
\end{tabular} & & & ${ }^{5}$ Total & ${ }^{6}$ perm \\
\hline Initial & 80,800 & 1,906 & 82,705 & 160 & \begin{tabular}{|l|l|}
1,147 \\
\end{tabular} & 1,307 & 0 & 0 & 0 & 0 & 0 & 0 & 84,012 & 1,650 & & \\
\hline 50 & 80,800 & 1,906 & 82,705 & 160 & \begin{tabular}{|l|l|}
1,147 \\
\end{tabular} & 1,307 & 161 & 1 & 162 & 2 & 1,207 & \begin{tabular}{|l|l|l}
1,209 \\
\end{tabular} & 85,384 & 677 & 800,000 & 3,098 \\
\hline
\end{tabular}

Global Warming Potential ( $\mathrm{kg}$ of $\mathrm{CO}_{2}$ eq.)

$\ddot{\infty}$

\begin{tabular}{|c|c|c|c|c|c|c|c|c|c|c|c|c|c|c|c|c|}
\hline \multirow{3}{*}{\begin{tabular}{|c} 
Lifespan \\
(Years)
\end{tabular}} & \multicolumn{14}{|c|}{ Embodied Global Warming Potential (GWP) } & \multirow{2}{*}{\multicolumn{2}{|c|}{\begin{tabular}{|c|} 
Difference in \\
Operating GWPP from \\
Baseline after \\
Lifespan
\end{tabular}}} \\
\hline & \multicolumn{3}{|c|}{ Manufacturing } & \multicolumn{3}{|c|}{ Construction } & \multicolumn{3}{|c|}{ Maintenance } & \multicolumn{3}{|c|}{ End of Life } & \multirow{2}{*}{$\begin{array}{l}{ }^{3} \text { Total } \\
\text { GWP }\end{array}$} & \multirow{2}{*}{ 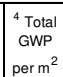 } & & \\
\hline & Material & ${ }^{2}$ Trans & 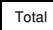 & Material & ${ }^{2}$ Trans. & Total & Material & ${ }^{2}$ Trans. & Total & Material & ${ }^{2}$ Trans. & Total & & & ${ }^{5}$ Total & ${ }_{6}^{6}$ perm $\mathrm{m}^{2}$ \\
\hline${ }^{1}$ Initial & 6,896 & 4 & 6,900 & 10 & 2 & 13 & 0 & 0 & 0 & 0 & 0 & 0 & 6,913 & 136 & & \\
\hline 50 & \begin{tabular}{|l|}
6,896 \\
\end{tabular} & 4 & \begin{tabular}{|l|l}
6,900 \\
\end{tabular} & 10 & 2 & 13 & 3 & 0 & 3 & 0 & 2 & 2 & 6,9918 & \begin{tabular}{|l|l|}
136 \\
\end{tabular} & 100,000 & 172 \\
\hline
\end{tabular}

ATHENA $®$ EIE Material List: (Includes all materials after 50 years)

\begin{tabular}{|l|c|c|}
\hline \multicolumn{1}{|c|}{ Material List } & Quantities & Unit \\
\hline 6 mil Polyethylene & 54.0 & $\mathrm{~m} 2$ \\
\hline Batt. Fiberglass & 289.8 & $\mathrm{~m} 2(25 \mathrm{~mm})$ \\
\hline Concrete $30 \mathrm{MPa}$ (flyash av) & 8.0 & $\mathrm{~m} 3$ \\
\hline Galvanized Sheet & 262.6 & $\mathrm{~kg}$ \\
\hline Galvanized Studs & 138.3 & $\mathrm{~kg}$ \\
\hline Nails & 3.1 & $\mathrm{~kg}$ \\
\hline Rebar, Rod, Light Sections & 347.6 & $\mathrm{~kg}$ \\
\hline Screws Nuts \& Bolts & 2.6 & $\mathrm{~kg}$ \\
\hline Solvent Based Alkyd Paint & 58.3 & $\mathrm{~L}$ \\
\hline Water Based Latex Paint & 6.7 & $\mathrm{~L}$ \\
\hline
\end{tabular}

Notes:
${ }^{1}$ Initial $=$ Time ' 0 ' (i.e. at the completion of initial construction)

${ }^{2}$ Trans. $=$ Transportation

Total EE (or Total GWP) = Total embodied energy (or total embodied GWP) of building component after lifespan (i.e. - total man
construction + total maintenance + total enc-oflife effects)

${ }^{4}$ Total EE (or Total GWP) per $\mathrm{m}^{2}=$ Total EE (or Total GWP) of building
component/ area of building component that was modelled in ATHENAQ EIE Total Difference in Operating Energy (or GWP) from Baseline after Lifespan = The difierence in the total life-cycle operating energy (or GWP)
from the baseline retail building atter lifespan, due to using this building
component instead of the baseline component

Total Difference in Operating Energy (or GWP) from Baseline after
Lifespan per $\mathrm{m}^{2}=$ Total difference in operating energy (or GWP) from Lifespan per $m^{2}=$ Total difference in operating energy
baseline after lifespan /net wall area of baseline retail building

* Total operating primary energy use of baseline retail building after 50 years =

"Total operating GWP of baseline reta

\section{Concrete Tilt-Up Wall \#4 (CTU-W4)}

Building Component Description:

\begin{tabular}{|c|c|c|c|c|}
\hline Category: & Exxerior Walls & & Assembly Layers & \\
\hline & & & $\begin{array}{l}\text { Outside } \\
\text { Akyd based paint }\end{array}$ & \\
\hline |Brief Description: & $\begin{array}{l}\text { Titt-upu insulate } \\
\text { wall with } 50 \mathrm{~mm}\end{array}$ & $\begin{array}{l}\text { cretes sandwich panel } \\
\text { lation }\end{array}$ & $\begin{array}{l}50 \mathrm{~mm} \text { concrete front wyhe (30MPa, 9\% flyash) } \\
\text { (WB) }\end{array}$ & \\
\hline & & & (includes $8 \mathrm{~m}$ of \#15Mperm${ }^{2}$ ) & \\
\hline Quick Numbers: & & & $50 \mathrm{~mm}$ extruded polystyrene rigid insulation & \\
\hline ASHRAE Standard 90. & (1) & 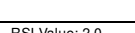 & 150mm concrete back wyhe (30MPa, 9\% flyash) & \\
\hline THERM 5.2: & R-Value: 11.2 & RSIVValue: 2.0 & (includes $8 \mathrm{~m}$ of $\# 15 \mathrm{Am}$ per $\mathrm{m}^{2}$ and steel angles) & \\
\hline Wall Thickness: & $250 \mathrm{~mm}$ & & & \\
\hline Total Embodied Energy: & $1,074 \quad \mathrm{MJ} / \mathrm{n}$ & & & \\
\hline Total Embodied GWP: & $\begin{array}{lll}84 & \mathrm{kgo}\end{array}$ & $\mathrm{eq} / \mathrm{m}^{2}$ & & \\
\hline
\end{tabular}

Life-Cycle Assessment Results:

Primary Energy Consumption (MJ)

\begin{tabular}{|c|c|c|c|c|c|c|c|c|c|c|c|c|c|c|c|c|}
\hline \multirow{3}{*}{ (Years } & \multicolumn{14}{|c|}{ Embodied Energy (EE) } & \multirow{2}{*}{\multicolumn{2}{|c|}{$\begin{array}{l}\text { Difference in } \\
\text { Operating Energy } \\
\text { from Baseline after } \\
\text { Lifiespan }\end{array}$}} \\
\hline & \multicolumn{3}{|c|}{ Manutacturing } & \multicolumn{3}{|c|}{ Construction } & \multicolumn{3}{|c|}{ Maintenance } & \multicolumn{3}{|c|}{ End of Lifie } & \multirow{2}{*}{$\begin{array}{c}3 \\
3 \\
\text { EE Total }\end{array}$} & \begin{tabular}{|c|}
${ }^{4}$ Total \\
EE
\end{tabular} & & \\
\hline & Material & \begin{tabular}{|l|l|}
${ }^{2}$ Trans. \\
\end{tabular} & Total & Material & ${ }^{2}$ Trans. & Total & Material & ${ }^{2}$ Trans. & Total & Materia & 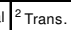 & Total & & & ${ }^{5}$ Tot: & ${ }^{6} \operatorname{perm}^{2}$ \\
\hline & 52,188 & 1,315 & 53,503 & 0 & 390 & 390 & 0 & 0 & 0 & 0 & 0 & 0 & 53,892 & 1,058 & & \\
\hline
\end{tabular}

Global Warming Potential (kg of $\mathrm{CO}_{2}$ eq.)

\begin{tabular}{|c|c|c|c|c|c|c|c|c|c|c|c|c|c|c|c|c|}
\hline \multirow{3}{*}{$\begin{array}{l}\text { Lifespan } \\
\text { (Years) }\end{array}$} & \multicolumn{14}{|c|}{ Embodied Global Warming Potential (GWP) } & \multirow{2}{*}{\multicolumn{2}{|c|}{$\begin{array}{l}\text { Difference in } \\
\text { Operating GWP fror } \\
\text { Baseline after } \\
\text { Lifespan }\end{array}$}} \\
\hline & \multicolumn{3}{|c|}{ Manufacturing } & \multicolumn{3}{|c|}{ Construction } & \multicolumn{3}{|c|}{ Maintenance } & \multicolumn{3}{|c|}{ End of L Life } & \multirow{2}{*}{$\begin{array}{l}{ }^{3} \text { Total } \\
\text { GWP }\end{array}$} & \multirow{2}{*}{$\begin{array}{l}{ }^{4} \text { Total } \\
\text { GWP } \\
\mathrm{perm}^{2}\end{array}$} & & \\
\hline & Material & ${ }^{2}$ Trans. & Total & Material & Trans. & Total & Material & Trans. & Total & Material & ${ }^{2}$ Trans. & Total & & & ${ }^{5}$ Total & ${ }^{6}{ }_{\text {per } m^{2}}$ \\
\hline Initial & 4,294 & 3 & 4,296 & 0 & 1 & 1 & $=$ & 0 & 0 & 0 & 0 & 0 & 4,297 & 84 & & \\
\hline 50 & 4,294 & 3 & 4,296 & 0 & 1 & 1 & 0 & 0 & 0 & 0 & 2 & 2 & 4,299 & 84 & 80,000 & 138 \\
\hline
\end{tabular}

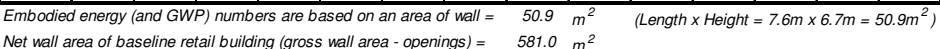

$\begin{aligned} & \text { ATHENA } \\
& \text { (Includes all materials after 50 50ears) }\end{aligned}$
\begin{tabular}{|l|c|c|}
\multicolumn{1}{|c|}{ Material List } & Quantities & Unit \\
\hline Concrete $30 \mathrm{MPa}$ (flyash av) & 10.7 & $\mathrm{~m} 3$ \\
\hline Extruded Polystyrene & 104.3 & $\mathrm{~m} 2(25 \mathrm{~mm})$ \\
\hline Nails & 3.1 & $\mathrm{~kg}$ \\
\hline Rebar, Rod, Light Sections & 994.0 & $\mathrm{~kg}$ \\
\hline Solvent Based Alkyd Paint & 116.5 & $\mathrm{~L}$ \\
\hline
\end{tabular}

Notes:
${ }^{1}$ Initial $=$ Time ' $O$ ' (i.e. at the completion of initial construction)

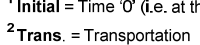

${ }^{3}$ Total EE (or Total GWP) $=$ Total embodied energy (or total embodied GWP)

of building component after lifespan (i.e. total man
construction + total maintenance + total end-of-life effects)

${ }^{4}$ Total EE (or Total GWP) per $\mathrm{m}^{2}=$ Total EE (or Total GWP) of building
component/area of building component that was modelled in ATHENAB EEE

Total Difference in Operating Energy (or GWP) from Baseline after Lifespan = The difference in the total life-cycle operating energy (or GWP)
from the baseline retali builing ater lifespan, due to using this building
component instead of the baseline component

${ }^{-}$Total Difference in Operating Energy (or GWP) from Baseline after Lifespan per $\mathrm{m}^{2}=$ Total difference in operating energy (or GWP) from

* Total operating primary energy use of baseline retail building after 50 years

of $\mathrm{CO}_{2} \mathrm{eq} .\left(80 \mathrm{~kg}\right.$ of $\mathrm{CO}_{2} \mathrm{eq} / \mathrm{m} \mathrm{m}^{2} \mathrm{yr}$. 


\section{Concrete Tilt-Up Wall \#5 (CTU-W5)}

Building Component Description:

\begin{tabular}{|c|c|c|c|c|}
\hline \multirow{3}{*}{\begin{tabular}{|l} 
Category: \\
Brief Description:
\end{tabular}} & \multicolumn{2}{|l|}{ Exerior Walls } & Assembly Layers & \multirow{10}{*}{5} \\
\hline & \multirow{2}{*}{\multicolumn{2}{|c|}{$\begin{array}{l}\text { Tilt-up insulated concrete sandwich panel } \\
\text { wall with } 100 \mathrm{~mm} \text { insulation }\end{array}$}} & $\begin{array}{l}\text { Outside } \\
\text { Akyd based paint }\end{array}$ & \\
\hline & & & $50 \mathrm{~mm}$ concrete front wyhe (30MPa, $9 \%$ flyash) & \\
\hline & & & (includes $8 \mathrm{~m}$ of $\# 15 \mathrm{Mper} \mathrm{m}^{2}$ ) & \\
\hline \multicolumn{3}{|l|}{ Quick Numbers: } & 100mm extruded polystyrene rigid insulation & \\
\hline ASHRAE Standard 90.1: & R.Value: 210 & RSI-Value 3.7 & $150 \mathrm{~mm}$ concrete back wyye (30MPa, 9\% filyash) & \\
\hline THERM 5.2: & R-Value: 21.0 & RSIVValue: 3.7 & (includes $8 \mathrm{~m}$ of \#1 $15 \mathrm{Mper} \mathrm{m}^{2}$ and steel angles) & \\
\hline Wall Thickness: & $300 \mathrm{~mm}$ & & & \\
\hline Total Embodied Energy: & $1,223 \mathrm{~m} / \mathrm{m}$ & & & \\
\hline Total Embodied GWP: & $92 \mathrm{~kg}$ of & $e q / \mathrm{m}^{2}$ & & \\
\hline
\end{tabular}

Life-Cycle Assessment Results:

Primary Energy Consumption (MJ)

\begin{tabular}{|c|c|c|c|c|c|c|c|c|c|c|c|c|c|c|c|c|}
\hline \multirow{3}{*}{\begin{tabular}{|l} 
Liffespan \\
(Years)
\end{tabular}} & \multicolumn{14}{|c|}{ Embodied Energy (EE) } & \multirow{2}{*}{\multicolumn{2}{|c|}{$\begin{array}{c}\text { Difference in } \\
\text { Operating Energy } \\
\text { from Baseline afte } \\
\text { Lifespan }\end{array}$}} \\
\hline & \multicolumn{3}{|c|}{ Manufacturing } & \multicolumn{3}{|c|}{ Construction } & \multicolumn{3}{|c|}{ Mainten } & \multicolumn{3}{|c|}{ End of Life } & \multirow{2}{*}{$\begin{array}{c}{ }^{3}{ }^{3} \text { Total } \\
\mathrm{EE}\end{array}$} & \multirow{2}{*}{\begin{tabular}{|c|}
${ }^{4} \mathrm{Total}$ \\
$\mathrm{EE}$ \\
per m
\end{tabular}} & & \\
\hline & Material & $\begin{array}{l}1{ }^{2} \text { Trans. } \\
\text {. }\end{array}$ & \begin{tabular}{|l|l|} 
Total \\
\end{tabular} & Materia & ${ }^{2}$ Trans. & Total & \begin{tabular}{|l|} 
Material \\
\end{tabular} & ${ }^{2}$ Trans. & \begin{tabular}{|l|l|} 
Total \\
\end{tabular} & Material & ${ }^{2}$ Trans. & \begin{tabular}{|l|l|} 
Total \\
\end{tabular} & & & ${ }^{5}$ Total & ${ }^{6}$ per m ${ }^{2}$ \\
\hline Initial & 59,771 & \begin{tabular}{|l|}
, 315 \\
\end{tabular} & \begin{tabular}{|l|l|}
61,086 \\
\end{tabular} & \begin{tabular}{|l|}
0 \\
\end{tabular} & \begin{tabular}{|l|}
390 \\
\end{tabular} & 390 & 0 & 0 & 0 & 0 & 0 & 0 & 61,476 & \begin{tabular}{|l|}
1,207 \\
\end{tabular} & & \\
\hline 50 & 59,771 & 1,315 & 661,086 & 0 & 390 & 390 & 0 & 0 & 0 & 2 & 810 & \begin{tabular}{|l|l|} 
& 811 \\
\end{tabular} & 62,287 & 11 & 000 & $-1,893$ \\
\hline
\end{tabular}

Global Warming Potential ( $\mathrm{kg}$ of $\mathrm{CO}_{2}$ eq.)

$\infty$

\begin{tabular}{|c|c|c|c|c|c|c|}
\hline \multirow{3}{*}{$\begin{array}{l}\text { Lifespan } \\
\text { (Years) }\end{array}$} & \\
\hline & \multicolumn{3}{|c|}{ Manutacturing } & \multicolumn{3}{|c|}{ Construction } \\
\hline & Material & ${ }^{2}$ Trans. & Total & Material & \begin{tabular}{|l|}
${ }^{2}$ Trans. \\
\end{tabular} & \begin{tabular}{|l|l|l|} 
Total \\
\end{tabular} \\
\hline \begin{tabular}{|l|}
${ }^{1}$ Initial \\
\end{tabular} & 4,682 & 3 & 4,684 & 0 & 1 & 1 \\
\hline 50 & 4,682 & 3 & \begin{tabular}{|l|l}
4,684 \\
\end{tabular} & \begin{tabular}{|l|l|}
0 & \\
\end{tabular} & 1 & \\
\hline \multicolumn{7}{|c|}{$\begin{array}{l}\text { Embodied energy (and GWP) numbers are based on an area of wa } \\
\text { Net wall area of baseline retail building (gross wall area - openings }\end{array}$} \\
\hline \multicolumn{7}{|c|}{$\begin{array}{c}\text { ATHENA } ® \text { EIE Material List: } \\
\text { (Includes all materials after } 50 \text { years) }\end{array}$} \\
\hline \multicolumn{4}{|c|}{ Material List } & \multicolumn{2}{|c|}{ Quantities } & Unit \\
\hline \multicolumn{4}{|c|}{ Concrete $30 \mathrm{MPa}$ (flyash av) } & & 10.7 & $\mathrm{~m} 3$ \\
\hline \multicolumn{4}{|c|}{ Extruded Polystyrene } & & 208.6 & m2 $2(25 \mathrm{~mm}$ \\
\hline \multicolumn{4}{|l|}{ Nails } & & 3.1 & $\mathrm{~kg}$ \\
\hline \multirow{2}{*}{\multicolumn{4}{|c|}{$\begin{array}{l}\text { Rebar, Rod, Light Sectic } \\
\text { Solvent Based Alkyd Pa }\end{array}$}} & & 994.0 & $\mathrm{~kg}$ \\
\hline & & & & & 116.5 & \\
\hline
\end{tabular}

Notes:

${ }^{1}$ 'Initial = Time 'o' (i.e. at

${ }^{3}$ Total EE (or Total GWP) $=$ Total embodied energy (or total embodied GWP) of building component after lifespan (i.e. total maal
construction + total maintenance + total end-oflife effects)

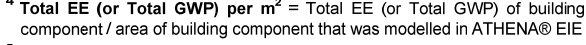

${ }^{5}$ Total Difference in Operating Energy (or GWP) from Baseline after

\section{Concrete Tilt-Up Wall \#6 (CTU-W6)}

Building Component Description:

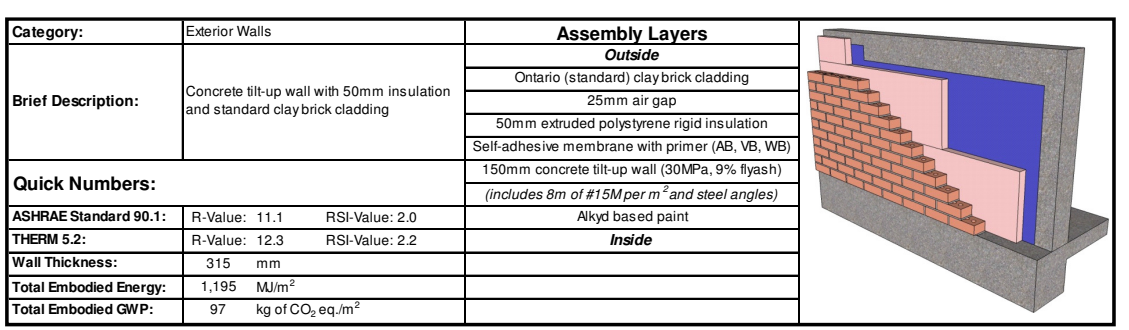

Life-Cycle Assessment Results:

Primary Energy Consumption (MJ)

\begin{tabular}{|c|c|c|c|c|c|c|c|c|c|c|c|c|c|c|c|c|}
\hline \multirow{3}{*}{$\begin{array}{l}\text { Lifespan } \\
\text { (Years) }\end{array}$} & \multicolumn{14}{|c|}{ Embodied Energy (EE) } & \multirow{2}{*}{\multicolumn{2}{|c|}{$\begin{array}{c}\text { Difference in } \\
\text { Operating Energy } \\
\text { from Baseline after } \\
\text { Lifespan }\end{array}$}} \\
\hline & \multicolumn{3}{|c|}{ Manutacturing } & \multicolumn{3}{|c|}{ Construction } & \multicolumn{3}{|c|}{ Maintenance } & \multicolumn{3}{|c|}{ End of Life } & \multirow{2}{*}{$\begin{array}{c}{ }^{3} \text { Total } \\
\mathrm{EE}\end{array}$} & \multirow{2}{*}{$\begin{array}{c}{ }^{4} \mathrm{Total}^{2} \\
\mathrm{EE} \\
\text { per } \mathrm{m}^{2}\end{array}$} & & \\
\hline & Material & \begin{tabular}{l|l|}
${ }^{2}$ Trans. \\
\end{tabular} & Total & Material & ${ }^{2}$ Trans. & Total & Material & 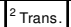 & Total & Material & $\begin{array}{l}2 \\
2\end{array}$ Trans. & Total & & & ${ }^{5}$ Total & ${ }^{6}{ }_{\mathrm{perm}}{ }^{2}$ \\
\hline Intural & 55,622 & 1,084 & 56,706 & 0 & 7 & 7 & 0 & 0 & 0 & 0 & 0 & 0 & 56,713 & 1,114 & & \\
\hline
\end{tabular}

Global Warming Potential (kg of $\mathrm{CO}_{2}$ eq.)

\begin{tabular}{|c|c|c|c|c|c|c|c|c|c|c|c|c|c|c|c|c|}
\hline \multirow{3}{*}{$\begin{array}{l}\text { Lifespan } \\
\text { (Years) }\end{array}$} & \multicolumn{14}{|c|}{ Embodied Global Warming Potential (GWP) } & \multirow{2}{*}{\multicolumn{2}{|c|}{$\begin{array}{c}\text { Difference in } \\
\text { Operating GWP fron } \\
\text { Baseline after } \\
\text { Lifespan }\end{array}$}} \\
\hline & \multicolumn{3}{|c|}{ Manufacturing } & \multicolumn{3}{|c|}{ Construction } & \multicolumn{3}{|c|}{ Maintenance } & \multicolumn{3}{|c|}{ End of Lifie } & \multirow{2}{*}{$\begin{array}{l}{ }^{3} \text { Total } \\
\text { GWP }\end{array}$} & \multirow{2}{*}{$\begin{array}{l}{ }^{4}{ }^{4} \text { Total } \\
\text { GWP } \\
\text { per m }\end{array}$} & & \\
\hline & Material & ${ }^{2}$ Trans & Total & Material & 2 Trans. & Total & Material & ${ }^{2}$ Trans. & Total & Material & | ${ }^{2}$ Trans. & Total & & & ${ }^{5}$ Total & ${ }^{6} \mathrm{perm}^{2}$ \\
\hline 1 Initial & 4,914 & 2 & 4,916 & 0 & 0 & 0 & 0 & 0 & 0 & 0 & 0 & 0 & 4,916 & 97 & & \\
\hline 50 & 4,914 & 2 & 4,916 & 0 & 0 & 0 & 44 & 0 & 44 & 0 & 2 & 2 & 4,962 & 97 & \begin{tabular}{|l|l|l}
50,000 \\
\end{tabular} & 86 \\
\hline
\end{tabular}

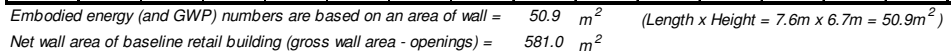

ATHENA $®$ EIE Material List: Lifespan = The difierence in the total lific-cycle operating energy (or GWP)
from the baseline retail building after lifespan, due to using this building Irom the baseline retali bulling atter litess
component instead of the baseline component

${ }^{6}$ Total Difference in Operating Energy (or GWP) from Baseline after Lifespan per $\mathrm{m}^{2}=$ Total difference in operating energy (or GWP) from

* Total operating primary energy use of baseline retail building after 50 years $=$

Total operating $\mathrm{GWP}$ of baseline retail building after 50 years $=2,310$ tonnes
of $\mathrm{CO}_{2} \mathrm{eq} .\left(80 \mathrm{~kg}\right.$ of $\left.\mathrm{CO}_{2} \mathrm{eq} / \mathrm{m}^{2} / \mathrm{yr}\right)$
(Includes all materials after 50 years)

\begin{tabular}{|l|c|c|}
\multicolumn{1}{|c|}{ Material List } & Quantities & Unit \\
\hline 3 mil Polyethylene & 54.0 & $\mathrm{~m} 2$ \\
\hline Cold Rolled Sheet & 10.3 & $\mathrm{~kg}$ \\
\hline Concrete 30 MPa (flyash av) & 8.0 & $\mathrm{~m} 3$ \\
\hline Extruded Polystyrene & 104.3 & $\mathrm{~m} 2(25 \mathrm{~mm})$ \\
\hline Modified Bitumen membrane & 68.2 & $\mathrm{~kg}$ \\
\hline Mortar & 1.5 & $\mathrm{~m} 3$ \\
\hline Nails & 3.1 & $\mathrm{~kg}$ \\
\hline Ontario (Standard) Brick & 53.5 & $\mathrm{~m} 2$ \\
\hline Rebar, Rod, Light Sections & 347.6 & $\mathrm{~kg}$ \\
\hline Solvent Based Alkyd Paint & 77.9 & $\mathrm{~L}$ \\
\hline
\end{tabular}

Notes:
1 Initial $=$ Time ' $O$ ' $($ i.e. at the completion of initial construction)

${ }^{2}$ Trans. $=$ Transportation

Total EE (or Total GWP) $=$ Total embodied energy (or total embodied GWP)

of building component after lifespan (i.e. total manu
construction + total maintenance + total end-o-f-life effects)

${ }^{4}$ Total EE (or Total GWP) per $\mathrm{m}^{2}=$ Total EE (or Total GWP) of building
component / area of building component that was modelled in ATHENAQ EIE

${ }^{5}$ Total Difference in Operating Energy (or GWP) from Baseline after fitespan = The difference in the total life-cycle operating energy (or GWP
from the baseline retail building after lifespan, due to using this building component instead of the baseline component

${ }^{6}$ Total Difference in Operating Energy (or GWP) from Baseline after Lifespan per $\mathrm{m}^{2}=$ Total difference in operating energy (or GWP) from

* Total operating primary energy use of baseline retail building after 50 years =
"Total operating $\mathrm{GWP}$ of baseline reta 


\section{Concrete Tilt-Up Wall \#7 (CTU-W7)}

Building Component Description:

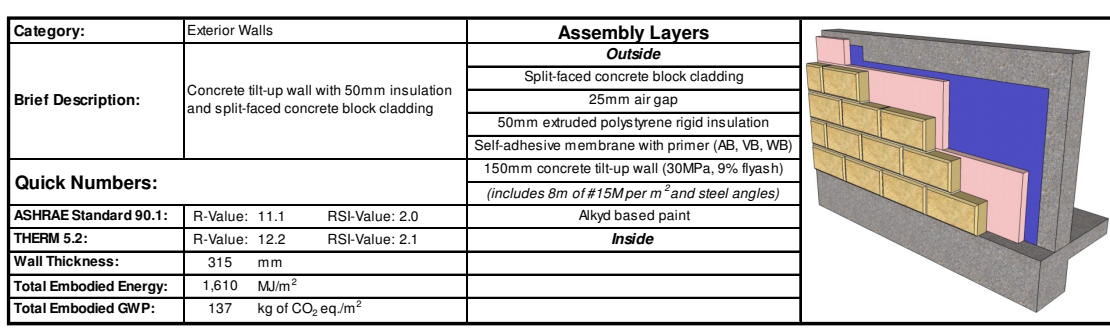

Life-Cycle Assessment Results:

Primary Energy Consumption (MJ)

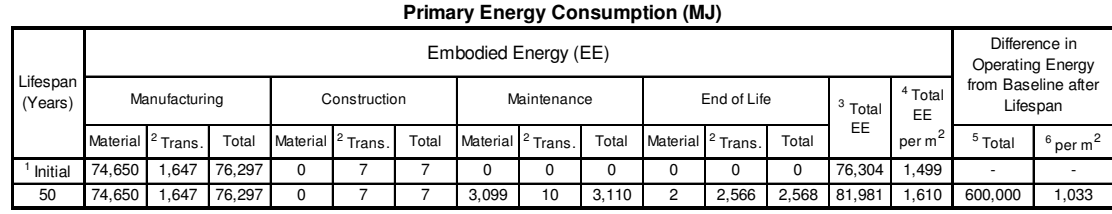

Global Warming Potential ( $k g$ of $\mathrm{CO}_{2}$ eq.)

$\varpi$

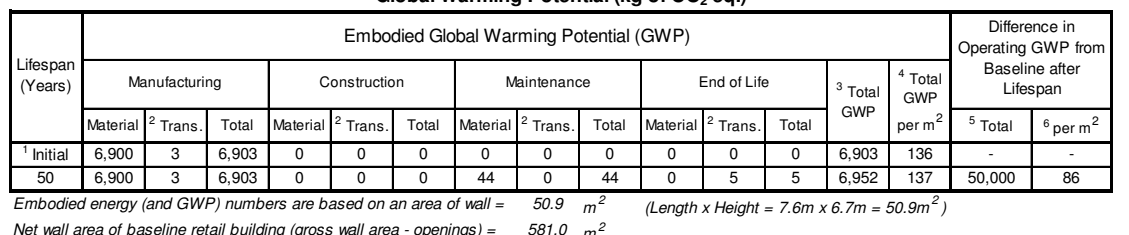

Net wall area of baseline retail building (gross wall area - openings) $=581.0$

ATHENA $®$ EIE Material List: (Includes all materials after 50 years

\begin{tabular}{|l|c|c|}
\hline \multicolumn{1}{|c|}{ Material List } & Quantities & Unit \\
\hline 3 mil Polyethylene & 54.0 & $\mathrm{~m} 2$ \\
\hline Cold Rolled Sheet & 10.3 & $\mathrm{~kg}$ \\
\hline Concrete 30 OPa (filyash av) & 8.0 & $\mathrm{~m} 3$ \\
\hline Extruded Polystyrene & 104.3 & $\mathrm{~m} 2(25 \mathrm{~mm})$ \\
\hline Modified Bitumen membrane & 40.1 .5 & $\mathrm{~kg}$ \\
\hline Mortar & 4.3 & $\mathrm{~m} 3$ \\
\hline Nails & 3.1 & $\mathrm{~kg}$ \\
\hline Rebar, Rod, Light Sections & 347.6 & $\mathrm{~kg}$ \\
\hline Solvent Based Alkyd Paint & 77.9 & $\mathrm{~L}$ \\
\hline Split-faced Concrete Block & $1,238.2$ & Blocks \\
\hline
\end{tabular}

Notes:
${ }^{1}$ Initial $=$ Time ' 0 ' (i.e. at the completion of initial construction)

${ }^{2}$ Trans. $=$ Transportation

Total EE (or Total GWP) = Total embodied energy (or total embodied GWP) of building component after lifespan (i.e. total manufacturing + tot

${ }^{4}$ Total EE (or Total GWP) per $\mathrm{m}^{2}=$ Total EE (or Total GWP) of building
component/ area of building component that was modelled in ATHENAQ EIE Total Difference in Operating Energy (or GWP) from Baseline after Lifespan $=$ The difference in the total lific-cycle operating energy (or GWP) from the baseline retail building after lifes
component instead of the baseline component

Total Difference in Operating Energy (or GWP) from Baseline aftor Lifespan per $\mathrm{m}^{2}=$ Total difference in operating energy (or GWP) from

* Total operating primary energy use of baseline retail building after 50 years
$50,700 \mathrm{GJ}\left(1,745 \mathrm{MJ} / \mathrm{m}^{2} \mathrm{yr}\right)$

Total operating $\mathrm{GWP}$ of baseline ref
of $\mathrm{CO}_{2}$ eq. $80 \mathrm{~kg}$ of $\mathrm{CO}_{2}$ eq. $\left./ \mathrm{m}^{2} \mathrm{yr}\right)$

\section{LCA Data for Wood Structural Insulated Panel Walls}

This section contains a detailed description of each wood structural insulated panel (WSIP) exterior infill wall that was examined in this study (12 in total). The assembly layers are listed for each wall, along with a detailed description of the material quantities from the ATHENA® Environmental Impact Estimator for Buildings.

A breakdown of the total primary energy consumption and the total global warming potential (GWP) for each wall is also included. In each case, the results were calculated for an area of wall equal to $50.9 \mathrm{~m}^{2}$, which represents a typical bay size for a single-storey retail building. The results are also expressed on a per $\mathrm{m}^{2}$ basis in each case. The data has been calculated for two different lifespans: at the completion of initial construction and after 50 years.

As a summary, the figure below illustrates a comparison of the total embodied energy (and GWP) after 50 years for the various WSIP walls in this section and the metal structural insulated panel (MSIP) exterior infill walls from the next section. For comparison purpose, the building component used in the baseline retail building has been shaded in grey.

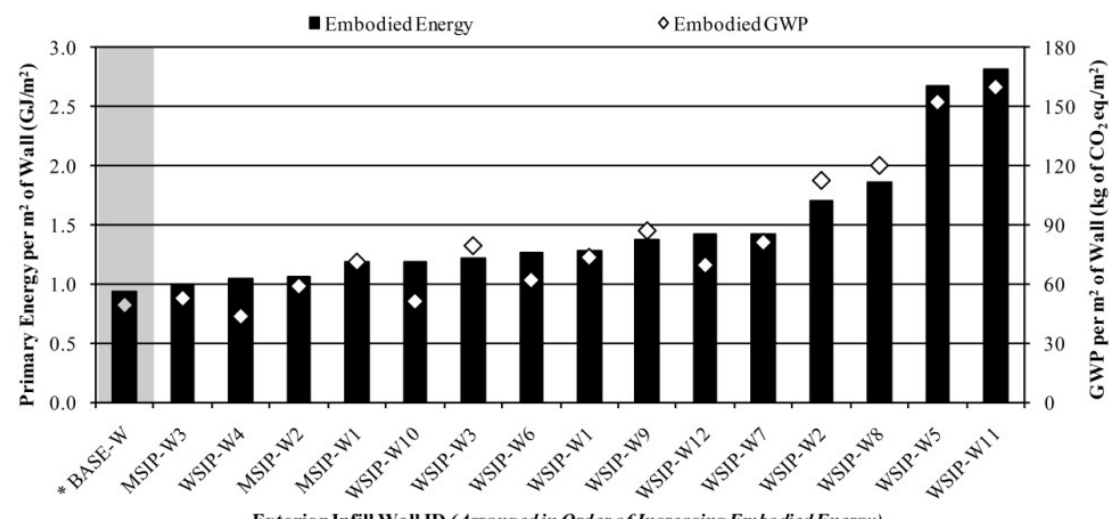


Wood Structural Insulated Panel Wall \#1 (WSIP-W1)

Building Component Description:

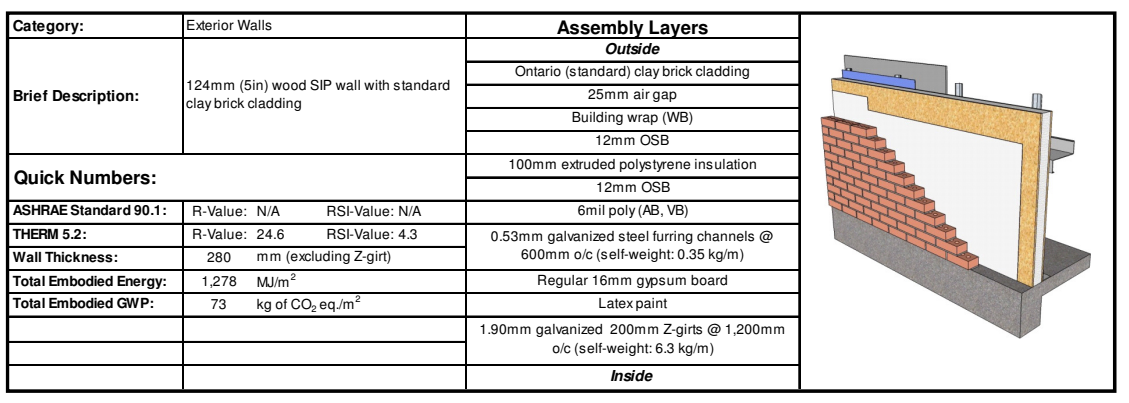

Life-Cycle Assessment Results:

Primary Energy Consumption ( $\mathrm{M}$

\begin{tabular}{|c|c|c|c|c|c|c|c|c|c|c|c|c|c|c|c|c|}
\hline \multirow{3}{*}{$\begin{array}{l}\text { Lifiespan } \\
\text { (Years) }\end{array}$} & \multicolumn{14}{|c|}{ Embodied Energy (EE) } & \multirow{2}{*}{\multicolumn{2}{|c|}{$\begin{array}{l}\text { Difference in } \\
\text { Operating Energy } \\
\text { from Baseline after } \\
\text { Lifespan }\end{array}$}} \\
\hline & \multicolumn{3}{|c|}{ Manufacturing } & \multicolumn{3}{|c|}{ Construction } & \multicolumn{3}{|c|}{ Maintenance } & \multicolumn{3}{|c|}{ End of Life } & \multirow{2}{*}{$\begin{array}{c}{ }^{3} \text { Total } \\
\mathrm{EE}\end{array}$} & \multirow{2}{*}{\begin{tabular}{|c|}
${ }^{4}$ Total \\
EE \\
per $\mathrm{m}^{2}$
\end{tabular}} & & \\
\hline & & \begin{tabular}{l|l|}
2 & Trans.
\end{tabular} & Total & \begin{tabular}{|l|} 
Material \\
\end{tabular} & Trans. & Total & \begin{tabular}{|l|} 
Material \\
\end{tabular} & \begin{tabular}{|l|}
2 Trans. \\
\end{tabular} & Total & Material & \begin{tabular}{|l|}
${ }^{2}$ Trans. \\
\end{tabular} & \begin{tabular}{|l|l|} 
Total \\
\end{tabular} & & & ${ }^{5}$ Total & ${ }^{6}$ per $\mathrm{m}^{2}$ \\
\hline & 62,680 & $5 \quad 502$ & 63,182 & 160 &, 203 & 1,363 & 0 & 0 & 0 & 0 & 0 & 0 & 64,545 & 1,268 & & \\
\hline
\end{tabular}

Global Warming Potential (kg of $\mathrm{CO}_{2}$ eq.)

\begin{tabular}{|c|c|c|c|c|c|c|c|c|c|c|c|c|c|c|c|c|}
\hline \multirow{4}{*}{$\begin{array}{l}\text { Lifespan } \\
\text { (Years) }\end{array}$} & \multicolumn{14}{|c|}{ Global Warming Potential ( $\mathrm{kg}$ of $\mathrm{CO}_{2}$ eq.) } & \multirow{3}{*}{\multicolumn{2}{|c|}{$\begin{array}{c}\text { Difference in } \\
\text { Operating GWPP from } \\
\text { Baseline affer } \\
\text { Lifespan }\end{array}$}} \\
\hline & \multicolumn{14}{|c|}{ Embodied Global Warming Potential (GWP) } & & \\
\hline & \multicolumn{3}{|c|}{ Manufacturing } & \multicolumn{3}{|c|}{ Construction } & \multicolumn{3}{|c|}{ Maintenance } & \multicolumn{3}{|c|}{ End of Life } & \multirow{2}{*}{$\begin{array}{l}{ }^{3} \text { Total } \\
\text { GWP }\end{array}$} & \multirow{2}{*}{\begin{tabular}{|l}
${ }^{4}{ }^{2}$ Total \\
GWW \\
per m
\end{tabular}} & & \\
\hline & Material & ${ }^{2}$ Trans. & \begin{tabular}{|l|l|l|l} 
Total & \\
\end{tabular} & Material & ${ }^{2}$ Trans. & Total & Material & ${ }^{2}$ Trans. & Total & Material & ||$^{2}$ Trans. & Total & & & ${ }^{5}$ Total & ${ }^{6}$ per m$^{2}$ \\
\hline \begin{tabular}{|l|l|} 
IInitial \\
\end{tabular} & 3,727 & 1 & 3,728 & 10 & 2 & 13 & 0 & 0 & 0 & 0 & 0 & 0 & 3,740 & 73 & & \\
\hline 50 & 3,727 & 1 & 3,728 & 10 & 2 & 13 & 0 & 0 & 0 & 0 & 1 & 1 & 3,742 & 73 & $-80,000$ & -138 \\
\hline
\end{tabular}

Embodied energy (and GWP) numbers are based on an area of wall $=\begin{aligned} 50.9 \mathrm{~m}^{2} \\ 581.0\end{aligned} \quad$ (Length $x$ Height $=7.6 \mathrm{~m} \times 6.7 \mathrm{~m}=50.9 \mathrm{~m}^{2}$ )

\section{ATHENA @ EIE Material List:}

\begin{tabular}{|l|c|c|}
\hline \multicolumn{1}{|c|}{ Material List } & Quantities & Unit \\
\hline 16mm Regular Gypsum Board & 56.0 & $\mathrm{~m} 2$ \\
\hline 6 mil Polyethylene & 108.0 & $\mathrm{~m} 2$ \\
\hline Cold Rolled Sheet & 10.3 & $\mathrm{~kg}$ \\
\hline Extruded Polystyrene & 208.6 & $\mathrm{~m} 2(25 \mathrm{~mm})$ \\
\hline Galvanized Sheet & 30.0 & $\mathrm{~kg}$ \\
\hline Galvanized Studs & 287.9 & $\mathrm{~kg}$ \\
\hline Joint Compound & 55.9 & $\mathrm{~kg}$ \\
\hline Mortar & 1.5 & $\mathrm{~m} 3$ \\
\hline Nails & 3.7 & $\mathrm{~kg}$ \\
\hline Ontario (Standard) Brick & 53.5 & $\mathrm{~m} 2$ \\
\hline Oriented Strand Board & 145.8 & $\mathrm{~m} 2(9 \mathrm{~mm})$ \\
\hline Paper Tape & 0.6 & $\mathrm{~kg}$ \\
\hline Screws Nuts \& Bolts & 2.6 & $\mathrm{~kg}$ \\
\hline Water Based Latex Paint & 66.3 & $\mathrm{~L}$ \\
\hline
\end{tabular}

$$
\begin{aligned}
& \text { Notes: } \\
& { }^{1} \text { Initial }=\text { Time 'o' (i.e. at the completion of initial construction) }
\end{aligned}
$$
Total EE (or Total GWP) $=$ Total embodied energy (or total embodied GWP
of building component after lifespan (i.e. total manufacturing + tota ${ }^{4}$ Total EE (or Total GWP) per $\mathrm{m}^{2}=$ Total EE (or Total GWP) of building
component / area of building component that was modelled in ATHENAQ EIE Total Difference in Operating Energy (or GWP) from Baseline after Lifespan $=$ The difference in the total life-cycle operating energy (or GWP)
from the baseline retail building after lifespan, due to using this building from the baseline retail building after lifespan,
component instead of the baseline component

Total Difference in Operating Energy (or GWP) from Baseline after Lifespan per $\mathrm{m}^{2}=$ Total difference in operating energy (or GWP) from

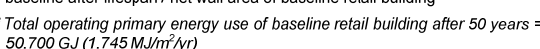

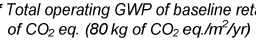

\section{Wood Structural Insulated Panel Wall \#2 (WSIP-W2)}

Building Component Description:

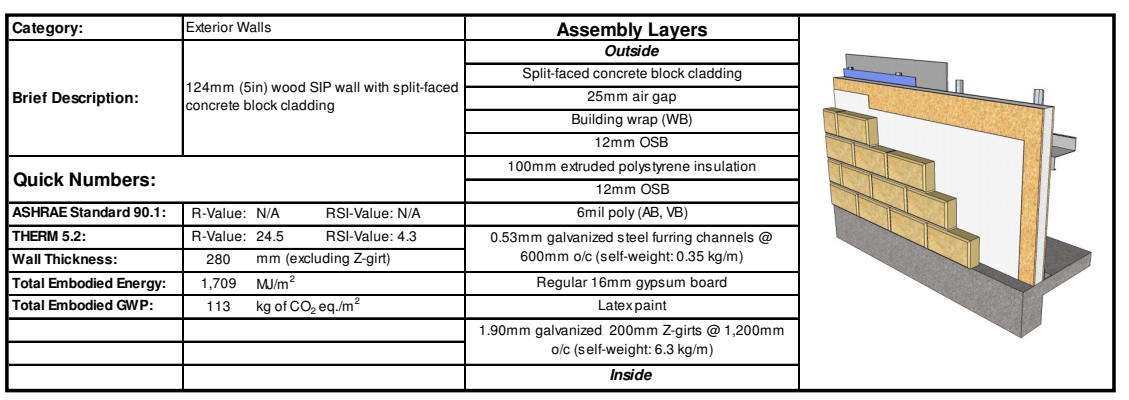

Life-Cycle Assessment Results:

\begin{tabular}{|c|c|c|c|c|c|c|c|c|c|c|c|c|c|c|c|c|}
\hline & \multicolumn{14}{|c|}{ y Energy Consun } & \multirow{3}{*}{\multicolumn{2}{|c|}{\begin{tabular}{|c} 
Difference in \\
Operating Energy \\
from Baseline after \\
Lifespan
\end{tabular}}} \\
\hline \multirow{3}{*}{ (Years) } & & & & & & & bodied & | Energy ( & & & & & & & & \\
\hline & \multicolumn{3}{|c|}{ Manutacturing } & \multicolumn{3}{|c|}{ Construction } & \multicolumn{3}{|c|}{ Maintena } & \multicolumn{3}{|c|}{ End of Life } & \multirow{2}{*}{$\begin{array}{l}{ }^{3} \text { Total } \\
\mathrm{EE}\end{array}$} & \multirow{2}{*}{$\begin{array}{c}{ }^{4} \text { Total } \\
\mathrm{E} \\
\text { per } \mathrm{m}^{2}\end{array}$} & & \\
\hline & \begin{tabular}{|l|} 
Material \\
\end{tabular} & ${ }^{2}$ Trans. & Total & Material & $1{ }^{2}{ }^{2}$ Trans. & Total & Naterial & al ${ }^{2}$ Trans. & Total & Material & 11 ${ }^{2}$ Trans. & Total & & & ${ }^{5}$ Total & ${ }^{6}{ }_{\text {per m}}^{2}$ \\
\hline Initia & $\begin{array}{l}11,708 \\
91709\end{array}$ & 1055 & 82,773 & 160 & & $\frac{2,173}{2,172}$ & 0 & & $\pi$ & 0 & 20 & 0 & $\begin{array}{ll}84,946 \\
80009\end{array}$ & 668 & & \\
\hline 50 & $\mid 181,708$ & $\mid 1,065$ & 82,773 & 160 & 2,013 & 2,173 & 0 & 0 & 0 & 1 & 2,061 & 2,062 & 87,008 & 1,709 & & .75 \\
\hline
\end{tabular}

\begin{tabular}{|c|c|c|c|c|c|c|c|c|c|c|c|c|c|c|c|c|}
\hline & \multicolumn{16}{|c|}{ Global Warming Potential ( $\mathrm{kg}$ of $\mathrm{CO}_{2}$ eq.) } \\
\hline \multirow{3}{*}{$\begin{array}{l}\text { Lifespan } \\
\text { (Years) }\end{array}$} & \multicolumn{14}{|c|}{ Embodied Global Warming Potential (GWP) } & \multirow{2}{*}{\multicolumn{2}{|c|}{$\begin{array}{l}\text { Difference in } \\
\text { Operating GWP from } \\
\text { Baseline a atter } \\
\text { Lifespan }\end{array}$}} \\
\hline & \multicolumn{3}{|c|}{ Manutacturing } & \multicolumn{3}{|c|}{ Construction } & \multicolumn{3}{|c|}{ Maintenance } & \multicolumn{3}{|c|}{ End of Life } & \multirow{2}{*}{$\begin{array}{l}{ }^{3} \text { Total } \\
\text { GWP }\end{array}$} & \multirow{2}{*}{$\begin{array}{l}{ }^{4} \text { Total } \\
\text { GWP } \\
\text { per m }{ }^{2}\end{array}$} & & \\
\hline & Material & ${ }^{2}$ Trans. & Total & Material & ${ }^{2}$ Trans. & Total & Material & ${ }^{2}$ Trans. & Total & Materia & al ${ }^{2}$ Trans. & Total & & & ${ }^{5}$ Total & ${ }^{6}{ }_{\text {perm }}^{2}$ \\
\hline${ }^{1}$ Initial & 5,713 & 2 & 5,715 & 10 & 4 & 14 & 0 & 0 & 0 & 0 & 0 & 0 & 5,729 & 113 & & \\
\hline 50 & 5,713 & 2 & 5,715 & 10 & 4 & 14 & 0 & 0 & 0 & 0 & 4 & 4 & 5,733 & $\begin{array}{lll}113 \\
\end{array}$ & $-80,000$ & -138 \\
\hline
\end{tabular}

Embodied energy (and GWP) numbers are based on an area of wall $=\begin{array}{rllll}50.9 & \mathrm{~m}^{2}\end{array} \quad$ (Length $x$ Height $=7.6 \mathrm{~m} \times 6.7 \mathrm{~m}=50.9 \mathrm{~m}^{2}$ ) Net wall area of baseline retail building (gross wall area - openings) $=581.0 \mathrm{~m}^{2}$

\section{ATHENA ${ }^{\circledR}$ EIE Material List:}

\begin{tabular}{|c|c|c|}
\hline Material List & Quantities & Unit \\
\hline $16 \mathrm{~mm}$ Regular Gypsum Board & 56.0 & $\mathrm{~m}^{\mathrm{m} 2}$ \\
\hline 6 mil Polyethylene & 108.0 & $\frac{\mathrm{m} 2}{\mathrm{~m} 2} \mathrm{Y}$ \\
\hline Cold Rolled Sheet & 10.3 & $\mathrm{~kg}$ \\
\hline Extruded Polystyrene & 208.6 & m2 (25mn \\
\hline Galvanized Sheet & 30.0 & $\mathrm{~kg}$ \\
\hline Galvanized Studs & 287.9 & $\mathrm{~kg}$ \\
\hline Joint Compound & 55.9 & $\mathrm{~kg}$ \\
\hline Modified Bitumen membrane & 333.3 & $\mathrm{~kg}$ \\
\hline Mortar & 4.3 & $\mathrm{~m} 3$ \\
\hline Nails & 3.7 & $\mathrm{~kg}$ \\
\hline Oriented Strand Board & 145.8 & $\mathrm{~m} 2(9 \mathrm{~mm}$ \\
\hline Paper Tape & 0.6 & $\mathrm{~kg}$ \\
\hline Screws Nuts \& Bolts & 2.6 & $\mathrm{~kg}$ \\
\hline Split-faced Concrete Block & $1,238.2$ & Blocks \\
\hline Water Based Latex Paint & 66.3 & L \\
\hline
\end{tabular}

Notes:
${ }^{1}$ Initial $=$ Time 'o' (i.e. at the completion of initial construction)

${ }^{2}$ Trans $=$ Transportation

"Total EE (or Total GWP) = Total embodied energy (or total embodied GWP

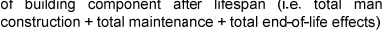

Total EE (or Total GWP) per $\mathrm{m}^{2}=$ Total EE (or Total GWP) of building component/ area of buidang component that was modelled in ATHENAQ EI Total Difference in Operating Energy (or GWP) from Baseline after
Lifespan $=$ The difference in the total lifecycle operating energy (or GWWP)

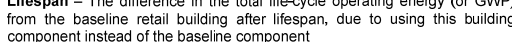
Total Difference in Operating Energy (or GWP) from Baseline after
Lifespan per $\mathrm{m}^{2}=$ Total difference in operating energy (or GWP) from

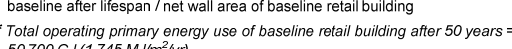

"Total operating GWP of baseline reta 
Wood Structural Insulated Panel Wall \#3 (WSIP-W3)

Building Component Description:

\begin{tabular}{|c|c|c|c|c|}
\hline \multirow{4}{*}{ Brief Description: } & \multicolumn{2}{|l|}{ Exterior Walls } & Assembly Layers & \\
\hline & \multirow{3}{*}{\multicolumn{2}{|c|}{$\begin{array}{l}124 \mathrm{~mm} \text { (5in) wood SIP wall with concrete } \\
\text { pre-cast cladding }\end{array}$}} & $\begin{array}{l}\text { Outside } \\
125 \mathrm{~mm} \text { concrete rec.cast claddina }\end{array}$ & \\
\hline & & & $25 \mathrm{~mm}$ air gap & \\
\hline & & & Building wrap (WB) & \\
\hline \multirow{2}{*}{\multicolumn{3}{|c|}{ Quick Numbers: }} & $100 \mathrm{~mm}$ extruded polystrene insulation & \\
\hline & & & $12 \mathrm{~mm}$ OSB & \\
\hline ASHRAE Standard 90.1: & R-Value: $N / A$ & RSI-Value: N/A & 6 mil poly $(A B, V B)$ & \\
\hline THERM 5.2: & R-Value: 24.5 & RSI-Value: 4.3 & $3 \mathrm{~mm}$ galvanized steel furring channels @ & \\
\hline Wall Thickness: & $315 \mathrm{~mm}$ & (ding z-girt) & $600 \mathrm{~mm}$ o/ (self-weight $0.35 \mathrm{~kg} / \mathrm{m}$ ) & \\
\hline \multirow{3}{*}{ Total Embodied GWP: } & $\begin{array}{ll}1,224 \quad \mathrm{~m} / \mathrm{m} \\
70\end{array}$ & & $\begin{array}{l}\text { Regular 16mm gypsum board } \\
\text { Latexpaint }\end{array}$ & \\
\hline & & & 1.90mm galvanized 200mm Z-girts @ 1,200mm & \\
\hline & & & 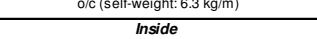 & \\
\hline
\end{tabular}

\section{Life-Cycle Assessment Results:}

Primary Energy Consumption (MJ)

\begin{tabular}{|c|c|c|c|c|c|c|c|c|c|c|c|c|c|c|c|c|}
\hline \multirow{3}{*}{$\begin{array}{l}\text { Lifespan } \\
\text { (Years) }\end{array}$} & \multicolumn{14}{|c|}{ Embodied Energy (EE) } & \multirow{2}{*}{\multicolumn{2}{|c|}{$\begin{array}{c}\text { Difference in } \\
\text { Operating Energy } \\
\text { from Baseline atte } \\
\text { Lifespan }\end{array}$}} \\
\hline & \multicolumn{3}{|c|}{ Manutacturing } & \multicolumn{3}{|c|}{ istruction } & \multicolumn{3}{|c|}{ Maintenance } & \multicolumn{3}{|c|}{ End of Life } & \multirow{2}{*}{${ }^{3}$ Total } & \multirow{2}{*}{$\begin{array}{c}{ }^{4} \text { Total } \\
\text { eE } \\
\text { per m }\end{array}$} & & \\
\hline & \begin{tabular}{|l|} 
Material \\
\end{tabular} & $1^{2}{ }^{2}$ Trans. & | Total & \begin{tabular}{|l|} 
Materia \\
\end{tabular} & ${ }^{2}$ Trans. & \begin{tabular}{|l|} 
Total \\
\end{tabular} & \begin{tabular}{|l|} 
Materia \\
\end{tabular} & ${ }^{2}$ Trans. & \begin{tabular}{|l|} 
Total \\
\end{tabular} & Material & 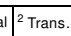 & Total & & & \begin{tabular}{|l|}
${ }^{5}$ Total \\
\end{tabular} & ${ }^{6} \operatorname{perm} \mathrm{m}^{2}$ \\
\hline Initial & 59,367 & 1,093 & 60,460 & 160 & 1,142 & \begin{tabular}{|l|l|}
1,302 \\
\end{tabular} & 0 & 0 & 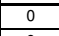 & 0 & 0 & 0 & 61,762 & 1,213 & & \\
\hline 50 & 59,367 & 1,093 & 60,460 & 160 & 1,142 & 1,302 & 0 & 0 & 0 & 2 & 568 & 570 & 62,332 & & $-1,600$ & $-2,76$ \\
\hline
\end{tabular}

Global Warming Potential ( $\mathrm{kg}$ of $\mathrm{CO}_{2}$ eq.)

\begin{tabular}{|c|c|c|c|c|c|c|c|c|c|c|c|c|c|c|c|c|}
\hline \multirow{3}{*}{$\begin{array}{l}\text { Lifespan } \\
\text { (Years) }\end{array}$} & \multicolumn{14}{|c|}{ Embodied Global Warming Potential (GWP) } & \multirow{2}{*}{\multicolumn{2}{|c|}{\begin{tabular}{|c} 
Difference in \\
Operating GWP from \\
Baseline after \\
Lifespan
\end{tabular}}} \\
\hline & \multicolumn{3}{|c|}{ Manufacturing } & \multicolumn{3}{|c|}{ Construction } & \multicolumn{3}{|c|}{ Maintenance } & \multicolumn{3}{|c|}{ End of Life } & \multirow{2}{*}{$\begin{array}{l}{ }^{3} \text { Total } \\
\text { GWP }\end{array}$} & \multirow{2}{*}{\begin{tabular}{|l}
${ }^{4}{ }^{\mathrm{T} o t a l}$ \\
GWP \\
per m
\end{tabular}} & & \\
\hline & Material & ${ }^{2}$ Trans & $\begin{array}{l}\text { Total } \\
\end{array}$ & Material & ${ }^{2}$ Trans. & Total & Material & \begin{tabular}{|l|}
${ }^{2}$ Trans. \\
\end{tabular} & Total & \begin{tabular}{|c|} 
Material \\
\end{tabular} & ${ }^{2}$ Trans. & Total & & & ${ }^{5}$ Total & ${ }^{6}$ per m ${ }^{2}$ \\
\hline Innitial & \begin{tabular}{|l|}
4,027 \\
\end{tabular} & 2 & 4,029 & 10 & 2 & 13 & 0 & 0 & 0 & 0 & 0 & 0 & $\begin{array}{l}4,042 \\
\end{array}$ & 79 & & \\
\hline 50 & \begin{tabular}{|l|l|}
4,027 \\
\end{tabular} & 2 & 4,029 & 10 & 2 & 13 & 0 & 0 & 0 & 0 & 1 & 1 & 4,043 & 79 & 80,000 & -13 \\
\hline
\end{tabular}

Embodied energy (and GWP) numbers are based on an area of wall $=50.9 \mathrm{~m}^{2} \quad$ (Length $\times$ Height $=7.6 \mathrm{~m} \times 6.7 \mathrm{~m}=50.9 \mathrm{~m}^{2}$ )

Net wall area of baseline retail building (gross wall area - openings) $=581.0 \mathrm{~m}^{2}$

ATHENA ® EIE Material List:

\begin{tabular}{|l|c|c|}
\hline \multicolumn{1}{|c|}{ Material List } & Quantities & Unit \\
\hline 16mm Regular Gypsum Board & 56.0 & $\mathrm{~m} 2$ \\
\hline 6 mil Polyythylene & 108.0 & $\mathrm{~m} 2$ \\
\hline Concrete 30 MPa (flyash av) & 6.7 & $\mathrm{~m} 3$ \\
\hline Extruded Polystyrene & 208.6 & $\mathrm{~m} 2(25 \mathrm{~mm})$ \\
\hline Galvanized Sheet & 30.0 & $\mathrm{~kg}$ \\
\hline Galvanized Studs & 287.9 & $\mathrm{~kg}$ \\
\hline Joint Compound & 55.9 & $\mathrm{~kg}$ \\
\hline Nails & 3.7 & $\mathrm{~kg}$ \\
\hline Oriented Strand Board & 145.8 & $\mathrm{~m} 2(9 \mathrm{~mm})$ \\
\hline Paper Tape & 0.6 & $\mathrm{~kg}$ \\
\hline Rebar, Rod, Light Sections & 404.0 & $\mathrm{~kg}$ \\
\hline Screws Nuts \& Bolts & 2.6 & $\mathrm{~kg}$ \\
\hline Water Based Latex Paint & 66.3 & $\mathrm{~L}$ \\
\hline
\end{tabular}

${ }^{1}$ Initial $=$ Time 'o' (i.e. at the cotes:

${ }^{2}$ Trans. $=$ Transportation

${ }^{3}$ Total EE (or Total GWP) $=$ Total embodied energy (or total embodied GWP) of building component after lifespan (i.e. total manufacturing + tota

${ }^{4}$ Total EE (or Total GWP) per $\mathrm{m}^{2}=$ Total EE (or Total GWP) of building
component/ area of building component that was modelled in ATHENAQ ${ }^{5}$ Total Difference in Operating Energy (or GWP) from Baseline after Lifespan $=$ The difference in the total lifecycle operating energy (or GWP) from the baseline retail building after lifespan, due to using this building

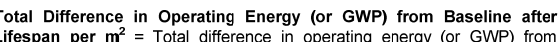
baseline after lifespan / net wall area of baseline retail building Total operating primary energy use of baseline retail building after 50 years $=$

"Total operating GWP of baseline retail building after 50 years $=2,310$ tonnes
Wood Structural Insulated Panel Wall \#4 (WSIP-W4)

Building Component Description:

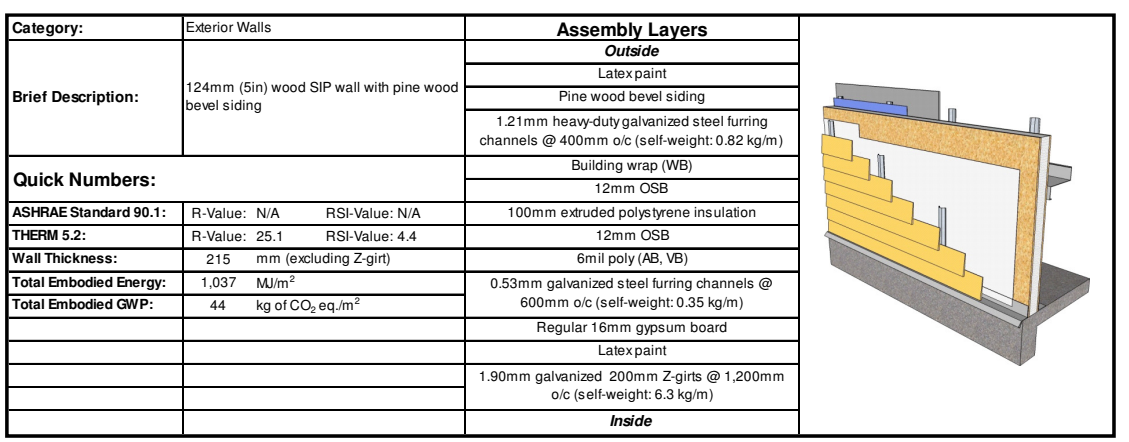

Life-Cycle Assessment Results:

Primary Energy Consumption (MJ)

\begin{tabular}{|c|c|c|c|c|c|c|c|c|c|c|c|c|c|c|c|c|}
\hline \multirow{3}{*}{\begin{tabular}{|l} 
Lifespan \\
(Years)
\end{tabular}} & \multicolumn{14}{|c|}{ Embodied Energy (EE) } & \multirow{2}{*}{\multicolumn{2}{|c|}{$\begin{array}{c}\text { Difference in } \\
\text { Operating Energy } \\
\text { from Baseline after } \\
\text { Lifespan }\end{array}$}} \\
\hline & \multicolumn{3}{|c|}{ Manufacturing } & \multicolumn{3}{|c|}{ Construction } & \multicolumn{3}{|c|}{ Maintenance } & \multicolumn{3}{|c|}{ End of Life } & \multirow{2}{*}{$\begin{array}{c}{ }^{3} \text { Total } \\
\mathrm{EE}\end{array}$} & \multirow{2}{*}{$\begin{array}{c}{ }^{4} \text { Total } \\
\text { EE } \\
\text { per m }{ }^{2}\end{array}$} & & \\
\hline & Material & ${ }^{2}$ Trans. & Total & Material & 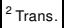 & Total & Material & ${ }^{2}$ Trans. & Total & Material & $\begin{array}{l}{ }^{2} \text { Trans. } \\
\text { The }\end{array}$ & Total & & & ${ }^{5}$ Total & ${ }^{6}$ perm $^{2}$ \\
\hline & 51,43 & 520 & 51,951 & 160 & 552 & 712 & 0 & 0 & 0 & 0 & 0 & 0 & 52,66 & 034 & & \\
\hline
\end{tabular}

Global Warming Potential (kg of $\mathrm{CO}_{2}$ eq.

\begin{tabular}{|c|c|c|c|c|c|c|c|c|c|c|c|c|c|c|}
\hline \multirow{3}{*}{$\begin{array}{l}\text { Lifespan } \\
\text { (rears) }\end{array}$} & \multicolumn{12}{|c|}{ Embodied Global Warming Potential (GWP) } & \multirow{2}{*}{\multicolumn{2}{|c|}{\begin{tabular}{|c} 
Difference in \\
Operating GWPP from \\
Baselinina after \\
Lifespan
\end{tabular}}} \\
\hline & Manufactur & & Constructic & & & Maintenanc & & & End of Life & & ${ }^{3} \mathrm{~T}$ & & & \\
\hline & \begin{tabular}{|l|l} 
Material & 2 \\
\end{tabular} & Total & 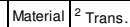 & Total & Material & $\begin{array}{l}2^{2} \text { Trans. } \\
\text {. }\end{array}$ & \begin{tabular}{|l|l|} 
Total \\
\end{tabular} & Material & 1. ${ }^{2}$ Trans. & Total & GWF & & & \begin{tabular}{|l|l}
${ }^{5}$ Total & ${ }^{6}$ per m ${ }^{2}$
\end{tabular} \\
\hline
\end{tabular}

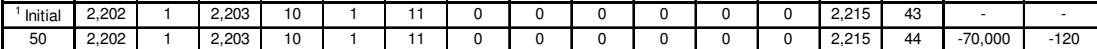
Embodied energy (and GWP) numbers are based on an area of wall $=50.9 \mathrm{~m}^{2} \quad$ (Length $x$ Height $=7.6 \mathrm{~m} \times 6.7 \mathrm{~m}=50.9 \mathrm{~m}^{2}$ ) Net wall area of baseline retal bulding (orss wall and-openings) $=581.0 \mathrm{~m}^{2}$

\begin{tabular}{|c|c|c|}
\hline Material List & Quantitites & Unit \\
\hline $6 \mathrm{~mm}$ Regular Gypsum Board & 56.0 & $\mathrm{~m} 2$ \\
\hline 6 mil Polyethylene & 108.0 & $\mathrm{~m} 2$ \\
\hline Extruded Polystyrene & 208.6 & $\mathrm{~m} 2(25 \mathrm{~mm})$ \\
\hline Galvanized Sheet & 136.4 & $\mathrm{~kg}$ \\
\hline Galvanized Studs & 287.9 & $\mathrm{~kg}$ \\
\hline Joint Compound & 55.9 & $\mathrm{~kg}$ \\
\hline $\begin{array}{ll}\text { Nails } \\
\text { a }\end{array}$ & 5.0 & $\mathrm{~kg}$ \\
\hline Oriented Strand Board & 145.8 & $\mathrm{~m} 2(9 \mathrm{~mm})$ \\
\hline Paper Tape & 0.6 & $\mathrm{~kg}$ \\
\hline Pine Wood Bevel Siding & 160.4 & $\mathrm{~m} 2$ \\
\hline S Nuts \& Bolts & 2.6 & $\mathrm{~kg}$ \\
\hline Based Latex Paint & 132.5 & L \\
\hline
\end{tabular}

Notes:
${ }^{1}$ Initial $=$ Time $O^{\prime}($ i.e. at the completion of initial construction) ${ }^{2}$ Trans. = Transportation

Total EE (or Total GWP) = Total embodied energy (or total embodied GWP of building component atter lifespan (i.e. total manu
construction + total maintenance + total end-oflife effects) Total EE (or Total GWP) per $\mathrm{m}^{2}=$ Total EE (or Total GWP) of building
component/ area of building component that was modelled in ATHENAE EIE Total Difference in Operating Energy (or GWP) from Baseline after

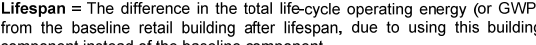
component instead of the beseline component
Total Difference in Operating Energy (or GWP) from Baseline after Total Difference in Operating Energy (or GWP) from Baseline after
Liffespan per $\mathrm{m}^{2}=$ Total difference in operating energy (or GWP) from baseline atter Ififspan / $n$ et wall area of baseline retail building Total operating primary energy use of baseline retail building affer 50 years
$50,700 \mathrm{GJ}\left(1.745 \mathrm{M} / \mathrm{Jm} \mathrm{m}^{2} \mathrm{yrd}\right.$ *Total operating $\mathrm{GWP}$ of baseline retail building after 50 years $=2,310$ tonnes 
Wood Structural Insulated Panel Wall \#5 (WSIP-W5)

Building Component Description:

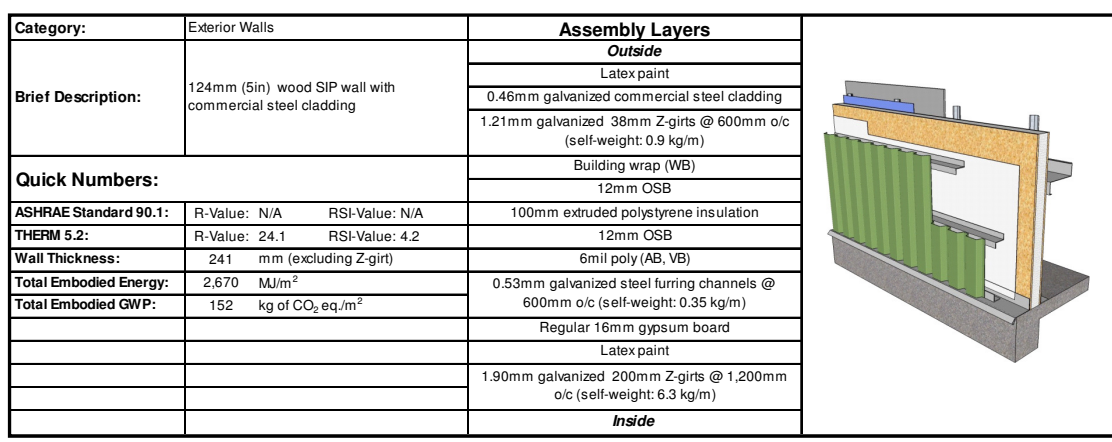

Life-Cycle Assessment Results:

Primary Energy Consumption (MJ)

\begin{tabular}{|c|c|c|c|c|c|c|c|c|c|c|c|c|c|c|c|c|}
\hline \multirow{3}{*}{\begin{tabular}{|c} 
Lifespan \\
(Years)
\end{tabular}} & \multicolumn{13}{|c|}{ Embodied Energy (EE) } & \multirow{2}{*}{\multicolumn{3}{|c|}{$\begin{array}{c}\text { Difference in } \\
\text { Operating Energy } \\
\text { from Baseline after } \\
\text { Lifespan }\end{array}$}} \\
\hline & \multicolumn{2}{|c|}{ Manufacturing } & \multicolumn{3}{|c|}{ Construction } & \multicolumn{3}{|c|}{ Maintenance } & \multicolumn{3}{|c|}{ End of Life } & \multirow{2}{*}{\begin{tabular}{|c|c}
${ }^{3}$ Total \\
EE
\end{tabular}} & \multirow{2}{*}{\begin{tabular}{|c}
${ }^{4}$ Tota \\
To \\
perm
\end{tabular}} & & & \\
\hline & $\mathrm{Ma}$ & Total & Material & 11 ${ }^{2}$ Trans. & Total & Material & ${ }^{2}$ Trans. & Total & Materia & ${ }^{2}$ Trans & Total & & & & ${ }^{5}$ Total & ${ }^{6}$ per m$^{2}$ \\
\hline Initial & \begin{tabular}{|l|l|}
134,780 & 471 \\
\end{tabular} & 135,251 & 160 & 450 & 610 & 0 & 0 & 0 & 0 & $\overline{0}$ & $\overline{0}$ & 1 & 2,8 & & & \\
\hline
\end{tabular}

Global Warming Potential ( $\mathrm{kg}$ of $\mathrm{CO}_{2}$ eq.)

\begin{tabular}{|c|c|c|c|c|c|c|c|c|c|c|c|c|c|c|c|c|}
\hline \multirow{3}{*}{$\begin{array}{l}\text { Lifespan } \\
\text { (Years) }\end{array}$} & \multicolumn{14}{|c|}{ Embodied Global Warming Potential (GWP) } & \multirow{2}{*}{\multicolumn{2}{|c|}{$\begin{array}{l}\text { Difference in } \\
\text { Operating GWP from } \\
\text { Baseline after } \\
\text { Lifespan }\end{array}$}} \\
\hline & \multicolumn{3}{|c|}{ Manufacturing } & \multicolumn{3}{|c|}{ Construction } & \multicolumn{3}{|c|}{ Maintenance } & \multicolumn{3}{|c|}{ End of Life } & \multirow{2}{*}{$\begin{array}{l}{ }^{3}{ }^{3} \text { Total } \\
\text { GWP }\end{array}$} & \multirow{2}{*}{\begin{tabular}{|l}
${ }^{4}$ Total \\
GoWP \\
per m
\end{tabular}} & & \\
\hline & Material & ${ }^{2}$ Trans. & Total & Material & 1) ${ }^{2}$ Trans. & Total & Material & ${ }^{2}$ Trans. & Total & Material & 1. ${ }^{2}$ Trans. & Total & & & ${ }^{5}$ Total & ${ }^{6}$ perm ${ }^{2}$ \\
\hline${ }^{1}$ Initial & 7,749 & 1 & 7,750 & 10 & 1 & 11 & 0 & 0 & 0 & 0 & 0 & 0 & 7,761 & 152 & & \\
\hline 50 & 7,749 & 1 & 7,750 & 10 & 1 & 11 & 0 & 0 & 0 & 0 & 0 & 0 & 7,761 & 152 & $-60,000$ & -103 \\
\hline
\end{tabular}

Net wall are of bello

\begin{tabular}{|c|c|c|}
\hline Material List & Quantities & Unit \\
\hline $16 \mathrm{~mm}$ Regular Gypsum Board & 56.0 & $\mathrm{~m} 2$ \\
\hline 6 mil Polyethylene & 108.0 & $\mathrm{~m} 2$ \\
\hline Commercial $0.46 \mathrm{~mm}$ Steel Cladding & 168.0 & $\mathrm{~m} 2$ \\
\hline Extruded Polystyrene & 208.6 & $\mathrm{~m} 2(25 \mathrm{~mm})$ \\
\hline Galvanized Sheet & 30.0 & $\mathrm{~kg}$ \\
\hline Galvanized Studs & 368.7 & $\mathrm{~kg}$ \\
\hline Joint Compound & 55.9 & $\mathrm{~kg}$ \\
\hline Nails & 3.7 & $\mathrm{~kg}$ \\
\hline Oriented Strand Board & 145.8 & $\mathrm{~m} 2(9 \mathrm{~mm})$ \\
\hline Paper Tape & 0.6 & $\mathrm{~kg}$ \\
\hline ews Nuts \& Bolts & 3.9 & tat \\
\hline Based LateX & 132.5 & \\
\hline
\end{tabular}

Notes:
${ }^{1}$ Initial $=$ Time $\mathrm{O}^{\prime}($ i.e. at the completion of initial construction)

${ }^{2}$ Trans. $=$ Transportation

Total EE (or Total GWP) = Total embodied energy (or total embodied GWP

of building component after lifespan (i.e. total man
construction +total maintenance + total end-ofifife effects)

Total EE (or Total GWP) per $\mathrm{m}^{2}=$ Total EE (or Total GWP) of building
component / area of building component that was modelled in ATHENAQ EIE

component / area of building component that was modelled in ATHENA@ EIE

Total Difference in Operating Energy (or GWP) from Baseline after
Lifespan = The difference in the total life-cycle operating energy (or GWP)
from the baseline retail building after lifiespan, due to using this building

component instead of the baseline component

Total Difference in Operating Energy (or GWP) from Baseline after

Lifespan per $\mathrm{m}^{2}=$ Total difference in operating energy (or GWP) from
baseline anter lifespan / net wall area of baseline retail building

* Total operating primary energy use of baseline retail building affer 50 years

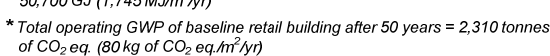

\section{Wood Structural Insulated Panel Wall \#6 (WSIP-W6)}

Building Component Description:

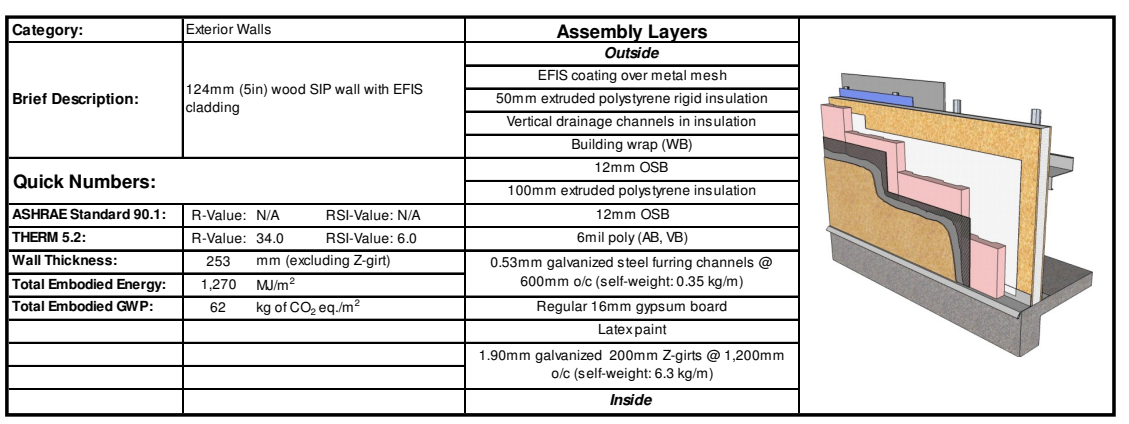

Life-Cycle Assessment Results:
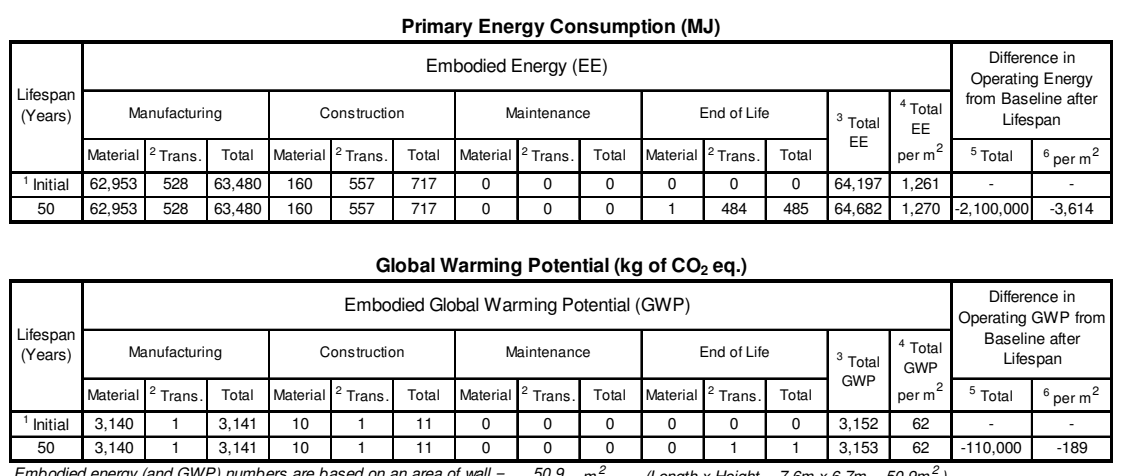

Embodied energy (and GWP) numbers are based on an area of wall $=50.9 \mathrm{~m}^{2} \quad \mathrm{~m}^{2} \quad 1 \quad 1 \quad 3,153 \quad 62$ Net wall area of baseline retail building (gross wall area - openings) $=581.0 \mathrm{~m}^{2}$

ATHENA ® EIE Material List:

(Includes all materials after 50 years)

${ }^{1}$ Initial $=$ Time ' 0 ' (i.e. at the completion of

\begin{tabular}{|l|c|c|}
\hline \multicolumn{1}{|c|}{ Material List } & Quantities & Unit \\
\hline \#15 Organic Felt & 219.4 & $\mathrm{~m} 2$ \\
\hline 16mm Regular Gypsum Board & 56.0 & $\mathrm{~m} 2$ \\
\hline 6 mil Polyethllene & 108.0 & $\mathrm{~m} 2$ \\
\hline Extruded & 313.0 & $\mathrm{~m} 2(25 \mathrm{~mm})$ \\
\hline Galvanizentyene & 81.4 & $\mathrm{~kg}$ \\
\hline Galvanized Stuet & 287.9 & $\mathrm{~kg}$ \\
\hline Joint Compound & 55.9 & $\mathrm{~kg}$ \\
\hline Nails & 8.3 & $\mathrm{~kg}$ \\
\hline Oriented Strand Board & 145.8 & $\mathrm{~m} 2(9 \mathrm{~mm})$ \\
\hline Paper Tape & 0.6 & $\mathrm{~kg}$ \\
\hline Screws Nuts \& Bolts & 2.6 & $\mathrm{~kg}$ \\
\hline Stucco over metal mesh & 136.0 & $\mathrm{~m} 2$ \\
\hline Water Based Latex Paint & 132.5 & $\mathrm{~L}$ \\
\hline
\end{tabular}

${ }^{2}$ Trans $=$ Transportation

otal EE (or Total GWP) = Total embodied energy (or total embodied GWP) of building component after lifespan (i.e. total man
construction t total maintenance t total end-of-life effects)

${ }^{4}$ Total EE (or Total GWP) per $\mathrm{m}^{2}=$ Total EE (or Total GWP) of building
component/ area of building component that was modelled in ATHENA@ EIE

${ }^{5}$ Total Difference in Operating Energy (or GWP) from Baseline after Lifespan $=$ The difference in the total life-cycle operating energy (or GWF
from the baseline retail building after lifespan, due to using this building component instead of the baseline component

Total Difference in Operating Energy (or GWP) from Baseline after Lifespan per $\mathrm{m}^{2}=$ Total difiference in operating energy (or GWP) from
baseline after lifespan $/$ net wall area of baseline retail building

"Total operating primary energy use of baseline retail building after 50 years
$50,700 \mathrm{GJ}\left(1,745 \mathrm{MJ} / \mathrm{m}^{\prime} \mathrm{yr}\right)$

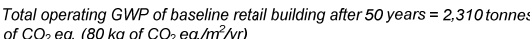


Wood Structural Insulated Panel Wall \#7 (WSIP-W7)

Building Component Description:

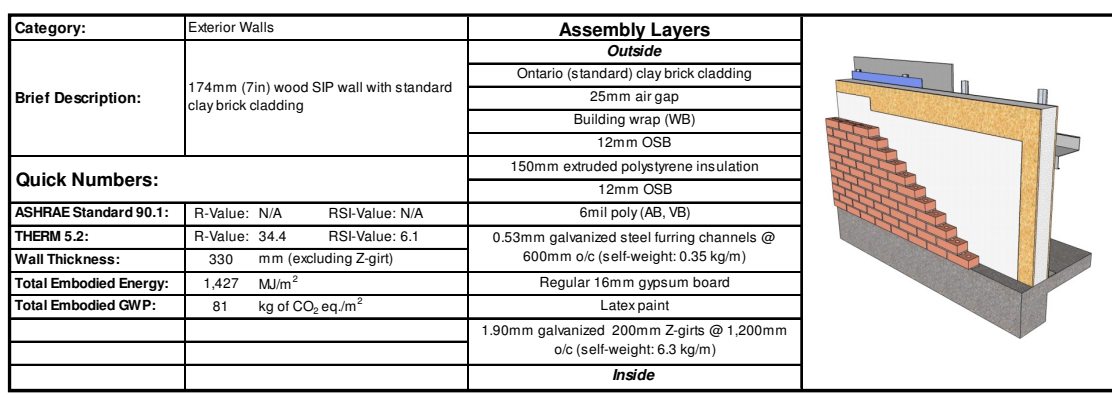

\section{Life-Cycle Assessment Results:}

Primary Energy Consumption (MJ)

\begin{tabular}{|c|c|c|c|c|c|c|c|c|c|c|c|c|c|c|c|c|}
\hline \multirow{3}{*}{$\begin{array}{l}\text { Lifespan } \\
\text { (Years) }\end{array}$} & \multicolumn{14}{|c|}{ Embodied Energy (EE) } & \multirow{2}{*}{\multicolumn{2}{|c|}{$\begin{array}{l}\text { Difiterence in } \\
\text { Operating Energy } \\
\text { from Baseline ate } \\
\text { Lifespan }\end{array}$}} \\
\hline & \multicolumn{3}{|c|}{ Manufacturing } & \multicolumn{3}{|c|}{ Construction } & \multicolumn{3}{|c|}{ Maintenance } & \multicolumn{3}{|c|}{ End of Life } & \multirow{2}{*}{$-{ }^{3}$ Total } & \multirow{2}{*}{$\begin{array}{c}{ }^{4} \mathrm{Total} \\
\mathrm{EE} \\
\mathrm{p}^{2} \mathrm{~m}^{2}\end{array}$} & & \\
\hline & Material & 11 ${ }^{2}$ Trans. & Total & Material & ${ }^{2}$ Trans. & Total & |Material & ${ }^{2}$ Trans. & Total & Material & ${ }^{2}$ Trans. & Total & & & ${ }^{5}$ Total & ${ }^{6}$ per m $^{2}$ \\
\hline |nitial & 70,263 & 502 & 70,765 & 160 & 1,215 & 1,375 & \begin{tabular}{l|l}
0 \\
\end{tabular} & 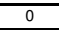 & 0 & 0 & 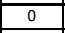 & 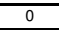 & 72,140 & 1,417 & & \\
\hline 50 & 70,263 & \begin{tabular}{l|l}
3 & 502 \\
3
\end{tabular} & 70,765 & 160 & \begin{tabular}{|l|l|l|} 
\\
\end{tabular} & $\begin{array}{l}1,375 \\
\end{array}$ & 0 & 0 & 0 & 1 & 545 & 546 & 72,686 & 1,427 & $-2,200,00$ & $-3,787$ \\
\hline
\end{tabular}

Global Warming Potential ( $\mathrm{kg}$ of $\mathrm{CO}_{2}$ eq.)

\begin{tabular}{|c|c|c|c|c|c|c|c|c|c|c|c|c|c|c|c|c|}
\hline \multirow{4}{*}{$\begin{array}{l}\text { Lifiesparars } \\
\text { (Years }\end{array}$} & \multirow{2}{*}{\multicolumn{14}{|c|}{ Embodied Global Warming Potential (GWP) }} & \multirow{3}{*}{\multicolumn{2}{|c|}{$\begin{array}{c}\text { Difference in } \\
\text { Operating GWW fron } \\
\text { Baseline after } \\
\text { Lifespan }\end{array}$}} \\
\hline & & & & & & & & & & & & & & & & \\
\hline & \multicolumn{3}{|c|}{ Manutacturing } & \multicolumn{3}{|c|}{ Construction } & \multicolumn{3}{|c|}{ Maintenance } & \multicolumn{3}{|c|}{ End of Life } & \multirow{2}{*}{$\begin{array}{l}{ }^{3} \text { Total } \\
\text { GWPP }\end{array}$} & \multirow{2}{*}{$\begin{array}{l}{ }^{4} \text { Total } \\
\text { GWP } \\
\text { per m }\end{array}$} & & \\
\hline & Material & ${ }^{2}$ Trans. & Total & Material & ${ }^{2}$ Trans & Total & Materia & 2 Trans. & Total & Material & 2 Trans. & Total & & & ${ }^{5}$ Total & ${ }^{6}$ perm $^{2}$ \\
\hline${ }^{1}$ Initial & 4,114 & 1 & 4,115 & 10 & 2 & 13 & 0 & 0 & 0 & 0 & 0 & 0 & 4,128 & 81 & & \\
\hline 50 & 4,114 & 1 & 4,115 & 10 & 2 & 13 & 0 & 0 & 0 & 0 & 1 & 1 & 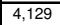 & 81 & $-110,000$ & -189 \\
\hline
\end{tabular}

Embodied energy (and GWP) numbers are based on an area of wall $=50.9 \mathrm{~m}^{2} \quad$ (Length $\times$ Height $=7.6 \mathrm{~m} \times 6.7 \mathrm{~m}=50.9 \mathrm{~m}^{2}$ )

Net wall area of baseline retail building (gross wall area - openings) $=581.0 \mathrm{~m}^{2}$

ATHENA $®$ EIE Material List:

\begin{tabular}{|l|c|c|}
\multicolumn{1}{|c|}{ Material List } & Quantities & Unit \\
\hline 16mm Regular Gypsum Board & 56.0 & $\mathrm{~m} 2$ \\
\hline 6 mil Polyethylene & 108.0 & $\mathrm{~m} 2$ \\
\hline Cold Rolled Sheet & 10.3 & $\mathrm{~kg}$ \\
\hline Extruded Polystyrene & 313.0 & $\mathrm{~m} 2(25 \mathrm{~mm})$ \\
\hline Galvanized Sheet & 30.0 & $\mathrm{~kg}$ \\
\hline Galvanized Studs & 287.9 & $\mathrm{~kg}$ \\
\hline Joint Compound & 55.9 & $\mathrm{~kg}$ \\
\hline Mortar & 1.5 & $\mathrm{~m} 3$ \\
\hline Nails & 3.7 & $\mathrm{~kg}$ \\
\hline Ontario (Standard) Brick & 53.5 & $\mathrm{~m} 2$ \\
\hline Oriented Strand Board & 145.8 & $\mathrm{~m} 2(9 \mathrm{~mm})$ \\
\hline Paper Tape & 0.6 & $\mathrm{~kg}$ \\
\hline Screws Nuts \& Bolts & 2.6 & $\mathrm{~kg}$ \\
\hline Water Based Latex Paint & 66.3 & $\mathrm{~L}$ \\
\hline
\end{tabular}

Notes:
${ }^{1}$ Initial $=$ Time 'o' (i.e. at the completion of initial onstruction)

${ }^{2}$ Trans $=$ Transportation

Total EE (or Total GWP) = Total embodied energy (or total embodied GWP) of building component after lifespan (i.e. total manufacturing + tota
construction + total maintenance+total end-ofifife effects

${ }^{4}$ Total EE (or Total GWP) per $\mathrm{m}^{2}=$ Total EE (or Total GWP) of building
component / area of building component that was modelled in ATHENAQ EIE Total Difference in Operating Energy (or GWP) from Baseline after Lifespan = The difference in the total life-cycle operating energy (or GWP
from the baseline retail building after lifespan, due to using this building

Total Difference in Operating Energy (or GWP) from Baseline after Lifespan per $\mathrm{m}^{2}=$ Total difference in operating energy (or GWP) from

Total operating primary energy use of baseline retail building after 50 years
$50,700 \mathrm{GJ}\left(1,745 \mathrm{MJ} \mathrm{m}^{2} / \mathrm{yr}\right)$

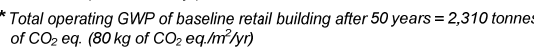

\section{Wood Structural Insulated Panel Wall \#8 (WSIP-W8)}

Building Component Description:

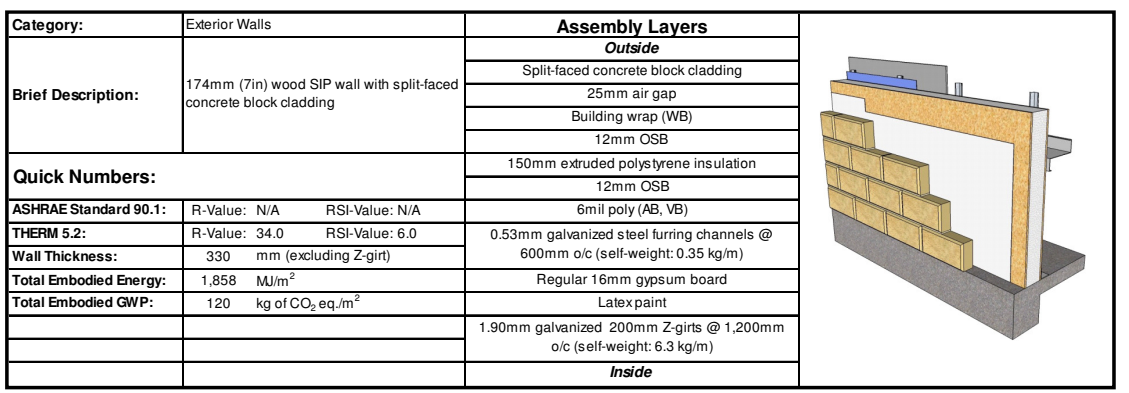

Life-Cycle Assessment Results:

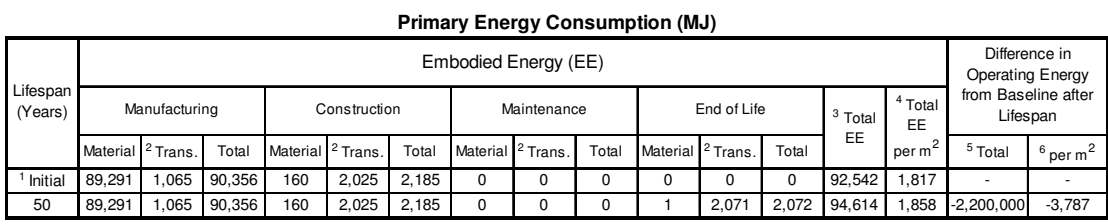

\begin{tabular}{|c|c|c|c|c|c|c|c|c|c|c|c|c|c|c|c|c|}
\hline & \multicolumn{16}{|c|}{ Global Warming Potential ( $\mathrm{kg}$ of $\mathrm{CO}_{2}$ eq.) } \\
\hline \multirow{3}{*}{ 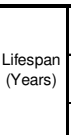 } & \multicolumn{14}{|c|}{ Embodied Global Warming Potential (GWP) } & \multirow{2}{*}{\multicolumn{2}{|c|}{$\begin{array}{l}\text { Difference in } \\
\text { Operating GWP Afror } \\
\text { Baseline after } \\
\text { Lifespan }\end{array}$}} \\
\hline & \multicolumn{3}{|c|}{ Manutacturing } & \multicolumn{3}{|c|}{ Construction } & \multicolumn{3}{|c|}{ Maintenance } & \multicolumn{3}{|c|}{ End of Life } & \multirow{2}{*}{$\begin{array}{l}{ }^{3} \text { Total } \\
\text { GWP }\end{array}$} & \multirow{2}{*}{$\begin{array}{l}{ }^{4} \text { Total } \\
\text { GWP } \\
\text { per m }{ }^{2}\end{array}$} & & \\
\hline & Material & ${ }^{2}$ Trans. & Total & Material & ${ }^{2}$ Trans. & Total & Material & ${ }^{2}$ Trans. & Total & Material & ${ }^{2}$ Trans. & Total & & & ${ }^{5}$ Total & ${ }_{6}^{6}$ perm $m^{2}$ \\
\hline${ }^{1}$ Initial & 6,100 & 2 & 6,102 & 10 & 4 & 14 & 0 & 0 & 0 & 0 & 0 & 0 & 6,117 & 120 & & \\
\hline 50 & $\begin{array}{ll}6,100 \\
\end{array}$ & 2 & 6,102 & 10 & 4 & 14 & 0 & 0 & 0 & 0 & 4 & 4 & 6,121 & $\begin{array}{l}120 \\
\end{array}$ & $-110,000$ & -189 \\
\hline
\end{tabular}

Embodied energy (and GWP) numbers are based on an area of wall $=\begin{array}{rlllll}50.9 & \mathrm{~m}^{2}\end{array} \quad$ (Length $x$ Height $=7.6 \mathrm{~m} \times 6.7 \mathrm{~m}=50.9 \mathrm{~m}^{2}$ ) Net wall area of baseline retail building (gross wall area - openings) $=581.0 \mathrm{~m}^{2}$

\section{ATHENA ® EIE Material List}

\begin{tabular}{|l|c|c|}
\hline \multicolumn{1}{|c|}{ Material List } & Quantities & Unit \\
\hline 16mm Regular Gypsum Board & 56.0 & $\mathrm{~m} 2$ \\
\hline 6 mil Polyyethylene & 108.0 & $\mathrm{~m} 2$ \\
\hline Cold Rolled Sheet & 10.3 & $\mathrm{~kg}$ \\
\hline Extruded Polystyrene & 313.0 & $\mathrm{~m} 2(25 \mathrm{~mm})$ \\
\hline Galvanized Sheet & 30.0 & $\mathrm{~kg}$ \\
\hline Gavanized Studs & 287.9 & $\mathrm{~kg}$ \\
\hline Joint Compound & 55.9 & $\mathrm{~kg}$ \\
\hline Modified Bitumen membrane & 333.3 & $\mathrm{~kg}$ \\
\hline Mortar & 4.3 & $\mathrm{~m} 3$ \\
\hline Nails & 3.7 & $\mathrm{~kg}$ \\
\hline Oriented Strand Board & 145.8 & $\mathrm{~m} 2(9 \mathrm{~mm})$ \\
\hline Paper Tape & 0.6 & $\mathrm{~kg}$ \\
\hline Screws Nuts \& Bolts & 2.6 & $\mathrm{~kg}$ \\
\hline Splitt-aced Concrete Block & $1,238.2$ & $\mathrm{Blocks}$ \\
\hline Water Based Latex Paint & 66.3 & $\mathrm{~L}$ \\
\hline
\end{tabular}

Notes:
${ }^{1}$ Initial $=$ Time 'o' (i.e. at the completion of initial construction)

${ }^{2}$ Trans $=$ Transportation

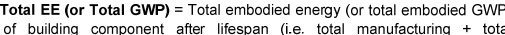
construction +otoal maintenance + total endo-oflifie effects)

Total EE (or Total GWP) per $\mathrm{m}^{2}=$ Total EE (or Total GWP) of building component/ area a I buidang component that was modelled in ATHENAQ EIE ${ }^{5}$ Total Difference in Operating Energy (or GWP) from Baseline after
Lifiespan $=$ The difference in the total lifec-cycle operating energy (or GWPP)
from the baseline retail building after lifespan due to using this building

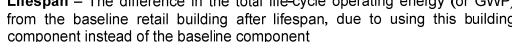
Total Difference in Operating Energy (or GWP) from Baseline after Thespan per $\mathrm{m}^{2}=$ Total difference in operating energy (or GWP) from * $T$ otalo operating primarn energy use of baseline retail building after 50 years $=$
$50,700 \mathrm{GJ}\left(1,745 \mathrm{MJ} / \mathrm{m}^{2} / \mathrm{yr}\right)$

"Total operating $\mathrm{GW}$ P of baseline reta 
Wood Structural Insulated Panel Wall \#9 (WSIP-W9)

Building Component Description:

\begin{tabular}{|c|c|c|c|}
\hline Category: & Exxerior Walls & Assembly Layers & \\
\hline \multirow{3}{*}{ |Brief Description: } & \multirow{3}{*}{$\begin{array}{l}174 m \text { m (7in) wood SIP wall with concrete } \\
\text { pre-castcladding }\end{array}$} & $\frac{\text { Outside }}{125 \mathrm{~mm} \text { concerete re-cast cladding }}$ & \\
\hline & & $25 \mathrm{~mm}$ air gap & \\
\hline & & Building wrap (WB) & \\
\hline \multicolumn{2}{|l|}{ Quick Numbers: } & $150 \mathrm{~mm}$ extruded polystryene insulation & \\
\hline ASHRAE Standard 90.1: & R-Value: N/A & $\frac{12 \mathrm{~mm} \text { OSB }}{6 \text { mil poly (AB, VB) }}$ & \\
\hline THERM 5.2: & $\begin{array}{ll}\text { R-Value: } 34.2 & \text { RSI-Value: } 6.0 \\
\end{array}$ & $0.53 \mathrm{~mm}$ galvanized steel furring channels @ & \\
\hline Wall Thickness: & $365 \mathrm{~mm}$ (excluding Z-girt) & $\begin{array}{l}600 m \text { mol/c (sell-weight: } 0.35 \mathrm{~kg} / \mathrm{k} / \mathrm{m} \text { ) } \\
\end{array}$ & \\
\hline Total Embodied Energy: & $1,373 \mathrm{~m} / \mathrm{m}^{2}$ & Regular $16 \mathrm{~mm}$ gypsum board & \\
\hline Total Embodied GWP: & $87 \mathrm{~kg} \mathrm{of} \mathrm{CO}_{2} \mathrm{eq} / \mathrm{m}^{2}$ & Latexpaint & \\
\hline & & $\begin{array}{c}1.90 \mathrm{~mm} \text { galvanized 2000m z.-girts @ } \\
\text { //c (self-weight: } 6.3 \mathrm{~kg} / \mathrm{m})\end{array}$ & \\
\hline & & Inside & \\
\hline
\end{tabular}

\section{Life-Cycle Assessment Results:}

Primary Energy Consumption (MJ)

\begin{tabular}{|c|c|c|c|c|c|c|c|c|c|c|c|c|c|c|c|c|}
\hline \multirow{3}{*}{$\begin{array}{l}\text { Lifespan } \\
\text { (Years) }\end{array}$} & \multicolumn{14}{|c|}{ Embodied Energy (EE) } & \multirow{2}{*}{\multicolumn{2}{|c|}{$\begin{array}{c}\text { Difference in } \\
\text { Operating Energy } \\
\text { from Baseline atte } \\
\text { Lifespan }\end{array}$}} \\
\hline & \multicolumn{3}{|c|}{ Manufacturing } & \multicolumn{3}{|c|}{ Constr } & \multicolumn{3}{|c|}{ Maintenance } & \multicolumn{3}{|c|}{ End of Life } & \multirow{2}{*}{$\begin{array}{c}{ }^{3} \text { Total } \\
\mathrm{EE}\end{array}$} & \multirow{2}{*}{$\begin{array}{c}{ }^{4} \text { Total } \\
\text { EE } \\
\text { per } \mathrm{m}^{2}\end{array}$} & & \\
\hline & \begin{tabular}{|l|} 
Material \\
\end{tabular} & 11 ${ }^{2}$ Trans. & \begin{tabular}{|l|l|} 
\\
\end{tabular} & \begin{tabular}{|l|} 
Materia \\
\end{tabular} & $\left.\right|^{2}$ Trans. & \begin{tabular}{|l|} 
Total \\
\end{tabular} & \begin{tabular}{|l|} 
Materia \\
\end{tabular} & ${ }^{2}$ Trans. & \begin{tabular}{|l|} 
Total \\
\end{tabular} & Material & i| ${ }^{2}$ Trans. & \begin{tabular}{|l|} 
\\
\end{tabular} & & & \begin{tabular}{|l|}
${ }^{5}$ Total \\
\end{tabular} & ${ }^{6} \operatorname{perm} \mathrm{m}^{2}$ \\
\hline Initial & 66,950 & 1,093 & 68,043 & 160 & 1,154 & \begin{tabular}{|l|l|}
1,314 \\
\end{tabular} & 0 & 0 & 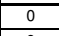 & 0 & 0 & 0 & 69,357 & \begin{tabular}{|l|l|}
1,362 \\
\end{tabular} & & \\
\hline 50 & 66,950 & \begin{tabular}{|l|l|} 
\\
\end{tabular} & 688,043 & 160 & \begin{tabular}{|l|l|l|} 
\\
154
\end{tabular} & 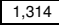 & 0 & 0 & 0 & 2 & 578 & 580 & \begin{tabular}{|l|l|}
69,937 \\
\end{tabular} & & $-2,200$ & $-3,7$ \\
\hline
\end{tabular}

Global Warming Potential ( $\mathrm{kg}$ of $\mathrm{CO}_{2}$ eq.)

\begin{tabular}{|c|c|c|c|c|c|c|c|c|c|c|c|c|c|c|c|c|}
\hline \multirow{3}{*}{$\begin{array}{l}\text { Lifespan } \\
\text { (Years) }\end{array}$} & \multicolumn{14}{|c|}{ Embodied Global Warming Potential (GWP) } & \multirow{2}{*}{\multicolumn{2}{|c|}{$\begin{array}{l}\text { Difference in } \\
\text { Operating GWP from } \\
\text { Baseline after } \\
\text { Lifespan }\end{array}$}} \\
\hline & \multicolumn{3}{|c|}{ Manufacturing } & \multicolumn{3}{|c|}{ Construction } & \multicolumn{3}{|c|}{ Maintenance } & \multicolumn{3}{|c|}{ End of Life } & \multirow{2}{*}{$\begin{array}{l}{ }^{3} \text { Total } \\
\text { GWP }\end{array}$} & \multirow{2}{*}{$\begin{array}{l}{ }^{4} \text { Total } \\
\text { GWP } \\
\text { per m }\end{array}$} & & \\
\hline & Material & Trans. & Total & Material & Trans. & Total & Material & Trans. & Total & Material & ${ }^{2}$ Trans. & Total & & & \begin{tabular}{ll|l}
${ }^{5}$ Total \\
\end{tabular} & ${ }^{6} \mathrm{perm}^{2}$ \\
\hline Initial & 4,414 & 2 & 4,417 & 10 & 2 & 13 & 0 & 0 & 0 & 0 & 0 & 0 & 4,429 & 87 & & \\
\hline 50 & 4,414 & 2 & 4,417 & 10 & 2 & 13 & 0 & 0 & 0 & 0 & 1 & 1 & 4,430 & 87 & $-110,000$ & -18 \\
\hline
\end{tabular}

Embodied energy (and GWP) numbers are based on an area of wall $=50.9 \mathrm{~m}^{2} \quad$ (Length $\times$ Height $=7.6 \mathrm{~m} \times 6.7 \mathrm{~m}=50.9 \mathrm{~m}^{2}$ ) Net wall area of baseline retail building (gross wall area - openings) $=581.0 \mathrm{~m}^{2}$

ATHENA ® EIE Material List:

\begin{tabular}{|l|c|c|}
\hline \multicolumn{1}{|c|}{ Material List } & Quantities & Unit \\
\hline 16mm Regular Gypsum Board & 56.0 & $\mathrm{~m} 2$ \\
\hline 6 mil Polyythylene & 108.0 & $\mathrm{~m} 2$ \\
\hline Concrete 30 MPa (flyash av) & 6.7 & $\mathrm{~m} 3$ \\
\hline Extruded Polystyrene & 313.0 & $\mathrm{~m} 2(25 \mathrm{~mm})$ \\
\hline Galvanized Sheet & 30.0 & $\mathrm{~kg}$ \\
\hline Galvanized Studs & 287.9 & $\mathrm{~kg}$ \\
\hline Joint Compound & 55.9 & $\mathrm{~kg}$ \\
\hline Nails & 3.7 & $\mathrm{~kg}$ \\
\hline Oriented Strand Board & 145.8 & $\mathrm{~m} 2(9 \mathrm{~mm})$ \\
\hline Paper Tape & 0.6 & $\mathrm{~kg}$ \\
\hline Rebar, Rod, Light Sections & 404.0 & $\mathrm{~kg}$ \\
\hline Screws Nuts \& Bolts & 2.6 & $\mathrm{~kg}$ \\
\hline Water Based Latex Paint & 66.3 & $\mathrm{~L}$ \\
\hline
\end{tabular}

${ }^{1}$ Initial $=$ Time 'o' (i.e. at the comples:

${ }^{2}$ Trans. = Transportation

${ }^{3}$ Total EE (or Total GWP) $=$ Total embodied energy (or total embodied GWP) of building component after lifespan (i.e. total manufacturing + tota construction + total maintenance + total end 0 of

${ }^{4}$ Total EE (or Total GWP) per $\mathrm{m}^{2}=$ Total EE (or Total GWP) of building
component/ area of building component that was modelled in ATHENAQ ${ }^{5}$ Total Difference in Operating Energy (or GWP) from Baselne a fer Lifespan $=$ The difference in the total life-cycle operating energy (or GWP) from the baseline retail building after lifespan, due to using this building (T)

Litespan Difference in Operating Energy (or GWP) from Baseline after
$\mathrm{m}^{2}=$ Total difference in operating energy (or GWP) ftom Lifespan per $\mathrm{m}^{2}=$ Total difierence in operating energy $(0 \mathrm{r}$
baseline after lifespan / net wall area of baseline retail building "Total operating primary energy use of baseline retail building after 50 years $=$

*Total operating $\mathrm{GWP}$ of baseline retail building after 50 years $=2,310$ tonnes
of $\mathrm{CO}_{2}$ eq. $88 \mathrm{~kg}$ of $\mathrm{CO}_{2}$ eq $/ \mathrm{hr}^{2} \mathrm{Mr}$ )
Wood Structural Insulated Panel Wall \#10 (WSIP-W10)

Building Component Description:

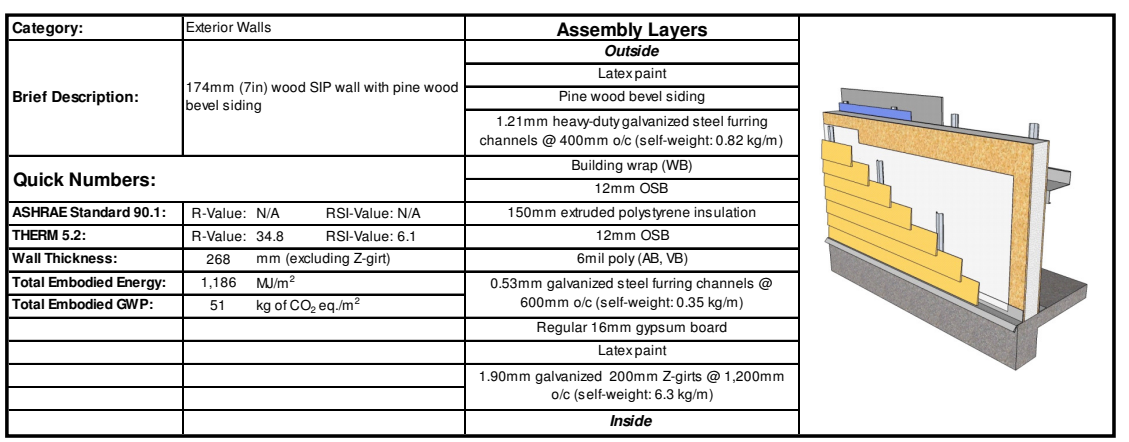

Life-Cycle Assessment Results:

Primary Energy Consumption (MJ)

\begin{tabular}{|c|c|c|c|c|c|c|c|c|c|c|c|c|c|c|c|c|}
\hline \multirow{3}{*}{$\begin{array}{l}\text { Lifespan } \\
\text { (Years) }\end{array}$} & \multicolumn{14}{|c|}{ Embodied Energy (EE) } & \multirow{2}{*}{\multicolumn{2}{|c|}{$\begin{array}{c}\text { Difference in } \\
\text { Operating Energy } \\
\text { from Baseline after } \\
\text { Lifespan }\end{array}$}} \\
\hline & \multicolumn{3}{|c|}{ Manutacturing } & \multicolumn{3}{|c|}{ Construction } & \multicolumn{3}{|c|}{ Maintenance } & \multicolumn{3}{|c|}{ End of Life } & \multirow{2}{*}{$\int_{\mathrm{3}}^{3}$ Total } & \multirow{2}{*}{$\begin{array}{c}{ }^{4} \text { Total } \\
\text { EE } \\
\operatorname{per} \mathrm{m}^{2}\end{array}$} & & \\
\hline & Material & ${ }^{2}$ Trans. & $\begin{array}{l}\text { Total } \\
\text { Sal }\end{array}$ & Material & ${ }^{2}$ Trans. & Total & Material & ${ }^{2}$ Trans. & $\begin{array}{c}\text { Total } \\
\end{array}$ & Material & 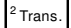 & Total & & & $\begin{array}{l}{ }^{5} \text { Total } \\
\end{array}$ & ${ }^{6}$ perm $^{2}$ \\
\hline & 9,015 & 520 & 59,535 & 160 & 564 & 724 & 0 & 0 & 0 & 0 & 0 & 0 & 60,259 & $\frac{118}{1.8}$ & & \\
\hline
\end{tabular}

Global Warming Potential (kg of $\mathrm{CO}_{2}$ eq.

\begin{tabular}{|c|c|c|c|c|c|c|c|c|c|c|c|c|c|c|}
\hline \multirow{3}{*}{$\begin{array}{l}\text { Lifespan } \\
\text { (Years) }\end{array}$} & \multicolumn{12}{|c|}{ Embodied Global Warming Potential (GWP) } & \multirow{2}{*}{\multicolumn{2}{|c|}{\begin{tabular}{|c} 
Difference in \\
Operating GWP from \\
Baseline after \\
Lifespan
\end{tabular}}} \\
\hline & Manutactur & & Constructio & & & Maintenanc & & & End of Life & & ${ }^{3}$ Tot & & & \\
\hline & \begin{tabular}{|l|l} 
Material & 2 \\
\end{tabular} & Total & \begin{tabular}{|l|l|} 
Material & 2 \\
\end{tabular} & Total & Material & $\begin{array}{l}2^{2} \text { Trans. } \\
\text {. }\end{array}$ & \begin{tabular}{|l|l} 
Total \\
\end{tabular} & Material & 1. ${ }^{2}$ Trans. & Total & & per & & \begin{tabular}{|l|l}
${ }^{5}$ Total & ${ }^{6}$ per m ${ }^{2}$
\end{tabular} \\
\hline
\end{tabular}
\begin{tabular}{|c|c|c|c|c|c|c|c|c|c|c|c|c|c|c|c|c|}
\hline 'Initial & 2,590 & 1 & 2,591 & 10 & 1 & 11 & 0 & 0 & 0 & 0 & 0 & 0 & 2,603 & 51 & - & - \\
\hline 50 & 2,590 & 1 & 2,591 & 10 & 1 & 11 & 0 & 0 & 0 & 0 & 0 & 0 & 2,603 & 51 & $-110,000$ & -189 \\
\hline
\end{tabular} Embodied energy (and GWP) numbers are based on an area of wall $=50.9 \mathrm{~m}^{2} \quad$ (Length $x$ Height $=7.6 \mathrm{~m} \times 6.7 \mathrm{~m}=50.9 \mathrm{~m}^{2}$ )

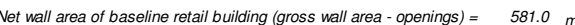

$\begin{aligned} & \text { ATHENA \& EIE Material List: } \\
& \text { (Includes all materials after 50 years) }\end{aligned}$
\begin{tabular}{|l|c|c|}
\multicolumn{1}{|c|}{ Material List } & Quantities & Unit \\
\hline 16mm Regular Gypsum Board & 56.0 & $\mathrm{~m} 2$ \\
\hline 6 mil Polyethylene & 108.0 & $\mathrm{~m} 2$ \\
\hline Extruded Polystyrene & 313.0 & $\mathrm{~m} 2(25 \mathrm{~mm})$ \\
\hline Galvanized Sheet & 136.4 & $\mathrm{~kg}$ \\
\hline Galvanized Studs & 287.9 & $\mathrm{~kg}$ \\
\hline Joint & 55.9 & $\mathrm{~kg}$ \\
\hline Nails & 5.0 & $\mathrm{~kg}$ \\
\hline Oriented Strand Board & 145.8 & $\mathrm{~m} 2(9 \mathrm{~mm})$ \\
\hline Paper Tape & 0.6 & $\mathrm{~kg}$ \\
\hline Pine Wood Bevel Siding & 160.4 & $\mathrm{~m} 2$ \\
\hline Screws Nuts \& Bolths & 2.6 & $\mathrm{~kg}$ \\
\hline Water Based Latex Paint & 132.5 & $\mathrm{~L}$ \\
\hline
\end{tabular}

Notes:
${ }^{1}$ Initial $=$ Time $0^{\prime}$ (i.e. at the completion of initial construction) ${ }^{2}$ Trans. = Transportation

${ }^{3}$ Total EE (or Total GWP) $=$ Total embodied energy (or total embodied GWP)
of building component after lifespan (i.e. 0 total manufacturing + tota consilding component after lifespan (i.e. total $\mathrm{mat}$
construction + total maintenance + total end-of-life effects) ${ }^{4}$ Total EE (or Total GWP) per $\mathrm{m}^{2}=$ Total EE (or Total GWP) of building
component/ area of building component that was modelled in ATHENAQ EIE 5 Total Difference in Operating Energy (or GWP) from Baseline after
Lifespan $=$ The difference in the total lifecycle operating energy (or GWP) Lifespan = The difference in the total lite-cycle operating energy (or GWP
from the baseline retail building after lifespan, due to using this building
component instead of the baseline component ${ }^{6}$ Total Difference in Operating Energy (or GWP) from Baseline after Lifespan per $\mathrm{m}^{2}=$ Tatal difference in operating energy
baseline atter lifespan $/ \mathrm{net}$ wall area of baseline retail building "Total operating primary energy use of baseline retail building after 50 years
$50,700 \mathrm{G}\left(1,745 \mathrm{MJ} / \mathrm{m}^{2} / \mathrm{yr}\right)$ * Total operating $\mathrm{GWP}$ of baseline retail building after 50 years $=2,310$ tonnes
of $\mathrm{CO}_{2} \mathrm{eq}$. $\left(80 \mathrm{~kg}\right.$ of $\left.\mathrm{CO}_{2} \mathrm{eq} / \mathrm{m}^{2} \mathrm{xr}\right)$ 


\section{Wood Structural Insulated Panel Wall \#11 (WSIP-W11)}

Building Component Description:

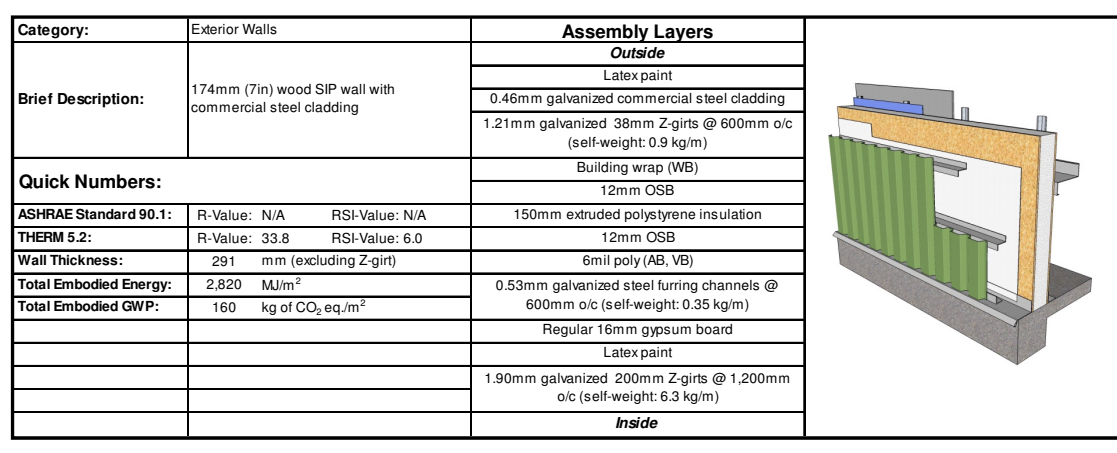

Life-Cycle Assessment Results:

Primary Energy Consumption (MJ)

\begin{tabular}{|c|c|c|c|c|c|c|c|c|c|c|c|c|c|c|c|}
\hline \multirow{3}{*}{$\begin{array}{l}\text { Lifiespan } \\
\text { (Years) }\end{array}$} & \multicolumn{13}{|c|}{ Embodied Energy (E } & \multirow{2}{*}{\multicolumn{2}{|c|}{$\begin{array}{l}\text { Difference in } \\
\text { Operating Energy } \\
\text { from Baseline after } \\
\text { Lifespan }\end{array}$}} \\
\hline & \multicolumn{2}{|c|}{ Manufacturing } & \multicolumn{3}{|c|}{ Construction } & \multicolumn{3}{|c|}{$\mathrm{Ma}$} & \multicolumn{3}{|c|}{ id of Life } & \multirow{2}{*}{$\begin{array}{c}{ }^{3} \text { Total } \\
\text { EE }\end{array}$} & \multirow{2}{*}{$\begin{array}{r}{ }^{2} \text { Total } \\
\text { EE } \\
\text { per } \mathrm{m}^{2}\end{array}$} & & \\
\hline & rial ${ }^{2}$ Trans. & Total & \begin{tabular}{|l|} 
Material \\
\end{tabular} & 2 Trans. & Total & \begin{tabular}{|l|} 
Material \\
\end{tabular} & \begin{tabular}{|l|}
${ }^{2}$ Trans. \\
\end{tabular} & Total & Material & \begin{tabular}{|l|l|}
${ }^{2}$ Trans. \\
\end{tabular} & Total & & & $\begin{array}{l}{ }^{5} \text { Total } \\
\text {. }\end{array}$ & ${ }^{6}$ per $\mathrm{m}^{2}$ \\
\hline itial & \begin{tabular}{|l|l|}
142,363 & 472 \\
\end{tabular} & 142,835 & 160 & 462 & 622 & 0 & 0 & 0 & $\overline{0}$ & 0 & 0 & $\frac{143.45}{10}$ & 2,817 & & \\
\hline
\end{tabular}

Global Warming Potential $\left(\mathbf{k g}\right.$ of $\left.\mathrm{CO}_{2} \mathrm{eq}\right)$

\begin{tabular}{|c|c|c|c|c|c|c|c|c|c|c|c|c|c|c|c|c|}
\hline \multirow{3}{*}{$\begin{array}{c}\text { Lifespan } \\
\text { (Years) }\end{array}$} & \multicolumn{14}{|c|}{ Embodied Global Warming Potential (GWP) } & \multirow{2}{*}{\multicolumn{2}{|c|}{$\begin{array}{l}\text { Difference in } \\
\text { Operating GWP from } \\
\text { Baseline after } \\
\text { Lifespan }\end{array}$}} \\
\hline & \multicolumn{3}{|c|}{ Manufacturing } & \multicolumn{3}{|c|}{ Construction } & \multicolumn{3}{|c|}{ Maintenance } & \multicolumn{3}{|c|}{ End of Life } & \multirow{2}{*}{$\begin{array}{l}{ }^{3} \text { Total } \\
\text { GWP }\end{array}$} & \multirow{2}{*}{ 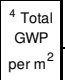 } & & \\
\hline & \begin{tabular}{|l|} 
Material \\
\end{tabular} & 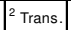 & \begin{tabular}{|l|} 
Total \\
\end{tabular} & Material & |2 Trans. & Total & \begin{tabular}{|l|} 
Materialal \\
\end{tabular} & 2 Trans. & \begin{tabular}{|l|} 
Total \\
\end{tabular} & \begin{tabular}{|l|} 
Material \\
\end{tabular} & \begin{tabular}{|l|}
2 Trans. \\
\end{tabular} & Total & & & ${ }^{5}$ Total & ${ }^{6}$ per m ${ }^{2}$ \\
\hline \begin{tabular}{|l|} 
\\
\end{tabular} & \begin{tabular}{|l|l|}
8,137 \\
\end{tabular} & 1 & 8,138 & 10 & 1 & 11 & 0 & 0 & 0 & 0 & 0 & 0 & 8,149 & 160 & & \\
\hline 50 & \begin{tabular}{|l}
8,137 \\
\end{tabular} & 1 & 8,138 & 10 & 1 & 11 & 0 & 0 & 0 & 0 & 0 & 0 & 8,149 & 160 & $-110,000$ & -189 \\
\hline
\end{tabular}

Net wall are of be

\begin{tabular}{|c|c|c|}
\hline Material List & Quantities & Unit \\
\hline $16 \mathrm{~mm}$ Regular Gypsum Board & 56.0 & $\mathrm{~m} 2$ \\
\hline 6 mil Polyethylene & 108.0 & $\mathrm{~m} 2$ \\
\hline Commercial $0.46 \mathrm{~mm}$ Steel Cladding & 168.0 & $\mathrm{~m} 2$ \\
\hline Extruded Polystyrene & 313.0 & $\mathrm{~m} 2(25 \mathrm{~mm}$ \\
\hline Gallvanized Sheet & 30.0 & $\mathrm{~kg}$ \\
\hline Galvanized Studs & 368.7 & $\mathrm{~kg}$ \\
\hline Joint Compound & 55.9 & $\mathrm{~kg}$ \\
\hline Nails & 3.7 & $\mathrm{~kg}$ \\
\hline Oriented Strand Board & 145.8 & $\mathrm{~m} 2(9 \mathrm{mr}$ \\
\hline Paper Tape & 0.6 & $\mathrm{~kg}$ \\
\hline Screws Nuts \& Bolts & 3.9 & $\mathrm{~kg}$ \\
\hline Water Based Latex Paint & 132.5 & $\mathrm{~L}$ \\
\hline
\end{tabular}

Notes:
${ }^{1}$ Initial $=$ Time $\mathrm{O}^{\prime}($ i.e. at the completion of initial construction)

${ }^{2}$ Trans. = Transportation

Total EE (or Total GWP) = Total embodied energy (or total embodied GWP of building component after lifespan (i.e. total manu
construction + total maintenance + total end-offife effects)

${ }^{4}$ Total EE (or Total GWP) per $\mathrm{m}^{2}=$ Total EE (or Total GWP) of building
component / area of building component that was modelled in ATHENAQ EIE component / area of building component that was modelled in ATHENA® EI ${ }^{5}$ Total Difference in Operating Energy (or GWP) from Baseline after
Lifespan = The eifference in the total life-cycle operating energy (or GWP)
from the baseline retail building after lifespan, due to using this building ${ }^{6}$ Total Difference in Operating Energy (or GWP) from Baseline after Total Difference in Operating Energy (or GWP) from Baseline after
Lifespan per $\mathrm{m}^{2}=$ = Total difierence in operating energy (or GWP) from
baseline atter lifespan / net wall area of baseline retail building Total operating primary energy use of baseline retail building after 50 years $=$ $50,700 \mathrm{GJ}\left(1,745 \mathrm{MJ} / \mathrm{m}^{2} \mathrm{YN}\right.$

* Total operating $\mathrm{GWP}$ of baseline retail building after 50 years $=2,310$ tonnes
of $\mathrm{CO}_{2}$ eq. $80 \mathrm{~kg}$ of $\mathrm{CO}_{2}$ eq, $/ \mathrm{m}^{2} \mathrm{y} r \mathrm{yr}$

Wood Structural Insulated Panel Wall \#12 (WSIP-W12)

Building Component Description:

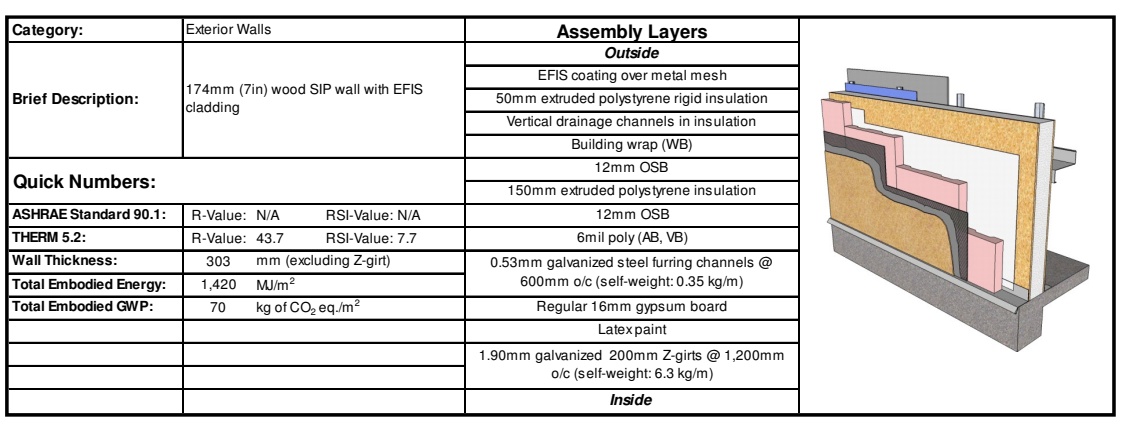

Life-Cycle Assessment Results:

\begin{tabular}{|c|c|c|c|c|c|c|c|c|c|c|c|c|c|c|c|c|}
\hline \multirow{4}{*}{$\begin{array}{c}\text { Lifespan } \\
\text { (Years) }\end{array}$} & \multicolumn{14}{|c|}{ Primary Energy Consumption (MJ) } & \multirow{3}{*}{\multicolumn{2}{|c|}{$\begin{array}{c}\text { Difference in } \\
\text { Operating Energy } \\
\text { from Baseline after } \\
\text { Lifespan }\end{array}$}} \\
\hline & \multicolumn{14}{|c|}{ Embodied Energy (EE) } & & \\
\hline & \multicolumn{3}{|c|}{ Manutacturing } & \multicolumn{3}{|c|}{ Construction } & \multicolumn{3}{|c|}{ Maintenance } & \multicolumn{3}{|c|}{ End of Life } & \multirow{2}{*}{$\begin{array}{c}{ }^{3} \text { Total } \\
\text { EE }\end{array}$} & \multirow{2}{*}{\begin{tabular}{|c|}
${ }^{4}$ Total \\
EE \\
per $\mathrm{m}^{2}$
\end{tabular}} & & \\
\hline & Material & ${ }^{2}$ Trans. & \begin{tabular}{|l|l|l|l|l} 
Total \\
\end{tabular} & Material & \begin{tabular}{|l|l|} 
& Trans. \\
\end{tabular} & Total & Material & \begin{tabular}{|l|}
${ }^{2}$ Trans. \\
\end{tabular} & Total & Material & ${ }^{2}$ Trans. & \begin{tabular}{|l|l|l|l} 
Total \\
\end{tabular} & & & ${ }^{5}$ Total & ${ }^{{ }^{\text {per m}} \mathrm{m}^{2}}$ \\
\hline itial & 70,536 & 528 & 71,064 & 160 & 569 & 729 & 0 & 0 & 0 & 0 & 0 & 0 & 71,793 & 1,410 & & \\
\hline
\end{tabular}

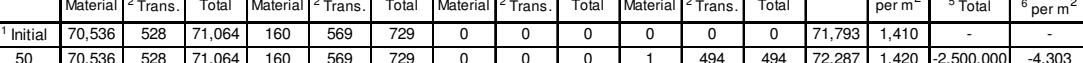

\begin{tabular}{|c|c|c|c|c|c|c|c|c|c|c|c|c|c|c|c|c|}
\hline \multirow{4}{*}{$\begin{array}{l}\text { Lifiespan } \\
\text { (Years) }\end{array}$} & \multicolumn{14}{|c|}{ Global Warming Potential $\left(\mathrm{kg}\right.$ of $\mathrm{CO}_{2} \mathrm{ec}$} & & \\
\hline & & & & & Embc & $\operatorname{died~G~}$ & bal War & $\operatorname{ming} \mathrm{Pc}$ & tential & (GWP) & & & & & \multirow{2}{*}{\multicolumn{2}{|c|}{$\begin{array}{l}\text { Difference in } \\
\text { Operating GWP } \\
\text { Baseline a ther } \\
\text { Lifespan }\end{array}$}} \\
\hline & \multicolumn{3}{|c|}{ Manutacturing } & \multicolumn{3}{|c|}{ Construction } & \multicolumn{3}{|c|}{ Maintenance } & \multicolumn{3}{|c|}{ End of Life } & \multirow{2}{*}{$\begin{array}{l}{ }^{3} \text { Total } \\
\text { GWWP }\end{array}$} & \multirow{2}{*}{$\begin{array}{l}{ }^{4} \text { Total } \\
\text { GWP } \\
\text { perm }\end{array}$} & & \\
\hline & Material & ${ }^{2}$ Trans. & Total & Material & Trans & Total & Material & ${ }^{2}$ Trans. & Total & Material & ${ }^{2}$ Trans. & Total & & & ${ }^{5}$ Total & ${ }^{6}$ per m ${ }^{2}$ \\
\hline \begin{tabular}{|l|l|} 
& Initial \\
\end{tabular} & 3,527 & 1 & 3,528 & 10 & 1 & 12 & 0 & 0 & 0 & 0 & 0 & 0 & \begin{tabular}{|l|l|}
3,540 \\
\end{tabular} & 70 & & \\
\hline 50 & \begin{tabular}{|l|l|}
3,527 \\
\end{tabular} & 1 & 3,528 & 10 & 1 & 12 & 0 & 0 & 0 & 0 & 1 & 1 & \begin{tabular}{|l|l|}
3,541 \\
\end{tabular} & 70 & $-130,000$ & -224 \\
\hline
\end{tabular}

Embodied energy (and GWP) numbers are based on an area of wall $=\begin{array}{llll}50.9 & \mathrm{~m}^{2}\end{array} \quad$ (Length $\times$ Height $=7.6 \mathrm{~m} \times 6.7 \mathrm{~m}=50.9 \mathrm{~m}^{2}$ ) Net wall area of baseline retail building (gross wall area - openings) $=581.0 \mathrm{~m}^{2}$

ATHENA $®$ EIE Material List:

$$
\text { (nncludes all materials after } 50 \text { years) }
$$

\begin{tabular}{|l|c|c|}
\hline \multicolumn{1}{|c|}{ Material List } & Quantities & Unit \\
\hline \#15 Organic Felt & 219.4 & $\mathrm{~m} 2$ \\
\hline 16mm Regular Gypsum Board & 56.0 & $\mathrm{~m} 2$ \\
\hline 6 mil Polyuthylenne & 108.0 & $\mathrm{~m} 2$ \\
\hline Extruded Polystyrene & 417.3 & $\mathrm{~m} 2(25 \mathrm{~mm})$ \\
\hline Galvanized Sheet & 81.4 & $\mathrm{~kg}$ \\
\hline Galvanized Studs & 287.9 & $\mathrm{~kg}$ \\
\hline Joint Compound & 55.9 & $\mathrm{~kg}$ \\
\hline Nails & 8.3 & $\mathrm{~kg}$ \\
\hline Oriented Strand Board & 145.8 & $\mathrm{~m} 2(9 \mathrm{~mm})$ \\
\hline Paper Tape & 0.6 & $\mathrm{~kg}$ \\
\hline Screws Nuts \& Bolts & 2.6 & $\mathrm{~kg}$ \\
\hline Stucco over metal mesh & 136.0 & $\mathrm{~m} 2$ \\
\hline Water Based Latex Paint & 132.5 & $\mathrm{~L}$ \\
\hline
\end{tabular}

${ }^{1}$ Initial $=$ Time ' 0 ' (i.e. at the completion:

${ }^{2}$ Trans. $=$ Transportation

Total EE (or Total GWP) $=$ Total embodied energy (or total embodied GWP) of building component after lifespan (i.e. total man
construction + total maintenance + total end-offlife effects)

${ }^{4}$ Total EE (or Total GWP) per $\mathrm{m}^{2}=$ Total EE (or Total GWP) of building
component/area of building component that was modelled in ATHENA@ EIE

Total Difference in Operating Energy (or GWP) from Baseline after Lifespan = The difference in the total life-cycle operating energy (or GWF
from the baseline retail building after lifespan, due to using this building component instead of the baseline component

Total Difference in Operating Energy (or GWP) from Baseline after Lifespan per $m^{2}=$ in Otal difference in operating energy (or GWP) from
baseline after lifespan "Total operating primary energy use of baseline retail building after 50 years
$50,700 \mathrm{GJ}\left(1,745 \mathrm{MJ} / \mathrm{m}^{\prime} \mathrm{yr}\right)$

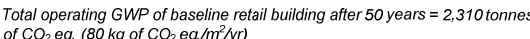




\section{LCA Data for Metal Structural Insulated Panel Walls}

This section contains a detailed description of each metal structural insulated panel (MSIP) exterior infill wall that was examined in this study (3 in total). The assembly layers are listed for each wall, along with a detailed description of the material quantities from the ATHENA® Environmental Impact Estimator for Buildings.

A breakdown of the total primary energy consumption and the total global warming potential (GWP) for each wall is also included. In each case, the results were calculated for an area of wall equal to $50.9 \mathrm{~m}^{2}$, which represents a typical bay size for a single-storey retail building. The results are also expressed on a per $\mathrm{m}^{2}$ basis in each case. The data has been calculated for two different lifespans: at the completion of initial construction and after 50 years.

As a summary, the figure below illustrates a comparison of the total embodied energy (and GWP) after 50 years for the various walls in this section as well as the WSIP walls from the previous section. For comparison purpose, the building component used in the baseline retail building has been shaded in grey.

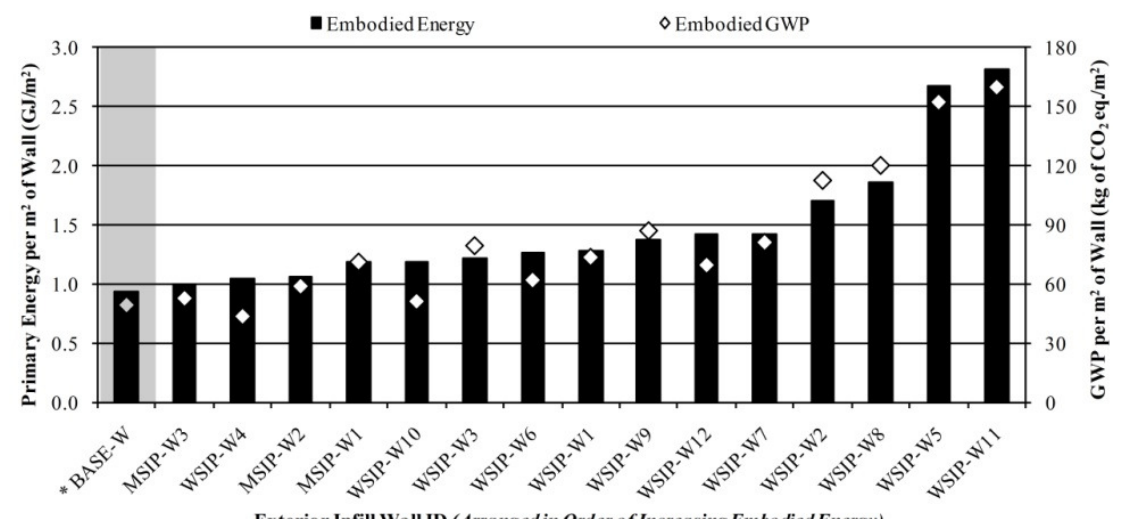

Metal Structural Insulated Panel Wall \#1 (MSIP-W1)

Building Component Description:

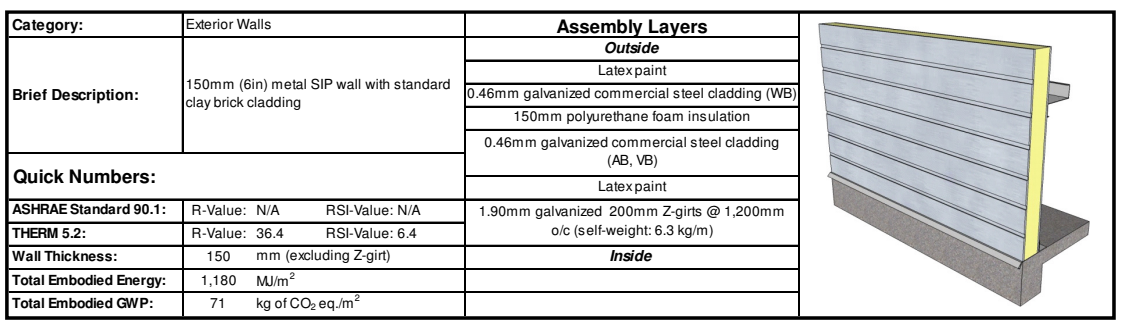

Life-Cycle Assessment Results:

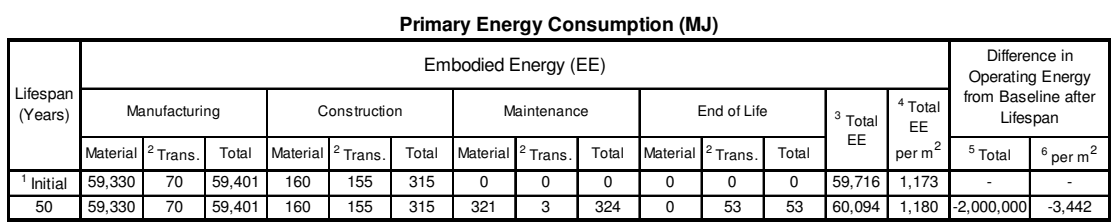

Global Warming Potential (kg of $\mathrm{CO}_{2}$ eq.)

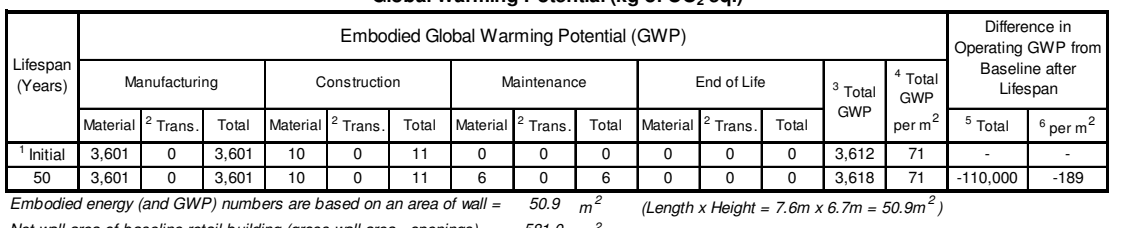

Net wall area of baseline retil buiding (aros wall area-openings) $=5810 \mathrm{~m}^{2}$

ATHENA ® EIE Material List:

(Includes all materials after 50 years

\begin{tabular}{|l|c|c|}
\hline \multicolumn{1}{|c|}{ Material List } & Quantities & Unit \\
\hline Foam Polyisocyanurate & 313.8 & $\mathrm{~m} 2(25 \mathrm{~mm})$ \\
\hline Galvanized Sheet & 529.2 & $\mathrm{~kg}$ \\
\hline Galvanized Studs & 287.9 & $\mathrm{~kg}$ \\
\hline Nails & 3.1 & $\mathrm{~kg}$ \\
\hline Screws Nuts \& Bolts & 2.6 & $\mathrm{~kg}$ \\
\hline Water Based Latex Paint & 13.5 & $\mathrm{~L}$ \\
\hline
\end{tabular}

Notes:

'Initial = Time 'O' (i.e. at the completion of initial construction)

${ }^{2}$ Trans. = Transportation

Total EE (or Total GWP) = Total embodied energy (or total embodied GWP) of building component after lifespan (i.e. total man
construction + total maintenance t total end-of-life effects)

${ }^{4}$ Total EE (or Total GWP) per $\mathrm{m}^{2}=$ Total EE (or Total GWP) of building
component / area of building component that was modelled in ATHENAQ EIE ${ }^{5}$ Total Difference in Operating Energy (or GWP) from Baseline after from the baseline retail building after lifescan operating energy (or GWP component instead of the baseline componen

${ }^{6}$ Total Difference in Operating Energy (or GWP) from Baseline after Lifespan per $\mathrm{m}^{2}=$ Total difference in operating energy (or GWP) from

* Total operating primary energy use of baseline retail building after 50 years

of $\mathrm{CO}_{2}$ eq. $\left(80 \mathrm{~kg}\right.$ of $\left.\mathrm{CO}_{2} \mathrm{eq} / \mathrm{h} \mathrm{m}^{2} \mathrm{yr}\right)$ 


\section{Metal Structural Insulated Panel Wall \#2 (MSIP-W2)}

Building Component Description:

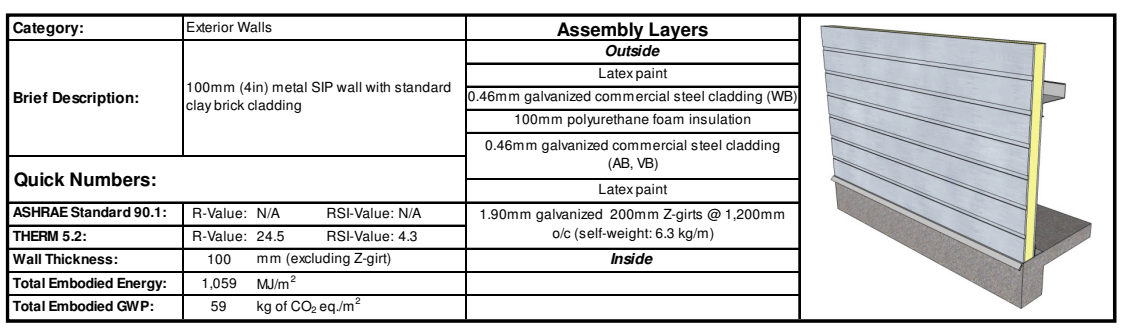

\section{Life-Cycle Assessment Results:}

Primary Energy Consumption (MJ)

\begin{tabular}{|c|c|c|c|c|c|c|c|c|c|c|c|c|c|c|c|c|}
\hline & \multirow{2}{*}{\multicolumn{14}{|c|}{ Embodied Energy (EE) }} & \multirow{3}{*}{\multicolumn{2}{|c|}{$\begin{array}{c}\text { Difference in } \\
\text { Operating Energy } \\
\text { from Baseline after } \\
\text { Lifespan }\end{array}$}} \\
\hline \multirow{3}{*}{$\begin{array}{l}\text { Lespart } \\
\text { (Years) }\end{array}$} & & & & & & & & & & & & & & & & \\
\hline & \multicolumn{3}{|c|}{ Manutacturing } & \multicolumn{3}{|c|}{ Construction } & \multicolumn{3}{|c|}{ Maintenance } & \multicolumn{3}{|c|}{ End of Life } & \multirow{2}{*}{$\begin{array}{c}{ }^{3} \text { Total } \\
\mathrm{EE}\end{array}$} & \multirow{2}{*}{$\mid \begin{array}{c}{ }^{4} \text { Total } \\
\text { EE } \\
\text { per m }\end{array}$} & & \\
\hline & Material & ${ }^{2}$ Trans. & Total & Material & ${ }^{2}$ Trans. & Total & Material & ${ }^{2}$ Trans. & Total & Material & Trans. & Total & & & ${ }^{5}$ Total & ${ }^{6}$ per $m^{2}$ \\
\hline 'Initial & 53,208 & 67 & 53,275 & 160 & 135 & 295 & 0 & 0 & 0 & 0 & 0 & 0 & \begin{tabular}{|c|c|}
53,569 \\
\end{tabular} & 1,052 & & \\
\hline 50 & 53,208 & 67 & 53,275 & 160 & \begin{tabular}{l|l}
135 \\
\end{tabular} & 295 & 321 & 3 & 324 & 0 & 46 & 46 & 53,940 & 1,059 & $\begin{array}{ll}1,300,000 \\
\end{array}$ & $-2,238$ \\
\hline
\end{tabular}

Global Warming Potential ( $\mathrm{kg}$ of $\mathrm{CO}_{2}$ eq.)

\begin{tabular}{|c|c|c|c|c|c|c|c|c|c|c|c|c|c|c|c|c|}
\hline \multirow{3}{*}{$\begin{array}{l}\text { Lifespan } \\
\text { (Years) }\end{array}$} & \multicolumn{14}{|c|}{ Embodied Global Warming Potential (GWP) } & \multirow{2}{*}{\multicolumn{2}{|c|}{$\begin{array}{c}\text { Difference in } \\
\text { Operating GWP from } \\
\text { Baseline after } \\
\text { Lifespan }\end{array}$}} \\
\hline & \multicolumn{3}{|c|}{ Manufacturing } & \multicolumn{3}{|c|}{ Construction } & \multicolumn{3}{|c|}{ Maintenance } & \multicolumn{3}{|c|}{ End of Life } & \multirow{2}{*}{$\begin{array}{l}{ }^{3} \text { Total } \\
\text { GWWP }\end{array}$} & \multirow{2}{*}{$\begin{array}{l}{ }^{4}{ }^{\mathrm{T} o t a l} \\
\mathrm{GWP} \\
\text { per m}\end{array}$} & & \\
\hline & Material & ${ }^{2}$ Trans. & 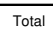 & Material & $\left.\right|^{2}$ Trans. & Total & Material & 2 Trans. & \begin{tabular}{|l|l} 
Total \\
\end{tabular} & Material & ${ }^{2}$ Trans. & Total & & & ${ }^{5}$ Total & ${ }^{6} \mathrm{perm}^{2}$ \\
\hline 1 Initial & 2,977 & 0 & 2,977 & 10 & 0 & 11 & 0 & 0 & 0 & 0 & 0 & 0 & 2,987 & 59 & & \\
\hline 50 & 2,977 & 0 & 2,977 & 10 & 0 & 11 & 6 & 0 & 6 & 0 & 0 & 0 & 2,994 & 59 & $-70,000$ & -120 \\
\hline
\end{tabular}

ATHENA ® EIE Material List:

\begin{tabular}{|l|c|c|}
\hline \multicolumn{1}{|c|}{ Material List } & Quantities & Unit \\
\hline Foam Polyisocyanurate & 209.2 & $\mathrm{~m} 2(25 \mathrm{~mm})$ \\
\hline Galvanized Sheet & 529.2 & $\mathrm{~kg}$ \\
\hline Galvanized Studs & 287.9 & $\mathrm{~kg}$ \\
\hline Nails & 3.1 & $\mathrm{~kg}$ \\
\hline Screws Nuts \& Bolts & 2.6 & $\mathrm{~kg}$ \\
\hline Water Based Latex Paint & 13.5 & $\mathrm{~L}$ \\
\hline
\end{tabular}

Notes:
${ }^{1}$ 'Initial = Time 'o' (i.e. at the completion of initial construction)

${ }^{2}$ Trans. $=$ Transportation

${ }^{3}$ Total EE (or Total GWP) $=$ Total embodied energy (or total embodied GWP) of building component after lifespan (i.e. total man
construction + total maintenance + total end-oflife effects)

${ }^{4}$ Total EE (or Total GWP) per $\mathrm{m}^{2}=$ Total EE (or Total GWP) of building
component / area of building component that was modelled in ATHENAQ EIE ${ }^{5}$ Total Difference in Operating Energy (or GWP) from Baseline after Lifespan = The difference in the total life-cycle operating energy (or GWP)
from the baseline retail butiding after lifespan, due to using this building
component instead of the baseline component

6 Total Difference in Operating Energy (or GWP) from Baseline after
Lifespan per $\mathrm{m}^{2}=$ Total difference in operating energy (or GWP) from Lifespan per $\mathrm{m}^{2}=$ Total difference in operating energy
baseline after lifespan $/$ net wall area of baseline retail building

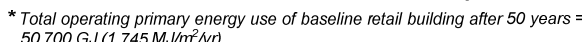

"Total operating $\mathrm{GWP}$ of baseline reta

\section{Metal Structural Insulated Panel Wall \#3 (MSIP-W3)}

Building Component Description:

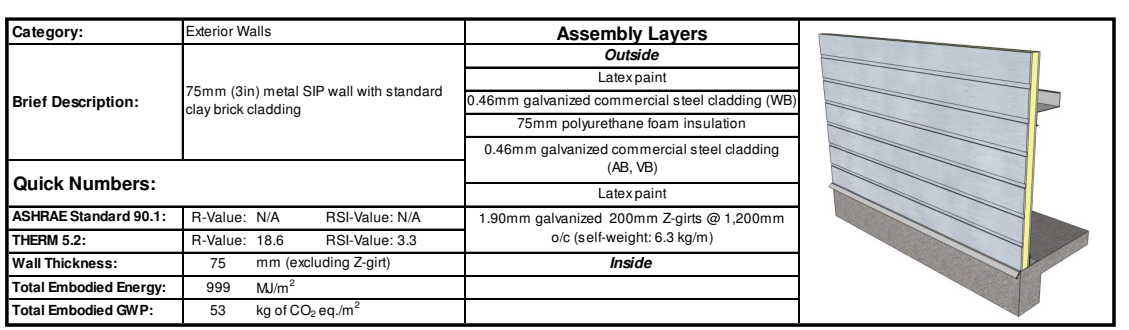

Life-Cycle Assessment Results:

Primary Energy Consumption (MJ)

\begin{tabular}{|c|c|c|c|c|c|c|c|c|c|c|c|c|c|c|c|c|}
\hline \multirow{3}{*}{$\begin{array}{l}\text { Lifespan } \\
\text { (Years) }\end{array}$} & \multicolumn{14}{|c|}{ Embodied Energy (EE) } & \multirow{2}{*}{\multicolumn{2}{|c|}{$\begin{array}{l}\text { Difference in } \\
\text { Operating Energy } \\
\text { from Baseline after } \\
\text { Lifespan }\end{array}$}} \\
\hline & \multicolumn{3}{|c|}{ Manufacturing } & \multicolumn{3}{|c|}{ Construction } & \multicolumn{3}{|c|}{ Maintenance } & \multicolumn{3}{|c|}{ End of Lifie } & \multirow{2}{*}{$\begin{array}{l}{ }^{3} \text { Total } \\
\mathrm{EE}\end{array}$} & \multirow{2}{*}{\begin{tabular}{|c|}
${ }^{4}$ Total \\
EE \\
per ${ }^{2}$
\end{tabular}} & & \\
\hline & \begin{tabular}{|l|} 
Material \\
\end{tabular} & ${ }^{2}$ Trans. & \begin{tabular}{|l|} 
Total \\
\end{tabular} & Materia & ${ }^{2}$ Trans. & Total & Materia & ${ }^{2}$ Trans. & Total & Material & ${ }^{2}$ Trans. & \begin{tabular}{|l|l|} 
Total \\
\end{tabular} & & & ${ }^{5}$ Total & ${ }^{6}$ per m ${ }^{2}$ \\
\hline & 50,147 & 65 & 50,212 & 160 & \begin{tabular}{|l|}
124 \\
\end{tabular} & 284 & 0 & 0 & 0 & 0 & 0 & 0 & 50,496 & 992 & & \\
\hline 50 & 50,147 & 65 & 50,212 & 160 & \begin{tabular}{|l|}
124 \\
\end{tabular} & 284 & 321 & 3 & 324 & 0 & 42 & 43 & 5 & 999 & 600,000 & $-1,033$ \\
\hline
\end{tabular}

Global Warming Potential (kg of $\mathrm{CO}_{2}$ eq.)

\begin{tabular}{|c|c|c|c|c|c|c|c|c|c|c|c|c|c|c|c|c|}
\hline \multirow{3}{*}{$\begin{array}{l}\text { Lifiesparar } \\
\text { (Years) }\end{array}$} & \multicolumn{14}{|c|}{ Embodied Global Warming Potential (GWP) } & \multirow{2}{*}{\multicolumn{2}{|c|}{$\begin{array}{l}\text { Difference in } \\
\text { Operating GWP from } \\
\text { Baseline after } \\
\text { Lifespan }\end{array}$}} \\
\hline & \multicolumn{3}{|c|}{ Manufacturing } & \multicolumn{3}{|c|}{ Construction } & \multicolumn{3}{|c|}{ Maintenance } & \multicolumn{3}{|c|}{ End of L Life } & \multirow{2}{*}{$\begin{array}{l}{ }^{3} \text { Total } \\
\text { GWP }\end{array}$} & \multirow{2}{*}{\begin{tabular}{|l}
${ }^{4} \mathrm{~T}_{\text {otal }}$ \\
GWPP \\
per m
\end{tabular}} & & \\
\hline & Material & ${ }^{2}$ Trans. & Total & Material & 2 Trans. & Total & Material & ${ }^{2}$ Trans. & Total & Material & $\mid{ }^{2}$ Trans. & Total & & & ${ }^{5}$ Total & ${ }^{6}$ perm $^{2}$ \\
\hline${ }^{1}$ Initial & 2,664 & 0 & 2,664 & 10 & 0 & 11 & 0 & 0 & 0 & 0 & 0 & 0 & 2,675 & 53 & & \\
\hline 50 & 2,664 & 0 & 2,664 & 10 & 0 & 11 & 6 & 0 & 6 & 0 & 0 & 0 & 2,682 & 53 & $-30,000$ & -52 \\
\hline
\end{tabular}
a - openings) $=581.0 \mathrm{~m}^{2}$

ATHENA ® EIE Material List:

(Includes all materials after 50 years)

\begin{tabular}{|l|c|c|}
\hline \multicolumn{1}{|c|}{ Material List } & Quantities & Unit \\
\hline Foam Polyisocyanurate & 156.9 & $\mathrm{~m} 2(25 \mathrm{~mm})$ \\
\hline Galvanized Sheet & 529.2 & $\mathrm{~kg}$ \\
\hline Galvanized Studs & 287.9 & $\mathrm{~kg}$ \\
\hline Nails & 3.1 & $\mathrm{~kg}$ \\
\hline Screws Nuts \& Bolts & 2.6 & $\mathrm{~kg}$ \\
\hline Water Based Latex Paint & 13.5 & $\mathrm{~L}$ \\
\hline
\end{tabular}

Notes:
1 Initial $=$ Time ' $O$ ' (i.e. at the completion of initial construction)

${ }^{2}$ Trans. $=$ Transportation

${ }^{3}$ Total EE (or Total GWP) = Total embodied energy (or total embodied GWP) of building component after lifespan (i.e. total man
construction + total maintenance + total end-of-life effects)

${ }^{4}$ Total EE (or Total GWP) per $\mathrm{m}^{2}=$ Total EE (or Total GWP) of building
component/ area of building component that was modelled in ATHENAB EEEE ${ }^{5}$ Total Difference in Operating Energy (or GWP) from Baseline after from the baseline retail building atter lifespan, due to using this building from the baseline retall bulding after lifesp
component instead of the baseline component

${ }^{6}$ Total Difference in Operating Energy (or GWP) from Baseline after Lifespan per $\mathrm{m}^{2}=$ Total difference in operating energy (or GWP) from * Total operating primary energy use of baseline retail building after 50 years =

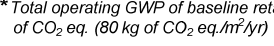




\section{LCA Data for Cold-Formed Steel Stud Walls}

This section contains a detailed description of each cold-formed steel stud (SS) exterior infill wall that was examined in this study (31 in total). The assembly layers are listed for each wall, along with a detailed description of the material quantities from the ATHENA ${ }^{\circledR}$ Environmental Impact Estimator for Buildings.

A breakdown of the total primary energy consumption and the total global warming potential (GWP) for each wall is also included. In each case, the results were calculated for an area of wall equal to $50.9 \mathrm{~m}^{2}$, which represents a typical bay size for a single-storey retail building. The results are also expressed on a per $\mathrm{m}^{2}$ basis in each case. The data has been calculated for two different lifespans: at the completion of initial construction and after 50 years.

As a summary, the figure below illustrates a comparison of the total embodied energy (and GWP) after 50 years for the various walls in this section. For comparison purpose, the building component used in the baseline retail building has been shaded in grey.

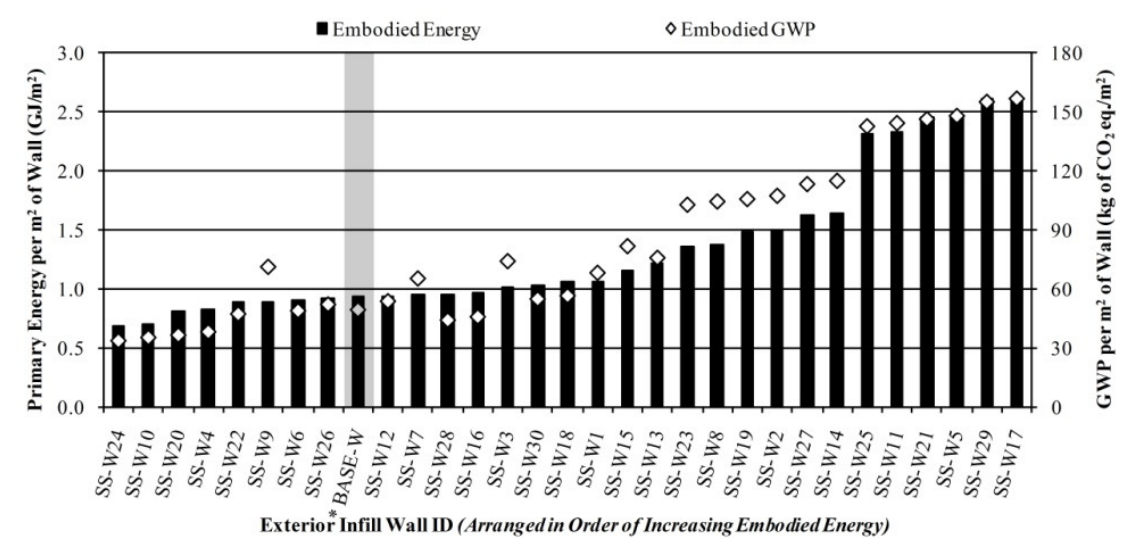

Baseline Retail Building Wall (BASE-W)

Building Component Description:

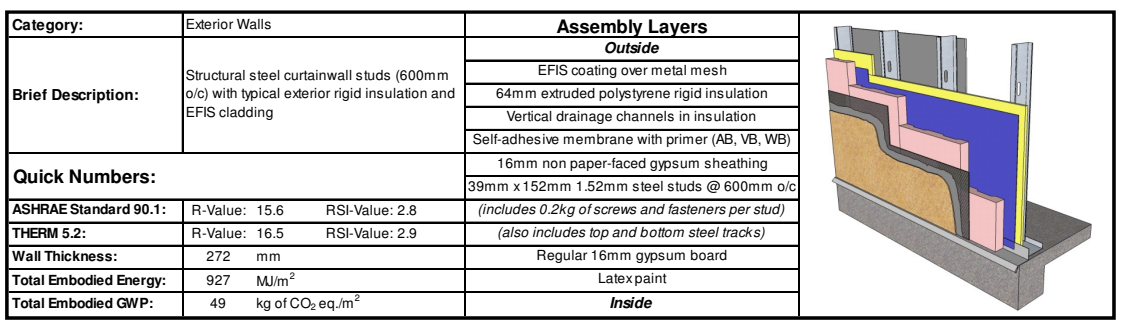

Life-Cycle Assessment Results:
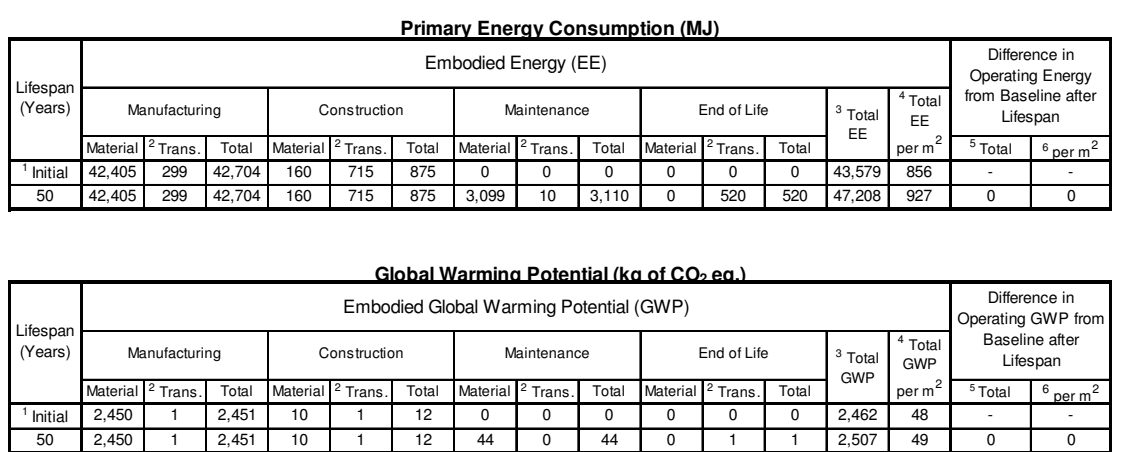

Embodied energy (and GWP) numbers are based on an area of wall $=\quad 50.9 \mathrm{~m}^{2} \quad$ (Length $x$ Height $=7.6 \mathrm{~m} \times 6.7 \mathrm{~m}=50.9 \mathrm{~m}^{2}$ )

Net wall area of baseline retail building (gross wall a rea - openings) -

ATHENA ® EIE Material List:

\begin{tabular}{|c|c|c|}
\hline Material List & Quantities & Unit \\
\hline$\# 15$ Organic Felt & 219.4 & $\mathrm{~m} 2$ \\
\hline 3 mil Polyethylene & 54.0 & $\mathrm{~m} 2$ \\
\hline $\begin{array}{l}16 \mathrm{~mm} \text { Moisture Resistant Gypsum } \\
\text { Board }\end{array}$ & 56.0 & $\mathrm{~m} 2$ \\
\hline $16 \mathrm{~mm}$ Regular Gypsum Board & 56.0 & $\mathrm{~m} 2$ \\
\hline Extruded Polystyrene & 133.5 & $\mathrm{~m} 2(25 \mathrm{~mm})$ \\
\hline Galvanized Sheet & 51.4 & $\mathrm{~kg}$ \\
\hline Galvanized Studs & 300.5 & $\mathrm{~kg}$ \\
\hline Joint Compound & 111.8 & $\mathrm{~kg}$ \\
\hline Modified Bitumen membrane & 68.2 & $\mathrm{~kg}$ \\
\hline Nails & 5.7 & $\mathrm{~kg}$ \\
\hline Paper Tape & 1.3 & $\mathrm{~kg}$ \\
\hline Screws Nuts \& Bolts & 2.6 & $\mathrm{~kg}$ \\
\hline Solvent Based Alkyd Paint & 19.6 & $\mathrm{~L}$ \\
\hline Stucco over metal mesh & 136.0 & $\mathrm{~m} 2$ \\
\hline Water Based Latex Paint & 132.5 & L \\
\hline
\end{tabular}

${ }^{1}$ IInitial $=$ Time 'o' (ie. at the completion os: mpletion of initial construction)

Total EE (or Total GWP) $=$ Total embodied energy (or total embodied GWP) of building component after lifespan (i.e. total manufacturing + tota ${ }^{4}$ Total EE (or Total GWP) per $\mathrm{m}^{2}=$ Total EE (or Total GWP) of building
component/area of building component that was modelled in ATHENA@EIE ${ }^{5}$ Total Difference in Operating Energy (or GWP) from Baselt Lifespan = The difference in the total life-cycle operating energy (or GWWP) from the baseline retail building after lifespan
component instead of the baseline component

${ }^{6}$ Total Difference in Operating Energy (or GWP) from Baseline after Total Difference in Operating Energy (or GWP) from Baseline after
Lifespan per $\mathrm{m}^{2}=$ Total difference in operating energy (or GWP) from baseline after lifespan / net wall area of baseline retail building "Total operating primary energy use of baseline retail building after 50 years =

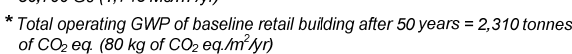

${ }^{1}$ 'Initial $=$ Time ' ${ }^{\prime}$ ' (i.e. at 


\section{Cold-Formed Steel Stud Wall \#1 (SS-W1)}

Building Component Description:

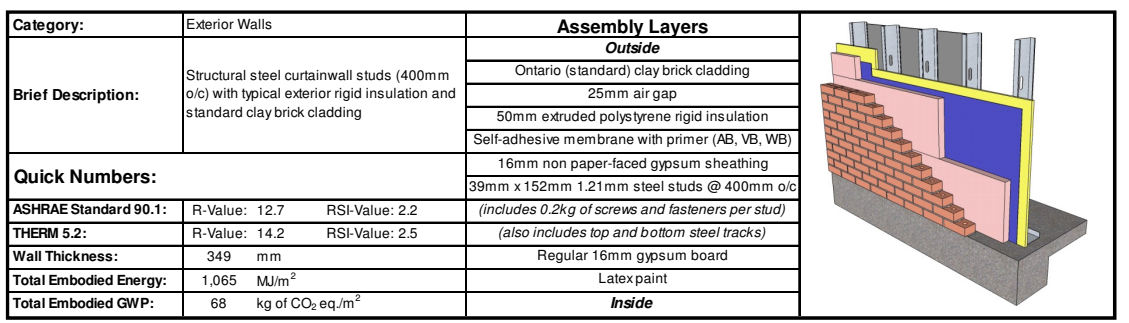

Life-Cycle Assessment Results:

Primary Energy Consumption (MJ)

\begin{tabular}{|c|c|c|c|c|c|c|c|c|c|c|c|c|c|c|c|c|}
\hline \multirow{3}{*}{$\begin{array}{l}\text { Lifespan } \\
\text { (Years) }\end{array}$} & \multicolumn{14}{|c|}{ Embodied Energy (EE) } & \multirow{2}{*}{\multicolumn{2}{|c|}{$\begin{array}{c}\text { Difference in } \\
\text { Operating Energy } \\
\text { from Baseline after } \\
\text { Lifespan }\end{array}$}} \\
\hline & \multicolumn{3}{|c|}{ Manufacturing } & \multicolumn{3}{|c|}{ Construction } & \multicolumn{3}{|c|}{ Maintenance } & \multicolumn{3}{|c|}{ End of Life } & \multirow{2}{*}{$\begin{array}{l}{ }^{3} \text { Total } \\
\mathrm{EE}\end{array}$} & \multirow{2}{*}{$\begin{array}{l}{ }^{4} \text { Total } \\
\text { EE } \\
\text { per m }\end{array}$} & & \\
\hline & \begin{tabular}{|l|l|} 
Material \\
\end{tabular} & ${ }^{2}$ Trans & Total & \begin{tabular}{|l|} 
Material \\
\end{tabular} & \begin{tabular}{|l}
${ }^{2}$ Trans. \\
(a)
\end{tabular} & \begin{tabular}{|l|} 
Total \\
\end{tabular} & Material & \begin{tabular}{|l|}
${ }^{2}$ Trans. \\
\end{tabular} & Total & \begin{tabular}{|l|} 
Material \\
\end{tabular} & ${ }^{2}$ Trans. & \begin{tabular}{|l|l|} 
Total \\
\end{tabular} & & & ${ }^{5}$ Total & ${ }^{6}$ per m ${ }^{2}$ \\
\hline 1 Initial & \begin{tabular}{|l|l|}
48,729 \\
\end{tabular} & 275 & \begin{tabular}{|l|l}
49,004 \\
\end{tabular} & 160 & 1,375 & 1,535 & 0 & 0 & 0 & 0 & 0 & 0 & 50,539 & 993 & & \\
\hline 50 & \begin{tabular}{|l|l|}
48,779 \\
\end{tabular} & \begin{tabular}{|l|l|} 
& 275 \\
\end{tabular} & 49,004 & 160 & \begin{tabular}{|l|l|}
1,375 \\
\end{tabular} & 1,535 & \begin{tabular}{|l|l|}
3,099 \\
\end{tabular} & 10 & \begin{tabular}{|l|l|}
3,110 \\
\end{tabular} & 0 & 580 & 580 & 54,228 & 1,065 & 300,000 & 516 \\
\hline
\end{tabular}

Global Warming Potential ( $k g$ of $\mathrm{CO}_{2}$ eq.)

\begin{tabular}{|c|c|c|c|c|c|c|c|c|c|c|c|c|c|c|c|c|}
\hline \multirow{3}{*}{$\begin{array}{l}\text { Lifespar } \\
\text { (Years) }\end{array}$} & \multicolumn{14}{|c|}{ Embodie } & \multirow{2}{*}{\multicolumn{2}{|c|}{$\begin{array}{l}\text { Difference in } \\
\text { Operating GWP from } \\
\text { Baseline after } \\
\text { Lifespan }\end{array}$}} \\
\hline & \multicolumn{3}{|c|}{ Manufacturing } & \multicolumn{3}{|c|}{ Construction } & \multicolumn{3}{|c|}{ Maintenance } & \multicolumn{3}{|c|}{ End of Life } & \multirow{2}{*}{$\begin{array}{l}{ }^{3} \text { Total } \\
\text { GWPP }\end{array}$} & \multirow{2}{*}{$\begin{array}{l}{ }^{4}{ }^{4} \text { Total } \\
\text { GWP } \\
\text { per m }\end{array}$} & & \\
\hline & Material & ${ }^{2}$ Trans. & \begin{tabular}{|l|l|} 
Total \\
\end{tabular} & Material & ${ }^{2}$ Trans. & Total & $\mid$\begin{tabular}{|l|} 
Material \\
\end{tabular} & ${ }^{2}$ Trans. & Total & \begin{tabular}{|l|} 
Material \\
\end{tabular} & $\mid{ }^{2}$ Trans. & Total & & & ${ }^{5}$ Total & ${ }^{6}$ per m$^{2}$ \\
\hline Initial & \begin{tabular}{|l|}
3,405 \\
\end{tabular} & 1 & 3,405 & 10 & 3 & 13 & 0 & 0 & 0 & 0 & 0 & 0 & 3,418 & 67 & & \\
\hline 50 & 3,405 & 1 & 3,405 & 10 & 3 & 13 & 44 & 0 & 44 & 0 & 1 & 1 & 3,463 & 68 & 20,000 & 34 \\
\hline
\end{tabular}

ATHENA ® EIE Material List: (Includes all materials after 50 years)

\begin{tabular}{|l|c|c|}
\hline \multicolumn{1}{|c|}{ Material List } & Quantities & Unit \\
\hline 3 mil Polyethylene & 54.0 & $\mathrm{~m} 2$ \\
\hline $\begin{array}{l}\text { 16mm Moisture Resistant Gypsum } \\
\text { Board }\end{array}$ & 56.0 & $\mathrm{~m} 2$ \\
\hline 16rmm Regular Gypsum Board & 56.0 & $\mathrm{~m} 2$ \\
\hline Cold Rolled Sheet & 10.3 & $\mathrm{~kg}$ \\
\hline Extruded Polystyrene & 104.3 & $\mathrm{~m} 2(25 \mathrm{~mm})$ \\
\hline Galvanized Studs & 342.5 & $\mathrm{~kg}$ \\
\hline Joint Compound & 111.8 & $\mathrm{~kg}$ \\
\hline Modified Bitumen membrane & 68.2 & $\mathrm{~kg}$ \\
\hline Mortar & 1.5 & $\mathrm{~m} 3$ \\
\hline Nails & 4.2 & $\mathrm{~kg}$ \\
\hline Ontario (Standard) Brick & 53.5 & $\mathrm{~m} 2$ \\
\hline Paper Tape & 1.3 & $\mathrm{~kg}$ \\
\hline Screws Nuts \& Bolts & 3.9 & $\mathrm{~kg}$ \\
\hline Solvent Based Alkyd Paint & 19.6 & $\mathrm{~L}$ \\
\hline Water Based Latex Paint & 66.3 & $\mathrm{~L}$ \\
\hline
\end{tabular}

\section{Cold-Formed Steel Stud Wall \#2 (SS-W2)}

Building Component Description:

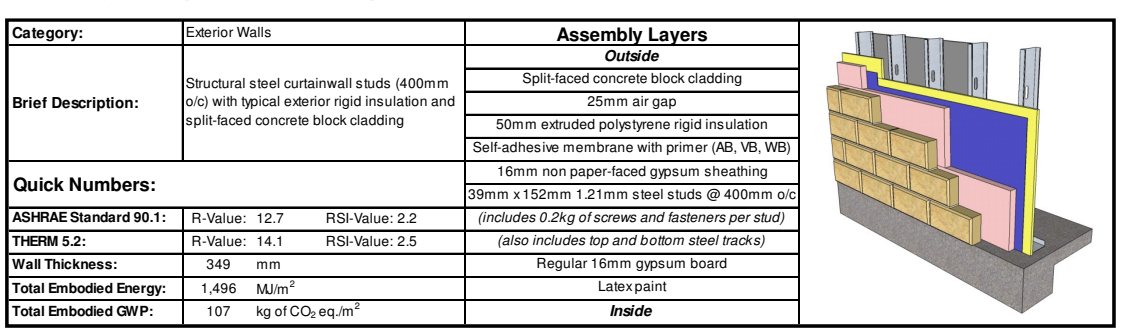

Life-Cycle Assessment Results:

Primary Energy Consumption (MJ)

\begin{tabular}{|c|c|c|c|c|c|c|c|c|c|c|c|c|c|c|c|}
\hline \multirow{3}{*}{$\begin{array}{l}\text { Yrespars } \\
\text { Years }\end{array}$} & \multicolumn{13}{|c|}{ Embodied Energy (EE) } & \multirow{2}{*}{\multicolumn{2}{|c|}{$\begin{array}{l}\text { Difference in } \\
\text { Operating Energy } \\
\text { from Baseline after } \\
\text { Lifespan }\end{array}$}} \\
\hline & \multicolumn{2}{|c|}{ Manutacturing } & \multicolumn{3}{|c|}{ Construction } & \multicolumn{3}{|c|}{ Maintenance } & \multicolumn{3}{|c|}{ End of Life } & \multirow{2}{*}{$\begin{array}{c}{ }^{3} \text { Total } \\
\text { EE }\end{array}$} & \multirow{2}{*}{$\begin{array}{l}{ }^{4}{ }^{4} \text { otal } \\
\text { EE } \\
\text { per m }\end{array}$} & & \\
\hline & 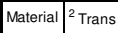 & Total & \begin{tabular}{|c|} 
Material \\
\end{tabular} & \begin{tabular}{l|l|}
1 & 2 \\
Trans.
\end{tabular} & Total & Material & ${ }^{2}$ Trans. & Total & Material & \begin{tabular}{|l|}
${ }^{2}$ Trans. \\
\end{tabular} & Total & & & ${ }^{5}$ Total & ${ }^{6} \mathrm{perm}^{2}$ \\
\hline tial & \begin{tabular}{ll|l}
7,757 & 839 \\
\end{tabular} & 68,595 & 160 & 2,185 & 2,345 & 0 & 0 & 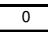 & 0 & 0 & 0 & 940 & 1,393 & & \\
\hline
\end{tabular}

Global Warming Potential (kg of $\mathrm{CO}_{2}$ eq.)

\begin{tabular}{|c|c|c|c|c|c|c|c|c|c|c|c|c|c|c|c|c|}
\hline \multirow{3}{*}{$\begin{array}{l}\text { Lifespan } \\
\text { (Years) }\end{array}$} & \multicolumn{14}{|c|}{ Embodied Global Warming Potential (GWP) } & \multirow{2}{*}{\multicolumn{2}{|c|}{$\begin{array}{l}\text { Difference in } \\
\text { Operating GWP from } \\
\text { Baselile anter } \\
\text { Lifespan }\end{array}$}} \\
\hline & \multicolumn{3}{|c|}{ Manutacturing } & \multicolumn{3}{|c|}{ Construction } & \multicolumn{3}{|c|}{ Maintenance } & \multicolumn{3}{|c|}{ End of Lite } & \multirow{2}{*}{$\begin{array}{l}{ }^{3} \text { Total } \\
\text { GWP }\end{array}$} & \multirow{2}{*}{$\begin{array}{c}{ }^{4} \text { Total } \\
\text { GWP } \\
\text { per m }{ }^{2}\end{array}$} & & \\
\hline & Material & ${ }^{2}$ Trans. & Total & Material: & Trans. & Total & Material & ${ }^{2}$ Trans. & Total & Material & $\mid 2$ Trans. & Total & & & ${ }^{5}$ Total & ${ }^{6}$ per $\mathrm{m}^{2}$ \\
\hline Initial & 5,390 & 2 & 5 & 10 & 4 & 15 & 0 & 0 & 0 & 0 & 0 & 0 & 5,407 & $\begin{array}{ll}106 \\
\end{array}$ & & \\
\hline 50 & 5390 & 2 & 5392 & 10 & 4 & 15 & 44 & 0 & 44 & 0 & 4 & 4 & 5.454 & 107 & 20,000 & 34 \\
\hline
\end{tabular}

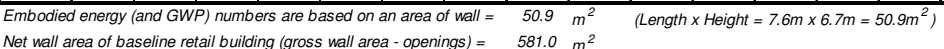

ATHENA $®$ EIE Material List:

Notes:
1 Initial $=$ Time ' $O$ ' (i.e. at the completion of initial construction)

${ }^{2}$ Trans. $=$ Transportation

${ }^{3}$ Total EE (or Total GWP) $=$ Total embodied energy (or total embodied GWP) of building component after lifespan (i.e. total manu
construction + total maintenance + total end-oflife effects)

${ }^{4}$ Total EE (or Total GWP) per $\mathrm{m}^{2}=$ Total EE (or Total GWP) of building
component/area of building component that was modelled in ATHENAQ EEE ${ }^{5}$ Total Difference in Operating Energy (or GWP) from Baseline after Lifespan = The difference in the total lifeccycle operating energy (or GWP)
from the baseline retail building after lifespan, due to using this building
component instead of the baseline component

${ }^{6}$ Total Difference in Operating Energy (or GWP) from Baseline after Lifespan per $\mathrm{m}^{2}=$ Total difference in operating energy (or GWP) from * Total operating primary energy use of baseline retail building after 50 years =

"Total operating $G$ WP of baseline retail building after 50 years $=2,310$ tonnes
of $\mathrm{CO}_{2}$ eq. $\left(80 \mathrm{~kg}\right.$ of $\mathrm{CO}_{2}$ eq. $\left./ \mathrm{m}^{2} \mathrm{yr}\right)$

\begin{tabular}{|l|c|c|}
\multicolumn{1}{|c|}{ (Includes all materials after 50 years) } \\
\hline \multicolumn{1}{|c|}{ Material List } & Quantities & Unit \\
\hline 3 mil Polyethylene & 54.0 & $\mathrm{~m} 2$ \\
\hline $16 \mathrm{~mm}$ Moisture Resistant Gypsum & 56.0 & $\mathrm{~m} 2$ \\
\hline Board & 56.0 & $\mathrm{~m} 2$ \\
\hline 16mm Regular Gypsum Board & 10.3 & $\mathrm{~kg}$ \\
\hline Cold Rolled Sheet & 104.3 & $\mathrm{~m} 2(25 \mathrm{~mm})$ \\
\hline Extruded Polystyrene & 342.5 & $\mathrm{~kg}$ \\
\hline Galvanized Studs & 111.8 & $\mathrm{~kg}$ \\
\hline Joint Compound & 401.5 & $\mathrm{~kg}$ \\
\hline Modified Bitumen membrane & 4.3 & $\mathrm{~m} 3$ \\
\hline Mortar & 4.2 & $\mathrm{~kg}$ \\
\hline Nails & 1.3 & $\mathrm{~kg}$ \\
\hline Paper Tape & 3.9 & $\mathrm{~kg}$ \\
\hline Screws Nuts \& Bolts & 19.6 & $\mathrm{~L}$ \\
\hline Solvent Based Alkyd Paint & $1,238.2$ & Blocks \\
\hline Split-faced Concrete Block & 66.3 & $\mathrm{~L}$ \\
\hline Water Based Latex Paint & 6 \\
\hline
\end{tabular}

Notes:
${ }^{1}$ Initial $=$ Time ' 0 ' (i.e. at the completion of initial construction)

${ }^{2}$ Trans. $=$ Transportation

${ }^{3}$ Total EE (or Total GWP) $=$ Total embodied energy (or total embodied GWP) of building component after lifespan (i.e. total man
construction + total maintenance + total end-of life effects)

${ }^{4}$ Total EE (or Total GWP) per $\mathrm{m}^{2}=$ Total EE (or Total GWP) of building
component / area of building component that was modelled in ATHENAQ EIE ${ }^{5}$ Total Difference in Operating Energy (or GWP) from Baseline after Lifespan = The difference in the total life-cycle operating energy (or GWP)
from the baseline retail builing after lifespan, due to using this building
component instead of the baseline component

${ }^{6}$ Total Difference in Operating Energy (or GWP) from Baseline after Lifespan per $\mathrm{m}^{2}=$ Total difference in operating energy (or GWP) from * Total operating primary energy use of baseline retail building after 50 years =

*Total operating GWP of baseline ret.
of $\mathrm{CO}_{2}$ eq. $\left(80 \mathrm{~kg}\right.$ of $\mathrm{CO}_{2}$ eq. $\left./ \mathrm{hr}^{2} \mathrm{yr}\right)$ 


\section{Cold-Formed Steel Stud Wall \#3 (SS-W3)}

Building Component Description:

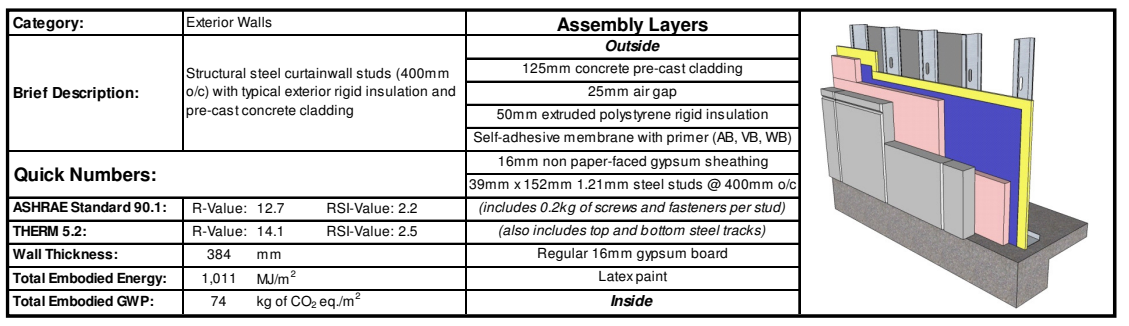

Life-Cycle Assessment Results:

Primary Energy Consumption (MJ)

\begin{tabular}{|c|c|c|c|c|c|c|c|c|c|c|c|c|c|c|c|c|}
\hline \multirow{3}{*}{$\mid \begin{array}{l}\text { Lifespan } \\
\text { (rears) }\end{array}$} & \multicolumn{14}{|c|}{ Embodied Energy (EE) } & \multirow{2}{*}{\multicolumn{2}{|c|}{$\begin{array}{c}\text { Difference in } \\
\text { Operating Energy } \\
\text { from Baseline after } \\
\text { Lifespan }\end{array}$}} \\
\hline & \multicolumn{3}{|c|}{ Manufacturing } & \multicolumn{3}{|c|}{ Construction } & \multicolumn{3}{|c|}{ Maintenance } & \multicolumn{3}{|c|}{ End of Life } & \multirow{2}{*}{$\begin{array}{l}{ }^{3} \text { Total } \\
\mathrm{EE}\end{array}$} & \multirow{2}{*}{$\begin{array}{c}{ }^{4} \text { Total } \\
\mathrm{EE} \\
\mathrm{perm}^{2}\end{array}$} & & \\
\hline & \begin{tabular}{|l|l|l|} 
Materia \\
\end{tabular} & ${ }^{2}$ Trans. & Total & \begin{tabular}{|l|l|} 
Material \\
\end{tabular} & \begin{tabular}{|l}
${ }^{2}$ Trans. \\
\end{tabular} & Total & Material & \begin{tabular}{|l|}
${ }^{2}$ Trans. \\
\end{tabular} & Total & \begin{tabular}{|l|} 
Material \\
\end{tabular} & ${ }^{2}$ Trans. & Total & & & ${ }^{5}$ Total & ${ }^{6}$ perm ${ }^{2}$ \\
\hline Initial & 45,416 & 866 & 46,282 & \begin{tabular}{|l|}
160 \\
\end{tabular} & \begin{tabular}{|l|l|}
1,314 \\
\end{tabular} & 1,474 & 0 & 0 & 0 & 0 & 0 & 0 & 47,756 & \begin{tabular}{|l|}
938 \\
\end{tabular} & & \\
\hline 50 & 45,416 & 866 & 46,282 & 160 & 1,314 & 1,474 & \begin{tabular}{|l}
3,099 \\
\end{tabular} & 10 & 3,110 & 1 & 613 & 614 & 51,479 & \begin{tabular}{|l|l|}
1,011 \\
\end{tabular} & 300,000 & 516 \\
\hline
\end{tabular}

Global Warming Potential ( $\mathrm{kg}$ of $\mathrm{CO}_{2}$ eq.)

\begin{tabular}{|c|c|c|c|c|c|c|c|c|c|c|c|c|c|c|c|c|}
\hline \multirow{3}{*}{$\begin{array}{l}\text { Lifespan } \\
\text { (Years) }\end{array}$} & \multicolumn{14}{|c|}{ Embodied Global Warming Potential (GWP) } & \multirow{2}{*}{\multicolumn{2}{|c|}{\begin{tabular}{|c|} 
Difference in \\
Operating GWP from \\
Baseline after \\
Lifespan
\end{tabular}}} \\
\hline & \multicolumn{3}{|c|}{ Manufacturing } & \multicolumn{3}{|c|}{ Construction } & \multicolumn{3}{|c|}{ Maintenance } & \multicolumn{3}{|c|}{ End of Life } & \multirow{2}{*}{$\begin{array}{l}{ }^{3} \text { Total } \\
\text { GWP }\end{array}$} & \multirow{2}{*}{$\begin{array}{l}{ }^{4}{ }^{4} \text { otal } \\
\text { GWP } \\
\text { perm }\end{array}$} & & \\
\hline & Material & ${ }^{2}$ Trans. & Total & Material & Trans. & Total & Material & ${ }^{2}$ Trans. & Total & Material & $\mid{ }^{2}$ Trans. & Total & & & \begin{tabular}{|c|}
${ }^{5}$ Total \\
\end{tabular} & ${ }^{6} \mathrm{perm}^{2}$ \\
\hline Initial & 3,705 & 5 & 3,706 & 10 & 3 & 13 & 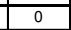 & 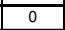 & 0 & 0 & 0 & 0 & 3,719 & 73 & & \\
\hline 50 & 3,705 & 2 & 3,706 & 10 & 3 & 13 & 44 & 0 & 44 & 0 & 1 & 1 & 3,764 & 74 & 20,000 & 34 \\
\hline
\end{tabular}

Net wall area of baseline retail building (gross wall area - openings) = $581.0 \mathrm{~m}^{2}$

ATHENA ® EIE Material List:

\begin{tabular}{|c|c|c|}
\hline Material List & Quantities & Unit \\
\hline 3 mil Polyethylene & 54.0 & $\mathrm{~m} 2$ \\
\hline $\begin{array}{l}16 \mathrm{~mm} \text { Moisture Resistant Gypsum } \\
\text { Board }\end{array}$ & 56.0 & $\mathrm{~m} 2$ \\
\hline 16mm Regular Gypsum Board & 56.0 & $\mathrm{~m} 2$ \\
\hline Concrete $30 \mathrm{MPa}$ (flyash av) & 6.7 & $\mathrm{~m} 3$ \\
\hline Extruded Polystyrene & 104.3 & m2 (25mm) \\
\hline Galvanized Studs & 342.5 & $\mathrm{~kg}$ \\
\hline Joint Compound & 111.8 & $\mathrm{~kg}$ \\
\hline Modified Bitumen membrane & 68.2 & $\mathrm{~kg}$ \\
\hline Nails & 4.2 & $\mathrm{~kg}$ \\
\hline $\begin{array}{l}\text { Paper Tape } \\
\end{array}$ & 1.3 & $\mathrm{~kg}$ \\
\hline Rebar, Rod, Light Sections & 404.0 & $\mathrm{~kg}$ \\
\hline Screws Nuts \& Bolts & 3.9 & $\mathrm{~kg}$ \\
\hline $\begin{array}{l}\text { Solvent Based Alkyd Paint } \\
\end{array}$ & 19.6 & 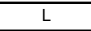 \\
\hline Water Based Latex Paint & 66.3 & $\mathrm{~L}$ \\
\hline
\end{tabular}

Notes:
${ }^{1}$ 'Initial = Time ' 0 ' (i.e. at the completion of nintial construction)

${ }^{2}$ Trans. $=$ Transportation

${ }^{3}$ Total EE (or Total GWP) $=$ Total embodied energy (or total embodied GWP) of building component after lifespan (i.e. total manu
construction + total maintenance + total end-oflifie effects)

${ }^{4}$ Total EE (or Total GWP) per $\mathrm{m}^{2}=$ Total EE (or Total GWP) of building
component/ area of building component that was modelled in ATHENAQ EEE ${ }^{5}$ Total Difference in Operating Energy (or GWP) from Baseline after
Lifespan $=$ The difference in the total life-cycle operating energy (or GWP) Lifespan = The difference in the total life-cycle operating energy (or GWP)
from the baseline retail builing after lifespan, due to using this building
component instead of the baseline component

${ }^{6}$ Total Difference in Operating Energy (or GWP) from Baseline after Lifespan per $\mathrm{m}^{2}=$ iotal difference in operating energy (or GWP) from

* Total operating primary energy use of baseline retail building after 50 years =
$50,700 \mathrm{G}\left(1,745 \mathrm{MJ} / \mathrm{m}^{2} / \mathrm{yr}\right)$

${ }^{*}$ Total operating GWP of baseline retain
of $\mathrm{CO}_{2}$ eq. $\left(80 \mathrm{~kg}\right.$ of $\left.\mathrm{CO}_{2} \mathrm{eq}, \mathrm{m} \mathrm{m}^{2} \mathrm{yr}\right)$

\section{Cold-Formed Steel Stud Wall \#4 (SS-W4)}

Building Component Description:

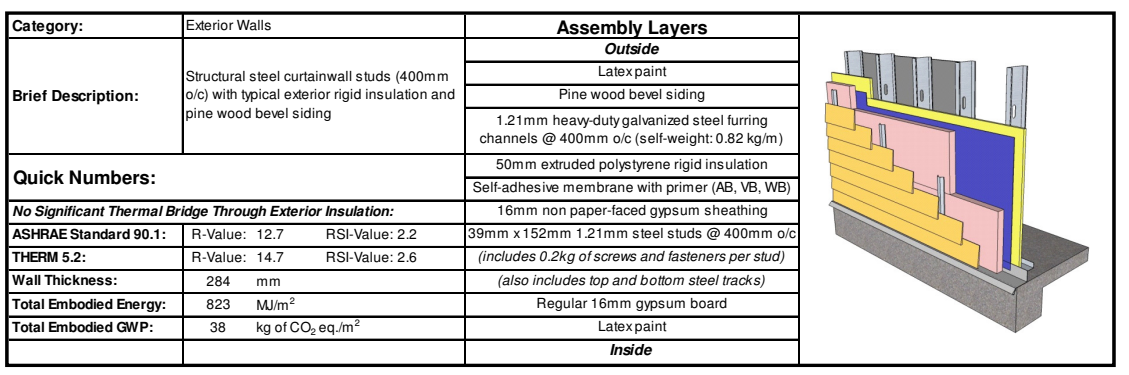

\section{Life-Cycle Assessment Results:}

\begin{tabular}{|c|c|c|c|c|c|c|c|c|c|c|c|c|c|c|c|c|}
\hline & & & & & & Primz & Ite & $y y<0$ & 100 & Jivit & & & & & & \\
\hline \multirow{3}{*}{ (Years) } & \multicolumn{14}{|c|}{ Embodied Energy (EE) } & \multirow{2}{*}{\multicolumn{2}{|c|}{$\begin{array}{l}\text { Difirenence in } \\
\text { Operating Energy } \\
\text { from Baseline aftel } \\
\text { Lifespan }\end{array}$}} \\
\hline & \multicolumn{3}{|c|}{ Manutacturing } & \multicolumn{3}{|c|}{ Construction } & \multicolumn{3}{|c|}{ Maintenance } & \multicolumn{3}{|c|}{ End of Life } & \multirow{2}{*}{$\mid \begin{array}{l}{ }^{3} \text { Total } \\
\mathrm{EE}\end{array}$} & \multirow{2}{*}{$\begin{array}{c}{ }^{4} \text { Total } \\
E \mathrm{E} \\
\mathrm{per} \mathrm{m}^{2}\end{array}$} & & \\
\hline & Material & [2Trans. & Total & Material & ${ }^{2}$ Trans. & Total & Material & Trans. & Total & Materia & Trans. & Total & & & ${ }^{5}$ Total & $6 \mathrm{per} \mathrm{m}^{2}$ \\
\hline itial & 37,461 & 293 & 37,754 & 160 & 724 & 884 & 0 & 0 & 0 & 0 & 0 & 0 & 38,638 & 759 & & \\
\hline & & & & & & & 100 & & 311 & & & 160 & & & 10,000 & \\
\hline
\end{tabular}

Global Warming Potential ( $k g$ of $\mathrm{CO}_{2}$ eq.)

\begin{tabular}{|c|c|c|c|c|c|c|c|c|c|c|c|c|c|c|c|c|}
\hline \multirow{3}{*}{$\begin{array}{l}\text { Litespan } \\
\text { (Years) }\end{array}$} & \multicolumn{14}{|c|}{ Embodied Global Warming Potential (GWP) } & \multirow{2}{*}{\multicolumn{2}{|c|}{\begin{tabular}{|c} 
Difference in \\
Operating GWPP from \\
Baseline after \\
Lifespan
\end{tabular}}} \\
\hline & \multicolumn{3}{|c|}{ Manufacturing } & \multicolumn{3}{|c|}{ Construction } & \multicolumn{3}{|c|}{ Maintenance } & \multicolumn{3}{|c|}{ End of Life } & \multirow{2}{*}{$\begin{array}{l}{ }^{3} \text { Total } \\
\text { GWP }\end{array}$} & \multirow{2}{*}{$\begin{array}{l}{ }^{4} \text { Total } \\
\text { GWP } \\
\text { per m }\end{array}$} & & \\
\hline & Material & ${ }^{2}$ Trans. & Total & Material & ${ }^{2}$ Trans. & Total & Material & ${ }^{2}$ Trans & Total & Material & ${ }^{2}$ Trans. & Total & & & ${ }^{5}$ Total & ${ }^{6}$ perm ${ }^{2}$ \\
\hline nitial & 1,880 & 1 & 1,880 & 10 & 1 & 12 & 0 & 0 & 0 & 0 & 0 & 0 & 1,892 & 37 & & \\
\hline 5 & 1,880 & 1 & 1,880 & 10 & 1 & 12 & 44 & 0 & 44 & 0 & 0 & 0 & 1,936 & 38 & 30,000 & 5 \\
\hline
\end{tabular}

Embodied energy (and GWP) numbers are based on an area of wall $=50.9 \mathrm{~m}^{2} \quad$ (Length $x$ Height $=7.6 \mathrm{~m} \times 6.7 \mathrm{~m}=50.9 \mathrm{~m}^{2}$ )

ATHENA ${ }^{\circledR}$ EIE Material List:

(Includes all materials after 50 years)

\begin{tabular}{|l|c|c|}
\hline \multicolumn{1}{|c|}{ Material List } & Quantities & Unit \\
\hline 3 mil Polyythylene & 54.0 & $\mathrm{~m} 2$ \\
\hline $\begin{array}{l}\text { 16mm Moisture Resistant Gypsum } \\
\text { Board }\end{array}$ & 56.0 & $\mathrm{~m} 2$ \\
\hline $16 \mathrm{~mm}$ Regular Gypsum Board & 56.0 & $\mathrm{~m} 2$ \\
\hline Extruded Polystyrene & 104.3 & $\mathrm{~m} 2(25 \mathrm{~mm})$ \\
\hline Galvanized Sheet & 106.1 & $\mathrm{~kg}$ \\
\hline Galvanized Studs & 342.5 & $\mathrm{~kg}$ \\
\hline Joint Compound & 111.8 & $\mathrm{~kg}$ \\
\hline Modified Bitumen membrane & 68.2 & $\mathrm{~kg}$ \\
\hline Nails & 5.5 & $\mathrm{~kg}$ \\
\hline Paper Tape & 1.3 & $\mathrm{~kg}$ \\
\hline Pine Wood Bevel Siding & 160.4 & $\mathrm{~m} 2$ \\
\hline Screws Nuts \& Bolts & 3.9 & $\mathrm{~kg}$ \\
\hline Solvent Based Alkyd Paint & 19.6 & $\mathrm{~L}$ \\
\hline Water Based Latex Paint & 132.5 & $\mathrm{~L}$ \\
\hline
\end{tabular}

Notes:
1 Initial $=$ Time
0

'Initial $=$ Time ' $O$ ' (i.e. at the

${ }^{3}$ Total EE (or Total GWP) $=$ Total embodied energy (or total embodied GWP)

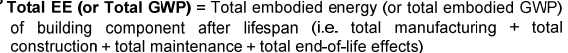
construction + total maintenance + total enc-of-life effects)
${ }^{4}$ Total EE (or Total GWP) per $\mathrm{m}^{2}=$ Total EE (or Total GWP) of building
component/ area of building component that was modelled in ATHENAQ EIE ${ }^{5}$ Total Differe in Opering Energ (or GWP) from Boseline after) Total Difference in Operating Energy (or GWP) from Baseline after
Lifespan = The difference in the total life cycle operating energy (or GWW)
from the baseline retail building after lifespan, due to using this building from the baseline retail building after lifespan, due to using this building
component instead of the baseline component

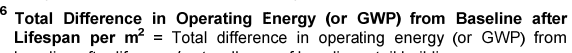
baseline after lifespan / net wall area of baseline retail building
*Total operating primary enengy use of baseline retail building after 50 years $=$
$50,700 \mathrm{GJ}\left(1,745 \mathrm{MJ} / \mathrm{m}^{2} \mathrm{y} / \mathrm{r}\right)$ "Total operating $G W$ P of baseline retail building affer 50 years $=2,310$ tonnes
of $\mathrm{CO}_{2} e q$. $80 \mathrm{Kg}$ of $\left.\mathrm{CO}_{2} e q / \mathrm{m}^{2} / \mathrm{yr}\right)$ 


\section{Cold-Formed Steel Stud Wall \#5 (SS-W5)}

Building Component Description:

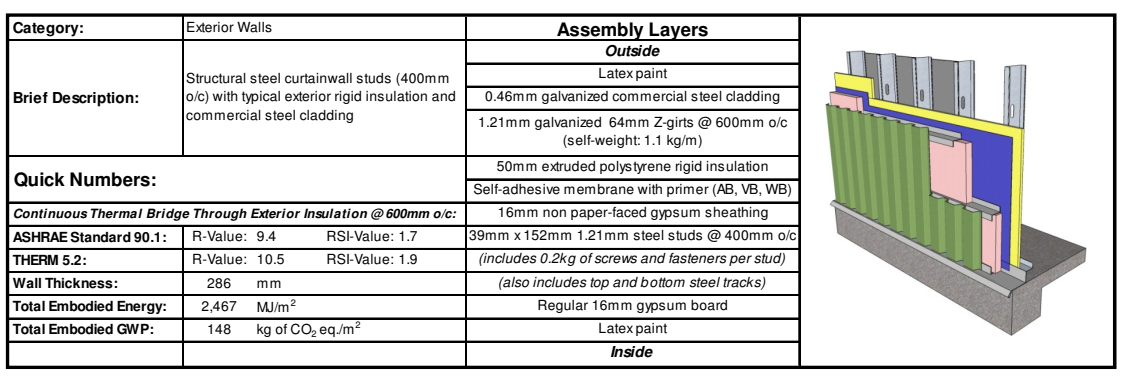

Life-Cycle Assessment Results:

Primary Energy Consumption (MJ)

\begin{tabular}{|c|c|c|c|c|c|c|c|c|c|c|c|c|c|c|c|c|}
\hline \multirow{3}{*}{$\begin{array}{l}\text { itespan } \\
\text { (Years) }\end{array}$} & \multicolumn{14}{|c|}{ Embodic } & \multirow{2}{*}{\multicolumn{2}{|c|}{$\begin{array}{c}\text { Difference in } \\
\text { Operating Energy } \\
\text { from Baseline afte } \\
\text { Lifespan }\end{array}$}} \\
\hline & \multicolumn{3}{|c|}{ Manutacturing } & \multicolumn{3}{|c|}{ Construction } & \multicolumn{3}{|c|}{ Maintenance } & \multicolumn{3}{|c|}{ End of Life } & \multirow{2}{*}{$\begin{array}{c}{ }^{3} \text { Total } \\
\mathrm{EE}\end{array}$} & \multirow{2}{*}{\begin{tabular}{|c}
${ }^{4}$ Total \\
$\mathrm{EE}$ \\
per m
\end{tabular}} & & \\
\hline & al & $2^{2}$ & Total & erial & Trans. & Total & \begin{tabular}{|l|} 
Material \\
\end{tabular} & ${ }^{2}$ Trans. & \begin{tabular}{|l|l|} 
Total \\
\end{tabular} & Material & \begin{tabular}{|l|l|}
${ }^{2}$ Trans. \\
\end{tabular} & \begin{tabular}{|l|l|} 
Total \\
\end{tabular} & & & ${ }^{5}$ Total & ${ }^{6}$ perm $\mathrm{m}^{2}$ \\
\hline itial & 11 & 246 & 121,55 & 160 & 624 & 784 & 0 & 0 & 0 & 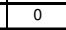 & I & 0 & & 2,403 & & \\
\hline & 311 & 246 & & & 024 & 184 & & & 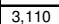 & & & 163 & & & & \\
\hline
\end{tabular}

Global Warming Potential ( $k g$ of $\mathrm{CO}_{2}$ eq.)

\begin{tabular}{|c|c|c|c|c|c|c|c|}
\hline \multirow{3}{*}{$\begin{array}{l}\text { Lifespan } \\
\text { (Years) }\end{array}$} & \multicolumn{7}{|c|}{ Embodied Global } \\
\hline & \multicolumn{3}{|c|}{ Manufacturing } & \multicolumn{3}{|c|}{ Construction } & \\
\hline & Material & Trans. & & Material & Trans. & \begin{tabular}{|l|l|l|} 
Total & \\
\end{tabular} & \\
\hline Initial & 7,466 & 0 & 7,466 & 10 & 1 & 12 & \\
\hline 50 & 7,466 & 0 & 7,466 & 10 & 1 & 12 & \\
\hline \multicolumn{8}{|c|}{$\begin{array}{l}\text { Embodied energy (and GWP) numbers are based on an area of wall } \\
\text { Net wall area of baseline retail building (gross wall area - openings) }\end{array}$} \\
\hline \multicolumn{8}{|c|}{$\begin{array}{l}\text { ATHENA @ EIE Material List: } \\
\text { (Includes all materials after } 50 \text { years) }\end{array}$} \\
\hline \multicolumn{4}{|c|}{ Material List } & \multicolumn{2}{|c|}{ Quantities } & \multicolumn{2}{|c|}{ Unit } \\
\hline \multicolumn{4}{|c|}{3 mil Polyethylene } & \multicolumn{2}{|r|}{54.0} & \multicolumn{2}{|c|}{$\mathrm{m} 2$} \\
\hline \multicolumn{4}{|c|}{$\begin{array}{l}\text { 16mm Moisture Resistant Gypsum } \\
\text { Board }\end{array}$} & & 56.0 & \multicolumn{2}{|c|}{$\mathrm{m} 2$} \\
\hline \multicolumn{4}{|c|}{$16 \mathrm{~mm}$ Regular Gypsum Board } & & 56.0 & \multicolumn{2}{|c|}{$\mathrm{m} 2$} \\
\hline \multicolumn{4}{|c|}{ Commercial $0.46 \mathrm{~mm}$ Steel Cladding } & & 168.0 & \multirow{2}{*}{\multicolumn{2}{|c|}{$\mathrm{m} 2$}} \\
\hline \multicolumn{4}{|c|}{ Extruded Polystyrene } & & 104.3 & & $\mathrm{~m} 2225 \mathrm{~mm}$ \\
\hline \multicolumn{4}{|c|}{ Galvanized Studs } & & 443.5 & \multicolumn{2}{|c|}{$\mathrm{kg}$} \\
\hline \multicolumn{4}{|l|}{ Join } & & 111.8 & \multicolumn{2}{|c|}{$\mathrm{kg}$} \\
\hline \multicolumn{4}{|c|}{ Modified Bitumen membrane } & & 68.2 & \multirow{2}{*}{\multicolumn{2}{|c|}{$\mathrm{kg}$}} \\
\hline \multicolumn{4}{|l|}{ Nails } & & & & \\
\hline \multicolumn{4}{|c|}{ Paper Tape } & & 4.2 & \multicolumn{2}{|c|}{$\mathrm{kg}$} \\
\hline \multicolumn{4}{|c|}{ Screws Nuts \& Bolts } & & 1.3 & \multicolumn{2}{|c|}{ ng } \\
\hline \multicolumn{4}{|c|}{ Solvent Based Alkyd Paint } & & 5.2 & \multirow{2}{*}{\multicolumn{2}{|c|}{2}} \\
\hline Water $\mathrm{B}$ & Based La & tex Pain & & & 1325 & & \\
\hline
\end{tabular}

\section{Cold-Formed Steel Stud Wall \#6 (SS-W6)}

Building Component Description:

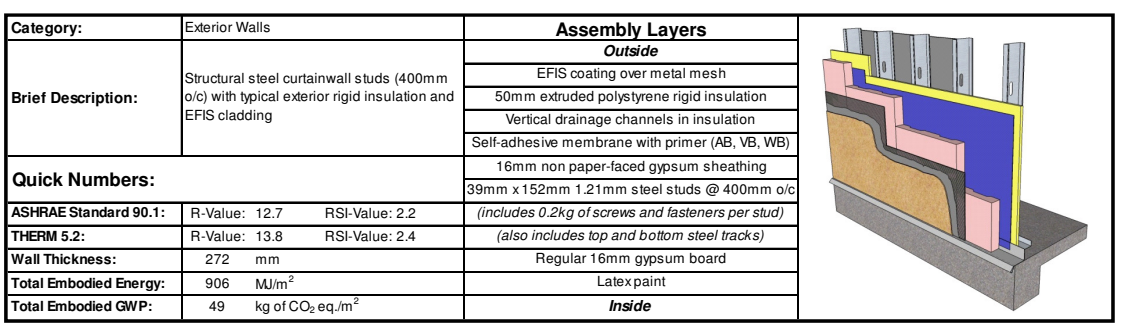

Life-Cycle Assessment Results:

Primary Energy Consumption (MJ)

\begin{tabular}{|c|c|c|c|c|c|c|c|c|c|c|c|c|c|c|c|c|}
\hline \multirow{3}{*}{$\begin{array}{l}\text { Lifespan } \\
\text { (Years) }\end{array}$} & \multicolumn{14}{|c|}{ Embodied Energy (EE) } & \multirow{2}{*}{\multicolumn{2}{|c|}{$\begin{array}{c}\text { Difference in } \\
\text { Operating Energy } \\
\text { from Baseline after } \\
\text { Lifespan }\end{array}$}} \\
\hline & \multicolumn{3}{|c|}{ Manufacturing } & \multicolumn{3}{|c|}{ Construction } & \multicolumn{3}{|c|}{ Maintenance } & \multicolumn{3}{|c|}{ End of Life } & \multirow{2}{*}{$\begin{array}{c}{ }^{3} \text { Total } \\
\mathrm{EE}\end{array}$} & \multirow{2}{*}{$\begin{array}{l}{ }^{4} \text { Total } \\
\mathrm{EE} \\
\text { per m }\end{array}$} & & \\
\hline & Material & ${ }^{2}$ Trans. & Total & Material & $\begin{array}{l}{ }^{2} \text { Trans. } \\
\end{array}$ & Total & Material & $\begin{array}{l}{ }^{2} \text { Trans. } \\
\end{array}$ & \begin{tabular}{|l|} 
Total \\
\end{tabular} & Material & \begin{tabular}{|l|}
${ }^{2}$ Trans. \\
\end{tabular} & Total & & & ${ }^{5}$ Total & ${ }^{6} \mathrm{perm}^{2}$ \\
\hline Initial & 41,328 & 301 & \begin{tabular}{|l|l|}
41,628 \\
\end{tabular} & 160 & \begin{tabular}{|l|}
717 \\
\end{tabular} & 876 & 0 & 0 & 0 & 0 & 0 & 0 & 42,505 & 835 & & \\
\hline 50 & 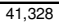 & 301 & 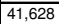 & 160 & 717 & 876 & 3,099 & 10 & 3,110 & 0 & 519 & 519 & 46,133 & 906 & 600,000 & 1,03 \\
\hline
\end{tabular}

Global Warming Potential (kg of $\mathrm{CO}_{2}$ eq.)

\begin{tabular}{|c|c|c|c|c|c|c|c|c|c|c|c|c|c|c|c|c|}
\hline \multirow{3}{*}{$\begin{array}{l}\text { Lifespan } \\
\text { (Years) }\end{array}$} & \multicolumn{14}{|c|}{ Embodied Global Warming Potential (GWP) } & \multirow{2}{*}{\multicolumn{2}{|c|}{$\begin{array}{l}\text { Difference in } \\
\text { Operating GWP fron } \\
\text { Baseline after } \\
\text { Lifespan } \\
\end{array}$}} \\
\hline & \multicolumn{3}{|c|}{ Manutacturing } & \multicolumn{3}{|c|}{ Construction } & \multicolumn{3}{|c|}{ Maintenance } & \multicolumn{3}{|c|}{ End of Life } & \multirow{2}{*}{$\begin{array}{l}{ }^{3} \text { Total } \\
\text { GWP }\end{array}$} & \multirow{2}{*}{$\begin{array}{l}{ }^{4}{ }^{4} \text { Total } \\
\text { GWP } \\
\text { per m }\end{array}$} & & \\
\hline & Material & ${ }^{2}$ Trans. & Total & Material & ${ }^{2}$ Trans. & Total & Material & ${ }^{2}$ Trans. & Total & Material & $\mid{ }^{2}$ Trans. & Total & & & ${ }^{5}$ Total & ${ }^{6}$ per m$^{2}$ \\
\hline${ }^{1}$ Initial & 2,425 & 1 & 2,426 & 10 & 1 & 12 & 0 & 0 & 0 & 0 & 0 & 0 & 2,438 & 48 & & \\
\hline 50 & 2,425 & 1 & 2,426 & 10 & 1 & 12 & 44 & 0 & 44 & 0 & 1 & 1 & 2,482 & 49 & 40,000 & 69 \\
\hline
\end{tabular}

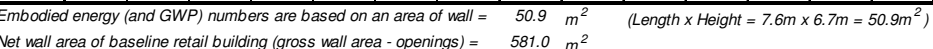

\begin{tabular}{|c|c|c|}
\hline Material List & Quantities & Unit \\
\hline \#15 Organic Felt & 219.4 & $\mathrm{~m} 2$ \\
\hline 3 mil Polyethylene & 54.0 & $\mathrm{~m} 2$ \\
\hline $\begin{array}{l}16 \mathrm{~mm} \text { Moisture Resistant Gypsum } \\
\text { Board }\end{array}$ & 56.0 & $\mathrm{~m} 2$ \\
\hline $16 \mathrm{~mm}$ Regular Gypsum Board & 56.0 & $\mathrm{~m} 2$ \\
\hline Extruded Polystyrene & 104.3 & $\mathrm{~m} 2(25 \mathrm{~mm}$ \\
\hline Galvanized Sheet & 51.4 & $\mathrm{~kg}$ \\
\hline Galvanized Studs & 342.5 & $\mathrm{~kg}$ \\
\hline Joint Compound & 111.8 & $\mathrm{~kg}$ \\
\hline Modified Bitumen membrane & 68.2 & $\mathrm{~kg}$ \\
\hline Nails & 5.7 & $\mathrm{~kg}$ \\
\hline Paper Tape & 1.3 & $\mathrm{~kg}$ \\
\hline Screws Nuts \& Bolts & 3.9 & $\mathrm{~kg}$ \\
\hline Solvent Based Alkyd Paint & 19.6 & $\mathrm{~L}$ \\
\hline Stucco over metal mesh & 136.0 & $\mathrm{~m} 2$ \\
\hline Water Based Latex Paint & 132.5 & $\bar{L}$ \\
\hline
\end{tabular}

Notes:
1 Initial = Time 'O' (i.e. at the completion of initial construction)

${ }^{2}$ Trans. = Transportation

${ }^{3}$ Total EE (or Total GWP) $=$ Total embodied energy (or total embodied GWP) of building component after lifespan (i.e. total man
construction + total maintenance + total end-of-life effects)

${ }^{4}$ Total EE (or Total GWP) per $\mathrm{m}^{2}=$ Total EE (or Total GWP) of building
component / area of building component that was modelled in ATHENAQ EIE ${ }^{5}$ Total Difference in Operating Energy (or GWP) from Baseline after Litespan = The difference in the total life-cycle operating energy (or GWP
from the baseline retail building after lifespan, due to using this building Derating Energy (or GWP) from Baseline after

Total operating primary energy use of baseline retail building after 50 years =

of $\mathrm{CO}_{2}$ eq, $\left(80 \mathrm{~kg}\right.$ of $\mathrm{CO}_{2}$ eq. $\left./ \mathrm{m}^{2} \mathrm{yr}\right)$

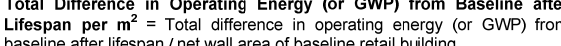




\section{Cold-Formed Steel Stud Wall \#7 (SS-W7)}

Building Component Description:
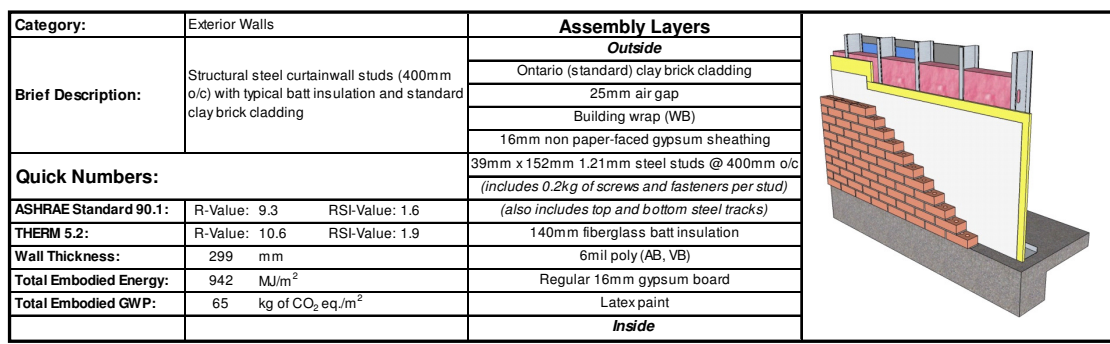

\section{Life-Cycle Assessment Results:}

Primary Energy Consumption (MJ)

\begin{tabular}{|c|c|c|c|c|c|c|c|c|c|c|c|c|c|c|c|c|}
\hline \multirow{3}{*}{\begin{tabular}{|c} 
Lifespan \\
(Years)
\end{tabular}} & \multicolumn{14}{|c|}{ Embodied Energy (EE) } & \multirow{2}{*}{\multicolumn{2}{|c|}{$\begin{array}{c}\text { Difference in } \\
\text { Operating Energy } \\
\text { from Baseline after } \\
\text { Lifespan }\end{array}$}} \\
\hline & \multicolumn{3}{|c|}{ Manufactu } & \multicolumn{3}{|c|}{ Constru } & \multicolumn{3}{|c|}{ Maintenance } & \multicolumn{3}{|c|}{ End of 1} & \multirow{2}{*}{$\begin{array}{c}{ }^{3}{ }^{\text {Total }} \\
\mathrm{EE}\end{array}$} & \multirow{2}{*}{$\begin{array}{c}{ }^{4} \text { Total } \\
\text { EE } \\
\text { per m}\end{array}$} & & \\
\hline & Materia & ${ }^{2}$ Trans. & Total & Materia & ${ }^{2}$ Trans. & Total & Material & \begin{tabular}{|l|}
${ }^{2}$ Trans. \\
\end{tabular} & Total & Material & \begin{tabular}{|l|}
${ }^{2}$ Trans. \\
\end{tabular} & \begin{tabular}{|l|l|l|} 
Total \\
\end{tabular} & & & ${ }^{5}$ Total & ${ }^{6}$ per $\mathrm{m}^{2}$ \\
\hline Initial & 45,549 & 301 & 45,850 & 160 & 1,373 & 1,533 & 0 & 0 & 0 & 0 & 0 & 0 & 47,383 & 931 & & \\
\hline 50 & 4 & 301 & 45,850 & 160 & 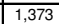 & 1,533 & 0 & 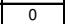 & 0 & 0 & 583 & \begin{tabular}{|l|l|}
5883 \\
\end{tabular} & \begin{tabular}{|l|l}
447,967 \\
\end{tabular} & 942 & $1,500,000$ & 2.582 \\
\hline
\end{tabular}

N

Global Warming Potential ( $k g$ of $\mathrm{CO}_{2}$ eq.)

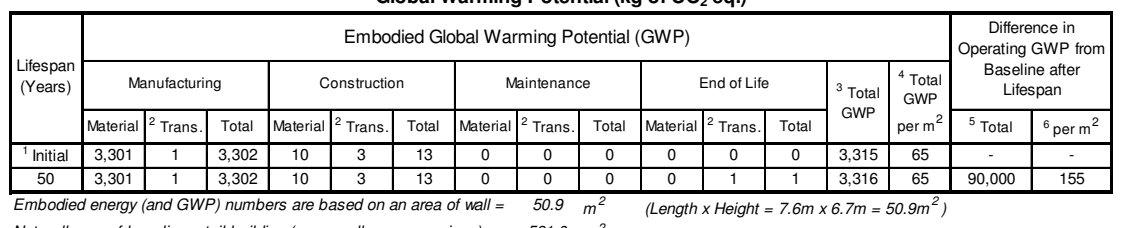

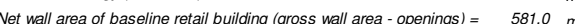

\section{ATHENA $®$ EIE Material List:}

\begin{tabular}{|c|c|c|}
\hline Material List & Quantities & Unit \\
\hline $\begin{array}{l}16 \mathrm{~mm} \text { Moisture Resistant Gypsum } \\
\text { Board }\end{array}$ & 56.0 & $\mathrm{~m} 2$ \\
\hline 16mm Regular Gypsum Board & 56.0 & $\mathrm{~m} 2$ \\
\hline 6 mil Polyethylene & 108.0 & $\mathrm{~m} 2$ \\
\hline Batt. Fiberglass & 289.8 & $\mathrm{~m} 2(25 \mathrm{~mm})$ \\
\hline Cold Rolled Sheet & 10.3 & $\mathrm{~kg}$ \\
\hline Galvanized Studs & 342.5 & $\mathrm{~kg}$ \\
\hline Joint Compound & $\begin{array}{l}111.8 \\
\end{array}$ & $\mathrm{~kg}$ \\
\hline Mortar & 1.5 & $\mathrm{~m} 3$ \\
\hline Nails & 4.2 & $\mathrm{~kg}$ \\
\hline Ontario (Standard) Brick & 53.5 & $\mathrm{~m} 2$ \\
\hline Paper Tape & 1.3 & $\mathrm{~kg}$ \\
\hline Screws Nuts \& Bolts & 3.9 & $\mathrm{~kg}$ \\
\hline
\end{tabular}

Notes:
${ }^{1}$ Initial $=$ Time 'o' (i.e. at the completion of initial construction)

${ }^{2}$ Trans. $=$ Transportation

Total EE (or Total GWP) = Total embodied energy (or total embodied GWP of building component after lifespan (i.e. total manu
construction + total maintenance + total enc-oflife effects)

${ }^{4}$ Total EE (or Total GWP) per $\mathrm{m}^{2}=$ Total EE (or Total GWP) of building
component/ area of building component that was modelled in ATHENAQ EIE Total Difference in Operating Energy (or GWP) from Baseline after (t) from the baseline retail building after lifespat
component instead of the baseline component

Total Difference in Operating Energy (or GWP) from Baseline after Lifespan per $\mathrm{m}^{2}=$ Total difference in operating energy (or GWP) from

"Total operating primary energy use of baseline retail building after 50 years =
$50.700 \mathrm{GJ}\left(1,745 \mathrm{MJ} / \mathrm{m}^{2} \mathrm{yr}\right)$

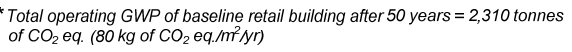

\section{Cold-Formed Steel Stud Wall \#8 (SS-W8)}

Building Component Description:

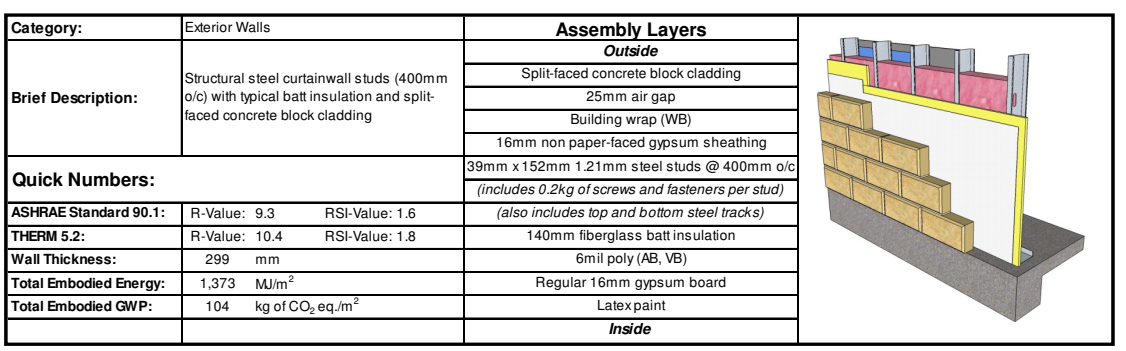

Life-Cycle Assessment Results:

Primary Energy Consumption (MJ)

\begin{tabular}{|c|c|c|c|c|c|c|c|c|c|c|c|c|c|c|c|c|c|}
\hline \multirow{3}{*}{\begin{tabular}{|c|} 
Litespan \\
(Years)
\end{tabular}} & \multicolumn{14}{|c|}{ Embodied Energy (EE) } & \multirow{2}{*}{\multicolumn{3}{|c|}{$\begin{array}{c}\text { Difference in } \\
\text { Operating Energy } \\
\text { from Baseline after } \\
\text { Lifespan }\end{array}$}} \\
\hline & \multicolumn{3}{|c|}{ Manufacturing } & \multicolumn{3}{|c|}{ Construction } & \multicolumn{3}{|c|}{ Maintenance } & \multicolumn{3}{|c|}{ End of Life } & \multirow{2}{*}{$\begin{array}{c}{ }^{3} \text { Total } \\
\text { EE }\end{array}$} & \multirow{2}{*}{$\begin{array}{c}{ }^{4}{ }^{\text {Totata }} \\
\mathrm{EE}\end{array}$} & & & \\
\hline & Material & $\left.\right|^{2}$ Trans. & Total & Material & ${ }^{2}$ Trans. & Total & Material & ${ }^{2}$ Trans. & Total & Material & $\begin{array}{l}2^{2} \text { Trans. } \\
\text { T. }\end{array}$ & Total & & & & & ${ }^{6}$ perm ${ }^{2}$ \\
\hline & & 864 & 65,441 & 0 & 2,184 & 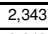 & 0 & 0 & 0 & 0 & 0 & 0 & 8. & $1,33^{1}$ & & & \\
\hline
\end{tabular}

Global Warming Potential (kg of $\mathrm{CO}_{2}$ eq.

\begin{tabular}{|c|c|c|c|c|c|c|c|c|c|c|c|c|c|c|c|c|}
\hline \multirow{3}{*}{$\begin{array}{l}\text { Lifespan } \\
\text { (Years) }\end{array}$} & \multicolumn{14}{|c|}{ Embodied Global Warming Potential (GWP) } & \multirow{2}{*}{\multicolumn{2}{|c|}{$\begin{array}{l}\text { Difference in } \\
\text { Operating GWP from } \\
\text { Baseline after } \\
\text { Lifespan }\end{array}$}} \\
\hline & \multicolumn{3}{|c|}{ nutacturing } & \multicolumn{3}{|c|}{ Construction } & \multicolumn{3}{|c|}{ Maintenance } & \multicolumn{3}{|c|}{ End of Life } & \multirow{2}{*}{$\begin{array}{l}{ }^{3} \text { Total } \\
\text { GWP }\end{array}$} & \multirow{2}{*}{$\begin{array}{c}{ }^{4} \text { Total } \\
\text { GWP } \\
\text { per } \mathrm{m}^{2}\end{array}$} & & \\
\hline & Material & ${ }^{2}$ Trans. & Total & Material & ${ }^{2}$ Trans. & Total & Material & ${ }^{2}$ Trans. & Total & Material & 1. 2 Trans. & Total & & & ${ }^{5}$ Total & ${ }_{6}^{6} \mathrm{perm}^{2}$ \\
\hline Initial & 5,287 & 2 & 5,289 & 10 & 4 & 15 & 0 & 0 & 0 & 0 & 0 & 0 & 3 & 104 & & \\
\hline 50 & \begin{tabular}{|l|l|}
5,287 \\
\end{tabular} & 2 & 5,589 & 10 & 4 & \begin{tabular}{l|l}
15 \\
\end{tabular} & 0 & 0 & 0 & 0 & 4 & 4 & \begin{tabular}{|l|l|l}
5,308 \\
\end{tabular} & $\begin{array}{ll}104 \\
\end{array}$ & 90,000 & 155 \\
\hline
\end{tabular}

Net wall a

ATHENA $®$ EIE Material List:

\begin{tabular}{|l|c|c|}
\multicolumn{1}{|c|}{ Materiales all materials after 50 years) } & Quantities & Unit \\
\hline $\begin{array}{l}\text { 16mm Moisture Resistant Gypsum } \\
\text { Board }\end{array}$ & 56.0 & $\mathrm{~m} 2$ \\
\hline 16mm Regular Gypsum Board & 56.0 & $\mathrm{~m} 2$ \\
\hline 6 mil Polyethylene & 108.0 & $\mathrm{~m} 2$ \\
\hline Batt. Fiberglass & 289.8 & $\mathrm{~m} 2(25 \mathrm{~mm})$ \\
\hline Cold Rolled Sheet & 10.3 & $\mathrm{~kg}$ \\
\hline Galvanized Studs & 342.5 & $\mathrm{~kg}$ \\
\hline Joint Compound & 111.8 & $\mathrm{~kg}$ \\
\hline Modified Bitumen membrane & 333.3 & $\mathrm{~kg}$ \\
\hline Mortar & 4.3 & $\mathrm{~m} 3$ \\
\hline Nails & 4.2 & $\mathrm{~kg}$ \\
\hline Paper Tape & 1.3 & $\mathrm{~kg}$ \\
\hline Screws Nuts \& Bolts & 3.9 & $\mathrm{~kg}$ \\
\hline Split-faced Concrete Block & $1,238.2$ & $\mathrm{Blocks}$ \\
\hline Water Based Latex Paint & 66.3 & $\mathrm{~L}$ \\
\hline
\end{tabular}

${ }^{2}$ Trans. $=$ Transportation

Total EE (or Total GWP) = Total embodied energy (or total embodied GWP) of building component after lifespan (i.e. total man
construction + total maintenance + total end-of-life effects) Total Difference in Operating Energy (or GWP) from Baseline after from the baseline retail building after lifespan, due to using this building component instead of the baseline component

Total Difference in Operating Energy (or GWP) from Baseline after Liffespan per $\mathrm{m}^{2}=$ Total difference in operating energy (or GWP) from

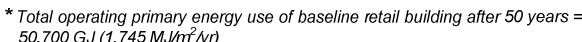

${ }^{2}$
of $\mathrm{CO}_{2}$ eq.
$\left(80 \mathrm{~kg}\right.$ of $\mathrm{CO}_{2}$ eq.
Notes:
'Initial = Time 'O' (i.e. at the completion of initial construction)

${ }^{4}$ Total EE (or Total GWP) per $\mathrm{m}^{2}=$ Total EE (or Total GWP) of building
component / area of building component that was modelled in ATHENAQ EIE 


\section{Cold-Formed Steel Stud Wall \#9 (SS-W9)}

Building Component Description:

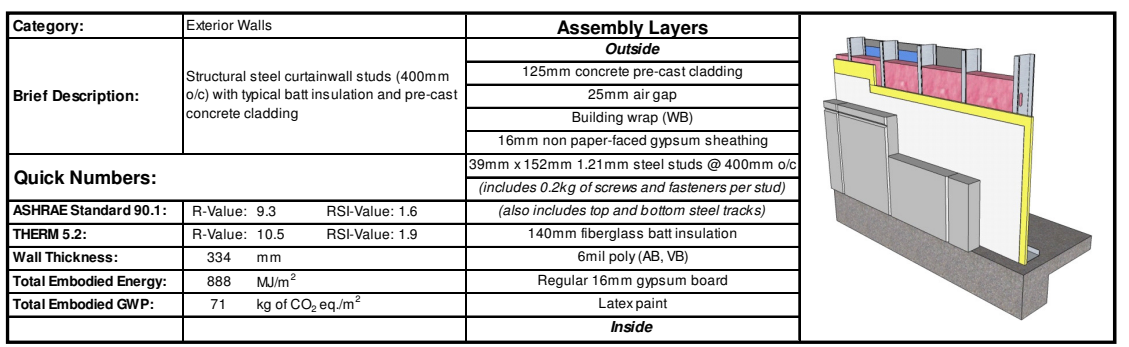

Life-Cycle Assessment Results:

Primary Energy Consumption (MJ)

\begin{tabular}{|c|c|c|c|c|c|c|c|c|c|c|c|c|c|c|c|c|}
\hline \multirow{3}{*}{$\begin{array}{l}\text { Lifespar } \\
\text { (Years) }\end{array}$} & \multicolumn{14}{|c|}{ Embodied Energy (EE) } & \multirow{2}{*}{\multicolumn{2}{|c|}{$\begin{array}{c}\text { Difference in } \\
\text { Operating Energy } \\
\text { from Baseline afte } \\
\text { Lifespan }\end{array}$}} \\
\hline & \multicolumn{3}{|c|}{ Manutacturing } & \multicolumn{3}{|c|}{ Construction } & \multicolumn{3}{|c|}{ Maintenance } & \multicolumn{3}{|c|}{ End of Life } & \multirow{2}{*}{$\begin{array}{c}{ }^{3} \text { Total } \\
\mathrm{EE}\end{array}$} & \multirow{2}{*}{$\begin{array}{l}{ }^{4} \text { Total } \\
\text { EE } \\
\text { per } \mathrm{m}^{2}\end{array}$} & & \\
\hline & Material & ${ }^{2}$ Trans. & \begin{tabular}{|l|} 
Total \\
\end{tabular} & \begin{tabular}{|l|} 
Materia \\
\end{tabular} & \begin{tabular}{|l|}
${ }^{2}$ Trans. \\
\end{tabular} & Total & \begin{tabular}{|l|} 
Material \\
\end{tabular} & \begin{tabular}{|l|}
${ }^{2}$ Trans. \\
\end{tabular} & \begin{tabular}{|l|} 
Total \\
\end{tabular} & Material & ${ }^{2}$ Trans. & \begin{tabular}{|l|l|} 
Total \\
\end{tabular} & & & ${ }^{5}$ Total & ${ }^{6}{ }^{6}$ per m ${ }^{2}$ \\
\hline 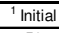 & 42,236 & \begin{tabular}{|l|l|}
892 \\
\end{tabular} & 43,128 & 160 & 1,313 & 1,472 & 0 & 0 & 0 & 0 & 0 & 0 & 44,600 & 876 & & \\
\hline 50 & \begin{tabular}{|l|l|l}
42,236 \\
\end{tabular} & 892 & 43,128 & 160 & 1,313 & 1,472 & 0 & 0 & 0 & 1 & 616 & 617 & 45,217 & \begin{tabular}{|l|l|}
888 \\
\end{tabular} & 500,000 & 2,582 \\
\hline
\end{tabular}

N

Global Warming Potential ( $k g$ of $\mathrm{CO}_{2}$ eq.)

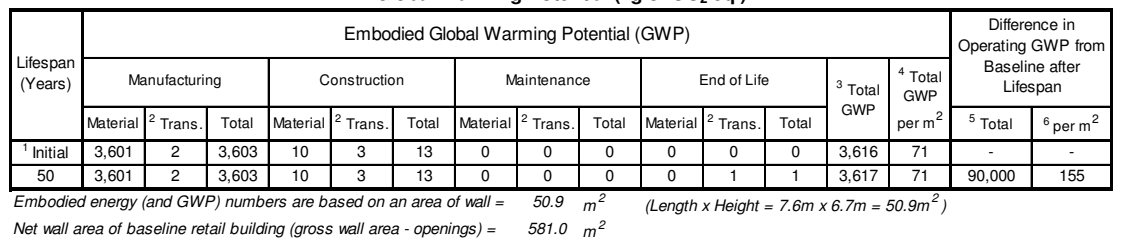

Net wall area of baseline retail building (gross wall area - openings) $=581.0$

ATHENA ® EIE Material List:

\begin{tabular}{|l|c|c|}
\hline \multicolumn{1}{|c|}{ Material List } & Quantities & Unit \\
\hline $\begin{array}{l}\text { 16mm Moisture Resistant Gypsum } \\
\text { Board }\end{array}$ & 56.0 & $\mathrm{~m} 2$ \\
\hline 16mm Regular Gypsum Board & 56.0 & $\mathrm{~m} 2$ \\
\hline 6 mil Polyuthylenpe & 108.0 & $\mathrm{~m} 2$ \\
\hline Batt. Fiberglass & 289.8 & $\mathrm{~m} 2(25 \mathrm{~mm})$ \\
\hline Concrete $30 \mathrm{MPa}$ (flyash av) & 6.7 & $\mathrm{~m} 3$ \\
\hline Galvanized Studs & 342.5 & $\mathrm{~kg}$ \\
\hline Joint Compound & 111.8 & $\mathrm{~kg}$ \\
\hline Nails & 4.2 & $\mathrm{~kg}$ \\
\hline Paper Tape & 1.3 & $\mathrm{~kg}$ \\
\hline Rebar, Rod, Light Sections & 404.0 & $\mathrm{~kg}$ \\
\hline Screws Nuts \& Bolts & 3.9 & $\mathrm{~kg}$ \\
\hline Water Based Latex Paint & 66.3 & $\mathrm{~L}$ \\
\hline
\end{tabular}

${ }^{1}$ Initial $=$ Time ' 0 ' (i.e. at the $\quad$ Notes

'Initial = Time 'o' (i.e. at the completion of initial construction)

${ }^{3}$ Total EE (or Total GWP) $=$ Total embodied energy (or total embodied GWP of building component after lifespan (i.e. total manu
construction + total maintenance + total end-of-life effects)

${ }^{4}$ Total EE (or Total GWP) per $\mathrm{m}^{2}=$ Total EE (or Total GWP) of building
component / area of building component that was modelled in ATHENAB EIE ${ }^{5}$ Total Difference in Operating Energy (or GWP) from Baseline after fifespan = The difierence in the total lific-cycle operating energy (or GWP)
from the baseline retail building after lifiespan, due to using this building component instead of the baseline component

Total Difference in Operating Energy (or GWP) from Baseline after
Lifespan per $\mathrm{m}^{2}=$ Total difference in operating energy (or GWP) from Lifespan per $m^{2}=$ Total difference in operating energy (or GWP) from
baseline after lifespan $/$ net wall area of baseline retail building

* Total operating primary energy use of baseline retail building after 50 years
$50,700 \mathrm{GJ}\left(1,745 \mathrm{MJ} / \mathrm{m}^{2} / \mathrm{yr}\right)$

"Total operating GWP of baseline reta
of $\mathrm{CO}_{2} \mathrm{eq}$. $\left(80 \mathrm{~kg}\right.$ of $\left.\mathrm{CO}_{2} e q . / \mathrm{m}^{2} \mathrm{yr}\right)$

\section{Cold-Formed Steel Stud Wall \#10 (SS-W10)}

Building Component Description:

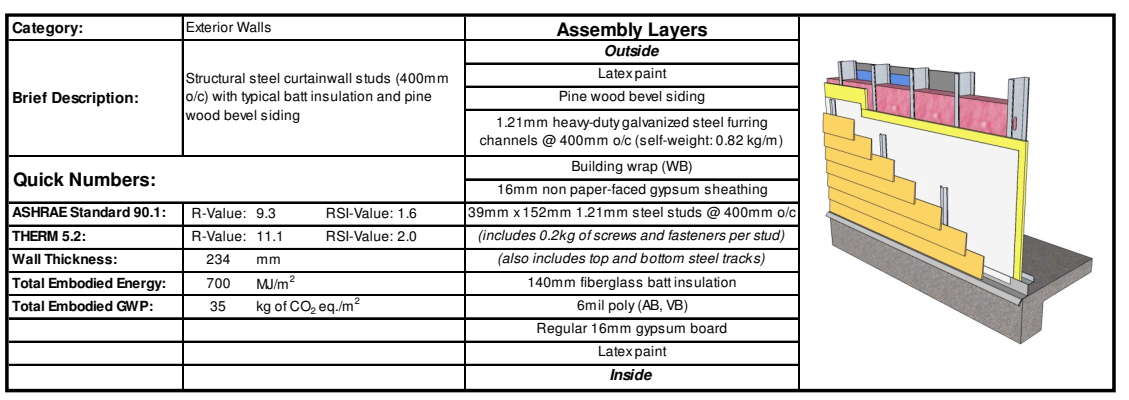

Life-Cycle Assessment Results:

\begin{tabular}{|c|c|c|c|c|c|c|c|c|c|c|c|c|c|c|c|c|}
\hline & \multirow{2}{*}{\multicolumn{14}{|c|}{$\begin{array}{l}\text { rimary Energy Consumption (MJ) } \\
\text { Embodied Energy (EE) }\end{array}$}} & & \\
\hline \multirow{3}{*}{$\begin{array}{l}\text { Lesespan } \\
\text { (Years) }\end{array}$} & & & & & & & & & & & & & & & \multirow{2}{*}{\multicolumn{2}{|c|}{$\begin{array}{l}\text { Difierence in } \\
\text { Operating Energy } \\
\text { from Baseline atter } \\
\text { Lifespan }\end{array}$}} \\
\hline & \multicolumn{3}{|c|}{ Manutacturing } & \multicolumn{3}{|c|}{ Construction } & \multicolumn{3}{|c|}{ Maintenance } & \multicolumn{3}{|c|}{ End of Life } & \multirow{2}{*}{$\begin{array}{c}{ }^{3} \text { Total } \\
\mathrm{EE}\end{array}$} & \multirow{2}{*}{$\left|\begin{array}{c}{ }^{4} \text { Total } \\
\text { EE } \\
\text { perm }\end{array}\right|$} & & \\
\hline & Material & ${ }^{2}$ Trans. & Total & \begin{tabular}{|l|l|} 
Material \\
\end{tabular} & al| ${ }^{2}$ Trans. & Total & \begin{tabular}{|l|} 
Material \\
\end{tabular} & $\begin{array}{l}1{ }^{1} \text { Trans. } \\
\text {. }\end{array}$ & Total & \begin{tabular}{|l|} 
Material \\
\end{tabular} & 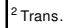 & \begin{tabular}{|l|l|} 
Total \\
\end{tabular} & & & 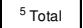 & ${ }^{6}$ perm $m^{2}$ \\
\hline |linitial & 34,281 & 319 & 34,600 & 160 & 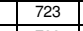 & 882 & 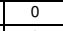 & 0 & 0 & 0 & & 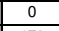 & 35,482 & 697 & & \\
\hline 50 & 34,281 & 319 & |34,600 & 160 & 723 & 882 & 0 & 0 & 0 & 0 & 172 & 173 & 35,655 & 700 & {$[1,500,00$} & 2,582 \\
\hline
\end{tabular}

Global Warming Potential (kg of $\mathrm{CO}_{2}$ eq.)

\begin{tabular}{|c|c|c|c|c|c|c|c|c|c|c|c|c|c|c|c|c|}
\hline & & & & & & bal & in & Poter & $(\mathbf{k}$ & $\mathrm{g}$ of $\mathrm{CO}_{2}$ & 2 eq.) & & & & & \\
\hline \multirow{3}{*}{$\begin{array}{l}\text { (Years) } \\
\text { (Leaph }\end{array}$} & \multicolumn{14}{|c|}{$\left(\mathrm{ng} 01 \mathrm{C}_{2}\right.$ eq. } & \multirow{2}{*}{\multicolumn{2}{|c|}{$\begin{array}{l}\text { Difference in } \\
\text { Operating GWP from } \\
\text { Baseline after } \\
\text { Lifespan }\end{array}$}} \\
\hline & \multicolumn{3}{|c|}{ Manutacturing } & \multicolumn{3}{|c|}{ Construction } & \multicolumn{3}{|c|}{ Maintenance } & \multicolumn{3}{|c|}{ End of L Life } & \multirow{2}{*}{$\mid \begin{array}{l}{ }^{3} \text { Total } \\
\text { GWP }\end{array}$} & \multirow{2}{*}{$\begin{array}{c}{ }^{4} \text { Total } \\
\text { GWP } \\
\text { per } \mathrm{m}^{2}\end{array}$} & & \\
\hline & \begin{tabular}{|l|l|} 
Material \\
\end{tabular} & 2 Trans. & Total & Material & $\mid{ }^{2}$ Trans & Total & Material & ${ }^{2}$ Trans. & Total & Material & 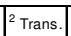 & Total & & & \begin{tabular}{|l|}
${ }^{5}$ Total \\
\end{tabular} & ${ }^{6} \operatorname{per} m^{2}$ \\
\hline |nitial & $1,7>$ & & & 10 & & 70 & 0 & & 0 & 0 & 0 & 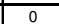 & & 35 & & \\
\hline 50 & $\begin{array}{l}1,776 \\
\end{array}$ & 1 & 1,777 & 10 & 1 & 12 & 0 & 0 & 0 & 0 & 0 & 0 & \begin{tabular}{|l|}
1,789 \\
\end{tabular} & 35 & 90,000 & 155 \\
\hline
\end{tabular}

Embodied energy (and GWP) numbers are based on an area of wall $=50.9 \mathrm{~m}^{2} \quad$ (Length $x$ Height $=7.6 \mathrm{~m} \times 6.7 \mathrm{~m}=50.9 \mathrm{~m}^{2}$ ) Net wall area of baseline retall building (gross wall area - openings) $=581.0 \mathrm{~m}^{2}$

ATHENA $®$ EIE Material List:

\begin{tabular}{|l|c|c|}
\hline \multicolumn{1}{|c|}{ Material List } & Quantities & Unit \\
\hline \begin{tabular}{l|c|} 
16mm Moisture Resistant Gypsum \\
Board
\end{tabular} & 56.0 & $\mathrm{~m} 2$ \\
\hline 16mm Regular Gypsum Board & 56.0 & $\mathrm{~m} 2$ \\
\hline 6 mil Polyethylene & 108.0 & $\mathrm{~m} 2$ \\
\hline Batt. Fiberglass & 289.8 & $\mathrm{~m} 2(25 \mathrm{~mm})$ \\
\hline Galvanized Sheet & 106.1 & $\mathrm{~kg}$ \\
\hline Galvanized Studs & 342.5 & $\mathrm{~kg}$ \\
\hline Joint Compound & 111.8 & $\mathrm{~kg}$ \\
\hline Nails & 5.5 & $\mathrm{~kg}$ \\
\hline Paper Tape & 1.3 & $\mathrm{~kg}$ \\
\hline Pine Wood Bevel Siding & 160.4 & $\mathrm{~m} 2$ \\
\hline Screws Nuts \& Bolts & 3.9 & $\mathrm{~kg}$ \\
\hline Water Based Latex Paint & 132.5 & $\mathrm{~L}$ \\
\hline
\end{tabular}

'Initial = Time 'o' (i.e. at the Notes:

${ }^{2}$ Trans. = Transportation

${ }^{3}$ Total EE (or Total GWP) $=$ Total embodied energy (or total embodied GWP of building component after lifespan (i.e. total manufacturing + tota
construction t total maintenance+ total end-of-life effects) ${ }^{4}$ Total EE (or Total GWP) per $\mathrm{m}^{2}=$ Total EE (or Total GWP) of building
component/ area of building component that was modelled in ATHENAB EIE Lifespan $=$ The difference in the total life-cycle operating energy (or GWP) from the baseline retail building after lifespan, due to using this building
component instead of the baseline component ${ }^{6}$ Total Difference in Operating Energy (or GWP) from Baseline after Total Difference in Operating Energy (or GWP) from Baseline after
Lifespan per $\mathrm{m}^{2}=$ Total difference in operating energy (or GWP) from
baseline after lifespan / $n$ et wall area of baseline retail building * Total operating primary energy use of baseline retail building after 50 years
$50,700 \mathrm{GJ}\left(1.745 \mathrm{MJ} / \mathrm{m}^{2} / \mathrm{yr}\right)$

$50,700 \mathrm{GJ}\left(1,745 \mathrm{MJ} / \mathrm{m}^{2} / \mathrm{yr}\right)$
"Total operating $\mathrm{GWP}$ of baseline retail building after 50 years $=2,310$ tonnes
of $\mathrm{CO}_{2}$ eq. $\left(80 \mathrm{~kg}\right.$ of $\mathrm{CO}_{2}$ eq. $/ \mathrm{m}^{2} / \mathrm{yr}$ ) 


\section{Cold-Formed Steel Stud Wall \#11 (SS-W11)}

Building Component Description:

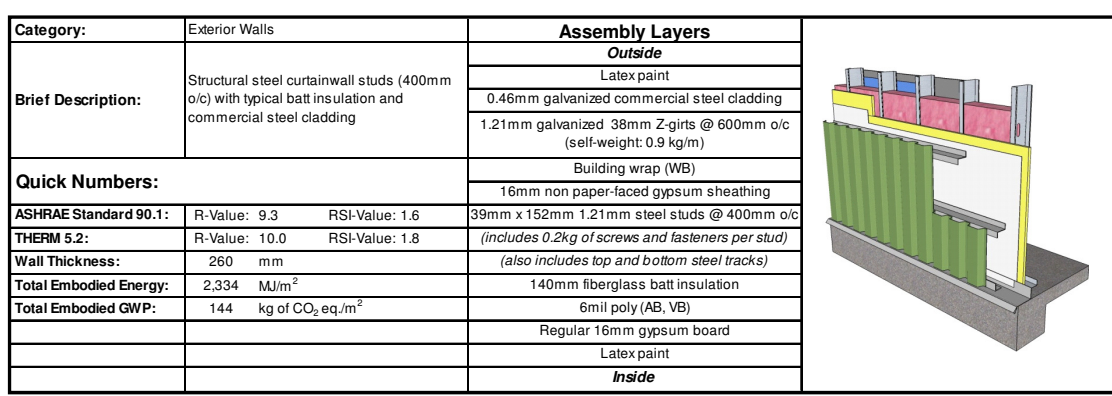

\section{Life-Cycle Assessment Results:}

Primary Energy Consumption (MJ)

\begin{tabular}{|c|c|c|c|c|c|c|c|c|c|c|c|c|c|c|c|}
\hline \multirow{3}{*}{\begin{tabular}{|l|l} 
Lifespan \\
(Years)
\end{tabular}} & \multicolumn{13}{|c|}{ Embodied Energy (EE) } & \multirow{2}{*}{\multicolumn{2}{|c|}{$\begin{array}{c}\text { Difference in } \\
\text { Operating Energy } \\
\text { from Baseline afte } \\
\text { Lifespan }\end{array}$}} \\
\hline & \multicolumn{2}{|c|}{ Manufacturing } & \multicolumn{3}{|c|}{ Construction } & \multicolumn{3}{|c|}{ Maintenance } & \multicolumn{3}{|c|}{ End of Life } & \multirow{2}{*}{$\begin{array}{c}{ }^{3} \text { Total } \\
\mathrm{EE}\end{array}$} & \multirow{2}{*}{\begin{tabular}{|c|}
${ }^{4}$ Total \\
EE \\
per $\mathrm{m}^{2}$
\end{tabular}} & & \\
\hline & \begin{tabular}{|l|l|} 
Material & 2 \\
Trans
\end{tabular} & Total & Materia & ${ }^{2}$ Trans. & Total & Materia & ${ }^{2}$ Trans & \begin{tabular}{|l|l|} 
Total \\
\end{tabular} & Material & | ${ }^{2}$ Trans. & Total & & & ${ }^{5}$ Total & ${ }^{6}$ perm \\
\hline I'Initial & \begin{tabular}{|l|l|}
117,649 & 270 \\
\end{tabular} & 117,922 & 160 & \begin{tabular}{|l|}
620 \\
\end{tabular} & 780 & 0 & 0 & 0 & 0 & 0 & 0 & 118,700 & 2,331 & & \\
\hline 50 & \begin{tabular}{|l|l|}
117,649 & 270 \\
\end{tabular} & 117,926 & 160 & 620 & 780 & 0 & 0 & 0 & 0 & 165 & 165 & & & $2,100,000$ & 3,61 \\
\hline
\end{tabular}

Global Warming Potential ( $k g$ of $\mathrm{CO}_{2}$ eq.)

\begin{tabular}{|c|c|c|c|c|c|c|c|c|c|c|c|c|c|c|c|c|}
\hline \multirow{3}{*}{$\begin{array}{l}\text { Lifespan } \\
\text { (Years) }\end{array}$} & \multicolumn{14}{|c|}{ Embodied Global Warming Potential (GWP) } & \multirow{2}{*}{\multicolumn{2}{|c|}{$\begin{array}{l}\text { Difference in } \\
\text { Operating GWP fror } \\
\text { Baseline after } \\
\text { Lifespan }\end{array}$}} \\
\hline & \multicolumn{3}{|c|}{ Manufacturing } & \multicolumn{3}{|c|}{ Construction } & \multicolumn{3}{|c|}{ Maintenance } & \multicolumn{3}{|c|}{ End of Life } & \multirow{2}{*}{ 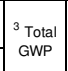 } & \multirow{2}{*}{$\begin{array}{l}{ }^{4} \text { Total } \\
\text { GWP } \\
\text { per m }\end{array}$} & & \\
\hline & Material & ${ }^{2}$ Trans & Total & Material & Trans. & Total & Material & ${ }^{2}$ Trans. & Total & Material & 2 Trans. & Total & & & \begin{tabular}{|c|}
${ }^{5}$ Total \\
\end{tabular} & ${ }^{6} \mathrm{perm}^{2}$ \\
\hline Initial & 7,324 & 0 & 7,324 & 10 & 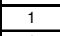 & 12 & 0 & 0 & 0 & 0 & 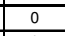 & 0 & 7,336 & 144 & & \\
\hline 50 & 7,324 & 0 & 7,324 & 10 & 1 & 12 & 0 & 0 & 0 & 0 & 0 & 0 & 7,336 & 144 & 120,00 & 20 \\
\hline
\end{tabular}

Embodied energy (and GWP) numbers are based on an area of wall $=50.9 \mathrm{~m}^{2} \quad$ (Length $\times$ Height $=7.6 \mathrm{~m} \times 6.7 \mathrm{~m}=50.9 \mathrm{~m}^{2}$ )

Net wall area of baseline retail building (gross wall area - openings) = $581.0 \mathrm{~m}^{2}$

\section{ATHENA $®$ EIE Material List:}

\begin{tabular}{|l|c|c|}
\hline \multicolumn{1}{|c|}{ Material List } & Quantities & Unit \\
\hline $\begin{array}{l}\text { 16mm Moisture Resistant Gypsum } \\
\text { Board }\end{array}$ & 56.0 & $\mathrm{~m} 2$ \\
\hline 16mm Regular Gypsum Board & 56.0 & $\mathrm{~m} 2$ \\
\hline 6 mil Polyethylene & 108.0 & $\mathrm{~m} 2$ \\
\hline Batt. Fiberglass & 289.8 & $\mathrm{~m} 2(25 \mathrm{~mm})$ \\
\hline Commercial 0.46mm Steel Cladding & 168.0 & $\mathrm{~m} 2$ \\
\hline Galvanized Studs & 423.3 & $\mathrm{~kg}$ \\
\hline Joint Compound & 111.8 & $\mathrm{~kg}$ \\
\hline Nails & 4.2 & $\mathrm{~kg}$ \\
\hline Paper Tape & 1.3 & $\mathrm{~kg}$ \\
\hline Screws Nuts \& Bolts & 5.2 & $\mathrm{~kg}$ \\
\hline Water Based Latex Paint & 132.5 & $\mathrm{~L}$ \\
\hline
\end{tabular}

Notes:
${ }^{1}$ Initial $=$ Time 'o' (i.e. at the completion of initial construction)

${ }^{2}$ Trans. $=$ Transportation

Total EE (or Total GWP) = Total embodied energy (or total embodied GWP) of building component after lifespan (i.e. total manufacturing + tota

Total EE (or Total GWP) per $\mathrm{m}^{2}=$ Total EE (or Total GWP) of building
component/ area of building component that was modelled in ATHENAQIEIE Lifespan = The difference in the total lifecycle operating energy (or GWP)

Total Difference in Operating Energy (or GWP) from Baseline after Lifespan per $\mathrm{m}^{2}=$ Total difference in operating energy (or
baseline after lifespan / net wall area of baseline retail building Total operating primary energy use of baseline retail building after 50 years

Total operating GWP of baseline retail building after 50 years $=2,310$ tonnes from the baseline retail building after lifespan, due to using this building

\section{Cold-Formed Steel Stud Wall \#12 (SS-W12)}

Building Component Description:

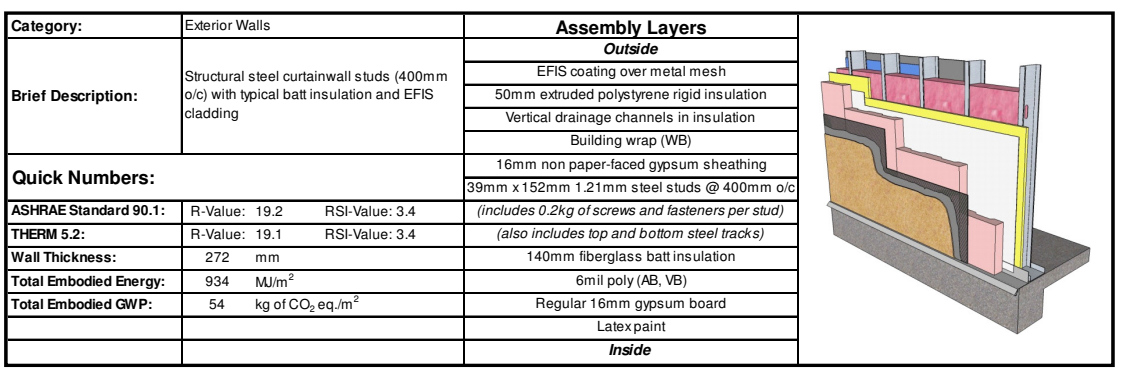

\section{Life-Cycle Assessment Results:}

Primary Energy Consumption (MJ)

\begin{tabular}{|c|c|c|c|c|c|c|c|c|c|c|c|c|c|c|c|c|}
\hline \multirow{3}{*}{\begin{tabular}{|c|} 
Litespan \\
(Years)
\end{tabular}} & \multicolumn{14}{|c|}{ Embodied Energy (EE) } & \multirow{2}{*}{\multicolumn{2}{|c|}{$\begin{array}{c}\text { Difference in } \\
\text { Operating Energy } \\
\text { from Baseline after } \\
\text { Lifespan }\end{array}$}} \\
\hline & \multicolumn{3}{|c|}{ Manufacturing } & \multicolumn{3}{|c|}{ Construction } & \multicolumn{3}{|c|}{ Maintenance } & \multicolumn{3}{|c|}{ End of Life } & \multirow{2}{*}{$\begin{array}{c}{ }^{3} \text { Total } \\
\text { EE }\end{array}$} & \multirow{2}{*}{$\begin{array}{c}{ }^{4}{ }^{\mathrm{T} o t a l} \\
\mathrm{EE} \\
\text { per m }\end{array}$} & & \\
\hline & Material & | ${ }^{2}$ Trans & Total & Material & ${ }^{2}$ Trans. & Total & Material & ${ }^{2}$ Trans. & Total & Material & \begin{tabular}{|l|}
${ }^{2}$ Trans. \\
\end{tabular} & Total & & & ${ }^{5}$ Total & ${ }^{6} \operatorname{perm}^{2}$ \\
\hline Initial & 45,822 & 327 & 46,149 & 160 & 728 & 887 & 0 & 0 & 0 & 0 & 0 & 0 & 47,036 & 924 & & \\
\hline 50 & 45,822 & $\begin{array}{l}327 \\
\end{array}$ & 46,149 & 160 & 728 & 887 & 0 & 0 & 0 & 0 & 532 & 532 & 47,568 & 934 & $-800,000$ & 1,3 \\
\hline
\end{tabular}

Global Warming Potential (kg of $\mathrm{CO}_{2}$ eq.)

\begin{tabular}{|c|c|c|c|c|c|c|c|c|c|c|c|c|c|c|c|c|}
\hline \multirow{3}{*}{\begin{tabular}{|l} 
Lifespan \\
(Years)
\end{tabular}} & \multicolumn{14}{|c|}{ Embodied Global Warming Potential (GWP) } & \multirow{2}{*}{\multicolumn{2}{|c|}{$\begin{array}{l}\text { Difference in } \\
\text { Operating GWP fron } \\
\text { Baseline after } \\
\text { Lifespan }\end{array}$}} \\
\hline & \multicolumn{3}{|c|}{ nufacturing } & \multicolumn{3}{|c|}{ Constru } & \multicolumn{3}{|c|}{ Maintenance } & \multicolumn{3}{|c|}{ End of Life } & \multirow{2}{*}{$\begin{array}{l}{ }^{3} \text { Total } \\
\text { GWPP }\end{array}$} & \multirow{2}{*}{$\begin{array}{l}{ }^{4} \text { Total } \\
\text { GWP } \\
\text { per }{ }^{2}\end{array}$} & & \\
\hline & Material & ${ }^{2}$ Trans. & Total & Material & ${ }^{2}$ Trans. & Total & Material & 2 Trans & Total & Material & ${ }^{2}$ Trans. & Total & & & ${ }^{5}$ Total & ${ }^{6}$ perm $^{2}$ \\
\hline nitial & 2,714 & 1 & 2,715 & 10 & 1 & 12 & 0 & 0 & 0 & 0 & 0 & 0 & 2,727 & 54 & & \\
\hline 50 & $\begin{array}{l}2,714 \\
\end{array}$ & 1 & 2,715 & 10 & 4 & 12 & 0 & 0 & 0 & 0 & 7 & 1 & 2,728 & 54 & 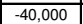 & -69 \\
\hline
\end{tabular}

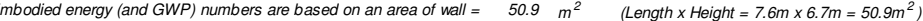

ATHENA $®$ EIE Material List:

(Includes all materials after 50 years) 'Initial = Time o' (i.e. at the completion of:

\begin{tabular}{|c|c|c|}
\hline Material List & Quantities & Unit \\
\hline$\# 15$ Organic Felt & 219.4 & $\mathrm{~m} 2$ \\
\hline $\begin{array}{l}16 \mathrm{~mm} \text { Moisture Resistant Gypsum } \\
\text { Board }\end{array}$ & 56.0 & $\mathrm{~m} 2$ \\
\hline 16mm Regular Gypsum Board & 56.0 & $\mathrm{~m} 2$ \\
\hline 6 mil Polyethylene & 108.0 & $\mathrm{~m} 2$ \\
\hline Batt. Fiberglass & 289.8 & m2 (25mm) \\
\hline Extruded Polystyrene & 104.3 & m2 (25mm) \\
\hline Galvanized Sheet & 51.4 & $\mathrm{~kg}$ \\
\hline Galvanized Studs & 342.5 & $\mathrm{~kg}$ \\
\hline Joint Compound & 111.8 & $\mathrm{~kg}$ \\
\hline Nails & 8.8 & $\mathrm{~kg}$ \\
\hline Paper Tape & 1.3 & $\mathrm{~kg}$ \\
\hline Screws Nuts \& Bolts & 3.9 & $\mathrm{~kg}$ \\
\hline Stucco over metal mesh & 136.0 & $\mathrm{~m} 2$ \\
\hline Water Based Latex Paint & 132.5 & L \\
\hline
\end{tabular}

'Initial $=$ Time 0 ' (i.e. at the

Total EE (or Total GWP) = Total embodied energy (or total embodied GWP)

${ }^{4}$ Total EE (or Total GWP per $\mathrm{m}^{2}=$ Total EE (or Total GWP) of building
component/ area of building commonent that was modeled in ATHENAD EIE

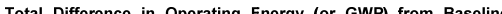
Lifespan $=$ The difference in the total lifecycle operating energy (or GWP from the baseline retail building after lifespan, due to using this building

Total Difference in Operating Energy (or GWP) from Baseline after Lifespan per $\mathrm{m}^{2}=$ Total difference in operating energy (or GWP) from

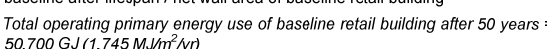

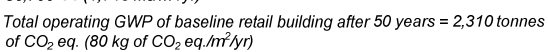
of building component after lifespan (i.e. total man
construction + total maintenance + total end-of-life effects) 


\section{Cold-Formed Steel Stud Wall \#13 (SS-W13)}

Building Component Description:

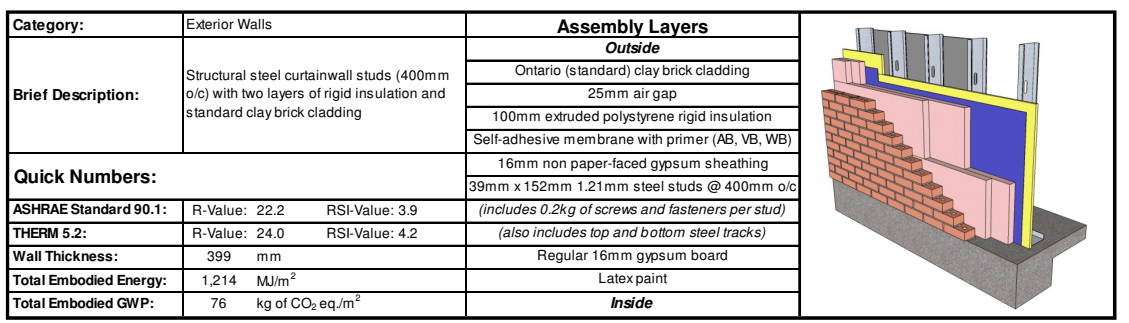

Life-Cycle Assessment Results:

Primary Energy Consumption (MJ)

\begin{tabular}{|c|c|c|c|c|c|c|c|c|c|c|c|c|c|c|c|c|}
\hline \multirow{3}{*}{$\mid$\begin{tabular}{|l} 
Lifespan \\
(Years)
\end{tabular}} & \multicolumn{14}{|c|}{ Embodied Energy (EE) } & \multirow{2}{*}{\multicolumn{2}{|c|}{$\begin{array}{c}\text { Difference in } \\
\text { Operating Energy } \\
\text { from Baseline after } \\
\text { Lifespan }\end{array}$}} \\
\hline & \multicolumn{3}{|c|}{ Manufacturing } & \multicolumn{3}{|c|}{ Construction } & \multicolumn{3}{|c|}{ Maintenance } & \multicolumn{3}{|c|}{ End of Life } & \multirow{2}{*}{$\begin{array}{c}{ }^{3} \text { Total } \\
\mathrm{EE}\end{array}$} & \multirow{2}{*}{$\begin{array}{l}{ }^{4} \text { Total } \\
\text { EE } \\
\text { per } \mathrm{m}^{2}\end{array}$} & & \\
\hline & Materia & ${ }^{2}$ Trans & Total & Materia & ${ }^{2}$ Trans. & \begin{tabular}{|l|} 
Total \\
\end{tabular} & \begin{tabular}{|l|} 
Material \\
\end{tabular} & $\begin{array}{l}{ }^{2} \text { Trans. } \\
\end{array}$ & \begin{tabular}{|l|l|} 
Total \\
\end{tabular} & Material & $\mid{ }^{2}$ Trans. & \begin{tabular}{|l|} 
Total \\
\end{tabular} & & & \begin{tabular}{|l|l|}
${ }^{5}$ Total \\
\end{tabular} & ${ }^{6}$ perm ${ }^{2}$ \\
\hline Initial & 56,312 & 276 & 56,58 & 160 & $\begin{array}{l}1,387 \\
\end{array}$ & \begin{tabular}{|l|l|l|}
1,547 \\
\end{tabular} & 0 & 0 & 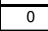 & 0 & 0 & 0 & 58,134 & 1,142 & & \\
\hline 50 & 56,312 & \begin{tabular}{|l|l|}
276 \\
\end{tabular} & (56,58 & 160 & \begin{tabular}{|l|l}
1,387 \\
\end{tabular} & $\mid \begin{array}{l}11,547 \\
\end{array}$ & 3,099 & 10 & 3,110 & 0 & 589 & 590 & $\mid$ & 1,214 & 1,6000, & $-2,75$ \\
\hline
\end{tabular}

Global Warming Potential ( $k g$ of $\mathrm{CO}_{2}$ eq.)

હ

\begin{tabular}{|c|c|c|c|c|c|c|c|c|c|c|c|c|c|c|c|c|}
\hline \multirow{3}{*}{$\begin{array}{c}\text { Lifespan } \\
\text { (Years) }\end{array}$} & \multicolumn{14}{|c|}{ Embodied Global Warming Potential (GWP) } & \multirow{2}{*}{\multicolumn{2}{|c|}{$\begin{array}{l}\text { Difference in } \\
\text { Operating GWP from } \\
\text { Baseline after } \\
\text { Lifespan }\end{array}$}} \\
\hline & \multicolumn{3}{|c|}{ Manufacturing } & \multicolumn{3}{|c|}{ Construction } & \multicolumn{3}{|c|}{ Maintenance } & \multicolumn{3}{|c|}{ End of Life } & \multirow{2}{*}{$\begin{array}{l}{ }^{3} \text { Total } \\
\text { GWP }\end{array}$} & \multirow{2}{*}{$\begin{array}{l}{ }^{4} \text { Total } \\
\text { GWP } \\
\text { per m}{ }^{2}\end{array}$} & & \\
\hline & Material & Trans. & Total & Material & ${ }^{2}$ Trans. & Total & Material & ${ }^{2}$ Trans. & Total & Material & $\left.\right|^{2}{ }^{2}$ Trans. & Total & & & ${ }^{5}$ Total & ${ }^{6} \operatorname{per~m}^{2}$ \\
\hline Initial & 3,792 & 1 & 3 & 10 & 3 & 13 & 0 & 0 & 0 & 0 & 0 & 0 & 3,806 & 75 & & \\
\hline 50 & 3,792 & 1 & 3,793 & 10 & 3 & 13 & 44 & 0 & 44 & 0 & 1 & 1 & 3,851 & 76 & $-80,000$ & -138 \\
\hline
\end{tabular}

ine retail building (gross wall area - openings) $=581.0 \mathrm{~m}^{2}$

ATHENA ® EIE Material List: (Includes all materials after 50 years)

\begin{tabular}{|l|c|c|}
\hline \multicolumn{1}{|c|}{ Material List } & Quantities & Unit \\
\hline 3 mil Polyethylene & 54.0 & $\mathrm{~m} 2$ \\
\hline 16mm Moisture Resistant Gypsum & 56.0 & $\mathrm{~m} 2$ \\
Board & 56.0 & $\mathrm{~m} 2$ \\
\hline 16mm Regular Gypsum Board & 56.0 & $\mathrm{~kg}$ \\
\hline Cold Rolled Sheet & 10.3 & materials after 50 years) \\
\hline Extruded Polystyrene & 208.6 & $\mathrm{~m} 2(25 \mathrm{~mm})$ \\
\hline Galvanized Studs & 342.5 & $\mathrm{~kg}$ \\
\hline Joint Compound & 111.8 & $\mathrm{~kg}$ \\
\hline Modiffed Bitumen membrane & 68.2 & $\mathrm{~kg}$ \\
\hline Mortar & 1.5 & $\mathrm{~m} 3$ \\
\hline Nails & 4.2 & $\mathrm{~kg}$ \\
\hline Ontario (Standard) Brick & 53.5 & $\mathrm{~m} 2$ \\
\hline Paper Tape & 1.3 & $\mathrm{~kg}$ \\
\hline Screws Nuts \& Bolts & 3.9 & $\mathrm{~kg}$ \\
\hline Solvent Based Alkyd Paint & 19.6 & $\mathrm{~L}$ \\
\hline Water Based Latex Paint & 66.3 & $\mathrm{~L}$ \\
\hline
\end{tabular}

\section{Cold-Formed Steel Stud Wall \#14 (SS-W14)}

Building Component Description:

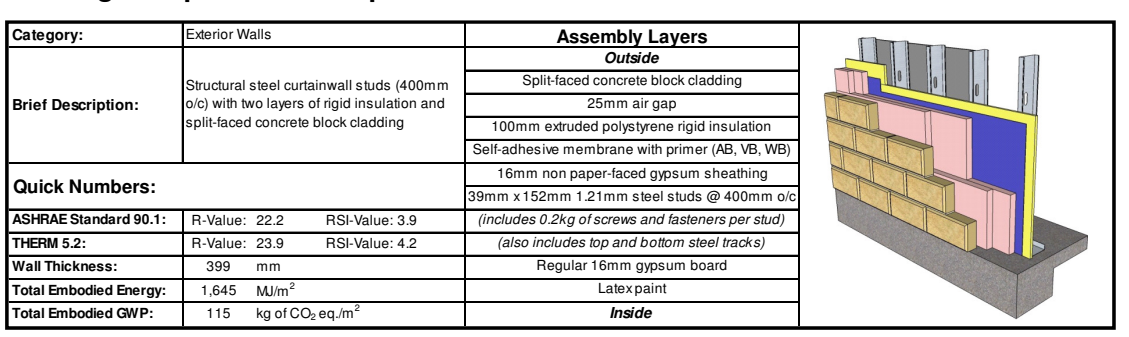

Life-Cycle Assessment Results:

Primary Energy Consumption (MJ)

\begin{tabular}{|c|c|c|c|c|c|c|c|c|c|c|c|c|c|c|c|c|}
\hline \multirow{3}{*}{\begin{tabular}{|c} 
Lifespan \\
(Years)
\end{tabular}} & \multicolumn{14}{|c|}{ Embodied Energy (EE) } & \multirow{2}{*}{\multicolumn{2}{|c|}{$\begin{array}{c}\text { Difference in } \\
\text { Operating Energy } \\
\text { from Baseline after } \\
\text { Lifespan }\end{array}$}} \\
\hline & \multicolumn{3}{|c|}{ Manufacturin } & \multicolumn{3}{|c|}{ Construction } & \multicolumn{3}{|c|}{ Maintenance } & \multicolumn{3}{|c|}{ End of Life } & \multirow{2}{*}{$\begin{array}{c}{ }^{3} \text { Total } \\
\text { EE }\end{array}$} & \multirow{2}{*}{\begin{tabular}{|l}
${ }^{4}{ }^{4}$ otal \\
$\mathrm{EE}$ \\
per $\mathrm{m}^{2}$
\end{tabular}} & & \\
\hline & Material & 1 ${ }^{2}$ Trans. & Total & Material & 1 ${ }^{2}$ Trans. & \begin{tabular}{|l|l} 
Total \\
\end{tabular} & Material & ${ }^{2}{ }^{2}$ Trans. & Total & Material & $\begin{array}{l}2 \\
2\end{array}$ Trans. & Total & & & ${ }^{5}$ Total & ${ }^{6}$ per $\mathrm{m}^{2}$ \\
\hline & 75,340 & 839 & 76,179 & 160 & 2,197 & 2,357 & 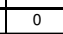 & 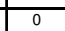 & 0 & 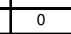 & 0 & 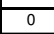 & 8,53 & 1,542 & & \\
\hline
\end{tabular}

Global Warming Potential (kg of $\mathrm{CO}_{2}$ eq)

\begin{tabular}{|c|c|c|c|c|c|c|c|c|c|c|c|c|c|c|c|c|}
\hline \multirow{3}{*}{ Lifespan } & \multicolumn{14}{|c|}{ Embodied Global Warming Potential (GWP) } & \multirow{2}{*}{\multicolumn{2}{|c|}{$\begin{array}{l}\text { Difference in } \\
\text { Operating GWP from } \\
\text { Baseline after } \\
\text { Lifespan }\end{array}$}} \\
\hline & \multicolumn{3}{|c|}{ Manutacturing } & \multicolumn{3}{|c|}{ Construction } & \multicolumn{3}{|c|}{ Maintenance } & \multicolumn{3}{|c|}{ End of Lifie } & \multirow{2}{*}{$\begin{array}{l}{ }^{3} \text { Total } \\
\text { GWP }\end{array}$} & \multirow{2}{*}{$\begin{array}{c}{ }^{4} \text { Total } \\
\text { GWP } \\
\text { per m }{ }^{2}\end{array}$} & & \\
\hline & Material & ${ }^{2}$ Trans. & Total & Material & Trans. & Total & Material & Trans. & Total & Material & ${ }^{2}$ Trans. & Total & & & ${ }^{5}$ Total & ${ }^{6}$ per $\mathrm{m}^{2}$ \\
\hline Initial & 5,778 & 2 & 5,780 & 10 & 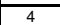 & 15 & 0 & 0 & 0 & 0 & 0 & 0 & 5,794 & $\begin{array}{l}114 \\
\end{array}$ & & \\
\hline 50 & \begin{tabular}{|l|}
5.778 \\
\end{tabular} & 2 & 5,780 & 10 & 4 & 15 & 44 & 0 & 44 & 0 & 4 & 4 & 5.842 & 1115 & 80,000 & -138 \\
\hline
\end{tabular}
Embodied energy (and GWP) numbers are based on an area of wall $=40.9 \mathrm{~m}^{2}$
Net wall area of baseline retail building (gross wall area - openings) $=581.0 \mathrm{~m}^{2}$ (Length $x$ Height $=7.6 \mathrm{~m} \times 6.7 \mathrm{~m}=50.9 \mathrm{~m}^{2}$ )

ATHENA $®$ EIE Material List:

Notes:
1 Initial $=$ Time ' $O$ ' (i.e. at the completion of initial construction)

${ }^{2}$ Trans. $=$ Transportation

${ }^{3}$ Total EE (or Total GWP) $=$ Total embodied energy (or total embodied GWP) of building component after lifespan (i.e. total man
construction + total maintenance + total end-of-life effects)

${ }^{4}$ Total EE (or Total GWP) per $\mathrm{m}^{2}=$ Total EE (or Total GWP) of building
component/area of building component that was modelled in ATHENAQ EEE ${ }^{5}$ Total Difference in Operating Energy (or GWP) from Baseline after Lifespan = The differerence in the total lifecycle operating energy (or GWP)
from the basaleline retail builing after lifespan, due to using this building
component instead of the baseline component

${ }^{6}$ Total Difference in Operating Energy (or GWP) from Baseline after

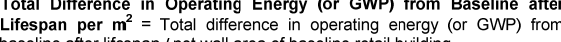

Total operating primary energy use of baseline retail building affer 50 years $=$
$50.700 \mathrm{GJ}\left(1.745 \mathrm{MJ}^{2} \mathrm{~m}^{2}\right.$ yr $)$

of $\mathrm{CO}_{2}$ eq. (80 kg of $\mathrm{CO}_{2}$ eq. $/ \mathrm{m}^{2} \mathrm{Yyr}$ )

\begin{tabular}{|c|c|c|}
\hline Material List & Quantities & Unit \\
\hline 3 mil Polyethylene & 54.0 & $\mathrm{~m} 2$ \\
\hline $\begin{array}{l}16 \mathrm{~mm} \text { Moisture Resistant Gypsum } \\
\text { Board }\end{array}$ & 56.0 & $\mathrm{~m} 2$ \\
\hline 16mm Regular Gypsum Board & 56.0 & $\mathrm{~m} 2$ \\
\hline Cold Rolled Sheet & 10.3 & $\mathrm{~kg}$ \\
\hline Extruded Polystyrene & 208.6 & $\mathrm{~m} 2(25 \mathrm{~mm})$ \\
\hline Galvanized Studs & 342.5 & $\mathrm{~kg}$ \\
\hline Joint Compound & 111.8 & $\mathrm{~kg}$ \\
\hline Modified Bitumen membrane & 401.5 & $\mathrm{~kg}$ \\
\hline Mortar & 4.3 & $\mathrm{~m} 3$ \\
\hline Nails & 4.2 & $\mathrm{~kg}$ \\
\hline Paper Tape & 1.3 & $\mathrm{~kg}$ \\
\hline Screws Nuts \& Bolts & 3.9 & $\mathrm{~kg}$ \\
\hline Solvent Based Alkyd Paint & 19.6 & $\mathrm{~L}$ \\
\hline Split-faced Concrete Block & $1,238.2$ & Blocks \\
\hline Water Based Latex Paint & 66.3 & L \\
\hline
\end{tabular}

Notes:

${ }^{2}$ Trans. $=$ Transportation

${ }^{3}$ Total EE (or Total GWP) $=$ Total embodied energy (or total embodied GWP of building component after lifespan (i.e. total man
construction + total maintenance + total enc-of-life effects)

${ }^{4}$ Total EE (or Total GWP) per $m^{2}=$ Total EE (or Total GWP) of building
component/area of building component that was modelled in ATHENAQ EIE ${ }^{5}$ Total Difference in Operating Energy (or GWP) from Baseline after Lifespan = The difference in the total life-cycle operating energy (or GWP)
from the baseline retail bualing after lifespan, due to using this building
component instead of the baseline component

Total Difference in Operating Energy (or GWP) from Baseline after Lifespan per $\mathrm{m}^{2}=$ Total difference in operating energy (or GWP) from * Total operating primary energy use of baseline retail building after 50 years =

*Total operating GWP of baseline ret.
of $\mathrm{CO}_{2}$ eq. $\left(80 \mathrm{~kg}\right.$ of $\mathrm{CO}_{2}$ eq. $\left./ \mathrm{hr}^{2} \mathrm{yr}\right)$ 


\section{Cold-Formed Steel Stud Wall \#15 (SS-W15)}

Building Component Description:

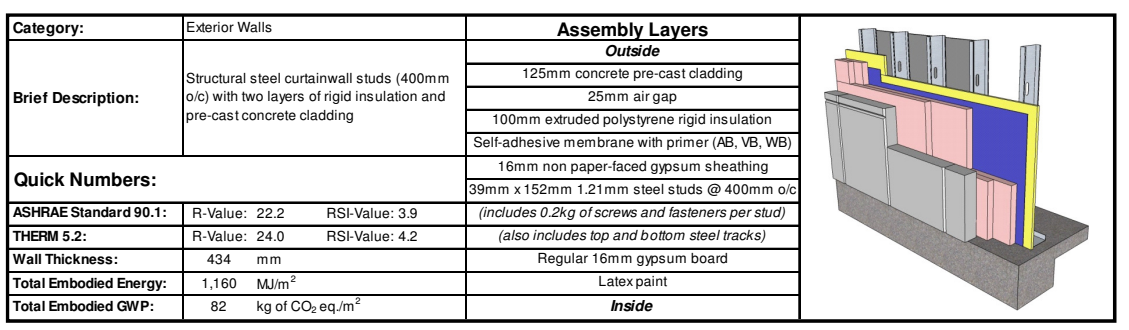

Life-Cycle Assessment Results:

Primary Energy Consumption (MJ)

\begin{tabular}{|c|c|c|c|c|c|c|c|c|c|c|c|c|c|c|c|c|}
\hline \multirow{3}{*}{$\begin{array}{l}\text { Lifespan } \\
\text { (Years) }\end{array}$} & \multicolumn{14}{|c|}{ Embodied } & \multirow{2}{*}{\multicolumn{2}{|c|}{$\begin{array}{c}\text { Difference in } \\
\text { Operating Energy } \\
\text { from Baseline after } \\
\text { Lifespan }\end{array}$}} \\
\hline & \multicolumn{3}{|c|}{ Manufacturing } & \multicolumn{3}{|c|}{ Construction } & \multicolumn{3}{|c|}{ Mainten } & \multicolumn{3}{|c|}{ End of Life } & \multirow{2}{*}{$\begin{array}{c}{ }^{3} \text { Total } \\
\mathrm{EE}\end{array}$} & \multirow{2}{*}{\begin{tabular}{|c|}
${ }^{4} \mathrm{Total}$ \\
$\mathrm{EE}$ \\
$\mathrm{Ee} \mathrm{m}^{2}$
\end{tabular}} & & \\
\hline & Material & ${ }^{2}$ Trans. & Total & \begin{tabular}{|l|} 
Material \\
\end{tabular} & ${ }^{2}$ Trans. & \begin{tabular}{|l|l|} 
Total \\
\end{tabular} & \begin{tabular}{|l|} 
Material \\
\end{tabular} & ${ }^{2}$ Trans. & Total & $\mid$ Material & \begin{tabular}{|l|}
${ }^{2}$ Trans. \\
\end{tabular} & Total & & & \begin{tabular}{|l|}
${ }^{5}$ Total \\
\end{tabular} & ${ }^{6} \mathrm{perm}^{2}$ \\
\hline Initial & 52,999 & 866 & 53,865 & 160 & 6 & $=$ & (5 & 0 & $x_{2}$ & 0 & 0 & 0 & 55,351 & \begin{tabular}{|l|}
1,087 \\
\end{tabular} & & \\
\hline 50 & 52,999 & 866 & 53,865 & 160 & 1,326 & 1,486 & $\begin{array}{l}3,099 \\
\end{array}$ & 10 & \begin{tabular}{|l|l|}
3,110 \\
\end{tabular} & 1 & \begin{tabular}{|l|l|}
622 \\
\end{tabular} & \begin{tabular}{|l|}
623 \\
\end{tabular} & \begin{tabular}{|l|l|}
59,084 \\
\end{tabular} & \begin{tabular}{|l|} 
\\
1,160
\end{tabular} & $\mid-1,600,000$ & $-2,754$ \\
\hline
\end{tabular}

Global Warming Potential ( $\mathrm{kg}$ of $\mathrm{CO}_{2}$ eq.)

\begin{tabular}{|c|c|c|c|c|c|c|c|c|c|c|c|c|c|c|c|c|}
\hline \multirow{3}{*}{$\mid \begin{array}{l}\text { Litespan } \\
\text { (Years) }\end{array}$} & \multicolumn{14}{|c|}{ Embodied Global Warming Potential (GWP) } & \multirow{2}{*}{\multicolumn{2}{|c|}{\begin{tabular}{|c|} 
Difference in \\
Operating GWP fron \\
Baseline after \\
Lifespan
\end{tabular}}} \\
\hline & \multicolumn{3}{|c|}{ Manufacturing } & \multicolumn{3}{|c|}{ Construction } & \multicolumn{3}{|c|}{ Maintenance } & \multicolumn{3}{|c|}{ End of Life } & \multirow{2}{*}{$\begin{array}{l}{ }^{3} \text { Total } \\
\text { GWP }\end{array}$} & \multirow{2}{*}{\begin{tabular}{|l}
${ }^{4}$ Total \\
GiWP \\
per m
\end{tabular}} & & \\
\hline & \begin{tabular}{|l|} 
Material \\
\end{tabular} & ${ }^{2}$ Trans. & Total & Material & ${ }^{2}$ Trans. & Total & Material & ${ }^{2}$ Trans. & Total & \begin{tabular}{|l|} 
Material \\
\end{tabular} & 列 ${ }^{2}$ Trans. & \begin{tabular}{|l|} 
Total \\
\end{tabular} & & & ${ }^{5}$ Total & $\begin{array}{l}{ }^{6} \text { per } \mathrm{m}^{2} \\
\end{array}$ \\
\hline${ }^{1}$ Initial & \begin{tabular}{|l|l}
4,092 \\
\end{tabular} & 2 & 4,094 & 10 & 3 & 13 & 0 & 0 & 0 & 0 & 0 & 0 & 4,107 & 81 & & \\
\hline 50 & \begin{tabular}{|l}
4,092 \\
\end{tabular} & 2 & 4,094 & 10 & 3 & 13 & 44 & 0 & 44 & 0 & 1 & 1 & 4,152 & 82 & $-80,000$ & -138 \\
\hline
\end{tabular}

ATHENA ® EIE Material List: (Includes all materials after 50 years)

\begin{tabular}{|l|c|c|}
\hline \multicolumn{1}{|c|}{ Material List } & Quantities & Unit \\
\hline 3 mil Polyethylene & 54.0 & $\mathrm{~m} 2$ \\
\hline $\begin{array}{l}\text { 16mm Moisture Resistant Gypsum } \\
\text { Board }\end{array}$ & 56.0 & $\mathrm{~m} 2$ \\
\hline 16mm Regular Gypsum Board & 56.0 & $\mathrm{~m} 2$ \\
\hline Concrete 30 MPa (flyash av) & 6.7 & $\mathrm{~m} 3$ \\
\hline Extruded Polystyrene & 208.6 & $\mathrm{~m} 2(25 \mathrm{~mm})$ \\
\hline Galvanized Studs & 342.5 & $\mathrm{~kg}$ \\
\hline Joint Compound & 111.8 & $\mathrm{~kg}$ \\
\hline Modified Bitumen membrane & 68.2 & $\mathrm{~kg}$ \\
\hline Nails & 4.2 & $\mathrm{~kg}$ \\
\hline Paper Tape & 1.3 & $\mathrm{~kg}$ \\
\hline Rebar, Rod, Light Sections & 404.0 & $\mathrm{~kg}$ \\
\hline Screws Nuts \& Bolts & 3.9 & $\mathrm{~kg}$ \\
\hline Solvent Based Alkyd Paint & 19.6 & $\mathrm{~L}$ \\
\hline Water Based Latex Paint & 66.3 & $\mathrm{~L}$ \\
\hline
\end{tabular}

\section{Cold-Formed Steel Stud Wall \#16 (SS-W16)}

Building Component Description:

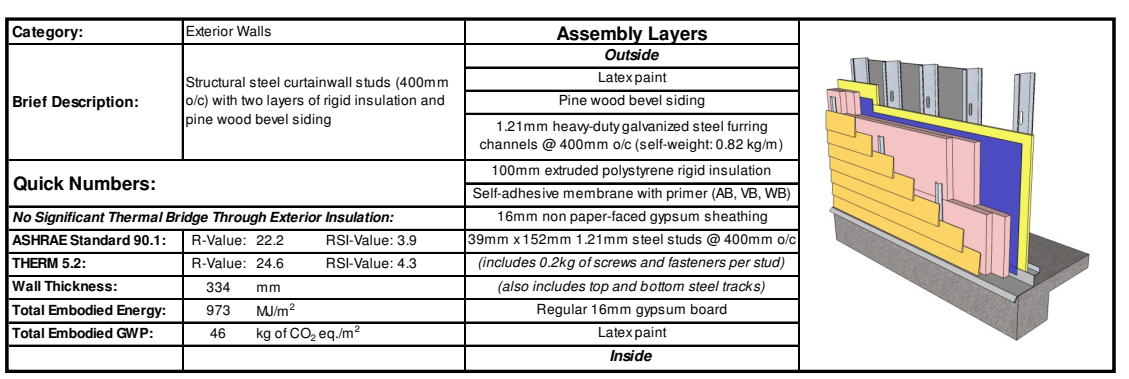

\section{Life-Cycle Assessment Results:}

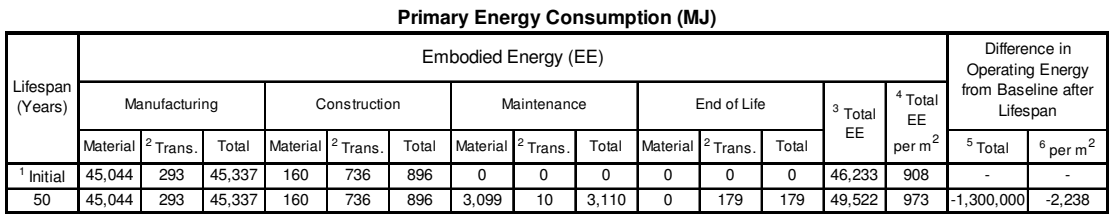

Global Warming Potential (kg of $\mathrm{CO}_{2}$ eq.)

\begin{tabular}{|c|c|c|c|c|c|c|c|c|c|c|c|c|c|c|c|c|}
\hline \multirow{3}{*}{$\begin{array}{l}\text { Lifespan } \\
\text { (Years) }\end{array}$} & \multicolumn{14}{|c|}{ Embodied Global Warming Potential (GWP) } & \multirow{2}{*}{\multicolumn{2}{|c|}{$\begin{array}{l}\text { Difference in } \\
\text { Operating GWP from } \\
\text { Baseline after } \\
\text { Lifespan }\end{array}$}} \\
\hline & \multicolumn{3}{|c|}{ Manutacturing } & \multicolumn{3}{|c|}{ Construction } & \multicolumn{3}{|c|}{ Maintenance } & \multicolumn{3}{|c|}{ End of Life } & \multirow{2}{*}{\begin{tabular}{|}
${ }^{3}$ Total \\
GWP
\end{tabular}} & \multirow{2}{*}{$\begin{array}{l}{ }^{4} \text { Total } \\
\text { GWP } \\
\text { per }{ }^{2}\end{array}$} & & \\
\hline & Material & ${ }^{2}$ Trans. & Total & \begin{tabular}{|l|} 
Material \\
\end{tabular} & ${ }^{2}$ Trans. & Total & Material & ${ }^{2}$ Trans. & Total & Material & ${ }^{2}$ Trans. & Total & & & ${ }^{5}$ Total & ${ }^{6}{ }_{\text {perm }}^{2}$ \\
\hline Initial & \begin{tabular}{|l|}
2,267 \\
\end{tabular} & 1 & 2,268 & 10 & 1 & 12 & 0 & 0 & 0 & 0 & 0 & 0 & 2,280 & 45 & & \\
\hline 50 & \begin{tabular}{|l|}
2,267 \\
\end{tabular} & 1 & 2.268 & 10 & 1 & 12 & 44 & 0 & 44 & 0 & 0 & 0 & 2,324 & 46 & $.70,000$ & -120 \\
\hline
\end{tabular}
Notes:
${ }^{1}{ }_{\text {Initial }}=T_{\text {Time }} \mathrm{O}^{\prime}$ (i.e. at the completion of initial construction) ${ }^{2}$ Trans $=$ Transportation

Total EE (or Total GWP) = Total embodied energy (or total embodied GWP) of building component after lifespan (i.e. total man
construction + total maintenance + total end-oflife effects)

${ }^{4}$ Total EE (or Total GWP) per $\mathrm{m}^{2}=$ Total EE (or Total GWP) of building
component / area of building component that was modelled in ATHENAQ EIE Total Difference in Operating Energy (or GWP) from Baseline after trom the base component instead of the baseline component

Total Difference in Operating Energy (or GWP) from Baseline after Lifespan per $m^{2}=$ Total difference in operating energy (or GWP) from

"Total operating primary energy use of baseline retail building after 50 years =
$50,700 \mathrm{G} J\left(1,745 \mathrm{MJ} / \mathrm{m}^{2} \mathrm{yr}\right)$ "Total operating GWP of baseline retail building after 50 years $=2,310$ tonnes "oftal operating $\mathrm{CO}_{2}$ eq. $\left(80 \mathrm{~kg}\right.$ of of $\mathrm{CO}_{2}$ eq. $\left./ \mathrm{m}^{2} / \mathrm{yr}\right)$

Net wall area of baseline retail building (gross wall area - openings)
ATHENA \& EIE Material List:
(Includes all materials after 50 50ears)
\begin{tabular}{|l|c|c|}
\hline \multicolumn{1}{|c|}{ Material List } & Quantities & Unit \\
\hline 3 mil Polyethylene & 54.0 & $\mathrm{~m} 2$ \\
\hline $\begin{array}{l}16 \mathrm{~mm} \text { Motisture Resistant Gypsum } \\
\text { Board }\end{array}$ & 56.0 & $\mathrm{~m} 2$ \\
\hline 16mm Regular Gypsum Board & 56.0 & $\mathrm{~m} 2$ \\
\hline Extruded Polystyrene & 208.6 & $\mathrm{~m} 2(25 \mathrm{~mm})$ \\
\hline Galvanized Sheet & 106.1 & $\mathrm{~kg}$ \\
\hline Galvanized Studs & 342.5 & $\mathrm{~kg}$ \\
\hline Joint Compound & 111.8 & $\mathrm{~kg}$ \\
\hline Modified Bitumen membrane & 68.2 & $\mathrm{~kg}$ \\
\hline Nails & 5.5 & $\mathrm{~kg}$ \\
\hline Paper Tape & 1.3 & $\mathrm{~kg}$ \\
\hline Pine Wood Bevel Siding & 160.4 & $\mathrm{~m} 2$ \\
\hline Screws Nuts \& Bolts & 3.9 & $\mathrm{~kg}$ \\
\hline Solvent Based Alkyd Paint & 19.6 & $\mathrm{~L}$ \\
\hline Water Based Latex Paint & 132.5 & $\mathrm{~L}$ \\
\hline
\end{tabular}

$$
\begin{aligned}
& \text { Notes: } \\
& 1 \text { Initial }=\text { Time ' } \\
& \text { ' (i.e. at the completion of initia }
\end{aligned}
$$

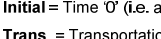

Trans = Transportation

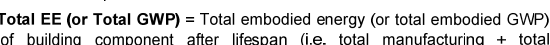

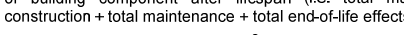

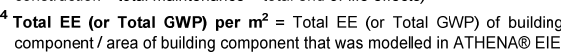

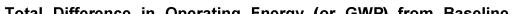

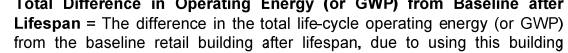

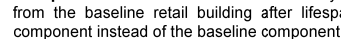

${ }^{6}$ Total Difference in Operating Energy (or GWP) from Baseline after

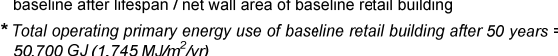

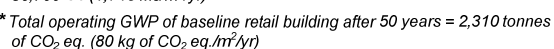

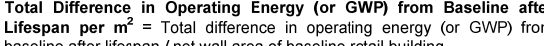




\section{Cold-Formed Steel Stud Wall \#17 (SS-W17)}

Building Component Description:

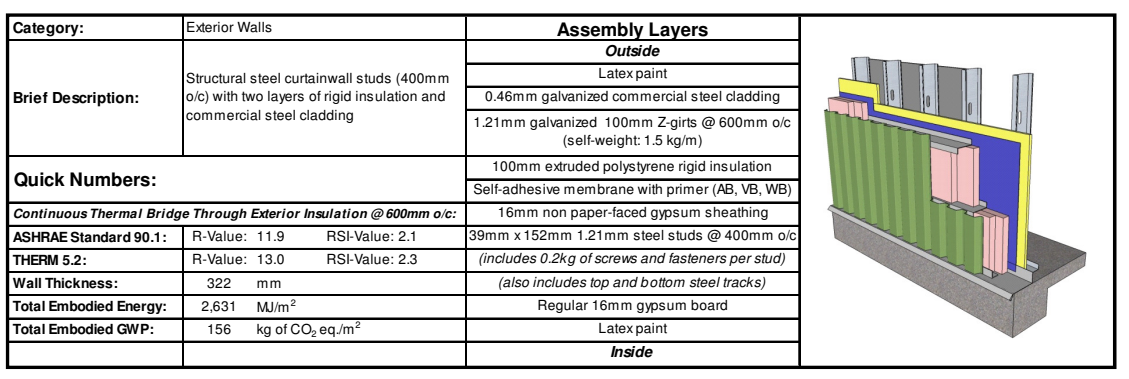

Life-Cycle Assessment Results:

Primary Energy Consumption (M

\begin{tabular}{|c|c|c|c|c|c|c|c|c|c|c|c|c|c|c|c|c|}
\hline \multirow{3}{*}{$\begin{array}{l}\text { Lifespan } \\
\text { (Yearss) }\end{array}$} & \multicolumn{14}{|c|}{ Embodied Energy (EE) } & \multirow{2}{*}{\multicolumn{2}{|c|}{$\begin{array}{l}\text { Difference in } \\
\text { OOperating Energy } \\
\text { from tasaseline after } \\
\text { Lifessan. }\end{array}$}} \\
\hline & \multicolumn{3}{|c|}{ Manufacturing } & \multicolumn{3}{|c|}{ Construction } & \multicolumn{3}{|c|}{ Maintenance } & \multicolumn{3}{|c|}{ End of Life } & \multirow{2}{*}{$\begin{array}{c}{ }^{3} \text { Total } \\
\mathrm{EE}\end{array}$} & \multirow{2}{*}{\begin{tabular}{|l|}
${ }^{4}$ Total \\
EE \\
per m² \\
\end{tabular}} & & \\
\hline & Material & ${ }^{2}$ Trans. & Total & Materia & ${ }^{2}$ Trans. & Total & Materia & \begin{tabular}{|l|}
${ }^{2}$ Trans. \\
\end{tabular} & Total & Material & Trans. & Total & & & ${ }^{5}$ Total & ${ }^{6}$ per m \\
\hline${ }^{\prime}$ Initial & 129,617 & 247 & 129,864 & 160 & 639 & 799 & 0 & 0 & 0 & 0 & 0 & 0 & 130,664 & 2,566 & 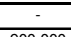 & \\
\hline 50 & 129,617 & 247 & 129,864 & 160 & 639 & 799 & & & & & & & & & 900,000 & 1,549 \\
\hline
\end{tabular}

Global Warming Potential $\left(\mathrm{kg}\right.$ of $\mathrm{CO}_{2}$ eq.)

\begin{tabular}{|c|c|c|c|c|c|c|c|}
\hline \multirow{3}{*}{\begin{tabular}{|c|} 
Lifespan \\
(Years)
\end{tabular}} & \\
\hline & \multicolumn{3}{|c|}{ Manufacturing } & \multicolumn{3}{|c|}{ Construction } & \\
\hline & Material & ${ }^{2}$ Trans. & \begin{tabular}{|l|} 
Total \\
\end{tabular} & Materia & 1) ${ }^{2}$ Trans. & Total & late \\
\hline${ }^{1}$ Initial & 7,912 & 0 & 7,913 & 10 & 1 & 12 & \\
\hline & 7,912 & 0 & & 10 & & 12 & \\
\hline \multicolumn{8}{|c|}{$\begin{array}{l}\text { Embodied energy (and GWP) numbers are based on an area of wall } \\
\text { Net wall area of baseline retail building (gross wall area - openings) = }\end{array}$} \\
\hline \multicolumn{8}{|c|}{$\begin{array}{c}\text { ATHENA @ EIE Material List: } \\
\text { (Includes all materials after 50 years) }\end{array}$} \\
\hline \multicolumn{4}{|c|}{ Material List } & \multicolumn{2}{|c|}{ Quantities } & \multicolumn{2}{|c|}{ Unit } \\
\hline \multicolumn{4}{|c|}{3 mil Polyethylene } & \multicolumn{2}{|r|}{54.0} & \multicolumn{2}{|c|}{$\mathrm{m} 2$} \\
\hline \multicolumn{4}{|l|}{$\begin{array}{l}16 \mathrm{~mm} 1 \\
\text { Board }\end{array}$} & & 56.0 & \multicolumn{2}{|c|}{$\mathrm{m} 2$} \\
\hline \multicolumn{4}{|c|}{ 16mm Regular Gypsum Board } & & 56.0 & \multicolumn{2}{|c|}{$\mathrm{m} 2$} \\
\hline \multicolumn{5}{|c|}{ 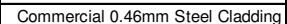 } & 168.0 & \\
\hline \multicolumn{4}{|c|}{ Extruded Polystyrene } & & 208.6 & & \\
\hline \multicolumn{4}{|c|}{ Galvanized Studs } & & 473.8 & \multicolumn{2}{|c|}{$\mathrm{m} 2(25 \mathrm{~mm})$} \\
\hline \multicolumn{4}{|c|}{ Joint Compound } & & 111.8 & \multicolumn{2}{|c|}{$\frac{\mathrm{kg}}{\mathrm{kg}}$} \\
\hline \multicolumn{4}{|c|}{ Modified Bitumen membrane } & & 68.2 & \multicolumn{2}{|c|}{$\mathrm{kg}$} \\
\hline \multicolumn{4}{|l|}{ Nails } & & 4.2 & \\
\hline \multicolumn{4}{|c|}{ Paper Tape } & & 1.3 & & \\
\hline \multicolumn{4}{|c|}{ Screws Nuts \& Bolts } & & 5.2 & \multicolumn{2}{|c|}{$\frac{\mathrm{gg}}{\mathrm{kg}}$} \\
\hline \multirow{2}{*}{\multicolumn{4}{|c|}{$\begin{array}{l}\text { Solvent Based Alkyd Paint } \\
\text { Water Based Latex Paint }\end{array}$}} & & 19.6 & \\
\hline & & & & & 132.5 & \multicolumn{2}{|c|}{$\frac{L}{L}$} \\
\hline
\end{tabular}

\section{Cold-Formed Steel Stud Wall \#18 (SS-W18)}

Building Component Description:

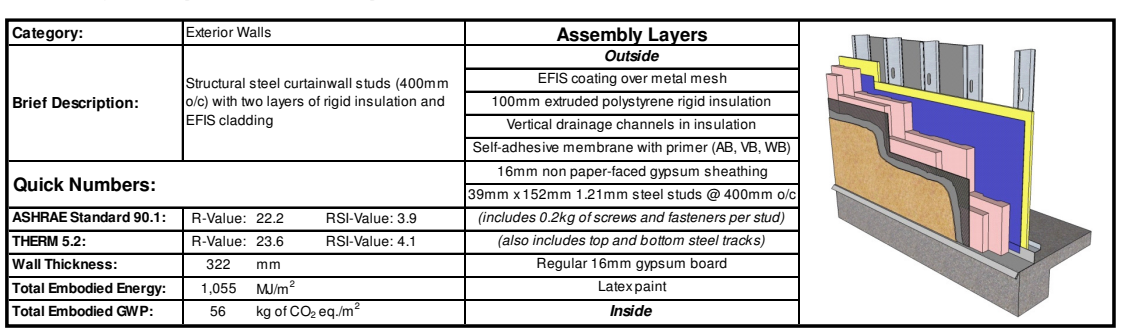

Life-Cycle Assessment Results:

Primary Energy Consumption (MJ)

\begin{tabular}{|c|c|c|c|c|c|c|c|c|c|c|c|c|c|c|c|c|}
\hline \multirow{3}{*}{$\mid \begin{array}{l}\text { Lifespan } \\
\text { (Years) }\end{array}$} & \multicolumn{14}{|c|}{ Embodied Energy (EE) } & \multirow{2}{*}{\multicolumn{2}{|c|}{$\begin{array}{c}\text { Difference in } \\
\text { Operating Energy } \\
\text { from Baseline after } \\
\text { Lifespan }\end{array}$}} \\
\hline & \multicolumn{3}{|c|}{ Manutacturing } & \multicolumn{3}{|c|}{ Construction } & \multicolumn{3}{|c|}{ Maintenance } & \multicolumn{3}{|c|}{ End of L Life } & \multirow{2}{*}{$\begin{array}{c}{ }^{3} \text { Total } \\
\text { EE }\end{array}$} & \multirow{2}{*}{\begin{tabular}{|c|c}
${ }^{4}$ Total \\
EE \\
per $m^{2}$
\end{tabular}} & & \\
\hline & Material & \begin{tabular}{|l|}
${ }^{2}$ Trans. \\
\end{tabular} & Total & \begin{tabular}{|l|} 
Material \\
\end{tabular} & ${ }^{2}$ Trans. & Total & Material & \begin{tabular}{|l|}
${ }^{2}$ Trans. \\
\end{tabular} & \begin{tabular}{|l|} 
Total \\
\end{tabular} & \begin{tabular}{|l|} 
Material \\
\end{tabular} & \begin{tabular}{|l|l|}
2 & Trans.
\end{tabular} & Total & & & ${ }^{5}$ Total & ${ }^{6}$ per $\mathrm{m}^{2}$ \\
\hline Initial & 48,911 & 301 & 49,212 & 160 & 729 & 889 & 0 & 0 & 0 & 0 & 0 & 0 & 50,100 & 984 & & \\
\hline 50 & 48,911 & 301 & 499,212 & 160 & \begin{tabular}{|l|}
729 \\
\end{tabular} & 889 & 3,099 & 10 & 3,110 & 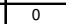 & 528 & 528 & & 55 & & \\
\hline
\end{tabular}

Global Warming Potential (kg of $\mathrm{CO}_{2}$ eq.)

\begin{tabular}{|c|c|c|c|c|c|c|c|c|c|c|c|c|c|c|c|c|}
\hline \multirow{3}{*}{\begin{tabular}{|l} 
Lifiespan \\
(Years)
\end{tabular}} & \multicolumn{14}{|c|}{ Embodied Global Warming Potential (GWP) } & \multirow{2}{*}{\multicolumn{2}{|c|}{$\begin{array}{l}\text { Difference in } \\
\text { Operating GWP from } \\
\text { Baseline after } \\
\text { Lifespan }\end{array}$}} \\
\hline & \multicolumn{3}{|c|}{ Manufacturing } & \multicolumn{3}{|c|}{ Construction } & \multicolumn{3}{|c|}{ Maintenance } & \multicolumn{3}{|c|}{ End of Life } & \multirow{2}{*}{$\begin{array}{l}{ }^{3} \text { Total } \\
\text { GWP }\end{array}$} & \multirow{2}{*}{$\begin{array}{l}{ }^{4}{ }^{4} \text { Total } \\
\text { GWP } \\
\text { per m }\end{array}$} & & \\
\hline & \begin{tabular}{|l|} 
Material \\
\end{tabular} & ${ }^{2}$ Trans. & \begin{tabular}{|l|l|} 
Total \\
\end{tabular} & Material & ${ }^{2}$ Trans. & Total & \begin{tabular}{|l|} 
Material \\
\end{tabular} & ${ }^{2}$ Trans. & Total & \begin{tabular}{|l|} 
Material \\
\end{tabular} & \begin{tabular}{|l|l|}
${ }^{2}$ Trans. \\
\end{tabular} & Total & & & ${ }^{5}$ Total & ${ }^{6}$ per $\mathrm{m}^{2}$ \\
\hline${ }^{1}$ nitial & \begin{tabular}{|l|}
2,813 \\
\end{tabular} & 1 & \begin{tabular}{|l|l|}
2,814 \\
\end{tabular} & 10 & 1 & 12 & 0 & 0 & 0 & 0 & 0 & 0 & 2,825 & 55 & & \\
\hline 50 & \begin{tabular}{|l|l|}
2,813 \\
\end{tabular} & 1 & 2,814 & 10 & 1 & 12 & 44 & 0 & 44 & 0 & 1 & 1 & 2,870 & 56 & 60,000 & -103 \\
\hline
\end{tabular}

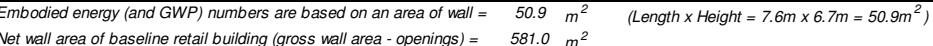

ATHENA $®$ EIE Material List:

(Includes all materials after 50 years)

Notes:
1 'Initial = Time 'o' (i.e. at the completion of nintial construction)

\begin{tabular}{|l|c|c|}
\hline \multicolumn{1}{|c|}{ Material List } & Quantities & Unit \\
\hline \#15 Organic Felt & 219.4 & $\mathrm{~m} 2$ \\
\hline 3 mil Polyethylene & 54.0 & $\mathrm{~m} 2$ \\
\hline $16 \mathrm{~mm}$ Moisture Resistant Gypsum & 56.0 & $\mathrm{~m} 2$ \\
\hline Board & 56.0 & $\mathrm{~m} 2$ \\
\hline $16 \mathrm{~mm}$ Regular Gypsum Board & 508.6 & $\mathrm{~m} 2(25 \mathrm{~mm})$ \\
\hline Extruded Polystyrene & 51.4 & $\mathrm{~kg}$ \\
\hline Galvanized Sheet & 342.5 & $\mathrm{~kg}$ \\
\hline Galvanized Studs & 111.8 & $\mathrm{~kg}$ \\
\hline Joint Compound & 68.2 & $\mathrm{~kg}$ \\
\hline Modified Bitumen membrane & 5.7 & $\mathrm{~kg}$ \\
\hline Naails & 1.3 & $\mathrm{~kg}$ \\
\hline Paper Tape & 3.9 & $\mathrm{~kg}$ \\
\hline Screws Nuts \& Bolts & 19.6 & $\mathrm{~L}$ \\
\hline Solvent Based Alkyd Paint & 136.0 & $\mathrm{~m} 2$ \\
\hline Stucco over metal mesh & 132.5 & $\mathrm{~L}$ \\
\hline Water Based Latex Paint & & \\
\hline & &
\end{tabular}

${ }^{2}$ Trans. = Transportation

${ }^{3}$ Total EE (or Total GWP) $=$ Total embodied energy (or total embodied GWP) of building component after lifespan (i.e. total manu
construction + total maintenance + total end-o-f-life effects)

${ }^{4}$ Total EE (or Total GWP) per $\mathrm{m}^{2}=$ Total EE (or Total GWP) of building
component / area of building component that was modelled in ATHENAQ EIE ${ }^{5}$ Total Difference in Operating Energy (or GWP) from Baseline after Lifespan = The difference in the total life-cycle operating energy (or GWP)
from the baseline retaib builing atter lifespan, due to using this building
component instead of the baseline component

Total Difference in Operating Energy (or GWP) from Baseline after * Total operating primary energy use of baseline retail building after 50 years =

of $\mathrm{CO}_{2}$ eq. $\left(80 \mathrm{~kg}\right.$ of $\mathrm{CO}_{2}$ eq. $\left./ \mathrm{m}^{2} \mathrm{yr}\right)$ Lifespan per $\mathrm{m}^{2}=$ Total difference in operating energy (or GWP) from 


\section{Cold-Formed Steel Stud Wall \#19 (SS-W19)}

Building Component Description:

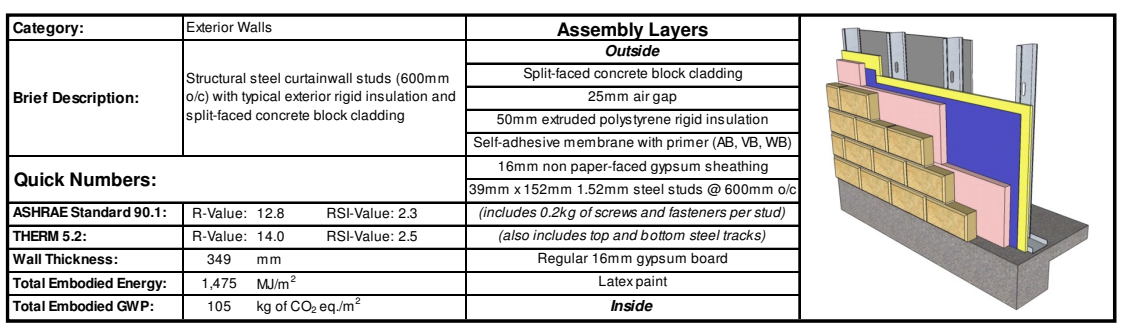

Life-Cycle Assessment Results:

Primary Energy Consumption (MJ)

\begin{tabular}{|c|c|c|c|c|c|c|c|c|c|c|c|c|c|c|c|c|}
\hline \multirow{3}{*}{$\begin{array}{l}\text { Lifespan } \\
\text { (Years) }\end{array}$} & \multicolumn{14}{|c|}{ Embodied Energy (EE) } & \multirow{2}{*}{\multicolumn{2}{|c|}{$\begin{array}{c}\text { Difference in } \\
\text { Operating Energy } \\
\text { from Baseline after } \\
\text { Lifespan }\end{array}$}} \\
\hline & \multicolumn{3}{|c|}{ Manufacturing } & \multicolumn{3}{|c|}{ Construction } & \multicolumn{3}{|c|}{ Maintenance } & \multicolumn{3}{|c|}{ End of Life } & \multirow{2}{*}{$\begin{array}{l}{ }^{3} \text { Total } \\
\mathrm{EE}\end{array}$} & \multirow{2}{*}{$\begin{array}{l}{ }^{4}{ }^{4} \text { Total } \\
\text { EE } \\
\text { per m }\end{array}$} & & \\
\hline & \begin{tabular}{|l|} 
Material \\
\end{tabular} & ${ }^{2}$ Trans. & Total & \begin{tabular}{|l|} 
Material \\
\end{tabular} & ${ }^{2}$ Trans. & Total & \begin{tabular}{|l|} 
Material \\
\end{tabular} & \begin{tabular}{|l}
${ }^{2}$ Trans. \\
\end{tabular} & Total & \begin{tabular}{|l|} 
Material \\
\end{tabular} & \begin{tabular}{|l|}
${ }^{2}$ Trans. \\
\end{tabular} & Total & & & ${ }^{5}$ Total & ${ }^{6}$ perm ${ }^{2}$ \\
\hline Initial & 66,710 & 837 & 67,547 & 160 & \begin{tabular}{|l|l|}
2,180 \\
\end{tabular} & 2,340 & 0 & 0 & 0 & 0 & 0 & 0 & \begin{tabular}{|l|}
69,887 \\
\end{tabular} & 1,372 & & \\
\hline 50 & \begin{tabular}{|l|l|l|}
66,710 \\
\end{tabular} & 837 & 67,547 & 160 & \begin{tabular}{|l|l|}
2,180 \\
\end{tabular} & 2,340 & \begin{tabular}{|l|l|}
3,099 \\
\end{tabular} & 10 & 3,110 & 1 & \begin{tabular}{|l|l|}
2,104 \\
\end{tabular} & 2,105 & 75,101 & 1,475 & 400,000 & 688 \\
\hline
\end{tabular}

Global Warming Potential ( $\mathrm{kg}$ of $\mathrm{CO}_{2}$ eq.)

\begin{tabular}{|c|c|c|c|c|c|c|c|c|c|c|c|c|c|c|c|c|}
\hline \multirow{3}{*}{$\begin{array}{l}\text { Lifespan } \\
\text { (Years) }\end{array}$} & \multicolumn{14}{|c|}{ Embodied Global Warming Potential (GWP) } & \multirow{2}{*}{\multicolumn{2}{|c|}{$\begin{array}{c}\text { Difierence in } \\
\text { Operating GWP from } \\
\text { Baseline after } \\
\text { Lifespan }\end{array}$}} \\
\hline & \multicolumn{3}{|c|}{ Manufacturing } & \multicolumn{3}{|c|}{ Construction } & \multicolumn{3}{|c|}{ Maintenance } & \multicolumn{3}{|c|}{ End of Life } & \multirow{2}{*}{$\begin{array}{l}{ }^{3} \text { Total } \\
\text { GWP }\end{array}$} & \multirow{2}{*}{$\begin{array}{l}{ }^{4}{ }^{4} \text { otal } \\
\text { GWP } \\
\text { perm }\end{array}$} & & \\
\hline & Material & ${ }^{2}$ Trans. & Total & Material & ${ }^{2}$ Trans. & Total & Material & ${ }^{2}$ Trans. & Total & Material & $\mid{ }^{2}$ Trans. & Total & & & ${ }^{5}$ Total & ${ }^{6} \mathrm{perm}^{2}$ \\
\hline Initial & 5,307 & 2 & 5,308 & 10 & - & 15 & 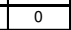 & 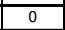 & 0 & 0 & 0 & 0 & 5,323 & 105 & & \\
\hline 50 & 5,307 & 2 & 5,308 & 10 & 4 & 15 & 44 & 0 & 44 & 0 & 4 & 4 & 5,370 & 105 & 20,000 & 34 \\
\hline
\end{tabular}

ATHENA $®$ EIE Material List:

\begin{tabular}{|c|c|c|}
\hline Material List & Quantities & Unit \\
\hline 3 mil Polyethylene & 54.0 & $\mathrm{~m} 2$ \\
\hline $\begin{array}{l}16 \mathrm{~mm} \text { Moisture Resistant Gypsum } \\
\text { Board }\end{array}$ & 56.0 & $\mathrm{~m} 2$ \\
\hline 16mm Regular Gypsum Board & 56.0 & $\mathrm{~m} 2$ \\
\hline Cold Rolled Sheet & 10.3 & $\mathrm{~kg}$ \\
\hline Extruded Polystyrene & 104.3 & m2 (25mm) \\
\hline Galvanized Studs & 300.5 & $\mathrm{~kg}$ \\
\hline Joint Compound & 111.8 & $\mathrm{~kg}$ \\
\hline Modified Bitumen membrane & 401.5 & $\mathrm{~kg}$ \\
\hline Mortar & 4.3 & $\mathrm{~m} 3$ \\
\hline Nails & 4.2 & $\mathrm{~kg}$ \\
\hline Paper Tape & 1.3 & $\mathrm{~kg}$ \\
\hline Screws Nuts \& Bolts & 2.6 & $\mathrm{~kg}$ \\
\hline Solvent Based Alkyd Paint & 19.6 & $\mathrm{~L}$ \\
\hline Split-faced Concrete Block & $1,238.2$ & Blocks \\
\hline Water Based Latex Paint & 66.3 & L \\
\hline
\end{tabular}

\section{Cold-Formed Steel Stud Wall \#20 (SS-W20)}

Building Component Description:

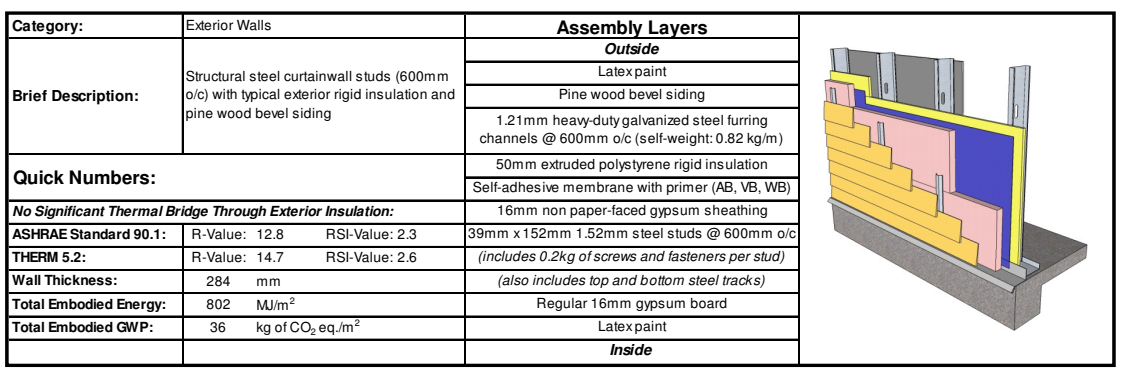

\section{Life-Cycle Assessment Results:}

\begin{tabular}{|c|c|c|c|c|c|c|c|c|c|c|c|c|c|c|c|c|}
\hline \multirow{4}{*}{$\begin{array}{l}\text { Lifespan } \\
\text { (Years) }\end{array}$} & \multicolumn{14}{|c|}{ sumption } & \multirow{3}{*}{\multicolumn{2}{|c|}{$\begin{array}{l}\text { Difference in } \\
\text { Operating Energy } \\
\text { from Baseline after } \\
\text { Lifespan }\end{array}$}} \\
\hline & \multicolumn{14}{|c|}{ Embodied Energy (EE) } & & \\
\hline & \multicolumn{3}{|c|}{ Manutacturing } & \multicolumn{3}{|c|}{ Construction } & \multicolumn{3}{|c|}{ Maintenance } & \multicolumn{3}{|c|}{ End of Life } & \multirow{2}{*}{\begin{tabular}{|c|}
${ }^{3}{ }^{3}$ otal \\
$\mathrm{EE}$
\end{tabular}} & \multirow{2}{*}{$\begin{array}{c}{ }^{4} \text { Total } \\
\mathrm{EE} \\
\text { per } \mathrm{m}^{2}\end{array}$} & & \\
\hline & Material & 整Trans. & \begin{tabular}{|l|} 
Total \\
\end{tabular} & Material & Trans. & \begin{tabular}{|l|l|} 
Total \\
\end{tabular} & Material & $\begin{array}{l}\text { Trans. } \\
\end{array}$ & Total & $\mid$\begin{tabular}{|c|} 
Material \\
a
\end{tabular} & ${ }^{2}$ Trans. & \begin{tabular}{|l|} 
Total \\
\end{tabular} & & & 每 ${ }^{5}$ total & $6^{6}$ per m ${ }^{2}$ \\
\hline Initial & 36,414 & 291 & 36,706 & 160 & 719 & 879 & 0 & 0 & 0 & 0 & 0 & 0 & 37,585 & \begin{tabular}{|l|l|}
738 & \\
\end{tabular} & & \\
\hline 50 & 36,414 & 291 & $\begin{array}{l}36,706 \\
\end{array}$ & 160 & 719 & 879 & 3,099 & 10 & 3,110 & 0 & 168 & 168 & \begin{tabular}{|l|l|}
40,862 \\
\end{tabular} & 802 & 400,000 & 688 \\
\hline
\end{tabular}

Global Warming Potential ( $k g$ of $\mathrm{CO}_{2}$ eq.)

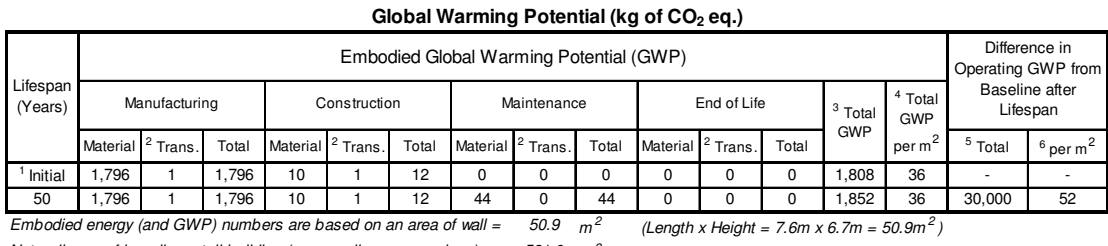

Notes:
${ }^{1}$ Initial = Time 'o' (i.e. at the completion of initial construction)

${ }^{2}$ Trans. $=$ Transportation

Total EE (or Total GWP) = Total embodied energy (or total embodied GWP) of building component after lifespan (i.e. total man
construction + total maintenance + total enc-oflife effects)

${ }^{4}$ Total EE (or Total GWP) per $\mathrm{m}^{2}=$ Total EE (or Total GWP) of building
component/ area of building component that was modelled in ATHENAQ EIE ${ }^{5}$ Total Difference in Operating Energy (or GWP) from Baseline after Lifespan = The difierence in the total life-cycle operating energy (or GWP)
from the baseline retail building after lifespan, due to using this building from the baseline retail building after lifespen,
component instead of the baseline component

Total Difference in Operating Energy (or GWP) from Baseline after Lifespan per $\mathrm{m}^{2}=$ Total difference in operating energy (or GWP) fro

* Total operating primary energy use of baseline retail building after 50 years =

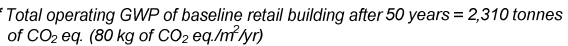

\begin{tabular}{|c|c|c|}
\hline Material List & Quantities & Unit \\
\hline 3 mil Polyethylene & 54.0 & $\mathrm{~m} 2$ \\
\hline $\begin{array}{l}16 \mathrm{~mm} \text { Moisture Resistant Gypsum } \\
\text { Board }\end{array}$ & 56.0 & $\mathrm{~m} 2$ \\
\hline 16mm Regular Gypsum Board & 56.0 & $\mathrm{~m} 2$ \\
\hline Extruded Polystyrene & $\frac{104.3}{1.3}$ & m2 $225 \mathrm{~mm})$ \\
\hline Galvanized Sheet & 106.1 & $\mathrm{~kg}$ \\
\hline Galvanized Studs & 300.5 & $\mathrm{~kg}$ \\
\hline Joint Compound & $\overline{111.8}$ & $\mathrm{~kg}$ \\
\hline Modified Bitumen membrane & 68.2 & $\mathrm{~kg}$ \\
\hline Nails & 5.5 & $\mathrm{~kg}$ \\
\hline Paper Tape & 1.3 & $\mathrm{~kg}$ \\
\hline Pine Wood Bevel Siding & 160.4 & $\mathrm{~m} 2$ \\
\hline Screws Nuts \& Bolts & 2.6 & $\mathrm{~kg}$ \\
\hline Solvent Based Alkyd Paint & 19.6 & $\mathrm{~L}$ \\
\hline Water Based Latex Paint & 132.5 & $\bar{L}$ \\
\hline
\end{tabular}

'Initial = Time ' $O$ ' $($ ie. at the completes:

Total EE (or Total GWP) = Total embodied energy (or total embodied GWP) of building component after lifespan (i.e. total manufacturing + total ${ }^{4}$ Total EE (or Total GWP) per $\mathrm{m}^{2}=$ Total EE (or Total GWP) of building Total Difference in Operating Energy (or GWP) from Baseline after
Lifespan = The edifference in the total life-cycle operating energy (or GWP)
from the baseline retail building after lifespan, due to using this building

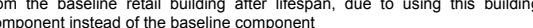
Total Difference in Operating Energy (or GWP) from Baseline after Lifespan per $\mathrm{m}^{2}=$ Total difference in operating energy (or GWP) from
baseline after lifespan / net wall area of baseline retail building * Total operating primary energy use of baseline retail building after 50 years =
$50,700 \mathrm{GJ}\left(1,745 \mathrm{MJ} / \mathrm{m}^{2} / \mathrm{yr}\right)$ * Total operating $\mathrm{GWP}$ of baseline retail building after 50 years $=2,310$ tonnes
of $\mathrm{CO}_{2}$ eq. $\left(80 \mathrm{~kg}\right.$ of $\mathrm{CO}_{2}$ eq/ $\left./ \mathrm{m}^{2} \mathrm{yrr}\right)$
'Initial $=$ Time 'o' (i.e. at 


\section{Cold-Formed Steel Stud Wall \#21 (SS-W21)}

Building Component Description:

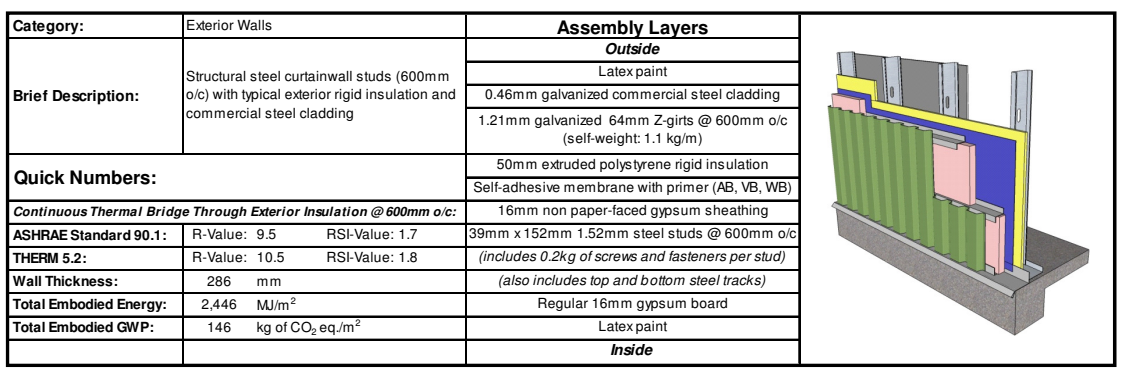

Life-Cycle Assessment Results:

Primary Energy Consumption (M)

\begin{tabular}{|c|c|c|c|c|c|c|c|c|c|c|c|c|c|c|}
\hline \multirow{3}{*}{$\begin{array}{c}\text { Lifespan } \\
\text { (Years) }\end{array}$} & \multicolumn{12}{|c|}{ Embodied Energy (EE) } & \multirow{2}{*}{\multicolumn{2}{|c|}{$\begin{array}{c}\text { Difference in } \\
\text { Operating Energy } \\
\text { from Baseline after } \\
\text { Lifespan }\end{array}$}} \\
\hline & Manufacturin & & Constructio & & & intenance & & & End of Life & & ${ }^{3}$ Tota & $\begin{array}{l}\text { To } \\
\text { Et }\end{array}$ & & \\
\hline & 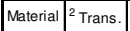 & Total & 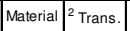 & Total & Material| 2 & ${ }^{2}$ Trans. & Total & Material & Trans. & Total & & per & & ${ }^{6}$ per $\mathrm{m}^{2}$ \\
\hline
\end{tabular}
\begin{tabular}{|c|c|c|c|c|c|c|c|c|c|c|c|c|c|c|c|c|}
\hline Initial & 120,264 & 244 & 120,508 & 160 & 619 & 779 & 0 & 0 & 0 & 0 & 0 & 0 & 121,287 & 2,382 & - & - \\
\hline 50 & 120,264 & 244 & 120,508 & 160 & 619 & 779 & 3,099 & 10 & 3,110 & 0 & 161 & 161 & 124,558 & 2,446 & $1,900,000$ & 3,270 \\
\hline
\end{tabular}

Global Warming Potential ( $\left(\mathrm{g}\right.$ of $\mathrm{CO}_{2}$ eq.)

\begin{tabular}{|c|c|c|c|c|c|c|c|c|c|c|c|c|c|c|c|c|}
\hline \multirow{3}{*}{$\begin{array}{l}\text { Lifespan } \\
\text { Years }\end{array}$} & \multicolumn{14}{|c|}{ Embodied Global Warming Potential (GWP) } & \multirow{2}{*}{\multicolumn{2}{|c|}{$\begin{array}{l}\text { Difference in } \\
\text { Operating GWP from } \\
\text { Baseline after } \\
\text { Lifespan }\end{array}$}} \\
\hline & \multicolumn{3}{|c|}{ Manufacturing } & \multicolumn{3}{|c|}{ Construction } & \multicolumn{3}{|c|}{ Maintenance } & \multicolumn{3}{|c|}{ End of Life } & \multirow{2}{*}{$\begin{array}{l}{ }^{3} \text { Total } \\
\text { GWP }\end{array}$} & \multirow{2}{*}{$\begin{array}{l}{ }^{4}{ }^{4} \text { otal } \\
\text { GWP } \\
\text { perm }\end{array}$} & & \\
\hline & Material & ${ }^{2}$ Trans. & Total & Material & ${ }^{2}$ Trans. & Total & Material & ${ }^{2}$ Trans. & Total & Material & $\mid{ }^{2}$ Trans. & Total & & & ${ }^{5}$ Total & ${ }^{6}$ per m ${ }^{2}$ \\
\hline Initial & 7,382 & 0 & 7,383 & 10 & 1 & 12 & 0 & 0 & 0 & 0 & 0 & 0 & 7,394 & 145 & & \\
\hline 50 & \begin{tabular}{|l|l}
7,382 \\
\end{tabular} & 0 & 7,383 & 10 & 1 & 12 & $\begin{array}{l}44 \\
\end{array}$ & 0 & 44 & 0 & 0 & 0 & \begin{tabular}{|l|l|l|l}
7,48 \\
\end{tabular} & 146 & $\mid$\begin{tabular}{|l|l|}
110,000 \\
\end{tabular} & 189 \\
\hline
\end{tabular}

Net wall area of baseline retail building (gross wall area - openings) $=581.0 \mathrm{~m}^{2}$

ATHENA ® EIE Material List:

\begin{tabular}{|l|c|c|}
\hline \multicolumn{1}{|c|}{ Material List } & Quantities & Unit \\
\hline 3 mil Polyethylene & 54.0 & $\mathrm{~m} 2$ \\
\hline 16mm Moisture Resistant Gypsum & 56.0 & $\mathrm{~m} 2$ \\
Board & 56.0 & $\mathrm{~m} 2$ \\
\hline 16mm Regular Gypsum Board & 56.0 & $\mathrm{~m} 2$ \\
\hline Commercial 0.46mm Steel Cladding & 168.0 & $\mathrm{~m}$ \\
\hline Extruded Polystyrene & 104.3 & $\mathrm{~m} 2(25 \mathrm{~mm})$ \\
\hline Galvanized Studs & 401.5 & $\mathrm{~kg}$ \\
\hline Joint Compound & 111.8 & $\mathrm{~kg}$ \\
\hline Modified Bitumen membrane & 68.2 & $\mathrm{~kg}$ \\
\hline Nails & 4.2 & $\mathrm{~kg}$ \\
\hline Paper Tape & 1.3 & $\mathrm{~kg}$ \\
\hline Screws Nuts \& Bolts & 3.9 & $\mathrm{~kg}$ \\
\hline Solvent Based Alkyd Paint & 19.6 & $\mathrm{~L}$ \\
\hline Water Based Latex Paint & 132.5 & $\mathrm{~L}$ \\
\hline
\end{tabular}

Notes:
${ }^{1}$ Initial $=$ Time ' ${ }^{\prime}$ (i.e. at the completion of initial construction)

${ }^{1}$ Initial $=$ Time ' $O$ ' (i.e. at

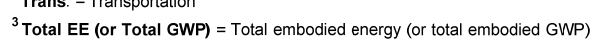
Total
of building component after lifespan (i.e. total manaufacturing + total
construction + total maintenance + total enc-oflife effects)

${ }^{4}$ Total EE (or Total GWP) per $\mathrm{m}^{2}=$ Total EE (or Total GWP) of building
component/ area of building component that was modelled in ATHENA@ EIE 5 Total Difference in Operating Energy (or GWP from Baseline after
Lifespan = The difference in the total lifecycle operating energy (or GWP) Lifespan = The difference in the total lite-cycle operating energy (or GWr
from the baseline retail building after lifespan, due to using this building ${ }^{6}$ Total Difference in Operating Energy (or GWP) from Baseline after Lifespan per $\mathrm{m}^{2}=$ Total difference in operating energy (or GWP) from * Total operating primary energy
$50,700 \mathrm{GJ}\left(1,745 \mathrm{MJ} / \mathrm{m}^{2} \mathrm{yr}\right)$

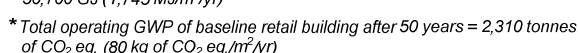

\section{Cold-Formed Steel Stud Wall \#22 (SS-W22)}

Building Component Description:

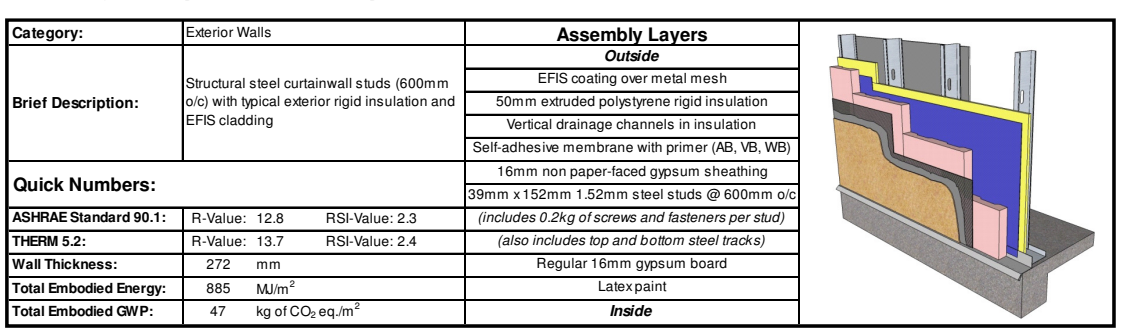

Life-Cycle Assessment Results:

Primary Energy Consumption (MJ)

\begin{tabular}{|c|c|c|c|c|c|c|c|c|c|c|c|c|c|c|c|c|}
\hline \multirow{3}{*}{\begin{tabular}{|l} 
Litespan \\
(Years)
\end{tabular}} & \multicolumn{14}{|c|}{ Embodied Energy (EE) } & \multirow{2}{*}{\multicolumn{2}{|c|}{$\begin{array}{c}\text { Difference in } \\
\text { Operating Energy } \\
\text { from Baseline after } \\
\text { Lifespan }\end{array}$}} \\
\hline & \multicolumn{3}{|c|}{ Manufacturing } & \multicolumn{3}{|c|}{ Construction } & \multicolumn{3}{|c|}{ Maintenance } & \multicolumn{3}{|c|}{ End of Life } & \multirow{2}{*}{$\begin{array}{c}{ }^{3}{ }^{3} \text { otal } \\
\mathrm{EE}\end{array}$} & \multirow{2}{*}{\begin{tabular}{c|}
${ }^{4} \mathrm{Total}$ \\
$\mathrm{EE}$ \\
$\mathrm{Eer} \mathrm{m}^{2}$
\end{tabular}} & & \\
\hline & Material & ${ }^{2}$ Trans. & \begin{tabular}{|l|l} 
Total \\
\end{tabular} & Material & ${ }^{2}$ Trans. & Total & Material & ${ }^{2}$ Trans. & Total & Material & $\begin{array}{ll}2 & 2 \\
2\end{array}$ & Total & & & ${ }^{5}$ Total & ${ }_{6}^{6} \operatorname{perm}^{2}$ \\
\hline Initial & 40,281 & 299 & 40,580 & 160 & 712 & 872 & 0 & 0 & 0 & 0 & 0 & 0 & 41,452 & 814 & & \\
\hline 50 & 40,281 & 299 & 40,580 & 160 & 712 & 872 & 3,099 & 10 & 3,110 & 0 & 517 & 517 & \begin{tabular}{|l|l}
445,079 \\
\end{tabular} & 885 & 500,000 & 1,033 \\
\hline
\end{tabular}

Global Warming Potential (kg of $\mathrm{CO}_{2}$ eq.)

\begin{tabular}{|c|c|c|c|c|c|c|c|c|c|c|c|c|c|c|c|c|}
\hline \multirow{3}{*}{ Lifespan } & \multicolumn{14}{|c|}{ Embodied Global Warming Potential (GWP) } & \multirow{2}{*}{\multicolumn{2}{|c|}{$\begin{array}{l}\text { Difference in } \\
\text { Operating GWP from } \\
\text { Baseline after } \\
\text { Lifespan }\end{array}$}} \\
\hline & \multicolumn{3}{|c|}{ Manutacturing } & \multicolumn{3}{|c|}{ Construction } & \multicolumn{3}{|c|}{ Maintenance } & \multicolumn{3}{|c|}{ End of Lifie } & \multirow{2}{*}{$\begin{array}{l}{ }^{3} \text { Total } \\
\text { GWP }\end{array}$} & \multirow{2}{*}{$\begin{array}{c}{ }^{4} \text { Total } \\
\text { GWP } \\
\text { per m }{ }^{2}\end{array}$} & & \\
\hline & Material & ${ }^{2}$ Trans. & Total & Material & ${ }^{2}$ Trans. & Total & Material & Trans. & Total & Material & 2 Trans. & Total & & & ${ }^{5}$ Total & ${ }^{6}$ per $\mathrm{m}^{2}$ \\
\hline Initial & 2,342 & 1 & 2,342 & 10 & 1 & 12 & 0 & 0 & 0 & 0 & 0 & 0 & 2,354 & 46 & & \\
\hline 50 & \begin{tabular}{|l|}
2.342 \\
\end{tabular} & 1 & 2.342 & 10 & 1 & 12 & 44 & 0 & 44 & 0 & 1 & 1 & 2,398 & 47 & 40,000 & 69 \\
\hline
\end{tabular}

Net wall are of boseline retal bulding (ross wall area-openings) $=581.0 \mathrm{~m}^{2}$

ATHENA ® EIE Material List:

\begin{tabular}{|c|c|c|}
\hline Material List & Quantities & Unit \\
\hline$\# 15$ Organic Felt & 219.4 & $\mathrm{~m} 2$ \\
\hline 3 mil Polyethylene & 54.0 & $\mathrm{~m} 2$ \\
\hline $\begin{array}{l}16 \mathrm{~mm} \text { Moisture Resistant Gypsum } \\
\text { Board }\end{array}$ & 56.0 & $\mathrm{~m} 2$ \\
\hline 16mm Regular Gypsum Board & 56.0 & $\mathrm{~m} 2$ \\
\hline Extruded Polystyrene & 104.3 & m2 (25mm) \\
\hline Galvanized Sheet & 51.4 & $\mathrm{~kg}$ \\
\hline Galvanized Studs & 300.5 & $\mathrm{~kg}$ \\
\hline Joint Compound & 111.8 & $\mathrm{~kg}$ \\
\hline Modified Bitumen membrane & 68.2 & $\mathrm{~kg}$ \\
\hline $\begin{array}{lll}\text { Nails } \\
\text { nats }\end{array}$ & 5.7 & $\mathrm{~kg}$ \\
\hline Paper Tape & 1.3 & $\mathrm{~kg}$ \\
\hline Screws Nuts \& Bolts & 2.6 & $\mathrm{~kg}$ \\
\hline Solvent Based Alkyd Paint & 19.6 & 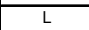 \\
\hline Stucco over metal mesh & 136.0 & $\mathrm{~m} 2$ \\
\hline Water Based Latex Paint & 132.5 & L \\
\hline
\end{tabular}

Notes:
1 'Initial = Time 'o' (i.e. at the completion of nintial construction)

${ }^{2}$ Trans. = Transportation

${ }^{3}$ Total EE (or Total GWP) $=$ Total embodied energy (or total embodied GWP) of building component after lifespan (i.e. total mal
construction + total maintenance + total end-oflife effects)

${ }^{4}$ Total EE (or Total GWP) per $\mathrm{m}^{2}=$ Total EE (or Total GWP) of building
component /area of building component that was modelled in ATHENAQ EIE ${ }^{5}$ Total Difference in Operating Energy (or GWP) from Baseline after Litespan = The difference in the total life-cycle operating energy (or GWP
from the baseline retail building after lifespan, due to using this building component instead of the baseline component

${ }^{6}$ Total Difference in Operating Energy (or GWP) from Baseline after Lifespan per $\mathrm{m}^{2}=$ Total difference in operating energy (or GWP) from * Total operating primary energy use of baseline retail building after 50 years =

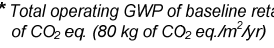




\section{Cold-Formed Steel Stud Wall \#23 (SS-W23)}

Building Component Description:

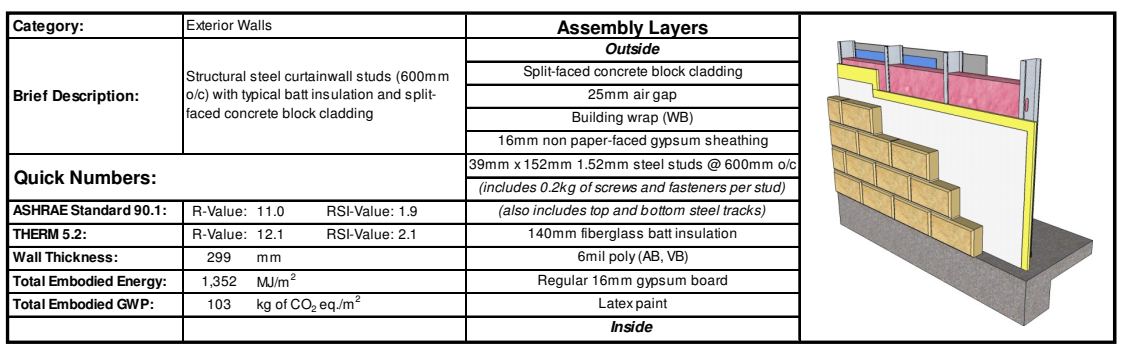

Life-Cycle Assessment Results:

Primary Energy Consumption (MJ)

\begin{tabular}{|c|c|c|c|c|c|c|c|c|c|c|c|c|c|c|c|c|}
\hline \multirow{3}{*}{$\begin{array}{l}\text { Lifespar } \\
\text { Yyears) }\end{array}$} & \multicolumn{14}{|c|}{ Embodied Energy (EE) } & \multirow{2}{*}{\multicolumn{2}{|c|}{$\begin{array}{c}\text { Difference in } \\
\text { Operatiting Energy } \\
\text { from Baseline atter } \\
\text { Liffessan }\end{array}$}} \\
\hline & \multicolumn{3}{|c|}{ Manufacturing } & \multicolumn{3}{|c|}{ Construction } & \multicolumn{3}{|c|}{ Maintenance } & \multicolumn{3}{|c|}{ End of Life } & \multirow{2}{*}{${ }^{3}{ }^{3}$ Total } & \multirow{2}{*}{\begin{tabular}{|l|l|}
${ }^{4}$ Total \\
EE \\
DE $^{2}$
\end{tabular}} & & \\
\hline & Material & ${ }^{2}$ Trans. & \begin{tabular}{|l|l} 
Total \\
\end{tabular} & Materia & ${ }^{2}$ Trans. & Total & \begin{tabular}{|l|l|} 
Materia \\
\end{tabular} & ${ }^{2}$ Trans. & \begin{tabular}{|l|l|l|} 
Total & \\
\end{tabular} & Material & ${ }^{2}$ Trans. & \begin{tabular}{|l|l|l|} 
Total \\
\end{tabular} & & & ${ }^{5}$ Total & ${ }^{6}$ perm ${ }^{2}$ \\
\hline Initial & 63,531 & 862 & 64,393 & 160 & 2,179 & \begin{tabular}{|l|l|}
2,339 \\
\end{tabular} & 0 & 0 & 0 & 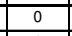 & 0 & 0 & 66,732 & \begin{tabular}{|l|}
1,311 \\
\end{tabular} & & \\
\hline 50 & 63,531 & 862 & 64,393 & 160 & \begin{tabular}{|l|l|} 
\\
\end{tabular} & \begin{tabular}{|l|l|}
2,339 \\
\end{tabular} & 0 & 0 & 0 & 1 & \begin{tabular}{|l|l|}
2,107 \\
\end{tabular} & 2,108 & $\begin{array}{ll}68,840 \\
\end{array}$ & 1,352 & 900,000 & 1,549 \\
\hline
\end{tabular}

Global Warming Potential ( $\mathrm{kg}$ of $\mathrm{CO}_{2}$ eq.)

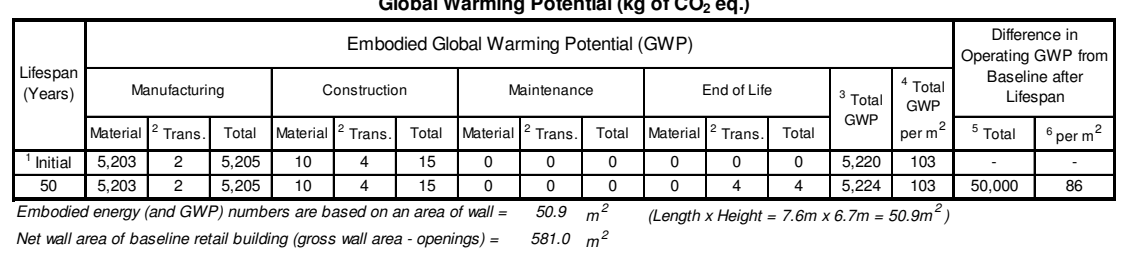

ATHENA $®$ EIE Material List:

\begin{tabular}{|c|c|c|}
\hline Material List & Quantities & Unit \\
\hline $\begin{array}{l}16 \mathrm{~mm} \text { Moisture Resistant Gypsum } \\
\text { Board }\end{array}$ & 56.0 & $\mathrm{~m} 2$ \\
\hline 16mm Regular Gypsum Board & 56.0 & 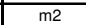 \\
\hline 6 mil Polyythylene & 108.0 & $\mathrm{~m} 2$ \\
\hline Batt. Fiberglass & 289.8 & m2 $25 \mathrm{~mm}$ \\
\hline Cold Rolled Sheet & 10.3 & $\mathrm{~kg}$ \\
\hline Galvanized Studs & 300.5 & $\mathrm{~kg}$ \\
\hline Joint Compound & 111.8 & $\mathrm{~kg}$ \\
\hline Modified Bitumen membrane & 333.3 & $\mathrm{~kg}$ \\
\hline Mortar & 4.3 & $\mathrm{~m} 33$ \\
\hline Nails & 4.2 & $\mathrm{~kg}$ \\
\hline Paper Tape & 1.3 & $\mathrm{~kg}$ \\
\hline Screws Nuts \& Bolts & 2.6 & $\mathrm{~kg}$ \\
\hline Split-faced Concrete Block & $1,238.2$ & Blocks \\
\hline Water Based Latex Paint & 66.3 & $\mathrm{~L}_{\mathrm{L}}$ \\
\hline
\end{tabular}

${ }^{1}$ Initial = Time '0' (i.e. at

${ }^{3}$ Total EE (or Total GWP) $=$ Total embodied energy (or total embodied GWP of building component after lifespan (i.e. total maal
construction + total maintenance + total end-of-life effects)

${ }^{4}$ Total EE (or Total GWP) per $\mathrm{m}^{2}=$ Total EE (or Total GWP) of building Lifespan = The difierence in the total life-cycle operating energy yor GWP)
from the baseline retail building after lifespan, due to using this building component instead of the baseline component

Total Difference in Operating Energy (or GWP) from Baseline aftor Lifespan per $\mathrm{m}^{2}=$ Total difference in operating energy (or GWP) fro

* Total operating primary energy use of baseline retail building after 50 years =

Total operating GWP of baseline retail building after 50 years $=2,310$ tonnes

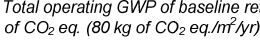

\section{Cold-Formed Steel Stud Wall \#24 (SS-W24)}

Building Component Description:

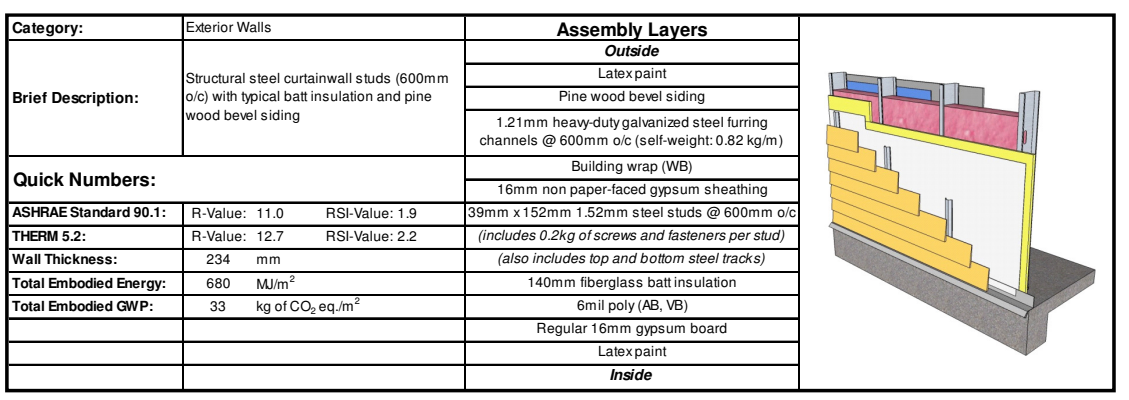

Life-Cycle Assessment Results:

Primary Energy Consumption (MJ)

\begin{tabular}{|c|c|c|c|c|c|c|c|c|c|c|c|c|c|c|c|}
\hline \multirow{3}{*}{$\begin{array}{l}\text { Lifespan } \\
\text { (Years) }\end{array}$} & \multicolumn{13}{|c|}{ Embodied Energy (EE) } & \multirow{2}{*}{\multicolumn{2}{|c|}{$\begin{array}{l}\text { Difference in } \\
\text { Operating Energy } \\
\text { from Baseline after } \\
\text { Lifespan }\end{array}$}} \\
\hline & \multicolumn{2}{|c|}{ Manufacturing } & \multicolumn{3}{|c|}{ Construction } & \multicolumn{3}{|c|}{ Maintenance } & \multicolumn{3}{|c|}{ End of Lifie } & \multirow{2}{*}{$\begin{array}{c}{ }^{3} \text { Total } \\
\text { EE }\end{array}$} & \multirow{2}{*}{\begin{tabular}{|l|l}
${ }^{4}{ }^{T}$ I \\
EE
\end{tabular}} & & \\
\hline & 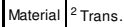 & Total & Material & |2 Trans. & Total & Material & ${ }^{2}$ Trans. & Total & Material & ${ }^{2}$ Trans. & Total & & & $\begin{array}{ll}{ }^{5} \text { Total } \\
\end{array}$ & ${ }^{6} \mathrm{perm}^{2}$ \\
\hline Initial & \begin{tabular}{l|l}
3,235 & 317 \\
\end{tabular} & 33,552 & 160 & 718 & 878 & 0 & 0 & 0 & 0 & 0 & 0 & 34,429 & 676 & & \\
\hline 50 & \begin{tabular}{|l|l|}
33,235 & 317 \\
\end{tabular} & 33,552 & 160 & 718 & 878 & 0 & $n^{2}+2$ & 0 & no & 171 & 171 & 34,600 & 680 & 900,000 & $\begin{array}{l}1,549 \\
\end{array}$ \\
\hline
\end{tabular}

Global Warming Potential (kg of $\mathrm{CO}_{2}$ eq.)

\begin{tabular}{|c|c|c|c|c|c|c|c|c|c|c|c|c|c|c|c|c|}
\hline & \multirow{2}{*}{\multicolumn{14}{|c|}{ Embodied Global Warming Potential (GWP) }} & & \\
\hline \multirow{3}{*}{$\begin{array}{l}\text { L Lestespan } \\
\text { (Years) }\end{array}$} & & & & & & & & & & & & & & & \multirow{2}{*}{\multicolumn{2}{|c|}{$\begin{array}{c}\text { Difference in } \\
\text { Operating GWP from } \\
\text { Baseline after } \\
\text { Lifespan }\end{array}$}} \\
\hline & \multicolumn{3}{|c|}{ Manutacturing } & \multicolumn{3}{|c|}{ Construction } & \multicolumn{3}{|c|}{ Maintenance } & \multicolumn{3}{|c|}{ End of Life } & \multirow{2}{*}{$\begin{array}{l}{ }^{3} \text { Total } \\
\text { GWPP }\end{array}$} & \multirow{2}{*}{$\begin{array}{c}{ }^{4} \text { Total } \\
\text { GWP } \\
\text { per } \mathrm{m}^{2}\end{array}$} & & \\
\hline & Material & ${ }^{2}$ Trans. & Total & Material & ${ }^{2}$ Trans. & Total & Material & $\begin{array}{l}{ }^{2} \text { Trans. } \\
\end{array}$ & Total & \begin{tabular}{|l|} 
Material \\
\end{tabular} & 2 Trans. & Total & & & ${ }^{5}$ Total & ${ }^{6}{ }_{\text {perm }}^{2}{ }^{2}$ \\
\hline |nitital & $\begin{array}{l}1,693 \\
\end{array}$ & 1 & \begin{tabular}{|l|l|}
1,6993 \\
\end{tabular} & $\overline{10}$ & 1 & 12 & 0 & 0 & 0 & 0 & 0 & 0 & 1,705 & 33 & & \\
\hline 50 & \begin{tabular}{|l|l|}
1,6993 \\
\end{tabular} & 1 & \begin{tabular}{|l|l|l|}
1,693 \\
\end{tabular} & 10 & 1 & 12 & 0 & 0 & 0 & 0 & 0 & 0 & 1,705 & 33 & 50,000 & 86 \\
\hline
\end{tabular}

Ecocied energy (and GWP) numbers are based on an area of wall $=50.9 \mathrm{~m}^{2} \quad$ (Length $\times$ Height $=7.6 \mathrm{~m} \times 6.7 \mathrm{~m}=50.9 \mathrm{~m}^{2}$ )

Net wall area of baseline retail building (gross wall area - openings) $=581.0 \mathrm{~m}^{2}$

\section{ATHENA $®$ EIE Material List:} component/ area of building component that was modelled in ATHENAQ EIE Total Difference in Operating Energy (or GWP) from Baseline after

\begin{tabular}{|c|c|c|}
\hline Material List & Quantities & Unit \\
\hline $\begin{array}{l}16 \mathrm{~mm} \text { Moisture Resistant Gypsum } \\
\text { Board }\end{array}$ & 56.0 & $\mathrm{~m} 2$ \\
\hline 16mm Regular Gypsum Board & 56.0 & $\mathrm{~m} 2$ \\
\hline 6 mil Polyethylene & 108.0 & $\mathrm{~m} 2$ \\
\hline Batt. Fiberglass & 289.8 & m2 (25mm) \\
\hline Galvanized Sheet & 106.1 & $\mathrm{~kg}$ \\
\hline Galvanized Studs & 300.5 & $\mathrm{~kg}$ \\
\hline Joint Compound & 111.8 & $\mathrm{~kg}$ \\
\hline Nails & 5.5 & $\mathrm{~kg}$ \\
\hline Paper Tape & 1.3 & $\mathrm{~kg}$ \\
\hline Pine Wood Bevel Siding & 160.4 & $\mathrm{~m} 2$ \\
\hline Screws Nuts \& Bolts & 2.6 & $\mathrm{~kg}$ \\
\hline Water Based Latex Paint & 132.5 & $\mathrm{~L}$ \\
\hline
\end{tabular}

Notes:
${ }^{1}$ Initial = Time 'o' (i.e. at the completion of initial construction)

${ }^{2}$ Trans. = Transportation construction + total maintenanoe + total end-of-life effects)

${ }^{4}$ Total EE (or Total GWP) per $\mathrm{m}^{2}=$ Total EE (or Total GWP) of building
component/ area of building component that was modelled in ATHENAQ EIE Lifespan = The difference in the total life-cycle operating energy (or GWP) from the baseline retail building after lifespan, due to using this building

${ }^{6}$ Total Difference in Operating Energy (or GWP) from Baseline after
Lifespan per $\mathrm{m}^{2}=$ Total difference in operating energy (or GWP) from Lifespan per $m^{2}=$ Total difference in operating energy
baseline atter lifespan / $n$ et wall area of baseline retail building " Total operating primary energy use of baseline retail building after 50 years $=$

* Total operating GWP of baseline retail building after 50 years $=2,310$ tonnes
${ }^{3}$ Total EE (or Total GWP) = Total embodied energy (or total embodied GWP)
of building component after lifespan (i.e. total manufacturing + tota 


\section{Cold-Formed Steel Stud Wall \#25 (SS-W25)}

Building Component Description:

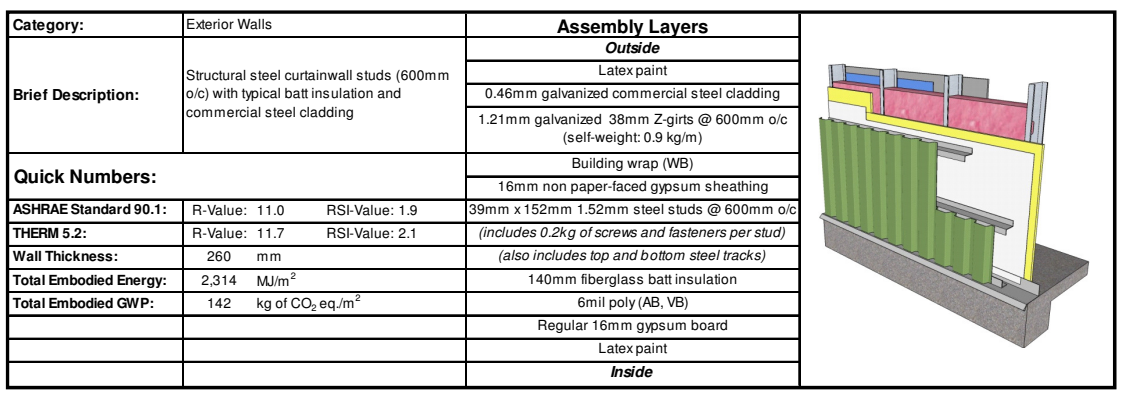

\section{Life-Cycle Assessment Results:}

Primary Energy Consumption (MJ)

\begin{tabular}{|c|c|c|c|c|c|c|c|c|c|c|c|c|c|c|c|c|}
\hline \multirow{3}{*}{$\begin{array}{l}\text { Lifespan } \\
\text { (Years) }\end{array}$} & \multicolumn{14}{|c|}{ Embod } & \multirow{2}{*}{\multicolumn{2}{|c|}{$\begin{array}{c}\text { Difference in } \\
\text { Operating Energy } \\
\text { from Baseline after } \\
\text { Lifespan }\end{array}$}} \\
\hline & \multicolumn{3}{|c|}{ Manufacturing } & \multicolumn{3}{|c|}{ struction } & \multicolumn{3}{|c|}{ intenance } & \multicolumn{3}{|c|}{ End of } & \multirow{2}{*}{$\begin{array}{c}{ }^{3} \text { Total } \\
\mathrm{EE}\end{array}$} & \multirow{2}{*}{$\begin{array}{c}{ }^{4} \text { Total } \\
\text { EE } \\
\text { per } \mathrm{m}^{2}\end{array}$} & & \\
\hline & Material & ${ }^{2}$ Trans. & Total & \begin{tabular}{|l|} 
Material \\
\end{tabular} & ${ }^{2}$ Trans. & Total & Material & ${ }^{2}$ Trans. & Total & Material & ${ }^{2}$ Trans. & \begin{tabular}{|l|} 
Total \\
\end{tabular} & & & ${ }^{5}$ Total & ${ }^{6}$ per m$^{2}$ \\
\hline \begin{tabular}{|l|l} 
Initial \\
\end{tabular} & 116,603 & 269 & 116,872 & 160 & 615 & 775 & 0 & $\pi$ & 0 & 0 & 0 & 0 & 117,647 & 2,310 & & \\
\hline 50 & 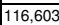 & 269 & \begin{tabular}{|l|l|l|}
11,87 \\
\end{tabular} & \begin{tabular}{|l|l|}
160 \\
\end{tabular} & 615 & 775 & 0 & 0 & 0 & 0 & 164 & 164 & \begin{tabular}{|l|l|l|}
117,81 \\
\end{tabular} & 2,314 & $1,300,00$ & 2,2 \\
\hline
\end{tabular}

Global Warming Potential ( $\mathrm{kg}$ of $\mathrm{CO}_{2}$ eq.)

\begin{tabular}{|c|c|c|c|c|c|c|c|c|c|c|c|c|c|c|c|c|}
\hline \multirow{3}{*}{$\begin{array}{l}\text { Lifespan } \\
\text { (Years) }\end{array}$} & \multicolumn{14}{|c|}{ Embodied Global Warming Potential (GWP) } & \multirow{2}{*}{\multicolumn{2}{|c|}{$\begin{array}{l}\text { Difference in } \\
\text { Operating GWPP fron } \\
\text { Baseline after } \\
\text { Lifespan }\end{array}$}} \\
\hline & \multicolumn{3}{|c|}{ Manufacturing } & \multicolumn{3}{|c|}{ Construction } & \multicolumn{3}{|c|}{ Maintenance } & \multicolumn{3}{|c|}{ End of Life } & \multirow{2}{*}{$\begin{array}{l}{ }^{3} \text { Total } \\
\text { GWP }\end{array}$} & \multirow{2}{*}{$\begin{array}{l}{ }^{4} \text { Total } \\
\text { GWP } \\
\text { per m }\end{array}$} & & \\
\hline & Material & ${ }^{2}$ Trans. & Total & Material & ${ }^{2}$ Trans. & Total & Material & ${ }^{2}$ Trans. & Total & Material & 2 Trans. & Total & & & 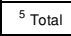 & ${ }^{6} \operatorname{perm}^{2}$ \\
\hline Initial & 7,240 & 0 & 7,2 & 10 & 1 & 12 & 0 & 0 & 0 & 0 & 0 & 0 & 52 & 142 & & \\
\hline 50 & 7,240 & 0 & 7,240 & 10 & 1 & 12 & 0 & 0 & 0 & 0 & 0 & 0 & 7,252 & 142 & 70,000 & 12 \\
\hline
\end{tabular}

Embodied energy (and GWP) numbers are based on an area of wall $=50.9 \mathrm{~m}^{2} \quad$ (Length $\times$ Height $=7.6 \mathrm{~m} \times 6.7 \mathrm{~m}=50.9 \mathrm{~m}^{2}$ )

Net wall area of baseline retall building (gross wall area - openings) $=581.0 \mathrm{~m}^{2}$

\section{ATHENA ® EIE Material List}

\begin{tabular}{|l|c|c|}
\hline \multicolumn{1}{|c|}{ Material List } & Quantitites & Unit \\
\hline \begin{tabular}{l|c|}
$16 \mathrm{~mm}$ Moisture Resistant Gypsum \\
Board
\end{tabular} & 56.0 & $\mathrm{~m} 2$ \\
\hline 16mm Regular Gypsum Board & 56.0 & $\mathrm{~m} 2$ \\
\hline 6 mil Polyethylene & 108.0 & $\mathrm{~m} 2$ \\
\hline Batt. Fiberglass & 289.8 & $\mathrm{~m} 2(25 \mathrm{~mm})$ \\
\hline Commercial $0.46 \mathrm{~mm}$ Steel Cladding & 168.0 & $\mathrm{~m} 2$ \\
\hline Galvanized Studs & 381.3 & $\mathrm{~kg}$ \\
\hline Joint Compound & 111.8 & $\mathrm{~kg}$ \\
\hline Nails & 4.2 & $\mathrm{~kg}$ \\
\hline Paper Tape & 1.3 & $\mathrm{~kg}$ \\
\hline Screws Nuts \& Bolts & 3.9 & $\mathrm{~kg}$ \\
\hline Water Based Latex Paint & 132.5 & $\mathrm{~L}$ \\
\hline
\end{tabular}

Notes:
${ }^{1}$ Initial = Time 'o' (i.e. at the completion of initial construction)

${ }^{2}$ Trans. $=$ Transportation

Total EE (or Total GWP) = Total embodied energy (or total embodied GWP) of building component after lifespan (i.e. total manufacturing + tota

${ }^{4}$ Total EE (or Total GWP) per $\mathrm{m}^{2}=$ Total EE (or Total GWP) of building
component/ area of building component that was modelled in ATHENAQ EIE Lifespan = The difference in the total lifi-cycle operating energy (or GWWP from the baseline retail building after lifespan,
component instead of the baseline component

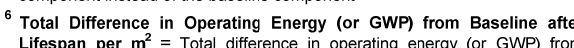
baseline after lifiespan / net wall area of baseline retail building * Total operating primary energy use of baseline retail building after 50 years *Total operating GWP of baseline

\section{Cold-Formed Steel Stud Wall \#26 (SS-W26)}

Building Component Description:

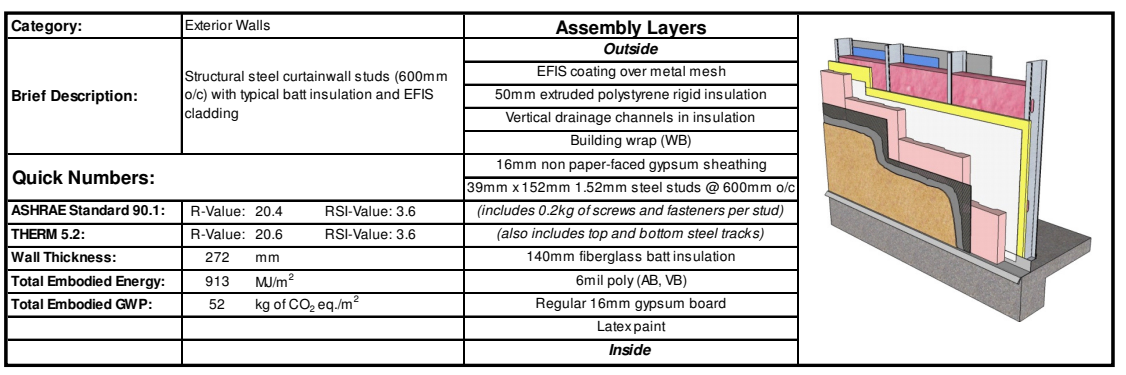

\section{Life-Cycle Assessment Results:}

Primary Energy Consumption (MJ)

\begin{tabular}{|c|c|c|c|c|c|c|c|c|c|c|c|c|c|c|c|c|}
\hline \multirow{3}{*}{$\mid$\begin{tabular}{|c|} 
Lifiespan \\
(Years)
\end{tabular}} & \multicolumn{14}{|c|}{ Embodied Energy (EE) } & \multirow{2}{*}{\multicolumn{2}{|c|}{$\begin{array}{c}\text { Difference in } \\
\text { Operating Energy } \\
\text { from Baseline after } \\
\text { Lifespan }\end{array}$}} \\
\hline & \multicolumn{3}{|c|}{ Manufacturing } & \multicolumn{3}{|c|}{ Construction } & \multicolumn{3}{|c|}{ Maintenance } & \multicolumn{3}{|c|}{ End of Life } & \multirow{2}{*}{$\begin{array}{c}{ }^{3} \text { Total } \\
\text { EE }\end{array}$} & \multirow{2}{*}{$\begin{array}{c}{ }^{4}{ }^{\mathrm{T} o t a l} \\
\mathrm{EE} \\
\text { per m }\end{array}$} & & \\
\hline & Material & 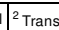 & Total & Material & ${ }^{2}$ Trans. & Total & Material & ${ }^{2}$ Trans. & Total & Material & $2{ }^{2}$ Trans. & Total & & & ${ }^{5}$ Total & ${ }^{6} \operatorname{perm}^{2}$ \\
\hline nitial & 44,776 & 325 & 45,101 & 160 & 723 & 883 & 0 & 0 & 0 & 0 & 0 & 0 & \begin{tabular}{|l|l|l|l|}
45,983 \\
\end{tabular} & 903 & & \\
\hline 50 & \begin{tabular}{|l|l}
44,776 \\
\end{tabular} & 325 & 45,101 & 160 & \begin{tabular}{|l|l|}
723 \\
\end{tabular} & 883 & 0 & 0 & 0 & 0 & 530 & 530 & \begin{tabular}{|l|l|}
46,513 \\
\end{tabular} & 913 & $-1,000,000$ & $-1,7$, \\
\hline
\end{tabular}

Global Warming Potential (kg of $\mathrm{CO}_{2}$ eq.)

\begin{tabular}{|c|c|c|c|c|c|c|c|c|c|c|c|c|c|c|c|c|}
\hline \multirow{3}{*}{$\begin{array}{l}\text { Lesearan } \\
\text { (Years) }\end{array}$} & \multicolumn{14}{|c|}{ Embodied Global Warming Potential (GWP) } & \multirow{2}{*}{\multicolumn{2}{|c|}{$\begin{array}{l}\text { Difference in } \\
\text { Operating GWP from } \\
\text { Baseline after } \\
\text { Lifespan }\end{array}$}} \\
\hline & \multicolumn{3}{|c|}{ Manufacturing } & \multicolumn{3}{|c|}{ Construction } & \multicolumn{3}{|c|}{ Maintenance } & \multicolumn{3}{|c|}{ End of Life } & \multirow{2}{*}{$\begin{array}{l}{ }^{3} \text { Total } \\
\text { GWPP }\end{array}$} & \multirow{2}{*}{$\begin{array}{l}{ }^{4}{ }^{\mathrm{T} o t a l} \\
\mathrm{GWP} \\
\text { per m}{ }^{2}\end{array}$} & & \\
\hline & Naterial & ${ }^{2}$ Trans. & Total & Materia & ${ }^{2}$ Trans. & Total & Material & \begin{tabular}{|c|}
${ }^{2}$ Trans. \\
\end{tabular} & Total & Vaterial & ${ }^{2}$ Trans. & Total & & & ${ }^{5}$ Total & ${ }^{6}{ }_{\text {per m}}^{2}$ \\
\hline nitial & 2,631 & 1 & 2,631 & 10 & 1 & 12 & 0 & 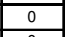 & 0 & 0 & 0 & 0 & 2,643 & 52 & & \\
\hline 50 & 2,631 & 1 & \begin{tabular}{|l|l|}
2,631 \\
\end{tabular} & 10 & 1 & 12 & 0 & 0 & 0 & 0 & 1 & 1 & \begin{tabular}{|l|l|} 
& 2,644 \\
\end{tabular} & 52 & $-50,000$ & -86 \\
\hline
\end{tabular}

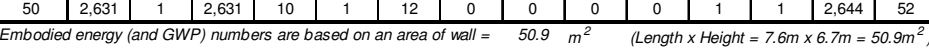

ATHENA $®$ EIE Material List:

(Includes all materials after 50 years)

\begin{tabular}{|l|c|c|}
\hline \multicolumn{1}{|c|}{ Material List } & Quantities & Unit \\
\hline \#15 Organic Felt & 219.4 & $\mathrm{~m} 2$ \\
\hline $\begin{array}{l}16 \mathrm{~mm} m \\
\text { Board Moisture Resistant Gypsum }\end{array}$ & 56.0 & $\mathrm{~m} 2$ \\
\hline $16 \mathrm{~mm}$ Regular Gypsum Board & 56.0 & $\mathrm{~m} 2$ \\
\hline 6 mil Polyethylene & 108.0 & $\mathrm{~m} 2$ \\
\hline Batt. Fiberglass & 289.8 & $\mathrm{~m} 2(25 \mathrm{~mm})$ \\
\hline Extruded Polystyrene & 104.3 & $\mathrm{~m} 2(25 \mathrm{~mm})$ \\
\hline Galvanized Sheet & 51.4 & $\mathrm{~kg}$ \\
\hline Galvanized Studs & 300.5 & $\mathrm{~kg}$ \\
\hline Joint Compound & 111.8 & $\mathrm{~kg}$ \\
\hline Nails & 8.8 & $\mathrm{~kg}$ \\
\hline Paper Tape & 1.3 & $\mathrm{~kg}$ \\
\hline Screws Nuts \& Bolts & 2.6 & $\mathrm{~kg}$ \\
\hline Stucco over metal mesh & 136.0 & $\mathrm{~m} 2$ \\
\hline Water Based Latex Paint & 132.5 & $\mathrm{~L}$ \\
\hline
\end{tabular}

$$
\begin{aligned}
& \text { Notes: } \\
& { }^{1} \text { Initial }=\text { Time 'o' (i.e. at the completion of initial construction) }
\end{aligned}
$$

'Initial $=$ Time ' '' ' (i.e. at

${ }^{3}$ Total EE (or Total GWP) $=$ Total embodied energy (or total embodied GWP) of building component after lifespan (i.e. total man
construction + total maintenance + total end-of-life effects)

“Total EE (or Total GWP) per $\mathrm{m}^{2}=$ Total EE (or Total GWP) of builing 5 Total Difference in Operating Energy (or GWP) from Baseline after
Lifespan $=$ The difference in the total life-cycle operating energy (or G GWP
from the baseline retail building atter licyser.

Total Difference in Operating Energy (or GWP) from Baseline after Lifespan per $\mathrm{m}^{2}=$ Total difterence in operating energy (or GWP) fro
baseline after Ififespan / net wall area of baseline retail building * Total operating primary energy use of baseline retail building after 50 years =
$50,700 \mathrm{GJ}\left(1,745 \mathrm{MV} / \mathrm{m}^{2} \mathrm{yr}\right)$

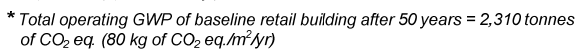
from the baseline retail buildiding after lifespan, due to using this building ${ }^{6}$ component instead of the baseline component 


\section{Cold-Formed Steel Stud Wall \#27 (SS-W27)}

Building Component Description:

\begin{tabular}{|c|c|c|c|c|}
\hline \multirow{4}{*}{$\begin{array}{l}\text { Category: } \\
\text { Brief Description: }\end{array}$} & \multicolumn{2}{|l|}{ Exterior Walls } & Assembly Layers & \\
\hline & & & $\begin{array}{l}\text { Outside } \\
\text { Split.taced concete block cladding }\end{array}$ & 101.1 \\
\hline & o/c) with two laye & f rigid insulation and & $25 \mathrm{~mm}$ airgap & \\
\hline & & & $100 \mathrm{~mm}$ extruded polys strene rigid insulation & \\
\hline & & & Self-adhesive membrane with primer (AB, VB, WB) & \\
\hline \multicolumn{3}{|l|}{ Quick Numbers: } & $16 \mathrm{~mm}$ non paper-faced gypsum sheathing & \\
\hline ASHRAE Standard 90.1: & R-Value: 22.2 & RSIVValue: 3.9 & 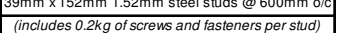 & \\
\hline THERM 5.2: & R-Value: 23.9 & RSIVValue: 4.2 & (also includes top and bottom steel tracks) & \\
\hline Wall Thickness: & $399 \mathrm{~mm}$ & & Regular $16 \mathrm{~mm}$ gyssum board & \\
\hline Total Embodied Energy: & $1,624 \quad \mathrm{MJ}^{2} \mathrm{~m}^{2}$ & & $\begin{array}{lll}\text { Latexpaint } \\
\end{array}$ & \\
\hline \begin{tabular}{|l|l|} 
Total Embodied GWP: \\
\end{tabular} & $113 \mathrm{~kg}$ of $\mathrm{S} \quad \mathrm{C}$ ( & eq $/ \mathrm{m}^{2}$ & Inside & \\
\hline
\end{tabular}

Life-Cycle Assessment Results:

Primary Energy Consumption (MJ)

\begin{tabular}{|c|c|c|c|c|c|c|c|c|c|c|c|c|c|c|c|c|}
\hline \multirow{3}{*}{$\begin{array}{l}\text { Lifespan } \\
\text { (Years) }\end{array}$} & \multicolumn{14}{|c|}{ Embodied Energy (EE) } & \multirow{2}{*}{\multicolumn{2}{|c|}{$\begin{array}{c}\text { Difference in } \\
\text { Operating Energy } \\
\text { from Baseline after } \\
\text { Lifespan }\end{array}$}} \\
\hline & \multicolumn{3}{|c|}{ Manufacturing } & \multicolumn{3}{|c|}{ Construction } & \multicolumn{3}{|c|}{ Maintenance } & \multicolumn{3}{|c|}{ End of Life } & \multirow{2}{*}{$\begin{array}{c}{ }^{3} \text { Total } \\
\mathrm{EE}\end{array}$} & \multirow{2}{*}{$\begin{array}{l}{ }^{4} \text { Total } \\
\text { EE } \\
\text { per } \mathrm{m}^{2}\end{array}$} & & \\
\hline & Material & ${ }^{2}$ Trans & Total & \begin{tabular}{|l|} 
Materia \\
\end{tabular} & ${ }^{2}$ Trans. & \begin{tabular}{|l|} 
Total \\
\end{tabular} & \begin{tabular}{|l|} 
Material \\
\end{tabular} & $\begin{array}{l}{ }^{2} \text { Trans. } \\
\end{array}$ & \begin{tabular}{|l|l|} 
Total \\
\end{tabular} & Material & $\mid{ }^{2}$ Trans. & \begin{tabular}{|l|} 
Total \\
\end{tabular} & & & \begin{tabular}{|l|l|}
${ }^{5}$ Total \\
\end{tabular} & ${ }^{6}$ perm ${ }^{2}$ \\
\hline Initial & \begin{tabular}{|l|l|}
74,294 \\
\end{tabular} & 837 & 75,13 & 160 & 2,192 & 2,352 & 0 & 0 & 0 & 0 & 0 & 0 & 77,483 & 1,522 & & \\
\hline 50 & \begin{tabular}{|l|l|}
74,294 \\
\end{tabular} & 837 & 75,13 & 160 & 2,192 & 2,352 & \begin{tabular}{|l|l|} 
& 3,099 \\
\end{tabular} & 10 & 3,110 & 1 & 2,114 & 2,114 & 82,707 & $\begin{array}{l}1,624 \\
\end{array}$ & 1,500, & $-2,5 \xi$ \\
\hline
\end{tabular}

Global Warming Potential ( $\mathrm{kg}$ of $\mathrm{CO}_{2}$ eq.)

\begin{tabular}{|c|c|c|c|c|c|c|c|c|c|c|c|c|c|c|c|c|}
\hline \multirow{3}{*}{$\begin{array}{c}\text { Lifespan } \\
\text { (Years) }\end{array}$} & \multicolumn{14}{|c|}{ Embodied Global Warming Potential (GWP) } & \multirow{2}{*}{\multicolumn{2}{|c|}{$\begin{array}{c}\text { Difference in } \\
\text { Operating GWP fron } \\
\text { Baseline after } \\
\text { Lifespan }\end{array}$}} \\
\hline & \multicolumn{3}{|c|}{ Manutacturing } & \multicolumn{3}{|c|}{ Construction } & \multicolumn{3}{|c|}{ Maintenance } & \multicolumn{3}{|c|}{ End of Life } & \multirow{2}{*}{$\begin{array}{l}{ }^{3} \text { Total } \\
\text { GWWP }\end{array}$} & \multirow{2}{*}{$\begin{array}{l}{ }^{4} \text { Total } \\
\text { GWP } \\
\text { per m}{ }^{2}\end{array}$} & & \\
\hline & Material & ${ }^{2}$ Trans. & Total & Material & ${ }^{2}$ Trans. & Total & Material & ${ }^{2}$ Trans. & Total & Material & $\left.\right|^{2}$ Trans. & Total & & & ${ }^{5}$ Total & ${ }^{6} \operatorname{per~m}^{2}$ \\
\hline 1 Initial & 5,694 & 2 & 5,696 & 10 & 4 & 15 & n & 0 & 0 & 0 & 0 & 0 & \begin{tabular}{|l}
5,710 \\
\end{tabular} & 112 & & \\
\hline 50 & 5,694 & 2 & 5,696 & 10 & 4 & 15 & 44 & 0 & 44 & 0 & 4 & 4 & 5,758 & 113 & $-80,000$ & -138 \\
\hline
\end{tabular}

ATHENA $®$ EIE Material List:

\begin{tabular}{|c|c|c|}
\hline Material List & Quantities & Unit \\
\hline 3 mil Polyethylene & 54.0 & $\mathrm{~m} 2$ \\
\hline $\begin{array}{l}16 \mathrm{~mm} \text { Moisture Resistant Gypsum } \\
\text { Board }\end{array}$ & 56.0 & $\mathrm{~m} 2$ \\
\hline 16mm Regular Gypsum Board & 56.0 & $\mathrm{~m} 2$ \\
\hline Cold Rolled Sheet & 10.3 & $\mathrm{~kg}$ \\
\hline Extruded Polystyrene & 208.6 & m2 (25mm) \\
\hline Galvanized Studs & 300.5 & $\mathrm{~kg}$ \\
\hline Joint Compound & 111.8 & $\mathrm{~kg}$ \\
\hline Modified Bitumen membrane & 401.5 & $\mathrm{~kg}$ \\
\hline Mortar & 4.3 & $\mathrm{~m} 3$ \\
\hline Nails & 4.2 & $\mathrm{~kg}$ \\
\hline Paper Tape & 1.3 & $\mathrm{~kg}$ \\
\hline Screws Nuts \& Bolts & 2.6 & $\mathrm{~kg}$ \\
\hline Solvent Based Alkyd Paint & 19.6 & $\mathrm{~L}$ \\
\hline Split-faced Concrete Block & $1,238.2$ & Blocks \\
\hline Water Based Latex Paint & 66.3 & L \\
\hline
\end{tabular}

\section{Cold-Formed Steel Stud Wall \#28 (SS-W28)}

Building Component Description:

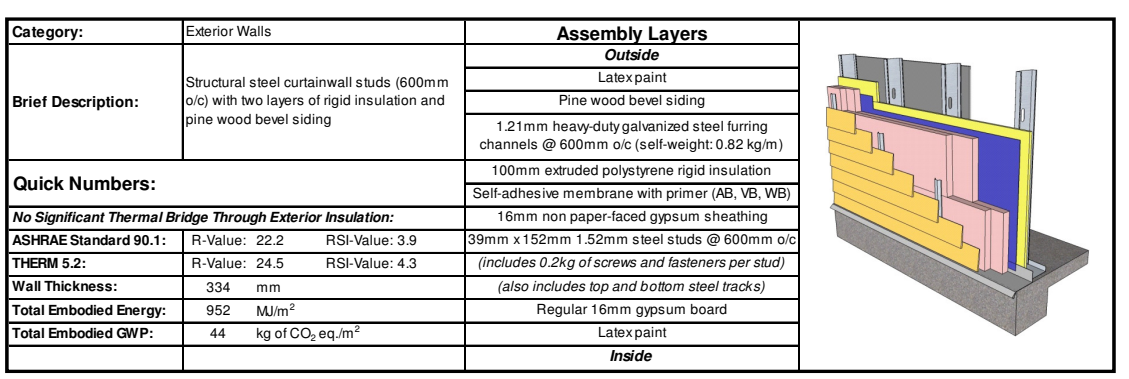

Life-Cycle Assessment Results:

\begin{tabular}{|c|c|c|c|c|c|c|c|c|c|c|c|c|c|c|c|c|}
\hline & \multicolumn{14}{|c|}{ nption (MJ) } & \multirow{3}{*}{\multicolumn{2}{|c|}{$\begin{array}{l}\text { Difference in } \\
\text { Operating Energy } \\
\text { from Baseline afte } \\
\text { Lifespan }\end{array}$}} \\
\hline \multirow{3}{*}{$\begin{array}{l}\text { Litesparars } \\
\text { (Years) }\end{array}$} & & & & & & & bodied E & Energy & & & & & & & & \\
\hline & \multicolumn{3}{|c|}{ Manufacturing } & \multicolumn{3}{|c|}{ Construction } & \multicolumn{3}{|c|}{ Maintenance } & \multicolumn{3}{|c|}{ End of Life } & \multirow{2}{*}{$\begin{array}{c}{ }^{3} \text { Total } \\
\text { EE }\end{array}$} & \multirow{2}{*}{$\begin{array}{c}{ }^{4}{ }^{4} \text { Total } \\
\mathrm{EE} \\
\text { eE } \mathrm{m}^{2}\end{array}$} & & \\
\hline & Material & ${ }^{2}$ Trans. & Total & \begin{tabular}{|l|} 
Material \\
\end{tabular} & 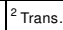 & Total & \begin{tabular}{|l|} 
Material \\
\end{tabular} & ${ }^{2}$ Trans. & Total & \begin{tabular}{|l|} 
Material \\
\end{tabular} & \begin{tabular}{|l|}
${ }^{2}$ Trans. \\
\end{tabular} & Total & & & ${ }^{5}$ Total & ${ }^{6}$ per $\mathrm{m}^{2}$ \\
\hline ititial & 88 & 29 & & 160 & 73 & 891 & & & 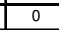 & S & & 0 & & 887 & & \\
\hline 50 & \begin{tabular}{|l|l|l} 
\\
\end{tabular} & 292 & 44,289 & 160 & 731 & 891 & 3,099 & 10 & 3,11 & 0 & 177 & 177 & 48,467 & 952 &, 300 & $-2,23$ \\
\hline
\end{tabular}

Global Warming Potential (kg of $\mathrm{CO}_{2}$ eq.)

\begin{tabular}{|c|c|c|c|c|c|c|c|c|c|c|c|c|c|c|c|c|}
\hline \multirow{3}{*}{$\begin{array}{c}\text { Liespan } \\
\text { (Years) }\end{array}$} & \multicolumn{14}{|c|}{ Embodied Global Warming Potential (GWP) } & \multirow{2}{*}{\multicolumn{2}{|c|}{$\begin{array}{l}\text { Difference in } \\
\text { Operating GWP fron } \\
\text { Baseline after } \\
\text { Lifespan }\end{array}$}} \\
\hline & \multicolumn{3}{|c|}{ Manutacturing } & \multicolumn{3}{|c|}{ Construction } & \multicolumn{3}{|c|}{ Maintenance } & \multicolumn{3}{|c|}{ End of Life } & \multirow{2}{*}{$\begin{array}{l}{ }^{3} \text { Total } \\
\text { GWP }\end{array}$} & \multirow{2}{*}{$\begin{array}{c}{ }^{4} \text { Total } \\
\text { GWP } \\
\text { per }{ }^{2}\end{array}$} & & \\
\hline & Material & ${ }^{2}$ Trans. & Total & Material & ${ }^{2}$ Trans. & Total & Material & ${ }^{2}$ Trans. & Total & Material & ${ }^{2}$ Trans. & Total & & & \begin{tabular}{l|l|l}
${ }^{5}$ Total \\
\end{tabular} & ${ }^{6}$ per $m^{2}$ \\
\hline Initial & 2,183 & 1 & 2,184 & 10 & 1 & 12 & 0 & 0 & 0 & 0 & 0 & 0 & 2,196 & 43 & & \\
\hline 50 & \begin{tabular}{|l|l|}
2,183 \\
\end{tabular} & 1 & 2,184 & 10 & 1 & $\begin{array}{l}12 \\
\end{array}$ & 44 & 0 & 44 & 0 & 0 & 0 & \begin{tabular}{|l|l|l}
2,240 \\
\end{tabular} & 44 & $-70,000$ & $\begin{array}{l}-120 \\
\end{array}$ \\
\hline
\end{tabular}

${ }^{1}$ Initial = Time

(.e. at he completion of initial construction)

${ }^{3}$ Total EE of buidding otal GWP) = Total embodied energy (or total embodied GWP of building component after lifespan (i.e. total mal
construction + total maintenance + total encof-flife effects)

${ }^{4}$ Total EE (or Total GWP) per $\mathrm{m}^{2}=$ Total EE (or Total GWP) of building
component/ area of building component that was modelled in ATHENA尺 EIE Total Difference in Operating Energy (or GWP) from Baseline after Lifespan = The difiererence in the total life-cycle operating energy (or GWP)
from the baseline retaib building after lifespan, due to using this building from the baseline retail building after lifess
component instead of the baseline component

Total Difference in Operating Energy (or GWP) from Baseline after Lifespan per $\mathrm{m}^{2}=$ Total difference in operating energy (or GWP) from

* Total operating primary energy use of baseline retail building after 50 years =

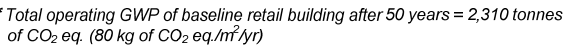

$\begin{gathered}\text { ATHENA @ EIE Material List: } \\
\text { (Includes all materials after 50years) }\end{gathered}$
\begin{tabular}{|l|c|c|}
\hline \multicolumn{1}{|c|}{ Material List } & Quantitities & Unit \\
\hline 3 mil Polyethylene & 54.0 & $\mathrm{~m} 2$ \\
\hline $\begin{array}{l}16 \mathrm{~mm} \text { Moisture Resistant Gypsum } \\
\text { Board }\end{array}$ & 56.0 & $\mathrm{~m} 2$ \\
\hline $16 \mathrm{~mm}$ Regular Gypsum Board & 56.0 & $\mathrm{~m} 2$ \\
\hline Extruded Polystyrene & 208.6 & $\mathrm{~m} 2(25 \mathrm{~mm})$ \\
\hline Galvanized Sheet & 106.1 & $\mathrm{~kg}$ \\
\hline Galvanized Studs & 300.5 & $\mathrm{~kg}$ \\
\hline Joint Compound & 111.8 & $\mathrm{~kg}$ \\
\hline Modified Bitumen membrane & 68.2 & $\mathrm{~kg}$ \\
\hline Nails & 5.5 & $\mathrm{~kg}$ \\
\hline Paper Tape & 1.3 & $\mathrm{~kg}$ \\
\hline Pine Wood Bevel Siding & 160.4 & $\mathrm{~m} 2$ \\
\hline Screws Nuts \& Bolts & 2.6 & $\mathrm{~kg}$ \\
\hline Solvent Based Alkyd Paint & 19.6 & $\mathrm{~L}$ \\
\hline Water Based Latex Paint & 132.5 & $\mathrm{~L}$ \\
\hline
\end{tabular}

Notes:
${ }^{1}$ Initial $=$ Time 'o' (i.e. at the completion of initial construction)

${ }^{2}$ 'Initial $=$ Time ' $O$ ' (i.e. at

${ }^{3}$ Total EE (or Total GWP) $=$ Total embodied energy (or total embodied GWP) of building component after lifespan (i.e. total man
construction + total maintenance + total end-of-life effects)

${ }^{4}$ Total EE (or Total GWP) per $\mathrm{m}^{2}=$ Total EE (or Total GWP) of building 5 Total Difference in Operating Energy (or GWP) from Baseline after
Lifespan $=$ The difference in the total life-cycle operating energy (or GWP)
from the baseline retail building atel firer lifespan due to using this building from the baseline retail building after lifespan, due to using this building
component instead of the baseline component ${ }^{6}$ Total Difference in Operating Energy (or GWP) from Baseline after Lifespan per $\mathrm{m}^{2}=$ Total difference in operating energy (or GWP) from
baseline atter lifespan / net wall area of baseline retail building * Total operating primary energy use of baseline retail building after 50 years =
$50,700 \mathrm{GJ}\left(1,745 \mathrm{MJMm}^{2} \mathrm{yr}\right)$

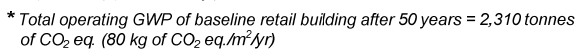




\section{Cold-Formed Steel Stud Wall \#29 (SS-W29)}

Building Component Description:

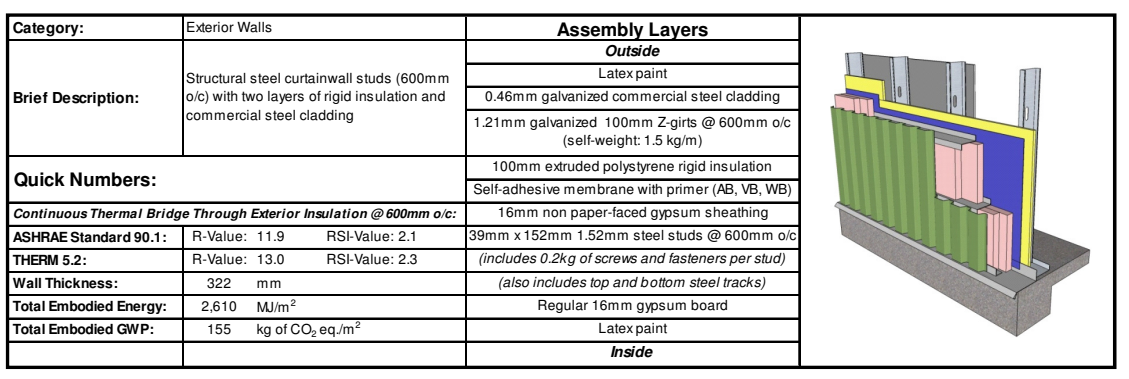

Life-Cycle Assessment Results:

Primary Energy Consumption (MJ)

\begin{tabular}{|c|c|c|c|c|c|c|c|c|c|c|c|c|c|c|c|c|}
\hline \multirow{3}{*}{$\begin{array}{l}\text { Lifespan } \\
\text { (Years) }\end{array}$} & \multicolumn{14}{|c|}{ Embodied Energy (EE) } & \multirow{2}{*}{\multicolumn{2}{|c|}{$\begin{array}{l}\text { Difference in } \\
\text { OOperating Energy } \\
\text { from tasaseline after } \\
\text { Lifessan. }\end{array}$}} \\
\hline & \multicolumn{3}{|c|}{ Manufacturing } & \multicolumn{3}{|c|}{ Construction } & \multicolumn{3}{|c|}{ Maintenance } & \multicolumn{3}{|c|}{ End of Life } & \multirow{2}{*}{${ }^{3}$ Total } & \multirow{2}{*}{$\begin{array}{c}{ }^{4} \text { Total } \\
\text { EE } \\
\text { per } \mathrm{m}^{2}\end{array}$} & & \\
\hline & Material & ${ }^{2}$ Trans. & Total & Materia & ${ }^{2}$ Trans. & Total & Materia & \begin{tabular}{|l|}
${ }^{2}$ Trans. \\
\end{tabular} & Total & Material & Trans. & Total & & & ${ }^{5}$ Total & ${ }^{6}$ per m \\
\hline${ }^{\prime}$ Initial & 128,571 & 245 & 128,816 & 160 & 635 & 794 & 0 & 0 & 0 & 0 & 0 & 0 & 129,611 & \begin{tabular}{|l|l|l|}
2,545 \\
\end{tabular} & r & \\
\hline 50 & 128,571 & 245 & $\mid 128,816$ & 160 & 635 & 794 & 3,099 & & & & & & & & 900,000 & 1,549 \\
\hline
\end{tabular}

Global Warming Potential ( $k g$ of $\mathrm{CO}_{2}$ eq.)

\begin{tabular}{|c|c|c|c|c|c|c|c|c|c|c|c|c|c|c|c|c|}
\hline \multirow{3}{*}{$\begin{array}{l}\text { Lifespan } \\
\text { (Years) }\end{array}$} & \multicolumn{14}{|c|}{ Embodied Global Warming Potential (GWP) } & \multirow{2}{*}{\multicolumn{2}{|c|}{$\begin{array}{l}\text { Difference in } \\
\text { Operating GWP from } \\
\text { Baseline after } \\
\text { Lifespan }\end{array}$}} \\
\hline & \multicolumn{3}{|c|}{ Manufacturing } & \multicolumn{3}{|c|}{ Construction } & \multicolumn{3}{|c|}{ Maintenance } & \multicolumn{3}{|c|}{ End of Life } & \multirow{2}{*}{$\begin{array}{l}{ }^{3} \text { Total } \\
\text { GWWP }\end{array}$} & \multirow{2}{*}{\begin{tabular}{|l}
${ }^{4}$ Total \\
GWP \\
per m
\end{tabular}} & & \\
\hline & Material & ${ }^{2}$ Trans & Total & Material & Trans. & Total & Material & 2 Trans. & Total & Material & $\left.\right|^{2}$ Trans. & Total & & & ${ }^{5}$ Total & ${ }^{6} \mathrm{perm}^{2}$ \\
\hline \begin{tabular}{|l|l|}
1 Initial \\
\end{tabular} & 7,829 & 0 & 7,829 & 10 & 1 & 12 & 0 & 0 & 0 & 0 & 0 & 0 & 7,841 & 154 & & \\
\hline 50 & 7,829 & 0 & 7,829 & 10 & 1 & 12 & 44 & 0 & 44 & 0 & 0 & 0 & 7,885 & 155 & 50,000 & 86 \\
\hline
\end{tabular}

Net wall area of baseline retail building (gross wall area - openings) = $581.0 \mathrm{~m}^{2}$

ATHENA ® EIE Material List:

\begin{tabular}{|c|c|c|}
\hline Material List & Quantities & Unit \\
\hline 3 mil Polyethylene & 54.0 & $\mathrm{~m} 2$ \\
\hline $\begin{array}{l}\text { 16mm Moisture Resistant Gypsum } \\
\text { Board }\end{array}$ & 56.0 & $\mathrm{~m} 2$ \\
\hline $16 \mathrm{~mm}$ Regular Gypsum Board & 56.0 & $\mathrm{~m} 2$ \\
\hline Commercial $0.46 \mathrm{~mm}$ Steel Cladding & 168.0 & $\mathrm{~m} 2$ \\
\hline Extruded Polystyrene & 208.6 & $\mathrm{~m} 2(25 \mathrm{~mm})$ \\
\hline Galvanized Studs & 431.8 & $\mathrm{~kg}$ \\
\hline Joint Compound & 111.8 & $\mathrm{~kg}$ \\
\hline Modified Bitumen membrane & 68.2 & $\mathrm{~kg}$ \\
\hline Nails & 4.2 & $\mathrm{~kg}$ \\
\hline Paper Tape & 1.3 & $\mathrm{~kg}$ \\
\hline Screws Nuts \& Bolts & 3.9 & $\mathrm{~kg}$ \\
\hline Solvent Based Alkyd Paint & 19.6 & $\mathrm{~L}$ \\
\hline Water Based Latex Paint & 132.5 & $\mathrm{~L}$ \\
\hline
\end{tabular}

\section{Cold-Formed Steel Stud Wall \#30 (SS-W30)}

Building Component Description:

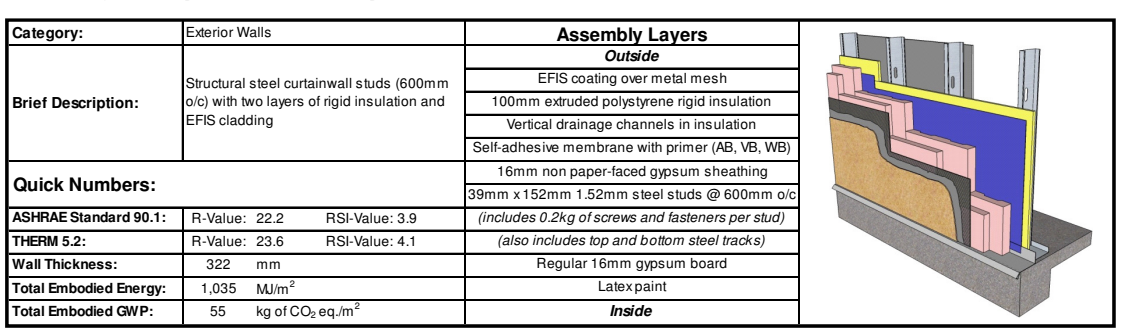

Life-Cycle Assessment Results:

Primary Energy Consumption (MJ)

\begin{tabular}{|c|c|c|c|c|c|c|c|c|c|c|c|c|c|c|c|c|}
\hline \multirow{3}{*}{$\begin{array}{l}\text { Litespan } \\
\text { (Years) }\end{array}$} & \multicolumn{14}{|c|}{ Embodied Energy (EE) } & \multirow{2}{*}{\multicolumn{2}{|c|}{$\begin{array}{c}\text { Difference in } \\
\text { Operating Energy } \\
\text { from Baseline after } \\
\text { Lifespan }\end{array}$}} \\
\hline & \multicolumn{3}{|c|}{ Manufacturing } & \multicolumn{3}{|c|}{ Construction } & \multicolumn{3}{|c|}{ Maintenance } & \multicolumn{3}{|c|}{ End of Life } & \multirow{2}{*}{$\mid \begin{array}{c}{ }^{3} \text { Total } \\
\mathrm{EE}\end{array}$} & \multirow{2}{*}{\begin{tabular}{|c|}
${ }^{4} \mathrm{~T}$ Total \\
$\mathrm{EE}$ \\
per
\end{tabular}} & & \\
\hline & Naterial & ${ }^{2}$ Trans. & Total & \begin{tabular}{|c|} 
Material \\
\end{tabular} & ${ }^{2}$ Trans. & Total & \begin{tabular}{|l|} 
Material \\
\end{tabular} & ${ }^{2}$ Trans. & Total & Material & \begin{tabular}{|l|}
${ }^{2}$ Trans. \\
\end{tabular} & Total & & & ${ }^{5}$ Total & ${ }^{6}$ perm $^{2}$ \\
\hline Initial & 47,865 & 299 & 48,164 & 160 & 724 & 884 & 0 & 0 & 0 & 0 & 0 & 0 & \begin{tabular}{|l|l}
49,048 \\
\end{tabular} & 963 & & \\
\hline 50 & 47,865 & 299 & 48,164 & 160 & 724 & 884 & 3,099 & 10 & 3,110 & 0 & 527 & 527 & 52,684 & 1.035 & 200,00 & 2.065 \\
\hline
\end{tabular}

Global Warming Potential (kg of $\mathrm{CO}_{2}$ eq.)

\begin{tabular}{|c|c|c|c|c|c|c|c|c|c|c|c|c|c|c|c|c|}
\hline \multirow{3}{*}{ 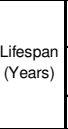 } & \multicolumn{14}{|c|}{ Embodied Global Warming Potential (GWP) } & \multirow{2}{*}{\multicolumn{2}{|c|}{$\begin{array}{l}\text { Difference in } \\
\text { Operating GWW Prom } \\
\text { Baseline after } \\
\text { Lifespan } \\
\end{array}$}} \\
\hline & \multicolumn{3}{|c|}{ Manufacturing } & \multicolumn{3}{|c|}{ Construction } & \multicolumn{3}{|c|}{ Maintenance } & \multicolumn{3}{|c|}{ End of Life } & \multirow{2}{*}{$\begin{array}{c}{ }^{3} \text { Total } \\
\text { GWP }\end{array}$} & \multirow{2}{*}{\begin{tabular}{|}
${ }^{4}$ Total \\
GWP \\
per m²
\end{tabular}} & & \\
\hline & Material & ${ }^{2}$ Trans. & Total & Material & ${ }^{2}$ Trans. & Total & Material & ${ }^{2}$ Trans. & Total & Material & ${ }^{2}$ Trans. & Total & & & ${ }^{5}$ Total & ${ }^{6} \mathrm{perm}^{2}$ \\
\hline Initial & 2,729 & 1 & 2,730 & 10 & 1 & 12 & 0 & 0 & 0 & 0 & 0 & 0 & 2,742 & 54 & & \\
\hline 0 & 2,729 & 1 & 2,730 & 10 & 1 & 12 & 44 & 0 & 44 & 0 & 1 & 1 & 2,786 & 55 & $-60,000$ & -103 \\
\hline
\end{tabular}

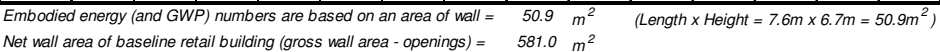

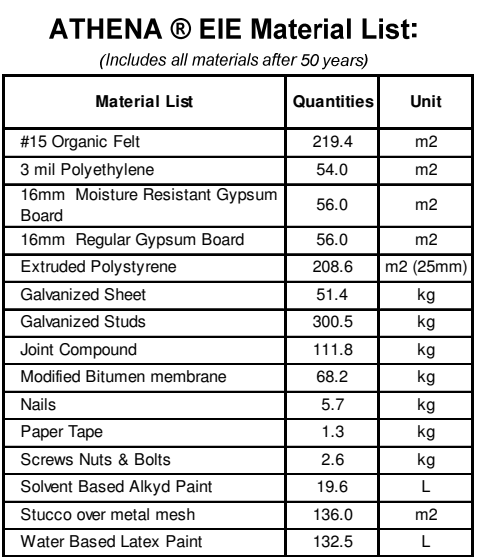

Notes:
${ }^{1}$ Initial $=$ Time 'o' (i.e. at the completion of initial construction)

${ }^{2}$ Trans. $=$ Transportation

${ }^{3}$ Total EE (or Total GWP) $=$ Total embodied energy (or total embodied GWP) of building component anter lifespan (i.e. total mat
construction + total maintenance + total end-ot life effects)

${ }^{4}$ Total EE (or Total GWP) per $\mathrm{m}^{2}=$ Total EE (or Total GWP) of building
component / area of building component that was modelled in ATHENAQ EIE ${ }^{5}$ Total Difference in Operating Energy (or GWP) from Baseline after the the component instead of the baseline component

${ }^{-}$Total Difference in Operating Energy (or GWP) from Baseline after Lifespan per $\mathrm{m}^{2}=$ Total difference in operating energy (or GWP) from

* Total operating primary energy use of baseline retail building after 50 years =

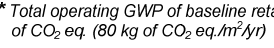




\section{LCA Data for Wood Stud Walls}

This section contains a detailed description of wood stud (WS) exterior infill wall that was examined in this study (30 in total). The assembly layers are listed for each wall, along with a detailed description of the material quantities from the ATHENA® Environmental Impact Estimator for Buildings.

A breakdown of the total primary energy consumption and the total global warming potential (GWP) for each wall is also included. In each case, the results were calculated for an area of wall equal to $50.9 \mathrm{~m}^{2}$, which represents a typical bay size for a single-storey retail building. The results are also expressed on a per $\mathrm{m}^{2}$ basis in each case. The data has been calculated for two different lifespans: at the completion of initial construction and after 50 years.

As a summary, the figure below illustrates a comparison of the total embodied energy (and GWP) after 50 years for the various walls in this comparison purpose, the building component used in the baseline retail building has been shaded in grey.

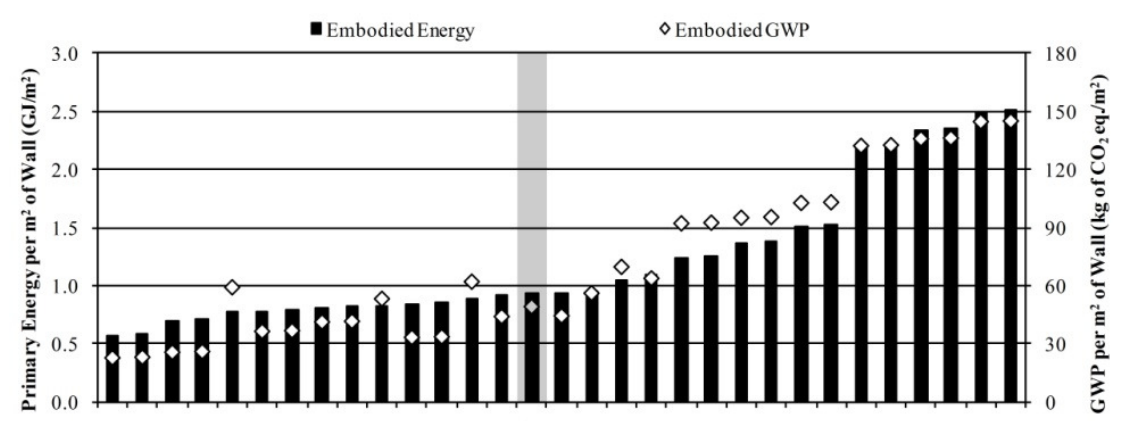

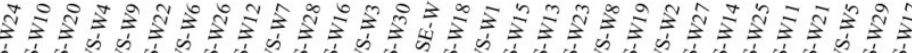

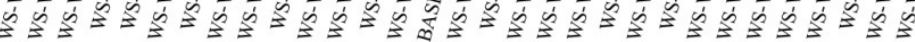

Exterior Infill Wall ID (Arranged in Order of Increasing Embodied Energy)

\section{Wood Stud Wall \#1 (WS-W1)}

Building Component Description:

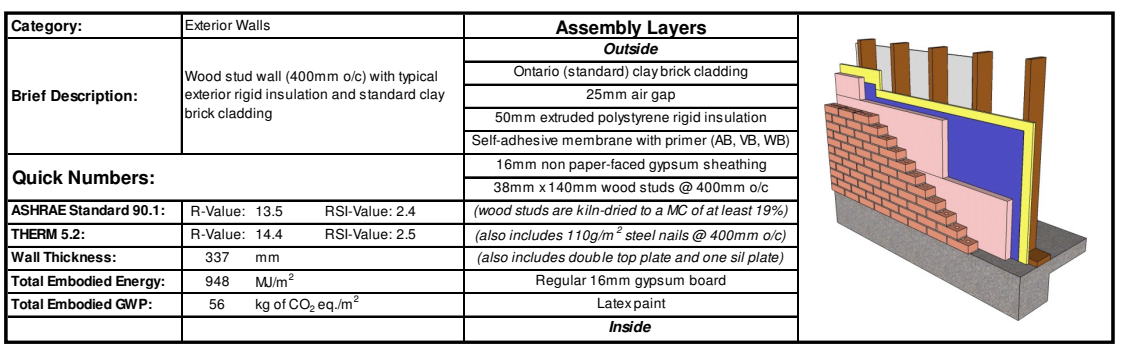

Life-Cycle Assessment Results:

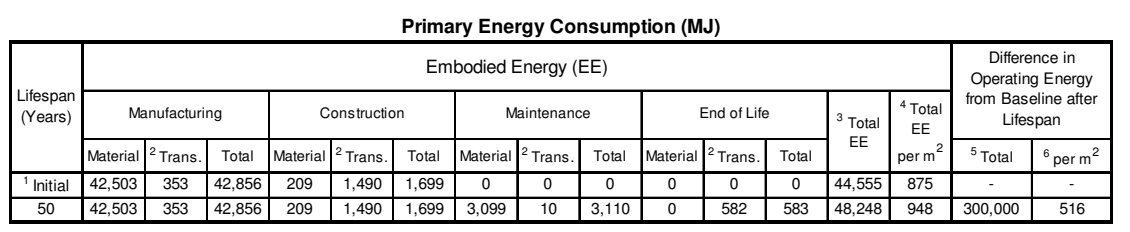

Global Warming Potential (kg of $\mathrm{CO}_{2}$ eq.)

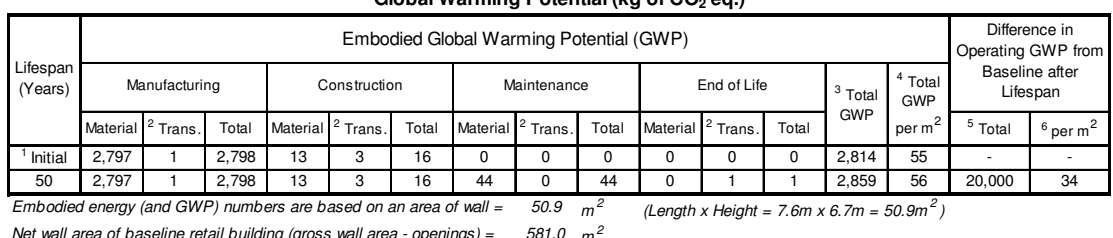

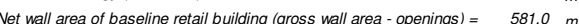

ATHENA $®$ EIE Material List: (Includes all materials after 50 years)

\begin{tabular}{|l|c|c|}
\hline \multicolumn{1}{|c|}{ Material List } & Quantities & Unit \\
\hline 3 mil Polyethylene & 54.0 & $\mathrm{~m} 2$ \\
\hline $\begin{array}{l}\text { 16mm Moisture Resistant Gypsum } \\
\text { Board }\end{array}$ & 56.0 & $\mathrm{~m} 2$ \\
\hline 16mm Regular Gypsum Board & 56.0 & $\mathrm{~m} 2$ \\
\hline Cold Rolled Sheet & 10.3 & $\mathrm{~kg}$ \\
\hline Extruded Polystyrene & 104.3 & $\mathrm{~m} 2(25 \mathrm{~mm})$ \\
\hline Joint Compound & 111.8 & $\mathrm{~kg}$ \\
\hline Modified Bitumen membrane & 68.2 & $\mathrm{~kg}$ \\
\hline Mortar & 1.5 & $\mathrm{~m} 3$ \\
\hline Nails & 9.0 & $\mathrm{~kg}$ \\
\hline Ontario (Standard) Brick & 53.5 & $\mathrm{~m} 2$ \\
\hline Paper Tape & 1.3 & $\mathrm{~kg}$ \\
\hline Small Inimension Sottwood Lumber, & 0.9 & $\mathrm{~m} 3$ \\
\hline kiln-dried & 0.9 & \\
\hline Solvent Based Alkyd Paint & 19.6 & $\mathrm{~L}$ \\
\hline Water Based Latex Paint & 66.3 & $\mathrm{~L}$ \\
\hline
\end{tabular}

Notes:
${ }^{1}$ Initial = Time '0' (i.e. at the completion of initial construction)

${ }^{2}$ Trans. $=$ Transportation

${ }^{3}$ Total EE (or Total GWP) $=$ Total embodied energy (or total embodied GWP) of building component after lifespan (i.e. total man
construction + total maintenance + total end-of-life effects)

${ }^{4}$ Total EE (or Total GWP) per $\mathrm{m}^{2}=$ Total EE (or Total GWP) of building
component / area of building component that was modelled in ATHENA@ EIE ${ }^{5}$ Total Difference in Operating Energy (or GWP) from Baseline after Lifespan = The difference in the Elotilife-cycle operating energy (or GWP
from the baseline retail building after lifespan, due to using this building component instead of the baseline componen

${ }^{6}$ Total Difference in Operating Energy (or GWP) from Baseline after Lifespan per $m^{2}=$ Total difference in operating energy (or GWP) from

"Total operating primary energy use of baseline retail building after 50 years =
$50,700 \mathrm{GJ}\left(1,745 \mathrm{MJ} / \mathrm{m}^{2} / \mathrm{yr}\right)$

of $\mathrm{CO}_{2}$ eq. $\left(80 \mathrm{~kg}\right.$ of $\left.\mathrm{CO}_{2} \mathrm{eq} . / \mathrm{m}^{2} / \mathrm{yr}\right)$ 


\section{Wood Stud Wall \#2 (WS-W2)}

Building Component Description:

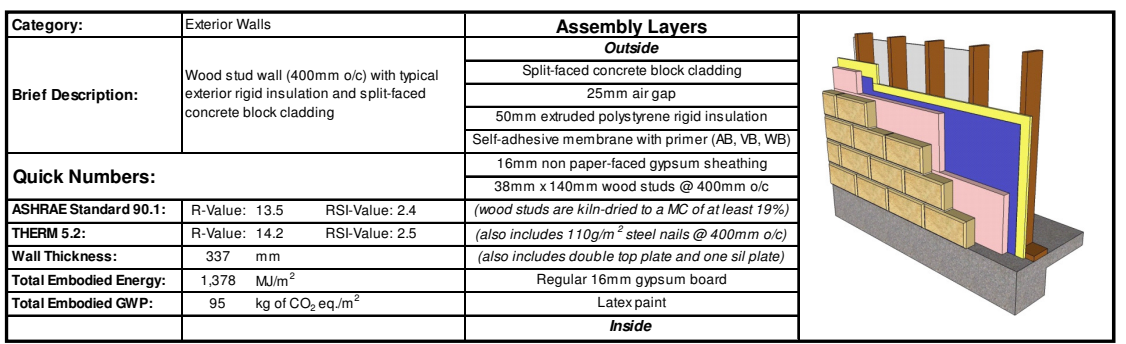

\section{Life-Cycle Assessment Results:}

Primary Energy Consumption (MJ)

\begin{tabular}{|c|c|c|c|c|c|c|c|c|c|c|c|c|c|c|c|c|}
\hline \multirow{3}{*}{\begin{tabular}{|l} 
Lifespan \\
(Years)
\end{tabular}} & \multicolumn{14}{|c|}{ Embodied Energy (EE) } & \multirow{2}{*}{\multicolumn{2}{|c|}{$\begin{array}{c}\text { Difference in } \\
\text { Operating Energy } \\
\text { from Baseline after } \\
\text { Lifespan }\end{array}$}} \\
\hline & \multicolumn{3}{|c|}{ Manufacturing } & \multicolumn{3}{|c|}{ Construction } & \multicolumn{3}{|c|}{ Maintenance } & \multicolumn{3}{|c|}{ End of L Life } & \multirow{2}{*}{$\begin{array}{c}{ }^{3} \text { Total } \\
\mathrm{EE}\end{array}$} & \multirow{2}{*}{$\begin{array}{c}{ }^{4}{ }^{4} \text { otal } \\
\text { EE } \\
\text { per m }\end{array}$} & & \\
\hline & Material & ${ }^{2}$ Trans. & Total & Materia & ${ }^{2}$ Trans. & Total & \begin{tabular}{|l|} 
Material \\
\end{tabular} & ${ }^{2}$ Trans. & \begin{tabular}{|l|l|} 
Total \\
\end{tabular} & Material & \begin{tabular}{|l|}
${ }^{2}$ Trans. \\
\end{tabular} & \begin{tabular}{|l|l|} 
Total \\
\end{tabular} & & & ${ }^{5}$ Total & ${ }^{6}$ per $\mathrm{m}^{2}$ \\
\hline \begin{tabular}{|c|} 
Initial \\
\end{tabular} & 61,531 & 916 & 62,447 & 209 & 2,300 & 2,509 & 0 & 0 & 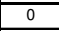 & 0 & 0 & 0 & $\begin{array}{l}64,957 \\
\end{array}$ & 1,276 & & \\
\hline 50 & \begin{tabular}{|l|l|}
61,531 \\
\end{tabular} & \begin{tabular}{|l|l|}
916 \\
\end{tabular} & \begin{tabular}{|l|l|}
62,47 \\
\end{tabular} & 209 & 2,300 & \begin{tabular}{|l|l|}
2,509 \\
\end{tabular} & $\begin{array}{l}3,099 \\
\end{array}$ & 10 & 3,110 & 1 & 2,108 & 2,109 & 70,175 & 1,378 & 300,000 & 516 \\
\hline
\end{tabular}

Global Warming Potential ( $\mathrm{kg}$ of $\mathrm{CO}_{2}$ eq.)

\begin{tabular}{|c|c|c|c|c|c|c|c|c|c|c|c|c|c|c|c|c|}
\hline \multirow{3}{*}{$\begin{array}{c}\text { Lifespan } \\
\text { (Years) }\end{array}$} & \multicolumn{14}{|c|}{ Embodied Global Warming Potential (GWP) } & \multirow{2}{*}{\multicolumn{2}{|c|}{$\begin{array}{l}\text { Difference in } \\
\text { Operating GWP from } \\
\text { Baseline after } \\
\text { Lifespan }\end{array}$}} \\
\hline & \multicolumn{3}{|c|}{ Manufacturing } & \multicolumn{3}{|c|}{ Construction } & \multicolumn{3}{|c|}{ Maintenance } & \multicolumn{3}{|c|}{ End of Life } & \multirow{2}{*}{$\begin{array}{l}3 \text { 桶 } \text { total } \\
\text { GWP }\end{array}$} & \multirow{2}{*}{$\begin{array}{c}{ }^{4} \text { Total } \\
\text { GWP } \\
\text { per m }\end{array}$} & & \\
\hline & Material & ${ }^{2}$ Trans. & Total & Material & ${ }^{2}$ Trans. & Total & Material & ${ }^{2}$ Trans. & Total & Naterial & $\mid{ }^{2}$ Trans. & Total & & & ${ }^{5}$ Total & ${ }^{6}$ per m ${ }^{2}$ \\
\hline IInitial & \begin{tabular}{|l|l|}
4,783 \\
\end{tabular} & 2 & 4,785 & 13 & $a_{1}$ & \begin{tabular}{|l|}
18 \\
\end{tabular} & 0 & 0 & 0 & 0 & 0 & 0 & 4,803 & 94 & & \\
\hline 50 & \begin{tabular}{|l|}
4,783 \\
\end{tabular} & 2 & 4,785 & 13 & 4 & $\begin{array}{ll}18 \\
\end{array}$ & 44 & 0 & 4 & $\overline{0}$ & 4 & $\begin{array}{ll}44 \\
\end{array}$ & 4,851 & 95 & 20,000 & 34 \\
\hline
\end{tabular}

Net wall a d o b

ATHENA $®$ EIE Material List:

\begin{tabular}{|c|c|c|}
\hline Material List & Quantities & Unit \\
\hline 3 mil Polyethylene & 54.0 & $\mathrm{~m} 2$ \\
\hline $\begin{array}{l}16 \mathrm{~mm} \text { Moisture Resistant Gypsum } \\
\text { Board }\end{array}$ & 56.0 & $\mathrm{~m} 2$ \\
\hline 16mm Regular Gypsum Board & 56.0 & $\mathrm{~m} 2$ \\
\hline Cold Rolled Sheet & 10.3 & $\mathrm{~kg}$ \\
\hline Extruded Polystyrene & 104.3 & $\mathrm{~m} 2(25 \mathrm{~mm})$ \\
\hline Joint Compound & 111.8 & $\mathrm{~kg}$ \\
\hline Modified Bitumen membrane & 401.5 & $\mathrm{~kg}$ \\
\hline Mortar & 4.3 & $\mathrm{~m} 3$ \\
\hline Nails & 9.0 & $\mathrm{~kg}$ \\
\hline Paper Tape & 1.3 & $\mathrm{~kg}$ \\
\hline $\begin{array}{l}\text { Small Dimension Softwood Lumber, } \\
\text { kiln-dried }\end{array}$ & 0.9 & m3 \\
\hline Solvent Based Alkyd Paint & 19.6 & $\mathrm{~L}$ \\
\hline Split-faced Concrete Block & $1,238.2$ & Blocks \\
\hline Water $\mathrm{t}$ & 6.3 & \\
\hline
\end{tabular}

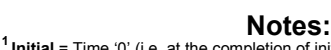

${ }^{1}$ Initial = Time ' 0 ' (i.e. at

${ }^{2}$ Trans. $=$ Transportation

Total EE (or Total GWP) = Total embodied energy (or total embodied GWP of building component after lifespan (i.e. total manu
construction + total maintenance + total end-of-life effects)

${ }^{4}$ Total EE (or Total GWP) per $\mathrm{m}^{2}=$ Total EE (or Total GWP) of building
component/ area of building component that was modelled in ATHENAQ EIEE Total Difference in Operating Energy (or GWP) from Baseline after Lifespan = The difference in the total life-cycle operating energy (or GWP)
from the baseline retail building after lifespan, due to using this building component instead of the baseline component Total Difference in Operating Energy (or GWP) from Baseline after
Lifespan per $\mathrm{m}^{2}=$ Total difference in operating energy (or GWP) from
baseline after lifespan $/$ net wall area of baseline retail building

"Total operating primary energy use of baseline retail building after 50 years =

"Total operating $\mathrm{GWP}$ of baseline retail building after 50 years $=2,310$ tonnes
of $\mathrm{CO}_{2}$ eq. $\left(80 \mathrm{~kg}\right.$ of $\mathrm{CO}_{2}$ eq. $\left./ \mathrm{m}^{2} / \mathrm{yr}\right)$

\section{Wood Stud Wall \#3 (WS-W3)}

Building Component Description:

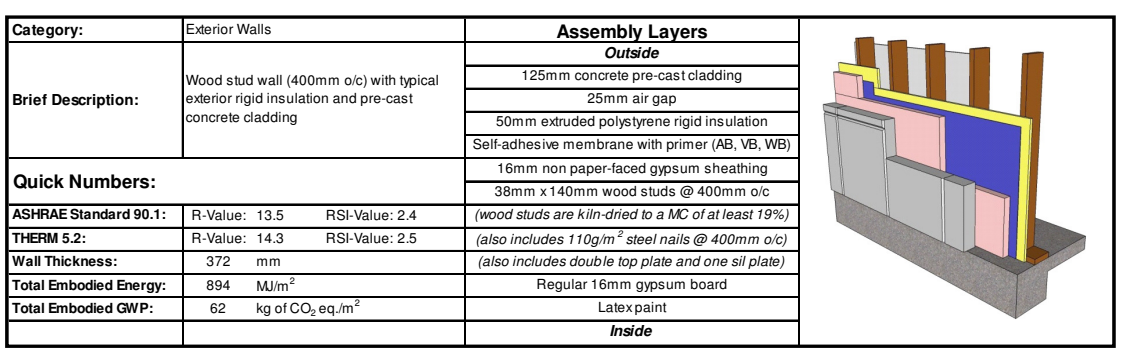

\section{Life-Cycle Assessment Results:}

Primary Energy Consumption (MJ)

\begin{tabular}{|c|c|c|c|c|c|c|c|c|c|c|c|c|c|c|c|c|}
\hline \multirow{3}{*}{$\begin{array}{l}\mid \begin{array}{l}\text { tisespan } \\
\text { (Years) }\end{array} \\
\text {. }\end{array}$} & \multicolumn{14}{|c|}{ Embodied Energy (EE) } & \multirow{2}{*}{\multicolumn{2}{|c|}{$\begin{array}{l}\text { Difference in } \\
\text { Operating Energy } \\
\text { from Baseline after } \\
\text { Lifespan }\end{array}$}} \\
\hline & \multicolumn{3}{|c|}{ Manufacturing } & \multicolumn{3}{|c|}{ Constru } & \multicolumn{3}{|c|}{ Maintenan } & \multicolumn{3}{|c|}{ End ofl } & \multirow{2}{*}{$\begin{array}{c}{ }^{3} \text { Total } \\
\text { EE }\end{array}$} & \multirow{2}{*}{\begin{tabular}{|c|c}
${ }^{4}$ Total \\
EE \\
EE ${ }^{2}$
\end{tabular}} & & \\
\hline & Material & ${ }^{2}$ Trans & 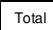 & Material & ${ }^{2}$ Trans. & Total & Materia & ${ }^{2}$ Trans. & \begin{tabular}{|l|l|l|} 
Total \\
\end{tabular} & Material & ${ }^{2}$ Trans. & Total & & & ${ }^{5}$ Total & ${ }^{6}$ per $\mathrm{m}^{2}$ \\
\hline & 39,190 & 944 & 40,134 & 209 & 1,429 & 1,638 & 0 & 0 & 0 & 0 & 0 & 0 & 41,772 & 820 & & \\
\hline 50 & 39,190 & \begin{tabular}{|l|l|l|}
9944 \\
\end{tabular} & 4 & 209 & \begin{tabular}{|l|l|} 
& 1,429 \\
\end{tabular} & 1,638 & 3.099 & 10 & 3,110 & 1 & 615 & 617 & \begin{tabular}{|l|l|}
45.498 \\
\end{tabular} & 894 & 300,000 & 516 \\
\hline
\end{tabular}

Global Warming Potential ( $k g$ of $\mathrm{CO}_{2}$ eq.)

\begin{tabular}{|c|c|c|c|c|c|c|c|c|c|c|c|c|c|c|c|c|}
\hline \multirow{3}{*}{\begin{tabular}{|l} 
Lifespan \\
(ears)
\end{tabular}} & \multicolumn{14}{|c|}{ Embodied Global Warming Potential (GWP) } & \multirow{2}{*}{\multicolumn{2}{|c|}{$\begin{array}{l}\text { Difference in } \\
\text { Operating GWP from } \\
\text { Baseline after } \\
\text { Lifespan }\end{array}$}} \\
\hline & \multicolumn{3}{|c|}{ Manufacturing } & \multicolumn{3}{|c|}{ Constru } & \multicolumn{3}{|c|}{ Maintenance } & \multicolumn{3}{|c|}{ End of Life } & \multirow{2}{*}{$\begin{array}{l}{ }^{3} \text { Total } \\
\text { GWP }\end{array}$} & \multirow{2}{*}{$\begin{array}{l}{ }^{4} \text { Total } \\
\text { GWP } \\
\text { per m }{ }^{2}\end{array}$} & & \\
\hline & Material & ${ }^{2}$ Trans. & Total & Material & ${ }^{2}$ Trans. & Total & Material & ${ }^{2}$ Trans. & Total & Material & ${ }^{2}$ Trans. & Total & & & ${ }^{5}$ Total & ${ }^{6}{ }_{\mathrm{perm}}^{2}{ }^{2}$ \\
\hline |nitial & \begin{tabular}{|l|l|l}
3,097 \\
\end{tabular} & 2 & 3,099 & 13 & 3 & 16 & 0 & 0 & 0 & 0 & 0 & 0 & 3,115 & 61 & & \\
\hline 50 & \begin{tabular}{|l|l|}
3,0097 \\
\end{tabular} & 2 & \begin{tabular}{|l|l|}
3,099 \\
\end{tabular} & 13 & 3 & \begin{tabular}{l|l}
16 \\
\end{tabular} & $\begin{array}{ll}44 \\
\end{array}$ & 0 & $\begin{array}{l}44 \\
\end{array}$ & 0 & 1 & 11 & 3,160 & 62 & 20,000 & 34 \\
\hline
\end{tabular}
Net well a

ATHENA $®$ EIE Material List:

(Includes all materials after 50 years)

\begin{tabular}{|l|c|c|}
\hline \multicolumn{1}{|c|}{ Material List } & Quantities & Unit \\
\hline 3 mil Polyyethylene & 54.0 & $\mathrm{~m} 2$ \\
\hline $\begin{array}{l}16 \mathrm{~mm} \text { Moisture Resistant Gypsum } \\
\text { Board }\end{array}$ & 56.0 & $\mathrm{~m} 2$ \\
\hline 16rmm Regular Gypsum Board & 56.0 & $\mathrm{~m} 2$ \\
\hline Concrete 30 MPa (flyash ay) & 6.7 & $\mathrm{~m} 3$ \\
\hline Extruded Polystyrene & 104.3 & $\mathrm{~m} 2(25 \mathrm{~mm})$ \\
\hline Joint Compound & 111.8 & $\mathrm{~kg}$ \\
\hline Modified Bitumen membrane & 68.2 & $\mathrm{~kg}$ \\
\hline Nails & 9.0 & $\mathrm{~kg}$ \\
\hline Paper Tape & 1.3 & $\mathrm{~kg}$ \\
\hline Rebar, Rod, Light Sections & 404.0 & $\mathrm{~kg}$ \\
\hline $\begin{array}{l}\text { Small Dimension Sottwood Lumber, } \\
\text { kiln-dried }\end{array}$ & 0.9 & $\mathrm{~m} 3$ \\
\hline Solvent Based Alkyd Paint & 19.6 & $\mathrm{~L}$ \\
\hline Water Based Latex Paint & 66.3 & $\mathrm{~L}$ \\
\hline
\end{tabular}

Notes:

'Initial $=$ Time ' 0 ' (i.e. at the

${ }^{3}$ Total EE (or Total GWP) = Total embodied energy (or total embodied GWP of building component after lifespan (i.e. total manu
construction + total maintenance + total end-of-life effects)

${ }^{4}$ Total EE (or Total GWP) per $\mathrm{m}^{2}=$ Total EE (or Total GWP) of building

component / area of building component that was modelled in ATHENA@ EIE Total Difference in Operating Energy (or GWP) from Baseline after from the baseline retail building after lifespan, due to using this building component instead of the baseline component

Total Difference in Operating Energy (or GWP) from Baseline after Lifespan per $\mathrm{m}^{2}=$ Total difference in operating energy (or GWP) from * Total operating primary energy use of baseline retail building after 50 years =

of $\mathrm{CO}_{2}$ eq. $\left(80 \mathrm{~kg}\right.$ of $\left.\mathrm{CO}_{2} e q . / \mathrm{m}^{2} / \mathrm{yr}\right)$ 


\section{Wood Stud Wall \#4 (WS-W4)}

Building Component Description:

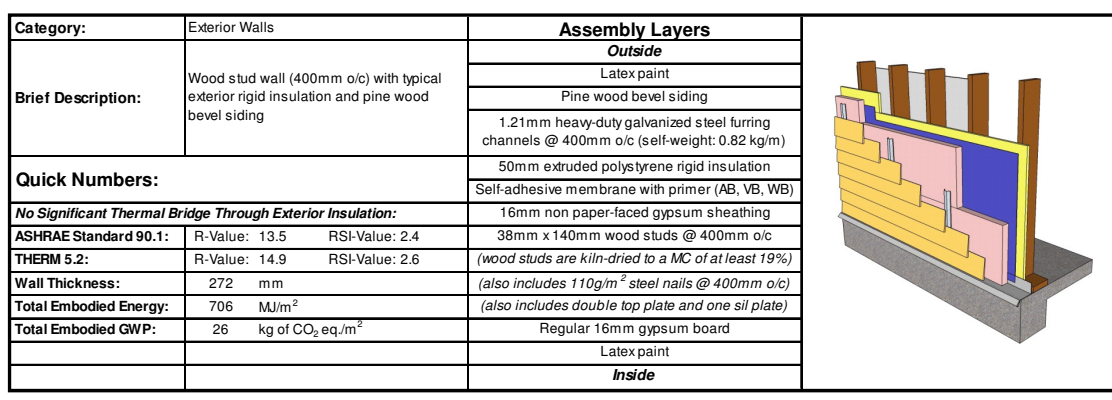

\section{Life-Cycle Assessment Results:}

Primary Energy Consumption (MJ)

\begin{tabular}{|c|c|c|c|c|c|c|c|c|c|c|c|c|c|c|c|c|}
\hline \multirow{3}{*}{\begin{tabular}{|l|l} 
Lifespan \\
(Years)
\end{tabular}} & \multicolumn{14}{|c|}{ Embodied Energy (EE) } & \multirow{2}{*}{\multicolumn{2}{|c|}{$\begin{array}{c}\text { Difference in } \\
\text { Operating Energy } \\
\text { from Baseline afte } \\
\text { Lifespan }\end{array}$}} \\
\hline & \multicolumn{3}{|c|}{ Manufacturing } & \multicolumn{3}{|c|}{ Construction } & \multicolumn{3}{|c|}{ Maintenance } & \multicolumn{3}{|c|}{ End of Life } & \multirow{2}{*}{$-{ }^{3}$ Total } & \multirow{2}{*}{\begin{tabular}{|c|}
${ }^{4}$ Total \\
EE \\
per m²
\end{tabular}} & & \\
\hline & \begin{tabular}{|l|l} 
Materia \\
\end{tabular} & $\begin{array}{l}1{ }^{2} \text { Trans. } \\
\text {. }\end{array}$ & \begin{tabular}{|l|l} 
Total \\
\end{tabular} & Materia & ${ }^{2}$ Trans. & \begin{tabular}{|l|l|l|l} 
Total & \\
\end{tabular} & Materia & ${ }^{2}$ Trans. & Total & Material & Trans. & Total & & & ${ }^{5}$ Total & ${ }^{6}$ per $\mathrm{m}^{2}$ \\
\hline Initial & 31,235 & 371 & 31,606 & 209 & 839 & 1,048 & 0 & 0 & 0 & 0 & 0 & 0 & 32,654 & $\begin{array}{l}641 \\
\end{array}$ & & \\
\hline 50 & 31,235 & \begin{tabular}{|l|l|}
371 \\
\end{tabular} & 31,606 & 209 & 839 & 1,048 & 3,099 & 10 & 3,110 & 0 & 172 & \begin{tabular}{|l|l|}
172 \\
\end{tabular} & 35,936 & 706 & 400,000 & 688 \\
\hline
\end{tabular}

Global Warming Potential ( $k g$ of $\mathrm{CO}_{2}$ eq.)

\begin{tabular}{|c|c|c|c|c|c|c|c|c|c|c|c|c|c|c|c|c|}
\hline \multirow{3}{*}{$\begin{array}{l}\text { Lifespan } \\
\text { (Years) }\end{array}$} & \multicolumn{14}{|c|}{ Embodied Global Warming Potential (GWP) } & \multirow{2}{*}{\multicolumn{2}{|c|}{\begin{tabular}{|c|} 
Difference in \\
Operating GWP fro \\
Baseline after \\
Lifespan
\end{tabular}}} \\
\hline & \multicolumn{3}{|c|}{ Manutacturing } & \multicolumn{3}{|c|}{ Construction } & \multicolumn{3}{|c|}{ Maintenance } & \multicolumn{3}{|c|}{ End of L Life } & \multirow{2}{*}{$\begin{array}{l}{ }^{3} \text { Total } \\
\text { GWP }\end{array}$} & \multirow{2}{*}{ 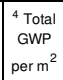 } & & \\
\hline & Materia & \begin{tabular}{|l|}
${ }^{2}$ Trans. \\
\end{tabular} & \begin{tabular}{|l|l|} 
Total \\
\end{tabular} & \begin{tabular}{|l|} 
Material \\
\end{tabular} & ${ }^{2}$ Trans & \begin{tabular}{|l|} 
Total \\
\end{tabular} & \begin{tabular}{|l|} 
Materia \\
\end{tabular} & ${ }^{2}$ Trans. & \begin{tabular}{|l|} 
Total \\
\end{tabular} & \begin{tabular}{|l|} 
Material \\
\end{tabular} & \begin{tabular}{l|l}
${ }^{2}$ Trans. \\
.
\end{tabular} & \begin{tabular}{|l|l|} 
Total \\
\end{tabular} & & & ${ }^{5}$ Total & ${ }^{6} \mathrm{per} \mathrm{m}^{2}$ \\
\hline 1 Initial & \begin{tabular}{|l|l|}
1,272 \\
\end{tabular} & 1 & 1,273 & 13 & 2 & 15 & 0 & 0 & 0 & 0 & 0 & 0 & 1,288 & 25 & & - \\
\hline 50 & \begin{tabular}{|l|l|}
1,272 \\
\end{tabular} & 1 & \begin{tabular}{|l|l|l|l}
1,273 \\
\end{tabular} & 13 & 2 & 15 & 44 & 0 & $\begin{array}{l}44 \\
\end{array}$ & 0 & 0 & 0 & $\mid 1,332$ & 26 & 20,000 & 34 \\
\hline
\end{tabular}

Embodied energy (and GWP) numbers are based on an area of wall $=\quad 50.9 \mathrm{~m}^{2} \quad$ (Length $\times$ Height $=7.6 \mathrm{~m} \times 6.7 \mathrm{~m}=50.9 \mathrm{~m}^{2}$ )

Net wall area of baseline retail building (grosss wall area - openings) $=581.0 \mathrm{~m}^{2}$

ATHENA $®$ EIE Material List:

\begin{tabular}{|c|c|c|}
\hline Material List & Quantities & Unit \\
\hline 3 mil Polyethylene & 54.0 & $\mathrm{~m} 2$ \\
\hline $\begin{array}{l}\text { 16mm Moisture Resistant Gypsum } \\
\text { Board }\end{array}$ & 56.0 & $\mathrm{~m} 2$ \\
\hline 16mm Regular Gypsum Board & 56.0 & $\mathrm{~m} 2$ \\
\hline Extruded Polystyrene & 104.3 & m2 $225 \mathrm{~mm}$ \\
\hline Galvanized Sheet & 106.1 & $\mathrm{~kg}$ \\
\hline Joint Compound & 111.8 & $\mathrm{~kg}$ \\
\hline Modified Bitumen membrane & 68.2 & $\mathrm{~kg}$ \\
\hline Nails & 10.3 & $\mathrm{~kg}$ \\
\hline $\begin{array}{l}\text { Paper Tape } \\
\end{array}$ & 1.3 & $\mathrm{~kg}$ \\
\hline Pine Wood Bevel Siding & 160.4 & $\mathrm{~m} 2$ \\
\hline $\begin{array}{l}\text { Small Dimension Softwood Lumber, } \\
\text { kilindried }\end{array}$ & 0.9 & $\mathrm{~m} 3$ \\
\hline Solvent Based Alkyd Paint & 19.6 & $\mathrm{~L}$ \\
\hline Water Based Latex Paint & 132.5 & L \\
\hline
\end{tabular}

Notes:
1 Initial $=$ Time ' $O$ ' (i.e. at the completion of initial construction)

${ }^{2}$ Trans. $=$ Transportation

Total EE (or Total GWP) = Total embodied energy (or total embodied GWP) of building component after lifespan (i.e. total mant
construction + total maintenance + total enc-of-life effects)

${ }^{4}$ Total EE (or Total GWP) per $\mathrm{m}^{2}=$ Total EE (or Total GWP) of building Total Difference in Operating Energy (or GWP) from Basello Lifespan = The difference in the total life-cycle operating energy (or GWP)
from the baseline retail building after lifespan, due to using this building trom the baseline retail building after lifespan, due to using this building
component instead of the baseline component ${ }^{6}$ Total Difference in Operating Energy (or GWP) from Baseline after
Lifespan per $\mathrm{m}^{2} \stackrel{\text { Total difference in operating energy (or GWP) from }}{ }$

* Total operating primary energy use of baseline retail building after 50 years

"Total operating GWP of baseline retal

Wood Stud Wall \#5 (WS-W5)

Building Component Description:

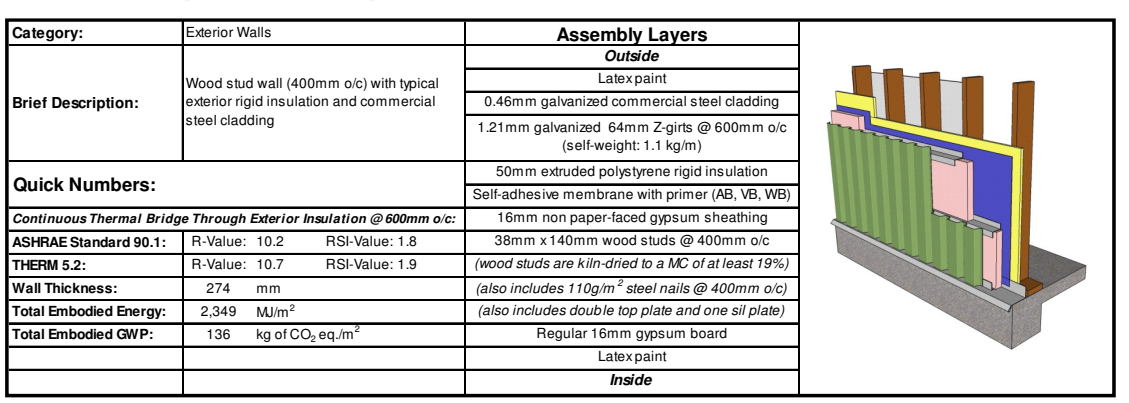

Life-Cycle Assessment Results:

Primary Energy Consumption (MJ)

\begin{tabular}{|c|c|c|c|c|c|c|c|c|c|c|c|c|c|c|c|}
\hline \multirow{3}{*}{$\begin{array}{c}\text { Lifespan } \\
\text { (Years) }\end{array}$} & \multicolumn{13}{|c|}{ Embodied Energy (EE) } & \multirow{2}{*}{\multicolumn{2}{|c|}{$\begin{array}{l}\text { Difiference in } \\
\text { Operating Energy } \\
\text { trom Baseline after } \\
\text { Lifiespan }\end{array}$}} \\
\hline & \multicolumn{2}{|c|}{ Manutacturing } & \multicolumn{3}{|c|}{ Construction } & \multicolumn{3}{|c|}{ Maintenance } & \multicolumn{3}{|c|}{ End of Life } & \multirow{2}{*}{$\begin{array}{c}{ }^{3} \text { Total } \\
\text { EE }\end{array}$} & \multirow{2}{*}{$\begin{array}{c}{ }^{4} \text { Total } \\
\text { EE } \\
\text { per } \mathrm{m}^{2}\end{array}$} & & \\
\hline & \begin{tabular}{|l|l} 
Material & ${ }^{2}$ Trans \\
\end{tabular} & Total & Material & ${ }^{2}$ Trans. & Total & Material & $\begin{array}{l}{ }^{2} \text { Trans. } \\
\end{array}$ & Total & Material & ${ }^{2}$ Trans. & Total & & & ${ }^{5}$ Total & ${ }^{6}$ perm m$^{2}$ \\
\hline Initial & \begin{tabular}{|l|l|}
115,085 & 324 \\
\end{tabular} & 115,409 & 209 & 739 & 948 & 0 & 0 & 0 & 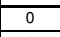 & 0 & 0 & 116,357 & 2,285 & & \\
\hline 50 & \begin{tabular}{|l|l|}
115,005 & 324 \\
\end{tabular} & $\overline{115,409}$ & 209 & 739 & $\begin{array}{lll}948 & \end{array}$ & $\begin{array}{lll}3,099 & \\
\end{array}$ & $\frac{10}{10}>(2)$ & 3,110 & no & 165 & 166 & 1199,63: & 2,349 & $\begin{array}{l}1,800,000 \\
\end{array}$ & 3,0 \\
\hline
\end{tabular}

Global Warming Potential (kg of $\mathrm{CO}_{2}$ eq.)

\begin{tabular}{|c|c|c|c|c|c|c|c|c|c|c|c|c|c|c|c|c|}
\hline & & & & & & bal & in & $g$ Poter & $(\mathbf{k}$ & $\mathrm{g}$ of $\mathrm{CO}_{2}$ & $2_{2}$ eq.) & & & & & \\
\hline \multirow{3}{*}{$\begin{array}{l}\text { Letespars } \\
\text { (Y Years) }\end{array}$} & \multicolumn{14}{|c|}{ 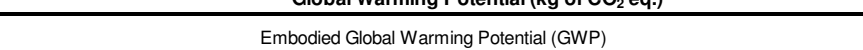 } & \multirow{2}{*}{\multicolumn{2}{|c|}{$\begin{array}{c}\text { Difference in } \\
\text { Operating GWP from } \\
\text { Baseline after } \\
\text { Lifespan }\end{array}$}} \\
\hline & \multicolumn{3}{|c|}{ Manufacturing } & \multicolumn{3}{|c|}{ Construction } & \multicolumn{3}{|c|}{ Maintenance } & \multicolumn{3}{|c|}{ End of L Life } & \multirow{2}{*}{$\begin{array}{l}{ }^{3} \text { Total } \\
\text { GWP }\end{array}$} & \multirow{2}{*}{$\begin{array}{c}{ }^{4} \text { Total } \\
\text { GWP } \\
\text { per m }{ }^{2}\end{array}$} & & \\
\hline & \begin{tabular}{|l|l|} 
Material \\
\end{tabular} & 2 Trans. & Total & Material & 1 ${ }^{2}$ Trans & Total & Material & ${ }^{2}$ Trans. & Total & \begin{tabular}{|l|} 
Material \\
\end{tabular} & 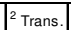 & Total & & & \begin{tabular}{|l|}
${ }^{5}$ Total \\
\end{tabular} & ${ }^{6}$ per $\mathrm{m}^{2}$ \\
\hline |nitial & $6,8>0$ & & & 13 & & 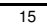 & 0 & 0 & 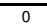 & 0 & 0 & 0 & & 135 & & \\
\hline 50 & \begin{tabular}{|c|c|c|} 
\\
\end{tabular} & 1 & 6,859 & 13 & 1 & 15 & 44 & 0 & 44 & 0 & 0 & 0 & \begin{tabular}{|l|l|}
6,918 \\
\end{tabular} & 136 & \begin{tabular}{|l|l|}
100,000 \\
\end{tabular} & 172 \\
\hline
\end{tabular}

Embodied energy (and GWP) numbers are based on an area of wall $=\begin{array}{rlll}50.9 & \mathrm{~m}^{2}\end{array} \quad$ (Length $x$ Height $=7.6 \mathrm{~m} \times 6.7 \mathrm{~m}=50.9 \mathrm{~m}^{2}$ )

ATHENA $®$ EIE Material List:

$$
\text { (ncludes all materials after } 50 \text { years) }
$$

\begin{tabular}{|c|c|c|}
\hline Material List & Quantities & Unit \\
\hline 3 mil Polyethylene & 54.0 & $\mathrm{~m} 2$ \\
\hline $16 \mathrm{~mm}$ Moisture Resistant Gypsum & 56.0 & $\mathrm{~m} 2$ \\
\hline $16 \mathrm{~mm}$ Regular Gypsum Board & 56.0 & $\mathrm{~m} 2$ \\
\hline Commercial $0.46 \mathrm{~mm}$ Steel Cladding & 168.0 & $\mathrm{~m} 2$ \\
\hline Extruded Polystyrene & 104.3 & $\mathrm{~m} 2(25 \mathrm{~mm})$ \\
\hline Galvanized Studs & 101.0 & $\mathrm{~kg}$ \\
\hline Joint Compound & 111.8 & $\mathrm{~kg}$ \\
\hline Modified Bitumen membrane & 68.2 & $\mathrm{~kg}$ \\
\hline Nails & 9.0 & \\
\hline Paper Tape & 1.3 & $\mathrm{~kg}$ \\
\hline Screws Nuts \& Bolts & 1.3 & $\mathrm{~kg}$ \\
\hline $\begin{array}{l}\text { Small Dimension Sottwood Lumber, } \\
\text { kiln-drie }\end{array}$ & 0.9 & m3 \\
\hline Solvent Based Alkyd Paint & 19.6 & $\mathrm{~L}$ \\
\hline Water Based Latex Paint & 132.5 & $\mathrm{~L}$ \\
\hline
\end{tabular}

$$
\begin{aligned}
& \text { Notes: } \\
& { }^{1} \text { Initial }=\text { Time } \mathrm{O}^{\prime} \text { (i.e. at the completion of initial construction) }
\end{aligned}
$$

Total EE (or Total GWP) $=$ Total embodied energy (or total embodied GWP

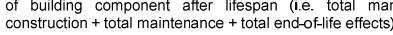

${ }^{4}$ Total EE (or Total GWP) per $\mathrm{m}^{2}=$ Total EE (or Total GWP) of building Total Difference in Operating Energy (or GWP) from Baseline after Lifespan = The difference in the total life-cycle operating energy (or GWP
from the baseline retail building atter fifespan, due to using this building
component instead of the baseline component, ${ }^{6}$ Total Difference in Operating Energy (or GWP) from Baseline after ${ }^{6}$ Total Difference in Operating Energy (or GWP) from Baseline after
Lifespan per $\mathrm{m}^{2}=$ Total difference in operating energy (or GWP) from
baseline after lifespan / net wall area of baseline retail building

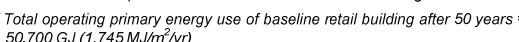

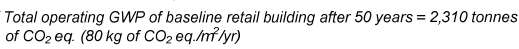




\section{Wood Stud Wall \#6 (WS-W6)}

Building Component Description:

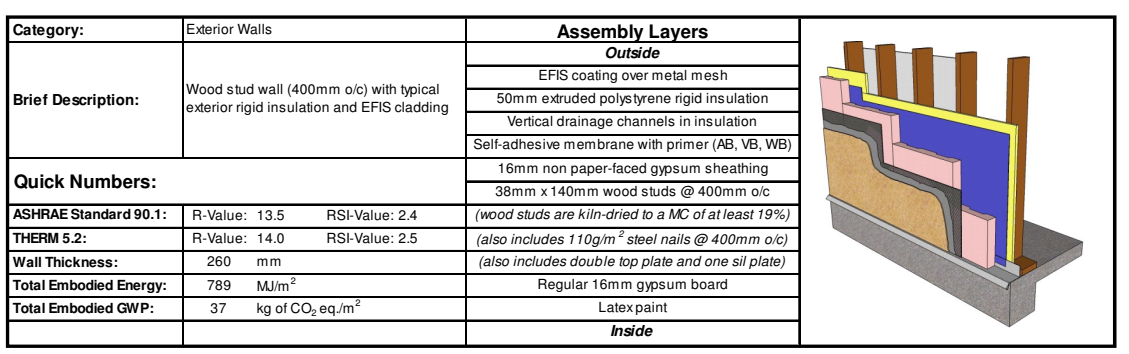

\section{Life-Cycle Assessment Results:}

Primary Energy Consumption (MJ)

\begin{tabular}{|c|c|c|c|c|c|c|c|c|c|c|c|c|c|c|c|c|}
\hline \multirow{3}{*}{$\begin{array}{l}\text { Lifespan } \\
\text { (Years) }\end{array}$} & \multicolumn{14}{|c|}{ Embodied Energy (EE) } & \multirow{2}{*}{\multicolumn{2}{|c|}{$\begin{array}{c}\text { Difference in } \\
\text { Operating Energy } \\
\text { from Baseline after } \\
\text { Lifespan }\end{array}$}} \\
\hline & \multicolumn{3}{|c|}{ Manufacturing } & \multicolumn{3}{|c|}{ onstruction } & \multicolumn{3}{|c|}{ Maintenance } & \multicolumn{3}{|c|}{ End of Life } & \multirow{2}{*}{$\begin{array}{c}{ }^{3} \text { Total } \\
\mathrm{EEE}\end{array}$} & \multirow{2}{*}{$\begin{array}{c}{ }^{4} \text { Total } \\
\text { EE } \\
\text { eer }{ }^{2}\end{array}$} & & \\
\hline & Material & ${ }^{2}$ Trans. & \begin{tabular}{|l|l|} 
Total \\
\end{tabular} & \begin{tabular}{|l|} 
Material \\
\end{tabular} & ${ }^{2}$ Trans. & Total & Material & $\begin{array}{l}{ }^{2} \text { Trans. } \\
\end{array}$ & \begin{tabular}{|l|l|} 
Total \\
\end{tabular} & \begin{tabular}{|l|} 
Material \\
\end{tabular} & ${ }^{2}$ Trans. & \begin{tabular}{|l|} 
Total \\
\end{tabular} & & & ${ }^{5}$ Total & ${ }^{6}{ }^{6}$ per m ${ }^{2}$ \\
\hline Initial & 35,102 & 378 & 35,481 & 209 & 831 & 1,041 & 0 & 0 & 0 & 0 & 0 & 0 & 36,521 & \begin{tabular}{|l|l|}
717 \\
\end{tabular} & & \\
\hline 50 & 35,102 & \begin{tabular}{|l|l|}
378 \\
\end{tabular} & 35,481 & \begin{tabular}{|l|l|} 
& 209 \\
\end{tabular} & \begin{tabular}{|l|l|}
831 \\
\end{tabular} & 1,041 & \begin{tabular}{|l|l|}
3,099 \\
\end{tabular} & 10 & \begin{tabular}{|l|l|l|}
3,110 \\
\end{tabular} & 0 & 521 & \begin{tabular}{|l|l|}
522 \\
\end{tabular} & \begin{tabular}{|l|l|}
40,153 \\
\end{tabular} & \begin{tabular}{|l|l|}
789 \\
\end{tabular} & 600,000 & 1,03 \\
\hline
\end{tabular}

Global Warming Potential $\left(\mathbf{k g}\right.$ of $\mathrm{CO}_{2}$ eq.)

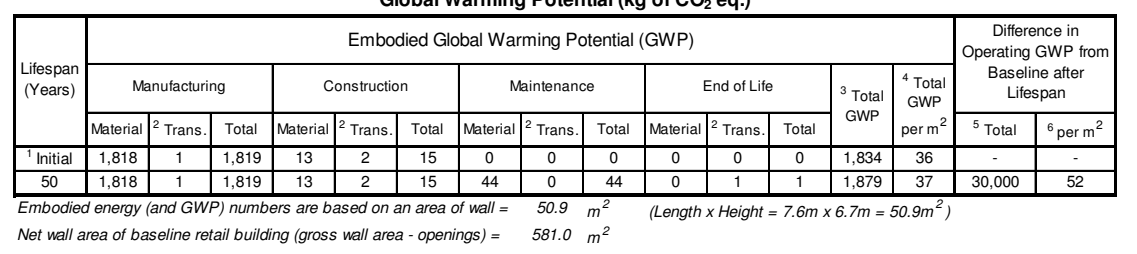

Net wall area of baseline retail building (gross wall area - openings) $=581.0 \mathrm{~m}^{2}$

ATHENA $®$ EIE Material List:

\begin{tabular}{|c|c|c|}
\hline $\begin{array}{l}\text { (Includes all } m \\
\text { Material List }\end{array}$ & \begin{tabular}{|l|} 
e 50 yearss \\
Quantitites
\end{tabular} & Unit \\
\hline$\# 15$ Organic Felt & 219.4 & $\mathrm{~m} 2$ \\
\hline 3 mil Polyethylene & 54.0 & $\mathrm{~m} 2$ \\
\hline $\begin{array}{l}16 \mathrm{~mm} \text { Moisture Resistant Gypsum } \\
\text { Board }\end{array}$ & 56.0 & $\mathrm{~m} 2$ \\
\hline $16 \mathrm{~mm}$ Regular Gypsum Board & 56.0 & $\mathrm{~m} 2$ \\
\hline Extruded Polystyrene & 104.3 & $\mathrm{~m} 2(25 \mathrm{~mm})$ \\
\hline Galvanized Sheet & 51.4 & $\mathrm{~kg}$ \\
\hline Joint Compound & 111.8 & $\mathrm{~kg}$ \\
\hline Modified Bitumen membrane & 68.2 & $\mathrm{~kg}$ \\
\hline Nails & 10.5 & $\mathrm{~kg}$ \\
\hline Paper Tape & 1.3 & $\mathrm{~kg}$ \\
\hline $\begin{array}{l}\text { Small Dimension Softwood Lumber, } \\
\text { kiln-dried }\end{array}$ & 0.9 & $\mathrm{~m} 3$ \\
\hline Solvent Based Alkyd Paint & 19.6 & $\mathrm{~L}$ \\
\hline Stucco over metal mesh & 136.0 & $\mathrm{~m} 2$ \\
\hline Water Based La & 132.5 & \\
\hline
\end{tabular}

Notes:
${ }^{1}$ Initial = Time 'o' (i.e. at the completion of initial construction)

${ }^{2}$ Trans. = Transportation

Total EE (or Total GWP) = Total embodied energy (or total embodied GWP) of building component after lifespan (i.e. total manu
construction + total maintenance + total end-of-life effects)

${ }^{4}$ Total EE (or Total GWP) per $\mathrm{m}^{2}=$ Total EE (or Total GWP) of building
component / area of building component that was modelled in ATHENAQ EIE Total Difference in Operating Energy (or GWP) from Baseline after Lifespan = The difiference in the total lifi-cycle operating energy (or GWP)
from the baseline retail building after lifespan, due to using this building trom the baseline retail bulling atter lifespan,
component instead of the baseline component

Total Difference in Operating Energy (or GWP) from Baseline after
Lifespan per $\mathrm{m}^{2}=$ Total difference in operating energy (or GWP) from Lifespan per $m^{2}=$ Total difference in operating energy
baseline after lifespan / net wall area of baseline retail building

"Total operating primary energy use of baseline retail building after 50 years
${ }^{*}$ Total operating $\mathrm{GWP}$ of baseline retail building after 50 years $=2,310$ tonnes
of $\mathrm{CO}_{2}$ eq. $\left(80 \mathrm{~kg}\right.$ of $\mathrm{CO}_{2}$ eq./m²/yr)

\section{Wood Stud Wall \#7 (WS-W7)}

Building Component Description:

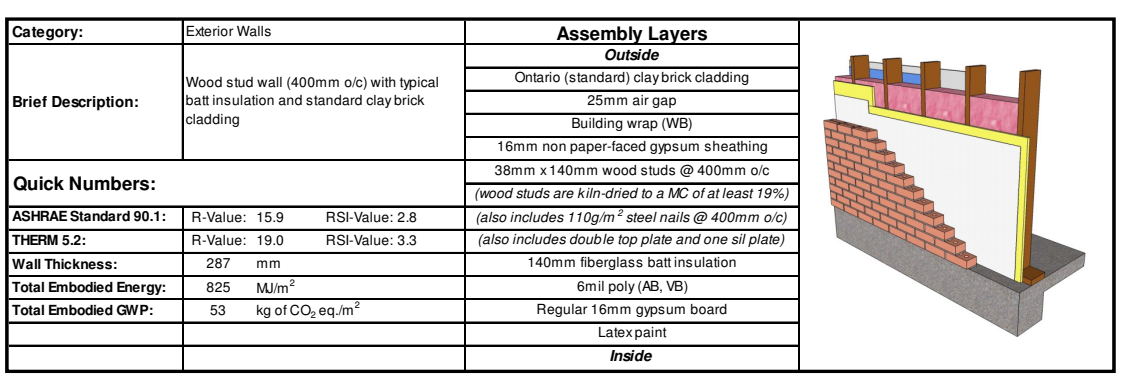

Life-Cycle Assessment Results:

Primary Energy Consumption (M

\begin{tabular}{|c|c|c|c|c|c|c|c|c|c|c|c|c|c|c|c|c|}
\hline \multirow{3}{*}{$\begin{array}{l}\text { Lifespan } \\
\text { (Years) }\end{array}$} & \multicolumn{14}{|c|}{ Embodied Energy (EE) } & \multirow{2}{*}{\multicolumn{2}{|c|}{$\begin{array}{c}\text { Difference in } \\
\text { Operating Energy } \\
\text { from Baseline afte } \\
\text { Lifespan }\end{array}$}} \\
\hline & \multicolumn{3}{|c|}{ Manutacturing } & \multicolumn{3}{|c|}{ Construction } & \multicolumn{3}{|c|}{ Maintenance } & \multicolumn{3}{|c|}{ End of Life } & \multirow{2}{*}{$\begin{array}{c}{ }^{3} \text { Total } \\
\text { EE }\end{array}$} & \multirow{2}{*}{\begin{tabular}{|l|}
${ }^{4} \mathrm{~T}$ otal \\
$\mathrm{EE}$ \\
per m
\end{tabular}} & & \\
\hline & \begin{tabular}{|l|l|} 
Material \\
\end{tabular} & ${ }^{2}$ Trans & \begin{tabular}{|l|l|} 
Total \\
\end{tabular} & Material & ${ }^{2}$ Trans. & Total & \begin{tabular}{|l|} 
Material \\
\end{tabular} & 2 Trans. & Total & \begin{tabular}{|l|} 
Material \\
\end{tabular} & \begin{tabular}{|l|l}
${ }^{2}$ Trans. \\
\end{tabular} & \begin{tabular}{|l|l|} 
Total \\
\end{tabular} & & & ${ }^{5}$ Total & ${ }^{6}$ per $\mathrm{m}^{2}$ \\
\hline Initial & 39,324 & 378 & 39,702 & \begin{tabular}{|l|}
209 \\
\end{tabular} & $\begin{array}{l}1,488 \\
\end{array}$ & 1,698 & 0 & 0 & 0 & 0 & 0 & 0 & 41,400 & 813 & & \\
\hline 50 & 399,324 & 378 & 39,702 & 209 & $\begin{array}{l}1,488 \\
\end{array}$ & 1,698 & 0 & 0 & 0 & 0 & 586 & 586 & 41,986 & 825 & $1,000,000$ & $-1,2$ \\
\hline
\end{tabular}

Global Warming Potential (kg of $\mathrm{CO}_{2}$ eq.)

\begin{tabular}{|c|c|c|c|c|c|c|c|c|c|c|c|c|c|c|c|c|}
\hline \multirow{3}{*}{$\begin{array}{l}\text { Lifespan } \\
\text { (Years) }\end{array}$} & \multicolumn{14}{|c|}{ Embodied Global Warming Potential (GWP) } & \multirow{2}{*}{\multicolumn{2}{|c|}{$\begin{array}{l}\text { Difference in } \\
\text { Operating GWP from } \\
\text { Baseline atter } \\
\text { Lifespan }\end{array}$}} \\
\hline & \multicolumn{3}{|c|}{ Manutacturing } & \multicolumn{3}{|c|}{ Construction } & \multicolumn{3}{|c|}{ Maintenance } & \multicolumn{3}{|c|}{ End of Life } & \multirow{2}{*}{\begin{tabular}{|l}
${ }^{3}$ Total \\
GWP
\end{tabular}} & \multirow{2}{*}{$\begin{array}{l}{ }^{4} \text { Total } \\
\text { GWP } \\
\text { per } \mathrm{m}^{2}\end{array}$} & & \\
\hline & Material & ${ }^{2}$ Trans. & Total & Material & ${ }^{2}$ Trans. & Total & Material & ${ }^{2}$ Trans. & Total & Material & ${ }^{2}$ Trans. & Total & & & ${ }^{5}$ Total & ${ }_{6}^{6}$ perm ${ }^{2}$ \\
\hline itial & 2,694 & 1 & 2,695 & 13 & 3 & 16 & 0 & 0 & 0 & 0 & 0 & 0 & 2,711 & 53 & & \\
\hline 50 & 2,694 & 1 & 2,695 & 13 & 3 & 16 & 0 & 0 & 0 & 0 & 1 & 1 & 2,712 & 53 & $-50,000$ & -86 \\
\hline
\end{tabular}

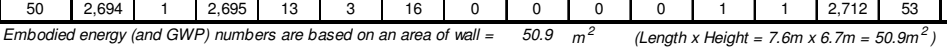

ATHENA $®$ EIE Material List:

(Includes all materials after 50 years)

\begin{tabular}{|l|c|c|}
\hline \multicolumn{1}{|c|}{ Material List } & Quantities & Unit \\
\hline \begin{tabular}{l|c|} 
16mm Moisture Resistant Gypsum \\
Board
\end{tabular} & 56.0 & $\mathrm{~m} 2$ \\
\hline 16mm Regular Gypsum Board & 56.0 & $\mathrm{~m} 2$ \\
\hline 6 mil Polyethylene & 108.0 & $\mathrm{~m} 2$ \\
\hline Batt. Fiberglass & 289.8 & $\mathrm{~m} 2(25 \mathrm{~mm})$ \\
\hline Cold Rolled Sheet & 10.3 & $\mathrm{~kg}$ \\
\hline Joint Compound & 111.8 & $\mathrm{~kg}$ \\
\hline Mortar & 1.5 & $\mathrm{~m} 3$ \\
\hline Nails & 9.0 & $\mathrm{~kg}$ \\
\hline Ontario (Standard) Brick & 53.5 & $\mathrm{~m} 2$ \\
\hline Paper Tape & 1.3 & $\mathrm{~kg}$ \\
\hline $\begin{array}{l}\text { Smanll Dimension Sottwood Lumber, } \\
\text { kilind-dried }\end{array}$ & 0.9 & $\mathrm{~m} 3$ \\
\hline Water Based Latex Paint & 66.3 & $\mathrm{~L}$ \\
\hline
\end{tabular}

Notes:
${ }^{1}$ Initial $=$ Time 'o' (i.e. at the completion of initial construction)

'Initial $=$ Time ' $O$ ' (i.e. at

${ }^{3}$ Total EE (or Total GWP) $=$ Total embodied energy (or total embodied GWP) of building component after lifespan (i.e. total man
construction + total maintenance + total end-of-life effects)

${ }^{4}$ Total EE (or Total GWP) per $\mathrm{m}^{2}=$ Total EE (or Total GWP) of building 5 Total Difference in Operating Energy (or GWP) from Baseline after
Lifespan $=$ The difference in the total lifec-cycle operating energy (or GWP) Lifiespan = The difference in the total life-cycle operating energy
from the baseline retail building after lifespan, due to using this building component instead of the baseline component

Total Difference in Operating Energy (or GWP) from Baseline after Lifespan per $m^{2}=$ Total difference in operating energy
baseline after lifespan $/ \mathrm{net}$ wall area of baseline retail building

* Total operating primary energ
$50,700 \mathrm{GJ}\left(1,745 \mathrm{MJ}^{2} \mathrm{~m}^{2} / \mathrm{yr}\right)$

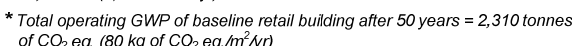




\section{Wood Stud Wall \#8 (WS-W8)}

Building Component Description:

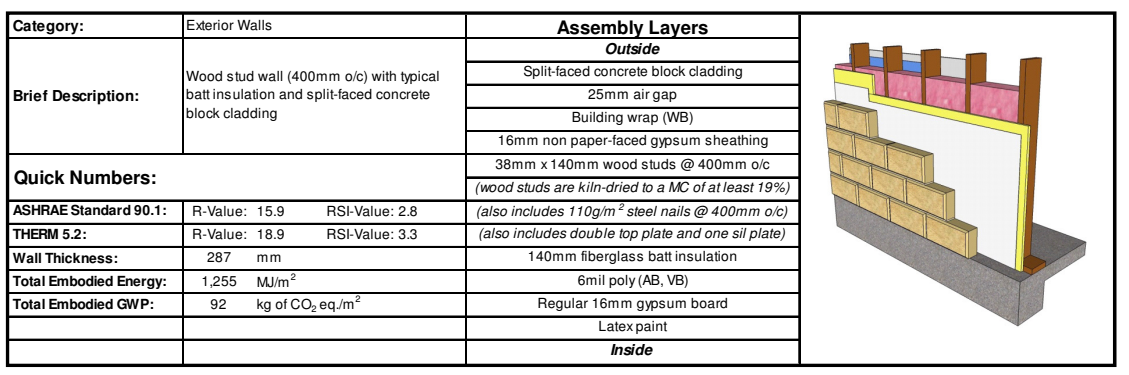

\section{Life-Cycle Assessment Results:}

Primary Energy Consumption (MJ)

\begin{tabular}{|c|c|c|c|c|c|c|c|c|c|c|c|c|c|c|c|c|}
\hline \multirow{3}{*}{\begin{tabular}{|l|l} 
Lifespan \\
(Years)
\end{tabular}} & \multicolumn{14}{|c|}{ Embodied Energy (EE) } & \multirow{2}{*}{\multicolumn{2}{|c|}{$\begin{array}{c}\text { Difference in } \\
\text { Operating Energy } \\
\text { from Baseline afte } \\
\text { Lifespan }\end{array}$}} \\
\hline & \multicolumn{3}{|c|}{ Manufacturing } & \multicolumn{3}{|c|}{ Construction } & \multicolumn{3}{|c|}{ Maintenance } & \multicolumn{3}{|c|}{ End of Life } & \multirow{2}{*}{\begin{tabular}{|l}
3 \\
3 \\
$\mathrm{EE}$
\end{tabular}} & \multirow{2}{*}{$\begin{array}{r}{ }^{4} \text { Total } \\
\mathrm{EE} \\
\text { perm }{ }^{2}\end{array}$} & & \\
\hline & Material & 2 Trans. & \begin{tabular}{|l|l|} 
Total \\
\end{tabular} & Materia & \begin{tabular}{|l|}
2 Trans. \\
\end{tabular} & Total & Material & 每Trans. & Total & Material & $\begin{array}{l}2 \text { Trans. } \\
\end{array}$ & Total & & & ${ }^{5}$ Tot: & ${ }^{6}$ per $m^{2}$ \\
\hline nitial & & 942 & 93 & 209 & , 250 & 08 & 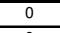 & 0 & 0 & 0 & 0 & 0 & 61,801 & 1,214 & & \\
\hline & & 942 & & & & & & & & & & & & & & \\
\hline
\end{tabular}

Global Warming Potential ( $k g$ of $\mathrm{CO}_{2}$ eq.)

\begin{tabular}{|c|c|c|c|c|c|c|c|c|c|c|c|c|c|c|c|c|}
\hline \multirow{3}{*}{$\begin{array}{l}\text { Lifespan } \\
\text { (Years) }\end{array}$} & \multicolumn{14}{|c|}{ Embodied Global Warming Potential (GWP) } & \multirow{2}{*}{\multicolumn{2}{|c|}{$\begin{array}{l}\text { Difference in } \\
\text { Operating GWP from } \\
\text { Baseline after } \\
\text { Lifespan }\end{array}$}} \\
\hline & \multicolumn{3}{|c|}{ Manufacturing } & \multicolumn{3}{|c|}{ Construction } & \multicolumn{3}{|c|}{ Maintenance } & \multicolumn{3}{|c|}{ End of Life } & \multirow{2}{*}{$\begin{array}{l}{ }^{3} \text { Total } \\
\text { GWP }\end{array}$} & \multirow{2}{*}{$\begin{array}{l}{ }^{4} \text { Total } \\
\text { GWP } \\
\text { per m}{ }^{2}\end{array}$} & & \\
\hline & Material & ${ }^{2}$ Trans. & Total & Material & ${ }^{2}$ Trans. & Total & Material & ${ }^{2}$ Trans. & Total & naterial & 12 2 Trans. & Total & & & ${ }^{5}$ Total & ${ }^{6}$ per m ${ }^{2}$ \\
\hline IIntitial & \begin{tabular}{|l|l|}
4,6880 \\
\end{tabular} & 2 & \begin{tabular}{|l|l|}
4,682 \\
\end{tabular} & 13 & 4 & \begin{tabular}{|l|l|}
18 \\
\end{tabular} & 0 & 0 & 0 & 0 & 0 & 0 & 4,700 & 92 & & \\
\hline 50 & \begin{tabular}{|l|l|}
4,680 \\
\end{tabular} & 2 & 4,6882 & 13 & 4 & $\begin{array}{ll}18 \\
\end{array}$ & 0 & $\overline{0}$ & 0 & 0 & 4 & 4 & 4,704 & 92 & $\begin{array}{ll}-50,000 \\
\end{array}$ & -86 \\
\hline
\end{tabular}

ATHENA ® EIE Material List:

\begin{tabular}{|l|c|c|}
\hline \multicolumn{1}{|c|}{ Material List } & Quantities & Unit \\
\hline $\begin{array}{l}\text { 16mm Moisture Resistant Gypsum } \\
\text { Board }\end{array}$ & 56.0 & $\mathrm{~m} 2$ \\
\hline 16mm Regular Gypsum Board & 56.0 & $\mathrm{~m} 2$ \\
\hline 6 mil Polyethylene & 108.0 & $\mathrm{~m} 2$ \\
\hline Batt. Fiberglass & 289.8 & $\mathrm{~m} 2(25 \mathrm{~mm})$ \\
\hline Cold Rolled Sheet & 10.3 & $\mathrm{~kg}$ \\
\hline Joint Compound & 111.8 & $\mathrm{~kg}$ \\
\hline Modified Bitumen membrane & 333.3 & $\mathrm{~kg}$ \\
\hline Mortar & 4.3 & $\mathrm{~m} 3$ \\
\hline Nails & 9.0 & $\mathrm{~kg}$ \\
\hline Paper Tape & 1.3 & $\mathrm{~kg}$ \\
\hline $\begin{array}{l}\text { Small Dimension Sottwood Lumber, } \\
\text { kiln-dried }\end{array}$ & 0.9 & $\mathrm{~m} 3$ \\
\hline Split-faced Concrete Block & $1,238.2$ & $\mathrm{Blocks}$ \\
\hline Water Based Latex Paint & 66.3 & $\mathrm{~L}$ \\
\hline
\end{tabular}

Notes:
${ }^{1}$ Initial = Time 'o' (i.e. at the completion of initial construction)

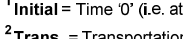

${ }^{3}$ Total EE (or Total GWP) $=$ Total embodied energy (or total embodied GWP) Total
of building component after lifespann (i.e. total manaufacturing + total
construction + total maintenance + total enc-oflife effects)

Total EE (or Total GWP) per $\mathrm{m}^{2}=$ Total EE (or Total GWP) of building
component/ area of building component that was modelled in ATHENA@ EIE component/ area of building component that was modelled in ATHENA® EIE
5 Total Difference in Operating Energy (or GWP) from Baseline after Total Difference in Operating Energy (or GWP) from Baseline after
Lifespan = The difference in the total life-cycle operating energy (or GWP Lifespan = The difiererence in the total lite-cycle operating energy (or GWP
from the basseline retail building after lifespan, due to using this building component instead of the baseline component

Total Difference in Operating Energy (or GWP) from Baseline after Lifespan per $\mathrm{m}^{2}=$ Total difference in operating energy
baseline after lifespan $/$ net wall area of baseline retail building

"Total operating primary energy use of baseline retail building after 50 years =
$50,700 \mathrm{GJ}\left(1,745 \mathrm{MJ} / \mathrm{m}^{2} \mathrm{yr}\right)$ otal operating GWP of baseline retail building after 50 years $=2,310$ tonnes
Wood Stud Wall \#9 (WS-W9)

Building Component Description:

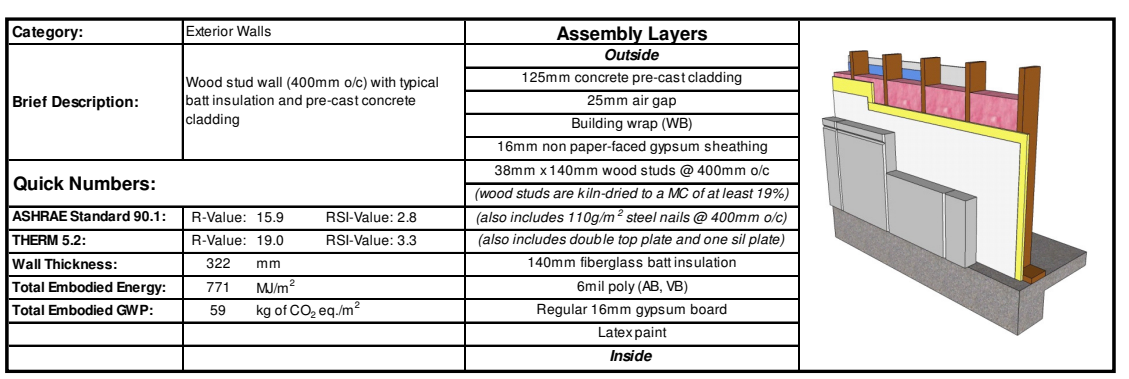

\section{Life-Cycle Assessment Results:}

Primary Energy Consumption (M

\begin{tabular}{|c|c|c|c|c|c|c|c|c|c|c|c|c|c|c|c|c|}
\hline \multirow{3}{*}{$\begin{array}{l}\text { Lifespan } \\
\text { (Years) }\end{array}$} & \multicolumn{14}{|c|}{ Embodied Energy (EE) } & \multirow{2}{*}{\multicolumn{2}{|c|}{$\begin{array}{c}\text { Difference in } \\
\text { Operating Energy } \\
\text { from Baseline atte } \\
\text { Lifespan }\end{array}$}} \\
\hline & \multicolumn{3}{|c|}{ Manufacturing } & \multicolumn{3}{|c|}{ Construction } & \multicolumn{3}{|c|}{ Maintenance } & \multicolumn{3}{|c|}{ End of Life } & \multirow{2}{*}{$\begin{array}{c}{ }^{3} \text { Total } \\
\text { EE }\end{array}$} & \multirow{2}{*}{\begin{tabular}{|l}
${ }^{4}$ Total \\
EE \\
per m ${ }^{2}$
\end{tabular}} & & \\
\hline & \begin{tabular}{|l|} 
Material \\
\end{tabular} & ${ }^{2}$ Trans & \begin{tabular}{|l|l|} 
Total \\
\end{tabular} & Material & 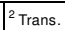 & Total & Material & $\mid{ }^{2}$ Trans. & \begin{tabular}{|l|l|} 
Total \\
\end{tabular} & \begin{tabular}{|l|} 
Material \\
\end{tabular} & \begin{tabular}{|l|l}
${ }^{2}$ Trans. \\
\end{tabular} & \begin{tabular}{|l|} 
Total \\
\end{tabular} & & & ${ }^{5}$ Total & ${ }^{6}$ per $\mathrm{m}^{2}$ \\
\hline Initial & 36,011 & \begin{tabular}{|l|}
969 \\
\end{tabular} & 36,980 & \begin{tabular}{|l|}
209 \\
\end{tabular} & \begin{tabular}{|l|l}
1,427 \\
\end{tabular} & 1,637 & 0 & 0 & 0 & 0 & 0 & 0 & 38,617 & 758 & & \\
\hline 50 & 36,011 & 969 & 36,980 & 209 & \begin{tabular}{|l|l|}
1,427 \\
\end{tabular} & 1,637 & 0 & 0 & 0 & 1 & 619 & 620 & 39,237 & 771 & $\begin{array}{ll}-1,000,000 \\
-\end{array}$ & $-1,7$ \\
\hline
\end{tabular}

Global Warming Potential (kg of $\mathrm{CO}_{2}$ eq.)

\begin{tabular}{|c|c|c|c|c|c|c|c|c|c|c|c|c|c|c|c|c|}
\hline \multirow{3}{*}{$\begin{array}{l}\text { Lifespan } \\
\text { (Years) }\end{array}$} & \multicolumn{14}{|c|}{ Embodied Global Warming Potential (GWP) } & \multirow{2}{*}{\multicolumn{2}{|c|}{$\begin{array}{c}\text { Difference in } \\
\text { Operating GWP from } \\
\text { Baseline after } \\
\text { Lifespan }\end{array}$}} \\
\hline & \multicolumn{3}{|c|}{ Manufacturing } & \multicolumn{3}{|c|}{ Construction } & \multicolumn{3}{|c|}{ Maintenance } & \multicolumn{3}{|c|}{ End of Life } & \multirow{2}{*}{$\begin{array}{l}{ }^{3} \text { Total } \\
\text { GWP }\end{array}$} & \multirow{2}{*}{$\begin{array}{l}{ }^{4} \text { Total } \\
\text { GWW } \\
\text { per m }\end{array}$} & & \\
\hline & Material & ${ }^{2}$ Trans. & Total & Material & ${ }^{2}$ Trans. & Total & Material & \begin{tabular}{|l|}
${ }^{2}$ Trans. \\
\end{tabular} & Total & Material & ${ }^{2}$ Trans. & Total & & & ${ }^{5}$ Total & ${ }^{6}$ perm $m^{2}$ \\
\hline Initial & 2,994 & 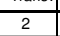 & 2,996 & 13 & 3 & 16 & 0 & 0 & 0 & 0 & 0 & 0 & 3,012 & 59 & & \\
\hline 50 & 2,994 & 2 & 2,996 & 13 & 3 & 16 & 0 & 0 & 0 & 0 & 1 & 1 & 3,014 & 59 & $-50,000$ & -86 \\
\hline
\end{tabular}

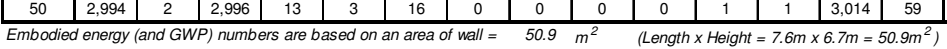
wall area of baseline retail building (gross wall area - openings) $=581.0 \mathrm{~m}^{2}$

ATHENA $®$ EIE Material List: (Includes all materials after 50 years)

\begin{tabular}{|l|c|c|}
\hline \multicolumn{1}{|c|}{ Material List } & Quantities & Unit \\
\hline $\begin{array}{l}16 \mathrm{~mm} \text { Moisture Resistant Gypsum } \\
\text { Board }\end{array}$ & 56.0 & $\mathrm{~m} 2$ \\
\hline $16 \mathrm{~mm}$ Regular Gypsum Board & 56.0 & $\mathrm{~m} 2$ \\
\hline 6 mil Polyethylene & 108.0 & $\mathrm{~m} 2$ \\
\hline Batt. Fiberglass & 289.8 & $\mathrm{~m} 2(25 \mathrm{~mm})$ \\
\hline Concrete $30 \mathrm{MPa}$ (flyash av) & 6.7 & $\mathrm{~m} 3$ \\
\hline Joint Compound & 111.8 & $\mathrm{~kg}$ \\
\hline Nails & 9.0 & $\mathrm{~kg}$ \\
\hline Paper Tape & 1.3 & $\mathrm{~kg}$ \\
\hline Rebar, Rod, Light Sections & 404.0 & $\mathrm{~kg}$ \\
\hline $\begin{array}{l}\text { Small Dimension Sottwood Lumber, } \\
\text { kiln-dried }\end{array}$ & 0.9 & $\mathrm{~m} 3$ \\
\hline Water Based Latex Paint & 66.3 & $\mathrm{~L}$ \\
\hline
\end{tabular}

Notes:
${ }^{1}$ Initial $=$ Time 'o' (i.e. at the completion of initial construction)

'Initial $=$ Time ' $O$ ' (i.e. at

${ }^{3}$ Total EE (or Total GWP) $=$ Total embodied energy (or total embodied GWP) of building component after lifespan (i.e. total man
construction + total maintenance + total end-of-life effects) ${ }^{4}$ Total EE (or Total GWP) per $\mathrm{m}^{2}=$ Total EE (or Total GWP) of building ${ }^{5}$ Total Difference in Operating Energy (or GWP) from Baseline after Lifespan = The difierence in the total life-cycle operating energy (or GWP
from the baseline retail building after lifespan, due to using this building ${ }_{6}^{6}$ component instead of the baseline component

Total Difference in Operating Energy (or GWP) from Baseline after Lifespan per $\mathrm{m}^{2}=$ Total difference in operating energy
baseline after lifespan / net wall area of baseline retail building

Total operating primary energy use of baseline retail building after 50 years
$50,700 \mathrm{GJ}\left(1,745 \mathrm{MJ} \mathrm{m}^{2} \mathrm{Y} y \mathrm{r}\right)$

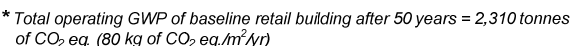

\begin{tabular}{l} 
Shili-dined \\
\hline Water Based Latex Paint \\
\hline
\end{tabular} 


\section{Wood Stud Wall \#10 (WS-W10)}

Building Component Description:

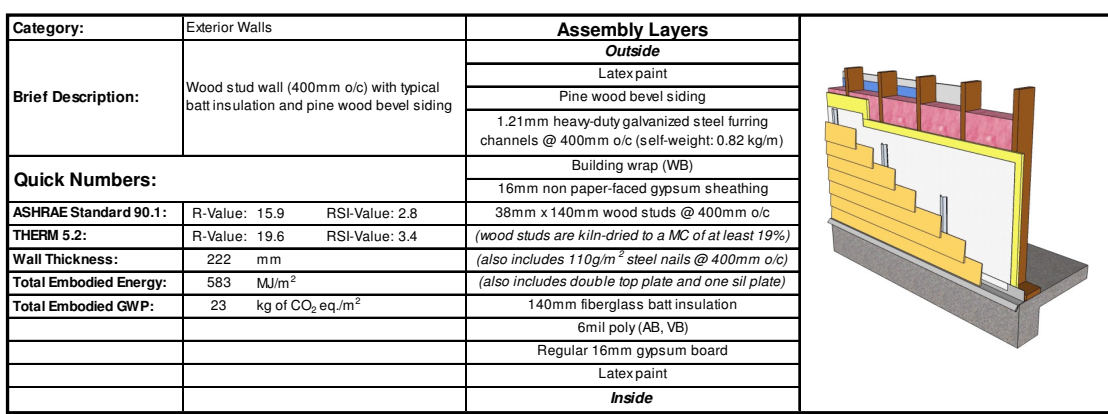

Life-Cycle Assessment Results:

\begin{tabular}{|c|c|c|c|c|c|c|c|c|c|c|c|c|c|c|c|c|}
\hline \multirow{4}{*}{$\mid \begin{array}{c}\text { Lifespan } \\
\text { (Vears) }\end{array}$} & \multicolumn{16}{|c|}{ Energy Consump } \\
\hline & \multicolumn{14}{|c|}{ Embodied Energy (EE) } & \multirow{2}{*}{\multicolumn{2}{|c|}{$\begin{array}{c}\text { Difference in } \\
\text { Operariting Energy } \\
\text { from Baseline after } \\
\text { Lifespan }\end{array}$}} \\
\hline & \multicolumn{3}{|c|}{ Manutacturing } & \multicolumn{3}{|c|}{ Construction } & \multicolumn{3}{|c|}{ Maintenance } & \multicolumn{3}{|c|}{ End of Life } & \multirow{2}{*}{$\begin{array}{l}{ }^{3} \text { Total } \\
\mathrm{EE}\end{array}$} & \multirow{2}{*}{\begin{tabular}{|c|}
${ }^{4}$ Total \\
EE \\
per $\mathrm{m}^{2}$
\end{tabular}} & & \\
\hline & \begin{tabular}{|l|} 
Materia \\
\end{tabular} & \begin{tabular}{l|l}
$1{ }^{2}$ Trans. \\
.
\end{tabular} & \begin{tabular}{|l|l|} 
Total \\
\end{tabular} & $\mid$\begin{tabular}{|l|} 
Material \\
\end{tabular} & ${ }^{2}$ Trans. & Total & Material & \begin{tabular}{ll|}
${ }^{2}$ Trans. \\
The
\end{tabular} & \begin{tabular}{|l|} 
Total \\
\end{tabular} & Material & $\mid{ }^{2}$ Trans. & Total & & & ${ }^{5}$ Total & ${ }^{6}{ }_{\text {per }} \mathrm{m}^{2}$ \\
\hline Initial & 28,056 & 396 & 28,452 & 209 & 837 & 1,047 & 0 & 0 & 0 & 0 & 0 & 0 & 29,499 & 579 & & \\
\hline
\end{tabular}

Global Warming Potential ( $\mathrm{kg}$ of $\mathrm{CO}_{2}$ eq.)

\begin{tabular}{|c|c|c|c|c|c|c|c|c|c|c|c|c|c|c|c|c|}
\hline & & & & & & bal & larming & g Potent & tial $(\mathbf{k}$ & of $\mathrm{CO}_{2}$ & ${ }_{2}$ eq.) & & & & & \\
\hline \multirow{3}{*}{$\begin{array}{l}\text { Litispan } \\
\text { (Years) }\end{array}$} & \multicolumn{14}{|c|}{ Embodied Global Warming Potential (GWP) } & \multirow{2}{*}{\multicolumn{2}{|c|}{$\begin{array}{l}\text { Difference in } \\
\text { Operating GWP from } \\
\text { Baseline after } \\
\text { Lifespan }\end{array}$}} \\
\hline & \multicolumn{3}{|c|}{ Manufacturing } & \multicolumn{3}{|c|}{ Construction } & \multicolumn{3}{|c|}{ Maintenance } & \multicolumn{3}{|c|}{ End of Life } & \multirow{2}{*}{$\begin{array}{l}3^{3} \text { otal } \\
\text { GWP }\end{array}$} & \multirow{2}{*}{$\mid \begin{array}{c}{ }^{4} \text { Total } \\
\text { GWP } \\
\text { per m }\end{array}$} & & \\
\hline & \begin{tabular}{|l|} 
Material \\
\end{tabular} & ${ }^{2}$ Trans. & \begin{tabular}{|l|} 
Total \\
\end{tabular} & Material & ${ }^{2}$ Trans. & Total & Material & 2 Trans. & Total & \begin{tabular}{|l|} 
Materiala \\
\end{tabular} & 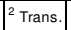 & Total & & & ${ }^{5}$ Total & ${ }^{6} \mathrm{per} \mathrm{m}^{2}$ \\
\hline$\overline{1}$ Initial & 1,169 & 1 & 1,170 & 13 & 2 & 15 & 0 & $\overline{0}$ & 0 & 0 & 0 & 0 & 1,185 & 23 & & \\
\hline 50 & \begin{tabular}{|l|l|}
1,169 \\
\end{tabular} & 1 & 1,170 & 13 & 2 & 15 & 0 & 0 & 0 & 0 & 0 & 0 & 1,185 & 23 & $-40,000$ & 69 \\
\hline
\end{tabular}

Net wall area of baseline retail building (gross wall area - openings) $=581.0 \mathrm{~m}^{2}$

\section{ATHENA ${ }^{\circledR}$ EIE Material List:}

\begin{tabular}{|l|c|c|}
\hline \multicolumn{1}{|c|}{ Material List } & Quantities & Unit \\
\hline $\begin{array}{l}\text { 16mm Moisture Resistant Gypsum } \\
\text { Board }\end{array}$ & 56.0 & $\mathrm{~m} 2$ \\
\hline 16mm Regular Gypsum Board & 56.0 & $\mathrm{~m} 2$ \\
\hline 6 mil Polyethylene & 108.0 & $\mathrm{~m} 2$ \\
\hline Batt. Fiberglass & 289.8 & $\mathrm{~m} 2(25 \mathrm{~mm})$ \\
\hline Galvanized Sheet & 106.1 & $\mathrm{~kg}$ \\
\hline Joint Compound & 111.8 & $\mathrm{~kg}$ \\
\hline Nails & 10.3 & $\mathrm{~kg}$ \\
\hline Paper Tape & 1.3 & $\mathrm{~kg}$ \\
\hline Pine Wood Bevel Siding & 160.4 & $\mathrm{~m} 2$ \\
\hline $\begin{array}{l}\text { Small } \\
\text { kiln-drimension Softwood Lumber, }\end{array}$ & 0.9 & $\mathrm{~m} 3$ \\
\hline Water Based Latex Paint & 132.5 & $\mathrm{~L}$ \\
\hline
\end{tabular}

$$
\begin{aligned}
& \text { Notes: } \\
& \text { ial }=\text { Time ' } 0 \text { ' (i.e. at the completion of in }
\end{aligned}
$$

${ }^{2}$ Trans. $=$ Transportation

Notes:
pletion of initial construction)

${ }^{3}$ Total EE (or Total GWP) = Total embodied energy (or total embodied GWP) of building component after lifespan (i.e. total manufacturing + tota

${ }^{4}$ Total EE (or Total GWP) per $\mathrm{m}^{2}=$ Total EE (or Total GWP) of building Total Difference in Operting Energy (or GWP) from Baseline after Lifespan $=$ The difference in the total life-cycle operating energy (or GWP)
from the baseline retail building after lifespan, due to using this building

Lifespan per $\mathrm{m}^{2}=$ Total difference in operating energy (or GWP) from baseline after lifiespan / net wall area of baseline retail building * Total operating primary energy use of baseline retail building after 50 years

* Total operating $\mathrm{GWP}$ of baseline retail building after 50 years $=2,310$ tonnes

\section{Wood Stud Wall \#11 (WS-W11)}

Building Component Description:

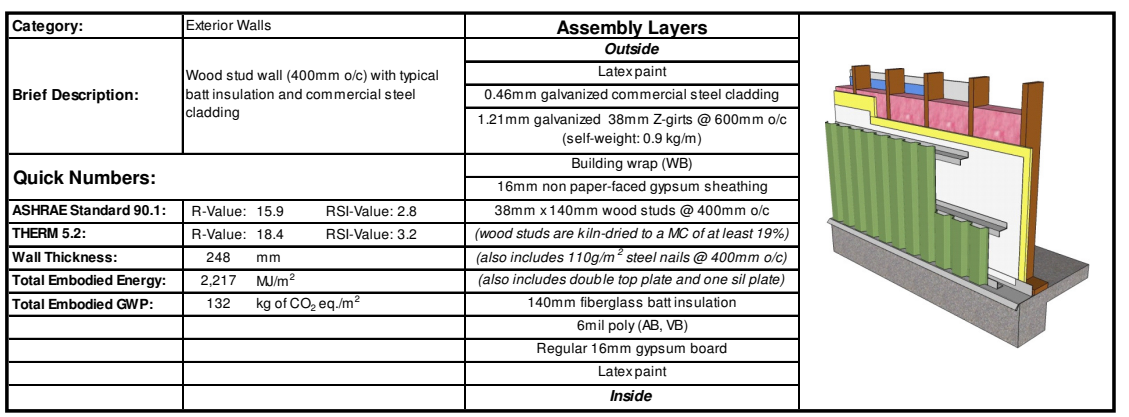

Life-Cycle Assessment Results:

\begin{tabular}{|c|c|c|c|c|c|c|c|c|c|c|c|c|c|c|c|}
\hline \multirow{4}{*}{$\begin{array}{l}\text { Lifespan } \\
\text { (Years) }\end{array}$} & \multicolumn{13}{|c|}{ ry Energy Consumption (MJ) } & \multirow{3}{*}{\multicolumn{2}{|c|}{$\begin{array}{l}\text { Difference in } \\
\text { Operating Energy } \\
\text { from Baseline after } \\
\text { Lifespan }\end{array}$}} \\
\hline & \multicolumn{13}{|c|}{ Embodied Energy (EE) } & & \\
\hline & \multicolumn{2}{|c|}{ Manutacturing } & \multicolumn{3}{|c|}{ Construction } & \multicolumn{3}{|c|}{ Mainten } & \multicolumn{3}{|c|}{ End of Life } & \multirow{2}{*}{ 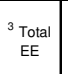 } & \multirow{2}{*}{\begin{tabular}{|c|}
${ }^{4}$ Total \\
EE \\
per m
\end{tabular}} & & \\
\hline & \begin{tabular}{|l|l|} 
Material & ${ }^{2}$ Trans. \\
\end{tabular} & Total & Material & ${ }^{2}$ Trans. & Total & Material & $2{ }^{2}$ Trans. & Total & Material & \begin{tabular}{|l|}
${ }^{2}$ Trans. \\
\end{tabular} & Total & & & ${ }^{5}$ Total & $6 \operatorname{perm}^{2}$ \\
\hline Initial & \begin{tabular}{|l|l|}
1,424 & 348 \\
\end{tabular} & 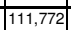 & 209 & - & 515 & - & 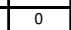 & 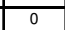 & 0 & 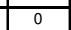 & $=$ & 2,716 & 2,214 & & \\
\hline 50 & \begin{tabular}{c|c|}
424 & 348
\end{tabular} & $\mid 111,772$ & 209 & \begin{tabular}{|l|l|}
735 \\
\end{tabular} & \begin{tabular}{|l|l|}
945 \\
\end{tabular} & 0 & 0 & 0 & 0 & 168 & 168 & 112,8 & 2,217 & 600,000 & $-1,03$ \\
\hline
\end{tabular}

Global Warming Potential ( $k g$ of $\mathrm{CO}_{2}$ eq)

\begin{tabular}{|c|c|c|c|c|c|c|c|c|c|c|c|c|c|c|c|c|}
\hline \multirow{3}{*}{$\begin{array}{l}\text { Lifespan } \\
\text { (Years) }\end{array}$} & \multicolumn{14}{|c|}{ Embodied Global Warming Potential (GWP) } & \multirow{2}{*}{\multicolumn{2}{|c|}{\begin{tabular}{|c|} 
Difference in \\
Operating GWP from \\
Baseline after \\
Lifespan
\end{tabular}}} \\
\hline & \multicolumn{3}{|c|}{ Manufacturing } & \multicolumn{3}{|c|}{ Construction } & \multicolumn{3}{|c|}{ Maint } & \multicolumn{3}{|c|}{ End of Life } & \multirow{2}{*}{$\begin{array}{l}3 \text { Total } \\
\text { GWP }\end{array}$} & \multirow{2}{*}{$\begin{array}{c}{ }^{4} \text { Total } \\
\text { GWP } \\
\text { per } \mathrm{m}^{2}\end{array}$} & & \\
\hline & Material & ${ }^{2}$ Trans. & Total & Material & ${ }^{2}$ Trans. & Total & Material & ${ }^{2}$ Trans. & Total & Material & ${ }^{2}$ Trans. & Total & & & ${ }^{5}$ Total & ${ }^{6}{ }_{\text {perm }}^{2}$ \\
\hline tial & 6,717 & 1 & 6,717 & 13 & 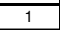 & 15 & 0 & 0 & 0 & 0 & n & 0 & & 132 & & \\
\hline 50 & 6,717 & 1 & 6,717 & 13 & 1 & 15 & 0 & 0 & 0 & 0 & 0 & 0 & 732 & 32 & 30,0 & -52 \\
\hline
\end{tabular}

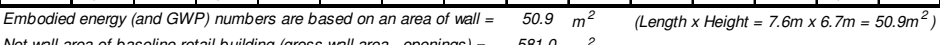
Net wall area of baseline retall building (gross wall area - openings) $=581.0 \mathrm{~m}^{2}$

ATHENA ${ }^{\circledR}$ EIE Material List:

\begin{tabular}{|c|c|c|}
\hline Material List & Quantities & Unit \\
\hline $\begin{array}{l}\text { 16mm Moisture Resistant Gypsum } \\
\text { Board }\end{array}$ & 56.0 & $\mathrm{~m} 2$ \\
\hline $16 \mathrm{~mm}$ Regular Gypsum Board & 56.0 & $\mathrm{~m} 2$ \\
\hline 6 mil Polyethylene & 108.0 & $\mathrm{~m} 2$ \\
\hline Batt. Fiberglass & 289.8 & $\mathrm{~m} 2(25 \mathrm{~mm}$ \\
\hline Commercial $0.46 \mathrm{~mm}$ Steel Cladding & 168.0 & $\mathrm{~m} 2$ \\
\hline Galvanized Studs & 80.8 & $\mathrm{~kg}$ \\
\hline Joint Compound & 111.8 & $\mathrm{~kg}$ \\
\hline Nails & 9.0 & $\mathrm{~kg}$ \\
\hline Paper Tape & 1.3 & $\mathrm{~kg}$ \\
\hline Screws Nuts \& Bolts & 1.3 & $\mathrm{~kg}$ \\
\hline $\begin{array}{l}\text { Small Dimension Sottwood Lumber, } \\
\text { kiln-dried }\end{array}$ & 0.9 & $\mathrm{~m} 3$ \\
\hline Water Based Latex Paint & 132.5 & $\mathrm{~L}$ \\
\hline
\end{tabular}

'Initial = Time 'o' (i.e. $\quad$ Notes:

${ }^{2}$ Trans. = Transportation

Total EE (or Total GWP) = Total embodied energy (or total embodied GWP) of building component after lifespan (i.e. total manufacturning + to

${ }^{4}$ Total EE (or Total GWP) per $\mathrm{m}^{2}=$ Total EE (or Total GWP) of building
component / area of building component that was modeled in ATHENAQ LIE ${ }^{5}$ Total Difference Lifespan = The difiterence in the total tive-cycle operating energy (or GWP) from the baseline retail building affer lifespan, due to using this building
component instead of the baseline component ${ }^{6}$ Total Difference in Operating Energy (or GWP) from Baseline after Lifiespan per $\mathrm{m}^{2}=$ Total difference in operating energy (or GWP) from
baseline after lifespan $/$ net wall area of baseline retail building

* Total operating primarn energy use of baseline retail building after 50 years
$50,700 \mathrm{GJ}\left(1,745 \mathrm{MJ} / \mathrm{m}^{2} / \mathrm{yr}\right)$

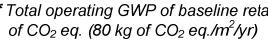


Wood Stud Wall \#12 (WS-W12)

Building Component Description:

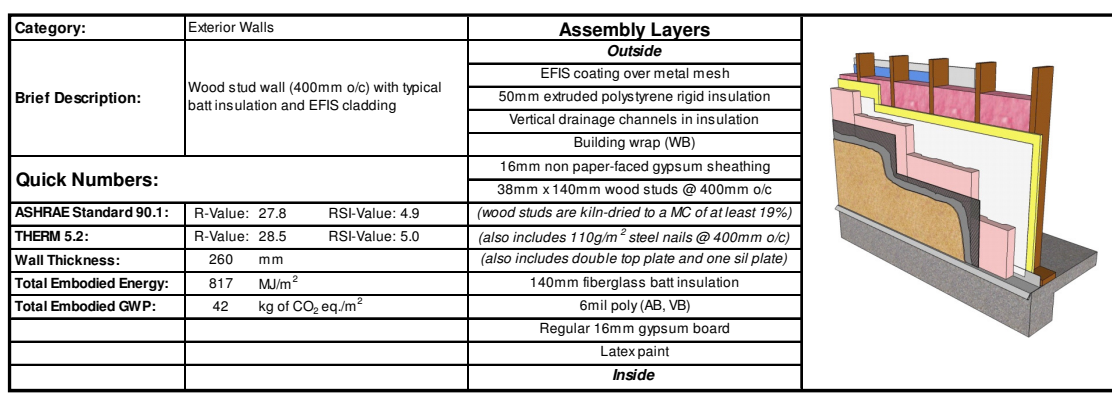

\section{Life-Cycle Assessment Results:}

Primary Energy Consumption (MJ)

\begin{tabular}{|c|c|c|c|c|c|c|c|c|c|c|c|c|c|c|c|c|}
\hline \multirow{3}{*}{$\begin{array}{l}\text { Lifespan } \\
\text { (Years) }\end{array}$} & \multicolumn{14}{|c|}{ Embodied Energy (EE) } & \multirow{2}{*}{\multicolumn{2}{|c|}{$\begin{array}{c}\text { Difference in } \\
\text { Operating Energy } \\
\text { from Baseline after } \\
\text { Lifespan }\end{array}$}} \\
\hline & \multicolumn{3}{|c|}{ Manutacturing } & \multicolumn{3}{|c|}{ Construction } & \multicolumn{3}{|c|}{ Maintenance } & \multicolumn{3}{|c|}{ End of Life } & \multirow{2}{*}{$\begin{array}{c}{ }^{3} \text { Total } \\
\mathrm{EE}\end{array}$} & \multirow{2}{*}{\begin{tabular}{|c|c}
${ }^{4}$ Total \\
$\mathrm{EE}$ \\
$\mathrm{ner}^{2}$
\end{tabular}} & & \\
\hline & Material & ${ }^{2}$ Trans. & Total & Materia & ${ }^{2}$ Trans. & Total & Material & ${ }^{2}$ Trans. & \begin{tabular}{|l|l|l|} 
Total & \\
\end{tabular} & Material & \begin{tabular}{|l|}
${ }^{2}$ Trans. \\
\end{tabular} & Total & & & ${ }^{5}$ Total & ${ }^{6}$ per m$^{2}$ \\
\hline |litial & 39,596 & 404 & 40,001 & 209 & 842 & 1,052 & 0 & 0 & 0 & 0 & 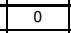 & 0 & 41,053 & 806 & & \\
\hline 50 & 39,596 & 404 & 40,001 & 209 & 842 & 1,052 & 0 & 0 & 0 & 0 & 534 & 535 & \begin{tabular}{|l|l|}
41,587 \\
\end{tabular} & 817 & $-1,900,00$ & 3,270 \\
\hline
\end{tabular}

Global Warming Potential ( $\left(\mathrm{gg}\right.$ of $\mathrm{CO}_{2}$ eq.)

\begin{tabular}{|c|c|c|c|c|c|c|c|c|c|c|c|c|c|c|c|c|}
\hline \multirow{4}{*}{$\begin{array}{l}\text { Lifespan } \\
\text { (Years) }\end{array}$} & \multirow{2}{*}{\multicolumn{14}{|c|}{ Embodied Global Warming Potential (GWP) }} & \multirow{3}{*}{\multicolumn{2}{|c|}{$\begin{array}{c}\text { Difference in } \\
\text { Operating GWPP fron } \\
\text { Baseline after } \\
\text { Lifespan } \\
\end{array}$}} \\
\hline & & & & & & & & & & & & & & & & \\
\hline & \multicolumn{3}{|c|}{ Manufacturing } & \multicolumn{3}{|c|}{ Construction } & \multicolumn{3}{|c|}{ Maintenance } & \multicolumn{3}{|c|}{ End of Life } & \multirow{2}{*}{$\begin{array}{l}{ }^{3} \text { Total } \\
\text { GWP }\end{array}$} & \multirow{2}{*}{$\begin{array}{c}{ }^{4} \text { Total } \\
\text { GWP } \\
\text { per m² }\end{array}$} & & \\
\hline & Material & ${ }^{2}$ Trans. & Total & Material & 2 Trans. & Total & Material & ${ }^{2}$ Trans. & Total & Material & 2 Trans. & Total & & & ${ }^{5}$ Total & ${ }^{6}$ pern \\
\hline Initial & 2,107 & 1 & 2, & 717 & 2 & 15 & 0 & 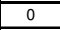 & 0 & 0 & 0 & 0 & 3 & 42 & & \\
\hline 50 & 2,107 & 1 & 2,1 & 13 & 2 & 15 & 0 & 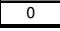 & 0 & 0 & 1 & 1 & 2,124 & 42 & -100, & $-1 / 2$ \\
\hline
\end{tabular}

Net wall area of baseline retail building (gross wall area - openings) $=50.9 \mathrm{~m}^{2}$

ATHENA ® EIE Material List:

\begin{tabular}{|c|c|c|}
\hline Material List & Quantities & Unit \\
\hline$\# 15$ Organic Felt & 219.4 & $\mathrm{~m} 2$ \\
\hline $\begin{array}{l}16 \mathrm{~mm} \text { Moisture Resistant Gypsum } \\
\text { Board }\end{array}$ & 56.0 & $\mathrm{~m} 2$ \\
\hline 16mm Regular Gypsum Board & 56.0 & $\mathrm{~m} 2$ \\
\hline 6 mil Polyethylene & 108.0 & $\mathrm{~m} 2$ \\
\hline Batt. Fiberglass & 289.8 & m2 (25mm) \\
\hline Extruded Polystyrene & 104.3 & m2 (25mm) \\
\hline Galvanized Sheet & 51.4 & $\mathrm{~kg}$ \\
\hline Joint Compound & 111.8 & $\mathrm{~kg}$ \\
\hline Nails & 13.6 & $\mathrm{~kg}$ \\
\hline Paper Tape & 1.3 & $\mathrm{~kg}$ \\
\hline 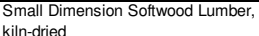 & 0.9 & $\mathrm{~m} 3$ \\
\hline Stucco over metal mesh & 136.0 & $\mathrm{~m} 2$ \\
\hline Water Based Latex Paint & 132.5 & $\mathrm{~L}$ \\
\hline
\end{tabular}

\section{Notes:}

${ }^{1}$ Initial $=$ Time '
${ }^{2}$ ' (i.e. at
${ }^{2}$ Trans. $=$ Transportation

${ }^{3}$ Total EE (or Total GWP) = Total embodied energy (or total embodied GWP) of building component after lifespan (i.e. total manufacturing + tota

${ }^{4}$ Total EE (or Total GWP) per $\mathrm{m}^{2}=$ Total EE (or Total GWP) of building
component/ area of building component that was modelled in ATHENAQEEE ${ }^{5}$ Tote Difference on Operating Energy (or GWP) from Barger Lifespan = The difference in the total life-cycle operating energy (or GWWP from the baseline retail building after lifespan, due to using this building

${ }^{6}$ Total Difference in Operating Energy (or GWP) from Baseline after Lifespan per $m^{2}=$ Total difference in operating energy
baseline after lifespan / net wall area of baseline retail building Total operating primary energy use of baseline retail building after 50 years

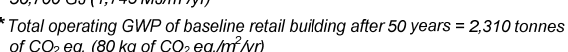

Wood Stud Wall \#13 (WS-W13)

Building Component Description:

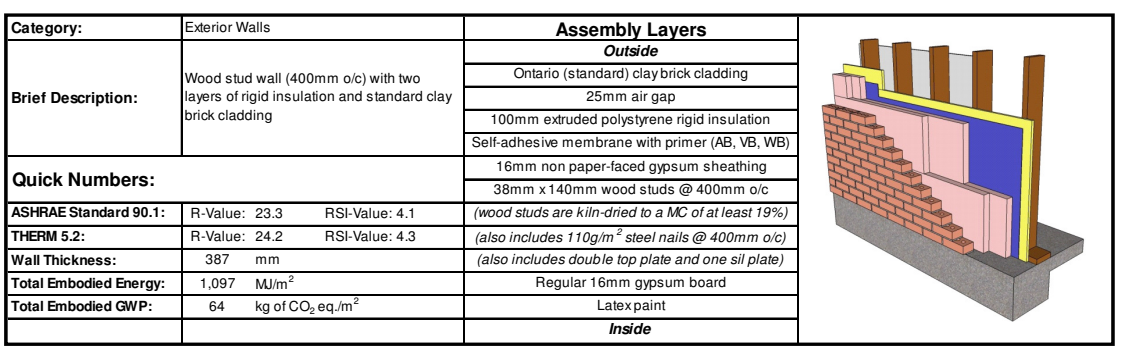

Life-Cycle Assessment Results:

Primary Energy Consumption (MJ)

\begin{tabular}{|c|c|c|c|c|c|c|c|c|c|c|c|c|c|c|c|c|}
\hline \multirow{3}{*}{$\begin{array}{l}\text { Lifespan } \\
\text { (Years) }\end{array}$} & \multicolumn{14}{|c|}{ Embodied Energy (EE) } & \multirow{2}{*}{\multicolumn{2}{|c|}{$\begin{array}{l}\text { Difference in } \\
\text { Operating Energy } \\
\text { from Baseline after } \\
\text { Lifespan }\end{array}$}} \\
\hline & \multicolumn{3}{|c|}{ Manufacturing } & \multicolumn{3}{|c|}{ Construction } & \multicolumn{3}{|c|}{ Maintenance } & \multicolumn{3}{|c|}{ End of Life } & \multirow{2}{*}{$\begin{array}{c}{ }^{3} \text { Total } \\
\text { EE }\end{array}$} & \multirow{2}{*}{$\begin{array}{l}{ }^{4} \text { Total } \\
\mathrm{EE} \\
\text { Der }{ }^{2}\end{array}$} & & \\
\hline & Material & ${ }^{2}$ Trans. & Total & Material & \begin{tabular}{|l|}
${ }^{2}$ Trans. \\
\end{tabular} & Total & Material & ${ }^{2}$ Trans. & Total & Material & 1 ${ }^{2}$ Trans. & Total & & & ${ }^{5}$ Total & erm $m^{2}$ \\
\hline |ltial & 50,0 & 353 & 50,440 & 209 & 1,502 & 1 & $\overline{0}$ & 0 & & 0 & 0 & 0 & $\overline{52,151}$ & 24 & & \\
\hline & & 253 & 5,440 & & & & & & & & & & & & & \\
\hline
\end{tabular}

Global Warming Potential (kg of $\mathrm{CO}_{2}$ eq.)

\begin{tabular}{|c|c|c|c|c|c|c|c|c|c|c|c|c|c|c|c|c|}
\hline \multirow{3}{*}{$\begin{array}{l}\text { Lifespan } \\
\text { (Years) }\end{array}$} & \multicolumn{14}{|c|}{ Embodied Global Warming Potential (GWP) } & \multirow{2}{*}{\multicolumn{2}{|c|}{$\begin{array}{l}\text { Difference in } \\
\text { Operating GWP from } \\
\text { Baseline after } \\
\text { Lifespan }\end{array}$}} \\
\hline & \multicolumn{3}{|c|}{ Manufacturing } & \multicolumn{3}{|c|}{ Construction } & \multicolumn{3}{|c|}{ Maintenance } & \multicolumn{3}{|c|}{ End of Lifie } & \multirow{2}{*}{$\begin{array}{l}{ }^{3} \text { Total } \\
\text { GWP }\end{array}$} & \multirow{2}{*}{\begin{tabular}{|l}
${ }^{4}$ Total \\
GWP
\end{tabular}} & & \\
\hline & Material & ${ }^{2}$ Trans. & Total & Material & Trans. & Total & Material & ${ }^{2}$ Trans. & Total & Material & 2 Trans. & Total & & & ${ }^{5}$ Total & ${ }^{6} \mathrm{perm}^{2}$ \\
\hline Intitial & 3,185 & 1 & 3,186 & 13 & 3 & 16 & 0 & 0 & 0 & 0 & 0 & 0 & 3,202 & 63 & & \\
\hline 50 & 3185 & 1 & 3.186 & 13 & 3 & 16 & 44 & 0 & 44 & 0 & 1 & 1 & 3 & 64 & 80,00 & -138 \\
\hline
\end{tabular}
(a)

ATHENA $®$ EIE Material List: (Includes all materials after 50 years

\begin{tabular}{|l|c|c|}
\hline \multicolumn{1}{|c|}{ Material List } & Quantities & Unit \\
\hline 3 mil Polyethylene & 54.0 & $\mathrm{~m} 2$ \\
\hline $\begin{array}{l}\text { 16mm Moisture Resistant Gypsum } \\
\text { Board }\end{array}$ & 56.0 & $\mathrm{~m} 2$ \\
\hline 16mm Regular Gypsum Board & 56.0 & $\mathrm{~m} 2$ \\
\hline Cold Rolled Sheet & 10.3 & $\mathrm{~kg}$ \\
\hline Extruded Polystyrene & 208.6 & $\mathrm{~m} 2(25 \mathrm{~mm})$ \\
\hline Joint Compound & 111.8 & $\mathrm{~kg}$ \\
\hline Modified Bitumen membrane & 68.2 & $\mathrm{~kg}$ \\
\hline Mortar & 1.5 & $\mathrm{~m} 3$ \\
\hline Nails & 9.0 & $\mathrm{~kg}$ \\
\hline Ontario (Standard) Brick & 53.5 & $\mathrm{~m} 2$ \\
\hline Paper Tape & 1.3 & $\mathrm{~kg}$ \\
\hline $\begin{array}{l}\text { Small Dimension Sottwood Lumber, } \\
\text { kiln-dried }\end{array}$ & 0.9 & $\mathrm{~m} 3$ \\
\hline Solvent Based Alkyd Paint & 19.6 & $\mathrm{~L}$ \\
\hline Water Based Latex Paint & 66.3 & $\mathrm{~L}$ \\
\hline
\end{tabular}
Notes:
${ }^{1}$ Initial $=$ Time 'o' (i.e. at the completion of initial construction) ${ }^{2}$ Trans. $=$ Transportation

Total EE (or Total GWP) = Total embodied energy (or total embodied GWP of building component after lifespan (i.e. total man
construction + total maintenance + total end-of-life effects)

${ }^{4}$ Total EE (or Total GWP) per $\mathrm{m}^{2}=$ Total EE (or Total GWP) of building
component / area of building component that was modelled in ATHENAB EIE ${ }^{5}$ Total Difference in Operating Energy (or GWP) from Baseline after (t) component instead of the baseline component

Total Difference in Operating Energy (or GWP) from Baseline after

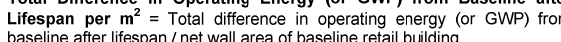

* Total operating primary energy use of baseline retail building after 50 years =

${ }^{2}$
of $\mathrm{CO}_{2}$ eq.
$\left(80 \mathrm{~kg}\right.$ of $\mathrm{CO}_{2}$ eq. 


\section{Wood Stud Wall \#14 (WS-W14)}

Building Component Description:

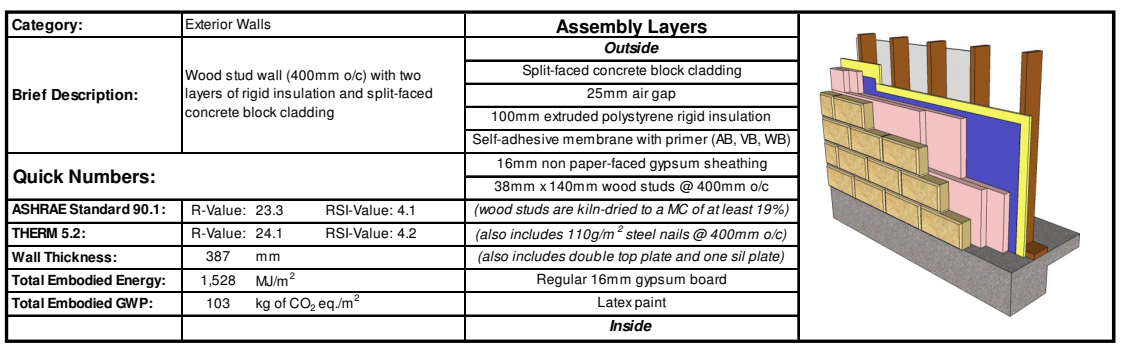

\section{Life-Cycle Assessment Results:}

Primary Energy Consumption (MJ)

\begin{tabular}{|c|c|c|c|c|c|c|c|c|c|c|c|c|c|c|c|c|}
\hline \multirow{3}{*}{$\begin{array}{l}\text { Lifespan } \\
\text { (ears) }\end{array}$} & \multicolumn{14}{|c|}{ Embodied Energy (EE) } & \multirow{2}{*}{\multicolumn{2}{|c|}{$\begin{array}{c}\text { Difference in } \\
\text { Operating Energy } \\
\text { from Baseline after } \\
\text { Lifespan }\end{array}$}} \\
\hline & \multicolumn{3}{|c|}{ Manufacturing } & \multicolumn{3}{|c|}{ Construc } & \multicolumn{3}{|c|}{ Maintenance } & \multicolumn{3}{|c|}{ End of $L$} & \multirow{2}{*}{$\begin{array}{c}{ }^{3} \text { Total } \\
\mathrm{EE}\end{array}$} & \multirow{2}{*}{$\begin{array}{l}{ }^{4}{ }^{4} \text { Total } \\
\text { EE } \\
\text { per m }\end{array}$} & & \\
\hline & Materia & ${ }^{2}$ Trans & Total & Materia & I Trans. & Total & Material & ${ }^{2}$ Trans. & Total & Material & 1. ${ }^{2}$ Trans. & 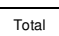 & & & ${ }^{5}$ Total & ${ }^{6} \operatorname{per~m}^{2}$ \\
\hline \begin{tabular}{|l|l} 
Initial \\
\end{tabular} & 69,114 & 917 & 70,031 & 209 & 2,312 & 2,521 & 0 & 0 & 0 & 0 & 0 & 0 & 72,552 & 1,425 & & \\
\hline 50 & 69,11 & 917 & 770,031 & 209 & 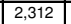 & 2,521 & \begin{tabular}{|l|l|}
3,099 \\
\end{tabular} & 10 & \begin{tabular}{|l|l|}
3,1110 \\
\end{tabular} & 1 & $\begin{array}{ll}2,118 \\
\end{array}$ & 2,119 & 77,781 & $\begin{array}{ll}1,528 \\
\end{array}$ & $-1,600,000$ & $\begin{array}{l}2,754 \\
\end{array}$ \\
\hline
\end{tabular}

Global Warming Potential ( $\mathrm{kg}$ of $\mathrm{CO}_{2}$ eq.)

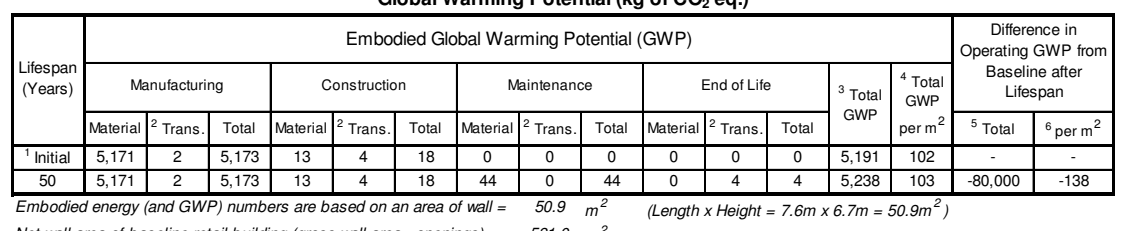

(a) $=581.0$

\section{ATHENA $®$ EIE Material List:} (Includes all materials after 50 years)

\begin{tabular}{|c|c|c|}
\hline Material List & Quantities & Unit \\
\hline 3 mil Polyethylene & 54.0 & $\mathrm{~m} 2$ \\
\hline $\begin{array}{l}\text { 16mm Moisture Resistant Gypsum } \\
\text { Board }\end{array}$ & 56.0 & $\mathrm{~m} 2$ \\
\hline 16mm Regular Gypsum Board & 56.0 & $\mathrm{~m} 2$ \\
\hline Cold Rolled Sheet & 10.3 & $\mathrm{~kg}$ \\
\hline Extruded Polystyrene & 208.6 & m2 (25mm) \\
\hline Joint Compound & 111.8 & $\mathrm{~kg}$ \\
\hline Modified Bitumen membrane & 401.5 & $\mathrm{~kg}$ \\
\hline Mortar & 4.3 & $\mathrm{~m} 3$ \\
\hline Nails & 9.0 & $\mathrm{~kg}$ \\
\hline Paper Tape & 1.3 & $\mathrm{~kg}$ \\
\hline $\begin{array}{l}\text { Small Dimension Softwood Lumber, } \\
\text { kiln-dried }\end{array}$ & 0.9 & $\mathrm{~m} 3$ \\
\hline Solvent Based Alkyd Paint & 19.6 & $\mathrm{~L}$ \\
\hline Split-faced Concrete Block & $1,238.2$ & Blocks \\
\hline Water Based Latex Paint & 66.3 & $\mathrm{~L}$ \\
\hline
\end{tabular}

\section{Wood Stud Wall \#15 (WS-W15)}

Building Component Description:

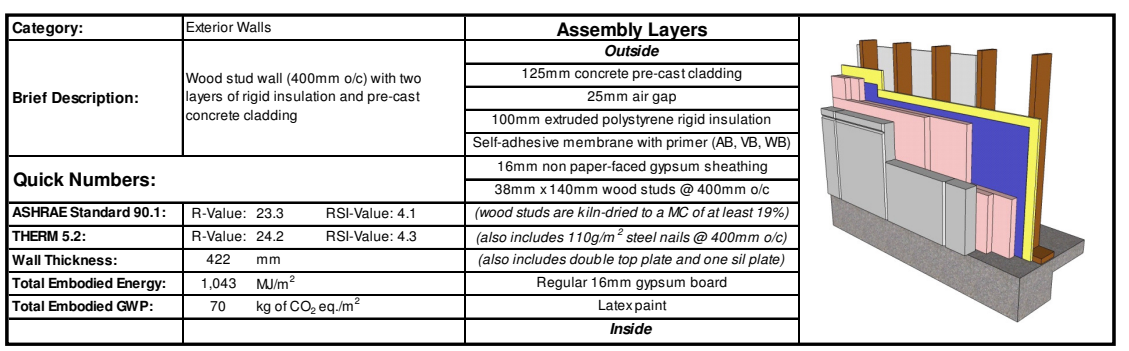

\section{Life-Cycle Assessment Results:}

Primary Energy Consumption (MJ)

\begin{tabular}{|c|c|c|c|c|c|c|c|c|c|c|c|c|c|c|c|c|}
\hline \multirow{3}{*}{$\begin{array}{c}\text { Lifespan } \\
\text { (Years) }\end{array}$} & \multicolumn{14}{|c|}{ Embodied Energy (EE) } & \multirow{2}{*}{\multicolumn{2}{|c|}{$\begin{array}{c}\text { Difference in } \\
\text { Operating Energy } \\
\text { from Baseline after } \\
\text { Lifespan }\end{array}$}} \\
\hline & \multicolumn{3}{|c|}{ Manufacturing } & \multicolumn{3}{|c|}{ Construction } & \multicolumn{3}{|c|}{ Maintenance } & \multicolumn{3}{|c|}{ End of Life } & \multirow{2}{*}{$\begin{array}{c}{ }^{3} \text { Total } \\
\text { EE }\end{array}$} & \multirow{2}{*}{$\begin{array}{c}{ }^{4} \text { Totat } \\
\text { EE } \\
\text { per m }\end{array}$} & & \\
\hline & Naterial & 1 ${ }^{2}$ Trans. & Total & Material & ${ }^{2}$ Trans. & Total & Material & ${ }^{2}$ Trans. & Total & Material & ${ }^{2}$ Trans. & Total & & & ${ }^{5} \mathrm{To}$ & ${ }^{6}$ per m ${ }^{2}$ \\
\hline & & 944 & 718 & & 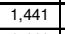 & 1,650 & 0 & 0 & 0 & 0 & 0 & 0 & 49,366 & 70 & & \\
\hline
\end{tabular}

Global Warming Potential ( $\mathrm{kg}$ of $\mathrm{CO}_{2}$ eq.)

\begin{tabular}{|c|c|c|c|c|c|c|c|c|c|c|c|c|c|c|c|c|}
\hline \multirow{3}{*}{\begin{tabular}{|l} 
Lifespan \\
(ears)
\end{tabular}} & \multicolumn{14}{|c|}{ Embodied Global Warming Potential (GWP) } & \multirow{2}{*}{\multicolumn{2}{|c|}{$\begin{array}{l}\text { Difference in } \\
\text { Operating GWP from } \\
\text { Baseline after } \\
\text { Lifespan }\end{array}$}} \\
\hline & \multicolumn{3}{|c|}{ Manufacturing } & \multicolumn{3}{|c|}{ Constru } & \multicolumn{3}{|c|}{ Maintenance } & \multicolumn{3}{|c|}{ End of Life } & \multirow{2}{*}{$\begin{array}{l}{ }^{3} \text { Total } \\
\text { GWP }\end{array}$} & \multirow{2}{*}{$\begin{array}{l}{ }^{4} \text { Total } \\
\text { GWP } \\
\text { per m }{ }^{2}\end{array}$} & & \\
\hline & Material & ${ }^{2}$ Trans. & Total & Material & ${ }^{2}$ Trans. & Total & Material & ${ }^{2}$ Trans. & Total & Material & ${ }^{2}$ Trans. & Total & & & ${ }^{5}$ Total & ${ }^{6}{ }_{\mathrm{perm}}^{2}{ }^{2}$ \\
\hline |litial & 3,485 & 2 & 3,487 & 13 & 3 & 16 & no & 0 & 0 & 0 & 0 & 0 & 3,4 & 69 & & \\
\hline 50 & \begin{tabular}{|l|l|l}
3,485 \\
\end{tabular} & 2 & \begin{tabular}{|l|l|l|}
3,487 \\
\end{tabular} & 13 & 3 & \begin{tabular}{l|l}
16 \\
\end{tabular} & $\begin{array}{ll}44 \\
\end{array}$ & 0 & $\begin{array}{l}44 \\
\end{array}$ & 0 & 1 & 11 & $\begin{array}{l}3,548 \\
\end{array}$ & 70 & 80,000 & \\
\hline
\end{tabular}
Net wall area of baseline retail building (gross wall area- openings) $=581.0 \mathrm{~m}^{2}$

ATHENA $®$ EIE Material List:

Notes:
${ }^{1}$ Initial $=$ Time 'o' (i.e. at the completion of initial construction)

${ }^{2}$ Trans. $=$ Transportation

Total EE (or Total GWP) = Total embodied energy (or total embodied GWP) of building component after lifespan (i.e. total manu
construction + total maintenance + total enc-oflife effects)

${ }^{4}$ Total EE (or Total GWP) per $\mathrm{m}^{2}=$ Total EE (or Total GWP) of building
component/ area of building component that was modelled in ATHENAQ EIE Total Difference in Operating Energy (or GWP) from Baseline after to from the baseline retali building after lifess
component instead of the baseline component

Total Difference in Operating Energy (or GWP) from Baseline after Lifespan per $\mathrm{m}^{2}=$ Total difference in operating energy (or GWP) from

"Total operating primary energy use of baseline retail building after 50 years =
$50,700 \mathrm{GJ}\left(1,745 \mathrm{MJ} / \mathrm{m}^{2} \mathrm{yr}\right)$

Total operating $\mathrm{GWP}$ of baseline retail building after 50 years $=2,310$ tonnes
of $\mathrm{CO}_{2} \mathrm{eq} .\left(80 \mathrm{~kg}\right.$ of $\left.\mathrm{CO}_{2} \mathrm{eq} / \mathrm{m}^{2} \mathrm{yr}\right)$

\begin{tabular}{|l|c|c|}
\multicolumn{1}{|c|}{ Matiluderial List } & Quantities & Unit \\
\hline 3 mil Polyethylene & 54.0 & $\mathrm{~m} 2$ \\
\hline $\begin{array}{l}16 \mathrm{~mm} \text { Moisture Resistant Gypsum } \\
\text { Board }\end{array}$ & 56.0 & $\mathrm{~m} 2$ \\
\hline 16mm Regular Gypsum Board & 56.0 & $\mathrm{~m} 2$ \\
\hline Concrete 30 MPa (flyash av) & 6.7 & $\mathrm{~m} 3$ \\
\hline Extruded Polystyrene & 208.6 & $\mathrm{~m} 2(25 \mathrm{~mm})$ \\
\hline Joint Compound & 111.8 & $\mathrm{~kg}$ \\
\hline Modified Bitumen membrane & 68.2 & $\mathrm{~kg}$ \\
\hline Nails & 9.0 & $\mathrm{~kg}$ \\
\hline Paper Tape & 1.3 & $\mathrm{~kg}$ \\
\hline Rebar, Rod, Light Sections & 404.0 & $\mathrm{~kg}$ \\
\hline $\begin{array}{l}\text { Small Dimension Sottwood Lumber, } \\
\text { kill-dried }\end{array}$ & 0.9 & $\mathrm{~m} 3$ \\
\hline Solvent Based Alkyd Paint & 19.6 & $\mathrm{~L}$ \\
\hline Water Based Latex Paint & 66.3 & $\mathrm{~L}$ \\
\hline
\end{tabular}

Notes:
IInitial = Time ' 0 ' (i.e. at the completion of initial construction)

${ }^{2}$ Trans. $=$ Transportation

${ }^{3}$ Total EE (or Total GWP) $=$ Total embodied energy (or total embodied GWP) of building component after lifespan (i.e. total mal
construction + total maintenance + total end-oflife effects)

${ }^{4}$ Total EE (or Total GWP) per $\mathrm{m}^{2}=$ Total EE (or Total GWP) of building
component / area of building component that was modelled in ATHENAQ EIE ${ }^{5}$ Total Difference in Operating Energy (or GWP) from Baseline after the component instead of the baseline component

Total Difference in Operating Energy (or GWP) from Baseline after Lifespan per $\mathrm{m}^{2}=$ Total difference in operating energy (or GWP) from * Total operating primary energy use of baseline retail building after 50 years =

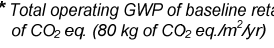


Wood Stud Wall \#16 (WS-W16)

Building Component Description:

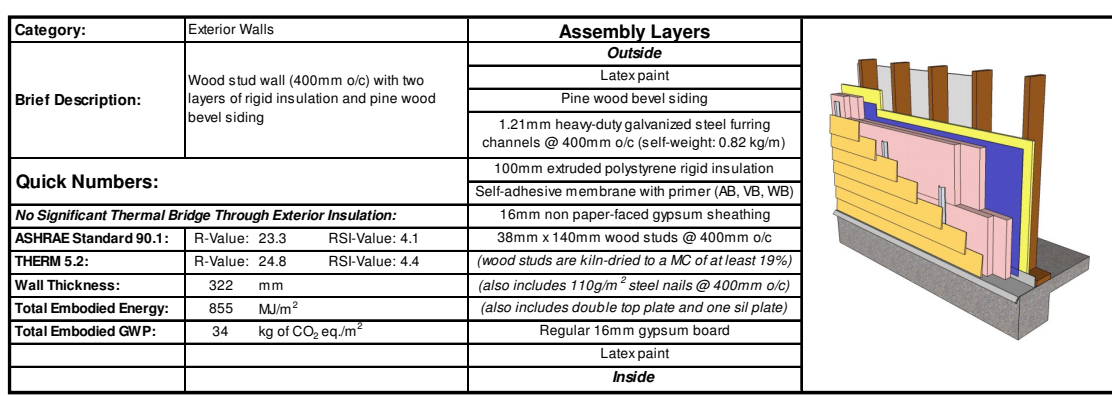

\section{Life-Cycle Assessment Results:}

Primary Energy Consumption (MJ)

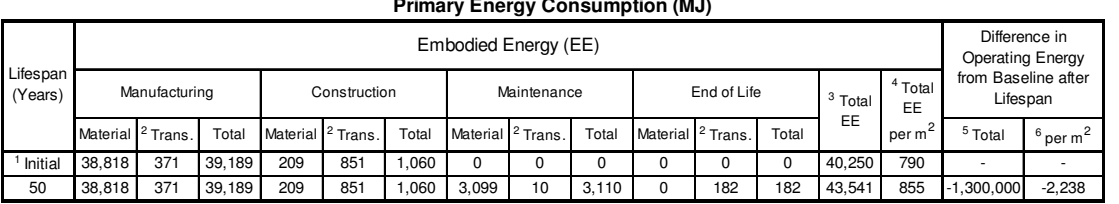

Global Warming Potential ( $\mathrm{kg}$ of $\mathrm{CO}_{2}$ eq.)

\begin{tabular}{|c|c|c|c|c|c|c|c|c|c|c|c|c|c|c|c|c|}
\hline \multirow{4}{*}{$\begin{array}{c}\text { Lifespan } \\
\text { (vears) }\end{array}$} & \multirow{2}{*}{\multicolumn{14}{|c|}{ Embodied Global Warming Potential (GWP) }} & \multirow{3}{*}{\multicolumn{2}{|c|}{$\begin{array}{c}\text { Difference in } \\
\text { Operating GWP from } \\
\text { Baseline after } \\
\text { Lifespan }\end{array}$}} \\
\hline & & & & & & & & & & & & & & & & \\
\hline & \multicolumn{3}{|c|}{ Manufacturing } & \multicolumn{3}{|c|}{ Construction } & \multicolumn{3}{|c|}{ Maintenance } & \multicolumn{3}{|c|}{ End $\mathrm{C}$} & \multirow{2}{*}{$\begin{array}{l}{ }^{3} \text { Total } \\
\text { GWP }\end{array}$} & \multirow{2}{*}{$\begin{array}{l}{ }^{4} \text { Total } \\
\text { GWP } \\
\text { per m }\end{array}$} & & \\
\hline & Material & ${ }^{2}$ Trans & \begin{tabular}{|l|l|} 
Total \\
\end{tabular} & Material & ${ }^{2}$ Trans. & Total & Material & ${ }^{2}$ Trans. & Total & \begin{tabular}{|l|} 
Material \\
\end{tabular} & ${ }^{2}$ Trans. & Total & & & ${ }^{5}$ Total & ${ }^{6}$ perm \\
\hline Initial & 1,660 & 1 & 1,661 & 13 & 2 & 15 & 0 & 0 & 0 & 0 & 0 & 0 & 6 & 33 & & \\
\hline 50 & 1,660 & 1 & $\begin{array}{ll}1,661 \\
\end{array}$ & 13 & 2 & 15 & 44 & 0 & 44 & 0 & 0 & 0 & 1,720 & 34 & 50 & -120 \\
\hline
\end{tabular}

Embodied energy (and GWP) numbers are based on an area of wall $=\quad 50.9 \mathrm{~m}^{2} \quad$ (Length $\times$ Height $=7.6 \mathrm{~m} \times 6.7 \mathrm{~m}=50.9 \mathrm{~m}^{2}$ )

Net wall area of baseline retail building (gross wall area - openings) $=581.0 \mathrm{~m}^{2}$

\section{ATHENA $®$ EIE Material List:}

\begin{tabular}{|c|c|c|}
\hline Material List & Quantities & Unit \\
\hline 3 mil Polyethylene & 54.0 & $\mathrm{~m}^{2} 2$ \\
\hline $\begin{array}{l}\text { 16mm Moisture Resistant Gypsum } \\
\text { Board }\end{array}$ & 56.0 & $\mathrm{~m} 2$ \\
\hline $16 \mathrm{~mm}$ Regular Gypsum Board & 56.0 & $\mathrm{~m} 2$ \\
\hline Extruded Polystyrene & 208.6 & m2 $225 \mathrm{~mm}$ \\
\hline Galvanized Sheet & 106.1 & $\mathrm{~kg}$ \\
\hline Joint Compound & 111.8 & $\mathrm{~kg}$ \\
\hline Modified Bitumen membrane & 68.2 & $\mathrm{~kg}$ \\
\hline Nails & 10.3 & $\mathrm{~kg}$ \\
\hline Paper Tape & 1.3 & $\mathrm{~kg}$ \\
\hline Pine Wood Bevel Siding & 160.4 & $\mathrm{~m} 2$ \\
\hline $\begin{array}{l}\text { Small Dimension Sottwood Lumber, } \\
\text { kiln-dred }\end{array}$ & 0.9 & $\mathrm{~m} 3$ \\
\hline Solvent Based Alkyd Paint & 19.6 & $L$ \\
\hline Nater E & 132.5 & \\
\hline
\end{tabular}

Notes:
'Initial $=$ Time 'o' (i.e. at the completion of initio

${ }^{2}$ Trans. = Transportation

${ }^{3}$ Total EE (or Total GWP) = Total embodied energy (or total embodied GWP) of building component after lifespan (i.e. total manufacturing + to

${ }^{4}$ Total EE (or Total GWP) per $\mathrm{m}^{2}=$ Total EE (or Total GWP) of building
component/ area of building component that was modelled in ATHENAQ EIE

${ }^{5}$ Total Difference in Operating Energy (or GWP) from Baseline after Lifespan = The difference in the total life-cycle operating energy (or GWP)
from the baseline retail building after lifespan, due to using this building component instead of the baseline component

Total Difference in Operating Energy (or GWP) from Baseline after Lifespan per $\mathrm{m}^{2}=$ Total difference in operating energy (or GWP) from

* Total operating primary energy use of baseline retail building after 50 years

"Total operating $G W$ Po b baseline retail building after 50 years $=2,310$ tonnes
of $\mathrm{CO}_{2}$ eq. $\left(80 \mathrm{~kg}\right.$ of $\mathrm{CO}_{2}$ eq./m/ $\left./ \mathrm{yr}\right)$

Wood Stud Wall \#17 (WS-W17)

Building Component Description:

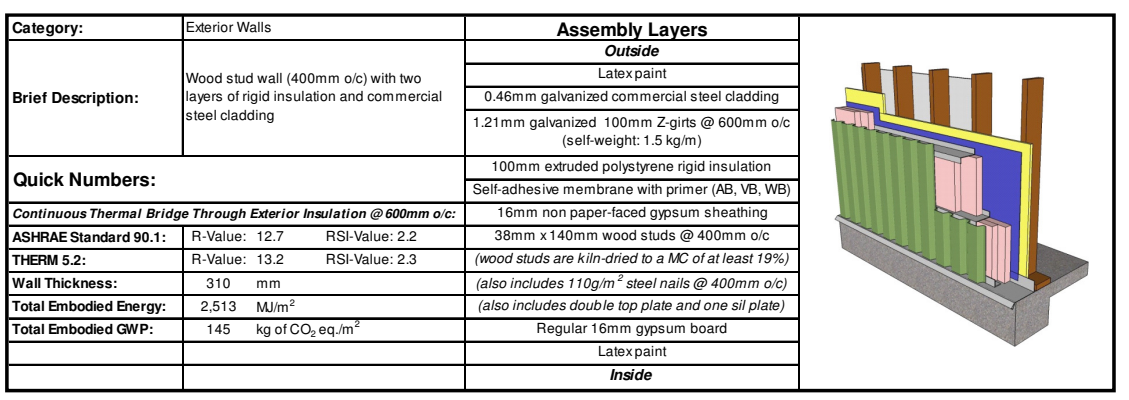

Life-Cycle Assessment Results:

Primary Energy Consumption (MJ)

\begin{tabular}{|c|c|c|c|c|c|c|c|c|c|c|c|c|c|c|c|}
\hline \multirow{3}{*}{$\begin{array}{l}\text { Lifiespan } \\
\text { (Years) }\end{array}$} & \multicolumn{13}{|c|}{ Embodied Energy (EE) } & \multirow{2}{*}{\multicolumn{2}{|c|}{$\begin{array}{l}\text { Difiference in } \\
\text { Operating Energy } \\
\text { trom Baseline after } \\
\text { Lifespana }\end{array}$}} \\
\hline & \multicolumn{2}{|c|}{ Manufacturing } & \multicolumn{3}{|c|}{ Construction } & \multicolumn{3}{|c|}{ Maintenance } & \multicolumn{3}{|c|}{ End of Lifie } & \multirow{2}{*}{$\begin{array}{c}{ }^{3} \text { Total } \\
\text { EE }\end{array}$} & \multirow{2}{*}{\begin{tabular}{|l|l}
${ }^{4}{ }^{T}$ I \\
EE
\end{tabular}} & & \\
\hline & 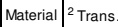 & Total & Material & |2 Trans. & Total & Material & ${ }^{2}$ Trans. & Total & Material & ${ }^{2}$ Trans. & Total & & & $\begin{array}{ll}{ }^{5} \text { Total } \\
\end{array}$ & ${ }^{6}$ per m$^{2}$ \\
\hline Initial & \begin{tabular}{|l|l|}
123,392 & 325 \\
\end{tabular} & 123,716 & 209 & 754 & 964 & 0 & 0 & 0 & 0 & 0 & 0 & 124,680 & 2,449 & & \\
\hline 50 & \begin{tabular}{|l|l|}
123,392 & 325 \\
\end{tabular} & 123,716 & 209 & 754 & 964 & 3,099 & 10 & 3,110 & no & 176 & 177 & 127,96 & 2,513 & 900,000 & 1,549 \\
\hline
\end{tabular}

Global Warming Potential (kg of $\mathrm{CO}_{2}$ eq.)

\begin{tabular}{|c|c|c|c|c|c|c|c|c|c|c|c|c|c|c|c|c|}
\hline \multirow{4}{*}{ (Years) } & \multirow{2}{*}{\multicolumn{14}{|c|}{ 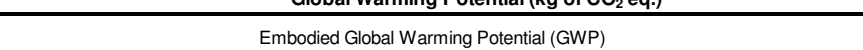 }} & & \\
\hline & & & & & & & & & & & & & & & \multirow{2}{*}{\multicolumn{2}{|c|}{$\begin{array}{l}\text { Difference in } \\
\text { Operating GWP from } \\
\text { Baseline atter } \\
\text { Lifespan }\end{array}$}} \\
\hline & \multicolumn{3}{|c|}{ Manufacturing } & \multicolumn{3}{|c|}{ Construction } & \multicolumn{3}{|c|}{ Maintenance } & \multicolumn{3}{|c|}{ End of Life } & \multirow{2}{*}{$\begin{array}{l}{ }^{3} \text { Total } \\
\text { GWP }\end{array}$} & \multirow{2}{*}{$\mid \begin{array}{l}{ }^{4} \text { Total } \\
\text { GWP } \\
\text { per m}{ }^{2}\end{array}$} & & \\
\hline & \begin{tabular}{|l|} 
Material \\
\end{tabular} & ${ }^{2}$ Trans & Total & Material & 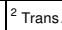 & Total & \begin{tabular}{|l|} 
Material \\
\end{tabular} & $\left.\right|^{2}$ Trans. & Total & Material & 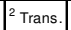 & Total & & & ${ }^{5}$ Total & ${ }^{6}{ }^{6}$ per m ${ }^{2}$ \\
\hline 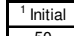 & \begin{tabular}{|l|l}
7,305 \\
7202
\end{tabular} & 1 & $\begin{array}{l}7,306 \\
7306\end{array}$ & $\overline{112}$ & & 15 & & 0 & $e^{-1}$ & & 0 & 0 & & 44 & & \\
\hline 50 & 7,305 & 1 & 7,306 & 13 & 1 & 15 & 44 & 0 & $\begin{array}{lllll}44 & \end{array}$ & 0 & 0 & 0 & 365 & 145 & 50,000 & 86 \\
\hline
\end{tabular}

Embodied energy (and GWP) numbers are based on an area of wall $=50.9 \mathrm{~m}^{2} \quad$ (Length $x$ Height $=7.6 \mathrm{~m} \times 6.7 \mathrm{~m}=50.9 \mathrm{~m}^{2}$ ) Net wall area of baseline retall building (gross wall area - openings) $=581.0 \mathrm{~m}^{2}$

ATHENA ® EIE Material List:

\begin{tabular}{|c|c|c|}
\hline Material List & Quantities & Unit \\
\hline 3 mil Polyethylene & 54.0 & $\mathrm{~m} 2$ \\
\hline $\begin{array}{l}16 \mathrm{~mm} \text { Moisture Resistant Gypsum } \\
\text { Bard }\end{array}$ & 56.0 & $\mathrm{~m} 2$ \\
\hline $16 \mathrm{~mm}$ Regular Gypsum Board & 56.0 & $\mathrm{~m} 2$ \\
\hline Commercial $0.46 \mathrm{~mm}$ Steel Cladding & 168.0 & $\mathrm{~m} 2$ \\
\hline Extruded Polystyrene & 208.6 & $\mathrm{~m} 2(25 \mathrm{~mm})$ \\
\hline Galvanized Studs & 131.3 & $\mathrm{~kg}$ \\
\hline Joint Compound & 111.8 & $\mathrm{~kg}$ \\
\hline Modified Bitumen membrane & 68.2 & $\mathrm{~kg}$ \\
\hline Nails & 9.0 & $\mathrm{~kg}$ \\
\hline Paper Tape & 1.3 & $\mathrm{~kg}$ \\
\hline Screws Nuts \& Bolts & 1.3 & $\mathrm{~kg}$ \\
\hline $\begin{array}{l}\text { Small Dimension Softwood Lumber } \\
\text { kiln-ried }\end{array}$ & 0.9 & $\mathrm{~m} 3$ \\
\hline Solvent Based Alkyd Paint & 19.6 & $\mathrm{~L}$ \\
\hline Water Based Latex Paint & 132.5 & \\
\hline
\end{tabular}

'Initial = Time 'o' '(i.e. at the completion of inition

${ }^{2}$ Trans. $=$ Transportation

Total EE (or Total GWP) = Total embodied energy (or total embodied GWP) of building component after lifespan (i.e. total man
construction + total maintenance + total end-offife effects)

${ }^{4}$ Total EE (or Total GWP) per $\mathrm{m}^{2}=$ Total EE (or Total GWP) of building
component/ area of building component that was modelled in ATHENAE EIE Total Difference in Operating Energy (or GWP) from Baseline atter Lirespan $=$ The ifirerence inthe
from the baseline retali building ater lifespan, due to using this building ${ }^{6}$ Total Difference in Operating Energy (or GWP) from Baseline after Lifespan per $\mathrm{m}^{2}=$ Total difference in operating energy (or GWP) from
baseline after lifespan / net wall area of baseline retail building * Total operating primary energy use of baseline retail building after 50 years $=$
$50,700 \mathrm{GJ}\left(1,745 \mathrm{MJ} / \mathrm{m}^{2} \mathrm{y}\right)$ * Total operating GWP of baseline retail building after 50 years $=2,310$ tonnes
of $\mathrm{CO}_{2}$ eq. $80 \mathrm{~kg}$ of $\mathrm{CO}_{2}$ eq.. $\left.\mathrm{m}^{2} \mathrm{yrr}\right)$ 


\section{Wood Stud Wall \#18 (WS-W18)}

Building Component Description:

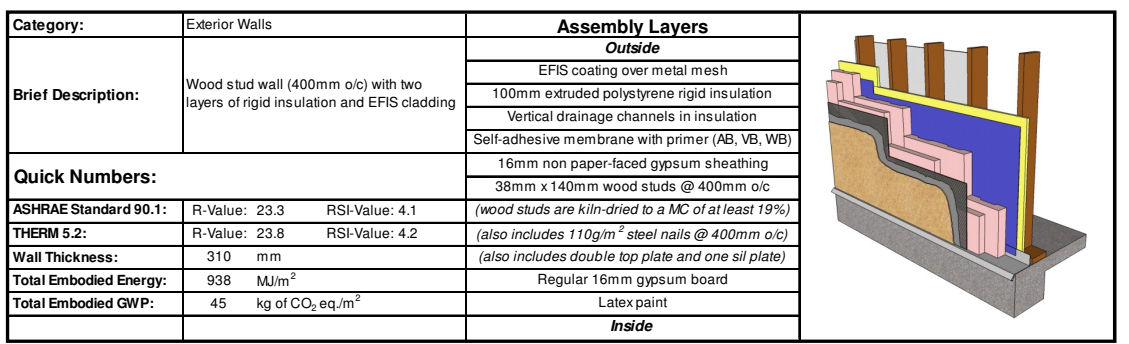

\section{Life-Cycle Assessment Results:}

Primary Energy Consumption (MJ)

\begin{tabular}{|c|c|c|c|c|c|c|c|c|c|c|c|c|c|c|c|c|}
\hline \multirow{3}{*}{\begin{tabular}{|c} 
Lifespan \\
(Years)
\end{tabular}} & \multicolumn{14}{|c|}{ Embodied Energy (EE) } & \multirow{2}{*}{\multicolumn{2}{|c|}{$\begin{array}{c}\text { Difference in } \\
\text { Operating Energy } \\
\text { from Baseline after } \\
\text { Lifespan }\end{array}$}} \\
\hline & \multicolumn{3}{|c|}{ Manufac } & \multicolumn{3}{|c|}{ Constrin } & \multicolumn{3}{|c|}{ Maintenance } & \multicolumn{3}{|c|}{ End 0} & \multirow{2}{*}{${ }^{3}$ Total } & \multirow{2}{*}{$\begin{array}{ll}{ }^{4} \text { Total } \\
\text { EE } \\
\text { per } \mathrm{m}^{2}\end{array}$} & & \\
\hline & Materia & 2 Trans. & Total & Materia & ${ }^{2}$ Trans. & Total & Material & ${ }^{2}$ Trans. & Total & Material & ${ }^{2}$ Trans. & Total & & & ${ }^{5}$ Total & ${ }^{6}$ per $\mathrm{m}^{2}$ \\
\hline Initial & 42,686 & 379 & 43,064 & 209 & 844 & 1,053 & 0 & 0 & 0 & 0 & 0 & 0 & 44,117 & 866 & & \\
\hline 50 & $\begin{array}{l}42,686 \\
\end{array}$ & 379 & 43,064 & 209 & $\begin{array}{l}844 \\
\end{array}$ & 1,053 & \begin{tabular}{|l|l|}
3.099 \\
\end{tabular} & 10 & \begin{tabular}{|l|l|}
3,110 \\
\end{tabular} & 0 & 531 & 531 & 47,758 & 938 & $-1,200,00$ & -2.065 \\
\hline
\end{tabular}

Global Warming Potential ( $\mathrm{kg}$ of $\mathrm{CO}_{2}$ eq.)

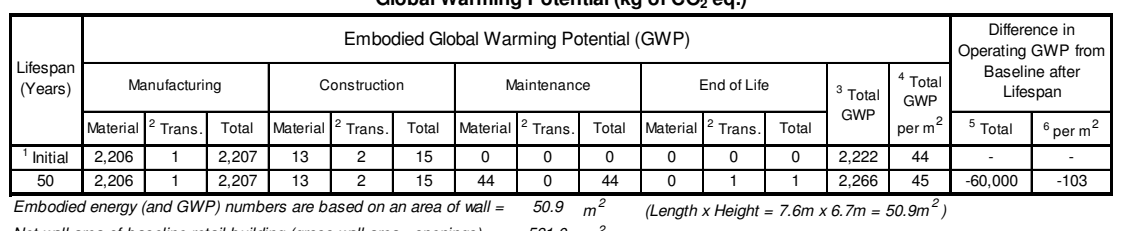

(a)

\section{ATHENA $®$ EIE Material List:} (Includes all materials after 50 years)

\begin{tabular}{|c|c|c|}
\hline Material List & Quantities & Unit \\
\hline \#15 Organic Felt & 219.4 & $\mathrm{~m} 2$ \\
\hline 3 mil Polyethylene & 54.0 & $\mathrm{~m} 2$ \\
\hline $\begin{array}{l}16 \mathrm{~mm} \text { Moisture Resistant Gypsum } \\
\text { Bapard }\end{array}$ & 56.0 & $\mathrm{~m} 2$ \\
\hline $16 \mathrm{~mm}$ Regular Gypsum Board & 56.0 & $\mathrm{~m} 2$ \\
\hline Extruded Polystyrene & 208.6 & m2 (25mm) \\
\hline Galvanized Sheet & 51.4 & $\mathrm{~kg}$ \\
\hline Joint Compound & 111.8 & $\mathrm{~kg}$ \\
\hline Modified Bitumen membrane & 68.2 & $\mathrm{~kg}$ \\
\hline Nails & 10.5 & $\mathrm{~kg}$ \\
\hline Paper Tape & 1.3 & $\mathrm{~kg}$ \\
\hline $\begin{array}{l}\text { Small Dimension Softwood Lumber, } \\
\text { kiln-dried }\end{array}$ & 0.9 & $\mathrm{~m} 3$ \\
\hline Solvent Based Alkyd Paint & 19.6 & $\mathrm{~L}$ \\
\hline Stucco over metal mesh & 136.0 & $\mathrm{~m} 2$ \\
\hline Water Based Latex Paint & 132.5 & -5 \\
\hline
\end{tabular}

\section{Wood Stud Wall \#19 (WS-W19)}

Building Component Description:

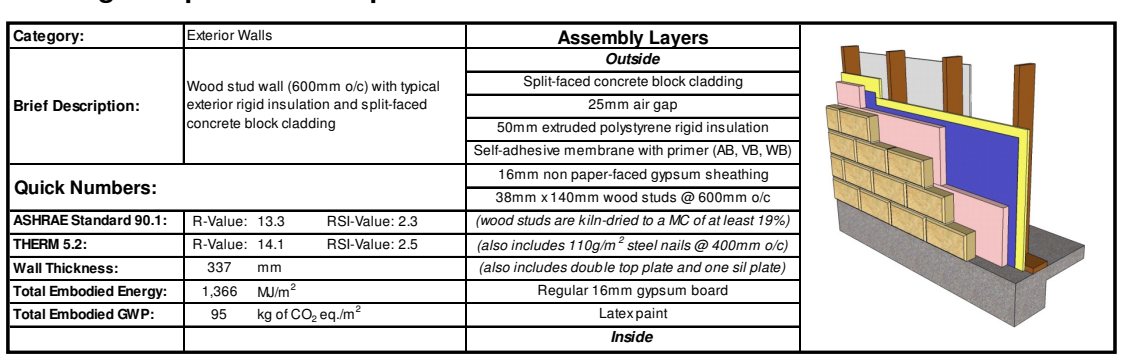

\section{Life-Cycle Assessment Results:}

Primary Energy Consumption (MJ)

\begin{tabular}{|c|c|c|c|c|c|c|c|c|c|c|c|c|c|c|c|c|}
\hline \multirow{3}{*}{\begin{tabular}{|} 
Lifespan \\
(Years)
\end{tabular}} & \multicolumn{14}{|c|}{ Embodied Energy (EE) } & \multirow{2}{*}{\multicolumn{2}{|c|}{$\begin{array}{l}\text { Difference in } \\
\text { Operating Energy } \\
\text { from Baseline after } \\
\text { Lifespan }\end{array}$}} \\
\hline & \multicolumn{3}{|c|}{ Manufacturing } & \multicolumn{3}{|c|}{ Construction } & \multicolumn{3}{|c|}{ Maintenance } & \multicolumn{3}{|c|}{ End of Life } & \multirow{2}{*}{$\begin{array}{c}{ }^{3} \text { Total } \\
\mathrm{EE}\end{array}$} & \multirow{2}{*}{$\begin{array}{c}{ }^{4} \text { Toto } \\
\text { EE } \\
\text { per m }\end{array}$} & & \\
\hline & \begin{tabular}{|l|l|} 
Material \\
\end{tabular} & \begin{tabular}{|l|}
${ }^{2}$ Trans. \\
\end{tabular} & Total & Material & ${ }^{2}$ Trans. & Total & Material & 2 Trans. & Total & Material & Trans. & Total & & & ${ }^{5} \mathrm{Tot}$ & per \\
\hline tial & 66,972 & \begin{tabular}{|l|l|}
892 \\
802
\end{tabular} & 61,864 & 209 & $\begin{array}{l}2,259 \\
\end{array}$ & 2,468 & 0 & 0 & 0 & 0 & 0 & 0 & 64,333 & 206 & & \\
\hline
\end{tabular}

Global Warming Potential ( $\mathrm{kg}$ of $\mathrm{CO}_{2}$ eq.)

\begin{tabular}{|c|c|c|c|c|c|c|c|c|c|c|c|c|c|c|c|c|}
\hline \multirow{3}{*}{$\begin{array}{l}\text { Lifespan } \\
\text { (Years) }\end{array}$} & \multicolumn{14}{|c|}{ Embodied Global Warming Potential (GWP) } & \multirow{2}{*}{\multicolumn{2}{|c|}{$\begin{array}{l}\text { Difference in } \\
\text { Operating GWP from } \\
\text { Baseline after } \\
\text { Lifespan }\end{array}$}} \\
\hline & \multicolumn{3}{|c|}{ Manufacturing } & \multicolumn{3}{|c|}{ Construction } & \multicolumn{3}{|c|}{ Maintenance } & \multicolumn{3}{|c|}{ End of Life } & \multirow{2}{*}{\begin{tabular}{|l|}
${ }^{3}$ Total \\
GWPP
\end{tabular}} & \multirow{2}{*}{$\begin{array}{l}{ }^{4}{ }^{4} \text { Total } \\
\text { GWP } \\
\text { per m }\end{array}$} & & \\
\hline & \begin{tabular}{|l|} 
Material \\
\end{tabular} & 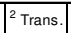 & \begin{tabular}{|l|l|} 
Total \\
\end{tabular} & Material & 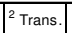 & $\begin{array}{l}\text { Total } \\
\end{array}$ & \begin{tabular}{|l|} 
Material \\
\end{tabular} & 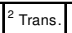 & \begin{tabular}{|l|l|} 
Total \\
\end{tabular} & \begin{tabular}{|l|} 
Material \\
\end{tabular} & ||$^{2}$ Trans. & Total & & & ${ }^{5}$ Total & ${ }^{6} \mathrm{perm} \mathrm{m}^{2}$ \\
\hline 'nitial & \begin{tabular}{|l|l|}
4,766 \\
\end{tabular} & 2 & \begin{tabular}{|l|l|}
4,768 \\
\end{tabular} & 13 & 4 & 18 & 0 & 0 & 0 & 0 & 0 & 0 & 4,785 & 94 & & \\
\hline 50 & \begin{tabular}{|l|l|}
4,766 \\
\end{tabular} & 2 & 4,768 & 13 & 4 & 18 & 44 & 0 & 44 & 0 & 4 & 4 & 4,833 & 95 & \begin{tabular}{|l|l|}
20,000 \\
\end{tabular} & 34 \\
\hline
\end{tabular}
apenings $)=581.0 \mathrm{~m}^{2}$

ATHENA $\circledast$ EIE Material List:

Notes:
${ }^{1}$ Initial $=$ Time ' $0^{\prime}$ (i.e. at the completion of initial construction)

${ }^{2}$ Trans. $=$ Transportation

Total EE (or Total GWP) = Total embodied energy (or total embodied GWP) of building component after lifespan (i.e. total manu
construction + total maintenance + total enc-oflife effects)

${ }^{4}$ Total EE (or Total GWP) per $\mathrm{m}^{2}=$ Total EE (or Total GWP) of building
component/ area of building component that was modelled in ATHENAQ EIE Total Difference in Operating Energy (or GWP) from Baseline after from the baseline retail building after lifespan, due to using this building
from from the baseline retali building after lifes
component instead of the baseline component

Total Difference in Operating Energy (or GWP) from Baseline after Lifespan per $\mathrm{m}^{2}=$ Total difference in operating energy (or GWP) from * Total operating primary energy use of baseline retail building affer 50 years =

${ }^{*}$ Total operating $\mathrm{GW}$ P of baseline retail building after 50 years $=2,310$ tonnes
of $\mathrm{CO}_{2} \mathrm{eq},\left(80 \mathrm{~kg}\right.$ of $\left.\mathrm{CO}_{2} \mathrm{eq} / / \mathrm{m}^{2} / \mathrm{yr}\right)$

\begin{tabular}{|c|c|c|}
\hline Material List & Quantities & Unit \\
\hline 3 mil Polyethylene & 54.0 & $\mathrm{~m} 2$ \\
\hline $\begin{array}{l}16 \mathrm{~mm} \text { Moisture Resistant Gypsum } \\
\text { Board }\end{array}$ & 56.0 & $\mathrm{~m} 2$ \\
\hline $16 \mathrm{~mm}$ Regular Gypsum Board & 56.0 & $\mathrm{~m} 2$ \\
\hline Cold Rolled Sheet & 10.3 & $\mathrm{~kg}$ \\
\hline Extruded Polystyrene & 104.3 & m2 (25mm) \\
\hline Joint Compound & 111.8 & $\mathrm{~kg}$ \\
\hline Modified Bitumen membrane & 401.5 & $\mathrm{~kg}$ \\
\hline Mortar & 4.3 & $\mathrm{~m} 3$ \\
\hline Nails & 7.4 & $\mathrm{~kg}$ \\
\hline Paper Tape & 1.3 & $\mathrm{~kg}$ \\
\hline on Sottwood Lumber, & 0.7 & $\mathrm{~m} 3$ \\
\hline Solvent Based Alkyd Paint & 19.6 & $\mathrm{~L}$ \\
\hline Split-faced Concrete Block & $1,238.2$ & Blocks \\
\hline Water Based Latex Paint & 66.3 & L \\
\hline
\end{tabular}

Notes:
IInitial $=T$ Time '
'' (i.e. at the completion of initial construction) ${ }^{2}$ Trans. $=$ Transportation

Total EE (or Total GWP) = Total embodied energy (or total embodied GWP) of building component after lifespan (i.e. total manu
construction + total maintenance + total end-of-life effects)

${ }^{4}$ Total EE (or Total GWP) per $\mathrm{m}^{2}=$ Total EE (or Total GWP) of building
component / area of building component that was modelled in ATHENAB EIE ${ }^{5}$ Total Difference in Operating Energy (or GWP) from Baseline after (t) component instead of the baseline component

Total Difference in Operating Energy (or GWP) from Baseline after

* Total operating primary energy use of baseline retail building after 50 years
$50,700 \mathrm{G} J\left(1.745 \mathrm{MJ}^{2} \mathrm{~m}^{2} \mathrm{yr}\right)$

"Total operating $\mathrm{GWP}$ of baseline retat
of $\mathrm{CO}_{2}$ eq $\left(80 \mathrm{~kg}\right.$ of $\mathrm{CO}_{2}$ eq. $\left./ \mathrm{m}^{2} \mathrm{Mr}\right)$ Lifespan per $\mathrm{m}^{2}=$ Total difference in operating energy (or GWP) from
baseline after lifespan / net wall area of baseline retail building 
Wood Stud Wall \#20 (WS-W20)

Building Component Description:

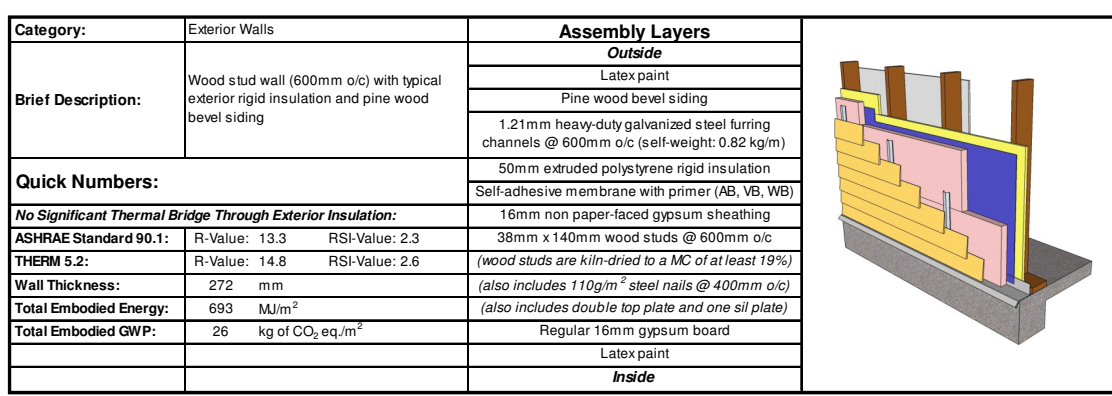

\section{Life-Cycle Assessment Results:}

Primary Energy Consumption (MJ)

\begin{tabular}{|c|c|c|c|c|c|c|c|c|c|c|c|c|c|c|c|c|}
\hline \multirow{3}{*}{$\begin{array}{l}\text { Lifiespan } \\
\text { (Years) }\end{array}$} & \multicolumn{14}{|c|}{ Embodied Energy (EE) } & \multirow{2}{*}{\multicolumn{2}{|c|}{$\begin{array}{c}\text { Difference in } \\
\text { Operating Energy } \\
\text { from Baseline after } \\
\text { Lifespan }\end{array}$}} \\
\hline & \multicolumn{3}{|c|}{ Manufacturing } & \multicolumn{3}{|c|}{ Construction } & \multicolumn{3}{|c|}{ Maintenance } & \multicolumn{3}{|c|}{ End of Life } & \multirow{2}{*}{$\begin{array}{c}{ }^{3} \text { Total } \\
\mathrm{EE}\end{array}$} & \multirow{2}{*}{\begin{tabular}{|c|}
${ }^{4}$ Total \\
EE \\
per m
\end{tabular}} & & \\
\hline & Material & ${ }^{2}$ Trans. & Total & Materia & ${ }^{2}$ Trans. & Total & Materia & ${ }^{2}$ Trans. & Total & Material & 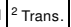 & Total & & & ${ }^{5}$ Total & ${ }^{6}$ per $\mathrm{m}^{2}$ \\
\hline 1 Initial & 30,676 & 347 & $31,02$. & 209 & \begin{tabular}{|l|l|}
798 \\
\end{tabular} & 1,007 & 0 & 0 & 0 & 0 & 0 & 0 & 32,030 & 629 & & \\
\hline 50 & 30,676 & \begin{tabular}{|l|} 
\\
\end{tabular} 47 & 311,02: & 209 & \begin{tabular}{|l|l|} 
& \\
\end{tabular} & 1,007 & 3,099 & 10 & 3,110 & 0 & 168 & 168 & 35,308 & 693 & 400,000 & 688 \\
\hline
\end{tabular}

Global Warming Potential ( $\mathrm{kg}$ of $\mathrm{CO}_{2}$ eq.)

\begin{tabular}{|c|c|c|c|c|c|c|c|c|c|c|c|c|c|c|c|c|}
\hline & & & & & & ob & & & & & & & & & & \\
\hline \multirow{3}{*}{$\begin{array}{l}\text { Lifiesparars } \\
\text { (Years }\end{array}$} & \multicolumn{14}{|c|}{ Embodied Global Warming Potential (GWP) } & \multirow{2}{*}{\multicolumn{2}{|c|}{$\begin{array}{l}\text { Difiterence in } \\
\text { Operating GWP } \\
\text { Baston } \\
\text { Baline after } \\
\text { Lifespan }\end{array}$}} \\
\hline & \multicolumn{3}{|c|}{ Manutacturing } & \multicolumn{3}{|c|}{ Construction } & \multicolumn{3}{|c|}{ Maintenance } & \multicolumn{3}{|c|}{ End of Life } & \multirow{2}{*}{$\begin{array}{l}{ }^{3} \text { Total } \\
\text { GWPP }\end{array}$} & \multirow{2}{*}{$\begin{array}{l}{ }^{4} \text { Total } \\
\text { GWP } \\
\text { per m }\end{array}$} & & \\
\hline & Material & ${ }^{2}$ Trans. & Total & Material & Trans & Total & Material & ${ }^{2}$ Trans. & Total & Material & 2 Trans. & Total & & & ${ }^{5}$ Total & ${ }^{6}$ perm $^{2}$ \\
\hline${ }^{\prime}$ Initial & 1,255 & 1 & 1,256 & 13 & 2 & 15 & 0 & 0 & 0 & 0 & 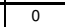 & 0 & 1,271 & 25 & & \\
\hline 50 & 1,255 & 1 & 1,256 & 13 & 2 & 15 & 44 & 0 & 44 & 0 & 0 & 0 & 1,315 & 26 & 20,000 & 34 \\
\hline
\end{tabular}

Embodied energy (and GWP) numbers are based on an area of wall $=50.9 \mathrm{~m}^{2} \quad$ (Length $x$ Height $=7.6 \mathrm{~m} \times 6.7 \mathrm{~m}=50.9 \mathrm{~m}^{2}$ )

Net wall area of baseline retall building (gross wall area - openings) $=581.0 \mathrm{~m}^{2}$

ATHENA $®$ EIE Material List:

\begin{tabular}{|c|c|c|}
\hline Material List & Quantities & Unit \\
\hline 3 mil Polyethylene & 54.0 & $\mathrm{~m} 2$ \\
\hline $\begin{array}{l}16 \mathrm{~mm} \text { Moisture Resistant Gypsum } \\
\text { Board }\end{array}$ & 56.0 & $\mathrm{~m} 2$ \\
\hline $16 \mathrm{~mm}$ Regular Gypsum Board & 56.0 & $\mathrm{~m} 2$ \\
\hline Extruded Polystyrene & 104.3 & $\mathrm{~m} 2(25 \mathrm{~mm}$ \\
\hline Galvanized Sheet & 106.1 & $\mathrm{~kg}$ \\
\hline Joint Compound & 111.8 & $\mathrm{~kg}$ \\
\hline Modified Bitumen membrane & 68.2 & $\mathrm{~kg}$ \\
\hline Nails & 8.7 & $\mathrm{~kg}$ \\
\hline Paper Tape & 1.3 & $\mathrm{~kg}$ \\
\hline Pine Wood Bevel Siding & 160.4 & $\mathrm{~m} 2$ \\
\hline $\begin{array}{l}\text { Small Dimension Sottwood Lumber, } \\
\text { kiln-dried }\end{array}$ & 0.7 & $\mathrm{~m} 3$ \\
\hline Solvent Based Alkyd Paint & 19.6 & \\
\hline & 132. & \\
\hline
\end{tabular}

${ }^{2}$ Trans. $=$ Transportation construction t total maintenance t total enco-filife effects

${ }^{4}$ Total EE (or Total GWP) per $\mathrm{m}^{2}=$ Total EE (or Total GWP) of building Lifespan $=$ The difference in the total life-cycle operating energy (or GWW) from the baseline retail building after lifespan, due to using this building
component instead of the baseline component ${ }^{6}$ Total Difference in Operating Energy (or GWP) from Baseline after Lifespan per $\mathrm{m}^{2}=$ Total difference in operating energy (or GWP) from * Total operating primary energy use of baseline retail building after 50 years $=$
$50,700 \mathrm{GJ}\left(1,745 \mathrm{MJ} \mathrm{m}^{2} \mathrm{~N} / \mathrm{r}\right)$

"Total operating GWP of baseline retail building after 50 years $=2,310$ tonnes
of $\mathrm{CO}_{2}$ eq. $\left(80 \mathrm{~kg}\right.$ of $\left.\mathrm{CO}_{2} \mathrm{eq} . / \mathrm{m}^{2} / \mathrm{yr}\right)$
Notes:
1 Initial = Time ' $O$ ' (i.e. at the completion of initial construction)

Total EE (or Total GWP) $=$ Total embodied energy (or total embodied GWP)
of building component after lifespan (i.e. total manufacturing + totat

Wood Stud Wall \#21 (WS-W21)

Building Component Description:

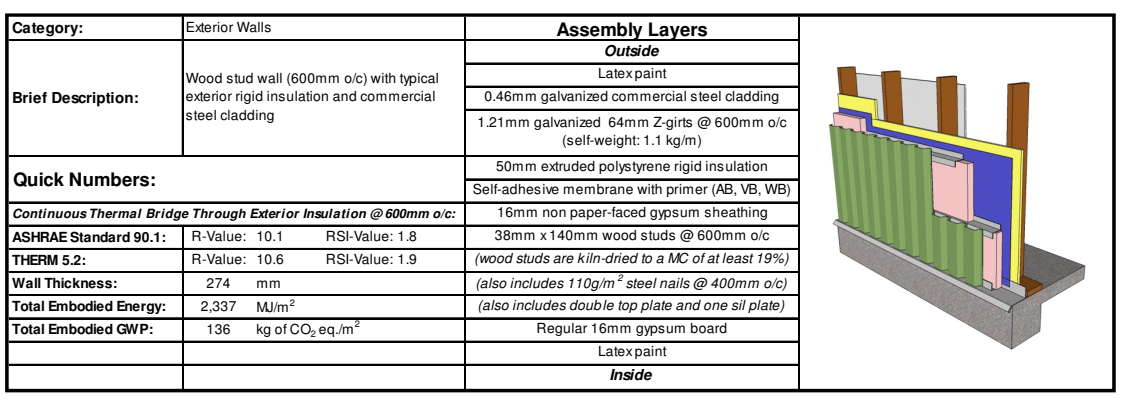

Life-Cycle Assessment Results:

Primary Energy Consumption (MJ)

\begin{tabular}{|c|c|c|c|c|c|c|c|c|c|c|c|c|c|c|c|c|}
\hline \multirow{3}{*}{$\begin{array}{l}\text { Lifespan } \\
\text { (Years) }\end{array}$} & \multicolumn{14}{|c|}{ Embodied Energy (EE) } & \multirow{2}{*}{\multicolumn{2}{|c|}{$\begin{array}{l}\text { Difiference in } \\
\text { Operating Energy } \\
\text { trom Baseline after } \\
\text { Lifiespan }\end{array}$}} \\
\hline & \multicolumn{3}{|c|}{ Manufacturing } & \multicolumn{3}{|c|}{ Construction } & \multicolumn{3}{|c|}{ Maintenance } & \multicolumn{3}{|c|}{ End of Lifie } & \multirow{2}{*}{$\begin{array}{c}{ }^{3} \text { Total } \\
\text { EE }\end{array}$} & \multirow{2}{*}{$\begin{array}{c}{ }^{4} \text { Total } \\
\text { EE } \\
\text { per } \mathrm{m}^{2}\end{array}$} & & \\
\hline & Material & ${ }^{2}$ Trans & Total & Material & ${ }^{2}$ Trans. & Total & Material & $\begin{array}{l}{ }^{2} \text { Trans. } \\
\end{array}$ & Total & Material & ${ }^{2}$ Trans. & Total & & & ${ }^{5}$ Total & ${ }^{6}$ perm m$^{2}$ \\
\hline Initial & 114,526 & & 114,825 & 209 & 698 & 907 & 0 & 0 & 0 & 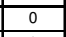 & 0 & 0 & 115,733 & 2,273 & & \\
\hline 50 & $\mid 114,526$ & 299 & 114,825 & 209 & 698 & 907 & \begin{tabular}{|l|l|}
3,0999 \\
\end{tabular} & $\frac{10}{10}$ & 3,110 & n & 161 & 161 & 119,00 & 2,337 & $1,800,000$ & 3,098 \\
\hline
\end{tabular}

Global Warming Potential (kg of $\mathrm{CO}_{2}$ eq.)

\begin{tabular}{|c|c|c|c|c|c|c|c|c|c|c|c|c|c|c|c|c|}
\hline \multirow{3}{*}{$\begin{array}{l}\text { Lifespan } \\
\text { (Years) }\end{array}$} & \multicolumn{14}{|c|}{ Embodied Global Warming Potential (GWP) } & \multirow{2}{*}{\multicolumn{2}{|c|}{$\begin{array}{c}\text { Difference in } \\
\text { Operating GWP from } \\
\text { Baseline after } \\
\text { Lifespan }\end{array}$}} \\
\hline & \multicolumn{3}{|c|}{ Manutacturing } & \multicolumn{3}{|c|}{ Construction } & \multicolumn{3}{|c|}{ Maintenance } & \multicolumn{3}{|c|}{ End of Life } & \multirow{2}{*}{$\begin{array}{l}{ }^{3} \text { Total } \\
\text { GWP }\end{array}$} & \multirow{2}{*}{ 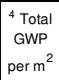 } & & \\
\hline & \begin{tabular}{|l|l|} 
Material \\
\end{tabular} & ${ }^{2}$ Trans. & \begin{tabular}{|l|l|} 
Total \\
\end{tabular} & Material & ${ }^{2}$ Trans & $\begin{array}{l}\text { Total } \\
\end{array}$ & Material & $\begin{array}{l}{ }^{2} \text { Trans. } \\
\end{array}$ & Total & \begin{tabular}{|l|} 
Material \\
\end{tabular} & $\begin{array}{l}{ }^{2} \text { Trans. } \\
\end{array}$ & \begin{tabular}{|l|l|} 
Total \\
\end{tabular} & & & \begin{tabular}{|l|}
${ }^{5}$ Total \\
\end{tabular} & ${ }^{6} \mathrm{per}$ \\
\hline \begin{tabular}{|l|l} 
Initial \\
\end{tabular} & \begin{tabular}{|l|}
6,842 \\
\end{tabular} & 0 & 6,842 & 13 & 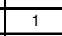 & 15 & 0 & 0 & 0 & 0 & 0 & 0 & $\begin{array}{l}6,857 \\
\end{array}$ & 135 & & \\
\hline 50 & \begin{tabular}{|l|l}
6,844 \\
\end{tabular} & 0 & 6,842 & 13 & 1 & 15 & 44 & 0 & 44 & 0 & 0 & 0 & 6,901 & 136 & 100,000 & 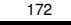 \\
\hline
\end{tabular}

Eocied energy (and GWP) numbers are based on an area of wall $=50.9 \mathrm{~m}^{2} \quad$ (Length $\times$ Height $\left.=7.6 \mathrm{~m} \times 6.7 \mathrm{~m}=50.9 \mathrm{~m}^{2}\right)$ Net wall area of baseline retall building (gross wall area - openings) $=581.0 \mathrm{~m}^{2}$

ATHENA $®$ EIE Material List:

\begin{tabular}{|c|c|c|}
\hline Material List & Quantities & Unit \\
\hline 3 mil Polyethylene & 54.0 & $\mathrm{~m} 2$ \\
\hline $\begin{array}{l}16 \mathrm{~mm} \text { Moisture Resistant Gypsum } \\
\text { Bard }\end{array}$ & 56.0 & $\mathrm{~m} 2$ \\
\hline $16 \mathrm{~mm}$ Regular Gypsum Board & 56.0 & $\mathrm{~m} 2$ \\
\hline Commercial $0.46 \mathrm{~mm}$ Steel Cladding & 168.0 & $\mathrm{~m} 2$ \\
\hline Extruded Polystyrene & 104.3 & $\mathrm{~m} 2(25 \mathrm{~mm})$ \\
\hline Galvanized Studs & 101.0 & $\mathrm{~kg}$ \\
\hline Joint Compound & 111.8 & $\mathrm{~kg}$ \\
\hline Modified Bitumen membrane & 68.2 & $\mathrm{~kg}$ \\
\hline Nails & 7.4 & $\mathrm{~kg}$ \\
\hline Paper Tape & 1.3 & $\mathrm{~kg}$ \\
\hline Screws Nuts \& Bolts & 1.3 & $\mathrm{~kg}$ \\
\hline $\begin{array}{l}\text { Small Dimension Softwood Lumber } \\
\text { kiln-ried }\end{array}$ & 0.7 & $\mathrm{~m} 3$ \\
\hline Solvent Based Alkyd Paint & 19.6 & $\mathrm{~L}$ \\
\hline Water Based Latex Paint & 132.5 & \\
\hline
\end{tabular}

'Initial = Time 'o' '(i.e. at the completion of initio

${ }^{2}$ Trans. = Transportation

Total EE (or Total GWP) = Total embodied energy (or total embodied GWP) of building component after lifespan (i.e. total man
construction + total maintenance + total end-offife effects)

${ }^{4}$ Total EE (or Total GWP) per $\mathrm{m}^{2}=$ Total EE (or Total GWP) of building
component/ area of building component that was modelled in ATHENAE EIE Total Difference in Operating Energy (or GWP) from Baseline after Lifespan $=$ The difference in the totar lecycle operating energy (or GWP)
from the baseline retail building ater lifespan, due to using this building ${ }^{6}$ Total Difference in Operating Energy (or GWP) from Baseline after Lifespan per $\mathrm{m}^{2}=$ Total difference in operating energy (or GWP) from
baseline after lifespan $/$ net wall area of baseline retail builing * Total operating primary energy use of baseline retail building after 50 years $=$
$50,700 \mathrm{GJ}\left(1,745 \mathrm{MJ} / \mathrm{m}^{2} / \mathrm{rr}\right)$ * Total operating GWP of baseline retail building after 50 years $=2,310$ tonnes
of $\mathrm{CO}_{2}$ eq. $80 \mathrm{~kg}$ of $\mathrm{CO}_{2}$ eq. $\left.\cdot \mathrm{m}^{2} \mathrm{yrr}\right)$ 
Wood Stud Wall \#22 (WS-W22)

Building Component Description:

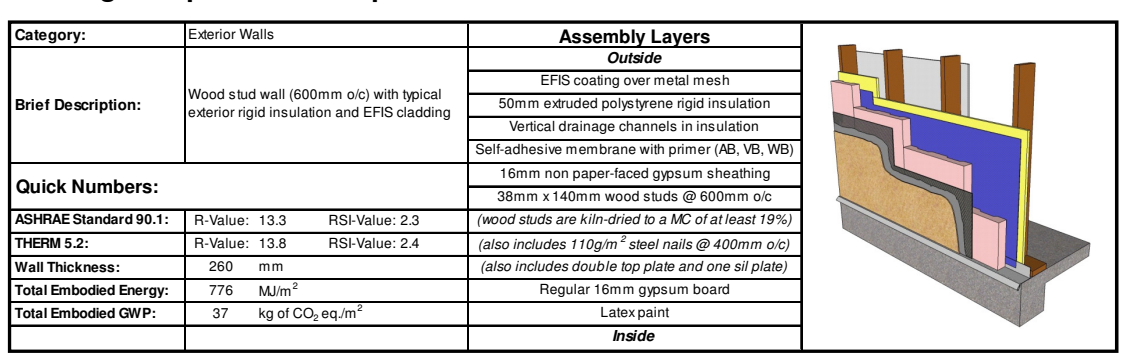

Life-Cycle Assessment Results:

Primary Energy Consumption (MJ)

\begin{tabular}{|c|c|c|c|c|c|c|c|c|c|c|c|c|c|c|c|c|}
\hline \multirow{3}{*}{$\begin{array}{l}\text { Lifespan } \\
\text { (Years) }\end{array}$} & \multicolumn{14}{|c|}{ Embodied Energy (EE) } & \multirow{2}{*}{\multicolumn{2}{|c|}{$\begin{array}{c}\text { Difference in } \\
\text { Operating Energy } \\
\text { from Baseline afte } \\
\text { Lifespan }\end{array}$}} \\
\hline & \multicolumn{3}{|c|}{ Manufacturing } & \multicolumn{3}{|c|}{ Construction } & \multicolumn{3}{|c|}{ Maintenance } & \multicolumn{3}{|c|}{ End of Life } & \multirow{2}{*}{$\begin{array}{c}{ }^{3}{ }^{3} \text { Total } \\
\mathrm{EE}\end{array}$} & \multirow{2}{*}{$\begin{array}{c}{ }^{4} \text { Total } \\
\text { EE } \\
\text { perm }\end{array}$} & & \\
\hline & Material & ${ }^{2}$ Trans & \begin{tabular}{|l|} 
Total \\
\end{tabular} & |Material & ${ }^{2}$ Trans. & Total & \begin{tabular}{|l|} 
Material \\
\end{tabular} & ${ }^{2}$ Trans. & \begin{tabular}{|l|} 
Total \\
\end{tabular} & Material & al| ${ }^{2}$ Trans. & \begin{tabular}{|l|l|} 
Total \\
\end{tabular} & & & ${ }^{5}$ Total & ${ }^{6}{ }_{\mathrm{per} \mathrm{m}}{ }^{2}$ \\
\hline |nitial & 34,543 & 354 & 97 & 209 & 791 & 1,000 & 0 & 0 & 0 & 0 & 0 & 0 & 35,897 & 705 & & \\
\hline 50 & 34,543 & 354 & \begin{tabular}{|l|l|}
34,897 \\
\end{tabular} & 209 & 791 & 1,000 & 3,099 & 10 & 3,110 & 0 & 517 & 517 & \begin{tabular}{|l|l|}
9,524 \\
\end{tabular} & 776 & 00,000 & 1,033 \\
\hline
\end{tabular}

N

Global Warming Potential ( $\mathrm{kg}$ of $\mathrm{CO}_{2}$ eq.)

\begin{tabular}{|c|c|c|c|c|c|}
\hline \multirow{3}{*}{$\begin{array}{l}\text { Lifespar } \\
\text { (Years) }\end{array}$} & \multicolumn{5}{|c|}{ Embodied Global } \\
\hline & \multicolumn{2}{|c|}{ Manufacturing } & \multicolumn{3}{|c|}{ Construction } \\
\hline & \begin{tabular}{|l|l|} 
Material & 2 \\
\end{tabular} & \begin{tabular}{|l|l} 
Total & $\mathrm{M}$ \\
\end{tabular} & Material & $\left.\right|^{2}{ }^{2}$ Trans. & Total \\
\hline Initial & \begin{tabular}{|l|l|}
1,801 & 1 \\
\end{tabular} & 1,802 & 13 & 1 & 15 \\
\hline 50 & \begin{tabular}{|l|l|}
1,801 & 1 \\
\end{tabular} & \begin{tabular}{|l|l|l|l}
1,802 \\
\end{tabular} & 13 & 1 & 15 \\
\hline \multicolumn{6}{|c|}{$\begin{array}{l}\text { Embodied energy (and GWP) numbers are based on an area of wal } \\
\text { Net wall area of baseline retail building (gross wall area - openings) }\end{array}$} \\
\hline \multicolumn{6}{|c|}{$\begin{array}{c}\text { ATHENA } ® \text { EIE Material List: } \\
\text { (Includes all materials after } 50 \text { years) }\end{array}$} \\
\hline \multicolumn{3}{|c|}{ Material List } & \multicolumn{2}{|c|}{ Quantities } & Unit \\
\hline \multicolumn{3}{|c|}{$\# 15$ Organic Felt } & \multicolumn{2}{|r|}{219.4} & $\mathrm{~m} 2$ \\
\hline \multicolumn{3}{|c|}{3 mil Polyethylene } & \multicolumn{2}{|r|}{54.0} & $\mathrm{~m} 2$ \\
\hline \multicolumn{3}{|c|}{$\begin{array}{l}16 \mathrm{~mm} \mathrm{M} \\
\text { Board }\end{array}$} & \multicolumn{2}{|r|}{56.0} & m2 \\
\hline \multicolumn{3}{|c|}{$16 \mathrm{~mm}$ Regular Gypsum Board } & & 56.0 & $\mathrm{~m} 2$ \\
\hline \multicolumn{3}{|c|}{ Extruded Polystyrene } & & 104.3 & $\mathrm{~m} 2(25 \mathrm{~mm})$ \\
\hline \multicolumn{3}{|c|}{ Galvanized Sheet } & & 51.4 & $\mathrm{~kg}$ \\
\hline \multicolumn{3}{|c|}{ Joint Compound } & & $\overline{1111.8}$ & $\mathrm{~kg}$ \\
\hline \multicolumn{3}{|c|}{ Modified Bitumen membrane } & & 68.2 & $\mathrm{~kg}$ \\
\hline \multicolumn{3}{|l|}{$\begin{array}{l}\text { Nails } \\
\end{array}$} & & 8.9 & $\mathrm{~kg}$ \\
\hline \multicolumn{3}{|c|}{ Paper Tape } & & 1.3 & $\mathrm{~kg}$ \\
\hline \multicolumn{3}{|c|}{$\begin{array}{l}\text { Small Dimension Softwoo } \\
\text { kiln-dried }\end{array}$} & & 0.7 & $\mathrm{~m} 3$ \\
\hline \multicolumn{3}{|c|}{ Solvent Based Alkyd Paint } & & 19.6 & 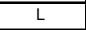 \\
\hline \multirow{2}{*}{\multicolumn{3}{|c|}{$\begin{array}{l}\text { Stucco over metal mesh } \\
\text { Water Based Latex Paint }\end{array}$}} & & 136.0 & $\mathrm{~m} 2$ \\
\hline & & & & 132.5 & 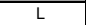 \\
\hline
\end{tabular}

Notes:
${ }^{1}$ Initial = Time 'o' (i.e. at the completion of initial construction)

${ }^{2}$ Trans. $=$ Transportation

Total EE (or Total GWP) = Total embodied energy (or total embodied GWP)

${ }^{4}$ Total EE (or Total GWP) per $\mathrm{m}^{2}=$ Total EE (or Total GWP) of building
component/ area of building component that was modelled in ATHENAQ EIE Total Difference in Operating Energy (or GWP) from Baseline after from the baseline retail building after lifespan, due to using this building
from from the baseline retail building after lifespa
component instead of the baseline component

Total Difference in Operating Energy (or GWP) from Baseline after

"Total operating primary energy use of baseline retail building after 50 years =
$50.700 \mathrm{GJ}\left(1,745 \mathrm{MJ} / \mathrm{m}^{2} \mathrm{yr}\right)$

"Total operating $\mathrm{GWP}$ of baseline retail building after 50 years $=2,310$ tonnes
of $\mathrm{CO}_{2}$ eq. $\left(80 \mathrm{~kg}\right.$ of $\mathrm{CO}_{2}$ eq, $\left./ \mathrm{n}^{2} \mathrm{yr}\right)$
Wood Stud Wall \#23 (WS-W23)

Building Component Description:

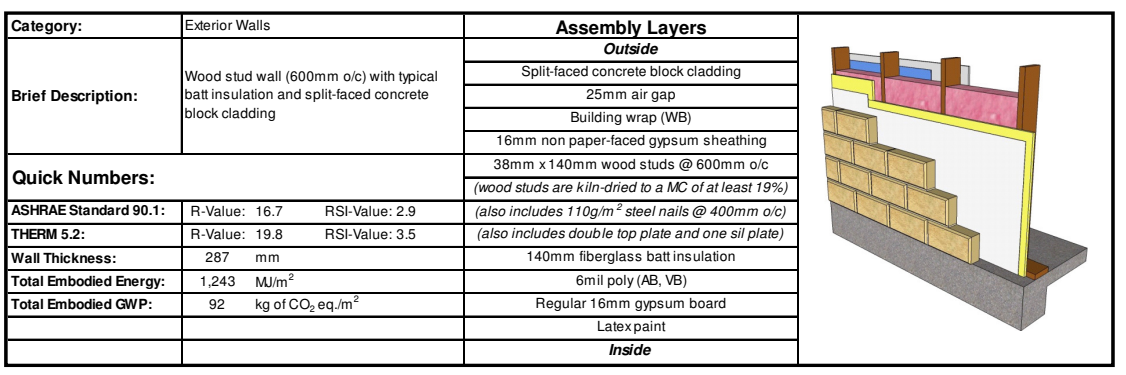

\section{Life-Cycle Assessment Results:}

Primary Energy Consumption (M

\begin{tabular}{|c|c|c|c|c|c|c|c|c|c|c|c|c|c|c|c|c|}
\hline \multirow{3}{*}{\begin{tabular}{|c|} 
Lifespan \\
(Years)
\end{tabular}} & \multicolumn{14}{|c|}{ Embodied Energy (EE) } & \multirow{2}{*}{\multicolumn{2}{|c|}{$\begin{array}{l}\text { Difierence in } \\
\text { Operating Energy } \\
\text { from Baseline ater } \\
\text { Lifespana }\end{array}$}} \\
\hline & \multicolumn{3}{|c|}{ Manutacturing } & \multicolumn{3}{|c|}{ Construction } & \multicolumn{3}{|c|}{ Maintenance } & \multicolumn{3}{|c|}{ End of Life } & \multirow{2}{*}{$\begin{array}{c}3 \\
3 \\
\text { Total } \\
\mathrm{EE}\end{array}$} & \multirow{2}{*}{\begin{tabular}{|l|}
${ }^{4}$ Total \\
EE \\
perm
\end{tabular}} & & \\
\hline & Material & ${ }^{2}$ Trans & \begin{tabular}{|l|l|l|l|} 
Total & \\
\end{tabular} & Material & ${ }^{2}$ Trans. & Total & Material & ${ }^{2}$ Trans & Total & Material & ${ }^{2}$ Trans. & \begin{tabular}{|l|l} 
Total \\
\end{tabular} & & & \begin{tabular}{|l|l|}
${ }^{5}$ Total \\
\end{tabular} & ${ }^{6}$ per $\mathrm{m}^{2}$ \\
\hline Initial & 57,793 & 918 & 58,710 & 209 & 2,258 & 2,467 & r & 0 & 0 & 0 & 0 & 0 & 61,177 & 1,201 & & \\
\hline & 57,793 & 918 & 58,710 & 209 & \begin{tabular}{|l|l|}
2,258 \\
\end{tabular} & $\begin{array}{l}2,467 \\
\end{array}$ & 0 & 0 & 0 & 1 & \begin{tabular}{|l|l|}
2,107 \\
\end{tabular} & 2,108 & 63,285 & \begin{tabular}{|l|l|}
1,243 \\
\end{tabular} & $\mid-1,100,000$ & $-1,893$ \\
\hline
\end{tabular}

Global Warming Potential (kg of $\mathrm{CO}_{2}$ eq.)

\begin{tabular}{|c|c|c|c|c|c|c|c|c|c|c|c|c|c|c|c|c|}
\hline & & & & & & & n & g Pote & rn & gorcos & $\partial_{2}$ eq.) & & & & & \\
\hline \multirow{3}{*}{ (Years) } & \multicolumn{14}{|c|}{ 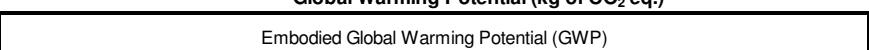 } & \multirow{2}{*}{\multicolumn{2}{|c|}{$\begin{array}{c}\text { Difference in } \\
\text { Operating GWP from } \\
\text { Baseline atter } \\
\text { Lifespan }\end{array}$}} \\
\hline & \multicolumn{3}{|c|}{ Manutacturing } & \multicolumn{3}{|c|}{ Construction } & \multicolumn{3}{|c|}{ Maintenance } & \multicolumn{3}{|c|}{ End of L Life } & \multirow{2}{*}{$\begin{array}{l}3 \\
\text { 3} \text { Total } \\
\text { GWP }\end{array}$} & \multirow{2}{*}{$\begin{array}{l}{ }^{4} \text { Total } \\
\text { GWP } \\
\text { per m }\end{array}$} & & \\
\hline & Material & $\begin{array}{l}{ }^{2} \text { Trans. } \\
\end{array}$ & \begin{tabular}{|l|} 
Total \\
\end{tabular} & Materia & Trans. & Total & Material & 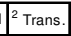 & Total & \begin{tabular}{|l|} 
Material \\
\end{tabular} & \begin{tabular}{|l|}
2 \\
\end{tabular} & Total & & & ${ }^{5}$ Total & \begin{tabular}{|l}
${ }^{6}$ per m \\
2
\end{tabular} \\
\hline Inite & \begin{tabular}{|l|l}
4,663 \\
\end{tabular} & 2 & & & & 18 & 0 & 0 & 0 & 0 & 0 & 0 & 32 & 92 & & \\
\hline 50 & 4,663 & 2 & 4,665 & 13 & 4 & 18 & 0 & 0 & 0 & 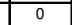 & 4 & 4 & 4,686 & 92 & $-50,000$ & \\
\hline
\end{tabular}

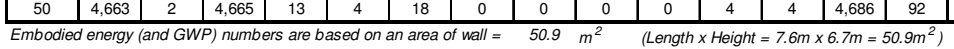

ATHENA $®$ EIE Material List: (Includes all materials after 50 years) of building component after lifespan (i.e. total man
construction + total maintenance t total enc-of-life effects) Lifespan per $\mathrm{m}^{2}=$ Total difference in operating energy (or GWP) from

\begin{tabular}{|l|c|c|}
\hline \multicolumn{1}{|c|}{ Material List } & Quantities & Unit \\
\hline $\begin{array}{l}\text { 16mm Moisture Resistant Gypsum } \\
\text { Board }\end{array}$ & 56.0 & $\mathrm{~m} 2$ \\
\hline 166mm Regular Gypsum Board & 56.0 & $\mathrm{~m} 2$ \\
\hline 6 mil Polyethylene & 108.0 & $\mathrm{~m} 2$ \\
\hline Batt. Fiberglass & 289.8 & $\mathrm{~m} 2(25 \mathrm{~mm})$ \\
\hline Cold Rolled Sheet & 10.3 & $\mathrm{~kg}$ \\
\hline Joint Compound & 111.8 & $\mathrm{~kg}$ \\
\hline Modiffed Bitumen membrane & 333.3 & $\mathrm{~kg}$ \\
\hline Mortar & 4.3 & $\mathrm{~m} 3$ \\
\hline Nails & 7.4 & $\mathrm{~kg}$ \\
\hline Paper Tape & 1.3 & $\mathrm{~kg}$ \\
\hline $\begin{array}{l}\text { Small } \\
\text { kilim-driension Sottwood Lumber, }\end{array}$ & 0.7 & $\mathrm{~m} 3$ \\
\hline Split-faced Concrete Block & $1,238.2$ & Blocks \\
\hline Water Based Latex Paint & 66.3 & $\mathrm{~L}$ \\
\hline
\end{tabular}

Notes:
1 'Initial = Time '

${ }^{2}$ Trans = Transportation

${ }^{3}$ Total EE (or Total GWP) $=$ Total embodied energy (or total embodied GWP) of building component after lifespan (i.e. total man
construction + total maintenance + total end-of-life effects)

${ }^{4}$ Total EE (or Total GWP) per $\mathrm{m}^{2}=$ Total EE (or Total GWP) of building Total Difference in Operating Energy (or GWP) from Baseline after Lifespan = The difference in the total life-cycle operating energy (or GWP)
from the baseline retail building atter lifespan, due to using this building component instead of the baseline component

Total Difference in Operating Energy (or GWP) from Baseline after Lifespan per $\mathrm{m}^{2}=$ Total difference in operating energy (or GWP) fro
baseline after lifespan / net wall area of baseline retail building * Total operating primary energy use of baseline retail building after 50 years =
$50,700 \mathrm{GJ}\left(1,745 \mathrm{MJ} / \mathrm{m}^{2} / \mathrm{yr}\right)$ * Total operating GWP of baseline retail building after 50 years $=2,310$ tonnes
of $\mathrm{CO}_{2}$ eq. $\left(80 \mathrm{~kg}\right.$ of $\mathrm{CO}_{2}$ eq. $\left./ \mathrm{m}^{2} / y r\right)$
${ }_{5}^{5}$ TOM D 
Wood Stud Wall \#24 (WS-W24)

Building Component Description:

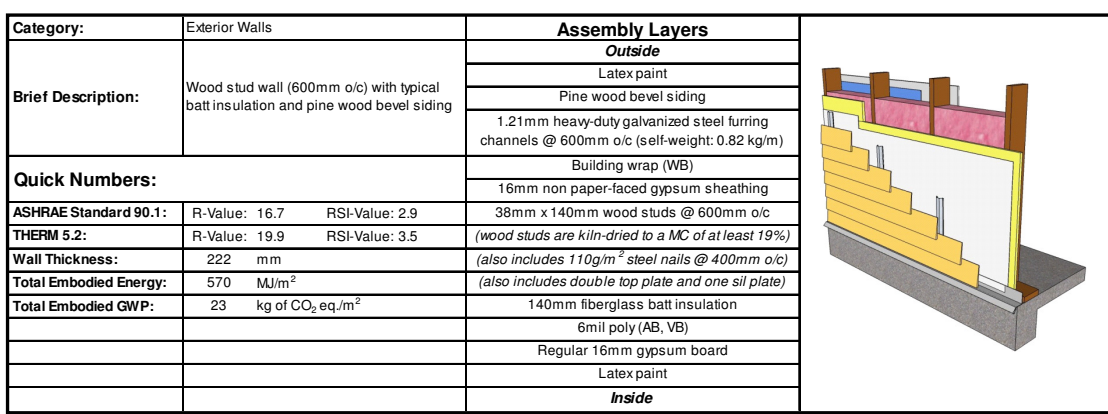

Life-Cycle Assessment Results:

\begin{tabular}{|c|c|c|c|c|c|c|c|c|c|c|c|c|c|c|c|c|}
\hline & \multicolumn{14}{|c|}{ Primary Energy Consumption (MJ) } & \multirow{3}{*}{\multicolumn{2}{|c|}{$\begin{array}{l}\text { Difference in } \\
\text { Operating Energy } \\
\text { from Baseline after } \\
\text { Lifespan }\end{array}$}} \\
\hline \multirow{3}{*}{$\begin{array}{l}\text { Lespan } \\
\text { (Years) }\end{array}$} & \multicolumn{14}{|c|}{ Embodied Energy (EE) } & & \\
\hline & \multicolumn{3}{|c|}{ Manufacturing } & \multicolumn{3}{|c|}{ Construction } & \multicolumn{3}{|c|}{ Maintenance } & \multicolumn{3}{|c|}{ End of Life } & \multirow{2}{*}{$\begin{array}{c}{ }^{3} \text { Total } \\
\text { EE }\end{array}$} & \multirow{2}{*}{\begin{tabular}{|cc}
4 & ${ }^{4}$ Total \\
EE \\
EE
\end{tabular}} & & \\
\hline & Material & Trans. & \begin{tabular}{|l} 
Total \\
\end{tabular} & Materia & \begin{tabular}{|l|l|}
${ }^{2}$ Trans. \\
\end{tabular} & $\begin{array}{c}\text { Total } \\
\end{array}$ & Material & \begin{tabular}{|l|l|}
${ }^{2}$ Trans. \\
\end{tabular} & Total & Material & | ${ }^{2}$ Trans. & Total & & & ${ }^{5}$ Total & ${ }^{6}$ per $\mathrm{m}^{2}$ \\
\hline Initial & 27,497 & 372 & 27,869 & 209 & 797 & 1,006 & 0 & 0 & 0 & 0 & 0 & 0 & 28,875 & 567 & & \\
\hline
\end{tabular}

Global Warming Potential (kg of $\mathrm{CO}_{2}$ eq.)

\begin{tabular}{|c|c|c|c|c|c|c|c|c|c|c|c|c|c|c|c|c|}
\hline \multirow{4}{*}{$\begin{array}{l}\text { Lifespan } \\
\text { (Years) }\end{array}$} & \multirow{2}{*}{\multicolumn{14}{|c|}{ Embodied Global Warming Potential (GWP) }} & \multirow{3}{*}{\multicolumn{2}{|c|}{$\begin{array}{c}\text { Difference in } \\
\text { Operatitig GWP fron } \\
\text { Baseline after } \\
\text { Lifespan }\end{array}$}} \\
\hline & & & & & & & & & & & & & & & & \\
\hline & \multicolumn{3}{|c|}{ Manufacturing } & \multicolumn{3}{|c|}{ Construction } & \multicolumn{3}{|c|}{ Maintenance } & \multicolumn{3}{|c|}{ End of Life } & \multirow{2}{*}{$\begin{array}{l}3 \text { Total } \\
\text { GWP }\end{array}$} & \multirow{2}{*}{$\begin{array}{l}{ }^{4} \text { Total } \\
\text { GWP } \\
\text { per }{ }^{2}\end{array}$} & & \\
\hline & Material & ${ }^{2}$ Trans. & Total & \begin{tabular}{|l|} 
Material \\
\end{tabular} & $\begin{array}{l}\text { Trans. } \\
\end{array}$ & $\begin{array}{l}\text { Total } \\
\end{array}$ & \begin{tabular}{|l|} 
Material \\
\end{tabular} & \begin{tabular}{|l|}
${ }^{2}$ Trans. \\
\end{tabular} & Total & |Material & ${ }^{2}$ Trans. & Total & & & ${ }^{5}$ Total & ${ }^{6}$ per m ${ }^{2}$ \\
\hline${ }^{1}$ Initial & 1,152 & 1 & 1,153 & 13 & 2 & 15 & 0 & 0 & 0 & 0 & 0 & 0 & 1,168 & 23 & & \\
\hline 50 & 1,152 & 1 & 1,153 & 13 & 2 & 15 & 0 & 0 & 0 & 0 & 0 & 0 & 1,168 & 23 & $-40,000$ & -69 \\
\hline
\end{tabular}
Net wall area of baseline retail building (gross wall area - openings) $=581.0 \mathrm{~m}^{2}$

\section{ATHENA ${ }^{\circledR}$ EIE Material List:}

(Includes all materials after 50 years)

\begin{tabular}{|l|c|c|}
\hline \multicolumn{1}{|c|}{ Material List } & Quantities & Unit \\
\hline $\begin{array}{l}16 \mathrm{~mm} \text { Moisture Resistant Gypsum } \\
\text { Board }\end{array}$ & 56.0 & $\mathrm{~m} 2$ \\
\hline 16mm Regular Gypsum Board & 56.0 & $\mathrm{~m} 2$ \\
\hline 6 m mil Polyethylene & 108.0 & $\mathrm{~m} 2$ \\
\hline Batt. Fiberglass & 289.8 & $\mathrm{~m} 2(25 \mathrm{~mm})$ \\
\hline Galvanized Sheet & 106.1 & $\mathrm{~kg}$ \\
\hline Joint Compound & 111.8 & $\mathrm{~kg}$ \\
\hline Nails & 8.7 & $\mathrm{~kg}$ \\
\hline Paper Tape & 1.3 & $\mathrm{~kg}$ \\
\hline Pine Wood Bevel Siding & 160.4 & $\mathrm{~m} 2$ \\
\hline $\begin{array}{l}\text { Small Dimension Sottwood Lumber, } \\
\text { kiln-dried }\end{array}$ & 0.7 & $\mathrm{~m} 3$ \\
\hline Water Based Latex Paint & 132.5 & $\mathrm{~L}$ \\
\hline
\end{tabular}

'1 Notes:

${ }^{2}$ Trans. = Transportation

${ }^{3}$ Total EE (or Total GWP) = Total embodied energy (or total embodied GWP) of building component after lifespan (i.e. total manufacturing + tota

${ }^{4}$ Total EE (or Total GWP) per $\mathrm{m}^{2}=$ Total EE (or Total GWP) of building ${ }^{5}$ Total Difference in Operating Energy (or GWP) from Baseline after Lifespan $=$ The difference in the total life-cycle operating energy (or GWWP)
from the baseline retail building after lifespan, due to using this building
component instead of the baseline component

Lifespan per $\mathrm{m}^{2}=$ Total difiference in operating energy (or GWP) from baseline after lifespan / net wall area of baseline retail buildinin Total operating primary energy use of baseline retail building after 50 years

* Total operating $\mathrm{GWP}$ of baseline retail building after 50 years $=2,310$ tonnes
of $\mathrm{CO}_{2}$ eq $\left(80 \mathrm{~kg}\right.$ of $\left.\mathrm{CO}_{2} \mathrm{eq} / \mathrm{m}^{2} / \mathrm{yr}\right)$

Wood Stud Wall \#25 (WS-W25)

Building Component Description:

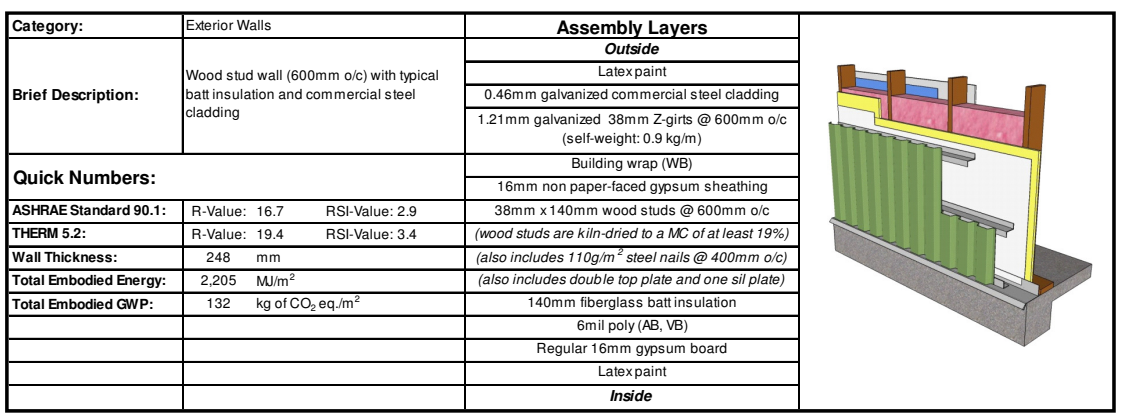

Life-Cycle Assessment Results:

\begin{tabular}{|c|c|c|c|c|c|c|c|c|c|c|c|c|c|c|c|c|}
\hline \multirow{4}{*}{\begin{tabular}{|c} 
Lifespan \\
(Years)
\end{tabular}} & \multicolumn{16}{|c|}{ nsumption (MJ) } \\
\hline & \multicolumn{14}{|c|}{ Embodied Energy (EE) } & \multirow{2}{*}{\multicolumn{2}{|c|}{$\begin{array}{c}\text { Difference in } \\
\text { Operating Energy } \\
\text { from Baseline after } \\
\text { Lifespan }\end{array}$}} \\
\hline & \multicolumn{3}{|c|}{ Manutacturing } & \multicolumn{3}{|c|}{ Construction } & \multicolumn{3}{|c|}{ Maintenance } & \multicolumn{3}{|c|}{ End of Life } & \multirow{2}{*}{$\begin{array}{l}{ }^{3} \text { Total } \\
\mathrm{EE}\end{array}$} & \multirow{2}{*}{$\begin{array}{c}{ }^{4} \text { Total } \\
\mathrm{E} \\
\mathrm{E} \\
\mathrm{C}^{2}\end{array}$} & & \\
\hline & $\mid$\begin{tabular}{|l|l|l|} 
Matrial \\
\end{tabular} & ${ }^{2}$ Trans. & \begin{tabular}{|l|l} 
Total \\
\end{tabular} & Materia & ${ }^{2}$ Trans. & Total & Material & ${ }^{2}$ Trans. & Total & Material & \begin{tabular}{|l|}
${ }^{2}$ Trans. \\
\end{tabular} & \begin{tabular}{|l|l|l|} 
Total \\
\end{tabular} & & & ${ }^{5}$ Total & ${ }^{6}$ per m$^{2}$ \\
\hline \begin{tabular}{|l|l} 
Initial \\
\end{tabular} & 110,865 & 324 & 111,189 & 209 & 694 & 904 & 0 & 0 & 0 & 0 & 0 & 0 & 112,092 & 2,201 & & \\
\hline
\end{tabular}

Global Warming Potential (kg of $\mathrm{CO}_{2}$ eq.)

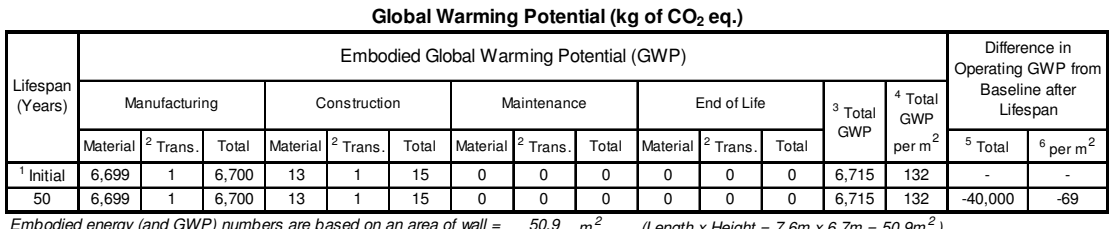
Embodied energy (and GWP) numbers are based on an area of wall $=50.9 \mathrm{~m}^{2} \quad$ (Length $x$ Height $=7.6 \mathrm{~m} \times 6.7 \mathrm{~m}=50.9 \mathrm{~m}^{2}$ ) Net wall area of baseline retail building (gross wall area - openings) $=581.0 \mathrm{~m}^{2}$

ATHENA ® EIE Material List:

\begin{tabular}{|c|c|c|}
\hline Material List & Quantities & Unit \\
\hline $\begin{array}{l}16 \mathrm{~mm} \text { Moisture Resistant Gypsum } \\
\text { Board }\end{array}$ & 56.0 & $\mathrm{~m} 2$ \\
\hline $16 \mathrm{~mm}$ Regular Gypsum Board & 56.0 & $\mathrm{~m} 2$ \\
\hline 6 mil Polyethylene & 108.0 & $\mathrm{~m} 2$ \\
\hline Batt. Fiberglass & 289.8 & $\mathrm{~m} 2(25 \mathrm{~mm}$ \\
\hline Commercial $0.46 \mathrm{~mm}$ Steel Cladding & 168.0 & $\mathrm{~m} 2$ \\
\hline Galvanized Studs & 80.8 & $\mathrm{~kg}$ \\
\hline Joint Compound & 111.8 & $\mathrm{~kg}$ \\
\hline Nails & 7.4 & $\mathrm{~kg}$ \\
\hline Paper Tape & 1.3 & $\mathrm{~kg}$ \\
\hline Screws Nuts \& Bolts & 1.3 & $\mathrm{~kg}$ \\
\hline $\begin{array}{l}\text { Small Dimension Softwood Lumber, } \\
\text { kiln-dried }\end{array}$ & 0.7 & $\mathrm{~m} 3$ \\
\hline Water Based Latex Paint & 132.5 & $\mathrm{~L}$ \\
\hline
\end{tabular}

Initial = Time $0 \cdot$ Notes:

${ }^{2}$ Trans. = Transportation

Total EE (or Total GWP) = Total embodied energy (or total embodied GWP) of building component after lifespan (i.e. total manufacturing + to

${ }^{4}$ Total EE (or Total GWP) per $\mathrm{m}^{2}=$ Total EE (or Total GWP) of building Lifespan = The difiterence in the total tire-cycle operating energy (or GWP) from the baseline retail building affer lifespan, due to using this building
component instead of the baseline component ${ }^{6}$ Total Difference in Operating Energy (or GWP) from Baseline after Lifiespan per $\mathrm{m}^{2}=$ Total difference in operating energy (or GWP) from
baseline after lifespan $/$ net wall area of baseline retail building

* Total operating primarn energy use of baseline retail building after 50 years
$50,700 \mathrm{GJ}\left(1,745 \mathrm{MJ} / \mathrm{m}^{2} / \mathrm{yr}\right)$

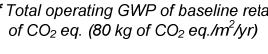


Wood Stud Wall \#26 (WS-W26)

Building Component Description:

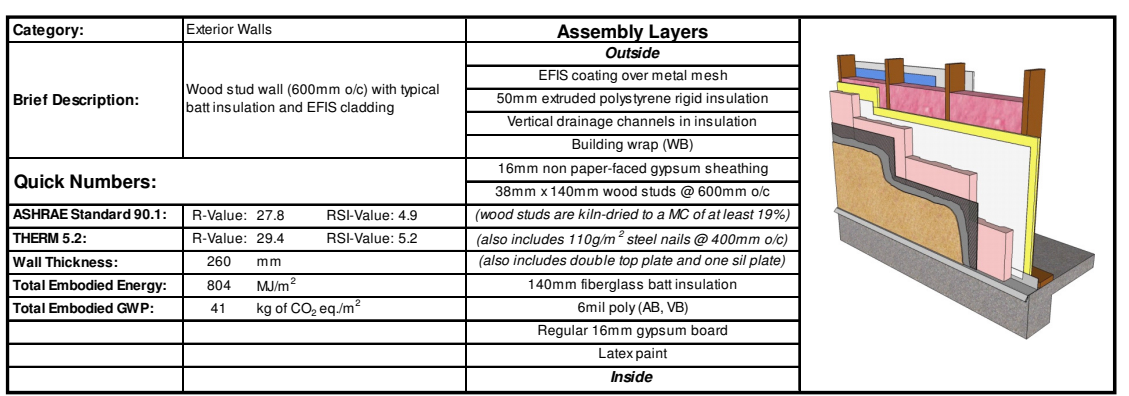

\section{Life-Cycle Assessment Results:}

Primary Energy Consumption (MJ)

\begin{tabular}{|c|c|c|c|c|c|c|c|c|c|c|c|c|c|c|c|c|}
\hline \multirow{3}{*}{$\begin{array}{l}\text { Lifespan } \\
\text { (Years) }\end{array}$} & \multicolumn{14}{|c|}{ Embodied Energy (EE) } & \multirow{2}{*}{\multicolumn{2}{|c|}{$\begin{array}{l}\text { Difference in } \\
\text { Operating Energy } \\
\text { from Baseline after } \\
\text { Lifespan }\end{array}$}} \\
\hline & \multicolumn{3}{|c|}{ Manufacturing } & \multicolumn{3}{|c|}{ istruction } & \multicolumn{3}{|c|}{ Maintenanc } & \multicolumn{3}{|c|}{ End of 1} & \multirow{2}{*}{$\begin{array}{l}{ }^{3} \text { Total } \\
\mathrm{EE}\end{array}$} & \multirow{2}{*}{$\begin{array}{c}{ }^{4} \text { Total } \\
\text { EE } \\
\text { per m }\end{array}$} & & \\
\hline & Material & ${ }^{2}$ Trans. & Total & Material & ${ }^{2}$ Trans. & $\begin{array}{l}\text { Total } \\
\text { Tol }\end{array}$ & Material & \begin{tabular}{|l|}
${ }^{2}$ Trans. \\
\end{tabular} & Total & Material & \begin{tabular}{|l|}
${ }^{2}$ Trans. \\
\end{tabular} & Total & & & ${ }^{5}$ Total & ${ }^{6}$ per m$^{2}$ \\
\hline \begin{tabular}{|l|l} 
Initial \\
\end{tabular} & 39,037 & 380 & 39,41 & 209 & 802 & 1,011 & 0 & 0 & 0 & 0 & 0 & 0 & \begin{tabular}{|l|}
40,429 \\
\end{tabular} & 794 & & \\
\hline 50 & \begin{tabular}{|l|l|}
39,037 \\
\end{tabular} & \begin{tabular}{|l|}
380 \\
\end{tabular} & \begin{tabular}{|l|l|}
39,417 \\
\end{tabular} & 209 & \begin{tabular}{|l|}
802 \\
\end{tabular} & 1,011 & 0 & 0 & 0 & 0 & 530 & 530 & 40,959 & \begin{tabular}{|l|}
804 \\
\end{tabular} & 900,0 & $-3,2$, \\
\hline
\end{tabular}

Global Warming Potential ( $\mathrm{kg}$ of $\mathrm{CO}_{2}$ eq.)

\begin{tabular}{|c|c|c|c|c|c|c|c|c|c|c|c|c|c|c|c|c|}
\hline \multirow{3}{*}{$\begin{array}{l}\text { Lifespan } \\
\text { (Years) }\end{array}$} & \multicolumn{14}{|c|}{ Embodied Global Warming Potential (GWP) } & \multirow{2}{*}{\multicolumn{2}{|c|}{$\begin{array}{l}\text { Difference in } \\
\text { Operating GWP fron } \\
\text { Baseline after } \\
\text { Lifespan }\end{array}$}} \\
\hline & \multicolumn{3}{|c|}{ Manutacturing } & \multicolumn{3}{|c|}{ Construction } & \multicolumn{3}{|c|}{ Maintenance } & \multicolumn{3}{|c|}{ End of Life } & \multirow{2}{*}{$\begin{array}{l}{ }^{3} \text { Total } \\
\text { GWP }\end{array}$} & \multirow{2}{*}{$\begin{array}{l}{ }^{4} \text { Total } \\
\text { GWP } \\
\text { per m }\end{array}$} & & \\
\hline & Material & ${ }^{2}$ Trans. & Total & Material & ${ }^{2}$ Trans. & Total & Material & ${ }^{2}$ Trans. & Total & Material & ${ }^{2}$ Trans. & Total & & & \begin{tabular}{|l|l|}
${ }^{5}$ Total \\
\end{tabular} & ${ }^{6} \operatorname{perm}^{2}$ \\
\hline Initial & 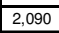 & 1 & & 13 & 2 & 15 & 0 & 0 & 0 & 0 & 0 & 0 & 2,106 & 41 & & \\
\hline 50 & 2,090 & 1 & 2,6 & 13 & 2 & 15 & 0 & 0 & 0 & 0 & 1 & 1 & 2,107 & 41 & -100, & -172 \\
\hline
\end{tabular}

Embodied energy (and GWP) numbers are based on an area of wall $=50.9 \mathrm{~m}^{2} \quad$ (Length $\times$ Height $=7.6 \mathrm{~m} \times 6.7 \mathrm{~m}=50.9 \mathrm{~m}^{2}$ )

Net wall area of baseline retail building (gross wall area - openings) $=581.0 \mathrm{~m}^{2}$

\section{ATHENA $®$ EIE Material List:}

\begin{tabular}{|c|c|c|}
\hline Material List & Quantities & Unit \\
\hline$\# 15$ Organic Felt & 219.4 & $\mathrm{~m}^{2} 2$ \\
\hline $\begin{array}{l}16 \mathrm{~mm} \text { Moisture Resistant Gypsum } \\
\text { Board }\end{array}$ & 56.0 & $\mathrm{~m} 2$ \\
\hline 16mm Regular Gypsum Board & 56.0 & $\mathrm{~m} 2$ \\
\hline 6 mil Polyethylene & 108.0 & $\mathrm{~m} 2$ \\
\hline Batt. Fiberglass & 289.8 & $\mathrm{~m} 2(25 \mathrm{~mm})$ \\
\hline Extruded Polystyrene & 104.3 & $\mathrm{~m} 2(25 \mathrm{~mm})$ \\
\hline Galvanized Sheet & 51.4 & $\mathrm{~kg}$ \\
\hline Joint Compound & 111.8 & $\mathrm{~kg}$ \\
\hline Nails & 12.0 & $\mathrm{~kg}$ \\
\hline Paper Tape & 1.3 & $\mathrm{~kg}$ \\
\hline $\begin{array}{l}\text { Small Dimension Softwood Lumber, } \\
\text {,inh-dipd }\end{array}$ & 0.7 & $\mathrm{~m} 3$ \\
\hline $\begin{array}{l}\text { Kiln-dried } \\
\text { Stucco over metal mesh }\end{array}$ & 36.0 & $\mathrm{~m} 2$ \\
\hline Nater Based Latex Paint & 132.5 & \\
\hline
\end{tabular}

Notes:
1 Initial $=$ Time 'o' 1 (i.e. at the completion of initio

${ }^{2}$ Trans. $=$ Transportation

Total EE (or Total GWP) = Total embodied energy (or total embodied GWP) of building component after lifespan (i.e. total man

${ }^{4}$ Total EE (or Total GWP) per $\mathrm{m}^{2}=$ Total EE (or Total GWP) of building
component/ area of building component that was modelled in ATHENAQ EIE

${ }^{5}$ Total Difference in Operating Energy (or GWP) from Baseline after Lifespan = The difference in the total life-cycle operating energy (or GWP)
from the baseline retail building after lifespan, due to using this building component instead of the baseline component

Total Difference in Operating Energy (or GWP) from Baseline after

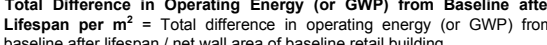
* Total operating primarn energy use of baseline retail building after 50 years

of $\mathrm{CO}_{2}$ eq. $\left(80 \mathrm{~kg}\right.$ of $\mathrm{CO}_{2}$ eq.

Wood Stud Wall \#27 (WS-W27)

Building Component Description:

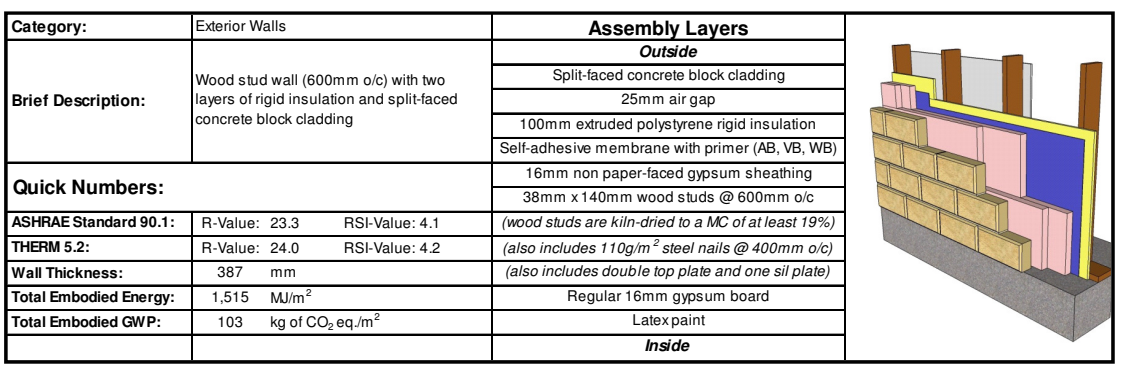

Life-Cycle Assessment Results:

Primary Energy Consumption (MJ)

\begin{tabular}{|c|c|c|c|c|c|c|c|c|c|c|c|c|c|c|c|c|}
\hline \multirow{3}{*}{$\begin{array}{l}\text { Lifespan } \\
\text { (Years) }\end{array}$} & \multicolumn{14}{|c|}{ Embodied Energy (EE) } & \multirow{2}{*}{\multicolumn{2}{|c|}{$\begin{array}{l}\text { Difference in } \\
\text { Operating Energy } \\
\text { from Baseline after } \\
\text { Lifespan }\end{array}$}} \\
\hline & \multicolumn{3}{|c|}{ Manufacturing } & \multicolumn{3}{|c|}{ Construction } & \multicolumn{3}{|c|}{ Maintenance } & \multicolumn{3}{|c|}{ End of Lifie } & \multirow{2}{*}{$\begin{array}{c}{ }^{3} \text { Total } \\
\mathrm{EE}\end{array}$} & \multirow{2}{*}{$\begin{array}{c}{ }^{4} \text { Totat } \\
\mathrm{EE} \\
\text { per } \mathrm{m}^{2}\end{array}$} & & \\
\hline & Material & ${ }^{2}$ Trans. & Total & Material & ${ }^{2}$ Trans. & Total & Material & ${ }^{2}$ Trans. & Total & Material & \begin{tabular}{|l|}
2 Trans. \\
\end{tabular} & Total & & & ${ }^{5}$ Total & ${ }^{6}$ perm ${ }^{2}$ \\
\hline Initial & 68,555 & 892 & 69,448 & 209 & 2,271 & 2,481 & 0 & 0 & 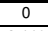 & 0 & 0 & 0 & \begin{tabular}{|c|}
71,928 \\
\end{tabular} & 1,413 & & \\
\hline 50 & 68,555 & 892 & \begin{tabular}{|l|l|l|l|}
69,448 \\
\end{tabular} & 209 & \begin{tabular}{|l|l|}
2,271 \\
\end{tabular} & 2,481 & 3,099 & 10 & 3,110 & 1 & 2,114 & 2,115 & 7,152 & $\begin{array}{l}1,515 \\
\end{array}$ & $-1,600,0$ & 2,7. \\
\hline
\end{tabular}

Global Warming Potential (kg of $\mathrm{CO}_{2}$ eq.)

\begin{tabular}{|c|c|c|c|c|c|c|c|c|c|c|c|c|c|c|c|c|}
\hline & & & & & & & & ote & & & & & & & & \\
\hline \multirow{3}{*}{ (Years) } & \multicolumn{14}{|c|}{ Embodied Global Warming Potential (GWP) } & \multirow{2}{*}{\multicolumn{2}{|c|}{\begin{tabular}{|c|} 
Difference in \\
Operatitig GWP from \\
Baseline after \\
Lifespan
\end{tabular}}} \\
\hline & \multicolumn{3}{|c|}{ Manufacturing } & \multicolumn{3}{|c|}{ Construction } & \multicolumn{3}{|c|}{ Maintenance } & \multicolumn{3}{|c|}{ End of Life } & \multirow{2}{*}{$\begin{array}{l}{ }^{3} \text { Total } \\
\text { GWP }\end{array}$} & \multirow{2}{*}{\begin{tabular}{|c|}
${ }^{4}$ Total \\
GWP \\
per m \\
2
\end{tabular}} & & \\
\hline & Material & ${ }^{2}$ Trans. & Total & Materia & Trans. & Total & Material & Trans. & Total & Material & Trans. & Total & & & ${ }^{5}$ Total & per $\mathrm{m}^{2}$ \\
\hline |nitial & 5,1 & 2 & 5,155 & 13 & & 18 & 0 & R & . & 0 & 0 & 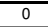 & 5,173 & 102 & & \\
\hline 50 & \begin{tabular}{|l|l|} 
\\
515
\end{tabular} & 2 & 5,155 & 13 & 4 & 18 & 44 & 0 & 44 & 0 & 4 & 4 & $\mid 5,221$ & 103 & $-80,0$ & -138 \\
\hline
\end{tabular}

Embodied energy (and GWP) numbers are based on an area of wall $=\quad 50.9 \mathrm{~m}^{2} \quad$ (Length $\times$ Height $=7.6 \mathrm{~m} \times 6.7 \mathrm{~m}=50.9 \mathrm{~m}^{2}$ ) Net wall area of baseline retail building (gross wall area - openings) $=581.0 \mathrm{~m}^{2}$

ATHENA $₫$ EIE Material List:

(Includes all materials after 50 years)

\begin{tabular}{|l|c|c|}
\hline \multicolumn{1}{|c|}{ Material List } & Quantitities & Unit \\
\hline 3 mil Polyethylene & 54.0 & $\mathrm{~m} 2$ \\
\hline $\begin{array}{l}16 \mathrm{~mm} \text { Moill materials after } 50 \text { years) } \\
\text { Board }\end{array}$ & 56.0 & $\mathrm{~m} 2$ \\
\hline 16rmm Regular Gypsum Board & 56.0 & $\mathrm{~m} 2$ \\
\hline Cold Rolled Sheet & 10.3 & $\mathrm{~kg}$ \\
\hline Extruded Polystyrene & 208.6 & $\mathrm{~m} 2(25 \mathrm{~mm})$ \\
\hline Joint Compound & 111.8 & $\mathrm{~kg}$ \\
\hline Modified Bitumen membrane & 401.5 & $\mathrm{~kg}$ \\
\hline Mortar & 4.3 & $\mathrm{~m} 3$ \\
\hline Nails & 7.4 & $\mathrm{~kg}$ \\
\hline Paper Tape & 1.3 & $\mathrm{~kg}$ \\
\hline $\begin{array}{l}\text { Small Dimension Sottwood Lumber, } \\
\text { kiln dried }\end{array}$ & 0.7 & $\mathrm{~m} 3$ \\
\hline Solvent Based Alkyd Paint & 19.6 & $\mathrm{~L}$ \\
\hline Split-taced Concrete Block & $1,238.2$ & Blocks \\
\hline Water Based Latex Paint & 66.3 & $\mathrm{~L}$ \\
\hline
\end{tabular}

\section{Notes:}

'Initial = Time ' 0 ' (i.e. at the

${ }^{2}$ Trans. = Transportation

Total EE (or Total GWP) = Total embodied energy (or total embodied GWP)
of building component after lifespan (i.e. total manufacturing + total of bulding component atter lifespan (i.e. total manu
construction + total maintenance + total end-of-life effects)

${ }^{4}$ Total EE (or Total GWP per $\mathrm{m}^{2}=$ Total EE (or Total GWP) of building
component/ area of building component that was modelled in ATHENAQ EIEE

${ }^{5}$ Total Difference in Operaling Enargy (or GWP) from Beseline after) Lifespan = The difference in the totat lific-cycle operating energy ( (or GWP)
from the baseline retail building after lifespan, due to using this building

Total Difference in Operating Energy (or GWP) from Baseline after
Lifespan per $\mathrm{m}^{2}=$ Total difference in operating energy (or GWP) from baseline after lifiespan / $n$ et wall area of baseline retail building * Total operating primary energy use of baseline retail building after 50 years =
$50,700 \mathrm{GJ}\left(1.745 \mathrm{MJ} / \mathrm{m}^{2} \mathrm{yr}\right)$

* Total operating $\mathrm{GWP}$ of baseline retail building after 50 years $=2,310$ tonnes
of $\mathrm{CO}_{2} e q .8 \mathrm{~kg}$ of $\mathrm{CO}_{2} e q . / \mathrm{m}^{2} \mathrm{yr}$ ) 
Wood Stud Wall \#28 (WS-W28)

Building Component Description:

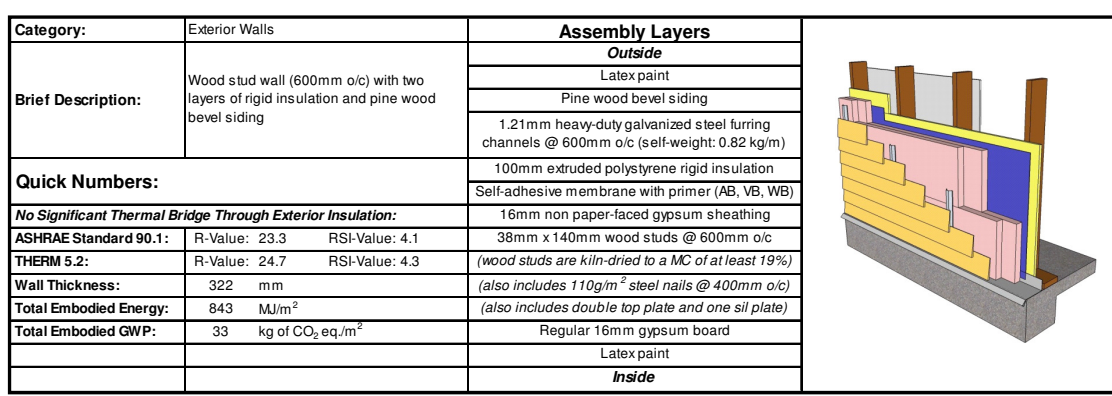

\section{Life-Cycle Assessment Results:}

Primary Energy Consumption (MJ)

\begin{tabular}{|c|c|c|c|c|c|c|c|c|c|c|c|c|c|c|c|c|}
\hline \multirow{3}{*}{$\mid$\begin{tabular}{|l} 
Lifespan \\
(Years)
\end{tabular}} & \multicolumn{14}{|c|}{ Embodied Energy (EE) } & \multirow{2}{*}{\multicolumn{2}{|c|}{$\begin{array}{c}\text { Difference in } \\
\text { Operating Energy } \\
\text { from Baseline after } \\
\text { Lifespan }\end{array}$}} \\
\hline & \multicolumn{3}{|c|}{ Manufacturing } & \multicolumn{3}{|c|}{ Construction } & \multicolumn{3}{|c|}{ Maintenance } & \multicolumn{3}{|c|}{ End of Life } & \multirow{2}{*}{$\begin{array}{l}{ }^{3} \text { Total } \\
\mathrm{EE}\end{array}$} & \multirow{2}{*}{$\begin{array}{l}{ }^{4} \text { Total } \\
\text { EE } \\
\text { per } \mathrm{m}^{2}\end{array}$} & & \\
\hline & Materia & 1 ${ }^{2}$ Trans & Total & Material & ${ }^{2}$ Trans. & \begin{tabular}{|l|l} 
Total \\
\end{tabular} & Materia & 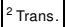 & Total & Material & al ${ }^{2}$ Trans. & Total & & & \begin{tabular}{|l|l|}
${ }^{5}$ Total \\
\end{tabular} & ${ }^{6}$ per $\mathrm{m}^{2}$ \\
\hline 1 Initial & 38,259 & 347 & 38,60 & 209 & 810 & 1,020 & 0 & 0 & 0 & 0 & 0 & 0 & 39,626 & 778 & & \\
\hline 50 & 38,259 & 347 & (88,60 & 209 & 810 & \begin{tabular}{|l|l}
1,020 \\
\end{tabular} & 3,099 & 10 & 3,110 & 0 & 177 & 178 & 42,913 & 843 & $-1,300$, & $-2,2$ \\
\hline
\end{tabular}

Global Warming Potential ( $\mathrm{kg}$ of $\mathrm{CO}_{2}$ eq.)

\begin{tabular}{|c|c|c|c|c|c|c|c|c|c|c|c|c|c|c|c|c|}
\hline \multirow{3}{*}{$\begin{array}{l}\text { Lifespar } \\
\text { (Years) }\end{array}$} & \multicolumn{14}{|c|}{ Embodied Global Warming Potential (GWP) } & \multirow{2}{*}{\multicolumn{2}{|c|}{$\begin{array}{c}\text { Difference in } \\
\text { Operating GWP from } \\
\text { Baseline after } \\
\text { Lifespan }\end{array}$}} \\
\hline & \multicolumn{3}{|c|}{ Manutacturing } & \multicolumn{3}{|c|}{ Construction } & \multicolumn{3}{|c|}{ Maintenance } & \multicolumn{3}{|c|}{ End of Life } & \multirow{2}{*}{$\begin{array}{l}{ }^{3} \text { Total } \\
\text { GWP }\end{array}$} & \multirow{2}{*}{ 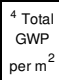 } & & \\
\hline & Materia & ${ }^{2}$ Trans. & \begin{tabular}{|l|l|} 
Total \\
\end{tabular} & \begin{tabular}{|l|} 
Material \\
\end{tabular} & \begin{tabular}{|l|}
${ }^{2}$ Trans. \\
\end{tabular} & \begin{tabular}{|l|} 
Total \\
\end{tabular} & \begin{tabular}{|l|} 
Materia \\
\end{tabular} & ${ }^{2}$ Trans. & \begin{tabular}{|l|} 
Total \\
\end{tabular} & \begin{tabular}{|l|} 
Materia \\
\end{tabular} & 2 Trans. & \begin{tabular}{|l|l|} 
Total \\
\end{tabular} & & & \begin{tabular}{|l|}
${ }^{5}$ Total \\
\end{tabular} & ${ }^{6}$ perm m$^{2}$ \\
\hline 1 Initial & 1,643 & 1 & $\begin{array}{ll}1,643 \\
\end{array}$ & 13 & 2 & 15 & 0 & 0 & 0 & 0 & 0 & 0 & 1,658 & 33 & & - \\
\hline 50 & \begin{tabular}{|l|l|}
1,643 \\
\end{tabular} & 1 & 1,643 & 13 & 2 & 15 & 44 & 0 & $\begin{array}{l}44 \\
\end{array}$ & 0 & 0 & 0 & 1,702 & 33 & $-70,000$ & -120 \\
\hline
\end{tabular}

Embodied energy (and GWP) numbers are based on an area of wall $=50.9 \mathrm{~m}^{2} \quad$ (Length $\times$ Height $=7.6 \mathrm{~m} \times 6.7 \mathrm{~m}=50.9 \mathrm{~m}^{2}$ )

Net wall area of baseline retail building (gross wall area - openings) $=581.0 \mathrm{~m}^{2}$

\section{ATHENA ${ }^{\circledR}$ EIE Material List:}

\begin{tabular}{|c|c|c|}
\hline Material List & Quantities & Unit \\
\hline 3 mil Polyethylene & 54.0 & $\mathrm{~m}^{2} 2$ \\
\hline $\begin{array}{l}\text { 16mm Moisture Resistant Gypsum } \\
\text { Board }\end{array}$ & 56.0 & $\mathrm{~m} 2$ \\
\hline $16 \mathrm{~mm}$ Regular Gypsum Board & 56.0 & $\mathrm{~m} 2$ \\
\hline Extruded Polystyrene & 208.6 & m2 $225 \mathrm{~mm}$ \\
\hline Galvanized Sheet & 106.1 & $\mathrm{~kg}$ \\
\hline Joint Compound & 111.8 & $\mathrm{~kg}$ \\
\hline Modified Bitumen membrane & 68.2 & $\mathrm{~kg}$ \\
\hline Nails & 8.7 & $\mathrm{~kg}$ \\
\hline $\begin{array}{l}\text { Paper Tape } \\
\end{array}$ & 1.3 & $\mathrm{~kg}$ \\
\hline Pine Wood Bevel Siding & 160.4 & $\mathrm{~m} 2$ \\
\hline $\begin{array}{l}\text { Small Dimension Sottwood Lumber, } \\
\text { kiln-dred }\end{array}$ & 0.7 & $\mathrm{~m} 3$ \\
\hline Solvent Based Alkyd Paint & 19.6 & $L$ \\
\hline Nater E & 132.5 & \\
\hline
\end{tabular}

Notes:
'Initial = Time 'o' (i.e. at the completion of initio

${ }^{2}$ Trans. $=$ Transportation

${ }^{3}$ Total EE (or Total GWP) = Total embodied energy (or total embodied GWP) of building component after lifespan (i.e. total manufacturing + to

${ }^{4}$ Total EE (or Total GWP) per $m^{2}=$ Total EE (or Total GWP) of building
component/ area of building component that was modelled in ATHENAQ EIE

${ }_{5}^{5}$ Total Difference in Operating Energy (or GWP) from Baseline after Lifespan = The difference in the total life-cycle operating energy (or GWP)
from the baseline retail building after lifespan, due to using this building component instead of the baseline component

Total Difference in Operating Energy (or GWP) from Baseline after Lifespan per $\mathrm{m}^{2}=$ Total difference in operating energy (or GWP) from

* Total operating primary energy use of baseline retail building after 50 years

"Total operating $G W$ Po baseline retail building after 50 years $=2,310$ tonnes
of $\mathrm{CO}_{2}$ eq. $\left(80 \mathrm{~kg}\right.$ of $\mathrm{CO}_{2}$ eq./m/ $\left./ \mathrm{yr}\right)$

Wood Stud Wall \#29 (WS-W29)

Building Component Description:

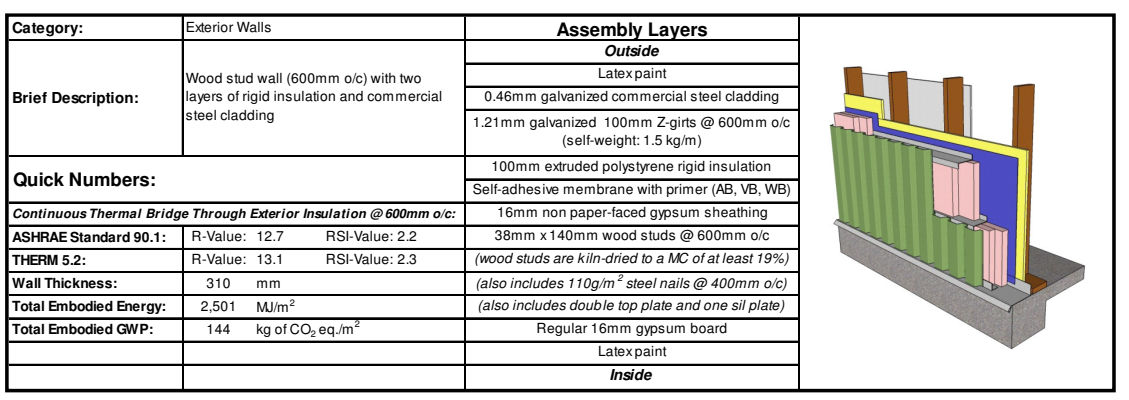

Life-Cycle Assessment Results:

Primary Energy Consumption (MJ)

\begin{tabular}{|c|c|c|c|c|c|c|c|c|c|c|c|c|c|c|c|}
\hline \multirow{3}{*}{$\begin{array}{l}\text { Lifiespan } \\
\text { (Years) }\end{array}$} & \multicolumn{13}{|c|}{ Embodied Energy (EE) } & \multirow{2}{*}{\multicolumn{2}{|c|}{$\begin{array}{c}\text { Difference in } \\
\text { Operating Energy } \\
\text { from Baseline after } \\
\text { Lifespan }\end{array}$}} \\
\hline & \multicolumn{2}{|c|}{ Manufacturing } & \multicolumn{3}{|c|}{ Construction } & \multicolumn{3}{|c|}{ Maintenance } & \multicolumn{3}{|c|}{ End of Lifie } & \multirow{2}{*}{$\begin{array}{c}{ }^{3} \text { Total } \\
\text { EE }\end{array}$} & \multirow{2}{*}{\begin{tabular}{|l|l}
${ }^{4}{ }^{T}$ I \\
EE
\end{tabular}} & & \\
\hline & 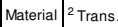 & Total & Material & |2 Trans. & Total & Material & ${ }^{2}$ Trans. & Total & Material & 1/2 Trans. & Total & & & $\begin{array}{ll}{ }^{5} \text { Total } \\
\end{array}$ & ${ }^{6}$ per m$^{2}$ \\
\hline Initial & \begin{tabular}{|l|l|}
122,833 & 301 \\
\end{tabular} & 123,133 & 209 & 714 & 923 & 0 & 0 & 0 & 0 & 0 & 0 & 124,05 & 2,436 & & \\
\hline 50 & \begin{tabular}{|l|l|}
122,833 & 301 \\
\end{tabular} & 123,133 & 209 & 714 & 923 & 3,099 & 10 & 3,110 & no & 172 & 172 & 127,33 & \begin{tabular}{|l|l|l|}
2,501 & \\
\end{tabular} & 900,000 & 1,549 \\
\hline
\end{tabular}

Global Warming Potential (kg of $\mathrm{CO}_{2}$ eq.)

\begin{tabular}{|c|c|c|c|c|c|c|c|c|c|c|c|c|c|c|c|c|}
\hline \multirow{3}{*}{$\begin{array}{l}\text { Lifespan } \\
\text { (Years) }\end{array}$} & \multicolumn{14}{|c|}{ Embodied Global Warming Potential (GWP) } & \multirow{2}{*}{\multicolumn{2}{|c|}{$\begin{array}{c}\text { Difference in } \\
\text { Operating GWP from } \\
\text { Baseline after } \\
\text { Lifespan }\end{array}$}} \\
\hline & \multicolumn{3}{|c|}{ Manutacturing } & \multicolumn{3}{|c|}{ Construction } & \multicolumn{3}{|c|}{ Maintenance } & \multicolumn{3}{|c|}{ End of Life } & \multirow{2}{*}{$\begin{array}{l}{ }^{3} \text { Total } \\
\text { GWP }\end{array}$} & \multirow{2}{*}{ 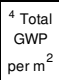 } & & \\
\hline & \begin{tabular}{|l|l|} 
Material \\
\end{tabular} & ${ }^{2}$ Trans. & $\begin{array}{l}\text { Total } \\
\end{array}$ & Material & ${ }^{2}$ Trans & $\begin{array}{l}\text { Total } \\
\end{array}$ & Material & $\begin{array}{l}{ }^{2} \text { Trans. } \\
\end{array}$ & Total & Material & $\begin{array}{l}{ }^{2} \text { Trans. } \\
\end{array}$ & \begin{tabular}{|l|l|} 
Total \\
\end{tabular} & & & \begin{tabular}{|l|}
${ }^{5}$ Total \\
\end{tabular} & ${ }^{6} \mathrm{per}$ \\
\hline \begin{tabular}{|l|l} 
Initial \\
\end{tabular} & \begin{tabular}{|l|}
7,288 \\
\end{tabular} & 0 & 7,288 & 13 & 4 & 15 & 0 & 0 & 0 & 0 & 0 & 0 & 7,303 & 143 & & \\
\hline 50 & 7,288 & 0 & 7,28 & 13 & 1 & 15 & 44 & 0 & 44 & 0 & 0 & 0 & 7 & 144 & 50,000 & \\
\hline
\end{tabular}

Embodied energy (and GWP) numbers are based on an area of wall $=\quad 50.9 \mathrm{~m}^{2} \quad$ (Length $x$ Height $=7.6 \mathrm{~m} \times 6.7 \mathrm{~m}=50.9 \mathrm{~m}^{2}$ ) Net wall area of baseline retail building (gross wall area - openings) = $581.0 \mathrm{~m}^{2}$

ATHENA $®$ EIE Material List:

\begin{tabular}{|c|c|c|}
\hline Material List & Quantities & Unit \\
\hline 3 mil Polyethylene & 54.0 & $\mathrm{~m} 2$ \\
\hline $\begin{array}{l}16 \mathrm{~mm} \text { Moisture Resistant Gypsum } \\
\text { Bard }\end{array}$ & 56.0 & $\mathrm{~m} 2$ \\
\hline $16 \mathrm{~mm}$ Regular Gypsum Board & 56.0 & $\mathrm{~m} 2$ \\
\hline Commercial $0.46 \mathrm{~mm}$ Steel Cladding & 168.0 & $\mathrm{~m} 2$ \\
\hline Extruded Polystyrene & 208.6 & $\mathrm{~m} 2(25 \mathrm{~mm})$ \\
\hline Galvanized Studs & 131.3 & $\mathrm{~kg}$ \\
\hline Joint Compound & 111.8 & $\mathrm{~kg}$ \\
\hline Modified Bitumen membrane & 68.2 & $\mathrm{~kg}$ \\
\hline Nails & 7.4 & $\mathrm{~kg}$ \\
\hline Paper Tape & 1.3 & $\mathrm{~kg}$ \\
\hline Screws Nuts \& Bolts & 1.3 & $\mathrm{~kg}$ \\
\hline $\begin{array}{l}\text { Small Dimension Softwood Lumber } \\
\text { kiln-ried }\end{array}$ & 0.7 & $\mathrm{~m} 3$ \\
\hline Solvent Based Alkyd Paint & 19.6 & $\mathrm{~L}$ \\
\hline Water Based Latex Paint & 132.5 & \\
\hline
\end{tabular}

'Initial = Time 'o' '(i.e. at the completion of :

${ }^{2}$ Trans. $=$ Transportation

Total EE (or Total GWP) = Total embodied energy (or total embodied GWP) of building component after lifespan (i.e. total man
construction + total maintenance + total end-offife effects)

${ }^{4}$ Total EE (or Total GWP) per $\mathrm{m}^{2}=$ Total EE (or Total GWP) of building
component/ area of building component that was modelled in ATHENAQ EIE Total Difference in Operating Energy (or GWP) from Baseline after

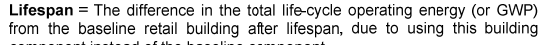
${ }^{6}$ Total Difference in Operating Energy (or GWP) from Baseline after Lifespan per $\mathrm{m}^{2}=$ Total difference in operating energy (or GWP) from
baseline after lifespan / net wall area of baseline retail building * Total operating primary energy use of baseline retail building atter 50 years $=$
$50,700 \mathrm{GJ}\left(1,745 \mathrm{MJ} / \mathrm{m}^{2} / \mathrm{rr}\right)$ * Total operating GWP of baseline retail building after 50 years $=2,310$ tonnes
of $\mathrm{CO}_{2}$ eq. $80 \mathrm{~kg}$ of $\mathrm{CO}_{2}$ eq. $\left./ \mathrm{m}^{2} \mathrm{yrr}\right)$ 
Wood Stud Wall \#30 (WS-W30)

Building Component Description:

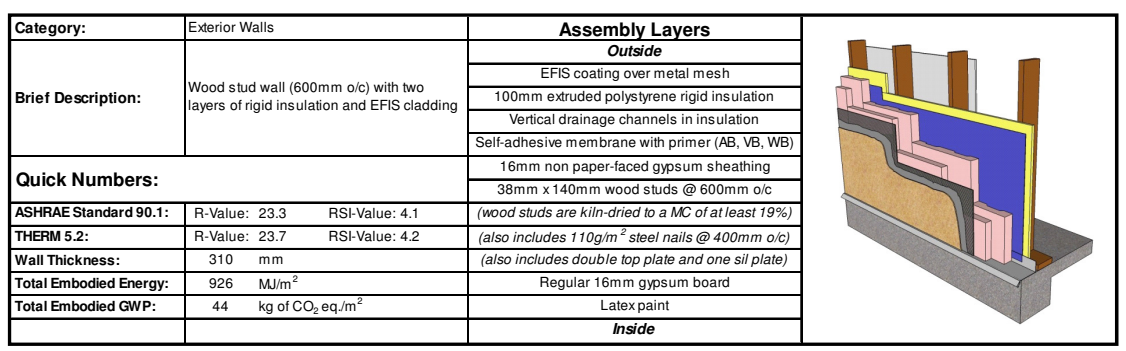

Life-Cycle Assessment Results:

Primary Energy Consumption (MJ)

\begin{tabular}{|c|c|c|c|c|c|c|c|c|c|c|c|c|c|c|c|c|}
\hline \multirow{3}{*}{$\begin{array}{l}\text { Lifespan } \\
\text { (Years) }\end{array}$} & \multicolumn{14}{|c|}{ Embodied Ene } & \multirow{2}{*}{\multicolumn{2}{|c|}{$\begin{array}{c}\text { Difference in } \\
\text { Operariting Energy } \\
\text { from Baseline afte } \\
\text { Lifespan }\end{array}$}} \\
\hline & \multicolumn{3}{|c|}{ Manutacturing } & \multicolumn{3}{|c|}{ Construction } & \multicolumn{3}{|c|}{ Maint } & \multicolumn{3}{|c|}{ End of Life } & \multirow{2}{*}{\begin{tabular}{|c|c}
${ }^{3}$ Total \\
$\mathrm{EE}$
\end{tabular}} & \multirow{2}{*}{$\begin{array}{ll}{ }^{4} \text { Total } \\
\text { EE } \\
\text { per } \mathrm{m}^{2}\end{array}$} & & \\
\hline & Materia & $11^{2}$ Trans. & \begin{tabular}{|l|} 
Total \\
\end{tabular} & Material & ${ }^{2}$ Trans. & Total & \begin{tabular}{|l|} 
Material \\
\end{tabular} & ${ }^{2}$ Trans. & \begin{tabular}{|l|} 
Total \\
\end{tabular} & Material & ${ }^{2}$ Trans. & \begin{tabular}{|l|} 
Total \\
\end{tabular} & & & 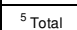 & ${ }^{6}$ perm ${ }^{2}$ \\
\hline Initial & \begin{tabular}{|l|}
42,126 \\
\end{tabular} & 355 & \begin{tabular}{|l|}
42,481 \\
\end{tabular} & tem & \begin{tabular}{|l|}
803 \\
\end{tabular} & 1,012 & $m_{3}$ & \begin{tabular}{|l}
0 \\
\end{tabular} & 0 & 0 & \begin{tabular}{|l|}
0 \\
\end{tabular} & 0 & \begin{tabular}{|l}
43,493 \\
\end{tabular} & 854 & & \\
\hline 50 & 42,126 & 355 & 42,481 & 209 & \begin{tabular}{|l|l|} 
& 803 \\
\end{tabular} & 1,012 & 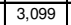 & 10 & 3,110 & 0 & 527 & \begin{tabular}{|l|l|}
527 \\
\end{tabular} & 47,130 & 926 & $-1,200,6$ & $-2,06$ \\
\hline
\end{tabular}

N

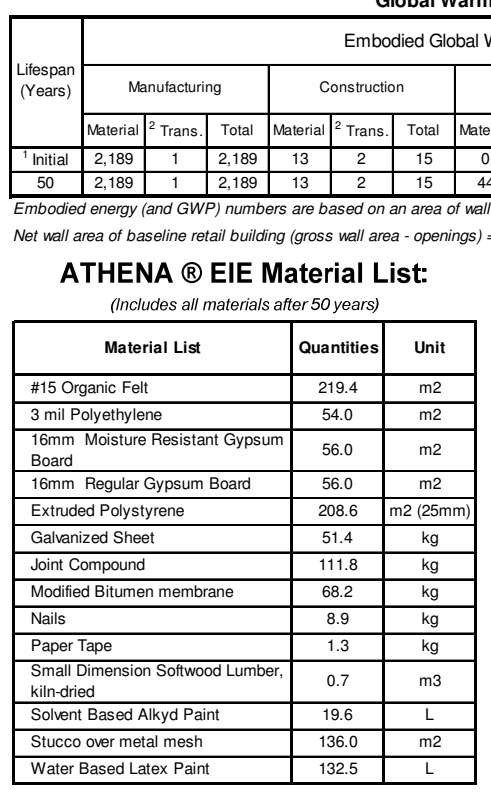

\section{LCA Data for Pre-Engineered Steel Building Exterior Walls}

This section contains a detailed description of each pre-engineered steel building (PENG) exterior infill wall that was examined in this study (4 in total). The assembly layers are listed for each wall, along with a detailed description of the material quantities from the ATHENA® Environmental Impact Estimator for Buildings.

A breakdown of the total primary energy consumption and the total global warming potential (GWP) for each wall is also included. In each case, the results were calculated for an area of wall equal to $50.9 \mathrm{~m}^{2}$, which represents a typical bay size for a single-storey retail building. The results are also expressed on a per $\mathrm{m}^{2}$ basis in each case. The data has been calculated for two different lifespans: at the completion of initial construction and after 50 years.

As a summary, the figure below illustrates a comparison of the total embodied energy (and GWP) after 50 years for the various walls in this section. For comparison purpose, the building component used in the baseline retail building has been shaded in grey.

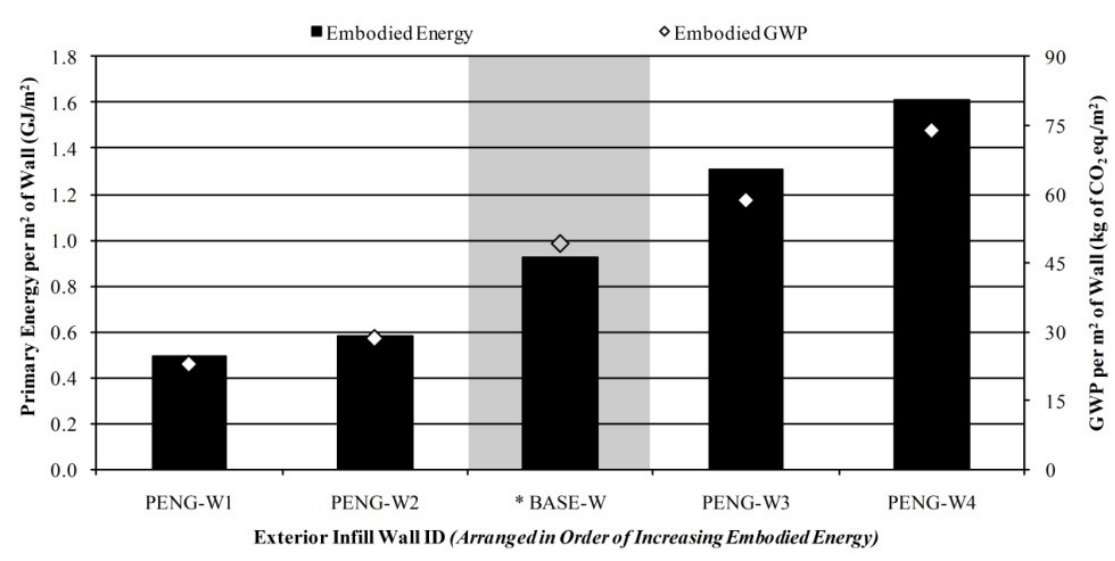




\section{Pre-Engineered Steel Building Exterior Wall \#1 (PENG-W1)}

Building Component Description:

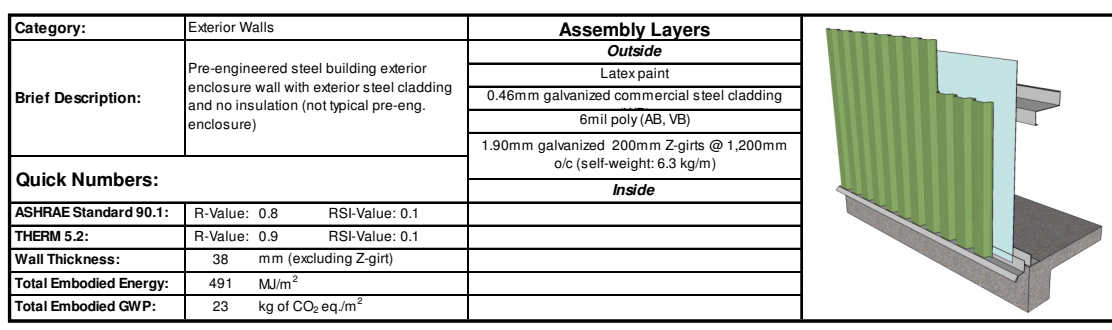

\section{Life-Cycle Assessment Results:}

Primary Energy Consumption (MJ)

\begin{tabular}{|c|c|c|c|c|c|c|c|c|c|c|c|c|c|c|c|c|}
\hline \multirow{3}{*}{\begin{tabular}{|l} 
Lifiespan \\
(Years)
\end{tabular}} & \multicolumn{14}{|c|}{ Embodied Energy (EE) } & \multirow{2}{*}{\multicolumn{2}{|c|}{$\begin{array}{c}\text { Difference in } \\
\text { Operating Energy } \\
\text { from Baseline afte } \\
\text { Lifespan }\end{array}$}} \\
\hline & \multicolumn{3}{|c|}{ Manufacturing } & \multicolumn{3}{|c|}{ Construction } & \multicolumn{3}{|c|}{ Maintenance } & \multicolumn{3}{|c|}{ End of Life } & \multirow{2}{*}{${ }^{3}$ Total } & \multirow{2}{*}{$\begin{array}{l}{ }^{4} \text { Total al } \\
\text { EE } \\
\text { per } \mathrm{m}^{2}\end{array}$} & & \\
\hline & Material & 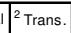 & \begin{tabular}{|l|} 
Total \\
\end{tabular} & \begin{tabular}{|l|} 
Materia| \\
\end{tabular} & \begin{tabular}{|l|}
${ }^{2}$ Trans. \\
\end{tabular} & Total & Material & \begin{tabular}{|l|}
${ }^{2}$ Trans. \\
\end{tabular} & \begin{tabular}{|l|l|} 
Total \\
\end{tabular} & Material & ||$^{2}$ Trans. & \begin{tabular}{|l|l|} 
Total \\
\end{tabular} & & & ${ }^{5}$ Total & ${ }^{6}$ per $\mathrm{m}^{2}$ \\
\hline \begin{tabular}{|l|l} 
nnitial \\
.
\end{tabular} & 24,579 & 35 & 24,614 & 160 & 64 & 223 & 0 & 0 & 0 & 0 & 0 & 0 & $\begin{array}{l}24,837 \\
\end{array}$ & 488 & & \\
\hline 50 & 24,579 & 35 & 24,61 & 160 & 64 & 223 & 161 & 1 & 162 & 0 & 22 & 22 & 25,021 & 491 & N/A & ${ }^{\circ} \mathrm{N} / \mathrm{A}$ \\
\hline
\end{tabular}

Global Warming Potential ( $\mathrm{kg}$ of $\mathrm{CO}_{2}$ eq.)

\begin{tabular}{|c|c|c|c|c|c|c|c|c|c|c|c|c|c|c|c|c|}
\hline \multirow{3}{*}{$\begin{array}{c}\text { Lifespana } \\
\text { (Years) }\end{array}$} & \multicolumn{14}{|c|}{ Embodied Global Warming Potential (GWP) } & \multirow{2}{*}{\multicolumn{2}{|c|}{$\begin{array}{c}\text { Difference in } \\
\text { Operating GWP from } \\
\text { Baseline after } \\
\text { Lifespan }\end{array}$}} \\
\hline & \multicolumn{3}{|c|}{ Manutacturing } & \multicolumn{3}{|c|}{ Construction } & \multicolumn{3}{|c|}{ Maintenance } & \multicolumn{3}{|c|}{ End of Life } & \multirow{2}{*}{$\begin{array}{l}{ }^{3} \text { Total } \\
\text { GWP }\end{array}$} & \multirow{2}{*}{$\begin{array}{l}{ }^{4}{ }^{\text {Total }} \\
\text { GWP } \\
\text { per m }\end{array}$} & & \\
\hline & Material & ${ }^{2}$ Trans. & Total & Material & ${ }^{2}$ Trans. & Total & Material & ${ }^{2}$ Trans. & Total & Material & 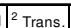 & Total & & & ${ }^{5}$ Total & $6{ }^{6}$ perm $^{2}$ \\
\hline${ }^{1}$ Initial & 1,154 & 0 & 1,154 & 10 & 0 & 11 & 0 & 0 & 0 & 0 & 0 & 0 & 1,165 & 23 & & \\
\hline 50 & 1,154 & 0 & 1,154 & 10 & 0 & 11 & 3 & 0 & 3 & 0 & 0 & 0 & 1,168 & 23 & ${ }^{*} \mathrm{~N} / \mathrm{A}$ & ${ }^{*} \mathrm{~N} / \mathrm{A}$ \\
\hline
\end{tabular}

Net wall area of baseline retail building (gross wall area - openings) = 581.0

(acturate evaluation of operating energy from computer simulations

\section{ATHENA $®$ EIE Material List:} (Includes all materials after 50 years)

\begin{tabular}{|l|c|c|}
\hline \multicolumn{1}{|c|}{ Material List } & Quantities & Unit \\
\hline 6 mil Polyethylene & 54.0 & $\mathrm{~m} 2$ \\
\hline Galvanized Sheet & 264.6 & $\mathrm{~kg}$ \\
\hline Galvanized Studs & 287.9 & $\mathrm{~kg}$ \\
\hline Screws Nuts \& Bolts & 2.6 & $\mathrm{~kg}$ \\
\hline Water Based Latex Paint & 6.7 & $\mathrm{~L}$ \\
\hline
\end{tabular}

Notes:

'Initial = Time ' 0 ' (i.e. at the completion of initial construction)

${ }^{2}$ Trans. $=$ Transportation

Total EE (or Total GWP) = Total embodied energy (or total embodied GWP) of building component after lifespan (i.e. total man
construction + total maintenance + total end-oflife effects)

${ }^{4}$ Total EE (or Total GWP) per $\mathrm{m}^{2}=$ Total EE (or Total GWP) of building
component/ area of building component that was modelled in ATHENAQ EIE Total Difference in Operating Energy (or GWP) from Baseline after rencerat (or GWP from the baseline retail bullining after lifescent
component instead of the baseline component

Total Difference in Operating Energy (or GWP) from Baseline after Lifespan per $\mathrm{m}^{2}=$ Total difference in operating energy (or GWP) fro

"Total operating primary energy use of baseline retail building after 50 years
$50,700 \mathrm{GJ}\left(1.745 \mathrm{MJ} / \mathrm{m}^{2} \mathrm{yr}\right)$

"Total operating GWP of baseline re

\section{Pre-Engineered Steel Building Exterior Wall \#2 (PENG-W2)} Building Component Description:

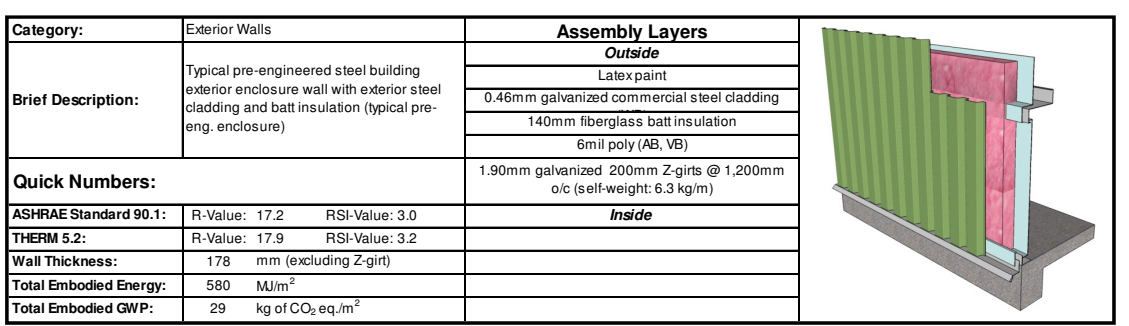

Life-Cycle Assessment Results:

Primary Energy Consumption (MJ)

\begin{tabular}{|c|c|c|c|c|c|c|c|c|c|c|c|c|c|c|c|c|}
\hline \multirow{3}{*}{$\begin{array}{l}\text { Lifespan } \\
\text { (Years) }\end{array}$} & \multicolumn{14}{|c|}{ Embodied Energy (EE) } & \multirow{2}{*}{\multicolumn{2}{|c|}{$\begin{array}{l}\text { Difference in } \\
\text { Operating Energy } \\
\text { from Baseline after } \\
\text { Lifespan }\end{array}$}} \\
\hline & \multicolumn{3}{|c|}{ Manufacturing } & \multicolumn{3}{|c|}{ Construction } & \multicolumn{3}{|c|}{ Mainte } & \multicolumn{3}{|c|}{ End of Life } & \multirow{2}{*}{$\begin{array}{c}{ }^{3} \text { Total } \\
\text { EE }\end{array}$} & \multirow{2}{*}{\begin{tabular}{|c|}
${ }^{4}$ Total \\
EE \\
per $m^{2}$
\end{tabular}} & & \\
\hline & Material & ${ }^{2}$ Trans & \begin{tabular}{|l|l|} 
Total \\
\end{tabular} & Material & ${ }^{2}$ Trans. & Total & Material & ${ }^{2}$ Trans. & Total & \begin{tabular}{|l|} 
Material \\
\end{tabular} & 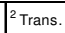 & Total & & & ${ }^{5}$ Total & ${ }^{6}$ per m ${ }^{2}$ \\
\hline Initial & 29,031 & 62 & 29,093 & 160 & 81 & 241 & 0 & 0 & 0 & 0 & 0 & 0 & 29,333 & 576 & & \\
\hline 50 & 29,031 & 62 & 29,093 & 160 & 81 & 241 & 161 & 1 & 162 & 0 & 36 & 36 & 29,531 & 580 & $-600,000$ & 1,03 \\
\hline
\end{tabular}

Global Warming Potential (kg of $\mathrm{CO}_{2}$ eq.)

\begin{tabular}{|c|c|c|c|c|c|c|c|c|c|c|c|c|c|c|c|c|}
\hline \multirow{3}{*}{$\begin{array}{l}\text { Lifespan } \\
\text { (Years) }\end{array}$} & \multicolumn{14}{|c|}{ Embodied Global Warming Potential (GWP) } & \multirow{2}{*}{\multicolumn{2}{|c|}{$\begin{array}{l}\text { Difference in } \\
\text { Operating GWP from } \\
\text { Baseline after } \\
\text { Lifespan }\end{array}$}} \\
\hline & \multicolumn{3}{|c|}{ Manutacturing } & \multicolumn{3}{|c|}{ Construction } & \multicolumn{3}{|c|}{ Maintenance } & \multicolumn{3}{|c|}{ End of Lifie } & \multirow{2}{*}{$\begin{array}{l}{ }^{3} \text { Total } \\
\text { GWP }\end{array}$} & \multirow{2}{*}{$\begin{array}{c}{ }^{4} \text { Total } \\
\text { GWP } \\
\text { per m }\end{array}$} & & \\
\hline & Material & ${ }^{2}$ Trans. & Total & Material & ${ }^{2}$ Trans. & Total & Material & Trans. & Total & Material & 2 Trans. & Total & & & ${ }^{5}$ Total & ${ }^{6} \mathrm{perm}^{2}$ \\
\hline Initial & 1,439 & 0 & 1,439 & 10 & 0 & 11 & 0 & 0 & 0 & 0 & 0 & 0 & 1,450 & 28 & & \\
\hline 50 & 1,439 & 0 & 1,439 & 10 & 0 & 11 & 3 & 0 & 3 & 0 & 0 & 0 & 1,453 & 29 & 30,000 & -52 \\
\hline
\end{tabular}

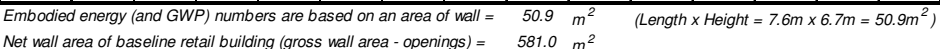

ATHENA ® EIE Material List:

\begin{tabular}{|l|c|c|}
\hline \multicolumn{1}{|c|}{ Material List } & Quantities & Unit \\
\hline 6 mil Polyethylene & 54.0 & $\mathrm{~m} 2$ \\
\hline Batt. Fiberglass & 289.8 & $\mathrm{~m} 2(25 \mathrm{~mm})$ \\
\hline Galvanized Sheet & 264.6 & $\mathrm{~kg}$ \\
\hline Galvanized Studs & 287.9 & $\mathrm{~kg}$ \\
\hline Nails & 3.1 & $\mathrm{~kg}$ \\
\hline Screws Nuts \& Bolts & 2.6 & $\mathrm{~kg}$ \\
\hline Water Based Latex Paint & 6.7 & $\mathrm{~L}$ \\
\hline
\end{tabular}

Notes:
1 Initial $=$ Time ' 0 ' (i.e. at the completion of initial construction)

${ }^{2}$ Trans. $=$ Transportation

${ }^{3}$ Total EE (or Total GWP) $=$ Total embodied energy (or total embodied GWP of building component after lifespan (i.e. total manufacturing + tota
construction + total maintenance + total enc-oflife effects)

Total EE (or Total GWP) per $\mathrm{m}^{2}=$ Total EE (or Total GWP) of building
component/ area of building component that was modelled in ATHENAQ EIE ${ }^{5}$ Total Difference in Operating Energy (or GWP) from Baseline after Lifespan = The difference in the total life-cycle operating energy (or GWP
from the baseline retail building after lifespan, due to using this building
component instead of the baseline component ${ }^{6}$ Total Difference in Operating Energy (or GWP) from Baseline after Lifespan per $\mathrm{m}^{2}=$ Total difference in operating energy (or GWP) from * Total operating primarn energy use of baseline retail building after 50 years =
$50,700 \mathrm{GJ}\left(1.745 \mathrm{MJ} / \mathrm{m}^{2} \mathrm{ND}\right)$

"Total operating GWP of baseline ret
of $\mathrm{CO}_{2}$ eq. $\left(80 \mathrm{~kg}\right.$ of $\mathrm{CO}_{2}$ eq. $\left./ \mathrm{m}^{2} \mathrm{yr}\right)$ 
Pre-Engineered Steel Building Exterior Wall \#3 (PENG-W3) Building Component Description:

\begin{tabular}{|c|c|c|c|}
\hline Category: & Exxerior Walls & Assembly Layers & \\
\hline & & $\begin{array}{l}\text { Outside } \\
\text { Latexpaint }\end{array}$ & \multirow{9}{*}{$\begin{array}{l}\text { Rendering \& R-value courtesy of on } \\
\text { BEHLENIndustries LP } \\
\text { http:L//www.behen.cal }\end{array}$} \\
\hline Brief Description: & 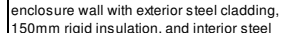 & $0.46 \mathrm{~mm}$ galvanized commercial steel cladding & \\
\hline & & 150mm extruded polystyrene rigid insulation & \\
\hline & channels @ $1,200 \mathrm{~mm}$ o/c (self-weight $0.82 \mathrm{~kg} / \mathrm{m}$ ) & \\
\hline Quick Numbers: & & $0.46 \mathrm{~mm}$ galvanized commercial steel cladding & \\
\hline 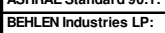 & 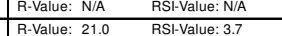 & $\begin{array}{c}\text { (AB, VB) } \\
\text { Latexpaint } \\
\end{array}$ & \\
\hline Wall Thickness: & $226 \mathrm{~mm}$ (excluding Z-girt) & 1.90mm galvanized $200 \mathrm{~mm}$ Z-girts @ 1. & \\
\hline Total Embodied Energy: & $\begin{array}{ll}1.312 \mathrm{M} / \mathrm{m}^{2} \\
\end{array}$ & 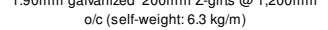 & \\
\hline Total Embodied GWP: & $59 \mathrm{~kg} \mathrm{of} \mathrm{CO}_{2} \mathrm{eq} / \mathrm{m}^{2}$ & Inside & \\
\hline
\end{tabular}

Life-Cycle Assessment Results:

\begin{tabular}{|c|c|c|c|c|c|c|c|c|c|c|c|c|c|c|c|c|}
\hline \multirow{4}{*}{$\begin{array}{c}\text { Lifespan } \\
\text { Years }\end{array}$} & \multicolumn{14}{|c|}{ Primary } & \multirow{3}{*}{\multicolumn{2}{|c|}{$\begin{array}{c}\text { Difference in } \\
\text { Operating Energy } \\
\text { from Baseline atter } \\
\text { Lifespan }\end{array}$}} \\
\hline & \multicolumn{14}{|c|}{ Embodied Energy (EE) } & & \\
\hline & \multicolumn{3}{|c|}{ Manutacturing } & \multicolumn{3}{|c|}{ Construction } & \multicolumn{3}{|c|}{ Maintenance } & \multicolumn{3}{|c|}{ End of Life } & \multirow{2}{*}{$\begin{array}{c}{ }^{3} \text { Total } \\
\mathrm{EE}\end{array}$} & \multirow{2}{*}{\begin{tabular}{|c|}
${ }^{4}$ Total \\
$E E$ \\
per m
\end{tabular}} & & \\
\hline & \begin{tabular}{|l|l|l|l|} 
Matrial \\
\end{tabular} & ${ }^{2}$ Trans. & Total & Material & ${ }^{2}$ Trans & Total & Material & ${ }^{2}$ Trans & Total & Material & ${ }_{2}^{2}$ Trans & Total & & & ${ }^{5}{ }^{5}$ otal & ${ }^{6} \mathrm{perm}^{2}$ \\
\hline 1 Initial & 66,041 & 64 & 66,105 & 160 & 134 & 294 & 0 & 0 & 0 & 0 & 0 & 0 & 66,399 & 1,304 & & \\
\hline 50 & 66,041 & 64 & 66,105 & 160 & 134 & 294 & 321 & 3 & 324 & 0 & 62 & 62 & 66,785 & 1,312 & $-900,000$ & $-1,549$ \\
\hline
\end{tabular}

Global Warming Potential ( $\mathrm{kg}$ of $\mathrm{CO}_{2}$ eq.)

$\stackrel{\omega}{\omega}$

\begin{tabular}{|c|c|c|c|c|c|c|c|}
\hline \multirow{3}{*}{$\begin{array}{l}\text {-ifespan } \\
\text { (Years) }\end{array}$} & \multicolumn{7}{|c|}{ Embodied Global $\mathrm{V}$} \\
\hline & \multicolumn{3}{|c|}{ Manufacturing } & \multicolumn{3}{|c|}{ Construction } & \\
\hline & \begin{tabular}{|l|l|} 
Material \\
\end{tabular} & ${ }^{2}$ Trans. & \begin{tabular}{|l|l|l|} 
Total \\
\end{tabular} & Materia & \begin{tabular}{l|l|}
${ }^{2}$ Trans. \\
\end{tabular} & \begin{tabular}{|l|l|l|} 
Total \\
\end{tabular} & \\
\hline Initial & 2,970 & 0 & 2,971 & 10 & 0 & 11 & \\
\hline 50 & \begin{tabular}{|l|l|}
2,970 \\
\end{tabular} & 0 & 2,971 & 10 & 0 & 11 & \\
\hline \multicolumn{8}{|c|}{$\begin{array}{l}\text { Embodied energy (and GWP) numbers are based on an area of wall } \\
\text { Net wall area of baseline retail building (gross wall area - openings) }\end{array}$} \\
\hline \multicolumn{8}{|c|}{$\begin{array}{c}\text { ATHENA @ EIE Material List: } \\
\text { (Includes all materials after } 50 \text { years) }\end{array}$} \\
\hline \multicolumn{4}{|c|}{ Material List } & \multicolumn{2}{|c|}{ Quantities } & \multicolumn{2}{|l|}{ Unit } \\
\hline \multicolumn{4}{|c|}{ Extruded Polystyrene } & \multicolumn{2}{|r|}{313.0} & \multicolumn{2}{|c|}{ m2 (25mm) } \\
\hline \multicolumn{4}{|c|}{ Galvanized Sheet } & \multicolumn{2}{|r|}{55.6} & \multirow{2}{*}{\multicolumn{2}{|c|}{$\mathrm{kg}$}} \\
\hline \multirow{2}{*}{\multicolumn{4}{|c|}{ Galvanized Studs }} & \multicolumn{2}{|r|}{287.9} & & \\
\hline & & & & \multicolumn{2}{|r|}{3.1} & \multicolumn{2}{|l|}{$\mathrm{kg}$} \\
\hline \multicolumn{4}{|c|}{ Screws Nuts \& Bolts } & & 2.6 & $\mathrm{~kg}$ & \\
\hline \multicolumn{4}{|c|}{$\begin{array}{l}\text { ccrews Nuts \& Bolts } \\
\end{array}$} & & 13.5 & L & \\
\hline
\end{tabular}

${ }^{6}$ Total Difference in Operating Energy (or GWP) from Baseline after Lifespan per $m^{2}=$ Total difference in operating energy (or GWP) from * Total operating primary energy use of baseline retail building after 50 years

Notes:

Total EE (or Total GWP) $=$ Total embodied energy (or total embodied GWP)
of building component after lifespan (i.e. total manufacturing + tot 作

Total EE (or Total GWP) per $\mathrm{m}^{2}=$ Total EE (or Total GWP) of building
Total operating $\mathrm{GWP}$ of baseline retail building after 50 years $=2,310$ tonnes

\section{Pre-Engineered Steel Building Exterior Wall \#4 (PENG-W4)} Building Component Description:

\begin{tabular}{|c|c|c|c|}
\hline \multirow{4}{*}{ Brief Description: } & \multirow{3}{*}{ 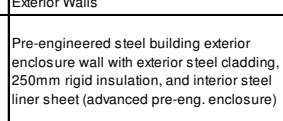 } & $\begin{array}{l}\text { Assembly Layers } \\
\text { outside }\end{array}$ & \\
\hline & & $\begin{array}{c}\text { Outside } \\
\text { Latexpint } \\
0.46 \mathrm{~mm} \text { galvaninged commerci }\end{array}$ & \multirow{9}{*}{$\begin{array}{l}\text { Rendering \& R-value courtesy of } \\
\text { BEHENEN Industries LP } \\
\text { http://www.behlen.ca/ }\end{array}$} \\
\hline & & 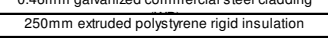 & \\
\hline & & 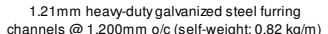 & \\
\hline \multicolumn{2}{|l|}{ Quick Numbers: } & $0.46 \mathrm{~mm}$ galvanized comm ercial Iteel cladding & \\
\hline ASHRAE Standard 90.1: & RSIVValue: NA & $(A B, v B)$ & \\
\hline BEHLENIndustries LP: & R-Value: $35.0 \quad$ RSI-Value: 6.2 & Latexpaint & \\
\hline Wall Thickness: & $326 \quad \mathrm{~mm}$ (excluding Z-git) & 1.90mm galvanized 200mm Z-girts @ 1,200mm & \\
\hline Total Embodied Energy: & $1,610 \mathrm{~m} / \mathrm{m}^{2}$ & ol/c (self-weight $6.3 \mathrm{~kg} / \mathrm{m}$ ) & \\
\hline Total Embodied GWP: & $74 \quad \mathrm{~kg}$ of $\mathrm{CO}_{2} \mathrm{eq} / \mathrm{m}^{2}$ & Inside & \\
\hline
\end{tabular}

Life-Cycle Assessment Results:

Primary Energy Consumption (MJ)

\begin{tabular}{|c|c|c|c|c|c|c|c|c|c|c|c|c|c|c|c|c|}
\hline \multirow{3}{*}{\begin{tabular}{|l} 
Lifespan \\
(vears)
\end{tabular}} & \multicolumn{14}{|c|}{ Embodied Energy (EE) } & \multirow{2}{*}{\multicolumn{2}{|c|}{$\begin{array}{c}\text { Difference in } \\
\text { Operating Energy } \\
\text { from Baseline after } \\
\text { Lifespan }\end{array}$}} \\
\hline & \multicolumn{3}{|c|}{ Manufacturing } & \multicolumn{3}{|c|}{ Construction } & \multicolumn{3}{|c|}{ Maintenance } & \multicolumn{3}{|c|}{ End of Life } & \multirow{2}{*}{$\begin{array}{c}{ }^{3}{ }^{3} \text { Total } \\
\mathrm{EE}\end{array}$} & \multirow{2}{*}{$\begin{array}{l}{ }^{4} \text { Total } \\
\mathrm{EE} \\
\mathrm{per}^{2}\end{array}$} & & \\
\hline & Material & ${ }^{2}$ Trans. & Total & Material & ${ }^{2}$ Trans. & Total & Material & ${ }^{2}$ Trans. & Total & \begin{tabular}{|l|} 
Material \\
\end{tabular} & \begin{tabular}{|l|}
${ }^{2}$ Trans. \\
\end{tabular} & Total & & & ${ }^{5}$ Total & ${ }^{6}{ }^{6}$ er m ${ }^{2}$ \\
\hline 1 Initial & 81,208 & 64 & 81,272 & 160 & 158 & 318 & 0 & 0 & 0 & 0 & 0 & 0 & 81,590 & \begin{tabular}{|l|l|}
1,602 \\
\end{tabular} & & \\
\hline 50 & 81,208 & 64 & 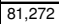 & 160 & 158 & 318 & 321 & 3 & 324 & 0 & 81 & 82 & 81,996 & 1,610 & 000,0 & 3,44 \\
\hline
\end{tabular}

Global Warming Potential (kg of $\mathrm{CO}_{2}$ eq.)

\begin{tabular}{|c|c|c|c|c|c|c|c|c|c|c|c|c|c|c|c|c|}
\hline \multirow{3}{*}{$\begin{array}{l}\text { Litespar } \\
\text { (Years) }\end{array}$} & \multicolumn{14}{|c|}{ Embodied Global Warming Potential (GWP) } & \multirow{2}{*}{\multicolumn{2}{|c|}{\begin{tabular}{|c|} 
Difference in \\
Operating GWP from \\
Baseline atter \\
Lifespan
\end{tabular}}} \\
\hline & \multicolumn{3}{|c|}{ Manufacturing } & \multicolumn{3}{|c|}{ Construction } & \multicolumn{3}{|c|}{ Maintenance } & \multicolumn{3}{|c|}{ End of Life } & \multirow{2}{*}{$\begin{array}{l}{ }^{3} \text { Total } \\
\text { GWP }\end{array}$} & \multirow{2}{*}{$\begin{array}{l}{ }^{4} \text { Total } \\
\text { GiWP } \\
\text { per m }\end{array}$} & & \\
\hline & Material & ${ }^{2}$ Trans. & \begin{tabular}{|l|l|} 
Total \\
\end{tabular} & Material & 2 Trans. & Total & \begin{tabular}{|l|} 
Material \\
\end{tabular} & \begin{tabular}{|l|l|}
${ }^{2}$ Trans. \\
\end{tabular} & Total & \begin{tabular}{|l|} 
Material \\
\end{tabular} & \begin{tabular}{l|l}
$2^{2}$ Trans. \\
.
\end{tabular} & Total & & & ${ }^{5}$ Total & ${ }^{6} \operatorname{perm}^{2}$ \\
\hline${ }^{1}$ Initial & \begin{tabular}{|l|}
3,746 \\
\end{tabular} & 0 & \begin{tabular}{|l|l|}
3,746 \\
\end{tabular} & 10 & 0 & 11 & 0 & 0 & 0 & 0 & 0 & 0 & \begin{tabular}{|l|l|}
3,757 \\
\end{tabular} & 74 & & \\
\hline 50 & 3,746 & 0 & $\begin{array}{l}3,746 \\
\end{array}$ & 10 & 0 & 11 & 6 & 0 & 6 & 0 & 0 & 0 & \begin{tabular}{|l|}
3,763 \\
\end{tabular} & 74 & $-110,000$ & -189 \\
\hline
\end{tabular}
Net wall area of tosedine retil building (oross wall area - openings) $=581.0 \mathrm{~m}^{2}$

$\begin{gathered}\text { ATHENA } \\
\text { (Includes all materials after 50 years) }\end{gathered}$
\begin{tabular}{|l|c|c|}
\multicolumn{1}{|c|}{ Material List } & Quantities & Unit \\
\hline Extruded Polystyrene & 521.6 & $\mathrm{~m} 2(25 \mathrm{~mm})$ \\
\hline Galvanized Sheet & 565.6 & $\mathrm{~kg}$ \\
\hline Galvanized Studs & 287.9 & $\mathrm{~kg}$ \\
\hline Nails & 3.1 & $\mathrm{~kg}$ \\
\hline Screws Nuts \& Bolts & 2.6 & $\mathrm{~kg}$ \\
\hline Water Based Latex Paint & 13.5 & $\mathrm{~L}$ \\
\hline
\end{tabular}

Notes:
${ }^{1}$ Initial $=$ Time ' 0 ' (i.e. at the completion of initial construction)

Water Based Latex Pain

1 Initial $=$ Time ' $O$ ' (i.e. at
${ }^{2}$ Trans. $=$ Transportation

${ }^{3}$ Total EE (or Total GWP) $=$ Total embodied energy (or total embodied GWP of building component after lifespan (i.e. total man
construction + total maintenance + total end-of life effects)

${ }^{4}$ Total EE (or Total GWP) per $\mathrm{m}^{2}=$ Total EE (or Total GWP) of building
component / area of building component that was modelled in ATHENAQ EIE ${ }^{5}$ Total Difference in Operating Energy (or GWP) from Baseline after Lifespan = The difference in the total lite-cycle operating energy (or GWP
from the baseline retail building after lifespan, due to using this building component instead of the baseline component

${ }^{6}$ Total Difference in Operating Energy (or GWP) from Baseline after Lifespan per $\mathrm{m}^{2}=$ Total difference in operating energy (or GWP) from * Total operating primary energy use of baseline retail building after 50 years =

"Total operating GWP of baseline ret
of $\mathrm{CO}_{2}$ eq. $\left(80 \mathrm{~kg}\right.$ of $\mathrm{CO}_{2}$ eq. $\left./ \mathrm{m}^{2} \mathrm{yr}\right)$ 


\section{LCA Data for Opaque Curtainwall Enclosures}

This section contains a detailed description of each opaque curtainwall enclosure (CWALL) that was examined in this study (4 in total). The assembly layers are listed for each wall, along with a detailed description of the material quantities from the ATHENA® Environmental Impact Estimator for Buildings.

A breakdown of the total primary energy consumption and the total global warming potential (GWP) for each wall is also included. In each case, the results were calculated for an area of wall equal to $50.9 \mathrm{~m}^{2}$, which represents a typical bay size for a single-storey retail building. The results are also expressed on a per $\mathrm{m}^{2}$ basis in each case. The data has been calculated for two different lifespans: at the completion of initial construction and after 50 years.

As a summary, the figure below illustrates a comparison of the total embodied energy (and GWP) after 50 years for the various walls in this section. For comparison purpose, the building component used in the baseline retail building has been shaded in grey.

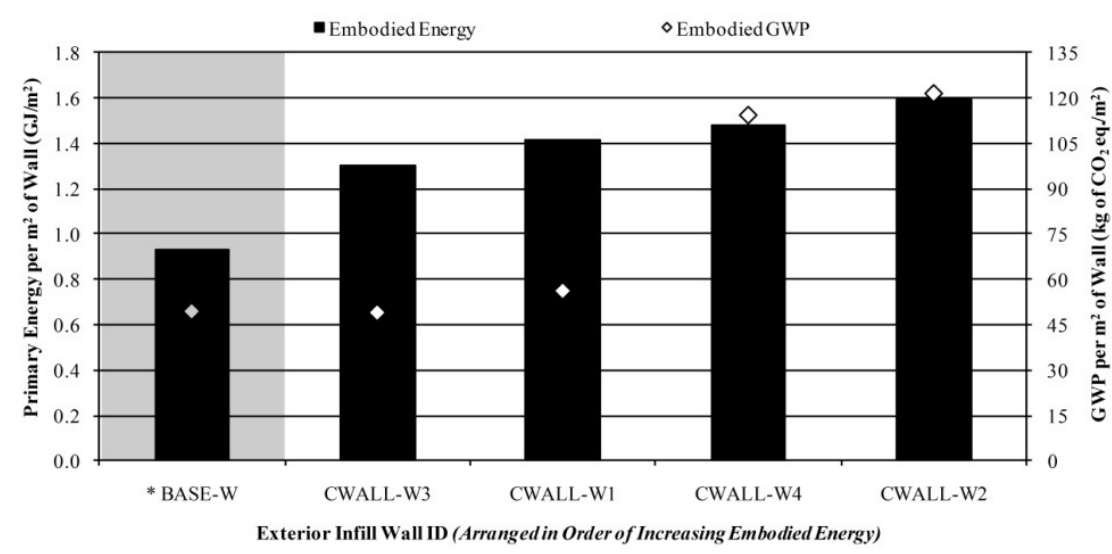

Opaque Curtainwall Enclosure \#1 (CWALL-W1)

Building Component Description:

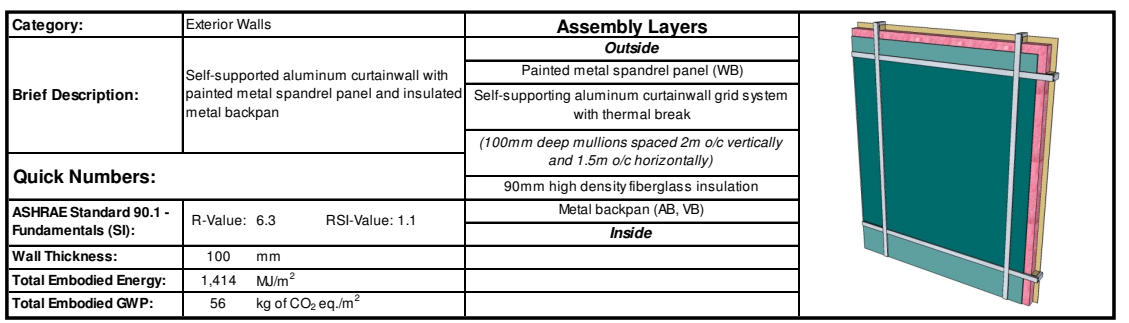

Life-Cycle Assessment Results:

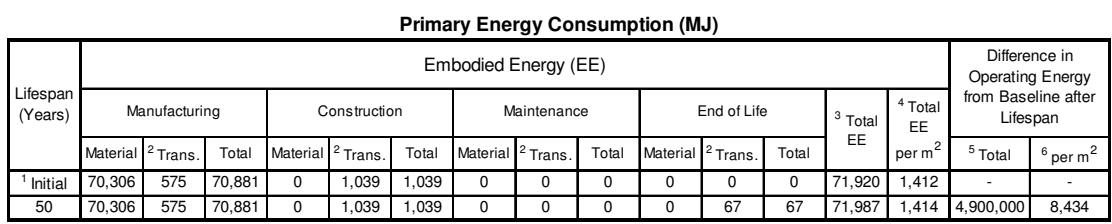

Global Warming Potential (kg of $\mathrm{CO}_{2}$ eq.)

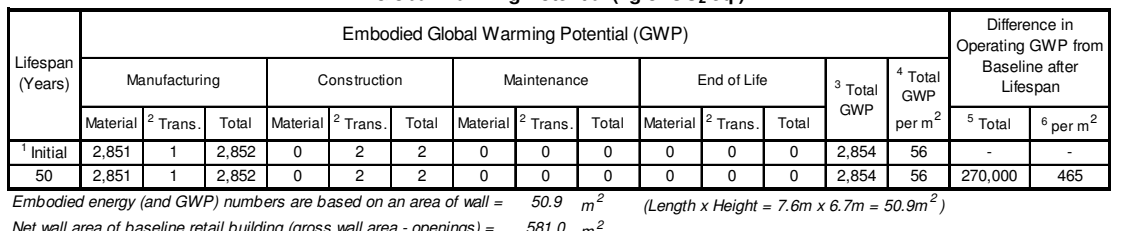

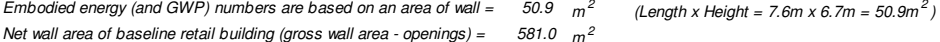

ATHENA $®$ EIE Material List: (Includes all materials after 50 years)

\begin{tabular}{|l|c|c|}
\hline \multicolumn{1}{|c|}{ Material Liales all materials after 50 years) } \\
\hline Aluminum & Quantities & Unit \\
\hline Batt. Fiberglass & 850.4 & $\mathrm{~kg}$ \\
\hline EPDM membrane & 378.9 & $\mathrm{~m} 2(25 \mathrm{~mm})$ \\
\hline Galvanized Sheet & 36.4 & $\mathrm{~kg}$ \\
\hline Screws Nuts \& Bolts & 346.6 & $\mathrm{~kg}$ \\
\hline
\end{tabular}

Notes:
${ }^{1}$ Initial $=$ Time 'o' (i.e. at the completion of initial construction)

${ }^{2}$ Trans. $=$ Transportation

${ }^{3}$ Total EE (or Total GWP) $=$ Total embodied energy (or total embodied GWP of building component after lifespan (i.e. total mat
construction + total maintenance + total end-ot-life effects)

${ }^{4}$ Total EE (or Total GWP) per $\mathrm{m}^{2}=$ Total EE (or Total GWP) of building
component / area of building component that was modelled in ATHENAB EIE ${ }_{5}^{5}$ Total Difference in Operating Energy (or GWP) from Baseline after from the baseline retail building after lifespan, due to using this building component instead of the baseline componen

${ }^{6}$ Total Difference in Operating Energy (or GWP) from Baseline after Lifespan per $\mathrm{m}^{2}=$ Total difference in operating energy (or GWP) from

* Total operating primary energy use of baseline retail building after 50 years =

of $\mathrm{CO}_{2}$ eq. $\left(80 \mathrm{~kg}\right.$ of $\mathrm{CO}_{2}$ eq. $\left./ \mathrm{m}^{2} \mathrm{yr}\right)$ 


\section{Opaque Curtainwall Enclosure \#2 (CWALL-W2)}

Building Component Description:

\begin{tabular}{|c|c|c|c|}
\hline Category: & Exterior Walls & Assembly Layers & $\overline{E B}$ \\
\hline \multirow[b]{2}{*}{ Brief Description: } & \multirow{2}{*}{$\begin{array}{l}\text { Sell-supported aluminum curtainwall with } \\
\text { opaquug glazing spandrel panel and } \\
\text { insulatelad metal backpan }\end{array}$} & $\begin{array}{l}\text { Ousside } \\
\text { Opaque glazing spandrel panel (WB) }\end{array}$ & \\
\hline & & $\begin{array}{l}\text { (one pane of } 6 \text { mmm glazing) } \\
\text { Sell-supporting aluminum uutainwall grid system } \\
\text { with thermal break }\end{array}$ & \\
\hline \multicolumn{2}{|l|}{ Quick Numbers: } & 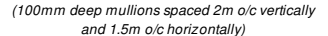 & \\
\hline $\begin{array}{l}\text { ASHAAE Standard 90.1- } \\
\text { Fundamentals (s): }\end{array}$ & R-Value: 6.3 & $\begin{array}{l}90 \mathrm{~mm} \text { high density fiberglass insulation } \\
\text { Metal backpan (AB, vB) }\end{array}$ & \\
\hline Wall Thickness: & $100 \mathrm{~mm}$ & Inside & \\
\hline Total Embodied Energy: & $1,590 \quad \mathrm{~m} / \mathrm{m}^{2}$ & & \\
\hline Total Embodied GWP: & $122 \mathrm{~kg}$ of $\mathrm{CO}_{2} \mathrm{eq} / \mathrm{m}^{2}$ & & \\
\hline
\end{tabular}

\section{Life-Cycle Assessment Results:}

Primary Energy Consumption (MJ)

\begin{tabular}{|c|c|c|c|c|c|c|c|c|c|c|c|c|c|c|c|c|}
\hline \multirow{4}{*}{$\begin{array}{l}\text { Lifespan } \\
\text { (Years) }\end{array}$} & \multirow{2}{*}{\multicolumn{14}{|c|}{ Embodied Energy (EE) }} & \multirow{3}{*}{\multicolumn{2}{|c|}{$\begin{array}{c}\text { Difference in } \\
\text { Operating Energy } \\
\text { from Baseline after } \\
\text { Lifespan }\end{array}$}} \\
\hline & & & & & & & & & & & & & & & & \\
\hline & \multicolumn{3}{|c|}{ Manufacturing } & \multicolumn{3}{|c|}{ Construction } & \multicolumn{3}{|c|}{ Maintenance } & \multicolumn{3}{|c|}{ End of Lifie } & \multirow{2}{*}{$\begin{array}{c}{ }^{3} \text { Total } \\
\text { EE }\end{array}$} & \multirow{2}{*}{$\mid \begin{array}{c}{ }^{4} \text { Total } \\
\text { EE } \\
\text { per m }\end{array}$} & & \\
\hline & Material & ${ }^{2}$ Trans. & \begin{tabular}{|l|l} 
Total \\
\end{tabular} & $\mid$ Material & ${ }^{2}$ Trans. & Total & Material & 2 Trans. & Total & Material & ${ }^{2}$ Trans. & Total & & & $\begin{array}{ll}{ }^{5} \text { Total } \\
\end{array}$ & ${ }^{6} \mathrm{perm}^{2}$ \\
\hline 1 Initial & 78,205 & 810 & 79,016 & n & 1,718 & 1,718 & 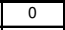 & 0 & 0 & 0 & 0 & 0 & 80,734 & \begin{tabular}{|l|l|}
1,5866 \\
\end{tabular} & & \\
\hline 50 & 78,205 & 810 & 799,016 & 0 & $\begin{array}{l}1,718 \\
\end{array}$ & $\begin{array}{l}1,718 \\
\end{array}$ & 0 & 0 & 0 & 0 & 205 & 205 & 80,939 & \begin{tabular}{|l|l|}
1,590 \\
\end{tabular} & 4,900,000 & 8,434 \\
\hline
\end{tabular}

Global Warming Potential ( $\mathrm{kg}$ of $\mathrm{CO}_{2}$ eq.)

\begin{tabular}{|c|c|c|c|c|c|c|c|}
\hline \multirow{3}{*}{$\begin{array}{l}\text { Lifespan } \\
\text { (Years) }\end{array}$} & \multicolumn{7}{|c|}{ Embodied Global } \\
\hline & \multicolumn{3}{|c|}{ Manufacturing } & \multicolumn{3}{|c|}{ Construction } & \\
\hline & Material & ${ }^{2}$ Trans. & Total & Materia & 1) ${ }^{2}$ Trans & \begin{tabular}{|c|c|} 
Total \\
\end{tabular} & \\
\hline${ }^{\prime}$ Initial & 6,189 & 1 & 6,190 & 0 & 3 & 3 & \\
\hline & \begin{tabular}{|l|l|}
6,189 \\
\end{tabular} & & 6,190 & 0 & 3 & 3 & \\
\hline \multicolumn{8}{|c|}{$\begin{array}{l}\text { Embodied energy (and GWP) numbers are based on an area of wa } \\
\text { Net wall area of baseline retail building (gross wall area - openings) }\end{array}$} \\
\hline \multicolumn{8}{|c|}{$\begin{array}{c}\text { ATHENA } ® \text { EIE Material List: } \\
\text { (Includes all materials after } 50 \text { years) }\end{array}$} \\
\hline \multicolumn{4}{|c|}{ Material List } & \multicolumn{2}{|c|}{\begin{tabular}{|l|} 
Quantities \\
\end{tabular}} & \multicolumn{2}{|c|}{ Unit } \\
\hline \multicolumn{4}{|c|}{ Aluminum } & \multicolumn{2}{|r|}{621.2} & \multicolumn{2}{|c|}{ 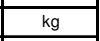 } \\
\hline \multicolumn{4}{|c|}{ Batt. Fiberglass } & \multicolumn{2}{|r|}{378.9} & \multicolumn{2}{|c|}{ m2 $225 \mathrm{~mm}$} \\
\hline \multicolumn{4}{|c|}{ EPDM membrane } & \multirow{2}{*}{\multicolumn{2}{|c|}{$\begin{array}{r}36.4 \\
2253\end{array}$}} & \\
\hline \multicolumn{4}{|c|}{ Galvanized Sheet } & & & \multicolumn{2}{|c|}{ is } \\
\hline \multicolumn{4}{|c|}{ Glazing Panel } & & 225.3 & \multicolumn{2}{|c|}{$\mathrm{kg}$} \\
\hline \multicolumn{4}{|c|}{ Screws Nuts \& Bolts } & & 22.0 & k & \\
\hline
\end{tabular}

Embodied Global Warming Potential (GWP)

\begin{tabular}{|c|c|c|c|c|c|}
\hline & \multirow{2}{*}{\multicolumn{2}{|c|}{$\begin{array}{c}\text { Difference in } \\
\text { Operating GWP fron } \\
\text { Baseline after } \\
\text { Lifespan }\end{array}$}} \\
\hline \multicolumn{2}{|c|}{ dof Life } & \multirow{2}{*}{$\begin{array}{l}{ }^{3} \text { Total } \\
\text { GWP }\end{array}$} & \multirow{2}{*}{\begin{tabular}{|c}
${ }^{4}$ Total \\
GWP \\
per m
\end{tabular}} & & \\
\hline Trass & Total & & & ${ }^{5}$ Total & ${ }^{6}{ }_{\text {per m }}^{2}$ \\
\hline & 0 & \begin{tabular}{|l|l|l}
6,193 \\
\end{tabular} & 122 & & \\
\hline & 0 & 6,194 & \begin{tabular}{|l|}
122 \\
\end{tabular} & \begin{tabular}{|l|l|}
270,000 \\
\end{tabular} & 465 \\
\hline
\end{tabular}

Notes:
initial $=$ Time 'o' (i.e. at the completion of initial construction)

${ }^{1}$ Initial $=$ Time ' $O$ ' (i.e. at the conpl
${ }^{2}$ Trans. = Transportation

${ }^{2}$ Trans. $=$ Transportation
${ }^{3}$ Total EE (or Total GWP) $=$ Total embodied energy (or total embodied GWP)

Total EE (or Total GWP) $=$ Total embodied
of building component after lifespan (i.e. total manufacturing + total

${ }^{4}$ Total EE (or Total GWP) per $\mathrm{m}^{2}=$ Total EE (or Total GWP) of building
component/ area of building component that was modelled in ATHENA@ EIE

${ }^{5}$ Total Difference in Operating Energy (or GWP) from Baseline after Lifespan = The difiference in the total life-cycle operating energy (or GWP)
from the baseline retail building after lifespan, due to using this building component instead of the baseline component ${ }^{6}$ Total Difference in Operating Energy (or GWP) from Baseline after
Lifespan per $\mathrm{m}^{2}=$ Total difference in operating energy (or GWP) from Lifespan per $\mathrm{m}^{2}=$ Total difference in operating energy (or GWP) from
baseline after lifespan / $\mathrm{net}$ wall area of baseline retail building

* Total operating primary energy use of baseline retail building after 50 years =

* Total operating GWP of baseline retion
of $\mathrm{CO}_{2}$ eq. $\left(80 \mathrm{~kg}\right.$ of $\mathrm{CO}_{2}$ eq. $\left./ \mathrm{m}^{2} \mathrm{yr}\right)$

\section{Opaque Curtainwall Enclosure \#3 (CWALL-W3)}

Building Component Description:

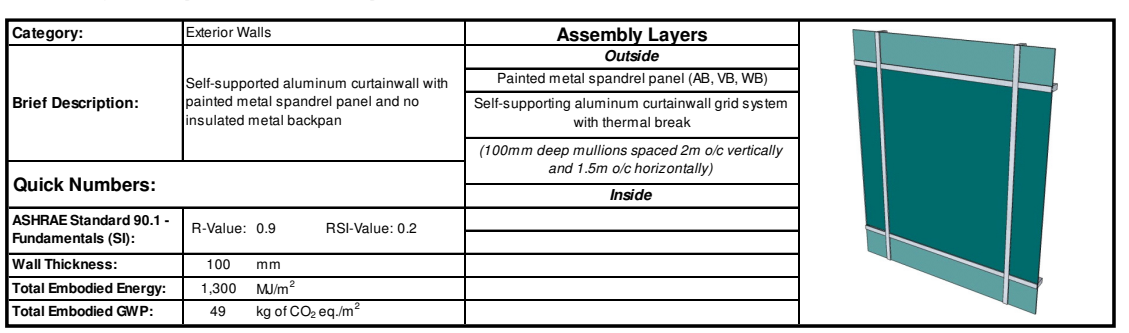

Life-Cycle Assessment Results:

Primary Energy Consumption (MJ)

\begin{tabular}{|c|c|c|c|c|c|c|c|c|c|c|c|c|c|c|c|c|}
\hline \multirow{3}{*}{$\begin{array}{l}\text { Years) } \\
\text { Years }\end{array}$} & \multicolumn{14}{|c|}{ Embodied Energy (EE) } & \multirow{2}{*}{\multicolumn{2}{|c|}{$\begin{array}{l}\text { Difference in } \\
\text { Operating Energy } \\
\text { from Baseline after } \\
\text { Lifespan }\end{array}$}} \\
\hline & \multicolumn{3}{|c|}{ Manufacturing } & \multicolumn{3}{|c|}{ Cons } & \multicolumn{3}{|c|}{ Maintenance } & \multicolumn{3}{|c|}{ End of Life } & \multirow{2}{*}{$\begin{array}{c}{ }^{3} \text { Total } \\
\mathrm{EE}\end{array}$} & \multirow{2}{*}{\begin{tabular}{|l}
${ }^{4}{ }^{4}$ otal \\
$\mathrm{EE}$ \\
per m
\end{tabular}} & & \\
\hline & Material & al ${ }^{2}$ Trans. & Total & Material & ${ }^{2}$ Trans. & Total & Material & $\begin{array}{l}{ }^{2} \text { Trans. } \\
\text { The }\end{array}$ & Total & Material & $\begin{array}{l}{ }^{2} \text { Trans. } \\
\end{array}$ & Total & & & ${ }^{5}$ Total & ${ }^{6}{ }^{6}$ er m ${ }^{2}$ \\
\hline tial & 64,602 & \begin{tabular}{|l|l|}
2 & 541 \\
\end{tabular} & 65,143 & 0 & \begin{tabular}{|l|l|}
1,017 \\
\end{tabular} & 1,017 & 0 & 0 & 0 & 0 & 0 & 0 & 66,160 & 1,299 & & \\
\hline
\end{tabular}

Global Warming Potential (kg of $\mathrm{CO}_{2}$ eq.)

\begin{tabular}{|c|c|c|c|c|c|c|c|c|c|c|c|c|c|c|c|c|}
\hline \multirow{3}{*}{$\begin{array}{l}\text { Lifespan } \\
\text { (Years) }\end{array}$} & \multicolumn{14}{|c|}{ Embodied Global Warming Potential (GWP) } & \multirow{2}{*}{\multicolumn{2}{|c|}{$\begin{array}{l}\text { Difference in } \\
\text { Operating GWP from } \\
\text { Baseline anter } \\
\text { Lifespan }\end{array}$}} \\
\hline & \multicolumn{3}{|c|}{ Manufacturing } & \multicolumn{3}{|c|}{ Construction } & \multicolumn{3}{|c|}{ Maintenance } & \multicolumn{3}{|c|}{ End of Life } & \multirow{2}{*}{$\begin{array}{l}{ }^{3} \text { Total } \\
\text { GWP }\end{array}$} & \multirow{2}{*}{$\begin{array}{c}{ }^{4} \text { Total } \\
\text { GWP } \\
\text { per m }{ }^{2}\end{array}$} & & \\
\hline & Material & \begin{tabular}{|l}
${ }^{2}$ Trans. \\
\end{tabular} & Total & \begin{tabular}{|l|} 
Material \\
\end{tabular} & $\begin{array}{l}\text { Trans. } \\
\end{array}$ & Total & Material & $\begin{array}{l}{ }^{2} \text { Trans. } \\
\end{array}$ & \begin{tabular}{|l|} 
Total \\
\end{tabular} & \begin{tabular}{|l|} 
Material \\
\end{tabular} & ${ }^{2}$ Trans. & Total & & & ${ }^{5}$ Total & ${ }^{6}$ perm ${ }^{2}$ \\
\hline ince & 33 & 1 & & 0 & 2 & 2 & 0 & 0 & 0 & 0 & 0 & 0 & & 49 & & \\
\hline 50 & 2,483 & 1 & \begin{tabular}{|l|}
2,484 \\
\end{tabular} & 0 & 2 & 2 & 0 & 0 & 0 & 0 & 0 & 0 & 2,486 & 49 & ${ }^{2} N / A$ & \\
\hline
\end{tabular}
Net wall area of baseline retail building (gross wall area - openings) $=581.0 \mathrm{~m}^{2}$

De

ATHENA ® EIE Material List:

(Includes all materials after 50 years)

\begin{tabular}{|l|c|c|}
\hline \multicolumn{1}{|c|}{ Material List } & Quantities & Unit \\
\hline Aluminum & 850.4 & $\mathrm{~kg}$ \\
\hline EPDM membrane & 36.4 & $\mathrm{~kg}$ \\
\hline Galvanized Sheet & 346.6 & $\mathrm{~kg}$ \\
\hline Screws Nuts \& Bolts & 22.0 & $\mathrm{~kg}$ \\
\hline
\end{tabular}

Notes:
'Initial $=$ Time ' $O$ ' (i.e. at the completion of initial construction)

${ }^{2}$ Trans. $=$ Transportation

${ }^{3}$ Total EE (or Total GWP) $=$ Total embodied energy (or total embodied GWP)

of building component after lifespan (i.e. total manu
construction + total maintenance + total end-of-life effects)

${ }^{4}$ Total EE (or Total GWP) per $\mathrm{m}^{2}=$ Total EE (or Total GWP) of building
component / area of building component that was modelled in ATHENAB EIE

${ }^{5}$ Total Difference in Operating Energy (or GWP) from Baseline after from the baseline retail building after lifespan, due to using this buidding trom the baseline retall bullining atter lifess
component instead of the baseline component

${ }^{6}$ Total Difference in Operating Energy (or GWP) from Baseline after Lifespan per $\mathrm{m}^{2}=$ Total difference in operating energy (or GWP) from

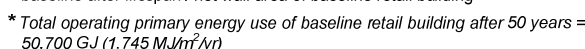

* Total operating GWP of baseline reti
of $\mathrm{CO}_{2}$ eq. $80 \mathrm{~kg}$ of $\mathrm{CO}_{2}$ eq. $\left./ \mathrm{m}^{2} \mathrm{yr}\right)$ 


\section{Opaque Curtainwall Enclosure \#4 (CWALL-W4)}

Building Component Description:

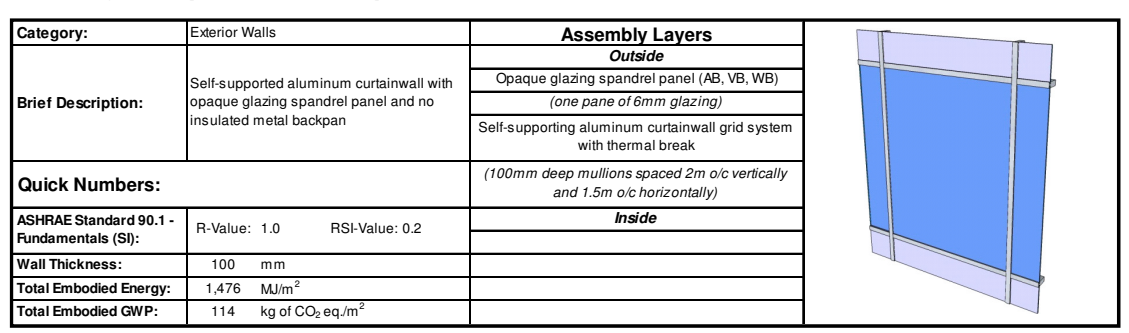

Life-Cycle Assessment Results:

Primary Energy Consumption (MJ)

\begin{tabular}{|c|c|c|c|c|c|c|c|c|c|c|c|c|c|c|c|c|}
\hline \multirow{3}{*}{$\begin{array}{c}\text { Lifespana } \\
\text { (Years) }\end{array}$} & \multicolumn{14}{|c|}{ Embodied Energy (EE) } & \multirow{2}{*}{\multicolumn{2}{|c|}{$\begin{array}{c}\text { Difference in } \\
\text { Operating Energy } \\
\text { from Baseline after } \\
\text { Lifespan }\end{array}$}} \\
\hline & \multicolumn{3}{|c|}{ Manutacturing } & \multicolumn{3}{|c|}{ Construction } & \multicolumn{3}{|c|}{ Maintenance } & \multicolumn{3}{|c|}{ End of Life } & \multirow{2}{*}{$\begin{array}{c}{ }^{3} \text { Total } \\
\mathrm{EE}\end{array}$} & \multirow{2}{*}{\begin{tabular}{|c|}
${ }^{4}$ Total \\
EE \\
per m
\end{tabular}} & & \\
\hline & \begin{tabular}{|l|l|} 
Material \\
\end{tabular} & ${ }^{2}$ Trans. & Total & Material & ${ }^{2}$ Trans. & Total & Material & ${ }^{2}$ Trans. & Total & Material & ${ }^{2}$ Trans. & Total & & & ${ }^{5}$ Total & ${ }^{6}$ per m ${ }^{2}$ \\
\hline 1 Initial & 72,502 & 776 & 73,277 & 0 & 1,696 & 1,696 & 0 & 0 & 0 & 0 & 0 & 0 & 74,973 & \begin{tabular}{|l|}
1,472 \\
\end{tabular} & & \\
\hline 50 & 72,502 & 776 & 73,277 & 0 & \begin{tabular}{|l|l|}
1,696 \\
\end{tabular} & $\begin{array}{ll}1,696 \\
\end{array}$ & 0 & 0 & 0 & 0 & 187 & \begin{tabular}{l|l|}
187 \\
\end{tabular} & 75,160 & \begin{tabular}{|l|l|}
1,476 \\
\end{tabular} & NNA & ${ }^{*} \mathrm{~N} / \mathrm{A}$ \\
\hline
\end{tabular}

Global Warming Potential ( $\mathrm{kg}$ of $\mathrm{CO}_{2}$ eq.)

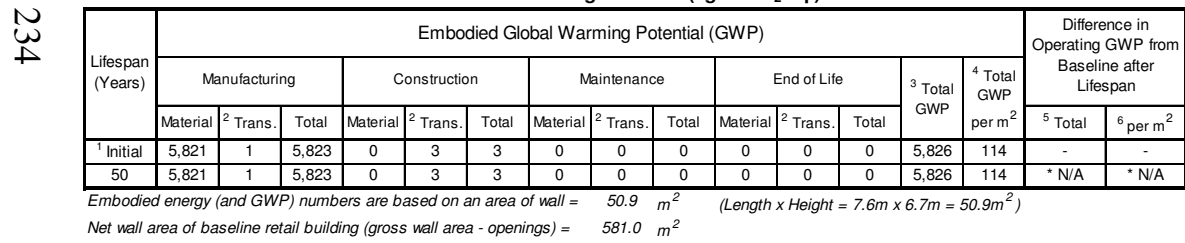

Net wall area of baseline retail building (gross wall area - openings) $=581.0 \mathrm{~m}^{2}$
*Thermal resistance and thermal mass of wall was too low to get an accurate evaluation of operating energy from computer simulations

ATHENA $®$ EIE Material List:

(Includes all materials after 50 years)

\begin{tabular}{|l|c|c|}
\hline \multicolumn{1}{|c|}{ (Includes all materials after 50 years) } \\
\hline Material List & Quantities & Unit \\
\hline Aluminum & 621.2 & $\mathrm{~kg}$ \\
\hline EPDM membrane & 36.4 & $\mathrm{~kg}$ \\
\hline Galvanized Sheet & 22.3 & $\mathrm{~kg}$ \\
\hline Glazing Panel & $1,997.9$ & $\mathrm{~kg}$ \\
\hline Screws Nuts \& Bolts & 22.0 & $\mathrm{~kg}$ \\
\hline
\end{tabular}

$$
\begin{aligned}
& \text { Notes: } \\
& { }^{1} \text { Initial }=\text { Time ' } 0 \text { ' (i.e. at the completion of initial construction) }
\end{aligned}
$$

${ }^{2}$ Trans. $=$ Transportation

Total EE (or Total GWP) = Total embodied energy (or total embodied GWP)

construction + total maintenance + total end-ofl-ifie effects)

${ }^{4}$ Total EE (or Total GWP) per $\mathrm{m}^{2}=$ Total EE (or Total GWP) of building
component/ area of building component that was modelled in ATHENA@ EIE

${ }^{5}$ Total Difference in Operating Energy (or GWP) from Baseline after Lifespan $=$ The difierence in the total life-cycle operating energy (or GWP
from the baseline retail building after lifespan, due to using this building component instead of the baseline component

${ }^{6}$ Total Difference in Operating Energy (or GWP) from Baseline after Lifespan per $m^{2}=$ Total difference in operating energy (or GWP) from

* Total operating primary energy use of baseline retail building after 50 years =
$50,700 \mathrm{GJ}\left(1,745 \mathrm{MJ} / \mathrm{m}^{2} / \mathrm{yr}\right)$

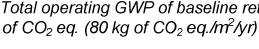


Appendix B-2

LCA Data for Roofs 


\section{LCA Data for Concrete Hollow Core Roofs}

This section contains a detailed description of each concrete hollow core $(\mathrm{CHC})$ roof that was examined in this study (10 in total). The assembly layers are listed for each roof, along with a detailed description of the material quantities from the ATHENA® Environmental Impact Estimator for Buildings.

A breakdown of the total primary energy consumption and the total global warming potential (GWP) for each roof is also included. In each case, the results were calculated for an area of roof equal to $57.8 \mathrm{~m}^{2}$, which represents a typical bay size for a single-storey retail building with this type of roof system. The results are also expressed on a per $\mathrm{m}^{2}$ basis in each case. The data has been calculated for two different lifespans: at the completion of initial construction and after 50 years.

As a summary, the figure below illustrates a comparison of the total embodied energy (and GWP) after 50 years for the various roofs in this section. For comparison purpose, the building component used in the baseline retail building has been shaded in grey.

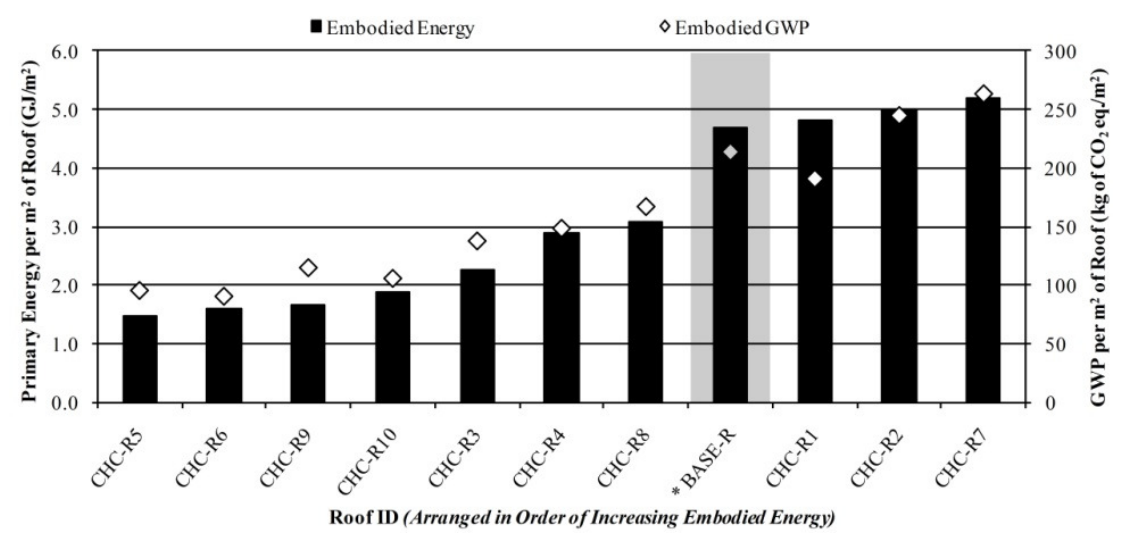

\section{Concrete Hollow Core Roof \#1 (CHC-R1)}

Building Component Description:

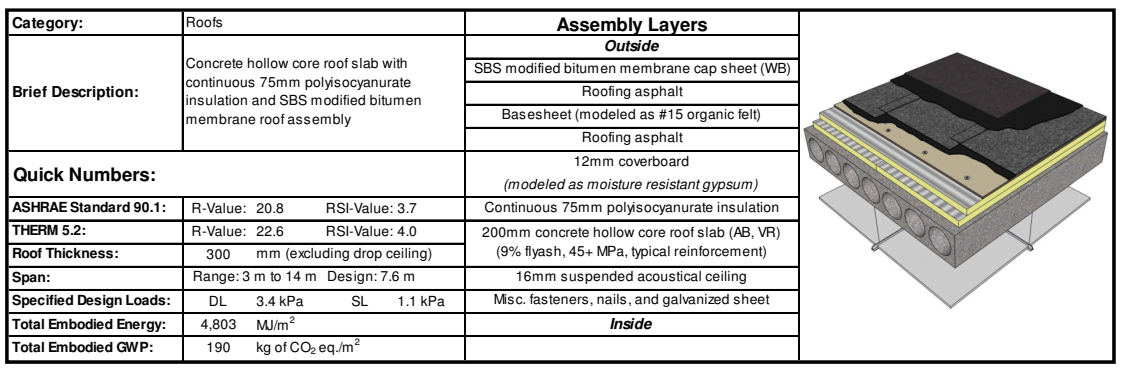

Life-Cycle Assessment Results:
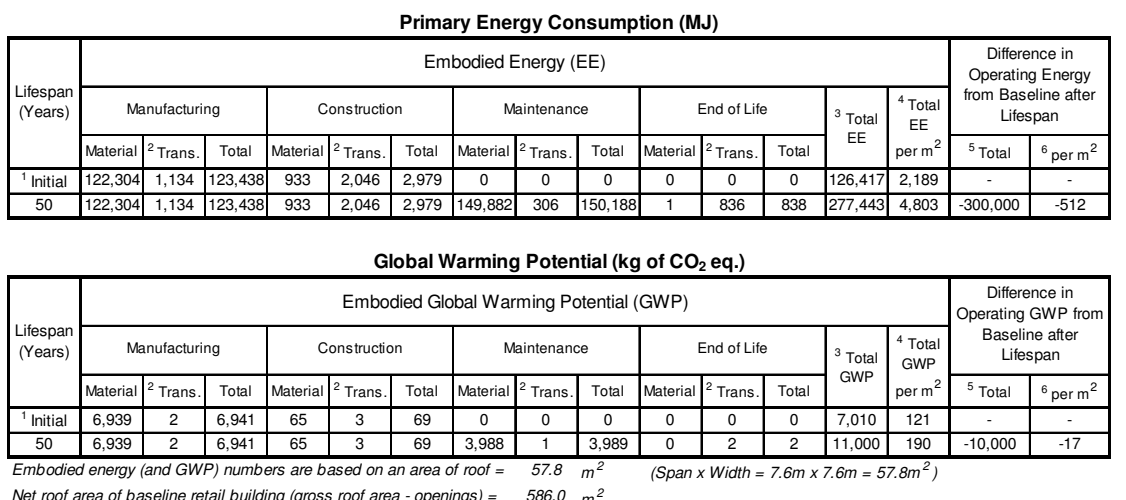

Net roof area of baseline retail building gross roof area - openings) $=586.0 \mathrm{~m}^{2} \quad$ (Span $\times$ Width $=7.6 \mathrm{~m} \times 7.6 \mathrm{~m}=57.8 \mathrm{~m}^{2}$ )

ATHENA $®$ EIE Material List:

\begin{tabular}{|c|c|c|}
\hline Material List & Quantities & Unit \\
\hline$\# 15$ Organic Felt & 521.6 & $\mathrm{~m} 2$ \\
\hline $\begin{array}{l}\text { 13mm Moisture Resistant Gypsum } \\
\text { Board }\end{array}$ & 63.5 & $\mathrm{~m} 2$ \\
\hline 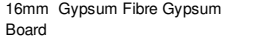 & 63.5 & m2 \\
\hline Concrete $20 \mathrm{MPa}$ (flyash av) & 3.0 & $\mathrm{~m} 3$ \\
\hline Concrete $60 \mathrm{MPa}$ (flyash av) & 5.7 & $m 3$ \\
\hline Galvanized Sheet & 155.7 & $\mathrm{~kg}$ \\
\hline Isocyanurate & 179.2 & $\mathrm{~m} 2(25 \mathrm{~mm})$ \\
\hline Joint Compound & 63.4 & $\mathrm{~kg}$ \\
\hline Modified Bitumen membrane & $2,101.2$ & $\mathrm{~kg}$ \\
\hline Nails & 30.3 & $\mathrm{~kg}$ \\
\hline Paper Tape & 0.7 & $\mathrm{~kg}$ \\
\hline Rebar, Rod, Light Sections & 197.2 & $\mathrm{~kg}$ \\
\hline Roofing Asphalt & $1,060.2$ & $\mathrm{~kg}$ \\
\hline Welded Wire Mesh / Ladder Wire & 88.4 & $\mathrm{~kg}$ \\
\hline
\end{tabular}

Notes:
${ }^{\prime}$ Initial $=$ Time ' $O$ ' (i.e. at the completion of initial construction)

'Initial $=$ Time ' $O$ ' (i.e. at

${ }^{3}$ Total EE (or Total GWP) $=$ Total embodied energy (or total embodied GWP) of building component after lifespan (i.e. total manufacturing + tota
construction + totel maintena

${ }^{4}$ Total EE (or Total GWP) per $\mathrm{m}^{2}=$ Total EE (or Total GWP) of building
component/ area of building component that was modelled in ATHENAQ EIE ${ }^{5}$ Total Difference in Operating Energy (or GWP) from Baseline after Lifespan = The difference in the total life-cycle operating energy (or GWWP)
from the baseline retail building after lifespan, due to using this building stead of the baseline component

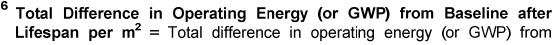

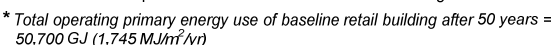

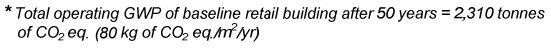




\section{Concrete Hollow Core Roof \#2 (CHC-R2)}

Building Component Description:

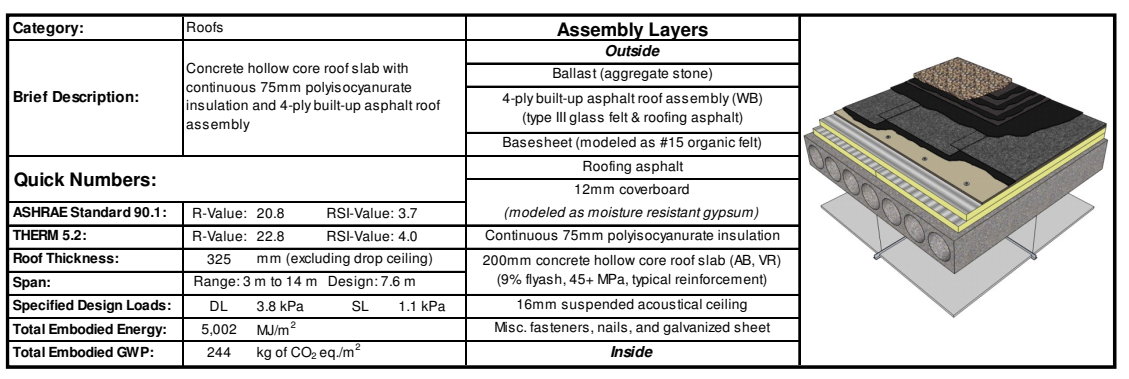

\section{Life-Cycle Assessment Results:}

Primary Energy Consumption (MJ)

\begin{tabular}{|c|c|c|c|c|c|c|c|c|c|c|c|c|c|c|c|}
\hline \multirow{3}{*}{$\begin{array}{l}\text { Lifespan } \\
\text { (Years) }\end{array}$} & \multicolumn{13}{|c|}{ Embodied Energy (EE) } & \multirow{2}{*}{\multicolumn{2}{|c|}{$\begin{array}{c}\text { Difference in } \\
\text { Operating Energy } \\
\text { from Baseline after } \\
\text { Lifespan }\end{array}$}} \\
\hline & \multicolumn{2}{|c|}{ Manutacturing } & \multicolumn{3}{|c|}{ Construction } & \multicolumn{3}{|c|}{ Maintenance } & \multicolumn{3}{|c|}{ End of Life } & \multirow{2}{*}{$\begin{array}{l}{ }^{3} \text { Total } \\
\mathrm{EE}\end{array}$} & \multirow{2}{*}{$\mid \begin{array}{c}{ }^{4} \text { Total } \\
\text { EE } \\
\text { per m}{ }^{2}\end{array}$} & & \\
\hline & 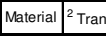 & Total & Material & \begin{tabular}{|l|}
2 Trans. \\
\end{tabular} & \begin{tabular}{|l|l} 
Total \\
\end{tabular} & Material & \begin{tabular}{|l|}
${ }^{2}$ Trans. \\
\end{tabular} & \begin{tabular}{|l|l|} 
Total \\
\end{tabular} & Material & Trans. & Total & & & ${ }^{5}$ Total & per $\mathrm{m}^{2}$ \\
\hline tial & 1,17 & & $\begin{array}{l}8933 \\
\end{array}$ & 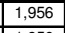 & 89 & & 0 & 0 & 0 & 0 & 0 & 145,81 & & & \\
\hline & 17 & & & 1,956 & & & & & & & & & & & \\
\hline
\end{tabular}

\begin{tabular}{|c|c|c|c|c|c|c|}
\hline \multirow{3}{*}{$\mid \begin{array}{l}\text { Lifespan } \\
\text { (Years) }\end{array}$} & \multicolumn{6}{|c|}{ Embodied G } \\
\hline & \multicolumn{3}{|c|}{ Manufacturing } & \multicolumn{3}{|c|}{ Construction } \\
\hline & Material & ${ }^{2}$ Trans. & Total & Material $^{2}$ & ${ }^{2}$ Trans. & Tot: \\
\hline Initial & 8,399 & 2 & \begin{tabular}{|l|l|}
8,401 & \\
\end{tabular} & 65 & 3 & \\
\hline 50 & \begin{tabular}{|l|l|}
8,399 \\
\end{tabular} & 2 & 8,401 & 65 & 3 & \\
\hline \multicolumn{7}{|c|}{$\begin{array}{l}\text { Embodied energy (and GWP) numbers are based on an area } \\
\text { Net roof area of baseline retail building (gross roof area - oper }\end{array}$} \\
\hline \multicolumn{7}{|c|}{$\begin{array}{c}\text { ATHENA } \circledast \text { EIE Material List: } \\
\text { (Includes all materials after } 50 \text { years) }\end{array}$} \\
\hline \multicolumn{4}{|c|}{ Material List } & Quantitie & \multicolumn{2}{|c|}{ Unit } \\
\hline \multicolumn{4}{|c|}{$\# 15$ Organic Felt } & 521.6 & \multicolumn{2}{|c|}{$\mathrm{m} 2$} \\
\hline \multicolumn{4}{|c|}{$\begin{array}{l}13 \mathrm{~mm} \text { Moisture Resistant Gypsum } \\
\text { Board }\end{array}$} & 63.5 & \multicolumn{2}{|r|}{ 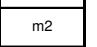 } \\
\hline \multicolumn{4}{|c|}{$\begin{array}{l}\text { Baard Gypsum Fibre Gypsum } \\
\text { Board }\end{array}$} & 63.5 & \\
\hline \multicolumn{4}{|c|}{ Ballast (aggregate stone) } & $4,993.0$ & \multicolumn{2}{|c|}{$\frac{\mathrm{m} 2}{\mathrm{~kg}}$} \\
\hline \multicolumn{4}{|c|}{ Concrete $20 \mathrm{MPa}$ (flyash av) } & 3.0 & \\
\hline \multicolumn{4}{|c|}{ Concrete $60 \mathrm{MPa}$ (flyash av) } & 5.7 & \\
\hline \multirow{2}{*}{\multicolumn{4}{|c|}{\begin{tabular}{|l} 
Galvanized Sheet \\
\end{tabular}}} & 203.2 & & \\
\hline \multicolumn{3}{|c|}{ Isocyanurate } & & 179.2 & \multicolumn{2}{|c|}{$\mathrm{kg}$} \\
\hline \multicolumn{4}{|c|}{ Joint Compound } & 63.4 & \\
\hline \multicolumn{4}{|l|}{ Nails } & 30.3 & & \\
\hline \multicolumn{4}{|c|}{ Paper Tape } & 0.7 & \\
\hline \multicolumn{4}{|c|}{ Rebar, Rod, Light S } & 197.2 & \\
\hline \multicolumn{4}{|c|}{$\begin{array}{l}\text { Roofing Asphalt } \\
\end{array}$} & $\begin{array}{l}1,927.6 \\
\end{array}$ & & \\
\hline Type IIII & Glass Felt & & & $\begin{array}{l}1,043.2 \\
\end{array}$ & & $\mathrm{~kg}$ \\
\hline & vire & & & 88.4 & & \\
\hline
\end{tabular}

Notes:
'Initial $=$ Time '
' (i.e. at the completion of initial construction)

Trans $=$ Transportation

Total EE (or Total GWP) = Total embodied energy (or total embodied GWP

Total EE (or Total GWP) per $\mathrm{m}^{2}=$ Total EE (or Total GWP) of building
component/ area of builing component that was modeled in ATHENAQ EIE Total Difference in Operating Energy (or GWP) from Baseline aft
Lifespan $=$ The difference in the total lifecycle operating energy (ar GW

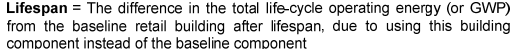
Total Difference in Operating Energy (or GWP) from Baseline after
Lifespan per $\mathrm{m}^{2}=$ Total difference in operating energy (or GWP) from baseline atter lifespan / net roof area of baseline retail build

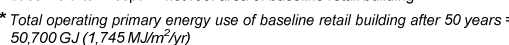
Total operating $\mathrm{GWP}$ of baseline retail building after 50 years $=2,310$ tonnes
of $\mathrm{CO}_{2} e q,\left(80 \mathrm{~kg}\right.$ of $\left.\mathrm{CO}_{2} e q, \mathrm{~m}^{2} / \mathrm{yl}\right)$ construction + total maintenance + total enco-otifife effects

\section{Concrete Hollow Core Roof \#3 (CHC-R3)}

Building Component Description:

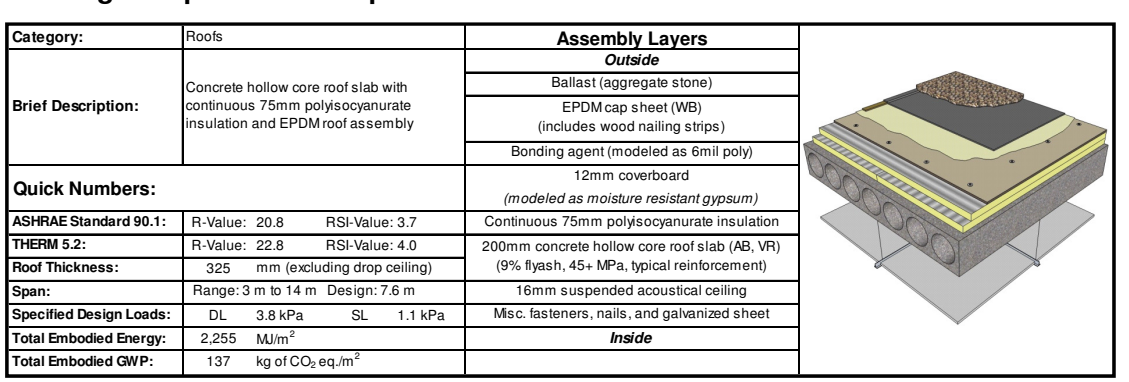

\section{Life-Cycle Assessment Results:}

Primary Energy Consumption (MJ)

\begin{tabular}{|c|c|c|c|c|c|c|c|c|c|c|c|c|c|c|c|c|}
\hline \multirow{3}{*}{$\begin{array}{l}\text { Litespar } \\
\text { (Years) }\end{array}$} & \multicolumn{14}{|c|}{ Embodied Energy (EE) } & \multirow{2}{*}{\multicolumn{2}{|c|}{$\begin{array}{l}\text { Difference in } \\
\text { Operating Energy } \\
\text { from Baseline after } \\
\text { Lifespan }\end{array}$}} \\
\hline & \multicolumn{3}{|c|}{ Manutacturing } & \multicolumn{3}{|c|}{ Construction } & \multicolumn{3}{|c|}{ Maintenance } & \multicolumn{3}{|c|}{ End of Life } & \multirow{2}{*}{\begin{tabular}{|c|}
${ }^{3}$ Total \\
$\mathrm{EE}$
\end{tabular}} & \multirow{2}{*}{$\begin{array}{c}{ }^{4} \text { Total } \\
\mathrm{E} \\
\text { per } \mathrm{m}^{2}\end{array}$} & & \\
\hline & Material & ${ }^{2}$ Trans. & Total & Material & ${ }^{2}$ Trans. & Total & Material & ${ }^{2}$ Trans. & Total & Material & ${ }^{2}$ Trans. & Total & & & ${ }^{5}$ Total & ${ }^{6}$ per $\mathrm{m}^{2}$ \\
\hline Initial & 66,358 & 1,178 & 67,53 & 933 & 2,235 & 3,167 & 0 & ת & 0 & 0 & 0 & 0 & 70,704 & 1,224 & & \\
\hline 50 & 66,358 & 1,178 & 67,53 & 933 & 2,235 & 3,167 & | & 689 & 58,611 & 1 & 910 & 911 & 130,22 & 2,255 & 300,000 & -512 \\
\hline
\end{tabular}

Global Warming Potential (kg of $\mathrm{CO}_{2}$ eq.)

\begin{tabular}{|c|c|c|c|c|c|c|c|c|c|c|c|c|c|c|c|c|}
\hline \multirow{4}{*}{$\left|\begin{array}{c}\text { Lifiespan } \\
\text { (vears) }\end{array}\right|$} & \multicolumn{14}{|c|}{ Global Warming Potential ( $\mathrm{kg}$ of $\mathrm{CO}_{2}$ eq.) } & \multirow{3}{*}{\multicolumn{2}{|c|}{$\begin{array}{c}\text { Difference in } \\
\text { Operating GWP fror } \\
\text { Baseline atter } \\
\text { Lifespan }\end{array}$}} \\
\hline & \multicolumn{14}{|c|}{ Embodied Global Warming Potential (GWP) } & & \\
\hline & \multicolumn{3}{|c|}{ Manutacturing } & \multicolumn{3}{|c|}{ Construction } & \multicolumn{3}{|c|}{ Maintenance } & \multicolumn{3}{|c|}{ End of Life } & \multirow{2}{*}{$\begin{array}{l}{ }^{3} \text { Total } \\
\text { GWP }\end{array}$} & \multirow{2}{*}{\begin{tabular}{|l}
${ }^{4}{ }^{T}$ total \\
GWWP \\
per m
\end{tabular}} & & \\
\hline & Material & ${ }^{2}$ Trans. & Total & Material & ${ }^{2}$ Trans. & Total & Material & 2 Trans. & Total & Material & 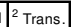 & Total & & & ${ }^{5}$ Total & ${ }^{6} \mathrm{perm}^{2}$ \\
\hline${ }^{1}$ Initial & 5,299 & 2 & 5,302 & 65 & 4 & 69 & 0 & 0 & 0 & 0 & 0 & 0 & 5,371 & 93 & & \\
\hline 50 & 5,299 & 2 & 5,302 & 65 & 4 & 69 & 2,560 & 1 & 2,562 & 0 & 2 & 2 & 7,934 & 137 & $-10,000$ & -17 \\
\hline
\end{tabular}

(Span $\times$ Width $=7.6 \mathrm{~m} \times 7.6 \mathrm{~m}=57.8 \mathrm{~m}^{2}$ )

Net roof area of baseline retail building (gross roof area - openings) $=586.0$

\begin{tabular}{|c|c|c|}
\hline Material List & Quantities & Unit \\
\hline $\begin{array}{l}13 \mathrm{~mm} \text { Moisture Resistant Gypsum } \\
\text { Board }\end{array}$ & 63.5 & $\mathrm{~m}^{2}$ \\
\hline $\begin{array}{l}\text { 16mm Gypsum Fibre Gypsum } \\
\text { Bacard }\end{array}$ & 63.5 & $\mathrm{~m} 2$ \\
\hline 6 mil Polyethylene & 58.9 & $\mathrm{~m} 2$ \\
\hline Ballast (aggregate stone) & $14,978.9$ & $\mathrm{~kg}$ \\
\hline Concrete $20 \mathrm{MPa}$ (flyash av) & 3.0 & $\mathrm{~m} 3$ \\
\hline Concrete $60 \mathrm{MPa}$ (flyash av) & 5.7 & $\mathrm{~m} 3$ \\
\hline EPDM membrane & 541.6 & $\mathrm{kg}$ \\
\hline Galvanized Sheet & 159.4 & $\mathrm{~kg}$ \\
\hline Isocyanurate & 179.2 & $2(25 n$ \\
\hline Joint Compound & 126.8 & $\mathrm{~kg}$ \\
\hline Nails & 5.9 & $\mathrm{~kg}$ \\
\hline Paper Tape & 1.5 & $\mathrm{~kg}$ \\
\hline Rebar, Rod, Light Sections & 197.2 & $\mathrm{~kg}$ \\
\hline $\begin{array}{l}\text { Small Dimension Sotwood Lumber, } \\
\text { kinndred }\end{array}$ & 0.1 & $\mathrm{~m} 3$ \\
\hline Softwood Plywood & 1.2 & $\mathrm{~m} 2(9 \mathrm{~mm})$ \\
\hline Welded Wire Mesh / Ladder & 88.4 & \\
\hline
\end{tabular}

\begin{tabular}{l} 
Notes: \\
${ }^{1}$ Initial $=T$ Time \\
\hline
\end{tabular}

${ }^{2}$ Initial $=$ Trme 0 ' (1.e. at

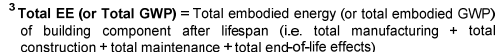

${ }^{4}$ Total EE (or Total GWP) per $m^{2}=$ Total EE (or Total GWP) of building
component / area of building component that was modelled in ATHENAQ EIE

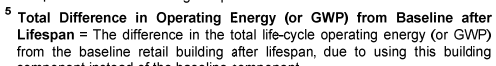
${ }^{6}$ Total Difference in operating Energy (or GWP) from Baseline after
Liftespan per $\mathrm{m}^{2}=$ Total difference in operating energy (or GWP) from

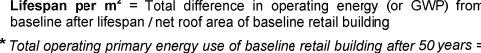
Total operating primary yne
$50,700 \mathrm{GJ}\left(1,745 \mathrm{MJ} / \mathrm{m}^{2} \mathrm{y}\right)$

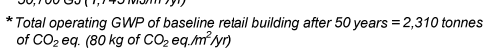




\section{Concrete Hollow Core Roof \#4 (CHC-R4)}

Building Component Description:

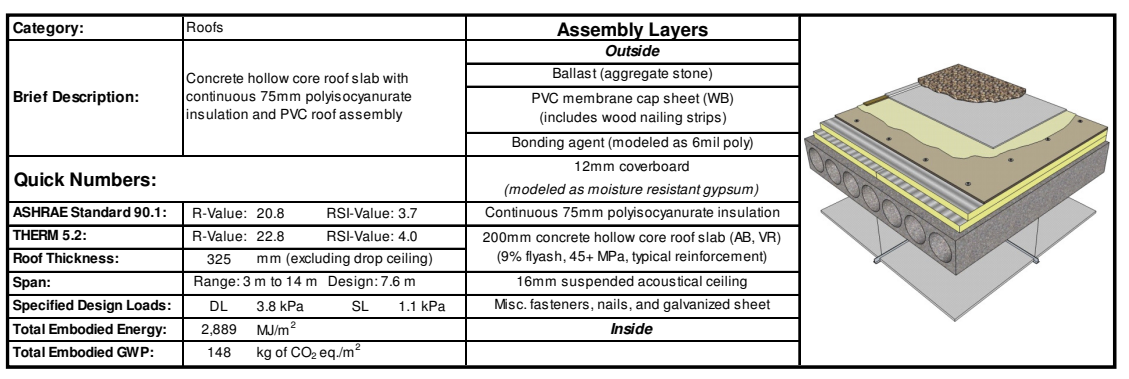

\section{Life-Cycle Assessment Results:}

Primary Energy Consumption (MJ)

\begin{tabular}{|c|c|c|c|c|c|c|c|c|c|c|c|c|c|c|c|c|}
\hline \multirow{3}{*}{$\begin{array}{l}\text { Lifespan } \\
\text { (Years) }\end{array}$} & \multicolumn{14}{|c|}{ Embodied Energy (EE) } & \multirow{2}{*}{\multicolumn{2}{|c|}{$\begin{array}{c}\text { Difference in } \\
\text { Operating Energy } \\
\text { from Baseline after } \\
\text { Lifespan }\end{array}$}} \\
\hline & \multicolumn{3}{|c|}{ Manutacturing } & \multicolumn{3}{|c|}{ Construction } & \multicolumn{3}{|c|}{ Maintenance } & \multicolumn{3}{|c|}{ End of Life } & \multirow{2}{*}{$\begin{array}{l}{ }^{3} \text { Total } \\
\mathrm{EE}\end{array}$} & \multirow{2}{*}{$\begin{array}{c}{ }^{4} \text { Total } \\
\text { EE } \\
\text { per } \mathrm{m}^{2}\end{array}$} & & \\
\hline & Material & ${ }^{2}$ Trans & \begin{tabular}{|l|l} 
Total \\
\end{tabular} & Material & \begin{tabular}{|l|}
2 Trans. \\
\end{tabular} & \begin{tabular}{|l|l} 
Total \\
\end{tabular} & Material & \begin{tabular}{|l|}
${ }^{2}$ Trans. \\
\end{tabular} & \begin{tabular}{|l|l|} 
Total \\
\end{tabular} & Material & Trans. & Total & & & ${ }^{5}$ Total & per $\mathrm{m}^{2}$ \\
\hline ial & & 1,208 & 20 & 933 & 然1 & 14 & & 0 & 0 & 0 & 0 & 0 & 77,934 & 7,349 & & \\
\hline & & 1,208 & & & 2.381 & & & & & & & & & & & \\
\hline
\end{tabular}

Global Warming Potential ( $\mathrm{kg}$ of $\mathrm{CO}_{2}$ eq.)

\begin{tabular}{|c|c|c|c|c|c|c|c|c|c|c|c|c|c|c|c|c|}
\hline \multirow{3}{*}{$\begin{array}{l}\text { Litespan } \\
\text { (Years) }\end{array}$} & \multicolumn{14}{|c|}{ Embodied Global Warming Potential (GWP) } & \multirow{2}{*}{\multicolumn{2}{|c|}{$\begin{array}{l}\text { Difference in } \\
\text { Operating GWP from } \\
\text { Baselile a atter } \\
\text { Lifespan }\end{array}$}} \\
\hline & \multicolumn{3}{|c|}{ Manufacturing } & \multicolumn{3}{|c|}{ Construction } & \multicolumn{3}{|c|}{ Maintenance } & \multicolumn{3}{|c|}{ End of Lifie } & \multirow{2}{*}{$\begin{array}{l}{ }^{3} \text { Total } \\
\text { GWP }\end{array}$} & \multirow{2}{*}{$\mid \begin{array}{c}{ }^{4} \text { Total } \\
\text { GWP } \\
\text { per m }\end{array}$} & & \\
\hline & Material & ${ }^{2}$ Trans. & Total & Material & ${ }^{2}$ Trans. & Total & Material & ${ }^{2}$ Trans. & Total & Material & 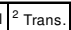 & Total & & & ${ }^{5}$ Total & ${ }^{6}$ per m$^{2}$ \\
\hline \begin{tabular}{|l|} 
'Initial \\
\end{tabular} & \begin{tabular}{|l}
5,415 \\
\end{tabular} & 2 & 5,418 & 65 & 4 & $\begin{array}{ll}69 \\
\end{array}$ & 0 & 0 & 0 & 0 & 0 & 0 & \begin{tabular}{|l|l|l|}
5,487 \\
\end{tabular} & 95 & & \\
\hline 50 & \begin{tabular}{|l|l|}
5,415 \\
\end{tabular} & 2 & 5,418 & 65 & 4 & 69 & \begin{tabular}{|l|}
3,063 \\
\end{tabular} & 2 & 3,065 & $\overline{0}$ & 2 & 2 & \begin{tabular}{|l|}
8,554 \\
\end{tabular} & 148 & $-10,000$ & -17 \\
\hline
\end{tabular}
Embodied energy (and GWP) numbers are based on an area of roof $=57.8 \mathrm{~m}^{2} \quad$ (Span $\times$ Width $=7.6 \mathrm{~m} \times 7.6 \mathrm{~m}=57.8 \mathrm{~m}^{2}$ )

\section{ATHENA $®$ EIE Material List:}

\begin{tabular}{|c|c|c|}
\hline Material List & Quantities & Unit \\
\hline \begin{tabular}{|l}
$13 \mathrm{~mm}$ Moisture Resistant Gypsum \\
\end{tabular} & 63.5 & $\mathrm{~m} 2$ \\
\hline $\begin{array}{l}\text { Domm Gypsum Fibre Gypsum } \\
\text { Bimard }\end{array}$ & 63.5 & $\mathrm{~m} 2$ \\
\hline \begin{tabular}{|l} 
Bodal molyethylene \\
\end{tabular} & 58.9 & $\mathrm{~m} 2$ \\
\hline Ballast (aggregate stone) & $14,978.9$ & $\mathrm{~kg}$ \\
\hline Concrete 20 & 3.0 & \\
\hline Concrete $60 \mathrm{MPa}$ a flyast & 5.1 & $\mathrm{~m} 3$ \\
\hline \begin{tabular}{|l} 
Galvanized Sheet \\
\end{tabular} & 153.5 & \\
\hline Isocyanurate & 179.2 & $22(25 \mathrm{~mm}$ \\
\hline \begin{tabular}{|l} 
Joint Compound \\
\end{tabular} & 126.8 & $\mathrm{~kg}$ \\
\hline Nails & 7.1 & \\
\hline Paper Tape & 1.5 & $\mathrm{k}$ \\
\hline c membran & $1,442.2$ & \\
\hline Rebar, Rod, L & 197.2 & $\mathrm{~kg}$ \\
\hline Vood Lumber, & 0.1 & $\mathrm{~m} 3$ \\
\hline \begin{tabular}{|l} 
kiln-dined \\
Softwood Plywoo
\end{tabular} & 1.2 & 20 \\
\hline Wire Mes & 88. & \\
\hline
\end{tabular}

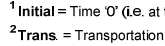

Notes:

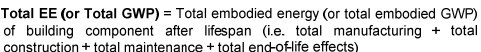

"ortal EE Gor Total GWPP per $\mathrm{m}^{2}=$ = Total EE Eor Total GWP of building
component area of builing component that was modelled in ATHENAQ EIE

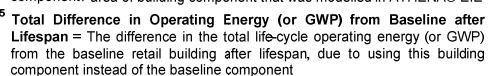

Total Difference in Operating Energy (or GWP) from Baseline after
Lifespan per $\mathbf{m}^{2}=$ Total difference in operating energy
Lifs GWP) from

"Total operating primary energy use of baseline retail building after 50 years =

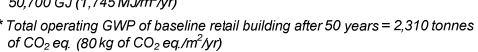

\section{Concrete Hollow Core Roof \#5 (CHC-R5)}

Building Component Description:

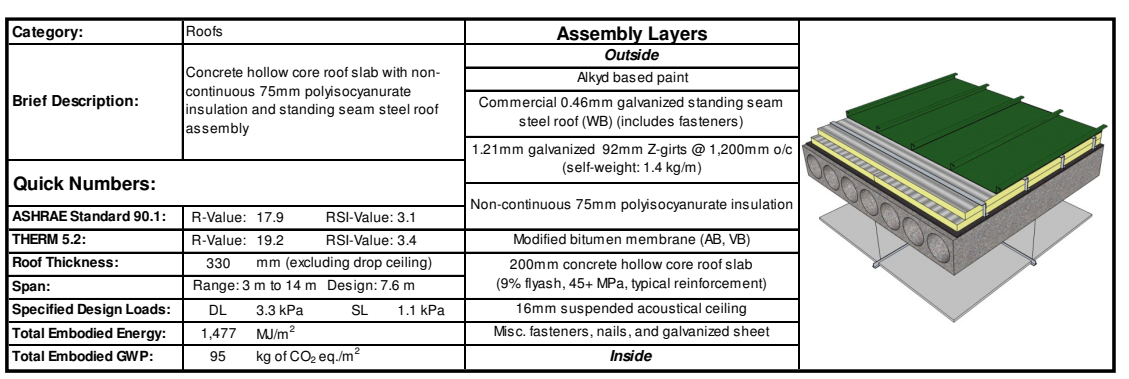

\section{Life-Cycle Assessment Results:}

Primary Energy Consumption (M

\begin{tabular}{|c|c|c|c|c|c|c|c|c|c|c|c|c|c|c|c|c|}
\hline \multirow{3}{*}{\begin{tabular}{|c} 
Lifespan \\
(Years)
\end{tabular}} & \multicolumn{14}{|c|}{ Embodied Energy (EE) } & \multirow{2}{*}{\multicolumn{2}{|c|}{$\begin{array}{l}\text { Difference in } \\
\text { Operating Energy } \\
\text { from Baseline after } \\
\text { Lifespan }\end{array}$}} \\
\hline & \multicolumn{3}{|c|}{ Manufacturing } & \multicolumn{3}{|c|}{ Construction } & \multicolumn{3}{|c|}{ Maintenance } & \multicolumn{3}{|c|}{ End of Lifie } & \multirow{2}{*}{\begin{tabular}{|c|c}
${ }^{3}$ Total \\
EE
\end{tabular}} & \multirow{2}{*}{$\begin{array}{c}{ }^{4}{ }^{4} \text { otal } \\
\mathrm{EE} \\
\text { per } \mathrm{m}^{2}\end{array}$} & & \\
\hline & Material & $\left.\right|^{2}$ Trans. & \begin{tabular}{|l|} 
Total \\
\end{tabular} & Material & ${ }^{2}$ Trans. & Total & Material & ${ }^{2}{ }^{2}$ Trans. & Total & Material & 1. ${ }^{2}$ Trans. & Total & & & ${ }^{5}$ Total & ${ }^{6}$ per $m^{2}$ \\
\hline Initial & 71,609 & 1,087 & 72,696 & 933 & 1,779 & 2,711 & 0 & 0 & 0 & 0 & 0 & 0 & 75,407 & 1,306 & & \\
\hline 50 & 71,609 & 1,087 & 72,696 & 933 & 1,779 & 2,711 & 9,162 & 29 & 9,191 & 1 & 731 & 733 & 85,331 & 1,477 & 100,000 & 171 \\
\hline
\end{tabular}

Global Warming Potential (kg of $\mathrm{CO}_{2}$ eq.)

\begin{tabular}{|c|c|c|c|c|c|c|c|c|c|c|c|c|c|c|c|c|}
\hline \multirow{3}{*}{$\begin{array}{l}\text { Lifespan } \\
\text { (Years) }\end{array}$} & \multicolumn{14}{|c|}{ Embodied Global Warming Potential (GWP) } & \multirow{2}{*}{\multicolumn{2}{|c|}{$\begin{array}{l}\text { Difference in } \\
\text { Operating GWP from } \\
\text { Baseline after } \\
\text { Lifespan }\end{array}$}} \\
\hline & \multicolumn{3}{|c|}{ Manutacturing } & \multicolumn{3}{|c|}{ Construction } & \multicolumn{3}{|c|}{ Maintenance } & \multicolumn{3}{|c|}{ End of L Life } & \multirow{2}{*}{$\begin{array}{l}{ }^{3} \text { Total } \\
\text { GWPP }\end{array}$} & \multirow{2}{*}{$\begin{array}{l}{ }^{4} \text { Total } \\
\mathrm{GWP} \\
\text { per m}{ }^{2}\end{array}$} & & \\
\hline & Material & ${ }^{2}$ Trans. & Total & Material & Trans. & Total & Material & ${ }^{2}$ Trans. & Total & Materia & $1{ }^{2}$ Trans. & Total & & & ${ }^{5}$ Total & ${ }^{6} \operatorname{perm}^{2}$ \\
\hline Initial & 5,297 & 2 & 5,299 & 65 & 3 & 68 & 0 & 0 & 0 & 0 & 0 & 0 & 5,367 & 93 & & \\
\hline 50 & 5,297 & 2 & 5,299 & 65 & 3 & 68 & 127 & 0 & 127 & 0 & 1 & 1 & 5,496 & 95 & 10,000 & 17 \\
\hline
\end{tabular}

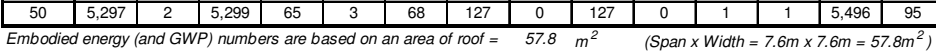

ATHENA $®$ EIE Material List:
Notes:
${ }^{1}$ Initial $=$ Time
0

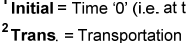

${ }^{3}$ Total EE (or Total GWP) $=$ Total embodied energy (or total embodied GWP)

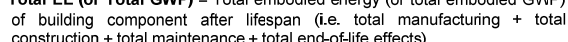
construction + total maintenance + total enc-ofilife effects)

${ }^{4}$ Total EE (or Total GWP) per $\mathrm{m}^{2}=$ Total EE (or Total GWP) of building
component / area of building component that was modelled in ATHENAQ EIE ${ }^{5}$ Total Difference in Operating Energy (or GWP) from Baseline after Total Difference in Operating Energy (or GWP) from Baseline after
Lifespan $=$ The eifference in the total life-cycle operating energy (or GWP)
from the baseline retail building after lifespan, due to using this building

Total Difference in Operating Energy (or GWP) from Baseline after
Lifespan per $\mathrm{m}^{2}=$ Total difference in operating) form (ar GWP) from baseline after lifespan / net roof area of baseline retail building * Total operating primary energy use of baseline retail building after 50 years =
$50,700 \mathrm{GJ}\left(1,745 \mathrm{MJ} / \mathrm{m}^{2} \mathrm{yr}\right)$ "Total operating GWP of baseline retail building after 50 years $=2,310$ tonnes

$\begin{gathered}\text { ATHENA @ EIE Material List: } \\
\text { (Includes all materials after } 50 \text { years) }\end{gathered}$
\begin{tabular}{|l|c|c|}
\hline \multicolumn{1}{|c|}{ Material List } & Quantities & Unit \\
\hline \begin{tabular}{l|c|}
$16 \mathrm{~mm}$ Gypsum Fibre Gypsum \\
Board
\end{tabular} & 63.5 & $\mathrm{~m} 2$ \\
\hline Concrete 20 MPa (flyash av) & 3.0 & $\mathrm{~m} 3$ \\
\hline Concrete $60 \mathrm{MPa}$ (flyash av) & 5.7 & $\mathrm{~m} 3$ \\
\hline Foam Polyisocyanurate & 178.0 & $\mathrm{~m} 2(25 \mathrm{~mm})$ \\
\hline Galvanized Sheet & 472.7 & $\mathrm{~kg}$ \\
\hline Galvanized Studs & 70.7 & $\mathrm{~kg}$ \\
\hline Joint Compound & 63.4 & $\mathrm{~kg}$ \\
\hline Modified Bitumen membrane & 203.3 & $\mathrm{~kg}$ \\
\hline Nails & 4.2 & $\mathrm{~kg}$ \\
\hline Paper Tape & 0.7 & $\mathrm{~kg}$ \\
\hline Rebar, Rod, Light Sections & 197.2 & $\mathrm{~kg}$ \\
\hline Screws Nuts \& Bolts & 0.6 & $\mathrm{~kg}$ \\
\hline Solvent Based Alkyd Paint & 48.4 & $\mathrm{~L}$ \\
\hline Welded Wire Mesh / Ladder Wire & 88.4 & $\mathrm{~kg}$ \\
\hline
\end{tabular}




\section{Concrete Hollow Core Roof \#6 (CHC-R6)}

Building Component Description:

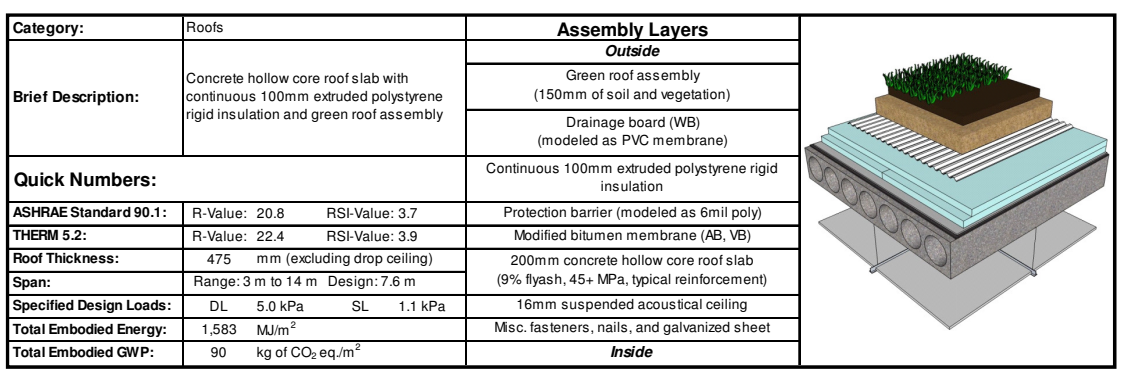

\section{Life-Cycle Assessment Results:}

Primary Energy Consumption (MJ)

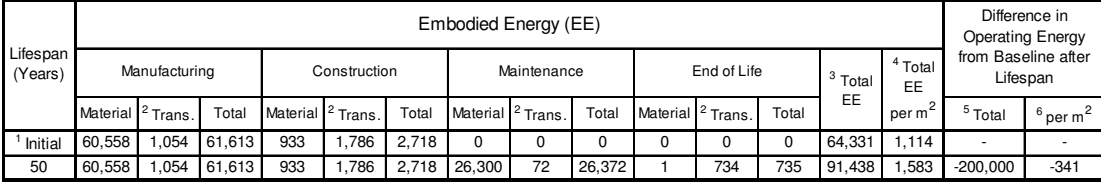

\begin{tabular}{|c|c|c|c|c|c|c|c|c|c|c|c|c|c|}
\hline \\
\hline \multirow{3}{*}{$\begin{array}{l}\text { (respanan } \\
\text { (Years) }\end{array}$} & \multicolumn{13}{|c|}{ Embodied Global } \\
\hline & \multicolumn{7}{|c|}{ Manutacturing } & & & & & & \\
\hline & Material & ${ }^{2}$ Trans. & Total & & & & & & & & ateria & ${ }^{2}$ Tran: & Total \\
\hline Initial & \begin{tabular}{|l|l}
4,407 \\
\end{tabular} & 2 & 4,409 & & & & & & & & 65 & 3 & 68 \\
\hline 50 & \begin{tabular}{|l|l|}
4,407 \\
\end{tabular} & 2 & 4,409 & & & & & & & & 65 & 3 & 68 \\
\hline \multicolumn{14}{|c|}{$\begin{array}{l}\text { Embodied energy (and GWP) numbers are based on an area of roc } \\
\text { Net roof area of baseline retail building (gross roof area - openings) }\end{array}$} \\
\hline \multicolumn{14}{|c|}{$\begin{array}{c}\text { ATHENA @ EIE Material List: } \\
\text { (Includes all materials after } 50 \text { years) }\end{array}$} \\
\hline \multicolumn{8}{|c|}{ Material List } & & & & & & Unit \\
\hline \multicolumn{8}{|c|}{$\begin{array}{l}16 \mathrm{~mm} \text { Gypsum Fibre G } \\
\text { Board }\end{array}$} & & & & & & $\mathrm{m} 2$ \\
\hline \multicolumn{8}{|c|}{6 mil Polyethylene } & & & & & 51.3 & $\mathrm{~m} 2$ \\
\hline \multicolumn{8}{|c|}{ Ballast (aggregate stone) } & & & & & 49.6 & $\mathrm{~kg}$ \\
\hline \multicolumn{8}{|c|}{ Concrete $20 \mathrm{MPa}$ (flyash av) } & & & & & 3.0 & $\mathrm{~m} 3$ \\
\hline \multirow{2}{*}{\multicolumn{8}{|c|}{ Concrete $60 \mathrm{MPa}$ (flyash av) }} & & & & & 5.7 & $\mathrm{~m} 3$ \\
\hline \multirow{2}{*}{\multicolumn{7}{|c|}{ Extruded Polystyrene }} & & & & & & 36.7 & $\mathrm{n2}(25 \mathrm{~mm})$ \\
\hline \multicolumn{7}{|c|}{ Galvanized Sheet } & & & & & & 34.3 & $\mathrm{~kg}$ \\
\hline \multicolumn{8}{|c|}{ Joint Compound } & & & & & 63.4 & $\mathrm{~kg}$ \\
\hline \multicolumn{8}{|c|}{ Modified Bitumen membrane } & & & & & 44.9 & $\mathrm{~kg}$ \\
\hline \multicolumn{8}{|l|}{ Nails } & & & & & 4.2 & $\mathrm{~kg}$ \\
\hline \multirow{2}{*}{\multicolumn{8}{|c|}{$\begin{array}{l}\text { Paper Tape } \\
\text { PVC membrane }\end{array}$}} & & & & & 0.7 & $\mathrm{~kg}$ \\
\hline \multicolumn{6}{|c|}{ PVC membrane } & & & & & & & 94.3 & $\mathrm{~kg}$ \\
\hline \multicolumn{8}{|c|}{ Rebar, Rod, Light Sections } & & & & & 97.2 & \\
\hline \multicolumn{8}{|c|}{ Welded Wire Mesh / Ladder Wire } & & & & & 38.4 & $\mathrm{~kg}$ \\
\hline
\end{tabular}

Notes:
${ }^{1}$ Initial $=$ Time 'o' (i.e. at the completion of initial construction)
${ }^{2}$ Trans $=$ Transpotation

${ }^{3}$ Total EE (or Total GWP) $=$ Total embodied energy (or total embodied GWP) of building component after lifespan (i.e. total man
construction + total maintenance + total end-of-life effects)

${ }^{4}$ Total EE (or Total GWP) per $\mathrm{m}^{2}=$ Total EE (or Total GWP) of building
component/ area of building component that was modeled in ATHENAB EIE

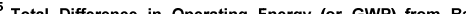

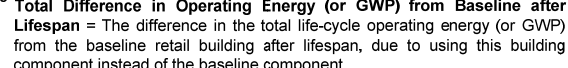

${ }^{6}$ Total Difference in Operating Energy (or GWP) from Baseline after
Lifespan per $\mathrm{m}^{2}=$ Total difference in operating energy (or GWP) from * Total operating primary energy use of baseline retail building after 50 years
$50,700 \mathrm{GJ}\left(1.745 \mathrm{M} / \mathrm{m}^{2} \mathrm{yr}\right)$ * Total operating $\mathrm{GWP}$ of baseline retail building after 50 years $=2,310$ tonnes
of $\mathrm{CO}_{2}$ eq. $8 \mathrm{kO} \mathrm{kg}$ of $\mathrm{CO}_{2}$ eq, $/ \mathrm{m}^{2} \mathrm{yr}$ ) baseline after lifespan $/ n$ et roof area of baseline retail building

\section{Concrete Hollow Core Roof \#7 (CHC-R7)}

Building Component Description:

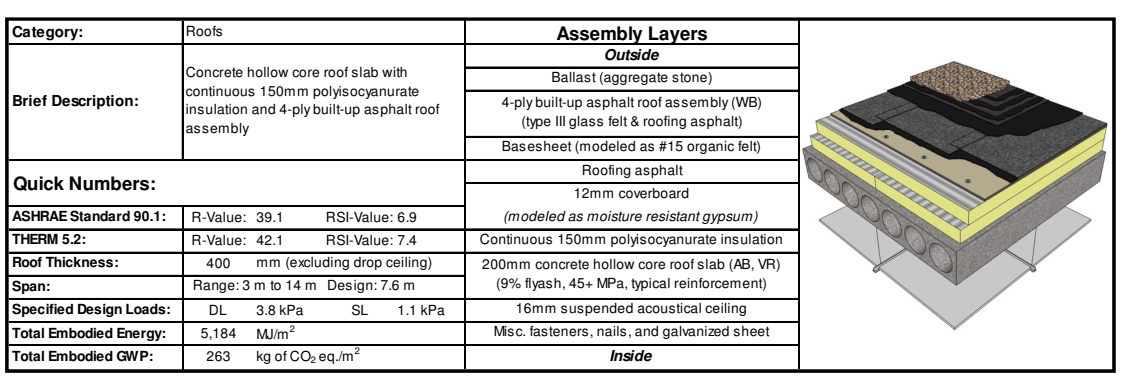

\section{Life-Cycle Assessment Results:}

Primary Energy Consumption (M

\begin{tabular}{|c|c|c|c|c|c|c|c|c|c|c|c|c|c|c|c|c|}
\hline \multirow{3}{*}{$\begin{array}{c}\text { Lifespan } \\
\text { (Years) }\end{array}$} & \multicolumn{14}{|c|}{ Embodied Energy (EE) } & \multirow{2}{*}{\multicolumn{2}{|c|}{$\begin{array}{l}\text { Difierence in } \\
\text { Operating Energy } \\
\text { from Baseline ater } \\
\text { Lifespan a }\end{array}$}} \\
\hline & \multicolumn{3}{|c|}{ Manutacturing } & \multicolumn{3}{|c|}{ Construction } & \multicolumn{3}{|c|}{ Maintenance } & \multicolumn{3}{|c|}{ End of Life } & \multirow{2}{*}{$\begin{array}{c}{ }^{3} \text { Total } \\
\mathrm{EE}\end{array}$} & \multirow{2}{*}{$\begin{array}{c}{ }^{4}{ }^{4} \text { Total } \\
\mathrm{EE} \\
\text { eE } \mathrm{m}^{2}\end{array}$} & & \\
\hline & Material & ${ }^{2}$ Trans & \begin{tabular}{|l|l|l|} 
Total \\
\end{tabular} & Material & ${ }^{2}$ Trans. & Total & Material & ${ }^{2}$ Trans. & Total & Material & ${ }^{2}$ Trans. & Total & & & ${ }^{5}$ Total & ${ }^{6}$ per m ${ }^{2}$ \\
\hline 'Initial & 152,239 & 1,184 & 153,423 & \begin{tabular}{|l|l|}
8933 \\
\end{tabular} & $\begin{array}{ll}1,991 \\
, 991\end{array}$ & 2,924 & $\frac{0}{0}$ & 0 & 0 & 0 & 0 & $\begin{array}{ll}0 \\
\end{array}$ & \begin{tabular}{|c|}
156,347 \\
$29044 \mid$
\end{tabular} & \begin{tabular}{|l|l|}
2,707 \\
5184
\end{tabular} & 120000 & \\
\hline 50 & 152,239 & 1,184 & 153,423 & B) 933 & 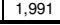 & 2,924 & $\mid 141,720$ & 490 & $\mid 142,2110$ & & & & & & & $-2,048$ \\
\hline
\end{tabular}

Global Warming Potential (kg of $\mathrm{CO}_{2}$ eq.)

\begin{tabular}{|c|c|c|c|c|c|c|c|c|c|c|c|c|c|c|c|c|}
\hline \multirow{4}{*}{\begin{tabular}{|l} 
Lifiespan \\
(Years)
\end{tabular}} & \multicolumn{16}{|c|}{$\mathrm{fco}$} \\
\hline & \multicolumn{14}{|c|}{ Embodied Global Warming Potential (GWP) } & \multirow{2}{*}{\multicolumn{2}{|c|}{\begin{tabular}{|c|} 
Difference in \\
Operating GWP from \\
Baseline atter \\
Lifespan \\
\end{tabular}}} \\
\hline & \multicolumn{3}{|c|}{ Manufacturing } & \multicolumn{3}{|c|}{ Construction } & \multicolumn{3}{|c|}{ Maintenance } & \multicolumn{3}{|c|}{ End of L Life } & \multirow{2}{*}{$\begin{array}{l}3 \text { Total } \\
\text { GWP }\end{array}$} & \multirow{2}{*}{$\begin{array}{l}{ }^{4} \text { Total } \\
\text { GiWP } \\
\text { per m }\end{array}$} & & \\
\hline & Material & 2 Trans. & Total & \begin{tabular}{|l|} 
Material \\
\end{tabular} & 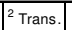 & Total & \begin{tabular}{|l|} 
Material \\
\end{tabular} & ${ }^{2}$ Trans. & Total & \begin{tabular}{|l|} 
Material \\
\end{tabular} & \begin{tabular}{|l|}
${ }^{2}$ Trans. \\
\end{tabular} & Total & & & ${ }^{5}$ Total & \begin{tabular}{|l}
${ }^{6} \operatorname{per} \mathrm{m}^{2}$ \\
\end{tabular} \\
\hline IInitial & 9,468 & 2 & 9,471 & 65 & 3 & 69 & 0 & 0 & 0 & 0 & 0 & 0 & 9,539 & 165 & & \\
\hline 50 & \begin{tabular}{|l}
9,468 \\
\end{tabular} & 2 & \begin{tabular}{|l|l|} 
\\
\end{tabular} & 65 & 3 & 69 & \begin{tabular}{|l|l|}
5,635 \\
\end{tabular} & 1 & 5,636 & 0 & 2 & 2 & 15,177 & 263 & \begin{tabular}{ll|l|}
$-60,000$ \\
\end{tabular} & -102 \\
\hline
\end{tabular}

Embodied energy (and GWP) numbers are based on an area of roof $=57.8 \mathrm{~m}^{2} \quad\left(\right.$ Span $\times$ Width $\left.=7.6 \mathrm{~m} \times 7.6 \mathrm{~m}=57.8 \mathrm{~m}^{2}\right)$

roof area of baseline retail building (gross roof area - openings) $=586.0 \mathrm{~m}^{2}$

ATHENA $®$ EIE Material List:

\begin{tabular}{|c|c|c|}
\hline Material List & Quantitites & Unit \\
\hline \#15 Organic Felt & 521.6 & $\frac{\mathrm{m}^{2}}{2}+\mathrm{C}^{2}$ \\
\hline $\begin{array}{l}\text { 13mm Moisture Resistant Gypsum } \\
\text { Board }\end{array}$ & 63.5 & $\mathrm{~m} 2$ \\
\hline $\begin{array}{l}16 \mathrm{~mm} \text { Gypsum Fibre Gypsum } \\
\text { Bopard }\end{array}$ & 63.5 & $\mathrm{~m} 2$ \\
\hline Ballast (aggregate stone) & $4,993.0$ & $\mathrm{~kg}$ \\
\hline Concretet $20 \mathrm{MPa}$ (tyash av) & 3.0 & $\begin{array}{l}\mathrm{m} 3 \\
3\end{array}$ \\
\hline Concrete $60 \mathrm{MPa}$ (flyash av) & 5.7 & $\mathrm{~m} 3$ \\
\hline Galvanized Sheet & 203.2 & $\mathrm{~kg}$ \\
\hline Isocyanurate & 358.4 & $\mathrm{~m} 2(25 \mathrm{~mm}) \mathrm{t}$ \\
\hline Joint Compound & 63.4 & $\mathrm{~kg}$ \\
\hline Nails & 30.3 & $\mathrm{~kg}$ \\
\hline Paper Tape & 0.7 & $\mathrm{~kg}$ \\
\hline Rebar, Rod, Light Sections & 197.2 & $\mathrm{~kg}$ \\
\hline Roofing Asphalt & $1,927.6$ & $\mathrm{~kg}$ \\
\hline Type III Glass Felt & $1,043.2$ & $\mathrm{~m} 2^{2}$ \\
\hline Welded Wire Mesh / Ladder Wire & 88.4 & $\mathrm{~kg}$ \\
\hline
\end{tabular}

$$
\begin{aligned}
& \text { Notes: } \\
& \text { 'Initial }=\text { Time ' } O \text { ' (i.e. at the completion of initial construction) }
\end{aligned}
$$

Trans. = Transportation

Total EE (or Total GWP) $=$ Total embodied energy (or total embodied GWP)
of building component after lifespan (i.e. total manufacturing + tota

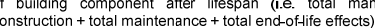

Total EE (or Total GWP) per $\mathrm{m}^{2}=$ Total EE (or Total GWP) of building
component / area of building component that was modelled in ATHENAQ EIE "Total Difference in Operating Energy (or GWP) from Baseline after from the baseline retail building after lifespan, due to using this building
component instead of the baseline component ${ }^{6}$ Total Difference in Operating Energy (or GWP) from Baseline after Lifespan per $\mathrm{m}^{2}=$ Total difference in operating energy (or GWP) from
baseline after lifespan $/$ net roof area of baseline retail building * Total operating primary energy use of baseline retail buididing after 50 years $=$
$50,700 \mathrm{Gs}\left(1,745 \mathrm{MJ} \mathrm{m}^{2} \mathrm{yr}\right)$ *Total operating $\mathrm{GWP}$ of baseline retail building affer 50 years $=2,310$ tonnes
of $\mathrm{CO}_{2}$ eq. $\left(80 \mathrm{~kg}\right.$ of $\left.\mathrm{CO}_{2} e q / \mathrm{m}^{2} / \mathrm{yr}\right)$ 


\section{Concrete Hollow Core Roof \#8 (CHC-R8)}

Building Component Description:

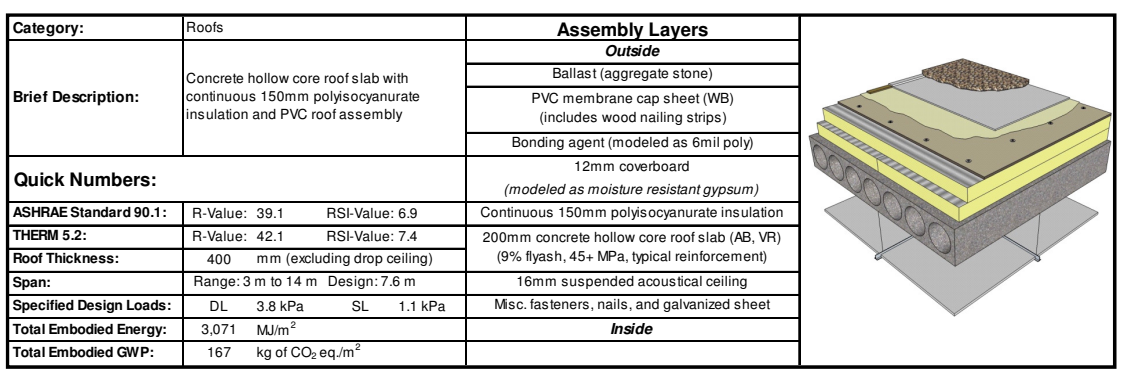

\section{Life-Cycle Assessment Results:}

Primary Energy Consumption (MJ)

\begin{tabular}{|c|c|c|c|c|c|c|c|c|c|c|c|c|c|c|c|c|}
\hline \multirow{3}{*}{ Years) } & \multicolumn{14}{|c|}{ Embodied Energy (EE) } & \multirow{2}{*}{\multicolumn{2}{|c|}{$\begin{array}{c}\text { Difference in } \\
\text { Operating Energy } \\
\text { from Baseline after } \\
\text { Lifespan }\end{array}$}} \\
\hline & \multicolumn{3}{|c|}{ Manutacturing } & \multicolumn{3}{|c|}{ Construction } & \multicolumn{3}{|c|}{ Maintenance } & \multicolumn{3}{|c|}{ End of Life } & \multirow{2}{*}{$\begin{array}{l}{ }^{3} \text { Total } \\
\mathrm{EE}\end{array}$} & \multirow{2}{*}{$\begin{array}{c}{ }^{4} \text { Total } \\
\text { EE } \\
\text { per } \mathrm{m}^{2}\end{array}$} & & \\
\hline & Material & ${ }^{2}$ Trans. & \begin{tabular}{|l|l|} 
Total \\
\end{tabular} & Material & \begin{tabular}{|l|}
2 Trans. \\
\end{tabular} & \begin{tabular}{|l|l} 
Total \\
\end{tabular} & Material & \begin{tabular}{|l|}
${ }^{2}$ Trans. \\
\end{tabular} & \begin{tabular}{|l|l|} 
Total \\
\end{tabular} & Material & \begin{tabular}{|l|}
${ }^{2}$ Trans. \\
\end{tabular} & Total & & & ${ }^{5}$ Total & per $m^{2}$ \\
\hline tial & & 1,214 & 85,115 & 933 & 11 & 50 & & 0 & 0 & 0 & 0 & 0 & 88,465 & 1,5 & & \\
\hline & & 1,214 & & & 2,417 & & & & & & & & & & & \\
\hline
\end{tabular}

\begin{tabular}{|c|c|c|c|c|c|c|c|c|c|c|c|c|c|c|c|c|}
\hline \multirow{4}{*}{$\mid$\begin{tabular}{|l} 
Lifiespan \\
(Vears)
\end{tabular}} & \multicolumn{14}{|c|}{$\mathrm{fco}$} & \multirow{3}{*}{\multicolumn{2}{|c|}{$\begin{array}{c}\text { Difference in } \\
\text { Operating GWP fron } \\
\text { Baseline after } \\
\text { Lifespan }\end{array}$}} \\
\hline & \multicolumn{14}{|c|}{ Embodied Global Warming Potential (GWP) } & & \\
\hline & \multicolumn{3}{|c|}{ Manufacturing } & \multicolumn{3}{|c|}{ Construction } & \multicolumn{3}{|c|}{ Maintenance } & \multicolumn{3}{|c|}{ End of Life } & \multirow{2}{*}{$\begin{array}{l}{ }^{3} \text { Total } \\
\text { GWP }\end{array}$} & \multirow{2}{*}{ 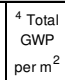 } & & \\
\hline & \begin{tabular}{|l|l|} 
Material \\
\end{tabular} & ${ }^{2}$ Trans. & Total & Material & ${ }^{2}$ Trans. & Total & Material & ${ }^{2}$ Trans. & Total & Material & ${ }^{2}$ Trans. & Total & & & ${ }^{5}$ Total & ${ }^{6} \operatorname{perm}^{2}$ \\
\hline${ }^{1}$ Initial & 6,485 & 2 & 6,488 & 65 & 4 & 69 & 0 & 0 & 0 & 0 & 0 & 0 & 6,557 & 114 & & \\
\hline 50 & 6,485 & 2 & 6,488 & 65 & 4 & 69 & 3,063 & 2 & 3,065 & 0 & 2 & 2 & 9,624 & 167 & $-60,000$ & -102 \\
\hline
\end{tabular}
Embodied energy (and GWP) numbers are based on an area of roof $=57.8 \quad \mathrm{~m}^{2} \quad$ (Span $\times$ Width $\left.=7.6 \mathrm{~m} \times 7.6 \mathrm{~m}=57 . \mathrm{sm}^{2}\right)$
Net roof

\begin{tabular}{|c|c|c|}
\hline \multicolumn{3}{|c|}{$\begin{array}{c}\text { ATHENA @ EIE Material List: } \\
\text { (nncludes all materials stere 50 years) }\end{array}$} \\
\hline Material List & \begin{tabular}{|l|} 
Quantities \\
\end{tabular} & Unit \\
\hline 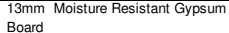 & 63.5 & $\mathrm{~m} 2$ \\
\hline $\begin{array}{l}16 m m \text { Gypsum Fibre Gypsum } \\
\text { Board }\end{array}$ & 63.5 & $\mathrm{~m} 2$ \\
\hline 6 mil Polyethylene & 58.9 & $\mathrm{~m} 2$ \\
\hline Ballast (aggregate stone) & \begin{tabular}{|l|l|}
14.978 .9 \\
\end{tabular} & $\mathrm{~kg}$ \\
\hline Concrete $20 \mathrm{MPa}$ (fiyash av) & 3.0 & $\mathrm{m3}$ \\
\hline Concrete $60 \mathrm{MPa}$ (flyash av) & 5.7 & $\mathrm{m3}$ \\
\hline Galvanized Sheet & 153.5 & $\mathrm{~kg}$ \\
\hline Isocyanurate & 358.4 & m2 (25mm \\
\hline Joint Compound & 126.8 & $\mathrm{~kg}$ \\
\hline Nalis & 7.1 & $\mathrm{~kg}$ \\
\hline $\begin{array}{l}\text { Paper Tape } \\
\end{array}$ & 1.5 & $\mathrm{~kg}$ \\
\hline PVC membrane & $1,442.2$ & $\mathrm{~kg}$ \\
\hline Rebar, Rod, Light Sections & 197.2 & $\mathrm{~kg}$ \\
\hline 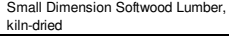 & 0.1 & $\mathrm{~m} 3$ \\
\hline Sottwood Plywood & 1.2 & m2 (9mm) \\
\hline Welded Wire Mesh / Ladder Wire & 88.4 & $\mathrm{~kg}$ \\
\hline
\end{tabular}

\section{Concrete Hollow Core Roof \#9 (CHC-R9)}

Building Component Description:

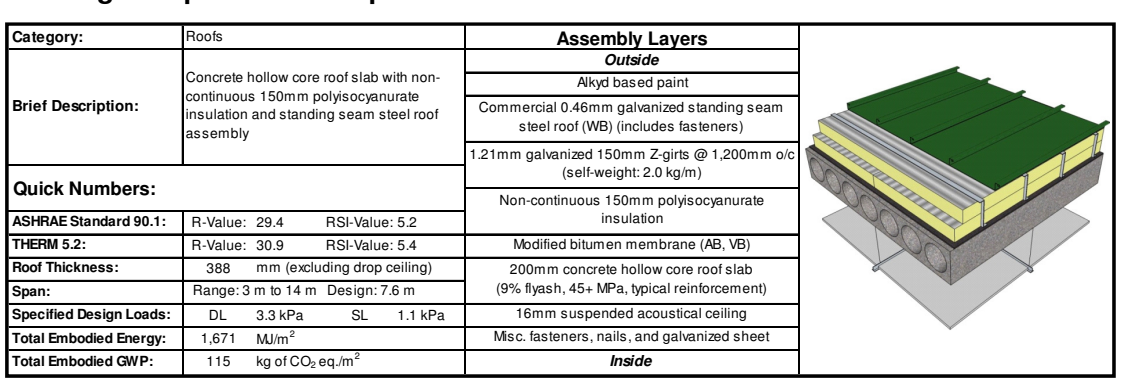

\section{Life-Cycle Assessment Results:}

Primary Energy Consumption (MJ)

\begin{tabular}{|c|c|c|c|c|c|c|c|c|c|c|c|c|c|c|c|c|}
\hline \multirow{3}{*}{$\begin{array}{l}\text { Litespar } \\
\text { (Years) }\end{array}$} & \multicolumn{14}{|c|}{ Embodied Energy (EE) } & \multirow{2}{*}{\multicolumn{2}{|c|}{$\begin{array}{l}\text { Difference in } \\
\text { Operating Energy } \\
\text { from Baseline after } \\
\text { Lifespan }\end{array}$}} \\
\hline & \multicolumn{3}{|c|}{ Manutacturing } & \multicolumn{3}{|c|}{ Construction } & \multicolumn{3}{|c|}{ Maintenance } & \multicolumn{3}{|c|}{ End of Life } & \multirow{2}{*}{$\begin{array}{c}{ }^{3} \text { Total } \\
\mathrm{EE}\end{array}$} & \multirow{2}{*}{$\begin{array}{c}{ }^{4} \text { Total } \\
\mathrm{E} \\
\text { per } \mathrm{m}^{2}\end{array}$} & & \\
\hline & Material & ${ }^{2}$ Trans. & Total & Material & |2 Trans. & Total & Material & ${ }^{2}$ Trans. & Total & Material & ${ }^{2}$ Trans. & 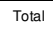 & & & ${ }^{5}$ Total & ${ }^{6}$ per $\mathrm{m}^{2}$ \\
\hline Initial & 82,750 & 1,094 & 83,843 & 933 & 1,817 & 2,750 & 0 & 0 & $\pi$ & 0 & 0 & 0 & 86,594 & 1,499 & & \\
\hline 50 & 82,750 & \begin{tabular}{|l|l}
1,094 \\
\end{tabular} & 83,843 & 933 & \begin{tabular}{|l|l|}
1,817 \\
\end{tabular} & 2,750 & 9,162 & 29 & 9,191 & 1 & 745 & 746 & 96,530 & 1,671 & 800,000 & $-1,36$ \\
\hline
\end{tabular}

Global Warming Potential (kg of $\mathrm{CO}_{2}$ eq.)

\begin{tabular}{|c|c|c|c|c|c|c|c|c|c|c|c|c|c|c|c|c|}
\hline \multirow{3}{*}{$\begin{array}{l}\text { Lifespan } \\
\text { (Years) }\end{array}$} & \multicolumn{14}{|c|}{ Embodied Global Warming Potential (GWP) } & \multirow{2}{*}{\multicolumn{2}{|c|}{\begin{tabular}{|c} 
Difference in \\
Operatiting GWP from \\
Baseline after \\
Lifespan
\end{tabular}}} \\
\hline & \multicolumn{3}{|c|}{ Manutacturing } & \multicolumn{3}{|c|}{ Construction } & \multicolumn{3}{|c|}{ Maintenance } & \multicolumn{3}{|c|}{ End of Life } & \multirow{2}{*}{ 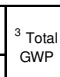 } & \multirow{2}{*}{$\begin{array}{l}{ }^{4} \text { Total } \\
\text { GWP } \\
\text { per m }\end{array}$} & & \\
\hline & Material & ${ }^{2}$ Trans. & Total & Material & ${ }^{2}$ Trans. & Total & Material & ${ }^{2}$ Trans. & Total & Material & ${ }^{2}$ Trans. & Total & & & 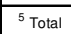 & ${ }^{6}$ perm $\mathrm{m}^{2}$ \\
\hline Initial & 6,418 & 2 & 6,420 & 65 & 3 & 68 & 0 & 0 & 0 & 0 & 0 & 0 & 6,488 & 112 & & \\
\hline 50 & 6,418 & 2 & 6,420 & 65 & 3 & 68 & 127 & 0 & 127 & 0 & 1 & 2 & 6,617 & 115 & $-40,000$ & -68 \\
\hline
\end{tabular}

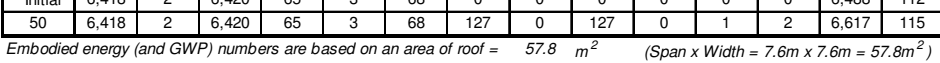
Net roof area of baseline retail building (gross roof area - openings) $=586.0 \mathrm{~m}^{2}$

\section{ATHENA $®$ EIE Material List:}

${ }^{-1}$ Initial $=$ Time
${ }^{2}$ '
Trans.

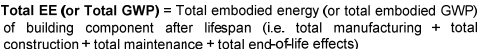

"ortal EE (or Total GWP per $m^{2}=$ Total EE E(r Total GWP of building
component/ area of builing component that was modelled in ATHENAQ EIE

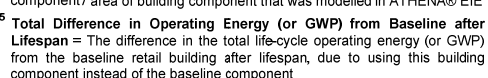

Total Difference in Operating Energy (or GWP) from Baseline after
Lifespan per ${ }^{2}=$ Total difference in operating energy (or GWP) from

"Total operating primary energy use of baseline retail building after 50 years =

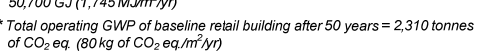

\begin{tabular}{|l|c|c|}
\hline \multicolumn{1}{|c|}{ Material List } & Quantitities & Unit \\
\hline $\begin{array}{l}\text { 16mm Gypsum Fibre Gypsum } \\
\text { Board }\end{array}$ & 63.5 & $\mathrm{~m} 2$ \\
\hline Concrete $20 \mathrm{MPa}$ (llyash av) & 3.0 & $\mathrm{~m} 3$ \\
\hline Concrete $60 \mathrm{MPa}$ (llyash av) & 5.7 & $\mathrm{~m} 3$ \\
\hline Foam Polyisocyanurate & 356.0 & $\mathrm{~m} 2(25 \mathrm{~mm})$ \\
\hline Galvanized Sheet & 472.7 & $\mathrm{~kg}$ \\
\hline Galvanized Studs & 101.0 & $\mathrm{~kg}$ \\
\hline Joint Compound & 63.4 & $\mathrm{~kg}$ \\
\hline Modified Bitumen membrane & 203.3 & $\mathrm{~kg}$ \\
\hline Nails & 4.2 & $\mathrm{~kg}$ \\
\hline Paper Tape & 0.7 & $\mathrm{~kg}$ \\
\hline Rebar, Rod, Light Sections & 197.2 & $\mathrm{~kg}$ \\
\hline Screws Nuts \& Bolts & 0.6 & $\mathrm{~kg}$ \\
\hline Solvent Based Alkyd Paint & 48.4 & $\mathrm{~L}$ \\
\hline Welded Wire Mesh / Ladder Wire & 88.4 & $\mathrm{~kg}$ \\
\hline
\end{tabular}

\begin{tabular}{l} 
Notes: \\
${ }^{1}$ Initial $=$ Time ' \\
\hline
\end{tabular}

'Initial $=$ Time ' 0 ' (i.e. at the

Total EE (or Total GWP) $=$ Total embodied energy (or total embodied GWP) of building component affer lifessan (i.e. total manufacturing + total
construction + total maintenance+total enc-oflife effects) ${ }^{4}$ Total EE (or Total GWP) per $\mathrm{m}^{2}=$ = Total EE (or Total GWP) of building Total Difference in Operating Energy (or GWP) from Baseline after
Lifespan = The difference in the total life-cycle operating energy (or GWP)
from the baseline retail building after lifespan, due to using this building from the baseline retail building after lifespan, due to using this building
component instead of the baseline component ${ }^{6}$ Total Difference in Operating Energy (or GWP) from Baseline after Lifespan per $\mathrm{m}^{2}=$ Total difiference in operating energy (or GWP) from
baseline after lifespan /net roof area of baseline retail building * Total operating primary energy use of baseline retail building after 50 years $=$
$50,700 \mathrm{GJ}\left(1,745 \mathrm{MJ} / \mathrm{m}^{2} \mathrm{yr}\right)$

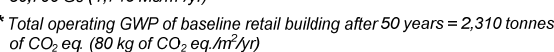




\section{Concrete Hollow Core Roof \#10 (CHC-R10)}

Building Component Description:

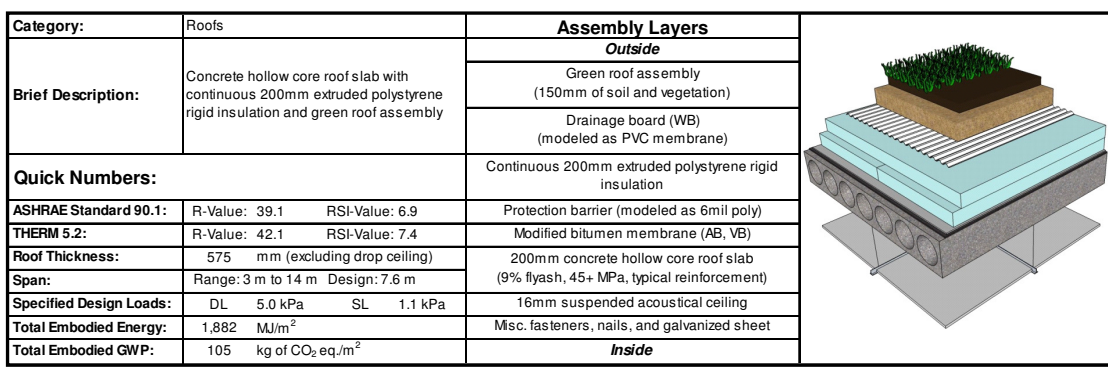

Life-Cycle Assessment Results:

Primary Energy Consumption (MJ)

\begin{tabular}{|c|c|c|c|c|c|c|c|c|c|c|c|c|c|c|c|c|}
\hline \multirow{3}{*}{\begin{tabular}{|l} 
Lifespan \\
(Years)
\end{tabular}} & \multicolumn{14}{|c|}{ Embodied Energy (EE) } & \multirow{2}{*}{\multicolumn{2}{|c|}{$\begin{array}{c}\text { Difference in } \\
\text { Operating Energy } \\
\text { from Baseline atte } \\
\text { Liffespan }\end{array}$}} \\
\hline & \multicolumn{3}{|c|}{ Manutacturing } & \multicolumn{3}{|c|}{ Construction } & \multicolumn{3}{|c|}{ Maintenance } & \multicolumn{3}{|c|}{ End of Life } & \multirow{2}{*}{$\begin{array}{c}{ }^{3} \text { Total } \\
\mathrm{EE}\end{array}$} & \multirow{2}{*}{\begin{tabular}{|l|}
${ }^{4}$ Total \\
EE \\
per m² \\
\end{tabular}} & & \\
\hline & Material & ${ }^{2}$ Trans. & Total & Material & ${ }^{2}$ Trans. & Total & Material & \begin{tabular}{|l}
${ }^{2}$ Trans. \\
\end{tabular} & Total & Material & \begin{tabular}{|l|l|}
${ }^{2}$ Trans. \\
\end{tabular} & \begin{tabular}{|l|l|l|} 
Total & \\
\end{tabular} & & & ${ }^{5}$ Total & ${ }^{6}$ per m$^{2}$ \\
\hline Initial & 77,762 & $\begin{array}{ll}1,055 \\
\end{array}$ & 78,817 & 933 & \begin{tabular}{|l|l|l|l|l}
1,813 \\
\end{tabular} & 2,746 & 0 & 0 & 0 & 0 & 0 & 0 & 81,563 & 1,412 & & \\
\hline 50 & 777,762 & 1,055 & 78,817 & 933 & $\mid 1,813$ & 2,746 & & 72 & & 1 & & 757 & 108,692 & & $-1,200$ & $-2,04$ \\
\hline
\end{tabular}

$\stackrel{N}{ \pm}$

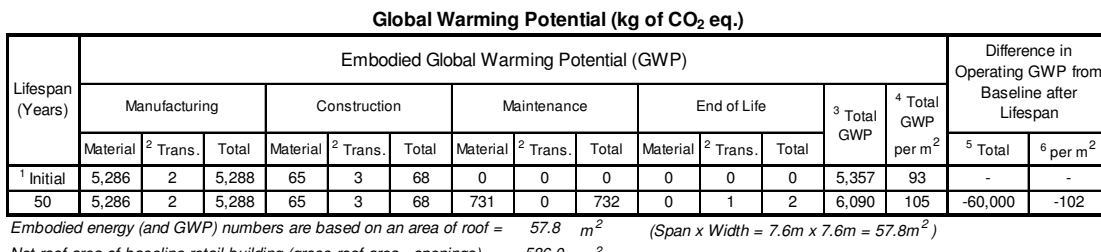

Net roof area of band GWP) numbers are based on an area of roof =

ATHENA ® EIE Material List:

\begin{tabular}{|c|c|c|}
\hline Material List & Quantities & Unit \\
\hline $\begin{array}{l}16 \mathrm{~mm} \text { Gypsum Fibre Gypsum } \\
\text { Board }\end{array}$ & 63.5 & $\mathrm{~m} 2$ \\
\hline 6 mil Polyethylene & 61.3 & $\mathrm{~m} 2$ \\
\hline Ballast (aggregate stone) & 249.6 & $\mathrm{~kg}$ \\
\hline Concrete $20 \mathrm{MPa}$ (flyash av) & 3.0 & $\mathrm{~m} 3$ \\
\hline Concrete $60 \mathrm{MPa}$ (flyasa av) & 5.7 & $\mathrm{~m} 3$ \\
\hline Extruded Polystyrene & 473.3 & $\mathrm{~m} 2(25 \mathrm{~mm})$ \\
\hline Galvanized Sheet & 134.3 & $\mathrm{~kg}$ \\
\hline Joint Compound & 63.4 & $\mathrm{~kg}$ \\
\hline Modified Bitumen membrane & 244.9 & $\mathrm{~kg}$ \\
\hline Nails & 4.2 & $\mathrm{~kg}$ \\
\hline Paper Tape & 0.7 & $\mathrm{~kg}$ \\
\hline PVC membrane & 294.3 & $\mathrm{~kg}$ \\
\hline Rebar, Rod, Light Sections & 197.2 & $\mathrm{~kg}$ \\
\hline Welded Wire Mesh / Ladder Wire & 88.4 & $\mathrm{~kg}$ \\
\hline
\end{tabular}

\section{LCA Data for Open Web Steel Joist Roofs}

This section contains a detailed description of each open web steel joist (OWSJ) roof that was examined in this study (11 in total). The assembly layers are listed for each roof, along with a detailed description of the material quantities from the ATHENA® Environmental Impact Estimator for Buildings.

A breakdown of the total primary energy consumption and the total global warming potential (GWP) for each roof is also included. In each case, the results were calculated for an area of roof equal to $69.2 \mathrm{~m}^{2}$, which represents a typical bay size for a single-storey retail building with this type of roof system. The results are also expressed on a per $\mathrm{m}^{2}$ basis in each case. The data has been calculated for two different lifespans: at the completion of initial construction and after 50 years.

As a summary, the figure below illustrates a comparison of the total embodied energy (and GWP) after 50 years for the various roofs in this section. For comparison purpose, the building component used in the baseline retail building has been shaded in grey.

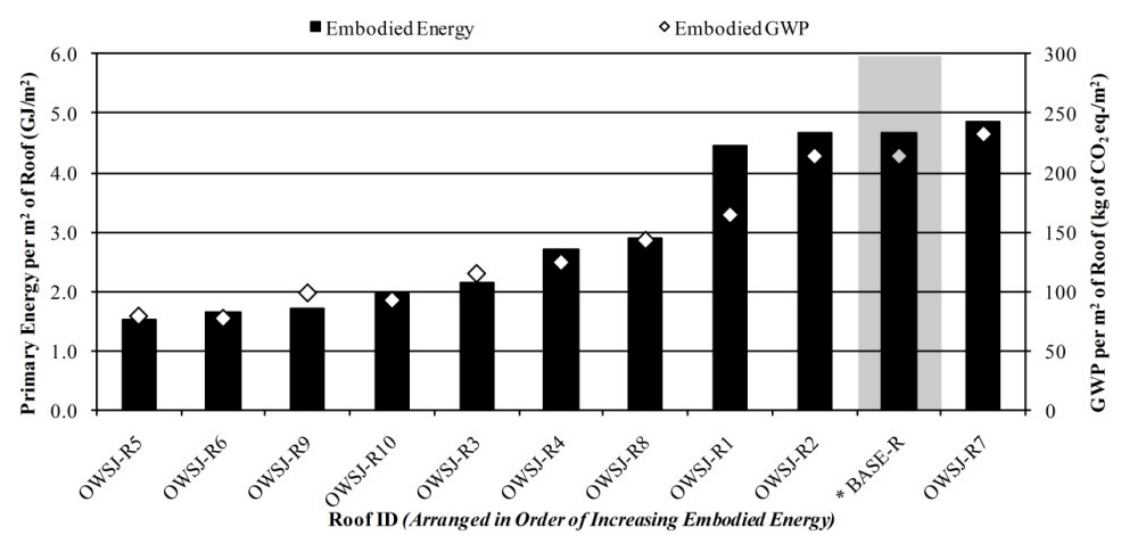




\section{Baseline Retail Building Roof (BASE-R)}

Building Component Description:

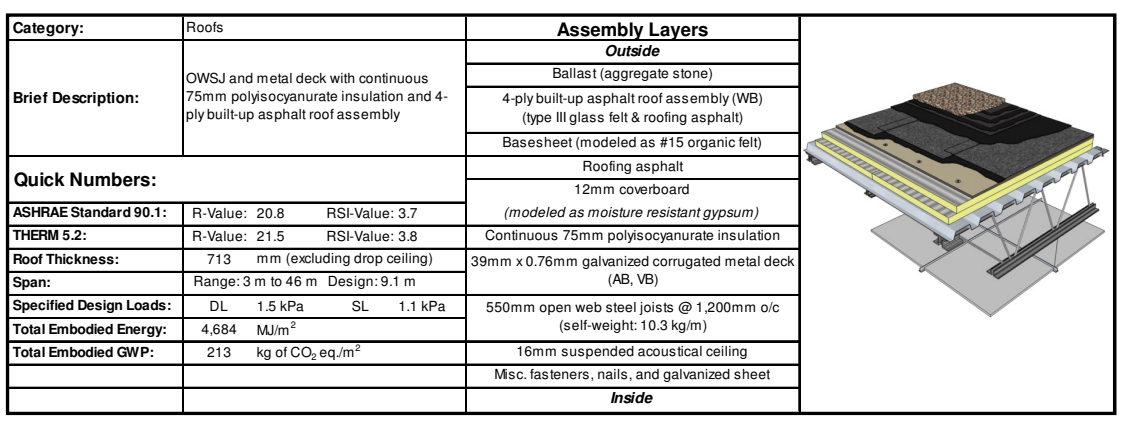

Life-Cycle Assessment Results:

Primary Energy Consumption (MJ)

\begin{tabular}{|c|c|c|c|c|c|c|c|c|c|c|c|c|c|c|c|c|}
\hline & \multirow{2}{*}{\multicolumn{14}{|c|}{ Embodied Energy (EE) }} & & \\
\hline \multirow{3}{*}{\begin{tabular}{|l} 
Lifespan \\
(Years)
\end{tabular}} & & & & & & & & & & & & & & & \multirow{2}{*}{\multicolumn{2}{|c|}{$\begin{array}{l}\text { Difference in } \\
\text { Operatitig Energy } \\
\text { from Baseline after } \\
\text { Lifespan }\end{array}$}} \\
\hline & \multicolumn{3}{|c|}{ Manutacturing } & \multicolumn{3}{|c|}{ Construction } & \multicolumn{3}{|c|}{ Maintenance } & \multicolumn{3}{|c|}{ End of Life } & \multirow{2}{*}{\begin{tabular}{|l|l|}
3 & Total \\
$\mathrm{EE}$ &
\end{tabular}} & \multirow{2}{*}{\begin{tabular}{|c|}
${ }^{4}$ Total \\
EE \\
Eer
\end{tabular}} & & \\
\hline & Material & ${ }^{2}$ Trans. & Total & \begin{tabular}{|l|l|} 
Material \\
\end{tabular} & ${ }^{2}$ Trans. & Total & Material & \begin{tabular}{|l}
2 Trans. \\
\end{tabular} & Total & Material & $\left.\right|^{2}$ Trans. & Total & & & ${ }^{5}$ Total & ${ }^{6}$ perm $m^{2}$ \\
\hline |nititial & 177,710 & 413 & 178,123 & 631 & \begin{tabular}{|l|l|}
1,051 \\
\end{tabular} & 1,683 & 0 & 0 & 0 & 0 & 0 & 0 & 179,805 & \begin{tabular}{|l|l|}
5 & 2,600 \\
\end{tabular} & & \\
\hline 50 & $|177,710|$ & \begin{tabular}{|l|l|}
413 \\
\end{tabular} & $\mid 178,122$. & \begin{tabular}{|l|}
631 \\
\end{tabular} & \begin{tabular}{|l|l|}
1,051 \\
\end{tabular} & \begin{tabular}{|l|l|}
1,683 \\
\end{tabular} & 143,295 & \begin{tabular}{|l|}
495 \\
\end{tabular} & \begin{tabular}{|l|l|}
143,790 \\
\end{tabular} & \begin{tabular}{l|l}
0 \\
0
\end{tabular} & 336 & 336 & 323,932 & \begin{tabular}{ll|}
24 & 4,684
\end{tabular} & 0 & 0 \\
\hline
\end{tabular}

Global Warming Potential ( $\mathrm{kg}$ of $\mathrm{CO}_{2}$ eq.)

\begin{tabular}{|c|c|c|c|c|c|c|c|c|c|c|c|c|c|c|c|c|}
\hline & \multirow{2}{*}{\multicolumn{14}{|c|}{ Embodied Global Warming Potential (GWP) }} & & \\
\hline \multirow{3}{*}{$\left|\begin{array}{c}\text { Lifespan } \\
\text { (Years) }\end{array}\right|$} & & & & & & & & & & & & & & & \multirow{2}{*}{\multicolumn{2}{|c|}{$\begin{array}{c}\text { Difference in } \\
\text { Operating GWP from } \\
\text { Baseline after } \\
\text { Lifespan }\end{array}$}} \\
\hline & \multicolumn{3}{|c|}{ Manufacturing } & \multicolumn{3}{|c|}{ Construction } & \multicolumn{3}{|c|}{ Maintenance } & \multicolumn{3}{|c|}{ End of Life } & \multirow{2}{*}{$\begin{array}{l}{ }^{3} \text { Total } \\
\text { GWP }\end{array}$} & \multirow{2}{*}{$\begin{array}{l}{ }^{4} \text { Total } \\
\text { GWP }\end{array}$} & & \\
\hline & \begin{tabular}{|l|l|} 
Material \\
\end{tabular} & Trans. & Total & aterial & Trans & Total & Material & Trans. & \begin{tabular}{|l|} 
Total \\
\end{tabular} & Material & Trans & Total & & & ${ }^{5}$ Total & 6 per m ${ }^{2}$ \\
\hline $\begin{array}{l}\text { 'Initial } \\
\end{array}$ & \begin{tabular}{|l|l|}
9,005 \\
\end{tabular} & 1 & 9,006 & 41 & 1 & 43 & 0 & 0 & 0 & 0 & 0 & 0 & 9,049 & 131 & & \\
\hline 50 & \begin{tabular}{|l|l|}
9,005 \\
\end{tabular} & 1 & $\begin{array}{l}9,006 \\
\end{array}$ & 41 & 1 & 43 & \begin{tabular}{|l|l|}
5,698 \\
\end{tabular} & 1 & \begin{tabular}{|l|l|}
5,6999 \\
\end{tabular} & 0 & 1 & 1 & 14,748 & 213 & 0 & 0 \\
\hline
\end{tabular}

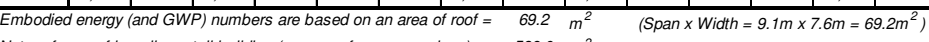

Net roof area of baseline retail building (gross roof area - openings) $=586.0 \mathrm{~m}^{2}$

ATHENA $₫$ EIE Material List:

\begin{tabular}{|c|c|c|}
\hline Material List & | Quantitites & Unit \\
\hline \#15 Organic Felt & 8551.9 & $\frac{\mathrm{m} 2}{\mathrm{~m} 2}$ \\
\hline 13mm Moisture Resistant Gypsum & 76.1 & $\mathrm{~m} 2$ \\
\hline $\begin{array}{l}\begin{array}{l}16 m m \\
\text { Board }\end{array} \\
\text { Bypsum Fibre Gypsum }\end{array}$ & 76.1 & m2 \\
\hline Ballast (aggregate stone) & $5,274.4$ & $\mathrm{~kg}$ \\
\hline Galvanized Decking & 684.5 & $\mathrm{~kg}$ \\
\hline Galvanized Sheet & 243.0 & $\mathrm{~kg}$ \\
\hline Isocyanurate & 214.6 & $\mathrm{~m} 2(25 \mathrm{~mm})$ \\
\hline Joint Compound & 75.9 & $\mathrm{~kg}$ \\
\hline Nails & 36.3 & $\mathrm{~kg}$ \\
\hline Open Web Joists & 596.4 & $\mathrm{~kg}$ \\
\hline Paper Tape & 0.9 & $\mathrm{~kg}$ \\
\hline Roofing Asphalt & $2,093.8$ & $\mathrm{~kg}$ \\
\hline Type III Glass Felt & $\begin{array}{ll}1,103.8 \\
\end{array}$ & $\mathrm{~m}^{2}$ \\
\hline
\end{tabular}

\section{Open Web Steel Joist Roof \#1 (OWSJ-R1)}

Building Component Description:

\begin{tabular}{|c|c|c|c|}
\hline Category: & Roofs & Assembly Layers & \\
\hline \multirow{3}{*}{ Brief Description: } & \multirow{3}{*}{ 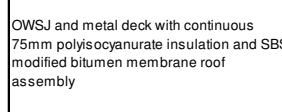 } & $\begin{array}{l}\text { Outside } \\
\text { SPS }\end{array}$ & \\
\hline & & \begin{tabular}{|ll} 
Roofing asphalt \\
\end{tabular} & \\
\hline & & Basesheet (modeled as \#15 organic felt) & \\
\hline \multirow{2}{*}{\multicolumn{2}{|c|}{ Quick Numbers: }} & $\overline{12 \mathrm{~mm} \text { coverboard }}$ & \\
\hline & & (modeled as moisture resistant typsum) & \\
\hline \begin{tabular}{|l|} 
ASHRAE Standard 90.1: \\
\end{tabular} & R-Value: 20.8 & Continuous $75 \mathrm{~mm}$ polyys ocyanurate insulation & \\
\hline THERM 5.2: & $\begin{array}{|ll|}\text { R-Value: } 21.3 & \text { RSI-Value: } 3.7 \\
\end{array}$ & $39 \mathrm{~mm} \times 0.76 \mathrm{~mm}$ galvanized corrugated metal deck & \\
\hline Roof Thickness: & $688 \quad \mathrm{~mm}$ (excluding drop ceiling) & (AB, VB) & \\
\hline $\begin{array}{l}\text { Span: } \\
\text { Specified Design Lads: }\end{array}$ & $\begin{array}{ll}\text { Range: } 3 \mathrm{~m} \text { to } 46 \mathrm{~m} \text { Design: } 9.1 \mathrm{~m} \\
\mathrm{SI}\end{array}$ & 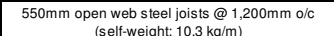 & \\
\hline Specified Design Loads: & $\begin{array}{ll}\mathrm{DL} & 1.1 \mathrm{kPa} \\
\end{array}$ & (sell-weight: $10.3 \mathrm{~kg} / \mathrm{m}$ ) & \\
\hline \begin{tabular}{|l} 
Total Embodied Energy: \\
\end{tabular} & $\begin{array}{ll}4.464 \quad \mathrm{~m} / \mathrm{m}^{2} \\
164\end{array}$ & 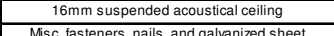 & \\
\hline Total Embodied GWP: & $164 \mathrm{~kg}$ of $\mathrm{CO}_{2} \mathrm{eq} / \mathrm{m}^{2}$ & $\begin{array}{l}\text { Misc. fasteners, nails, and galvanized sheet } \\
\text { Inside }\end{array}$ & \\
\hline
\end{tabular}

\section{Life-Cycle Assessment Results:}

\begin{tabular}{|c|c|c|c|c|c|c|c|c|c|c|c|c|c|c|c|}
\hline & \multicolumn{13}{|c|}{ Primary Energy Consumption (MJ) } & \multirow{3}{*}{\multicolumn{2}{|c|}{$\begin{array}{l}\text { Difference in } \\
\text { Operating Energy } \\
\text { from Baseline after } \\
\text { Lifespan }\end{array}$}} \\
\hline \multirow{3}{*}{$\begin{array}{l}\text { Lifespa } \\
\text { (Years) }\end{array}$} & & & & & & bodied & Energy & & & & & & & & \\
\hline & \multicolumn{2}{|c|}{ Manutacturing } & \multicolumn{3}{|c|}{ Construction } & \multicolumn{3}{|c|}{ Maintenance } & \multicolumn{3}{|c|}{ End of Lifie } & \multirow{2}{*}{$\begin{array}{c}{ }^{3} \text { Total } \\
\text { EE }\end{array}$} & \multirow{2}{*}{\begin{tabular}{|c|}
${ }^{4} \mathrm{Total}$ \\
$\mathrm{EE}$ \\
per m
\end{tabular}} & & \\
\hline & 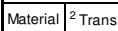 & Total & Material & ${ }^{2}$ Trans. & Total & Material & ${ }^{2}$ Trans. & Total & Material & $\left.1\right|^{2}$ Trans. & Total & & & ${ }^{5}$ Total & ${ }^{6} \operatorname{perm}^{2}$ \\
\hline${ }^{1}$ Initial & \begin{tabular}{|l|l|}
154,426 & 360 \\
\end{tabular} & 154,786 & 631 & 1,160 & 1,791 & 0 & 0 & 0 & 0 & 0 & 0 & 156,577 & \begin{tabular}{r|r|}
7,264 \\
\end{tabular} & & \\
\hline 50 & \begin{tabular}{|l|l|}
154,426 & 360 \\
\end{tabular} & $\mid 154,786$ & \begin{tabular}{|l|l|}
631 \\
\end{tabular} & 1,160 & 1,791 & 151,547 & 309 & 151,856 & 0 & 292 & 292 & 308,726 & \begin{tabular}{|l|l|}
6 & 4,464 \\
\end{tabular} & - & -171 \\
\hline
\end{tabular}

\begin{tabular}{|c|c|c|c|c|c|c|c|c|c|c|c|c|c|c|c|c|}
\hline \multirow{4}{*}{$\begin{array}{l}\text { Lestespan } \\
\text { (Years) }\end{array}$} & \multicolumn{14}{|c|}{ Global Warming Potential $\left(\mathrm{kg}\right.$ of $\mathrm{CO}_{2} \mathrm{e}$} & & \\
\hline & & & & & Embo & iled Gl & bal War & ming $\mathrm{Po}$ & tential & (GWP) & & & & & \multirow{2}{*}{\multicolumn{2}{|c|}{$\begin{array}{c}\text { Difference in } \\
\text { Operating GWP from } \\
\text { Baseline after } \\
\text { Lifespan }\end{array}$}} \\
\hline & \multicolumn{3}{|c|}{ Manutacturing } & \multicolumn{3}{|c|}{ Construction } & \multicolumn{3}{|c|}{ Maintenance } & \multicolumn{3}{|c|}{ End of Life } & \multirow{2}{*}{$\begin{array}{l}{ }^{3} \text { Total } \\
\text { GWP }\end{array}$} & \multirow{2}{*}{$\begin{array}{l}{ }^{4} \text { Total } \\
\text { GWP } \\
\text { per m}\end{array}$} & & \\
\hline & Material & ${ }^{2}$ Trans. & Total & Material & ${ }^{2}$ Trans. & Total & Material & ${ }^{2}$ Trans. & $\begin{array}{c}\text { Total } \\
\end{array}$ & Material & ${ }^{2}$ Trans. & Total & & & ${ }^{5}$ Total & $\begin{array}{ll}{ }^{6} \mathrm{perm}^{2} \\
\end{array}$ \\
\hline${ }^{1 \text { Initial }}$ & 7,258 & 1 & 7,259 & 41 & 1 & 43 & 0 & 0 & 0 & 0 & 0 & 0 & 7,301 & 106 & & \\
\hline 50 & \begin{tabular}{|l|l|}
7,258 \\
\end{tabular} & 1 & 7,259 & 41 & 1 & 43 & $\begin{array}{l}4,032 \\
\end{array}$ & 1 & 4,033 & 0 & 1 & 1 & 11,335 & 164 & 0 & 0 \\
\hline
\end{tabular}

Embodied energy (and GWP) numbers are based on an area of roof $=\begin{array}{lllllllll}69.2 & \mathrm{~m}^{2} & 0 & 1 & 1 & 11,335 & 164 \\ \text { (Span } \times \text { Width }=9.1 \mathrm{~m} \times 7.6 \mathrm{~m}=69.2 \mathrm{~m}^{2} \text { ) }\end{array}$ Net roof area of baseline retal bullaing (gross roof area - openings) $=586.0 \mathrm{~m}^{2}$

\section{ATHENA $®$ EIE Material List:}

${ }^{1}$ Initial $=$ Time 'o' (i.e. at
${ }^{2}$ Trans. $=$ Transportation
${ }^{2}$ Tol

${ }^{3}$ Total
of (of (or Total GWP) $=$ Total embodided energy (or total embodied GWP

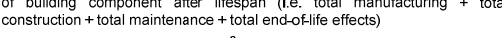

Total EE (or Total GWP) per $\mathrm{m}^{2}=$ Total EE (or Total GWP) of building
component/ area of builing component that was modelled in ATHENAQ EIE ${ }^{5}$ Total Difference in Operating Energy (or GWP) from Baseline after Total Difference in operating Energy (or GWP) from Baseline after
Lifespan = The eifference in the total lifecyle operating energy (or GWP)
from the baseline retail building after lifespan, due to using this building Total Difference in operating Energy (or GWP) from Baseline after

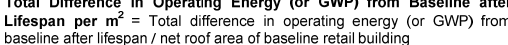

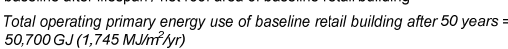

"Total operating GWP of baseline retal

\begin{tabular}{|l|c|c|}
\hline \multicolumn{1}{|c|}{ Material List } & Quantities & Unit \\
\hline \#15 Organic Felt & 551.9 & $\mathrm{~m} 2$ \\
\hline $\begin{array}{l}13 \mathrm{~mm} \text { Moisture Resistant Gypsum } \\
\text { Board }\end{array}$ & 76.1 & $\mathrm{~m} 2$ \\
\hline $\begin{array}{l}\text { 16mm Gypsum Fibre Gypsum } \\
\text { Board }\end{array}$ & 76.1 & $\mathrm{~m} 2$ \\
\hline Galvanized Decking & 684.5 & $\mathrm{~kg}$ \\
\hline Galvanized Sheet & 186.2 & $\mathrm{~kg}$ \\
\hline Isocyanurate & 214.6 & $\mathrm{~m} 2(25 \mathrm{~mm})$ \\
\hline Joint Compound & 75.9 & $\mathrm{~kg}$ \\
\hline Modified Bitumen membrane & $2,219.6$ & $\mathrm{~kg}$ \\
\hline Nails & 36.3 & $\mathrm{~kg}$ \\
\hline Open Web Joists & 596.4 & $\mathrm{~kg}$ \\
\hline Paper Tape & 0.9 & $\mathrm{~kg}$ \\
\hline Roofing Asphalt & $1,151.6$ & $\mathrm{~kg}$ \\
\hline
\end{tabular}

'Initial = Time 'o' (ie. at the cotes:

${ }^{2}$ Trans. = Transportation

${ }^{3}$ Total EE (or Total GWP) $=$ Total embodied energy (or total embodied GWP) of building component after lifespan (i.e. total manufacturing + to

${ }^{4}$ Total EE (or Total GWP) per $\mathrm{m}^{2}=$ Total EE (or Total GWP) of building
component /area of building component that was modelled in ATHENAQ EIE ${ }^{5}$ Total Difference in Operating Energy (or GWP) from Baseline after Lifespan = The difference in the total life-cycle operating energy (or GWP)
from the baseline retail building after lifespan, due to using this building

Lotal Difference in Operating Energy (or GWP) from Baseline after
Lifespan per $\mathrm{m}^{2}=$ Total difference in operating energy (or GWP) from Lifespan per $\mathrm{m}^{2}=$ Total difiterence in operating energy (or
baseline after lifespan / net rof area of baseline retail building Total operating primary energy use of baseline retail building after 50 years $=$ * Total operating $\mathrm{GWP}$ of baseline retail building after 50 years $=2,310$ tonnes
of $\mathrm{CO}_{2} \mathrm{eq}\left(8 \mathrm{~kg}\right.$ of $\left.\mathrm{CO}_{2} \mathrm{eq} / \mathrm{m}^{2} \mathrm{Ar}\right)$ 
Open Web Steel Joist Roof \#2 (OWSJ-R2)

Building Component Description:

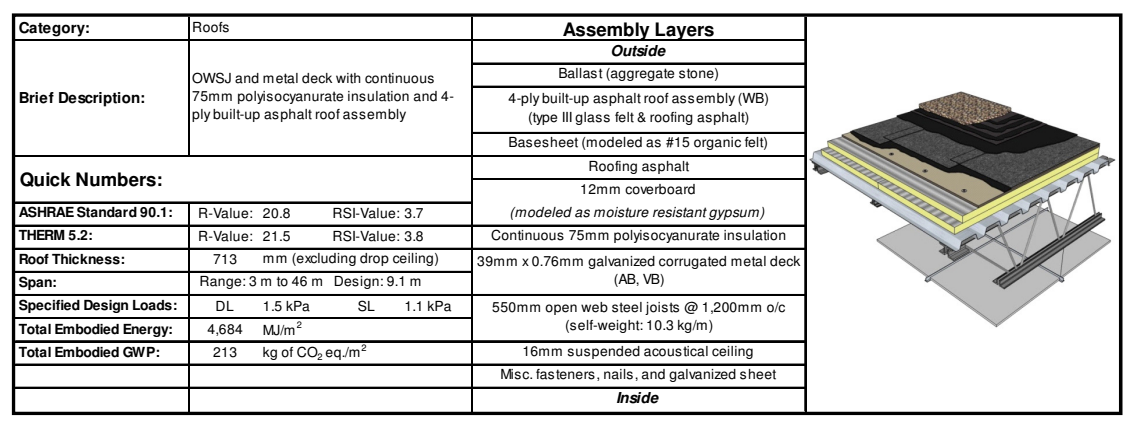

Life-Cycle Assessment Results:

Primary Energy Consumption (MJ)

\begin{tabular}{|c|c|c|c|c|c|c|c|c|c|c|c|c|c|c|c|}
\hline \multirow{3}{*}{ 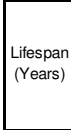 } & \multicolumn{13}{|c|}{ Embodied Energy (EE) } & \multirow{2}{*}{\multicolumn{2}{|c|}{$\begin{array}{c}\text { Difference in } \\
\text { Operating Energy } \\
\text { from Baseline afte } \\
\text { Lifespan }\end{array}$}} \\
\hline & \multicolumn{2}{|c|}{ Manufacturing } & \multicolumn{3}{|c|}{ Construction } & \multicolumn{3}{|c|}{ Maintenance } & \multicolumn{3}{|c|}{ End of L Life } & \multirow{2}{*}{$\begin{array}{c}{ }^{3} \text { Total } \\
\mathrm{EEE}\end{array}$} & \multirow{2}{*}{\begin{tabular}{|c|}
${ }^{4}$ Total \\
EE \\
per $\mathrm{m}^{2}$
\end{tabular}} & & \\
\hline & \begin{tabular}{|l|l|} 
Material & ${ }^{2}$ Trans. \\
\end{tabular} & Total & Materia & ${ }^{2}$ Trans. & Total & Material & ${ }^{2}$ Trans. & \begin{tabular}{|l|l|} 
Total \\
\end{tabular} & Material & \begin{tabular}{|l|}
${ }^{2}$ Trans. \\
\end{tabular} & Total & & & ${ }^{5}$ Total & ${ }^{6}$ per $\mathrm{m}^{2}$ \\
\hline IInitial & \begin{tabular}{|c|c|c|}
177,710 & 413
\end{tabular} & $\begin{array}{l}178,123 \\
\end{array}$ & 631 & \begin{tabular}{|l|}
1,051 \\
\end{tabular} & 1,683 & 0 & 0 & 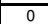 & 0 & 0 & 0 & $\begin{array}{l}179,805 \\
\end{array}$ & $\begin{array}{l}52,600 \\
5\end{array}$ & & \\
\hline
\end{tabular}

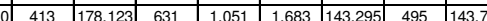

\begin{tabular}{|c|c|c|c|c|c|c|c|c|c|c|c|c|c|c|c|c|}
\hline \multirow{4}{*}{\begin{tabular}{|} 
Litespan \\
(Years)
\end{tabular}} & \multicolumn{16}{|c|}{ Gl } \\
\hline & \multicolumn{14}{|c|}{ Embodied Global Warming Potential (GWP) } & \multirow{2}{*}{\multicolumn{2}{|c|}{$\begin{array}{c}\text { Difference in } \\
\text { Operating GWP fron } \\
\text { Baseline after } \\
\text { Lifespan }\end{array}$}} \\
\hline & \multicolumn{3}{|c|}{ Manutacturing } & \multicolumn{3}{|c|}{ Construction } & \multicolumn{3}{|c|}{ Maintenance } & \multicolumn{3}{|c|}{ End of Life } & \multirow{2}{*}{$\mid \begin{array}{l}3 \\
3 \\
\text { Total } \\
\text { GWP }\end{array}$} & \multirow{2}{*}{$\begin{array}{l}{ }^{4} \text { Total } \\
\text { GWP } \\
\text { per m }\end{array}$} & & \\
\hline & Material & ${ }^{2}$ Trans. & Total & Material & 1) ${ }^{2}$ Trans. & Total & Material & ${ }^{2}$ Trans. & Total & Material & 1. ${ }^{2}$ Trans. & Total & & & ${ }^{5}$ Total & ${ }^{6} \mathrm{perm}^{2}$ \\
\hline 1 Initial & 9,005 & 1 & 9,006 & 41 & 1 & 43 & 0 & 0 & 0 & 0 & 0 & 0 & 9,049 & 131 & & \\
\hline 50 & 9,005 & 1 & 9,006 & 41 & 1 & 43 & 5,698 & 1 & 5,699 & 0 & 1 & 1 & 14,748 & 213 & 0 & 0 \\
\hline
\end{tabular}

Embodied energy (and GWP) numbers are based on an area of roof $=69.2 \mathrm{~m}^{2} \quad\left(\right.$ Span $\times$ Width $=9.1 \mathrm{~m} \times 7.6 \mathrm{~m}=69.2 \mathrm{~m}^{2}$ )

\section{ATHENA $®$ EIE Material List:}

\begin{tabular}{|c|c|c|}
\hline Material List & Quantities & Unit \\
\hline$\# 15$ Organic Felt & 551.9 & $\mathrm{~m} 2$ \\
\hline $\begin{array}{l}\text { 13mm Moisture Resistant Gypsum } \\
\text { Bagrd }\end{array}$ & 76.1 & $\mathrm{~m} 2$ \\
\hline $\begin{array}{l}16 \text { mimm Gypsum Fibre Gypsum } \\
\text { Board }\end{array}$ & 76.1 & m2 \\
\hline Ballast (aggregate stone) & $5,274.4$ & $\mathrm{~kg}$ \\
\hline Galvanized Decking & 684.5 & $\mathrm{~kg}$ \\
\hline Galvanized Sheet & 243.0 & $\mathrm{~kg}$ \\
\hline Isocyanurate & 214.6 & $\mathrm{~m} 2(25 \mathrm{~mm})$ \\
\hline Joint Compound & 75.9 & $\mathrm{~kg}$ \\
\hline $\begin{array}{lll}\text { Nalls } \\
\end{array}$ & 36.3 & $\mathrm{~kg}$ \\
\hline Open Web Joists & 596.4 & $\mathrm{kg}$ \\
\hline Paper Tape & 0.9 & $\mathrm{~kg}$ \\
\hline Roofing Asphalt & $2,093.8$ & $\mathrm{~kg}$ \\
\hline Type III Glass Felt & $1,103.8$ & $\mathrm{~m} 2$ \\
\hline
\end{tabular}

\section{Open Web Steel Joist Roof \#3 (OWSJ-R3)}

Building Component Description:

\begin{tabular}{|c|c|c|c|}
\hline \multirow{4}{*}{ Brief Description: } & Roots & Assembly Layers & \\
\hline & \multirow{3}{*}{$\begin{array}{l}\text { OWSJ and metal deck with continuous } \\
75 m m \text { polysisocyanurate insulation and } \\
\text { EPDM roof assembly }\end{array}$} & $\begin{array}{l}\text { Outside } \\
\text { Ballast tagargate stone) }\end{array}$ & \\
\hline & & $\begin{array}{l}\text { EPDM cap sheet (WB) } \\
\text { Encter }\end{array}$ & \\
\hline & & Bonding agent (modeled as 6 mil poly) & \\
\hline \multicolumn{2}{|l|}{ Quick Numbers: } & $12 \mathrm{~mm}$ coverboard & \\
\hline ASHRAE Standard 90.1: & & (modeled as moisture resistant gypsum) & \\
\hline & 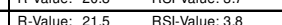 & Continuous $/ 5 \mathrm{~mm}$ polys ocoyanurateit insulation & \\
\hline \begin{tabular}{|l} 
Roof Thickness: \\
\end{tabular} & 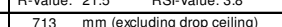 & $39 \mathrm{~mm} \times 0.76 \mathrm{~mm}$ galvanaized corrugated metal deck & \\
\hline Span: & Range: $3 \mathrm{~m}$ to $46 \mathrm{~m}$ Design: $9.1 \mathrm{~m}$ & 550mm open web steel joists @ @1,200mm o/c & \\
\hline Specified Design Loads: & $\begin{array}{ll}\mathrm{DL} & 1.5 \mathrm{kPa} \\
\end{array}$ & (self-weight: $10.3 \mathrm{~kg} / \mathrm{m}$ ) & \\
\hline & $2,162 \quad \mathrm{~m} / \mathrm{m}^{2}$ & $16 \mathrm{~mm}$ suspended acoustical celiling & \\
\hline Total Embodied GWP: & $115 \mathrm{~kg}$ of $\mathrm{CO}_{2} \mathrm{eq} / \mathrm{m}^{2}$ & Misc. fasteners, nails, and gavanized sheet & \\
\hline
\end{tabular}

\section{Life-Cycle Assessment Results:}

\begin{tabular}{|c|c|c|c|c|c|c|c|c|c|c|c|c|c|c|c|c|}
\hline \multirow{4}{*}{\begin{tabular}{|} 
Lifiespan \\
(Years)
\end{tabular}} & \multicolumn{14}{|c|}{ Primary Energy Consumption (MJ) } & \multirow{3}{*}{\multicolumn{2}{|c|}{$\begin{array}{l}\text { Difference in } \\
\text { Operating Energy } \\
\text { from Baseline after } \\
\text { Lifespan }\end{array}$}} \\
\hline & & & & & & & bodied E & Energy ( & & & & & & & & \\
\hline & \multicolumn{3}{|c|}{ Manufacturing } & \multicolumn{3}{|c|}{ Construction } & \multicolumn{3}{|c|}{ Maintenance } & \multicolumn{3}{|c|}{ End of Lifie } & \multirow{2}{*}{$\begin{array}{c}{ }^{3} \text { Total } \\
\text { EE }\end{array}$} & \multirow{2}{*}{\begin{tabular}{|c|}
${ }^{4}$ Total \\
$E E$ \\
$E E$
\end{tabular}} & & \\
\hline & 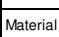 & ${ }^{2}$ Trans. & Total & Material & $\begin{array}{ll}2 & 2 \\
\text { Trans. }\end{array}$ & Total & Material & 1. ${ }^{2}$ Trans. & Total & Material & al ${ }^{2}$ Trans. & Total & & & ${ }^{5}$ Total & ${ }^{6}$ per m$^{2}$ \\
\hline${ }^{1}$ Initial & 87,438 & 413 & 87,852 & 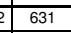 & 1,385 & 2,016 & 0 & 0 & 0 & 0 & 0 & 0 & 89,868 & 1,299 & & \\
\hline 50 & 87,438 & 413 & 87,852 & 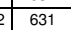 & 1,385 & 2,016 & 58,565 & 696 & 59,261 & 1 & 380 & 381 & 149,510 & 2,162 & $-100,000$ & -171 \\
\hline
\end{tabular}

\begin{tabular}{|c|c|c|c|c|c|c|c|c|c|c|c|c|c|c|c|c|}
\hline & \multirow{2}{*}{\multicolumn{14}{|c|}{$\begin{array}{l}\text { Global Warming Potential (kg of } \mathrm{CO}_{2} \text { eq.) } \\
\text { Embodied Global Warming Potential (GWP) }\end{array}$}} & & \\
\hline \multirow{3}{*}{$\begin{array}{l}\text { Lifespan } \\
\text { (Yyars) }\end{array}$} & & & & & & & & & & & & & & & \multirow{2}{*}{\multicolumn{2}{|c|}{\begin{tabular}{|c|} 
Difference in \\
Operatitig GWP from \\
Baseline after \\
Lifespan
\end{tabular}}} \\
\hline & \multicolumn{3}{|c|}{ Manufacturing } & \multicolumn{3}{|c|}{ Construction } & \multicolumn{3}{|c|}{ Maintenance } & \multicolumn{3}{|c|}{ End of Life } & \multirow{2}{*}{$\begin{array}{l}{ }^{3} \text { Total } \\
\text { GWP }\end{array}$} & \multirow{2}{*}{$\begin{array}{c}{ }^{4} \text { Total } \\
\text { GWP } \\
\text { per } \mathrm{m}^{2}\end{array}$} & & \\
\hline & \begin{tabular}{|l|} 
Material \\
\end{tabular} & ${ }^{2}$ Trans. & Total & Material & ${ }^{2}$ Trans. & Total & \begin{tabular}{|l|l|} 
Material \\
\end{tabular} & Trans. & Total & \begin{tabular}{|l|} 
Material \\
\end{tabular} & ${ }^{2}$ Trans. & \begin{tabular}{|l|l|} 
Total \\
\end{tabular} & & & ${ }^{5}$ Total & ${ }^{6}{ }^{6}$ erm ${ }^{2}$ \\
\hline${ }^{1}$ Initial & 5,294 & 1 & 5,295 & 41 & 2 & 43 & 0 & 0 & 0 & 0 & 0 & 0 & 5,338 & 77 & & \\
\hline 50 & \begin{tabular}{|l|l|}
5,294 \\
\end{tabular} & 1 & 5,295 & 41 & 2 & 43 & 2,589 & 1 & 2,590 & 0 & 1 & 1 & \begin{tabular}{|l|l}
7,929 \\
\end{tabular} & 115 & 0 & 0 \\
\hline
\end{tabular}

Embodied energy (and GWP) numbers are based on an area of roof $=69.2 \mathrm{~m}^{2} \quad$ (Span $\times$ Width $=9.1 \mathrm{~m} \times 7.6 \mathrm{~m}=69.2 \mathrm{~m}^{2}$ )

Net roof area of baseline retail building (gross roof area - openings) $=586.0 \mathrm{~m}^{2}$
ATHENA $₫$ EIE Material List:

\begin{tabular}{|c|c|c|}
\hline Material List & \begin{tabular}{|l|l} 
Quantities \\
\end{tabular} & Unit \\
\hline $\begin{array}{l}13 \mathrm{~mm} \text { Moisture Resistant Gy } \\
\text { Baard }\end{array}$ & 76.1 & $\mathrm{~m} 2$ \\
\hline $16 \mathrm{~mm}$ Gypsum Fibre Gypsum & 76.1 & $\mathrm{~m} 2$ \\
\hline 6 mil Polyethylene & 70.5 & $\mathrm{~m} 2$ \\
\hline Ballast (aggregate stone) & $\begin{array}{ll}15,823.1 \\
\end{array}$ & $\mathrm{~kg}$ \\
\hline EPDM membrane & 568.0 & $\mathrm{~kg}$ \\
\hline Galvanized Decking & 684.5 & $\mathrm{~kg}$ \\
\hline Galvanized Sheet & 190.6 & $\mathrm{~kg}$ \\
\hline Isocyanurate & 214.6 & $2(25 \mathrm{mr}$ \\
\hline Joint Compound & 151.9 & $\mathrm{~kg}$ \\
\hline Nails & 7.1 & $\mathrm{~kg}$ \\
\hline Open Web Joists & 596.4 & $\mathrm{~kg}$ \\
\hline Paper Tape & 1.7 & $\mathrm{~kg}$ \\
\hline $\begin{array}{l}\text { Small Dimension Sottwood Lumber, } \\
\text { kiln-dred }\end{array}$ & 0.2 & $\mathrm{~m} 3$ \\
\hline Softwood Plywood & 1.4 & $n 2(9 n$ \\
\hline
\end{tabular}

$$
\begin{aligned}
& \text { Notes: } \\
& { }^{1} \text { Initial }=T \text { Time ' } O^{\prime} \text { (i.e. at the completion of initial construction) }
\end{aligned}
$$

${ }^{2}$ Trans $=$ Transportation

Total EE (or Total GWP) $=$ Total embodied energy (or total embodied GWP)
of building component after lifespan (i.e. total manufacturing + total

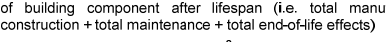
Total EE (or Total GWP) per $\mathrm{m}^{2}=$ Total EE (or Total GWP) of building
component/ area of building component that was modedled in ATHENA@ EIE ${ }^{5}$ Total Difference in Operating Energy (or GWP) from Baseline after
Lifespan = The difference in the total ile-cycle operating energy (or GWPP)
from the baseline retail building after fifespan, due to using this building ${ }^{6}$ Total Difference in Operating Energy (or GWP) from Baseline after
Lifespan per $\mathrm{m}^{2} \stackrel{\text { Ontal }}{=}$ Total difference in operating energy (or GWP) from Lifespan per $\mathrm{m}^{2}=$ Total difference in operating energy (or $\mathrm{GWP}$ ) from
baseline affer lifespan $/$ net roof area of baseline retail building * Total operating primary energy use of baseline retail building after 50 years =
$50,700 \mathrm{GJ}\left(1,745 \mathrm{MJ} / \mathrm{m}^{2} \mathrm{y} r\right)$

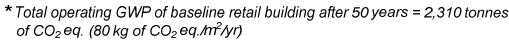

* Total operating primary energy use of baseline retail building after 50 years $=$
$50,700 \mathrm{GJ}\left(1,745 \mathrm{MJ} / \mathrm{m}^{2} \mathrm{y} y \mathrm{r}\right)$

*Total operating $\mathrm{GWP}$ of baseline retail building after 50 years $=2,310$ tonnes
of $\mathrm{CO}_{2}$ eq. $\left(8 \mathrm{Bg}\right.$ of $\left.\mathrm{CO}_{2} e q / \mathrm{h}^{2} / \mathrm{yr}\right)$
'Initial $=$ Time $0^{\prime}$ (i.e. at 1 .
${ }^{2}$ Trans. $=$ Transportation

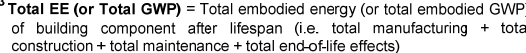

Total EE (or Total GWP) per $\mathrm{m}^{2}=$ Total EE (or Total GWP) of building
component / area of building component that was modelled in ATHENAB EIE from the baseline retail building after lifespan, due to using this building 


\section{Open Web Steel Joist Roof \#4 (OWSJ-R4)}

Building Component Description:

\begin{tabular}{|c|c|c|c|}
\hline \multirow{4}{*}{ Brief Description: } & Roofs & Assembly Layers & \\
\hline & \multirow{2}{*}{$\begin{array}{l}\text { owSJ and metal deck with continuous } \\
75 m m \text { polysisocyanurate insulation and PVV } \\
\text { roof assemmly }\end{array}$} & $\begin{array}{c}\text { Outside } \\
\text { Ballast (agregate stone) }\end{array}$ & \\
\hline & & $\begin{array}{l}\text { PVC membrane cap sheet(WB) } \\
\text { (unculdes oood naling strip) }\end{array}$ & \\
\hline & & Bonding agent (modeled as 6mil poly) & \\
\hline \multicolumn{2}{|l|}{ Quick Numbers: } & $\begin{array}{l}12 \mathrm{~mm} \text { coverboard } \\
\text { (modeled as o oistur ersistant gyosum) }\end{array}$ & \\
\hline \begin{tabular}{|l} 
ASHRAE Standard 90.1: \\
\end{tabular} & R-Value: 20.8 & Continuous $75 \mathrm{~mm}$ polyisocyanurate insulation & \\
\hline THERM 5.2: & $\begin{array}{|lr|}\text { R-Value: } 21.5 & \text { RSIVValue } 3.8 \\
\end{array}$ & $39 \mathrm{~mm} \times 0.76 \mathrm{~mm}$ galvanized corrugated metal dech & \\
\hline Roof Thickness: & $713 \mathrm{~mm}$ (excluding drop ceiling) & (AB, VB) & \\
\hline \begin{tabular}{|l} 
Span: \\
Specified Design Loads:
\end{tabular} & 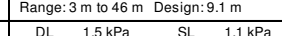 & $\begin{array}{c}550 \mathrm{~mm} \text { open web tseet jiosts @ @ 1,200mm o/c } \\
\text { (self-weight } 1.0 .3 \mathrm{~kg} / \mathrm{m} \text { ) }\end{array}$ & \\
\hline $\begin{array}{l}\text { Total Embodied Energy: } \\
\end{array}$ & $2,717 \quad \mathrm{M} / \mathrm{m}^{2}$ & $16 \mathrm{~mm}$ suspended acoustical ceiling & \\
\hline Total Embodied GWP: & $124 \quad \mathrm{~kg}$ of $\mathrm{CO}_{2} \mathrm{eq} / \mathrm{m}^{2}$ & Misc. fasteners, nails, and galvanized sheet & \\
\hline
\end{tabular}

\section{Life-Cycle Assessment Results:}

Primary Energy Consumption (MJ)

\begin{tabular}{|c|c|c|c|c|c|c|c|c|c|c|c|c|c|c|c|c|}
\hline \multirow{3}{*}{$\begin{array}{l}\text { LLessan } \\
\text { (Years) }\end{array}$} & \multicolumn{14}{|c|}{ Embodied Energy (EE) } & \multirow{2}{*}{\multicolumn{2}{|c|}{$\begin{array}{c}\text { Difference in } \\
\text { Operating Energy } \\
\text { from Baseline atte } \\
\text { Lifespan }\end{array}$}} \\
\hline & \multicolumn{3}{|c|}{ Manufacturing } & \multicolumn{3}{|c|}{ Construction } & \multicolumn{3}{|c|}{ Maintenance } & \multicolumn{3}{|c|}{ End of Life } & \multirow{2}{*}{${ }^{3}{ }^{3}$ Total } & \multirow{2}{*}{$\begin{array}{c}{ }^{4} \text { Total } \\
\text { EE } \\
\text { per } \mathrm{m}^{2}\end{array}$} & & \\
\hline & Material & ${ }^{2}$ Trans. & \begin{tabular}{|l|} 
Total \\
\end{tabular} & Materia & ${ }^{2}$ Trans. & \begin{tabular}{|l|} 
Total \\
\end{tabular} & Material & $\left.\right|^{2}$ Trans. & Total & Material & al| $\left.\right|^{2}$ Trans. & Total & & & ${ }^{5}$ Total & ${ }^{6}$ per $\mathrm{m}^{2}$ \\
\hline Initial & 95,885 & 448 & 96,333 & 631 & 1,561 & 2,192 & 0 & 0 & 0 & 0 & 0 & 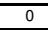 & 98,525 & 1,425 & & \\
\hline 50 & 95,885 & \begin{tabular}{|l|l|l|}
448 \\
\end{tabular} & $\begin{array}{l}996,333 \\
\end{array}$ & 631 & 1,561 & \begin{tabular}{|l|l|}
2,192 \\
\end{tabular} & 88,088 & 870 & 88,958 & 1 & 397 & 397 & 187,880 & 2,717 & $\begin{array}{l}-100,000 \\
\end{array}$ & 171. \\
\hline
\end{tabular}

Global Warming Potential ( $\mathrm{kg}$ of $\mathrm{CO}_{2}$ eq.)

\begin{tabular}{|c|c|c|c|c|c|c|c|c|c|c|c|c|c|c|c|c|}
\hline \multirow{3}{*}{$\begin{array}{l}\text { Lifespan } \\
\text { (Years) }\end{array}$} & \multicolumn{14}{|c|}{ Embodied Global Warming Potential (GWP) } & \multirow{2}{*}{\multicolumn{2}{|c|}{$\begin{array}{l}\text { Difference in } \\
\text { Operating GWP } \\
\text { Baseline after } \\
\text { Lifespan } \\
\end{array}$}} \\
\hline & \multicolumn{3}{|c|}{ Manufacturing } & \multicolumn{3}{|c|}{ Construction } & \multicolumn{3}{|c|}{ Maintenance } & \multicolumn{3}{|c|}{ End of Life } & \multirow{2}{*}{$\begin{array}{l}{ }^{3} \text { Total } \\
\text { GWPP }\end{array}$} & \multirow{2}{*}{$\begin{array}{l}{ }^{4}{ }^{4} \text { Total } \\
\text { GWP } \\
\text { per m }\end{array}$} & & \\
\hline & Material & ${ }^{2}$ Trans. & Total & Material & ${ }^{2}$ Trans. & Total & Material & ${ }^{2}$ Trans. & Total & Material & $\left.\right|^{2}$ Trans. & Total & & & ${ }^{5}$ Total & ${ }^{6}$ perm $^{2}$ \\
\hline 1 Initial & 5,433 & 1 & 5,434 & 41 & 2 & 44 & 0 & 0 & 0 & 0 & 0 & 0 & 5,478 & 79 & - & \\
\hline 50 & 5,433 & 1 & 5,434 & 41 & 2 & 44 & 3,097 & 2 & 3,099 & 0 & 1 & 1 & 8,577 & 124 & 0 & 0 \\
\hline
\end{tabular}

Embodied energy (and GWP) numbers are based on an area of roof $=69.2 \mathrm{~m}^{2} \quad$ (Span $\times$ Width $=9.1 \mathrm{~m} \times 7.6 \mathrm{~m}=69.2 \mathrm{~m}^{2}$ )

Net roof area of baseline retail building (gross roof area - openings) $=586.0 \mathrm{~m}^{2}$

ATHENA $₫$ EIE Material List:

\begin{tabular}{|c|c|c|}
\hline Material List & \begin{tabular}{|l|l} 
Quantitites \\
\end{tabular} & Unit \\
\hline $\begin{array}{l}13 \mathrm{~mm} \text { Moisture Resistant Gypsum } \\
\text { Board }\end{array}$ & 76.1 & $\mathrm{~m} 2$ \\
\hline $16 \mathrm{~mm}$ Gypsum Fibre Gypsum & 76.1 & $\mathrm{~m} 2$ \\
\hline 6 mil Polyethylene & 70.5 & $\mathrm{~m} 2$ \\
\hline Ballast (aggregate stone) & $\begin{array}{ll}15,823.1 \\
\end{array}$ & $\mathrm{~kg}$ \\
\hline Galvanized Decking & 684.5 & $\mathrm{~kg}$ \\
\hline Galvanized Sheet & 183.5 & $\mathrm{~kg}$ \\
\hline Isocyanurate & 214.6 & $\mathrm{~m} 2(25 \mathrm{~mm}$ \\
\hline Joint Compound & 151.9 & $\mathrm{~kg}$ \\
\hline Nails & 8.5 & $\mathrm{~kg}$ \\
\hline Open Web Joists & 596.4 & $\mathrm{~kg}$ \\
\hline Paper Tape & 1.7 & $\mathrm{~kg}$ \\
\hline PVC membrane & $1,512.6$ & $\mathrm{~kg}$ \\
\hline Small Dimension Sottwood Lumber, & 0.2 & $\mathrm{~m} 3$ \\
\hline $\begin{array}{l}\text { Kint-anea } \\
\text { Softwood Plywood }\end{array}$ & 1.4 & $\mathrm{~m} 2(9 \mathrm{~mm})$ \\
\hline
\end{tabular}

\section{Open Web Steel Joist Roof \#5 (OWSJ-R5)}

Building Component Description:

\begin{tabular}{|c|c|c|c|}
\hline \multirow{4}{*}{ Brief Description: } & Roofs & $\begin{array}{l}\text { Assembly Layers } \\
\text { Outside }\end{array}$ & \\
\hline & \multirow{2}{*}{ 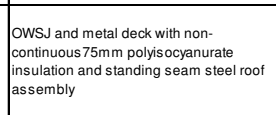 } & $\begin{array}{l}\text { Outside } \\
\text { Alkyd based paint }\end{array}$ & \\
\hline & & \begin{tabular}{|l|} 
Commercial $0.46 \mathrm{~mm}$ galvanized standing seam \\
steel root (WB) (includes tasteners)
\end{tabular} & \\
\hline & & $\begin{array}{l}1.21 \mathrm{~mm} \text { galvanized } 92 \mathrm{~mm} z-\text {-girts @ } 1,200 \mathrm{~mm} \text { o/c } \\
\text { (sell-weight: 1.4 kg/m) }\end{array}$ & \\
\hline \multicolumn{2}{|l|}{ Quick Numbers: } & Non-continuous $75 \mathrm{~mm}$ polys ocyanurate insulation & \\
\hline $\begin{array}{l}\text { ASHRAE Standard 90.1: } \\
\text { THERM 5.2: }\end{array}$ & $\begin{array}{|ll|}\text { R-Value: } 17.9 & \text { RSIVValue } 3.1 \\
\text { R-Value: } 17.8 & \text { RSI-Value }: 3.1 \\
\end{array}$ & 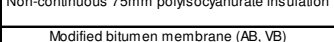 & \\
\hline \begin{tabular}{|l} 
IHoof Thickness: \\
Rof Tin
\end{tabular} & 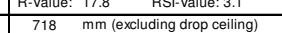 & 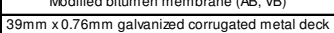 & \\
\hline Span: & Range: $3 \mathrm{~m}$ to $46 \mathrm{~m}$ Design: $9.1 \mathrm{~m}$ & 550mm open web steel joists @ $1,200 \mathrm{~mm}$ o/c & \\
\hline Spectified Design Loads: & $\begin{array}{llll}D L & 1.0 \mathrm{kPa} & \mathrm{SL} & 1.1 \mathrm{kPa} \\
\end{array}$ & (selt-weight: $10.3 \mathrm{~kg} / \mathrm{m})$ & \\
\hline Total Embodied Energy: & $1.516 \mathrm{~m} / \mathrm{m}^{2}$ & $16 \mathrm{~mm}$ suspended acoustical ceiling & \\
\hline Total Emboodied GWP: & $79 \mathrm{~kg} \mathrm{ofC \textrm {C } _ { 2 } \mathrm { eq } / \mathrm { m } ^ { 2 }}$ & $\begin{array}{l}\text { Misc. fasteners, nails, and galvanized sheet } \\
\text { Inside }\end{array}$ & \\
\hline
\end{tabular}

\section{Life-Cycle Assessment Results:}

Primary Energy Consumption (MJ)

\begin{tabular}{|c|c|c|c|c|c|c|c|c|c|c|c|c|c|c|c|c|}
\hline \multirow{3}{*}{$\left|\begin{array}{c}\text { Lifiespan } \\
\text { (Years) }\end{array}\right|$} & \multicolumn{14}{|c|}{ Embodied Energy (EE) } & \multirow{2}{*}{\multicolumn{2}{|c|}{$\begin{array}{c}\text { Difference in } \\
\text { Operating Energy } \\
\text { from Baseline after } \\
\text { Lifespan }\end{array}$}} \\
\hline & \multicolumn{3}{|c|}{ Manutacturing } & \multicolumn{3}{|c|}{ Construction } & \multicolumn{3}{|c|}{ Maintenance } & \multicolumn{3}{|c|}{ End of Lifie } & \multirow{2}{*}{$\begin{array}{c}{ }^{3} \text { Total } \\
\text { EE }\end{array}$} & \multirow{2}{*}{\begin{tabular}{|l}
${ }^{4} \mathrm{Total}$ \\
$\mathrm{EE}$ \\
per m $\mathrm{m}^{2}$
\end{tabular}} & & \\
\hline & \begin{tabular}{|l|} 
Material \\
\end{tabular} & ${ }^{2}$ Trans. & \begin{tabular}{|l|l|} 
Total \\
\end{tabular} & Material & ${ }^{2}$ Trans. & Total & \begin{tabular}{|l|l|} 
Material \\
\end{tabular} & ${ }^{2}$ Trans. & Total & Material & $\left.\right|^{2}{ }^{2}$ Trans. & Total & & & $\begin{array}{l}{ }^{5} \text { Total } \\
\end{array}$ & ${ }^{6}$ per m $^{2}$ \\
\hline 1 Initial & 93,634 & 303 & 93,937 & 631 & 839 & 1,470 & 0 & 0 & 0 & 0 & 0 & 0 & \begin{tabular}{|l|l}
95,407 \\
\end{tabular} & 1,380 & & \\
\hline 50 & \begin{tabular}{|l|l|}
33,634 \\
\end{tabular} & 303 & 93,937 & 631 & 839 & 1,470 & \begin{tabular}{|l|l|}
9,263 \\
\end{tabular} & 29 & $\begin{array}{l}, 293 \\
\end{array}$ & 1 & 166 & $\begin{array}{l}167 \\
\end{array}$ & 104,866 & \begin{tabular}{|l|l|}
1,516 \\
\end{tabular} & 300,000 & 512 \\
\hline
\end{tabular}

Global Warming Potential (kg of $\mathrm{CO}_{2}$ eq.)

\begin{tabular}{|c|c|c|c|c|c|c|c|c|c|c|c|c|c|c|c|c|}
\hline & & & & & & & & & & & & & & & & \\
\hline \multirow{3}{*}{ (Years) } & \multicolumn{14}{|c|}{ (GWP) } & \multirow{2}{*}{\multicolumn{2}{|c|}{\begin{tabular}{|c|} 
Difference in \\
Operating GWP from \\
Baseline after \\
Lifespan
\end{tabular}}} \\
\hline & \multicolumn{3}{|c|}{ Manutacturing } & \multicolumn{3}{|c|}{ Construction } & \multicolumn{3}{|c|}{ Maintenance } & \multicolumn{3}{|c|}{ End of Life } & \multirow{2}{*}{$\begin{array}{l}{ }^{3} \text { Total } \\
\text { GWP }\end{array}$} & \multirow{2}{*}{$\begin{array}{l}{ }^{4} \text { Total } \\
\text { GWP } \\
\text { per m }\end{array}$} & & \\
\hline & Material & ${ }^{2}$ Trans. & Total & Material & ${ }^{2}$ Trans. & Total & \begin{tabular}{|l|} 
Material \\
\end{tabular} & Trans. & Total & Material & ${ }^{2}$ Trans. & Total & & & ${ }^{5}$ Total & ${ }^{6}{ }_{\text {per m}}^{2}$ \\
\hline 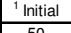 & \begin{tabular}{|l|l|}
5,284 \\
5284
\end{tabular} & 1 & & 41 & & & & & & 0 & & & & & & \\
\hline 50 & 5,284 & 1 & 5,285 & 41 & 1 & 42 & 129 & 0 & 129 & 0 & 0 & 0 & 5,456 & 79 & 20,000 & 34 \\
\hline
\end{tabular}

Embodied energy (and GWP) numbers are based on an area of roof $=69.2 \mathrm{~m}^{2} \quad$ (Span $\times$ Width $=9.1 \mathrm{~m} \times 7.6 \mathrm{~m}=69.2 \mathrm{~m}^{2}$ )

\section{ATHENA $®$ EIE Material List:}

${ }^{2}$ Trans. $=$ Transportation

Total EE (or Total GWP) $)$ Total embodied energy (or total embodied GWP)
of building component after lifespan (i.e. total manufacturing + total

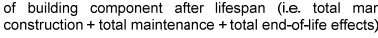

Total EE (or Total GWP) per $\mathrm{m}^{2}=$ Total EE (or Total GWP) of building
component / area of builing component that was modeled in ATHENAQ EIE

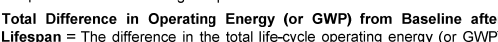

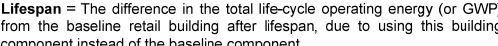

Total Difference in Operating Energy (or GWP) from Baseline atter
Lifespan per $\mathrm{m}^{2}=$ Total difference in operating energy (or GWP) from

Total operating primary energy use of baseline retail building affer 50 years $=$
$50,700 \mathrm{GJ}\left(1,745 \mathrm{MJ} / \mathrm{H}^{2} \mathrm{y} r \mathrm{r}\right)$

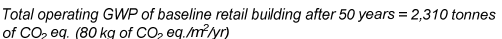

ATHENA $@$ EIE Material List:

\begin{tabular}{|l|c|c|}
\multicolumn{1}{|c|}{ Material List } & Quantities & Unit \\
\hline $\begin{array}{l}\text { 16mm Gypsum Fibre Gypsum } \\
\text { Board }\end{array}$ & 76.1 & $\mathrm{~m} 2$ \\
\hline Foam Polyisocyanurate & 213.1 & $\mathrm{~m} 2(25 \mathrm{~mm})$ \\
\hline Galvanized Decking & 684.5 & $\mathrm{~kg}$ \\
\hline Galvanized Sheet & 565.7 & $\mathrm{~kg}$ \\
\hline Galvanized Studs & 80.8 & $\mathrm{~kg}$ \\
\hline Joint Compound & 75.9 & $\mathrm{~kg}$ \\
\hline Modified Bitumen membrane & 214.7 & $\mathrm{~kg}$ \\
\hline Nails & 5.0 & $\mathrm{~kg}$ \\
\hline Open Web Joists & 596.4 & $\mathrm{~kg}$ \\
\hline Paper Tape & 0.9 & $\mathrm{~kg}$ \\
\hline Screws Nuts \& Bolts & 0.7 & $\mathrm{~kg}$ \\
\hline Solvent Based Alkyd Paint & 49.7 & $\mathrm{~L}$ \\
\hline
\end{tabular}

Notes:

'Initial $=$ Time ' 0 ' (i.e. at
${ }^{2}$ Trans. $=$ Transportation

${ }^{3}$ Total EE (or Total GWP) = Total embodied energy (or total embodied GWP)

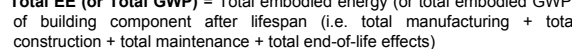
${ }^{4}$ Total EE (or Total GWP) per $\mathrm{m}^{2}=$ Total EE (or Total GWP) of building
component/ area of building component that was modelled in ATHENAB EIE Lifespan = The difference in the total life-cycle operating energy (or GWP from the baseline retail building after lifespan, due to using this building ${ }^{6}$ Total Difference in Operating Energy (or GWP) from Baseline after Tifaspan per $\mathrm{m}^{2}=$ in Total difference in operating energy (or GWP) from
Laseline after lifespan * Total operating primary energy use of baseline retail building after 50 years

*Total operatitig $\mathrm{GWP}$ of baseline retail building after 50 years $=2,310$ tonnes
of $\mathrm{CO}_{2}$ eq. $\left(80 \mathrm{~kg}\right.$ of $\mathrm{CO}_{2}$ eq. $\left./ \mathrm{m}^{2} / \mathrm{yr}\right)$ 
Open Web Steel Joist Roof \#6 (OWSJ-R6)

Building Component Description:

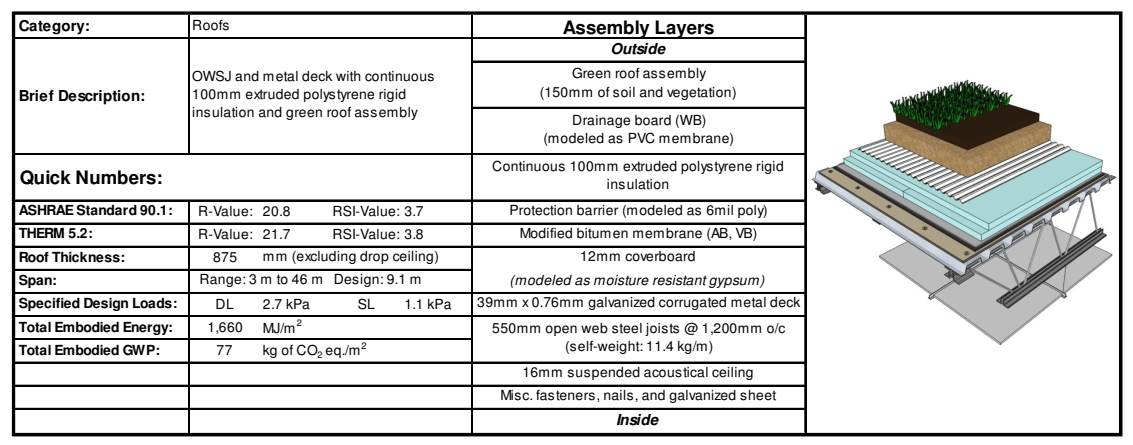

Life-Cycle Assessment Results:

Primary Energy Consumption (MJ)

\begin{tabular}{|c|c|c|c|c|c|c|c|c|c|c|c|c|c|c|c|c|}
\hline \multirow{3}{*}{$\begin{array}{l}\text { Lifespan } \\
\text { (vears) }\end{array}$} & \multicolumn{13}{|c|}{ Embodied Energy (EE) } & \multirow{2}{*}{\multicolumn{3}{|c|}{$\begin{array}{l}\text { Difference in } \\
\text { Operating Energy } \\
\text { from Baseline after } \\
\text { Lifespan }\end{array}$}} \\
\hline & \multicolumn{2}{|c|}{ Manutacturing } & \multicolumn{3}{|c|}{ Construction } & \multicolumn{3}{|c|}{ Maintenance } & \multicolumn{3}{|c|}{ End of Life } & \multirow{2}{*}{$\begin{array}{c}{ }^{3} \text { Total } \\
\mathrm{EE}\end{array}$} & \multirow{2}{*}{\begin{tabular}{|c|}
${ }^{4}$ Total \\
EE \\
per $\mathrm{m}^{2}$
\end{tabular}} & & & \\
\hline & 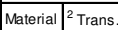 & Total & Materia & ${ }^{2}$ Trans. & \begin{tabular}{|l|l|} 
Total \\
\end{tabular} & \begin{tabular}{|l|l|} 
Material \\
\end{tabular} & ${ }^{2}$ Trans & \begin{tabular}{|l|l|} 
Total \\
\end{tabular} & Material & \begin{tabular}{l|l}
2 & 2 \\
2
\end{tabular} & Total & & & & ${ }^{5}$ Total & ${ }^{6}$ per m$^{2}$ \\
\hline & \begin{tabular}{|l|l|}
85,939 & 318
\end{tabular} & 86,257 & 631 & 1,059 & $\frac{1,690}{1,690}$ & & \begin{tabular}{|l|l}
0 \\
\end{tabular} & 0 & 0 & & & 87.94 & $\bar{T}+2+2$ & & & \\
\hline
\end{tabular}

Global Warming Potential ( $k g$ of $\mathrm{CO}_{2}$ eq.)

\begin{tabular}{|c|c|c|c|c|c|c|c|c|c|c|c|c|c|c|c|c|}
\hline \multirow{3}{*}{$\begin{array}{l}\text { Lifespan } \\
\text { (Years) }\end{array}$} & \multicolumn{14}{|c|}{ Embodied Global Warming Potential (GWP) } & \multirow{2}{*}{\multicolumn{2}{|c|}{$\begin{array}{l}\text { Difference in } \\
\text { Operating GWP from } \\
\text { Baseline after } \\
\text { Lifespan }\end{array}$}} \\
\hline & \multicolumn{3}{|c|}{ Manufacturing } & \multicolumn{3}{|c|}{ Construction } & \multicolumn{3}{|c|}{ Maintenance } & \multicolumn{3}{|c|}{ End of Life } & \multirow{2}{*}{$\begin{array}{l}{ }^{3}{ }^{3} \text { Total } \\
\text { GWP }\end{array}$} & \multirow{2}{*}{\begin{tabular}{|l}
${ }^{4}$ Total \\
GoWP \\
per m
\end{tabular}} & & \\
\hline & Material & ${ }^{2}$ Trans. & Total & Material & 1) ${ }^{2}$ Trans. & Total & Material & $2{ }^{2}$ Trans. & Total & Material & 1. ${ }^{2}$ Trans. & Total & & & ${ }^{5}$ Total & ${ }^{6}$ perm ${ }^{2}$ \\
\hline 1 Initial & 4,544 & 1 & 4,544 & 41 & 1 & 43 & 0 & 0 & 0 & 0 & 0 & 0 & 4,587 & 66 & & \\
\hline 50 & 4,544 & 1 & 4,544 & 41 & 1 & 43 & 740 & 0 & 740 & 0 & 0 & 0 & 5,327 & 77 & 0 & 0 \\
\hline
\end{tabular}
Net roof act

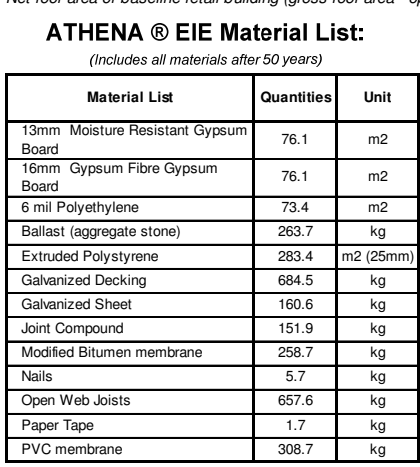

\section{Open Web Steel Joist Roof \#7 (OWSJ-R7)}

Building Component Description:

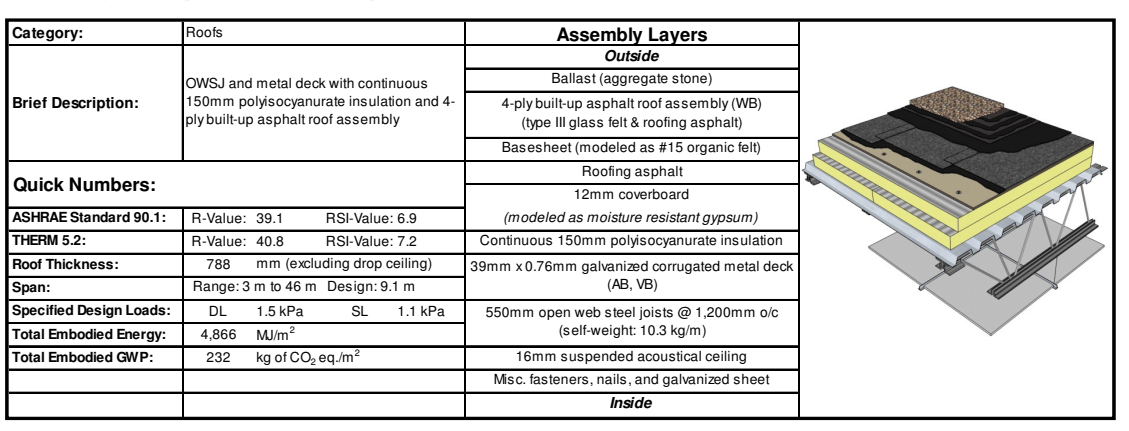

Life-Cycle Assessment Results:

\begin{tabular}{|c|c|c|c|c|c|c|c|c|c|c|c|c|c|c|c|}
\hline \multirow{4}{*}{$\begin{array}{l}\text { Litiespan } \\
\text { (Years) }\end{array}$} & \multicolumn{15}{|c|}{ Primary Energy Consumption (MJ) } \\
\hline & \multicolumn{13}{|c|}{ Embodied Energy (EE) } & \multirow{2}{*}{\multicolumn{2}{|c|}{$\begin{array}{c}\text { Difference in } \\
\text { Operating Energy } \\
\text { from Baseline after } \\
\text { Lifespan }\end{array}$}} \\
\hline & \multicolumn{2}{|c|}{ Manufacturing } & \multicolumn{3}{|c|}{ Construction } & \multicolumn{3}{|c|}{ Maintenance } & \multicolumn{3}{|c|}{ End of Life } & \multirow{2}{*}{$\begin{array}{c}{ }^{3} \text { Total } \\
\mathrm{EE}\end{array}$} & \multirow{2}{*}{\begin{tabular}{|c|}
${ }^{4}$ Total \\
EE \\
per $m^{2}$
\end{tabular}} & & \\
\hline & Material $\mid{ }^{2}$ Trans. & Total & Materia & $\begin{array}{ll}\text { al } & 2 \\
\end{array}$ & $\begin{array}{c}\text { Total } \\
\end{array}$ & Material & \begin{tabular}{|l|}
${ }^{2}$ Trans. \\
\end{tabular} & \begin{tabular}{|l|l} 
Total \\
\end{tabular} & Material & \begin{tabular}{l|l}
$1{ }^{2}$ Trans. \\
.
\end{tabular} & \begin{tabular}{|l|l|l|} 
Total \\
\end{tabular} & & & \begin{tabular}{|l}
${ }^{5}$ Total \\
\end{tabular} & ${ }^{6}$ perm ${ }^{2}$ \\
\hline |ititial & \begin{tabular}{|l|l|}
190,269 & 420 \\
\end{tabular} & $190,68 \mathrm{~S}$ & \begin{tabular}{l|l}
9631 \\
9
\end{tabular} & 1,094 & 1,725 & 0 & 0 & 0 & 0 & 0 & 0 & 192,4 & \begin{tabular}{|l|l|}
4 & 2,782 \\
\end{tabular} & & \\
\hline
\end{tabular}

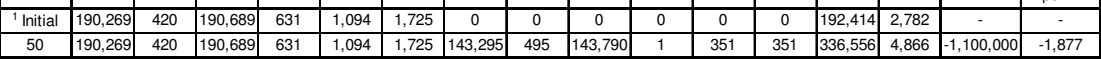

\begin{tabular}{|c|c|c|c|c|c|c|c|c|c|c|c|c|c|c|c|c|}
\hline \multirow{4}{*}{$\begin{array}{l}\text { Lifespar } \\
\text { (Years) }\end{array}$} & \multirow{2}{*}{\multicolumn{14}{|c|}{ Global Warming Potential (kg of $\mathrm{CO}_{2}$ eq.) }} & \multirow{3}{*}{\multicolumn{2}{|c|}{$\begin{array}{l}\text { Difference in } \\
\text { Operating GWP fro } \\
\text { Baseline aatter } \\
\text { Lifespan }\end{array}$}} \\
\hline & \multicolumn{2}{|c|}{ Embodied Global Warming Potential (GWP) } & & & & & & & & & & & & & & \\
\hline & \multicolumn{3}{|c|}{ Manufacturing } & \multicolumn{3}{|c|}{ Construction } & \multicolumn{3}{|c|}{ Maintenance } & \multicolumn{3}{|c|}{ End of L Life } & \multirow{2}{*}{$\begin{array}{l}{ }^{3} \text { Total } \\
\text { GWP }\end{array}$} & \multirow{2}{*}{$\begin{array}{l}{ }^{4}{ }^{\mathrm{T} o t a l} \\
\text { GWW } \\
\text { per m }\end{array}$} & & \\
\hline & Material & ${ }^{2}$ Trans. & Total & Material & ${ }^{2}$ Trans. & Total & Material & ${ }^{2}$ Trans. & Total & Material & $\begin{array}{l}{ }^{2} \text { Trans. } \\
\text {. }\end{array}$ & Total & & & ${ }^{5}$ Total & ${ }^{6}$ per $\mathrm{m}^{2}$ \\
\hline${ }^{\prime}$ Initial & 10,286 & 1 & 10,287 & 41 & 1 & 43 & 0 & 0 & 0 & 0 & 0 & 0 & 10,330 & 149 & & \\
\hline 50 & 10,286 & 1 & 10,28 & 41 & 1 & 43 & 5,698 & 1 & 5,699 & 0 & 1 & 1 & 16,029 & 232 & $-60,000$ & -102 \\
\hline
\end{tabular}

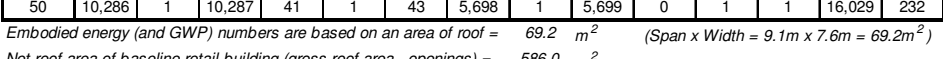
Net roof area of baseline retail building (gross roof area - openings) $=586.0 \mathrm{~m}^{2}$

ATHENA @ EIE Material List:

\begin{tabular}{|c|c|c|}
\hline & & \\
\hline \#15 Oranic Fol & & \\
\hline 13mm Moisture Resistant Gypsum & 551.9 & $\mathrm{~m} 2$ \\
\hline Board & 76.1 & $\mathrm{~m} 2$ \\
\hline $\begin{array}{l}16 \mathrm{~mm} \text { Gypsum Fibre Gypsum } \\
\text { Board }\end{array}$ & 76.1 & $\mathrm{~m} 2$ \\
\hline Ballast (aggregate stone) & $5,274.4$ & $\mathrm{~kg}$ \\
\hline Galvanized Decking & 684.5 & $\mathrm{~kg}$ \\
\hline Galvanized Sheet & 243.0 & $\mathrm{~kg}$ \\
\hline Isocyanurate & 429.2 & $\mathrm{~m} 2(25 \mathrm{~mm})$ \\
\hline Joint Compound & 75.9 & $\mathrm{~kg}$ \\
\hline Nails & 36.3 & $\mathrm{~kg}$ \\
\hline Open Web Joists & 596.4 & \\
\hline Paper Tape & 0.9 & $\mathrm{~kg}$ \\
\hline Roofing Asphalt & $2,093.8$ & $\mathrm{~kg}$ \\
\hline Type III Glass Felt & $1,103.8$ & $\mathrm{~m} 2$ \\
\hline
\end{tabular}

${ }^{1}$ Initial $=$ Time ' ${ }^{\prime}$ (i.e. at the completion of:

${ }^{2}$ Trans. $=$ Transportation

Total EE (or Total GWP) $)$ Total embodied energy (or total embodied GWP)
of building component after lifespan ( ie of building component after lifespan (i.e. total manu
construction t total maintenance t total end-ofifife effects) ${ }^{4}$ Total EE (or Total GWP) per $\mathrm{m}^{2}=$ Total EE (or Total GWP) of building
component/ area of building component that was modelled in ATHENAQ EIE 5 Total Difference in Operating Energy (or GWP) from Baseline after
Liffespan = The differenee in the total Iif-cycle operating energy (or GWWP)
from the baseline retail building after lifespan, due to using this building from the baseline retail building after lifespan, due to using this building
component instead of the baseline component ${ }^{6}$ Total Difference in Operating Energy (or GWP) from Baseline after
Lifespan per $\mathrm{m}^{2}=$ Total difference in operating energy (or GWP) from * Total operating primary energy use of baseline retail building after 50 years = * Total operating $\mathrm{GWP}$ of baseline retail building affer 50 years $=2,310$ tonnes
of $\mathrm{CO}_{2}$ eq. $\mathrm{kg}$ of $\left.\mathrm{CO}_{2} \mathrm{eq} / \mathrm{m} \mathrm{m}^{2} \mathrm{yr}\right)$ 


\section{Open Web Steel Joist Roof \#8 (OWSJ-R8)}

Building Component Description:

\begin{tabular}{|c|c|c|c|}
\hline \multirow{4}{*}{ |Brief Description: } & Roots & Assembly Layers & \\
\hline & \multirow{3}{*}{$\begin{array}{l}\text { OWSS and metal deck with continuous } \\
150 \mathrm{~mm} \text { polysisocyanurate insulation and } \\
\text { PVC root assemmly }\end{array}$} & $\begin{array}{l}\text { Outside } \\
\text { Ballast (agragate stone) }\end{array}$ & \\
\hline & & 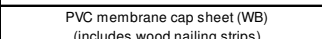 & \\
\hline & & Bonding agent (modeled as Gmil poly) & \\
\hline \multicolumn{2}{|l|}{ Quick Numbers: } & (modeled as moistur resistant grosum & \\
\hline ASHRAE Standard 90.1: & RSI-Value: 6.9 & Continuous $150 \mathrm{~mm}$ polysisocyanurate insulation & \\
\hline THERM 5.2: & $\begin{array}{|ll|}\text { R-Value: } 40.8 & \text { RSI-Value: } 7.2 \\
\end{array}$ & $39 \mathrm{~mm} \times 0.76 \mathrm{~mm}$ galvanized corrugated metal dec & \\
\hline Roof Thickness: & $788 \quad \mathrm{~mm}$ (excluding drop ceiling) & (AB, VB) & \\
\hline Span: & \begin{tabular}{|l|l|l|} 
Range: $3 \mathrm{~m}$ to $46 \mathrm{~m}$ Design: $9.1 \mathrm{~m}$ \\
\end{tabular} & $550 \mathrm{~mm}$ open web steel joists @ @ 1,200mm o/c & \\
\hline Specififed Design Loads: & $\begin{array}{llll}D L & 1.5 \mathrm{kPa} & \mathrm{SL} & 1.1 \mathrm{kPa} \\
\end{array}$ & (sell-weight: $10.3 \mathrm{~kg} / \mathrm{m}$ ) & \\
\hline Total Embodied Energy: & $2,899 \mathrm{~m} / \mathrm{m}^{2}$ & $16 \mathrm{~mm}$ suspended acoustical celiling & \\
\hline Total Embodied GWP: & $143 \quad \mathrm{~kg}$ of $\mathrm{CO}_{2} \mathrm{eq} / \mathrm{m}^{2}$ & $\begin{array}{l}\text { Misc. fasteners, nails, and galvanized sheet } \\
\text { Inside }\end{array}$ & \\
\hline
\end{tabular}

\section{Life-Cycle Assessment Results:}

Primary Energy Consumption (MJ)

\begin{tabular}{|c|c|c|c|c|c|c|c|c|c|c|c|c|c|c|c|c|}
\hline \multirow{3}{*}{$\begin{array}{l}\text { LLessan } \\
\text { (Years) }\end{array}$} & \multicolumn{14}{|c|}{ Embodied Energy (EE) } & \multirow{2}{*}{\multicolumn{2}{|c|}{$\begin{array}{c}\text { Difference in } \\
\text { Operating Energy } \\
\text { from Baseline atte } \\
\text { Lifespan }\end{array}$}} \\
\hline & \multicolumn{3}{|c|}{ Manufacturing } & \multicolumn{3}{|c|}{ Construction } & \multicolumn{3}{|c|}{ Maintenance } & \multicolumn{3}{|c|}{ End of Lifie } & \multirow{2}{*}{${ }^{3}{ }^{3}$ Total } & \multirow{2}{*}{$\begin{array}{c}{ }^{4} \text { Total } \\
\text { EE } \\
\text { per } \mathrm{m}^{2}\end{array}$} & & \\
\hline & Material & ${ }^{2}$ Trans. & \begin{tabular}{|l|} 
Total \\
\end{tabular} & Materia & ${ }^{2}$ Trans. & \begin{tabular}{|l|} 
Total \\
\end{tabular} & |Material & $\left.\right|^{2}$ Trans. & Total & Material & al| $\left.\right|^{2}$ Trans. & Total & & & \begin{tabular}{|l|}
${ }^{5}$ Total \\
\end{tabular} & ${ }^{6}{ }^{6}$ perm ${ }^{2}$ \\
\hline Initial & \begin{tabular}{|l|}
108,444 \\
\end{tabular} & & 108,899 & 631 & 1,603 & 2,235 & 0 & $=0$ & 0 & 0 & 0 & 8 & 1111,134 & 41,607 & & \\
\hline 50 & 108,444 & \begin{tabular}{|l|l|}
455 \\
\end{tabular} & 108,899 & 631 & 1,603 & $\begin{array}{ll}2,235 \\
\end{array}$ & 88,088 & 870 & 88,958 & 1 & 412 & 412 & 200,504 & 42,899 & $-1,100,0$ & $-1,87$ \\
\hline
\end{tabular}

Global Warming Potential ( $\left(\mathrm{gg}\right.$ of $\mathrm{CO}_{2}$ eq.)

\begin{tabular}{|c|c|c|c|c|c|c|c|c|c|c|c|c|c|c|c|c|}
\hline \multirow{3}{*}{$\begin{array}{l}\text { Lifespan } \\
\text { (Years) }\end{array}$} & \multicolumn{14}{|c|}{ Embodied Global Warming Potential (GWP) } & \multirow{2}{*}{\multicolumn{2}{|c|}{$\begin{array}{c}\text { Difference in } \\
\text { Operating GWP fro } \\
\text { Baseline after } \\
\text { Lifespan }\end{array}$}} \\
\hline & \multicolumn{3}{|c|}{ Manutacturing } & \multicolumn{3}{|c|}{ Construction } & \multicolumn{3}{|c|}{ Maintenance } & \multicolumn{3}{|c|}{ End of Life } & \multirow{2}{*}{$\mid \begin{array}{l}3 \text { Total } \\
\text { GWP }\end{array}$} & \multirow{2}{*}{\begin{tabular}{|l}
${ }^{4}$ Total \\
GWP \\
per m
\end{tabular}} & & \\
\hline & \begin{tabular}{|l|} 
Material \\
\end{tabular} & ${ }^{2}$ Trans & \begin{tabular}{|l|} 
Total \\
\end{tabular} & Material & ${ }^{2}$ Trans. & \begin{tabular}{|l|} 
Total \\
\end{tabular} & \begin{tabular}{|l|} 
Material \\
\end{tabular} & ${ }^{2}$ Trans. & Total & \begin{tabular}{|l|} 
Material \\
\end{tabular} & $\left.\right|^{2}$ Trans. & \begin{tabular}{|l|l|} 
Total \\
\end{tabular} & & & ${ }^{5}$ Total & ${ }^{6}$ per $\mathrm{m}^{2}$ \\
\hline 1 Initial & $\begin{array}{ll}6,714 \\
\end{array}$ & 1 & 6,715 & 41 & 2 & 44 & 0 & 0 & 0 & 0 & 0 & 0 & 6,759 & 98 & & - \\
\hline 50 & \begin{tabular}{|l}
6,714 \\
\end{tabular} & 11 & 6,715 & 41 & 2 & 44 & 3,097 & 2 & 3,099 & 0 & 1 & 1 & 9,858 & 143 & $-60,000$ & -102 \\
\hline
\end{tabular}

Embodied energy (and GWP) numbers are based on an area of roof $=69.2 \mathrm{~m}^{2} \quad$ (Span $\times$ Width $=9.1 \mathrm{~m} \times 7.6 \mathrm{~m}=69.2 \mathrm{~m}^{2}$ )

Net roof area of baseline retail building (gross roof area - openings) $=586.0 \mathrm{~m}^{2}$

\section{ATHENA ® EIE Material List}

\begin{tabular}{|c|c|c|}
\hline Material List & \begin{tabular}{|l|l} 
Quantitites \\
\end{tabular} & Unit \\
\hline $\begin{array}{l}13 \mathrm{~mm} \text { Moisture Resistant Gypsum } \\
\text { Board }\end{array}$ & 76.1 & $\mathrm{~m} 2$ \\
\hline $16 \mathrm{~mm}$ Gypsum Fibre Gypsum & 76.1 & $\mathrm{~m} 2$ \\
\hline 6 mil Polyethylene & 70.5 & $\mathrm{~m} 2$ \\
\hline Ballast (aggregate stone) & $\begin{array}{ll}15,823.1 \\
\end{array}$ & $\mathrm{~kg}$ \\
\hline Galvanized Decking & 684.5 & $\mathrm{~kg}$ \\
\hline Galvanized Sheet & 183.5 & $\mathrm{~kg}$ \\
\hline Isocyanurate & 429.2 & $\mathrm{~m} 2(25 \mathrm{~mm}$ \\
\hline Joint Compound & 151.9 & $\mathrm{~kg}$ \\
\hline Nails & 8.5 & $\mathrm{~kg}$ \\
\hline Open Web Joists & 596.4 & $\mathrm{~kg}$ \\
\hline Paper Tape & 1.7 & $\mathrm{~kg}$ \\
\hline PVC membrane & $1,512.6$ & $\mathrm{~kg}$ \\
\hline Small Dimension Sottwood Lumber, & 0.2 & $\mathrm{~m} 3$ \\
\hline $\begin{array}{l}\text { Kint-anea } \\
\text { Softwood Plywood }\end{array}$ & 1.4 & $\mathrm{~m} 2(9 \mathrm{~mm})$ \\
\hline
\end{tabular}

\section{Open Web Steel Joist Roof \#9 (OWSJ-R9)}

Building Component Description:

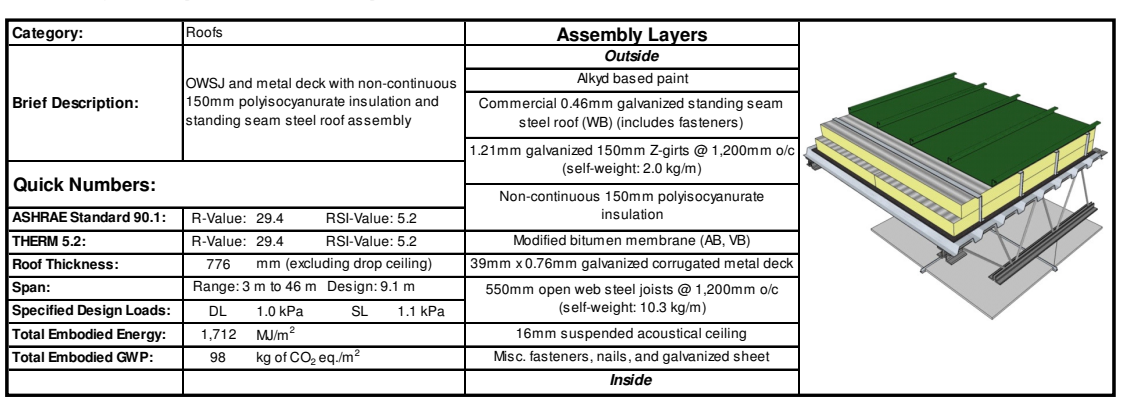

\section{Life-Cycle Assessment Results:}

Primary Energy Consumption (MJ)

\begin{tabular}{|c|c|c|c|c|c|c|c|c|c|c|c|c|c|c|c|c|}
\hline \multirow{3}{*}{$\left|\begin{array}{c}\text { Lifiespan } \\
\text { (Years) }\end{array}\right|$} & \multicolumn{14}{|c|}{ Embodied Energy (EE) } & \multirow{2}{*}{\multicolumn{2}{|c|}{$\begin{array}{c}\text { Difference in } \\
\text { Operating Energy } \\
\text { from Baseline after } \\
\text { Lifespan }\end{array}$}} \\
\hline & \multicolumn{3}{|c|}{ Manutacturing } & \multicolumn{3}{|c|}{ Construction } & \multicolumn{3}{|c|}{ Maintenance } & \multicolumn{3}{|c|}{ End of Life } & \multirow{2}{*}{$\begin{array}{c}{ }^{3} \text { Total } \\
{ }_{\mathrm{EE}}\end{array}$} & \multirow{2}{*}{\begin{tabular}{|l}
${ }^{4} \mathrm{Total}$ \\
$\mathrm{EE}$ \\
per m
\end{tabular}} & & \\
\hline & \begin{tabular}{|l|} 
Material \\
\end{tabular} & ${ }^{2}$ Trans. & Total & Material & ${ }^{2}$ Trans. & \begin{tabular}{|l|} 
Total \\
\end{tabular} & \begin{tabular}{|l|l|} 
Material \\
\end{tabular} & ${ }^{2}$ Trans. & Total & Material & \begin{tabular}{|l|}
${ }^{2}$ Trans. \\
\end{tabular} & Total & & & ${ }^{5}{ }^{5}$ Total & ${ }^{6}$ per m $^{2}$ \\
\hline 1 Initial & 107,071 & 312 & 107,38 & 631 & 885 & 1,517 & 0 & 0 & 0 & 0 & 0 & 0 & 108,900 & \begin{tabular}{|l|}
1,575 \\
\end{tabular} & & \\
\hline 50 & 107,071 & 312 & 107,38 & \begin{tabular}{|l|l|}
631 \\
\end{tabular} & 885 & \begin{tabular}{ll|}
1,517 \\
\end{tabular} & 9,263 & 29 & $\begin{array}{l}, 293 \\
\end{array}$ & 1 & 182 & 183 & $|118,376|$ & \begin{tabular}{|l|l}
1,712 \\
\end{tabular} & 700,000 & $-1,19$ \\
\hline
\end{tabular}

Global Warming Potential (kg of $\mathrm{CO}_{2}$ eq.)

\begin{tabular}{|c|c|c|c|c|c|c|c|c|c|c|c|c|c|c|c|c|}
\hline & & & & & & & & & & & & & & & & \\
\hline \multirow{3}{*}{ (Years) } & \multicolumn{14}{|c|}{ (GWP) } & \multirow{2}{*}{\multicolumn{2}{|c|}{$\begin{array}{l}\text { Difierence in } \\
\text { Operating GWP from } \\
\text { Baseline atter } \\
\text { Lifespan }\end{array}$}} \\
\hline & \multicolumn{3}{|c|}{ Manutacturing } & \multicolumn{3}{|c|}{ Construction } & \multicolumn{3}{|c|}{ Maintenance } & \multicolumn{3}{|c|}{ End of Life } & \multirow{2}{*}{$\begin{array}{l}{ }^{3} \text { Total } \\
\text { GWP }\end{array}$} & \multirow{2}{*}{$\begin{array}{l}{ }^{4} \text { Total } \\
\text { GWP } \\
\text { per m }\end{array}$} & & \\
\hline & Materia & $\left.1\right|^{2}$ Trans & Total & Material & ${ }^{2}$ Trans. & Total & \begin{tabular}{|l|} 
Material \\
\end{tabular} & Trans. & Total & Material & ${ }^{2}$ Trans. & Total & & & ${ }^{5}$ Total & ${ }^{6}{ }_{\text {per m }}^{2}$ \\
\hline $\begin{array}{l}\text { |lintial } \\
50 \text { s. }\end{array}$ & 6,6 & 1 & & 11 & & 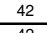 & & & & 0 & & 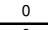 & & & & \\
\hline 50 & 6,635 & 1 & 6,635 & 41 & 1 & 42 & 129 & 0 & 129 & 0 & 0 & 0 & 6,807 & 98 & $-30,000$ & \\
\hline
\end{tabular}

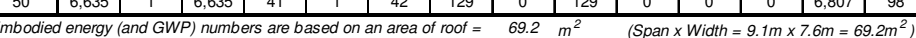
Net roof area of baseline retail building (gross roof area- openings) $=586.0 \mathrm{~m}^{2}$

\section{ATHENA $®$ EIE Material List:}

${ }^{2}$ Trans. $=$ Transportation

Total EE (or Total GWP) $=$ Total embodied energy (or total embodied GWP)
of building component after lifespan (i.e. total manufacturing + total

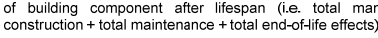

${ }^{4}$ Total EE (or Total GWP) per $\mathrm{m}^{2}=$ Total EE (or Total GWP) of building
component/ area of building component that was modeled in ATHENAQ EIE ${ }^{5}$ Total Difference in Operating Energy (or GWP) from Baseline after
Lifespan $=$ The difference in the total life-cycle operating energy (or GWP)

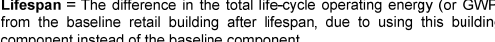
Total Difference in Operating Energy (or GWP) from Baseline after
Lifespan per $\mathrm{m}^{2}=$ Total difference in operating energy (or GWP) from

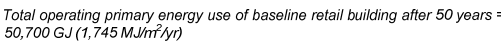

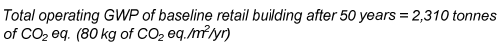

\begin{tabular}{|l|c|c|}
\hline \multicolumn{1}{|c|}{ Material List } & Quantities & Unit \\
\hline \begin{tabular}{l|c|}
$16 \mathrm{~mm}$ Gypsum Fibre Gypsum \\
Board
\end{tabular} & 76.1 & $\mathrm{~m} 2$ \\
\hline Foam Polyisocyanurate & 426.2 & $\mathrm{~m} 2(25 \mathrm{~mm})$ \\
\hline Galvanized Decking & 684.5 & $\mathrm{~kg}$ \\
\hline Galvanized Sheet & 565.7 & $\mathrm{~kg}$ \\
\hline Galvanized Studs & 121.2 & $\mathrm{~kg}$ \\
\hline Joint Compound & 75.9 & $\mathrm{~kg}$ \\
\hline Modified Bitumen membrane & 214.7 & $\mathrm{~kg}$ \\
\hline Nails & 5.0 & $\mathrm{~kg}$ \\
\hline Open Web Joists & 596.4 & $\mathrm{~kg}$ \\
\hline Paper Tape & 0.9 & $\mathrm{~kg}$ \\
\hline Screws Nuts \& Bolts & 0.7 & $\mathrm{~kg}$ \\
\hline Solvent Based Alkyd Paint & 49.7 & $\mathrm{~L}$ \\
\hline
\end{tabular}

Notes:
${ }^{1}$ Initial $=$ Time ' 0 ' (i.e. at the completion of initial construction

${ }^{2}$ Trans. = Transportation

${ }^{3}$ Total EE (or Total GWP) = Total embodied energy (or total embodied GWP

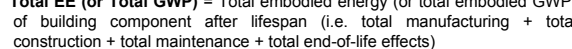
${ }^{4}$ Total EE (or Total GWP) per $\mathrm{m}^{2}=$ Total EE (or Total GWP) of building
component/ area of building component that was modelled in ATHENAB EIE Lifespan = The difference in the total life-cycle operating energy (or GWP from the baseline retail building after lifespan, due to using this building ${ }^{6}$ Total Difference in Operating Energy (or GWP) from Baseline after Total Difference in Operating Energy (or
Lifespan per $\mathrm{m}^{2}=$ Total difference in operating energy (or GWP) from
baseline after lifespan * Total operating primary energy use of baseline retail building after 50 years $50,700 \mathrm{GJ}\left(1,745 \mathrm{MJ} / \mathrm{m}^{2} / \mathrm{y}\right)$

* Total operating GWP of baseline retail building after 50 years $=2,310$ tonnes
of $\mathrm{CO}_{2}$ eq. $\left(80 \mathrm{~kg}\right.$ of $\mathrm{CO}_{2}$ eq. $\left./ \mathrm{m}^{2} / \mathrm{yr}\right)$ 
Open Web Steel Joist Roof \#10 (OWSJ-R10)

Building Component Description:

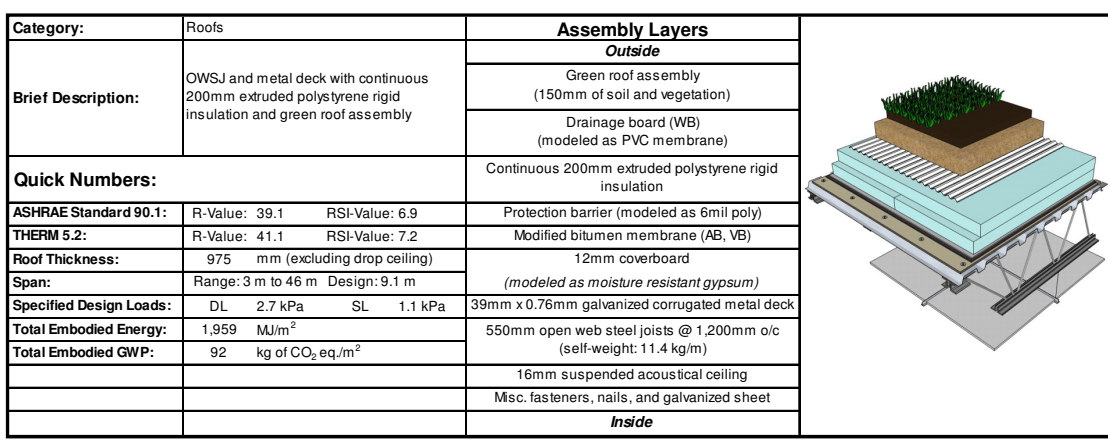

Life-Cycle Assessment Results:

Primary Energy Consumption (MJ)

\begin{tabular}{|c|c|c|c|c|c|c|c|c|c|c|c|c|c|c|c|c|}
\hline \multirow{3}{*}{$\begin{array}{l}\text { Lifiespanan } \\
\text { (Years) }\end{array}$} & \multicolumn{14}{|c|}{ Embodied Energy (EE) } & \multirow{2}{*}{\multicolumn{2}{|c|}{$\begin{array}{l}\text { Difference in } \\
\text { Operating Energy } \\
\text { from Baseline after } \\
\text { Lifespan }\end{array}$}} \\
\hline & \multicolumn{3}{|c|}{ Manufacturing } & \multicolumn{3}{|c|}{ Construction } & \multicolumn{3}{|c|}{ Maintenance } & \multicolumn{3}{|c|}{ End of Life } & \multirow{2}{*}{$-{ }^{3} \begin{array}{c}\text { Total } \\
\mathrm{EE}\end{array}$} & \multirow{2}{*}{$\begin{array}{c}{ }^{4}{ }^{\mathrm{T} o t a l} \\
\mathrm{EE} \\
\text { per m} \mathrm{m}^{2}\end{array}$} & & \\
\hline & Material & ${ }^{2}$ Trans. & Total & Materia & $\left.\right|^{2}$ Trans. & Total & Material & ${ }^{2}$ Trans. & \begin{tabular}{|l} 
Total \\
\end{tabular} & Material & 2 Trans. & Total & & & ${ }^{5}$ Total & ${ }^{6}{ }_{\text {per m}}^{2}$ \\
\hline Initial & 106,538 & & 106,85 & 631 & 1,092 & 1,723 & 0 & 0 & 0 & 0 & 0 & 0 & 108,580 & 1,570 & & \\
\hline 50 & 106,538 & 318 & 106,85 & 631 & 1,092 & 1,723 & 26,592 & 73 & 26,665 & 0 & 254 & 254 & $\mid 135,499$ & $\begin{array}{l}1,959 \\
\end{array}$ & $1,100,000$ & $-1,877$ \\
\hline
\end{tabular}

Global Warming Potential (kg of $\mathrm{CO}_{2}$ eq.)

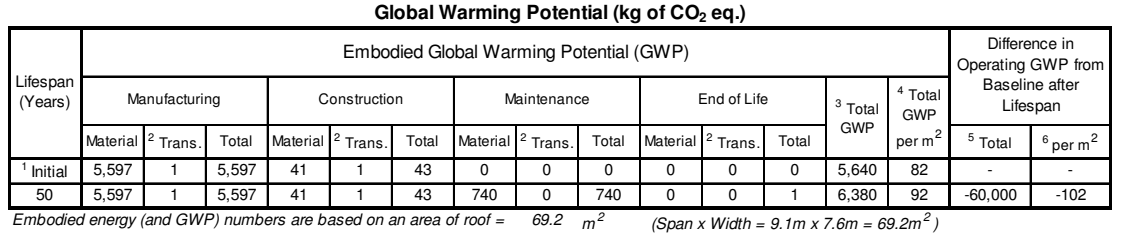

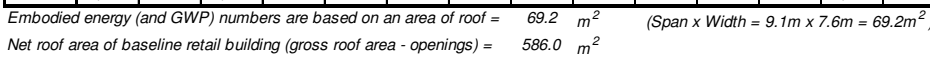

ATHENA $®$ EIE Material List:

\begin{tabular}{|c|c|c|}
\hline Material List & Quantities & Unit \\
\hline $\begin{array}{l}13 \mathrm{~mm} \text { Moisture Resistant Gypsum } \\
\text { Board }\end{array}$ & 76.1 & $\mathrm{~m} 2$ \\
\hline $\begin{array}{l}16 \mathrm{~mm} \text { Gypsum Fibre Gypsum } \\
\text { Bapard }\end{array}$ & 76.1 & $\mathrm{~m} 2$ \\
\hline 6 mil Polyethylene & 73.4 & $\mathrm{~m} 2$ \\
\hline Ballast (aggregate stone) & 263.7 & $\mathrm{~kg}$ \\
\hline $\begin{array}{l}\text { Extruded Polystyrene } \\
\end{array}$ & 566.8 & $\mathrm{~m} 2(25 \mathrm{~mm}$ \\
\hline Galvanized Decking & 684.5 & $\mathrm{~kg}$ \\
\hline Galvanized Sheet & 160.6 & $\mathrm{~kg}$ \\
\hline Joint Compound & 151.9 & $\mathrm{~kg}$ \\
\hline Modified Bitumen membrane & 258.7 & $\mathrm{~kg}$ \\
\hline Nails & 5.7 & $\mathrm{~kg}$ \\
\hline Open Web Joists & 657.6 & $\mathrm{~kg}$ \\
\hline Paper Tape & 1.7 & $\mathrm{~kg}$ \\
\hline PVC membrane & 308.7 & $\mathrm{~kg}$ \\
\hline
\end{tabular}

\section{LCA Data for Cold-Formed Steel Roofs}

This section contains a detailed description of each cold-formed steel (CFS) roof that was examined in this study (13 in total). The assembly layers are listed for each roof, along with a detailed description of the material quantities from the ATHENA ${ }^{\circledR}$ Environmental Impact Estimator for Buildings.

A breakdown of the total primary energy consumption and the total global warming potential (GWP) for each roof is also included. In general, the results were calculated for an area of roof equal to $38.0 \mathrm{~m}^{2}$, which represents a typical bay size for a single-storey retail building with this type of roof system (with the exception of the double joist system and the cold-formed steel truss systems which were calculated for $57.8 \mathrm{~m}^{2}$ and $69.2 \mathrm{~m}^{2}$ respectfully). The results are also expressed on a per $\mathrm{m}^{2}$ basis in each case. The data has been calculated for two different lifespans: at the completion of initial construction and after 50 years.

As a summary, the figure below illustrates a comparison of the total embodied energy (and GWP) after 50 years for the various roofs in this section. For comparison purpose, the building component used in the baseline retail building has been shaded in grey.

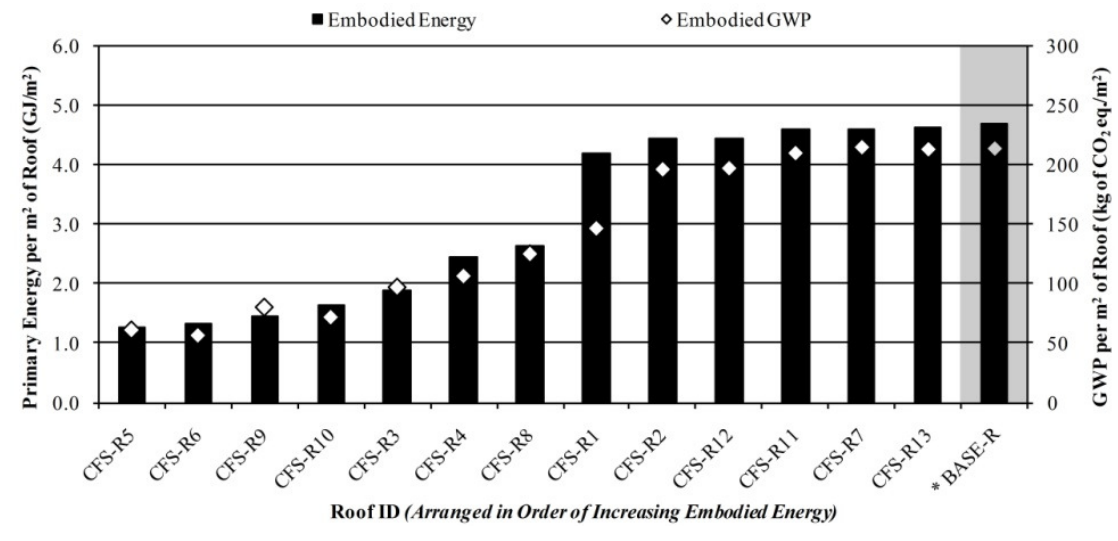




\section{Cold-Formed Steel Roof \#1 (CFS-R1)}

Building Component Description:

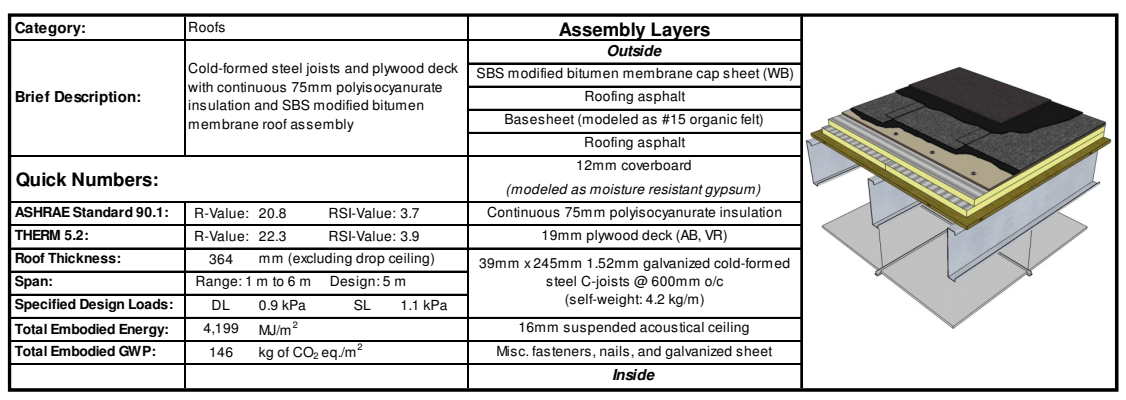

\section{Life-Cycle Assessment Results:}

Primary Energy Consumption (MJ)

\begin{tabular}{|c|c|c|c|c|c|c|c|c|c|c|c|c|c|c|c|c|}
\hline \multirow{3}{*}{$\begin{array}{l}\text { Lifespan } \\
\text { (Years) }\end{array}$} & \multicolumn{14}{|c|}{ Embodied Energy (EE) } & \multirow{2}{*}{\multicolumn{2}{|c|}{$\begin{array}{c}\text { Difference in } \\
\text { Operating Energy } \\
\text { from Baseline after } \\
\text { Lifespan }\end{array}$}} \\
\hline & \multicolumn{3}{|c|}{ Manutacturing } & \multicolumn{3}{|c|}{ Construction } & \multicolumn{3}{|c|}{ Maintenance } & \multicolumn{3}{|c|}{ End of Life } & \multirow{2}{*}{$\begin{array}{c}{ }^{3} \text { Total } \\
\mathrm{EE}\end{array}$} & \multirow{2}{*}{\begin{tabular}{|c|}
${ }^{4}$ Total \\
$\mathrm{EE}$ \\
\end{tabular}} & & \\
\hline & \begin{tabular}{|l|} 
Material \\
\end{tabular} & ${ }^{2}$ Trans. & Total & Material & ${ }^{2}$ Trans. & Total & Material & ${ }^{2}$ Trans. & Total & Material & $\left.\right|^{2}{ }^{2}$ Trans. & Total & & & ${ }^{5}$ Total & ${ }^{6} \mathrm{perm}^{2}$ \\
\hline Initial & & & 75,379 & 0 & 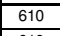 & 610 & 0 & n & 0 & 0 & $\pi$ & 0 & 5,989 & 2,000 & & \\
\hline 50 & 75,163 & 216 & 75,379 & 0 & \begin{tabular}{|l|}
610 \\
\end{tabular} & 610 & 83,268 & 170 & 83,438 & $\frac{1}{1}$ & 152 & 152 & 9.,579 & $\begin{array}{l}4,4009 \\
4,199 \\
\end{array}$ & $-2000,000$ & -34 \\
\hline
\end{tabular}

Global Warming Potential ( $k g$ of $\mathrm{CO}_{2}$ eq.)

\begin{tabular}{|c|c|c|c|c|c|c|c|c|c|c|c|c|c|c|c|c|}
\hline & & & & & & obal & Varmin & $g$ Poter & tial $(\mathbf{k}$ & $\mathrm{g}$ of $\mathrm{CO}$ & $2_{2}$ eq.) & & & & & \\
\hline \multirow{3}{*}{$\begin{array}{l}\text { Litisparars } \\
\text { (Years }\end{array}$} & \multicolumn{14}{|c|}{ Embodied Global Warming Potential (GWP) } & \multirow{2}{*}{\multicolumn{2}{|c|}{$\begin{array}{l}\text { Difference in } \\
\text { Operating GWP fron } \\
\text { Baseline after } \\
\text { Lifespan }\end{array}$}} \\
\hline & \multicolumn{3}{|c|}{ Manufacturing } & \multicolumn{3}{|c|}{ Construction } & \multicolumn{3}{|c|}{ Maintenance } & \multicolumn{3}{|c|}{ End of Life } & \multirow{2}{*}{$\begin{array}{l}{ }^{3} \text { Total } \\
\text { GWP }\end{array}$} & \multirow{2}{*}{$\begin{array}{l}{ }^{4} \text { Total } \\
\text { GWP } \\
\text { per m }\end{array}$} & & \\
\hline & \begin{tabular}{|l|} 
Material \\
\end{tabular} & \begin{tabular}{|l|}
${ }^{2}$ Trans. \\
\end{tabular} & \begin{tabular}{|l|l|} 
Total \\
\end{tabular} & Material & ${ }^{2}$ Trans. & Total & Material & $\mid 2$ Trans. & Total & Material & 2 Trans. & \begin{tabular}{|l|l|} 
Total \\
\end{tabular} & & & ${ }^{5}$ Total & ${ }^{6}$ per m ${ }^{2}$ \\
\hline Initial & 3,349 & 0 & 3,350 & 0 & 1 & 1 & 0 & 0 & 0 & 0 & 0 & 0 & 3,350 & 88 & & \\
\hline 50 & 3,349 & 0 & 3,350 & 0 & 1 & 1 & 2,216 & 0 & 2,216 & 0 & 0 & 0 & 5,566 & 146 & 0 & \\
\hline
\end{tabular}

Embodied energy (and GWP) numbers are based on an area of roof $=38.0 \mathrm{~m}^{2} \quad\left(\right.$ Span $\times$ Width $=5.0 \mathrm{~m} \times 7.6 \mathrm{~m}=38.0 \mathrm{~m}^{2}$ )

Net roof area of baseline retail building (gross roof area - openings) = $586.0 \mathrm{~m}^{2}$

\section{ATHENA ${ }^{\circledR}$ EIE Material List:}

\begin{tabular}{|c|c|c|}
\hline Material List & Quantities & Unit \\
\hline$\# 15$ Organic Felt & 303.2 & $\mathrm{~m} 2$ \\
\hline $\begin{array}{l}\text { 13mm Moisture Resistant Gypsum } \\
\text { Board }\end{array}$ & 41.8 & $\mathrm{~m} 2$ \\
\hline $\begin{array}{l}\begin{array}{l}16 m m \\
\text { Board }\end{array} \\
\text { Gypsum Fibre Gypsum }\end{array}$ & 41.8 & $\mathrm{~m} 2$ \\
\hline Galvanized Sheet & 102.3 & $\mathrm{~kg}$ \\
\hline Galvanized Studs & 266.0 & $\mathrm{~kg}$ \\
\hline Isocyanurate & 117.9 & $\mathrm{~m} 2(25 \mathrm{~mm})$ \\
\hline Joint Compound & $\begin{array}{lll}41.7 \\
\end{array}$ & $\mathrm{~kg}$ \\
\hline Modified Bitumen membrane & $1,219.6$ & $\mathrm{~kg}$ \\
\hline Nails & 20.0 & $\mathrm{~kg}$ \\
\hline Paper Tape & 0.5 & $\mathrm{~kg}$ \\
\hline Roofing Asphalt & 632.7 & $\mathrm{~kg}$ \\
\hline Screws Nuts \& Bolts & 5.5 & $\mathrm{~kg}$ \\
\hline Softwood Plywood & 79.8 & m2 (9mm) \\
\hline
\end{tabular}

\section{Cold-Formed Steel Roof \#2 (CFS-R2)}

Building Component Description:

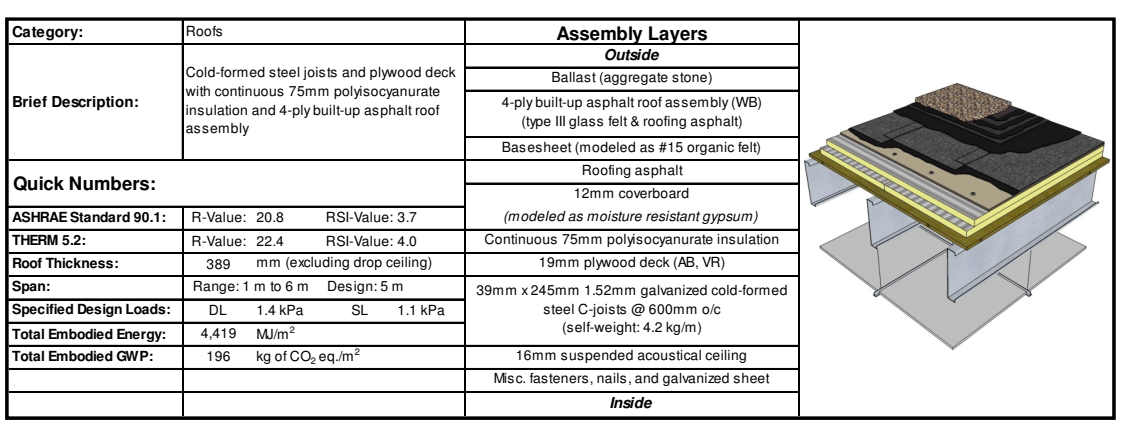

Life-Cycle Assessment Results:

\begin{tabular}{|c|c|c|c|c|c|c|c|c|c|c|c|c|c|c|c|c|}
\hline & \multicolumn{14}{|c|}{ Primary Energy Consumption (MJ) } & \multirow{3}{*}{\multicolumn{2}{|c|}{$\begin{array}{l}\text { Difference in } \\
\text { Operating Energy } \\
\text { from Baseline after } \\
\text { Lifespan }\end{array}$}} \\
\hline \multirow{3}{*}{$\begin{array}{l}\text { Lifespan } \\
\text { (Years) }\end{array}$} & & & & & & & oodied E & nergy ( & & & & & & & & \\
\hline & \multicolumn{3}{|c|}{ Manufacturing } & \multicolumn{3}{|c|}{ Construction } & \multicolumn{3}{|c|}{ Maintenance } & \multicolumn{3}{|c|}{ End of Life } & \multirow{2}{*}{$\begin{array}{c}3 \text { Total } \\
\text { EE }\end{array}$} & \multirow{2}{*}{$\begin{array}{c}{ }^{4} \text { Total } \\
\text { EE } \\
\text { EE }\end{array}$} & & \\
\hline & Material & ${ }^{2}$ Trans. & Total & Material & i1 ${ }^{2}$ Trans. & Total & Material & ${ }^{2}$ Trans. & Total & Material & \begin{tabular}{l|l|l|}
2 & 2 \\
\end{tabular} & Total & & & ${ }^{5}$ Total & ${ }^{6}$ perm $m^{2}$ \\
\hline itial & 87,956 & 245 & 88,201 & 0 & 551 & 551 & 0 & 0 & 0 & 0 & 0 & 0 & 88,752 & 2,336 & & \\
\hline
\end{tabular}
\begin{tabular}{|c|c|c|c|c|c|c|c|c|c|c|c|c|c|c|c|c|c|}
50 & 87,956 & 245 & 88,201 & 0 & 551 & 551 & 78,734 & 272 & 79,006 & 1 & 176 & 176 & 167,934 & 2,3419 & $-200,000$ & -341 \\
\hline
\end{tabular}

\begin{tabular}{|c|c|c|c|c|c|c|c|c|c|c|c|c|c|c|c|c|}
\hline \multirow{4}{*}{\begin{tabular}{|l} 
Lifiespan \\
(Years)
\end{tabular}} & \multicolumn{14}{|c|}{ Global Warming Potential ( $\mathrm{kg}$ of $\mathrm{CO}_{2}$ eq.) } & \multirow{3}{*}{\multicolumn{2}{|c|}{$\begin{array}{c}\text { Difference in } \\
\text { Operating GWP fror } \\
\text { Baseline after } \\
\text { Lifespan }\end{array}$}} \\
\hline & \multicolumn{14}{|c|}{ Embodied Global Warming Potential (GWP) } & & \\
\hline & \multicolumn{3}{|c|}{ Manutacturing } & \multicolumn{3}{|c|}{ Construction } & \multicolumn{3}{|c|}{ Maintenance } & \multicolumn{3}{|c|}{ End of L Life } & \multirow{2}{*}{\begin{tabular}{|l|}
${ }^{3}$ Total \\
GWP
\end{tabular}} & \multirow{2}{*}{$\begin{array}{c}{ }^{4} \mathrm{Cotal}^{\mathrm{G}} \\
\mathrm{GWP} \\
\text { per }{ }^{2}\end{array}$} & & \\
\hline & Materia & ${ }^{2}$ Trans & Total & Materia & $\begin{array}{ll}\text { al }{ }^{2} \text { Trans. } & \end{array}$ & Total & Material & ${ }^{2}$ Trans & Total & Material & $1^{2}$ Trans. & Total & & & ${ }^{5}$ Total & ${ }^{6}$ per m² ${ }^{2}$ \\
\hline Initial & 4,309 & 0 & 4,310 & 0 & 1 & 1 & 0 & 0 & 0 & 0 & 0 & 0 & 4,310 & 113 & 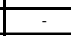 & - \\
\hline 50 & 4,309 & 0 & 4,310 & 0 & 1 & 1 & 3,131 & 1 & 3,131 & 0 & 0 & 0 & 7,442 & 196 & 0 & 0 \\
\hline
\end{tabular}

Embodied energy (and GWP) numbers are based on an area of roof $=38.0 \mathrm{~m}^{2} \quad$ (Span $\times$ Width $=5.0 \mathrm{~m} \times 7.6 \mathrm{~m}=38.0 \mathrm{~m}^{2}$ )

\begin{tabular}{|c|c|c|}
\hline Material List & \begin{tabular}{|l|l|} 
Quantitites \\
\end{tabular} & Unit \\
\hline$\# 15$ Organic Felt & 303.2 & $\mathrm{~m} 2$ \\
\hline $\begin{array}{l}13 \mathrm{~mm} \text { Moisture Resistant Gypsum } \\
\text { Board }\end{array}$ & 41.8 & $\mathrm{~m} 2$ \\
\hline $16 \mathrm{~mm}$ Gypsum Fibre Gypsum & 41.8 & m2 \\
\hline Ballast (aggregate stone) & $2,898.0$ & $\mathrm{~kg}$ \\
\hline Galvanized Sheet & 133.6 & $\mathrm{~kg}$ \\
\hline Galvanized Studs & 266.0 & $\mathrm{~kg}$ \\
\hline Isocyanurate & 117.9 & $\mathrm{~m} 2(25 \mathrm{mn}$ \\
\hline Joint Compound & 41.7 & $\mathrm{~kg}$ \\
\hline Nails & 20.0 & $\mathrm{~kg}$ \\
\hline Paper Tape & 0.5 & $\mathrm{~kg}$ \\
\hline Roofing Asphatt & $\begin{array}{l}1,150.4 \\
\text {. }\end{array}$ & $\mathrm{kg}$ \\
\hline Screws Nuts \& B B & 5.5 & $\mathrm{~kg}$ \\
\hline Softwood Plywood & 79.8 & $\mathrm{~m} 2(9 \mathrm{gmm}$ \\
\hline Type III Glass Felt & 600.5 & $\mathrm{~m} 2$ \\
\hline
\end{tabular}

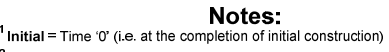

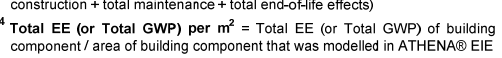

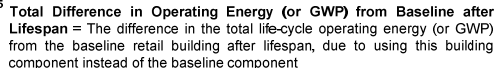
Total Difference in Operating Energy (or GWP) from Baseline after Lifespan per $m^{2}=$ Total difiterence in operating energy (or GWP) from
baseline after lifespan / net roof area of baseline retail building

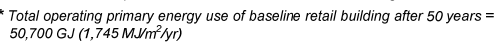

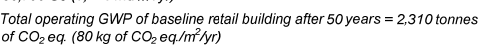

Total EE (or Total GWP) $=$ Total embodied energy (or total embodied GWP)
of building component after lifespan (ie.e. total manufacturing + total

net roof area of baseline retail building

"Total operating GWP of baseline retail building after 50 years $=2,310$ tonnes 


\section{Cold-Formed Steel Roof \#3 (CFS-R3)}

Building Component Description:

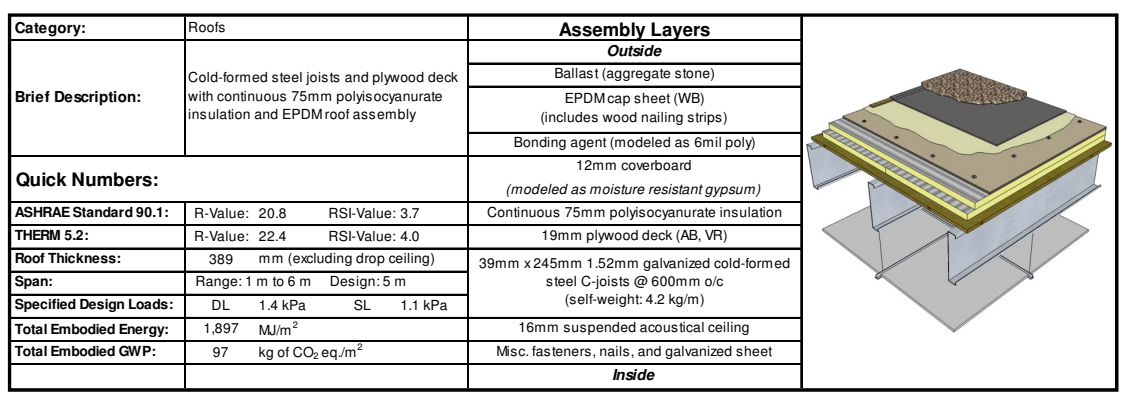

\section{Life-Cycle Assessment Results:}

Primary Energy Consumption (MJ)

\begin{tabular}{|c|c|c|c|c|c|c|c|c|c|c|c|c|c|c|c|c|}
\hline \multirow{3}{*}{$\begin{array}{l}\text { Lifespan } \\
\text { (Years) }\end{array}$} & \multicolumn{14}{|c|}{ Embodied Energy (EE) } & \multirow{2}{*}{\multicolumn{2}{|c|}{$\begin{array}{c}\text { Difference in } \\
\text { Operating Energy } \\
\text { from Baseline atte } \\
\text { Lifespan }\end{array}$}} \\
\hline & \multicolumn{3}{|c|}{ Manufacturing } & \multicolumn{3}{|c|}{ nstru } & \multicolumn{3}{|c|}{ Maintenance } & \multicolumn{3}{|c|}{ End of Life } & \multirow{2}{*}{${ }^{3}$ Total } & \multirow{2}{*}{$\begin{array}{c}{ }^{4} \text { Total } \\
\text { EE } \\
\text { per } \mathrm{m}^{2}\end{array}$} & & \\
\hline & \begin{tabular}{|l|} 
Material \\
\end{tabular} & ${ }^{2}$ Trans. & Total & \begin{tabular}{|l|} 
Materia \\
\end{tabular} & $\left.\right|^{2}$ Trans. & \begin{tabular}{|l|l|} 
\\
\end{tabular} & \begin{tabular}{|l|} 
Material \\
\end{tabular} & ${ }^{2}$ Trans. & Total & Material & 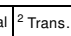 & \begin{tabular}{|l|} 
\\
\end{tabular} & & & \begin{tabular}{|l|}
${ }^{5}$ Total \\
\end{tabular} & ${ }^{6}{ }^{6} \mathrm{er} \mathrm{m}^{2}{ }^{2}$ \\
\hline Inititial & 38,357 & \begin{tabular}{|l|}
245 \\
\end{tabular} & 38,602 & 0 & 734 & \begin{tabular}{|l|}
734 \\
\end{tabular} & 0 & 0 & 0 & 0 & 0 & 0 & 39,336 & 1,035 & & \\
\hline 50 & 38,357 & 245 & $\mid 38,602$ & 0 & 734 & \begin{tabular}{|l|}
734 \\
\end{tabular} & & \begin{tabular}{|l|}
383 \\
\end{tabular} & & 1 & & & 72,097 & & $\mid-200,000$ & -34 \\
\hline
\end{tabular}

Global Warming Potential ( $\mathrm{kg}$ of $\mathrm{CO}_{2}$ eq.)

\begin{tabular}{|c|c|c|c|c|c|c|c|c|c|c|c|c|c|c|c|c|}
\hline \multirow{3}{*}{$\begin{array}{c}\text { Lifespan } \\
\text { (Years) }\end{array}$} & \multicolumn{14}{|c|}{ Embodied Global Warming Potential (GWP) } & \multirow{2}{*}{\multicolumn{2}{|c|}{$\begin{array}{l}\text { Difference in } \\
\text { Operating GWP from } \\
\text { Baseline after } \\
\text { Lifespan }\end{array}$}} \\
\hline & \multicolumn{3}{|c|}{ Manufacturing } & \multicolumn{3}{|c|}{ Construction } & \multicolumn{3}{|c|}{ Maintenance } & \multicolumn{3}{|c|}{ End of Life } & \multirow{2}{*}{\begin{tabular}{l|l|}
${ }^{3}$ Total \\
GWP
\end{tabular}} & \multirow{2}{*}{$\begin{array}{l}{ }^{4} \text { Total } \\
\text { GWP } \\
\text { per m}^{2}\end{array}$} & & \\
\hline & Material & \begin{tabular}{|c|}
${ }^{2}$ Trans. \\
\end{tabular} & Total & Material $\left.\right|^{2}$ & ${ }^{2}$ Trans. & Total & Material & ${ }^{2}$ Trans. & Total & Material & ${ }^{2}$ Trans. & Total & & & ${ }^{5}$ Total & ${ }^{6} \operatorname{perm}^{2}$ \\
\hline 1 Initial & 2,270 & 0 & 2,271 & 0 & 1 & 1 & 0 & 0 & 0 & 0 & 0 & 0 & 2,272 & 60 & - & \\
\hline 50 & 2,270 & 0 & 2,271 & 0 & 1 & 1 & 1,422 & 1 & 1,423 & 0 & 0 & 0 & 3,695 & 97 & 0 & 0 \\
\hline
\end{tabular}

Embodied energy (and GWP) numbers are based on an area of roof $=38.0 \mathrm{~m}^{2} \quad$ (Span $\times$ With $\left.=5.0 \mathrm{~m} \times 7.6 \mathrm{~m}=38.0 \mathrm{~m}^{2}\right)$

Net roof area of baseline retail building (gross roof area - openings) $=586.0 \mathrm{~m}^{2}$

\section{ATHENA $\circledast$ EIE Material Lis}

\begin{tabular}{|c|c|c|}
\hline Material List & Quantities & Unit \\
\hline $\begin{array}{l}13 m \mathrm{~mm} \text { Moisture Resistant Gypsum } \\
\text { Board }\end{array}$ & 41.8 & $\mathrm{~m} 2$ \\
\hline $\begin{array}{l}16 \mathrm{~mm} \text { Gypsum Fibre Gypsum } \\
\text { Board }\end{array}$ & 41.8 & $\mathrm{~m} 2$ \\
\hline 6 mil Polyethylene & 38.8 & $\mathrm{~m} 2$ \\
\hline Ballast (aggregate stone) & $8,694.0$ & $\mathrm{~kg}$ \\
\hline EPDM membrane & 312.1 & $\mathrm{~kg}$ \\
\hline Galvanized Sheet & 104.8 & $\mathrm{~kg}$ \\
\hline Galvanized Studs & 266.0 & $\mathrm{~kg}$ \\
\hline $\begin{array}{l}\text { Isocyanurate } \\
\end{array}$ & 117.9 & $\mathrm{m2} 225 \mathrm{~mm}$ \\
\hline Joint Compound & 83.4 & $\mathrm{~kg}$ \\
\hline Nails & 3.9 & $\mathrm{~kg}$ \\
\hline Paper Tape & 1.0 & $\mathrm{~kg}$ \\
\hline Screws Nuts \& Bolts & 5.5 & $\mathrm{~kg}$ \\
\hline $\begin{array}{l}\text { Small Dimension Sottwood Lumber, } \\
\text { kiln-dred }\end{array}$ & 0.1 & m3 3 \\
\hline Sottwood Plywood & 80.6 & $\mid$ \\
\hline
\end{tabular}

\section{Cold-Formed Steel Roof \#4 (CFS-R4)}

Building Component Description:

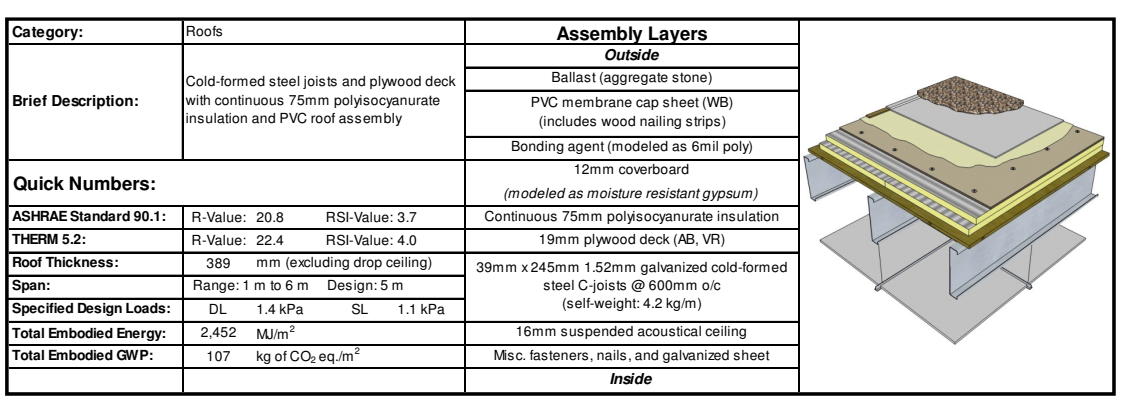

\section{Life-Cycle Assessment Results:}

\begin{tabular}{|c|c|c|c|c|c|c|c|c|c|c|c|c|c|c|c|c|}
\hline & \multirow{2}{*}{\multicolumn{14}{|c|}{ Embodied Energy (EE) }} & & \\
\hline \multirow{3}{*}{$\begin{array}{l}\text { Lestespan } \\
\text { (Years) }\end{array}$} & & & & & & & & & & & & & & & \multirow{2}{*}{\multicolumn{2}{|c|}{$\begin{array}{c}\text { Difference in } \\
\text { Operating Energy } \\
\text { from Baseline afte } \\
\text { Lifespan }\end{array}$}} \\
\hline & \multicolumn{3}{|c|}{ Manutacturing } & \multicolumn{3}{|c|}{ Construction } & \multicolumn{3}{|c|}{ Maintenance } & \multicolumn{3}{|c|}{ End of Life } & \multirow{2}{*}{$\begin{array}{c}{ }^{3} \text { Total } \\
\mathrm{EE}\end{array}$} & \multirow{2}{*}{$\begin{array}{c}{ }^{4} \text { Total } \\
\mathrm{E} \\
\text { per } \mathrm{m}^{2}\end{array}$} & & \\
\hline & Material & ${ }^{2}$ Trans. & Total & Material & ${ }^{2}$ Trans. & Total & \begin{tabular}{|l|} 
Material \\
\end{tabular} & ${ }^{2}$ Trans. & Total & Material & $\mid{ }^{2}$ Trans. & \begin{tabular}{|l|l|} 
Total \\
\end{tabular} & & & ${ }^{5}$ Total & ${ }^{6}$ per m ${ }^{2}$ \\
\hline Initia & \begin{tabular}{|l|l|}
22,997 \\
1007
\end{tabular} & 264 & 43,262 & 0 & 831 & \begin{tabular}{|l|l|}
831 \\
821
\end{tabular} & 1800 & & $1897-72-7$ & 0 & 0 & & \begin{tabular}{|l|l|l}
44,092 \\
2,90
\end{tabular} & $\frac{1,160}{2,450}$ & & \\
\hline 50 & |42,997 & 264 & $\mid 43,262$ & 0 & 831 & 831 & 48,400 & 478 & 48,878 & 1 & 209 & 210 & 933,180 & 2,452 & 00,000 & -341 \\
\hline
\end{tabular}

\begin{tabular}{|c|c|c|c|c|c|c|c|c|c|c|c|c|c|c|c|c|}
\hline & \multirow{2}{*}{\multicolumn{14}{|c|}{$\begin{array}{l}\text { Global Warming Potential ( } \mathrm{kg} \text { of } \mathrm{CO}_{2} \text { eq.) } \\
\text { Embodied Global Warming Potential (GWP) }\end{array}$}} & & \\
\hline \multirow{3}{*}{$\begin{array}{l}\text { Lestespan } \\
\text { (Years) }\end{array}$} & & & & & & & & & & & & & & & \multirow{2}{*}{\multicolumn{2}{|c|}{$\begin{array}{c}\text { Difference in } \\
\text { Operatiting GWP fron } \\
\text { Baseline after } \\
\text { Lifespan }\end{array}$}} \\
\hline & \multicolumn{3}{|c|}{ Manufacturing } & \multicolumn{3}{|c|}{ Construction } & \multicolumn{3}{|c|}{ Maintenance } & \multicolumn{3}{|c|}{ End of Life } & \multirow{2}{*}{$\begin{array}{l}{ }^{3} \text { Total } \\
\text { GWP }\end{array}$} & \multirow{2}{*}{$\begin{array}{l}{ }^{4} \mathrm{~T}^{4} \text { otal } \\
\text { GWP } \\
\text { per } \mathrm{m}^{2}\end{array}$} & & \\
\hline & \begin{tabular}{|l|} 
Material \\
\end{tabular} & ${ }^{2}$ Trans & Total & Material & 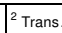 & Total & \begin{tabular}{|l|} 
Material \\
\end{tabular} & 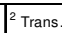 & Total & Materia & $\left.\right|^{2}{ }^{2}$ Trans. & Total & & & ${ }^{5}$ Total & ${ }^{6}$ per m ${ }^{2}$ \\
\hline${ }^{1}$ Initial & \begin{tabular}{|l|l|}
2,347 \\
\end{tabular} & 1 & 2,347 & 0 & 1 & 1 & 0 & 0 & 0 & 0 & 0 & 0 & \begin{tabular}{|l|l|}
2,348 \\
\end{tabular} & 62 & & \\
\hline 50 & \begin{tabular}{|l|l|}
2,3447 \\
\end{tabular} & 1 & 2,347 & 0 & 1 & 1 & \begin{tabular}{|l|l|}
1,702 \\
\end{tabular} & 1 & 1,703 & 0 & 0 & 0 & 4,051 & 107 & 0 & 0 \\
\hline
\end{tabular}

Embodied energy (and GWP) numbers are based on an area of roof $=38.0 \mathrm{~m}^{2} \quad\left(\right.$ Span $\times$ Width $\left.=5.0 \mathrm{~m} \times 7.6 \mathrm{~m}=38.0 \mathrm{~m}^{2}\right)$

Net roof area of baseline retail building (gross roof area - openings) $=586.0 \mathrm{~m}^{2}$
ATHENA $₫$ EIE Material List:

${ }^{2}$ Trans. = Transportatio

${ }^{3}$ Total EE (or Total GWP) $=$ Total embodided energy (or total embodied GWP
of buiding comonent of building component after lifiespan (i.e. total $\mathrm{mat}$
construction t total maintenance total

${ }^{4}$ Total EE (or Total GWP) per $\mathrm{m}^{2}=$ Total EE (or Total GWP) of building
component/ area of building component that was modeled in ATHENAQ EIE

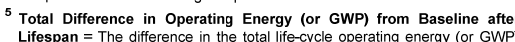

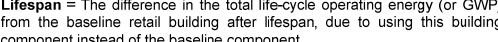
Total Difference in Operating Energy (or GWP) from Baseline after
Lifespan per $\mathrm{m}^{2}=$ Total difference in operating energy (or GWP) from

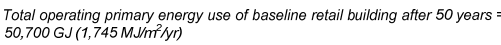

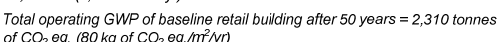

$$
\begin{aligned}
& \text { Notes: } \\
& { }^{1} \text { Initial }=\text { Time ' }
\end{aligned}
$$

${ }^{2}$ Trans. $=$ Transportation

of of building component after lifespan (i.e. total mal
construction +total maintenance + total endo-ofifife effects)

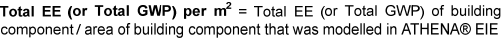

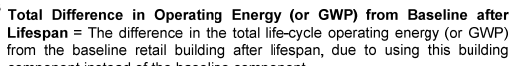

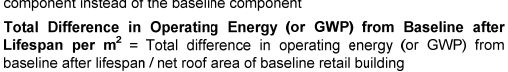

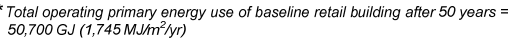

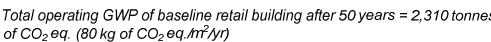

\begin{tabular}{l}
\hline Paper Tape \\
\hline PVC
\end{tabular}

\begin{tabular}{|l}
\hline PVC membrane \\
\hline Screws Nuts \& Bo \\
\hline
\end{tabular}

Snall Dimension Sotwood Lumber

kill-dried
Softwood Plywood 


\section{Cold-Formed Steel Roof \#5 (CFS-R5)}

Building Component Description:

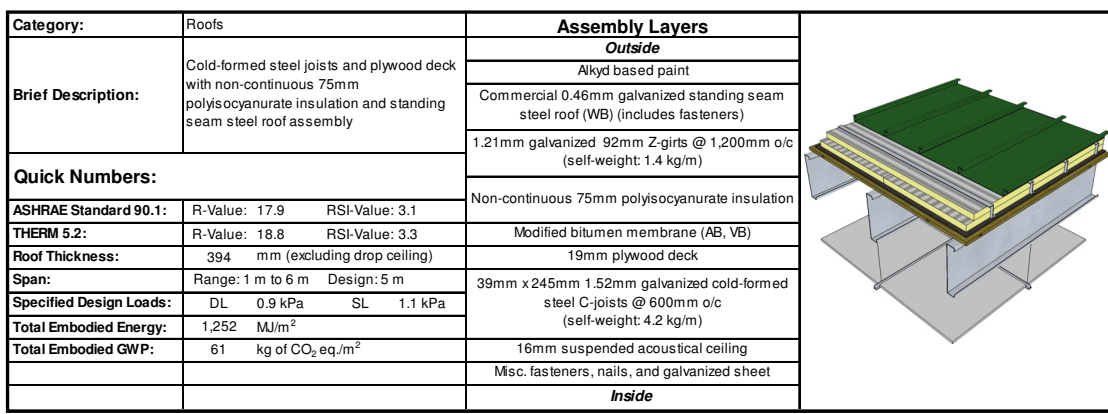

Life-Cycle Assessment Results:

Primary Energy Consumption (MJ)

\begin{tabular}{|c|c|c|c|c|c|c|c|c|c|c|c|c|c|c|c|}
\hline \multirow{3}{*}{\begin{tabular}{|c} 
Lifespan \\
(Years)
\end{tabular}} & \multicolumn{13}{|c|}{ Embodied Energy (EE) } & \multirow{2}{*}{\multicolumn{2}{|c|}{$\begin{array}{c}\text { Difference in } \\
\text { Operating Energy } \\
\text { from Baseline after } \\
\text { Lifespan }\end{array}$}} \\
\hline & \multicolumn{2}{|c|}{ Manufacturing } & \multicolumn{3}{|c|}{ Construction } & \multicolumn{3}{|c|}{ Maintenance } & \multicolumn{3}{|c|}{ End of Life } & \multirow{2}{*}{${ }_{-3}^{3}$ Total } & \multirow{2}{*}{\begin{tabular}{|c|}
${ }^{4} \mathrm{Total}$ \\
$\mathrm{EE}$ \\
perm
\end{tabular}} & & \\
\hline & \begin{tabular}{l|l} 
Material & 2 \\
2
\end{tabular} & \begin{tabular}{|l|} 
Total \\
\end{tabular} & Materia & al ${ }^{2}$ Tras & \begin{tabular}{|l|l} 
Total \\
\end{tabular} & Materia & ${ }^{2}{ }^{2}$ Trans & \begin{tabular}{|l|} 
Total \\
\end{tabular} & Material & ${ }^{2}$ Trans & \begin{tabular}{|l|} 
Total \\
\end{tabular} & & & ${ }^{5}$ Total & ${ }_{6}^{6} \mathrm{perm}^{2}$ \\
\hline & 1,7 & 944 & & 434 & $\overline{434}$ & 0 & 0 & 0 & 0 & 0 & 0 & 42,378 & 1,115 & & \\
\hline
\end{tabular}

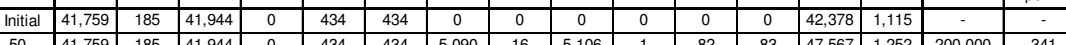

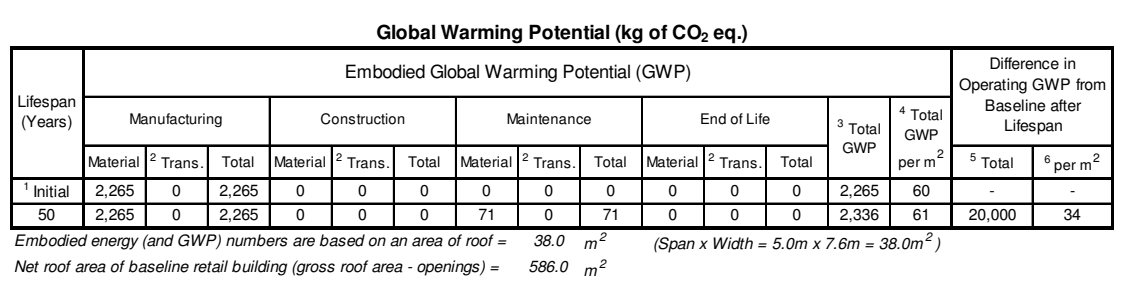

(Span $\times$ Width $\left.=5.0 \mathrm{~m} \times 7.6 \mathrm{~m}=38.0 \mathrm{~m}^{2}\right)$

\section{ATHENA $®$ EIE Material List:}

\begin{tabular}{|l|c|c|}
\multicolumn{1}{|c|}{ Material List } & Quantities & Unit \\
\hline \begin{tabular}{l|c|c|}
\hline $16 \mathrm{~mm}$ Gypsum Fibre Gypsum \\
Board
\end{tabular} & 41.8 & $\mathrm{~m} 2$ \\
\hline Foam Polyisocyanurate & 117.1 & $\mathrm{~m} 2(25 \mathrm{~mm})$ \\
\hline Galvanized Sheet & 310.9 & $\mathrm{~kg}$ \\
\hline Galvanized Studs & 310.4 & $\mathrm{~kg}$ \\
\hline Joint Compound & 41.7 & $\mathrm{~kg}$ \\
\hline Modififed Bitumen membrane & 118.0 & $\mathrm{~kg}$ \\
\hline Nails & 2.7 & $\mathrm{~kg}$ \\
\hline Paper Tape & 0.5 & $\mathrm{~kg}$ \\
\hline Screws Nuts \& Bolts & 5.9 & $\mathrm{~kg}$ \\
\hline Softwood Plywood & 79.8 & $\mathrm{~m} 2(9 \mathrm{~mm})$ \\
\hline Solvent Based Alkyd Paint & 27.3 & $\mathrm{~L}$ \\
\hline
\end{tabular}

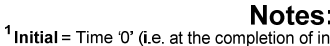

${ }^{2}$ Trans. $=$ Transportation

Total EE (or Total GWP) = Total embodied energy (or total embodied GWP) of building component after lifespan (i.e. total manufacturing + to

Total EE (or Total GWP) per $\mathrm{m}^{2}=$ Total EE (or Total GWP) of building
component/ area of building component that was modelled in ATHENAQIEE Total Difference in Operating Energy (or GWP) from Beseline after) Lifespan = The difference in the total life-cycle operating energy (or GWP)
from the baseline retail building after lifespan, due to using this building from the baseline retail building after lifespan,
component instead of the baseline component

Tor Lifespan per $\mathrm{m}^{2}=$ Total difference in operating energy (or GWP) from baseline after lifespan / net roof area of baseline retail building

* Total operating primary energy use of baseline retail building after 50 years

Total operating GWP of baseline retail building after 50 years $=2,310$ tonnes

\section{Cold-Formed Steel Roof \#6 (CFS-R6)}

Building Component Description:

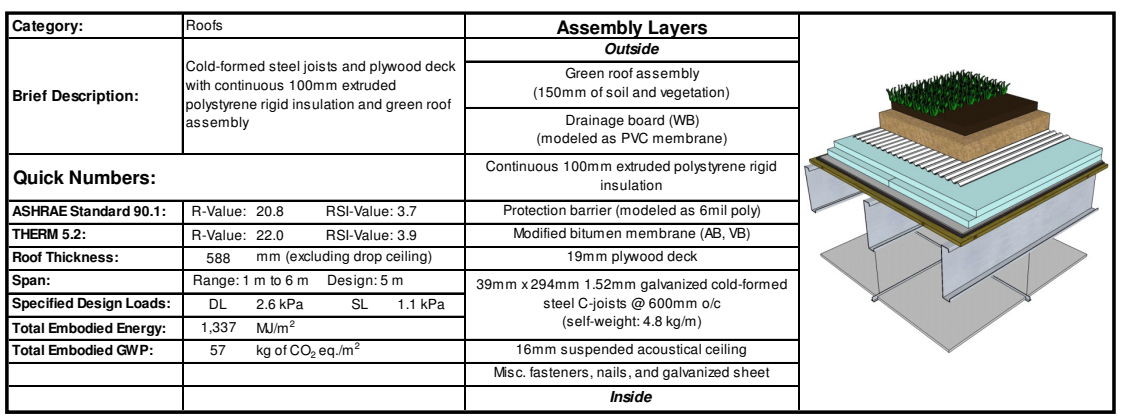

Life-Cycle Assessment Results:

\begin{tabular}{|c|c|c|c|c|c|c|c|c|c|c|c|c|c|c|c|c|}
\hline & \multicolumn{14}{|c|}{ Primary } & & \\
\hline \multirow{3}{*}{$\begin{array}{l}\text { Lifespan } \\
\text { (Years) }\end{array}$} & \multicolumn{14}{|c|}{ Embodied Energy (EE) } & \multirow{2}{*}{\multicolumn{2}{|c|}{$\begin{array}{l}\text { Difference in } \\
\text { Operating Energy } \\
\text { trom Baseline after } \\
\text { Liefspan }\end{array}$}} \\
\hline & \multicolumn{3}{|c|}{ Manutacturing } & \multicolumn{3}{|c|}{ Construction } & \multicolumn{3}{|c|}{ Maintenance } & \multicolumn{3}{|c|}{ End of Life } & \multirow{2}{*}{$\begin{array}{l}{ }^{3} \text { Total } \\
\text { EE }\end{array}$} & \multirow{2}{*}{\begin{tabular}{|c|}
${ }^{4}$ Total \\
$\mathrm{EE}$ \\
$\mathrm{EE}$
\end{tabular}} & & \\
\hline & Material & Trans. & Total & Material & ${ }^{2}$ Trans. & Total & Material & ${ }^{2}$ Trans. & \begin{tabular}{|l|l|} 
Total \\
\end{tabular} & Material & Tians. & Total & & & ${ }^{5}$ Total & ${ }^{6}$ perm ${ }^{2}$ \\
\hline Initial & 35,457 & 165 & 35,622 & 0 & 443 & 443 & 0 & 0 & 0 & 0 & 0 & 0 & 36,065 & 949 & & \\
\hline
\end{tabular}
\begin{tabular}{|c|c|c|c|c|c|c|c|c|c|c|c|c|c|c|c|c|}
\hline 50 & 35,457 & 165 & 35,622 & 0 & 443 & 443 & 14,611 & 40 & 14,651 & 1 & 85 & 86 & 50,802 & 1,337 & $-200,000$ & -341 \\
\hline
\end{tabular}

\begin{tabular}{|c|c|c|c|c|c|c|c|c|c|c|c|c|c|c|c|c|}
\hline \multirow{4}{*}{$\begin{array}{l}\text { Lifespan } \\
\text { (Years) }\end{array}$} & \multicolumn{16}{|c|}{ Global Warming Potential ( $\mathrm{kg}$ of $\mathrm{CO}_{2}$ eq.) } \\
\hline & \multicolumn{14}{|c|}{ Embodied Global Warming Potential (GWP) } & \multirow{2}{*}{\multicolumn{2}{|c|}{$\begin{array}{l}\text { Difference in } \\
\text { Operating GWP from } \\
\text { Baseline after } \\
\text { Lifespan }\end{array}$}} \\
\hline & \multicolumn{3}{|c|}{ Manutacturing } & \multicolumn{3}{|c|}{ Construction } & \multicolumn{3}{|c|}{ Maintenance } & \multicolumn{3}{|c|}{ End of Life } & \multirow{2}{*}{$\begin{array}{l}{ }^{3} \text { Total } \\
\text { GWWP }\end{array}$} & \multirow{2}{*}{$\begin{array}{c}{ }^{4} \text { Total } \\
\text { GWP } \\
\text { per } \mathrm{m}^{2}\end{array}$} & & \\
\hline & Material & ${ }^{2}$ Trans. & Total & Material & ${ }^{2}$ Trans. & Total & Material & ${ }^{2}$ Trans. & Total & Material & ${ }^{2}$ Trans. & \begin{tabular}{|l|} 
Total \\
\end{tabular} & & & ${ }^{5}$ Total & ${ }^{6}$ perm ${ }^{2}$ \\
\hline Initial & 1,758 & 0 & 1,758 & 0 & 0 & 0 & 0 & 0 & 0 & 0 & 0 & 0 & 1,758 & 46 & & \\
\hline 50 & 1,758 & 0 & 1,758 & 0 & 0 & 0 & 406 & 0 & 406 & 0 & 0 & 0 & 2,165 & 57 & 0 & 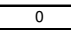 \\
\hline
\end{tabular}

ATHENA $₫$ EIE Material List:

\begin{tabular}{|c|c|c|}
\hline Material List & Quantities & Unit \\
\hline $\begin{array}{l}16 \mathrm{~mm} \text { Gypsum Fibre Gypsum } \\
\text { Board }\end{array}$ & 41.8 & $\mathrm{~m} 2$ \\
\hline 6 mil Polyethylene & 40.3 & $\mathrm{~m} 2$ \\
\hline Ballast (aggregate stone) & 144.9 & $\mathrm{~kg}$ \\
\hline Extruded Polystyrene & 155.7 & $\mathrm{~m} 2(25 \mathrm{~mm})$ \\
\hline Galvanized Sheet & 88.3 & $\mathrm{~kg}$ \\
\hline Galvanized Studs & 304.4 & $\mathrm{~kg}$ \\
\hline Joint Compound & 41.7 & $\mathrm{~kg}$ \\
\hline Modified Bitumen membrane & 142.1 & $\mathrm{~kg}$ \\
\hline Nails & 2.7 & $\mathrm{~kg}$ \\
\hline $\begin{array}{l}\text { Paper Tape } \\
\end{array}$ & 0.5 & $\mathrm{~kg}$ \\
\hline PVC membrane & 169.6 & $\mathrm{~kg}$ \\
\hline Screws Nuts \& Bolts & 5.5 & $\mathrm{~kg}$ \\
\hline Softwood Plywood & 79.8 & $29 \mathrm{mn}$ \\
\hline
\end{tabular}

Notes:
${ }^{1}{ }^{\prime}$ Initial $=$ Time $\mathrm{O}^{\prime}($ i.e. at the completion of initial construction)

${ }^{2}$ Trans. = Transportation

Total EE (or Total GWP) = Total embodied energy (or total embodied GWP of building component after lifespan (i.e. total man
construction + total maintenance + total end-oflife effects)

${ }^{4}$ Total EE (or Total GWP) per $\mathrm{m}^{2}=$ Total EE (or Total GWP) of building
component / area of building component that was modelled in ATHENAQEIE 5 Tomponent/ area of building component that was modelled in ATHENAQ ELE Total Difference in Operating Energy (or GWP) from Baseline after
Lifespan = The difference in the total life-cycle operating energy (or GWPP) from the baseline retail building ater lifespan, due to using this building
component instead of the baseline component ${ }^{6}$ Total Difference in Operating Energy (or GWP) from Baseline after Lifespan per $\mathrm{m}^{2}=$ Total difference in operating energy (or GWP) from
baseline after lifespan $/$ net roof area of baseline retail building

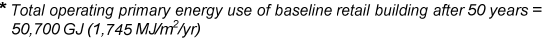
* Total operating GWP of baseline retail building after 50 years $=2,310$ tonnes
of $\mathrm{CO}_{2}$ eq. $\left(80 \mathrm{~kg}\right.$ of $\mathrm{CO}_{2}$ eq. $\left.\mathrm{m}^{2} / \mathrm{yr}\right)$ 


\section{Cold-Formed Steel Roof \#7 (CFS-R7)}

Building Component Description:

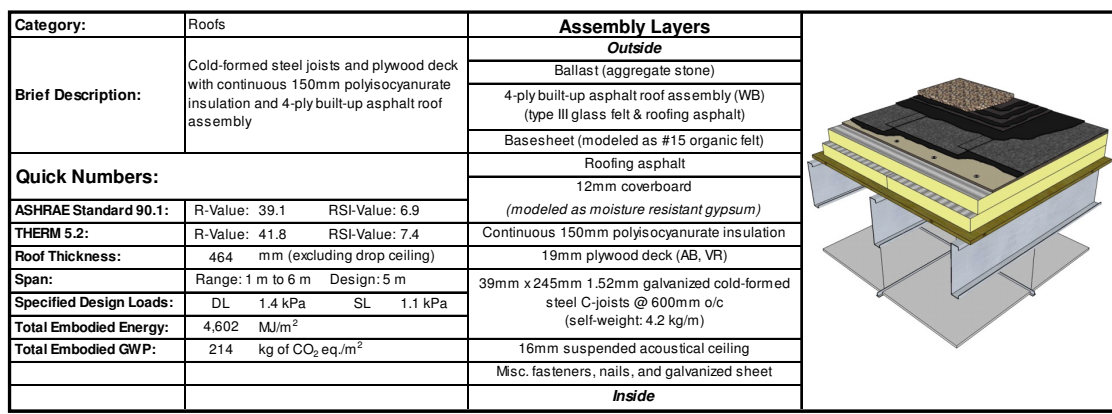

Life-Cycle Assessment Results:

\begin{tabular}{|c|c|c|c|c|c|c|c|c|c|c|c|c|c|c|c|c|}
\hline \multirow{4}{*}{$\mid \begin{array}{c}\text { Lifespan } \\
\text { (Years) }\end{array}$} & \multicolumn{16}{|c|}{ Energy Consump } \\
\hline & \multicolumn{14}{|c|}{ Embodied Energy (EE) } & \multirow{2}{*}{\multicolumn{2}{|c|}{$\begin{array}{l}\text { Difference in } \\
\text { Operating Energy } \\
\text { from Baseline after } \\
\text { Lifespan }\end{array}$}} \\
\hline & \multicolumn{3}{|c|}{ Manutacturing } & \multicolumn{3}{|c|}{ Construction } & \multicolumn{3}{|c|}{ Maintenance } & \multicolumn{3}{|c|}{ End of Life } & \multirow{2}{*}{$\begin{array}{l}{ }^{3} \text { Total } \\
\mathrm{EE}^{2}\end{array}$} & \multirow{2}{*}{$\begin{array}{c}{ }^{4} \text { Total } \\
\text { EE } \\
\text { per m }\end{array}$} & & \\
\hline & \begin{tabular}{|l|l|} 
Materia \\
\end{tabular} & $\begin{array}{l}1{ }^{2} \text { Trans. } \\
\text {. }\end{array}$ & Total & Material & \begin{tabular}{|l|}
${ }^{2}$ Trans. \\
\end{tabular} & Total & Material & $\begin{array}{l}{ }^{2} \text { Trans. } \\
\text {. }\end{array}$ & \begin{tabular}{|l|l|} 
Total \\
\end{tabular} & Material & \begin{tabular}{|l|}
${ }^{2}$ Trans. \\
\end{tabular} & Total & & & ${ }^{5}$ Total & ${ }^{6}$ per $^{2}$ \\
\hline Initial & 94,857 & 249 & 95,106 & 0 & 574 & 574 & 0 & 0 & 0 & 0 & 0 & 0 & 95,680 & 2,518 & & \\
\hline
\end{tabular}

\begin{tabular}{|c|cc|c|c|c|c|c|c|c|c|c|c|c|c|c|c|}
\hline 'Initial & 94,857 & 249 & 95,106 & 0 & 574 & 574 & 0 & 0 & 0 & 0 & 0 & 0 & 95,680 & 2,518 & - & - \\
\hline 50 & 94,857 & 249 & 95,106 & 0 & 574 & 574 & 78,734 & 272 & 79,006 & 1 & 184 & 184 & 174,870 & 4,602 & $-1,100,000$ & $-1,877$ \\
\hline
\end{tabular}

\begin{tabular}{|c|c|c|c|c|c|c|c|c|c|c|c|c|c|c|c|c|}
\hline \multirow{4}{*}{$\begin{array}{c}\text { Lifespan } \\
\text { (Years) }\end{array}$} & \multicolumn{16}{|c|}{ Global Warming Potential ( $\mathrm{kg}$ of $\mathrm{CO}_{2}$ eq.) } \\
\hline & \multicolumn{14}{|c|}{ Embodied Global Warming Potential (GWP) } & \multirow{2}{*}{\multicolumn{2}{|c|}{$\begin{array}{l}\text { Difference in } \\
\text { Operating GWP fron } \\
\text { Baseline after } \\
\text { Lifespan }\end{array}$}} \\
\hline & \multicolumn{3}{|c|}{ Manutacturing } & \multicolumn{3}{|c|}{ Construction } & \multicolumn{3}{|c|}{ Maintenance } & \multicolumn{3}{|c|}{ End of Life } & \multirow{2}{*}{$\begin{array}{l}{ }^{3} \text { Total } \\
\text { GWP }\end{array}$} & \multirow{2}{*}{$\begin{array}{l}{ }^{4}{ }^{\mathrm{T} o t a l} \\
\mathrm{GWW} \\
\text { per m}\end{array}$} & & \\
\hline & Material & ${ }^{2}$ Trans. & Total & Material & 1| ${ }^{2}$ Trans. & Total & Material & ${ }^{2}$ Trans. & Total & Material & $\left.\right|^{2}$ Trans. & Total & & & ${ }^{5}$ Total & ${ }^{6}$ per m$^{2}$ \\
\hline \begin{tabular}{|l|} 
'Initial \\
\end{tabular} & 5,013 & 0 & 5,013 & 0 & 1 & 1 & 0 & 0 & 0 & 0 & 0 & 0 & 5,014 & 132 & & \\
\hline 50 & 5,013 & 0 & 5,013 & 0 & 1 & 1 & 3,131 & 1 & 3,131 & 0 & 0 & 0 & 8,146 & 214 & $-60,000$ & -102 \\
\hline
\end{tabular}

Net roof area of baseline retail building (gross roof area - openings) $=586.0 \mathrm{~m}^{2}$

ATHENA $₫$ EIE Material List:

\begin{tabular}{|c|c|c|}
\hline Material List & Quantities & Unit \\
\hline \#15 Organic Felt & 303.2 & $\mathrm{~m} 2$ \\
\hline $\begin{array}{l}\text { 13mm Moisture Resistant Gypsum } \\
\text { Board }\end{array}$ & 41.8 & $\mathrm{~m} 2$ \\
\hline $\begin{array}{l}16 \mathrm{~mm} \text { Gypsum Fibre Gypsum } \\
\text { Board }\end{array}$ & 41.8 & $\mathrm{~m} 2$ \\
\hline Ballast (aggregate stone) & $2,898.0$ & $\mathrm{~kg}$ \\
\hline $\begin{array}{l}\text { Galvanized Sheet } \\
\end{array}$ & 133.6 & $\mathrm{~kg}$ \\
\hline $\begin{array}{l}\text { Galvanized Studs } \\
\end{array}$ & 266.0 & $\mathrm{~kg}$ \\
\hline Isocyanurate & 235.8 & $2(25)$ \\
\hline \begin{tabular}{|l|} 
Joint Compound \\
\end{tabular} & 41.7 & $\mathrm{~kg}$ \\
\hline Nails & 20.0 & $\mathrm{~kg}$ \\
\hline $\begin{array}{l}\text { Paper Tape } \\
\end{array}$ & 0.5 & $\mathrm{~kg}$ \\
\hline $\begin{array}{l}\text { Roofing Asphatt } \\
\end{array}$ & 1,150.4 & $\mathrm{kg}$ \\
\hline Screws Nuts \& Bolts & 5.5 & $\mathrm{~kg}$ \\
\hline Soltwood Plywood & 79.8 & $m 2$ 2 $9 n$ \\
\hline Type III Glass Felt & 606.5 & $\mathrm{~m}^{2}$ \\
\hline
\end{tabular}

Cold-Formed Steel Roof \#8 (CFS-R8)

Building Component Description:

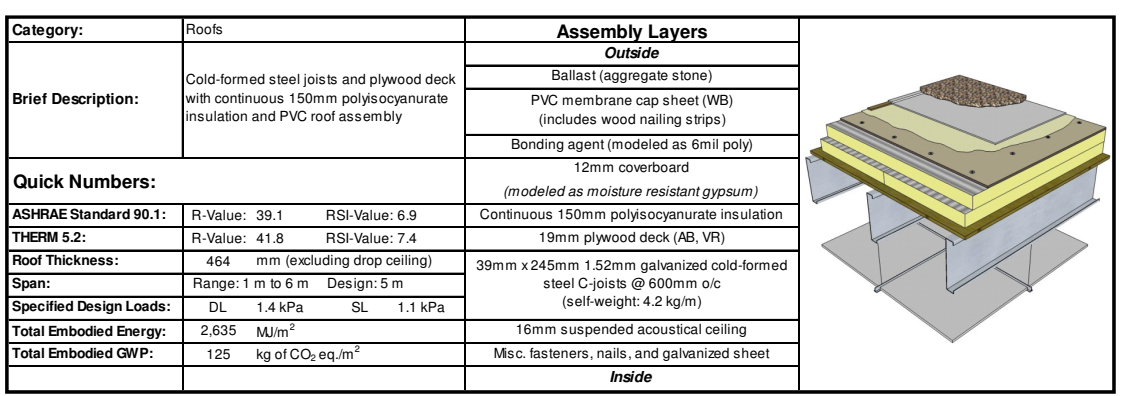

Life-Cycle Assessment Results:

Primary Energy Consumption (MJ)

\begin{tabular}{|c|c|c|c|c|c|c|c|c|c|c|c|c|c|c|c|c|}
\hline \multirow{3}{*}{\begin{tabular}{|l} 
Lifespan \\
(Years)
\end{tabular}} & \multicolumn{14}{|c|}{ Embodied Energy (EE) } & \multirow{2}{*}{\multicolumn{2}{|c|}{$\begin{array}{c}\text { Difference in } \\
\text { Operating Energy } \\
\text { from Baseline afte } \\
\text { Lifespan }\end{array}$}} \\
\hline & \multicolumn{3}{|c|}{ Manutacturing } & \multicolumn{3}{|c|}{ Construction } & \multicolumn{3}{|c|}{ Maintenance } & \multicolumn{3}{|c|}{ End of Life } & \multirow{2}{*}{$-{ }^{3}$ Total } & \multirow{2}{*}{$\begin{array}{c}{ }^{4} \text { Total } \\
\text { EE } \\
\text { per m }{ }^{2}\end{array}$} & & \\
\hline & Material & ${ }^{2}$ Trans. & Total & \begin{tabular}{|l|} 
Material \\
\end{tabular} & ${ }^{2}$ Trans. & Total & \begin{tabular}{|l|} 
Material \\
\end{tabular} & 1/2 Trans. & Total & \begin{tabular}{|l|} 
Material \\
\end{tabular} & ${ }^{2}$ Trans. & \begin{tabular}{|l|} 
Total \\
\end{tabular} & & & ${ }^{5}$ Total & ${ }^{6}$ perm $^{2}$ \\
\hline \begin{tabular}{|l} 
nnitial \\
nation
\end{tabular} & 49,898 & $\begin{array}{l}268 \\
\end{array}$ & 50,166 & 0 & 854 & 854 & 0 & 0 & 0 & 0 & 0 & 0 & \begin{tabular}{|l|l|}
51,020 \\
\end{tabular} & 1,343 & & \\
\hline 50 & |49,898 & 268 & 50,1166 & 0 & 854 & 854 & 48,400 & 478 & \begin{tabular}{|l|l|} 
\\
\end{tabular} & 1 & 217 & 218 & & & 4,100, & $-1,8$ \\
\hline
\end{tabular}

Global Warming Potential (kg of $\mathrm{CO}_{2}$ eq.)

\begin{tabular}{|c|c|c|c|c|c|c|c|c|c|c|c|c|c|c|c|c|}
\hline \multirow{4}{*}{ (Years) } & & & \\
\hline & & & & & & & & & & & & & & & \multirow{2}{*}{\multicolumn{2}{|c|}{\begin{tabular}{|c} 
Difference in \\
Operating GWP from \\
Baseline after \\
Lifespan
\end{tabular}}} \\
\hline & \multicolumn{3}{|c|}{ Manutacturing } & \multicolumn{3}{|c|}{ Construction } & \multicolumn{3}{|c|}{ Maintenance } & \multicolumn{3}{|c|}{ End of Life } & \multirow{2}{*}{$\begin{array}{l}{ }^{3} \text { Total } \\
\text { GWP }\end{array}$} & \multirow{2}{*}{$\begin{array}{c}{ }^{4} \text { Total } \\
\mathrm{GWP} \\
\text { per } \mathrm{m}^{2}\end{array}$} & & \\
\hline & \begin{tabular}{|l|} 
Material \\
\end{tabular} & ${ }^{2}$ Trans & otal & Material & 2 Trans. & \begin{tabular}{|l|l|} 
Total \\
\end{tabular} & \begin{tabular}{|l|} 
Material \\
\end{tabular} & 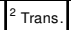 & Total & Material & 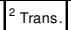 & Total & & & ${ }^{5}$ Total & ${ }^{6}{ }^{6}$ per m ${ }^{2}$ \\
\hline 50 & \begin{tabular}{|l|l|}
3,050 \\
\end{tabular} & 1 & & 0 & & 1 & 0 & & & & 8 & $\pi$ & & & & \\
\hline 50 & ,050 & 1 & 3,051 & 0 & 1 & 1 & 1,702 & 1 & 1,703 & 0 & 0 & 0 & 4,755 & 125 & $-60,000$ & \\
\hline
\end{tabular}

Embocied energy (and GWP) numbers are based on an area of roof $=38.0 \mathrm{~m}^{2} \quad\left(\right.$ Span $\times$ Width $\left.=5.0 \mathrm{~m} \times 7.6 \mathrm{~m}=38.0 \mathrm{~m}^{2}\right)$

Net roof area of baseline retail building (gross root area - openings) $=586.0 \mathrm{~m}^{2}$

ATHENA ® EIE Material List:

\begin{tabular}{|c|c|c|}
\hline Material List & Quantities & Unit \\
\hline 13mm Moisture Resistant Gypsum & 41.8 & $\mathrm{~m} 2$ \\
\hline $\begin{array}{l}\text { Doanm Gypsum Fibre Gypsum } \\
\text { Board } \\
\text { Board }\end{array}$ & 41.8 & $\mathrm{~m} 2$ \\
\hline 6 mil Polyethylene & 38.8 & $\mathrm{~m} 2$ \\
\hline Ballast (aggregate stone) & $8,694.0 \quad-\quad 2$ & $\mathrm{~kg}$ \\
\hline Galvanized Sheet & 100.9 & $\mathrm{~kg}$ \\
\hline Galvanized Studs & 266.0 & \\
\hline Isocyanurate & 235.8 & $\mathrm{~m} 2(25 \mathrm{~mm}$ \\
\hline Joint Compound & 83.4 & $\mathrm{~kg}$ \\
\hline Nails & 4.7 & $\mathrm{~kg}$ \\
\hline Paper Tape & 1.0 & $\mathrm{~kg}$ \\
\hline PVC membrane & 831.1 & $\mathrm{~kg}$ \\
\hline Screws Nuts \& Bolts & 5.5 & $\mathrm{~kg}$ \\
\hline Soffwood Lumber. & 0.1 & $\mathrm{~m} 3$ \\
\hline & 80.6 & m2 (9mm) \\
\hline
\end{tabular}

Notes:

${ }^{2}$ Tritial $=$ Time $0^{\prime}$ (i.e. at

Total EE (or Total GWP) $=$ Total embodied energy (or total embodied GWP)
of building component after lifespan (i.e. total manufacturing + total

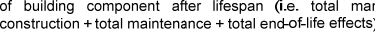

Total EE (or Total GWP) per $m^{2}=$ Total EE (or Total GWP) of builing
component/ area of builing component that was modeled in ATHENAQEIE ${ }^{5}$ Total Difference in Operating Energy (or GWP) from Baseline after

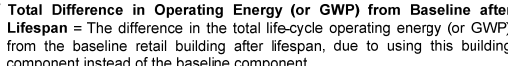
Total Difference in Operating Energy (or GWP) from Baseline after
tifespan per $\mathrm{m}^{2} \stackrel{\text { Total }}{=}$ Tifference in operating energy (or GWP) from

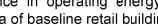

- Total operating primary energy use of baseline retail building affer 50 years

"Total operating GWP of baseline retail building affer 50 years $=2,310$ tonnes

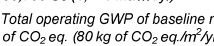

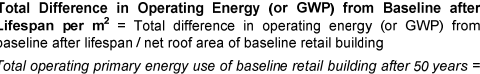

${ }^{1}$ Initial $=$ Time $\mathrm{O}^{\prime}$ (i.e. at th
${ }^{2}$ Trans. $=$ Transportation

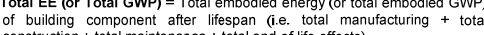

Cotal EE (or Total GWP) per $\mathrm{m}^{2}=$ Total EE (or Total GWP) of building
component area of building component that was modelled in ATHENAQ EIE

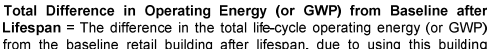

component instead of the baseline component 


\section{Cold-Formed Steel Roof \#9 (CFS-R9)}

Building Component Description:

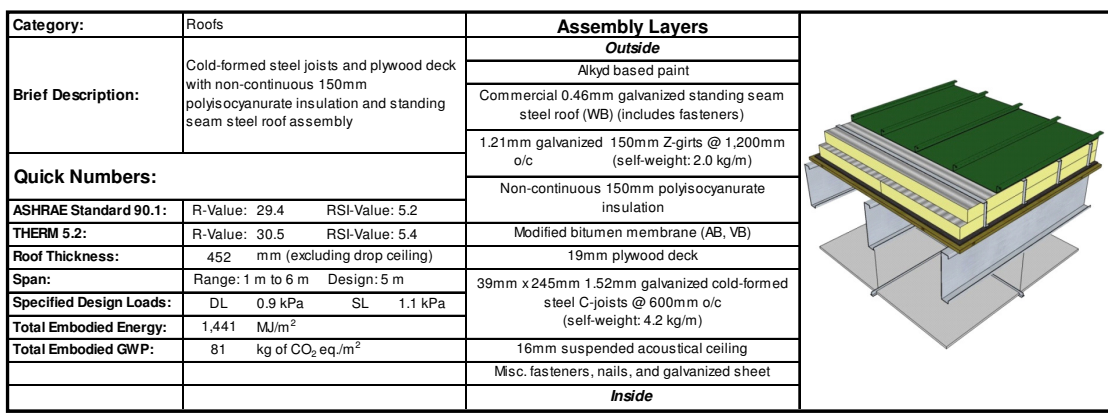

Life-Cycle Assessment Results:

\begin{tabular}{|c|c|c|c|c|c|c|c|c|c|c|c|c|c|c|c|c|}
\hline \multirow{4}{*}{$\begin{array}{l}\text { Lifespan } \\
\text { (Years) }\end{array}$} & \multicolumn{14}{|c|}{ Primary Energy Consumption (MJ) } & \multirow{3}{*}{\multicolumn{2}{|c|}{$\begin{array}{c}\text { Difference in } \\
\text { Operating Energy } \\
\text { from Baseline after } \\
\text { Lifespan }\end{array}$}} \\
\hline & & & & & & & bodied $\mathrm{E}$ & Energy ( & & & & & & & & \\
\hline & \multicolumn{3}{|c|}{ Manutacturing } & \multicolumn{3}{|c|}{ Construction } & \multicolumn{3}{|c|}{ Maintenance } & \multicolumn{3}{|c|}{ End of Life } & \multirow{2}{*}{$\begin{array}{c}{ }^{3} \text { Total } \\
\text { EE }\end{array}$} & \multirow{2}{*}{$\mid \begin{array}{c}{ }^{4} \text { Total } \\
\mathrm{EE} \\
\text { per } \mathrm{m}^{2}\end{array}$} & & \\
\hline & Materia & 11 ${ }^{2}$ Trans. & Total & Material & 2 Trans. & Total & Material & al ${ }^{2}$ Trans. & Total & Material & $\left.\right|^{2}$ Trans. & Total & & & ${ }^{5}$ Total & ${ }^{6}$ per m$^{2}$ \\
\hline |initial & 48,926 & 189 & 49,115 & 0 & 458 & 458 & 0 & 0 & 0 & 0 & 0 & 0 & 49,574 & 1,305 & & \\
\hline
\end{tabular}

Global Warming Potential ( $\mathrm{kg}$ of $\mathrm{CO}_{2}$ eq.)

\begin{tabular}{|c|c|c|c|c|c|c|c|c|c|c|c|c|c|c|c|c|}
\hline & & & & & & bal Y & Varming & Poten & tial ( $\mathbf{k}$ & $\mathrm{g}$ of $\mathrm{CO}_{2}$ & ${ }_{2}$ eq.) & & & & & \\
\hline \multirow{3}{*}{$\begin{array}{l}\text { Lifespan } \\
\text { (Years) }\end{array}$} & \multicolumn{14}{|c|}{ Embodied Global Warming Potential (GWP) } & \multirow{2}{*}{\multicolumn{2}{|c|}{$\begin{array}{l}\text { Difference in } \\
\text { Operating GWP fron } \\
\text { Baseline after } \\
\text { Lifespan }\end{array}$}} \\
\hline & \multicolumn{3}{|c|}{ Manufacturing } & \multicolumn{3}{|c|}{ Construction } & \multicolumn{3}{|c|}{ Maintenance } & \multicolumn{3}{|c|}{ End of Lifie } & \multirow{2}{*}{$\begin{array}{l}3^{3} \text { otal } \\
\text { GWP }\end{array}$} & \multirow{2}{*}{$\begin{array}{l}{ }^{4} \text { Total } \\
\text { GWP } \\
\text { per m }\end{array}$} & & \\
\hline & Material & ${ }^{2}$ Trans. & \begin{tabular}{|l|} 
Total \\
\end{tabular} & Material & ${ }^{2}$ Trans. & Total & \begin{tabular}{|l|} 
Material \\
\end{tabular} & ${ }^{2}$ Trans. & Total & \begin{tabular}{|l|} 
Material \\
\end{tabular} & 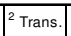 & Total & & & \begin{tabular}{|l|}
${ }^{5}$ Total \\
\end{tabular} & ${ }^{6}$ per m ${ }^{2}$ \\
\hline \begin{tabular}{|l|} 
Innitial \\
\end{tabular} & \begin{tabular}{|l|}
2,989 \\
\end{tabular} & 0 & $\begin{array}{ll}2,989 \\
\end{array}$ & 0 & 0 & 0 & 0 & 0 & 0 & 0 & 0 & 0 & 2,990 & 79 & & \\
\hline 50 & \begin{tabular}{|l|l|}
2,989 \\
\end{tabular} & 0 & 2,989 & 0 & 0 & 0 & 71 & 0 & 71 & 0 & 0 & 0 & 3,061 & 81 & $-30,000$ & -51 \\
\hline
\end{tabular}
Net roof area of baseline retail building (gross roof area - openings) $=586.0 \mathrm{~m}^{2}$

\section{ATHENA $₫$ EIE Material List:}

\begin{tabular}{|l|c|c|}
\multicolumn{1}{|c|}{ Material List } & Quantities & Unit \\
\hline $\begin{array}{l}\text { 16mm Gypsum Fibre Gypsum } \\
\text { Board }\end{array}$ & 41.8 & $\mathrm{~m} 2$ \\
\hline Foam Polyisocyanurate & 234.2 & $\mathrm{~m} 2(25 \mathrm{~mm})$ \\
\hline Galvanized Sheet & 310.9 & $\mathrm{~kg}$ \\
\hline Galvanized Studs & 323.5 & $\mathrm{~kg}$ \\
\hline Joint Compound & 41.7 & $\mathrm{~kg}$ \\
\hline Modified Bitumen membrane & 118.0 & $\mathrm{~kg}$ \\
\hline Nails & 2.7 & $\mathrm{~kg}$ \\
\hline Paper Tape & 0.5 & $\mathrm{~kg}$ \\
\hline Screws Nuts \& Bolts & 5.9 & $\mathrm{~kg}$ \\
\hline Softwood Plywood & 79.8 & $\mathrm{~m} 2(9 \mathrm{~mm})$ \\
\hline Solvent Based Alkyd Paint & 27.3 & $\mathrm{~L}$ \\
\hline
\end{tabular}

Notes:

'Initial $=$ Time '
${ }^{2}$ '
Trae. at at

Total EE (or Total GWP) = Total embodied energy (or total embodied GWP) of building component after lifespan (i.e. total manufacturing + tot

${ }^{4}$ Total EE (or Total GWP) per $\mathrm{m}^{2}=$ Total EE (or Total GWP) of building Total Difference in Operating Energy (or GWP) from Baseline after) Lifespan = The difference in the total lificycycle operating energy (or GWWP)
from the baseline retail building after lifespan, due to using this building from the baseline retail building after lifes
component instead of the baseline component

Total Difference in Operating Energy (or GWP) from Baseline after Lifespan per $\mathrm{m}^{2}=$ Total difference in operating energy (or GWP) from
baseline after lifespan / net roof area of baseline retail building * Total operating primary energy use of baseline retail building after 50 years

"Total operating GWP of baseline retail building after 50 years $=2,310$ tonnes

Cold-Formed Steel Roof \#10 (CFS-R10)

Building Component Description:

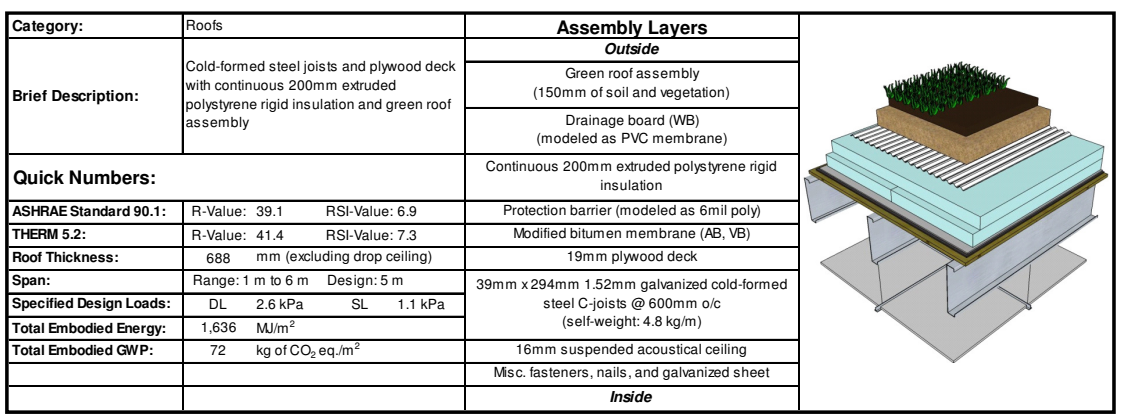

Life-Cycle Assessment Results:

Primary Energy Consumption (MJ)

\begin{tabular}{|c|c|c|c|c|c|c|c|c|c|c|c|c|c|c|c|c|c|}
\hline \multirow{3}{*}{$\begin{array}{l}\text { LCespan } \\
\text { (Years) }\end{array}$} & \multicolumn{14}{|c|}{ Embodied Energy (EE) } & \multirow{2}{*}{\multicolumn{3}{|c|}{$\begin{array}{c}\text { Difference in } \\
\text { Operating Energy } \\
\text { from Baseline after } \\
\text { Lifespan }\end{array}$}} \\
\hline & \multicolumn{3}{|c|}{ Manutacturing } & \multicolumn{3}{|c|}{ Construction } & \multicolumn{3}{|c|}{ Maintenance } & \multicolumn{3}{|c|}{ End of Life } & \multirow{2}{*}{\begin{tabular}{|c|}
${ }^{3}$ Total \\
$\mathrm{EE}$
\end{tabular}} & \multirow{2}{*}{$\begin{array}{l}{ }^{4} \mathrm{To} \\
\mathrm{E} \\
\mathrm{Eer}\end{array}$} & & & \\
\hline & Material & 1/2 Trans. & Total & Material & al ${ }^{2}$ Trans. & Total & Material & in ${ }^{2}$ Trans. & Total & Material & $\mathbb{1}^{2}$ Trans. & Total & & & & ${ }^{5}$ Total & ${ }^{6}$ perm ${ }^{2}$ \\
\hline itial & \begin{tabular}{|l|l|}
46,775 \\
\end{tabular} & 165 & 46,94 & 0 & 461 & 461 & 0 & 0 & 0 & 0 & & & 47,4 & & & & \\
\hline
\end{tabular}

Global Warming Potential (kg of $\mathrm{CO}_{2}$ eq.)

\begin{tabular}{|c|c|c|c|c|c|c|c|c|c|c|c|c|c|c|c|c|}
\hline \multirow{4}{*}{$\begin{array}{l}\text { Lestespan } \\
\text { (Years) }\end{array}$} & \multicolumn{14}{|c|}{ Narming Potential ( $\mathrm{kg}$ of $\mathrm{CO}_{2}$ eq.) } & \multirow{3}{*}{\multicolumn{2}{|c|}{\begin{tabular}{|c} 
Difference in \\
Operating GWPP from \\
Baseline after \\
Lifespan
\end{tabular}}} \\
\hline & \multicolumn{14}{|c|}{ Embodied Global Warming Potential (GWP) } & & \\
\hline & \multicolumn{3}{|c|}{ Manutacturing } & \multicolumn{3}{|c|}{ Construction } & \multicolumn{3}{|c|}{ Maintenance } & \multicolumn{3}{|c|}{ End of Life } & \multirow{2}{*}{$\begin{array}{l}{ }^{3} \text { Total } \\
\text { GWW }\end{array}$} & \multirow{2}{*}{$\begin{array}{l}{ }^{4} \text { Total } \\
\text { GWP } \\
\text { per m }\end{array}$} & & \\
\hline & Materia & ${ }^{2}$ Trans. & Total & Material & ${ }^{2}$ Trans. & Total & Material & ${ }^{2}$ Trans. & Total & Material & 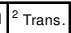 & Total & & & ${ }^{5}$ Total & ${ }^{6} \operatorname{perm}^{2}$ \\
\hline Initic & a & 0 & 2,3 & n & 0 & 0 & R & 0 & ne & - & $\Omega_{1}$ & $\pi$ & & 61 & & \\
\hline 50 & 2,336 & 0 & 2,4 & 0 & 0 & 0 & 406 & 0 & 40 & 0 & 0 & 0 & 2,744 & 2 & $-60,000$ & -102 \\
\hline
\end{tabular}

Embodied energy (and GWP) numbers are based on an area of roof $=\begin{array}{rll}38.0 & \mathrm{~m}^{2}\end{array} \quad$ (Span $\times$ Width $=5.0 \mathrm{~m} \times 7.6 \mathrm{~m}=38 . \mathrm{m}^{2}$ )

ATHENA $₫$ EIE Material List:

(Includes all materials atter 50 years

\begin{tabular}{|c|c|c|}
\hline Material List & Quantities & Unit \\
\hline $\begin{array}{l}16 \mathrm{~mm} \text { Gypsum Fibre Gypsum } \\
\text { Board }\end{array}$ & 41.8 & $\mathrm{~m} 2$ \\
\hline 6 mil Polyethylene & 40.3 & $\mathrm{~m} 2$ \\
\hline Ballast (aggregate stone) & 144.9 & $\mathrm{~kg}$ \\
\hline Extruded Polystyrene & 311.4 & $\mathrm{~m} 2(25 \mathrm{~mm})$ \\
\hline Galvanized Sheet & 88.3 & $\mathrm{~kg}$ \\
\hline Galvanized Studs & 304.4 & $\mathrm{~kg}$ \\
\hline Joint Compound & 41.7 & $\mathrm{~kg}$ \\
\hline Modified Bitumen membrane & 142.1 & $\mathrm{~kg}$ \\
\hline Nails & 2.7 & $\mathrm{~kg}$ \\
\hline Paper Tape & 0.5 & $\mathrm{~kg}$ \\
\hline PVC membrane & 169.6 & $\mathrm{~kg}$ \\
\hline Screws Nuts \& Bolts & 5.5 & $\mathrm{~kg}$ \\
\hline Softwood Plywood & 79.8 & m2 $29 \mathrm{~mm}$ \\
\hline
\end{tabular}

\begin{tabular}{l} 
Notes: \\
${ }^{\prime}$ Initial $=$ Time \\
\hline
\end{tabular}

${ }^{2}$ Trans. = Transportation

${ }^{3}$ Total EE (or Total GWP) $=$ Total embodied energy (or total embodied GWP) of building component after lifespan (i.e. total mat
construction + total maintenance t+total end-ofifife effects)

${ }^{4}$ Total EE (or Total GWP) per $\mathrm{m}^{2}=$ Total EE (or Total GWP) of building
component / area of building component that was modelled in ATHENAQ EIE component/ area of building component that was modelled in ATHENA®EEE Total Difference in Operating Energy (or GWP) from Baseline after
Lifespan = The difference in the total life-cycle operating energy (or GWPP) trom the baseline retail builining ater lifecespan, operating energy to using this building
component instead of the baseline component ${ }^{6}$ Total Difference in Operating Energy (or GWP) from Baseline after Lifespan per $\mathrm{m}^{2}=$ Total difference in operating energy (or GWP) from
baseline after lifespan $/$ net roof area of baseline retail building * Total operating primary energy use of baseline retail building after 50 years =
$50,700 \mathrm{GJ}\left(1,745 \mathrm{M} \mathrm{Mm}^{2} \mathrm{yr}\right)$

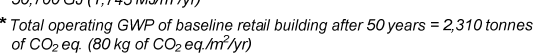




\section{Cold-Formed Steel Roof \#11 (CFS-R11)}

Building Component Description:

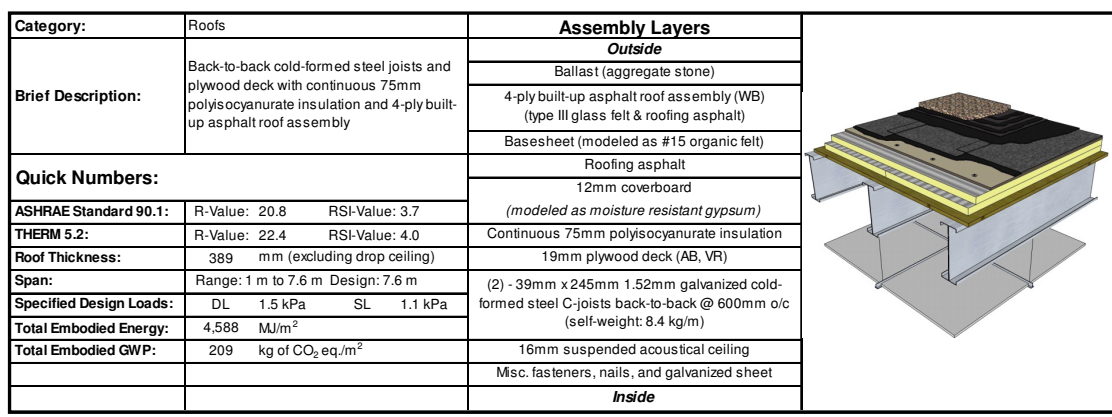

Life-Cycle Assessment Results:

\begin{tabular}{|c|c|c|c|c|c|c|c|c|c|c|c|c|c|c|c|}
\hline \multirow{4}{*}{$\mid \begin{array}{c}\text { Lifespan } \\
\text { (Years) }\end{array}$} & \multicolumn{15}{|c|}{ Energy Consumption (N } \\
\hline & \multicolumn{13}{|c|}{ Embodied Energy (EE) } & \multirow{2}{*}{\multicolumn{2}{|c|}{$\begin{array}{l}\text { Difference in } \\
\text { Operating Energy } \\
\text { from Baseline after } \\
\text { Lifespan }\end{array}$}} \\
\hline & \multicolumn{2}{|c|}{ Manutacturing } & \multicolumn{3}{|c|}{ Construction } & \multicolumn{3}{|c|}{ Maintenance } & \multicolumn{3}{|c|}{ End of Life } & \multirow{2}{*}{\begin{tabular}{|c|}
${ }^{3}$ Total \\
$\mathrm{EE}$
\end{tabular}} & \multirow{2}{*}{\begin{tabular}{|c|}
${ }^{4} \mathrm{Total}$ \\
$\mathrm{EE}$ \\
perm
\end{tabular}} & & \\
\hline & \begin{tabular}{|l|l|} 
Material & 2 \\
\end{tabular} & Total & Material & ${ }^{2}$ Trans. & \begin{tabular}{c|c|c|} 
Total \\
\end{tabular} & Material & \begin{tabular}{|l|}
${ }^{2}$ Trans. \\
\end{tabular} & \begin{tabular}{|l|l|} 
Total \\
\end{tabular} & Material & \begin{tabular}{|l|}
${ }^{2}$ Trans. \\
\end{tabular} & Total & & & ${ }^{5}$ Total & ${ }^{6}$ per $^{2}$ \\
\hline Initial & \begin{tabular}{|l|l|}
143,368 & 387 \\
\end{tabular} & 143,755 & 0 & \begin{tabular}{|l|l|}
883 \\
\end{tabular} & 883 & 0 & 0 & 0 & 0 & 0 & 0 & 144,638 & 2,504 & & \\
\hline
\end{tabular}

\begin{tabular}{|c|c|c|c|c|c|c|c|c|c|c|c|c|c|c|c|c|}
\hline Initial & 143,368 & 387 & 143,755 & 0 & 883 & 883 & 0 & 0 & 0 & 0 & 0 & 0 & 144,638 & 2,504 & - & - \\
\hline 50 & 143,368 & 387 & 143,755 & 0 & 883 & 883 & 119,654 & 414 & 120,068 & 1 & 282 & 283 & 264,990 & 4,588 & $-200,000$ & -341 \\
\hline
\end{tabular}

\begin{tabular}{|c|c|c|c|c|c|c|c|c|c|c|c|c|c|c|c|c|}
\hline \multirow{4}{*}{\begin{tabular}{|l} 
Lifespan \\
(Years)
\end{tabular}} & \multicolumn{16}{|c|}{ Global Warming Potential ( $\mathrm{kg}$ of $\mathrm{CO}_{2}$ eq.) } \\
\hline & \multicolumn{14}{|c|}{ Embodied Global Warming Potential (GWP) } & \multirow{2}{*}{\multicolumn{2}{|c|}{$\begin{array}{c}\text { Difference in } \\
\text { Operating GWP from } \\
\text { Baseline after } \\
\text { Lifespan }\end{array}$}} \\
\hline & \multicolumn{3}{|c|}{ Manufacturing } & \multicolumn{3}{|c|}{ Construction } & \multicolumn{3}{|c|}{ Maintenance } & \multicolumn{3}{|c|}{ End of Life } & \multirow{2}{*}{\begin{tabular}{|c|}
${ }^{3}$ Total \\
GWP
\end{tabular}} & \multirow{2}{*}{$\begin{array}{l}{ }^{4} \text { Total } \\
\text { GWP } \\
\text { p }\end{array}$} & & \\
\hline & Material & ${ }^{2}$ Trans. & Total & Material & 2 Trans. & Total & Material & $\left.\right|^{2}$ Trans. & Total & Material & 1. ${ }^{2}$ Trans. & Total & & & ${ }^{5}$ Total & ${ }^{6}$ per m $m^{2}$ \\
\hline 1 Initial & 7,336 & 1 & 7,336 & 0 & 1 & 1 & 0 & 0 & 0 & 0 & 0 & 0 & 7,337 & 127 & & \\
\hline 50 & 7,336 & 1 & 7,336 & 0 & 1 & 1 & 4,758 & 1 & 4,759 & 0 & 1 & 1 & 12,097 & 209 & 0 & 0 \\
\hline
\end{tabular}

Embodied energy (and GWP) numbers are based on an area of roof $=57.8 \mathrm{~m}^{2} \quad$ (Span $\times$ Width $\left.=7.6 \mathrm{~m} \times 7.6 \mathrm{~m}=57.8 \mathrm{~m}^{2}\right)$

Net roof area of baseline retail building (gross roof area - openings) $=586.0 \mathrm{~m}^{2}$

ATHENA $\circledast$ EIE Material List:

\begin{tabular}{|c|c|c|}
\hline Material List & Quantities & Unit \\
\hline \#15 Organic Felt & 460.9 & $\mathrm{~m} 2$ \\
\hline 13mm Moisture Resistant Gypsum & 63.5 & $\mathrm{~m} 2$ \\
\hline \begin{tabular}{|l|}
$16 m m$ Gypsum Fibre Gypsum \\
Bagrd
\end{tabular} & 63.5 & $\mathrm{~m} 2$ \\
\hline Ballast (aggregate stone) & 4,404.2 & $\mathrm{kg}$ \\
\hline $\begin{array}{l}\text { Galvanized Sheet } \\
\end{array}$ & 203.1 & $\mathrm{~kg}$ \\
\hline $\begin{array}{l}\text { Galvanized Studs } \\
\end{array}$ & 810.1 & $\mathrm{~kg}$ \\
\hline \begin{tabular}{|l|} 
Isocyanurate \\
\end{tabular} & 179.2 & 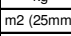 \\
\hline \begin{tabular}{|l|} 
Joint Compound \\
\end{tabular} & 63.4 & $\mathrm{~kg}$ \\
\hline Nails & 30.3 & ta \\
\hline \begin{tabular}{|l|} 
Paper Tape \\
\end{tabular} & 0.7 & $\mathrm{~kg}$ \\
\hline Roofing Asphalt & 1,748.4 & $\mathrm{kg}$ \\
\hline Screws $\mathrm{N}$ & 8.3 & $\mathrm{~kg}$ \\
\hline Soltwood Ply & 121.3 & \\
\hline \begin{tabular}{|l|l|} 
Type III Glass Feett \\
\end{tabular} & $\begin{array}{l}9221.7 \\
\end{array}$ & $\mathrm{~m}^{2}$ \\
\hline
\end{tabular}

${ }^{2}$ Trans. $=$ Transportation

Total EE (or Total GWP) $=$ Total embodied energy (or total embodied GWP)
of buiding component after lifespan (i.e. total manufacturing + total

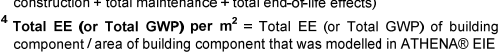

${ }^{5}$ Total Difference in Operating Energy (or GWP) from Baseline atter
Lifespan $=$ The difference in the total life-cycle operating energy (or GWP)

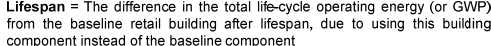

Total Difference in Operating Energy (or GWP) from Baseline after
Lifiespan per $\mathrm{m}^{2}=$ Total difference in operating energy (or GWP) from

Lifespan per $\mathrm{m}^{2}=$ Total difference in operating energy
baseline after lifespan $/$ net roof area of baseline retail builing

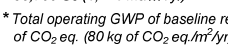

Notes:
'Initial $=T$ Time $\mathcal{O}^{\prime}($ (ie. at the completion of initial construction)

Total operating primary energy use of baseline retail building after 50 years $=$
$50,700 \mathrm{GJ}\left(1,745 \mathrm{MJ} / \mathrm{m}^{2} \gamma \mathrm{\gamma r}\right)$

\section{Cold-Formed Steel Roof \#12 (1CFS-R12)}

Building Component Description:

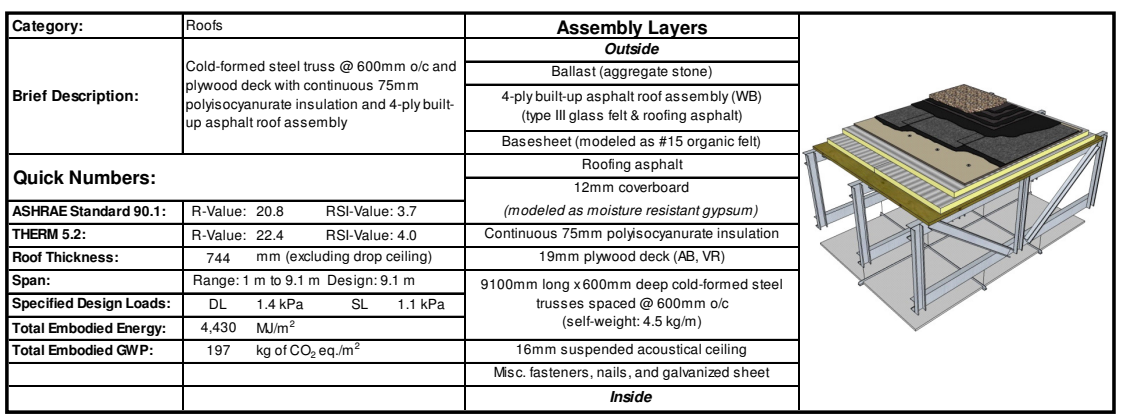

Life-Cycle Assessment Results:

\begin{tabular}{|c|c|c|c|c|c|c|c|c|c|c|c|c|c|c|c|c|}
\hline \multirow{4}{*}{$\begin{array}{l}\text { Lifespan } \\
\text { (Years) }\end{array}$} & \multicolumn{14}{|c|}{ Primary Energy Consumption (MJ) } & \multirow{3}{*}{\multicolumn{2}{|c|}{$\begin{array}{c}\text { Difference in } \\
\text { Operating Energy } \\
\text { from Baseline afte } \\
\text { Lifespan }\end{array}$}} \\
\hline & \multicolumn{14}{|c|}{ Embodied Energy (EE) } & & \\
\hline & \multicolumn{3}{|c|}{ Manutacturing } & \multicolumn{3}{|c|}{ Construction } & \multicolumn{3}{|c|}{ Maintenance } & \multicolumn{3}{|c|}{ End of Life } & \multirow{2}{*}{$\begin{array}{c}{ }^{3} \text { Total } \\
\mathrm{EE}\end{array}$} & \multirow{2}{*}{\begin{tabular}{|c|}
${ }^{4} \mathrm{Total}$ \\
$\mathrm{EE}$ \\
per m
\end{tabular}} & & \\
\hline & \begin{tabular}{|l|} 
Material \\
\end{tabular} & ${ }^{2}$ Trans. & Total & Material & 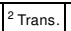 & Total & Material & ${ }^{2}$ Trans. & Total & Material & \begin{tabular}{ll|l|}
2 & 2 Trans. \\
\end{tabular} & Total & & & ${ }^{5}$ Total & ${ }^{6}{ }_{\text {per m}}^{2}$ \\
\hline itial & 160,867 & 447 & 161,314 & \begin{tabular}{l|l}
4 & 0 \\
4
\end{tabular} & 1,006 & 1,006 & 0 & 0 & 0 & 0 & 0 & 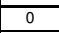 & 162,32 & \begin{tabular}{l|l|}
0 & 2,347 \\
\end{tabular} & & \\
\hline
\end{tabular}

\begin{tabular}{|c|c|c|c|c|c|c|c|c|c|c|c|c|c|c|c|c|}
\hline 50 & 160,867 & 447 & 161,314 & 0 & 1,006 & 1,006 & 0 & 0 & 0 & 0 & 0 & 0 & 162,320 & 2,347 & - & - \\
\hline
\end{tabular}

\begin{tabular}{|c|c|c|c|c|c|c|c|c|c|c|c|c|c|c|c|c|}
\hline \multirow{4}{*}{$\begin{array}{l}\text { Lifespan } \\
\text { (Years) }\end{array}$} & \multicolumn{14}{|c|}{ Global Warming Potential $\left(\mathrm{kg}\right.$ of $\mathrm{CO}_{2}$} & \multirow{3}{*}{\multicolumn{2}{|c|}{$\begin{array}{c}\text { Difference in } \\
\text { Operating GWP fron } \\
\text { Baseline after } \\
\text { Lifespan }\end{array}$}} \\
\hline & \multicolumn{14}{|c|}{ Embodied Global Warming Potential (GWP) } & & \\
\hline & \multicolumn{3}{|c|}{ Manutacturing } & \multicolumn{3}{|c|}{ Construction } & \multicolumn{3}{|c|}{ Maintenance } & \multicolumn{3}{|c|}{ End of Life } & \multirow{2}{*}{$\begin{array}{l}{ }^{3} \text { Total } \\
\text { GWP }\end{array}$} & \multirow{2}{*}{$\begin{array}{l}{ }^{4} \text { Total } \\
\text { GWP } \\
\text { per m }\end{array}$} & & \\
\hline & Material & 2 Trans. & Total & Material & ${ }^{2}$ Trans. & Total & Material & Trans. & Total & Material & ${ }^{2}$ Trans. & Total & & & ${ }^{5}$ Total & ${ }^{6}$ perm $m^{2}-3{ }^{2}$ \\
\hline${ }^{1}$ Initial & 7,909 & 1 & 7,909 & 0 & 1 & 1 & 0 & 0 & 0 & 0 & 0 & 0 & 7,910 & 114 & & \\
\hline 50 & 7,909 & 1 & 7,909 & 0 & 1 & 1 & 5,696 & 1 & 5,697 & 0 & 1 & 1 & 13,608 & 197 & 0 & 0 \\
\hline
\end{tabular}

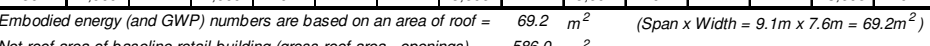

Net roof area of baseline retail building (gross roof area - openings) $=586.0 \mathrm{~m}^{2}$

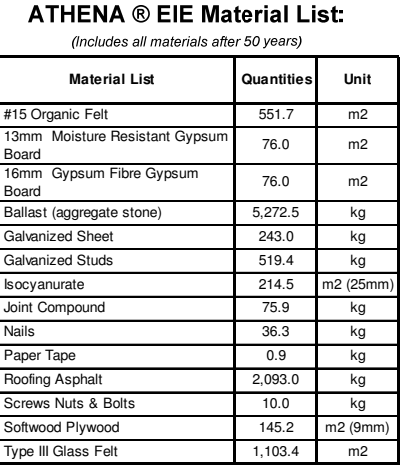

$$
\begin{aligned}
& \text { Notes: } \\
& \text { Initial }=\text { Time } \\
& O P(\text { (i.e. at the completion of initial construction) }
\end{aligned}
$$

Trans = Transportation

Total EE (or Total GWP) $=$ Total embodied energy (or total embodied GWP)
of building component after lifespan (ie.e. fotal manufacturing + total

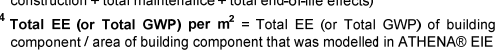

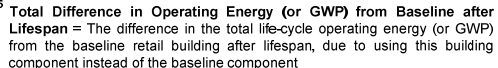
Total Difference in Operating Energy (or GWP) from Baseline after Lifespan per $m^{2}=$ Total difiterence in operating energy (or GWP) from
baseline after lifespan / net roof area of baseline retail building

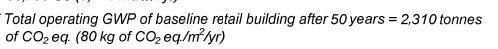

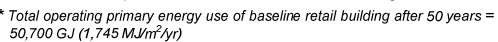




\section{Cold-Formed Steel Roof \#13 (CFS-R13)}

Building Component Description:

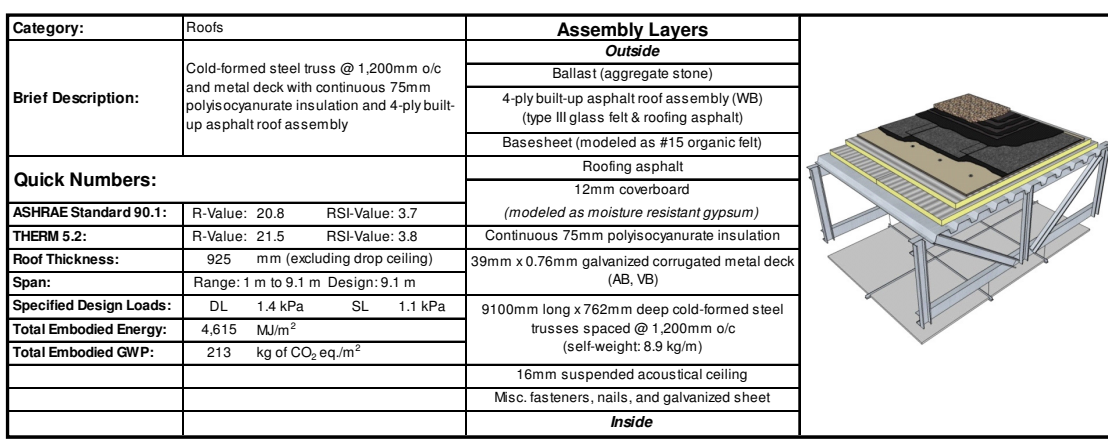

Life-Cycle Assessment Results:

Primary Energy Consumption (MJ)

\begin{tabular}{|c|c|c|c|c|c|c|c|c|c|c|c|c|c|c|c|}
\hline \multirow{3}{*}{$\begin{array}{l}\text { Litiespan } \\
\text { (Years) }\end{array}$} & \multicolumn{13}{|c|}{ Embodied Energy (EE) } & \multirow{2}{*}{\multicolumn{2}{|c|}{$\begin{array}{l}\text { Difference in } \\
\text { Operating Energy } \\
\text { from Baseline after } \\
\text { Lifespan }\end{array}$}} \\
\hline & \multicolumn{2}{|c|}{ Manutacturing } & \multicolumn{3}{|c|}{ Construction } & \multicolumn{3}{|c|}{ Maintenance } & \multicolumn{3}{|c|}{ End of Life } & \multirow{2}{*}{$\begin{array}{c}3^{3} \text { Total } \\
\mathrm{EE}\end{array}$} & \multirow{2}{*}{$\begin{array}{c}{ }^{4} \text { Total } \\
\text { EE } \\
\text { EE }\end{array}$} & & \\
\hline & Material ${ }^{2}$ Trans & Total & |Material & ${ }^{2}$ Trans. & Total & \begin{tabular}{|l|} 
Material \\
\end{tabular} & 1) 2 Trans. & Total & |Material & $\left.\right|^{2}$ Trans. & Total & & & ${ }^{5}$ Total & ${ }^{6}$ per m ${ }^{2}$ \\
\hline Initial & \begin{tabular}{|l|l|}
173,625 & 367 \\
\end{tabular} & 173,992 & 0 & 1,076 & 1,076 & 0 & 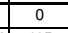 & 0 & 0 & Th & 0 & 175,06 & \begin{tabular}{|l|l|}
8 & 2,531 \\
\end{tabular} & & \\
\hline 50 & $\begin{array}{lll}1773,625 & 367 \\
\end{array}$ & 1773,999; & 0 & $\begin{array}{llll} & 1,076 \\
\end{array}$ & 1,076 & {$[143,243$} & 495 & 143,738 & $\begin{array}{lll}8 & 0 \\
\end{array}$ & 346 & 347 & 319,15 & 4,615 & $\begin{array}{c}100,000 \\
1000\end{array}$ & -171 \\
\hline
\end{tabular}

Global Warming Potential $\left(\mathbf{k g}\right.$ of $\mathrm{CO}_{2}$ eq.

\begin{tabular}{|c|c|c|c|c|c|}
\hline \multirow{3}{*}{$\begin{array}{l}\text { Lifiespar } \\
\text { (Years) }\end{array}$} & \multicolumn{5}{|r|}{$\mathrm{Er}$} \\
\hline & \multicolumn{3}{|c|}{ Manufacturing } & \multicolumn{2}{|c|}{ Construction } \\
\hline & Material & ${ }^{2}$ Trans. & \begin{tabular}{|l|l|} 
Total \\
\end{tabular} & Material & |2 2 Trans. \\
\hline Initial & \begin{tabular}{|l|l}
9,005 \\
\end{tabular} & 1 & \begin{tabular}{|l|l}
9,006 \\
\end{tabular} & 0 & 1 \\
\hline 50 & 9,000 & 1 & \begin{tabular}{|l|}
9,006 \\
\end{tabular} & & 1 \\
\hline \multicolumn{6}{|c|}{$\begin{array}{l}\text { Embodied energy (and GWP) numbers are based on an } \\
\text { Net roof area of baseline retail building (gross roof area }\end{array}$} \\
\hline \multicolumn{6}{|c|}{$\begin{array}{c}\text { ATHENA } ® \text { EIE Material List: } \\
\text { (Includes all materials after } 50 \text { years) }\end{array}$} \\
\hline & Materia & & & Quantities & Unit \\
\hline \begin{tabular}{|l|l|l}
15 Org \\
\end{tabular} & nic Felt & & & 551.7 & m2 \\
\hline \begin{tabular}{|l|}
$\begin{array}{l}13 m \mathrm{~mm} \\
\text { Board }\end{array}$ \\
\end{tabular} & Doisture R & esistant Gy & & 76.0 & $\mathrm{~m} 2$ \\
\hline \begin{tabular}{|l|}
$\begin{array}{l}16 \mathrm{~mm} \\
\text { Board }\end{array}$ \\
\end{tabular} & iypsum Fi & bre Gyssum & & 76.0 & $\mathrm{~m} 2$ \\
\hline \begin{tabular}{|l|} 
Ballast \\
\end{tabular} & aggregate & stone) & & $\begin{array}{l}5,272.5 \\
\end{array}$ & $\mathrm{~kg}$ \\
\hline & & & & 684.8 & $\mathrm{~kg}$ \\
\hline \begin{tabular}{|l|l|} 
Galvaniz \\
\end{tabular} & ed Sheet & & & 243.0 & $\mathrm{~kg}$ \\
\hline & ed Studs & & & 513.4 & $\mathrm{~kg}$ \\
\hline 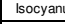 & & & & 214.5 & $2(25 \mathrm{~mm}$ \\
\hline & & & & & $\mathrm{kg}$ \\
\hline \begin{tabular}{|l} 
Nalis \\
\end{tabular} & & & & 36.3 & $\mathrm{~kg}$ \\
\hline \begin{tabular}{|l|} 
Paper T \\
\end{tabular} & & & & & $\mathrm{kg}$ \\
\hline \begin{tabular}{|l|} 
Roofing \\
Sofi
\end{tabular} & Asphalt & & & $2,093.0$ & $\mathrm{~kg}$ \\
\hline \begin{tabular}{|l|l} 
Screws \\
Treally
\end{tabular} & Vuts \& Bo & & & $\begin{array}{r}10.0 \\
11034\end{array}$ & $\mathrm{~kg}$ \\
\hline & & & & & \\
\hline
\end{tabular}

\section{LCA Data for Glulam Joist Roofs}

This section contains a detailed description of each glulam (GLU) roof that was examined in this study (10 in total). The assembly layers are listed for each roof, along with a detailed description of the material quantities from the ATHENA ${ }^{\circledR}$ Environmental Impact Estimator for Buildings.

A breakdown of the total primary energy consumption and the total global warming potential (GWP) for each roof is also included. In each case, the results were calculated for an area of roof equal to $69.2 \mathrm{~m}^{2}$, which represents a typical bay size for a single-storey retail building with this type of roof system. The results are also expressed on a per $\mathrm{m}^{2}$ basis in each case. The data has been calculated for two different lifespans: at the completion of initial construction and after 50 years.

As a summary, the figure below illustrates a comparison of the total embodied energy (and GWP) after 50 years for the various roofs in this section. For comparison purpose, the building component used in the baseline retail building has been shaded in grey.

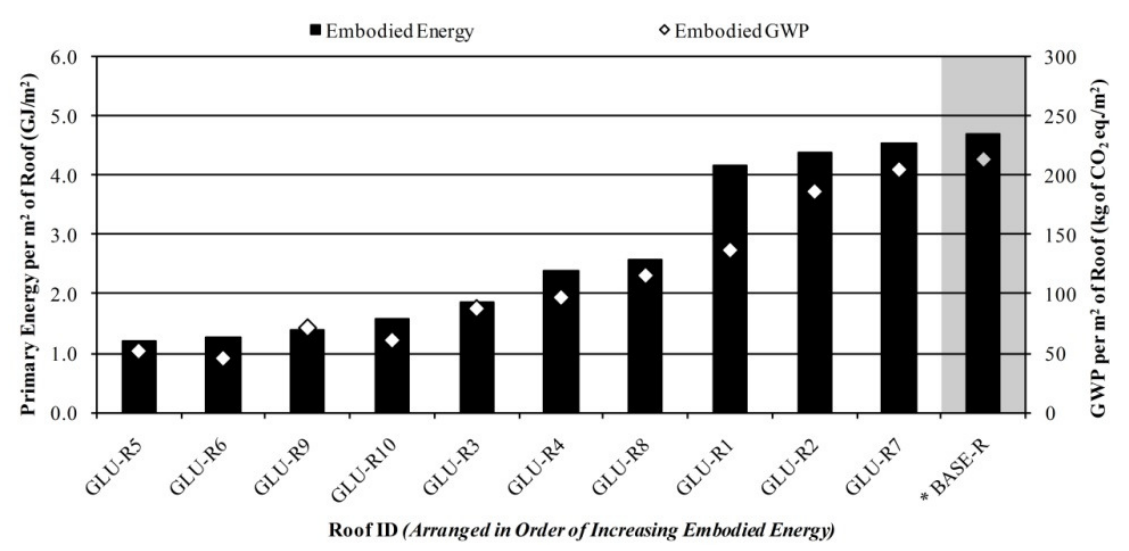




\section{Glulam Roof \#1 (GLU-R1)}

Building Component Description:

\begin{tabular}{|c|c|c|c|}
\hline Category: & Roofs & Assembly Layers & \\
\hline \multirow{4}{*}{ Brief Description: } & \multirow{4}{*}{ 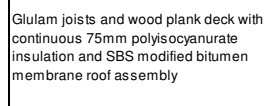 } & $\begin{array}{c}\text { Outside } \\
\text { SBS modified bitumen membrane cap sheet (WB) }\end{array}$ & \\
\hline & & Roofing asphalt & \\
\hline & & Basesheet (modeled as \#15 organic felt) & \\
\hline & & Roofing asphatt & \\
\hline \multicolumn{2}{|l|}{ Quick Numbers: } & $\begin{array}{l}12 \mathrm{~mm} \text { coverboard } \\
\text { (mododed as moisture resistant gyosum) }\end{array}$ & \\
\hline ASHRAE Standard 90.1: & \begin{tabular}{|l|} 
R-Value: 20.8 \\
\end{tabular} & Continuous $75 \mathrm{~mm}$ polyisocyanurate insulation & \\
\hline THERM 5.2: & \begin{tabular}{|lr} 
R-Value: 22.9 & RSI-Value: 4.0 \\
\end{tabular} & 38mm SPF tongue \& groove solid wood plank & \\
\hline Roof Thickness: & $632 \mathrm{~mm}$ (excluding drop ceiling) & decking (AB, VR) & \\
\hline Span: & Range: $2 \mathrm{~m}$ to $12 \mathrm{~m}$ Design: $9.1 \mathrm{~m}$ & 80mm x494mm 241-E glulam joists @ 1800m o/c & \\
\hline Specified Design Loads: & $\mathrm{DL} \quad 1.2 \mathrm{kPa}$ & $16 \mathrm{~mm}$ suspended acoustical celling & \\
\hline Total Embodied Energy: & $4,149 \quad \mathrm{M} / \mathrm{m}^{2}$ & Msc. fasteners, nails, and galuanized sheet & \\
\hline & $137 \quad \mathrm{~kg}$ of $\mathrm{CO}_{2} \mathrm{eq} / \mathrm{m}^{2}$ & Inside & \\
\hline
\end{tabular}

\section{Life-Cycle Assessment Results:}

Primary Energy Consumption (MJ)

\begin{tabular}{|c|c|c|c|c|c|c|c|c|c|c|c|c|c|c|c|}
\hline \multirow{3}{*}{$\begin{array}{l}\text { Lifiespan } \\
\text { (Years) }\end{array}$} & \multicolumn{13}{|c|}{ Embodied Energy (EE) } & \multirow{2}{*}{\multicolumn{2}{|c|}{$\begin{array}{c}\text { Difference in } \\
\text { Operating Energy } \\
\text { from Baseline atte } \\
\text { Lifespan }\end{array}$}} \\
\hline & \multicolumn{2}{|c|}{ Manufacturing } & \multicolumn{3}{|c|}{ nstrm } & \multicolumn{3}{|c|}{ Maintenance } & \multicolumn{3}{|c|}{ End of Life } & \multirow{2}{*}{$\begin{array}{c}{ }^{3} \text { Total } \\
\mathrm{EE}\end{array}$} & \multirow{2}{*}{$\begin{array}{c}{ }^{4} \text { Total } \\
\text { EE } \\
\text { per } \mathrm{m}^{2}\end{array}$} & & \\
\hline & \begin{tabular}{|l|l|} 
Material & 2 \\
& Trans.
\end{tabular} & \begin{tabular}{|l|l} 
Total \\
\end{tabular} & \begin{tabular}{|l|l|} 
Materia \\
\end{tabular} & 2 Trans. & Total & \begin{tabular}{|l|l|} 
Material \\
\end{tabular} & ${ }^{2}$ Trans. & \begin{tabular}{|l|l|l|} 
Total \\
\end{tabular} & Material & 1 ${ }^{2}$ Trans. & \begin{tabular}{|l|l|} 
Total \\
\end{tabular} & & & ${ }^{5}$ Total & ${ }^{6}$ per $\mathrm{m}^{2}$ \\
\hline Initial & \begin{tabular}{|l|l|}
131,645 & 731 \\
\end{tabular} & 132,377 & 461 & 1,903 & \begin{tabular}{|l|l|}
2,364 \\
\end{tabular} & 0 & 0 & 0 & 0 & 0 & 0 & 134,740 & 1,948 & & \\
\hline 50 & \begin{tabular}{|l|l|l|}
131,645 & 731 \\
\end{tabular} & 132,377 & 461 & 1,903 & 2,364 & $\mid 151,547$ & \begin{tabular}{|l|l|}
309 & \\
\end{tabular} & 151,856 & 2 & 333 & 335 & 286,93 & 4,149 & -300, & 年 \\
\hline
\end{tabular}

Global Warming Potential ( $\mathrm{kg}$ of $\mathrm{CO}_{2}$ eq.)

\begin{tabular}{|c|c|c|c|c|c|c|c|c|c|c|c|c|c|c|c|c|}
\hline & & & & & & & rming & Poten & & & & & & & & \\
\hline \multirow{3}{*}{$\begin{array}{l}\text { Lifespan } \\
\text { (Years) }\end{array}$} & \multicolumn{14}{|c|}{ abodied Global Warming Potential (GWP) } & \multirow{2}{*}{\multicolumn{2}{|c|}{$\begin{array}{c}\text { Difference in } \\
\text { Operating GWP from } \\
\text { Baseline after } \\
\text { Lifespan }\end{array}$}} \\
\hline & \multicolumn{3}{|c|}{ Manufacturing } & \multicolumn{3}{|c|}{ Construction } & \multicolumn{3}{|c|}{ Maintenance } & \multicolumn{3}{|c|}{ End of Life } & \multirow{2}{*}{$\begin{array}{l}3 \\
3 \\
\text { Totala } \\
\text { GWP }\end{array}$} & \multirow{2}{*}{$\begin{array}{l}{ }^{4}{ }^{4} \text { Tal } \\
\text { GWP } \\
\text { per m }\end{array}$} & & \\
\hline & Material & ${ }^{2}$ Trans. & \begin{tabular}{|l|} 
Total \\
\end{tabular} & Material & Trans. & Total & Material & ${ }^{2}$ Trans. & Total & Material & | ${ }^{2}$ Trans. & Total & & & ${ }^{5}$ Total & 6 per \\
\hline |litial & 5,389 & 1 & & 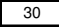 & 2 & 33 & & 0 & 0 & 0 & 0 & 0 & 5,423 & 78 & & \\
\hline 50 & 5,389 & 1 & 5,390 & 30 & 2 & 33 & 4,032 & 1 & 4,033 & 0 & 1 & 1 & \begin{tabular}{|l|l|}
9,457 \\
\end{tabular} & 137 & \begin{tabular}{|l|l|}
$-10,000$ \\
\end{tabular} & \\
\hline
\end{tabular}

(Span $\times$ Width $\left.=9.1 \mathrm{~m} \times 7.6 \mathrm{~m}=69.2 \mathrm{~m}^{2}\right)$

Net roof area of baseline retail building (gross roof area - openings) = $586.0 \mathrm{~m}^{2}$

\section{ATHENA ${ }^{\circledR}$ EIE Material List:}

\begin{tabular}{|l|c|c|}
\multicolumn{1}{|c|}{ Material List } & Quantities & Unit \\
\hline \#15 Organic Felt & 551.9 & $\mathrm{~m} 2$ \\
\hline $\begin{array}{l}13 \mathrm{~mm} \text { Moisture Resistant Gypsum } \\
\text { Board }\end{array}$ & 76.1 & $\mathrm{~m} 2$ \\
\hline $16 \mathrm{~mm}$ Gypsum Fibre Gypsum & 76.1 & $\mathrm{~m} 2$ \\
\hline $\begin{array}{l}\text { Board } \\
\text { Gavanized Sheet }\end{array}$ & 186.2 & $\mathrm{~kg}$ \\
\hline GluLam Sections & 1.5 & $\mathrm{~m} 3$ \\
\hline Isocyanurate & 214.6 & $\mathrm{~m} 2(25 \mathrm{~mm})$ \\
\hline Joint Compound & 75.9 & $\mathrm{~kg}$ \\
\hline Modified Bitumen membrane & $2,219.6$ & $\mathrm{~kg}$ \\
\hline Nails & 49.2 & $\mathrm{~kg}$ \\
\hline Paper Tape & 0.9 & $\mathrm{~kg}$ \\
\hline Roofing Asphalt & $1,151.6$ & $\mathrm{~kg}$ \\
\hline $\begin{array}{l}\text { Small Dimension Softwood Lumber, } \\
\text { kiln-dried }\end{array}$ & 3.5 & $\mathrm{~m} 3$ \\
\hline
\end{tabular}

Notes:
1 Initial = Time ' 0 ' (i.e. at the completion of initial construction)

${ }^{2}$ Trans. = Transportation

Total EE (or Total GWP) = Total embodied energy (or total embodied GWP) of building component after lifespan (i.e. total manufacturing + tota

Total EE (or Total GWP) per $\mathrm{m}^{2}=$ Total EE (or Total GWP) of building
component/ area of building component that was modeled in ATHENAQ EIE Total Difference in Operating Energy (or GWP) from Baseline after Lifespan = The difference in the total life-cycle operating energy (or GWP)
from the baseline retail building after lifespan, due to using this building component instead of the baseline component

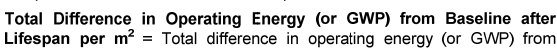
Dof "Total operating primary energy use of baseline retail building after 50 years =

"Total operating $\mathrm{SWP}$ of baseline retal

\section{Glulam Roof \#2 (GLU-R2)}

Building Component Description:

\begin{tabular}{|c|c|c|c|}
\hline \multirow{4}{*}{ Brief Description: } & Roofs & Assembly Layers & \\
\hline & \multirow{3}{*}{ 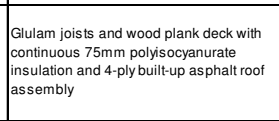 } & $\begin{array}{c}\text { Outside } \\
\text { Ballast (aggregate stone) }\end{array}$ & \\
\hline & & 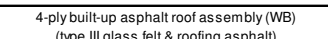 & \\
\hline & & Basesheet(modeled as $\# 15$ organic felt) & \\
\hline \multicolumn{2}{|l|}{ Quick Numbers: } & Roofing asphalt & \\
\hline ASHRAE Standard 90.1: & R-Value: 20.8 & $\begin{array}{l}12 \mathrm{~mm} \text { coverboard } \\
\text { a }\end{array}$ & \\
\hline THERM 5.2: & R-Value: 23.1 & Continuous $75 \mathrm{~mm}$ polysisocyanurate insulation & \\
\hline Roof Thickness: & $657 \mathrm{~mm}$ (excluding drop ceiling) & 38mm SPF tongue \& groove solid wood plank & \\
\hline Span: & Range: $2 \mathrm{~m}$ to $12 \mathrm{~m}$ Design: $9.1 \mathrm{~m}$ & decking (AB, VR) & \\
\hline Specified Design Loads: & $\begin{array}{ll}\mathrm{SL} & 1.1 \mathrm{kPa} \\
\end{array}$ & 80mm x494mm 24f:E glulam joists @ 1800m o/c & \\
\hline Total Embodied Energy: & $4,369 \quad \mathrm{M} / \mathrm{m}^{2}$ & 16mm suspended acoustical ceiling & \\
\hline Total Emboodied GWP: & $186 \mathrm{~kg}$ of $\mathrm{CO}_{2} \mathrm{eq} / \mathrm{m}^{2}$ & $\begin{array}{l}\text { Misc. fasteners, nails, and galvanized sheet } \\
\end{array}$ & \\
\hline
\end{tabular}

\section{Life-Cycle Assessment Results:}

Primary Energy Consumption (MJ)

\begin{tabular}{|c|c|c|c|c|c|c|c|c|c|c|c|c|c|c|c|}
\hline \multirow{3}{*}{$\left|\begin{array}{c}\text { Lifiespan } \\
\text { (Years) }\end{array}\right|$} & \multicolumn{13}{|c|}{ Embodied Energy (EE) } & \multirow{2}{*}{\multicolumn{2}{|c|}{$\begin{array}{c}\text { Difference in } \\
\text { Operating Energy } \\
\text { from Baseline after } \\
\text { Lifespan }\end{array}$}} \\
\hline & \multicolumn{2}{|c|}{ Manufacturing } & \multicolumn{3}{|c|}{ Construction } & \multicolumn{3}{|c|}{ Maintenance } & \multicolumn{3}{|c|}{ End of Life } & \multirow{2}{*}{$\begin{array}{c}{ }^{3} \text { Total } \\
{ }_{\mathrm{EE}}\end{array}$} & \multirow{2}{*}{\begin{tabular}{|l}
${ }^{4} \mathrm{Total}$ \\
$\mathrm{EE}$ \\
per m
\end{tabular}} & & \\
\hline & 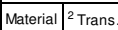 & Total & Material & 2 Trans. & Total & |Material & ${ }^{2}$ Trans. & Total & Material & \begin{tabular}{|l|}
${ }^{2}$ Trans. \\
\end{tabular} & Total & & & ${ }^{5}$ Total & ${ }^{6}$ per m$^{2}$ \\
\hline 1 Initial & \begin{tabular}{|l|l|}
154,929 & 785 \\
\end{tabular} & 155,713 & 461 & 1,795 & 2,255 & 0 & 0 & 0 & 0 & 0 & 0 & 157,969 & 2,284 & & \\
\hline 50 & \begin{tabular}{|l|l|}
154,929 & 785 \\
\end{tabular} & $\mid 155,713$ & 461 & 1,795 & 2,255 & 43,295 & 495 & 143,790 & 2 & 377 & 378 & 302,138 & \begin{tabular}{|l|l|}
4,369 \\
\end{tabular} & $-300,000$ & -512 \\
\hline
\end{tabular}

Global Warming Potential (kg of $\mathrm{CO}_{2}$ eq.)

\begin{tabular}{|c|c|c|c|c|c|c|c|c|c|c|c|c|c|c|c|c|}
\hline \multirow{4}{*}{$\begin{array}{l}\text { Lifespan } \\
\text { (Years) }\end{array}$} & \multirow{2}{*}{\multicolumn{14}{|c|}{ iontial (GWP) }} & \multirow{3}{*}{\multicolumn{2}{|c|}{$\begin{array}{c}\text { Difference in } \\
\text { Operating GWW Pfron } \\
\text { Baseline after } \\
\text { Lifespan }\end{array}$}} \\
\hline & & & & & & & & & & & & & & & & \\
\hline & \multicolumn{3}{|c|}{ nufacturing } & \multicolumn{3}{|c|}{ Construction } & \multicolumn{3}{|c|}{ Maintenance } & \multicolumn{3}{|c|}{ End of } & \multirow{2}{*}{$\begin{array}{l}{ }^{3} \text { Total } \\
\text { GWP }\end{array}$} & \multirow{2}{*}{$\begin{array}{l}{ }^{4} \text { Total } \\
\text { GWP } \\
\text { per }{ }^{2}\end{array}$} & & \\
\hline & Material & ${ }^{2}$ Trans. & Total & Materia & | $\left.\right|^{2}$ Trans. & Total & Material & ${ }^{2}$ Trans. & Total & Material & 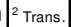 & Total & & & \begin{tabular}{|l|}
${ }^{5}$ Total \\
\end{tabular} & ${ }^{6}$ per $\mathrm{m}^{2}$ \\
\hline Initial & 136 & 1 & 7, & 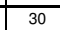 & 2 & 33 & & 0 & 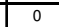 & 0 & 0 & 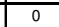 & & 104 & & \\
\hline 50 & 7,136 & 1 & 138 & 30 & 2 & 33 & 5,698 & 1 & 699 & 0 & 1 & 1 & $2,87.7-3$ & 186 & $-10,000$ & -17 \\
\hline
\end{tabular}

Embodied energy (and GWP) numbers are based on an area of roof $=69.2 \mathrm{~m}^{2} \quad$ (Span $\times$ Width $=9.1 \mathrm{~m} \times 7.6 \mathrm{~m}=69.2 \mathrm{~m}^{2}$ )

\section{ATHENA ${ }^{\circledR}$ EIE Material List:}

$$
\text { (nncludes all materials afer } 50 \text { y years) }
$$

\begin{tabular}{|c|c|c|}
\hline Material List & Quantities & Unit \\
\hline$\# 15$ Organic Felt & 551.9 & $\mathrm{~m} 2$ \\
\hline 13mm Moisture Resistant Gypsum & 76.1 & $\mathrm{~m} 2$ \\
\hline $\begin{array}{l}16 \mathrm{~mm} \text { Gypsum Fibre Gypsum } \\
\text { Board }\end{array}$ & 76.1 & $\mathrm{~m} 2$ \\
\hline Bollast (aggregate stone) & $5,274.4$ & $\mathrm{~kg}$ \\
\hline Galvanized Sheet & 243.0 & $\mathrm{~kg}$ \\
\hline GluLam Sections & $\begin{array}{l}1.5 \\
\end{array}$ & $\begin{array}{l}\mathrm{m} 33 \\
3\end{array}$ \\
\hline Isocyanurate & 214.6 & m2 $225 \mathrm{~mm}$ \\
\hline Joint Compound & 75.9 & $\mathrm{~kg}$ \\
\hline Nails & 49.2 & $\mathrm{~kg}$ \\
\hline $\begin{array}{l}\text { Paper Tape } \\
\end{array}$ & 0.9 & $\mathrm{~kg}$ \\
\hline Roofing Asphalt & $2,093.8$ & $\mathrm{~kg}$ \\
\hline 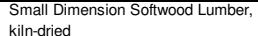 & 3.5 & $\mathrm{~m} 3$ \\
\hline Type III G & $\begin{array}{ll}1,103.8 \\
\end{array}$ & $\mathrm{~m} 2$ \\
\hline
\end{tabular}

$$
\begin{aligned}
& \text { Notes: } \\
& \text { 'Initial }=\text { Time ' } O \text { ' (i.e. at the completion of initial construction) }
\end{aligned}
$$

Total EE (or Total GWP) = Total embodied energy (or total embodied GWP) of building component after lifespan (i.e. total manu
construction + total maintenance t total end-of-life effects)

Total EE (or Total GWP) per $\mathrm{m}^{2}=$ Total EE (or Total GWP) of build

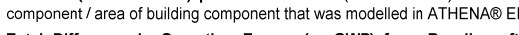
Total Difference in Operating Energy (or GWP) from Baseline after
Lifespan $=$ The difference in the total lific-cycle operating energy (or GWP Lifespan = The difference in the total life-cycle operating energy (or GWP
from the baseline retail buildiding afer lifespan, due to using this building
component instead of the baseline component Total Difference in Operating Energy (or GWP) from Baseline after Lifespan per $\mathrm{m}^{2}=$ Total difference in operating energy (or
baseline atter Ififspan / net roof area of baseline retail building "Total operating primary energy use of baseline retail building after 50 years
$50,700 \mathrm{G} J\left(1.745 \mathrm{M} / \mathrm{Jm} \mathrm{m}^{2} / \mathrm{rr}\right)$ "Total operating GWP of baseline retail building after 50 years $=2,310$ tonnes
of $\mathrm{CO}_{2}$ eq $\left(80 \mathrm{~kg}\right.$ of $\left.\mathrm{CO}_{2} \mathrm{eq} / \mathrm{m}^{2} \mathrm{Yrr}\right)$ 


\section{Glulam Roof \#3 (GLU-R3)}

Building Component Description:

\begin{tabular}{|c|c|c|c|}
\hline \multirow{4}{*}{ Brief Description: } & Roofs & Assembly Layers & \\
\hline & \multirow{3}{*}{ 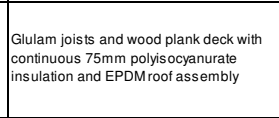 } & $\begin{array}{l}\text { Outside } \\
\text { Ballast tagragate stone) }\end{array}$ & \\
\hline & & EPDM cap sheet (WB) & \\
\hline & & & \\
\hline \multirow{2}{*}{\multicolumn{2}{|c|}{ Quick Numbers: }} & & \\
\hline & & 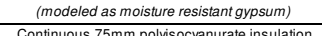 & \\
\hline THERM 5.2: & R-Value: 23.1 & 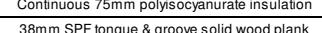 & \\
\hline Roof Thickness: & $657 \mathrm{~mm}$ (excluding drop ceiling) & $\begin{array}{l}\text { 38mm SPF Fotogue \& \& roovev solid wood plank } \\
\text { decking (AB, VR) }\end{array}$ & \\
\hline Span: & Range: $2 \mathrm{~m}$ to $12 \mathrm{~m}$ Design: $9.1 \mathrm{~m}$ & $80 \mathrm{~mm} \times 494 \mathrm{~mm}$ 24-E glulam joists @ 1800 o/c & \\
\hline \begin{tabular}{|l} 
Specilied Design Loads: \\
\end{tabular} & SL $\quad 1.1 \mathrm{kPa}$ & $16 \mathrm{~mm}$ suspended acoustical ceiling & \\
\hline Total Embodied Energy: & $1,847 \mathrm{MN} / \mathrm{m}^{2}$ & Misc. fasteners, nails, and galvanized sheet & \\
\hline Total Embodied GWP: & $87 \mathrm{~kg}$ of $\mathrm{CO}_{2} \mathrm{eq} / \mathrm{m}^{2}$ & Inside & \\
\hline
\end{tabular}

\section{Life-Cycle Assessment Results:}

Primary Energy Consumption (MJ)

\begin{tabular}{|c|c|c|c|c|c|c|c|c|c|c|c|c|c|c|c|c|}
\hline \multirow{3}{*}{$\begin{array}{l}\text { Lifespan } \\
\text { (Years) }\end{array}$} & \multicolumn{14}{|c|}{ Embodied Energy (EE) } & \multirow{2}{*}{\multicolumn{2}{|c|}{$\begin{array}{c}\text { Difference in } \\
\text { Operating Energy } \\
\text { from Baseline after } \\
\text { Lifespan }\end{array}$}} \\
\hline & \multicolumn{3}{|c|}{ Manufacturing } & \multicolumn{3}{|c|}{ Construction } & \multicolumn{3}{|c|}{ Maintenance } & \multicolumn{3}{|c|}{ End of Life } & \multirow{2}{*}{$\begin{array}{l}{ }^{3} \text { Total } \\
\mathrm{EE}\end{array}$} & \multirow{2}{*}{$\begin{array}{l}{ }^{4} \text { Total } \\
\\
\mathrm{EE} \\
\text { per } \mathrm{m}^{2}\end{array}$} & & \\
\hline & \begin{tabular}{|l} 
Materia \\
\end{tabular} & $\left.1\right|^{2}$ Trans & Total & \begin{tabular}{|l|l|} 
Materia \\
\end{tabular} & ${ }^{2}$ Trans. & \begin{tabular}{|l|l|} 
Total \\
\end{tabular} & \begin{tabular}{|l|l|} 
Material \\
\end{tabular} & ${ }^{2}$ Trans. & Total & Material & \begin{tabular}{l|l|}
${ }^{2}$ Trans. \\
.
\end{tabular} & \begin{tabular}{|l|l|} 
Total \\
\end{tabular} & & & ${ }^{5}$ Total & $6^{6}$ perm \\
\hline Initial & 64,658 & 785 & 65,442 & 461 & 2,128 & \begin{tabular}{|l|}
2,589 \\
\end{tabular} & 0 & 0 & 0 & 0 & 0 & 0 & 688,031 & 984 & & \\
\hline 50 & 64,658 & 3785 & 655,44 & $\begin{array}{l}461 \\
\end{array}$ & 2,128 & \begin{tabular}{|l|l|}
2,589 \\
\end{tabular} & 58,565 & 696 & 59,261 & 2 & 421 & 423 & 127,715 & \begin{tabular}{|l|l|}
5 & 1,847
\end{tabular} & $-300,000$ & -5 \\
\hline
\end{tabular}

Global Warming Potential ( $k g$ of $\mathrm{CO}_{2}$ eq.)

\begin{tabular}{|c|c|c|c|c|c|c|c|c|c|c|c|c|c|c|c|c|}
\hline \multirow{3}{*}{$\begin{array}{l}\text { Lifespan } \\
\text { (Years) }\end{array}$} & \multicolumn{14}{|c|}{ Embodied Global Warming Potential (GWP) } & \multirow{2}{*}{\multicolumn{2}{|c|}{$\begin{array}{c}\text { Difference in } \\
\text { Operating GWP from } \\
\text { Baseline after } \\
\text { Lifespan }\end{array}$}} \\
\hline & \multicolumn{3}{|c|}{ Manutacturing } & \multicolumn{3}{|c|}{ Construction } & \multicolumn{3}{|c|}{ Maintenance } & \multicolumn{3}{|c|}{ End of L Lite } & \multirow{2}{*}{$\begin{array}{l}{ }^{3} \text { Total } \\
\text { GWP }\end{array}$} & \multirow{2}{*}{ 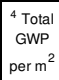 } & & \\
\hline & Materia & ${ }^{2}$ Trans. & 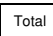 & Materia & ${ }^{2}$ Trans & 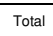 & Material & ${ }^{2}$ Trans. & Total & Material & $\left.\right|^{2}$ Trans. & Total & & & ${ }^{5}$ Total & ${ }^{6}$ perm ${ }^{2}$ \\
\hline Initial & 3,425 & 1 & 3,427 & 30 & 3 & 33 & 0 & 0 & 0 & 0 & 0 & 0 & 3,460 & 50 & & - \\
\hline 50 & 3,425 & 1 & 3,427 & 30 & 3 & 33 & 2,589 & 1 & 2,590 & 0 & 1 & 1 & 6,051 & 87 & $-10,000$ & -17 \\
\hline
\end{tabular}

Embodied energy (and GWP) numbers are based on an area of roof $=69.2 \mathrm{~m}^{2} \quad\left(\right.$ Span $\times$ Width $\left.=9.1 \mathrm{~m} \times 7.6 \mathrm{~m}=69.2 \mathrm{~m}^{2}\right)$

Net roof area of baseline retail building (gross roof area - openings) = $586.0 \mathrm{~m}^{2}$

\section{ATHENA $®$ EIE Material List}

\begin{tabular}{|l|c|c|}
\hline \multicolumn{1}{|c|}{ Material List } & Quantities & Unit \\
\hline $\begin{array}{l}\text { 13mm Moisture Resistant Gypsum } \\
\text { Board }\end{array}$ & 76.1 & $\mathrm{~m} 2$ \\
\hline $\begin{array}{l}16 \mathrm{~mm} \text { Gypsum Fibre Gypsum } \\
\text { Board }\end{array}$ & 76.1 & $\mathrm{~m} 2$ \\
\hline 6 mil Polyethylene & 70.5 & $\mathrm{~m} 2$ \\
\hline Ballast (aggreagate stone) & $15,823.1$ & $\mathrm{~kg}$ \\
\hline EPDM membrane & 568.0 & $\mathrm{~kg}$ \\
\hline Galvanized Sheet & 190.6 & $\mathrm{~kg}$ \\
\hline GluLam Sections & 1.5 & $\mathrm{~m} 3$ \\
\hline Isocyanurate & 214.6 & $\mathrm{~m} 2(25 \mathrm{~mm})$ \\
\hline Joint Compound & 151.9 & $\mathrm{~kg}$ \\
\hline Nails & 19.9 & $\mathrm{~kg}$ \\
\hline Paper Tape & 1.7 & $\mathrm{~kg}$ \\
\hline $\begin{array}{l}\text { Small Dimension Sottwood Lumber, } \\
\text { kiln-dried }\end{array}$ & 3.7 & $\mathrm{~m} 3$ \\
\hline Softwood Plywood & 1.4 & $\mathrm{~m} 2(9 \mathrm{~mm})$ \\
\hline
\end{tabular}

$$
\begin{aligned}
& \text { Notes: } \\
& \text { 'Initial = Time 'o' (i.e. at the completion of inits }
\end{aligned}
$$

${ }^{2}$ Trans. $=$ Transportation

${ }^{3}$ Total EE (or Total GWP) = Total embodied energy (or total embodied GWP) of building component after lifespan (i.e. total manufacturing + total
construction t total maintenance + total end-oflifie effects) ${ }^{4}$ Total EE (or Total GWP) per $\mathrm{m}^{2}=$ Total EE (or Total GWP) of building
component/ area of building component that was modelled in ATHENAA EIE Total Difference in Operating Energy (or GWP) from Baseline after Lifespan = The difference in the total life-cycle operating energy (or GWP)
from the baseline retail building atter lifespan, due to using this building ${ }^{6}$ Total Difference in Operating Energy (or GWP) from Baseline after Liffespan per $\mathrm{m}^{2}=$ Total difierence in operating energy (or GWP) from
baseline after lifespan /net roof area of baseline retail building $*$ *otal operating primary energy use of baseline retail building after 50 years $=$
$50,700 \mathrm{GJ}\left(1,745 \mathrm{MJ} / \mathrm{m}^{2} \mathrm{y}\right)$

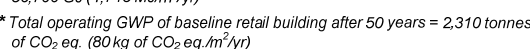

\section{Glulam Roof \#4 (GLU-R4)}

Building Component Description:

\begin{tabular}{|c|c|c|c|}
\hline \multirow{4}{*}{ Brief Description: } & Roofs & Assembly Layers & \\
\hline & \multirow{3}{*}{ 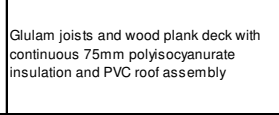 } & $\begin{array}{c}\text { Outside } \\
\text { Ballast (aggregate stone) }\end{array}$ & \\
\hline & & $\begin{array}{l}\text { PVC membrane cap sheet (WB) } \\
\text { (includes wood naling strips) }\end{array}$ & \\
\hline & & Bonding agent(modeled as 6mil poly) & \\
\hline \multicolumn{2}{|l|}{ Quick Numbers: } & $\begin{array}{l}12 \mathrm{~mm} \text { coverboard } \\
\text { (modeled as moisture resistant gyosum) }\end{array}$ & \\
\hline ASHRAE Standard 90.1: & \begin{tabular}{|l|} 
R-Value: 20.8 \\
\end{tabular} & Continuous $75 \mathrm{~mm}$ polysisocyanurate insulation & \\
\hline THERM 5.2: & $\begin{array}{|lr|}\text { R-Value: } 23.1 & \text { RSI-Value: } 4.1 \\
\end{array}$ & 38mm SPF tongue \& groove solid wood plank & \\
\hline Roof Thickness: & $657 \quad \mathrm{~mm}$ (excluding drop ceiling) & decking (AB, VR) & \\
\hline Span: & Range: $2 \mathrm{~m}$ to $12 \mathrm{~m}$ Design: $9.1 \mathrm{~m}$ & 80mm x494mm 24f-E glulam joists @ 1800 olc & \\
\hline \begin{tabular}{|l} 
Specified Design Loads: \\
\end{tabular} & $\mathrm{SL} \quad 1.1 \mathrm{kPa}$ & $16 \mathrm{~mm}$ suspended acoustical celling & \\
\hline Total Embodied Energy: & $2,401 \mathrm{MN} / \mathrm{m}^{2}$ & Misc. fasteners, nails, and galvanized sheet & \\
\hline Total Embodied GWP: & 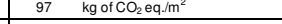 & Inside & \\
\hline
\end{tabular}

\section{Life-Cycle Assessment Results:}

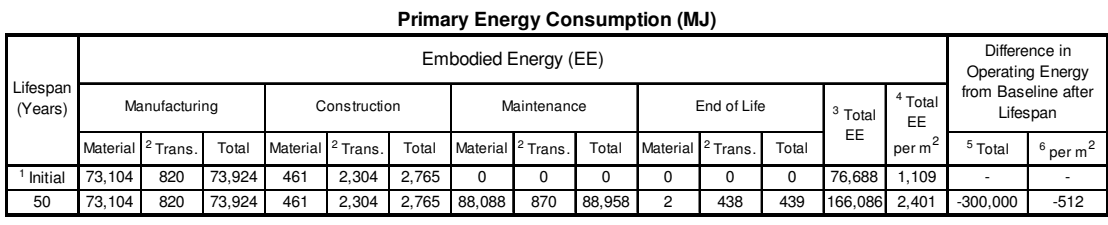

\begin{tabular}{|c|c|c|c|c|c|c|c|c|c|c|c|c|c|c|c|c|}
\hline & \multirow{2}{*}{\multicolumn{14}{|c|}{$\begin{array}{l}\text { Global Warming Potential ( } \mathbf{k g} \text { of } \mathrm{CO}_{2} \mathbf{~ e q} \text {.) } \\
\text { Embodied Global Warming Potential (GWP) }\end{array}$}} & & \\
\hline \multirow{3}{*}{$\begin{array}{l}\text { Lifespanan } \\
\text { (Year) }\end{array}$} & & & & & & & & & & & & & & & \multirow{2}{*}{\multicolumn{2}{|c|}{$\begin{array}{c}\text { Difference in } \\
\text { Operatiting GWP fron } \\
\text { Baseline after } \\
\text { Lifespan }\end{array}$}} \\
\hline & \multicolumn{3}{|c|}{ Manutacturing } & \multicolumn{3}{|c|}{ Construction } & \multicolumn{3}{|c|}{ Maintenance } & \multicolumn{3}{|c|}{ End of Life } & \multirow{2}{*}{$\begin{array}{l}{ }^{3} \text { Total } \\
\text { GWP }\end{array}$} & \multirow{2}{*}{$\mid \begin{array}{c}4 \\
{ }^{4} \text { Total } \\
\text { GWP } \\
\text { perm }\end{array}$} & & \\
\hline & Material & ${ }^{2}$ Trans. & Total & Materia & Trans & Total & Materia & $\mid 2$ Trans. & Total & \begin{tabular}{|l|} 
Materia \\
\end{tabular} & Trans. & Total & & & \begin{tabular}{|l|}
${ }^{5}$ Total \\
\end{tabular} & ${ }^{6} \operatorname{perm}^{2}$ \\
\hline Initial & 3,565 & 2 & 3,566 & 30 & 3 & 34 & 0 & 0 & 0 & 0 & 0 & 0 & 3,600 & 52 & & \\
\hline 50 & 3,565 & 2 & 3,566 & 30 & 3 & 34 & 3,097 & 2 & 3,099 & 0 & 1 & 1 & \begin{tabular}{|l|l|}
6,699 \\
\end{tabular} & 97 & $-10,000$ & -17 \\
\hline
\end{tabular}

Embodied energy (and GWP) numbers are based on an area of roof $=69.2 \mathrm{~m}^{2} \quad\left(\right.$ Span $\times$ Width $\left.=9.1 \mathrm{~m} \times 7.6 \mathrm{~m}=69.2 \mathrm{~m}^{2}\right)$

\section{ATHENA $®$ EIE Material List:}

$$
\text { (Includes all materials affer } 50 \text { years) }
$$

\begin{tabular}{|l|c|c|}
\hline \multicolumn{1}{|c|}{ Material List } & Quantities & Unit \\
\hline $\begin{array}{l}\text { 13mm Moisture Resistant Gypsum } \\
\text { Board }\end{array}$ & 76.1 & $\mathrm{~m} 2$ \\
\hline $\begin{array}{l}\text { 16mm Gypsum Fibre Gypsum } \\
\text { Board }\end{array}$ & 76.1 & $\mathrm{~m} 2$ \\
\hline 6 mil Polyethylene & 70.5 & $\mathrm{~m} 2$ \\
\hline Ballast (aggregate stone) & $15,823.1$ & $\mathrm{~kg}$ \\
\hline Galvanized Sheet & 183.5 & $\mathrm{~kg}$ \\
\hline GluLam Sections & 1.5 & $\mathrm{~m} 3$ \\
\hline Isocyanurate & 214.6 & $\mathrm{~m} 2(25 \mathrm{~mm})$ \\
\hline Joint Compound & 151.9 & $\mathrm{~kg}$ \\
\hline Nails & 21.4 & $\mathrm{~kg}$ \\
\hline Paper Tape & 1.7 & $\mathrm{~kg}$ \\
\hline PVC membrane & 1.512 .6 & $\mathrm{~kg}$ \\
\hline $\begin{array}{l}\text { Small Dimension Sottwood Lumber, } \\
\text { kiln-dried }\end{array}$ & 3.7 & $\mathrm{~m} 3$ \\
\hline Softwood Plywood & 1.4 & $\mathrm{~m} 2(9 \mathrm{~mm})$ \\
\hline
\end{tabular}

Notes:
${ }^{\prime}$ Initial $=$ Time
$O^{\prime}$ ' (i.e. at the completion of initial construction)

${ }^{2}$ Trans. $=$ Transportation

${ }^{3}$ Total EE (or Total GWP) = Total embodied energy (or total embodied GW of building component after lifespan (i.e. total manu
construction + total maintenance t total end-of-life effects)

${ }^{4}$ Total EE (or Total GWP) per $\mathrm{m}^{2}=$ Total EE (or Total GWP) of building
component / area of building component that was modelled in ATHENA@ EIE Total Difference in Operating Energy (Oo GWP) from Baseline after
Lifespan $=$ The difference in the total lifecycle operating energy (or GWP) Lifespan $=$ The difference in the total lifecycle operating energy (or GWP)
from the baseline retail builing ater fifespan, due to using this building
component instead of the baseline component Total Difference in Operating Energy (or GWP) from Baseline after Lifespan per $\mathrm{m}^{2}=$ Total difference in operating energy (or GWP) from
baseline after Ififespan/net roof area of baseline retail building * Total operating primary energy use of baseline retail building after 50 years =
$50,700 \mathrm{GJ}\left(1,745 \mathrm{MJ} / \mathrm{m}^{2} \mathrm{yr}\right)$

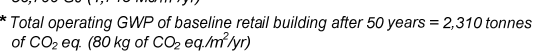




\section{Glulam Roof \#5 (GLU-R5)}

Building Component Description:

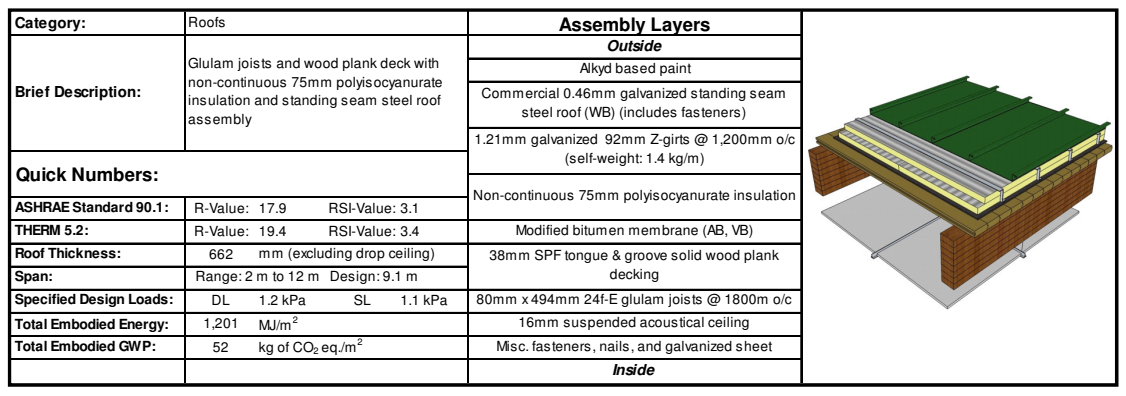

\section{Life-Cycle Assessment Results:}

Primary Energy Consumption (MJ)

\begin{tabular}{|c|c|c|c|c|c|c|c|c|c|c|c|c|c|c|c|c|}
\hline \multirow{3}{*}{\begin{tabular}{|l} 
Lifespan \\
(Years)
\end{tabular}} & \multicolumn{14}{|c|}{ Embodied Energy (EE) } & \multirow{2}{*}{\multicolumn{2}{|c|}{$\begin{array}{c}\text { Difference in } \\
\text { Operating Energy } \\
\text { from Baseline afte } \\
\text { Lifespan }\end{array}$}} \\
\hline & \multicolumn{3}{|c|}{ Manufacturing } & \multicolumn{3}{|c|}{ Construction } & \multicolumn{3}{|c|}{ Maintenance } & \multicolumn{3}{|c|}{ End of Life } & \multirow{2}{*}{$\begin{array}{c}{ }^{3} \text { Total } \\
\mathrm{EE}\end{array}$} & \multirow{2}{*}{\begin{tabular}{|c|}
${ }^{4}$ Total \\
EE \\
per $\mathrm{m}^{2}$
\end{tabular}} & & \\
\hline & \begin{tabular}{|l|} 
Materia \\
\end{tabular} & \begin{tabular}{l|l}
$1{ }^{2}$ Trans. \\
.
\end{tabular} & \begin{tabular}{|l|l|} 
Total \\
\end{tabular} & \begin{tabular}{|l|} 
Materia \\
\end{tabular} & \begin{tabular}{|l|}
${ }^{2}$ Trans. \\
\end{tabular} & Total & Material & ${ }^{2}$ Trans. & Total & \begin{tabular}{|l|} 
Material \\
\end{tabular} & | ${ }^{2}$ Trans. & Total & & & ${ }^{5}$ Total & ${ }^{6}$ per $\mathrm{m}^{2}$ \\
\hline Initial & 70,853 & 675 & 71,528 & 461 & \begin{tabular}{|l|l|}
1,582 \\
\end{tabular} & 2,042 & 0 & 0 & 0 & 0 & 0 & 0 & 73,570 & 1,064 & & \\
\hline 50 & 70,853 & \begin{tabular}{|l|l|}
3 & 675 \\
\end{tabular} & 771,528 & 461 & \begin{tabular}{|l|l|}
1,582 \\
\end{tabular} & 2,042 & \begin{tabular}{|l|l|}
9,263 \\
\end{tabular} & 29 & \begin{tabular}{|l|l|}
9,293 \\
\end{tabular} & 2 & 207 & \begin{tabular}{|l|}
209 \\
\end{tabular} & & & \begin{tabular}{|l|l|l}
100,000 \\
\end{tabular} & 17 \\
\hline
\end{tabular}

Global Warming Potential ( $k g$ of $\mathrm{CO}_{2}$ eq.)

\begin{tabular}{|c|c|c|c|c|c|c|c|c|c|c|c|c|c|c|c|c|}
\hline \multirow{4}{*}{$\begin{array}{c}\text { Lifispan } \\
\text { (Years) }\end{array}$} & \multirow{2}{*}{\multicolumn{14}{|c|}{ Embodied Global Warming Potential (GWP) }} & \multirow{3}{*}{\multicolumn{2}{|c|}{$\begin{array}{l}\text { Difference in } \\
\text { Operating GWP from } \\
\text { Baseline after } \\
\text { Lifiespan }\end{array}$}} \\
\hline & & & & & & & & & & & & & & & & \\
\hline & \multicolumn{3}{|c|}{ Manufacturing } & \multicolumn{3}{|c|}{ Construction } & \multicolumn{3}{|c|}{ Maintenance } & \multicolumn{3}{|c|}{ End of Lite } & \multirow{2}{*}{$\begin{array}{l}{ }^{3} \text { Total } \\
\text { GWP }\end{array}$} & \multirow{2}{*}{$\begin{array}{l}{ }^{4} \text { Total } \\
\text { GWP } \\
\text { per m }\end{array}$} & & \\
\hline & Material & Trans. & Total & Material & ${ }^{2}$ Trans. & Total & Material $\left.\right|^{2}$ & ${ }^{2}$ Trans. & Total & Material & \begin{tabular}{|c|}
${ }^{2}$ Trans. \\
\end{tabular} & Total & & & ${ }^{5}$ Total & ${ }^{6} \mathrm{perm}^{2}$ \\
\hline Intitial & 3,415 & 1 & 3,416 & 30 & 2 & 32 & 0 & 0 & 0 & 0 & 0 & 0 & 3,449 & 50 & & \\
\hline 50 & 3,415 & 1 & 3,416 & 30 & 2 & 32 & 129 & 0 & 129 & 0 & 0 & 1 & 3,578 & 52 & 10,000 & 17 \\
\hline
\end{tabular}

Embodied energy (and GWP) numbers are based on an area of roof $=69.2 \mathrm{~m}^{2} \quad\left(\right.$ Span $\times$ Wiath $\left.=9.1 \mathrm{~m} \times 7.6 \mathrm{~m}=69.2 \mathrm{~m}^{2}\right)$

Net roof area of baseline retall building (gross roof area - openings) = $586.0 \mathrm{~m}^{2}$

\section{ATHENA ® EIE Material List}

\begin{tabular}{|l|c|c|}
\hline \multicolumn{1}{|c|}{ Material List } & Quantities & Unit \\
\hline $\begin{array}{l}\text { 16mm Gypsum Fibre Gypsum } \\
\text { Board }\end{array}$ & 76.1 & $\mathrm{~m} 2$ \\
\hline Foam Polyisocyanurate & 213.1 & $\mathrm{~m} 2(25 \mathrm{~mm})$ \\
\hline Galvanized Sheet & 0.6 & $\mathrm{~kg}$ \\
\hline Galvanized Studs & 80.8 & $\mathrm{~kg}$ \\
\hline GluLam Sections & 1.5 & $\mathrm{~m} 3$ \\
\hline Joint Compound & 75.9 & $\mathrm{~kg}$ \\
\hline Modififed Bitumen membrane & 214.7 & $\mathrm{~kg}$ \\
\hline Nails & 17.8 & $\mathrm{~kg}$ \\
\hline Paper Tape & 0.9 & $\mathrm{~kg}$ \\
\hline Screws Nuts \& Bolts & 0.7 & $\mathrm{~kg}$ \\
\hline $\begin{array}{l}\text { Small Dimension Softwood Lumber, } \\
\text { kiln-dried }\end{array}$ & 3.5 & $\mathrm{~m} 3$ \\
\hline Solvent Based Allkyd Paint & 49.7 & $\mathrm{~L}$ \\
\hline
\end{tabular}

$$
\begin{aligned}
& \text { Notes: } \\
& \text { 'Initial }=\text { Time 'o' (i.e. at the completion of initia }
\end{aligned}
$$

${ }^{2}$ Trans. $=$ Transportation

Total EE (or Total GWP) $=$ Total embodied energy (or total embodied GWP) of building component after lifespan (i.e. total manufacturing + to

Total EE (or Total GWP) per $\mathrm{m}^{2}=$ Total EE (or Total GWP) of building
component/ area of building component that was modelled in ATHENAQ EIE Total Diferce in Operaing Energy (or GWP) fiom Baseline a Lifespan $=$ The difference in the total life-cycle operating energy (or GWP)
from the baseline retail building after lifespan, due to using this building

Total Difference in Operating Energy (or GWP) from Baseline after
Lifespan per $\mathrm{m}^{2}=$ Total difference in operating energy (or GWP) from Lifespan per $\mathrm{m}^{2}=$ Total difterence in ooerating energy
baseline after lifespan / net roof area of baseline retail building Total operating primary energy use of baseline retail building after 50 years

"Total operating $\mathrm{GWP}$ of baseline retail building after 50 years $=2,310$ tonnes
of $\mathrm{CO}_{2}$ eq. $\left(80 \mathrm{~kg}\right.$ of $\left.\mathrm{CO}_{2} \mathrm{eq} / / \mathrm{m}^{2} \mathrm{Yrr}\right)$
Glulam Roof \#6 (GLU-R6)

Building Component Description:

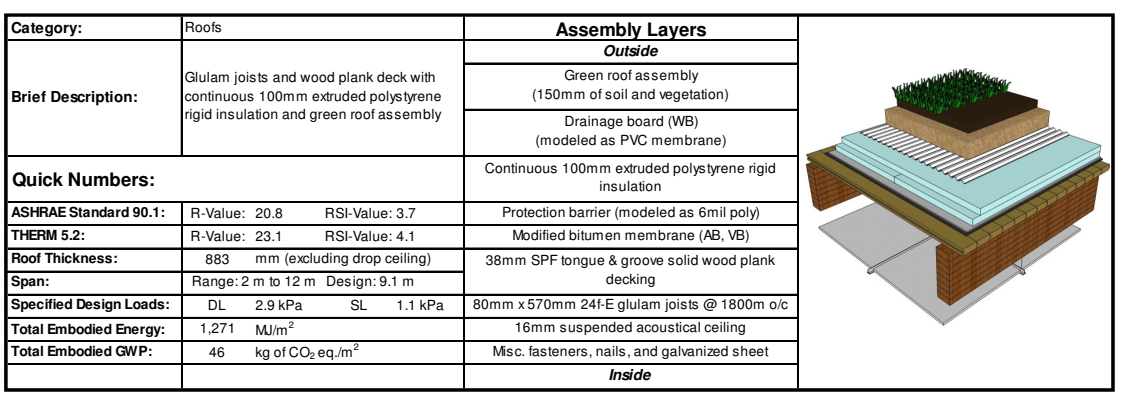

Life-Cycle Assessment Results:

Primary Energy Consumption (MJ)

\begin{tabular}{|c|c|c|c|c|c|c|c|c|c|c|c|c|c|c|c|c|}
\hline \multirow{3}{*}{$\mid \begin{array}{l}\text { Litiespan } \\
\text { (Years) }\end{array}$} & \multicolumn{14}{|c|}{ Embodied Energy (EE) } & \multirow{2}{*}{\multicolumn{2}{|c|}{$\begin{array}{c}\text { Difference in } \\
\text { Operating Energy } \\
\text { from Baseline after } \\
\text { Lifespan }\end{array}$}} \\
\hline & \multicolumn{3}{|c|}{ Manufacturing } & \multicolumn{3}{|c|}{ Construction } & \multicolumn{3}{|c|}{ Maintenance } & \multicolumn{3}{|c|}{ End of Life } & \multirow{2}{*}{$\begin{array}{c}{ }^{3} \text { Total } \\
\text { EE }\end{array}$} & \multirow{2}{*}{\begin{tabular}{|c|}
${ }^{4} \mathrm{~T}$ Total \\
$\mathrm{EE}$ \\
per m
\end{tabular}} & & \\
\hline & \begin{tabular}{|l|} 
Material \\
\end{tabular} & ${ }^{2}$ Trans. & Total & Material & ${ }^{2}$ Trans. & Total & Material & $\begin{array}{l}{ }^{2} \text { Trans. } \\
\text { Thens. }\end{array}$ & Total & \begin{tabular}{|l|l|} 
Material \\
\end{tabular} & \begin{tabular}{|l|}
${ }^{2}$ Trans. \\
\end{tabular} & Total & & & ${ }^{5}$ Total & ${ }^{6 \text { per m}{ }^{2}}$ \\
\hline Initial & 58,297 & 659 & 58,957 & 461 & 1,633 & 2,093 & 0 & 0 & 0 & 0 & 0 & 0 & 61,050 & 883 & & \\
\hline 50 & 58,297 & 659 & \begin{tabular}{|l|l|l|l}
58,957 &
\end{tabular} & 461 & \begin{tabular}{|l|l|}
1,633 \\
\end{tabular} & $\begin{array}{l}2,093 \\
\end{array}$ & 26,592 & 73 & 26,665 & 2 & 212 & 214 & 87,930 & \begin{tabular}{|l|l|}
1,271 \\
\end{tabular} & $-300,000$ & 5 \\
\hline
\end{tabular}

Global Warming Potential (kg of $\mathrm{CO}_{2}$ eq.)

\begin{tabular}{|c|c|c|c|c|c|c|c|c|c|c|c|c|c|c|c|c|}
\hline \multirow{4}{*}{$\begin{array}{l}\text { Llespan } \\
\text { (Years) }\end{array}$} & \multirow{2}{*}{\multicolumn{14}{|c|}{ of $\mathrm{CO}_{2}$ eq.) }} & \multirow{3}{*}{\multicolumn{2}{|c|}{\begin{tabular}{|c} 
Difference in \\
Operating GWP from \\
Baseline affer \\
Lifespan
\end{tabular}}} \\
\hline & & & & & & & & & & & & & & & & \\
\hline & \multicolumn{3}{|c|}{ Manutacturing } & \multicolumn{3}{|c|}{ Construction } & \multicolumn{3}{|c|}{ Maintenance } & \multicolumn{3}{|c|}{ End of Life } & \multirow{2}{*}{$\begin{array}{l}{ }^{3} \text { Total } \\
\text { GWP }\end{array}$} & \multirow{2}{*}{$\begin{array}{l}{ }^{4} \text { Total } \\
\text { GWP } \\
\text { per m }\end{array}$} & & \\
\hline & Material & ${ }^{2}$ Trans. & Total & Material & Trans. & Total & \begin{tabular}{|l|} 
Material \\
\end{tabular} & ${ }^{2}$ Trans. & Total & \begin{tabular}{|l|} 
Material \\
\end{tabular} & \begin{tabular}{|l|l|}
${ }^{2}$ Trans. \\
\end{tabular} & Total & & & \begin{tabular}{|l|}
${ }^{5}$ Total \\
\end{tabular} & ${ }^{6} \operatorname{perm}^{2}$ \\
\hline |nitial & 2,382 & 1 & 2,000 & 30 & 2 & 32 & 0 & 0 & $n_{1}$ & 0 & 0 & S & & 35 & & \\
\hline 50 & 2,382 & 1 & 2,383 & 30 & 2 & 32 & 740 & 0 & 740 & 0 & 0 & 1 & 156 & 46 & 10,000 & \\
\hline
\end{tabular}

Embodied energy (and GWP) numbers are based on an area of roof $=69.2 \mathrm{~m}^{2} \quad$ (Span $\times$ Width $=9.1 \mathrm{~m} \times 7.6 \mathrm{~m}=69.2 \mathrm{~m}^{2}$ )

(n) $=586.0 \mathrm{~m}^{2}$
ATHENA ${ }^{\circledR}$ EIE Material List:

\begin{tabular}{|l|c|c|}
\hline \multicolumn{1}{|c|}{ Material List } & Quantities & Unit \\
\hline $\begin{array}{l}\text { 16mm Gypsum Fibre Gypsum } \\
\text { Board }\end{array}$ & 76.1 & $\mathrm{~m} 2$ \\
\hline 6 mil Polyethylene & 73.4 & $\mathrm{~m} 2$ \\
\hline Ballast (aggregate stone) & 263.7 & $\mathrm{~kg}$ \\
\hline Extruded Polystyrene & 283.4 & $\mathrm{~m} 2(25 \mathrm{~mm})$ \\
\hline Galvanized Sheet & 160.6 & $\mathrm{~kg}$ \\
\hline GluLam Sections & 1.8 & $\mathrm{~m} 3$ \\
\hline Joint Compound & 75.9 & $\mathrm{~kg}$ \\
\hline Modified Bitumen membrane & 258.7 & $\mathrm{~kg}$ \\
\hline Nails & 17.8 & $\mathrm{~kg}$ \\
\hline Paper Tape & 0.9 & $\mathrm{~kg}$ \\
\hline PVC membrane & 308.7 & $\mathrm{~kg}$ \\
\hline $\begin{array}{l}\text { Small Dimension Sottwood Lumber, } \\
\text { kill-dried }\end{array}$ & 3.5 & $\mathrm{~m} 3$ \\
\hline
\end{tabular}

'Initial = Time 'o' (ie ates:

${ }^{2}$ Trans. = Transportation

${ }^{3}$ Total EE (or Total GWP) = Total embodied energy (or total embodied GWP)

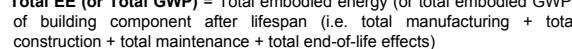
${ }^{4}$ Total EE (or Total GWP) per $\mathrm{m}^{2}=$ Total EE (or Total GWP) of building
component/ area of building component that was modelled in ATHENAQ EIE Lifespan $=$ The difference in the total life-cycle operating energy (or GWP from the baseline retail building after lifespan, due to using this building
component ${ }^{6}$ Total Difference in Operating Energy (or GWP) from Baseline after Total Difference in Operating Energy (or GWP) from Baseline after
Lifespan per $\mathrm{m}^{2}=$ Total difference in operating energy (or GWP) from
basaline after lifespan / net roof area of baseline retail building * Total operating primary energy use of baseline retail building after 50 years $50,700 \mathrm{GJ}\left(1,745 \mathrm{MJ} / \mathrm{m}^{2} / \mathrm{yr}\right)$

* Total operating GWP of baseline retail building after 50 years $=2,310$ tonnes
of $\mathrm{CO}_{2}$ eq. $\left(80 \mathrm{~kg}\right.$ of $\mathrm{CO}_{2}$ eq. $\left./ \mathrm{m}^{2} \mathrm{yrl}\right)$ 


\section{Glulam Roof \#7 (GLU-R7)}

Building Component Description:

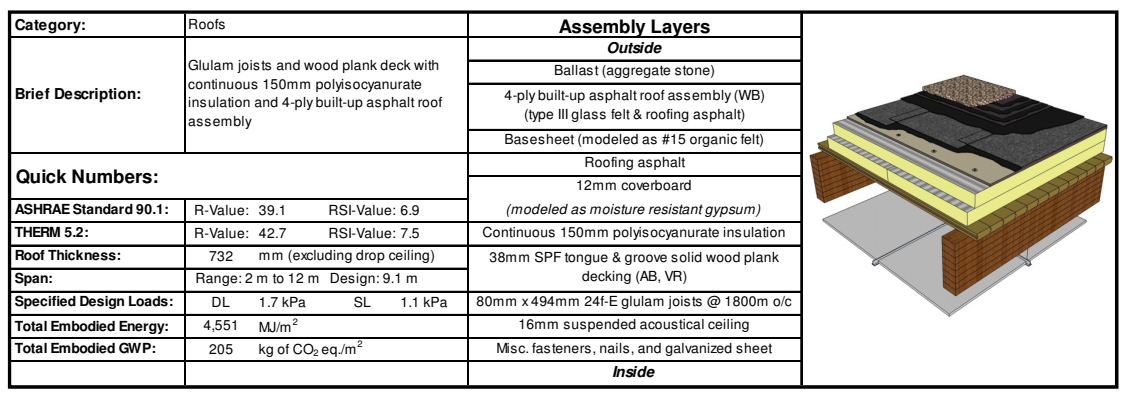

\section{Life-Cycle Assessment Results:}

Primary Energy Consumption (MJ)

\begin{tabular}{|c|c|c|c|c|c|c|c|c|c|c|c|c|c|c|c|}
\hline \multirow{3}{*}{\begin{tabular}{|l} 
Lifespan \\
(Years)
\end{tabular}} & \multicolumn{13}{|c|}{ Embodied Energy (EE) } & \multirow{2}{*}{\multicolumn{2}{|c|}{$\begin{array}{c}\text { Difference in } \\
\text { Operating Energy } \\
\text { from Baseline atte } \\
\text { Lifespan }\end{array}$}} \\
\hline & \multicolumn{2}{|l|}{ Manufac } & \multicolumn{3}{|c|}{ onstri } & \multicolumn{3}{|c|}{ Maintenance } & \multicolumn{3}{|c|}{ End of Life } & \multirow{2}{*}{$\begin{array}{c}{ }^{3} \text { Total } \\
\mathrm{EE}\end{array}$} & \multirow{2}{*}{\begin{tabular}{|c|}
${ }^{4}$ Total \\
EE \\
per m
\end{tabular}} & & \\
\hline & Material $\mid{ }^{2}$ Trans & \begin{tabular}{|l|l} 
Total \\
\end{tabular} & Materia & ${ }^{2}$ Trans. & \begin{tabular}{|l|l|} 
Total \\
\end{tabular} & \begin{tabular}{|l|} 
Material \\
\end{tabular} & ${ }^{2}$ Trans. & \begin{tabular}{|l|l|} 
Total \\
\end{tabular} & Material & $\begin{array}{ll}2 & 2 \\
2\end{array}$ & \begin{tabular}{|l|l|} 
Total \\
\end{tabular} & & & ${ }^{5}$ Total & ${ }^{6}{ }_{\mathrm{perm}}^{2}{ }^{2}$ \\
\hline Initial & \begin{tabular}{|l|l|}
167,488 & 792 \\
\end{tabular} & 168,280 & 461 & $\begin{array}{ll}1,837 \\
\end{array}$ & \begin{tabular}{|l|l|}
2,298 \\
\end{tabular} & 0 & 0 & 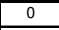 & 0 & 0 & Th & 170,577 & 2,466 & & \\
\hline 50 & $\begin{array}{lll}167,488 & 792 \\
\end{array}$ & 168,280 & | 461 & $\begin{array}{llll} & 1,837 \\
\end{array}$ & $\begin{array}{llll}2,298 & & \\
\end{array}$ & $\mid 143,295$ & 495 & 143,790 & 2 & 392 & 393 & 314,7 & 4,551 & $\begin{array}{l}-1,200 \\
.\end{array}$ & $-2,0$ \\
\hline
\end{tabular}

Global Warming Potential ( $\mathrm{kg}$ of $\mathrm{CO}_{2}$ eq.)

\begin{tabular}{|c|c|c|c|c|c|c|c|c|c|c|c|c|c|c|c|c|}
\hline \multirow{3}{*}{$\begin{array}{l}\text { Lifespan } \\
\text { (Years) }\end{array}$} & \multicolumn{14}{|c|}{ Embodied Global Warming Potential (GWP) } & \multirow{2}{*}{\multicolumn{2}{|c|}{$\begin{array}{l}\text { Difference in } \\
\text { Operating GWP from } \\
\text { Baseline after } \\
\text { Lifespan }\end{array}$}} \\
\hline & \multicolumn{3}{|c|}{ Manutacturing } & \multicolumn{3}{|c|}{ Construction } & \multicolumn{3}{|c|}{ Maintenance } & \multicolumn{3}{|c|}{ End of Life } & \multirow{2}{*}{$\begin{array}{l}{ }^{3} \text { Total } \\
\text { GWP }\end{array}$} & \multirow{2}{*}{$\begin{array}{l}{ }^{4} \text { Total } \\
\text { GWP } \\
\text { per m }\end{array}$} & & \\
\hline & Material & ${ }^{2}$ Trans. & Total & Material & ${ }^{2}$ Trans. & Total & Material & ${ }^{2}$ Trans. & Total & Material & ${ }^{2}$ Trans. & Total & & & ${ }^{5}$ Total & ${ }^{6} \mathrm{perm}^{2}$ \\
\hline "Initial & 8,417 & $\frac{2}{2}$ & 8,419 & 30 & $\frac{2}{2}$ & 33 & 0 & 0 & 0 & 0 & 0 & 0 & \begin{tabular}{|c|}
8,451 \\
1451
\end{tabular} & 122 & & \\
\hline & 8,417 & 2 & 8,419 & 30 & 2 & 33 & 5,698 & 1 & 5,699 & 0 & 1 & & 14,151 & 205 & $-60,000$ & \\
\hline
\end{tabular}

Embodied energy (and GWP) numbers are based on an area of roof $=69.2 \mathrm{~m}^{2} \quad\left(\right.$ Span $\times$ Width $\left.=9.1 \mathrm{~m} \times 7.6 \mathrm{~m}=69.2 \mathrm{~m}^{2}\right)$

Net roof area of baseline retail building (gross roof area - openings) $=586.0 \mathrm{~m}^{2}$

\section{ATHENA $\otimes$ EIE Material List:}

\begin{tabular}{|c|c|c|}
\hline \multicolumn{3}{|c|}{50 years) } \\
\hline Material List & Quantities & Unit \\
\hline \#15 Organic Felt & 551.9 & $\frac{\mathrm{m} 2}{2}$ \\
\hline $\begin{array}{l}\text { 13mm Moisture Resistant Gypsum } \\
\text { Board }\end{array}$ & 76.1 & $\mathrm{~m} 2$ \\
\hline $\begin{array}{l}16 \mathrm{~mm} \text { Gypsum Fibre Gypsum } \\
\text { Board }\end{array}$ & 76.1 & $\mathrm{~m} 2$ \\
\hline Ballast (aggregate stone) & $5,274.4$ & $\mathrm{~kg}$ \\
\hline Galvanized Sheet & 243.0 & $\mathrm{~kg}$ \\
\hline GluLam Sections & 1.5 & $\mathrm{~m} 3$ \\
\hline Isocyanurate & 429.2 & $\mathrm{~m} 2(25 \mathrm{mr}$ \\
\hline Joint Compound & 75.9 & $\mathrm{~kg}$ \\
\hline Nails & 49.2 & $\mathrm{~kg}$ \\
\hline $\begin{array}{l}\text { Paper Tape } \\
\end{array}$ & 0.9 & $\mathrm{~kg}$ \\
\hline Roofing Asphalt & $\begin{array}{l}2,093.8 \\
\end{array}$ & $\mathrm{~kg}$ \\
\hline $\begin{array}{l}\text { Small Dimension Softwood Lumber, } \\
\text { killdried }\end{array}$ & 3.5 & m3 \\
\hline Type III Glass Felt & $1,103.8$ & $\mathrm{~m} 2$ \\
\hline
\end{tabular}
* Total operating primary energy use of baseline retail building after 50 years = *Total operating $\mathrm{GWP}$ of baseline retail building after 50 years $=2,310$ tonnes
of $\mathrm{CO}_{2}$ eq. $80 \mathrm{~kg}$ of $\mathrm{CO}_{2}$ eq. $\left.\mathrm{m}^{2} / \mathrm{yr}\right)$

\section{Glulam Roof \#8 (GLU-R8)}

Building Component Description:

\begin{tabular}{|c|c|c|c|}
\hline Category: & Roofs & Assembly Layers & \\
\hline \multirow{3}{*}{ Brief Description: } & \multirow{3}{*}{ 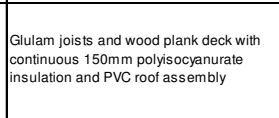 } & \begin{tabular}{|c|c|} 
Outside \\
Ballast (tagragate stone)
\end{tabular} & \\
\hline & & 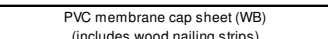 & \\
\hline & & Bonding agent (modeled as 6mil poly) & \\
\hline \multicolumn{2}{|l|}{ Quick Numbers: } & $12 \mathrm{~mm}$ coverboard & \\
\hline ASHRAE Standard 90.1: & RSIVValue: 6.9 & $\begin{array}{l}\text { (modeled as mosisture resistant gyposum) } \\
\text { Continuous } 150 \mathrm{~mm} \text { polyis ocyanurate insulation }\end{array}$ & \\
\hline THERM 5.2: & 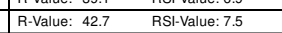 & 38mm SPF tongue \& grove solid wood plank & \\
\hline Roof Thickness: & $732 \mathrm{~mm}$ (excluding drop ceiling) & decking (AB, VR) & \\
\hline Span: & \begin{tabular}{|l} 
Range: $2 \mathrm{~m}$ to $12 \mathrm{~m}$ Design: $9.1 \mathrm{~m}$ \\
\end{tabular} & 80mm x494mm 24f:E glulam joists @ $@ 1800 \mathrm{~m} \mathrm{o/c}$ & \\
\hline Specified Design Loads: & DL $\quad 1.7 \mathrm{kPa}$ & $16 \mathrm{~mm}$ suspended acoustical celling & \\
\hline \begin{tabular}{|l} 
Total Embodied Energy: \\
\end{tabular} & $2.584 \mathrm{~m} / \mathrm{m}^{2}$ & Misc. fasteners, nails, and galvanized sheet & \\
\hline Total Embodied GWP: & $115 \mathrm{~kg} \mathrm{of} \mathrm{CO} 2 \mathrm{eq} / \mathrm{m}^{2}$ & $\begin{array}{lll}\text { Inside } \\
\text {. }\end{array}$ & \\
\hline
\end{tabular}

\section{Life-Cycle Assessment Results:}

Primary Energy Consumption (MJ)

\begin{tabular}{|c|c|c|c|c|c|c|c|c|c|c|c|c|c|c|c|c|}
\hline \multirow{3}{*}{$\left|\begin{array}{c}\text { Lifiespan } \\
\text { (Years) }\end{array}\right|$} & \multicolumn{14}{|c|}{ Embodied Energy (EE) } & \multirow{2}{*}{\multicolumn{2}{|c|}{$\begin{array}{c}\text { Difference in } \\
\text { Operating Energy } \\
\text { from Baseline after } \\
\text { Lifespan }\end{array}$}} \\
\hline & \multicolumn{3}{|c|}{ Manutacturing } & \multicolumn{3}{|c|}{ Construction } & \multicolumn{3}{|c|}{ Maintenance } & \multicolumn{3}{|c|}{ End of Lifie } & \multirow{2}{*}{$\begin{array}{c}{ }^{3} \text { Total } \\
\text { EE }\end{array}$} & \multirow{2}{*}{\begin{tabular}{|l}
${ }^{4} \mathrm{Total}$ \\
$\mathrm{EE}$ \\
per m${ }^{2}$
\end{tabular}} & & \\
\hline & \begin{tabular}{|l|l|} 
Material \\
\end{tabular} & ${ }^{2}$ Trans. & \begin{tabular}{|l|l|} 
Total \\
\end{tabular} & Material & 2 Trans. & Total & \begin{tabular}{|l|l|} 
Material \\
\end{tabular} & ${ }^{2}$ Trans. & Total & Material & \begin{tabular}{|l|}
${ }^{2}$ Trans. \\
\end{tabular} & Total & & & ${ }^{5}$ Total & ${ }^{6}$ per $m^{2}$ \\
\hline 1 Initial & 85,663 & 827 & 86,490 & 461 & 2,347 & 2,807 & 0 & 0 & 0 & 0 & 0 & 0 & 89,297 & \begin{tabular}{|l|l|}
1,291 \\
\end{tabular} & & \\
\hline 50 & \begin{tabular}{|l|l|}
85,663 \\
\end{tabular} & 827 & 86,490 & 461 & 2,347 & $\begin{array}{l}2,807 \\
\end{array}$ & 88,088 & 870 & 88,958 & 2 & 453 & $\begin{array}{l}454 \\
\end{array}$ & 178,710 & \begin{tabular}{|l|l|} 
\\
\end{tabular} & ,200,0000 & $-2,02$ \\
\hline
\end{tabular}

Global Warming Potential (kg of $\mathrm{CO}_{2}$ eq.)

\begin{tabular}{|c|c|c|c|c|c|c|c|c|c|c|c|c|c|c|c|c|}
\hline \multirow{4}{*}{$\begin{array}{l}\text { Lifespan } \\
\text { (Years) }\end{array}$} & \multicolumn{16}{|c|}{ rming Pote } \\
\hline & \multicolumn{14}{|c|}{ Embodied Global Warming Potential (GWP) } & \multirow{2}{*}{\multicolumn{2}{|c|}{\begin{tabular}{|c|} 
Difference in \\
Operating GWPP from \\
Baseline after \\
Lifespan
\end{tabular}}} \\
\hline & \multicolumn{3}{|c|}{ Manufacturing } & \multicolumn{3}{|c|}{ Construction } & \multicolumn{3}{|c|}{ Maintenance } & \multicolumn{3}{|c|}{ End of } & \multirow{2}{*}{$\begin{array}{l}{ }^{3} \text { Total } \\
\text { GWP }\end{array}$} & \multirow{2}{*}{$\begin{array}{l}{ }^{4} \text { Total } \\
\text { GWP } \\
\text { per m }\end{array}$} & & \\
\hline & \begin{tabular}{|l|} 
Material \\
\end{tabular} & ${ }^{2}$ Trans & \begin{tabular}{|l|} 
Total \\
\end{tabular} & Material & ${ }^{2}$ Trans & \begin{tabular}{|l|l|} 
Total \\
\end{tabular} & \begin{tabular}{|l|} 
Material \\
\end{tabular} & 1/ ${ }^{2}$ Trans. & Total & \begin{tabular}{|l|} 
Material \\
\end{tabular} & 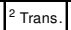 & Total & & & ${ }^{5}$ Total & ${ }^{6}$ per $\mathrm{m}^{2}$ \\
\hline |nitial & 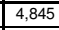 & 2 & 4,847 & 30 & 3 & 34 & 0 & 0 & 8 & 7 & - & 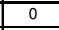 & & 77 & & \\
\hline 50 & 845 & 2 & 847 & 30 & 3 & 34 & 3,097 & 2 & 3,099 & & $\overline{1}$ & & 7,980 & 15 & 60,000 & 102 \\
\hline
\end{tabular}

Embodied energy (and GWP) numbers are based on an area of roof $=69.2 \mathrm{~m}^{2} \quad$ (Span $\times$ Width $=9.1 \mathrm{~m} \times 7.6 \mathrm{~m}=69.2 \mathrm{~m}^{2}$ )

\section{ATHENA ${ }^{\circledR}$ EIE Material List:}

(Includes all materials after 50 years)

\begin{tabular}{|l|c|c|}
\hline \multicolumn{1}{|c|}{ Material List } & Quantities & Unit \\
\hline \begin{tabular}{l|c|}
\hline $13 \mathrm{~mm}$ Moisture Resistant Gypsum \\
Board
\end{tabular} & 76.1 & $\mathrm{~m} 2$ \\
\hline $16 \mathrm{~mm}$ Gypsum Fibre Gypsum & 76.1 & $\mathrm{~m} 2$ \\
\hline Board & 70.5 & $\mathrm{~m} 2$ \\
\hline 6 mil Polyethylene & $15,823.1$ & $\mathrm{~kg}$ \\
\hline Ballast (aggregate stone) & 183.5 & $\mathrm{~kg}$ \\
\hline Galvanized Sheet & 1.5 & $\mathrm{~m} 3$ \\
\hline GluLam Sections & 429.2 & $\mathrm{~m} 2(25 \mathrm{~mm})$ \\
\hline Isocyanurate & 151.9 & $\mathrm{~kg}$ \\
\hline Joint Compound & 21.4 & $\mathrm{~kg}$ \\
\hline Nails & 1.7 & $\mathrm{~kg}$ \\
\hline Paper Tape & $1,512.6$ & $\mathrm{~kg}$ \\
\hline PVC membrane & 3.7 & $\mathrm{~m} 3$ \\
\hline $\begin{array}{l}\text { Small Dimrension Softwood Lumber, } \\
\text { kiln-dried }\end{array}$ & 1.4 & $\mathrm{~m} 2(9 \mathrm{~mm})$ \\
\hline Softwood Plywood & &
\end{tabular}

$$
\begin{aligned}
& \text { Notes: } \\
& \text { 'Initial }=\text { Time 'o' (i.e. at the completion of init }
\end{aligned}
$$

${ }^{2}$ Trans = = Transportation

Total EE (or Total GWP) $=$ Total embodied energy (or total embodied GWP)
of building component after lifespan (i.e. total manufacturing + tota of building component after lifespan (i.e. total mant
construction + total maintenance + total end-of-life effects)

${ }^{4}$ Total EE (or Total GWP) per $\mathrm{m}^{2}=$ Total EE (or Total GWP) of building
component/ area of building component that was modelled in ATHENAQ EIE Total Difference in Operating Energy (or GWP) from Baseline aff

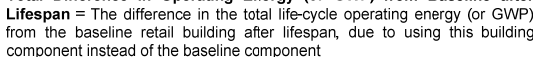

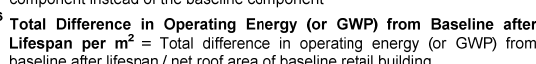
baseline after lifespan / net roof area of baseline retail building

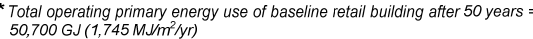
${ }^{*}$ Total operatiting GWP of baseline retail building after 50 years $=2,310$ tonnes
of $\mathrm{CO}_{2}$ eq. $\left(80 \mathrm{~kg}\right.$ of $\left.\mathrm{CO}_{2} 2 \mathrm{eq} / \mathrm{m}^{2} \mathrm{yrr}\right)$ 


\section{Glulam Roof \#9 (GLU-R9)}

Building Component Description:

\begin{tabular}{|c|c|c|c|}
\hline Category: & Roots & Assembly Layers & \\
\hline \multirow{3}{*}{ Brief Description: } & \multirow{2}{*}{ 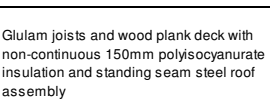 } & $\begin{array}{l}\text { Outside } \\
\text { Akyd based paint }\end{array}$ & \\
\hline & & $\begin{array}{l}\text { Commercial 0.46mm galvanized standing seam } \\
\text { steel roof (WB) (includes tasteners) }\end{array}$ & \\
\hline \multirow{2}{*}{\multicolumn{2}{|c|}{ Quick Numbers: }} & 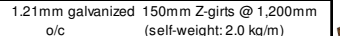 & \\
\hline & & Non-continuous $150 \mathrm{~mm}$ polyis occlanurate & \\
\hline ASHRAE Standard 90.1: & \begin{tabular}{|l|l|} 
R-Value: 29.4 \\
\end{tabular} & insulation & \\
\hline THERM 5.2: & $\begin{array}{|ll|}\text { R-Value: } 31.0 & \text { RSI-Value: } 5.5 \\
\end{array}$ & Modified bitumen membrane ( $A B, V B)$ & \\
\hline Root Thickness: & $\begin{array}{rl}720 & \mathrm{~mm} \text { (excluding drop ceiling) }\end{array}$ & 38mm SPF tongue \& groveve solid wood plar & \\
\hline Span: & Range: $2 \mathrm{~m}$ to $12 \mathrm{~m}$ Design: $9.1 \mathrm{~m}$ & 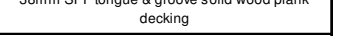 & \\
\hline Specifited Design Loads: & SL $\quad 1.1 \mathrm{kPa}$ & 80mm x 494mm 24-E glulam joists @ 1800m o/c & \\
\hline Total Embodied Energy: & $1,396 \quad \mathrm{M} / \mathrm{m}^{2}$ & $16 \mathrm{~mm}$ suspended acoustical ceiling & \\
\hline Total Embodied GWP: & $71 \quad \mathrm{~kg} \mathrm{of} \mathrm{CO}_{2} \mathrm{eq} / \mathrm{m}^{2}$ & $\begin{array}{l}\text { Misc. fasteners, nails, and galvanized sheet } \\
\text { Inside }\end{array}$ & \\
\hline
\end{tabular}

\section{Life-Cycle Assessment Results:}

\begin{tabular}{|c|c|c|c|c|c|c|c|c|c|c|c|c|c|c|c|c|}
\hline \multirow{4}{*}{$\begin{array}{l}\text { Lifespan } \\
\text { (Years) }\end{array}$} & \multicolumn{16}{|c|}{ inn } \\
\hline & \multicolumn{14}{|c|}{ Embodied Energy (EE) } & \multirow{2}{*}{\multicolumn{2}{|c|}{$\begin{array}{c}\text { Difference in } \\
\text { Operating Energy } \\
\text { from Baseline after } \\
\text { Lifespan }\end{array}$}} \\
\hline & \multicolumn{3}{|c|}{ Manufacturing } & \multicolumn{3}{|c|}{ Construction } & \multicolumn{3}{|c|}{ Maintenance } & \multicolumn{3}{|c|}{ End of Lifie } & \multirow{2}{*}{$\begin{array}{c}{ }^{3} \text { Total } \\
\mathrm{EE}\end{array}$} & \multirow{2}{*}{$\begin{array}{c}{ }^{4} \text { Total } \\
\mathrm{EE} \\
\text { per }{ }^{2}\end{array}$} & & \\
\hline & \begin{tabular}{|l|l|l} 
Material \\
\end{tabular} & ${ }^{2}$ Trans & Total & Material & ${ }^{2}$ Trans. & \begin{tabular}{|l|} 
Total \\
\end{tabular} & Material & ${ }^{2}$ Trans. & Total & Material & ${ }^{2}$ Trans. & Total & & & ${ }^{5}$ Total & per $\mathrm{m}^{2}$ \\
\hline Initial & 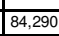 & 683 & 7 & \begin{tabular}{|l|}
461 \\
\end{tabular} & 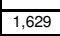 & & 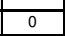 & 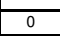 & 0 & 0 & 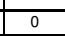 & 0 & 87,063 & 1,259 & & \\
\hline & 84 & 683 & 84,974 & 461 & 1,629 & 2,089 & 9,263 & 29 & 9,293 & 2 & 223 & 225 &, 581 & 1,396 & 700,0 & $-1,195$ \\
\hline
\end{tabular}

\begin{tabular}{|c|c|c|c|c|c|c|c|c|c|c|c|c|c|c|c|c|}
\hline \multirow{4}{*}{$\begin{array}{l}\text { Lifespan } \\
\text { (Years) }\end{array}$} & \multicolumn{16}{|c|}{ Glo } \\
\hline & \multicolumn{14}{|c|}{ Embodied Global Warming Potential (GWP) } & \multirow{2}{*}{\multicolumn{2}{|c|}{$\begin{array}{c}\text { Difference in } \\
\text { Operating GWP fror } \\
\text { Baseline after } \\
\text { Lifespan }\end{array}$}} \\
\hline & \multicolumn{3}{|c|}{ Manutacturing } & \multicolumn{3}{|c|}{ Construction } & \multicolumn{3}{|c|}{ Maintenance } & \multicolumn{3}{|c|}{ End of Lite } & \multirow{2}{*}{\begin{tabular}{|}
${ }^{3}$ Total \\
GWWP
\end{tabular}} & \multirow{2}{*}{$\begin{array}{l}{ }^{4} \text { Total } \\
\text { GWP } \\
\text { per m }\end{array}$} & & \\
\hline & Material & ${ }^{2}$ Trans. & Total & Material & ${ }^{2}$ Trans. & Total & Material & ${ }^{2}$ Trans. & Total & Material & | ${ }^{2}$ Trans. & Total & & & ${ }^{5}$ Total & ${ }_{6}^{6}$ perm ${ }^{2}$ \\
\hline${ }^{1}$ Initial & 4,766 & 1 & \begin{tabular}{|l|l|l}
4,767 \\
\end{tabular} & 30 & 2 & 32 & 0 & 0 & 0 & 0 & 0 & 0 & 4,799 & 69 & & \\
\hline 50 & \begin{tabular}{|l|l} 
\\
\end{tabular} & 1 & \begin{tabular}{|l|l|} 
\\
\end{tabular} & 30 & 2 & 32 & 129 & 0 & 129 & 0 & 0 & 1 & 4,929 & 71 & - 30,000 & -51 \\
\hline
\end{tabular}

$\begin{gathered}\text { ATHENA @ EIE Material List: } \\
\text { (Includes all materials after 50 years) }\end{gathered}$
\begin{tabular}{|l|c|c|}
\multicolumn{1}{|c|}{ Material List } & Quantities & Unit \\
\hline $\begin{array}{l}\text { 16mm Gypsum Fibre Gypsum } \\
\text { Board }\end{array}$ & 76.1 & $\mathrm{~m} 2$ \\
\hline Foam Polyisocyanurate & 426.2 & $\mathrm{~m} 2(25 \mathrm{~mm})$ \\
\hline Galvanized Sheet & 565.7 & $\mathrm{~kg}$ \\
\hline Galvanized Studs & 121.2 & $\mathrm{~kg}$ \\
\hline GluLam Sections & 1.5 & $\mathrm{~m} 3$ \\
\hline Joint Compound & 75.9 & $\mathrm{~kg}$ \\
\hline Modified Bitumen membrane & 214.7 & $\mathrm{~kg}$ \\
\hline Nails & 17.8 & $\mathrm{~kg}$ \\
\hline Paper Tape & 0.9 & $\mathrm{~kg}$ \\
\hline Screws Nuts \& Bolts & 0.7 & $\mathrm{~kg}$ \\
\hline $\begin{array}{l}\text { Small Dimension Sottwood Lumber, } \\
\text { kiln-dried }\end{array}$ & 3.5 & $\mathrm{~m} 3$ \\
\hline Solvent Based Alkyd Paint & 49.7 & $\mathrm{~L}$ \\
\hline
\end{tabular}

Notes:
${ }_{1}$ Initial $=$ Time '
0

${ }^{2}$ Trans. $=$ Transportation

Total EE (or Total GWP) = Total embodied energy (or total embodied GWP) of building component after lifespan (i.e. total manufacturing + to

Total EE (or Total GWP) per $\mathrm{m}^{2}=$ Total EE (or Total GWP) of builing

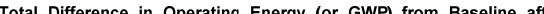
Lifespan $=$ The difference in the total life-cycle operating energy (or GWP)
trom the baseline retail building after lifespan, due to using this building from the baseline retail building after lifespan
component instead of the baseline component ${ }^{6}$ Total Difference in Operating Energy (or GWP) from Baseline after
Lifespan per $\mathrm{m}^{2}=$ Total difference in operating energy (or GWP) from Lifespan per $\mathrm{m}^{2}=$ Total difterencec in operating enerey
baseline after lifespan / net roof area of baseline retail building Total operating primary energy use of baseline retail building after 50 years =

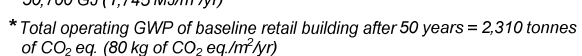

\section{Glulam Roof \#10 (GLU-R10)}

Building Component Description:

\begin{tabular}{|c|c|c|c|}
\hline \multirow{3}{*}{ Brief Description: } & \multirow{3}{*}{ 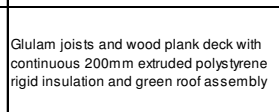 } & $\begin{array}{l}\text { Assembly Layers } \\
\text { outside }\end{array}$ & \\
\hline & & $\begin{array}{l}\text { Outside } \\
\text { Green roof assembly } \\
\text { (150mm of soiland vegatition }\end{array}$ & \\
\hline & & $\begin{array}{l}\text { Drainage board (WB) } \\
\text { (modeled as PVC membrane) }\end{array}$ & \\
\hline \multicolumn{2}{|l|}{ Quick Numbers: } & $\begin{array}{l}\text { Continuous } 200 \mathrm{~mm} \text { extruded polystyrene rigid } \\
\text { insulation }\end{array}$ & \\
\hline ASHRAE Standard 90.1: & $\begin{array}{ll}\text { R-Value: } 39.1 \\
\end{array}$ & Protection barrier (modeled as 6mil poly) & \\
\hline THERM 5.2: & $\begin{array}{|ll|}\text { R-Value: } 42.4 & \text { RSI-Value: } 7.5 \\
\end{array}$ & Moditied bitumen membrane (AB, VB) & \\
\hline Roof Thickness: & $983 \mathrm{~mm}$ (excluding drop ceiling) & 38mm SPF tongue \& groove solid wood pla & \\
\hline Span: & Range: $2 \mathrm{~m}$ to $12 \mathrm{~m}$ Design: $9.1 \mathrm{~m}$ & decking & \\
\hline Spectified Design Loads: & $\begin{array}{ll}\mathrm{DL} & 2.9 \mathrm{kPa} \\
\end{array}$ & $80 \mathrm{~mm} \times 570 \mathrm{~mm}$ 24F-E glulam joists @ $1800 \mathrm{~m}$ olc & \\
\hline Total Embodied Energy: & $1,570 \quad \mathrm{MN} / \mathrm{m}^{2}$ & $16 \mathrm{~mm}$ suspended acoustical ceiling & \\
\hline Total Embodied GWP: & $\begin{array}{ll}61 & \mathrm{~kg} \text { of } \mathrm{CO}_{2} \mathrm{eq} / \mathrm{m}^{2} \\
\end{array}$ & $\begin{array}{l}\text { Misc. fasteners, nails, and galvanized sheet } \\
\text { Inside }\end{array}$ & \\
\hline
\end{tabular}

\section{Life-Cycle Assessment Results:}

Primary Energy Consumption (MJ)

\begin{tabular}{|c|c|c|c|c|c|c|c|c|c|c|c|c|c|c|c|c|}
\hline \multirow{3}{*}{$\begin{array}{l}\text { Lifespan } \\
\text { (Years) }\end{array}$} & \multicolumn{14}{|c|}{ Embodied Energy (EE) } & \multirow{2}{*}{\multicolumn{2}{|c|}{$\begin{array}{c}\text { Difference in } \\
\text { Operating Energy } \\
\text { from Baseline after } \\
\text { Lifespan }\end{array}$}} \\
\hline & \multicolumn{3}{|c|}{ Manutacturing } & \multicolumn{3}{|c|}{ Construction } & \multicolumn{3}{|c|}{ Maintenance } & \multicolumn{3}{|c|}{ End of Life } & \multirow{2}{*}{$\begin{array}{c}{ }^{3} \text { Total } \\
\text { EE }\end{array}$} & \multirow{2}{*}{$\begin{array}{l}{ }^{4} \text { Total } \\
\mathrm{EE} \\
\text { per } \mathrm{m}^{2}\end{array}$} & & \\
\hline & \begin{tabular}{|l|l|} 
Material \\
\end{tabular} & ${ }^{2}$ Trans. & Total & Material & 2 Trans. & Total & Material & ${ }^{2}$ Trans. & Total & \begin{tabular}{|l|} 
Material \\
\end{tabular} & 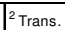 & Total & & & ${ }^{5}$ Total & ${ }^{6}{ }_{\text {per }}{ }^{2}$ \\
\hline 1 Initial & \begin{tabular}{|l|l|}
78,897 \\
\end{tabular} & 660 & 79,557 & 461 & 1,666 & 2,126 & 0 & 0 & 0 & 0 & 0 & 0 & \begin{tabular}{|l|l|}
81,683 \\
\end{tabular} & 1,181 & & \\
\hline 50 & $\begin{array}{ll}78,897 \\
\end{array}$ & 660 & 79,557 & 461 & 1,666 & 2,126 & \begin{tabular}{|l|l|}
26,592 \\
\end{tabular} & 73 & 26,665 & 2 & 239 & 240 & 108,589 & 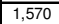 & $, 200,000$ & $-2,0$ \\
\hline
\end{tabular}

Global Warming Potential (kg of $\mathrm{CO}_{2}$ eq.)

\begin{tabular}{|c|c|c|c|c|c|c|c|c|c|c|c|c|c|c|c|c|}
\hline \multirow{4}{*}{$\begin{array}{l}\text { Lifiespan } \\
\text { (Years) }\end{array}$} & \multirow{2}{*}{\multicolumn{14}{|c|}{ Embadiod flabalWorming Potontial (CIMP) }} & \multirow{3}{*}{\multicolumn{2}{|c|}{\begin{tabular}{|c} 
Difference in \\
Operating GWPP from \\
Baseline after \\
Lifespan
\end{tabular}}} \\
\hline & & & & & & & & & & & & & & & & \\
\hline & \multicolumn{3}{|c|}{ Manufacturing } & \multicolumn{3}{|c|}{ Construction } & \multicolumn{3}{|c|}{ Maintenar } & \multicolumn{3}{|c|}{ End of Life } & \multirow{2}{*}{$\begin{array}{l}{ }^{3} \text { Total } \\
\text { GWP }\end{array}$} & \multirow{2}{*}{\begin{tabular}{|l}
${ }^{4}$ Total \\
GWP \\
Tor
\end{tabular}} & & \\
\hline & Material & ${ }^{2}$ Trans. & Total & Material & ${ }^{2}$ Trans. & Total & Material & ${ }^{2}$ Trans. & Total & \begin{tabular}{|l|} 
Material \\
\end{tabular} & 1. ${ }^{2}$ Trans. & Total & & & \begin{tabular}{|l|}
${ }^{5}$ Total \\
\end{tabular} & 6 per m \\
\hline Initial & 135 & 1 & 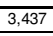 & $\bar{T}$ & 2 & 32 & 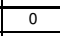 & 0 & 0 & $\pi$ & 0 & 0 & & 50 & & \\
\hline 50 & 3,435 & 1 & , 437 & 30 & 2 & 32 & 740 & 0 & 740 & 0 & 0 & 1 & 209 & 61 & $-60,000$ & -102 \\
\hline
\end{tabular}
Embodied energy (and GWP) numbers are based on an area of roof $=69.2 \mathrm{~m}^{2} \quad\left(\right.$ Span $\times$ Width $\left.=9.1 \mathrm{~m} \times 7.6 \mathrm{~m}=69.2 \mathrm{~m}^{2}\right)$ Net roof area of baseline retail building (gross roof area - openings) $=586.0 \mathrm{~m}^{2}$

\section{ATHENA $®$ EIE Material List:}

\begin{tabular}{|c|c|c|}
\hline Material List & Quantities & Unit \\
\hline $\begin{array}{l}16 \mathrm{~mm} \text { Gypsum Fibre Gypsum } \\
\text { Board }\end{array}$ & 76.1 & $\mathrm{~m} 2$ \\
\hline 6 mil Polyethylene & 73.4 & $\mathrm{~m} 2$ \\
\hline Ballast (aggregate stone) & 263.7 & $\mathrm{~kg}$ \\
\hline Extruded Polystyrene & 566.8 & $\mathrm{~m} 2(25 \mathrm{~mm})$ \\
\hline Galvanized Sheet & 160.6 & $\mathrm{~kg}$ \\
\hline GluLam Sections & 1.8 & $\mathrm{~m} 3$ \\
\hline Joint Compound & 75.9 & $\mathrm{~kg}$ \\
\hline Modified Bitumen membrane & 258.7 & $\mathrm{~kg}$ \\
\hline Nails & 17.8 & $\mathrm{~kg}$ \\
\hline Paper Tape & 0.9 & $\mathrm{~kg}$ \\
\hline PVC membrane & 308.7 & $\mathrm{~kg}$ \\
\hline Small Dimension Softwood Lun & 3.5 & $\mathrm{~m} 3$ \\
\hline
\end{tabular}

Notes:

$$
{ }^{1} \text { Initial }=\text { Time ' } O \text { ' (i.e. at the completion of initial construction }
$$

${ }^{2}$ Trans. = Transportation ${ }^{3}$ Total EE (or Total GWP) $=$ Total embodied energy (or total embodied GWP)
of building component after lifespan (i.e. total manufacturing + total
construction + total maintenance + total end-of-life effects)

${ }^{4}$ Total EE (or Total GWP) per $\mathrm{m}^{2}=$ Total EE (or Total GWP) of building
component / area of building component that was modelled in ATHENAQ EIE

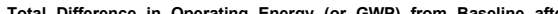
Total Difference in Operating Energy (or GWP) from Baseline after
Liffespan = The difference in the total life-cycle operating energy (or GWWP)
from the baseline retail building affer lifespan, due to using this building

0
0 Total Difference in Operating Energy (or GWP) from Baseline after
Lifespan per $\mathrm{m}^{2}=$ Total difference in operating energy (or GWP) from Lifespan per $\mathrm{m}^{2}=$ Total lifference in operating energy (or GWP) from
baseline after lifespan / net roof area of baseline retail building * Total operating primary energy use of baseline retail building after 50 years =
$50,700 \mathrm{GJ}\left(1,745 \mathrm{MJ} / \mathrm{m}^{2} / \mathrm{yr}\right)$ * Total operatiting $G W$ P o b baseline retail building affer 50 years $=2,310$ tonnes
of $\mathrm{CO}_{2}$ eq. $80 \mathrm{~kg}$ of $\mathrm{CO}_{2}$ eq. $\left./ \mathrm{m}^{2} \mathrm{yrr}\right)$ 


\section{LCA Data for Wood Structural Insulated Panel Roofs}

This section contains a detailed description of each wood structural insulated panel (WSIP) roof that was examined in this study (6 in total). The assembly layers are listed for each roof, along with a detailed description of the material quantities from the ATHENA® Environmental Impact Estimator for Buildings.

A breakdown of the total primary energy consumption and the total global warming potential (GWP) for each roof is also included. In each case, the results were calculated for an area of roof equal to $38.0 \mathrm{~m}^{2}$, which represents a typical bay size for a single-storey retail building with this type of roof system. The results are also expressed on a per $\mathrm{m}^{2}$ basis in each case. The data has been calculated for two different lifespans: at the completion of initial construction and after 50 years.

As a summary, the figure below illustrates a comparison of the total

embodied energy (and GWP) after 50 years for the various roofs in this section as well as the metal structural insulated panel (MSIP) roofs from the next section. For comparison purpose, the building component used in the baseline retail building has been shaded in grey.

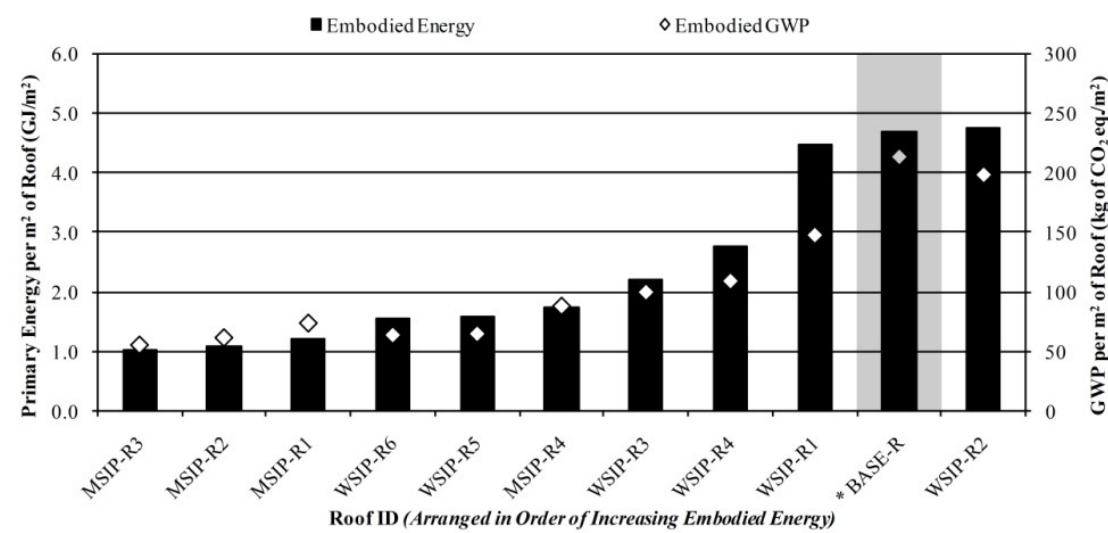

Wood Structural Insulated Panel Roof \#1 (WSIP-R1)

Building Component Description:

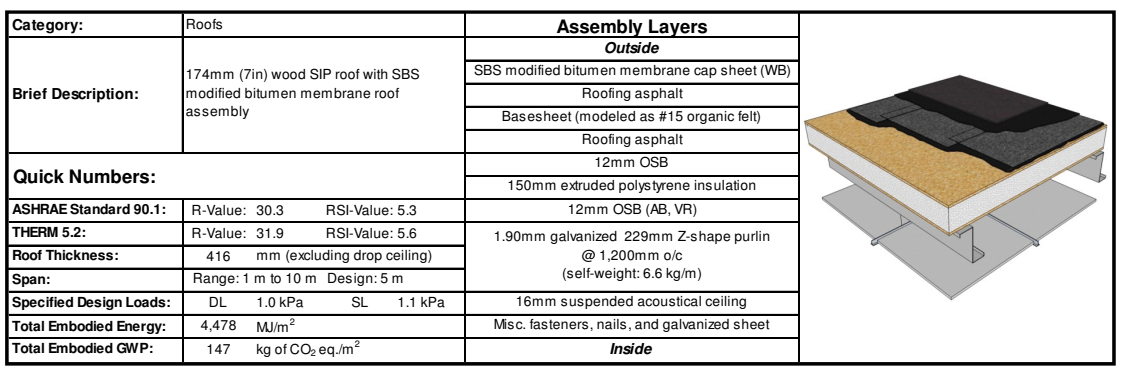

Life-Cycle Assessment Results:

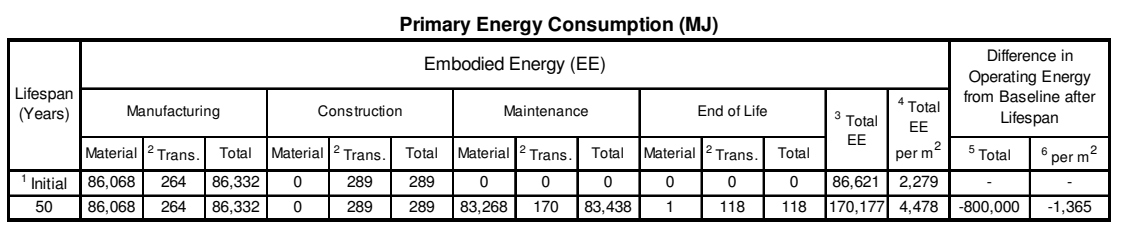

Global Warming Potential ( $k g$ of $\mathrm{CO}_{2}$ eq.)

\begin{tabular}{|c|c|c|c|c|c|c|c|c|c|c|c|c|c|c|c|c|}
\hline \multirow{3}{*}{$\begin{array}{l}\text { Lifespan } \\
\text { (Years) }\end{array}$} & \multicolumn{14}{|c|}{ Embodied Global Warming Potential (GWP) } & \multirow{2}{*}{\multicolumn{2}{|c|}{$\begin{array}{l}\text { Difference in } \\
\text { Operating GW GW from } \\
\text { Baseline atter } \\
\text { Lifespan }\end{array}$}} \\
\hline & \multicolumn{3}{|c|}{ Manutacturing } & \multicolumn{3}{|c|}{ Construction } & \multicolumn{3}{|c|}{ Maintenance } & \multicolumn{3}{|c|}{ End of Life } & \multirow{2}{*}{$\begin{array}{l}{ }^{3} \text { Total } \\
\text { GWP }\end{array}$} & \multirow{2}{*}{$\left|\begin{array}{c}4 \\
{ }^{4} \text { otal } \\
\text { GWP } \\
\text { perm }\end{array}\right|$} & & \\
\hline & Material & 2 Trans. & Total & Material & ${ }^{2}$ Trans. & Total & Material & Trans. & Total & Material & ${ }^{2}$ Trans. & Total & & & ${ }^{5}$ Total & ${ }^{6}$ per m² \\
\hline Initial & 3,386 & 1 & 3,386 & 0 & 1 & 1 & 0 & 0 & 0 & 0 & 0 & 0 & $\begin{array}{ll}3,387 \\
\end{array}$ & 89 & & \\
\hline 50 & 3,386 & 1 & 3,386 & 0 & 1 & 1 & 2,216 & 0 & 2,216 & 0 & 0 & 0 & \begin{tabular}{|l|}
5,603 \\
\end{tabular} & 147 & $-40,000$ & -68 \\
\hline \multicolumn{15}{|c|}{$\begin{array}{l}\text { Embodied energy (and GWP) numbers are based on an area of roof }=\begin{array}{rll}38.0 & \mathrm{~m}^{2}\end{array} \quad\left(\text { Span } \times \text { Width }=5.0 \mathrm{~m} \times 7.6 \mathrm{~m}=38.0 \mathrm{~m}^{2}\right) \\
\text { Net roof area of baseline retail building (gross roof area }- \text { openings) }=586.0 \mathrm{~m}^{2}\end{array}$} & & \\
\hline \multicolumn{8}{|c|}{$\begin{array}{l}\text { ATHENA @ EIE Material List: } \\
\text { (Includes all materials after 50 years) }\end{array}$} & \multirow{3}{*}{\multicolumn{9}{|c|}{$\begin{array}{l}\text { Notes: } \\
{ }^{1} \text { Initial = Time 'o' (i.e. at the completion of initial construction) } \\
{ }^{2} \text { Trans. }=\text { Transportation } \\
{ }^{3} \text { Total EE (or Total GWP) = Total embodied energy (or total embodied GWP) } \\
\text { of building component } \text { after lifespan (i.e. total manufacturing + total } \\
\text { construction + total maintenance + total end-of-life effects) }\end{array}$}} \\
\hline \multicolumn{4}{|c|}{ Material List } & \multicolumn{2}{|c|}{ Quantities } & \multicolumn{2}{|c|}{ Unit } & & & & & & & & & \\
\hline \multicolumn{4}{|c|}{$\# 15$ Organic Felt } & & 303.2 & \multicolumn{2}{|c|}{ m2 } & & & & & & & & & \\
\hline \multirow{2}{*}{\multicolumn{5}{|c|}{$\begin{array}{l}\text { 13mm Moisture Resistant Gypsum } \\
\text { Board } \\
\text { Extruded Polystyrene }\end{array}$}} & 41.8 & \multicolumn{2}{|c|}{ m2 } & \multicolumn{9}{|c|}{$\begin{array}{l}{ }^{4} \text { Total EE (or Total GWP) per } \mathrm{m}^{2}=\text { Total EE (or Total GWP) of building } \\
\text { component / area of building component that was modelled in ATHENAQ EIE }\end{array}$} \\
\hline \multirow{2}{*}{\multicolumn{4}{|c|}{ Galvanized Sheet }} & & 235.8 & \multicolumn{2}{|c|}{ m2 (25mm) } & \multirow{3}{*}{\multicolumn{9}{|c|}{$\begin{array}{l}{ }^{5} \text { Total Difference in Operating Energy (or GWP) from Baseline after } \\
\text { Lifespan }=\text { The difference in the total life-cycle operating energy (or GWP) } \\
\text { from the baseline retail building after lifescan, due to using this building } \\
\text { component instead of the baseline component }\end{array}$}} \\
\hline & & & & & 102.3 & \multirow{2}{*}{\multicolumn{2}{|c|}{$\frac{\mathrm{kg}}{\mathrm{kg}}$}} & & & & & & & & & \\
\hline \multicolumn{4}{|c|}{ Galvanized Studs } & & 262.9 & & & & & & & & & & & \\
\hline \multicolumn{4}{|c|}{ Modified Bitumen membrane } & & $1,219.6$ & \multicolumn{2}{|c|}{$\frac{\mathrm{kg}}{\mathrm{kg}}$} & \multirow{3}{*}{\multicolumn{9}{|c|}{$\begin{array}{l}{ }^{6} \text { Total Difference in Operating Energy (or GWP) from Baseline after } \\
\text { Lifespan per } \mathrm{m}^{2}=\text { Total difference in operating energy (or GWP) from } \\
\text { baseline after lifespan / net roof area of baseline retail building }\end{array}$}} \\
\hline \multicolumn{4}{|l|}{ Nails } & & 19.6 & \multicolumn{2}{|c|}{$\frac{\mathrm{kg}}{\mathrm{kg}}$} & & & & & & & & & \\
\hline \multicolumn{4}{|c|}{ Oriented Strand Board } & & 103.3 & \multicolumn{2}{|c|}{$\mathrm{m} 2(9 \mathrm{~mm})$} & & & & & & & & & \\
\hline Roofing & Asphall & & & & 632.7 & $\mathrm{~kg}$ & & & & ng primary & ry energy & use of $b$ & & & ding after 5 & 50 years $=$ \\
\hline Screws & Nuts \& & Bolts & & & 5.5 & $\mathrm{~kg}$ & & & & & & & & & & \\
\hline
\end{tabular}


Wood Structural Insulated Panel Roof \#2 (WSIP-R2)

Building Component Description:

\begin{tabular}{|c|c|c|c|}
\hline Category: & Roots & Assembly Layers & \\
\hline \multirow{3}{*}{ Brief Description: } & \multirow{3}{*}{$\begin{array}{l}174 \mathrm{~mm} \text { (7in) wood SIP roof with 4-ply built } \\
\text { up asphalt roof assembly }\end{array}$} & $\begin{array}{l}\text { Outside } \\
\text { Ballasttagareate stone) }\end{array}$ & \\
\hline & & 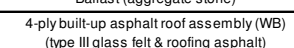 & \\
\hline & & Basesheet (modeled as \#15 organic felt) & \\
\hline \multicolumn{2}{|l|}{ Quick Numbers: } & Roofing asphalt & \\
\hline ASHRAE Standard 90.1: & R-Value: 303 & $\begin{array}{l}12 \mathrm{~mm} \text { OSB } \\
150 \mathrm{~mm} \text { extuded olysthrene insulation }\end{array}$ & \\
\hline THERM 5.2: & R-Value: 32.1 & $12 \mathrm{~mm}$ OSB (AB, VR) & \\
\hline Roof Thickness: & $441 \mathrm{~mm}$ (excluding drop ceiling) & Ivanized 229mm Z-shape pu & \\
\hline Span: & Range: $1 \mathrm{~m}$ to $10 \mathrm{~m}$ Design: $5 \mathrm{~m}$ & & \\
\hline Specififed Design Loads: & SL $\quad 1.1 \mathrm{kPa}$ & (self-weight: $6.6 \mathrm{~kg} / \mathrm{m}$ ) & \\
\hline $\begin{array}{l}\text { Total Embodied Energy: } \\
\text { Total Embodied GWP: }\end{array}$ & $4,735 \quad \mathrm{MJ} / \mathrm{m}^{2}$ & $16 \mathrm{~mm}$ suspended acoustical ceiling & \\
\hline Total Embodied GWP: & $198 \mathrm{~kg} \mathrm{of} \mathrm{CO}_{2} \mathrm{eq} / \mathrm{m}^{2}$ & $\begin{array}{l}\text { Misc. fasteners, nails, and galvanized sheet } \\
\text { Inside }\end{array}$ & \\
\hline
\end{tabular}

\section{Life-Cycle Assessment Results:}

Primary Energy Consumption (MJ)

\begin{tabular}{|c|c|c|c|c|c|c|c|c|c|c|c|c|c|c|c|c|}
\hline \multirow{4}{*}{$\begin{array}{l}\text { Lifespan } \\
\text { (Years) }\end{array}$} & \multirow{2}{*}{\multicolumn{14}{|c|}{ Embodied Energy (EE) }} & \multirow{3}{*}{\multicolumn{2}{|c|}{$\begin{array}{l}\text { Difference in } \\
\text { Operarating Energy } \\
\text { from Baseline afte } \\
\text { Lifespan }\end{array}$}} \\
\hline & \multirow{2}{*}{\multicolumn{3}{|c|}{ Manutacturing }} & & & & & & & & & & & & & \\
\hline & & & & \multicolumn{3}{|c|}{ Construction } & \multicolumn{3}{|c|}{ Mainter } & \multicolumn{3}{|c|}{ End of Life } & \multirow{2}{*}{\begin{tabular}{|c|}
${ }^{3}$ Total \\
$\mathrm{EE}$
\end{tabular}} & \multirow{2}{*}{$\begin{array}{c}{ }^{4} \text { Total } \\
\mathrm{EE} \\
\text { per m }\end{array}$} & & \\
\hline & \begin{tabular}{|l|l|} 
Material \\
\end{tabular} & ${ }^{2}$ Trans. & \begin{tabular}{|l|l} 
Total \\
\end{tabular} & Materia & 2 Trans. & Total & Material & ${ }^{2}$ Trans. & Total & Material & |' ${ }^{2}$ Trans. & Total & & & \begin{tabular}{|l|}
${ }^{5}$ Total \\
\end{tabular} & ${ }^{6}{ }_{\text {per }}^{2}{ }^{2}$ \\
\hline Initial & & 333 & & 0 & \begin{tabular}{|l|}
484 \\
\end{tabular} & 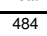 & 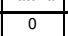 & 0 & 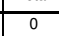 & 0 & 0 & 0 & 100,760 & 50 & & \\
\hline 50 & 99,943 & 333 & 100,276 & 0 & 484 & 484 & 78,734 & 272 & 79,006 & 1 & 161 & 162 & $\mid 179,928$ & 4,73 & $-800,000$ & $-1,36$ \\
\hline
\end{tabular}

Global Warming Potential ( $\mathrm{kg}$ of $\mathrm{CO}_{2}$ eq.)

\begin{tabular}{|c|c|c|c|c|c|c|c|c|c|c|c|c|c|c|c|c|}
\hline \multirow{4}{*}{$\begin{array}{l}\text { Lifespan } \\
\text { (Years) }\end{array}$} & \multirow{2}{*}{\multicolumn{14}{|c|}{ Embodied Global Warming Potential (GWP) }} & \multirow{3}{*}{\multicolumn{2}{|c|}{$\begin{array}{c}\text { Difference in } \\
\text { Operating GWP fron } \\
\text { Baseline after } \\
\text { Lifespan }\end{array}$}} \\
\hline & & & & & & & & & & & & & & & & \\
\hline & \multicolumn{3}{|c|}{ Manutacturing } & \multicolumn{3}{|c|}{ Construction } & \multicolumn{3}{|c|}{ Maintenance } & \multicolumn{3}{|c|}{ End of Life } & \multirow{2}{*}{$\begin{array}{l}{ }^{3} \text { Total } \\
\text { GWP }\end{array}$} & \multirow{2}{*}{$\begin{array}{l}{ }^{4} \text { Total } \\
\text { GWP } \\
\text { per m}{ }^{2}\end{array}$} & & \\
\hline & Material & ${ }^{2}$ Trans. & Total & Material & 2 Trans. & Total & Material & ${ }^{2}$ Trans. & Total & Material & 2 Trans. & Total & & & ${ }^{5}$ Total & ${ }^{6}$ pern \\
\hline Initial & 4,397 & 1 & 4,3 & 0 & 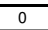 & 0 & 0 & 0 & 8 & 0 & 0 & 0 & 398 & 116 & & \\
\hline 50 & \begin{tabular}{|l|l}
4,397 \\
\end{tabular} & 1 & 4,3 & 0 & 0 & 0 & 3,131 & & 3,131 & 0 & 0 & 0 & 7,529 & & $-40,0$ & \\
\hline
\end{tabular}

Embodied energy (and GWP) numbers are based on an area of roof $=38.0 \mathrm{~m}^{2} \quad$ (Span $\times$ Width $=5.0 \mathrm{~m} \times 7.6 \mathrm{~m}=38.0 \mathrm{~m}^{2}$ )

Net roof area of baseline retail building (gross roof area - openings) $=586.0 \mathrm{~m}^{2}$

ATHENA $₫$ EIE Material List:

\begin{tabular}{|c|c|c|}
\hline Material List & Quantities & Unit \\
\hline$\# 15$ Organic Felt & 303.2 & $\mathrm{~m} 2$ \\
\hline $\begin{array}{l}16 \mathrm{~mm} \text { Gypsum Fibre Gypsum } \\
\text { Board }\end{array}$ & 41.8 & $\mathrm{~m} 2$ \\
\hline Ballast (aggregate stone) & $2,898.0$ & $\mathrm{~kg}$ \\
\hline Extruded Polystyrene & 235.8 & m2 (25mm) \\
\hline Galvanized Sheet & 133.6 & $\mathrm{~kg}$ \\
\hline Galvanized Studs & 262.9 & $\mathrm{~kg}$ \\
\hline Joint Compound & $\begin{array}{lll}41.7 \\
\end{array}$ & $\mathrm{~kg}$ \\
\hline Nails & 20.0 & $\mathrm{~kg}$ \\
\hline Oriented Strand Board & 103.3 & m2 (9mm) \\
\hline Paper Tape & 0.5 & $\mathrm{~kg}$ \\
\hline Roofing Asphalt & $1,150.4$ & $\mathrm{~kg}$ \\
\hline Screws Nuts \& Bolts & 5.5 & $\mathrm{~kg}$ \\
\hline Type III Glass Felt & 606.5 & $\frac{\mathrm{m} 2}{2} \mathrm{C}$ \\
\hline
\end{tabular}

Notes:
Initial $=$ Time 'o' (i.e. at the completion of in

${ }^{2}$ Trans. = Transportation

Total EE (or Total GWP) = Total embodied energy (or total embodied GWP) of building component after lifespan (i.e. total manufacturing + to

${ }^{4}$ Total EE (or Total GWP) per $\mathrm{m}^{2}=$ Total EE (or Total GWP) of building
component/ area of building component that was modelled in ATHENAQ EIE

Total Difference in Operating Energy (or GWP) from Baseline after Lifespan = The difference in the total life-cycle operating energy (or GWP)
from the baseline retail building after lifespan, due to using this building component instead of the baseline component

Total Difference in Operating Energy (or GWP) from Baseline after
Lifospan per $\mathrm{m}^{2}=$ Total difference in operating energy (or GWP) from Total

${ }^{*}$ Total operating primary energy use of baseline retail building after 50 years $=$
$50,700 \mathrm{G}\left(1,745 \mathrm{M} / \mathrm{m}^{2} \mathrm{Arr}\right)$

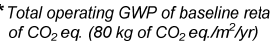

\section{Wood Structural Insulated Panel Roof \#3 (WSIP-R3)}

Building Component Description:

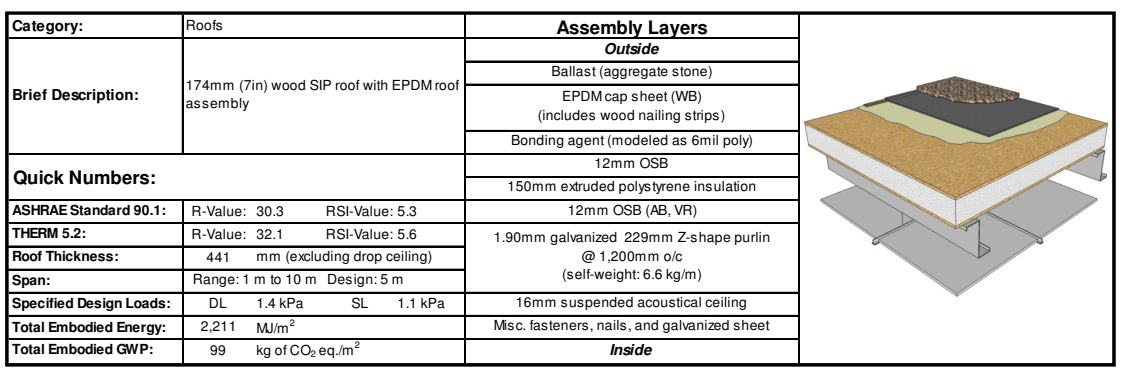

\section{Life-Cycle Assessment Results:}

Primary Energy Consumption (MJ)

\begin{tabular}{|c|c|c|c|c|c|c|c|c|c|c|c|c|c|c|c|c|}
\hline \multirow{3}{*}{\begin{tabular}{|l} 
Lifespan \\
(Years)
\end{tabular}} & \multicolumn{14}{|c|}{ Embodied Energy (EE) } & \multirow{2}{*}{\multicolumn{2}{|c|}{$\begin{array}{l}\text { Difference in } \\
\text { Operating Energy } \\
\text { from Baseline after } \\
\text { Lifespan }\end{array}$}} \\
\hline & \multicolumn{3}{|c|}{ Manufacturing } & \multicolumn{3}{|c|}{ Construction } & \multicolumn{3}{|c|}{ Maintenance } & \multicolumn{3}{|c|}{ End of Life } & \multirow{2}{*}{$\begin{array}{c}{ }^{3} \text { Total } \\
\text { EE }\end{array}$} & \multirow{2}{*}{$\begin{array}{c}{ }^{4} \text { Total } \\
\text { E } \\
\text { per m }\end{array}$} & & \\
\hline & Material & ${ }^{2}$ Trans. & Total & Material & ${ }^{2}$ Trans. & Total & Material & 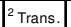 & Total & \begin{tabular}{|l|} 
Materialal \\
\end{tabular} & | ${ }^{2}$ Trans. & Total & & & ${ }^{5}$ Total & ${ }^{6} \mathrm{per} \mathrm{m}^{2}$ \\
\hline 'Initial & 50,309 & 332 & 50,641 & 0 & $\begin{array}{ll}622 \\
\end{array}$ & 622 & 0 & 0 & 0 & 0 & 0 & 0 & 51,263 & 1,349 & & \\
\hline 50 & 50,309 & 332 & 50,641 & 0 & 622 & 622 & 32,178 & 383 & 32,561 & 1 & 182 & 183 & 84,007 & \begin{tabular}{|l|l|}
2,211 \\
\end{tabular} & $-800,000$ & 1,365 \\
\hline
\end{tabular}

Global Warming Potential ( $k g$ of $\mathrm{CO}_{2}$ eq.)

\begin{tabular}{|c|c|c|c|c|c|c|c|c|c|c|c|c|c|c|c|c|}
\hline \multirow{3}{*}{$\begin{array}{l}\text { Lifespan } \\
\text { (Years) }\end{array}$} & \multicolumn{14}{|c|}{ Embodied Global Warming Potential (GWP) } & \multirow{2}{*}{\multicolumn{2}{|c|}{\begin{tabular}{|c|} 
Difference in \\
Operating GWPP from \\
Baseline after \\
Lifespan
\end{tabular}}} \\
\hline & \multicolumn{3}{|c|}{ Manutacturing } & \multicolumn{3}{|c|}{ Construction } & \multicolumn{3}{|c|}{ Maintenance } & \multicolumn{3}{|c|}{ End of Life } & \multirow{2}{*}{$\begin{array}{l}{ }^{3} \text { Total } \\
\text { GWWP }\end{array}$} & \multirow{2}{*}{\begin{tabular}{|l}
${ }^{4}$ Total \\
GWP \\
per $\mathrm{m}^{2}$
\end{tabular}} & & \\
\hline & Material & ${ }^{2}$ Trans. & Total & \begin{tabular}{|l|} 
Material \\
\end{tabular} & ${ }^{2}$ Trans. & Total & \begin{tabular}{|l|} 
Material \\
\end{tabular} & ${ }^{2}$ Trans. & Total & \begin{tabular}{|l|} 
Material \\
\end{tabular} & \begin{tabular}{l|l}
$1{ }^{2}$ Trans. \\
.
\end{tabular} & \begin{tabular}{|l|l|} 
Total \\
\end{tabular} & & & ${ }^{5}$ Total & per \\
\hline Initial & 2,356 & 1 & 2,357 & 0 & 1 & 1 & 0 & 0 & 0 & 0 & 0 & 0 & 2,357 & 62 & & \\
\hline 50 & 2,356 & 1 & \begin{tabular}{|l|l|} 
\\
\end{tabular} & 0 & 1 & 1 & \begin{tabular}{ll|}
1,422 \\
\end{tabular} & 1 & 1,423 & 0 & 0 & 0 & 3,781 & 99 & $-40,000$ & -68 \\
\hline
\end{tabular}

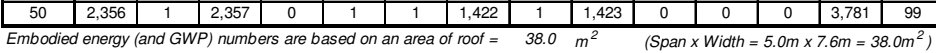

\section{ATHENA $®$ EIE Material List:}

\begin{tabular}{|c|c|c|}
\hline Material List & Quantities & Unit \\
\hline $\begin{array}{l}16 \mathrm{~mm} \text { Gypsum Fibre Gypsum } \\
\text { Board }\end{array}$ & 41.8 & $\mathrm{~m} 2$ \\
\hline 6 mil Polyethylene & 38.8 & $\mathrm{~m} 2$ \\
\hline Ballast (aggregate stone) & $8,694.0$ & $\mathrm{~kg}$ \\
\hline EPDM membrane & 312.1 & $\mathrm{~kg}$ \\
\hline Extruded Polystyrene & 235.8 & $\mathrm{~m} 2(25 \mathrm{~mm})$ \\
\hline Galvanized Sheet & 104.8 & $\mathrm{~kg}$ \\
\hline Galvanized Studs & 262.9 & $\mathrm{~kg}$ \\
\hline Joint Compound & 41.7 & $\mathrm{~kg}$ \\
\hline Nails & 3.5 & \\
\hline Oriented Strand Board & 103.3 & m2 (9mm) \\
\hline Paper Tape & 0.5 & $\mathrm{~kg}$ \\
\hline ews Nuts \& Bolts & 5.5 & $\mathrm{~kg}$ \\
\hline $\begin{array}{l}\text { Small Iimension Softwood Lumber } \\
\text { kiln-dried }\end{array}$ & 0.1 & $\mathrm{~m} 3$ \\
\hline oftwood & 0.8 & $\mathrm{~m} 2(9 \mathrm{~mm}$ \\
\hline
\end{tabular}

Notes:
${ }^{1}$ Initial $=$ Time
$O^{\prime}($ i.e. at the completion of initial construction)

${ }^{2}$ Tritial $=$ Time $\mathbf{O}^{\prime}$ (i.e. at

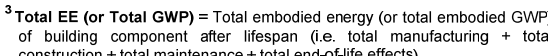
${ }^{4}$ Total EE (or Total GWP) per $\mathrm{m}^{2}=$ Total EE (or Total GWP) of building ${ }^{5}$ Total Difference in Operating Energy (or GWP) from Baseline after Lifespan = The ofiterence in the total lifiec-cycle operating energy (or GWP component instead of the baseline component Total Difference in Operating Energy (or GWP) from Baseline after
Lifispan per $\mathrm{m}^{2}=$ Total difference in operatitig energy (or GWP) from
baseline after lifespan / net roof area of baseline retail building "Total operating primary energy use of baseline retail building affer 50 years
$50,700 \mathrm{GJ}\left(1,745 \mathrm{MJ} / \mathrm{m}^{2} \mathrm{yr}\right)$ Total operating $\mathrm{GW}$ of baseline retail building after 50 years $=2,310$ tonnes
of $\mathrm{CO}_{2} \mathrm{eq}$. $\left(80 \mathrm{~kg}\right.$ of $\mathrm{CO}_{2}$ eq. $\left./ \mathrm{m}^{2} \mathrm{yr}\right)$ 
Wood Structural Insulated Panel Roof \#4 (WSIP-R4)

Building Component Description:

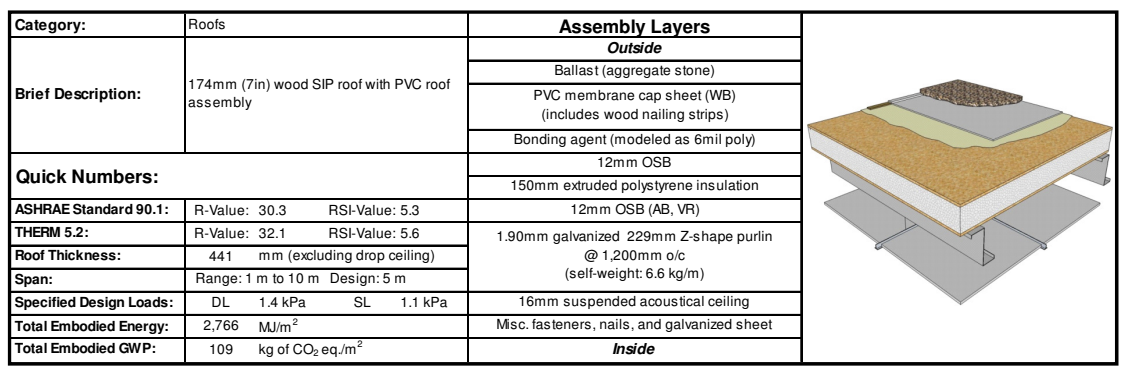

\section{Life-Cycle Assessment Results:}

Primary Energy Consumption (MJ)

\begin{tabular}{|c|c|c|c|c|c|c|c|c|c|c|c|c|c|c|c|c|}
\hline \multirow{3}{*}{ s) } & \multicolumn{14}{|c|}{ mbodi } & \multirow{2}{*}{\multicolumn{2}{|c|}{$\begin{array}{c}\text { Difference in } \\
\text { Operating Energy } \\
\text { from Baseline atte } \\
\text { Lifespan }\end{array}$}} \\
\hline & \multicolumn{3}{|c|}{ Manufacturing } & \multicolumn{3}{|c|}{ Construction } & \multicolumn{3}{|c|}{ Maintenance } & \multicolumn{3}{|c|}{ End of Life } & \multirow{2}{*}{$\begin{array}{c}{ }^{3}{ }^{3} \text { Total } \\
\mathrm{EE}\end{array}$} & \multirow{2}{*}{\begin{tabular}{|l|}
${ }^{4}$ Total \\
EE \\
perm
\end{tabular}} & & \\
\hline & \begin{tabular}{|l|l|} 
Material \\
\end{tabular} & Trans. & Total & Materia & 2 Trans. & Total & Material & ${ }^{2}$ Trans & Total & Material & $\mid{ }^{2}$ Trans. & \begin{tabular}{|l|l|} 
Total \\
\end{tabular} & & & ${ }^{5}$ Total & ${ }^{6}{ }_{\text {per }}{ }^{2}{ }^{2}$ \\
\hline & 54,9 & 35 & 55,3 & 0 & 71 & 719 & 0 & & 0 & 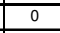 & 0 & 0 & & 1 & & \\
\hline & 54,950 & 352 & & & 719 & 719 & & 478 & 48,87 & & & 192 & & & & \\
\hline
\end{tabular}

\begin{tabular}{|c|c|c|c|c|c|}
\hline \multirow{3}{*}{$\mid \begin{array}{l}\text { Lifespan } \\
\text { (Years) }\end{array}$} & \multicolumn{5}{|c|}{ Embodied Global } \\
\hline & \multicolumn{2}{|c|}{ Manufacturing } & \multicolumn{3}{|c|}{ Construction } \\
\hline & 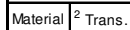 & Total & Material & ${ }^{2}$ Trans & Total \\
\hline Initial & 2,432 & 2,433 & 0 & 1 & 1 \\
\hline 50 & \begin{tabular}{|l|l}
2,432 & 1 \\
\end{tabular} & 2,433 & 0 & 1 & 1 \\
\hline \multicolumn{6}{|c|}{$\begin{array}{l}\text { Embodied energy (and GWP) numbers are based on an area of } \mathrm{r} \\
\text { Net roof area of baseline retail building (gross roof area - opening }\end{array}$} \\
\hline \multicolumn{6}{|c|}{$\begin{array}{c}\text { ATHENA @ EIE Material List: } \\
\text { (ncludes all materials atter } 50 \text { years) }\end{array}$} \\
\hline \multicolumn{3}{|c|}{ Material List } & \multicolumn{2}{|c|}{ Quantities } & Unit \\
\hline \multicolumn{3}{|c|}{$\begin{array}{l}16 \mathrm{~mm} \text { Gypsum Fibre Gypsum } \\
\text { Board }\end{array}$} & \multicolumn{2}{|c|}{41.8} & $\mathrm{~m} 2$ \\
\hline \multirow{2}{*}{\multicolumn{3}{|c|}{\begin{tabular}{l|l}
6 mil Polyethylene \\
Ballast (agaregate stone)
\end{tabular}}} & & 38.8 & m2 \\
\hline & & & & 694.0 & $\mathrm{~kg}$ \\
\hline \multicolumn{3}{|c|}{ Extruded Polystyrene } & & 35.8 & $\mid$\begin{tabular}{|l|l|}
$\mathrm{m} 2(25 \mathrm{~mm})$ \\
\end{tabular} \\
\hline \multicolumn{3}{|c|}{$\begin{array}{l}\text { Galvanized Sheet } \\
\end{array}$} & & 00.9 & $\mathrm{~kg}$ \\
\hline \multirow{2}{*}{\multicolumn{3}{|c|}{$\begin{array}{l}\text { Galvanized Studs } \\
\text { Joint Compound }\end{array}$}} & & 62.9 & $\mathrm{~kg}$ \\
\hline & & & & 41.7 & $\mathrm{~kg}$ \\
\hline \multicolumn{3}{|c|}{ Nails } & & $\begin{array}{l}4.3 \\
\end{array}$ & $\mathrm{~kg}$ \\
\hline \multicolumn{3}{|c|}{\begin{tabular}{|l|} 
Oriented Strand Board \\
\end{tabular}} & & 03.3 & m2 (9mm) \\
\hline \multicolumn{3}{|c|}{$\begin{array}{l}\text { Paper Tape } \\
\end{array}$} & & 0.5 & $\mathrm{~kg}$ \\
\hline \multicolumn{3}{|c|}{ PVC membrane } & & 331.1 & $\mathrm{~kg}$ \\
\hline \multicolumn{3}{|c|}{ Screws Nuts \& Bolts } & & 5.5 & $\mathrm{~kg}$ \\
\hline \multicolumn{3}{|c|}{$\begin{array}{l}\text { Small Dimension Sott } \\
\text { kiln-dried }\end{array}$} & & 0.1 & $\mathrm{~m} 3$ \\
\hline \multicolumn{3}{|c|}{ Softwood Plywood } & & 0.8 & $\mathrm{~m} 2(9 \mathrm{~mm})$ \\
\hline
\end{tabular}

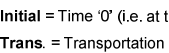

Notes:

Total EE (or Total GWP) = Total embodied energy (or total embodied GWP)

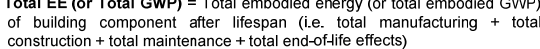

${ }^{4}$ Total EE (or Total GWP) per $\mathrm{m}^{2}=$ Total EE (or Total GWP) of building
component/ area of building component that was modelled in ATHENAQ EIE

Total Difference in Operating Energy (or GWP) from Baseline after Lifespan = The difference in the totat life-cycle operating enerery (or GWP
from the baseline retail building atter lifespan, due to using this building Nrom the baseline retail building atter lifespan,
component instead of the baseline component Total Difference in Operating Energy (or GWP) from Baseline after
Lifespan per $\mathrm{m}^{2}=$ Total difference in operatitg energy (or GWP) from
baseline after lifspan $n$ net roof area of baseline retail bwilding

${ }^{*}$ Total o perating primary energy use of baseline retail building after 50 years $=$
$50,700 \mathrm{G}\left(1,745 \mathrm{MJ} / \mathrm{m}^{2} / \mathrm{yr}\right)$

Total operating GWP of baseline retail building after 50 years $=2,310$ tonnes
Wood Structural Insulated Panel Roof \#5 (WSIP-R5)

Building Component Description:

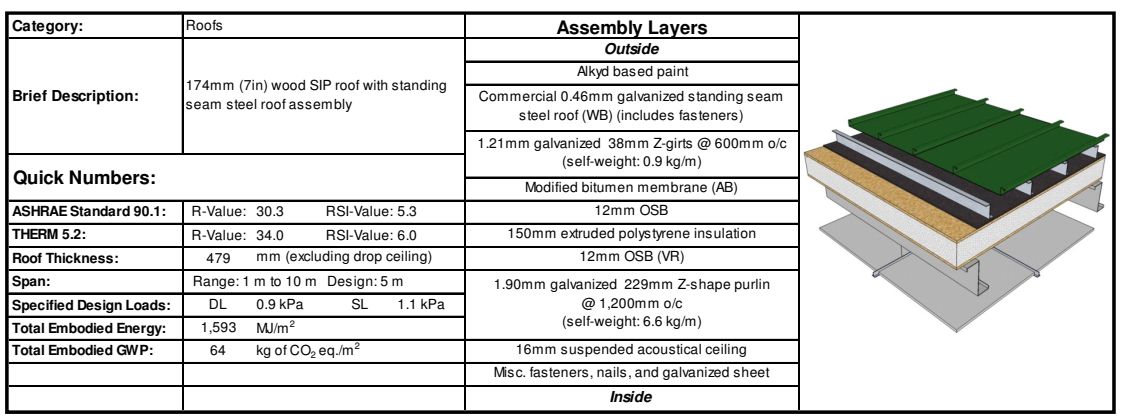

Life-Cycle Assessment Results:

\begin{tabular}{|c|c|c|c|c|c|c|c|c|c|c|c|c|c|c|c|c|}
\hline \multirow{4}{*}{\begin{tabular}{|l} 
Liespann \\
(Years)
\end{tabular}} & \multicolumn{14}{|c|}{ Primary Energy Consumption (MJ) } & \multirow{3}{*}{\multicolumn{2}{|c|}{$\begin{array}{l}\text { Difference in } \\
\text { Operating Energy } \\
\text { from Baseline after } \\
\text { Lifespan }\end{array}$}} \\
\hline & & & & & & & bodied & Energy $(\mathrm{E}$ & & & & & & & & \\
\hline & \multicolumn{3}{|c|}{ Manutacturing } & \multicolumn{3}{|c|}{ Construction } & \multicolumn{3}{|c|}{ Maintenance } & \multicolumn{3}{|c|}{ End of Life } & ${ }^{3}$ Total & \multirow{2}{*}{$\begin{array}{c}{ }^{4} \text { Total } \\
\mathrm{EE} \\
\mathrm{EE}\end{array}$} & & \\
\hline & \begin{tabular}{|l|l} 
Material \\
\end{tabular} & \begin{tabular}{|l|l|}
${ }^{2}$ Trans. \\
\end{tabular} & Total & Material & 11 ${ }^{2}$ Trans. & Total & Material & \begin{tabular}{|l|}
${ }^{2}$ Trans. \\
\end{tabular} & Total & Material & 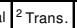 & Total & & & ${ }^{5}$ Total & ${ }^{6}$ per $\mathrm{m}^{2}$ \\
\hline itial & 54,592 & 296 & 54,888 & 0 & 431 & 431 & 0 & 0 & 0 & 0 & 0 & 0 & 55,319 & 1,456 & & \\
\hline
\end{tabular}

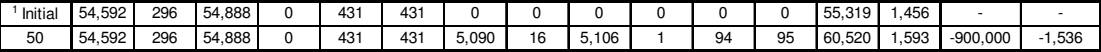

\begin{tabular}{|c|c|c|c|c|c|c|c|c|c|c|c|c|c|c|c|c|}
\hline \multicolumn{17}{|c|}{ obal Warming Potential (kg of $\mathrm{CO}_{2}$ eq.) } \\
\hline \multirow{3}{*}{\begin{tabular}{|l} 
Lifespan \\
(Years)
\end{tabular}} & \multicolumn{14}{|c|}{ Embodied Global Warming Potential (GWP) } & \multirow{2}{*}{\multicolumn{2}{|c|}{\begin{tabular}{|c} 
Difference in \\
Operatiting GWP from \\
Baseline after \\
Lifespan
\end{tabular}}} \\
\hline & \multicolumn{3}{|c|}{ Manufacturing } & \multicolumn{3}{|c|}{ Construction } & \multicolumn{3}{|c|}{ Maintenance } & \multicolumn{3}{|c|}{ End of Life } & \multirow{2}{*}{\begin{tabular}{|l}
${ }^{3}$ Total \\
GWP
\end{tabular}} & \multirow{2}{*}{$\begin{array}{l}{ }^{4} \text { Total } \\
\text { GWP } \\
\text { Ger m }\end{array}$} & & \\
\hline & Material & 2 Trans. & \begin{tabular}{|l|l|} 
Total \\
\end{tabular} & \begin{tabular}{|l|} 
Material \\
\end{tabular} & ${ }^{2}$ Trans. & Total & \begin{tabular}{|l|} 
Material \\
\end{tabular} & ${ }^{2}$ Trans. & Total & Material & \begin{tabular}{l|l}
${ }^{2}$ Trans. \\
.
\end{tabular} & Total & & & ${ }^{5}$ Total & ${ }^{6}$ perm $\mathrm{m}^{2}$ \\
\hline Initial & \begin{tabular}{|l|l|}
2,378 \\
\end{tabular} & 1 & \begin{tabular}{|l|}
2,379 \\
\end{tabular} & 0 & 0 & 0 & 0 & 0 & 0 & 0 & 0 & 0 & \begin{tabular}{|l|l|}
2,379 \\
\end{tabular} & 63 & & \\
\hline 50 & 2,378 & 1 & \begin{tabular}{|l|l|}
2,379 \\
\end{tabular} & 0 & 0 & 0 & 71 & 0 & 71 & 0 & 0 & 0 & 2,450 & 64 & $-40,000$ & -68 \\
\hline
\end{tabular}

(Span $\times$ Width $\left.=5.0 \mathrm{~m} \times 7.6 \mathrm{~m}=38.0 \mathrm{~m}^{2}\right)$

\section{ATHENA ${ }^{\circledR}$ EIE Material List: \\ Notes: \\ ${ }^{2}$ Trans. $=$ Transportation}

\begin{tabular}{|l|c|c|}
\multicolumn{1}{|c|}{ Material List } & Quantities & Unit \\
\hline \begin{tabular}{l|c|}
$16 \mathrm{~mm}$ Gypsum Fibre Gypsum \\
Board
\end{tabular} & 41.8 & $\mathrm{~m} 2$ \\
\hline Extruded Polystyrene & 233.6 & $\mathrm{~m} 2(25 \mathrm{~mm})$ \\
\hline Galvanized Sheet & 310.9 & $\mathrm{~kg}$ \\
\hline Galvanized Studs & 262.9 & $\mathrm{~kg}$ \\
\hline Joint Commound & 41.7 & $\mathrm{~kg}$ \\
\hline Modififed Bitumen membrane & 118.0 & $\mathrm{~kg}$ \\
\hline Naill & 2.7 & $\mathrm{~kg}$ \\
\hline Oriented Strand Board & 103.3 & $\mathrm{~m} 2(9 \mathrm{~mm})$ \\
\hline Paper Tape & 0.5 & $\mathrm{~kg}$ \\
\hline Screws Nuts \& Bolts & 5.9 & $\mathrm{~kg}$ \\
\hline Solvent Based Alkyd Paint & 27.3 & $\mathrm{~L}$ \\
\hline
\end{tabular}

${ }^{3}$ Total EE (or Total GWP) $=$ Total embodied energy (or total embodied GWP) of building component after lifespan (i.e. total manufacturing + tota
construction + total maintenance + total end-of-life effects) ${ }^{4}$ Total EE (or Total GWP) per $\mathrm{m}^{2}=$ Total EE (or Total GWP) of building ${ }^{5}$ Total Difference in Operating Energy (or GWP) from Baseline after Lifespan = The difference in the total life-cycle operating energy (or GWP)
from the baseline retail building after lifespan, due to using this building from the baseline retail building after lifespan,
component instead of the baseline component

${ }^{6}$ Total Difference in Operting Energy (or GWP) from Boserine ator Lifespan per $\mathrm{m}^{2}=$ Total difference in operating energy (or GWP) from baseline after lifespan / net roof area of baseline retail building * Total operating primary energy use of baseline retail building after 50 years $=$
$50,700 \mathrm{GJ}\left(1,745 \mathrm{MJm} \mathrm{T}^{2} \mathrm{yI}\right)$ * Total operating GWP of baseline 
Wood Structural Insulated Panel Roof \#6 (WSIP-R6)

Building Component Description:

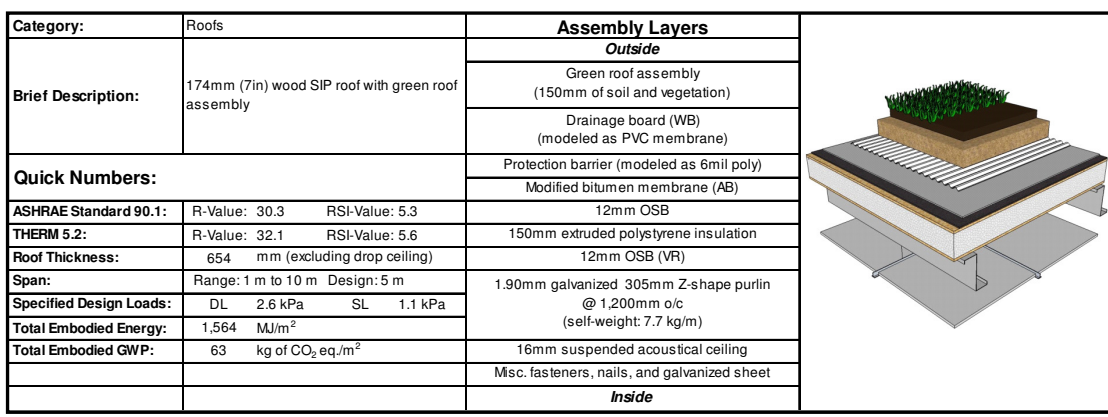

Life-Cycle Assessment Results:

Primary Energy Consumption (MJ)

\begin{tabular}{|c|c|c|c|c|c|c|c|c|c|c|c|c|c|c|c|c|}
\hline \multirow{3}{*}{\begin{tabular}{|l} 
Lifespan \\
(Years)
\end{tabular}} & \multicolumn{14}{|c|}{ Embodied Energy (EE) } & \multirow{2}{*}{\multicolumn{2}{|c|}{$\begin{array}{c}\text { Difference in } \\
\text { Operating Energy } \\
\text { from Baseline after } \\
\text { Lifespan }\end{array}$}} \\
\hline & \multicolumn{3}{|c|}{ Manutacturing } & \multicolumn{3}{|c|}{ Construction } & \multicolumn{3}{|c|}{ Maintenance } & \multicolumn{3}{|c|}{ End of Life } & \multirow{2}{*}{$\begin{array}{l}{ }^{3} \text { Total } \\
\mathrm{EE}\end{array}$} & \multirow{2}{*}{\begin{tabular}{|c|}
${ }^{4}$ Total \\
EE \\
per m
\end{tabular}} & & \\
\hline & \begin{tabular}{|l|l} 
Material \\
\end{tabular} & \begin{tabular}{l|l|}
${ }^{2}$ Trans. \\
\end{tabular} & Total & Material & ${ }^{2}$ Trans. & Total & Material & \begin{tabular}{|l|}
${ }^{2}$ Trans. \\
\end{tabular} & Total & Material & \begin{tabular}{|l|}
${ }^{2}$ Trans. \\
\end{tabular} & Total & & & \begin{tabular}{|l|l|}
${ }^{5}$ Total \\
\end{tabular} & ${ }^{6}$ per $\mathrm{m}^{2}$ \\
\hline Initial & 43,967 & 280 & 44,247 & 0 & \begin{tabular}{|c|}
44 \\
4
\end{tabular} & 446 & 0 & 0 & 0 & 0 & 0 & 0 & 44,693 & 1,176 & & \\
\hline 50 & 443,967 & 280 & 44,247 & 0 & $\begin{array}{lll}446 \\
\end{array}$ & 4466 & 14,611 & 40 & $\mid 14,651$ & 1 & 91 & 92 & 59,436 & 1 & $-800,000$ & 1,00 \\
\hline
\end{tabular}

Global Warming Potential $\left(\mathrm{kg}\right.$ of $\mathrm{CO}_{2}$ eq.)

\begin{tabular}{|c|c|c|c|c|c|c|c|c|c|c|c|c|c|c|c|c|}
\hline \multirow{3}{*}{$\begin{array}{l}\text { Lifespan } \\
\text { (Years) }\end{array}$} & \multicolumn{14}{|c|}{ Embodied Global Warming Potential (GWP) } & \multirow{2}{*}{\multicolumn{2}{|c|}{$\begin{array}{c}\text { Difference in } \\
\text { Operating GWP fron } \\
\text { Baseline after } \\
\text { Lifespan }\end{array}$}} \\
\hline & \multicolumn{3}{|c|}{ Manutacturing } & \multicolumn{3}{|c|}{ Constru } & \multicolumn{3}{|c|}{ Maintenance } & \multicolumn{3}{|c|}{ End of Life } & \multirow{2}{*}{$\begin{array}{l}{ }^{3} \text { Total } \\
\text { GWP }\end{array}$} & \multirow{2}{*}{$\begin{array}{l}{ }^{4}{ }^{\mathrm{T} o t a l} \\
\text { GWP } \\
\text { per m }\end{array}$} & & \\
\hline & \begin{tabular}{|l|} 
Material \\
\end{tabular} & ${ }^{2}$ Trans. & \begin{tabular}{|l|l|} 
Total \\
\end{tabular} & Material & ${ }^{2}$ Trans. & Total & \begin{tabular}{|l|} 
Material \\
\end{tabular} & \begin{tabular}{|l|}
${ }^{2}$ Trans. \\
(a)
\end{tabular} & Total & \begin{tabular}{|l|} 
Material \\
\end{tabular} & 2 Trans. & Total & & & ${ }^{5}$ Total & ${ }^{6}$ per m ${ }^{2}$ \\
\hline Initial & \begin{tabular}{|l|l|}
2,003 \\
\end{tabular} & 1 & 2,004 & 0 & 0 & 0 & 0 & 0 & 0 & 0 & 0 & 0 & \begin{tabular}{|l|}
2,004 \\
\end{tabular} & 53 & & \\
\hline 50 & \begin{tabular}{|l|}
2,003 \\
\end{tabular} & 1 & 2,004 & 0 & 0 & 0 & 406 & 0 & 406 & 0 & 0 & 0 & 2,411 & 63 & $-40,6$ & -68 \\
\hline
\end{tabular}

Embodied energy (and GWP) numbers are based on an area of roof $=38.0 \mathrm{~m}^{2} \quad\left(\right.$ Span $\times$ Width $\left.=5.0 \mathrm{~m} \times 7.6 \mathrm{~m}=38.0 \mathrm{~m}^{2}\right)$
ATHENA ® EIE Material List:

\begin{tabular}{|c|c|c|}
\hline Material List & Quantities & Unit \\
\hline $\begin{array}{l}16 \mathrm{~mm} \text { Gypsum Fibre Gypsum } \\
\text { Baprd }\end{array}$ & 41.8 & $\mathrm{~m} 2$ \\
\hline 6 mil Polyethylene & 40.3 & $\mathrm{~m} 2$ \\
\hline Ballast (aggregate stone) & 144.9 & $\mathrm{~kg}$ \\
\hline Extruded Polystyrene & 233.6 & $\mathrm{~m} 2(25 \mathrm{~mm})$ \\
\hline Galvanized Sheet & 88.3 & $\mathrm{~kg}$ \\
\hline Galvanized Studs & 262.9 & $\mathrm{~kg}$ \\
\hline Joint Compound & 41.7 & $\mathrm{~kg}$ \\
\hline Modified Bitumen membrane & 142.1 & $\mathrm{~kg}$ \\
\hline Nails & 2.7 & \\
\hline Oriented Strand Board & 103.3 & $\mathrm{~m} 2(9 \mathrm{~mm})$ \\
\hline Paper Tape & 0.5 & $\mathrm{~kg}$ \\
\hline PVC membrane & 169.6 & $\mathrm{~kg}$ \\
\hline Screws Nuts \& Bolts & 5.5 & $\mathrm{~kg}$ \\
\hline
\end{tabular}

\section{LCA Data for Metal Structural Insulated Panel Roofs}

This section contains a detailed description of each metal structural insulated panel (MSIP) roof that was examined in this study (4 in total). The assembly layers are listed for each roof, along with a detailed description of the material quantities from the ATHENA® Environmental Impact Estimator for Buildings.

A breakdown of the total primary energy consumption and the total global warming potential (GWP) for each roof is also included. In each case, the results were calculated for an area of roof equal to $38.0 \mathrm{~m}^{2}$, which represents a typical bay size for a single-storey retail building with this type of roof system. The results are also expressed on a per $\mathrm{m}^{2}$ basis in each case. The data has been calculated for two different lifespans: at the completion of initial construction and after 50 years.

As a summary, the figure below illustrates a comparison of the total embodied energy (and GWP) after 50 years for the various roofs in this section as well as the WSIP roofs from the previous section. For comparison purpose, the building component used in the baseline retail building has been shaded in grey.

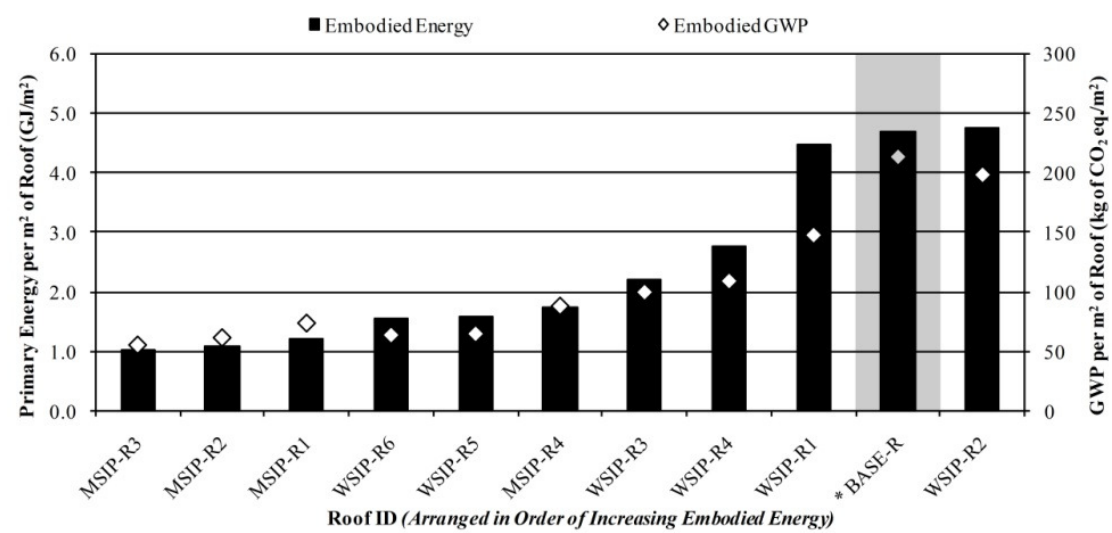

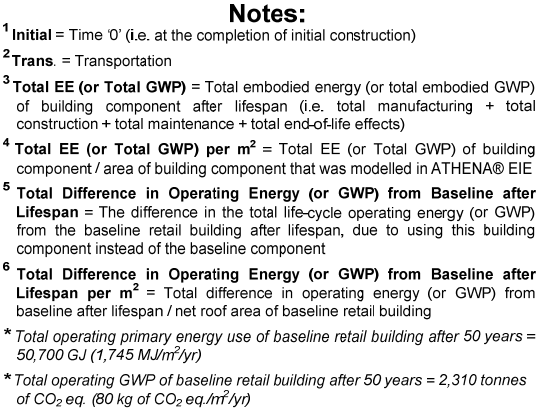

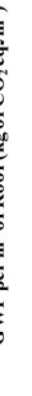




\section{Metal Structural Insulated Panel Roof \#1 (MSIP-R1)}

Building Component Description:

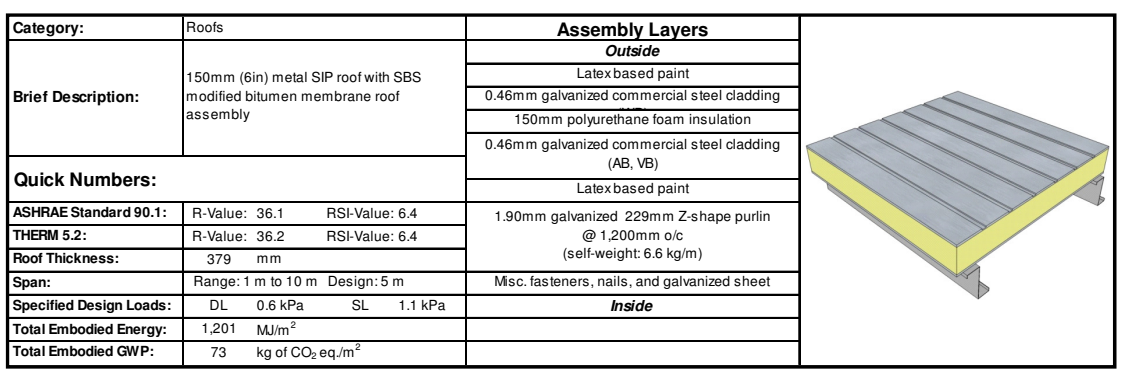

\section{Life-Cycle Assessment Results:}

Primary Energy Consumption (MJ)

\begin{tabular}{|c|c|c|c|c|c|c|c|c|c|c|c|c|c|c|c|c|}
\hline \multirow{3}{*}{$\begin{array}{l}\text { Lifespan } \\
\text { (Years) }\end{array}$} & \multicolumn{14}{|c|}{ Embodied Energy (EE) } & \multirow{2}{*}{\multicolumn{2}{|c|}{$\begin{array}{c}\text { Difference in } \\
\text { Operating Energy } \\
\text { from Baseline after } \\
\text { Lifespan }\end{array}$}} \\
\hline & \multicolumn{3}{|c|}{ Manufacturing } & \multicolumn{3}{|c|}{ Construction } & \multicolumn{3}{|c|}{ Maintenance } & \multicolumn{3}{|c|}{ End of Life } & \multirow{2}{*}{$\begin{array}{c}{ }^{3} \text { Total } \\
{ }^{E E}\end{array}$} & \multirow{2}{*}{$\begin{array}{c}{ }^{4} \text { Total } \\
\text { EE } \\
\text { per } \mathrm{m}^{2}\end{array}$} & & \\
\hline & Material & ${ }^{2}$ Trans. & Total & Materia & ${ }^{2}$ Trans. & Total & Material & ${ }^{2}$ Trans. & Total & Material & ${ }^{2}$ Trans. & Total & & & ${ }^{5}$ Total & ${ }_{6}^{6}$ per m ${ }^{2}$ \\
\hline Initial & 45,534 & 55 & 45,589 & 0 & 122 & 122 & 0 & 0 & 0 & 0 & 0 & 0 & 45,710 & 1,203 & & \\
\hline 50 & 45,534 & 55 & \begin{tabular}{|l|l|} 
\\
\end{tabular} & 0 & 122 & 122 & 241 & 2 & 243 & 0 & 42 & 42 & \begin{tabular}{|l|l}
$\mid 45,996$ \\
\end{tabular} & $\begin{array}{l}1,210 \\
\end{array}$ & $-1,000$ & $-1,70$ \\
\hline
\end{tabular}

Global Warming Potential ( $\mathrm{kg}$ of $\mathrm{CO}_{2}$ eq.)

\begin{tabular}{|c|c|c|c|c|c|c|c|}
\hline \multirow{3}{*}{$\begin{array}{l}\text { Lifespan } \\
\text { (Years) }\end{array}$} & \multicolumn{7}{|c|}{ Embodied Global Wa } \\
\hline & \multicolumn{3}{|c|}{ Manufacturing } & \multicolumn{3}{|c|}{ Construction } & \multirow[b]{2}{*}{ Mater } \\
\hline & Material & ${ }^{2}$ Trans & \begin{tabular}{|l|l|l|} 
Total \\
\end{tabular} & Materia & $\begin{array}{l}1{ }^{2} \text { Trans. } \\
\text {. }\end{array}$ & \begin{tabular}{|l|l} 
Total \\
\end{tabular} & \\
\hline 1 Initial & 2,787 & 0 & 2,787 & 0 & 0 & 0 & 0 \\
\hline 50 & $\begin{array}{ll}2,787 \\
\end{array}$ & 0 & \begin{tabular}{|l}
, 787 \\
\end{tabular} & 0 & 0 & 0 & 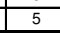 \\
\hline \multicolumn{8}{|c|}{$\begin{array}{l}\text { Embodied energy (and GWP) numbers are based on an area of roof = } \\
\text { Net roof area of baseline retail building (gross roof area - openings) = }\end{array}$} \\
\hline \multicolumn{8}{|c|}{$\begin{array}{c}\text { ATHENA ® EIE Material List: } \\
\text { (Includes all materials after 50 years) }\end{array}$} \\
\hline \multicolumn{4}{|c|}{ Material List } & \multicolumn{2}{|c|}{ Quantities } & \multicolumn{2}{|c|}{ Unit } \\
\hline \multicolumn{4}{|c|}{$\begin{array}{l}\text { Foam Polyisocyanurate } \\
\end{array}$} & \multicolumn{2}{|r|}{2342} & \multicolumn{2}{|c|}{ m2 (25mm) } \\
\hline \multirow{2}{*}{\multicolumn{4}{|c|}{$\begin{array}{l}\text { Galvanized Sheet } \\
\text { Galvanized Studs }\end{array}$}} & \multirow{2}{*}{\multicolumn{2}{|c|}{394.9}} & \multicolumn{2}{|c|}{$\mathrm{kg}$} \\
\hline & & & & & & \multirow{2}{*}{\multicolumn{2}{|c|}{$\frac{\mathrm{kg}}{\mathrm{kg}}$}} \\
\hline \multicolumn{4}{|l|}{ Nails } & & 2.3 & & \\
\hline \multicolumn{4}{|c|}{ Screws Nuts \& Bolts } & & 5.5 & \multicolumn{2}{|c|}{$\mathrm{kg}$} \\
\hline \multicolumn{4}{|c|}{ Water Based Latex Paint } & & 10.1 & & \\
\hline
\end{tabular}
from the baseline retail building after lifespan, due to using this building

\begin{tabular}{|c|c|c|c|c|c|c|c|c|}
\hline & \multirow{2}{*}{\multicolumn{2}{|c|}{$\begin{array}{l}\text { Operating GWP from } \\
\text { Baseline after } \\
\text { Lifespan }\end{array}$}} \\
\hline \multicolumn{2}{|l|}{ Mainte } & \multicolumn{3}{|c|}{ End of Lifie } & \multirow{2}{*}{$\begin{array}{l}{ }^{3} \text { Total } \\
\text { GWP }\end{array}$} & \multirow{2}{*}{$\begin{array}{l}{ }^{4}{ }^{4} \text { Total } \\
\text { GWP } \\
\text { per m }\end{array}$} & & \\
\hline Trans. & Total & \begin{tabular}{|l|} 
Material \\
\end{tabular} & | ${ }^{2}$ Trans. & Total & & & ${ }^{5}$ Total & ${ }^{6}$ per m ${ }^{2}$ \\
\hline & 0 & 0 & & 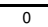 & & 73 & & \\
\hline 0 & 5 & 0 & 0 & 0 & 2,792 & 73 & $-50,000$ & -85 \\
\hline
\end{tabular}

$38.0 \mathrm{~m}^{2}$
$586.0 \mathrm{~m}^{2}$

(Span $\times$ Width $=5.0 \mathrm{~m} \times 7.6 \mathrm{~m}=38.0 \mathrm{~m}^{2}$ )

$$
\begin{aligned}
& \text { Notes: } \\
& { }^{1} \text { Initial }=\text { Time ' } O \text { ' (i.e. at the completion of init }
\end{aligned}
$$

'Initial = Time ' 0 ' (i.e. at to

Total EE (or Total GWP) = Total embodied energy (or total embodied GWP) of building component after lifespan (i.e. total manufacturing + total
of total construction + total maintenance + total end-oflifie effects)

Total EE (or Total GWP) per $\mathrm{m}^{2}=$ Total EE (or Total GWP) of building
component/ area of building component that was modelled in ATHENAQ EIE ${ }^{5}$ Total Difference in Operating Energy (or GWP) from Baseline after component instead of the baseline component

Total Difference in Operating Energy (or GWP) from Baseline after Lifespan per $\mathrm{m}^{2}=$ Total difference in operating energy
baseline after lifespan $/$ net roof area of baseline retail building

* Total operating primary energy use of baseline retail building after 50 years

$50,700 \mathrm{GJ}\left(1,745 \mathrm{MJ} / \mathrm{m}^{2} \mathrm{y}\right)$

"Total operating GWP of baseline retail building after 50 years $=2,310$ tonnes

\section{Metal Structural Insulated Panel Roof \#2 (MSIP-R2)}

Building Component Description:

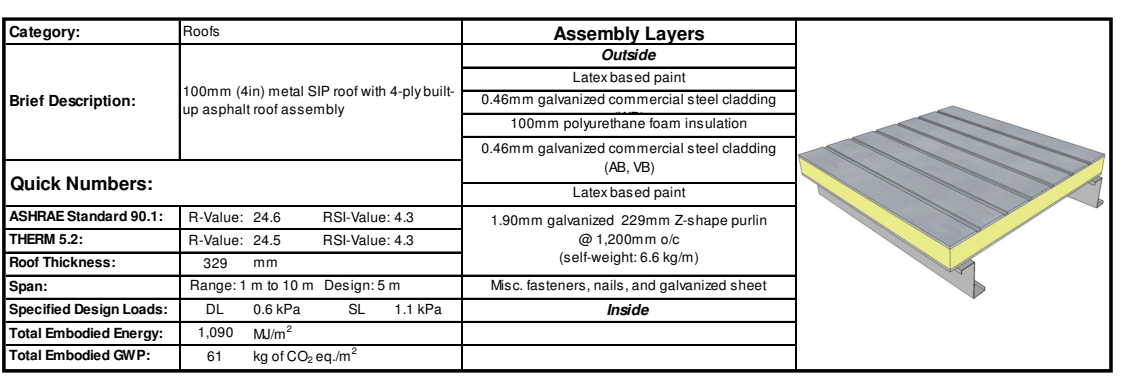

\section{Life-Cycle Assessment Results:}

Primary Energy Consumption (MJ)

\begin{tabular}{|c|c|c|c|c|c|c|c|c|c|c|c|c|c|c|c|c|}
\hline \multirow{3}{*}{$\mid \begin{array}{l}\text { Lifiespan } \\
\text { (Years) }\end{array}$} & \multicolumn{14}{|c|}{ Embodied Energy (EE) } & \multirow{2}{*}{\multicolumn{2}{|c|}{$\begin{array}{c}\text { Difference in } \\
\text { Operating Energy } \\
\text { from Baseline after } \\
\text { Lifespan }\end{array}$}} \\
\hline & \multicolumn{3}{|c|}{ Manufacturing } & \multicolumn{3}{|c|}{ Construction } & \multicolumn{3}{|c|}{ Maintenance } & \multicolumn{3}{|c|}{ End of Life } & \multirow{2}{*}{$\begin{array}{c}{ }^{3} \text { Total } \\
\text { EE }\end{array}$} & \multirow{2}{*}{\begin{tabular}{|c|}
${ }^{4}$ Total \\
EE \\
per m
\end{tabular}} & & \\
\hline & \begin{tabular}{|l|} 
Material \\
\end{tabular} & \begin{tabular}{|l|}
${ }^{2}$ Trans \\
\end{tabular} & Total & \begin{tabular}{|l|} 
Material \\
\end{tabular} & ${ }^{2}$ Trans. & Total & Material & ${ }^{2}$ Trans. & Total & \begin{tabular}{|l|} 
Material \\
\end{tabular} & \begin{tabular}{|l|l}
${ }^{2}$ Trans. \\
\end{tabular} & Total & & & ${ }^{5}$ Total & ${ }^{6}{ }^{6}$ per $m^{2}$ \\
\hline Initial & 40,965 & 52 & 41,017 & 0 & 106 & 106 & 0 & 0 & 0 & 0 & 0 & 0 & 41,123 & \begin{tabular}{|l|}
1,082 \\
\end{tabular} & & \\
\hline 50 & \begin{tabular}{|l}
40,965 \\
\end{tabular} & \begin{tabular}{|l|l|} 
& 52 \\
\end{tabular} & 41,017 & 0 & 106 & 106 & 241 & 2 & 243 & 0 & 36 & 36 & 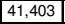 & \begin{tabular}{|l|l|}
1,090 \\
\end{tabular} & $-300,000$ & -512 \\
\hline
\end{tabular}

Global Warming Potential ( $k g$ of $\mathrm{CO}_{2}$ eq.)

\begin{tabular}{|c|c|c|c|c|c|c|c|c|c|c|c|c|c|c|c|c|}
\hline \multirow{3}{*}{$\begin{array}{l}\text { Lifespan } \\
\text { (Years) }\end{array}$} & \multicolumn{14}{|c|}{ Embodied Global Warming Potential (GWP) } & \multirow{2}{*}{\multicolumn{2}{|c|}{$\begin{array}{c}\text { Difference in } \\
\text { Operating GWP from } \\
\text { Baseline after } \\
\text { Lifespan }\end{array}$}} \\
\hline & \multicolumn{3}{|c|}{ Manufacturing } & \multicolumn{3}{|c|}{ Construction } & \multicolumn{3}{|c|}{ Maintenance } & \multicolumn{3}{|c|}{ End of Life } & \multirow{2}{*}{$\begin{array}{l}{ }^{3} \text { Total } \\
\text { GWP }\end{array}$} & \multirow{2}{*}{$\begin{array}{c}{ }^{4} \text { Total } \\
\text { GWP } \\
\text { per m }\end{array}$} & & \\
\hline & Material & 2 Trans. & Total & Material & ${ }^{2}$ Trans. & Total & Material & ${ }^{2}$ Trans. & Total & Material & $\left.\right|^{2}$ Trans. & Total & & & ${ }^{5}$ Total & ${ }^{6} \mathrm{perm}^{2}$ \\
\hline Initial & 2,321 & 0 & 2,321 & 0 & 0 & 0 & 0 & 0 & 0 & 0 & 0 & 0 & 2,321 & 61 & & \\
\hline 50 & 2,321 & 0 & 2,321 & 0 & 0 & 0 & 5 & 0 & 5 & 0 & 0 & 0 & 2,326 & 61 & $-10,000$ & -17 \\
\hline
\end{tabular}
Embodied energy (and GWP) numbers are based on an area of roof $=38.0 \mathrm{~m}^{2} \quad$ (Span $\times$ Width $=5.0 \mathrm{~m} \times 7.6 \mathrm{~m}=38 . \mathrm{om}^{2}$ )

ATHENA ® EIE Material List:

(Includes all materials after 50 years)

${ }^{1}$ Initial $=$ Time 0 ' (i.e at the comples:

\begin{tabular}{|l|c|c|}
\hline \multicolumn{1}{|c|}{ Material List } & Quantities & Unit \\
\hline Foam Polyisocyanurate & 156.1 & $\mathrm{~m} 2(25 \mathrm{~mm})$ \\
\hline Galvanized Sheet & 394.9 & $\mathrm{~kg}$ \\
\hline Galvanized Studs & 26.9 & $\mathrm{~kg}$ \\
\hline Nails & 2.3 & $\mathrm{~kg}$ \\
\hline Screws Nuts \& Bolts & 5.5 & $\mathrm{~kg}$ \\
\hline Water Based Latex Paint & 10.1 & $\mathrm{~L}$ \\
\hline
\end{tabular}

${ }^{2}$ Trans. $=$ Transportation

${ }^{3}$ Total EE (or Total GWP) $=$ Total embodied energy (or total embodied GWP) of bulding component after lifespan (i.e. total manufacturing + tota
construction + total maintenance + total end-of-life effects) ${ }^{4}$ Total EE (or Total GWP) per $\mathrm{m}^{2}=$ Total EE (or Total GWP) of building
component/area of building component that was modelled in ATHENAQ EIE ${ }^{5}$ Toponent/area o buiding component that was modelled in ATHENA@ EIE ${ }^{5}$ Total Difference in Operating Energy (Or GWP) from Baseline after
Lifiespan $=$ The difference in the total life-cycle operating energy (or GWPP)
from the baseline retal building ater lifespan, due to using this building from the baseline retail building after lifespan, due to using this building component instead of the baseline component
${ }^{6}$ Total Difference in Operating Energy (or GWP) from Baseline after ${ }^{6}$ Total Difference in Operating Energy (or GWP) from Baseline after
Lifespan per $\mathrm{m}^{2} \stackrel{\text { Total difference in operating energy (or GWP) from }}{\text { baseline after lifespan } / \text { net roof area of baseline retail building }}$

* Total operating primary energy use of baseline retail building after 50 years =
$50,700 \mathrm{GJ}\left(1,745 \mathrm{M} / \mathrm{m}^{2} \mathrm{H}(\mathrm{r})\right.$ * Total operating $\mathrm{GWP}$ of baseline retail building after 50 years $=2,310$ tonnes
of $\mathrm{CO}_{2}$ eq. (80 kg of $\mathrm{CO}_{2}$ eq. $\left./ \mathrm{m}^{2} / \mathrm{yr}\right)$ 


\section{Metal Structural Insulated Panel Roof \#3 (MSIP-R3)}

Building Component Description:

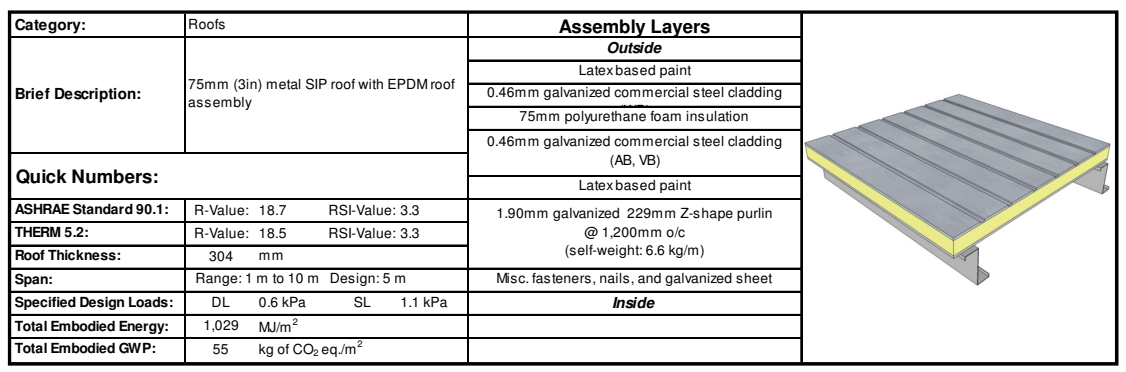

\section{Life-Cycle Assessment Results:}

Primary Energy Consumption (MJ)

\begin{tabular}{|c|c|c|c|c|c|c|c|c|c|c|c|c|c|c|c|c|}
\hline \multirow{3}{*}{$\begin{array}{l}\text { Lifespan } \\
\text { (Years) }\end{array}$} & \multicolumn{14}{|c|}{ Embodied Energy (EE) } & \multirow{2}{*}{\multicolumn{2}{|c|}{$\begin{array}{c}\text { Difference in } \\
\text { Operating Energy } \\
\text { from Baseline after } \\
\text { Lifespan }\end{array}$}} \\
\hline & \multicolumn{3}{|c|}{ Manutacturing } & \multicolumn{3}{|c|}{ Construction } & \multicolumn{3}{|c|}{ Maintenance } & \multicolumn{3}{|c|}{ End of Life } & \multirow{2}{*}{$\begin{array}{l}{ }^{3} \text { Total } \\
\text { EE }\end{array}$} & \multirow{2}{*}{\begin{tabular}{|c|c}
${ }^{4}$ Total \\
$\mathrm{EE}$ \\
$\mathrm{perm}^{2}$
\end{tabular}} & & \\
\hline & Material & ${ }^{2}$ Trans & Total & Materia & 2 Trans. & Total & Materia & ${ }^{2}$ Trans. & Total & Materia & ${ }^{2}$ Trans. & Total & & & ${ }^{5}$ Total & ${ }^{6}$ per m ${ }^{2}$ \\
\hline${ }^{1}$ Initial & 38,681 & 51 & 38,732 & 0 & 98 & 98 & 0 & 0 & 0 & 0 & 0 & 0 & 38,830 & 1,022 & & \\
\hline 50 & 388,681 & 51 & 38,732 & 0 & 98 & 98 & 241 & 2 & 243 & 0 & 34 & 34 & \begin{tabular}{|l|l|}
39,107 \\
\end{tabular} & 1,029 & 2000,000 & 341 \\
\hline
\end{tabular}

Global Warming Potential ( $\mathrm{kg}$ of $\mathrm{CO}_{2}$ eq.)

\begin{tabular}{|c|c|c|c|c|c|c|c|}
\hline \multirow{3}{*}{$\begin{array}{l}\text { Lifespan } \\
\text { (Years) }\end{array}$} & \multicolumn{7}{|c|}{ Embodied Global Wa } \\
\hline & \multicolumn{3}{|c|}{ Manufacturing } & \multicolumn{3}{|c|}{ Construction } & \multirow[b]{2}{*}{ Mater } \\
\hline & Material & ${ }^{2}$ Trans & \begin{tabular}{|l|l|l|} 
Total \\
\end{tabular} & \begin{tabular}{|l|} 
Materia \\
\end{tabular} & \begin{tabular}{l|l|}
2 & 2 \\
2
\end{tabular} & \begin{tabular}{|l|l} 
Total \\
\end{tabular} & \\
\hline 1 Initial & 2,088 & 0 & 2,088 & 0 & 0 & 0 & 0 \\
\hline 50 & 2,088 & 0 & \begin{tabular}{|l}
2,088 \\
\end{tabular} & 0 & 0 & 0 & 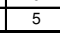 \\
\hline \multicolumn{8}{|c|}{$\begin{array}{l}\text { Embodied energy (and GWP) numbers are based on an area of roof = } \\
\text { Net roof area of baseline retail building (gross roof area - openings) = }\end{array}$} \\
\hline \multicolumn{8}{|c|}{$\begin{array}{c}\text { ATHENA ® EIE Material List: } \\
\text { (Includes all materials after 50 years) }\end{array}$} \\
\hline \multicolumn{4}{|c|}{ Material List } & \multicolumn{2}{|c|}{ Quantities } & \multicolumn{2}{|c|}{ Unit } \\
\hline \multicolumn{4}{|c|}{ Foam Polyisocyanurate } & \multicolumn{2}{|r|}{$\overline{117.1}$} & \multicolumn{2}{|c|}{ m2 (25mm) } \\
\hline \multirow{2}{*}{\multicolumn{4}{|c|}{$\begin{array}{l}\text { Galvanized Sheet } \\
\text { Galvanized Studs }\end{array}$}} & \multirow{2}{*}{\multicolumn{2}{|c|}{394.9}} & \multicolumn{2}{|c|}{$\mathrm{kg}$} \\
\hline & & \multicolumn{2}{|c|}{ Galvanized Studs } & \multirow{2}{*}{\multicolumn{2}{|c|}{$\frac{262.9}{2.3}$}} & \multirow{2}{*}{\multicolumn{2}{|c|}{$\mathrm{kg}$}} \\
\hline \multicolumn{4}{|l|}{ Nails } & & & & \\
\hline \multirow{2}{*}{\multicolumn{4}{|c|}{ Screws Nuts \& Bolts }} & & 5.5 & \multicolumn{2}{|c|}{$\mathrm{kg}$} \\
\hline & & & & & 10.1 & \multicolumn{2}{|c|}{$\frac{\mathrm{L}}{\mathrm{L}}$} \\
\hline
\end{tabular}

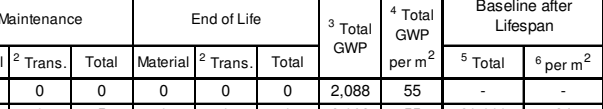
$\begin{array}{cccccc}38.0 \mathrm{~m}^{2} & 0 & 0 & 0 & 2,093 & 55 \\ (\text { Span } \times \text { With } & \left.=5.0 \mathrm{~m} \times 7.6 \mathrm{~m}=38.0 \mathrm{~m}^{2}\right)\end{array}$

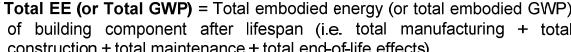

Warming Potential (GWP) 1 Notes: 'Initial = Time ' 0 ' (i.e. a

${ }^{3}$ Total EE (or Total GWP) = Total embodied energy (or total embodied GWP) construction + total maintenance + total end-oflifie effects)

Total EE (or Total GWP) per $\mathrm{m}^{2}=$ Total EE (or Total GWP) of building
component/ area of building component that was modelled in ATHENAQ EIE 5 Total Difference in Operating Energy (or GWP) from Baseline after
Lifespan = The difference in the total life cycle operating energy (or GWP) from the baseline retail building after lifespan, due to using this building component instead of the baseline component
${ }^{6}$ Total Difference in Operating Energy (or GWP) from Baseline after Lifespan per $\mathrm{m}^{2}=$ Total difference in operating energy (or
baseline after lifespan / net roof area of baseline retail building

"Total operating primary energy use of baseline retail building after 50 years $=$ $50,700 \mathrm{GJ}\left(1,745 \mathrm{MJ} / \mathrm{m}^{2} \mathrm{yr}\right)$

* Total operating GWP of baseline retail building after 50 years $=2,310$ tonnes

\section{Metal Structural Insulated Panel Roof \#4 (MSIP-R4)}

Building Component Description:

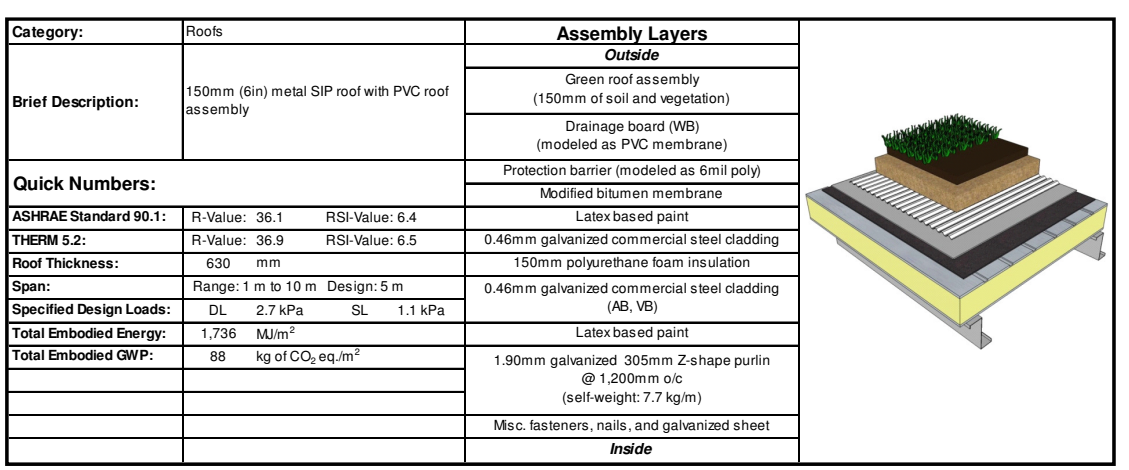

Life-Cycle Assessment Results:

Primary Energy Consumption (MJ)

\begin{tabular}{|c|c|c|c|c|c|c|c|c|c|c|c|c|c|c|c|c|}
\hline \multirow{3}{*}{$\begin{array}{l}\text { Lifiesparn } \\
\text { (Years) }\end{array}$} & \multicolumn{14}{|c|}{ Embodied Energy (EE) } & \multirow{2}{*}{\multicolumn{2}{|c|}{$\begin{array}{c}\text { Difference in } \\
\text { Operating Energy } \\
\text { from Baseline after } \\
\text { Lifespan }\end{array}$}} \\
\hline & \multicolumn{3}{|c|}{ Manufacturing } & \multicolumn{3}{|c|}{ Construction } & \multicolumn{3}{|c|}{ Maintena } & \multicolumn{3}{|c|}{ Life } & \multirow{2}{*}{${ }^{3}$ Total } & \multirow{2}{*}{\begin{tabular}{|c|}
${ }^{4}$ Total \\
EE \\
per m
\end{tabular}} & & \\
\hline & al & ${ }^{2}$ Trans. & Total & Material & \begin{tabular}{|l|}
${ }^{2}$ Trans. \\
. Thans.
\end{tabular} & Total & \begin{tabular}{|l|l|} 
Material \\
\end{tabular} & \begin{tabular}{|l|}
${ }^{2}$ Trans \\
\end{tabular} & $\begin{array}{l}\text { Total } \\
\end{array}$ & \begin{tabular}{|l|} 
Material \\
\end{tabular} & 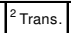 & Total & & & ${ }^{5}$ Total & ${ }^{6}$ per m ${ }^{2}$ \\
\hline & 50,797 & 61 & 50,858 & 0 & 174 & 174 & 0 & 0 & 0 & 0 & 0 & 0 & 51,032 & 1,343 & & \\
\hline
\end{tabular}
\begin{tabular}{|c|c|c|c|c|c|c|c|c|c|c|c|c|c|c|c|c|}
\hline 'Initial & 50,797 & 61 & 50,858 & 0 & 174 & 174 & 0 & 0 & 0 & 0 & 0 & 0 & 51,032 & 1,343 & - & - \\
\hline 50 & 50,797 & 61 & 50,858 & 0 & 174 & 174 & 14.852 & 42 & 14,894 & 0 & 50 & 50 & 65.976 & 1,736 & $-1,000,000$ & $-1,706$ \\
\hline
\end{tabular}

Global Warming Potential (kg of $\mathrm{CO}_{2}$ eq.)

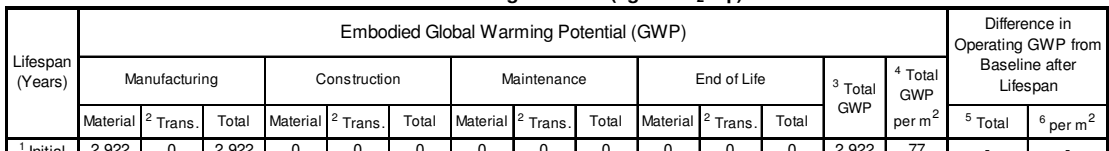
\begin{tabular}{|c|c|c|c|c|c|c|c|c|c|c|c|c|c|c|c|c|}
\hline${ }^{1}$ Initial & 2,922 & 0 & 2,922 & 0 & 0 & 0 & 0 & 0 & 0 & 0 & 0 & 0 & 2,922 & 77 & - & - \\
\hline 50 & 2,922 & 0 & 2,922 & 0 & 0 & 0 & 411 & 0 & 411 & 0 & 0 & 0 & 3,333 & 88 & $-50,000$ & -85 \\
\hline
\end{tabular} Embodied energy (and GWP) numbers are based on an area of roof $=38.0 \quad \mathrm{~m}^{2} \quad\left(\right.$ Span $\times$ Width $\left.=5.0 \mathrm{~m} \times 7.6 \mathrm{~m}=38.0 \mathrm{~m}^{2}\right)$ (1)
ATHENA $\circledast$ EIE Material List:
(Includes all materials after 50 years)

$$
\begin{aligned}
& \text { Notes: } \\
& { }^{1} \text { Initial }=\text { Time } O^{\prime}(\text { i.e. at the completion of initial construction) } \\
& { }^{2} \text { Trans. }=\text { Transportation }
\end{aligned}
$$

\begin{tabular}{|l|c|c|}
\hline \multicolumn{1}{|c|}{ Material List } & Quantities & Unit \\
\hline 6 mil Polyythylene & 40.3 & $\mathrm{~m} 2$ \\
\hline Ballast (agriegate stone) & 144.9 & $\mathrm{~kg}$ \\
\hline Foam PPlyisocyanurate & 234.2 & $\mathrm{~m} 2(25 \mathrm{~mm})$ \\
\hline Galvanized Sheet & 394.9 & $\mathrm{~kg}$ \\
\hline Galvanized Studs & 262.9 & $\mathrm{~kg}$ \\
\hline Modified Bitumen membrane & 142.1 & $\mathrm{~kg}$ \\
\hline Nails & 2.3 & $\mathrm{~kg}$ \\
\hline PVC membrane & 169.6 & $\mathrm{~kg}$ \\
\hline Screws Nuts \& Bolts & 5.5 & $\mathrm{~kg}$ \\
\hline Water Based Latex Paint & 10.1 & $\mathrm{~L}$ \\
\hline
\end{tabular}

Thans. $=$ Transportation
${ }^{3}$ Total EE (or Total GWP) $=$ Total embodied energy (or total of building component ater lifespan (i.e. total manufacturing + tota ${ }^{4}$ Total EE (or Total GWP per $\mathrm{m}^{2}=$ T Total EE (or Total GWP) of building
component/ area of building component that was modelled in ATHENAQ EIE ${ }^{5}$ Total Difference in Operating Energy (or GWP) from Baseline after

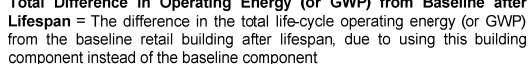
6 Total Difference in Operating Energy (or GWP) from Baseline after
Lifespan per $\mathrm{m}^{2}=$ Total difference in (operating energy (or GWP) from
baseline after lifespan $/$ net roof area of baseline retail building

* Total operating primary energy use of baseline retail building after 50 years =
$50,700 \mathrm{GJ}\left(1,745 \mathrm{MJ} / \mathrm{m}^{2} / \mathrm{yr}\right)$ * Total operating $\mathrm{GWP}$ of baseline retail building after 50 years $=2,310$ tonnes
of $\mathrm{CO}_{2}$ eq. (80 kg of $\left.\mathrm{CO}_{2} \mathrm{eqq} / \mathrm{m}^{2} \mathrm{yr}\right)$ 


\section{LCA Data for Pre-Engineered Steel Building Roofs}

This section contains a detailed description of each pre-engineered steel building (PENG) roof that was examined in this study (4 in total). The assembly layers are listed for each roof, along with a detailed description of the material quantities from the ATHENA® Environmental Impact Estimator for Buildings.

A breakdown of the total primary energy consumption and the total global warming potential (GWP) for each roof is also included. In each case, the results were calculated for an area of roof equal to $57.8 \mathrm{~m}^{2}$, which represents a typical bay size for a single-storey retail building with this type of roof system. The results are also expressed on a per $\mathrm{m}^{2}$ basis in each case. The data has been calculated for two different lifespans: at the completion of initial construction and after 50 years.

As a summary, the figure below illustrates a comparison of the total embodied energy (and GWP) after 50 years for the various roofs in this section. For comparison purpose, the building component used in the baseline retail building has been shaded in grey.

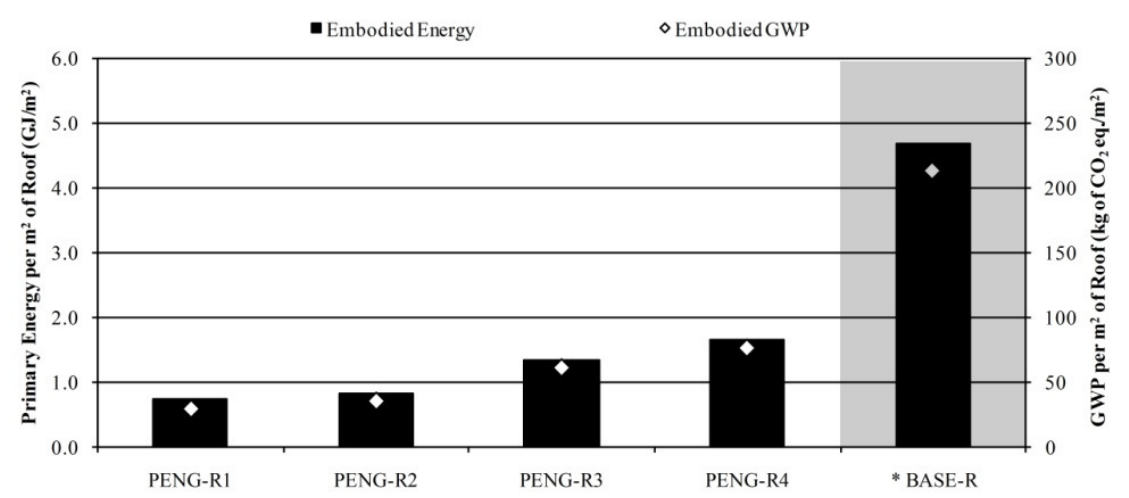

Pre-Engineered Steel Building Roof \#1 (PENG-R1)

Building Component Description:

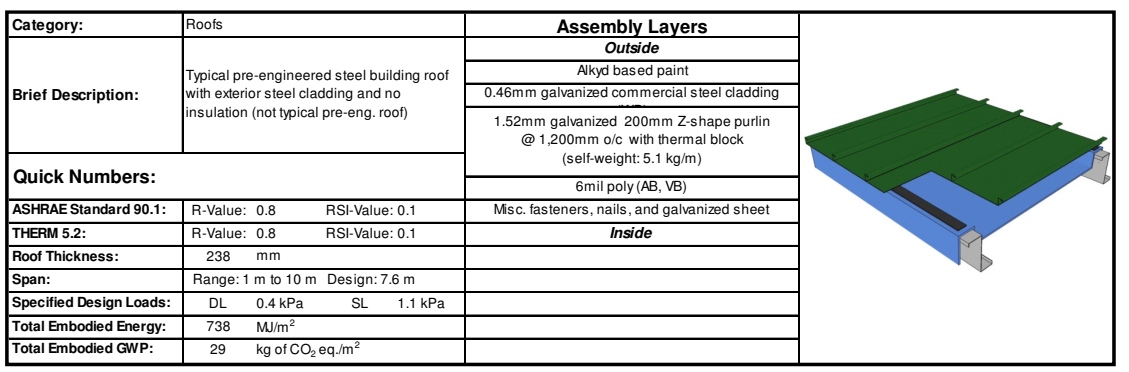

Life-Cycle Assessment Results:

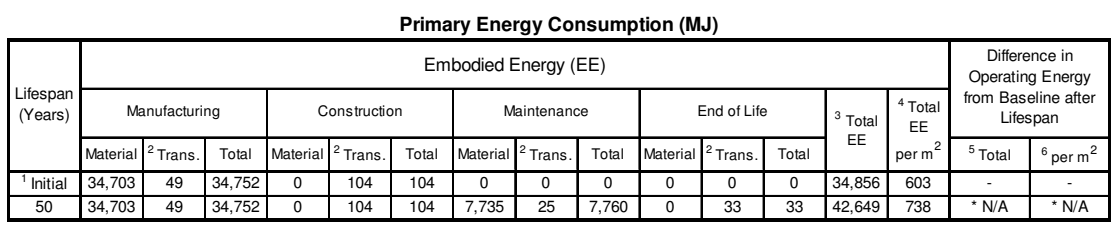

Global Warming Potential (kg of $\mathrm{CO}_{2}$ eq.)

\begin{tabular}{|c|c|c|c|c|c|c|c|c|c|c|c|c|c|c|c|c|}
\hline \multirow{3}{*}{$\begin{array}{l}\text { Litespan } \\
\text { (Years) }\end{array}$} & \multicolumn{14}{|c|}{ Embodied Global Warming Potential (GWP) } & \multirow{2}{*}{\multicolumn{2}{|c|}{$\begin{array}{l}\text { Difference in } \\
\text { Operating GWP from } \\
\text { Baseline after } \\
\text { Lifespan }\end{array}$}} \\
\hline & \multicolumn{3}{|c|}{ Manufacturing } & \multicolumn{3}{|c|}{ Construction } & \multicolumn{3}{|c|}{ Maintenance } & \multicolumn{3}{|c|}{ End of Life } & \multirow{2}{*}{$\begin{array}{l}{ }^{3} \text { Total } \\
\text { GWPP }\end{array}$} & \multirow{2}{*}{$\begin{array}{c}{ }^{4} \text { Total } \\
\text { GWP } \\
\text { per m }\end{array}$} & & \\
\hline & \begin{tabular}{|l|} 
Material \\
\end{tabular} & ${ }^{2}$ Trans. & \begin{tabular}{|l|} 
Total \\
\end{tabular} & \begin{tabular}{|l|} 
Material \\
\end{tabular} & \begin{tabular}{|l|}
${ }^{2}$ Trans. \\
\end{tabular} & Total & \begin{tabular}{|l|} 
Material \\
\end{tabular} & Trans. & Total & \begin{tabular}{|l|}
$\mid$ Material \\
\end{tabular} & \begin{tabular}{|l|}
${ }^{2}$ Trans. \\
\end{tabular} & Total & & & ${ }^{5}$ Total & ${ }^{6} \mathrm{per} \mathrm{m}^{2}$ \\
\hline Initial & 1,577 & 0 & \begin{tabular}{|l|}
1,577 \\
\end{tabular} & 0 & 0 & 0 & 0 & 0 & 0 & 0 & 0 & 0 & 1,577 & 27 & & \\
\hline 50 & \begin{tabular}{|l|l|}
1,577 \\
\end{tabular} & 0 & \begin{tabular}{|l|l|}
1,577 \\
\end{tabular} & 0 & 0 & 0 & 108 & 0 & 108 & 0 & 0 & 0 & \begin{tabular}{|l|l|}
1,685 \\
\end{tabular} & 29 & ${ }^{*} \mathrm{~N} / \mathrm{A}$ & ${ }^{*} \mathrm{~N} / \mathrm{A}$ \\
\hline
\end{tabular}

ion of operating energy from computer simulations

$\begin{gathered}\text { ATHENA } \\
\text { (Includes all materials after 50 50ears) }\end{gathered}$
\begin{tabular}{|l|c|c|}
\multicolumn{1}{|c|}{ Material List } & Quantities & Unit \\
\hline 6 mil Polyethylene & 61.3 & $\mathrm{~m} 2$ \\
\hline Galvanized SSheet & 338.3 & $\mathrm{~kg}$ \\
\hline Galvanized Studs & 399.5 & $\mathrm{~kg}$ \\
\hline Modified Bitumen membrane & 179.3 & $\mathrm{~kg}$ \\
\hline Screws Nuts \& Bolts & 8.9 & $\mathrm{~kg}$ \\
\hline Solvent Based Alkyd Paint & 41.5 & $\mathrm{~L}$ \\
\hline
\end{tabular}

Notes:
${ }^{1}$ Initial $=$ Time ' 0 ' (i.e. at the completion of inition

1 'Initial $=$ Time 'O' (i.e. at
${ }^{2}$ Trans. $=$ Transportation

etion of initial construction)

otal EE (or Total GWP) = Total embodied energy (or total embodied GWP) of building component after lifespan (i.e. total man
construction + total maintenance + total end-of-life effects)

${ }^{4}$ Total EE (or Total GWP) per $\mathrm{m}^{2}=$ Total EE (or Total GWP) of building
component /area of building component that was modelled in ATHENAB EIE 5 Total Difference in Operating Energy (or GWP) from Baseline after
Lifispan = The difference in the total lifeccycle operating energy (or GWP)
from the baseline retail building after lifespan, due to using this building component instead of the baseline componen

${ }^{6}$ Total Difference in Operating Energy (or GWP) from Baseline after
Lifespan per $\mathrm{m}^{2}=$ Total difference in operating energy (or GWP) from Lifespan per $\mathrm{m}^{2}=$ Total difference in operating energy (or GWP) from
baseline after lifespan $/$ net roof area of baseline retail building * Total operating primary energy use of baseline retail building after 50 years =
$50,700 \mathrm{GJ}\left(1,745 \mathrm{MJ} \mathrm{m}^{2} \mathrm{y} r\right)$ * Total operating $G W P$ of baseline retail building after 50 years $=2,310$ tonnes 


\section{Pre-Engineered Steel Building Roof \#2 (PENG-R2)}

Building Component Description:

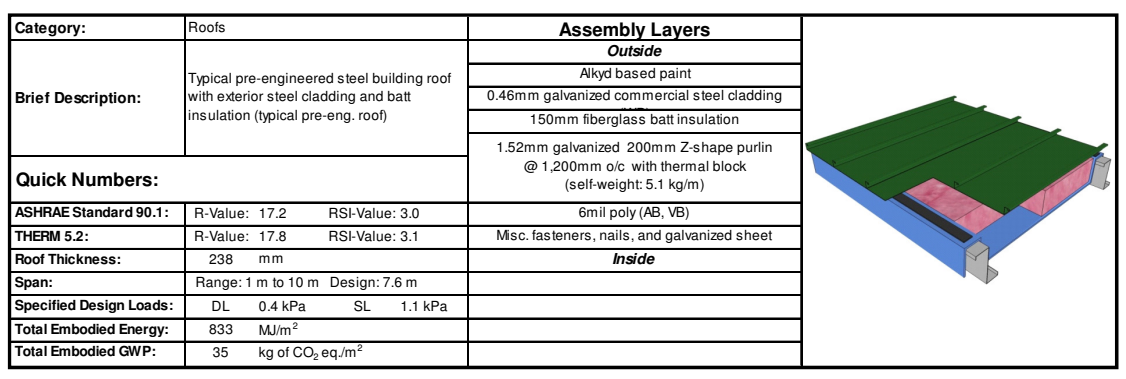

\section{Life-Cycle Assessment Results:}

Primary Energy Consumption (MJ)

\begin{tabular}{|c|c|c|c|c|c|c|c|c|c|c|c|c|c|c|c|c|}
\hline \multirow{3}{*}{ Years) } & \multicolumn{14}{|c|}{ Embodied Energy (EE) } & \multirow{2}{*}{\multicolumn{2}{|c|}{$\begin{array}{c}\text { Difference in } \\
\text { Operating Energy } \\
\text { from Baseline afte } \\
\text { Lifespan }\end{array}$}} \\
\hline & \multicolumn{3}{|c|}{ Manutacturing } & \multicolumn{3}{|c|}{ Construction } & \multicolumn{3}{|c|}{ Maintenance } & \multicolumn{3}{|c|}{ End of Life } & \multirow{2}{*}{$\begin{array}{c}{ }^{3} \text { Total } \\
\mathrm{EE}\end{array}$} & \multirow{2}{*}{$\begin{array}{c}{ }^{4}{ }^{4} \text { otal } \\
\mathrm{EE} \\
\text { per m}\end{array}$} & & \\
\hline & Materia & 2 Trans. & Total & \begin{tabular}{|c|} 
Material \\
\end{tabular} & \begin{tabular}{|l|}
2 Trans. \\
\end{tabular} & Total & Material & $\begin{array}{l}{ }^{2} \text { Trans. } \\
\end{array}$ & \begin{tabular}{|l|l|l|} 
Total \\
\end{tabular} & Material & ${ }^{2}$ Trans. & Total & & & ${ }^{5}$ Total & per $m^{2}$ \\
\hline tial & & 81 & & 0 & 124 & 124 & $\begin{array}{ll} \\
\end{array}$ & 0 & 0 & $\overline{0}$ & 0 & 0 & 40,312 & 698 & & \\
\hline & 40,1 & 81 & 40,187 & & 124 & & & & & & & & & & & \\
\hline
\end{tabular}

Global Warming Potential ( $\left(\mathrm{g}\right.$ of $\mathrm{CO}_{2}$ eq.)

\begin{tabular}{|c|c|c|c|c|c|c|c|c|c|c|c|c|c|c|c|c|}
\hline \multirow{3}{*}{$\begin{array}{l}\text { Lifespan } \\
\text { (Years) }\end{array}$} & \multicolumn{14}{|c|}{ Embodied Global Warming Potential (GWP) } & \multirow{2}{*}{\multicolumn{2}{|c|}{$\begin{array}{l}\text { Difference in } \\
\text { Operating GWP fron } \\
\text { Baseline after } \\
\text { Lifespan }\end{array}$}} \\
\hline & \multicolumn{3}{|c|}{ Manutacturing } & \multicolumn{3}{|c|}{ Construction } & \multicolumn{3}{|c|}{ Maintenance } & \multicolumn{3}{|c|}{ End of Life } & \multirow{2}{*}{$\begin{array}{l}{ }^{3} \text { Total } \\
\text { GWP }\end{array}$} & \multirow{2}{*}{$\begin{array}{l}{ }^{4}{ }^{\mathrm{T} o t a l} \\
\text { GWP } \\
\text { perm }\end{array}$} & & \\
\hline & Material & ${ }^{2}$ Trans & 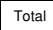 & Material & ${ }^{2}$ Trans. & Total & Material & 2 Trans. & Total & Material & 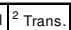 & Total & & & ${ }^{5}$ Total & ${ }^{6}$ per m$^{2}$ \\
\hline 1 Initial & 1,923 & 0 & 1,923 & 0 & 0 & 0 & 0 & 0 & 0 & 0 & 0 & 0 & 1,923 & 33 & & \\
\hline 50 & \begin{tabular}{|l|l}
1,923 \\
\end{tabular} & 0 & \begin{tabular}{|l|l|l|}
1,923 \\
\end{tabular} & 0 & 0 & 0 & $\begin{array}{ll}108 \\
\end{array}$ & 0 & 108 & 0 & 0 & 0 & \begin{tabular}{|l|}
2,031 \\
\end{tabular} & 35 & \begin{tabular}{|l|}
20,000 \\
\end{tabular} & 34 \\
\hline
\end{tabular}
Net roof area of baseline retail building (gross roof area - openings) $=586.0 \mathrm{~m}^{2}$

ATHENA ® EIE Material List:

(Includes all materials after 50 years)

\begin{tabular}{|l|c|c|}
\hline \multicolumn{1}{|c|}{ Material List } & Quantities & Unit \\
\hline 6 mil Polyethylene & 61.3 & $\mathrm{~m} 2$ \\
\hline Batt. Fiberglass & 352.1 & $\mathrm{~m} 2(25 \mathrm{~mm})$ \\
\hline Galvanized Sheet & 338.3 & $\mathrm{~kg}$ \\
\hline Galvanized Studs & 399.5 & $\mathrm{~kg}$ \\
\hline Modifed Bitumen membrane & 179.3 & $\mathrm{~kg}$ \\
\hline Nails & 3.6 & $\mathrm{~kg}$ \\
\hline Screws Nuts \& Bolts & 8.9 & $\mathrm{~kg}$ \\
\hline Solvent Based Alkyd Paint & 41.5 & $\mathrm{~L}$ \\
\hline
\end{tabular}

Notes:
${ }^{1}$ IInitial = Time ' $O$ ' (i.e. at the completion of initial construction) Trans. $=$ Transportation
${ }^{3}$ Total EE $($ or Total GWP) $=$ Total embodied energy (or total embodied GWP)
of building component after lifespan (i.e. total manufacturing + total construction + total maintenance + total end-of-life effects)

${ }^{4}$ Total EE (or Total GWP) per $\mathrm{m}^{2}=$ Total EE (or Total GWP) of building
component/ area of building component that was modelled in ATHENAQ EIE 5 component/area of bulling component that was modelled in ATHENA
Total Difference in Operating Energy (or GWP) from Baseline after
Lifespan $=$ The difference in the total life-cycle operating energy (or GWP) frrom the baseline retail building after lifespan, due to using this building component instead of the baseline component

Total Difference in Operating Energy (or GWP) from Baseline after
Lifespan per $\mathrm{m}^{2}=$ Total difference in operating energy (ar GWP) from Lifespan per $\mathrm{m}^{2}=$ Total difference in operating energy
baseline after lifespan / net roof area of baseline retail building

* Total operating primary energy use of baseline retail building after 50 years $=$ $50,700 \mathrm{GJ}\left(1,745 \mathrm{MJ} / \mathrm{m}^{2} \mathrm{YIN}\right.$

* Total operating $\mathrm{GWP}_{\mathrm{P}}$ of baseline retail building after 50 years $=2,310$ tonnes
of $\mathrm{CO}_{2}$ eq. $\left(80 \mathrm{~kg}\right.$ of $\mathrm{CO}_{2}$ eq. $\left./ \mathrm{m}^{2} \mathrm{yr}\right)$

\section{Pre-Engineered Steel Building Roof \#3 (PENG-R3)}

Building Component Description:

\begin{tabular}{|c|c|c|c|c|}
\hline \multirow[t]{2}{*}{ Category: } & \multicolumn{2}{|l|}{ Roots } & Assembly Layers & \multirow{13}{*}{$\begin{array}{l}\text { Rendering \& R-value courtesy of } \\
\text { BEHLEN Industries LP } \\
\text { http://www.behlen.cal }\end{array}$} \\
\hline & \multirow{3}{*}{\multicolumn{2}{|c|}{ 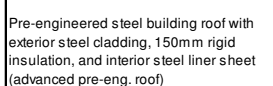 }} & $\begin{array}{l}\text { Outside } \\
\text { Alkd based paint }\end{array}$ & \\
\hline \multirow[t]{2}{*}{ Brief Description: } & & & $0.46 \mathrm{~mm}$ galvanized comm ercial steel cladding & \\
\hline & & & $150 \mathrm{~mm}$ extruded polystyrene rigid insulation & \\
\hline & 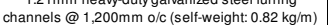 & \\
\hline & & & $0.46 \mathrm{~mm}$ galvanized commercial s & \\
\hline \multicolumn{3}{|l|}{ Quick Numbers: } & (AB, VB) & \\
\hline BEHLENIndustries LP: & R-Value: 21.0 & RSIVValue: 3.7 & Alkyd based paint & \\
\hline Roof Thickness: & $\begin{array}{ll}426 & \mathrm{~mm} \\
\end{array}$ & & $1.52 \mathrm{~mm}$ galvanized $200 \mathrm{~mm}$ Z-shape purlin & \\
\hline Span: & Range: $1 \mathrm{~m}$ to $10 \mathrm{~m}$ & Design: $7.6 \mathrm{~m}$ & @1,200mm o/c with thermal block & \\
\hline Specified Design Loads: & $\mathrm{DL} \quad 0.5 \mathrm{kPa}$ & SL $\quad 1.1 \mathrm{kPa}$ & (self-weight: $5.1 \mathrm{~kg} / \mathrm{m}$ ) & \\
\hline Total Embodied Energy: & $1,341 \quad \mathrm{~m} / \mathrm{m}^{2}$ & & Inside & \\
\hline Total Embodied GWP: & $61 \quad \mathrm{~kg} \mathrm{ofCO}_{2}$ & $\mathrm{eq} / \mathrm{m}^{2}$ & & \\
\hline
\end{tabular}

\section{Life-Cycle Assessment Results:}

Primary Energy Consumption (MJ)

\begin{tabular}{|c|c|c|c|c|c|c|c|c|c|c|c|c|c|c|c|c|}
\hline \multirow{3}{*}{$\mid \begin{array}{l}\text { Lifiespan } \\
\text { (Years) }\end{array}$} & \multicolumn{14}{|c|}{ Embodied Energy (EE) } & \multirow{2}{*}{\multicolumn{2}{|c|}{$\begin{array}{c}\text { Difference in } \\
\text { Operating Energy } \\
\text { from Baseline after } \\
\text { Lifespan }\end{array}$}} \\
\hline & \multicolumn{3}{|c|}{ Manufacturing } & \multicolumn{3}{|c|}{ Construction } & \multicolumn{3}{|c|}{ Maintenance } & \multicolumn{3}{|c|}{ End of Life } & \multirow{2}{*}{$\begin{array}{c}{ }^{3} \text { Total } \\
\text { EE }\end{array}$} & \multirow{2}{*}{\begin{tabular}{|c|c}
${ }^{4}$ Total \\
EE \\
per m
\end{tabular}} & & \\
\hline & \begin{tabular}{|l|} 
Material \\
\end{tabular} & \begin{tabular}{l|l}
$\left.\right|^{2}$ Trans. \\
.
\end{tabular} & Total & Material & ${ }^{2}$ Trans. & Total & Material & ${ }^{2}$ Trans. & Total & \begin{tabular}{|l|} 
Material \\
\end{tabular} & 1 ${ }^{2}$ Trans. & Total & & & ${ }^{5}$ Total & ${ }^{6}{ }^{6}$ per $m^{2}$ \\
\hline Initial & 76,510 & \begin{tabular}{|l|l|}
5 & 75 \\
\end{tabular} & 76,585 & 0 & $\begin{array}{l}160 \\
\end{array}$ & 160 & 0 & 0 & 0 & 0 & 0 & 0 & 76,745 & \begin{tabular}{|l|}
1,329 \\
\end{tabular} & & \\
\hline 50 & 76,510 & $\begin{array}{l} \\
\end{array}$ & 76,585 & 0 & 160 & 160 & 621 & 4 & 626 & 0 & 73 & 73 & 77,444 & \begin{tabular}{|l|l|l|} 
\\
\end{tabular} & 0 & 0 \\
\hline
\end{tabular}

Global Warming Potential ( $\mathrm{kg}$ of $\mathrm{CO}_{2}$ eq.)

\begin{tabular}{|c|c|c|c|c|c|c|c|c|c|c|c|c|c|c|c|c|}
\hline \multirow{3}{*}{$\begin{array}{c}\text { Liespan } \\
\text { (Years) }\end{array}$} & \multicolumn{14}{|c|}{ Embodied Global Warming Potential (GWP) } & \multirow{2}{*}{\multicolumn{2}{|c|}{$\begin{array}{l}\text { Difference in } \\
\text { Operating GWP from } \\
\text { Baseline after } \\
\text { Lifespan }\end{array}$}} \\
\hline & \multicolumn{3}{|c|}{ Manufacturing } & \multicolumn{3}{|c|}{ Construction } & \multicolumn{3}{|c|}{ Maintenance } & \multicolumn{3}{|c|}{ End of Life } & \multirow{2}{*}{$\begin{array}{l}{ }^{3} \text { Total } \\
\text { GWP }\end{array}$} & \multirow{2}{*}{ 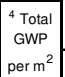 } & & \\
\hline & Material & ${ }^{2}$ Trans. & Total & Material & ${ }^{2}$ Trans. & Total & Material & ${ }^{2}$ Trans. & Total & Material & ${ }^{2}$ Trans. & Total & & & ${ }^{5}$ Total & ${ }^{6}$ per $m^{2}$ \\
\hline Initial & 3,509 & 0 & 3,509 & 0 & 0 & 0 & 0 & 0 & 0 & 0 & 0 & 0 & 3,509 & 61 & & \\
\hline 50 & 3,509 & 0 & 3,509 & 0 & 0 & 0 & 11 & 0 & 11 & 0 & 0 & 0 & 3,520 & 61 & 0 & 0 \\
\hline
\end{tabular}
(Span $\times$ Width $\left.=7.6 \mathrm{~m} \times 7.6 \mathrm{~m}=57.8 \mathrm{~m}^{2}\right)$

ATHENA ® EIE Material List:

(Includes all materials after 50 years)

Notes:
${ }^{1}$ Initial = Time 'o' (i.e. at the completion of initial construction)

\begin{tabular}{|l|c|c|}
\hline \multicolumn{1}{|c|}{ Material List } & Quantities & Unit \\
\hline Extruded Polystyrene & 355.0 & $\mathrm{~m} 2(25 \mathrm{~mm})$ \\
\hline Galvanized Sheet & 636.3 & $\mathrm{~kg}$ \\
\hline Gavvanized Studs & 399.5 & $\mathrm{~kg}$ \\
\hline Naills & 3.6 & $\mathrm{~kg}$ \\
\hline Screws Nuts \& Bolts & 8.3 & $\mathrm{~kg}$ \\
\hline Solvent Based Alkyd Paint & 15.3 & $\mathrm{~L}$ \\
\hline
\end{tabular}

${ }^{2}$ Trans. $=$ Transportation

${ }^{3}$ Total EE (or Total GWP) $=$ Total embodied energy (or total embodied GWP)

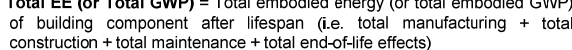
construction + total maintenance + total end-of-life effects) ${ }^{4}$ Total EE (or Total GWP) per $\mathrm{m}^{2}=$ Total EE (or Total GWP) of building
component / area of building component that was modelled in ATHENAB EIE ${ }^{5}$ Total Difference in Operating Energy (or GWP) from Baseline after from the baseline retail builining after lifespan, due to using this building

component instead of the baseline component
Total Difference in Operating Energy (or GWP) from Baseline after
Lifespan per $\mathrm{m}^{2}=$ Ootal difference in operating energy (or GWP) from Lifespan per $\mathrm{m}^{2}=$ Total difference in operating energy (or GWP) from
baseline after lifespan / net roof area of baseline retail building "Total operating primary energy use of baseline retail building after 50 years $=$
$50,700 \mathrm{GJ}\left(1,745 \mathrm{MJm}^{2} \mathrm{yr}\right)$ * Total operating $G W \mathrm{~W}$ of baseline retail building after 50 years $=2,310$ tonnes
of $\mathrm{CO}_{2}$ eq, (80 kg of $\mathrm{CO}_{2}$ eq, $\left./ \mathrm{m}^{2} \mathrm{yrr}\right)$ 


\section{Pre-Engineered Steel Building Roof \#4 (PENG-R4)}

Building Component Description:

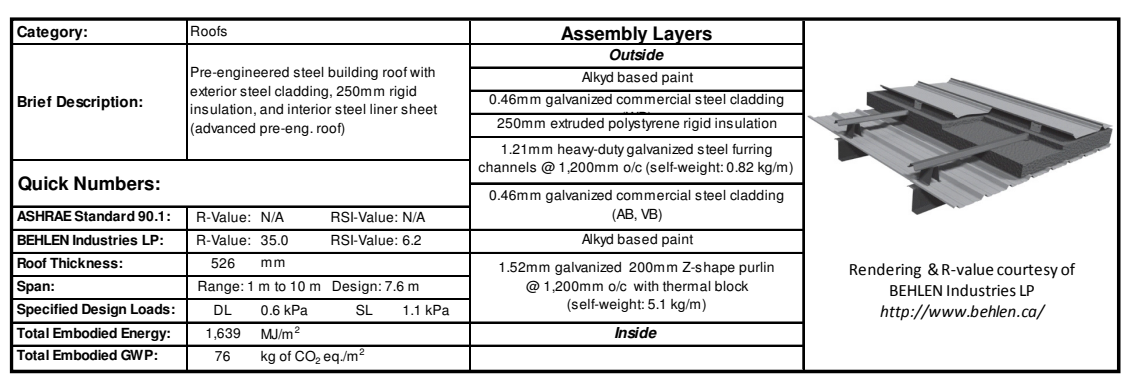

\section{Life-Cycle Assessment Results:}

Primary Energy Consumption (MJ)

\begin{tabular}{|c|c|c|c|c|c|c|c|c|c|c|c|c|c|c|c|c|}
\hline \multirow{3}{*}{$\begin{array}{l}\text { Lifespan } \\
\text { (vears) }\end{array}$} & \multicolumn{14}{|c|}{ Embodied Energy (EE) } & \multirow{2}{*}{\multicolumn{2}{|c|}{$\begin{array}{l}\text { Difference in } \\
\text { Operating Energy } \\
\text { from Baseline after } \\
\text { Lifespan }\end{array}$}} \\
\hline & \multicolumn{3}{|c|}{ Manufacturing } & \multicolumn{3}{|c|}{ Construction } & \multicolumn{3}{|c|}{ Maintenance } & \multicolumn{3}{|c|}{ End of Life } & \multirow{2}{*}{$\begin{array}{c}{ }^{3} \text { Total } \\
\text { EE }\end{array}$} & \multirow{2}{*}{$\begin{array}{c}{ }^{4} \text { Total } \\
\text { EE } \\
\text { per m }{ }^{2}\end{array}$} & & \\
\hline & Material & 11 ${ }^{2}$ Trans. & \begin{tabular}{|l|l} 
Total \\
\end{tabular} & Material & $\begin{array}{l}{ }^{2} \text { Trans. } \\
\text { Tran }\end{array}$ & Total & Material & $\begin{array}{l}{ }^{2} \text { Trans. } \\
\text { Tran }\end{array}$ & \begin{tabular}{|l|l|l|l} 
Total \\
\end{tabular} & Material & ${ }^{2}$ Trans. & Total & & & ${ }^{5}$ Total & ${ }^{6}{ }_{\text {per m}}^{2}$ \\
\hline 'Initial & 93,711 & 76 & 93,787 & 0 & 187 & 187 & 0 & 0 & 0 & 0 & 0 & 0 & \begin{tabular}{|l|l|}
33,974 \\
\end{tabular} & \begin{tabular}{|l|}
1,627 \\
\end{tabular} & & \\
\hline 50 & 93,711 & 76 & \begin{tabular}{|l|l|}
93,787 \\
\end{tabular} & 0 & 187 & 187 & 621 & 4 & 626 & 0 & 95 & 95 & 94,695 & 1,639 & $-900,000$ & \begin{tabular}{|l|l|} 
\\
\end{tabular} \\
\hline
\end{tabular}

Global Warming Potential ( $\mathrm{kg}$ of $\mathrm{CO}_{2}$ eq.)

\begin{tabular}{|c|c|c|c|c|c|c|c|c|c|c|c|c|c|c|c|c|}
\hline \multirow{3}{*}{$\begin{array}{l}\text { Lifespan } \\
\text { (Years) }\end{array}$} & \multicolumn{14}{|c|}{ Embodied Global Warming Potential (GWP) } & \multirow{2}{*}{\multicolumn{2}{|c|}{$\begin{array}{c}\text { Difference in } \\
\text { Operating GWP from } \\
\text { Baseline after } \\
\text { Lifespan }\end{array}$}} \\
\hline & \multicolumn{3}{|c|}{ Manutacturing } & \multicolumn{3}{|c|}{ Construction } & \multicolumn{3}{|c|}{ Maintenance } & \multicolumn{3}{|c|}{ End of Lifie } & \multirow{2}{*}{$\begin{array}{l}{ }^{3} \text { Total } \\
\text { GWP }\end{array}$} & \multirow{2}{*}{$\begin{array}{l}{ }^{4}{ }^{\mathrm{T} o t a l} \\
\text { GWP } \\
\text { perm }\end{array}$} & & \\
\hline & Material & ${ }^{2}$ Trans. & 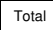 & Material & ${ }^{2}$ Trans. & Total & Material & ${ }^{2}$ Trans. & Total & Material & 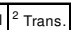 & Total & & & ${ }^{5}$ Total & ${ }^{6} \mathrm{perm}^{2}$ \\
\hline Initial & 4,388 & 0 & 4,388 & 0 & 0 & 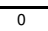 & $\pi$ & 0 & 0 & 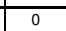 & $\pi$ & 0 & & 76 & & \\
\hline 50 & 4,388 & 0 & 4,3 & 0 & 0 & 0 & 11 & 0 & 11 & 0 & 0 & 0 & 400 & 76 & $-50,000$ & -85 \\
\hline
\end{tabular}

ings $=586.0 \mathrm{~m}^{2}$

ATHENA ® EIE Material List:

$$
\begin{aligned}
& \text { (Includes all materials after } 50 \text { years) } \\
& \text { (n) }
\end{aligned}
$$

\begin{tabular}{|l|c|c|}
\hline \multicolumn{1}{|c|}{ Material List } & Quantities & Unit \\
\hline Extruded Polystyrene & 591.6 & $\mathrm{~m} 2(25 \mathrm{~mm})$ \\
\hline Galvanized Sheet & 636.3 & $\mathrm{~kg}$ \\
\hline Galvanized Studs & 399.5 & $\mathrm{~kg}$ \\
\hline Nails & 3.6 & $\mathrm{~kg}$ \\
\hline Screws Nuts \& Bolts & 8.3 & $\mathrm{~kg}$ \\
\hline Solvent Based Alkyd Paint & 15.3 & $\mathrm{~L}$ \\
\hline
\end{tabular}

Notes:
${ }^{1}$ Initial $=$ Time 'o' (i.e. at the completion of initial construction)

${ }^{2}$ Initial $=$ Time ${ }^{2}($ i.e. at

${ }^{3}$ Total EE (or Tos GWP) = Total embodied energy (or total embodied GWP of building component after lifespan (i.e. total manufacturing + total construction + total maintenance + total end-oflife effects)

${ }^{4}$ Total EE (or Total GWP) per $\mathrm{m}^{2}=$ Total EE (or Total GWP) of building 5 Total Difference in Operating Energy (or GWP) from Baseline after
Lifespan $=$ The difference in the total life-cycle operating energy (or GWP) from the baseline retail builiding after lifespan, due to using this building component instead of the baseline componen

${ }^{6}$ Total Difference in Operating Energy (or GWP) from Baseline after

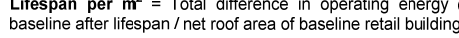

* Total operating primary energy use of baseline retail building after 50 years = $50,700 \mathrm{GJ}\left(1,745 \mathrm{MJ} / \mathrm{m}^{2} \mathrm{yr}\right)$

* *otal operating GWP of baseline retail building after 50 years $=2,310$ tonnes 
Appendix B-3

LCA Data for Structural Systems 


\section{LCA Data for Structural Systems}

This section contains a detailed description of structural systems that were examined in this study ( 3 in total). A summary of the important elements of each system are listed, along with a detailed description of the material quantities from the ATHENA ${ }^{\circledR}$ Environmental Impact Estimator for Buildings.

A breakdown of the total primary energy consumption and the total global warming potential (GWP) for each structural system is also included. In each case, the results were calculated for an entire retail building. The results are also expressed per $\mathrm{m}^{2}$ of structural system in each case. The data has been calculated for two different lifespans: at the completion of initial construction and after 50 years.

As a summary, the figure below illustrates a comparison of the total embodied energy (and GWP) after 50 years for the various structural systems in this section. For comparison purpose, the building component used in the baseline retail building has been shaded in grey.

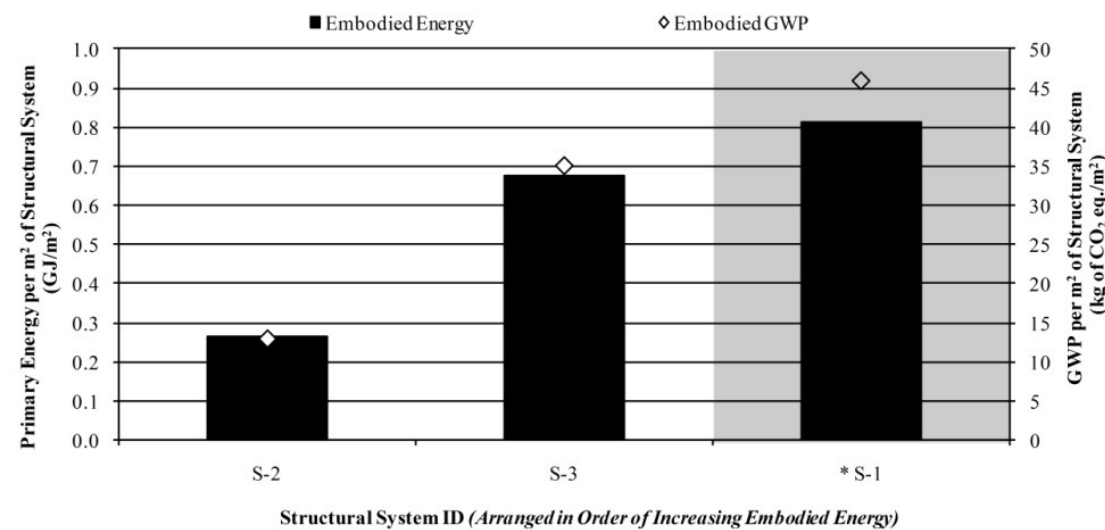

Structural System ID (Arranged in Order of Increasing Embodied Energy)

\section{Structural System \#1 (S-1)}

Building Component Description:

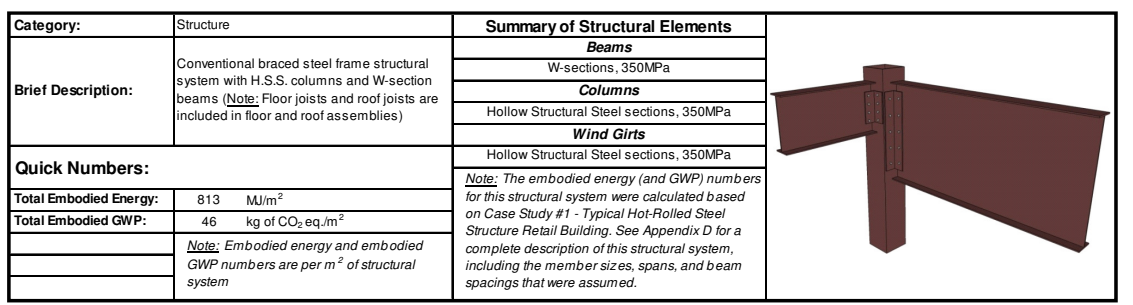

Life-Cycle Assessment Results:

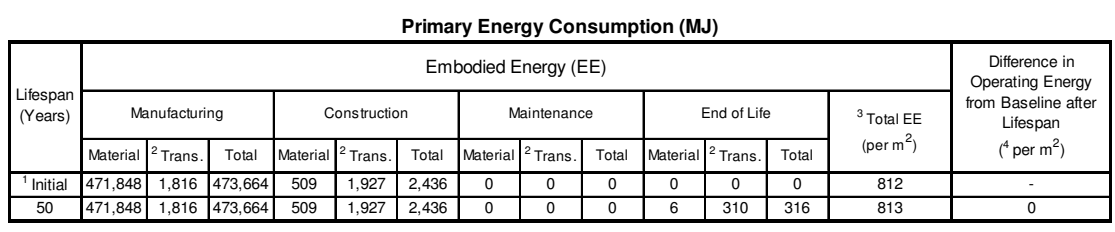

Global Warming Potential $\left(\mathrm{kg}\right.$ of $\mathrm{CO}_{2}$ eq.)

\begin{tabular}{|c|c|c|c|c|c|c|c|c|c|c|c|c|c|c|}
\hline \multirow{3}{*}{$\begin{array}{l}\text { Lifespan } \\
\text { (Years) }\end{array}$} & \multicolumn{13}{|c|}{ Embodied Global Warming Potential (GWP) } & \multirow{3}{*}{\begin{tabular}{|c|} 
Difference in \\
Operating GWP from \\
Baseline after \\
Lifespan \\
$\left({ }^{4}\right.$ per $\left.\mathrm{m}^{2}\right)$
\end{tabular}} \\
\hline & \multicolumn{3}{|c|}{ Manufacturing } & \multicolumn{3}{|c|}{ Construction } & \multicolumn{3}{|c|}{ Maintenance } & \multicolumn{3}{|c|}{ End of Life } & \multirow{2}{*}{$\begin{array}{l}{ }^{3} \text { Total GWP } \\
\left.\text { (per } \mathrm{m}^{2}\right)\end{array}$} & \\
\hline & Material & 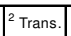 & Total & Material & 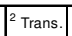 & Total & \begin{tabular}{|l|l|} 
Material \\
\end{tabular} & 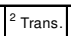 & Total & \begin{tabular}{|l|} 
Material \\
\end{tabular} & \begin{tabular}{|l|} 
\\
\end{tabular} & Total & & \\
\hline 1 Initial & 26,830 & 4 & 26,833 & 33 & 4 & 37 & 0 & 0 & 0 & 0 & 0 & 0 & 46 & \\
\hline 50 & 26,830 & 4 & 26,833 & 33 & 4 & 37 & 0 & 0 & 0 & 0 & 1 & 1 & 46 & 0 \\
\hline
\end{tabular}
Floor area of Case Study Building \#1 = $\quad 586.0 \mathrm{~m}^{2}$

ATHENA ® EIE Material List:

\begin{tabular}{|l|c|c|}
\hline \multicolumn{1}{|c|}{ (Includes all materials after 50 years) } \\
\hline Material List & Quantities & Unit \\
\hline Hollow Structural Steel & $3,307.4$ & $\mathrm{~kg}$ \\
\hline Hot Rolled Sheet & $1,515.0$ & $\mathrm{~kg}$ \\
\hline Screws Nuts \& Bolts & 626.7 & $\mathrm{~kg}$ \\
\hline Wide Flange Sections & $11,822.9$ & $\mathrm{~kg}$ \\
\hline
\end{tabular}

Notes:

${ }^{2}$ Trans. $=$ Transportation

${ }^{3}$ Total EE (or Total GWP) per $\mathrm{m}^{2}=$ Total embodied energy (or total embodied GWP) of building component after lifespan (i.e. total manufacturing + totad baseline building

${ }^{4}$ Total Difference in Operating Energy (or GWP) from Baseline after Lifespan per $m^{2}=$ The difference in the total life-cycle operating energy (or GWP) from the baseline retail building after lifespan, due to using this
building component instead of the buseline commonent/flioor area of baseline building
building

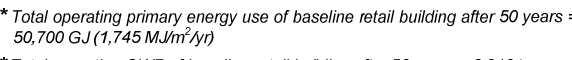

* Total operating $\mathrm{GWP}$ of baseline retail building after 50 years $=2,310$ tonnes
of $\mathrm{CO}_{2}$ eq. $\left(80 \mathrm{~kg}\right.$ of $\mathrm{CO}_{2}$ eq. $\left./ \mathrm{m}^{2} / \mathrm{yr}\right)$ 


\section{Structural System \#2 (S-2)}

Building Component Description:

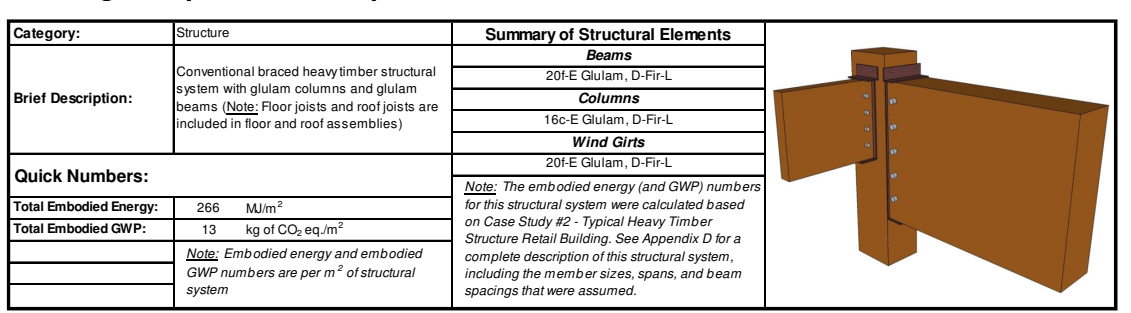

Life-Cycle Assessment Results:

Primary Energy Consumption (MJ)

\begin{tabular}{|c|c|c|c|c|c|c|c|c|c|c|c|c|c|c|}
\hline \multirow{3}{*}{$\begin{array}{c}\text { Lifespana } \\
\text { (Years) }\end{array}$} & \multicolumn{13}{|c|}{ Embodied Energy (EE) } & \multirow{3}{*}{$\begin{array}{l}\text { Difference in } \\
\text { Operating Energy } \\
\text { from Baseline after } \\
\text { Lifespan } \\
\left({ }^{4} \text { per } \mathrm{m}^{2}\right)\end{array}$} \\
\hline & \multicolumn{3}{|c|}{ Manufacturing } & \multicolumn{3}{|c|}{ Construction } & \multicolumn{3}{|c|}{ Maintenance } & \multicolumn{3}{|c|}{ End of Life } & \multirow{2}{*}{$\begin{array}{l}{ }^{3} \text { Total EE } \\
\left(\text { per m }{ }^{2}\right)\end{array}$} & \\
\hline & Material & ${ }^{2}$ Trans. & \begin{tabular}{|l|l|} 
Total \\
\end{tabular} & \begin{tabular}{|l|} 
Material \\
\end{tabular} & ${ }^{2}$ Trans. & Total & Material & ${ }^{2}$ Trans. & Total & Material & 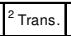 & Total & & \\
\hline Initial & 144,531 & \begin{tabular}{|l|l|}
3,510 \\
\end{tabular} & 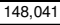 & 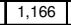 & $\begin{array}{ll}6,306 \\
\end{array}$ & \begin{tabular}{|l|l|}
7,472 \\
\end{tabular} & 0 & 0 & 0 & 0 & 0 & 0 & 265 & \\
\hline 50 & 144,531 & \begin{tabular}{|l|l}
3,510 \\
\end{tabular} & 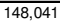 & 1,166 & $\begin{array}{ll}6,306 \\
\end{array}$ & $\begin{array}{l}7,472 \\
\end{array}$ & 0 & 0 & 0 & 16 & 442 & 458 & 266 & 0 \\
\hline
\end{tabular}

Global Warming Potential (kg of $\mathrm{CO}_{2}$ eq.)

\begin{tabular}{|c|c|c|c|c|c|c|c|c|c|c|c|c|c|c|}
\hline \multirow{3}{*}{\begin{tabular}{|} 
Lifespan \\
(Years)
\end{tabular}} & \multicolumn{13}{|c|}{ Embodied Global Warming Potential (GWP) } & \multirow{3}{*}{$\begin{array}{l}\text { Difference in } \\
\text { Operating GWP from } \\
\text { Baseline after } \\
\text { Lifespan } \\
\left({ }^{4} \text { per } \mathrm{m}^{2}\right)\end{array}$} \\
\hline & \multicolumn{3}{|c|}{ Manutacturing } & \multicolumn{3}{|c|}{ Construction } & \multicolumn{3}{|c|}{ Maintenance } & \multicolumn{3}{|c|}{ End of Life } & \multirow{2}{*}{$\begin{array}{c}{ }^{3} \text { Total GWP } \\
\left(\text { per m }{ }^{2} \text { ) }\right.\end{array}$} & \\
\hline & Material & ${ }^{2}$ Trans. & Total & Material & ${ }^{2}$ Trans. & Total & Material & ${ }^{2}$ Trans. & Total & Material & 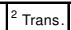 & Total & & \\
\hline 1 Initial & 7,468 & 7 & 7,475 & 75 & 4 & 79 & 0 & 0 & 0 & 0 & 0 & 0 & 13 & \\
\hline 50 & 7,468 & 7 & 7,475 & 75 & 4 & 79 & 0 & 0 & 0 & 1 & 1 & 2 & 13 & 0 \\
\hline
\end{tabular}
Floor area of Case Study Building \#2 $586.0 \mathrm{~m}^{2}$

ATHENA ® EIE Material List:

\begin{tabular}{|l|c|c|}
\hline \multicolumn{1}{|c|}{ (Includes all materials after 50 years) } \\
\hline Material List & Quantities & Unit \\
\hline GluLam Sections & 33.5 & $\mathrm{~m} 3$ \\
\hline Hollow Structural Steel & 780.7 & $\mathrm{~kg}$ \\
\hline Hot Rolled Sheet & $1,515.0$ & $\mathrm{~kg}$ \\
\hline Screws Nuts \& Bolts & 206.0 & $\mathrm{~kg}$ \\
\hline
\end{tabular}

Notes:
${ }^{1}$ Initial $=$ Time 'o' (i.e. at the completion of in

${ }^{2}$ Trans. $=$ Transportation

${ }^{3}$ Total EE (or Total GWP) per $\mathrm{m}^{2}=$ Total embodied energy (or total embodied GWP) of building component after lifespan (i.e. total manufacturing + total
construction + total maintenance + total enc-of-life effects) / floor area of construction +
baseline building

${ }^{4}$ Total Difference in Operating Energy (or GWP) from Baseline after Lifespan per $\mathrm{m}^{2}$ = The difference in the total life-cycle operating energy (or GWP) from the baseline retail building after lifespan, due to using this
building component instead of the baseline component/floor area of baseling building

* Total operating primary energy use of baseline retail building after 50 years $50,700 \mathrm{GJ}\left(1,745 \mathrm{MJ} / \mathrm{m}^{2} \mathrm{yr}\right)$

"Total operating GWP of baseline retail building after 50 years $=2,310$ tonnes

\section{Structural System \#3 (S-3)}

Building Component Description:

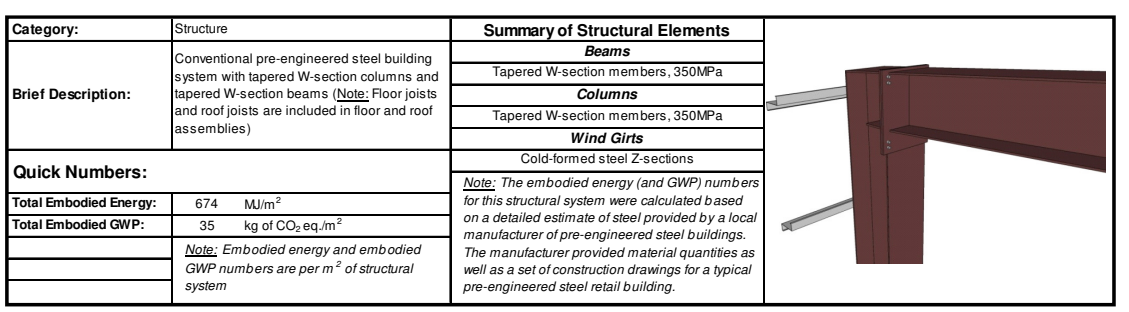

Life-Cycle Assessment Results:

Primary Energy Consumption (MJ)

\begin{tabular}{|c|c|c|c|c|c|c|c|c|c|c|c|c|c|c|}
\hline \multirow{3}{*}{$\begin{array}{l}\text { Lifespan } \\
\text { (years) }\end{array}$} & \multicolumn{13}{|c|}{ Embodied Energy (EE) } & \multirow{3}{*}{$\begin{array}{c}\text { Difference in } \\
\text { Operating Energy } \\
\text { from Baseline after } \\
\text { Lifespan } \\
\left({ }^{4} \text { per } \mathrm{m}^{2}\right) \\
\end{array}$} \\
\hline & \multicolumn{3}{|c|}{ Manutacturing } & \multicolumn{3}{|c|}{ Construction } & \multicolumn{3}{|c|}{ Maintenance } & \multicolumn{3}{|c|}{ End of Lite } & \multirow{2}{*}{$\begin{array}{l}{ }^{3} \text { Total EE } \\
\left(\operatorname{per} \mathrm{m}^{2}\right)\end{array}$} & \\
\hline & Material & ${ }^{2}$ Trans. & Total & Materia & 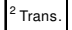 & Total & Material & $\begin{array}{l}{ }^{2} \text { Trans. } \\
\end{array}$ & Total & Material & \begin{tabular}{|l|}
${ }^{2}$ Trans. \\
\end{tabular} & Total & & \\
\hline${ }^{1}$ Initial & 390,970 & 1,701 & 392,671 & 509 & 1,521 & 2,030 & 0 & 0 & 0 & 0 & 0 & 0 & 674 & \\
\hline 50 & [390,970 & 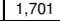 & 392,671 & \begin{tabular}{|l|l|}
509 \\
\end{tabular} & 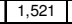 & 2,030 & 0 & 0 & 0 & 4 & 222 & 226 & 674 & 0 \\
\hline
\end{tabular}

Global Warming Potential ( $\mathrm{kg}$ of $\mathrm{CO}_{2}$ eq)

\begin{tabular}{|c|c|c|c|c|c|c|c|c|c|c|c|c|c|c|}
\hline \multirow{3}{*}{$\begin{array}{l}\text { Lifespan } \\
\text { (Years) }\end{array}$} & \multicolumn{13}{|c|}{ Embodied Global Warming Potential (GWP) } & \multirow{3}{*}{\begin{tabular}{|c|} 
Difference in \\
Operating GWP from \\
Baseline effer \\
Lifespan \\
$\left({ }^{4}\right.$ per $\left.\mathrm{m}^{2}\right)$ \\
\end{tabular}} \\
\hline & \multicolumn{3}{|c|}{ Manutacturing } & \multicolumn{3}{|c|}{ Construction } & \multicolumn{3}{|c|}{ Maintenance } & \multicolumn{3}{|c|}{ End of Life } & \multirow{2}{*}{$\begin{array}{l}{ }^{3} \text { Total GWP } \\
\left(\text { per m }{ }^{2}\right)\end{array}$} & \\
\hline & Material & 2 Trans. & Total & Material & \begin{tabular}{|l|}
${ }^{2}$ Trans. \\
\end{tabular} & Total & Material & ${ }^{2}$ Trans. & Total & \begin{tabular}{|c|} 
Material \\
\end{tabular} & 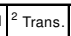 & \begin{tabular}{|l|l|} 
Total \\
\end{tabular} & & \\
\hline Initial & 20,477 & 3 & 20,481 & 33 & 3 & 36 & 0 & 0 & 0 & 0 & 0 & 0 & 35 & \\
\hline 50 & 20,477 & 3 & 20,481 & 33 & 3 & 36 & 0 & 0 & 0 & 0 & 0 & 1 & 35 & 0 \\
\hline
\end{tabular}
Embodied energy (and GWP) numbers weroch $586.0 \mathrm{~m}^{2}$

ATHENA $®$ EIE Material List:

(Includes all materials after 50 years)

Notes:
${ }^{1}$ Initial $=$ Time 'o' (i.e. at the completion of initial construction)

\begin{tabular}{|l|c|c|}
\hline \multicolumn{1}{|c|}{ Material List } & Quantities & Unit \\
\hline Screws Nuts \& Bolts & 626.7 & $\mathrm{~kg}$ \\
\hline Wide Flange Sections & $13,077.9$ & $\mathrm{~kg}$ \\
\hline
\end{tabular}

${ }^{2}$ Trans. $=$ Transportation

Total EE (or Total GWP) per $\mathrm{m}^{2}=$ Total embodied energy (or total embodied GWP) of building component atter lifespan (i.e. total manufacturing + tota baseline building

Difference in Operating Energy (or GWP) from Baseline after LWespan per $\mathrm{m}^{2}=$ The difference in the total life-cycle operating energy (or
GWP) from the baseline retail building after lifespan, due to using this uuiling component instead of the baseline component / floor area of baseline

* Total operating primary energy
$50,700 \mathrm{GJ}\left(1,745 \mathrm{M} / \mathrm{Mn}^{2} / y\right)$

* ${ }^{*}$ Total operating GWP of baseline retail building after 50 years $=2,310$ tonnes 
Appendix B-4

LCA Data for Floors 


\section{LCA Data for Floors}

This section contains a detailed description of each floor (FL) assembly that was examined in this study (5 in total). The assembly layers are listed for each floor, along with a detailed description of the material quantities from the ATHENA ${ }^{\circledR}$ Environmental Impact Estimator for Buildings.

A breakdown of the total primary energy consumption and the total global warming potential (GWP) for each floor is also included. In each case, the results were calculated for a different area of mezzanine floor, depending on the typical spans of each system. The results are also expressed on a per $\mathrm{m}^{2}$ basis in each case. The data has been calculated for two different lifespans: at the completion of initial construction and after 50 years.

As a summary, the figure below illustrates a comparison of the total embodied energy (and GWP) after 50 years for the various floors in this section. For comparison purpose, the building component used in the baseline retail building has been shaded in grey.

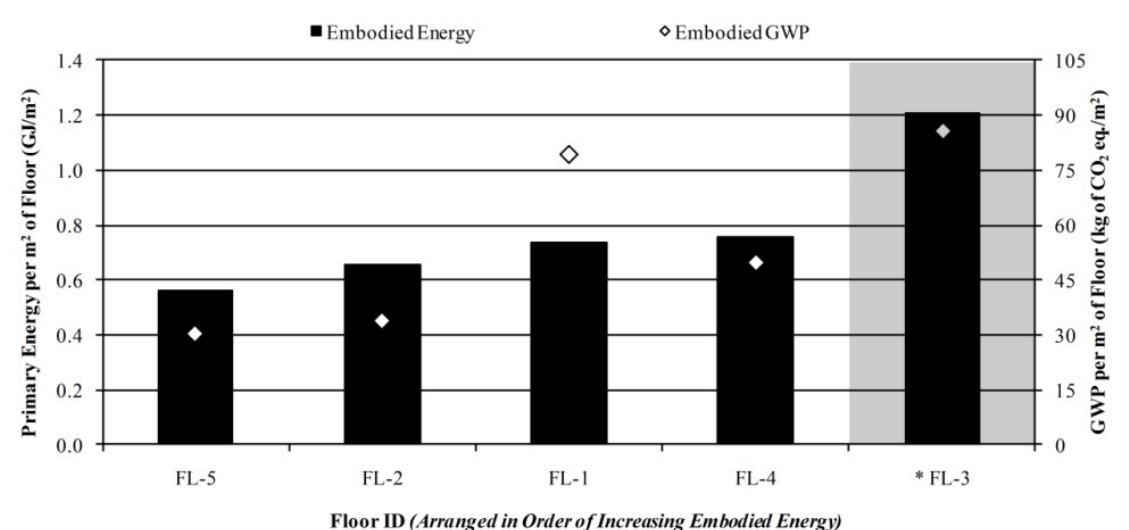

Floor \#1 (FL-1)

Building Component Description:

\begin{tabular}{|c|c|c|}
\hline \multirow{4}{*}{ |Brief Description: } & \begin{tabular}{|l} 
Mezzanine Floors \\
\end{tabular} & Assembly Layers \\
\hline & \multirow{3}{*}{$\begin{array}{l}\text { Floor assembly: : 200mmm concrete hollow } \\
\text { cores slab with viny tile finish }\end{array}$} & $\frac{\text { Top }}{\text { Viny floor tile }}$ \\
\hline & & Adhesive (modeled as alkyd based paint) \\
\hline & & $\begin{array}{l}50 \mathrm{~mm} \text { concrete topping } \\
\text { (modeled as 50mm of mortar) }\end{array}$ \\
\hline \multicolumn{2}{|l|}{ Quick Numbers: } & $\begin{array}{c}\text { (concrete topping is reinforced with } 150 \mathrm{~mm} x \\
150 \mathrm{~mm} \text { \#10M steel mesh } 11.0 \mathrm{~kg} / \mathrm{m}^{2} \text { ) }\end{array}$ \\
\hline \begin{tabular}{|l|} 
Foor Thickness: \\
Span:
\end{tabular} & $\begin{array}{ll}256 \quad \mathrm{~mm} \\
\text { Range: } 3 \mathrm{~m} \text { to } 14 \mathrm{~m} \text { Design: } 7.6\end{array}$ & 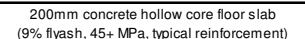 \\
\hline $\begin{array}{l}\text { Span: } \\
\text { Specified Design Loads: }\end{array}$ & 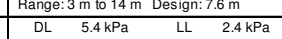 & Bottom \\
\hline Total Embodied Energy: & $739 \quad \mathrm{M} / \mathrm{m}^{2}$ & \\
\hline
\end{tabular}

Life-Cycle Assessment Results:

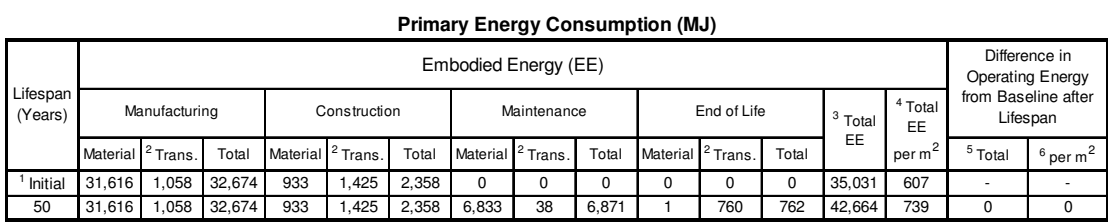

Global Warming Potential ( $\mathrm{kg}$ of $\mathrm{CO}_{2}$ eq.)

\begin{tabular}{|c|c|c|c|c|c|c|c|c|c|c|c|c|c|c|c|c|}
\hline \multirow{3}{*}{$\begin{array}{l}\text { Lifespan } \\
\text { (Years) }\end{array}$} & \multicolumn{14}{|c|}{ Embodied Global Warming Potential (GWP) } & \multirow{2}{*}{\multicolumn{2}{|c|}{\begin{tabular}{|c|} 
Difference in \\
Operating GWP from \\
Baseline after \\
Lifespan \\
\end{tabular}}} \\
\hline & \multicolumn{3}{|c|}{ Manufacturing } & \multicolumn{3}{|c|}{ Construction } & \multicolumn{3}{|c|}{ Maintenance } & \multicolumn{3}{|c|}{ End of Life } & \multirow{2}{*}{$\begin{array}{l}{ }^{3} \text { Total } \\
\text { GWP }\end{array}$} & \multirow{2}{*}{$\begin{array}{l}{ }^{4} \text { Total } \\
\text { GWP } \\
\text { per m }{ }^{2}\end{array}$} & & \\
\hline & \begin{tabular}{|l|} 
Material \\
\end{tabular} & ${ }^{2}$ Trans. & \begin{tabular}{|l|l|} 
Total \\
\end{tabular} & \begin{tabular}{|l|} 
Material \\
\end{tabular} & \begin{tabular}{|l}
${ }^{2}$ Trans. \\
\end{tabular} & Total & Materia & ${ }^{2}$ Trans. & \begin{tabular}{|l|} 
Total \\
\end{tabular} & Materia & 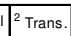 & \begin{tabular}{|l|} 
Total \\
\end{tabular} & & & ${ }^{5}$ Total & ${ }^{6}$ perm $m^{2}$ \\
\hline Initial & 4,016 & 2 & 4,018 & 65 & 3 & 68 & 0 & 0 & 0 & 0 & 0 & 0 & 4,086 & 71 & & 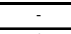 \\
\hline 50 & 4,016 & 2 & 4,018 & 65 & 3 & 68 & 498 & 0 & 498 & 0 & 1 & 2 & & & 0 & 0 \\
\hline
\end{tabular}

Embodied energy (and GWP) numbers are based on a mezzanine floor area = $57.8 \mathrm{~m}^{2} \quad$ (Length $x$ Height $=7.6 \mathrm{~m} \times 7.6 \mathrm{~m}=57.8 \mathrm{~m}^{2}$ )

ATHENA ® EIE Material List:

(Includes all materials after 50 years)

${ }^{1}$ Initial = Time ' $O$ ' (i.e. at the completion of initial construction)

\begin{tabular}{|l|c|c|}
\hline \multicolumn{1}{|c|}{ Material List } & Quantities & Unit \\
\hline Concrete $20 \mathrm{MPa}$ (flyash av) & 3.0 & $\mathrm{~m} 3$ \\
\hline Concrete $60 \mathrm{MPa}$ (flyash av) & 5.7 & $\mathrm{~m} 3$ \\
\hline Mortar & 3.3 & $\mathrm{~m} 3$ \\
\hline Rebar, Rod, Light Sections & 197.2 & $\mathrm{~kg}$ \\
\hline Solvent Based Alkyd Paint & 12.3 & $\mathrm{~L}$ \\
\hline Vinyl Siding & 119.5 & $\mathrm{~m} 2$ \\
\hline Welded Wire Mesh / Ladder Wire & 98.6 & $\mathrm{~kg}$ \\
\hline
\end{tabular}

${ }^{2}$ Trans. $=$ Transportation

${ }^{3}$ Total EE (or Total GWP) $=$ Total embodied energy (or total embodied GWP of building component after lifespan (i.e. total mat
construction + total maintenance + total end-of-life effects)

${ }^{4}$ Total EE (or Total GWP) per $\mathrm{m}^{2}=$ Total EE (or Total GWP) of building
component /area of building component that was modelled in ATHENA® EIE ${ }^{5}$ Total Difference in Operating Energy (or GWP) from Baseline after Lifespan = The difierence in the total lifiec-cycle operating energy (or GWP)
from the baseline retail building after lifiespan, due to using this building component instead of the baseline component

${ }^{6}$ Total Difference in Operating Energy (or GWP) from Baseline after Lifespan per $\mathrm{m}^{2}=$ Total difference in operating energy (or GWP) from * Total operating primary energy use of baseline retail building after 50 years =

"Total operating $\mathrm{GW}$ of of baseline ref 


\section{Floor \#2 (FL-2)}

Building Component Description:

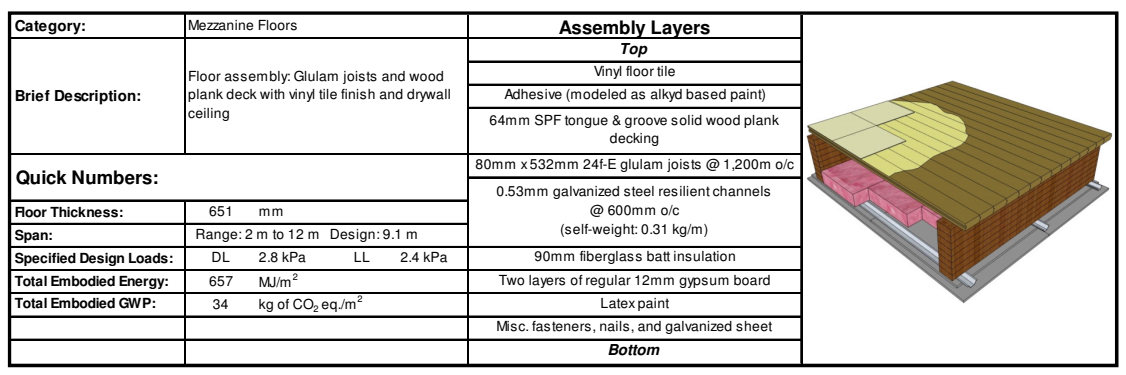

\section{Life-Cycle Assessment Results:}

Primary Energy Consumption (MJ)

\begin{tabular}{|c|c|c|c|c|c|c|c|c|c|c|c|c|c|c|c|c|}
\hline \multirow{3}{*}{\begin{tabular}{|l|l} 
Lifespan \\
(Years)
\end{tabular}} & \multicolumn{14}{|c|}{ Embodied Energy (EE) } & \multirow{2}{*}{\multicolumn{2}{|c|}{$\begin{array}{c}\text { Difference in } \\
\text { Operating Energy } \\
\text { from Baseline after } \\
\text { Lifespan }\end{array}$}} \\
\hline & \multicolumn{3}{|c|}{ Manufacturing } & \multicolumn{3}{|c|}{ Construction } & \multicolumn{3}{|c|}{ Maintenance } & \multicolumn{3}{|c|}{ End of L Life } & \multirow{2}{*}{${ }^{3}$ Total } & \multirow{2}{*}{${ }^{4}$ Total } & & \\
\hline & Material & ${ }^{2}$ Trans. & Total & Materia & Trans. & Total & Material & 每Trans. & \begin{tabular}{|l|l|l|} 
Total \\
\end{tabular} & Material & Trans & Total & & & ${ }^{5}$ Total & ${ }^{6}$ per $\mathrm{m}^{2}$ \\
\hline ititial & 32,233 & \begin{tabular}{|l|l|}
738 \\
738 \\
\end{tabular} & 971 971 & \begin{tabular}{|l|l|}
343 \\
333
\end{tabular} & 1,504 & $\frac{1,84 \prime}{1847}$ & 0 & 0 & 0 & 0 & $\frac{0}{019}$ & 0 & \begin{tabular}{|l|l|l|l|l|}
34,818 \\
4545
\end{tabular} & 503 & & \\
\hline
\end{tabular}

Global Warming Potential ( $k g$ of $\mathrm{CO}_{2}$ eq.)

\begin{tabular}{|c|c|c|c|c|c|c|c|}
\hline \multirow{3}{*}{$\begin{array}{l}\text { Lifespan } \\
\text { (Years) }\end{array}$} & \multicolumn{7}{|c|}{$\mathrm{Em}$} \\
\hline & \multicolumn{3}{|c|}{ Manufacturing } & \multicolumn{3}{|c|}{ Construction } & \\
\hline & Material & ${ }^{2}$ Trans. & \begin{tabular}{|l|} 
Total \\
\end{tabular} & Materia & al ${ }^{2}$ Trans. & \begin{tabular}{|l|l} 
Total \\
S
\end{tabular} & \\
\hline Initial & 1,685 & & 1,686 & 22 & 2 & 25 & \\
\hline 50 & 1,685 & 1 & 86 & 22 & 2 & 25 & \\
\hline \multirow{2}{*}{\multicolumn{8}{|c|}{$\begin{array}{l}\text { mbodied energy (and GWP) numbers are based on a mezzz } \\
\text { ATHENA @ EIE Material List: } \\
\text { (Includes all materials after 50 years) }\end{array}$}} \\
\hline & & & & & & & \\
\hline \multicolumn{4}{|c|}{ Material List } & \multicolumn{2}{|c|}{ Quantities } & \multicolumn{2}{|c|}{ Unit } \\
\hline \multicolumn{4}{|c|}{ 13mm Regular Gypsum Board } & \multicolumn{2}{|r|}{152.2} & \\
\hline \multirow{2}{*}{\multicolumn{4}{|c|}{ Batt. Fiberglass }} & & 253.0 & & \\
\hline \multicolumn{3}{|c|}{ Galvanized Studs } & & & 40.4 & \multicolumn{2}{|c|}{\begin{tabular}{|l|l|}
$\mathrm{m} 2(25 \mathrm{~mm})$ \\
\end{tabular}} \\
\hline \multirow{2}{*}{\multicolumn{4}{|c|}{ GluLam Sections }} & & 2.8 & \multicolumn{2}{|c|}{$\frac{\mathrm{kg}}{\mathrm{m} 3}$} \\
\hline & & & Joint Compound & & 151.9 & \multicolumn{2}{|c|}{ m3 } \\
\hline \multicolumn{4}{|l|}{ Nails } & & 18.5 & \multicolumn{2}{|c|}{$\mathrm{kg}$} \\
\hline & & 1.7 & \multicolumn{2}{|c|}{ kg } \\
\hline \multicolumn{5}{|c|}{$\begin{array}{l}\text { Small Dimension Softwood Lumber, } \\
\text { kill-dried }\end{array}$} & 3.5 & \multicolumn{2}{|c|}{ m3 } \\
\hline \multicolumn{4}{|c|}{ Solvent Based Alkyd Paint } & & 14.6 & \\
\hline \multicolumn{4}{|c|}{ Vinyl Siding } & & 142.9 & \multirow{2}{*}{\multicolumn{2}{|c|}{$\mathrm{m} 2$}} \\
\hline \multicolumn{4}{|c|}{ Water Based Latex Paint } & & 90.0 & & \\
\hline
\end{tabular}

\section{Floor \#3 (FL-3)}

Building Component Description:

\begin{tabular}{|c|c|c|c|}
\hline Category: & Mezzanine Floors & Assembly Layers & \\
\hline \multirow{4}{*}{ Brief Description: } & \multirow{3}{*}{ 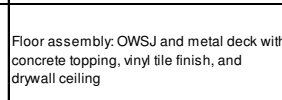 } & $\begin{array}{c}\text { Top } \\
\text { Viny flloor tile }\end{array}$ & \\
\hline & & Achesive (modeled as alkyd based paint) & \\
\hline & & $89 \mathrm{~mm}$ concretet topping & \\
\hline & & 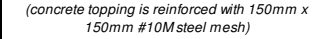 & \\
\hline \multicolumn{2}{|l|}{ Quick Numbers: } & $39 \mathrm{~mm} \times 0.76 \mathrm{~mm}$ galvanized corrugated metal deck & \\
\hline Foor Thickness: & $732 \mathrm{~mm}$ & $550 \mathrm{~mm}$ open web steel joists @ $1,200 \mathrm{~mm}$ o/c & \\
\hline Span: & Range: $3 \mathrm{~m}$ to $46 \mathrm{~m}$ Design: $9.1 \mathrm{~m}$ & (self-weight: 15.4 kg/m) & \\
\hline Specified Design Loads: & $\begin{array}{ll}\mathrm{DL} & 4.4 \mathrm{kPa} \\
\end{array}$ & galvanized steel resilient c & \\
\hline Total Embodied Energy: & & & \\
\hline \multirow[t]{6}{*}{ Total Embodied GWP: } & $86 \mathrm{~kg}$ of $\mathrm{CO}_{2} \mathrm{eq} / \mathrm{m}^{2}$ & (self-weight: $0.31 \mathrm{~kg} /$ & \\
\hline & & $90 \mathrm{~mm}$ fiberglass batt insulation & \\
\hline & & Two layers of regular $12 \mathrm{~mm}$ gypsum bo & \\
\hline & & Latexpaint & \\
\hline & & Misc. fasteners, nails, and galvanized sheet & \\
\hline & & Bottom & \\
\hline
\end{tabular}

\section{Life-Cycle Assessment Results:}

Primary Energy Consumption (MJ)

\begin{tabular}{|c|c|c|c|c|c|c|c|c|c|c|c|c|c|c|c|c|}
\hline \multirow{3}{*}{\begin{tabular}{|l} 
Lifespan \\
(Years)
\end{tabular}} & \multicolumn{14}{|c|}{ Embodied Energy (EE) } & \multirow{2}{*}{\multicolumn{2}{|c|}{$\begin{array}{c}\text { Difference in } \\
\text { Operating Energy } \\
\text { from Baseline after } \\
\text { Lifespan }\end{array}$}} \\
\hline & \multicolumn{3}{|c|}{ Manutacturing } & \multicolumn{3}{|c|}{ Construction } & \multicolumn{3}{|c|}{ Maintenance } & \multicolumn{3}{|c|}{ End of Life } & \multirow{2}{*}{$\begin{array}{l}{ }^{3} \text { Total } \\
\mathrm{EE}\end{array}$} & \multirow{2}{*}{$\begin{array}{c}{ }^{4}{ }^{\mathrm{T} o t a l} \\
\mathrm{EE} \\
\text { per m} \mathrm{m}^{2}\end{array}$} & & \\
\hline & Material & 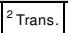 & Total & Material & ${ }^{2}$ Trans. & $\begin{array}{l}\text { Total } \\
\end{array}$ & Material & ${ }^{2}$ Trans. & $\begin{array}{l}\text { Total } \\
\end{array}$ & Material & \begin{tabular}{|l|}
${ }^{2}$ Trans. \\
\end{tabular} & Total & & & $\begin{array}{l}{ }^{5} \text { Total } \\
\end{array}$ & ${ }^{6}{ }_{\text {per m}}^{2}$ \\
\hline & 69,423 & 852 & 70,275 & 669 & 1,398 & 2,066 & 0 & 0 & 0 & 0 & 0 & 0 & 72,34 & 1,046 & & \\
\hline
\end{tabular}
\begin{tabular}{|c|c|c|c|c|c|c|c|c|c|c|c|c|c|c|c|c|c|}
\hline${ }^{1}$ Initial & 69,423 & 852 & 70,275 & 669 & 1,398 & 2,066 & 0 & 0 & 0 & 0 & 0 & 0 & 72,341 & 1,046 & - & - \\
\hline 50 & 69,423 & 852 & 70,275 & 669 & 1,398 & 2,066 & 10,312 & 64 & 10,377 & 1 & 621 & 622 & 83,339 & 1,205 & 0 & 0 \\
\hline
\end{tabular}

Global Warming Potential (kg of $\mathrm{CO}_{2}$ eq.)

\begin{tabular}{|c|c|c|c|c|c|c|c|c|c|c|c|c|c|c|c|}
\hline \multirow{3}{*}{\begin{tabular}{|} 
Lifiespan \\
(Years)
\end{tabular}} & \multicolumn{13}{|c|}{ Embodied Global Warming Potential (GWP) } & \multirow{2}{*}{\multicolumn{2}{|c|}{$\begin{array}{l}\text { Difference in } \\
\text { Operating GWP from } \\
\text { Baseline after } \\
\text { Lifespan }\end{array}$}} \\
\hline & Manufacturin & & & Eonstructior & & & aintenance & & & End of Life & & ${ }^{3}$ Totat & \begin{tabular}{|l|l|}
${ }^{4}$ Total \\
\end{tabular} & & \\
\hline & \begin{tabular}{|l|l|} 
Material ${ }^{2}$ Trans. \\
\end{tabular} & Total & Material & ${ }^{2}$ Trans. & Total & Material & ${ }^{2}$ Trans. & Total & Material $\left.\right|^{2}$ & ${ }^{2}$ Trans & Total & & per $\mathrm{m}$ & & Total \\
\hline
\end{tabular}

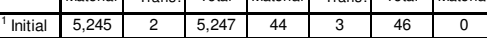

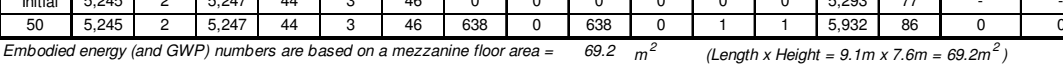

ATHENA $\circledast$ EIE Material List:

${ }^{2}$ Trans. $=$ Transportation

Total EE (or Total GWP) = Total embodied energy (or total embodied GWP) of building component after lifespan (i.e. total manufacturing + tota
construction + total maintenance + total end-oflifife effects) Total EE (or Total GWP) per $\mathrm{m}^{2}=$ Total EE (or Total GWP) of building - component/ area or billang componenthat was modeled in ATHENA® EI Total Difference in Operating Energy (or GWP) from Baseline after
Lifespan $=$ The difference in the total life-cycle operating energy (or GWP) Lifespan = The difiference in the total life-cycle operating energy (or GWP
from the basseline retail building after lifespan, due to using this building
component instead of the baseline component. component instead of the baseline component

Total Difference in Operating Energy (or GWP) from Baseline after Lifespan per $m^{2}=$ Total difference in operating energy (or GWP) from
baseline after lifespan / floor area of baseline retail building * Total operating primary energy use of baseline retail building after 50 years =
$50,700 \mathrm{GJ}\left(1,745 \mathrm{MJ} / \mathrm{m}^{2} \mathrm{yr}\right)$ "Total operating $G W$ P of baseline retail building after 50 years $=2,310$ tonnes
of $\mathrm{CO}_{2}$ eq. $\left(80 \mathrm{~kg}\right.$ of $\mathrm{CO}_{2}$ eq. $\left./ \mathrm{m}^{2} \mathrm{Ar}\right)$
(Includes all materials after 50 years)

\begin{tabular}{|l|c|c|}
\hline \multicolumn{1}{|c|}{ Material List } & Quantities & Unit \\
\hline 13mm Regular Gypsum Board & 152.2 & $\mathrm{~m} 2$ \\
\hline Batt. Fiberglass & 253.0 & $\mathrm{~m} 2(25 \mathrm{~mm})$ \\
\hline Concrete 20 MPa (flyash av) & 6.4 & $\mathrm{m3}$ \\
\hline Galvanized Decking & 684.5 & $\mathrm{~kg}$ \\
\hline Galvanized Studs & 40.4 & $\mathrm{~kg}$ \\
\hline Joint Compound & 151.9 & $\mathrm{~kg}$ \\
\hline Nails & 5.7 & $\mathrm{~kg}$ \\
\hline Open Web Joists & 891.6 & $\mathrm{~kg}$ \\
\hline Paper Tape & 1.7 & $\mathrm{~kg}$ \\
\hline Rebar, Rod, Light Sections & 62.5 & $\mathrm{~kg}$ \\
\hline Solvent Based Alkyd Paint & 14.6 & $\mathrm{~L}$ \\
\hline Vinyl Siding & 142.9 & $\mathrm{~m} 2$ \\
\hline Water Based Latex Paint & 90.0 & $\mathrm{~L}$ \\
\hline
\end{tabular}

\begin{tabular}{l} 
Notes: \\
'Initial $=T$ Time \\
\hline
\end{tabular}

${ }^{2}$ Trans. $=$ Transportation

Total EE (or Total GWP) = Total embodied energy (or total embodied GWP) of building component after lifespan (i.e. $t$ total mal mat
construction + total maintenance + total end-otlife effects)

${ }^{4}$ Total EE (or Total GWP) per $\mathrm{m}^{2}=$ Total EE (or Total GWP) of building 作 Total Difference in Operating Energy (or GWP) from Baseline after Lifespan = The difererence in the total life-cycle operating energy (or GWP,
from the baseline retail building after lifespan, due to using this building component instead of the baseline component 6 Total Difference in Operating Energy (or GWP) from Baseline after
Lifespan per $\mathrm{cm}^{2}=$ Total difference in operating energy (or GWP) from
baseline after lififspan / floor area of baseline retail building

* Total operating primary energy use of baseline retail building after 50 years
$50,700 \mathrm{GJ}\left(1,745 \mathrm{MJ} / \mathrm{m}^{2} \mathrm{yr}\right)$ "Total operating GWP of baseline retail building after 50 years $=2,310$ tonnes
of $\mathrm{CO}_{2}$ eq. $\left(80 \mathrm{~kg}\right.$ of $\mathrm{CO}_{2}$ eq/ $\left./ \mathrm{m}^{2} / \mathrm{yr}\right)$ 


\section{Floor \#4 (FL-4)}

Building Component Description:

\begin{tabular}{|c|c|c|c|}
\hline Category: & Mezzanine Floors & Assembly Layers & \\
\hline \multirow{4}{*}{ |Brief Description: } & \multirow{4}{*}{ 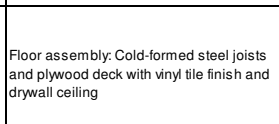 } & & \\
\hline & & Viny flloor tile & \\
\hline & & $\begin{array}{l}\text { Adhesive (modeled as allkyd based paint) } \\
\text { 19mm plwwood deck }\end{array}$ & \\
\hline & & $245 \mathrm{~mm} 1.52 \mathrm{~mm}$ galvanized cold-form & \\
\hline \multirow{2}{*}{\multicolumn{2}{|c|}{ Quick Numbers: }} & veight $4.2 \mathrm{~kg} / \mathrm{m}$ & \\
\hline & & $0.53 \mathrm{~mm}$ galvanized steel resilient channels & \\
\hline \begin{tabular}{|l} 
Foor Thickness: \\
\end{tabular} & $319 \mathrm{~mm}$ & $\begin{array}{l}\text { @ } 600 \mathrm{~mm} / \mathrm{oc} \\
\text { (self-weight } 0.31 \mathrm{~kg} / \mathrm{m} \text { ) }\end{array}$ & \\
\hline Span: & $\begin{array}{ll}\text { Range: } 1 \mathrm{~m} \text { to } 6 \mathrm{~m} & \text { Design: } 5 \mathrm{~m} \\
\mathrm{DL} & 22 \mathrm{LPa} \\
\end{array}$ & 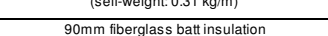 & \\
\hline Specified Design Loads: & 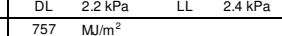 & 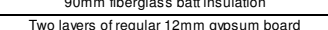 & \\
\hline \multirow{3}{*}{ Total Embodied GWP: } & $50 \mathrm{~kg}$ of $\mathrm{CO}_{2} \mathrm{eg} / \mathrm{m}^{2}$ & Latexpaint & \\
\hline & & Misc. fasteners, nails, and gavanized sheet & \\
\hline & & Bottom & \\
\hline
\end{tabular}

\section{Life-Cycle Assessment Results:}

Primary Energy Consumption (MJ)

\begin{tabular}{|c|c|c|c|c|c|c|c|c|c|c|c|c|c|c|c|c|}
\hline \multirow{3}{*}{$\begin{array}{l}\text { Lifiespan } \\
\text { (Years) }\end{array}$} & \multicolumn{14}{|c|}{ Embodied Energy (EE) } & \multirow{2}{*}{\multicolumn{2}{|c|}{$\begin{array}{c}\text { Difference in } \\
\text { Operating Energy } \\
\text { from Baseline after } \\
\text { Lifespan }\end{array}$}} \\
\hline & \multicolumn{3}{|c|}{ Manufacturing } & \multicolumn{3}{|c|}{ Construction } & \multicolumn{3}{|c|}{ Maintenance } & \multicolumn{3}{|c|}{ End of Life } & \multirow{2}{*}{$\begin{array}{l}{ }^{3} \text { Total } \\
\mathrm{EE}\end{array}$} & \multirow{2}{*}{$\begin{array}{c}{ }^{4} \text { Total } \\
\text { EE } \\
\text { per m }\end{array}$} & & \\
\hline & Material & ${ }^{2}$ Trans. & Total & Material & ${ }^{2}$ Trans. & Total & Material & \begin{tabular}{|l|}
${ }^{2}$ Trans. \\
\end{tabular} & Total & Material & |i Trans. & Total & & & ${ }^{5}$ Total & ${ }^{6}$ per $\mathrm{m}^{2}$ \\
\hline Initial & 22,541 & $\begin{array}{l}157 \\
\end{array}$ & 22,698 & 0 & 284 & 24 & 0 & 0 & 0 & 0 & 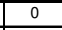 & 0 & 22,982 & 605 & & \\
\hline 50 & 22,541 & 157 & 22,698 & 0 & 284 & 284 & 5,680 & 35 & 5,715 & 1 & 87 & 88 & 28,785 & 757 & 0 & 0 \\
\hline
\end{tabular}

Global Warming Potential ( $k g$ of $\mathrm{CO}_{2}$ eq.)

\begin{tabular}{|c|c|c|c|c|c|c|c|c|c|c|c|c|c|c|c|c|}
\hline \multirow{4}{*}{\begin{tabular}{|l} 
Lifespan \\
(Years)
\end{tabular}} & \multirow{2}{*}{\multicolumn{14}{|c|}{ Embodied Global Warming Potential (GWP) }} & \multirow{3}{*}{\multicolumn{2}{|c|}{$\begin{array}{c}\text { Difference in } \\
\text { Operating GWP from } \\
\text { Baseline after } \\
\text { Lifespan }\end{array}$}} \\
\hline & & & & & & & & & & & & & & & & \\
\hline & \multicolumn{3}{|c|}{ Manufacturing } & \multicolumn{3}{|c|}{ Construction } & \multicolumn{3}{|c|}{ Maintenance } & \multicolumn{3}{|c|}{ End of Life } & \multirow{2}{*}{\begin{tabular}{|l}
${ }^{3}$ Total \\
GWPP
\end{tabular}} & \multirow{2}{*}{$\begin{array}{l}{ }^{4} \text { Total } \\
\text { GWP } \\
\text { per } \mathrm{m}^{2}\end{array}$} & & \\
\hline & \begin{tabular}{|l|} 
Materia \\
\end{tabular} & 1. ${ }^{2}$ Trans. & \begin{tabular}{|l|l|} 
Total \\
\end{tabular} & Materia & \begin{tabular}{|l|}
${ }^{2}$ Trans. \\
\end{tabular} & Total & \begin{tabular}{|l|} 
Material \\
\end{tabular} & \begin{tabular}{|l|}
${ }^{2}$ Trans. \\
\end{tabular} & \begin{tabular}{|l|} 
Total \\
\end{tabular} & \begin{tabular}{|l|} 
Material \\
\end{tabular} & \begin{tabular}{l|l|}
2 & 2 \\
2
\end{tabular} & Total & & & \begin{tabular}{|l|}
${ }^{5}$ Total \\
\end{tabular} & ${ }^{6}$ per $\mathrm{m}^{2}$ \\
\hline${ }^{1}$ Initial & \begin{tabular}{|l|l|}
1,542 \\
\end{tabular} & 0 & 1,542 & 0 & 1 & 1 & 0 & 0 & 0 & 0 & 0 & 0 & 1,543 & 41 & & \\
\hline 50 & 1,542 & 0 & 1,542 & 0 & 1 & 1 & 351 & 0 & 351 & 0 & 0 & 0 & $\begin{array}{l}1,895 \\
\end{array}$ & 50 & 0 & 0 \\
\hline
\end{tabular}

Embodied energy (and GWP) numbers are based on a mezzanine floor area = $38.0 \mathrm{~m}^{2} \quad$ (Length $x$ Height $=5.0 \mathrm{~m} \times 7.6 \mathrm{~m}=38.0 \mathrm{~m}^{2}$ )

ATHENA ® EIE Material List:

\begin{tabular}{|l|c|c|}
\hline \multicolumn{1}{|c|}{ Material List } & Quantities & Unit \\
\hline 13mm Regular Gypsum Board & 83.6 & $\mathrm{~m} 2$ \\
\hline Batt. Fiberglass & 139.0 & $\mathrm{~m} 2(25 \mathrm{~mm})$ \\
\hline Galvanized Studs & 444.3 & $\mathrm{~kg}$ \\
\hline Joint Compound & 83.4 & $\mathrm{~kg}$ \\
\hline Nails & 3.1 & $\mathrm{~kg}$ \\
\hline Paper Tape & 1.0 & $\mathrm{~kg}$ \\
\hline Screws Nuts \& Bolts & 5.5 & $\mathrm{~kg}$ \\
\hline Sottwood Plywood & 79.8 & $\mathrm{~m} 2(9 \mathrm{~mm})$ \\
\hline Solvent Based Alkyd Paint & 8.1 & $\mathrm{~L}$ \\
\hline Vinyl Siding & 78.7 & $\mathrm{~m} 2$ \\
\hline Water Based Latex Paint & 49.5 & $\mathrm{~L}$ \\
\hline
\end{tabular}

$$
\begin{aligned}
& \text { Notes: } \\
& { }^{1} \text { Initial }=\text { Time 'o' (i.e. at the completion of initial construction) }
\end{aligned}
$$

${ }^{1}$ Initial $=$ Time ' $O$ ' $($ i.e. at
${ }^{2}$ Trans. $=$ Transportation

${ }^{3}$ Total EE (or Total GWP) $=$ Total embodied energy (or total embodied GWP) of building component after lifespan (i.e. total manufacturing + tota
onstion construction + total maintenance + total end-ofilife effects)

${ }^{4}$ Total EE (or Total GWP) per $\mathrm{m}^{2}=$ Total EE (or Total GWP) of building ${ }^{5}$ Tote Differen in Operting Enry (or GWP) from Lifespan = The difference in the total life-cycle operating energy (or GWP from the baseline retaili building alter lifespant, due to using this building
component instead of the baseline component

${ }^{6}$ component instead of the baseline component
Tifference in Operating Energy (or GWP) from Baseline after
Lifespan per $m^{2}=$ Total difference in operating energy (or GWP) from Lifespan per $\mathrm{m}^{2}=$ Total difierence in operating energ
baseline after lifespan / floor area of baseline retail building "Total operating primary energy use of baseline retail building affer 50 years $=$

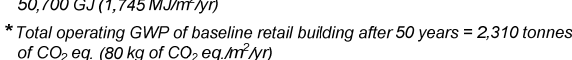

\section{Floor \#5 (FL-5)}

Building Component Description:

\begin{tabular}{|c|c|c|c|}
\hline Category: & Mezzanine Floors & Assembly Layers & \\
\hline \multirow{4}{*}{ Brief Description: } & \multirow{4}{*}{$\begin{array}{l}\text { Floor assembly: Traditional wood joists an } \\
\text { pilwwood deck with winy tile finish and } \\
\text { drywwall celling }\end{array}$} & $\begin{array}{ll}\text { Top } \\
\text { Vinylfoor tile }\end{array}$ & \\
\hline & & Achesive (modeleld as alkyd based paint) & \\
\hline & & $19 \mathrm{~mm}$ plywood deck & \\
\hline & & $\begin{array}{l}38 \mathrm{~mm} \times 286 \mathrm{~mm} \mathrm{SPF} \text { No. } 1 / \text { No. } 2 \text { solid wood } \\
\text { joists } @ 400 \mathrm{~mm} \text { occ }\end{array}$ & \\
\hline \multicolumn{2}{|l|}{ Quick Numbers: } & (Includes solid lumber bridging at $2,000 \mathrm{~mm}$ o/c) & \\
\hline \begin{tabular}{|l} 
Foor Thickness: \\
\end{tabular} & $360 \mathrm{~mm}$ & \multirow{3}{*}{$\begin{array}{l}0.53 \mathrm{~mm} \text { galvanized steel resilient channels } \\
\text { @6 600mm oc/ } \\
\text { (self-weight: } 0.31 \mathrm{~kg} / \mathrm{m} \text { ) }\end{array}$} & \\
\hline Span: & $\begin{array}{ll}\text { Range: } 1 \mathrm{~m} \text { to } 6 \mathrm{~m} & \text { Design: } 5 \mathrm{~m} \\
\end{array}$ & & \\
\hline Specified Design Loads: & $\begin{array}{llll}\mathrm{DL} & 2.3 \mathrm{kPa} & \mathrm{LL} & 2.4 \mathrm{kPa} \\
\end{array}$ & & \\
\hline \begin{tabular}{|l|} 
Total Embodied Energy: \\
Total Embodied GWP: \\
\end{tabular} & $\begin{array}{ll}559 & \mathrm{M} / \mathrm{m}^{2} \\
30 & \mathrm{~kg} \text { of } \mathrm{CO}_{2} \mathrm{eq} / \mathrm{m}^{2}\end{array}$ & 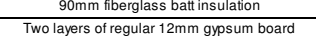 & \\
\hline \multirow[t]{3}{*}{\begin{tabular}{|l|} 
Total Embodied GWP: \\
\end{tabular}} & $30 \mathrm{~kg}$ of $\mathrm{CO}_{2} \mathrm{eq} / \mathrm{m}^{2}$ & $\begin{array}{c}\text { Two layers of regular 12mm gypsum board } \\
\text { Latexpaint }\end{array}$ & \\
\hline & & Misc. fasteners, nails, and galvanized sheet & \\
\hline & & Bottom & \\
\hline
\end{tabular}

\section{Life-Cycle Assessment Results:}

Primary Energy Consumption (MJ)

\begin{tabular}{|c|c|c|c|c|c|c|c|c|c|c|c|c|c|c|c|c|}
\hline \multirow{3}{*}{\begin{tabular}{|c} 
Lifespan \\
(Years)
\end{tabular}} & \multicolumn{14}{|c|}{ Embodied Energy (EE) } & \multirow{2}{*}{\multicolumn{2}{|c|}{$\begin{array}{l}\text { Difference in } \\
\text { Operating Energy } \\
\text { trom Baseline after } \\
\text { Litespan }\end{array}$}} \\
\hline & \multicolumn{3}{|c|}{ Manutacturing } & \multicolumn{3}{|c|}{ Constr } & \multicolumn{3}{|c|}{ Maintenance } & \multicolumn{3}{|c|}{ End of Life } & \multirow{2}{*}{$-{ }^{3}$ Total } & \multirow{2}{*}{$\begin{array}{c}{ }^{4} \text { Total } \\
\text { EE } \\
\text { per m }{ }^{2}\end{array}$} & & \\
\hline & Material & ${ }^{2}$ Trans & Total & Material & ${ }^{2}$ Trans. & Total & Material & ${ }^{2}$ Trans. & Total & Material & \begin{tabular}{|l|}
${ }^{2}$ Trans. \\
\end{tabular} & Total & & & ${ }^{{ }^{5} \text { Total }}$ & ${ }^{6}$ per m ${ }^{2}$ \\
\hline Initial & $\overline{14,264}$ & 238 & 14,502 & 238 & 684 & 922 & 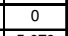 & 0 & 0 & 0 & 0 & 0 & 15,425 & 406 & & \\
\hline 50 & 14,264 & 238 & 14,502 & 238 & 684 & 922 & 5,679 & 35 & 5,715 & 1 & 85 & 86 & 21,225 & 559 & 0 & \\
\hline
\end{tabular}

Global Warming Potential (kg of $\mathrm{CO}_{2}$ eq.)

\begin{tabular}{|c|c|c|c|c|c|c|c|c|c|c|c|c|c|c|c|c|}
\hline & \multirow{2}{*}{\multicolumn{14}{|c|}{ Embodied Global Warming Potential (GWP) }} & & \\
\hline \multirow{3}{*}{$\begin{array}{l}\text { Lifespan } \\
\text { (Years) }\end{array}$} & & & & & & & & & & & & & & & \multirow{2}{*}{\multicolumn{2}{|c|}{$\begin{array}{l}\text { Difference in } \\
\text { Dperating GWP from } \\
\text { Baseline after } \\
\text { Lifespan }\end{array}$}} \\
\hline & \multicolumn{3}{|c|}{ Manutacturing } & \multicolumn{3}{|c|}{ Construction } & \multicolumn{3}{|c|}{ Maintenance } & \multicolumn{3}{|c|}{ End of Life } & \multirow{2}{*}{$\begin{array}{l}{ }^{3} \text { Total } \\
\text { GWP }\end{array}$} & \multirow{2}{*}{$\begin{array}{c}{ }^{4} \text { Total } \\
\mathrm{GWP} \\
\text { per } \mathrm{m}^{2}\end{array}$} & & \\
\hline & \begin{tabular}{|l|} 
Material \\
\end{tabular} & ${ }^{2}$ Trans. & Total & \begin{tabular}{|l|} 
Material \\
\end{tabular} & \begin{tabular}{|l|}
${ }^{2}$ Trans. \\
\end{tabular} & Total & Material & Trans. & $\begin{array}{l}\text { Total } \\
\end{array}$ & \begin{tabular}{|l|} 
Material \\
\end{tabular} & \begin{tabular}{|l|}
${ }^{2}$ Trans. \\
\end{tabular} & $\begin{array}{l}\text { Total } \\
\end{array}$ & & & ${ }^{5}{ }^{5}$ total & ${ }^{6}$ per $\mathrm{m}^{2}$ \\
\hline Initial & 788 & 0 & 789 & 16 & 1 & 17 & 0 & $\overline{0}$ & 0 & $\overline{0}$ & 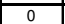 & 0 & 805 & 21 & & \\
\hline 50 & 788 & 0 & 789 & 16 & 1 & 17 & 351 & 0 & 351 & 0 & 0 & 0 & 1,157 & 30 & 0 & 0 \\
\hline
\end{tabular}

Embodied energy (and GWP) numbers are based on a mezzanine floor area = $38.0 \mathrm{~m}^{2} \quad$ (Length $\times$ Height $=5.0 \mathrm{~m} \times 7.6 \mathrm{~m}=38.0 \mathrm{~m}^{2}$ )
ATHENA ${ }^{\circledR}$ EIE Material List:

(Includes all materials after 50 years)

\begin{tabular}{|l|c|c|}
\hline \multicolumn{1}{|c|}{ Material List } & Quantities & Unit \\
\hline 13mm Regular Gypsum Board & 83.6 & $\mathrm{~m} 2$ \\
\hline Batt. Fiberglass & 138.9 & $\mathrm{~m} 2(25 \mathrm{~mm})$ \\
\hline Galvanized Sheet & 0.0 & $\mathrm{~kg}$ \\
\hline Galvanized Studs & 36.4 & $\mathrm{~kg}$ \\
\hline Joint Compound & 83.4 & $\mathrm{~kg}$ \\
\hline Large Dimension Softwood Lumber, \\
kiln-dried & 0.9 & $\mathrm{~m} 3$ \\
\hline Nails & 8.6 & $\mathrm{~kg}$ \\
\hline Paper Tape & 1.0 & $\mathrm{~kg}$ \\
\hline Softwood Plywood & 79.8 & $\mathrm{~m} 2(9 \mathrm{~mm})$ \\
\hline Solvent Based Alkyd Paint & 8.1 & $\mathrm{~L}$ \\
\hline Vinyl Siding & 78.7 & $\mathrm{~m} 2$ \\
\hline Water Based Latex Paint & 49.4 & $\mathrm{~L}$ \\
\hline
\end{tabular}

$$
\text { 'Initial = Time ' } O \text { ' (i.e. at the completion of init }
$$

$$
\text { Notes: }
$$

${ }^{3}$ Total EE (or Total GWP) $=$ Total embodied energy (or total embodied GWP) of building component after lifespan (i.e. total man
construction t total maintenance + total end-oflifife effects)

${ }^{4}$ Total EE (or Total GWP) per $\mathrm{m}^{2}=$ Total EE (or Total GWP) of building
component/ area of building component that was modelled in ATHENAB EEE 5 Total Difference in Operating Energy (or GWP) from Baseline after
Lifespan = The difference in the total life-cycle operating energy (or GWPP)
from the baseline retail butiding a atter lifespan, due to using this building
component instead of the baseline component ${ }^{6}$ Total Difference in Operating Energy (or GWP) from Baseline after Total Difference in Operating Energy (or GWP) from Baseline after
Lifespan per $\mathrm{m}^{2}=$ Total difference in operating energy (or GWP) from
baseline after lifespan / floor area of baseline retail building * Total operating primary energy use of baseline retail building after 50 years = $50,700 \mathrm{GJ}\left(1,745 \mathrm{MJ} / \mathrm{m}^{2} / \mathrm{rr}\right)$

* Total operating GWP of baseline retail building after 50 years $=2,310$ tonnes
of $\mathrm{CO}_{2}$ eq. $\left(80 \mathrm{~kg}\right.$ of $\mathrm{CO}_{2}$ eq. $\left./ \mathrm{m}^{2} / \mathrm{yr}\right)$ 


\section{Appendix B-5}

LCA Data for Windows and Doors 


\section{LCA Data for Windows}

This section contains a detailed description of each window that was examined in this study ( 9 in total). The assembly layers are listed for each window, along with a detailed description of the material quantities from the ATHENA ${ }^{\circledR}$ Environmental Impact Estimator for Buildings.

A breakdown of the total primary energy consumption and the total global warming potential (GWP) for each window is also included. In each case, the results were calculated for a window area equal to 2.9 $\mathrm{m}^{2}$, which represents a typical window size for a single-storey retail building. The results are also expressed on a per $\mathrm{m}^{2}$ basis in each case. The data has been calculated for two different lifespans: at the completion of initial construction and after 50 years.

I As a summary, the figure below illustrates a comparison of the total embodied energy (and GWP) after 50 years for the various windows in this section. For comparison purpose, the building component used in the baseline retail building has been shaded in grey.

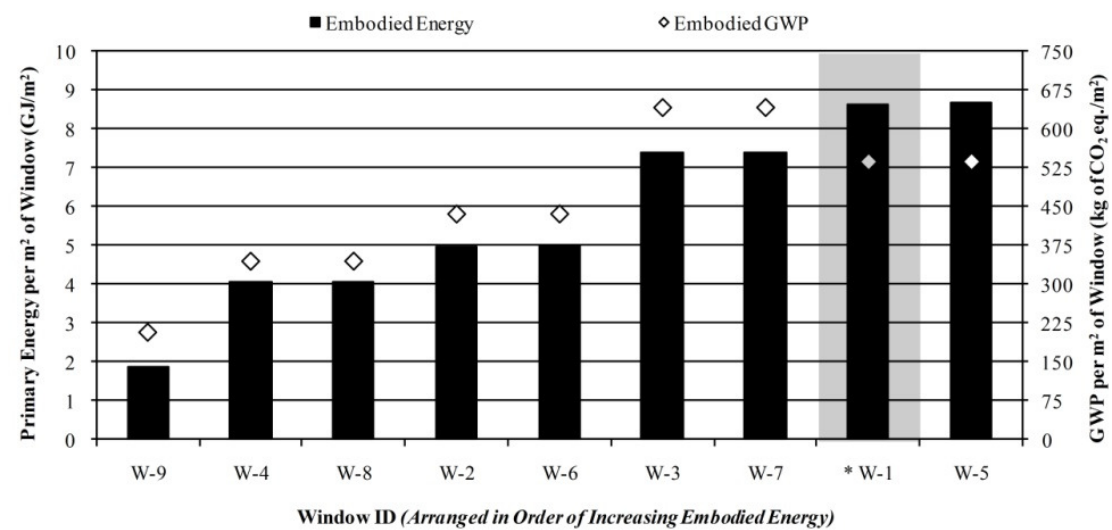

\section{Window \#1 (W-1)}

Building Component Description:

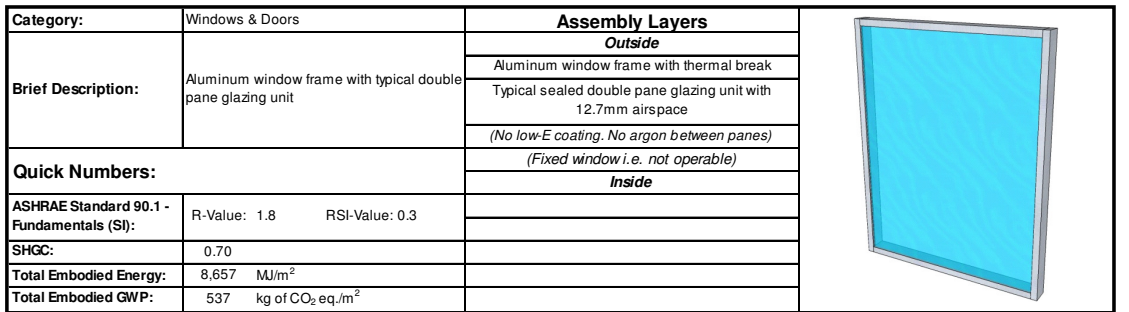

Life-Cycle Assessment Results:

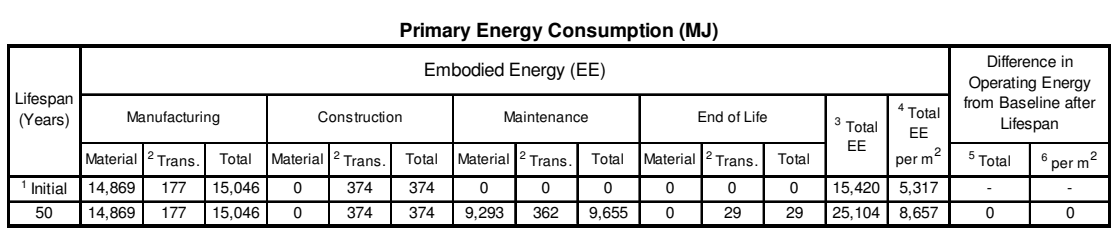

Global Warming Potential $\left(\mathrm{kg}\right.$ of $\mathrm{CO}_{2}$ eq $)$

\begin{tabular}{|c|c|c|c|c|c|c|c|c|c|c|c|c|c|c|c|c|}
\hline \multirow{3}{*}{$\begin{array}{l}\text { Lifespan } \\
\text { (Years) }\end{array}$} & \multicolumn{14}{|c|}{ Embodied Global Warming Potential (GWP) } & \multirow{2}{*}{\multicolumn{2}{|c|}{$\begin{array}{c}\text { Difierence in } \\
\text { Operating GWP from } \\
\text { Baseline after } \\
\text { Lifespan }\end{array}$}} \\
\hline & \multicolumn{3}{|c|}{ Manufacturing } & \multicolumn{3}{|c|}{ Construction } & \multicolumn{3}{|c|}{ Maintenance } & \multicolumn{3}{|c|}{ End of Life } & \multirow{2}{*}{$\begin{array}{l}{ }^{3} \text { Total } \\
\text { GWP }\end{array}$} & \multirow{2}{*}{\begin{tabular}{|l|}
${ }^{4}$ Total \\
GWP \\
per m
\end{tabular}} & & \\
\hline & Material & ${ }^{2}$ Trans. & Total & Material & 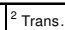 & Total & \begin{tabular}{|l|} 
Material \\
\end{tabular} & \begin{tabular}{|l|}
${ }^{2}$ Trans. \\
\end{tabular} & Total & Material & \begin{tabular}{|l|l|}
${ }^{2}$ & Trans.
\end{tabular} & \begin{tabular}{|l|l|} 
Total \\
\end{tabular} & & & ${ }^{5}$ Total & ${ }^{6}$ per $m^{2}$ \\
\hline${ }^{1}$ Initial & 958 & 0 & 958 & 0 & 1 & 1 & 0 & 0 & 0 & 0 & 0 & 0 & 959 & 331 & & \\
\hline 50 & \begin{tabular}{|l|}
958 \\
\end{tabular} & 0 & 958 & 0 & 1 & 1 & 599 & 1 & \begin{tabular}{|l|}
599 \\
\end{tabular} & 0 & 0 & 0 & 1,558 & 537 & 0 & 0 \\
\hline
\end{tabular}

Total window area of baseline retail building (total fenestration area) $=$

ATHENA ® EIE Material List:

(Includes all materials after 50 years)

\begin{tabular}{|l|c|c|}
\hline \multicolumn{1}{|c|}{ Material List } & Quantities & Unit \\
\hline Aluminum & 179.0 & $\mathrm{~kg}$ \\
\hline EPDM membrane & 11.8 & $\mathrm{~kg}$ \\
\hline Nails & 10.2 & $\mathrm{~kg}$ \\
\hline Standard Glazing & 12.7 & $\mathrm{~m} 2$ \\
\hline
\end{tabular}

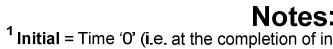

1 Initial $=$ Time ' $O$ ' (i.e. at the
${ }^{2}$ Trans $=$ Transportation
${ }^{3}$ Totis

Total EE (or Total GWP) $=$ Total embodied energy (or total embodied GWP of building component after lifespan (i.e. total manuffacturing + tota

${ }^{4}$ Total EE (or Total GWP) per $\mathrm{m}^{2}=$ Total EE (or Total GWP) of building
component / area of building component that was modelled in ATHENAB EIE ${ }^{5}$ Total Difference in Operating Energy (or GWP) from Baseline after Lifespan = The difference in the total life-cycle operating energy (or GWP)
from the baseline retail building after lifespan, due to using this building rrom the baseline retail building after lifespent
component instead of the baseline component

${ }^{6}$ Total Difference in Operating Energy (or GWP) from Baseline after Lifespan per $\mathrm{m}^{2}=$ Total difference in operating energy (or GWP) from * Total operating primary energy use of baseline retail building after 50 years
$50,700 \mathrm{GJ}\left(1,745 \mathrm{M} \mathrm{Vm}^{2} \mathrm{Mr}\right)$ "Total operating $\mathrm{GWP}^{\mathrm{N}}$ of baseline ret) 
Window \#2 (W-2)

Building Component Description:

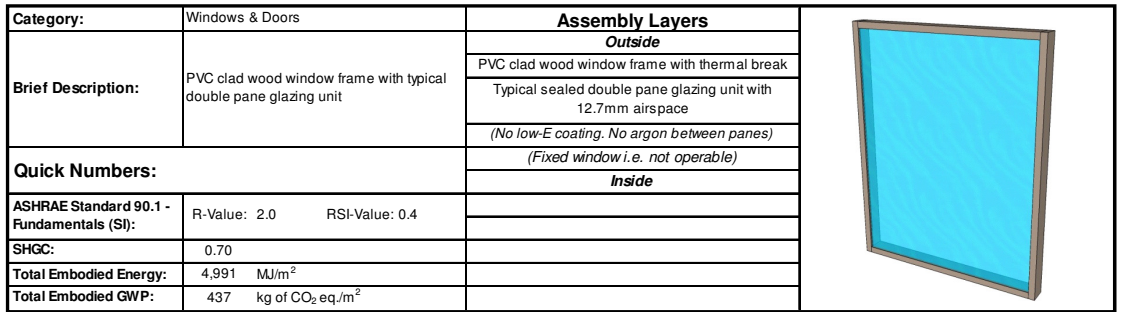

\section{Life-Cycle Assessment Results:}

Primary Energy Consumption (MJ)

\begin{tabular}{|c|c|c|c|c|c|c|c|c|c|c|c|c|c|c|c|c|}
\hline \multirow{3}{*}{\begin{tabular}{|l} 
Lifespan \\
(Years)
\end{tabular}} & \multicolumn{14}{|c|}{ Embodied Energy (EE) } & \multirow{2}{*}{\multicolumn{2}{|c|}{$\begin{array}{c}\text { Difference in } \\
\text { Operating Energy } \\
\text { from Baseline afte } \\
\text { Lifespan }\end{array}$}} \\
\hline & \multicolumn{3}{|c|}{ Manufactur } & \multicolumn{3}{|c|}{ onstruc } & \multicolumn{3}{|c|}{ Maintenance } & \multicolumn{3}{|c|}{ End of Life } & \multirow{2}{*}{$\begin{array}{c}{ }^{3} \text { Total } \\
\mathrm{EE}\end{array}$} & \multirow{2}{*}{\begin{tabular}{|c|}
${ }^{4}$ Total \\
EE \\
per $\mathrm{m}^{2}$
\end{tabular}} & & \\
\hline & Materiz & ${ }^{2}$ Trans & \begin{tabular}{|l|} 
Total \\
\end{tabular} & |Materia & ${ }^{2}$ Trans. & Total & \begin{tabular}{|l|} 
Materialal \\
\end{tabular} & ${ }^{2}$ Trans. & \begin{tabular}{|l|l|} 
Total \\
\end{tabular} & Material & $\begin{array}{l}2 \\
2\end{array}$ Trans. & \begin{tabular}{|l|l|} 
Total \\
\end{tabular} & & & \begin{tabular}{|l|}
${ }^{5}$ Total \\
\end{tabular} & ${ }^{6}$ perm \\
\hline |litial & \begin{tabular}{|l|l|}
8,858 \\
\end{tabular} & 85 & 8, & 0 & 179 & 179 & 0 & 0 & 0 & 0 & n & 0 & 9,122 & 3,146 & & \\
\hline 50 & 8,858 & 85 & 8,943 & 0 & \begin{tabular}{|l|l|}
179 \\
\end{tabular} & 179 & 5,150 & 171 & 5,321 & 0 & 30 & 30 & \begin{tabular}{|l|l|l|}
1474 \\
\end{tabular} & 4,991 & $-200,000$ & $-1,351$ \\
\hline
\end{tabular}

Global Warming Potential ( $\mathrm{kg}$ of $\mathrm{CO}_{2}$ eq.)

\begin{tabular}{|c|c|c|c|c|c|c|c|}
\hline \multirow{3}{*}{$\begin{array}{l}\text { Lifespar } \\
\text { (Years) }\end{array}$} & \multicolumn{7}{|c|}{ Embodied Global $\mathrm{V}$} \\
\hline & \multicolumn{3}{|c|}{ Manufacturing } & \multicolumn{3}{|c|}{ Construction } & \\
\hline & Material & ${ }^{2}$ Trans. & \begin{tabular}{|l|l|} 
Total \\
\end{tabular} & Materia & ${ }^{2}$ Trans. & Total & \\
\hline 1 Initial & & 0 & 800 & 0 & 0 & 0 & \\
\hline 50 & 800 & 0 & 800 & 0 & 0 & 0 & \\
\hline \multicolumn{8}{|c|}{$\begin{array}{l}\text { Embodied energy (and GWP) numbers are based on a window area } \\
\text { Total window area of baseline retall building (total fenestration area) }\end{array}$} \\
\hline \multicolumn{8}{|c|}{$\begin{array}{l}\text { ATHENA @ EIE Material List: } \\
\text { (Includes all materials atter 50 years) }\end{array}$} \\
\hline \multicolumn{4}{|c|}{ Material List } & \multicolumn{2}{|c|}{ Quantities } & \multicolumn{2}{|c|}{ Unit } \\
\hline \multicolumn{4}{|c|}{ Batt. Fiberglass } & \multicolumn{2}{|r|}{0.6} & \multicolumn{2}{|c|}{$\mathrm{m} 2(25 \mathrm{~mm}$} \\
\hline \multicolumn{4}{|c|}{ EPDM membrane } & & 10.3 & \multicolumn{2}{|c|}{$\mathrm{kg}$} \\
\hline \multicolumn{4}{|l|}{ Nails } & & 8.9 & \multicolumn{2}{|c|}{$\mathrm{kg}$} \\
\hline \multicolumn{4}{|c|}{$\begin{array}{l}\text { Small Dimension Softwood Lumber, } \\
\text { kiln-dried }\end{array}$} & & 0.3 & \multicolumn{2}{|c|}{$\mathrm{m} 3$} \\
\hline \multicolumn{4}{|c|}{ Standard Glazing } & & 11.1 & \multirow{2}{*}{\multicolumn{2}{|c|}{$\mathrm{m} 2$}} \\
\hline \multicolumn{4}{|c|}{ Vinyl Siding } & & 29.9 & & \\
\hline
\end{tabular}

Vinyl Siding

Notes:
${ }^{1}$ Initial $=$ Time '
2 ' (i.e. at the completion of initial construction)

Window \#3 (W-3)

Building Component Description:

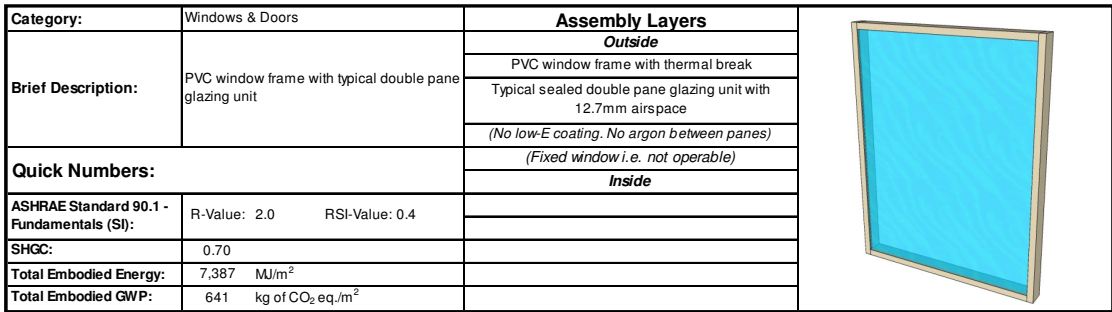

\section{Life-Cycle Assessment Results:}

\begin{tabular}{|c|c|c|c|c|c|c|c|c|c|c|c|c|c|c|c|c|}
\hline \multirow{4}{*}{$\begin{array}{l}\text { Lifespan } \\
\text { (Years) }\end{array}$} & \multicolumn{14}{|c|}{ ry Energy Consumption (MJ) } & \multirow{3}{*}{\multicolumn{2}{|c|}{$\begin{array}{l}\text { Difference in } \\
\text { Operating Energy } \\
\text { from Baseline after } \\
\text { Lifespan }\end{array}$}} \\
\hline & \multicolumn{14}{|c|}{ Embodied Energy (EE) } & & \\
\hline & \multicolumn{3}{|c|}{ Manutacturing } & \multicolumn{3}{|c|}{ Construction } & \multicolumn{3}{|c|}{ Main } & \multicolumn{3}{|c|}{ End of Life } & \multirow{2}{*}{$\mid \begin{array}{c}{ }^{3} \text { Total } \\
\mathrm{EE}\end{array}$} & \multirow{2}{*}{\begin{tabular}{|c}
${ }^{4}$ Total \\
EE \\
per m
\end{tabular}} & & \\
\hline & Material & ${ }^{2}$ Trans. & Total & Material & ${ }^{2}$ Trans. & Total & Material & $2^{2}$ Trans. & Total & Material & $2^{2}$ Trans. & Total & & & ${ }^{5}$ Total & 6 perm ${ }^{2}$ \\
\hline Initial & 13,302 & 71 & 13,373 & 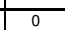 & 137 & 137 & $\pi$ & 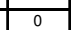 & - & 0 & 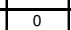 & - & 13,511 & 4,659 & & \\
\hline 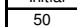 & 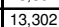 & 71 & 13, & 0 & 137 & 137 & 7,734 & 142 & 376 & 0 & 35 & 35 & 4 & 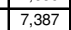 & $-200,000$ & $-1,35$ \\
\hline
\end{tabular}

Global Warming Potential ( $k g$ of $\mathrm{CO}_{2}$ eq.)

\begin{tabular}{|c|c|c|c|c|c|c|c|c|c|c|c|c|c|c|c|c|}
\hline \multirow{3}{*}{$\begin{array}{l}\text { Lifespan } \\
\text { (Years) }\end{array}$} & \multicolumn{14}{|c|}{ died Global Warming Potential (GWP) } & \multirow{2}{*}{\multicolumn{2}{|c|}{$\begin{array}{c}\text { Difference in } \\
\text { Operating GWP from } \\
\text { Baseline after } \\
\text { Lifespan }\end{array}$}} \\
\hline & \multicolumn{3}{|c|}{ Manufacturing } & \multicolumn{3}{|c|}{ tion } & \multicolumn{3}{|c|}{ Maintenance } & \multicolumn{3}{|c|}{ End of 1} & \multirow{2}{*}{$\begin{array}{l}{ }^{3} \text { Total } \\
\text { GWPP }\end{array}$} & \multirow{2}{*}{$\begin{array}{l}{ }^{4} \text { Total } \\
\text { GWP } \\
\text { per m }\end{array}$} & & \\
\hline & Material & ${ }^{2}$ Trans. & Total & Material & ${ }^{2}$ Trans. & Total & Material & ${ }^{2}$ Trans. & Total & Material & ${ }^{2}$ Trans. & Total & & & ${ }^{5}$ Total & ${ }^{6}$ perm $^{2}$ \\
\hline tial & 76 & 0 & 1,176 & 0 & 0 & 0 & 0 & 0 & 0 & 0 & 0 & 0 & 1,176 & 406 & & \\
\hline 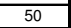 & 1,176 & 0 & \begin{tabular}{|l|l}
1,176 \\
\end{tabular} & 0 & $\overline{0}$ & 0 & 684 & $\overline{0}$ & 684 & 0 & $\overline{0}$ & $\overline{0}$ & 1,860 & 641 & & -68 \\
\hline
\end{tabular}

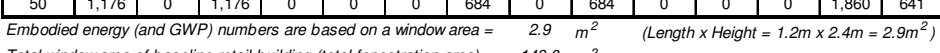
Total window area of baseline retail building (total fenestration area) $=148.0 \mathrm{~m}^{2}$

ATHENA ® EIE Material List:

${ }^{2}$ Trans. $=$ Transportation

${ }^{3}$ Total EE (or Total GWP) $=$ Total embodied energy (or total embodied GWP) of building component after lifespan (i.e. total manufacturing + tota ${ }^{4}$ Total EE (or Total GWP) per $\mathrm{m}^{2}=$ Total EE (or Total GWP) of building ${ }^{5}$ Total Difference in Opering Energy (or GWP) from Baselne aWr) Lifespan $=$ The difference in the total life-cycle operating energy (or GWP)
from the baseline retail building after lifespan, due to using this building from the baseline retail building after lifespan, due to using this building
component instead of the baseline component ${ }^{6}$ Total Difference in Operating Energy (or GWP) from Baseline after Total Difference in Operating Energy (or GWP) from Baseline after
Lifespan per $\mathrm{m}^{2}=$ Total difference in operating energy (or GWP) from
baseline after lifespan / window area of baseline retail building baseline after lifespan / window area of baseline retail builling
* Total operating primary energy use of baseline retail building after 50 years $=$

* Total operating GWP of baseline retail building after 50 years $=2,310$ tonnes

\begin{tabular}{|l|c|c|}
\hline \multicolumn{1}{|c|}{ (Includes all materials after 50 years) } \\
\hline \multicolumn{1}{|c|}{ Material List } & Quantities & Unit \\
\hline Batt. Fiberglass & 0.6 & $\mathrm{~m} 2(25 \mathrm{~mm})$ \\
\hline EPDM membrane & 10.3 & $\mathrm{~kg}$ \\
\hline Nails & 8.9 & $\mathrm{~kg}$ \\
\hline Standard Glazing & 11.1 & $\mathrm{~m} 2$ \\
\hline Vinyl Siding & 74.9 & $\mathrm{~m} 2$ \\
\hline
\end{tabular}

Notes:

${ }^{2}$ Trans. $=$ Transportation

${ }^{3}$ Total EE (or Total GWP) $=$ Total embodied energy (or total embodied GWP) of building component after lifespan (i.e. total manufacturing + tota ${ }^{4}$ Total EE (or Total GWP) per $\mathrm{m}^{2}=$ Total EE (or Total GWP) of building
component / area of building component that was modelled in ATHENAQ EIE ${ }^{5}$ Total Difference in Operating Energy (or GWP) from Baseline after Lifespan = The difference in the total life-cycle operating energy (or GWP)
from the basaline retail building after lifespan, due to using this building
component instead of the baseline component

Lifespan per $\mathrm{m}^{2}=$ Total difference in operating energy (or GWP) from baseline after ilitespan/window area of baseline retail building ${ }^{*}$ Total operating primary energy use of baseline retail building after 50 years
$50,700 \mathrm{G}\left(1,745 \mathrm{MU} / \mathrm{m}^{2} / \mathrm{yr}\right)$

${ }^{*}$ Total operating $\mathrm{GWP}$ of baseline retail building after 50 years $=2,310$ tonnes
of $\mathrm{CO}_{2} \mathrm{eq}\left(8 \mathrm{~kg}\right.$ of $\left.\mathrm{CO}_{2} \mathrm{eq} / \mathrm{m}^{2} \mathrm{Ar}\right)$ 


\section{Window \#4 (W-4)}

Building Component Description:

\begin{tabular}{|c|c|c|}
\hline \multirow{4}{*}{ Brief Description: } & \multirow{4}{*}{$\begin{array}{l}\text { Wood window frame with ypical double } \\
\text { pane glazing unit }\end{array}$} & Assembly Layers \\
\hline & & $\begin{array}{l}\text { Outside } \\
\text { Wood window frame with thermal break }\end{array}$ \\
\hline & & $\begin{array}{l}\text { Typical sealed double pane glazing unit with } \\
12.7 \mathrm{~mm} \text { airspace }\end{array}$ \\
\hline & & (No low-E coating. No argon between panes) \\
\hline \multicolumn{2}{|l|}{ Quick Numbers: } & $\begin{array}{l}\text { (Fixed windowi.e. not operable) } \\
\text { (Iside }\end{array}$ \\
\hline \begin{tabular}{|l|} 
ASHRAE Standard 90.1- \\
\end{tabular} & R.Value: 2.0 & \\
\hline $\begin{array}{l}\text { fundamentals (SI): } \\
\text { SH@C: }\end{array}$ & 0.70 & \\
\hline Total Embodied Energy: & $4,084 \quad \mathrm{~m} / \mathrm{m}^{2}$ & \\
\hline Total Embodied GWP & 346 & \\
\hline
\end{tabular}

\section{Life-Cycle Assessment Results:}

Primary Energy Consumption (MJ)

\begin{tabular}{|c|c|c|c|c|c|c|c|c|c|c|c|c|c|c|c|c|}
\hline \multirow{3}{*}{\begin{tabular}{|l} 
Lifespan \\
(Years)
\end{tabular}} & \multicolumn{14}{|c|}{ Embodied Energy (EE) } & \multirow{2}{*}{\multicolumn{2}{|c|}{$\begin{array}{c}\text { Difference in } \\
\text { Operating Energy } \\
\text { from Baseline afte } \\
\text { Lifespan }\end{array}$}} \\
\hline & \multicolumn{3}{|c|}{ Manufacturing } & \multicolumn{3}{|c|}{ Construction } & \multicolumn{3}{|c|}{ Maintenance } & \multicolumn{3}{|c|}{ End of Lifie } & \multirow{2}{*}{$\begin{array}{c}{ }^{3} \text { Total } \\
\mathrm{EE}\end{array}$} & \multirow{2}{*}{$\begin{array}{c}{ }^{4} \text { Total } \\
\text { EE } \\
\text { per m }\end{array}$} & & \\
\hline & Materia & ${ }^{2}$ Trans & \begin{tabular}{|l|l} 
Total \\
\end{tabular} & Materia & ${ }^{2}$ Trans. & \begin{tabular}{|l|l|l|} 
Total & \\
\end{tabular} & Material & ${ }^{2}$ Trans. & \begin{tabular}{|l|l|l|} 
Total & \\
\end{tabular} & Material & 1. ${ }^{2}$ Trans. & Total & & & ${ }^{5}$ Total & ${ }^{6}$ per m$^{2}$ \\
\hline |nitial & 7,025 & 94 & 7,119 & 0 & 213 & 213 & 0 & 0 & 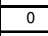 & 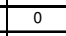 & 0 & 0 & 7,331 & 2,528 & & \\
\hline 50 & 7,025 & 94 & $\begin{array}{l}7,119 \\
\end{array}$ & 0 & 213 & \begin{tabular}{|l|l|}
213 \\
\end{tabular} & 4,284 & 203 & \begin{tabular}{|l|l|}
4,487 \\
\end{tabular} & 0 & 27 & 27 & 111,844 & 4,084 & $-200,000$ & $-1,35$ \\
\hline
\end{tabular}

Global Warming Potential ( $k g$ of $\mathrm{CO}_{2}$ eq.)

\begin{tabular}{|c|c|c|c|c|c|c|c|}
\hline \multirow{3}{*}{$\mid$\begin{tabular}{|c} 
Lifespan \\
(Years)
\end{tabular}} & \multicolumn{7}{|c|}{ Embodi } \\
\hline & \multicolumn{3}{|c|}{ Manufacturing } & \multicolumn{3}{|c|}{ Construction } & \\
\hline & Material & | ${ }^{2}$ Trans. & \begin{tabular}{|l|l|l|} 
Total \\
\end{tabular} & Materiz & 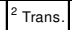 & Total & \\
\hline${ }^{1}$ Initial & 622 & 0 & 623 & 0 & 0 & 0 & \\
\hline & & & & & & & \\
\hline \multicolumn{8}{|c|}{$\begin{array}{l}\text { Embodied energy (and GWP) numbers are based on a window warea } \\
\text { Total window area of baseline retail building (total fenestration area) }\end{array}$} \\
\hline \multicolumn{8}{|c|}{$\begin{array}{l}\text { ATHENA @ EIE Material List: } \\
\text { (Includes all materials after } 50 \text { years) }\end{array}$} \\
\hline \multicolumn{4}{|c|}{ Material List } & \multicolumn{2}{|c|}{ Quantities } & \multicolumn{2}{|c|}{ Unit } \\
\hline \multicolumn{5}{|c|}{ Aluminum } & 20.9 & $\mathrm{~kg}$ & \\
\hline \multicolumn{5}{|c|}{ Batt. Fiberglass } & 0.6 & I $\mathrm{m} 2_{2}^{25}$ & \\
\hline \multicolumn{5}{|c|}{ EPDM membrane } & 11.2 & $\mathrm{~kg}$ & \\
\hline \multicolumn{5}{|l|}{ Nails } & 10.0 & $\mathrm{~kg}$ & \\
\hline \multicolumn{5}{|c|}{$\begin{array}{l}\text { Small Dimen } \\
\text { kiln-dried }\end{array}$} & 0.3 & $\mathrm{~m}$ & \\
\hline \multicolumn{5}{|c|}{ Standard Glazing } & 12.1 & $\mathrm{~m}$ & \\
\hline \multicolumn{4}{|l|}{ - } & & 1.2 & & \\
\hline
\end{tabular}

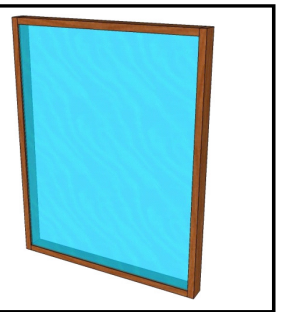

\section{ATHENA ® EIE Material List:}

Notes:
${ }^{1}$ Initial $=$ Time 'o' (i.e. at the completion of initial construction)

${ }^{2}$ Trans. $=$ Transportation

Total EE (or Total GWP) = Total embodied energy (or total embodied GWP) of building component after lifespan (i.e. total manufacturing + to ${ }^{4}$ Total EE (or Total GWP) per $\mathrm{m}^{2}=$ Total EE (or Total GWP) of building
component/ area of building component that was modeled in ATHENAQIEE ${ }^{5}$ Total Difference in Operating Energy (or GWP) from Baselne after) Lifespan = The difference in the total life-cycle operatiting energy (or GWP)
from the baseline retail building after lifespan, due to using this building from the baseline retail building after lifespen,
component instead of the baseline component

${ }^{6}$ Total Difference in Operting Energy (or GWP) from Boser Lifespan per $\mathrm{m}^{2}=$ Total difference in operating energy (or GWP) from baseline after lifespan $/$ window area of baseline retail building * Total operating primary energy use of baseline retail building after 50 years

"Total operating GWP of baseline retail building after 50 years $=2,310$ tonnes

\section{Life-Cycle Assessment Results:}

\begin{tabular}{|c|c|c|c|c|c|c|c|c|c|c|c|c|c|c|c|c|}
\hline & & & & & & Prime & ry Ene & $\mathrm{rgy}$ Co & sump & tion (M & & & & & & \\
\hline \multirow{3}{*}{$\begin{array}{l}\text { Lifespan } \\
\text { (Years) }\end{array}$} & & & & & & & bodied & Energy ( & & & & & & & \multirow{2}{*}{\multicolumn{2}{|c|}{$\begin{array}{l}\text { Difference in } \\
\text { Operatitg Energy } \\
\text { from Baseline after } \\
\text { Lifessan }\end{array}$}} \\
\hline & \multicolumn{3}{|c|}{ Manutacturing } & \multicolumn{3}{|c|}{ Construction } & \multicolumn{3}{|c|}{ Maintenance } & \multicolumn{3}{|c|}{ End of Life } & \multirow{2}{*}{$\begin{array}{c}{ }^{3} \text { Total } \\
\mathrm{EE}\end{array}$} & \multirow{2}{*}{$\begin{array}{c}{ }^{4} \mathrm{Total}^{2} \\
\mathrm{EE} \\
\mathrm{Der}^{2}\end{array}$} & & \\
\hline & Naterial & 2 Trans. & Total & Materia & Trans. & Total & Material & ${ }^{2}$ Trans. & Total & Material & ${ }^{2}$ Trans. & Total & & & ${ }^{5}$ Total & ${ }^{6}$ perm ${ }^{2}$ \\
\hline Initial & 14,874 & 178 & 15,053 & $\overline{0}$ & 374 & 374 & $\overline{0}$ & $\overline{0}$ & $\overline{0}$ & $\overline{0}$ & n & - & 15,427 & 5,320 & & \\
\hline 50 & 14,874 & 178 & 15,053 & 0 & 374 & 374 & 9,297 & 363 & 9,660 & 0 & 29 & 29 & 25,116 & \begin{tabular}{|l|l|}
8.661 \\
\end{tabular} & $1,900,000$ & $-12,838$ \\
\hline
\end{tabular}

\begin{tabular}{|c|c|c|c|c|c|c|c|c|c|c|c|c|c|c|c|c|}
\hline \multirow{4}{*}{$\begin{array}{c}\text { Lifespan } \\
\text { (Years) }\end{array}$} & \multicolumn{14}{|c|}{ Global Warming Potential ( $\mathrm{kg}$ of $\mathrm{CO}_{2}$ eq.) } & \multirow{3}{*}{\multicolumn{2}{|c|}{$\begin{array}{c}\text { Difference in } \\
\text { Operating GWW fror } \\
\text { Baseline after } \\
\text { Lifespan }\end{array}$}} \\
\hline & \multicolumn{14}{|c|}{ Embodied Global Warming Potential (GWP) } & & \\
\hline & \multicolumn{3}{|c|}{ Manufacturing } & \multicolumn{3}{|c|}{ Construction } & \multicolumn{3}{|c|}{ Maintenance } & \multicolumn{3}{|c|}{ End of Life } & \multirow{2}{*}{$\begin{array}{l}{ }^{3} \text { Total } \\
\text { GWP }\end{array}$} & \multirow{2}{*}{$\begin{array}{l}{ }^{4}{ }^{4} \text { Total } \\
\text { GWP } \\
\text { perm }\end{array}$} & & \\
\hline & Material & $2^{2}$ Trans & Total & Material & 1. ${ }^{2}$ Trans. & Total & Material & ${ }^{2}$ Trans. & Total & Material & 1. ${ }^{2}$ Trans. & Total & & & ${ }^{5}$ Total & ${ }^{6}$ per $\mathrm{m}^{2}$ \\
\hline${ }^{\prime}$ Initial & \begin{tabular}{|c|}
958 \\
\end{tabular} & 0 & 958 & 0 & 1 & 1 & 0 & 0 & 0 & 0 & 0 & 0 & 959 & 331 & & \\
\hline 50 & 958 & 0 & 958 & 0 & 1 & 1 & 599 & 1 & 599 & 0 & 0 & 0 & 1,559 & 537 & $-100,000$ & -676 \\
\hline
\end{tabular}

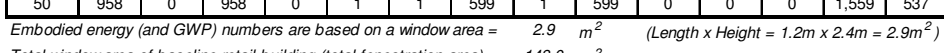
Total window area of baseline retail building (total fenestration area) $=148.0 \mathrm{~m}^{2}$

\section{Window \#5 (W-5)}

Building Component Description:

\begin{tabular}{|l|c|c|}
\hline \multicolumn{1}{|c|}{ Material List } & Quantities & Unit \\
\hline Aluminum & 179.0 & $\mathrm{~kg}$ \\
\hline EDDM membrane & 11.8 & $\mathrm{~kg}$ \\
\hline Low E Tin Argon Filled Glazing & 12.7 & $\mathrm{~m} 2$ \\
\hline Nails & 10.2 & $\mathrm{~kg}$ \\
\hline
\end{tabular}

'Initial = Time 'o' (ie. at the completion of:

${ }^{2}$ Trans. $=$ Transportation

${ }^{3}$ Total EE (or Total GWP) $=$ Total embodied energy (or total embodied GWP) of building component after lifespan (i.e. total manufacturing + tot
construction + total maintenance + total end-of-life effects) ${ }^{4}$ Total EE (or Total GWP) per $\mathrm{m}^{2}=$ Total EE (or Total GWP) of building
component / area of building component that was modelled in ATHENAQEIE ${ }^{5}$ Total Difference in Operating Energy (or GWP) from Baseline after Lifespan = The difference in the total life-cycle operating energy (or GWP)
from the baseline retail builing after fifespan, due to using this building
componentinstead of the baseline component ${ }^{6}$ Total Difference in Operating Energy (or

Lifespan per $\mathrm{m}^{2}=$ Total difference in operating energy (or GWP) from baseline anter Ilifspan/wincow area of baseline retail building * Total operating primary energy use of baseline retail building after 50 years
$50,700 \mathrm{G} J\left(1,745 \mathrm{M} / \mathrm{Jn}^{2} \mathrm{yr}\right)$

* Total operating GWP of baseline retail building after 50 years $=2,310$ tonnes 
Window \#6 (W-6)

Building Component Description:

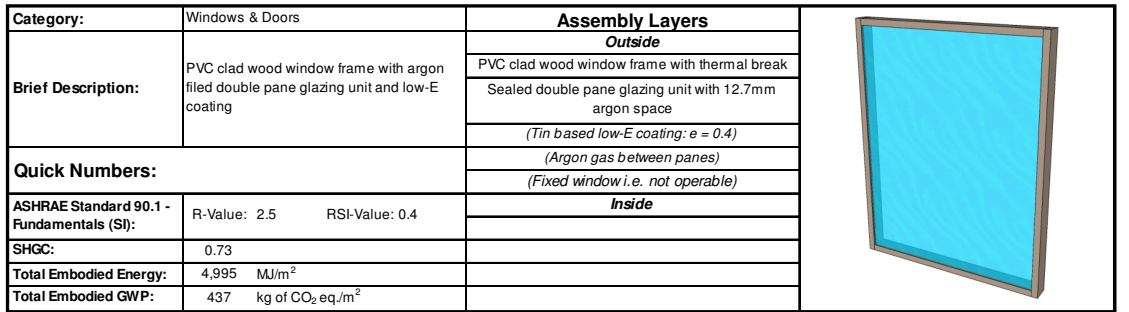

\section{Life-Cycle Assessment Results:}

Primary Energy Consumption (MJ)

\begin{tabular}{|c|c|c|c|c|c|c|c|c|c|c|c|c|c|c|c|c|}
\hline \multirow{3}{*}{\begin{tabular}{|l} 
Lifespan \\
(Years)
\end{tabular}} & \multicolumn{14}{|c|}{ Embodied Energy (EE) } & \multirow{2}{*}{\multicolumn{2}{|c|}{$\begin{array}{c}\text { Difference in } \\
\text { Operating Energy } \\
\text { from Baseline after } \\
\text { Lifespan }\end{array}$}} \\
\hline & \multicolumn{3}{|c|}{ Manufacturing } & \multicolumn{3}{|c|}{ Construction } & \multicolumn{3}{|c|}{ Maintenance } & \multicolumn{3}{|c|}{ End of Life } & \multirow{2}{*}{$\begin{array}{c}{ }^{3} \text { Total } \\
\mathrm{EE}\end{array}$} & \multirow{2}{*}{\begin{tabular}{|c}
${ }^{4}$ Total \\
EE \\
per m
\end{tabular}} & & \\
\hline & Materia & ${ }^{2}$ Trans & \begin{tabular}{|l|l|l|} 
Total \\
\end{tabular} & Materia & ${ }^{2}$ Trans. & Total & Material & ${ }^{2}$ Trans. & \begin{tabular}{|l|l|l|l} 
Total & \\
\end{tabular} & Material & 1. ${ }^{2}$ Trans. & Total & & & \begin{tabular}{|l|l|}
${ }^{5}$ Total \\
\end{tabular} & ${ }^{6}$ perm ${ }^{2}$ \\
\hline Initial & 8,863 & 86 & 8,849 & 0 & 179 & 179 & 0 & 0 & 0 & 0 & 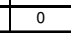 & 0 & 9,129 & 3,148 & & \\
\hline 50 & 8,863 & 86 & \begin{tabular}{|l|l|}
8,949 \\
\end{tabular} & 0 & 179 & 179 & 5,153 & 172 & 5,325 & 0 & 30 & 30 & \begin{tabular}{|l|l|l|} 
\\
\end{tabular} & 4,995 & $\mid-2,000$ & $-13,5$ \\
\hline
\end{tabular}

Global Warming Potential $\left(\mathrm{kg}\right.$ of $\mathrm{CO}_{2}$ eq.)

\begin{tabular}{|c|c|c|c|c|c|c|c|}
\hline \multirow{3}{*}{$\begin{array}{l}\text { Lifespar } \\
\text { (Years) }\end{array}$} & \multicolumn{7}{|c|}{ Embodied } \\
\hline & \multicolumn{3}{|c|}{ Manufacturing } & \multicolumn{3}{|c|}{ Construction } & \\
\hline & Material & ${ }^{2}$ Trans. & Total & Material & Trans. & \begin{tabular}{|l} 
Tota \\
\end{tabular} & \\
\hline 1 Initial & 801 & 0 & 801 & 0 & 0 & 0 & \\
\hline 50 & 801 & 0 & 801 & 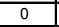 & 0 & 0 & 46 \\
\hline \multicolumn{8}{|c|}{$\begin{array}{l}\text { Embodied energy (and GWP) numbers are based on a window area } \\
\text { Total window area of b baseline retail building (total fenestration area }\end{array}$} \\
\hline \multicolumn{8}{|c|}{$\begin{array}{l}\text { ATHENA } @ \text { EIE Material List: } \\
\text { (Includes all materials after } 50 \text { years) }\end{array}$} \\
\hline \multicolumn{4}{|c|}{ Material List } & \multicolumn{2}{|c|}{ Quantities } & \multicolumn{2}{|c|}{ Unit } \\
\hline \multicolumn{4}{|c|}{ Batt. Fiberglass } & \multicolumn{2}{|r|}{0.6} & \multicolumn{2}{|c|}{ m2 (25m) } \\
\hline \multicolumn{4}{|c|}{ EPDM membrane } & \multicolumn{2}{|r|}{10.3} & \multicolumn{2}{|c|}{$\mathrm{kg}$} \\
\hline \multicolumn{4}{|c|}{ Low E Tin Argon Filled Glazing } & \multirow{2}{*}{\multicolumn{2}{|c|}{11.1}} & \multicolumn{2}{|c|}{$\mathrm{m} 2$} \\
\hline \multicolumn{4}{|l|}{ Nails } & \multirow{2}{*}{\multicolumn{2}{|c|}{8.9}} & \multirow{2}{*}{\multicolumn{2}{|c|}{$\mathrm{kg}$}} \\
\hline \multicolumn{4}{|c|}{ 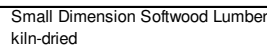 } & & & & \\
\hline \multicolumn{4}{|c|}{ Vinyl Siding } & \multicolumn{2}{|r|}{29.9} & \multicolumn{2}{|c|}{$\mathrm{m} 2$} \\
\hline
\end{tabular}

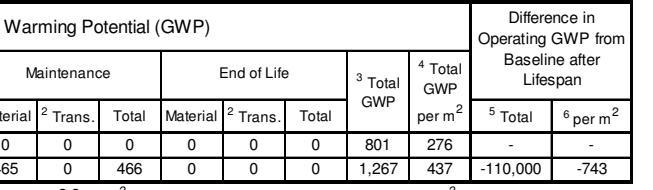

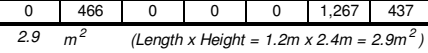

$148.0 \mathrm{~m}^{2}$

Notes:

${ }^{2}$ 'Initial $=$ Time ' 0 ' $($ i.e. at

${ }^{3}$ Total EE (or Total GWP) $=$ Total embodied energy (or total embodied GWP) of building component after lifespan (i.e. total manufacturing + tota ${ }^{5}$ Total Difference in Operating Energy (or GWP) from Baselime after Lifespan = The difference in the total life-cycle operating energy (or GWP)
from the baseline retail building after lifespan, due to using this building from the baseline retail building after lifesp
component instead of the baseline component

${ }^{6}$ Total Difference in Operating Energy (or GWP) from Baseline after Lotal Difference in Operating Energy (or GWP) from Baseline after
Lifespan per $\mathrm{m}^{2}=$ Total difference in operating energy (or GWP) from
baseline after lifespan / window area of baseline retail building baseline after lifespan / window area of baseline retail builling
* Total operating primary energy use of baseline retail building after 50 years $=$

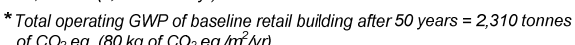

Notes:
pletion of initial construction)

${ }^{4}$ Total EE (or Total GWP) per $\mathrm{m}^{2}=$ Total EE (or Total GWP) of building

\section{Window \#7 (W-7)}

Building Component Description:

\begin{tabular}{|c|c|c|c|}
\hline \multirow{4}{*}{\begin{tabular}{|l} 
Category: \\
Brief Description:
\end{tabular}} & \multirow{4}{*}{$\begin{array}{l}\text { Windows \& Doors } \\
\text { PVC window frame with argon filed double } \\
\text { pane glazing unit and low-E coating }\end{array}$} & Assembly Layers & \multirow{9}{*}{ 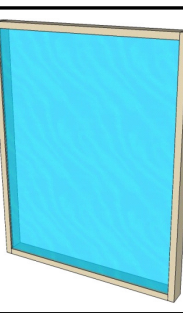 } \\
\hline & & $\begin{array}{l}\text { Outside } \\
\text { PVC window frame with thermal break }\end{array}$ & \\
\hline & & $\begin{array}{l}\text { Sealed double pane glazing unit with } 12.7 \mathrm{~mm} \\
\text { argon space }\end{array}$ & \\
\hline & & $($ Tin based low-E coating: $e=0.4)$ & \\
\hline \multicolumn{2}{|l|}{ Quick Numbers: } & $\begin{array}{l}\text { (Argon gas between panes) } \\
\end{array}$ & \\
\hline $\begin{array}{l}\text { ASHRAE Standard 90.1- } \\
\text { Fundamentals (SI): }\end{array}$ & R-Value: 2.5 & Inside & \\
\hline SHGC: & 0.73 & & \\
\hline Total Embodied Energy: & $7,390 \quad \mathrm{~N} / \mathrm{m}^{2}$ & & \\
\hline Total Embodied GWP: & $642 \mathrm{~kg}$ of $\mathrm{CO}_{2} \mathrm{eq} / \mathrm{m}^{2}$ & & \\
\hline
\end{tabular}

\section{Life-Cycle Assessment Results:}

\begin{tabular}{|c|c|c|c|c|c|c|c|c|c|c|c|c|c|c|c|c|}
\hline \multirow{4}{*}{$\begin{array}{l}\text { Lifespan } \\
\text { (Years) }\end{array}$} & \multicolumn{14}{|c|}{ y Energy Consumption (MJ) } & \multirow{3}{*}{\multicolumn{2}{|c|}{$\begin{array}{c}\text { Difiference in } \\
\text { Operating Energy } \\
\text { from Baseline after } \\
\text { Lifespan }\end{array}$}} \\
\hline & \multicolumn{14}{|c|}{ Embodied Energy (EE) } & & \\
\hline & \multicolumn{3}{|c|}{ Manutacturing } & \multicolumn{3}{|c|}{ Construction } & \multicolumn{3}{|c|}{ Main } & \multicolumn{3}{|c|}{ End of Life } & \multirow{2}{*}{$\mid \begin{array}{c}{ }^{3} \text { Total } \\
\mathrm{EE}\end{array}$} & \multirow{2}{*}{$\mid \begin{array}{c}{ }^{4} \mathrm{~T} \text { Total } \\
\mathrm{EE} \\
\text { per m }{ }^{2}\end{array}$} & & \\
\hline & Material & ${ }^{2}$ Trans. & Total & Material & ${ }^{2}$ Trans. & Total & Material & $2^{2}$ Trans. & Total & Material & ${ }^{2}$ Trans. & Total & & & ${ }^{5}$ Total & per $m^{2}$ \\
\hline Initial & 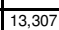 & 73 & 13,38 & 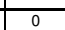 & & 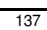 & $\pi$ & - & - & 0 & 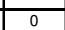 & - & 13,518 & 4,661 & & \\
\hline 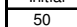 & 13 & 73 & 13,380 & 0 & 137 & 137 & 737 & 142 & 379 & 0 & 35 & 35 & 32 & + & $2,000,000$ & $-13,5$ \\
\hline
\end{tabular}

Global Warming Potential ( $\mathrm{kg}$ of $\mathrm{CO}_{2}$ eq.)

\begin{tabular}{|c|c|c|c|c|c|c|c|c|c|c|c|c|c|c|c|c|}
\hline \multirow{4}{*}{$\begin{array}{l}\text { Lifespar } \\
\text { (Years) }\end{array}$} & \multirow{2}{*}{\multicolumn{14}{|c|}{ Embodied Global Warming Potential (GWP) }} & \multirow{3}{*}{\multicolumn{2}{|c|}{$\begin{array}{c}\text { Difference in } \\
\text { Operating GWP from } \\
\text { Baseline after } \\
\text { Lifespan }\end{array}$}} \\
\hline & & & & & & & & & & & & & & & & \\
\hline & \multicolumn{3}{|c|}{ Manutacturing } & \multicolumn{3}{|c|}{ Construction } & \multicolumn{3}{|c|}{ Maintenance } & \multicolumn{3}{|c|}{ End of Life } & \multirow{2}{*}{$\begin{array}{l}{ }^{3} \text { Total } \\
\text { GWPP }\end{array}$} & \multirow{2}{*}{$\begin{array}{l}{ }^{4} \text { Total } \\
\text { GWP } \\
\text { per m }\end{array}$} & & \\
\hline & Materia & ${ }^{2}$ Trans. & Total & Material & Trans. & Total & Material & ${ }^{2}$ Trans. & Total & Material & ${ }^{2}$ Trans. & Total & & & ${ }^{5}$ Total & perm \\
\hline & & 0 & & 0 & 0 & 0 & 8 & 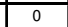 & 0 & 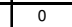 & 0 & 0 & 1,177 & 406 & & \\
\hline 5 & 1,176 & 0 & 1,176 & 0 & 0 & 0 & 684 & 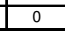 & 684 & 0 & 0 & 0 & 1,861 & 642 & $-110,000$ & -743 \\
\hline
\end{tabular}
Embodied energy (and GWP) numbers are based on a window area = $2.9 \mathrm{~m}^{2} \quad$ (Length $x$ Height $=1.2 \mathrm{~m} \times 2.4 \mathrm{~m}=2.9 \mathrm{~m}^{2}$ ) Total window area of baseline retail building (total fenestration area) $=148.0 \mathrm{~m}^{2}$

ATHENA $®$ EIE Material List:

\begin{tabular}{|l|c|c|}
\hline \multicolumn{1}{|c|}{ Material List } & Quantities & Unit \\
\hline Batt. Fiberglass & 0.6 & $\mathrm{~m} 2(25 \mathrm{~mm})$ \\
\hline EPDM membrane & 10.3 & $\mathrm{~kg}$ \\
\hline Low E Tin Argon Filled Glazing & 11.1 & $\mathrm{~m} 2$ \\
\hline Nails & 8.9 & $\mathrm{~kg}$ \\
\hline Vinyl Siding & 74.9 & $\mathrm{~m} 2$ \\
\hline
\end{tabular}

Vinyl Siding
${ }^{1}$ Initial $=$ Time 'o' (i.e. at the completion of ${ }^{2}$ Trans. $=$ Transportation

${ }^{3}$ Total EE (or Total GWP) $=$ Total embodied energy (or total embodied GWP) of building component after lifespan (i.e. total manufacturing + tota
construction + total maintenance + total end-oflife effects)

${ }^{4}$ Total EE (or Total GWP) per $\mathrm{m}^{2}=$ Total EE (or Total GWP) of building
component / area of building component that was modelled in ATHENAQ EIE ${ }^{5}$ Total Difference in Operating Energy (or GWP) from Baseline after Lifespan = The difference in the total life-cycle operating energy (or GWP)
from the basaline retail building after lifespan, due to using this building
component instead of the baseline component ${ }^{6}$ Total Difference in Operating Energy (or

Lifespan per $\mathrm{m}^{2}=$ Total difference in operating energy (or GWP) from baseline after lifespan/window area of baseline retail building * Total operating primary energy use of baseline retail building after 50 years
$50,700 \mathrm{GJ}\left(1,745 \mathrm{M} / \mathrm{N}^{2} \mathrm{y} r\right)$

* Total operating GWP of baseline retail building after 50 years $=2,310$ tonnes 
Window \#8 (W-8)

Building Component Description:

\begin{tabular}{|c|c|c|}
\hline Category: & Windows \& Doors & Assembly Layers \\
\hline \multirow{4}{*}{ Brief Description: } & \multirow{4}{*}{$\begin{array}{l}\text { Wood window frame with argon flifd double } \\
\text { pane glazing unit and low-E co coating }\end{array}$} & Outside \\
\hline & & Wood window frame with thermal break \\
\hline & & $\begin{array}{l}\text { Sealed double pane glazing unit with } 12.7 \mathrm{~mm} \\
\text { argon space }\end{array}$ \\
\hline & & (Tin based low-E coating: $e=0.4)$ \\
\hline \multirow{2}{*}{\multicolumn{2}{|c|}{ Quick Numbers: }} & $\begin{array}{l}\text { (Argon gas between panes) } \\
\end{array}$ \\
\hline & & (Fixed windowie. not operablele \\
\hline $\begin{array}{l}\text { ASHRAE Standard 90.1- } \\
\text { Fundamentals (SI): }\end{array}$ & R-Value: 2.5 & Inside \\
\hline SHGC: & 0.73 & \\
\hline \begin{tabular}{|l|} 
Total Embodied Energy: \\
\end{tabular} & $4,088 \quad \mathrm{~m} / \mathrm{m}^{2}$ & \\
\hline Total Embodied GI & $346 \quad \mathrm{~kg} \mathrm{of} \mathrm{CO}_{2} \mathrm{eq} / \mathrm{m}^{2}$ & \\
\hline
\end{tabular}

\section{Life-Cycle Assessment Results:}

Primary Energy Consumption (MJ)

\begin{tabular}{|c|c|c|c|c|c|c|c|c|c|c|c|c|c|c|c|c|}
\hline \multirow{3}{*}{$\begin{array}{l}\text { Lifespan } \\
\text { Yrears) }\end{array}$} & \multicolumn{14}{|c|}{ Embodied Energy (EE) } & \multirow{2}{*}{\multicolumn{2}{|c|}{$\begin{array}{c}\text { Difference in } \\
\text { Operating Energy } \\
\text { from Baseline after } \\
\text { Lifespan }\end{array}$}} \\
\hline & \multicolumn{3}{|c|}{ Manufacturing } & \multicolumn{3}{|c|}{ Construction } & \multicolumn{3}{|c|}{ Maintenance } & \multicolumn{3}{|c|}{ End of Life } & \multirow{2}{*}{$\begin{array}{c}{ }^{3} \text { Total } \\
\mathrm{EE}\end{array}$} & \multirow{2}{*}{\begin{tabular}{|c|}
${ }^{4}{ }^{4}$ Total \\
EE \\
perm
\end{tabular}} & & \\
\hline & Material & ${ }^{2}$ Trans. & Total & Material & ${ }^{2}$ Trans. & Total & Material & ${ }^{2}$ Trans. & Total & Material & Trans. & Total & & & ${ }^{5}$ Total & ${ }^{6}$ per m ${ }^{2}$ \\
\hline Initial & 7,031 & 95 & 7,126 & 0 & 213 & 213 & 0 & 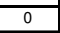 & 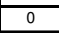 & 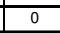 & 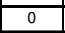 & 8 & 7,338 & 2,530 & & \\
\hline 50 & \begin{tabular}{|l|l|}
7,031 \\
\end{tabular} & 95 & 7,126 & 0 & \begin{tabular}{|l|}
213 \\
\end{tabular} & 213 & 4,287 & 204 & \begin{tabular}{|l|l|}
4,491 \\
\end{tabular} & 0 & 27 & 27 & 111,856 & 4,088 & $-2,000$ & $-13,514$ \\
\hline
\end{tabular}

Global Warming Potential (kg of $\mathrm{CO}_{2}$ eq.)

\begin{tabular}{|c|c|c|c|c|c|c|c|}
\hline \multirow{3}{*}{$\begin{array}{l}\text { Lifespar } \\
\text { (Years) }\end{array}$} & \multicolumn{7}{|c|}{ Embodied Global } \\
\hline & \multicolumn{3}{|c|}{ Manutacturing } & \multicolumn{3}{|c|}{ Construction } & \\
\hline & Material & ${ }^{2}$ Trans. & \begin{tabular}{|l|l} 
Total \\
\end{tabular} & Material & $\left.\right|^{2}$ Trans. & \begin{tabular}{|l|l} 
Total & r l \\
\end{tabular} & \\
\hline Initial & 623 & 0 & 623 & 0 & Th & 0 & \\
\hline & & & & & & 0 & \\
\hline \multicolumn{8}{|c|}{$\begin{array}{l}\text { Embodied energy (and GWP) numbers are based on a window area } \\
\text { Total window area of baseline retail building (total fenestration area) }\end{array}$} \\
\hline \multicolumn{8}{|c|}{$\begin{array}{c}\text { ATHENA @ EIE Material List: } \\
\text { (Includes all materials after } 50 \text { years) }\end{array}$} \\
\hline \multicolumn{4}{|c|}{ Material List } & \multicolumn{2}{|c|}{ Quantities } & \multicolumn{2}{|c|}{ Unit } \\
\hline \multicolumn{4}{|c|}{ Aluminum } & & 20.9 & $\mathrm{~kg}$ & \\
\hline \multirow{2}{*}{\multicolumn{4}{|c|}{ Batt. Fiberglass }} & & 0.6 & \multirow{2}{*}{\multicolumn{2}{|c|}{$\mathrm{m} 2(25 \mathrm{~mm}$}} \\
\hline & & \multicolumn{2}{|c|}{ EPDM membrane } & & 11.2 & & \\
\hline \multicolumn{4}{|c|}{ Low ET } & & 12.1 & \\
\hline \multicolumn{4}{|l|}{ Nalis } & & 10.0 & \multicolumn{2}{|l|}{$\mathrm{kg}$} \\
\hline \multicolumn{4}{|c|}{$\begin{array}{l}\text { Smalll Dimension Softwood Lumber, } \\
\text { kill-dried }\end{array}$} & & 0.3 & \multicolumn{2}{|l|}{$\mathrm{m} 3$} \\
\hline \multicolumn{4}{|c|}{ Nater Based Latex Paint } & & 1.2 & & \\
\hline
\end{tabular}
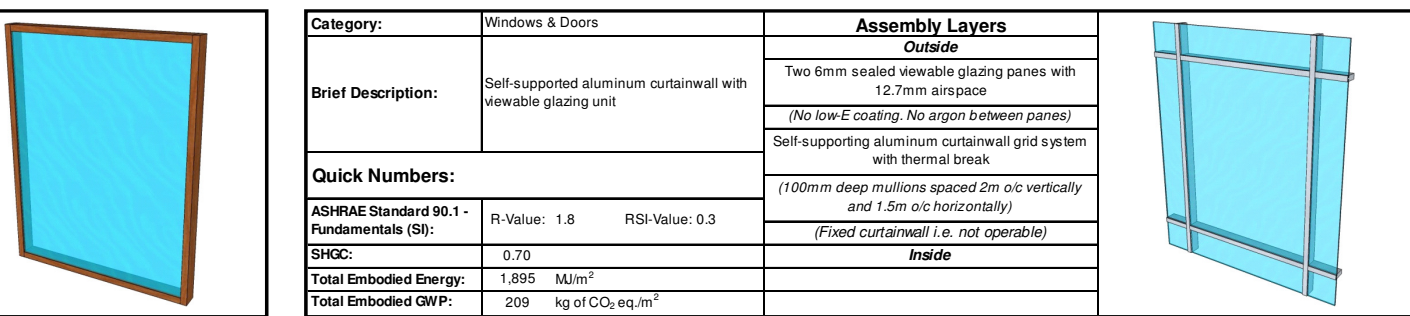

\section{Life-Cycle Assessment Results:}

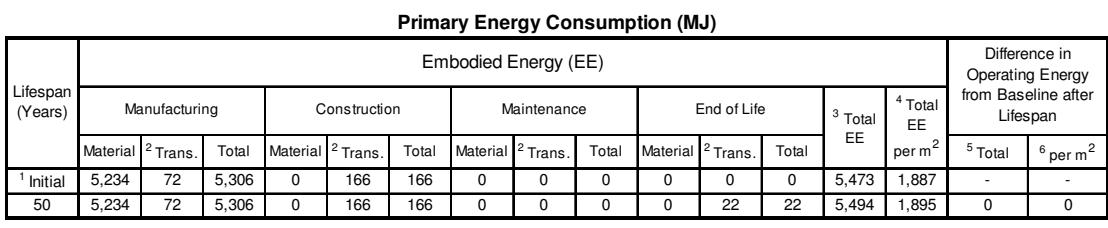

Global Warming Potential ( $k g$ of $\mathrm{CO}_{2}$ eq.)

\begin{tabular}{|c|c|c|c|c|c|c|c|c|c|c|c|c|c|c|c|c|}
\hline \multirow{3}{*}{$\mid$\begin{tabular}{|l} 
Lifespan \\
(Years)
\end{tabular}} & \multicolumn{14}{|c|}{ Embodied Global Warming Potential (GWP) } & \multirow{2}{*}{\multicolumn{2}{|c|}{\begin{tabular}{|c|} 
Difference in \\
Operating GWPP from \\
Baseline after \\
Lifespan
\end{tabular}}} \\
\hline & \multicolumn{3}{|c|}{ Manufacturing } & \multicolumn{3}{|c|}{ Construction } & \multicolumn{3}{|c|}{ Maintenance } & \multicolumn{3}{|c|}{ End of Life } & \multirow{2}{*}{$\begin{array}{l}{ }^{3} \text { Total } \\
\text { GWP }\end{array}$} & \multirow{2}{*}{$\begin{array}{l}{ }^{4} \text { Total } \\
\text { GWP } \\
\text { eer } \mathrm{m}^{2}\end{array}$} & & \\
\hline & Material & ${ }^{2}$ Trans & 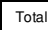 & Material & ${ }^{2}$ Trans & Total & Materia & 1. 2 Trans. & Total & Material & $1{ }^{2}$ Trans. & Total & & & ${ }^{5}$ Total & ${ }^{6}$ perm ${ }^{2}$ \\
\hline & 606 & 0 & 606 & 4 & 0 & 0 & 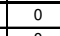 & 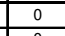 & 0 & 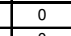 & 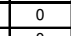 & 0 & 606 & \begin{tabular}{|l|l|}
209 \\
\end{tabular} & & \\
\hline & 606 & 0 & 606 & 0 & 0 & 0 & 0 & 0 & 0 & 0 & 0 & 0 & 606 & 209 & 0 & \\
\hline
\end{tabular}
Embodied energy (and GWP) numbers are based on a window area = $2.9 \mathrm{~m}^{2} \quad$ (Length $\times$ Height $=1.2 \mathrm{~m} \times 2.4 \mathrm{~m}=2.9 \mathrm{~m}^{2}$ ) Total mndow area of baseline relail building (total fenestration area) $=148.0 \mathrm{~m}^{2}$

ATHENA ® EIE Material List:

Notes:
${ }^{1}$ Initial $=$ Time '0' (i.e. at the completion of initial construction)

${ }^{2}$ Trans. $=$ Transportation

Total EE (or Total GWP) = Total embodied energy (or total embodied GWP) of building component after lifespan (i.e. total manufacturing + tota

${ }^{4}$ Total EE (or Total GWP) per $\mathrm{m}^{2}=$ Total EE (or Total GWP) of building
component/ area of building component that was modelled in ATHENAPEIE ${ }^{5}$ Total Difference in Operating Energy (or GWP) from Baseline after) Lifespan = The difference in the total life-cycle operating energy (or GWWP)
from the baseline retail building after lifespan, due to using this building from the baseline retail building after lifesp
component instead of the baseline component

Totol Diff Lifespan per $\mathrm{m}^{2}=$ Total difference in operating energy (or GWP) from baseline after lifespan / window area of baseline retail building * Total operating primary energy use of baseline retail building after 50 years
$50,700 \mathrm{G}\left(1.745 \mathrm{MJ} / \mathrm{m}^{2} \mathrm{yrr}\right)$

"Total operating GWP of baseline retail building after 50 years $=2,310$ tonnes

\begin{tabular}{|l|c|c|}
\hline \multicolumn{1}{|c|}{ Material List } & Quantities & Unit \\
\hline Aluminum & 35.1 & $\mathrm{~kg}$ \\
\hline EPDM membrane & 2.1 & $\mathrm{~kg}$ \\
\hline Glazing Panel & 265.1 & $\mathrm{~kg}$ \\
\hline Screws Nuts \& Bolts & 1.2 & $\mathrm{~kg}$ \\
\hline
\end{tabular}

Notes:

${ }^{2}$ Trans. $=$ Transportation

${ }^{3}$ Total EE (or Total GWP) $=$ Total embodied energy (or total embodied GWP) of building component after lifespan (i.e. total manufacturing + tot
construction + total maintenance + total end-of-life effects) ${ }^{4}$ Total EE (or Total GWP) per $\mathrm{m}^{2}=$ Total EE (or Total GWP) of building
component / area of building component that was modelled in ATHENAQ EIE ${ }^{5}$ Total Difference in Operating Energy (or GWP) from Baseline after Lifespan = The difference in the total life-cycle operating energy (or GWP)
from the baseline retail builing after fifespan, due to using this building
componentinstead of the baseline component ${ }^{6}$ Total Difference in Operating Energy (or

Lifespan per $\mathrm{m}^{2}=$ Total difference in operating energy (or GWP) from baseline after ifiespan/window area of baseline retail building * Total operating primary energy use of baseline retail building after 50 years
$50,700 \mathrm{GJ}\left(1,745 \mathrm{M} \mathrm{M}^{2} \mathrm{~T}^{2} \mathrm{yr}\right)$

* ${ }^{*}$ Total operating GWP of baseline retail building after 50 years $=2,310$ tonnes 


\section{LCA Data for Doors}

This section contains a detailed description of each door that was examined in this study (6 in total). The assembly layers are listed for each door, along with a detailed description of the material quantities from the ATHENA® Environmental Impact Estimator for Buildings.

A breakdown of the total primary energy consumption and the total global warming potential (GWP) for each door is also included. In each case, the results were calculated for a single door. The data has been calculated for two different lifespans: at the completion of initial construction and after 50 years.

As a summary, the figure below illustrates a comparison of the total embodied energy (and GWP) after 50 years for the various doors in this section. For comparison purpose, the building component used in the baseline retail building has been shaded in grey.

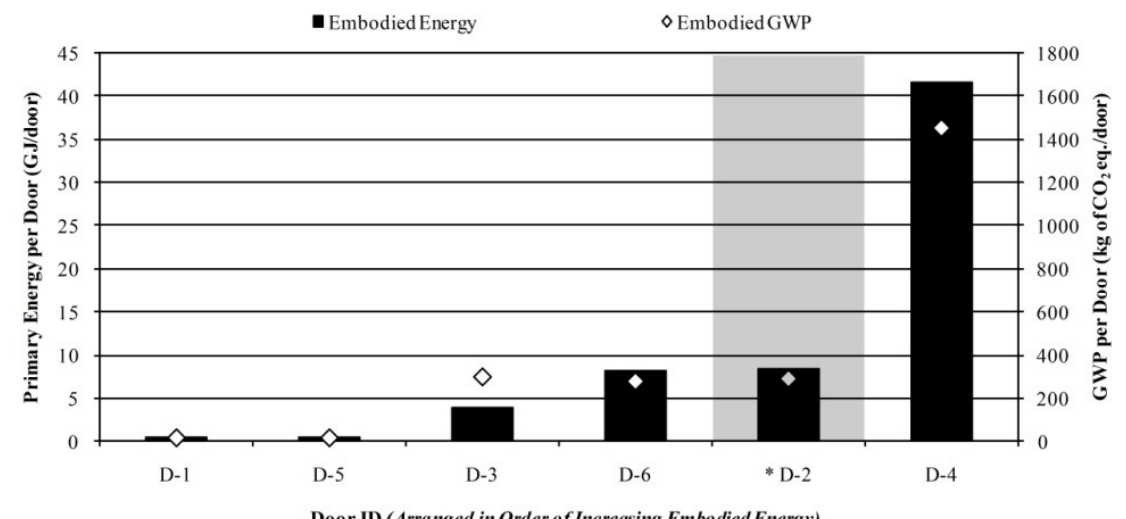

\section{Door \#1 (D-1)}

Building Component Description:

\begin{tabular}{|c|c|c|c|}
\hline \multirow{4}{*}{ Brief Description: } & \multirow{4}{*}{$\begin{array}{l}\text { Standard size solid wood exterior door with } \\
\text { no glazing }\end{array}$} & Assembly Layers & \multirow{9}{*}{1} \\
\hline & & $\begin{array}{l}\text { Ootside } \\
\text { Solid wood exterior door with no glazing }\end{array}$ & \\
\hline & & (Includes latex paint) & \\
\hline & & (Standard size: $813 \mathrm{~mm} \times 2134 \mathrm{~mm}$ ) & \\
\hline \multirow{2}{*}{\multicolumn{2}{|c|}{ Quick Numbers: }} & istue & \\
\hline & & & \\
\hline \begin{tabular}{|l} 
ASHRAE Standard 90.1- \\
Fundamentals (SI):
\end{tabular} & RSI-Value: 0.3 & & \\
\hline Total Embodied Energy: & 539 $\quad$ M/unit & & \\
\hline Total Embodied GWP: & $\begin{array}{cc}16 & \mathrm{~kg} \text { of } \mathrm{CO}_{2} \text { eq/unit } \\
\end{array}$ & & \\
\hline
\end{tabular}

\section{Life-Cycle Assessment Results:}

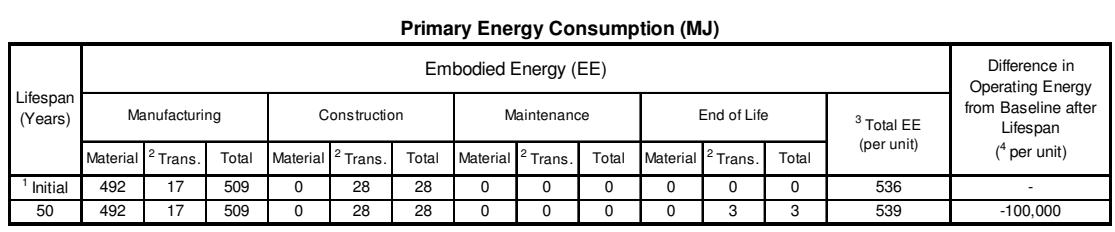

Global Warming Potential ( $\mathrm{kg}$ of $\left.\mathrm{CO}_{2} \mathrm{eq}\right)$

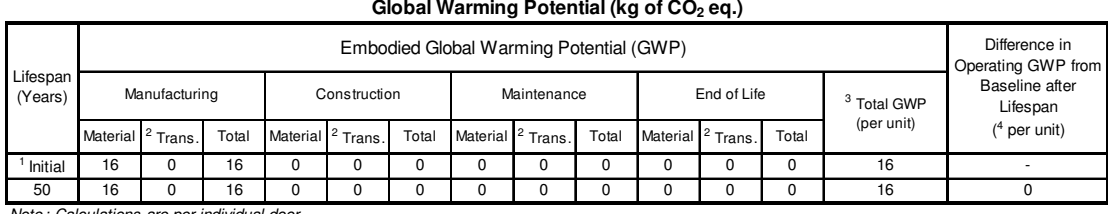

ATHENA $®$ EIE Material List:

\begin{tabular}{|l|c|c|}
\hline \multicolumn{1}{|c|}{ Material List } & Quantities & Unit \\
\hline Nails & 2.0 & $\mathrm{~kg}$ \\
\hline $\begin{array}{l}\text { Small Dimension Sottwood Lumber, } \\
\text { kill-dried }\end{array}$ & 0.1 & $\mathrm{~m} 3$ \\
\hline Water Based Latex Paint & 0.7 & $\mathrm{~L}$ \\
\hline
\end{tabular}

Notes:

${ }^{2}$ Trans. $=$ Transportation

${ }^{3}$ Total EE (or Total GWP) per unit $=$ Total embodied energy (or total embodied GWP) of building component after lifespan (i.e. total manufacturing

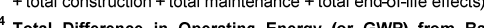

(itos GWP) from the baseline retail building atter lifespan, due to using this * Total operating primary energy use of baseline retail building after 50 years

* Total operating GWP of baseline retail building after 50 years $=2,310$ tonnes
of $\mathrm{CO}_{2} \mathrm{eq} .\left(8 \mathrm{~kg}\right.$ of $\left.\mathrm{CO}_{2} \mathrm{eq} / \mathrm{m}^{2} \mathrm{Yrt}\right)$ 


\section{Door \#2 (D-2)}

Building Component Description:

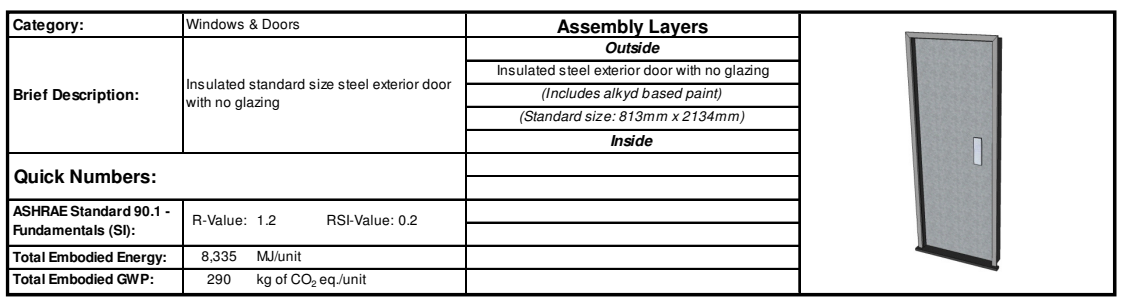

\section{Life-Cycle Assessment Results:}

Primary Energy Consumption (MJ)

\begin{tabular}{|c|c|c|c|c|c|c|c|c|c|c|c|c|c|c|}
\hline & & & & & & Pri & ry Ene & rgy con & & tion (M. & & & & \\
\hline \multirow{3}{*}{$\begin{array}{l}\text { Lifespan } \\
\text { (Years) }\end{array} \mid$} & \multicolumn{13}{|c|}{ Embodied Energy (EE) } & \multirow{3}{*}{$\begin{array}{l}\text { Difference in } \\
\text { Operating Energy } \\
\text { from Baseline after } \\
\text { Lifespan } \\
\text { (' }{ }^{4} \text { per unit) }\end{array}$} \\
\hline & \multicolumn{3}{|c|}{ Manufacturing } & \multicolumn{3}{|c|}{ Construction } & \multicolumn{3}{|c|}{ Maintenance } & \multicolumn{3}{|c|}{ End of Life } & \multirow{2}{*}{$\begin{array}{l}{ }^{3} \text { Total EE } \\
\text { (per unit) }\end{array}$} & \\
\hline & Materia & ${ }^{2}$ Trans & \begin{tabular}{|l|l|l|} 
Total \\
\end{tabular} & Material & ${ }^{2}$ Trans. & Total & Material & ${ }^{2}$ Trans. & Total & Material & ${ }^{2}$ Trans. & \begin{tabular}{|l|} 
Total \\
\end{tabular} & & \\
\hline${ }^{1}$ Initial & 8,303 & 12 & 8,315 & 0 & 15 & 15 & 0 & 0 & 0 & 0 & 0 & 0 & 8,33 & \\
\hline 50 & 8,303 & 12 & 8,315 & 0 & 15 & 15 & 0 & 0 & 0 & 0 & 5 & 5 & 8,335 & 0 \\
\hline
\end{tabular}

Global Warming Potential ( $k g$ of $\mathrm{CO}_{2}$ eq.)

$\infty$

\begin{tabular}{|c|c|c|c|c|c|c|c|c|c|c|c|c|c|c|}
\hline \multirow{3}{*}{$\begin{array}{c}\text { Lifespan } \\
\text { (Years) }\end{array}$} & \multicolumn{13}{|c|}{ Embodied Global Warming Potential (GWP) } & \multirow{3}{*}{$\begin{array}{c}\text { Difference in } \\
\text { Operating GWW fron } \\
\text { Baseline after } \\
\text { Lifespan } \\
\left({ }^{4} \text { per unit }\right)\end{array}$} \\
\hline & \multicolumn{3}{|c|}{ Manufacturing } & \multicolumn{3}{|c|}{ Construction } & \multicolumn{3}{|c|}{ Maintenance } & \multicolumn{3}{|c|}{ End of Life } & \multirow{2}{*}{$\begin{array}{l}{ }^{3} \text { Total GWP } \\
\text { (per unit) }\end{array}$} & \\
\hline & \begin{tabular}{|l|} 
Material \\
\end{tabular} & ${ }^{2}$ Trans. & \begin{tabular}{|l|l|} 
Total \\
\end{tabular} & Material & ${ }^{2}{ }^{2}$ Tans. & Total & \begin{tabular}{|l|} 
Material \\
\end{tabular} & $\left.\right|^{2}$ Trans. & Total & Material & $\left.\right|^{2}$ Trans. & Total & & \\
\hline 'Initial & 290 & 0 & 2900 & 0 & 0 & 0 & 0 & 0 & 0 & 0 & 0 & 0 & 290 & \\
\hline 50 & 290 & 0 & \begin{tabular}{|l|l|}
290 \\
\end{tabular} & 0 & 0 & 0 & 0 & 0 & 0 & 0 & 0 & 0 & 290 & 0 \\
\hline
\end{tabular}

ATHENA $®$ EIE Material List:

(Includes all materials after 50 years

\begin{tabular}{|l|c|c|}
\hline \multicolumn{1}{|c|}{ Material List } & Quantities & Unit \\
\hline Expanded Polystyrene & 3.3 & $\mathrm{~m} 2(25 \mathrm{~mm})$ \\
\hline Galvanized Sheet & 61.9 & $\mathrm{~kg}$ \\
\hline Nails & 2.0 & $\mathrm{~kg}$ \\
\hline Solvent Based Alkyd Paint & 0.3 & $\mathrm{~L}$ \\
\hline
\end{tabular}

Notes:

${ }^{4}$ Initial $=$ Time $0^{\prime}($ i.e. 2 a

${ }^{3}$ Total EE (or Total GWP) per unit = Total embodied energy (or tota embodied GWP) of building component affer lifespan (i.e. total manufactun ${ }_{4}^{4}$ total construction + total maintenance + total end-ofilife effects

Lifespan per unit $=$ The difference in the tort GWP) from Baseline after

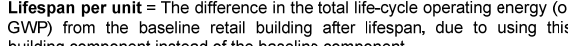

* Total operating primary energy use of baseline retail building after 50 years =

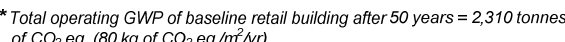

\section{Door \#3 (D-3)}

Building Component Description:

\begin{tabular}{|c|c|c|c|}
\hline \multirow{4}{*}{ |Brief Description: } & \multirow{4}{*}{$\begin{array}{l}\text { Uninsulated standard size aluminum } \\
\text { exterior door with } 80 \% \text { glazing }\end{array}$} & $\begin{array}{l}\text { Assembly Layers } \\
\text { Outside }\end{array}$ & \\
\hline & & $\begin{array}{c}\text { Uninsulated aluminum exterior door with } 80 \% \\
\text { glazing }\end{array}$ & \\
\hline & & (Typical sealed double pane glazing unit) & \\
\hline & & (No low-E coating. No argon between panes) & \\
\hline \multicolumn{2}{|l|}{ Quick Numbers: } & $\begin{array}{l}\text { (Standard size: } 813 \mathrm{~mm} \times 2134 \mathrm{~mm} \text { ) } \\
\text { Inside }\end{array}$ & \\
\hline 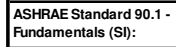 & R-Value: 1.1 & & \\
\hline SHGC: & 0.76 & & \\
\hline Total Embodied Energy: & $\begin{array}{ll}3,806 \text { M/unit } \\
, 208\end{array}$ & & \\
\hline
\end{tabular}

Life-Cycle Assessment Results:

Primary Energy Consumption (MJ)

\begin{tabular}{|c|c|c|c|c|c|c|c|c|c|c|c|c|c|c|}
\hline \multirow{3}{*}{$\begin{array}{l}\text { Lifespan } \\
\text { (Years) }\end{array}$} & \multicolumn{13}{|c|}{ Embodied Energy (EE) } & \multirow{3}{*}{$\begin{array}{c}\text { Difference in } \\
\text { Operating Energy } \\
\text { from Baseline after } \\
\text { Lifespan } \\
\left({ }^{4} \text { per unit }\right)\end{array}$} \\
\hline & \multicolumn{3}{|c|}{ Manufacturing } & \multicolumn{3}{|c|}{ Construction } & \multicolumn{3}{|c|}{ Maintenance } & \multicolumn{3}{|c|}{ End of Life } & \multirow{2}{*}{$\begin{array}{l}{ }^{3} \text { Total EE } \\
\text { (per unit) }\end{array}$} & \\
\hline & Materia & ${ }^{2}$ Trans & Total & \begin{tabular}{|l|} 
Material \\
\end{tabular} & ${ }^{2}$ Trans. & Total & Material & ${ }^{2}$ Trans. & \begin{tabular}{|l|} 
Total \\
\end{tabular} & \begin{tabular}{|l|} 
Material \\
\end{tabular} & \begin{tabular}{|l|}
${ }^{2}$ Trans. \\
\end{tabular} & \begin{tabular}{|l|l|} 
Total \\
\end{tabular} & & \\
\hline 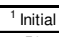 & $\overline{3,641}$ & 51 & \begin{tabular}{|l}
3,691 \\
\end{tabular} & 0 & \begin{tabular}{|l|}
105 \\
\end{tabular} & 105 & 0 & 0 & 0 & 0 & 0 & 0 & 3,796 & \\
\hline 50 & \begin{tabular}{|l|}
3,641 \\
\end{tabular} & 51 & \begin{tabular}{|l|l|} 
\\
3,69
\end{tabular} & 0 & 105 & 105 & 0 & 0 & 0 & 0 & 10 & 10 & 3,806 & 0 \\
\hline
\end{tabular}

Global Warming Potential (kg of $\mathrm{CO}_{2}$ eq.)

\begin{tabular}{|c|c|c|c|c|c|c|c|c|c|c|c|c|c|c|}
\hline \multirow{3}{*}{\begin{tabular}{|c} 
Lifespan \\
(Years)
\end{tabular}} & \multicolumn{13}{|c|}{ Embodied Global Warming Potential (GWP) } & \multirow{3}{*}{\begin{tabular}{|c} 
Difference in \\
Operating GWP from \\
Baseline after \\
Lifespan \\
$\left({ }^{4}\right.$ per unit)
\end{tabular}} \\
\hline & \multicolumn{3}{|c|}{ Manufacturing } & \multicolumn{3}{|c|}{ Construction } & \multicolumn{3}{|c|}{ Maintenance } & \multicolumn{3}{|c|}{ End of Life } & \multirow{2}{*}{$\begin{array}{l}{ }^{3} \text { Total GWP } \\
\text { (per unit) }\end{array}$} & \\
\hline & Material & ${ }^{2}$ Trans. & Total & \begin{tabular}{|l|} 
Material \\
\end{tabular} & ${ }^{2}$ Trans. & Total & \begin{tabular}{|l|} 
Material \\
\end{tabular} & ${ }^{2}$ Trans. & Total & \begin{tabular}{|l|} 
Material \\
\end{tabular} & $1{ }^{2}$ Trans. & \begin{tabular}{|l|l|} 
Total \\
\end{tabular} & & \\
\hline Initial & 298 & 0 & 298 & 0 & 0 & 0 & 0 & 0 & 0 & 0 & 0 & 0 & & 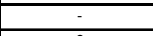 \\
\hline 50 & 298 & 0 & 298 & 0 & 0 & 0 & 0 & 0 & 0 & 0 & 0 & 0 & 298 & 0 \\
\hline
\end{tabular}

ATHENA ® EIE Material List:

(Includes all materials after 50 years)

\begin{tabular}{|l|c|c|}
\hline \multicolumn{1}{|c|}{ Material List } & Quantities & Unit \\
\hline Aluminum & 25.7 & $\mathrm{~kg}$ \\
\hline Glazing Panel & 50.7 & $\mathrm{~kg}$ \\
\hline Nails & 2.0 & $\mathrm{~kg}$ \\
\hline
\end{tabular}

'Initial = Time 'o' (i.e. at the Notes:

${ }^{2}$ Trans. = Transportation

Total EE (or Total GWP) per unit = Total embodied energy (or total + total construction + total maintenance + total enc-offlife effects)

4otal Difference in Operating Energy (or GWP) from B

Lifespan per unit $=$ The difference in the total lifecycle operating energy (of GWP) from the baseline retail building after lifespan, due to using this

* Total operating primary energy use of baseline retail building after 50 years

${ }^{*}$ Total operating GWP of baseline retat
of $\mathrm{CO}_{2}$ eq. (80 kg of $\mathrm{CO}_{2}$ eq. $\left./ \mathrm{m}^{2} \mathrm{Yyr}\right)$ 


\section{Door \#4 (D-4)}

Building Component Description:

\begin{tabular}{|c|c|c|}
\hline Category: & Windows \& Doors & $\begin{array}{l}\text { Assembly Layers } \\
\text { Outside }\end{array}$ \\
\hline \multirow[t]{2}{*}{ Brief Description: } & \multirow{2}{*}{$\begin{array}{l}\text { Insulated sectional overhead steel door } \\
\text { with no glazing }\end{array}$} & $\begin{array}{c}\text { Insulated sectional overhead steel door with no } \\
\text { glazing }\end{array}$ \\
\hline & & $\begin{array}{l}\text { (modeled in ATHENA as equivalent area of } \\
\text { insulated steel exterior door with no glazing) }\end{array}$ \\
\hline \multicolumn{2}{|l|}{ Quick Numbers: } & (50mm extruded polystyrene insulation assumed) \\
\hline $\begin{array}{l}\text { ASHRAE Standard 90.1- } \\
\text { Fundamentals (S): }\end{array}$ & RSIVValue: 0.2 & $\frac{\text { (Standard size: } 3050 \mathrm{~mm} \times 3050 \mathrm{~mm})}{\text { Inside }}$ \\
\hline Total Embodied Energy: & 41,677 M/unit & \\
\hline
\end{tabular}

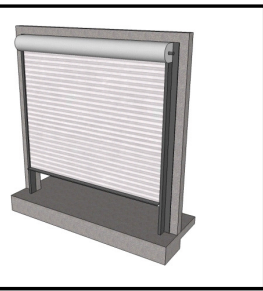

\section{Life-Cycle Assessment Results:}

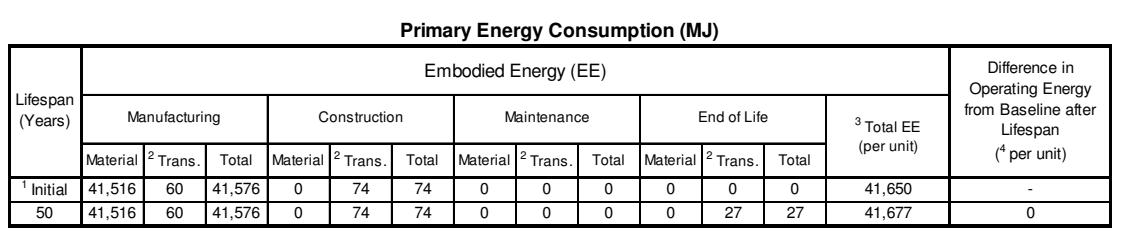

Global Warming Potential ( $\mathrm{kg}$ of $\mathrm{CO}_{2}$ eq.)

$\stackrel{N}{+}$

\begin{tabular}{|c|c|c|c|c|c|c|c|c|c|c|c|c|c|c|}
\hline \multirow{3}{*}{$\begin{array}{l}\text { Litesparar } \\
\text { (Years) }\end{array}$} & \multicolumn{13}{|c|}{ Embodied Global Warming Potential (GWP) } & \multirow{3}{*}{$\begin{array}{c}\text { Difference in } \\
\text { Operating GWP from } \\
\text { Baseline after } \\
\text { Lifespan } \\
\left({ }^{4} \text { per unit) }\right.\end{array}$} \\
\hline & \multicolumn{3}{|c|}{ Manutacturing } & \multicolumn{3}{|c|}{ Construction } & \multicolumn{3}{|c|}{ Maintenance } & \multicolumn{3}{|c|}{ End of Life } & \multirow{2}{*}{$\begin{array}{l}{ }^{3} \text { Total GWP } \\
\text { (per unit) }\end{array}$} & \\
\hline & Material & ${ }^{2}$ Trans & Total & Material & 2 Trans. & Total & Material & ${ }^{2}$ Trans. & Total & Material & ${ }^{2}$ Trans. & Total & & \\
\hline 'Initial & 1,448 & 0 & 1,448 & $\overline{0}$ & $\overline{0}$ & 0 & 0 & $\overline{0}$ & 0 & $\overline{0}$ & 0 & 0 & 1,448 & \\
\hline 50 & \begin{tabular}{|l|l|l|} 
\\
\end{tabular} & 0 & 1,448 & 0 & 0 & 0 & 0 & 0 & 0 & 0 & 0 & 0 & 1,448 & 0 \\
\hline
\end{tabular}

\section{ATHENA ® EIE Material List:}

(Includes all materials after 50 years)

Notes:

\begin{tabular}{|l|c|c|}
\hline \multicolumn{1}{|c|}{ Material List } & Quantities & Unit \\
\hline Expanded Polystyrene & 16.3 & $\mathrm{~m} 2(25 \mathrm{~mm})$ \\
\hline Galvanized Sheet & 309.6 & $\mathrm{~kg}$ \\
\hline Nails & 9.8 & $\mathrm{~kg}$ \\
\hline Solvent Based Alkyd Paint & 1.5 & $\mathrm{~L}$ \\
\hline
\end{tabular}

${ }^{2}$ Trans. $=$ Transportation

Total EE (or Total GWP) per unit = Total embodied energy (or tota embodied GWP) of building component affer lifespan (i.e. total manufacturing total consiricion to

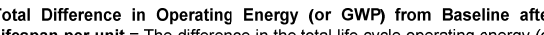
Lifespan per unit = The difference in the total life-cycle operating energy (or
GWP) from the baseline retail building after lifespan, due to using this

* Total operating primary energy use of baseline retail building after 50 years $=$

${ }^{*}$ Total operating GWP of baseline retail building after 50 years $=2,310$ tonnes

\section{Door \#5 (D-5)}

Building Component Description:

\begin{tabular}{|c|c|c|c|}
\hline Category: & Windows \& Doors & $\begin{array}{l}\text { Assembly Layers } \\
\text { Outside }\end{array}$ & \multirow{7}{*}{ ] } \\
\hline \multirow{3}{*}{ Brief Description: } & \multirow{3}{*}{$\begin{array}{l}\text { Standard size solid wood interior door with } \\
\text { no glazing }\end{array}$} & $\begin{array}{l}\text { Outside } \\
\text { Solid wood interior door with no glazing } \\
\text { (Includes late w paint) }\end{array}$ & \\
\hline & & $\begin{array}{c}\text { (Includes Satex paint) } \\
\text { Standard }\end{array}$ & \\
\hline & & Inside & \\
\hline \multicolumn{2}{|l|}{ Quick Numbers: } & & \\
\hline Total Embodied Energy & 539 W/unit & & \\
\hline Total Embodied GWP: & $16 \mathrm{~kg}$ of $\mathrm{CO}_{2}$ eq/unit & & \\
\hline
\end{tabular}

Life-Cycle Assessment Results:

\begin{tabular}{|c|c|c|c|c|c|c|c|c|c|c|c|c|c|c|}
\hline \multirow{4}{*}{$\begin{array}{l}\text { Lifespan } \\
\text { (Years) }\end{array}$} & \multicolumn{13}{|c|}{ Primary Energy Consumption (MJ) } & \multirow{4}{*}{$\begin{array}{c}\text { Difference in } \\
\text { Operating Energy } \\
\text { from Baseline afte } \\
\text { Lifespan } \\
\text { ( }{ }^{4} \text { per unit) }\end{array}$} \\
\hline & \multicolumn{13}{|c|}{ Embodied Energy (EE) } & \\
\hline & \multicolumn{3}{|c|}{ Manutacturing } & \multicolumn{3}{|c|}{ Construction } & \multicolumn{3}{|c|}{ Maintenance } & \multicolumn{3}{|c|}{ End of Life } & \multirow{2}{*}{$\begin{array}{l}{ }^{3} \text { Total EE } \\
\text { (per unit) }\end{array}$} & \\
\hline & Material & ${ }^{2}$ Trans. & Total & Material & ${ }^{2}$ Trans. & Total & Material & ${ }^{2}$ Trans & \begin{tabular}{|c|c|} 
Total \\
\end{tabular} & Material & \begin{tabular}{|l|}
${ }^{2}$ Trans. \\
\end{tabular} & \begin{tabular}{|l} 
Total \\
\end{tabular} & & \\
\hline Initial & 492 & 17 & 509 & 0 & 28 & 28 & 0 & 0 & 0 & 0 & 0 & 0 & & \\
\hline 50 & 492 & 17 & 509 & 0 & 28 & 28 & 0 & 0 & 0 & 0 & 3 & 3 & 539 & \\
\hline
\end{tabular}

Global Warming Potential (kg of $\mathrm{CO}_{2}$ eq.)

\begin{tabular}{|c|c|c|c|c|c|c|c|c|c|c|c|c|c|c|}
\hline \multirow{3}{*}{$\begin{array}{l}\text { Lifespan } \\
\text { (Years) }\end{array}$} & \multicolumn{13}{|c|}{ Embodied Global Warming Potential (GWP) } & \multirow{3}{*}{\begin{tabular}{|c} 
Difference in \\
Operating GWP from \\
Baseline after \\
Lifespan \\
$\left({ }^{4}\right.$ per unit $)$
\end{tabular}} \\
\hline & \multicolumn{3}{|c|}{ Manutacturing } & \multicolumn{3}{|c|}{ Construction } & \multicolumn{3}{|c|}{ Maintenance } & \multicolumn{3}{|c|}{ End of Life } & \multirow{2}{*}{$\begin{array}{l}{ }^{3} \text { Total GWP } \\
\text { (per unit) }\end{array}$} & \\
\hline & Material & ${ }^{2}$ Trans. & Total & Material & ${ }^{2}$ Trans. & Total & Material & ${ }^{2}$ Trans. & \begin{tabular}{|l|l} 
Total \\
\end{tabular} & Material & ${ }^{2}$ Trans. & Total & & \\
\hline \begin{tabular}{|l|l} 
nitial \\
\end{tabular} & 16 & 0 & 16 & 0 & 0 & 0 & 0 & 0 & 0 & 0 & 0 & 0 & & \\
\hline 50 & 16 & 0 & 16 & 0 & 0 & 0 & 0 & 0 & 0 & 0 & 0 & 0 & 16 & 0 \\
\hline
\end{tabular}

ATHENA $®$ EIE Material List:

(Includes all materials after 50 years)

\begin{tabular}{|l|c|c|}
\hline \multicolumn{1}{|c|}{ Material List } & Quantities & Unit \\
\hline Nails & 2.0 & $\mathrm{~kg}$ \\
\hline $\begin{array}{l}\text { Small Dimension Softwood Lumber, } \\
\text { kiln-dried }\end{array}$ & 0.1 & $\mathrm{~m} 3$ \\
\hline Water Based Latex Paint & 0.7 & $\mathrm{~L}$ \\
\hline
\end{tabular}
Notes:
= Time 'o' (i.e. at the completion of initial construction) ${ }^{2}$ Trans. $=$ Transportation

${ }^{3}$ Total EE (or Total GWP) per unit = Total embodied energy (or total embodied GWP) of building component affer lifespan (i.e. total manufacturing

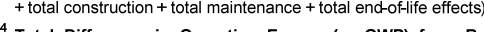

${ }^{4}$ Total Difference in Operating Energy (or GWP) from Baseline after
Lifespan per unit = The difference in the total life-cycle operating energy (or GWP) from the baseline retail building after lifespan, due to using this

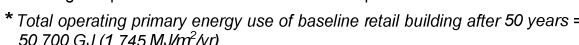

* Total operating $\mathrm{GWP}_{\mathrm{W}}$ of baseline retail building after 50 years $=2,310$ tonnes
of $\mathrm{CO}_{2}$ eq. $\left(80 \mathrm{~kg}\right.$ of $\mathrm{CO}_{2}$ eq. $\left.\mathrm{m}^{2} \mathrm{yr}\right)$ 


\section{Door \#6 (D-6)}

Building Component Description:

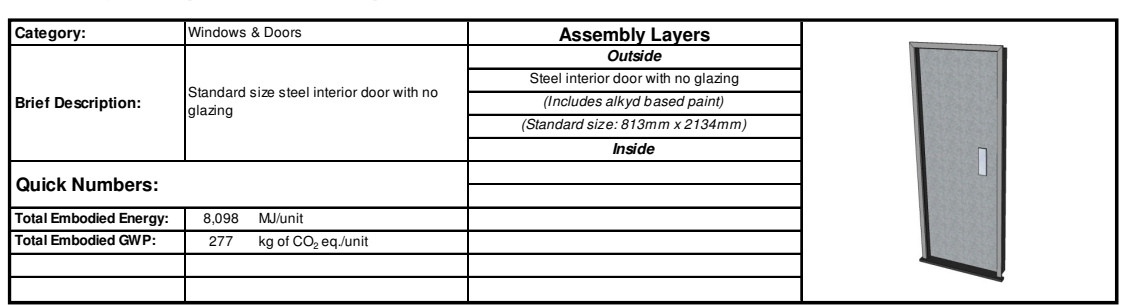

Life-Cycle Assessment Results:

\begin{tabular}{|c|c|c|c|c|c|c|c|c|c|c|c|c|c|c|}
\hline \multirow{4}{*}{$\begin{array}{l}\text { Litespas) } \\
\text { (Years) }\end{array}$} & \multicolumn{14}{|c|}{ Primary Energy Consumption (MJ) } \\
\hline & \multicolumn{13}{|c|}{ Embodied Energy (EE) } & \multirow{3}{*}{$\begin{array}{c}\text { Difference in } \\
\text { Operating Energy } \\
\text { from Baseline after } \\
\text { Lifespan } \\
\left({ }^{4} \text { per unit) }\right.\end{array}$} \\
\hline & \multicolumn{3}{|c|}{ Manufacturing } & \multicolumn{3}{|c|}{ Construction } & \multicolumn{3}{|c|}{ Maintenance } & \multicolumn{3}{|c|}{ End of Life } & \multirow{2}{*}{$\begin{array}{l}{ }^{3} \text { Total EE } \\
\text { (per unit) }\end{array}$} & \\
\hline & Material & ${ }^{2}$ Trans. & Total & Material & ${ }^{2}$ Trans. & Total & Material & ${ }^{2}$ Trans. & Total & Material & ${ }^{2}$ Trans. & Total & & \\
\hline${ }^{\prime}$ Initial & 8,067 & 12 & 8,079 & 0 & 14 & 14 & 0 & 0 & 0 & 0 & 0 & 0 & 8,093 & \\
\hline 50 & \begin{tabular}{|l|l|}
8,067 \\
\end{tabular} & 12 & 8,079 & 0 & 14 & 14 & 0 & 0 & 0 & 0 & 5 & 5 & 8,098 & 0 \\
\hline
\end{tabular}

Global Warming Potential $\left(\mathrm{kg}\right.$ of $\mathrm{CO}_{2}$ eq.)

$\stackrel{\infty}{\infty}$

\begin{tabular}{|c|c|c|c|c|c|c|c|c|c|c|c|c|c|c|}
\hline \multirow{3}{*}{$\begin{array}{l}\text { Lifespan } \\
\text { (Years) }\end{array}$} & \multicolumn{13}{|c|}{ Embodied Global Warming Potential (GWP) } & \multirow{3}{*}{$\begin{array}{c}\text { Difference in } \\
\text { Operating GWP from } \\
\text { Baseline after } \\
\text { Lifespan } \\
\left({ }^{4} \text { per unit }\right)\end{array}$} \\
\hline & \multicolumn{3}{|c|}{ Manufacturing } & \multicolumn{3}{|c|}{ Construction } & \multicolumn{3}{|c|}{ Maintenance } & \multicolumn{3}{|c|}{ End of Life } & \multirow{2}{*}{$\begin{array}{l}{ }^{3} \text { Total GWP } \\
\text { (per unit) }\end{array}$} & \\
\hline & Material & ${ }^{2}$ Trans. & Total & Material & ${ }^{2}$ Trans. & Total & Material & ${ }^{2}$ Trans. & Total & Material & ${ }^{2}$ Trans. & Total & & \\
\hline Initial & 277 & 0 & 277 & 0 & 0 & 0 & 0 & 0 & 0 & 0 & 0 & 0 & 277 & 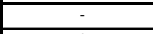 \\
\hline & & & & 0 & & & & & 0 & & & & & 0 \\
\hline
\end{tabular}

Note: Calculations ar

ATHENA ® EIE Material List:

(Includes all materiats arer 50 years

Notes:
1 Initial $=$ Time ' 0 ' (i.e. at the completion of nitial construction)

\begin{tabular}{|l|c|c|}
\hline \multicolumn{1}{|c|}{ Material List } & Quantities & Unit \\
\hline Galvanized Sheet & 61.9 & $\mathrm{~kg}$ \\
\hline Nails & 2.0 & $\mathrm{~kg}$ \\
\hline Solvent Based Alkyd Paint & 0.3 & $\mathrm{~L}$ \\
\hline
\end{tabular}

${ }^{3}$ Total EE (or Total GWP) per unit $=$ Total embodied energy (or total

embodied GWP) of building component affer lifespan (i.e. total manufacturing
+ total construction + total maintenance + total end-of-life effects)

4 total construction + total mantenance + total end-of-ifie effects)

${ }^{4}$ Total Diference in Operating Energy (or GWP) from Baseline after GWP from the baseline retail building after lifespan, due to using this
building componentinstear of the besline componet (t) baseline component

* Total operating primary energy use of baseline retail building after 50 years $=$

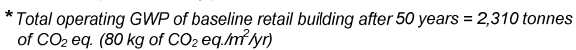


Appendix B-6

LCA Data for Foundations 


\section{LCA Data for Isolated Footings and Concrete Piers}

This section contains a detailed description of each isolated footing and concrete pier combination that was examined in this study ( 7 in total). The assembly layers are listed for each case, along with a detailed description of the material quantities from the ATHENA® Environmental Impact Estimator for Buildings.

A breakdown of the total primary energy consumption and the total global warming potential (GWP) for each footing and pier is also included. In each case, the results were calculated for one isolated footing with one concrete pier. The data has been calculated for two different lifespans: at the completion of initial construction and after 50 years.

As a summary, the figure below illustrates a comparison of the total embodied energy (and GWP) after 50 years for the various footing and pier combinations in this section. For comparison purpose, the building component used in the baseline retail building has been shaded in grey.

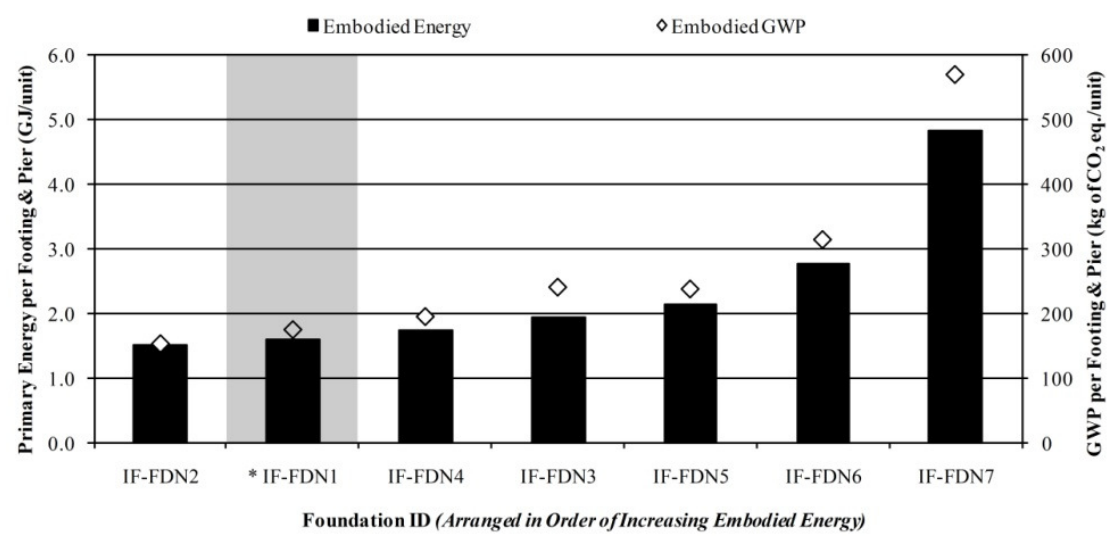

Isolated Concrete Footing and Pier \#1 (IF-FDN1)

Building Component Description:

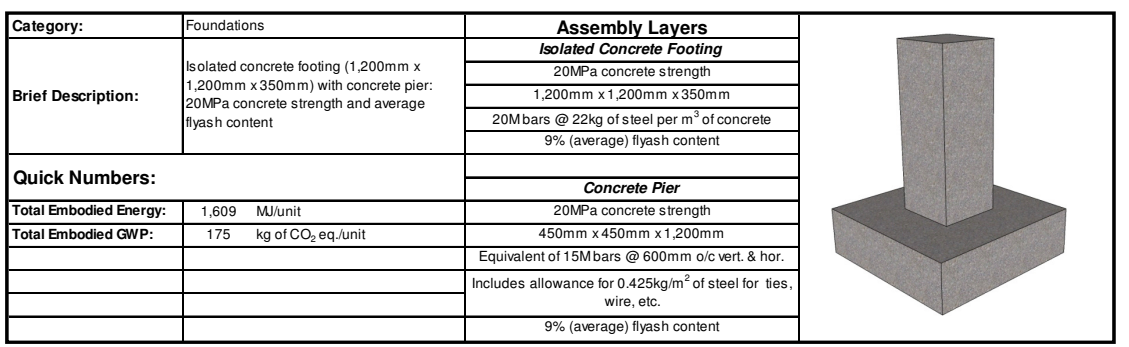

Life-Cycle Assessment Results:

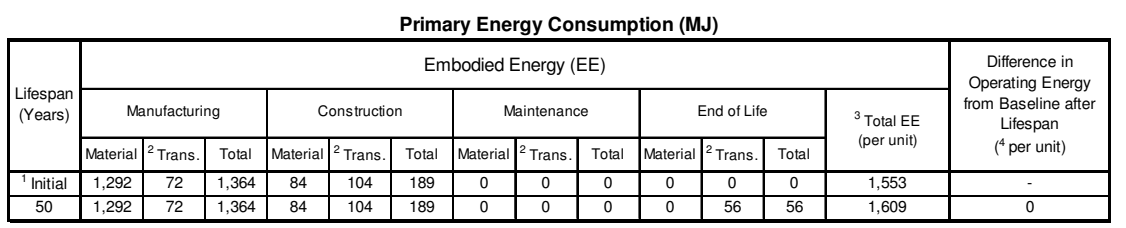

Global Warming Potential (kg of $\mathrm{CO}_{2}$ eq.)

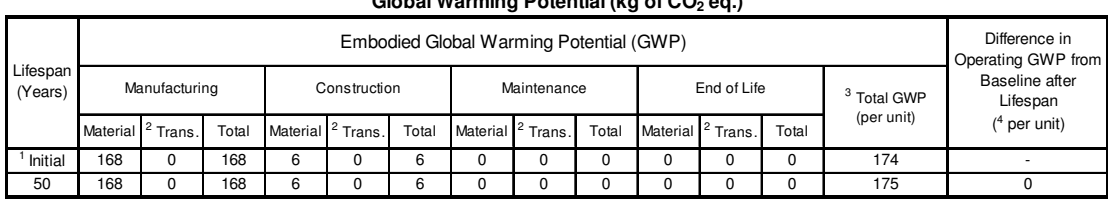

Note: Calculations are for one isolated footing and one concrete pier combination

ATHENA $₫$ EIE Material List:

(Includes all materials after 50 years)

$$
\begin{aligned}
& \text { Notes: } \\
& { }^{1} \text { Initial }=\text { Time ' } 0 \text { ' (i.e. at the completion of initial construction) }
\end{aligned}
$$

\begin{tabular}{|l|c|c|}
\hline \multicolumn{1}{|c|}{ Material List } & Quantities & Unit \\
\hline Concrete $20 \mathrm{MPa}$ (flyash av) & 0.8 & $\mathrm{~m} 3$ \\
\hline Rebar, Rod, Light Sections & 14.4 & $\mathrm{~kg}$ \\
\hline
\end{tabular}

${ }^{2}$ Trans. = Transportation

${ }^{3}$ Total EE (or Total GWP) per unit $=$ Total embodied energy (or total embodied GWP) of building component after lifespan (i.e. total manufacturing + total construction + total mantenance + total end-of-life effects) ${ }^{4}$ Total Difference in Operating Energy (or GWP) from Baseline after
Lifespan per unit $=$ The difference in the total life-cycle operating energy (o Lifespan per unit $=$ The difference in the total life-cycle operating energy (or
GWP) from the baseline retail building after lifespan, due to using this building component instead of the baseline componen * Total operating primary energy use of baseline retail building after 50 years =

* Total operating $\mathrm{GWP}$ of baseline retail building after 50 years $=2,310$ tonnes
of $\mathrm{CO}_{2}$ eq. $80 \mathrm{~kg}$ of $\mathrm{CO}_{2}$ eq. $\left./ \mathrm{m}^{2} / \mathrm{yr}\right)$ 


\section{Isolated Concrete Footing and Pier \#2 (IF-FDN2)}

Building Component Description:

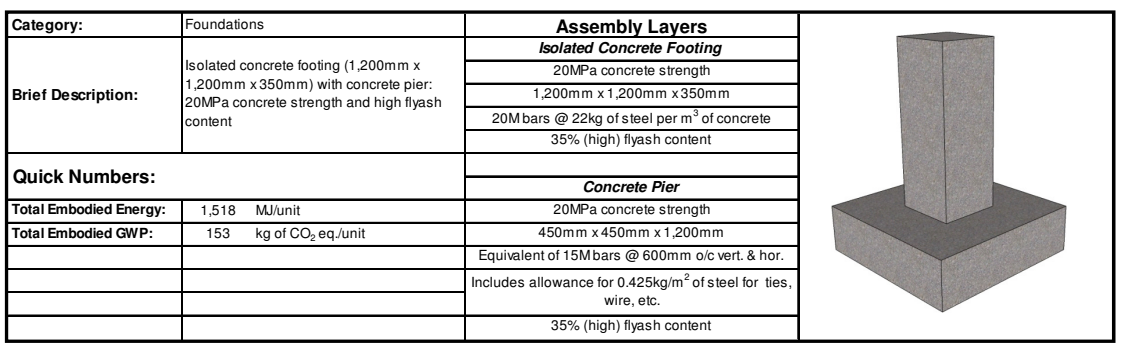

Life-Cycle Assessment Results:

Primary Energy Consumption (MJ)

\begin{tabular}{|c|c|c|c|c|c|c|c|c|c|c|c|c|c|c|}
\hline \multirow{3}{*}{$\begin{array}{l}\text { Lifiesparars } \\
\text { (Years }\end{array}$} & \multicolumn{13}{|c|}{ Embodied Energy (EE) } & \multirow{3}{*}{\begin{tabular}{|c|} 
Difference in \\
Operating Energy \\
from Baseline after \\
Lifespan \\
$\left({ }^{4}\right.$ per unit)
\end{tabular}} \\
\hline & \multicolumn{3}{|c|}{ Manufacturing } & \multicolumn{3}{|c|}{ Construction } & \multicolumn{3}{|c|}{ Maintenance } & \multicolumn{3}{|c|}{ End of L Life } & \multirow{2}{*}{$\begin{array}{l}{ }^{3} \text { Total EE } \\
\text { (per unit) }\end{array}$} & \\
\hline & Material & ${ }^{2}$ Trans. & \begin{tabular}{|l|l|l|} 
Total \\
\end{tabular} & Material & ${ }^{2}$ Trans. & Total & Material & ${ }^{2}$ Trans. & Total & Material & ${ }^{2}$ Trans. & Total & & \\
\hline Initial & 1,179 & 94 & 1,274 & 84 & 104 & 189 & 0 & 0 & 8 & 0 & 0 & 0 & & \\
\hline 50 & \begin{tabular}{|l|}
1,179 \\
\end{tabular} & $\begin{array}{ll}94 \\
\end{array}$ & \begin{tabular}{|l|l|l|}
1,274 \\
\end{tabular} & 84 & 104 & 189 & 0 & 0 & 0 & 0 & 56 & 56 & 1,51 & \\
\hline
\end{tabular}

Global Warming Potential ( $\mathrm{kg}$ of $\mathrm{CO}_{2}$ eq. $)$

$\infty$

\begin{tabular}{|c|c|c|c|c|c|c|c|c|c|c|c|c|c|c|}
\hline \multirow{3}{*}{$\begin{array}{l}\text { Lifespan } \\
\text { (Years) }\end{array}$} & \multicolumn{13}{|c|}{ Embodied Global Warming Potential (GWP) } & \multirow{3}{*}{\begin{tabular}{|c} 
Difference in \\
Operating GWP from \\
Baseline after \\
Lifespan \\
$\left({ }^{4}\right.$ per unit $)$
\end{tabular}} \\
\hline & \multicolumn{3}{|c|}{ Manufacturing } & \multicolumn{3}{|c|}{ Construction } & \multicolumn{3}{|c|}{ Maintenance } & \multicolumn{3}{|c|}{ End of Life } & \multirow{2}{*}{$\begin{array}{l}{ }^{3} \text { Total GWP } \\
\text { (per unit) }\end{array}$} & \\
\hline & Material & ${ }^{2}$ Trans. & Total & Material & \begin{tabular}{|l}
${ }^{2}$ Trans. \\
\end{tabular} & Total & Material & ${ }^{2}$ Trans. & \begin{tabular}{|l|} 
Total \\
\end{tabular} & \begin{tabular}{|c|} 
Material \\
\end{tabular} & $\begin{array}{ll}2 & 2 \\
2\end{array}$ & \begin{tabular}{|l|l|} 
Total \\
\end{tabular} & & \\
\hline${ }^{1}$ Initial & 146 & 0 & 147 & 6 & 0 & 6 & 0 & 0 & 0 & 0 & 0 & 0 & 153 & \\
\hline 50 & 146 & 0 & 147 & 6 & 0 & 6 & 0 & 0 & 0 & 0 & 0 & 0 & 153 & 0 \\
\hline
\end{tabular}

Note: Calculations are for one isolated footing and one concrete pier combination

ATHENA ® EIE Material List:
(Includes all materials after 50 years)
\begin{tabular}{|l|c|c|}
\hline Material List & Quantities & Unit \\
\hline Concrete 20 MPa (flyash 35\%) & 0.8 & $\mathrm{~m} 3$ \\
\hline Rebar, Rod, Light Sections & 14.4 & $\mathrm{~kg}$ \\
\hline
\end{tabular}

Notes:

${ }^{2}$ Trans. = Transportation

${ }^{3}$ Total EE (or Total GWP) per unit $=$ Total embodied energy (or total embodied GWP) of building component after lifespan (i.e. total manufacturing

+ total construction + total maintenance + total end-of-ifie effects)

Total Difference in Operating Energy (or GWP) from Baseline after Lifespan per unit $=$ The difference in the total life-cycle operating energy (or
GWP from the baseline retail building after lifespan, due to using this building component instead of the baseline component

"Total operating primary energy use of baseline retail building after 50 years

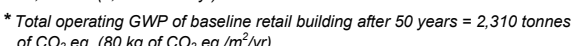

\section{Isolated Concrete Footing and Pier \#3 (IF-FDN3)}

Building Component Description:

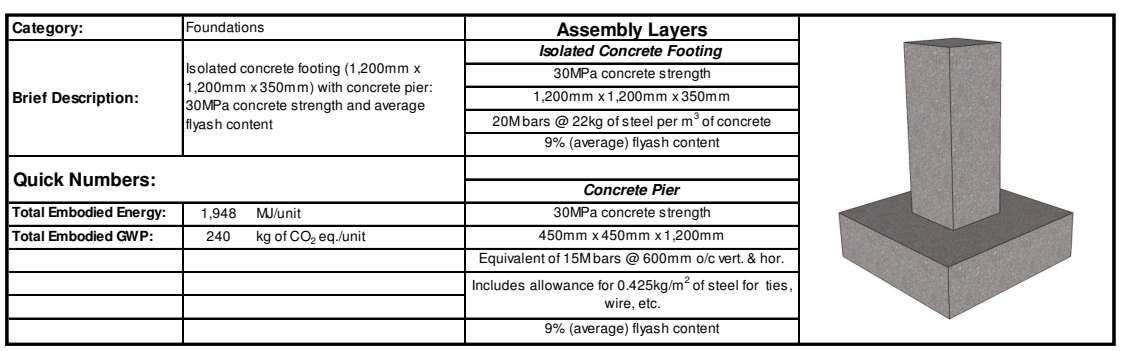

Life-Cycle Assessment Results:

Primary Energy Consumption (MJ)

\begin{tabular}{|c|c|c|c|c|c|c|c|c|c|c|c|c|c|c|}
\hline \multirow{3}{*}{$\begin{array}{l}\text { Lifespanan } \\
\text { (Years) }\end{array}$} & \multicolumn{13}{|c|}{ Embodied Energy (EE) } & \multirow{3}{*}{$\begin{array}{l}\text { Difference in } \\
\text { Operating Energy } \\
\text { from Baseline after } \\
\text { Lifespan } \\
\left({ }^{4} \text { per unit) }\right.\end{array}$} \\
\hline & \multicolumn{3}{|c|}{ Manufacturing } & \multicolumn{3}{|c|}{ Construction } & \multicolumn{3}{|c|}{ Maintenance } & \multicolumn{3}{|c|}{ End of Life } & \multirow{2}{*}{$\begin{array}{l}{ }^{3} \text { Total EE } \\
\text { (per unit) }\end{array}$} & \\
\hline & Material & ${ }^{2}$ Trans. & Total & Materia & ${ }^{2}$ Trans. & Total & Material & ${ }^{2}$ Trans. & Total & Material & ${ }^{2}$ Trans. & \begin{tabular}{|l|l} 
Total \\
\end{tabular} & & \\
\hline 'Initial & 1,619 & 84 & 1,703 & 84 & 104 & 189 & 0 & 0 & 0 & 0 & 0 & 0 & $\frac{1,8}{1,8}$ & F \\
\hline 50 & 1,619 & 84 & 1,703 & 84 & $\begin{array}{l}104 \\
\end{array}$ & $\begin{array}{l}189 \\
\end{array}$ & 0 & 0 & 0 & 0 & 56 & 56 & 1,948 & 0 \\
\hline
\end{tabular}

Global Warming Potential (kg of $\mathrm{CO}_{2}$ eq.)

\begin{tabular}{|c|c|c|c|c|c|c|c|c|c|c|c|c|c|c|}
\hline \multirow{3}{*}{$\begin{array}{l}\text { Lifespan } \\
\text { (Years) }\end{array}$} & \multicolumn{13}{|c|}{ Embodied Global Warming Potential (GWP) } & \multirow{3}{*}{\begin{tabular}{|l} 
Difference in \\
Operating GWP from \\
Baseline after \\
Lifespan \\
$\left({ }^{4}\right.$ per unit)
\end{tabular}} \\
\hline & \multicolumn{3}{|c|}{ Manutacturing } & \multicolumn{3}{|c|}{ Construction } & \multicolumn{3}{|c|}{ Maintenance } & \multicolumn{3}{|c|}{ End of Life } & \multirow{2}{*}{$\begin{array}{l}{ }^{3} \text { Total GWP } \\
\text { (per unit) }\end{array}$} & \\
\hline & Materia & ${ }^{2}$ Trans. & Total & Material & ${ }^{2}$ Trans. & Total & Materia & ${ }^{2}$ Trans & Total & Material & ${ }^{2}$ Trans. & Total & & \\
\hline Initial & 234 & 0 & 234 & 6 & 0 & 6 & 0 & 0 & 0 & 0 & 0 & 0 & 240 & \\
\hline 50 & 234 & 0 & 234 & 6 & 0 & 6 & 0 & 0 & 0 & 0 & 0 & 0 & 240 & 0 \\
\hline
\end{tabular}
Note: Calculations are for one isolated footing and one concrete pier combination

\section{ATHENA ${ }^{\circledR}$ EIE Material List: \\ ${ }^{1}$ Initial $=$ Time ' $O$ ' (i.e. at the completes:}

(Includes all materials after 50 years)

\begin{tabular}{|l|c|c|}
\hline \multicolumn{1}{|c|}{ Material List } & Quantities & Unit \\
\hline Concrete $30 \mathrm{MPa}$ (flyash av) & 0.8 & $\mathrm{~m} 3$ \\
\hline Rebar, Rod, Light Sections & 14.4 & $\mathrm{~kg}$ \\
\hline
\end{tabular}

${ }^{3}$ Total EE (or Total GWP) per unit = Total embodied energy (or total embodied GWP) of building component after lifespan (i.e. total manufacturing
+ total construction + total maintenance + total end-of-life effects) ${ }^{4}$ Total Difference in Operating Energy (or GWP) from Baseline after Lifespan per unit = The difference in the total life-cycle operating energy (or
GWP) from the baseline retail building after lifespan, due to using this building component instead of the baseline component * Total operating primary energy use of baseline retail building after 50 years

* Total operatiting $\mathrm{GWP}$ of baseline retail building after 50 years $=2,310$ tonnes
of $\mathrm{CO}_{2}$ eq. $80 \mathrm{~kg}$ of $\mathrm{CO}_{2}$ eq. $\left./ \mathrm{m}^{2} \mathrm{Yyr}\right)$ 


\section{Isolated Concrete Footing and Pier \#4 (IF-FDN4)}

Building Component Description:

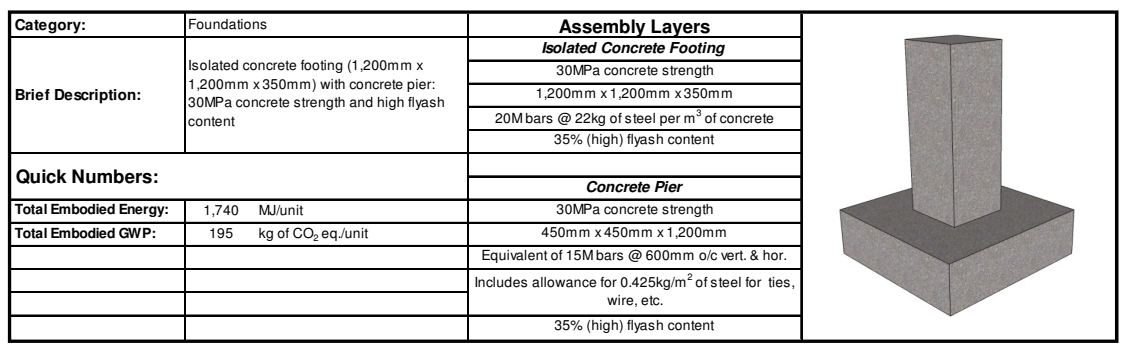

Life-Cycle Assessment Results:

Primary Energy Consumption (MJ)

\begin{tabular}{|c|c|c|c|c|c|c|c|c|c|c|c|c|c|c|}
\hline \multirow{3}{*}{$\begin{array}{l}\text { Lifesparan } \\
\text { (Years) }\end{array}$} & \multicolumn{13}{|c|}{ Embodied Energy (EE) } & \multirow{3}{*}{\begin{tabular}{|c|} 
Difference in \\
Operating Energy \\
from Baseline after \\
Lifespan \\
$\left({ }^{4}\right.$ per unit)
\end{tabular}} \\
\hline & \multicolumn{3}{|c|}{ Manutacturing } & \multicolumn{3}{|c|}{ Construction } & \multicolumn{3}{|c|}{ Maintenance } & \multicolumn{3}{|c|}{ End of L Life } & \multirow{2}{*}{$\begin{array}{l}{ }^{3} \text { Total EE } \\
\text { (per unit) }\end{array}$} & \\
\hline & Material & ${ }^{2}$ Trans. & \begin{tabular}{|l|l|l|} 
Total & \\
\end{tabular} & Material & ${ }^{2}$ Trans. & Total & Material & ${ }^{2}$ Trans. & Total & Material & ${ }^{2}$ Trans. & Total & & \\
\hline Initial & 1,383 & 111 & 1,495 & 84 & 104 & 189 & 0 & 0 & 0 & 0 & 0 & 8 & & \\
\hline 50 & \begin{tabular}{|l|}
1,383 \\
\end{tabular} & \begin{tabular}{|l|l|}
111 \\
\end{tabular} & \begin{tabular}{|l|l|}
1,495 \\
\end{tabular} & 84 & 104 & 189 & 0 & 0 & 0 & 0 & 56 & \begin{tabular}{|l|}
56 \\
\end{tabular} & 1,7 & \\
\hline
\end{tabular}

Global Warming Potential ( $\mathrm{kg}$ of $\mathrm{CO}_{2}$ eq.)

$\underset{\wp}{\infty}$

\begin{tabular}{|c|c|c|c|c|c|c|c|c|c|c|c|c|c|c|}
\hline \multirow{3}{*}{$\begin{array}{l}\text { Lifespan } \\
\text { (Years) }\end{array}$} & \multicolumn{13}{|c|}{ Embodied Global Warming Potential (GWP) } & \multirow{3}{*}{\begin{tabular}{|c|} 
Difference in \\
Operating GWP from \\
Baseline after \\
Lifespan \\
$\left({ }^{4}\right.$ per unit $)$ \\
\end{tabular}} \\
\hline & \multicolumn{3}{|c|}{ Manufacturing } & \multicolumn{3}{|c|}{ Construction } & \multicolumn{3}{|c|}{ Maintenance } & \multicolumn{3}{|c|}{ End of Life } & \multirow{2}{*}{$\begin{array}{l}{ }^{3} \text { Total GWP } \\
\text { (per unit) }\end{array}$} & \\
\hline & \begin{tabular}{|l|} 
Material \\
\end{tabular} & ${ }^{2}$ Trans. & Total & \begin{tabular}{|l|} 
Material \\
\end{tabular} & ${ }^{2}$ Trans. & $\begin{array}{l}\text { Total } \\
\end{array}$ & Material & 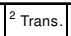 & \begin{tabular}{|l|} 
Total \\
\end{tabular} & \begin{tabular}{|l|} 
Material \\
\end{tabular} & 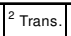 & Total & & \\
\hline${ }^{1}$ Initial & 188 & 0 & 188 & 6 & 0 & 6 & 0 & 0 & 0 & 0 & 0 & 0 & & \\
\hline 50 & 188 & 0 & 188 & 6 & 0 & 6 & 0 & 0 & 0 & 0 & 0 & 0 & 195 & 0 \\
\hline
\end{tabular}

Note: Calculations are for one isolated footing and one concrete pier combination

ATHENA ® EIE Material List:
(Includes all materials after 50 years)
\begin{tabular}{|l|c|c|}
\hline Material List & Quantities & Unit \\
\hline Concrete 30 MPa (flyash $35 \%)$ & 0.8 & $\mathrm{~m} 3$ \\
\hline Rebar, Rod, Light Sections & 14.4 & $\mathrm{~kg}$ \\
\hline
\end{tabular}

Notes:

${ }^{2}$ Trans. = Transportation

${ }^{3}$ Total EE (or Total GWP) per unit $=$ Total embodied energy (or total embodied GWP) of building component after lifespan (i.e. total manufacturing

+ total construction + total maintenance + total end-of-ifie effects)

Total Difference in Operating Energy (or GWP) from Baseline after Lifespan per unit $=$ The difference in the total life-cycle operating energy (or
GWP) from the baseline retail building after lifespan, due to using this building component instead of the baseline component

"Total operating primary energy use of baseline retail building after 50 years

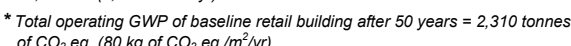

Isolated Concrete Footing and Pier \#5 (IF-FDN5)

Building Component Description:

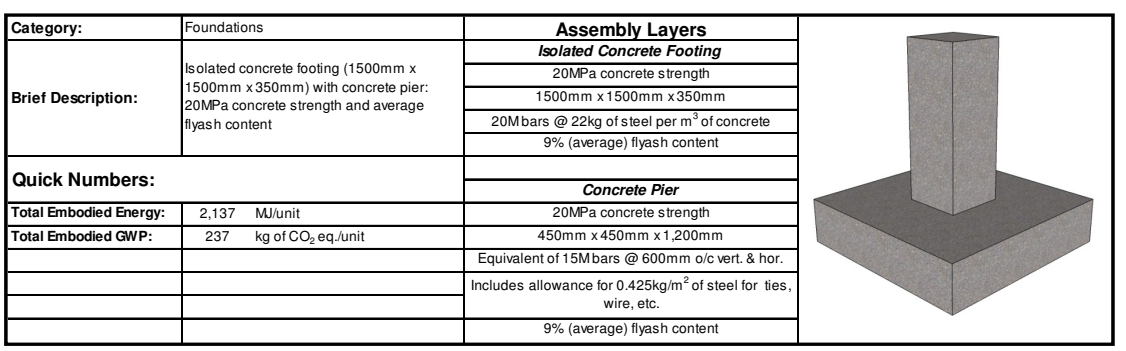

Life-Cycle Assessment Results:

Primary Energy Consumption (MJ)

\begin{tabular}{|c|c|c|c|c|c|c|c|c|c|c|c|c|c|c|}
\hline \multirow{3}{*}{$\begin{array}{l}\text { Lifespan } \\
\text { (Years) }\end{array}$} & \multicolumn{13}{|c|}{ Embodied Energy (EE) } & \multirow{3}{*}{\begin{tabular}{|c|} 
Difference in \\
Operating Energy \\
from Baseline after \\
Lifespan \\
$\left({ }^{4}\right.$ per unit)
\end{tabular}} \\
\hline & \multicolumn{3}{|c|}{ Manufacturing } & \multicolumn{3}{|c|}{ Construction } & \multicolumn{3}{|c|}{ Maintenance } & \multicolumn{3}{|c|}{ End of Life } & \multirow{2}{*}{$\begin{array}{l}{ }^{3} \text { Total EE } \\
\text { (per unit) }\end{array}$} & \\
\hline & Material & $\left.1\right|^{2}$ Trans. & \begin{tabular}{|l|l|l|} 
Total \\
\end{tabular} & Materia & ${ }^{2}$ Trans. & Total & Materia & ${ }^{2}$ Trans & Total & Material & ${ }^{2}$ Trans. & \begin{tabular}{|l|l} 
Total \\
\end{tabular} & & \\
\hline 'Initial & 1,718 & 99 & 1,817 & 99 & 144 & 243 & 0 & 0 & 0 & 0 & 0 & 0 & 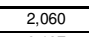 & F \\
\hline 50 & 1,718 & 99 & 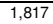 & $\begin{array}{l}99 \\
\end{array}$ & 144 & 243 & 0 & 0 & 0 & 0 & 77 & $\begin{array}{l}77 \\
\end{array}$ & $\begin{array}{l}2,137 \\
\end{array}$ & 0 \\
\hline
\end{tabular}

Global Warming Potential (kg of $\mathrm{CO}_{2}$ eq.)

\begin{tabular}{|c|c|c|c|c|c|c|c|c|c|c|c|c|c|c|}
\hline \multirow{3}{*}{$\begin{array}{l}\text { Lifespan } \\
\text { (Years) }\end{array}$} & \multicolumn{13}{|c|}{ Embodied Global Warming Potential (GWP) } & \multirow{3}{*}{\begin{tabular}{|l} 
Difference in \\
Operating GWP from \\
Baseline after \\
Lifespan \\
$\left({ }^{4}\right.$ per unit)
\end{tabular}} \\
\hline & \multicolumn{3}{|c|}{ Manutacturing } & \multicolumn{3}{|c|}{ Construction } & \multicolumn{3}{|c|}{ Maintenance } & \multicolumn{3}{|c|}{ End of Life } & \multirow{2}{*}{$\begin{array}{l}{ }^{3} \text { Total GWP } \\
\text { (per unit) }\end{array}$} & \\
\hline & Materia & ${ }^{2}$ Trans. & Total & Material & ${ }^{2}$ Trans. & Total & Materia & ${ }^{2}$ Trans & Total & Material & ${ }^{2}$ Trans. & Total & & \\
\hline Initial & 230 & 0 & 230 & 7 & 0 & 7 & 0 & 0 & 0 & 0 & 0 & 0 & 23 & 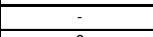 \\
\hline 50 & 230 & 0 & 230 & 7 & 0 & 7 & 0 & 0 & 0 & 0 & 0 & 0 & 237 & 0 \\
\hline
\end{tabular}
Note: Calculations are for one isolated footing and one concrete pier combination

\section{ATHENA $₫$ EIE Material List: \\ ${ }^{1}$ nitial $=$ Time ' $O$ ' (i.e. at the completes:}

(Includes all materials after 50 years)

\begin{tabular}{|l|c|c|}
\hline \multicolumn{1}{|c|}{ (Includes all materials after 50 years) } \\
\hline Concrete 20 MPa Lifiyash av) & Quantities & Unit \\
\hline Rebar, Rod, Light Sections & 1.1 & $\mathrm{~m} 3$ \\
\hline
\end{tabular}

${ }^{3}$ Total EE (or Total GWP) per unit $=$ Total embodied energy (or total embodied GWP) of building component after lifespan (i.e. total manufacturing
+ total construction + total maintenance + total end-of-life effects) ${ }^{4}$ Total Difference in Operating Energy (or GWP) from Baseline after Lifespan per unit = The difference in the total life-cycle operating energy (or
GWP) from the baseline retail building after lifespan, due to using this building component instead of the baseline component * Total operating primary energy use of baseline retail building after 50 years

* Total operatiting $\mathrm{GWP}$ of baseline retail building after 50 years $=2,310$ tonnes
of $\mathrm{CO}_{2}$ eq. $80 \mathrm{~kg}$ of $\mathrm{CO}_{2}$ eq. $\left./ \mathrm{m}^{2} \mathrm{Yyr}\right)$ 


\section{Isolated Concrete Footing and Pier \#6 (IF-FDN6)}

Building Component Description:

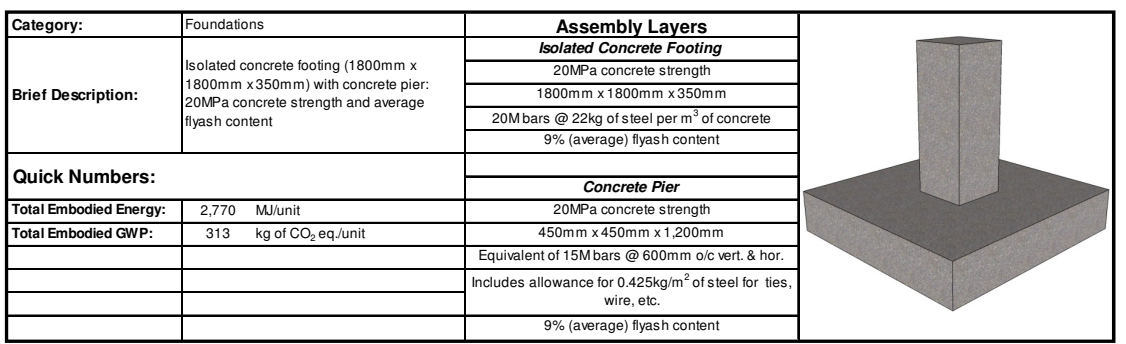

\section{Life-Cycle Assessment Results:}

Primary Energy Consumption (MJ)

\begin{tabular}{|c|c|c|c|c|c|c|c|c|c|c|c|c|c|c|}
\hline & & & & & & Prim & ry Ene & rgy Co & nsum & otion (M & & & & \\
\hline \multirow{3}{*}{ Years) } & \multicolumn{13}{|c|}{ Embodied Energy (EE) } & \multirow{3}{*}{$\begin{array}{c}\text { Difference in } \\
\text { Operating Energy } \\
\text { from Baseline after } \\
\text { Liffespan } \\
\left({ }^{4} \text { per unit }\right)\end{array}$} \\
\hline & \multicolumn{3}{|c|}{ Manutacturing } & \multicolumn{3}{|c|}{ Construction } & \multicolumn{3}{|c|}{ Maintenance } & \multicolumn{3}{|c|}{ End of Life } & \multirow{2}{*}{$\begin{array}{l}{ }^{3} \text { Total EE } \\
\text { (per unit) }\end{array}$} & \\
\hline & Material & $\begin{array}{l}1^{2} \text { Trans. } \\
\text { The }\end{array}$ & \begin{tabular}{|l|l|} 
Total \\
\end{tabular} & \begin{tabular}{|l|} 
Material \\
\end{tabular} & \begin{tabular}{|l|}
${ }^{2}$ Trans. \\
\end{tabular} & Total & Material & \begin{tabular}{|l}
${ }^{2}$ Trans. \\
\end{tabular} & \begin{tabular}{|l|l|} 
Total \\
\end{tabular} & \begin{tabular}{|l|} 
Material \\
\end{tabular} & $\mid{ }^{2}$ Trans. & \begin{tabular}{|l|} 
Total \\
\end{tabular} & & \\
\hline nitial & 2,227 & 131 & 2,358 & 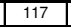 & 192 & 309 & 0 & 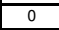 & 0 & 0 & 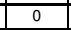 & 0 & 2,667 & \\
\hline 50 & $\mid 2,227$ & 131 & \begin{tabular}{|l|}
2,358 \\
\end{tabular} & 117 & \begin{tabular}{|l|}
192 \\
\end{tabular} & 309 & 0 & 0 & 0 & 0 & 102 & \begin{tabular}{|l|}
103 \\
\end{tabular} & 2,770 & 0 \\
\hline
\end{tabular}

Global Warming Potential ( $\mathrm{kg}$ of $\mathrm{CO}_{2}$ eq.)

\begin{tabular}{|c|c|c|c|c|c|c|c|c|c|c|c|c|c|c|}
\hline \multirow{3}{*}{$\begin{array}{c}\text { Lifiespan } \\
\text { (Years) }\end{array}$} & \multicolumn{13}{|c|}{ Embodied Global Warming Potential (GWP) } & \multirow{3}{*}{$\begin{array}{l}\text { Difference in } \\
\text { Operating GWP from } \\
\text { Baseline after } \\
\text { Lifespan } \\
\left({ }^{4} \text { per unit }\right)\end{array}$} \\
\hline & \multicolumn{3}{|c|}{ Manufacturing } & \multicolumn{3}{|c|}{ Construction } & \multicolumn{3}{|c|}{ Maintenance } & \multicolumn{3}{|c|}{ End of Life } & \multirow{2}{*}{$\begin{array}{l}{ }^{3} \text { Total GWP } \\
\text { (per unit) }\end{array}$} & \\
\hline & Materia & ${ }^{2}$ Trans. & Total & Material & Trans. & Total & Material & ${ }^{2}$ Trans. & Total & Material & 2 Trans. & Total & & \\
\hline Initial & 304 & 0 & 305 & 8 & 0 & 8 & 0 & 0 & 0 & 0 & 0 & 0 & 313 & \\
\hline 50 & 304 & 0 & 305 & 8 & 0 & 8 & 0 & 0 & 0 & 0 & 0 & 0 & 313 & 0 \\
\hline
\end{tabular}

Note: Calculations are for one isolated footing and one concrete pier combination

ATHENA ® EIE Material List:
(Includes all materials after 50 years)
\begin{tabular}{|l|c|c|}
\hline Material List & Quantities & Unit \\
\hline Concrete 20 MPa (flyash av) & 1.4 & $\mathrm{~m} 3$ \\
\hline Rebar, Rod, Light Sections & 19.4 & $\mathrm{~kg}$ \\
\hline
\end{tabular}

Notes:

${ }^{2}$ Trans. = Transportation

${ }^{3}$ Total EE (or Total GWP) per unit $=$ Total embodied energy (or total embodied GWP) of building component after lifespan (i.e. total manufacturing

+ total construction + total maintenance + total end-of-ifie effects)

Total Difference in Operating Energy (or GWP) from Baseline after Lifespan per unit = The difference in the total life-cycle operating energy (or
GWP) from the baseline retail building after lifespan, due to using this building component instead of the baseline component

"Total operating primary energy use of baseline retail building after 50 years

"Total operating $\mathrm{GW}$ of baseline retail building after 50 years $=2,310$ tonnes

\section{Isolated Concrete Footing and Pier \#7 (IF-FDN7)}

Building Component Description:

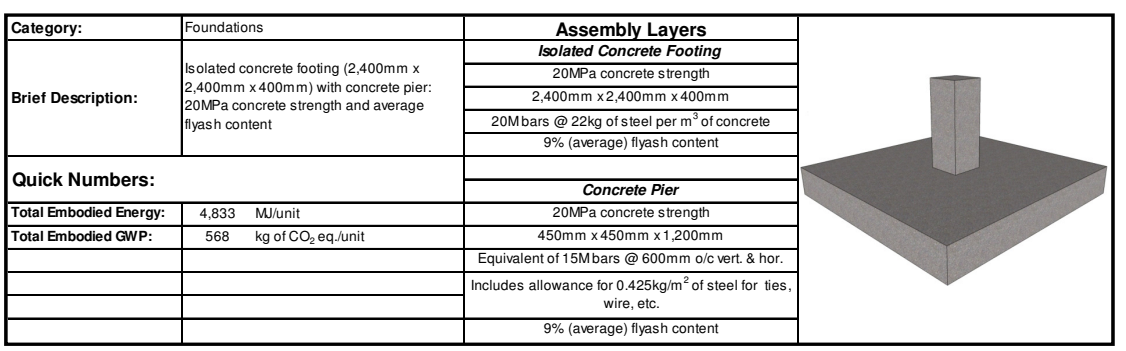

Life-Cycle Assessment Results:

Primary Energy Consumption (MJ)

\begin{tabular}{|c|c|c|c|c|c|c|c|c|c|c|c|c|c|c|}
\hline \multirow{3}{*}{$\begin{array}{l}\text { Lifespan } \\
\text { (Years) }\end{array}$} & \multicolumn{13}{|c|}{ Embodied Energy (EE) } & \multirow{3}{*}{$\begin{array}{l}\text { Difference in } \\
\text { Operating Energy } \\
\text { from Baseline after } \\
\text { Lifespan } \\
\left({ }^{4} \text { per unit) }\right.\end{array}$} \\
\hline & \multicolumn{3}{|c|}{ Manufacturing } & \multicolumn{3}{|c|}{ Construction } & \multicolumn{3}{|c|}{ Maintenance } & \multicolumn{3}{|c|}{ End of Life } & \multirow{2}{*}{$\begin{array}{l}{ }^{3} \text { Total EE } \\
\text { (per unit) }\end{array}$} & \\
\hline & Material & ${ }^{2}$ Trans. & \begin{tabular}{|l|l|l|} 
Total \\
\end{tabular} & Materia & ${ }^{2}$ Trans. & Total & Materia & ${ }^{2}$ Trans & Total & Material & ${ }^{2}$ Trans. & \begin{tabular}{|l|l} 
Total \\
\end{tabular} & & \\
\hline 'Initial & 3,869 & 241 & 4,111 & 178 & 355 & 533 & 0 & 0 & 0 & 0 & 0 & 0 & 4,644 & F \\
\hline 50 & 3,869 & 241 & 4,111 & $\begin{array}{ll}178 \\
\end{array}$ & 355 & 533 & 0 & 0 & 0 & 0 & $\begin{array}{l}189 \\
\end{array}$ & \begin{tabular}{|l|l|}
189 \\
\end{tabular} & $\begin{array}{l}4,833 \\
\end{array}$ & 0 \\
\hline
\end{tabular}

Global Warming Potential (kg of $\mathrm{CO}_{2}$ eq.)

\begin{tabular}{|c|c|c|c|c|c|c|c|c|c|c|c|c|c|c|}
\hline \multirow{3}{*}{$\begin{array}{l}\text { Lifespan } \\
\text { (Years) }\end{array}$} & \multicolumn{13}{|c|}{ Embodied Global Warming Potential (GWP) } & \multirow{3}{*}{\begin{tabular}{|l} 
Difference in \\
Operating GWP from \\
Baseline after \\
Lifespan \\
$\left({ }^{4}\right.$ per unit)
\end{tabular}} \\
\hline & \multicolumn{3}{|c|}{ Manutacturing } & \multicolumn{3}{|c|}{ Construction } & \multicolumn{3}{|c|}{ Maintenance } & \multicolumn{3}{|c|}{ End of Life } & \multirow{2}{*}{$\begin{array}{l}{ }^{3} \text { Total GWP } \\
\text { (per unit) }\end{array}$} & \\
\hline & Material & ${ }^{2}$ Trans. & Total & Materia & ${ }^{2}$ Trans. & Total & Materia & ${ }^{2}$ Trans & Total & Material & ${ }^{2}$ Trans. & Total & & \\
\hline Initial & 554 & 0 & 555 & 12 & 1 & 13 & 0 & 0 & 0 & 0 & 0 & 0 & 568 & \\
\hline 50 & 554 & 0 & 555 & 12 & 1 & 13 & 0 & 0 & 0 & 0 & 0 & 0 & 568 & 0 \\
\hline
\end{tabular}
Note: Calculations are for one isolated footing and one concrete pier combination

\section{ATHENA $®$ EIE Material List: \\ Notes:}

(Includes all materials after 50 years)

\begin{tabular}{|l|c|c|}
\hline \multicolumn{1}{|c|}{ Material List } & Quantities & Unit \\
\hline Concrete 20 MPa (flyash av) & 2.7 & $\mathrm{~m} 3$ \\
\hline Rebar, Rod, Light Sections & 24.4 & $\mathrm{~kg}$ \\
\hline
\end{tabular}

${ }^{3}$ Total EE (or Total GWP) per unit = Total embodied energy (or total embodied GWP) of building component after lifespan (i.e. total manufacturing
+ total construction + total maintenance + total end-of-life effects) 4
4 Total Difference in Operating Energy (or GWP) from Baseline after Lifespan per unit = The difference in the total life-cycle operating energy (or
GWP) from the baseline retail building after lifespan, due to using this building component instead of the baseline componen * Total operating primary energy use of baseline retail building after 50 years

* Total operatiting $\mathrm{GWP}$ of baseline retail building after 50 years $=2,310$ tonnes
of $\mathrm{CO}_{2}$ eq. $880 \mathrm{~kg}$ of $\mathrm{CO}_{2}$ eq. $\left./ \mathrm{m}^{2} / \mathrm{yr}\right)$ 


\section{LCA Data for Concrete Strip Footings and Foundation Walls}

This section contains a detailed description of each concrete strip footing and concrete foundation wall combination that was examined in this study (8 in total). The assembly layers are listed for each case, along with a detailed description of the material quantities from the ATHENA® Environmental Impact Estimator for Buildings.

A breakdown of the total primary energy consumption and the total global warming potential (GWP) for each footing and wall is also included. In each case, the results were calculated per linear meter of strip footing and foundation wall. The data has been calculated for two different lifespans: at the completion of initial construction and after 50 years.

As a summary, the figure below illustrates a comparison of the total embodied energy (and GWP) after 50 years for the various footing and wall combinations in this section. For comparison purpose, the building component used in the baseline retail building has been shaded in grey.

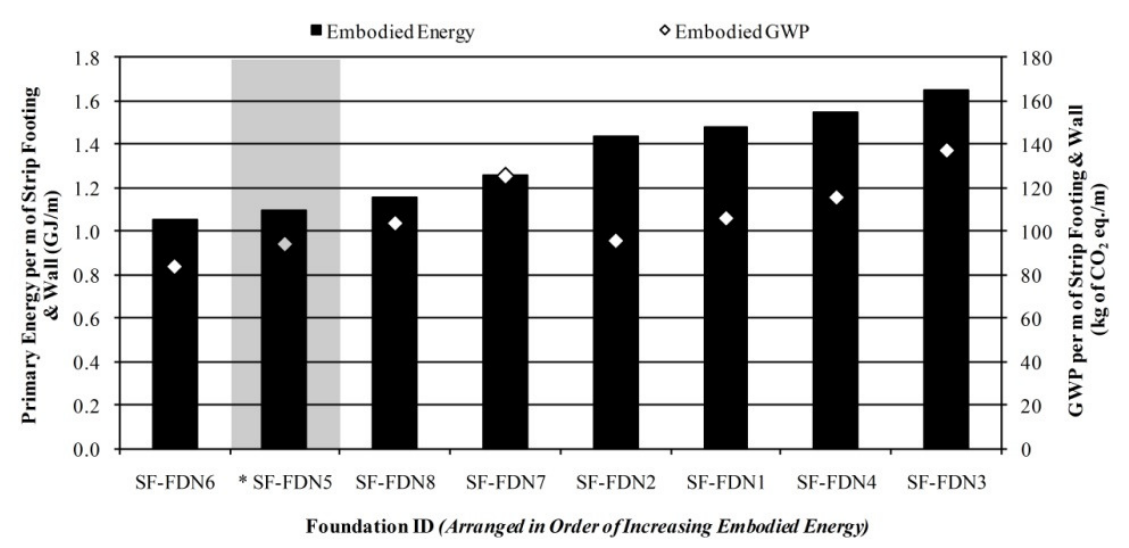

\section{Concrete Strip Footing and Foundation Wall \#1 (SF-FDN1)}

Building Component Description:

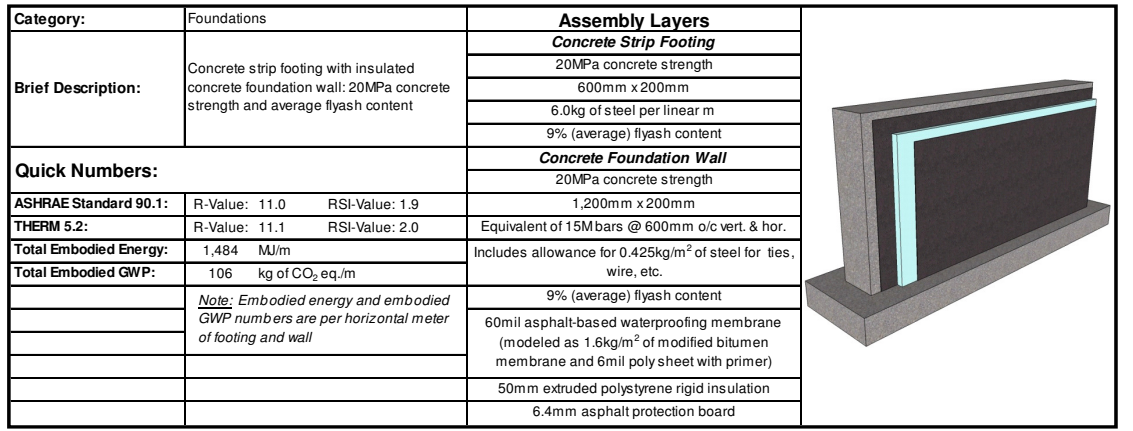

Life-Cycle Assessment Results:

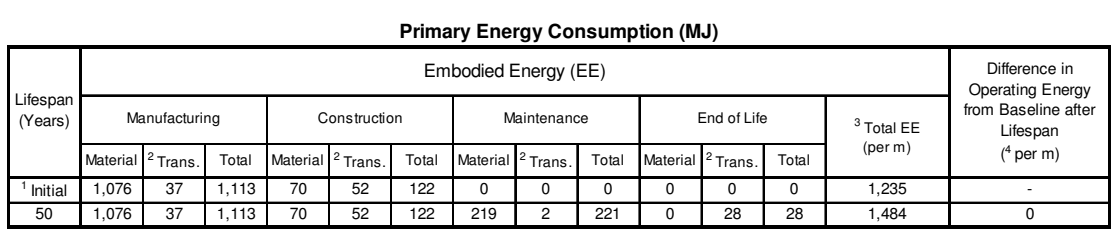

Global Warming Potential (kg of $\mathrm{CO}_{2}$ eq.)

\begin{tabular}{|c|c|c|c|c|c|c|c|c|c|c|c|c|c|c|}
\hline \multirow{3}{*}{$\begin{array}{l}\text { Litespanan } \\
\text { (Years) }\end{array}$} & \multicolumn{13}{|c|}{ Embodied Global Warming Potential (GWP) } & \multirow{3}{*}{\begin{tabular}{|c} 
Difference in \\
Operating GWP from \\
Baseline after \\
Lifespan \\
$\left({ }^{4}\right.$ per $\left.\mathrm{m}\right)$
\end{tabular}} \\
\hline & \multicolumn{3}{|c|}{ Manutacturing } & \multicolumn{3}{|c|}{ Construction } & \multicolumn{3}{|c|}{ Maintenance } & \multicolumn{3}{|c|}{ End of Life } & \multirow{2}{*}{$\begin{array}{l}{ }^{3} \text { Total GWP } \\
\quad(\text { per } m)\end{array}$} & \\
\hline & Material & ${ }^{2}$ Trans & Total & Material & ${ }^{2}$ Trans. & Total & Material & ${ }^{2}$ Trans & Total & Material & ${ }^{2}$ Trans. & Total & & \\
\hline 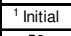 & 98 & 0 & 98 & 5 & 0 & 5 & 0 & 0 & 0 & 0 & 0 & 0 & & \\
\hline 50 & 98 & 0 & 98 & 5 & 0 & 5 & 3 & 0 & 3 & 0 & 0 & 0 & 106 & 0 \\
\hline
\end{tabular}

Note: Calculations are per linear meter of strip footing $(600 \mathrm{~mm} \times 200 \mathrm{~m})$ and foundation wall (1200mm $\times 200 \mathrm{~mm})$ combination

$\begin{aligned} & \text { ATHENA @ EIE Material List: } \\
& \text { (Includes all materials after 50 years) }\end{aligned}$
\begin{tabular}{|l|c|c|}
\multicolumn{1}{|c|}{ Material List } & Quantities & Unit \\
\hline 6 mil Polyethylene & 1.3 & $\mathrm{~m} 2$ \\
\hline Concrete $20 \mathrm{MPa}$ (flyash av) & 0.4 & $\mathrm{~m} 3$ \\
\hline Extruded Polystyrene & 2.5 & $\mathrm{~m} 2(25 \mathrm{~mm})$ \\
\hline Modified Bitumen membrane & 2.1 & $\mathrm{~kg}$ \\
\hline Nails & 0.1 & $\mathrm{~kg}$ \\
\hline Organic Felt shingles 25yr & 1.3 & $\mathrm{~m} 2$ \\
\hline Rebar, Rod, Light Sections & 14.9 & $\mathrm{~kg}$ \\
\hline Solvent Based Alkyd Paint & 0.1 & $\mathrm{~L}$ \\
\hline
\end{tabular}

Notes:
${ }^{1}$ Initial $=$ Time ' $\mathrm{O}^{\prime}$ (i.e. at the completion of initial construction)

'Initial $=$ Time $0^{\prime}$ ' (i.e. at

${ }^{3}$ Total EE (or Totration $\mathrm{GWP}$ ) per $m=$ Total embodied energy (or total eng GWP) of building component after lifespan (i.e. total manufacturing + tota construction + total maintenance + total end-of-life effects) Total Difference in Operating Energy (or GWP) from Baseline after GWP) from the baseline retail building after lifespan, due to using this building component instead of the baseline component Total operating primary energy use of baseline retail building after 50 years $=$

"Total operating GWP of baseline retail building after 50 years $=2,310$ tonnes Total operating $\mathrm{GWP}$ of baseline reta
of $\mathrm{CO}_{2}$ eq. $\left(80 \mathrm{~kg}\right.$ of $\mathrm{CO}_{2}$ eq. $\left.\mathrm{m}^{2} \mathrm{Ar}\right)$ 


\section{Concrete Strip Footing and Foundation Wall \#2 (SF-FDN2)}

Building Component Description:

\begin{tabular}{|c|c|c|c|}
\hline Category: & Foundations & Assembly Layers & \\
\hline & & $\begin{array}{l}\text { Concrete Strip Footing } \\
\text { 20MPa concrete strenth }\end{array}$ & \\
\hline Brief Description: & 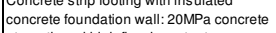 & $600 \mathrm{~mm} \times 200 \mathrm{~mm}$ & \\
\hline & strtength and high hlyash content & $6.0 \mathrm{~kg}$ of steel per linear $\mathrm{m}$ & \\
\hline & & $35 \%$ (high) filyash content & \\
\hline Quick Numbers: & & $\begin{array}{l}\text { Concrete Foundation Wall } \\
20 \mathrm{MPa} \text { concrete strength }\end{array}$ & \\
\hline ASHRAE Standard 90.1: & R-Value: 11.0 & $1,200 \mathrm{~mm} \times 200 \mathrm{~mm}$ & \\
\hline THERM 5.2: & R-Value: $11.1 \quad$ RSI-Value 2.0 & Equivalent of $15 \mathrm{MM}$ bars @ $600 \mathrm{~mm}$ o/c vert. \& hor. & \\
\hline Total Embodied Energy: & $1,440 \mathrm{~m} / \mathrm{m}$ & Includes allowance for $0.425 \mathrm{~kg} / \mathrm{m}^{2}$ of steel for tites & \\
\hline Total Embodied GWP: & $96 \quad \mathrm{~kg}$ of $\mathrm{CO}_{2} \mathrm{eq} / \mathrm{m}$ & wire, etc. & \\
\hline & $\begin{array}{l}\text { Notete: Embodied energy and embodied } \\
G W P \text { numbers are per horizontal meter }\end{array}$ & $\begin{array}{l}\text { 35\%\% (high) flyash content } \\
60 \text { il assholatbased waterorofing membrane }\end{array}$ & \\
\hline & $\begin{array}{l}\text { GWP numbers are eer horizontal meter } \\
\text { of footing and wall }\end{array}$ & $\begin{array}{l}\text { 60mil asphall-based waterproofing membrane } \\
\text { (modeled as } 1.6 \mathrm{~kg} / \mathrm{m}^{2} \text { of modified bitumen }\end{array}$ & \\
\hline & & membrane and 6mil poly sheet with primer) & \\
\hline & & $50 \mathrm{~mm}$ extruded polystryene rigid insulation & \\
\hline & & $\overline{6.4 m m}$ asphalt protection board & \\
\hline
\end{tabular}

Life-Cycle Assessment Results:

Primary Energy Consumption (MJ)

\begin{tabular}{|c|c|c|c|c|c|c|c|c|c|c|c|c|c|c|}
\hline \multirow{3}{*}{\begin{tabular}{|} 
Lifiespan \\
(Years)
\end{tabular}} & \multicolumn{13}{|c|}{ Embodied Energy (EE) } & \multirow{3}{*}{$\begin{array}{l}\text { Difference in } \\
\text { Operating Energy } \\
\text { from Baseline after } \\
\text { Lifespan } \\
\left({ }^{4} \text { per } \mathrm{m}\right)\end{array}$} \\
\hline & \multicolumn{3}{|c|}{ Manufacturing } & \multicolumn{3}{|c|}{ Construction } & \multicolumn{3}{|c|}{ Maintenance } & \multicolumn{3}{|c|}{ End of Life } & \multirow{2}{*}{$\begin{array}{l}{ }^{3} \text { Total EE } \\
\text { (per } \mathrm{m})\end{array}$} & \\
\hline & Material & ${ }^{2}$ Trans. & \begin{tabular}{|l|l|l|} 
Total \\
\end{tabular} & Material & Trans. & Total & Material & 2 Trans. & Total & Material & \begin{tabular}{|l|}
${ }^{2}$ Trans. \\
\end{tabular} & Total & & \\
\hline Initial & 1,022 & 47 & 1,069 & 70 & 52 & 122 & 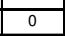 & 0 & 0 & 0 & 0 & 0 & $\overline{1,1}$ & \\
\hline 50 & \begin{tabular}{|l|l}
1,022 \\
\end{tabular} & 47 & \begin{tabular}{|l|l}
1,069 \\
\end{tabular} & 70 & 52 & 122 & 219 & 2 & 221 & 0 & 28 & 28 & 1,440 & 0 \\
\hline
\end{tabular}

Global Warming Potential ( $k g$ of $\mathrm{CO}_{2}$ eq.)

\begin{tabular}{|c|c|c|c|c|c|c|c|c|c|c|c|c|c|c|}
\hline \multirow{3}{*}{$\begin{array}{c}\text { Lifespan } \\
\text { (Years) }\end{array}$} & \multicolumn{13}{|c|}{ Embodied Global Warming Potential (GWP) } & \multirow{3}{*}{$\begin{array}{l}\text { Difference in } \\
\text { Operating GWP from } \\
\text { Baseline after } \\
\text { Lifespan } \\
\left({ }^{4} \text { per } \mathrm{m}\right)\end{array}$} \\
\hline & \multicolumn{3}{|c|}{ Manutacturing } & \multicolumn{3}{|c|}{ Construction } & \multicolumn{3}{|c|}{ Maintenance } & \multicolumn{3}{|c|}{ End of Life } & \multirow{2}{*}{$\begin{array}{c}{ }^{3} \text { Total GWP } \\
\text { (per m) }\end{array}$} & \\
\hline & Material & ${ }^{2}$ Trans & Total & Material & ${ }^{2}$ Trans. & Total & Material & ${ }^{2}$ Trans. & Total & Material & Ti ${ }^{2}$ Trans. & Total & & \\
\hline${ }^{1}$ Initial & 87 & 0 & 88 & 5 & 0 & 5 & 0 & 0 & 0 & 0 & 0 & 0 & 92 & \\
\hline 50 & 87 & 0 & 88 & 5 & 0 & 5 & 3 & 0 & 3 & 0 & 0 & 0 & 96 & $\overline{0}$ \\
\hline
\end{tabular}

Note: Calculations are per linear meter of strip footing $(600 \mathrm{~mm} \times 200 \mathrm{~m})$ and foundation wall $(1200 \mathrm{~mm} \times 200 \mathrm{~mm})$ combination

ATHENA $®$ EIE Material List: (Includes all materials after 50 years)

\begin{tabular}{|l|c|c|}
\hline \multicolumn{1}{|c|}{ Material List } & Quantities & Unit \\
\hline 6 mil Polyethylene & 1.3 & $\mathrm{~m} 2$ \\
\hline Concrete 20 MPa (flyash $35 \%)$ & 0.4 & $\mathrm{~m} 3$ \\
\hline Extruded Polystyrene & 2.5 & $\mathrm{~m} 2(25 \mathrm{~mm})$ \\
\hline Modified Bitumen membrane & 2.1 & $\mathrm{~kg}$ \\
\hline Nails & 0.1 & $\mathrm{~kg}$ \\
\hline Organic Felt shingles 25yr & 1.3 & $\mathrm{~m} 2$ \\
\hline Rebar, Rod, Light Sections & 14.9 & $\mathrm{~kg}$ \\
\hline Solvent Based Alkyd Paint & 0.1 & $\mathrm{~L}$ \\
\hline
\end{tabular}

Notes:
${ }^{1}$ Initial $=$ Time 'o' (i.e. at the completion of initial construction) 'Initial $=$ Time ' $O$ ' (i.e. at the
${ }^{2}$ Trans. = Transportation

${ }^{3}$ Total EE (or Total GWP) per $m=$ Total embodied energy (or total embodied GWP) of building component after lifespan (i.e. total manufacturing + total construction + total maintenance + total enc-oflifie effects)

Total Difference in Operating Energy (or GWP) from Baseline afte GWP) from the baseline retil bulding afer lifcespan operating energy (or building component instead of the baseline component

Total operating primary energy use of baseline retail building after 50 years

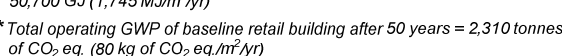

\section{Concrete Strip Footing and Foundation Wall \#3 (SF-FDN3)} Building Component Description:

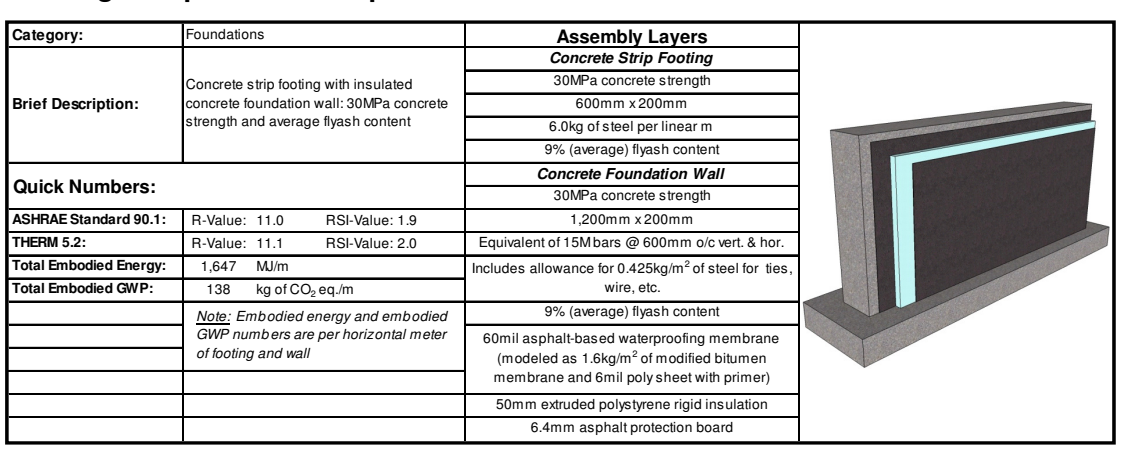

Life-Cycle Assessment Results:

Primary Energy Consumption (MJ)

\begin{tabular}{|c|c|c|c|c|c|c|c|c|c|c|c|c|c|c|}
\hline \multirow{3}{*}{\begin{tabular}{|l} 
Lifespan \\
(Years)
\end{tabular}} & \multicolumn{13}{|c|}{ Embodied Energy (EE) } & \multirow{3}{*}{\begin{tabular}{|c} 
Difference in \\
Operating Energy \\
from Baseline after \\
Lifespan \\
$\left({ }^{4}\right.$ per $\left.\mathrm{m}\right)$
\end{tabular}} \\
\hline & \multicolumn{3}{|c|}{ Manufacturing } & \multicolumn{3}{|c|}{ Construction } & \multicolumn{3}{|c|}{ Maintenance } & \multicolumn{3}{|c|}{ End of Life } & \multirow{2}{*}{$\begin{array}{l}{ }^{3} \text { Total EE } \\
\text { (per } \mathrm{m})\end{array}$} & \\
\hline & \begin{tabular}{|l|} 
Material \\
\end{tabular} & 2 Trans. & Total & Material & ${ }^{2}$ Trans. & Total & \begin{tabular}{|l|} 
Material \\
\end{tabular} & \begin{tabular}{|l|}
${ }^{2}$ Trans. \\
\end{tabular} & Total & Material & \begin{tabular}{|l|}
${ }^{2}$ Trans. \\
\end{tabular} & Total & & \\
\hline${ }^{1}$ Initial & 1,233 & 43 & 1,276 & 70 & 52 & 122 & 0 & 0 & 0 & 0 & 0 & 0 & 1,398 & \\
\hline 50 & $\mid 1,233$ & 43 & 1,276 & 70 & 52 & 122 & 219 & 2 & 221 & 0 & 28 & 28 & 1,647 & 0 \\
\hline
\end{tabular}

Global Warming Potential (kg of $\mathrm{CO}_{2}$ eq.)

\begin{tabular}{|c|c|c|c|c|c|c|c|c|c|c|c|c|c|c|}
\hline \multirow{3}{*}{$\begin{array}{l}\text { Lifespan } \\
\text { (Years) }\end{array}$} & \multicolumn{13}{|c|}{ Embodied Global Warming Potential (GWP) } & \multirow{3}{*}{$\begin{array}{l}\text { Difference in } \\
\text { Operating GWP from } \\
\text { Baseline atter } \\
\text { Lifespan } \\
\left({ }^{4} \text { per } \mathrm{m}\right)\end{array}$} \\
\hline & \multicolumn{3}{|c|}{ Manutacturing } & \multicolumn{3}{|c|}{ Construction } & \multicolumn{3}{|c|}{ Maintenance } & \multicolumn{3}{|c|}{ End of Life } & \multirow{2}{*}{$\begin{array}{l}{ }^{3} \text { Total GWP } \\
\text { (per m) }\end{array}$} & \\
\hline & Material & ${ }^{2}$ Trans. & Total & Material & ${ }^{2}$ Trans. & Total & Material & ${ }^{2}$ Trans. & Total & Material & \begin{tabular}{|l|}
${ }^{2}$ Trans. \\
\end{tabular} & Total & & \\
\hline Initial & 130 & 0 & 130 & 5 & 0 & 5 & 0 & 0 & 0 & 0 & 0 & 0 & 135 & \\
\hline 50 & 130 & 0 & 130 & 5 & 0 & 5 & 3 & 0 & 3 & 0 & 0 & 0 & 138 & 0 \\
\hline
\end{tabular}

Note: Calculations are per linear meter of strip footing $(600 \mathrm{~mm} \times 200 \mathrm{~m})$ and foundation wall $(1200 \mathrm{~mm} \times 200 \mathrm{~mm})$ combination

$\begin{gathered}\text { ATHENA } \text { @ EIE Material List: } \\
\text { (Includes all materials after 50 50ars) }\end{gathered}$
\begin{tabular}{|l|c|c|}
\multicolumn{1}{|c|}{ Material List } & Quantities & Unit \\
\hline 6 mil Polyethylene & 1.3 & $\mathrm{~m} 2$ \\
\hline Concrete $30 \mathrm{MPa}$ (flyash av) & 0.4 & $\mathrm{~m} 3$ \\
\hline Extruded Polystyrene & 2.5 & $\mathrm{~m} 2(25 \mathrm{~mm})$ \\
\hline Modified Bitumen membrane & 2.1 & $\mathrm{~kg}$ \\
\hline Nails & 0.1 & $\mathrm{~kg}$ \\
\hline Organic Felt shingles 25yr & 1.3 & $\mathrm{~m} 2$ \\
\hline Rebar, Rod, Light Sections & 14.9 & $\mathrm{~kg}$ \\
\hline Solvent Based Alkyd Paint & 0.1 & $\mathrm{~L}$ \\
\hline
\end{tabular}

Notes:
${ }^{1}$ Initial $=$ Time 'o' (i.e. at the completion of initial construction)

'Initial $=$ Time ' $O$ ' (i.e. at

${ }^{3}$ Total EE (or Total GWP) per $m=$ Total embodied energy (or total embodied ${ }^{3}$ Total EE (or Total GWP) per $\mathrm{m}=$ Total embodied energy (or total embodied
GWP) of building component after lifespana (i.e. totalal manufacturing + total
construction + total maintenance + total end-of-life effects)

Total Difference in Operating Energy (or GWP) from Baseline after building component instead of the baseline component

Total operating primary energy use of baseline retail building after 50 years =

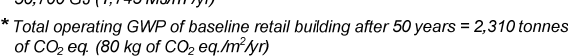

( $180 \mathrm{~kg}$ of $\mathrm{CO}_{2}$ eq. $\left./ \mathrm{m}^{2} \mathrm{ym}\right)$ GWP) from the baseline retail building after lifespan, due to using this

Rebar, Rod, Light Section 


\section{Concrete Strip Footing and Foundation Wall \#4 (SF-FDN4)}

Building Component Description:

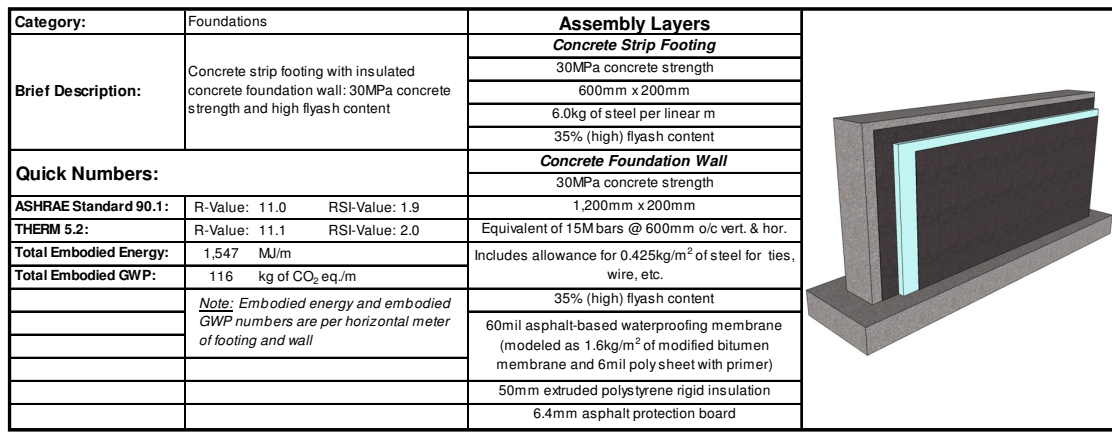

Life-Cycle Assessment Results:

\begin{tabular}{|c|c|c|c|c|c|c|c|c|c|c|c|c|c|c|}
\hline \multirow{4}{*}{$\begin{array}{l}\text { Lifespan } \\
\text { (Years) }\end{array}$} & \multicolumn{13}{|c|}{ Primary Energy Consumption (MJ) } & \multirow{4}{*}{$\begin{array}{l}\text { Difference in } \\
\text { Operating Energy } \\
\text { from Baseline afte } \\
\text { Lififspan } \\
{ }^{4} \text { per } m \text { ) }\end{array}$} \\
\hline & \multicolumn{13}{|c|}{ Embodied Energy (EE) } & \\
\hline & \multicolumn{3}{|c|}{ Manutacturing } & \multicolumn{3}{|c|}{ Construction } & \multicolumn{3}{|c|}{ Maintenance } & \multicolumn{3}{|c|}{ End of Life } & \multirow{2}{*}{$\begin{array}{l}{ }^{3} \text { Total EE } \\
\text { (per } m \text { ) }\end{array}$} & \\
\hline & Material & ${ }^{2}$ Trans. & Total & Material & $\begin{array}{l}1{ }^{2} \text { Trans. } \\
\text {. }\end{array}$ & 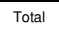 & Material & ${ }^{2}$ Trans. & $\begin{array}{c}\text { Total } \\
\end{array}$ & Material & ${ }^{2}$ Trans. & Total & & \\
\hline Initial & 1,120 & 56 & 1,176 & 70 & 52 & 122 & 0 & 0 & 0 & 0 & 0 & 0 & 298 & \\
\hline 50 & $\frac{1,120}{1,10}$ & & 1,176 & 70 & 52 & 122 & 219 & 2 & 221 & & 28 & 28 & 547 & \\
\hline
\end{tabular}

Global Warming Potential $\left(\mathrm{kg}\right.$ of $\left.\mathrm{CO}_{2} \mathrm{eq}\right)$

\begin{tabular}{|c|c|c|c|c|c|c|c|c|c|c|c|c|c|c|}
\hline \multirow{3}{*}{ (Years) } & \multicolumn{13}{|c|}{ Embodied Global Warming Potential (GWP) } & \multirow{3}{*}{\begin{tabular}{|c} 
Difference in \\
Operating GWPP from \\
Baseline atter \\
Lifespan \\
$\left({ }^{4}\right.$ per $\left.\mathrm{m}\right)$ \\
\end{tabular}} \\
\hline & \multicolumn{3}{|c|}{ Manutacturing } & \multicolumn{3}{|c|}{ Construction } & \multicolumn{3}{|c|}{ Maintenance } & \multicolumn{3}{|c|}{ End of Life } & \multirow{2}{*}{$\begin{array}{l}{ }^{3} \text { Total GWP } \\
\text { (per m) }\end{array}$} & \\
\hline & Material & ${ }^{2}$ Trans. & Total & Material & | ${ }^{2}$ Trans. & Total & Material & 2 Trans. & Total & Material & ${ }^{2}$ Trans. & Total & & \\
\hline 'Initial & 108 & 0 & 108 & 5 & 0 & 5 & 0 & 0 & 0 & 0 & 0 & 0 & ra & \\
\hline 50 & 108 & 0 & 108 & 5 & 0 & 5 & 3 & 0 & 3 & 0 & 0 & 0 & 116 & 0 \\
\hline
\end{tabular}

Note: Calculations are per linear meter of strip footing $(600 \mathrm{~mm} \times 200 \mathrm{~m})$ and foundation wall (1200mm $\times 200 \mathrm{~mm})$ combination

\section{ATHENA $®$ EIE Material List}

(Includes all materials after 50 years)

\begin{tabular}{|l|c|c|}
\hline \multicolumn{1}{|c|}{ Material List } & Quantities & Unit \\
\hline 6 mil Polyethylene & 1.3 & $\mathrm{~m} 2$ \\
\hline Concrete $30 \mathrm{MPa}$ (flyash $35 \%)$ & 0.4 & $\mathrm{~m} 3$ \\
\hline Extruded Polystrene & 2.5 & $\mathrm{~m} 2(25 \mathrm{~mm})$ \\
\hline Modified Bitumen membrane & 2.1 & $\mathrm{~kg}$ \\
\hline Nails & 0.1 & $\mathrm{~kg}$ \\
\hline Organic Felt shingles 25yr & 1.3 & $\mathrm{~m} 2$ \\
\hline Rebar, Rod, Light Sections & 14.9 & $\mathrm{~kg}$ \\
\hline Solvent Based Alkyd Paint & 0.1 & $\mathrm{~L}$ \\
\hline
\end{tabular}

Notes:
${ }^{\prime}$ Initial $=$ Time ' 0 ' (i.e. at the completion of initial construction) 'Initial $=$ Time 'o' (i.e. at
${ }^{2}$ Trans. $=$ Transportation

Total EE (or Total GWP) per $m=$ Total embodied energy (or total embodied GWP) of building component after lifespan (i.e. total manufacturing + tota construction + total maintenance + total end-oflifie effects)

Total Difference in Operating Energy (or GWP) from Baseline after Lifespan per $m=$ The difference in the total life-cycle operating energy ( building component instead of the baseline componen

"Total operating primary energy use of baseline retail building after 50 years $50,700 \mathrm{GJ}\left(1,745 \mathrm{MJ} / \mathrm{m}^{2} \mathrm{M}\right)$

* Total operating GWP of baseline retail building after 50 years $=2,310$ tonnes

Concrete Strip Footing and Foundation Wall \#5 (SF-FDN5) Building Component Description:

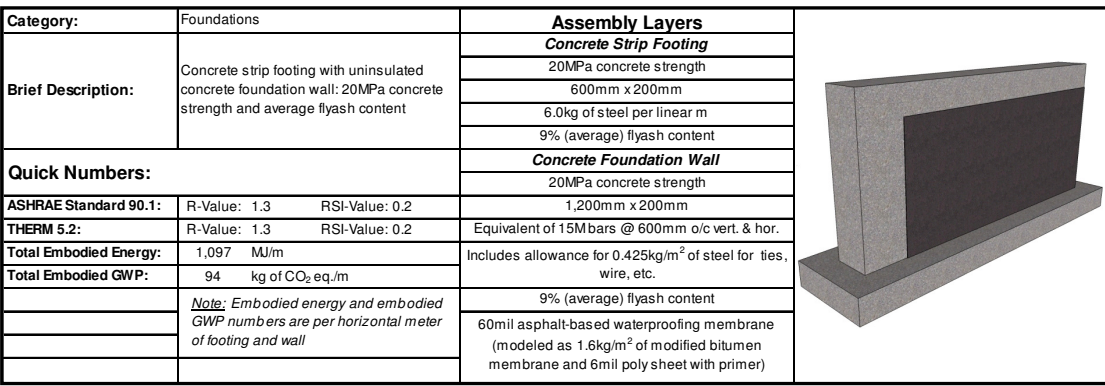

Life-Cycle Assessment Results:

\begin{tabular}{|c|c|c|c|c|c|c|c|c|c|c|c|c|c|c|}
\hline \multirow{4}{*}{$\begin{array}{l}\text { Lifespan } \\
\text { (Years) }\end{array}$} & \multicolumn{13}{|c|}{ Primary Energy Consumption (MJ) } & \multirow{4}{*}{$\begin{array}{c}\text { Difference in } \\
\text { Operating Energy } \\
\text { from Baseline afte } \\
\text { Lifespan } \\
\left(^{4} \text { per } \mathrm{m}\right)\end{array}$} \\
\hline & \multicolumn{13}{|c|}{ Embodied Energy (EE) } & \\
\hline & \multicolumn{3}{|c|}{ Manutacturing } & \multicolumn{3}{|c|}{ Construction } & \multicolumn{3}{|c|}{ Maintenance } & \multicolumn{3}{|c|}{ End of Life } & \multirow{2}{*}{$\begin{array}{l}{ }^{3} \text { Total EE } \\
\text { (per } \mathrm{m} \text { ) }\end{array}$} & \\
\hline & Material & ${ }^{2}$ Trans & Total & Material & ${ }^{2}$ Trans. & Total & Material & \begin{tabular}{|c|}
${ }^{2}$ Trans. \\
\end{tabular} & Total & Material & ${ }^{2}$ Trans. & Total & & \\
\hline Initial & 837 & 36 & 874 & 70 & 51 & 121 & 0 & 0 & 0 & 0 & 0 & 0 & & \\
\hline 50 & 837 & 36 & 874 & 70 & 51 & 121 & 75 & 0 & 75 & 0 & 27 & 27 & ,097 & \\
\hline
\end{tabular}

Global Warming Potential (kg of $\mathrm{CO}_{2}$ eq.)

\begin{tabular}{|c|c|c|c|c|c|c|c|c|c|c|c|c|c|c|}
\hline \multirow{3}{*}{$\begin{array}{l}\text { Lifespan } \\
\text { (Years) }\end{array}$} & \multicolumn{13}{|c|}{ Embodied Global Warming Potential (GWP) } & \multirow{3}{*}{\begin{tabular}{|c} 
Difference in \\
Operating GWPP from \\
Baseline after \\
Lifespan \\
$\left({ }^{4}\right.$ per $\left.\mathrm{m}\right)$
\end{tabular}} \\
\hline & \multicolumn{3}{|c|}{ Manufacturing } & \multicolumn{3}{|c|}{ Construction } & \multicolumn{3}{|c|}{ Maintenance } & \multicolumn{3}{|c|}{ End of Life } & \multirow{2}{*}{$\begin{array}{l}{ }^{3} \text { Total GWP } \\
\text { (per m) }\end{array}$} & \\
\hline & Materia & ${ }^{2}$ Trans. & Total & \begin{tabular}{|l|l|} 
Material \\
\end{tabular} & ${ }^{2}$ Trans. & Total & Material & ${ }^{2}$ Trans & \begin{tabular}{|l|} 
Total \\
\end{tabular} & Material & $11^{2}$ Trans. & \begin{tabular}{|l|l|} 
Total \\
\end{tabular} & & \\
\hline 'Initial & 88 & 0 & 88 & 5 & 0 & 5 & 0 & 0 & 0 & 0 & 0 & 0 & 93 & \\
\hline 50 & 88 & 0 & 88 & 5 & 0 & 5 & 1 & 0 & 1 & 0 & 0 & 0 & 94 & 0 \\
\hline
\end{tabular}

\begin{tabular}{|lll}
50 & 10 \\
\hline
\end{tabular}

ATHENA $\circledast$ EIE Material List:

(Includes all materials after 50 years)

\begin{tabular}{|l|c|c|}
\hline \multicolumn{1}{|c|}{ Material List } & Quantities & Unit \\
\hline 6 mil Polyethylene & 1.3 & $\mathrm{~m} 2$ \\
\hline Concrete 20 MPa (flyash av) & 0.4 & $\mathrm{~m} 3$ \\
\hline Modified Bitumen membrane & 2.1 & $\mathrm{~kg}$ \\
\hline Rebar, Rod, Light Sections & 14.9 & $\mathrm{~kg}$ \\
\hline Solvent Based Alkyd Paint & 0.1 & $\mathrm{~L}$ \\
\hline
\end{tabular}

Notes:

${ }^{2}$ Trans. = Transportation

${ }^{3}$ Total EE (or Total GWP) per $m=$ Total embodied energy (or total embodied GWP) of building component after lifespan (i.e. total manufacturing + tota ${ }^{4}$ Total Difference in Operating Energy (or GWP) from Baseline after
Lifespan per $m=$ The difference in the total life-cycle operating energy (or GWP from the baseline retail building after lifespan, due to using this * Total operating primary energy use of baseline retail building after 50 years =

* Total operating $\mathrm{GWP}$ of baseline retail building affer 50 years $=2,310$ tonnes
of $\mathrm{CO}_{2}$ eq. $\left(80 \mathrm{~kg}\right.$ of $\mathrm{CO}_{2}$ eq. $\left./ \mathrm{m}^{2} \mathrm{Yrt}\right)$ 
Concrete Strip Footing and Foundation Wall \#6 (SF-FDN6)

Building Component Description:

\begin{tabular}{|c|c|c|c|}
\hline Category: & Foundations & Assembly Layers & \\
\hline & & 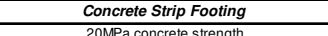 & \\
\hline Brief Description: & concrete foundation wall: : $20 \mathrm{MP}$ Pa concrerete & $600 \mathrm{~mm} \times 200 \mathrm{~mm}$ & \\
\hline & & $6.0 \mathrm{~kg}$ of steel per linear $\mathrm{m}$ & \\
\hline & & Concrete Foundation Wall & \\
\hline Quick Numbers: & & 20MPa concrete strength & \\
\hline ASHRAE Standard 90.1: & RSI-Value: 0.2 & $1,200 \mathrm{~mm} \times 200 \mathrm{~mm}$ & \\
\hline THERM 5.2: & RSI-Value: 0.2 & Equivalent of $15 \mathrm{Mbars} @ 6600 \mathrm{~mm}$ o/c vert. \& hor. & \\
\hline Total Embodied Energy: & $1,053 \mathrm{~m} / \mathrm{m}$ & ncludes allowance for $0.425 \mathrm{~kg} / \mathrm{m}^{2}$ of s & \\
\hline Total Embodied GWP: & $83 \mathrm{~kg} \mathrm{of} \mathrm{CO}_{2} \mathrm{eq} / \mathrm{m}$ & $\begin{array}{l}\text { wire, etc. } \\
35 \% \text { (high) Hitash content }\end{array}$ & \\
\hline & $\begin{array}{l}\text { Note: } \text { Embodied energy and embodied } \\
\text { GWP numbers are per horizontal meter } \\
\text { of footing and wall }\end{array}$ & $\begin{array}{l}\text { 60mil asphall-based waterprofofing membrane } \\
\text { (modeled as } 1.6 \mathrm{~kg} / \mathrm{m}^{2} \text { of modified bitumen }\end{array}$ & \\
\hline & & & \\
\hline
\end{tabular}

Life-Cycle Assessment Results:

Primary Energy Consumption (MJ)

\begin{tabular}{|c|c|c|c|c|c|c|c|c|c|c|c|c|c|c|}
\hline \multirow{3}{*}{$\begin{array}{l}\text { Lifespan } \\
\text { (Years) }\end{array}$} & \multicolumn{13}{|c|}{ Embodied Energy (EE) } & \multirow{3}{*}{$\begin{array}{c}\text { Difference in } \\
\text { Operating Energy } \\
\text { from Baseline afte } \\
\text { Lifespan } \\
\left({ }^{4} \text { per } \mathrm{m}\right)\end{array}$} \\
\hline & \multicolumn{3}{|c|}{ Manufacturing } & \multicolumn{3}{|c|}{ Construction } & \multicolumn{3}{|c|}{ Maintenance } & \multicolumn{3}{|c|}{ End of Life } & \multirow{2}{*}{$\begin{array}{l}{ }^{3} \text { Total EE } \\
\text { (per } m \text { ) }\end{array}$} & \\
\hline & \begin{tabular}{|l|} 
Materia \\
\end{tabular} & ${ }^{2}$ Trans & \begin{tabular}{|l|l|l|} 
Total & \\
\end{tabular} & Materia & ${ }^{2}$ Trans. & Total & \begin{tabular}{|l|l|} 
Material \\
\end{tabular} & ${ }^{2}$ Trans. & \begin{tabular}{|l|l|} 
Total \\
\end{tabular} & Material & \begin{tabular}{|l|}
${ }^{2}$ Trans. \\
\end{tabular} & Total & & \\
\hline Initial & 783 & 47 & 830 & 70 & 51 & 121 & 0 & 0 & 0 & 0 & 0 & 0 & & \\
\hline 50 & 783 & 47 & 830 & 70 & 51 & 121 & 75 & 0 & 75 & 0 & 27 & 27 & 1,053 & 0 \\
\hline
\end{tabular}

Global Warming Potential ( $\mathrm{kg}$ of $\mathrm{CO}_{2}$ eq.)

\begin{tabular}{|c|c|c|c|c|c|c|c|c|c|c|c|c|c|c|}
\hline \multirow{3}{*}{$\begin{array}{l}\text { Lifespars } \\
\text { (Year) }\end{array}$} & \multicolumn{13}{|c|}{ Embodied Global Warming Potential (GWP) } & \multirow{3}{*}{$\begin{array}{c}\text { Difference in } \\
\text { Operating GWP fron } \\
\text { Baseline after } \\
\text { Lifispan } \\
\left({ }^{4} \text { per } \mathrm{m}\right)\end{array}$} \\
\hline & \multicolumn{3}{|c|}{ Manufacturing } & \multicolumn{3}{|c|}{ Construction } & \multicolumn{3}{|c|}{ Maintenance } & \multicolumn{3}{|c|}{ End of Life } & \multirow{2}{*}{$\begin{array}{l}{ }^{3} \text { Total GWP } \\
\text { (per m) }\end{array}$} & \\
\hline & 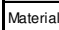 & ${ }^{2}$ Trans. & Total & Materia & ${ }^{2}$ Trans. & Total & Materia & ${ }^{2}$ Trans. & Total & Material & ${ }^{2}$ Trans. & Total & & \\
\hline 'Initial & 77 & 0 & 78 & 5 & 0 & 5 & 0 & 0 & 0 & 0 & 0 & 0 & 82 & \\
\hline 50 & 77 & 0 & 78 & 5 & 0 & 5 & 1 & 0 & 1 & 0 & 0 & 0 & 83 & 0 \\
\hline
\end{tabular}

Note: Calculations are per linear meter of strip footing $600 \mathrm{~mm} \times 200 \mathrm{~m})$ and 10 (1)

ATHENA @ EIE Material List: (Includes all materials after 50 years)

\begin{tabular}{|l|c|c|}
\hline \multicolumn{1}{|c|}{ Material List } & Quantities & Unit \\
\hline 6 mil Polyethylene & 1.3 & $\mathrm{~m} 2$ \\
\hline Concrete 20 MPa (flyash 35\%) & 0.4 & $\mathrm{~m} 3$ \\
\hline Modified Bitumen membrane & 2.1 & $\mathrm{~kg}$ \\
\hline Rebar, Rod, Light Sections & 14.9 & $\mathrm{~kg}$ \\
\hline Solvent Based Alkyd Paint & 0.1 & $\mathrm{~L}$ \\
\hline
\end{tabular}

'Initial = Time ' 0 ' (i.e. at the comples:

${ }^{2}$ Trans. = Transportation

${ }^{3}$ Total EE (or Total GWP) per $m=$ Total embodied energy (or total embodied GWP) of building component atter lifespan (i.e. total manufacturing + tola

Total Difference in Operating Energy (or GWP) from Baseline after Lifespan per $m=$ The difference in the total life-cycle operating energy y
GWP from the baseline retail buildieng affer lifespan, due to using this * Total operating primary energy use of baseline retail building after 50 years

* Total operating $\mathrm{GWP}$ of baseline retail building after 50 years $=2,310$ tonnes
of $\mathrm{CO}_{2}$ eq, $80 \mathrm{~kg}$ of $\mathrm{CO}_{2}$ eq. $\left.\mathrm{m}^{2} \mathrm{yr}\right)$

Concrete Strip Footing and Foundation Wall \#7 (SF-FDN7) Building Component Description:

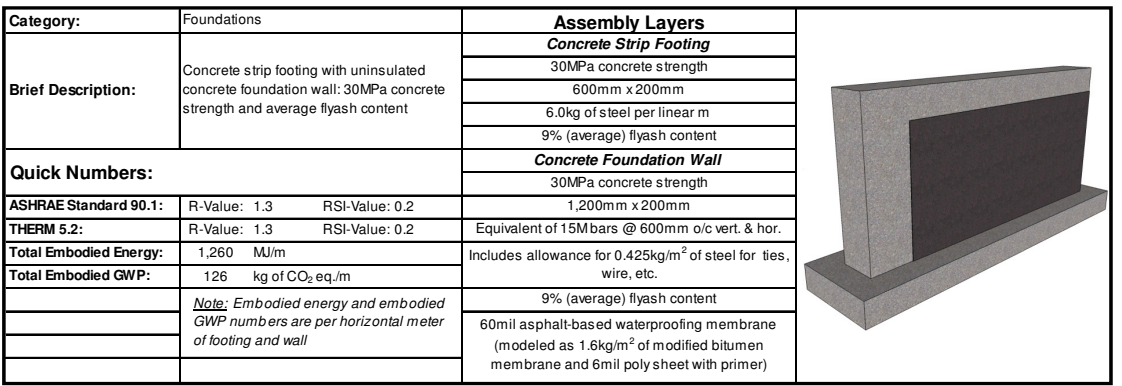

Life-Cycle Assessment Results:

Primary Energy Consumption (MJ)

\begin{tabular}{|c|c|c|c|c|c|c|c|c|c|c|c|c|c|c|}
\hline \multirow{3}{*}{$\begin{array}{l}\text { Lifespan } \\
\text { (Years) }\end{array}$} & \multicolumn{13}{|c|}{ Embodied Energy (EE) } & \multirow{3}{*}{$\begin{array}{l}\text { Difference in } \\
\text { Operating Energy } \\
\text { from Baseline after } \\
\text { Lifespan } \\
\left({ }^{4} \mathrm{per} \mathrm{m}\right)\end{array}$} \\
\hline & \multicolumn{3}{|c|}{ Manufacturing } & \multicolumn{3}{|c|}{ Construction } & \multicolumn{3}{|c|}{ Maintenance } & \multicolumn{3}{|c|}{ End of Life } & \multirow{2}{*}{$\begin{array}{l}{ }^{3} \text { Total EE } \\
\text { (per } m \text { ) }\end{array}$} & \\
\hline & Material & ${ }^{2}$ Trans & \begin{tabular}{|l|l|l|l|} 
Total & \\
\end{tabular} & Material & ${ }^{2}$ Trans & Total & Material & ${ }^{2}$ Trans & \begin{tabular}{|l|l|l|} 
Total \\
\end{tabular} & Material & ${ }^{2}$ Trans. & Total & & \\
\hline & 995 & 42 & 1,037 & 70 & 51 & 121 & 0 & 0 & 0 & 0 & 0 & 0 & & \\
\hline 50 & 995 & 42 & 1,037 & 70 & 51 & 121 & 75 & 0 & 75 & 0 & 27 & 27 & 1,260 & 0 \\
\hline
\end{tabular}

Global Warming Potential ( $\mathrm{kg}$ of $\mathrm{CO}_{2}$ eq.)

\begin{tabular}{|c|c|c|c|c|c|c|c|c|c|c|c|c|c|c|}
\hline \multirow{3}{*}{\begin{tabular}{|l} 
Lifespan \\
(Years)
\end{tabular}} & \multicolumn{13}{|c|}{ Embodied Global Warming Potential (GWP) } & \multirow{3}{*}{\begin{tabular}{|c} 
Difference in \\
Operating GWP from \\
Baseline after \\
Lifespan \\
$\left({ }^{4}\right.$ per $\left.\mathrm{m}\right)$
\end{tabular}} \\
\hline & \multicolumn{3}{|c|}{ Manutacturing } & \multicolumn{3}{|c|}{ Construction } & \multicolumn{3}{|c|}{ Maintenance } & \multicolumn{3}{|c|}{ End of Life } & \multirow{2}{*}{$\begin{array}{l}{ }^{3} \text { Total GWP } \\
(\text { per m) }\end{array}$} & \\
\hline & Material & ${ }^{2}$ Trans & Total & Material & ${ }^{2}$ Trans. & Total & Material & ${ }^{2}$ Trans. & Total & Material & ${ }^{2}$ Trans. & Total & & \\
\hline 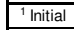 & 120 & 0 & 120 & 5 & 0 & 5 & 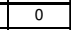 & 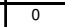 & 0 & 0 & 0 & 0 & & \\
\hline 50 & 120 & 0 & 120 & 5 & 0 & 5 & 1 & 0 & 1 & 0 & 0 & 0 & 126 & 0 \\
\hline
\end{tabular}

Note: Calculations are per linear meter of strip footing $(600 \mathrm{~mm} \times 200 \mathrm{~m})$ ad for 100

ATHENA ® EIE Material List:

(Includes all materials after 50 years)

\begin{tabular}{|l|c|c|}
\hline \multicolumn{1}{|c|}{ Material List } & Quantities & Unit \\
\hline 6 mil Polyyethylene & 1.3 & $\mathrm{~m} 2$ \\
\hline Concrete 30 MPa (lfyash av) & 0.4 & $\mathrm{~m} 3$ \\
\hline Modified Bitumen membrane & 2.1 & $\mathrm{~kg}$ \\
\hline Rebar, Rod, Light Sections & 14.9 & $\mathrm{~kg}$ \\
\hline Solvent Based Alkyd Paint & 0.1 & $\mathrm{~L}$ \\
\hline
\end{tabular}

Notes:

${ }^{2}$ Trans. = Transportation

${ }^{3}$ Total EE (or Total GWP) per $m=$ Total embodied energy (or total embodied GWP) of building component after lifiespan (i.e. total manufacturing + tota ${ }^{4}$ Total Difference in Operating Energy (or GWP) from Baseline after
Lifespan per $m=$ The difierence in the total life-cycle operating energy (or
GWP from the baseline retail building after lifespan, due to using this Lifespan per $m=$ The difference in the total life-cycle operating energy (or
$G W P$ f from the baseline retail building after lifespan, due to using this
building * Total operating primary energy use of baseline retail building after 50 years =

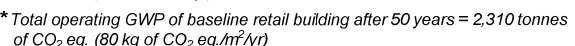


Concrete Strip Footing and Foundation Wall \#8 (SF-FDN8) Building Component Description:

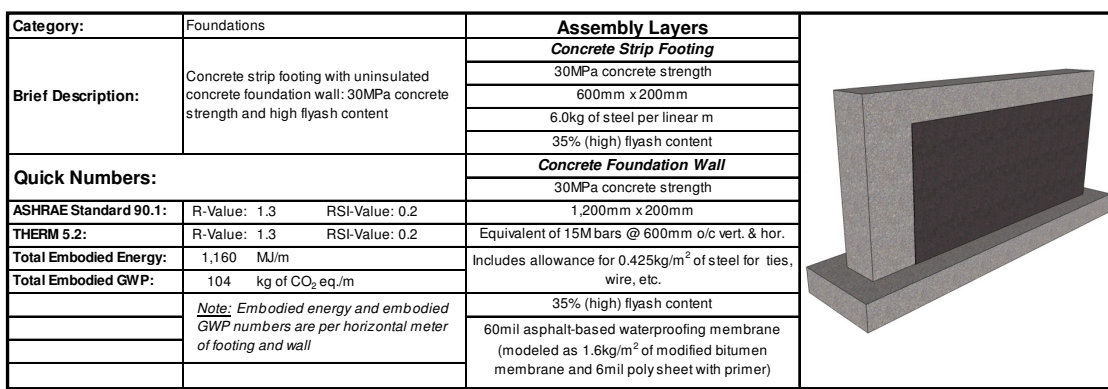

Life-Cycle Assessment Results:

Primary Energy Consumption (MJ)

\begin{tabular}{|c|c|c|c|c|c|c|c|c|c|c|c|c|c|c|}
\hline \multirow{3}{*}{$\begin{array}{c}\text { Lifespan } \\
\text { (vears) }\end{array}$} & \multicolumn{13}{|c|}{ Embodied Energy (EE) } & \multirow{3}{*}{$\begin{array}{l}\text { Difference in } \\
\text { Operating Energy } \\
\text { from Baseline after } \\
\text { Lifespan } \\
\left({ }^{4} \text { per } \mathrm{m}\right)\end{array}$} \\
\hline & \multicolumn{3}{|c|}{ Manufacturing } & \multicolumn{3}{|c|}{ Construction } & \multicolumn{3}{|c|}{ Maintenance } & \multicolumn{3}{|c|}{ End of Life } & \multirow{2}{*}{$\begin{array}{l}{ }^{3} \text { Total EE } \\
\text { (per } \mathrm{m})\end{array}$} & \\
\hline & Material & ${ }^{2}$ Trans. & Total & Material & ${ }^{2}$ Trans. & Total & Material & \begin{tabular}{|l|}
2 \\
\end{tabular} & Total & Material & \begin{tabular}{|l|l|}
${ }^{2}$ Trans. \\
\end{tabular} & Total & & \\
\hline \begin{tabular}{|l|} 
'Initial \\
\end{tabular} & 881 & $\begin{array}{ll}55 \\
\end{array}$ & \begin{tabular}{|l|}
937 \\
\end{tabular} & 70 & 51 & $\begin{array}{ll}121 \\
\end{array}$ & 0 & 0 & 0 & 0 & 0 & 0 & $\begin{array}{l}1,0 \\
0\end{array}$ & \\
\hline 50 & \begin{tabular}{|l|}
881 \\
\end{tabular} & 55 & \begin{tabular}{|l|}
937 \\
\end{tabular} & 70 & 51 & 121 & 75 & 0 & 75 & 0 & 27 & $\begin{array}{l}27 \\
\end{array}$ & 1,160 & 0 \\
\hline
\end{tabular}

Global Warming Potential ( $\mathrm{kg}$ of $\mathrm{CO}_{2}$ eq.)

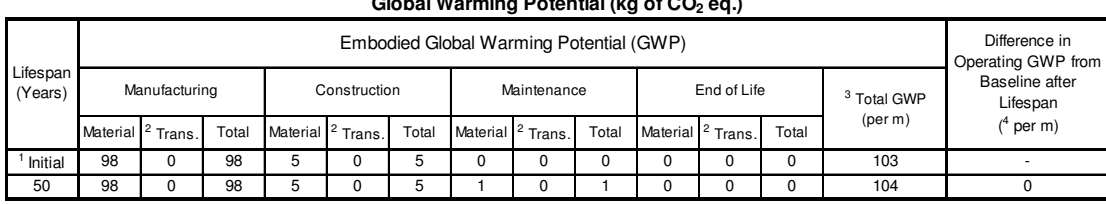

Note: Calculations are per linear meter of strip footing (600 $\mathrm{mm} \times 200 \mathrm{~m})$ and foundation wall (1200 $\mathrm{mm} \times 200 \mathrm{~mm})$ combination ATHENA ® EIE Material List:

\begin{tabular}{|l|c|c|}
\hline \multicolumn{1}{|c|}{ Matiludes all materials List } & Quantitities & Un years) \\
\hline 6 mil Polyethylene & 1.3 & $\mathrm{~m} 2$ \\
\hline Concrete 30 MPa (flyash 35\%) & 0.4 & $\mathrm{~m} 3$ \\
\hline Modified Bitumen membrane & 2.1 & $\mathrm{~kg}$ \\
\hline Rebar, Rod, Light Sections & 14.9 & $\mathrm{~kg}$ \\
\hline Solvent Based Alkyd Paint & 0.1 & $\mathrm{~L}$ \\
\hline
\end{tabular}

${ }^{3}$ Total EE (or Total GWP) per $m=$ Total embodied energy (or total embodied

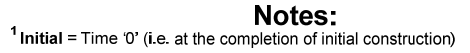

${ }^{2}$ Trans. = Transportation GWP) of building component after lifespan (i.e. total manufacturing + total Total Difference in Operating Energy (or GWP) for GWP) from the baseline retail building after lifespan, due to using this

"Total operating primary energy use of baseline retail building after 50 years =
$50,700 \mathrm{GJ}\left(1.745 \mathrm{MJ} / \mathrm{m}^{2} / \mathrm{rr}\right)$ * $T$ otal operating $\mathrm{GWP}$ of baseline retail building after 50 years $=2,310$ tonnes
of $\mathrm{CO}_{2}$ eq. ( $80 \mathrm{~kg}$ of $\mathrm{CO}_{2}$ eq, $\left./ \mathrm{m}^{2} / \mathrm{yr}\right)$

\section{LCA Data for Concrete Slab-on-Grades}

This section contains a detailed description of each concrete slab-ongrade that was examined in this study (6 in total). The assembly layers are listed for each case, along with a detailed description of the material quantities from the ATHENA® Environmental Impact Estimator for Buildings.

A breakdown of the total primary energy consumption and the total global warming potential (GWP) for each slab-on-grade is also included. In each case, the results were calculated for a slab-on-grade area of $610 \mathrm{~m}^{2}$, which represents a typical size for a single-storey retail building. The results are also expressed per $\mathrm{m}^{2}$ of slab-on-grade. The data has been calculated for two different lifespans: at the completion of initial construction and after 50 years.

As a summary, the figure below illustrates a comparison of the total embodied energy (and GWP) after 50 years for the various slab-ongrades in this section. For comparison purpose, the building component used in the baseline retail building has been shaded in grey.

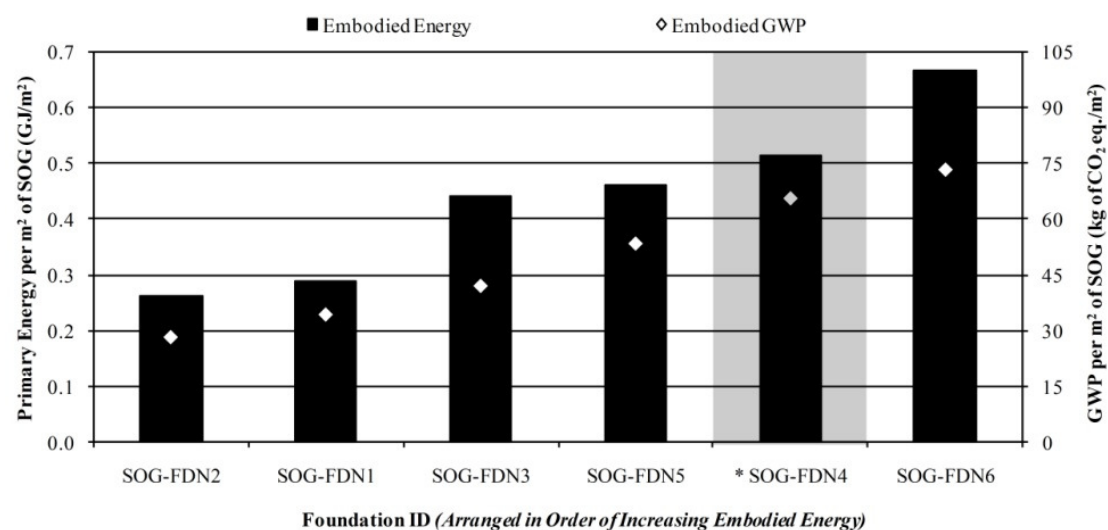




\section{Concrete Slab-On-Grade \#1 (SOG-FDN1)}

Building Component Description:

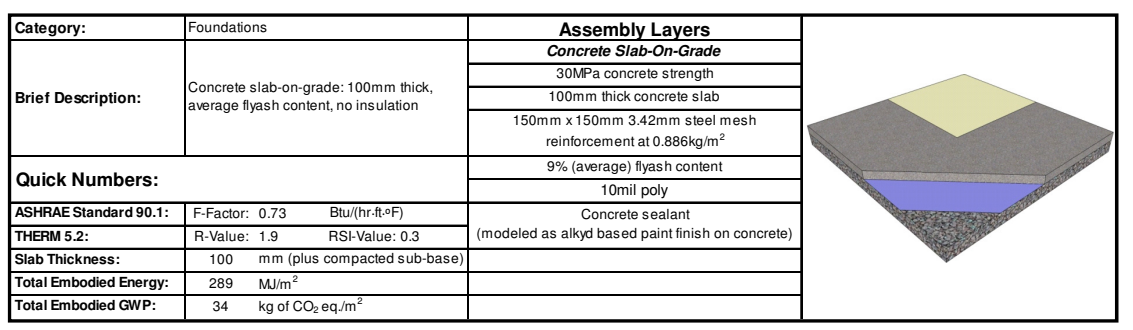

\section{Life-Cycle Assessment Results:}

Primary Energy Consumption (MJ)

\begin{tabular}{|c|c|c|c|c|c|c|c|c|c|c|c|c|c|c|c|}
\hline \multirow{4}{*}{$\begin{array}{l}\text { Lifespana } \\
\text { (Years) }\end{array}$} & \multicolumn{13}{|c|}{ Embodied E } & \multirow{3}{*}{\multicolumn{2}{|c|}{$\begin{array}{c}\text { Difference in } \\
\text { Operating Energy } \\
\text { from Baseline after } \\
\text { Lifespan }\end{array}$}} \\
\hline & \multirow{2}{*}{\multicolumn{2}{|c|}{ Manufacturing }} & \multirow{2}{*}{\multicolumn{3}{|c|}{ Construction }} & \multirow{2}{*}{\multicolumn{3}{|c|}{ Mainten }} & \multirow{2}{*}{\multicolumn{3}{|c|}{ End of Life }} & \multirow{3}{*}{$\begin{array}{c}{ }^{3} \text { Total } \\
\mathrm{EE}\end{array}$} & \multirow{3}{*}{$\begin{array}{c}{ }^{4} \text { Total } \\
\mathrm{EE} \\
\text { per } \mathrm{m}^{2}\end{array}$} & & \\
\hline & & & & & & & & & & & & & & & \\
\hline & 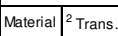 & \begin{tabular}{|l|l|} 
Total \\
\end{tabular} & Material & ${ }^{2} \mathrm{Tra}$ & \begin{tabular}{|l|l|} 
Total \\
\end{tabular} & Material & ${ }^{2}$ Trans. & Total & Material & \begin{tabular}{|l|}
${ }^{2}$ Trans. \\
\end{tabular} & Total & & & ${ }^{5}$ Total & ${ }^{6}$ per $\mathrm{m}^{2}$ \\
\hline Initial & \begin{tabular}{|l|l|}
137,399 & 6,6 \\
\end{tabular} & 144,063 & 16,495 & 8, & 25,002 & 0 & 0 & 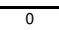 & 0 & 0 & 0 & 169,065 & 277 & & \\
\hline 50 & \begin{tabular}{|l|l}
137,399 & 6,664 \\
\end{tabular} & 144,063 & & \begin{tabular}{l|l|l|}
8,507 & \\
\end{tabular} & 25,002 & 2,740 & 20 & 2,760 & 8 & \begin{tabular}{|l|l|}
4,524 & \\
\end{tabular} & 4,532 & & 289 & 0 & 0 \\
\hline
\end{tabular}

Global Warming Potential ( $k g$ of $\mathrm{CO}_{2}$ eq.)

\begin{tabular}{|c|c|c|c|c|c|c|c|c|c|c|c|c|c|c|c|c|}
\hline \multirow{3}{*}{$\begin{array}{l}\text { Lefespan } \\
\text { (Years) }\end{array}$} & \multicolumn{14}{|c|}{ Embodied Global Warming Potential (GWP) } & \multirow{2}{*}{\multicolumn{2}{|c|}{\begin{tabular}{|c} 
Difterence in \\
Operating GWPP from \\
Baseline after \\
Lifespan
\end{tabular}}} \\
\hline & \multicolumn{3}{|c|}{ Manufacturing } & \multicolumn{3}{|c|}{ Construction } & \multicolumn{3}{|c|}{ Maintenance } & \multicolumn{3}{|c|}{ End of Llife } & \multirow{2}{*}{$\begin{array}{l}{ }^{3} \text { Total } \\
\text { GWP }\end{array}$} & \multirow{2}{*}{$\begin{array}{l}{ }^{4} \text { Total } \\
\text { GWP } \\
\text { per m }\end{array}$} & & \\
\hline & Material & 1' 2 Trans. & Total & Material & ${ }^{2}$ Trans. & Total & Material & ${ }^{2}$ Trans. & Total & Material & ${ }^{2}$ Trans. & Total & & & ${ }^{5}$ Total & ${ }^{6}{ }^{6}$ er m ${ }^{2}$ \\
\hline |nitial & \begin{tabular}{|l|l|}
19,766 \\
\end{tabular} & 13 & \begin{tabular}{|l|l|l|l}
19,778 \\
\end{tabular} & \begin{tabular}{|l|}
1,071 \\
\end{tabular} & 16 & \begin{tabular}{|l|}
1,088 \\
\end{tabular} & 0 & 0 & 0 & 0 & 0 & 0 & \begin{tabular}{|l|l|}
20,866 \\
\end{tabular} & 34 & & \\
\hline 50 & 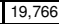 & 13 & $\begin{array}{l}19,778 \\
\end{array}$ & \begin{tabular}{|l|l|}
1,071 \\
\end{tabular} & \begin{tabular}{l|l}
16 \\
\end{tabular} & \begin{tabular}{|l|l|}
1,088 \\
\end{tabular} & 47 & 0 & 47 & 0 & 9 & 9 & \begin{tabular}{|l|l|}
20,922 \\
\end{tabular} & 34 & 10,000 & 17 \\
\hline
\end{tabular}
Area of SOG in baseline retail building = $586.0 \mathrm{~m}^{2}$

\section{ATHENA ® EIE Material List:} (Includes all materials after 50 years)

\begin{tabular}{|l|c|c|}
\hline \multicolumn{1}{|c|}{ Material List } & Quantities & Unit \\
\hline 6 mil Polyethylene & 645.4 & $\mathrm{~m} 2$ \\
\hline Concrete 30 MPa (flyash av) & 63.9 & $\mathrm{~m} 3$ \\
\hline Solvent Based Alkyd Paint & 67.3 & $\mathrm{~L}$ \\
\hline Welded Wire Mesh / Ladder Wire & 549.9 & $\mathrm{~kg}$ \\
\hline
\end{tabular}

Notes:
${ }^{1}$ Initial = Time 'o' (i.e. at the completion of initial construction)

${ }^{2}$ Trans. $=$ Transportation

Total EE (or Total GWP) = Total embodied energy (or total embodied GWP) of building component after lifespan (i.e. - total man
construction + total maintenance + total enc-oflife effects)

${ }^{4}$ Total EE (or Total GWP) per $\mathrm{m}^{2}=$ Total EE (or Total GWP) of building
component/ area of building component that was modelled in ATHENAQ EIE Total Difference in Operating Energy (or GWP) from Baseline after Lifespan = The difference in the total life-cycle operating energy (or GWP)
from the baseline retail building after lifespan, due to using this building
component instead of the baseline component

Total Difference in Operating Energy (or GWP) from Baseline after
Lifespan per $\mathrm{m}^{2}=$ Total difference in operating energy (or GWP) from Lifespan per $\mathrm{m}^{2}=$ Total difference in operating energy (or
baseline after lifespan / baseline retail building slab-on-grade area

* Total operating primary energy use of baseline retail building after 50 years =
$50,700 \mathrm{GJ}\left(1,745 \mathrm{MJ} / \mathrm{m}^{2} / \mathrm{yr}\right)$

Total operating GWP of baseline rets
of $\mathrm{CO}_{2}$ eq. $\left(80 \mathrm{~kg}\right.$ of $\left.\mathrm{CO}_{2} \mathrm{eq} / \mathrm{m} \mathrm{m}^{2} \mathrm{yr}\right)$

\section{Concrete Slab-On-Grade \#2 (SOG-FDN2)}

Building Component Description:

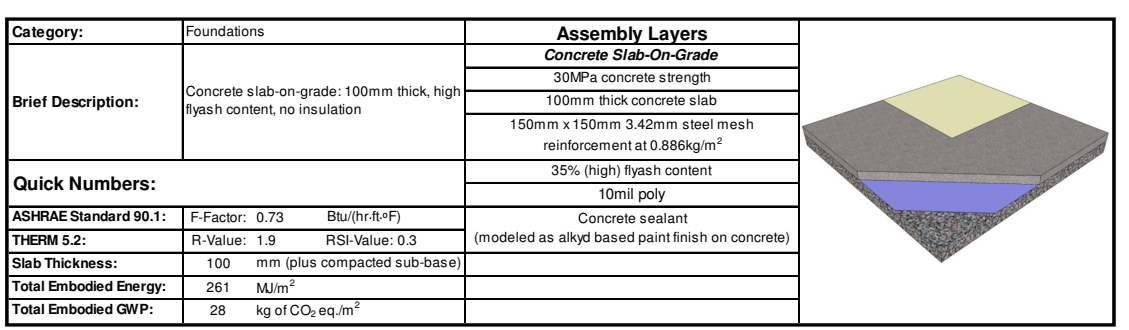

\section{Life-Cycle Assessment Results:}

Primary Energy Consumption (MJ)

\begin{tabular}{|c|c|c|c|c|c|c|c|c|c|c|c|c|c|c|c|c|}
\hline \multirow{3}{*}{$\begin{array}{l}\text { Lifespan } \\
\text { (Years) }\end{array}$} & \multicolumn{14}{|c|}{ Embodied Energy (EE) } & \multirow{2}{*}{\multicolumn{2}{|c|}{$\begin{array}{c}\text { Difference in } \\
\text { Operating Energy } \\
\text { from Baseline after } \\
\text { Lifespan }\end{array}$}} \\
\hline & \multicolumn{3}{|c|}{ Manufacturing } & \multicolumn{3}{|c|}{ Construction } & \multicolumn{3}{|c|}{ Maintenance } & \multicolumn{3}{|c|}{ End of L Life } & \multirow{2}{*}{$\begin{array}{c}{ }^{3} \text { Total } \\
\text { EE }\end{array}$} & \multirow{2}{*}{\begin{tabular}{|c|c}
${ }^{4}$ Total \\
EE \\
per $m^{2}$
\end{tabular}} & & \\
\hline & Material & ${ }^{2}$ Trans. & \begin{tabular}{|l|l|} 
Total \\
\end{tabular} & Material & ${ }^{2}$ Trans. & Total & \begin{tabular}{|l|} 
Material \\
\end{tabular} & ${ }^{2}$ Trans. & \begin{tabular}{|l|} 
Total \\
\end{tabular} & Material & \begin{tabular}{|l|}
${ }^{2}$ Trans. \\
\end{tabular} & Total & & & ${ }^{5}$ Total & ${ }^{6} \mathrm{perm}^{2}$ \\
\hline Initial & 118,196 & 8,905 & 127,100 & 16,495 & \begin{tabular}{l|l|}
8,507 \\
\end{tabular} & 25,002 & 0 & 0 & 0 & 0 & 0 & 0 & 152,103 & 249 & & \\
\hline 50 & 118,196 & 8,905 & 127,100 & \begin{tabular}{|l|l|l|l} 
\\
\end{tabular} & 8,507 & 25,002 & 2,740 & 20 & 2,760 & 8 & 4,524 & 4,532 & 159,395 & 5261 & 0 & 0 \\
\hline
\end{tabular}

Global Warming Potential (kg of $\mathrm{CO}_{2}$ eq.)

\begin{tabular}{|c|c|c|c|c|c|c|c|c|c|c|c|c|c|c|c|c|}
\hline \multirow{3}{*}{$\begin{array}{l}\text { Litespan } \\
\text { (Years) }\end{array}$} & \multicolumn{14}{|c|}{ Embodied Global Warming Potential (GWP) } & \multirow{2}{*}{\multicolumn{2}{|c|}{$\begin{array}{l}\text { Difference in } \\
\text { Operating GWP from } \\
\text { Baseline after } \\
\text { Lifespan }\end{array}$}} \\
\hline & \multicolumn{3}{|c|}{ Manutacturing } & \multicolumn{3}{|c|}{ Construction } & \multicolumn{3}{|c|}{ Maintenance } & \multicolumn{3}{|c|}{ End of Lite } & \multirow{2}{*}{$\begin{array}{l}{ }^{3} \text { Total } \\
\text { GWPP }\end{array}$} & \multirow{2}{*}{$\begin{array}{l}{ }^{4} \text { Total } \\
\text { GWr } \\
\text { perm }{ }^{2}\end{array}$} & & \\
\hline & Material & ${ }^{2}$ Trans. & Total & Material & ${ }^{2}$ Trans. & Total & Material & ${ }^{2}$ Trans. & Total & Material & ${ }^{2}$ Trans. & Total & & & ${ }^{5}$ Total & ${ }^{6}$ perm $^{2}$ \\
\hline 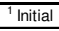 & 16,064 & 17 & 16,081 & 1,071 & 16 & 1,088 & 0 & 0 & 0 & 0 & 0 & 0 & 17,169 & 28 & & \\
\hline 50 & 16,064 & 17 & 16,081 & \begin{tabular}{|l|l|} 
& 1,071 \\
\end{tabular} & 16 & $\begin{array}{ll}1,088 \\
\end{array}$ & 47 & 0 & $\begin{array}{l}47 \\
\end{array}$ & 0 & 9 & 9 & 17,225 & 28 & $\begin{array}{c}10,000 \\
\end{array}$ & $\begin{array}{l}17 \\
\end{array}$ \\
\hline
\end{tabular}

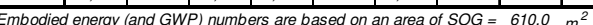

Area of SOG in baseline retail building = $586.0 \mathrm{~m}^{2}$

ATHENA ® EIE Material List: (Includes all materials after 50 years)

\begin{tabular}{|l|c|c|}
\hline \multicolumn{1}{|c|}{ Material List } & Quantities & Unit \\
\hline 6 mil Polyethylene & 645.4 & $\mathrm{~m} 2$ \\
\hline Concrete 30 MPa (flyash $35 \%)$ & 63.9 & $\mathrm{~m} 3$ \\
\hline Solvent Based Alkyd Paint & 67.3 & $\mathrm{~L}$ \\
\hline Welded Wire Mesh / Ladder Wire & 549.9 & $\mathrm{~kg}$ \\
\hline
\end{tabular}

Notes:
${ }^{1}$ Initial = Time 'O' (i.e. at the completion of initial construction) ${ }^{2}$ Trans. $=$ Transportation

${ }^{3}$ Total EE (or Total GWP) $=$ Total embodied energy (or total embodied GWP) of building component after lifespan (i.e. total manu
construction + total maintenance + total end-of-life effects)

${ }^{4}$ Total EE (or Total GWP) per $\mathrm{m}^{2}=$ Total EE (or Total GWP) of building
component / area of building component that was modelled in ATHENAQ EIE ${ }^{5}$ Total Difference in Operating Energy (or GWP) from Baseline after from the baseline retail building after lifespan, due to using this buildiing component instead of the baseline component

${ }^{6}$ Total Difference in Operating Energy (or GWP) from Baseline after Lifespan per $\mathrm{m}^{2}=$ Total difference in operating energy (or GWP) from * Total operating primary energy use of baseline retail building after 50 years =

* Total operating $\mathrm{GWP}$ of baseline reta
of $\mathrm{CO}_{2}$ eq. $\left(80 \mathrm{~kg}\right.$ of $\mathrm{CO}_{2}$ eq. $\left./ \mathrm{m}^{2} \mathrm{yr}\right)$ 


\section{Concrete Slab-On-Grade \#3 (SOG-FDN3)}

Building Component Description:

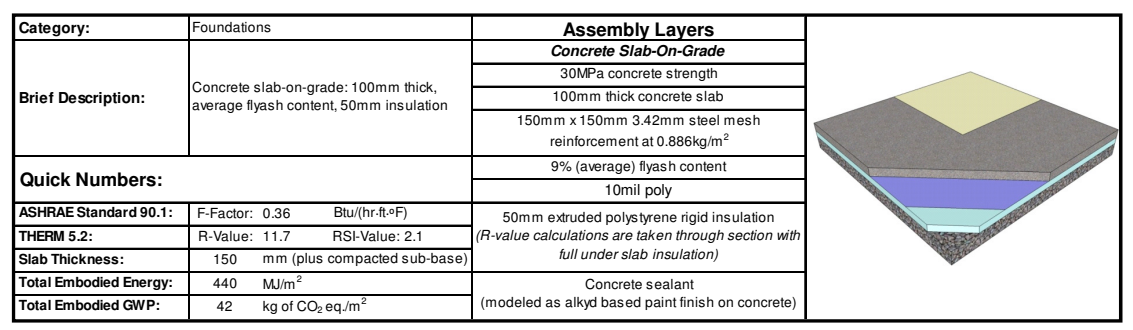

Life-Cycle Assessment Results:

Primary Energy Consumption (MJ)

\begin{tabular}{|c|c|c|c|c|c|c|c|c|c|c|c|c|c|c|c|}
\hline \multirow{3}{*}{\begin{tabular}{|l} 
Lifespan \\
(Years)
\end{tabular}} & \multicolumn{13}{|c|}{ Embodied Energy (EE) } & \multirow{2}{*}{\multicolumn{2}{|c|}{$\begin{array}{c}\text { Difference in } \\
\text { Operating Energy } \\
\text { from Baseline afte } \\
\text { Lifespan }\end{array}$}} \\
\hline & \multicolumn{2}{|c|}{ Manutacturing } & \multicolumn{3}{|c|}{ Construction } & \multicolumn{3}{|c|}{ Mainte } & \multicolumn{3}{|c|}{ End of Life } & \multirow{2}{*}{$\begin{array}{l}3 \text { Total } \\
\mathrm{EE}\end{array}$} & \multirow{2}{*}{\begin{tabular}{|l}
${ }^{4}$ Total \\
EE \\
per $\mathrm{m}^{2}$
\end{tabular}} & & \\
\hline & \begin{tabular}{|l|l} 
Material & 2 \\
& Trans.
\end{tabular} & \begin{tabular}{|l|l} 
Total \\
\end{tabular} & Materia & \begin{tabular}{|l|}
2 \\
\end{tabular} & \begin{tabular}{|l|l|} 
Total \\
\end{tabular} & Material & \begin{tabular}{|l|}
${ }^{2}$ Trans. \\
\end{tabular} & Total & Material & | ${ }^{2}$ Trans. & Total & & & ${ }^{5}$ Total & ${ }^{6}{ }_{\mathrm{perm}}^{2}{ }^{2}$ \\
\hline Initial & \begin{tabular}{|l|l|}
229,088 & 6,671 \\
\end{tabular} & 235,759 & 16,495 & 655 & 25,150 & 0 & 0 & 0 & 0 & 0 & 0 & 260,909 & \begin{tabular}{|l|l|}
428 \\
\end{tabular} & & \\
\hline 50 & \begin{tabular}{|l|l|}
229,088 & 6,671 \\
\end{tabular} & 235,759 & 16,495 & 8,655 & 255,150 & 2,740 & 20 & 2,760 & 8 & 4,643 & 4,651 & 268,320 & D 440 & $\sqrt{, 000}$ & $-2,901$ \\
\hline
\end{tabular}

Global Warming Potential ( $\mathrm{kg}$ of $\mathrm{CO}_{2}$ eq.)

\begin{tabular}{|c|c|c|c|c|c|c|c|c|c|c|c|c|c|c|c|c|}
\hline \multirow{3}{*}{$\begin{array}{l}\text { Lespanan } \\
\text { (Years) }\end{array}$} & \multicolumn{14}{|c|}{ Embodied Global Warming Potential (GWP) } & \multirow{2}{*}{\multicolumn{2}{|c|}{\begin{tabular}{|c} 
Difterence in \\
Operating GWPP from \\
Baseline after \\
Lifespan
\end{tabular}}} \\
\hline & \multicolumn{3}{|c|}{ Manufacturing } & \multicolumn{3}{|c|}{ Construction } & \multicolumn{3}{|c|}{ Maintenance } & \multicolumn{3}{|c|}{ End of Llife } & \multirow{2}{*}{$\begin{array}{l}{ }^{3} \text { Total } \\
\text { GWP }\end{array}$} & \multirow{2}{*}{$\begin{array}{l}{ }^{4} \text { Total } \\
\text { GWP } \\
\text { per m}{ }^{2}\end{array}$} & & \\
\hline & Material & 12 ${ }^{2}$ Trans. & Total & Material & ${ }^{2}$ Trans. & Total & Material & ${ }^{2}$ Trans. & Total & Material & ${ }^{2}$ Trans. & Total & & & ${ }^{5}$ Total & ${ }^{6}{ }^{6}$ er m ${ }^{2}$ \\
\hline |litital & 24,451 & 13 & \begin{tabular}{|l|l|l}
24,464 \\
\end{tabular} & \begin{tabular}{|l|}
1,071 \\
\end{tabular} & 17 & \begin{tabular}{|l|}
1,088 \\
\end{tabular} & 0 & 0 & 0 & 0 & 0 & 0 & 25,552 & 42 & & \\
\hline 50 & 24,451 & 13 & \begin{tabular}{|l|l|l}
24,464 \\
\end{tabular} & \begin{tabular}{|l|l|}
1,071 \\
\end{tabular} & \begin{tabular}{l|l}
17 \\
\end{tabular} & \begin{tabular}{|l|l|l|l|}
1,088 \\
\end{tabular} & 47 & 0 & 47 & 0 & 9 & 9 & 25,608 & 42 & \begin{tabular}{|l|l|}
$-100,000$ \\
\end{tabular} & -171 \\
\hline
\end{tabular}
Area of SOG in baseline retail building = $586.0 \mathrm{~m}^{2}$

\section{ATHENA $®$ EIE Material List:} (Includes all materials after 50 years)

\begin{tabular}{|l|c|c|}
\hline \multicolumn{1}{|c|}{ Material List } & Quantities & Unit \\
\hline 6 mil Polyethylene & 645.4 & $\mathrm{~m} 2$ \\
\hline Concrete 30 MPa (flyash av) & 63.9 & $\mathrm{~m} 3$ \\
\hline Extruded Polystyrene & $1,246.6$ & $\mathrm{~m} 2(25 \mathrm{~mm})$ \\
\hline Nails & 37.6 & $\mathrm{~kg}$ \\
\hline Solvent Based Alkyd Paint & 67.3 & $\mathrm{~L}$ \\
\hline Welded Wire Mesh / Ladder Wire & 549.9 & $\mathrm{~kg}$ \\
\hline
\end{tabular}

Notes:
${ }^{1}$ Initial = Time 'o' (i.e. at the completion of initial construction)

${ }^{2}$ Trans. $=$ Transportation

Total EE (or Total GWP) = Total embodied energy (or total embodied GWP) of building component after lifespan (i.e. total man
construction + total maintenance + total end-of-life effects)

Total EE (or Total GWP) per $\mathrm{m}^{2}=$ Total EE (or Total GWP) of building

component/ area of building component that was modelled in ATHENAQ EIE

Total Difference in Operating Energy (or GWP) from Baseline after
Lifespan $=$ The difference in the total lifecycle operating energy (or GWP) Lifespan = The difference in the total lifec cycle operating energy (or GWP)
from the basaline retail builiding arter lifespan, due to using this building
component instead of the baseline component

${ }^{6}$ Total Difference in Operating Energy (or GWP) from Baseline after
Lifespan per $\mathrm{m}^{2}=$ Total difference in operating energy (or GWP) from Lifespan per $\mathrm{m}^{2}=$ Total difference in operating energy (or
baseline after lifespan / baseline retail building slab-on-grade area

* Total operating primary energy use of baseline retail building after 50 years =
$50,700 \mathrm{GJ}\left(1,745 \mathrm{MJ} / \mathrm{m}^{2} / \mathrm{yr}\right)$

"Total operating $\mathrm{GWP}$ of baseline ret)

\section{Concrete Slab-On-Grade \#4 (SOG-FDN4)}

Building Component Description:

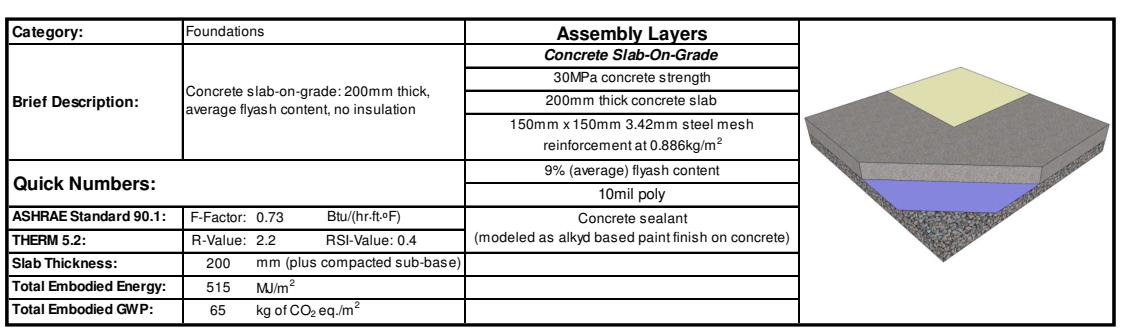

\section{Life-Cycle Assessment Results:}

Primary Energy Consumption (MJ)

\begin{tabular}{|c|c|c|c|c|c|c|c|c|c|c|c|c|c|c|c|}
\hline \multirow{3}{*}{$\begin{array}{l}\text { Lifespan } \\
\text { (Years) }\end{array}$} & \multicolumn{13}{|c|}{ Embodied Energy (EE) } & \multirow{2}{*}{\multicolumn{2}{|c|}{$\begin{array}{c}\text { Difference in } \\
\text { Operating Energy } \\
\text { from Baseline after } \\
\text { Lifespan }\end{array}$}} \\
\hline & \multicolumn{2}{|c|}{ Manufacturing } & \multicolumn{3}{|c|}{ Construction } & \multicolumn{3}{|c|}{ Maintenance } & \multicolumn{3}{|c|}{ End of L Life } & \multirow{2}{*}{$\begin{array}{c}{ }^{3} \text { Total } \\
\text { EE }\end{array}$} & \multirow{2}{*}{\begin{tabular}{|c|}
${ }^{4} \mathrm{~T}$ otal \\
$\mathrm{EE}$ \\
per m
\end{tabular}} & & \\
\hline & 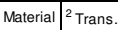 & Total & \begin{tabular}{|l|} 
Material \\
\end{tabular} & ${ }^{2}$ Trans. & Total & Material & ${ }^{2}$ Trans. & \begin{tabular}{|l|} 
Total \\
\end{tabular} & Material & \begin{tabular}{|l|}
${ }^{2}$ Trans. \\
\end{tabular} & Total & & & ${ }^{5}$ Total & ${ }^{6}$ perm ${ }^{2}$ \\
\hline Initial & \begin{tabular}{|l|l|}
243,853 & 13,299 \\
\end{tabular} & 257,152 & 28,420 & 16,945 & 45,365 & 0 & 0 & 0 & 0 & 0 & 0 & 302,517 & 496 & & \\
\hline 50 & \begin{tabular}{|l|l|l|l|}
243,853 & 13,299 \\
\end{tabular} & 257,152 & 28,420 & 16,945 & 45,365 & \begin{tabular}{|l|l|}
2,740 \\
\end{tabular} & 20 & 2,760 & 15 & $\begin{array}{ll}9,024 & \\
\end{array}$ & $\begin{array}{l}9,039 \\
\end{array}$ & 314,316 & 515 & 0 & 0 \\
\hline
\end{tabular}

Global Warming Potential (kg of $\mathrm{CO}_{2}$ eq.)

\begin{tabular}{|c|c|c|c|c|c|c|c|c|c|c|c|c|c|c|c|c|}
\hline \multirow{3}{*}{$\begin{array}{l}\text { Litespan } \\
\text { (Years) }\end{array}$} & \multicolumn{14}{|c|}{ Embodied Global Warming Potential (GWP) } & \multirow{2}{*}{\multicolumn{2}{|c|}{\begin{tabular}{|c} 
Difference in \\
Operating GWP from \\
Baseline after \\
Lifespan
\end{tabular}}} \\
\hline & \multicolumn{3}{|c|}{ Manufacturing } & \multicolumn{3}{|c|}{ Construction } & \multicolumn{3}{|c|}{ Maintenance } & \multicolumn{3}{|c|}{ End of Life } & \multirow{2}{*}{$\mid \begin{array}{l}{ }^{3} \text { Total } \\
\text { GWPP }\end{array}$} & \multirow{2}{*}{$\begin{array}{l}{ }^{4} \text { Total } \\
\text { GWP } \\
\text { per m }\end{array}$} & & \\
\hline & Material & ${ }^{2}$ Trans. & Total & Material & ${ }^{2}$ Trans. & Total & Material & ${ }^{2}$ Trans. & Total & Material & 2 Trans. & Total & & & ${ }^{5}$ Total & ${ }^{6}$ perm $m^{2}$ \\
\hline nititial & 37,878 & 25 & 37,903 & 1,915 & 33 & 1,948 & 0 & 0 & 0 & 0 & 0 & 0 & 39,851 & 65 & & \\
\hline 50 & 37,878 & \begin{tabular}{|l|}
25 \\
\end{tabular} & 37,903 & 1,995 & 33 & 1,948 & 47 & 0 & $\begin{array}{l}47 \\
\end{array}$ & 1 & 17 & $\begin{array}{l}18 \\
\end{array}$ & 39,916 & 65 & 0 & 0 \\
\hline
\end{tabular}

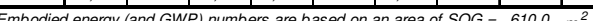

Area of SOG in baseline retail building $=586.0 \mathrm{~m}^{2}$

ATHENA ® EIE Material List: (Includes all materials after 50 years)

\begin{tabular}{|l|c|c|}
\hline \multicolumn{1}{|c|}{ Material List } & Quantities & Unit \\
\hline 6 mil Polyyethylene & 645.4 & $\mathrm{~m} 2$ \\
\hline Concrete 30 MPa (flyash av) & 127.8 & $\mathrm{~m} 3$ \\
\hline Solvent Based Alkyd Paint & 67.3 & $\mathrm{~L}$ \\
\hline Welded Wire Mesh / Ladder Wire & 549.9 & $\mathrm{~kg}$ \\
\hline
\end{tabular}

Notes:
${ }^{1}$ Initial = Time 'O' (i.e. at the completion of initial construction) ${ }^{2}$ Trans. $=$ Transportation

${ }^{3}$ Total EE (or Total GWP) $=$ Total embodied energy (or total embodied GWP) of building component after lifespan (i.e. total man
construction + total maintenance + + total end-oflife effects)

${ }^{4}$ Total EE (or Total GWP) per $\mathrm{m}^{2}=$ Total EE (or Total GWP) of building
component / area of building component that was modelled in ATHENAQ EIE ${ }^{5}$ Total Difference in Operating Energy (or GWP) from Baseline after from the baseline retail building after lifespan, due to using this buildiing component instead of the baseline component

${ }^{6}$ Total Difference in Operating Energy (or GWP) from Baseline after Lifespan per $\mathrm{m}^{2}=$ Total difference in operating energy (or GWP) from

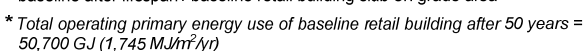

* Total operating $\mathrm{GWP}$ of baseline reta
of $\mathrm{CO}_{2}$ eq. $\left(80 \mathrm{~kg}\right.$ of $\mathrm{CO}_{2}$ eq. $\left./ \mathrm{m}^{2} \mathrm{yr}\right)$ 


\section{Concrete Slab-On-Grade \#5 (SOG-FDN5)}

Building Component Description:

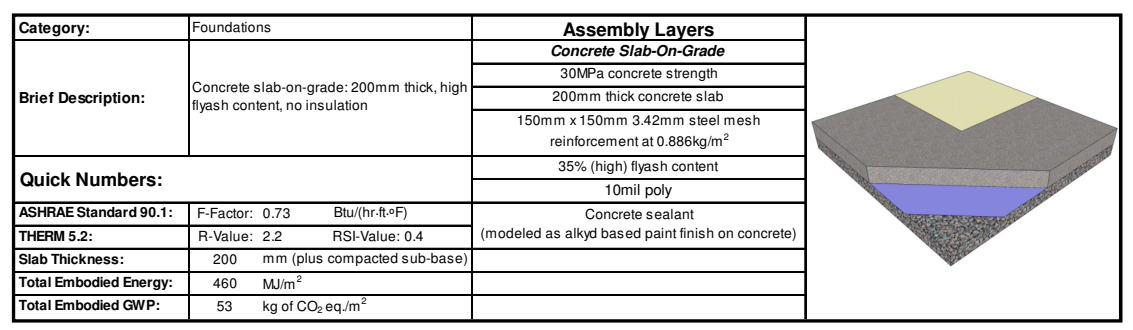

\section{Life-Cycle Assessment Results:}

Primary Energy Consumption (MJ)

\begin{tabular}{|c|c|c|c|c|c|c|c|c|c|c|c|c|c|c|c|}
\hline \multirow{3}{*}{$\begin{array}{l}\text { Lifiespan } \\
\text { (Years) }\end{array}$} & \multicolumn{13}{|c|}{ Embodied Energy (EE) } & \multirow{2}{*}{\multicolumn{2}{|c|}{$\begin{array}{c}\text { Difference in } \\
\text { Operating Energy } \\
\text { from Baseline after } \\
\text { Lifespan }\end{array}$}} \\
\hline & \multicolumn{2}{|c|}{ Manutacturing } & \multicolumn{3}{|c|}{ Construction } & \multicolumn{3}{|c|}{ Maintenar } & \multicolumn{3}{|c|}{ End of Life } & \multirow{2}{*}{$\begin{array}{c}{ }^{3} \text { Total } \\
\mathrm{EE}\end{array}$} & \multirow{2}{*}{\begin{tabular}{|c|}
${ }^{4}$ Total \\
EE \\
per m${ }^{2}$
\end{tabular}} & & \\
\hline & \begin{tabular}{|l|l|} 
Material & 2 \\
\end{tabular} & \begin{tabular}{|l|l|} 
Total \\
\end{tabular} & Material & || ${ }^{2}$ Trans. & \begin{tabular}{|l|l|} 
Total \\
\end{tabular} & Material & ${ }^{2}$ Trans. & Total & Material & ${ }^{2}$ Trans. & Total & & & ${ }^{5}$ Total & ${ }^{6}$ per $\mathrm{m}^{2}$ \\
\hline |lititial & \begin{tabular}{|l|l|l|l|l|l|}
205,447 & 1781 \\
\end{tabular} & 223,228 & 28,420 & 16,945 & 45,365 & 0 & 0 & 0 & 0 & 0 & 0 & 33 & $3 \longdiv { 4 4 0 }$ & & \\
\hline 50 & \begin{tabular}{|l|l|l|l|l|l|}
205,447 & 17,78 \\
\end{tabular} & 223,228 & 28,4 & 16,945 & 45,365 & 2,740 & 20 & 2,760 & 15 & 9,024 & 9,039 & 280,392 & 460 & 0 & 0 \\
\hline
\end{tabular}

Global Warming Potential ( $k g$ of $\mathrm{CO}_{2}$ eq.)

\begin{tabular}{|c|c|c|c|c|c|c|c|c|c|c|c|c|c|c|c|c|}
\hline \multirow{3}{*}{$\begin{array}{l}\text { Lifespan } \\
\text { (Years }\end{array}$} & \multicolumn{14}{|c|}{ Embodied Global Warming Potential (GWP) } & \multirow{2}{*}{\multicolumn{2}{|c|}{$\begin{array}{l}\text { Difference in } \\
\text { Operating GWP from } \\
\text { Baseline after } \\
\text { Lifespan }\end{array}$}} \\
\hline & \multicolumn{3}{|c|}{ Manufacturing } & \multicolumn{3}{|c|}{ Construction } & \multicolumn{3}{|c|}{ Maintenance } & \multicolumn{3}{|c|}{ End of Life } & \multirow{2}{*}{$\begin{array}{l}{ }^{3} \text { Total } \\
\text { GWP }\end{array}$} & \multirow{2}{*}{$\begin{array}{l}{ }^{4} \text { Total } \\
\text { GWP } \\
\text { per m }\end{array}$} & & \\
\hline & \begin{tabular}{|l|} 
Material \\
\end{tabular} & ${ }^{2}$ Trans. & \begin{tabular}{|l|} 
Total \\
\end{tabular} & Material & ${ }^{2}$ Trans. & Total & Material & ${ }^{2}$ Trans. & Total & \begin{tabular}{|l|} 
Material \\
\end{tabular} & \begin{tabular}{|l|}
$\left.\right|^{2}$ Trans. \\
\end{tabular} & Total & & & ${ }^{5}$ Total & ${ }^{6} \mathrm{perm}^{2}$ \\
\hline 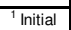 & 30,474 & 34 & 30,508 & \begin{tabular}{|l|l|}
1,915 \\
\end{tabular} & 33 & 1,948 & 0 & 0 & 0 & 0 & 0 & 0 & \begin{tabular}{|l|l|}
32,456 \\
\end{tabular} & 53 & & \\
\hline 50 & 30,474 & 34 & 30,508 & $\begin{array}{ll}1,915 \\
\end{array}$ & 33 & \begin{tabular}{l|l|}
1,948 \\
\end{tabular} & 47 & 0 & 47 & 1 & 17 & 18 & \begin{tabular}{|l|l|}
32,521 \\
\end{tabular} & 53 & 0 & 0 \\
\hline
\end{tabular}
Area of SOG in baseline retail building = 586.0

\section{ATHENA ® EIE Material List:} (Includes all materials after 50 years)

\begin{tabular}{|l|c|c|}
\hline \multicolumn{1}{|c|}{ Material List } & Quantities & Unit \\
\hline 6 mil Polyethylene & 645.4 & $\mathrm{~m} 2$ \\
\hline Concrete 30 MPa (flyash $35 \%)$ & 127.8 & $\mathrm{~m} 3$ \\
\hline Solvent Based Alkyd Paint & 67.3 & $\mathrm{~L}$ \\
\hline Welded Wire Mesh / Ladder Wire & 549.9 & $\mathrm{~kg}$ \\
\hline
\end{tabular}

Notes:
'Initial = Time 'o' (i.e. at the completion of initial construction)

${ }^{2}$ Trans. $=$ Transportation

Total EE (or Total GWP) = Total embodied energy (or total embodied GWP) of building component after lifespan (i.e. total man
construction + total maintenance + total end-offlife effects)

${ }^{4}$ Total EE (or Total GWP per $\mathrm{m}^{2}=$ Total EE (or Total GWP) of building
component/ area of building component that was modelled in ATHENAQ EIE Total Difference in Operating Energy (or GWP) from Baseline after Lifespan = The differerece in the total life-cycle operating energy (or GWP)
from the basaleline retail builing after lifespan, due to using this building
component instead of the baseline component

Total Difference in Operating Energy (or GWP) from Baseline after
Lifespan per $\mathrm{m}^{2}=$ Total difference in operating energy (or GWP) from Lifespan per $\mathrm{m}^{2}=$ Total difference in operating energy (or
baseline after lifespan / baseline retail building slab-on-grade area

* Total operating primary energy use of baseline retail building after 50 years =
$50,700 \mathrm{GJ}\left(1,745 \mathrm{MJ} / \mathrm{m}^{2} \mathrm{yr}\right)$

"Total operating GWP of baseline rets

\section{Concrete Slab-On-Grade \#6 (SOG-FDN6)}

Building Component Description:

\begin{tabular}{|c|c|c|c|}
\hline \multirow{3}{*}{ Category: } & \multicolumn{2}{|l|}{ Foundations } & Assembly Layers \\
\hline & \multirow{3}{*}{\multicolumn{2}{|c|}{\begin{tabular}{|l} 
Concrete slab-on-grade: $200 \mathrm{~mm}$ thick, \\
average flyash content, $50 \mathrm{~mm}$ insulation
\end{tabular}}} & $\begin{array}{l}\text { Concrete Slab-On-Grade } \\
\text { 30MPa concrete strength }\end{array}$ \\
\hline & & & $200 \mathrm{~mm}$ thick concrete slab \\
\hline Brief Description: & & & 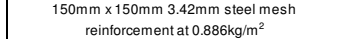 \\
\hline \multicolumn{3}{|l|}{ Quick Numbers: } & $9 \%$ (average) flyash content \\
\hline ASHRAE Standard 90.1: & F.Factor: 0.36 & But/(hr.t.t.F) & 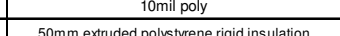 \\
\hline THERM 5.2: & R-Value: 12.0 & RSIVValue: 2.1 & 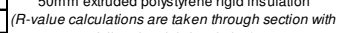 \\
\hline Slab Thickness: & $250 \mathrm{~mm}$ & us compacted sub-base) & full under slab insulation) \\
\hline $\begin{array}{l}\text { Total Embodided Energy: } \\
\text { Total Embodiled GW: }\end{array}$ & $\begin{array}{ll}666 & \mathrm{M} / \mathrm{m} \\
73 & \mathrm{~kg} 01\end{array}$ & $\mathrm{O}_{2} \mathrm{eq} / \mathrm{m}^{2}$ & $\begin{array}{l}\text { Concrete sealant } \\
\text { (modeled as alkyd based paint finish on concretete) }\end{array}$ \\
\hline
\end{tabular}

Life-Cycle Assessment Results:

Primary Energy Consumption (MJ)

\begin{tabular}{|c|c|c|c|c|c|c|c|c|c|c|c|c|c|c|}
\hline \multirow{3}{*}{$\begin{array}{l}\text { L Liespan } \\
\text { (Years) }\end{array}$} & \multicolumn{12}{|c|}{ Embodied Energy (EE) } & \multirow{2}{*}{\multicolumn{2}{|c|}{$\begin{array}{l}\text { Difference in } \\
\text { Operating Energy } \\
\text { from Baseline after } \\
\text { Lifespan }\end{array}$}} \\
\hline & \multicolumn{2}{|c|}{ Manufacturing } & \multicolumn{2}{|c|}{ Construction } & \multicolumn{3}{|c|}{ Mainte } & \multicolumn{3}{|c|}{ End of Life } & \multirow{2}{*}{$\begin{array}{c}{ }^{3} \text { Total } \\
\text { EE }\end{array}$} & \multirow{2}{*}{\begin{tabular}{|c|c}
${ }^{4}$ Total \\
EE \\
per m
\end{tabular}} & & \\
\hline & 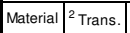 & Total & 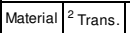 & Total & Material & ${ }^{2}$ Trans. & Total & \begin{tabular}{|l|} 
Material \\
\end{tabular} & \begin{tabular}{|l|}
2 \\
\end{tabular} & Total & & & ${ }^{5}$ Total & ${ }^{6}$ per m ${ }^{2}$ \\
\hline Initial & \begin{tabular}{|l|l|l|l|l|l}
335,542 & 13,30 \\
\end{tabular} & 348,849 & \begin{tabular}{|l|l|}
28,420 & 17,092 \\
\end{tabular} & 45,512 & 0 & 0 & 0 & 0 & 0 & 0 & 394,361 & 646 & & \\
\hline 50 & \begin{tabular}{|l|l|}
335,542 & 13,307 \\
\end{tabular} & 348,849 & \begin{tabular}{|l|l|}
28,420 & 17,092 \\
\end{tabular} & 45,512 & 2,740 & 20 & $\begin{array}{lll}2,760 & \\
\end{array}$ & 15 & $\begin{array}{l}9,143 \\
\end{array}$ & 9,158 & 406,279 & 666 & $.500,01$ & 2,560 \\
\hline
\end{tabular}

Global Warming Potential (kg of $\mathrm{CO}_{2}$ eq.)

\begin{tabular}{|c|c|c|c|c|c|c|c|c|c|c|c|c|c|c|c|c|}
\hline \multirow{3}{*}{$\begin{array}{l}\text { Lifespan } \\
\text { (Years) }\end{array}$} & \multicolumn{14}{|c|}{ Embodied Global Warming Potential (GWP) } & \multirow{2}{*}{\multicolumn{2}{|c|}{$\begin{array}{l}\text { Difference in } \\
\text { Operating GWP from } \\
\text { Baseline after } \\
\text { Lifespan }\end{array}$}} \\
\hline & \multicolumn{3}{|c|}{ Manufacturing } & \multicolumn{3}{|c|}{ Construction } & \multicolumn{3}{|c|}{ Maintenance } & \multicolumn{3}{|c|}{ End of Life } & \multirow{2}{*}{$\mid \begin{array}{l}{ }^{3} \text { Total } \\
\text { GWWP }\end{array}$} & \multirow{2}{*}{$\begin{array}{l}{ }^{4} \text { Total } \\
\text { GWP } \\
\text { per } \mathrm{m}^{2}\end{array}$} & & \\
\hline & Material & ${ }^{2}$ Trans. & Total & Material & Trans. & Total & Material & Trans. & Total & Material & ${ }^{2}$ Trans. & Total & & & ${ }^{5}$ Total & ${ }^{6}$ per m ${ }^{2}$ \\
\hline Initial & 42,563 & 25 & 42,589 & 1,915 & 33 & 1,948 & 0 & 0 & 0 & 0 & 0 & 0 & 44,537 & 73 & & \\
\hline 50 & 42,563 & 25 & 42,589 & 1,915 & 33 & 1,948 & 47 & 0 & 47 & 1 & 18 & 19 & 44,602 & 73 & 90,000 & 154 \\
\hline
\end{tabular}

ilding $=586.0 \mathrm{~m}^{2}$

ATHENA $®$ EIE Material List:

\begin{tabular}{|l|c|c|}
\multicolumn{1}{|c|}{ (Includes all materials after 50 years) } \\
\hline \multicolumn{1}{|c|}{ Material List } & Quantities & Unit \\
\hline 6 mil Polyethylene & 645.4 & $\mathrm{~m} 2$ \\
\hline Concrete $30 \mathrm{MPa}$ (flyash av) & 127.8 & $\mathrm{~m} 3$ \\
\hline Extruded Polystyrene & $1,246.6$ & $\mathrm{~m} 2(25 \mathrm{~mm})$ \\
\hline Nails & 37.6 & $\mathrm{~kg}$ \\
\hline Solvent Based Alkyd Paint & 67.3 & $\mathrm{~L}$ \\
\hline Welded Wire Mesh / Ladder Wire & 549.9 & $\mathrm{~kg}$ \\
\hline
\end{tabular}

Notes:
1 Initial $=$ Time ' 0 ' (i.e. at the completion of initial construction)

${ }^{2}$ Trans. = Transportation

${ }^{3}$ Total EE (or Total GWP) = Total embodied energy (or total embodied GWP) of building component after lifespan (i.e. total manufacturing + tota
construction + total maintenance + total enc-oflifife effects)

${ }^{4}$ Total EE (or Total GWP) per $\mathrm{m}^{2}=$ Total EE (or Total GWP) of building
component/ area of building component that was modelled in ATHENAQ EIE ${ }^{5}$ Total Difference in Operating Energy (or GWP) from Baseline after from the baseline retail building after lifespan, due to using this building component instead of the baseline component

${ }^{6}$ Total Difference in Operating Energy (or GWP) from Baseline after Lifespan per $\mathrm{m}^{2}=$ Total difference in operating energy (or GWP) from

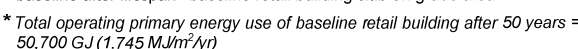

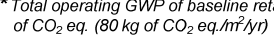




\section{Appendix B-7}

\section{LCA Data for Interior Partitions}




\section{LCA Data for Interior Partition Walls}

This section contains a detailed description of each interior partition wall that was examined in this study ( 9 in total). The assembly layers are listed for each wall, along with a detailed description of the material quantities from the ATHENA ${ }^{\circledR}$ Environmental Impact Estimator for Buildings.

A breakdown of the total primary energy consumption and the total global warming potential (GWP) for each wall is also included. In each case, the results were calculated for an area of partition wall equal to $50.9 \mathrm{~m}^{2}$, which represents a typical bay size for a single-storey retail building. The results are also expressed on a per $\mathrm{m}^{2}$ basis in each case. The data has been calculated for two different lifespans: at the completion of initial construction and after 50 years.

As a summary, the figure below illustrates a comparison of the total embodied energy (and GWP) after 50 years for the various interior partition walls in this section. For comparison purpose, the building component used in the baseline retail building has been shaded in grey.

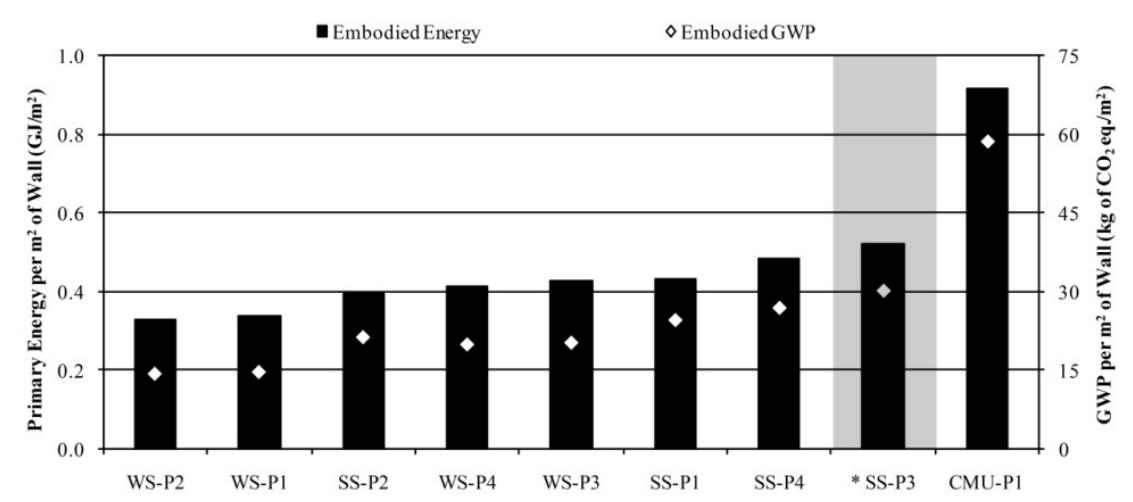

Interior Partition Wall ID (Arranged in Order of Increasing Embodied Energy)

Concrete Masonry Unit Interior Partition Wall \#1 (CMU-P1)

Building Component Description:

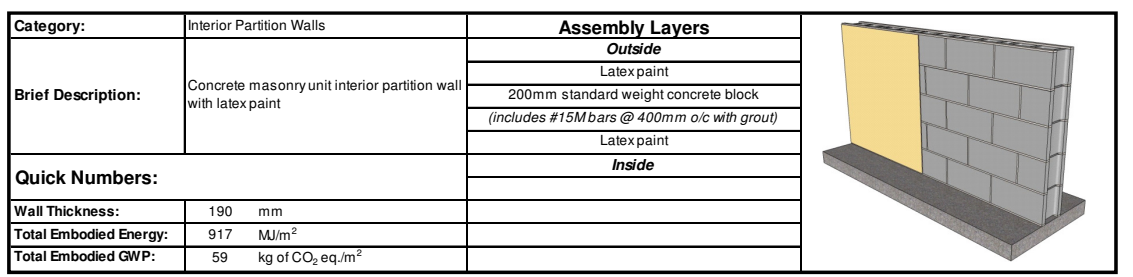

Life-Cycle Assessment Results:

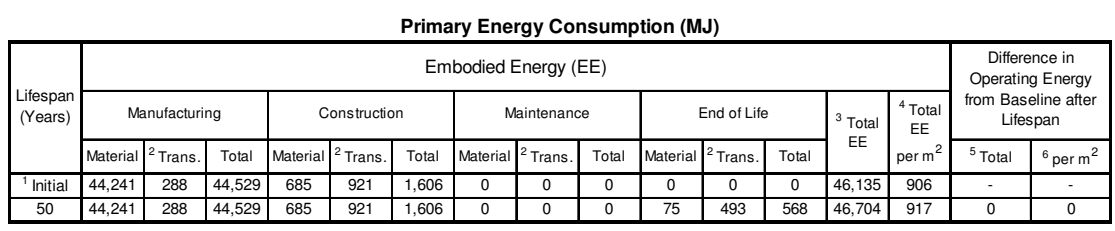

Global Warming Potential (kg of $\mathrm{CO}_{2}$ eq.

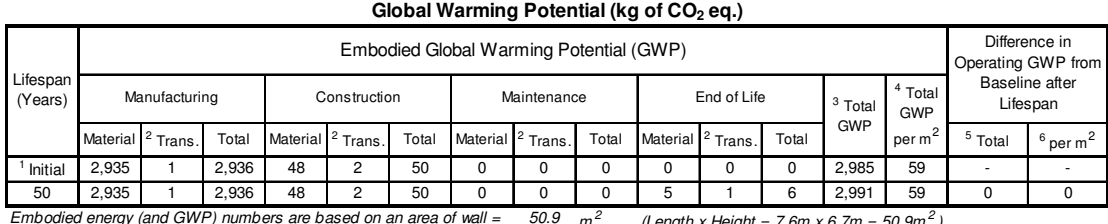

Embodied energy (and GWP) numbers are based on an area of wall $=50.9 \mathrm{~m}^{2} \quad$ (Length $\times$ Height $=7.6 \mathrm{~m} \times 6.7 \mathrm{~m}=50.9 \mathrm{~m}^{2}$ )

ATHENA $®$ EIE Material List:

(Includes all materials after 50 years)

\begin{tabular}{|l|c|c|}
\hline \multicolumn{1}{|c|}{ Material List } & Quantities & Unit \\
\hline Concrete Blocks & 648.0 & Blocks \\
\hline Mortar & 2.1 & $\mathrm{~m} 3$ \\
\hline Rebar, Rod, Light Sections & $1,092.8$ & $\mathrm{~kg}$ \\
\hline Water Based Latex Paint & 132.5 & $\mathrm{~L}$ \\
\hline
\end{tabular}

${ }^{1}$ Initial $=$ Time 'o' (i.e. at the completion of

${ }^{2}$ Trans. $=$ Transportation

${ }^{3}$ Total EE (or Total GWP) $=$ Total embodied energy (or total embodied GWP) of builing component after lifespan (i.e. total manufacturing + total ${ }^{4}$ Total EE (or Total GWP) per $\mathrm{m}^{2}=$ Total EE (or Total GWP) of building Lifespan $=$ The difference in the total life-cycle operating energy (or GWP from the baseline retail building after lifespan, due to using this building

(or GWP) from Baseline after Lifespan per $\mathrm{m}^{2}=$ Total difference in operating energy (or GWP) from
baseline after lifespan /interior partition wall area of baseline retail building * Total operating primary energy use of baseline retail building after 50 years
$50,700 \mathrm{GJ}\left(1.745 \mathrm{MJMm}^{2} \mathrm{Xr}\right)$

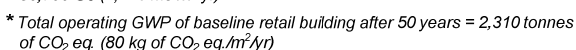




\section{Cold-Formed Steel Stud Interior Partition Wall \#1 (SS-P1)}

Building Component Description:

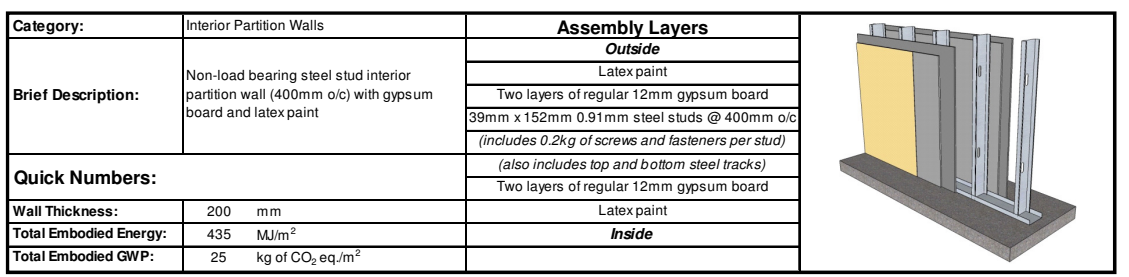

\section{Life-Cycle Assessment Results:}

\begin{tabular}{|c|c|c|c|c|c|c|c|c|c|c|c|c|c|c|c|c|}
\hline & \multirow{2}{*}{\multicolumn{14}{|c|}{ Primary Energy Consumption (MJ) }} & \multirow{3}{*}{\multicolumn{2}{|c|}{$\begin{array}{c}\text { Difference in } \\
\text { Operating Energy } \\
\text { from Baseline after } \\
\text { Lifespan }\end{array}$}} \\
\hline \multirow{3}{*}{$\begin{array}{l}\text { Lifespan } \\
\text { (Years) }\end{array}$} & & & & & & & bodied E & Energy ( & & & & & & & & \\
\hline & \multicolumn{3}{|c|}{ Manufacturing } & \multicolumn{3}{|c|}{ Construction } & \multicolumn{3}{|c|}{ Maintenance } & \multicolumn{3}{|c|}{ End of Life } & \multirow{2}{*}{$\begin{array}{c}{ }^{3} \text { Total } \\
\text { EE }\end{array}$} & \multirow{2}{*}{\begin{tabular}{|c|}
${ }^{4}$ Total \\
EE \\
per m
\end{tabular}} & & \\
\hline & Material & 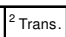 & Total & $\mid$ Material & ${ }^{2}$ Trans. & \begin{tabular}{|l|} 
Total \\
\end{tabular} & \begin{tabular}{|l|l|} 
Material \\
\end{tabular} & 2 Trans. & Total & Material & \begin{tabular}{|l|}
${ }^{2}$ Trans. \\
nat
\end{tabular} & Total & & & ${ }^{5}$ Total & ${ }^{6}$ per m ${ }^{2}$ \\
\hline |nitial & 21,0 & 146 & 21,183 & 460 & 616 & 776 & $\overline{0}$ & 0 & 0 & 0 & $\overline{0}$ & $\pi$ & 21,960 & 431 & & \\
\hline 50 & 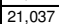 & 146 & 21,183 & 160 & 616 & 776 & 0 & 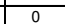 & 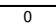 & 0 & 173 & 173 & 22,132 & 435 & 0 & \\
\hline
\end{tabular}

Global Warming Potential ( $\mathrm{kg}$ of $\mathrm{CO}_{2}$ eq.)

\begin{tabular}{|c|c|c|c|c|c|c|c|c|c|c|c|c|c|c|c|c|}
\hline \multirow{3}{*}{$\begin{array}{c}\text { Lifespan } \\
\text { (Years) }\end{array}$} & \multicolumn{14}{|c|}{ Embodied Global Warming Potential (GWP) } & \multirow{2}{*}{\multicolumn{2}{|c|}{$\begin{array}{c}\text { Difference in } \\
\text { Operating GWP from } \\
\text { Baseline after } \\
\text { Lifespan }\end{array}$}} \\
\hline & \multicolumn{3}{|c|}{ Manufacturing } & \multicolumn{3}{|c|}{ Construction } & \multicolumn{3}{|c|}{ Maintenance } & \multicolumn{3}{|c|}{ End of Lite } & \multirow{2}{*}{$\begin{array}{l}{ }^{3} \text { Total } \\
\text { GWWP }\end{array}$} & \multirow{2}{*}{$\begin{array}{l}{ }^{4} \text { Total } \\
\text { GWP } \\
\text { per m }\end{array}$} & & \\
\hline & Material & ${ }^{2}$ Trans. & Total & Material & ${ }^{2}$ Trans. & $\begin{array}{c}\text { Total } \\
\end{array}$ & Material & ${ }^{2}$ Trans. & Total & Material & ${ }^{2}$ Trans. & Total & & & \begin{tabular}{|l|l|}
${ }^{5}$ Total \\
\end{tabular} & ${ }^{6}{ }_{\text {per m}}^{2}$ \\
\hline${ }^{\prime}$ Initial & 1,240 & 0 & 1,241 & 10 & 1 & 12 & 0 & 0 & 0 & 0 & 0 & 0 & 1,252 & 25 & & \\
\hline 50 & 1,240 & 0 & 1,241 & 10 & 1 & 12 & 0 & 0 & 0 & 0 & 0 & 0 & 1,253 & 25 & 0 & 0 \\
\hline
\end{tabular}

\section{ATHENA ${ }^{\circledR}$ EIE Material List:}

\begin{tabular}{|l|c|c|}
\hline \multicolumn{1}{|c|}{ Material List } & Quantities & Unit \\
\hline 13mm Regular Gypsum Board & 224.0 & $\mathrm{~m} 2$ \\
\hline Galvanized Studs & 290.4 & $\mathrm{~kg}$ \\
\hline Joint Compound & 223.6 & $\mathrm{~kg}$ \\
\hline Nails & 2.1 & $\mathrm{~kg}$ \\
\hline Paper Tape & 2.6 & $\mathrm{~kg}$ \\
\hline Screws Nuts \& Bolts & 3.9 & $\mathrm{~kg}$ \\
\hline Water Based Latex Paint & 132.5 & $\mathrm{~L}$ \\
\hline
\end{tabular}

Notes:

${ }^{2}$ Trans. $=$ Transportation

${ }^{3}$ Total EE (or Total GWP) $=$ Total embodied energy (or total embodied GWP) of building component after lifespan (i.e. total manufacturing + tota

${ }^{4}$ Total EE (or Total GWP) per $\mathrm{m}^{2}=$ Total EE (or Total GWP) of building Lifespan $=$ The difference in the total life-cycle operating energy (or GWPP from the baseline retail buildiding after lifespan, due to using this building

${ }^{6}$ Total Difference in Operating Energy (or GWP) from Baseline after Lifespan per $\mathrm{m}^{2}=$ Total difference in operating energy (or GWP) from
baseline after lifespan / interior partition wall area of baseline retail building * Total operating primary energy use of baseline retail building after 50 years
$50,700 \mathrm{G}\left(1.745 \mathrm{MJ}^{2} \mathrm{~m}^{2} \mathrm{Mr}\right)$

* Total operating $\mathrm{GWP}$ of baseline retail building after 50 years $=2,310$ tonnes
of $\mathrm{CO}_{2}$ eq. $\left(80 \mathrm{~kg}\right.$ of $\left.\mathrm{CO}_{2} \mathrm{eq} / \mathrm{m}^{2} \mathrm{Yrr}\right)$

\section{Cold-Formed Steel Stud Interior Partition Wall \#2 (SS-P2)}

Building Component Description:

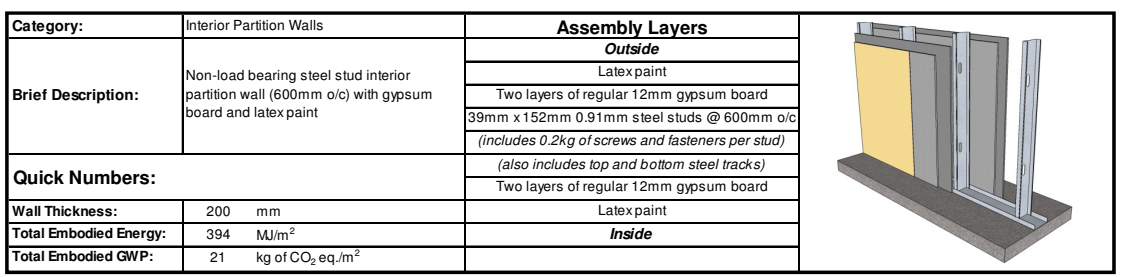

Life-Cycle Assessment Results:

\begin{tabular}{|c|c|c|c|c|c|c|c|c|c|c|c|c|c|c|c|c|}
\hline \multirow{4}{*}{$\begin{array}{l}\text { Lifespan } \\
\text { (Years) }\end{array}$} & \multicolumn{16}{|c|}{ Primary Energy Consumption (MJ) } \\
\hline & \multicolumn{14}{|c|}{ Embodied Energy (EE) } & \multirow{2}{*}{\multicolumn{2}{|c|}{$\begin{array}{l}\text { Difference in } \\
\text { Operating Energy } \\
\text { from Baseline after } \\
\text { Lifespan }\end{array}$}} \\
\hline & \multicolumn{3}{|c|}{ Manutacturing } & \multicolumn{3}{|c|}{ Construction } & \multicolumn{3}{|c|}{ Maintenance } & \multicolumn{3}{|c|}{ End of Life } & \multirow{2}{*}{\begin{tabular}{|c|}
${ }^{3}$ Total \\
$\mathrm{EE}$
\end{tabular}} & \multirow{2}{*}{\begin{tabular}{|c|c|}
${ }^{4}$ Total \\
EE \\
EE
\end{tabular}} & & \\
\hline & Materia & ${ }^{2}$ Trans. & Total & Material & ${ }^{2}$ Trans. & Total & Material & ${ }^{2}$ Trans. & Total & Material & $\begin{array}{l}2 \\
2\end{array}$ Trans. & Total & & & ${ }^{5}$ Total & ${ }^{6}$ per $\mathrm{m}^{2}$ \\
\hline${ }^{1}$ Initial & 18,969 & 143 & 19,111 & 160 & \begin{tabular}{|l|l|}
607 \\
\end{tabular} & 767 & 0 & 0 & 0 & 0 & 0 & 0 & 19,878 & 390 & & \\
\hline 50 & 18,969 & 143 & 19,111 & 160 & 607 & 767 & 0 & 0 & 0 & 0 & 169 & 170 & 20,048 & 394 & 0 & 0 \\
\hline
\end{tabular}

\begin{tabular}{|c|c|c|c|c|c|c|c|}
\hline \multirow{3}{*}{$\begin{array}{l}\text { Lifespar } \\
\text { (Years) }\end{array}$} & \multicolumn{7}{|c|}{ Embodied Global } \\
\hline & \multicolumn{3}{|c|}{ Manufacturing } & \multicolumn{3}{|c|}{ Construction } & \\
\hline & Material & ${ }^{2}$ Trans. & \begin{tabular}{|l|l|l|} 
Total \\
\end{tabular} & Materia & ${ }^{2}$ Trans. & Tota & \\
\hline${ }^{1}$ Initial & 1,074 & 0 & 1,074 & 10 & 1 & 12 & \\
\hline 50 & 1,074 & 0 & 1,074 & 10 & 1 & 12 & \\
\hline \multicolumn{8}{|c|}{$\begin{array}{c}\text { ATHENA @ EIE Material List: } \\
\text { (Includes all materials after 50 years) }\end{array}$} \\
\hline \multicolumn{4}{|c|}{ Material List } & \multicolumn{2}{|c|}{ Quantities } & \multicolumn{2}{|c|}{ Unit } \\
\hline \multicolumn{4}{|c|}{ 13mm Regular Gypsum Board } & & 224.0 & \multicolumn{2}{|c|}{$\mathrm{m} 2$} \\
\hline \multicolumn{4}{|c|}{ Galvanized Studs } & & 205.5 & \multicolumn{2}{|c|}{$\mathrm{kg}$} \\
\hline \multicolumn{4}{|c|}{ Joint Compound } & & 223.6 & \multirow{2}{*}{\multicolumn{2}{|c|}{$\mathrm{kg}$}} \\
\hline \multicolumn{4}{|l|}{ Nails } & & 2.1 & & \\
\hline \multicolumn{4}{|c|}{ Paper Tape } & & 2.6 & \multicolumn{2}{|c|}{$\mathrm{kg}$} \\
\hline \multicolumn{4}{|c|}{ Screws Nuts \& Bolts } & & 2.6 & \multicolumn{2}{|c|}{$\mathrm{kg}$} \\
\hline \multicolumn{4}{|c|}{ Water Based Latex Paint } & & 132.5 & \multicolumn{2}{|c|}{$\mathrm{L}$} \\
\hline
\end{tabular}

Global Warming Potential ( $\mathrm{kg}$ of $\mathrm{CO}_{2}$ eq

mbodied Global Warming Potential (GWP)

Water Based Latex Pain \begin{tabular}{l|l} 
Maintenance & End of Lit
\end{tabular} Easeline atter \begin{tabular}{|l|l|l|l|l|l|l|}
\hline & & & \\
\hline
\end{tabular} $50.9 \mathrm{~m}^{2} \quad$ (Length $\times$ Height $=7.6 \mathrm{~m} \times 6.7 \mathrm{~m}=50.9 \mathrm{~m}^{2}$ ) Notes: ${ }^{2}$ Trans. $=$ Transportation

${ }^{3}$ Total EE (or Total GWP) = Total embodied energy (or total embodied GWP) of building component after lifespan (i.e. total manuffacturing + tota
construction + total maintenance + total enc-oflife effects ${ }^{4}$ Total EE (or Total GWP) per $\mathrm{m}^{2}=$ Total EE (or Total GWP) of building ${ }^{5}$ Total Differce in Operating Energy (or GWP) from Baseline after Lifespan = The difference in the total life-cycle operating energy (or GWP) from the baseline retail building affer lifespan, due to using this building
component instead of the baseline component ${ }^{6}$ Total Difference in Operating Energy (or GWP) from Baseline after Total Difference in Operating Energy (or GWP) from Baseline after
Lifespan per $\mathrm{m}^{2}=$ Total difference in operating energy (or GWPP from
baseline after lifespan / interior partition wall area of baseline retail building * Total operating primary energy use of baseline retail building after 50 years
$50,700 \mathrm{G}\left(1.745 \mathrm{M} / \mathrm{ym} \mathrm{m}^{2} / \mathrm{yr}\right)$

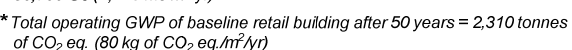


Cold-Formed Steel Stud Interior Partition Wall \#3 (SS-P3) Building Component Description:

\begin{tabular}{|c|c|c|c|}
\hline Category: & Interior Partition Walls & Assembly Layers & Denter \\
\hline \multirow{3}{*}{ Brief Description: } & \multirow{4}{*}{$\begin{array}{l}\text { Non-load bearing steel stud interior } \\
\text { partition wall (400 omm oflc) with gypsum } \\
\text { board, fiberglass batt insulation, and latex } \\
\text { paint }\end{array}$} & $\begin{array}{c}\text { Outside } \\
\text { Latexpaint } \\
\end{array}$ & \\
\hline & & Two layers of regular $12 \mathrm{~mm}$ gypsum board & \\
\hline & & $9 \mathrm{~mm} \times 152 \mathrm{~mm} 0.91 \mathrm{~mm}$ steel studs $@ 400 \mathrm{~mm}$ olc & \\
\hline \multirow{2}{*}{\multicolumn{2}{|c|}{ Quick Numbers: }} & (includes $0.22 \mathrm{~g}$ of screws and fasteners per stud) & \\
\hline & & 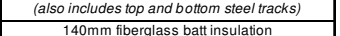 & \\
\hline Wall Thickness: & $200 \mathrm{~mm}$ & Two layers of regular $12 \mathrm{~mm}$ gypsum board & \\
\hline Total Embodied Energy: & $523 \quad \mathrm{M} / \mathrm{m}^{2}$ & Latexpaint & \\
\hline Total Embodied GWP: & $\mathrm{kg}$ of C & Inside & \\
\hline
\end{tabular}

\section{Life-Cycle Assessment Results:}

\begin{tabular}{|c|c|c|c|c|c|c|c|c|c|c|c|c|c|c|c|c|}
\hline & \multirow{2}{*}{\multicolumn{14}{|c|}{ Primary Energy Consumption (MJ) }} & \multirow{3}{*}{\multicolumn{2}{|c|}{$\begin{array}{c}\text { Difference in } \\
\text { Operating Energy } \\
\text { from Baseline after } \\
\text { Lifespan }\end{array}$}} \\
\hline \multirow{3}{*}{$\begin{array}{l}\text { Lifespan } \\
\text { (Years) }\end{array}$} & & & & & & & bodied E & Energy ( & & & & & & & & \\
\hline & \multicolumn{3}{|c|}{ Manufacturing } & \multicolumn{3}{|c|}{ Construction } & \multicolumn{3}{|c|}{ Maintenance } & \multicolumn{3}{|c|}{ End of Life } & \multirow{2}{*}{$\begin{array}{c}{ }^{3} \text { Total } \\
\text { EE }\end{array}$} & \multirow{2}{*}{\begin{tabular}{|c|}
${ }^{4}$ Total \\
EE \\
per m
\end{tabular}} & & \\
\hline & \begin{tabular}{|l|} 
Material \\
\end{tabular} & 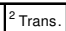 & \begin{tabular}{|l|} 
Total \\
\end{tabular} & \begin{tabular}{|l|} 
Material \\
\end{tabular} & ${ }^{2}$ Trans. & Total & Material & 2 Trans. & Total & \begin{tabular}{|l|} 
Material \\
\end{tabular} & $\mid{ }^{2}$ Trans. & Total & & & ${ }^{5}$ Total & ${ }^{6}$ per m ${ }^{2}$ \\
\hline |nitial & 25,489 & 173 & $\begin{array}{lll}25,662 \\
\end{array}$ & 460 & $\begin{array}{l}634 \\
64\end{array}$ & 794 & $\overline{0}$ & 0 & 0 & 0 & $\overline{0}$ & $\pi$ & 26,456 & 520 & & \\
\hline 50 & \begin{tabular}{|l|}
25,489 \\
\end{tabular} & 173 & 25,662 & 160 & 634 & 794 & 0 & 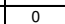 & 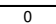 & 0 & 187 & 187 & 26,642 & 523 & & \\
\hline
\end{tabular}

Global Warming Potential ( $\mathrm{kg}$ of $\mathrm{CO}_{2}$ eq.)

\begin{tabular}{|c|c|c|c|c|c|c|c|c|c|c|c|c|c|c|c|c|}
\hline \multirow{3}{*}{$\mid$\begin{tabular}{|c} 
Lifespan \\
(Vears)
\end{tabular}} & \multicolumn{14}{|c|}{ Embodied Global Warming Potential (GWP) } & \multirow{2}{*}{\multicolumn{2}{|c|}{$\begin{array}{c}\text { Difference in } \\
\text { Operating GWP from } \\
\text { Baseline after } \\
\text { Lifespan }\end{array}$}} \\
\hline & \multicolumn{3}{|c|}{ Manutacturing } & \multicolumn{3}{|c|}{ Construction } & \multicolumn{3}{|c|}{ Maintenance } & \multicolumn{3}{|c|}{ End of Life } & \multirow{2}{*}{$\begin{array}{l}{ }^{3} \text { Total } \\
\text { GWP }\end{array}$} & \multirow{2}{*}{$\begin{array}{l}{ }^{4} \text { Total } \\
\text { GWP } \\
\text { per m }\end{array}$} & & \\
\hline & Material & ${ }^{2}$ Trans. & 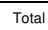 & Material & ${ }^{2}$ Trans. & Total & Material & ${ }^{2}$ Trans. & Total & Material & ${ }^{2}$ Trans. & Total & & & \begin{tabular}{|c|c|c|}
${ }^{5}$ Total \\
\end{tabular} & ${ }_{6}^{6} \mathrm{perm}^{2}$ \\
\hline \begin{tabular}{|l|l|}
${ }^{\prime}$ Initial \\
\end{tabular} & 1,526 & 0 & 1,526 & 10 & 1 & 12 & 0 & 0 & 0 & 0 & 0 & 0 & 1,538 & 30 & & \\
\hline 50 & 1,526 & 0 & 1,526 & 10 & 1 & 12 & 0 & 0 & 0 & 0 & 0 & 0 & 1,538 & 30 & 0 & 0 \\
\hline
\end{tabular}

\section{ATHENA ${ }^{\circledR}$ EIE Material List:}

(Includes all materials after 50 years)

\begin{tabular}{|l|c|c|}
\hline \multicolumn{1}{|c|}{ Material List } & Quantities & Unit \\
\hline 13mm Regular Gypsum Board & 224.0 & $\mathrm{~m} 2$ \\
\hline Batt. Fiberglass & 289.8 & $\mathrm{~m} 2(25 \mathrm{~mm})$ \\
\hline Gaavanized Studs & 290.4 & $\mathrm{~kg}$ \\
\hline Joint Compound & 223.6 & $\mathrm{~kg}$ \\
\hline Nails & 5.2 & $\mathrm{~kg}$ \\
\hline Paper Tape & 2.6 & $\mathrm{~kg}$ \\
\hline Screws Nuts \& Bolts & 3.9 & $\mathrm{~kg}$ \\
\hline Water Based Latex Paint & 132.5 & $\mathrm{~L}$ \\
\hline
\end{tabular}

Notes:

'Initial $=$ Time ' 0 ' (i.e. at the

${ }^{3}$ Total EE (or Total GWP) $=$ Total embodied energy (or total embodied GWP) of building component after lifespan (i.e. total manufacturing + tota

${ }^{4}$ Total EE (or Total GWP) per $\mathrm{m}^{2}=$ Total EE (or Total GWP) of building

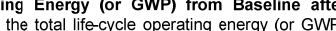
trom the baseline retail building after lifespan, due to using this building

${ }^{6}$ Total Difference in Operating Energy (or GWP) from Baseline after Lifespan per $m^{2}=$ Total difference in operating energy (or GWP) from
baseline after lifespan / interior partition wall area of baseline retail building * Total operating primary energy use of baseline retail building after 50 years
$50,700 \mathrm{GJ}\left(1,745 \mathrm{MJ}^{2} \mathrm{~m}^{2} \mathrm{yr}\right)$ * Total operating GWP of baseline

\section{Cold-Formed Steel Stud Interior Partition Wall \#4 (SS-P4)}

Building Component Description:

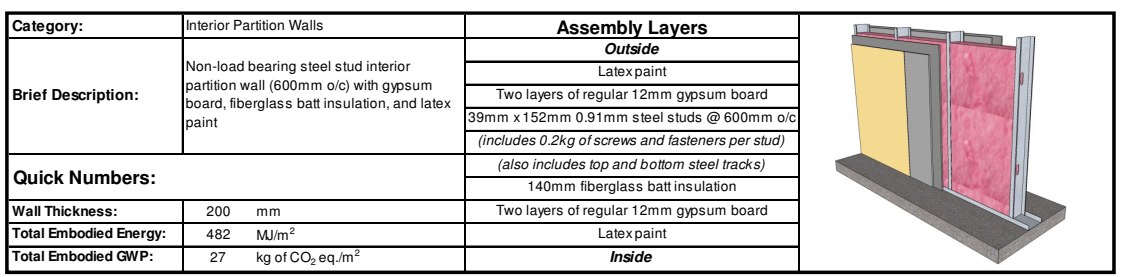

Life-Cycle Assessment Results:

\begin{tabular}{|c|c|c|c|c|c|c|c|c|c|c|c|c|c|c|c|c|}
\hline \multirow{4}{*}{$\begin{array}{l}\text { Lespanan } \\
\text { (Years) }\end{array}$} & \multicolumn{14}{|c|}{ Primary Energy Consumption (MJ) } & \multirow{3}{*}{\multicolumn{2}{|c|}{$\begin{array}{c}\text { Difference in } \\
\text { Operating Energy } \\
\text { from Baseline after } \\
\text { Lifespan }\end{array}$}} \\
\hline & \multicolumn{14}{|c|}{ Embodied Energy (EE) } & & \\
\hline & \multicolumn{3}{|c|}{ Manutacturing } & \multicolumn{3}{|c|}{ Construction } & \multicolumn{3}{|c|}{ Maintenance } & \multicolumn{3}{|c|}{ End of Lifie } & \multirow{2}{*}{$\begin{array}{c}{ }^{3} \text { Total } \\
\mathrm{EE}\end{array}$} & \multirow{2}{*}{$\begin{array}{c}{ }^{4} \text { Total } \\
\mathrm{EE} \\
\text { Der } \mathrm{m}^{2}\end{array}$} & & \\
\hline & Material & 1 ${ }^{2}$ Trans & Total & Material & ${ }^{2}$ Trans. & Total & Material & ${ }^{2}$ Trans & Total & Material & ${ }^{2}$ Trans. & Total & & & ${ }^{5}$ Total & ${ }^{6}{ }_{\text {perm }}^{2}$ \\
\hline |litial & 23,420 & 170 & 23,590 & 160 & 624 & 784 & n & 0 & 0 & 0 & 0 & 0 & 24,374 & 479 & & \\
\hline 50 & 420 & 1770 & & 100 & 624 & 784 & 0 & 0 & 0 & 0 & 183 & 183 & & & & \\
\hline
\end{tabular}

\begin{tabular}{|c|c|c|c|c|c|c|c|}
\hline \multirow{3}{*}{$\begin{array}{l}\text { Lifiespar } \\
\text { (Years) }\end{array}$} & \multicolumn{7}{|c|}{ Embodied Global V } \\
\hline & \multicolumn{3}{|c|}{ Manutacturing } & \multicolumn{3}{|c|}{ Construction } & \\
\hline & Material & ${ }^{2}$ Trans. & Total & Materia & ${ }^{2}$ Trans. & Tot: & \\
\hline \multirow{2}{*}{$\frac{{ }^{\prime} \text { Initial }}{50}$} & $\begin{array}{ll}1,359 \\
\end{array}$ & 0 & $\begin{array}{ll}, 359 \\
, 250\end{array}$ & 10 & 1 & $\frac{12}{12}$ & \\
\hline & 1,359 & 0 & 1,359 & 10 & 1 & 12 & \\
\hline \multicolumn{8}{|c|}{$\begin{array}{c}\text { ATHENA } @ \text { EIE Material List: } \\
\text { (Includes all materials after } 50 \text { years) }\end{array}$} \\
\hline \multicolumn{4}{|c|}{ Material List } & \multicolumn{2}{|c|}{ Quantities } & \multicolumn{2}{|c|}{ Unit } \\
\hline \multicolumn{4}{|c|}{ 13mm Regular Gypsum Board } & \multicolumn{2}{|r|}{224.0} & \multicolumn{2}{|c|}{$\mathrm{m} 2$} \\
\hline \multicolumn{4}{|c|}{ Batt. Fiberglass } & \multicolumn{2}{|r|}{289.8} & \multicolumn{2}{|c|}{ m2 (25mm) } \\
\hline \multirow{2}{*}{\multicolumn{4}{|c|}{ Galvanized Studs }} & \multicolumn{2}{|r|}{205.5} & \multicolumn{2}{|c|}{$\mathrm{kg}$} \\
\hline \multirow{2}{*}{\multicolumn{4}{|c|}{\begin{tabular}{|l|} 
Joint Compound \\
\end{tabular}}} & \multirow{2}{*}{\multicolumn{2}{|c|}{$\begin{array}{c}223.6 \\
5.2\end{array}$}} & \multicolumn{2}{|c|}{$\mathrm{kg}$} \\
\hline & & & & & & \multirow{2}{*}{\multicolumn{2}{|c|}{$\frac{\mathrm{kg}}{\mathrm{kg}}$}} \\
\hline \multicolumn{4}{|c|}{ Paper Tape } & & 2.6 & & \\
\hline \multicolumn{4}{|c|}{ Screws Nuts \& Bolts } & & 2.6 & \multicolumn{2}{|r|}{$\mathrm{kg}$} \\
\hline \multicolumn{4}{|c|}{ Water Based Latex Paint } & & 132.5 & \multicolumn{2}{|c|}{$\mathrm{L}$} \\
\hline
\end{tabular}

Global Warming Potential $\left(\mathrm{kg}_{\mathrm{f}} \mathrm{CO}_{2}\right.$ eq $)$

mbodied Global Warming Potential (GWP)

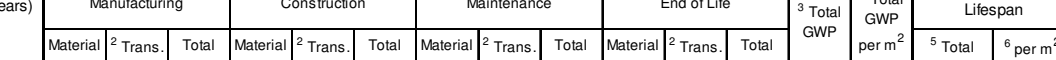
\begin{tabular}{|c|c|c|c|c|c|c|c|c|c|c|}
\hline 12 & 0 & 0 & 0 & 0 & 0 & 0 & 1,371 & 27 & - & - \\
\hline
\end{tabular} (Length $x$ Height $=7.6 \mathrm{~m} \times 6.7 \mathrm{~m}=50.9 \mathrm{~m}^{2}$ ) Notes: ${ }^{1}$ Initial $=$ Trime $0^{\circ}($ i.e. at the $=$ Transportation

${ }^{3}$ Total EE (or Total GWP) $=$ Total embodied energy (or total embodied GWP

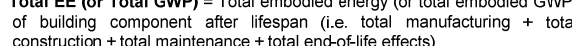

${ }^{4}$ Total EE (or Total GWP) per $\mathrm{m}^{2}=$ Total EE (or Total GWP) of building
component/area of building component that was modelled in ATHENAQ EIE ${ }^{5}$ Total Difference in Operating Energy (or GWP) from Baseline after Total Difference in Operating Energy (or GWP) from Baseline after
Lifespan = The difference in the total life-cycle operating energy (or GWP)
from the baseline retail butiling atter lifessan, due to using this building
component instead of the baseline component ${ }^{6}$ Total Difference in Operating Energy (or GWP) from Baseline after Lotal Difference in Operating Energy (or GWP) from Baseline after
Lifespan per $\mathrm{m}^{2}=$ Total difference in operatiti energy (or GWP) from
baseline after lifespan / interiof partition wall area of baseline retail building baseline after lifespan / interior partition wall area of baseline retail building
* Total operating primary energy use of baseline retail building after 50 years

* Total operating GWP of baseline retail building after 50 years $=2,310$ tonnes 


\section{Wood Stud Interior Partition Wall \#1 (WS-P1)}

Building Component Description:

\begin{tabular}{|c|c|c|}
\hline Category: & Interior Partition Walls & Assembly Layers \\
\hline & & $\begin{array}{l}\text { Outside } \\
\text { Latexpaint } \\
\end{array}$ \\
\hline Brief Description: & $\begin{array}{l}\text { Nonolload bearangin wood stud interiror } \\
\text { partition wall (4000mm o/c) with yypsum }\end{array}$ & Two layers of fregular $12 \mathrm{~mm}$ gypsum board \\
\hline & & $38 \mathrm{~mm} \times 140 \mathrm{~mm}$ wood studs $@ 400 \mathrm{~mm}$ o/c \\
\hline & & 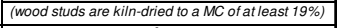 \\
\hline Quick Numbers: & & 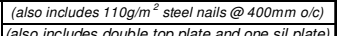 \\
\hline Wall Thickness: & $188 \mathrm{~mm}$ & Two layers of regular $12 \mathrm{~mm}$ gypsum board \\
\hline Total Embodied Energy: & $340 \quad \mathrm{~m} / \mathrm{m}^{2}$ & Latexpaint \\
\hline
\end{tabular}

\section{Life-Cycle Assessment Results:}

\begin{tabular}{|c|c|c|c|c|c|c|c|c|c|c|c|c|c|c|c|c|}
\hline & \multirow{2}{*}{\multicolumn{14}{|c|}{ Primary Energy Consumption (MJ) }} & \multirow{3}{*}{\multicolumn{2}{|c|}{$\begin{array}{c}\text { Difference in } \\
\text { Operating Energy } \\
\text { from Baseline after } \\
\text { Lifespan }\end{array}$}} \\
\hline \multirow{3}{*}{$\begin{array}{l}\text { Lifespan } \\
\text { (Years) }\end{array}$} & & & & & & & bodied E & Energy ( & & & & & & & & \\
\hline & \multicolumn{3}{|c|}{ Manufacturing } & \multicolumn{3}{|c|}{ Construction } & \multicolumn{3}{|c|}{ Maintenance } & \multicolumn{3}{|c|}{ End of Life } & \multirow{2}{*}{$\begin{array}{c}{ }^{3} \text { Total } \\
\text { EE }\end{array}$} & \multirow{2}{*}{\begin{tabular}{|c|}
${ }^{4}$ Total \\
EE \\
per m
\end{tabular}} & & \\
\hline & Material & 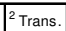 & \begin{tabular}{|l|} 
Total \\
\end{tabular} & \begin{tabular}{|l|} 
Material \\
\end{tabular} & ${ }^{2}$ Trans. & Total & Material & 2 Trans. & Total & \begin{tabular}{|l|} 
Material \\
\end{tabular} & ||$^{2}$ Trans. & Total & & & ${ }^{5}$ Total & ${ }^{6} \mathrm{perm} \mathrm{m}^{2}$ \\
\hline |nitial & $\mid$ & 222 & 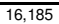 & 209 & 730 & 939 & $\overline{0}$ & 0 & 0 & 0 & $\overline{0}$ & $\pi$ & 17,125 & 336 & & \\
\hline 50 & 15,964 & 222 & $\mid 16,185$ & 209 & 730 & 939 & 0 & 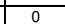 & 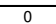 & 0 & 177 & 177 & 17,301 & 340 & & \\
\hline
\end{tabular}

Global Warming Potential ( $\mathrm{kg}$ of $\mathrm{CO}_{2}$ eq.)

\begin{tabular}{|c|c|c|c|c|c|c|c|c|c|c|c|c|c|c|c|c|}
\hline \multirow{3}{*}{$\begin{array}{c}\text { Lifespan } \\
\text { (Years) }\end{array}$} & \multicolumn{14}{|c|}{ Embodied Global Warming Potential (GWP) } & \multirow{2}{*}{\multicolumn{2}{|c|}{$\begin{array}{c}\text { Difference in } \\
\text { Operating GWP from } \\
\text { Baseline after } \\
\text { Lifespan }\end{array}$}} \\
\hline & \multicolumn{3}{|c|}{ Manufacturing } & \multicolumn{3}{|c|}{ Construction } & \multicolumn{3}{|c|}{ Maintenance } & \multicolumn{3}{|c|}{ End of Life } & \multirow{2}{*}{$\begin{array}{l}{ }^{3} \text { Total } \\
\text { GWP }\end{array}$} & \multirow{2}{*}{$\begin{array}{l}{ }^{4}{ }^{\text {Total }} \\
\text { GWP } \\
\text { per m }\end{array}$} & & \\
\hline & \begin{tabular}{|l|l} 
Material \\
\end{tabular} & ${ }^{2}$ Trans. & Total & Material & ${ }^{2}$ Trans. & Total & Material & ${ }^{2}$ Trans. & Total & Material & ${ }^{2}$ Trans. & Total & & & ${ }^{5}$ Total & ${ }^{6}$ perm m$^{2}$ \\
\hline Initial & \begin{tabular}{|l|l|l}
732 \\
\end{tabular} & 0 & 732 & 13 & 1 & 15 & 0 & 0 & 0 & 0 & 0 & 0 & 747 & 15 & & \\
\hline 50 & 732 & 0 & 732 & 13 & 1 & 15 & 0 & 0 & 0 & 0 & 0 & 0 & 747 & 15 & 0 & 0 \\
\hline
\end{tabular}

\section{ATHENA ${ }^{\circledR}$ EIE Material List:}

\begin{tabular}{|l|c|c|}
\hline \multicolumn{1}{|c|}{ Material List } & Quantities & Unit \\
\hline 13mm Regular Gypsum Board & 224.0 & $\mathrm{~m} 2$ \\
\hline Joint Compound & 223.6 & $\mathrm{~kg}$ \\
\hline Nails & 6.9 & $\mathrm{~kg}$ \\
\hline Paper Tape & 2.6 & $\mathrm{~kg}$ \\
\hline $\begin{array}{l}\text { Small Dimension Sottwood Lumber, } \\
\text { kiln-dried }\end{array}$ & 0.9 & $\mathrm{~m} 3$ \\
\hline Water Based Latex Paint & 132.5 & $\mathrm{~L}$ \\
\hline
\end{tabular}

Notes:
${ }^{1}$ Initial $=$ Time '

${ }^{2}$ Trans. $=$ Transportation

${ }^{3}$ Total EE (or Total GWP) $=$ Total embodied energy (or total embodied GWP) of building component after lifespan (i.e. total manufacturing + tota

${ }^{4}$ Total EE (or Total GWP) per $\mathrm{m}^{2}=$ Total EE (or Total GWP) of building Lifespan $=$ The difference in the total life-cycle operating energy (or GWP from the baseline retail building after lifespan, due to using this building

Lifespan per $\mathrm{m}^{2}=$ in Operating Energy (or GWP) from Baseline after Lifespan per $\mathrm{m}^{2}=$ Total difference in operating energy (or GWP) from
baseline after lifiespan /interior partition wall area of baseline retail building * Total operating primary energy use of baseline retail building after 50 years
$50,700 \mathrm{G} J\left(1,745 \mathrm{MJ} / \mathrm{m}^{2} \mathrm{YMr}\right)$

$*$
$*$
Wood Stud Interior Partition Wall \#2 (WS-P2)

Building Component Description:

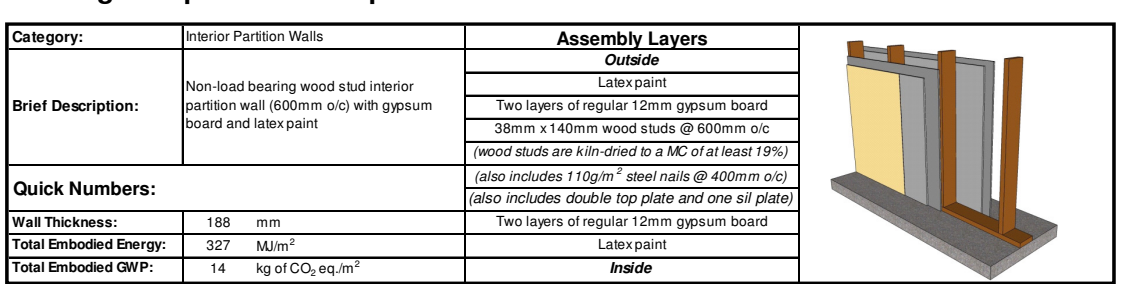

Life-Cycle Assessment Results:

\begin{tabular}{|c|c|c|c|c|c|c|c|c|c|c|c|c|c|c|c|c|}
\hline \multirow{4}{*}{$\begin{array}{l}\text { Lifespan } \\
\text { (Years) }\end{array}$} & \multicolumn{16}{|c|}{ Prima } \\
\hline & \multicolumn{14}{|c|}{ Embodied Energy (EE) } & \multirow{2}{*}{\multicolumn{2}{|c|}{$\begin{array}{l}\text { Difference in } \\
\text { Operating Energy } \\
\text { from Baseline after } \\
\text { Lifespan }\end{array}$}} \\
\hline & \multicolumn{3}{|c|}{ Manutacturing } & \multicolumn{3}{|c|}{ Construction } & \multicolumn{3}{|c|}{ Maintenance } & \multicolumn{3}{|c|}{ End of Life } & \multirow{2}{*}{$\mid \begin{array}{c}{ }^{3} \text { Total } \\
\mathrm{EE}\end{array}$} & \multirow{2}{*}{\begin{tabular}{|c|}
${ }^{4}$ Total \\
EE \\
per m
\end{tabular}} & & \\
\hline & Material & ${ }^{2}$ Trans. & Total & Material & ${ }^{2}$ Trans. & Total & Material & $\left.\right|^{2}{ }^{2}$ Trans. & Total & Material & ${ }^{2}$ Trans. & Total & & & ${ }^{5}$ Total & ${ }^{6}$ per m ${ }^{2}$ \\
\hline Initial & 15,404 & 197 & 15,602 & 209 & \begin{tabular}{|l|}
689 \\
\end{tabular} & 899 & 0 & T & 0 & 0 & 0 & 0 & 16,501 & 324 & & \\
\hline 50 & 15,404 & 197 & 15,602 & 209 & 689 & 899 & 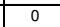 & 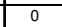 & 0 & 0 & 172 & 173 & 16,673 & 327 & 0 & \\
\hline
\end{tabular}

\begin{tabular}{|c|c|c|c|c|c|c|c|}
\hline \multirow{3}{*}{$\mid \begin{array}{l}\text { Lifespar } \\
\text { (Years) }\end{array}$} & \multicolumn{7}{|c|}{ Embodied Global W } \\
\hline & \multicolumn{3}{|c|}{ Manufacturing } & \multicolumn{3}{|c|}{ Construction } & \\
\hline & \begin{tabular}{|l|} 
Material \\
\end{tabular} & \begin{tabular}{|l|}
${ }^{2}$ Trans. \\
\end{tabular} & \begin{tabular}{|l|l|l|} 
Total & 1 \\
\end{tabular} & Material & ${ }^{2}$ Trans. & Total & \\
\hline$\frac{1}{1}$ Initial & 714 & 0 & 715 & 13 & 1 & 15 & \\
\hline 50 & 714 & & 715 & 13 & 1 & 15 & \\
\hline \multicolumn{8}{|c|}{$\begin{array}{c}\text { ATHENA @ EIE Material List: } \\
\text { (lncludes all materials after } 50 \text { years) }\end{array}$} \\
\hline \multicolumn{4}{|c|}{ Material List } & \multicolumn{2}{|c|}{ Quantities } & \multicolumn{2}{|c|}{ Unit } \\
\hline \multicolumn{4}{|c|}{ 13mm Regular Gypsum Board } & & 224.0 & $\mathrm{~m} 2$ & \\
\hline \multirow{2}{*}{\multicolumn{4}{|c|}{ Joint Cor }} & & 223.6 & $\mathrm{~kg}$ & \\
\hline & & & & & 5.3 & $\mathrm{~kg}$ & \\
\hline \multicolumn{4}{|c|}{ Paper Tape } & & 2.6 & $\mathrm{~kg}$ & \\
\hline \multicolumn{4}{|c|}{$\begin{array}{l}\text { Small Dimension Sottwo } \\
\text { kiln-dried }\end{array}$} & & 0.6 & $\mathrm{~ms}$ & \\
\hline \multicolumn{4}{|c|}{ Water Based Latex Paint } & & 132.5 & $\mathrm{~L}$ & \\
\hline
\end{tabular}

Global Warming Potential ( $\mathrm{kg}$ of $\mathrm{CO}_{2}$ eq $)$

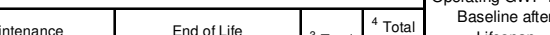
Notes: ${ }^{2}$ Trans. = Transportation

${ }^{3}$ Total EE (or Total GWP) $=$ Total embodied energy (or total embodied GWP of building component affer lifespan (i.e. total manufacturing + tota
construction + total maintenance+t total enc-oflife effects) ${ }^{4}$ Total EE (or Total GWP) per $\mathrm{m}^{2}=$ Total EE (or Total GWP) of building 5 TOtat Diffrence in Operating Energy (or GWP) from Baseline after Total Difference in Operating Energy (or GWP) from Baseline after
Lifespan = The difference in the total life-cycle operating energy (or GWP)
from the baseline retail bubiling atfer lifespan, due to using this building
component instead of the baseline component ${ }^{6}$ Total Difference in Operating Energy (or GWP) from Baseline after Lifiespan per $\mathrm{m}^{2}=$ Total difference in operating energy (or GWPP from * Total operating primary energy use of baseline retail building after 50 years
$50,700 \mathrm{G}\left(1.745 \mathrm{M} / \mathrm{Mm} \mathrm{m}^{2} / \mathrm{yr}\right)$

* Total operating $G W P$ of baseline retail building after 50 years $=2,310$ tonnes 


\section{Wood Stud Interior Partition Wall \#3 (WS-P3)}

Building Component Description:

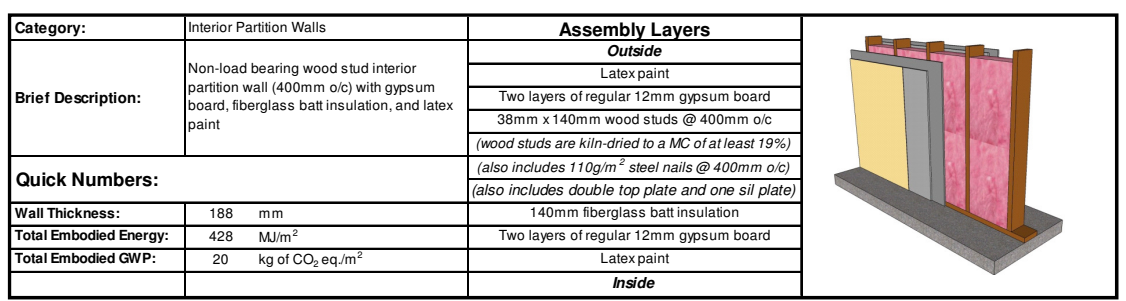

Life-Cycle Assessment Results:

\begin{tabular}{|c|c|c|c|c|c|c|c|c|c|c|c|c|c|c|c|c|}
\hline \multirow{4}{*}{$\begin{array}{l}\text { Lifespan } \\
\text { (Years) }\end{array}$} & \multicolumn{16}{|c|}{ Primary Energy Consumption (MJ) } \\
\hline & \multicolumn{14}{|c|}{ Embodied Energy (EE) } & \multirow{2}{*}{\multicolumn{2}{|c|}{$\begin{array}{l}\text { Difference in } \\
\text { Operating Energy } \\
\text { from Baseline after } \\
\text { Lifespan }\end{array}$}} \\
\hline & \multicolumn{3}{|c|}{ Manufacturing } & \multicolumn{3}{|c|}{ Construction } & \multicolumn{3}{|c|}{ Maintenance } & \multicolumn{3}{|c|}{ End of Life } & \multirow{2}{*}{$\begin{array}{l}{ }^{3} \text { Total } \\
\mathrm{EE}\end{array}$} & \multirow{2}{*}{\begin{tabular}{|l|}
${ }^{4}$ Total \\
EE \\
per m
\end{tabular}} & & \\
\hline & Material & ${ }^{2}$ Trans. & Total & Material & $\left.\right|^{2}$ Trans. & Total & |Material & ${ }^{2}$ Trans. & Total & Material & ${ }^{2}$ Trans. & Total & & & ${ }^{5}$ Total & ${ }^{6}$ per m ${ }^{2}$ \\
\hline${ }^{1}$ Initial & 20,416 & 248 & 20,664 & 209 & 747 & 957 & 0 & 0 & 0 & 0 & 0 & 0 & 21,620 & 425 & & \\
\hline 50 & 20,416 & 248 & 20,664 & 209 & 747 & 957 & 0 & 0 & 0 & 0 & 191 & 191 & 21,811 & 428 & 0 & 0 \\
\hline
\end{tabular}

Global Warming Potential $\left(\mathrm{kg}\right.$ of $\mathrm{CO}_{2}$ eq.)

$\stackrel{\omega}{\not}$

\begin{tabular}{|c|c|c|c|c|c|c|c|c|c|c|c|c|c|c|c|c|}
\hline \multirow{3}{*}{$\begin{array}{l}\text { Lifespan } \\
\text { (Years) }\end{array}$} & \multicolumn{14}{|c|}{ Embodied Global Warming Potential (GWP) } & \multirow{2}{*}{\multicolumn{2}{|c|}{$\begin{array}{l}\text { Difference in } \\
\text { Operating GWP from } \\
\text { Baseline atter } \\
\text { Lifespan }\end{array}$}} \\
\hline & \multicolumn{3}{|c|}{ Manutacturing } & \multicolumn{3}{|c|}{ Construction } & \multicolumn{3}{|c|}{ Maintenance } & \multicolumn{3}{|c|}{ End of Life } & \multirow{2}{*}{$\begin{array}{l}{ }^{3} \text { Total } \\
\text { GWP }\end{array}$} & \multirow{2}{*}{$\begin{array}{c}{ }^{4} \text { Total } \\
G \mathrm{GWP} \\
\text { per m }\end{array}$} & & \\
\hline & \begin{tabular}{|l|l|l|l|} 
Material \\
\end{tabular} & $\left.1\right|^{2}$ Trans. & Total & Material & ${ }^{2}$ Trans. & 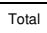 & Material & ${ }^{2}$ Trans. & Total & Material & ${ }^{2}$ Trans. & Total & & & ${ }^{5}$ Total & ${ }^{6} \operatorname{perm}^{2}$ \\
\hline Initial & & 0 & & 13 & & 10 & 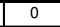 & 0 & 0 & 0 & 0 & 0 & 1,032 & 20 & & - \\
\hline 50 & 1,017 & 0 & 1,017 & 13 & 1 & 15 & 0 & 0 & 0 & 0 & 0 & 0 & 1,033 & 20 & 0 & 0 \\
\hline
\end{tabular}

\section{ATHENA $®$ EIE Material List:}

(Includes all materials after 50 years)

\begin{tabular}{|l|c|c|}
\hline \multicolumn{1}{|c|}{ Material List } & Quantities & Unit \\
\hline 13mm Regular Gypsum Board & 224.0 & $\mathrm{~m} 2$ \\
\hline Batt. Fiberglass & 289.8 & $\mathrm{~m} 2(25 \mathrm{~mm})$ \\
\hline Joint Compound & 223.6 & $\mathrm{~kg}$ \\
\hline Nails & 10.1 & $\mathrm{~kg}$ \\
\hline Paper Tape & 2.6 & $\mathrm{~kg}$ \\
\hline $\begin{array}{l}\text { Small Dimension Sottwood Lumber, } \\
\text { kill-dried }\end{array}$ & 0.9 & $\mathrm{~m} 3$ \\
\hline Water Based Latex Paint & 132.5 & $\mathrm{~L}$ \\
\hline
\end{tabular}

Notes:
${ }^{1}$ Initial $=$ Time ' $O$ ' (i.e. at the completion of initial construction)

${ }^{2}$ Trans. $=$ Transportation

${ }^{3}$ Total EE (or Total GWP) $=$ Total embodied energy (or total embodied GWP) of building component after lifespan (i.e. total manufacturing + tot

${ }^{4}$ Total EE (or Total GWP) per $\mathrm{m}^{2}=$ Total EE (or Total GWP) of building ${ }^{5}$ Total Difference in Operating Energy (or GWP) from Baseline after Lifespan = The difference in the total life-cycle operating energy (or GWP)
from the baseline retail building after lifespan, due to using this building from the baseline retail building after lifesp
component instead of the baseline component

${ }^{6}$ Total Difference in Operating Energy (or GWP) from Bar Lifespan per $\mathrm{m}^{2}=$ Total difference in operating energy (or GWP) from baseline atter If ifespan/interior partition Wall area of baseline retail building * Total operating primary energy use of baseline retail building after 50 years
$50,700 \mathrm{G}\left(1,745 \mathrm{MJ} / \mathrm{m}^{2} \mathrm{Mr}\right)$

* Total operating GWP of baseline retail building after 50 years $=2,310$ tonnes
of $\mathrm{CO}_{2}$ eq. $180 \mathrm{~kg}$ of $\mathrm{CO}_{2}$ eq. $/ \mathrm{m}^{2} \mathrm{Yrl}$ )

Wood Stud Interior Partition Wall \#4 (WS-P4)

Building Component Description:

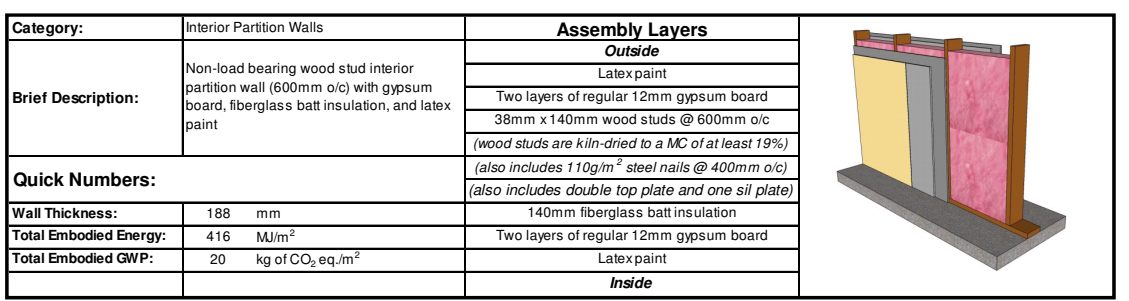

Life-Cycle Assessment Results:

\begin{tabular}{|c|c|c|c|c|c|c|c|c|c|c|c|c|c|c|c|c|}
\hline \multirow{4}{*}{ (Years) } & \multicolumn{14}{|c|}{ Primary Energy Consumption (MJ) } & \multirow{3}{*}{\multicolumn{2}{|c|}{$\begin{array}{c}\text { Difference in } \\
\text { Operating Energy } \\
\text { from Baseline afte } \\
\text { Lifespan }\end{array}$}} \\
\hline & & & & & & & ibodied & Energy ( & & & & & & & & \\
\hline & \multicolumn{3}{|c|}{ Manutacturing } & \multicolumn{3}{|c|}{ Construction } & \multicolumn{3}{|c|}{ Maintenance } & \multicolumn{3}{|c|}{ End of Life } & \multirow{2}{*}{${ }^{3}$ Total } & \multirow{2}{*}{$\begin{array}{c}{ }^{4} \text { Total } \\
\text { EE } \\
\text { per } m^{2}\end{array}$} & & \\
\hline & Material & ${ }^{2}$ Trans & Total & Material & ${ }^{2}$ Trans. & Total & Material & ${ }^{2}$ Trans. & Total & \begin{tabular}{|l|} 
Material \\
\end{tabular} & 2 Trans. & Total & & & ${ }^{5}$ Total & per m \\
\hline \begin{tabular}{|l} 
nnitial \\
\end{tabular} & 19,856 & 224 & 20,081 & 209 & 706 & & 0 & 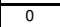 & 0 & 0 & 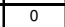 & 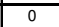 & 20,996 & 112 & & \\
\hline & 8.856 & 224 & \begin{tabular}{|l|l|}
20,081 \\
\end{tabular} & 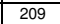 & 706 & 916 & & 0 & 0 & & 186 & 878 & 21,183 & 416 & & \\
\hline
\end{tabular}

\section{Global Warming Potential $\left(\mathrm{kg}_{\mathrm{g}}\right.$ of $\left.\mathrm{CO}_{2} \mathrm{eq}\right)$}

\begin{tabular}{|c|c|c|c|c|c|c|c|c|c|c|c|c|c|c|c|c|}
\hline \multirow{3}{*}{\begin{tabular}{|c} 
Lifespan \\
(Years)
\end{tabular}} & \multicolumn{14}{|c|}{ Embodied Global Warming Potential (GWP) } & \multirow{2}{*}{\multicolumn{2}{|c|}{$\begin{array}{c}\text { Difference in } \\
\text { Operating GWP from } \\
\text { Baseline after } \\
\text { Lifespan }\end{array}$}} \\
\hline & \multicolumn{3}{|c|}{ Manutacturing } & \multicolumn{3}{|c|}{ Construction } & \multicolumn{3}{|c|}{ Maintenance } & \multicolumn{3}{|c|}{ End of Life } & \multirow{2}{*}{$\begin{array}{l}{ }^{3} \text { Total } \\
\text { GWP }\end{array}$} & \multirow{2}{*}{ 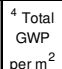 } & & \\
\hline & Material & 1/ ${ }^{2}$ Trans. & Total & Material & ${ }^{2}$ Trans. & Total & Material & 2 Trans. & Total & Materia & ${ }^{2}$ Trans. & Total & & & 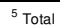 & ${ }^{6}$ perm m$^{2}$ \\
\hline 'Initial & $\frac{1,000}{1,000}$ & 0 & $\frac{1,000}{1000}$ & 13 & 1 & $\frac{15}{15}$ & 0 & 0 & 8 & 0 & 0 & 0 & 1,015 & 20 & , & O \\
\hline
\end{tabular}

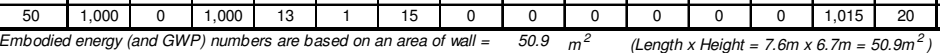

ATHENA $®$ EIE Material List:

(Includes all materials after 50 years)

\begin{tabular}{|l|c|c|}
\hline \multicolumn{1}{|c|}{ Material List } & Quantities & Unit \\
\hline 13mm Regular Gypsum Board & 224.0 & $\mathrm{~m} 2$ \\
\hline Batt. Fiberglass & 289.8 & $\mathrm{~m} 2(25 \mathrm{~mm})$ \\
\hline Joint Compound & 223.6 & $\mathrm{~kg}$ \\
\hline Nails & 8.4 & $\mathrm{~kg}$ \\
\hline Paper Tape & 2.6 & $\mathrm{~kg}$ \\
\hline $\begin{array}{l}\text { Small Dimension Sottwood Lumber, } \\
\text { kill-dried }\end{array}$ & 0.6 & $\mathrm{~m} 3$ \\
\hline Water Based Latex Paint & 132.5 & $\mathrm{~L}$ \\
\hline
\end{tabular}

Notes:

${ }^{2}$ Trans. $=$ Transportation

Total EE (or Total GWP) = Total embodied energy (or total embodied GWP) of building component after lifespan (i.e. total manufacturing + tota ${ }^{4}$ Total EE (or Total GWP) per $\mathrm{m}^{2}=$ Total EE (or Total GWP) of building ${ }_{5}^{5}$ Total Difference in Operating Energy (or GWP) from Baseline after Lifespan = The difference in the total life-cycle operating energy (or GWP)
from the baseline retail building after lifespan, due to using this building

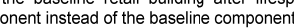

${ }^{6}$ Total Difference in Operating Energy (or GWP) from Baseline after Lifespan per $\mathrm{m}^{2}$ = Total difference in operating energy (or GWP) from *aseline after lifespan $/$ interior partition wall area of baseline retail building
* Total operating primary energy use of baseline retail building after 50 years
$50,700 \mathrm{GJ}\left(1,745 \mathrm{MJ} / \mathrm{m}^{2} / \mathrm{yr}\right.$ )

* Total operating GWP of baseline retail building after 50 years $=2,310$ tonnes
of $\mathrm{CO}_{2}$ eq. $\left(80 \mathrm{~kg}\right.$ of $\mathrm{CO}_{2}$ eq. $\left./ \mathrm{h}^{2} \mathrm{Yr}\right)$ 


\section{Appendix C Life-Cycle Assessment Results for Case Study Buildings}

Appendix C contains a breakdown of the LCA results for the five case study buildings. This includes a summary of the various building components for each building, the estimated quantity of each component, and the corresponding life-cycle energy use and GWP. 


\section{Case Study \#1: Typical Hot-Rolled Steel Structure Retail Building (Baseline Retail Building)}

Total Life-Cycle Energy of Typical Hot-Rolled Steel Structure Retail Building after 50 Year Lifespan in Toronto (Case Study \#1 a.k.a. Baseline Building)

\begin{tabular}{|c|c|c|c|c|}
\hline \multirow[b]{2}{*}{ Building Component } & \multicolumn{3}{|c|}{ Building Component Quantities } & \multirow{2}{*}{\begin{tabular}{|c|} 
Total Embodied Energy of \\
Building Components after \\
50 Years (MJ)
\end{tabular}} \\
\hline & ID & $\begin{array}{c}\text { Estimated } \\
\text { Quantity }\end{array}$ & Unit & \\
\hline Exterior Infill Wall Enclosure & BASE-W & 581.0 & sq.m & 535,776 \\
\hline Roof Enclosure (Includes Roof Joists, JOIST-1) & BASE-R & 586.0 & sq.m & $2,728,802$ \\
\hline Structural System - 350W Hot-Rolled Steel & - & - & - & - \\
\hline Beams (Includes BM-1, BM-2, BM-3, GIRT-1) & S-1 & 11.8 & tonnes & \multirow{5}{*}{476,416} \\
\hline Columns (Includes COL-A) & S-1 & 3.3 & tonnes & \\
\hline Hot-Rolled Steel Connection Plates & N/A & 0.3 & tonnes & \\
\hline Fasteners & N/A & 0.2 & tonnes & \\
\hline Miscellaneous Hot-Rolled Steel & N/A & 1.2 & tonnes & \\
\hline Mezzanine Floor (Includes Floor Joists, JOIST-1) & FL-3 & 48.0 & sq.m & 57,882 \\
\hline Windows & - & - & - & - \\
\hline Curtainwall (Façade) & W-9 & 128.0 & sq.m & 242,560 \\
\hline Curtainwall (Interior Vestibule) & W-9 & 34.5 & sq.m & 65,378 \\
\hline Windows & $\mathrm{W}-1$ & 20.3 & sq.m & 175,737 \\
\hline Doors & - & - & - & - \\
\hline Overhead Doors & D-4 & 1.0 & doors & 41,677 \\
\hline Exterior Doors - Opaque & D-2 & 1.0 & doors & 8,335 \\
\hline Exterior Doors - Glazing & D-3 & 6.0 & doors & 22,836 \\
\hline Interior Doors & D-6 & 9.0 & doors & 72,882 \\
\hline Interior Partitions & - & - & - & - \\
\hline Fire Rated Stair Tower & CMU-P1 & 84.0 & sq.m & 76,815 \\
\hline Insulated Interior Stud Wall Partition & SS-P3 & 75.0 & sq.m & 39,138 \\
\hline Uninsulated Interior Stud Wall Partition & SS-P1 & 52.0 & sq.m & 22,593 \\
\hline 6mm Tempered Glass & $\mathrm{N} / \mathrm{A}$ & 5.7 & sq.m & 1,132 \\
\hline Foundations & - & - & - & - \\
\hline Slab-On-Grade & SOG-FDN4 & 586.0 & sq.m & 302,448 \\
\hline Isolated Footings with Concrete Piers & IF-FDN1 & 15.0 & units & 200,874 \\
\hline Strip Footings with Concrete Foundation Wall & \begin{tabular}{|l|l|} 
SF-FDN5 \\
\end{tabular} & 160.0 & $\mathrm{~m}$ & 175,641 \\
\hline Total Embodied Energy of Entire Building (GJ) & & & & 5,247 \\
\hline Total Operating Energy of Entire Building (GJ) & Annual $=$ & 1,014 & & $\mathbf{5 0 , 7 0 0}$ \\
\hline Total Energy of Entire Building (GJ) & & & & 55,947 \\
\hline
\end{tabular}

Total Life-Cycle GWP of Typical Hot-Rolled Steel Structure Retail Building after 50 Year Lifespan in Toronto (Case Study \#1 a.k.a. Baseline Building)

\begin{tabular}{|c|c|c|c|c|}
\hline \multirow[b]{2}{*}{ Building Component } & \multicolumn{3}{|c|}{ Building Component Quantities } & \multirow{2}{*}{\begin{tabular}{|c|} 
Total Embodied GWP of \\
Building Components after \\
50 Years (kg of $\mathrm{CO}_{2}$ eq.)
\end{tabular}} \\
\hline & ID & $\begin{array}{c}\text { Estimated } \\
\text { Quantity }\end{array}$ & Unit & \\
\hline Exterior Infill Wall Enclosure & BASE-W & 581.0 & sq.m & 28,375 \\
\hline 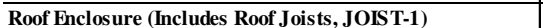 & BASE-R & 586.0 & sq.m & 124,234 \\
\hline $\begin{array}{l}\text { Structural System - 350W Hot-Rolled Steel } \\
\end{array}$ & - & - & - & - \\
\hline Beams (Includes BM-1, BM-2, BM-3, GIRT-1) & S-1 & 11.8 & tonnes & \multirow{5}{*}{26,872} \\
\hline Columns (Includes COL-A) & S-1 & 3.3 & tonnes & \\
\hline Hot-Rolled Steel Connection Plates & N/A & 0.3 & tonnes & \\
\hline Fasteners & N/A & 0.2 & tonnes & \\
\hline Miscellaneous Hot-Rolled Steel & N/A & 1.2 & tonnes & \\
\hline Mezzanine Floor (Includes Floor Joists, JOIST-1) & FL-3 & 48.0 & sq.m & 4,120 \\
\hline Windows & - & - & - & - \\
\hline Curtainwall (Façade) & W-9 & 128.0 & sq.m & 26,752 \\
\hline Curtainwall (Interior Vestibule) & W-9 & 34.5 & sq.m & 7,211 \\
\hline Windows & $\mathrm{W}-1$ & 20.3 & sq.m & 10,901 \\
\hline Doors & - & - & - & - \\
\hline Overhead Doors & D-4 & 1.0 & doors & 1,448 \\
\hline Exterior Doors - Opaque & D-2 & 1.0 & doors & 290 \\
\hline Exterior Doors - Glazing & D-3 & 6.0 & doors & 1,788 \\
\hline Interior Doors & D-6 & 9.0 & doors & 2,493 \\
\hline Interior Partitions & - & - & - & - \\
\hline Fire Rated Stair Tower & CMU-P1 & 84.0 & sq.m & 4,919 \\
\hline Insulated Interior Stud Wall Partition & SS-P3 & 75.0 & sq.m & 2,256 \\
\hline Uninsulated Interior Stud Wall Partition & SS-P1 & 52.0 & sq.m & 1,279 \\
\hline 6mm Tempered Glass & N/A & 5.7 & sq.m & 172 \\
\hline Foundations & - & - & - & - \\
\hline Slab-On-Grade & SOG-FDN4 & 586.0 & sq.m & 38,410 \\
\hline Isolated Footings with Concrete Piers & IF-FDN1 & 15.0 & units & 25,291 \\
\hline Strip Footings with Concrete Foundation Wall & SF-FDN5 & 160.0 & $\mathrm{~m}$ & 15,033 \\
\hline Total Embodied GWP of Entire Building (tonnes of $\mathrm{CO}_{2}$ eq.) & & & & 322 \\
\hline Total Operating GWP of Entire Building (tonnes of $\mathrm{CO}_{2}$ eq.) & Annual = & 46 & & 2,310 \\
\hline Total GWP of Entire Building (tonnes of $\mathrm{CO}_{2}$ eq.) & & & & 2,632 \\
\hline
\end{tabular}




\section{Case Study \#2: Typical Heavy Timber Structure Retail Building}

Total Life-Cycle Energy of Typical Heavy Timber Structure Retail Building after 50 Year Lifespan in Toronto (Case Study \#2)

\begin{tabular}{|c|c|c|c|c|}
\hline \multirow[b]{2}{*}{ Building Component } & \multicolumn{3}{|c|}{ Building Component Quantities } & \multirow{2}{*}{$\begin{array}{c}\text { Total Embodied Energy of } \\
\text { Building Components after } \\
50 \text { Years (MJ) }\end{array}$} \\
\hline & ID & $\begin{array}{l}\text { Es timated } \\
\text { Quantity }\end{array}$ & Unit & \\
\hline Exterior Infill Wall Enclosure & BASE-W & 581.0 & sq.m & 535,776 \\
\hline Roof Enclosure (Includes Roof Joists, JOIST-1) & BASE-R & 586.0 & sq.m & $2,728,802$ \\
\hline Structural System - 24f-EGlulam Timber & - & - & - & - \\
\hline Beams (Includes BM-1, BM-2, BM-3, GIRT-1) & S-2 & 25.4 & cu.m & \multirow{5}{*}{155,972} \\
\hline Columns (Includes COL-A) & S-2 & 8.0 & cu.m & \\
\hline Hot-Rolled Steel Connection Plates & $\mathrm{N} / \mathrm{A}$ & 0.3 & tonnes & \\
\hline Fasteners & N/A & 0.2 & tonnes & \\
\hline Miscellaneous Hot-Rolled Steel & N/A & 1.2 & tonnes & \\
\hline Mezzanine Floor (Includes Floor Joists, JOIST-1) & FL-3 & 48.0 & sq.m & 57,882 \\
\hline Windows & - & - & - & - \\
\hline Curtainwall (Façade) & W-9 & 128.0 & sq.m & 242,560 \\
\hline Curtainwall (Interior Vestibule) & W-9 & 34.5 & sq.m & 65,378 \\
\hline Windows & W-1 & 20.3 & sq.m & 175,737 \\
\hline Doors & - & - & - & - \\
\hline Overhead Doors & $\mathrm{D}-4$ & 1.0 & doors & 41,677 \\
\hline Exterior Doors - Opaque & $\mathrm{D}-2$ & 1.0 & doors & 8,335 \\
\hline Exterior Doors - Glazing & D-3 & 6.0 & doors & 22,836 \\
\hline Interior Doors & D-6 & 9.0 & doors & 72,882 \\
\hline Interior Partitions & - & - & - & - \\
\hline Fire Rated Stair Tower & CMU-P1 & 84.0 & sq.m & 76,815 \\
\hline Insulated Interior Stud Wall Partition & SS-P3 & 75.0 & sq.m & 39,138 \\
\hline Uninsulated Interior Stud Wall Partition & SS-P1 & 52.0 & sq.m & 22,593 \\
\hline $6 \mathrm{~mm}$ Tempered Glass & N/A & 5.7 & sq.m & 1,132 \\
\hline Foundations & - & - & - & - \\
\hline Slab-On-Grade & SOG-FDN4 & 586.0 & sq.m & 302,448 \\
\hline Isolated Footings with Concrete Piers & IF-FDN1 & 15.0 & units & 200,874 \\
\hline Strip Footings with Concrete Foundation Wall & \begin{tabular}{|l|} 
SF-FDN5 \\
\end{tabular} & 160.0 & $\mathrm{~m}$ & 175,641 \\
\hline Total Embodied Energy of Entire Building (GJ) & & & & 4,926 \\
\hline Total Operating Energy of Entire Building (GJ) & Annual $=$ & 1,014 & & 50,700 \\
\hline Total Energy of Entire Building (GJ) & & & & 55,626 \\
\hline
\end{tabular}

Total Life-Cycle GWP of Typical Heavy Timber Structure Retail Building after 50 Year Lifespan in Toronto (Case Study \#2)

\begin{tabular}{|c|c|c|c|c|}
\hline \multirow[b]{2}{*}{ Building Component } & \multicolumn{3}{|c|}{ Building Component Quantities } & \multirow{2}{*}{$\begin{array}{l}\text { Total Embodied GWP of } \\
\text { Building Components after } \\
50 \text { Years (kg of } \mathrm{CO}_{2} \mathrm{eq} \text { ) }\end{array}$} \\
\hline & ID & \begin{tabular}{|c|} 
Estimated \\
Quantity \\
\end{tabular} & Unit & \\
\hline Exterior Infill Wall Enclosure & \begin{tabular}{l|l} 
BASE-W \\
\end{tabular} & 581.0 & sq.m & 28,375 \\
\hline Roof Enclosure (Includes Roof Joists, JOIST-1) & BASE-R & 586.0 & sq.m & 124,234 \\
\hline Structural System - 24f-E Glulam Timber & - & - & - & - \\
\hline Beams (Includes BM-1, BM-2, BM-3, GIRT-1) & $\mathrm{S}-2$ & 25.4 & cu.m & \multirow{5}{*}{7,556} \\
\hline Columns (Includes COL-A) & S-2 & 8.0 & cu.m & \\
\hline Hot-Rolled Steel Connection Plates & N/A & 0.3 & tonnes & \\
\hline Fasteners & N/A & 0.2 & tonnes & \\
\hline Miscellaneous Hot-Rolled Steel & N/A & 1.2 & tonnes & \\
\hline Mezzanine Floor (Includes Floor Joists, JOIST-1) & FL-3 & 48.0 & sq.m & 4,120 \\
\hline Windows & - & - & - & - \\
\hline Curtainwall (Façade) & W-9 & 128.0 & sq.m & 26,752 \\
\hline Curtainwall (Interior Vestibule) & W-9 & 34.5 & sq.m & 7,211 \\
\hline Windows & $\mathrm{W}-1$ & 20.3 & sq.m & 10,901 \\
\hline Doors & - & - & - & - \\
\hline Overhead Doors & D-4 & 1.0 & doors & 1,448 \\
\hline Exterior Doors - Opaque & D-2 & 1.0 & doors & 290 \\
\hline Exterior Doors - Glazing & D-3 & 6.0 & doors & 1,788 \\
\hline Interior Doors & D-6 & 9.0 & doors & 2,493 \\
\hline Interior Partitions & - & - & - & - \\
\hline Fire Rated Stair Tower & CMU-P1 & 84.0 & sq.m & 4,919 \\
\hline Insulated Interior Stud Wall Partition & SS-P3 & 75.0 & sq.m & 2,256 \\
\hline Uninsulated Interior Stud Wall Partition & SS-P1 & 52.0 & sq.m & 1,279 \\
\hline 6mm Tempered Glass & N/A & 5.7 & sq.m & 172 \\
\hline Foundations & - & - & - & - \\
\hline Slab-On-Grade & SOG-FDN4 & 586.0 & sq.m & 38,410 \\
\hline Isolated Footings with Concrete Piers & IF-FDN1 & 15.0 & units & 25,291 \\
\hline Strip Footings with Concrete Foundation Wall & SF-FDN5 & 160.0 & $\mathrm{~m}$ & 15,033 \\
\hline Total Embodied GWP of Entire Building (tonnes of $\mathrm{CO}_{2}$ eq.) & & & & 303 \\
\hline Total Operating GWP of Entire Building (tonnes of $\mathrm{CO}_{2}$ eq.) & Annual $=$ & & & 2,310 \\
\hline Total GWP of Entire Building (tonnes of $\mathrm{CO}_{2}$ eq.) & & & & 2,613 \\
\hline
\end{tabular}




\section{Case Study \#3: Typical Pre-Engineered Steel Retail Building}

Total Life-Cycle Energy of Typical Pre-Engineered Steel Retail Building after 50 Year Lifespan in Toronto (Case Study \#3)

\begin{tabular}{|c|c|c|c|c|}
\hline \multirow[b]{2}{*}{ Building Component } & \multicolumn{3}{|c|}{ Building Component Quantities } & \multirow{2}{*}{\begin{tabular}{|c|} 
Total Embodied Energy of \\
Building Components after \\
50 Years (MJ)
\end{tabular}} \\
\hline & ID & $\begin{array}{c}\text { Es timated } \\
\text { Quantity }\end{array}$ & Unit & \\
\hline Exterior Infill Wall Enclosure (Includes Girts) & PENG-W2 & 581.0 & sq.m & 394,629 \\
\hline Roof Enclosure (Includes Roof Joists) & PENG-R2 & 586.0 & sq.m & 544,654 \\
\hline Structural System - Pre-Engineered Steel & - & - & - & - \\
\hline Beams and Columns (Hot-Rolled Steel) & N/A & 13.1 & tonnes & \multirow{2}{*}{394,927} \\
\hline Fasteners & N/A & 0.2 & tonnes & \\
\hline \multirow{2}{*}{$\begin{array}{l}\text { Additional Hot-Rolled Steel } \\
\text { (Including Hot-Rolled Steel Connection Plates) }\end{array}$} & N/A & 1.3 & tonnes & \multirow{2}{*}{36,549} \\
\hline & N/A & 1.2 & tonnes & \\
\hline Additional Cold-Formed Steel & N/A & 1.8 & tonnes & 45,568 \\
\hline Mezzanine Floor (Includes Floor Joists) & N/A & 48.0 & sq.m & 62,143 \\
\hline Windows & - & - & - & - \\
\hline Curtainwall (Façade) & W-9 & 128.0 & sq.m & 242,560 \\
\hline Curtainwall (Interior Vestibule) & W-9 & 34.5 & sq.m & 65,378 \\
\hline Windows & $\mathrm{W}-1$ & 20.3 & sq.m & 175,737 \\
\hline Doors & - & - & - & - \\
\hline Overhead Doors & $\mathrm{D}-4$ & 1.0 & doors & 41,677 \\
\hline Exterior Doors - Opaque & $\mathrm{D}-2$ & 1.0 & doors & 8,335 \\
\hline Exterior Doors - Glazing & D-3 & 6.0 & doors & 22,836 \\
\hline Interior Doors & D-6 & 9.0 & doors & 72,882 \\
\hline Interior Partitions & - & - & - & - \\
\hline Fire Rated Stair Tower & CMU-P1 & 84.0 & sq.m & 76,815 \\
\hline Insulated Interior Stud Wall Partition & SS-P3 & 75.0 & sq.m & 39,138 \\
\hline Uninsulated Interior Stud Wall Partition & SS-P1 & 52.0 & sq.m & 22,593 \\
\hline 6mm Tempered Glass & N/A & 5.7 & sq.m & 1,132 \\
\hline Foundations & - & - & - & - \\
\hline Slab-On-Grade & SOG-FDN4 & 586.0 & sq.m & 302,448 \\
\hline Isolated Footings with Concrete Piers & IF-FDN1 & 15.0 & units & 200,874 \\
\hline Strip Footings with Concrete Foundation Wall & \begin{tabular}{|l|l|} 
SF-FDN5 \\
\end{tabular} & 160.0 & $\mathrm{~m}$ & 175,641 \\
\hline Total Embodied Energy of Entire Building (GJ) & & & & 2,927 \\
\hline Total Operating Energy of Entire Building (GJ) & Annual $=$ & 1,009 & & $\mathbf{5 0 , 4 7 0}$ \\
\hline Total Energy of Entire Building (GJ) & & & & 53,396 \\
\hline
\end{tabular}

Total Life-Cycle GWP of Typical Pre-Engineered Steel Retail Building after 50 Year Lifespan in Toronto (Case Study \#3)

\begin{tabular}{|c|c|c|c|c|}
\hline \multirow[b]{2}{*}{ Building Component } & \multicolumn{3}{|c|}{ Building Component Quantities } & \multirow{2}{*}{\begin{tabular}{|c|} 
Total Embodied GWP of \\
Building Components after \\
50 Years (kg of $\mathrm{CO}_{2}$ eq.)
\end{tabular}} \\
\hline & ID & $\begin{array}{l}\text { Estimated } \\
\text { Quantity }\end{array}$ & Unit & \\
\hline $\begin{array}{l}\text { Exterior Infill Wall Enclosure (Includes Girts) } \\
\end{array}$ & PENG-W2 & 581.0 & sq.m & 18,406 \\
\hline Roof Enclosure (Includes Roof Joists) & PENG-R2 & 586.0 & sq.m & 22,498 \\
\hline $\begin{array}{l}\text { Structural System - Pre-Engineered Steel } \\
\text { S }\end{array}$ & - & - & - & - \\
\hline Beams and Columns (Hot-Rolled Steel) & $\mathrm{N} / \mathrm{A}$ & 13.1 & tonnes & \multirow{2}{*}{20,518} \\
\hline Fasteners & N/A & 0.2 & tonnes & \\
\hline \multirow{2}{*}{$\begin{array}{l}\text { Additional Hot-Rolled Steel } \\
\text { (Including Hot-Rolled Steel Connection Plates) }\end{array}$} & N/A & 1.3 & tonnes & \multirow{2}{*}{2,097} \\
\hline & N/A & 1.2 & tonnes & \\
\hline Additional Cold-Formed Steel & N/A & 1.8 & tonnes & 3,034 \\
\hline Mezzanine Floor (Includes Floor Joists) & $\mathrm{N} / \mathrm{A}$ & 48.0 & sq.m & 3,285 \\
\hline Windows & - & - & - & - \\
\hline Curtainwall (Façade) & W-9 & 128.0 & sq.m & 26,752 \\
\hline Curtainwall (Interior Vestibule) & W-9 & 34.5 & sq.m & 7,211 \\
\hline Windows & $\mathrm{W}-1$ & 20.3 & sq.m & 10,901 \\
\hline Doors & - & - & - & - \\
\hline Overhead Doors & D-4 & 1.0 & doors & 1,448 \\
\hline Exterior Doors - Opaque & D-2 & 1.0 & doors & 290 \\
\hline Exterior Doors - Glazing & D-3 & 6.0 & doors & 1,788 \\
\hline Interior Doors & D-6 & 9.0 & doors & 2,493 \\
\hline Interior Partitions & - & - & - & - \\
\hline Fire Rated Stair Tower & CMU-P1 & 84.0 & sq.m & 4,919 \\
\hline Insulated Interior Stud Wall Partition & SS-P3 & 75.0 & sq.m & 2,256 \\
\hline Uninsulated Interior Stud Wall Partition & SS-P1 & 52.0 & sq.m & 1,279 \\
\hline 6mm Tempered Glass & N/A & 5.7 & sq.m & 172 \\
\hline Foundations & - & - & - & - \\
\hline Slab-On-Grade & SOG-FDN4 & 586.0 & sq.m & 38,410 \\
\hline Isolated Footings with Concrete Piers & IF-FDN1 & 15.0 & units & 25,291 \\
\hline Strip Footings with Concrete Foundation Wall & SF-FDN5 & 160.0 & $\mathrm{~m}$ & 15,033 \\
\hline Total Embodied GWP of Entire Building (tonnes of $\mathrm{CO}_{2}$ eq.) & & & & 208 \\
\hline Total Operating GWP of Entire Building (tonnes of $\mathrm{CO}_{2}$ eq.) & Annual = & & & 2,300 \\
\hline Total GWP of Entire Building (tonnes of $\mathrm{CO}_{2}$ eq.) & & & & 2,508 \\
\hline
\end{tabular}




\section{Case Study \#4: Predominately Steel Retail Building}

Total Life-Cycle Energy of Predominately Steel Retail Building after 50 Year Lifespan in Toronto (Case Study \#4)

\begin{tabular}{|c|c|c|c|c|}
\hline \multirow[b]{2}{*}{ Building Component } & \multicolumn{3}{|c|}{ Building Component Quantities } & \multirow{2}{*}{$\begin{array}{c}\text { Total Embodied Energy of } \\
\text { Building Components after } \\
50 \text { Years (MJ) }\end{array}$} \\
\hline & ID & $\begin{array}{l}\text { Es timated } \\
\text { Quantity }\end{array}$ & Unit & \\
\hline Exterior Infill Wall Enclosure & SS-W17 & 581.0 & sq.m & $1,525,301$ \\
\hline Roof Enclosure (Includes Roof Joists, JOIST-1) & OWSJ-R5 & 586.0 & sq.m & 888,710 \\
\hline Structural System - 350W Hot-Rolled Steel & - & - & - & - \\
\hline Beams (Includes BM-1, BM-2, BM-3, GIRT-1) & S-1 & 11.8 & tonnes & \multirow{5}{*}{476,416} \\
\hline Columns (Includes COL-A) & S-1 & 3.3 & tonnes & \\
\hline Hot-Rolled Steel Connection Plates & N/A & 0.3 & tonnes & \\
\hline Fasteners & N/A & 0.2 & tonnes & \\
\hline Miscellaneous Hot-Rolled Steel & $\mathrm{N} / \mathrm{A}$ & 1.2 & tonnes & \\
\hline Mezzanine Floor (Includes Floor Joists, JOIST-1) & FL-3 & 48.0 & sq.m & 57,882 \\
\hline Windows & - & - & - & - \\
\hline Curtainwall (Façade) & W-9 & 128.0 & sq.m & 242,560 \\
\hline Curtainwall (Interior Vestibule) & W-9 & 34.5 & sq.m & 65,378 \\
\hline Windows & $\mathrm{W}-1$ & 20.3 & sq.m & 175,737 \\
\hline Doors & - & - & - & - \\
\hline Overhead Doors & $\mathrm{D}-4$ & 1.0 & doors & 41,677 \\
\hline Exterior Doors - Opaque & $\mathrm{D}-2$ & 1.0 & doors & 8,335 \\
\hline Exterior Doors - Glazing & D-3 & 6.0 & doors & 22,836 \\
\hline Interior Doors & D-6 & 9.0 & doors & 72,882 \\
\hline Interior Partitions & - & - & - & - \\
\hline Fire Rated Stair Tower & CMU-P1 & 84.0 & sq.m & 76,815 \\
\hline Insulated Interior Stud Wall Partition & SS-P3 & 75.0 & sq.m & 39,138 \\
\hline Uninsulated Interior Stud Wall Partition & SS-P1 & 52.0 & sq.m & 22,593 \\
\hline $6 \mathrm{~mm}$ Tempered Glass & N/A & 5.7 & sq.m & 1,132 \\
\hline Foundations & - & - & - & - \\
\hline Slab-On-Grade & SOG-FDN4 & 586.0 & sq.m & 302,448 \\
\hline Isolated Footings with Concrete Piers & IF-FDN1 & 15.0 & units & 200,874 \\
\hline Strip Footings with Concrete Foundation Wall & \begin{tabular}{|l|} 
SF-FDN5 \\
\end{tabular} & 160.0 & $\mathrm{~m}$ & 175,641 \\
\hline Total Embodied Energy of Entire Building (GJ) & & & & 4,396 \\
\hline Total Operating Energy of Entire Building (GJ) & Annual $=$ & 1,040 & & 51,981 \\
\hline Total Energy of Entire Building (GJ) & & & & 56,377 \\
\hline
\end{tabular}

Total Life-Cycle GWP of Predominately Steel Retail Building after 50 Year Lifespan in Toronto (Case Study \#4)

\begin{tabular}{|c|c|c|c|c|}
\hline \multirow[b]{2}{*}{ Building Component } & \multicolumn{3}{|c|}{ Building Component Quantities } & \multirow{2}{*}{$\begin{array}{l}\text { Total Embodied GWP of } \\
\text { Building Components after } \\
50 \text { Years (kg of } \mathrm{CO}_{2} \mathrm{eq} \text { ) }\end{array}$} \\
\hline & ID & $\begin{array}{c}\text { Estimated } \\
\text { Quantity }\end{array}$ & Unit & \\
\hline Exterior Infill Wall Enclos ure & SS-W17 & 581.0 & sq.m & 90,679 \\
\hline Roof Enclosure (Includes Roof Joists, JOIST-1) & OWSJ-R5 & 586.0 & sq.m & 46,236 \\
\hline Structural System - 350 W Hot-Rolled Steel & - & - & - & - \\
\hline Beams (Includes BM-1, BM-2, BM-3, GIRT-1) & S-1 & 11.8 & tonnes & \multirow{5}{*}{26,872} \\
\hline Columns (Includes COL-A) & S-1 & 3.3 & tonnes & \\
\hline Hot-Rolled Steel Connection Plates & N/A & 0.3 & tonnes & \\
\hline Fasteners & N/A & 0.2 & tonnes & \\
\hline Miscellaneous Hot-Rolled Steel & N/A & 1.2 & tonnes & \\
\hline Mezzanine Floor (Includes Floor Joists, JOIST-1) & FL-3 & 48.0 & sq.m & 4,120 \\
\hline Windows & - & - & - & - \\
\hline Curtainwall (Façade) & W-9 & 128.0 & sq.m & 26,752 \\
\hline Curtainwall (Interior Vestibule) & W-9 & 34.5 & sq.m & 7,211 \\
\hline Windows & $\mathrm{W}-1$ & 20.3 & sq.m & 10,901 \\
\hline Doors & - & - & - & - \\
\hline Overhead Doors & D-4 & 1.0 & doors & 1,448 \\
\hline Exterior Doors - Opaque & $\mathrm{D}-2$ & 1.0 & doors & 290 \\
\hline Exterior Doors - Glazing & D-3 & 6.0 & doors & 1,788 \\
\hline Interior Doors & D-6 & 9.0 & doors & 2,493 \\
\hline Interior Partitions & - & - & - & - \\
\hline Fire Rated Stair Tower & CMU-P1 & 84.0 & sq.m & 4,919 \\
\hline Insulated Interior Stud Wall Partition & SS-P3 & 75.0 & sq.m & 2,256 \\
\hline Uninsulated Interior Stud Wall Partition & SS-P1 & 52.0 & sq.m & 1,279 \\
\hline 6mm Tempered Glass & N/A & 5.7 & sq.m & 172 \\
\hline Foundations & - & - & - & - \\
\hline Slab-On-Grade & SOG-FDN4 & 586.0 & sq.m & 38,410 \\
\hline Isolated Footings with Concrete Piers & \begin{tabular}{|l|} 
IF-FDN1 \\
\end{tabular} & 15.0 & units & 25,291 \\
\hline Strip Footings with Concrete Foundation Wall & SF-FDN5 & 160.0 & $\mathrm{~m}$ & 15,033 \\
\hline Total Embodied GWP of Entire Building (tonnes of $\mathrm{CO}_{2}$ eq.) & & & & 306 \\
\hline Total Operating GWP of Entire Building (tonnes of $\mathrm{CO}_{2}$ eq.) & Annual = & 48 & & 2,381 \\
\hline Total GWP of Entire Building (tonnes of $\mathrm{CO}_{2}$ eq.) & & & & 2,687 \\
\hline
\end{tabular}




\section{Case Study \#5: Predominately Timber Retail Building}

Total Life-Cycle Energy of Predominately Timber Retail Building after 50 Year Lifespan in Toronto

(Case Study \#5)

\begin{tabular}{|c|c|c|c|c|}
\hline \multirow[b]{2}{*}{ Building Component } & \multicolumn{3}{|c|}{ Building Component Quantities } & \multirow{2}{*}{$\begin{array}{c}\text { Total Embodied Energy of } \\
\text { Building Components after } \\
50 \text { Years (MJ) }\end{array}$} \\
\hline & ID & \begin{tabular}{|c|} 
Estimated \\
Quantity
\end{tabular} & Unit & \\
\hline Exterior Infill Wall Enclosure & WS-W4 & 581.0 & sq.m & 410,119 \\
\hline Roof Enclosure (Includes Roof Joists, JOIST-1) & GLU-R2 & 586.0 & sq.m & $2,560,210$ \\
\hline Structural System - 24f-E Glulam Timber & - & - & - & - \\
\hline Beams (Includes BM-1, BM-2, BM-3, GIRT-1) & S-2 & 25.4 & cu.m & \multirow{5}{*}{155,972} \\
\hline Columns (Includes COL-A) & $\mathrm{S}-2$ & 8.0 & cu.m & \\
\hline Hot-Rolled Steel Connection Plates & N/A & 0.3 & tonnes & \\
\hline Fasteners & N/A & 0.2 & tonnes & \\
\hline Miscellaneous Hot-Rolled Steel & N/A & 1.2 & tonnes & \\
\hline Mezzanine Floor (Includes Floor Joists, JOIST-1) & FL-2 & 48.0 & sq.m & 31,527 \\
\hline Windows & - & - & - & - \\
\hline Curtainwall (Façade) & W-9 & 128.0 & sq.m & 242,560 \\
\hline Curtainwall (Interior Vestibule) & W-9 & 34.5 & sq.m & 65,378 \\
\hline Windows & $\mathrm{W}-4$ & 20.3 & sq.m & 82,905 \\
\hline Doors & - & - & - & - \\
\hline Overhead Doors & $\mathrm{D}-4$ & 1.0 & doors & 41,677 \\
\hline Exterior Doors - Opaque & D-1 & 1.0 & doors & 539 \\
\hline Exterior Doors - Glazing & D-3 & 6.0 & doors & 22,836 \\
\hline Interior Doors & D-5 & 9.0 & doors & 4,851 \\
\hline Interior Partitions & - & - & - & - \\
\hline Fire Rated Stair Tower & CMU-P1 & 84.0 & sq.m & 76,815 \\
\hline Insulated Interior Stud Wall Partition & WS-P3 & 75.0 & sq.m & 32,143 \\
\hline Uninsulated Interior Stud Wall Partition & WS-P1 & 52.0 & sq.m & 17,666 \\
\hline 6mm Tempered Glass & N/A & 5.7 & sq.m & 1,132 \\
\hline Foundations & - & - & - & - \\
\hline Slab-On-Grade & SOG-FDN4 & 586.0 & sq.m & 302,448 \\
\hline Isolated Footings with Concrete Piers & IF-FDN1 & 15.0 & units & 200,874 \\
\hline Strip Footings with Concrete Foundation Wall & \begin{tabular}{|l|l|} 
SF-FDN5 \\
\end{tabular} & 160.0 & $\mathrm{~m}$ & 175,641 \\
\hline Total Embodied Energy of Entire Building (GJ) & & & & 4,425 \\
\hline Total Operating Energy of Entire Building (GJ) & Annual $=$ & 1,016 & & $\mathbf{5 0 , 8 2 2}$ \\
\hline Total Energy of Entire Building (GJ) & & & & 55,247 \\
\hline
\end{tabular}

Total Life-Cycle GWP of Predominately Timber Retail Building after 50 Year Lifespan in Toronto (Case Study \#5)

\begin{tabular}{|c|c|c|c|c|}
\hline \multirow[b]{2}{*}{ Building Component } & \multicolumn{3}{|c|}{ Building Component Quantities } & \multirow{2}{*}{\begin{tabular}{|c|} 
Total Embodied GWP of \\
Building Components after \\
50 Years (kg of $\mathrm{CO}_{2}$ eq. $)$
\end{tabular}} \\
\hline & ID & $\begin{array}{c}\text { Estimated } \\
\text { Quantity }\end{array}$ & Unit & \\
\hline Exterior Infill Wall Enclosure & WS-W4 & 581.0 & sq.m & 15,202 \\
\hline Roof Enclosure (Includes Roof Joists, JOIST-1) & GLU-R2 & 586.0 & sq.m & 109,056 \\
\hline $\begin{array}{l}\text { Structural System - 24f-E Glulam Timber } \\
\end{array}$ & - & - & - & - \\
\hline Beams (Includes BM-1, BM-2, BM-3, GIRT-1) & S-2 & 25.4 & cu.m & \multirow{5}{*}{7,556} \\
\hline Columns (Includes COL-A) & S-2 & 8.0 & cu.m & \\
\hline Hot-Rolled Steel Connection Plates & N/A & 0.3 & tonnes & \\
\hline Fasteners & N/A & 0.2 & tonnes & \\
\hline Miscellaneous Hot-Rolled Steel & N/A & 1.2 & tonnes & \\
\hline Mezzanine Floor (Includes Floor Joists, JOIST-1) & FL-2 & 48.0 & sq.m & 1,633 \\
\hline Windows & - & - & - & - \\
\hline Curtainwall (Façade) & W-9 & 128.0 & sq.m & 26,752 \\
\hline Curtainwall (Interior Vestibule) & W-9 & 34.5 & sq.m & 7,211 \\
\hline Windows & $\mathrm{W}-4$ & 20.3 & sq.m & 7,024 \\
\hline Doors & - & - & - & - \\
\hline Overhead Doors & D-4 & 1.0 & doors & 1,448 \\
\hline Exterior Doors - Opaque & D-1 & 1.0 & doors & 16 \\
\hline Exterior Doors - Glazing & D-3 & 6.0 & doors & 1,788 \\
\hline Interior Doors & D-5 & 9.0 & doors & 144 \\
\hline Interior Partitions & - & - & - & - \\
\hline Fire Rated Stair Tower & CMU-P1 & 84.0 & sq.m & 4,919 \\
\hline Insulated Interior Stud Wall Partition & WS-P3 & 75.0 & sq.m & 1,522 \\
\hline Uninsulated Interior Stud Wall Partition & WS-P1 & 52.0 & sq.m & 763 \\
\hline 6mm Tempered Glass & N/A & 5.7 & sq.m & 172 \\
\hline Foundations & - & - & - & - \\
\hline Slab-On-Grade & SOG-FDN4 & 586.0 & sq.m & 38,410 \\
\hline Isolated Footings with Concrete Piers & IF-FDN1 & 15.0 & units & 25,291 \\
\hline Strip Footings with Concrete Foundation Wall & SF-FDN5 & 160.0 & $\mathrm{~m}$ & 15,033 \\
\hline Total Embodied GWP of Entire Building (tonnes of $\mathrm{CO}_{2}$ eq.) & & & & 264 \\
\hline Total Operating GWP of Entire Building (tonnes of $\mathrm{CO}_{2}$ eq.) & Annual = & & & 2,319 \\
\hline Total GWP of Entire Building (tonnes of $\mathrm{CO}_{2}$ eq.) & & & & 2,583 \\
\hline
\end{tabular}

